Prepared in cooperation with the Central Colorado Water Conservancy District

\title{
Estimates of Deep-Percolation Return Flow Beneath a Flood- and a Sprinkler-Irrigated Site in Weld County, Colorado, 2008-2009
}
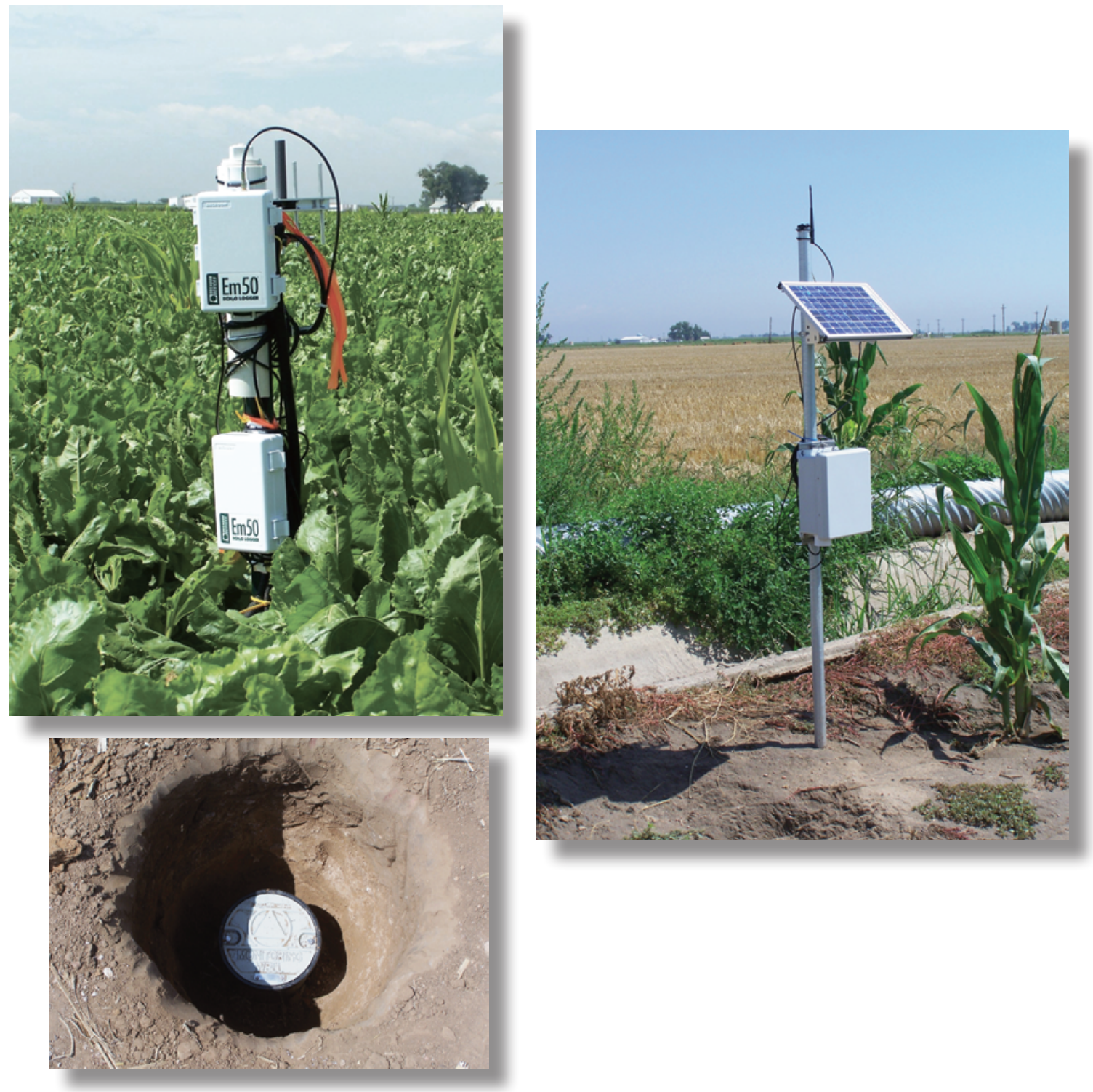

Scientific Investigations Report 2011-5001 



\title{
Estimates of Deep-Percolation Return Flow Beneath a Flood- and a Sprinkler-Irrigated Site in Weld County, Colorado, 2008-2009
}

\author{
By L.R. Arnold
}

Prepared in cooperation with the Central Colorado Water Conservancy District

Scientific Investigations Report 2011-5001 


\title{
U.S. Department of the Interior \\ KEN SALAZAR, Secretary \\ U.S. Geological Survey \\ Marcia K. McNutt, Director
}

\section{U.S. Geological Survey, Reston, Virginia: 2011}

\author{
For more information on the USGS — the Federal source for science about the Earth, its natural and living resources, \\ natural hazards, and the environment, visit http://www.usgs.gov or call 1-888-ASK-USGS \\ For an overview of USGS information products, including maps, imagery, and publications, \\ visit http://www.usgs.gov/pubprod \\ To order this and other USGS information products, visit http://store.usgs.gov
}

\begin{abstract}
Any use of trade, product, or firm names is for descriptive purposes only and does not imply endorsement by the U.S. Government.

Although this report is in the public domain, permission must be secured from the individual copyright owners to reproduce any copyrighted materials contained within this report.
\end{abstract}

Suggested citation:

Arnold, L.R., 2011, Estimates of deep-percolation return flow beneath a flood- and a sprinkler-irrigated site in Weld County, Colorado, 2008-2009: U.S. Geological Survey Scientific Investigations Report 2011-5001, 225 p. 


\section{Contents}

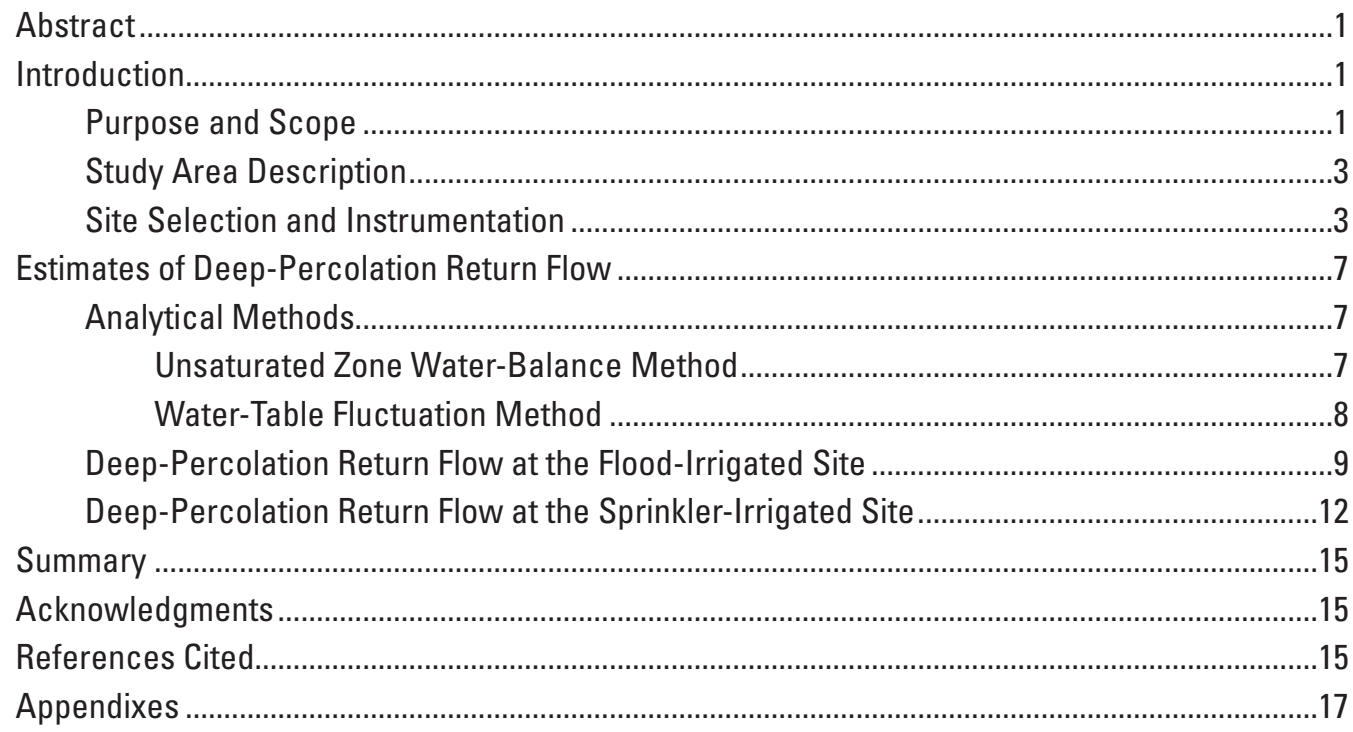

\section{Figures}

1. Location of study area, Weld County, Colorado …………...................................................

2. Installation design of soil-moisture sensors and observation wells used to monitor deep-percolation return flow at the flood- and sprinkler-irrigated sites, Weld County, Colorado.

3. Lithologic descriptions of sediments at the flood-and sprinkler-irrigated sites, Weld County, Colorado

4. Estimated change in soil-water storage in response to the deep-percolation event on June 25, 2008, at the flood-irrigated site, Weld County, Colorado ......................9

5. Volumetric water content, depth to water, irrigation, and precipitation at the flood-irrigated site during 2008, Weld County, Colorado ........................................10

6. Volumetric water content, depth to water, irrigation, and precipitation at the flood-irrigated site during 2009, Weld County, Colorado

7. Volumetric water content, depth to water, irrigation, and precipitation at the sprinkler-irrigated site during 2008, Weld County, Colorado.

8. Volumetric water content, depth to water, irrigation, and precipitation at the sprinkler-irrigated site during 2009, Weld County, Colorado.

\section{Tables}

1. Unsaturated gravimetric water content, bulk density, saturated and unsaturated volumetric water content, and specific yield of sediments at the flood- and sprinkler-irrigated sites, Weld County, Colorado 
2. Locations, construction information, and depth to water of observation wells used to monitor groundwater levels at the flood- and sprinkler-irrigated sites, Weld County, Colorado.

3. Net irrigation, precipitation, and deep percolation estimated by using the unsaturated zone water balance and water-table fluctuation methods at the flood- and sprinkler-irrigated sites, Weld County, Colorado.

\section{Conversion Factors}

Inch/Pound to SI

\begin{tabular}{|c|c|c|}
\hline Multiply & By & To obtain \\
\hline \multicolumn{3}{|c|}{ Length } \\
\hline inch (in.) & 2.54 & centimeter $(\mathrm{cm})$ \\
\hline foot $(\mathrm{ft})$ & 0.3048 & meter $(\mathrm{m})$ \\
\hline mile (mi) & 1.609 & kilometer $(\mathrm{km})$ \\
\hline \multicolumn{3}{|c|}{ Flow rate } \\
\hline inch per year (in/yr) & 25.4 & millimeter per year $(\mathrm{mm} / \mathrm{yr})$ \\
\hline \multicolumn{3}{|c|}{ Hydraulic conductivity* } \\
\hline foot per day (ft/d) & 0.3048 & meter per day $(\mathrm{m} / \mathrm{d})$ \\
\hline inch per hour (in/h) & 0.0254 & meter per hour $(\mathrm{m} / \mathrm{h})$ \\
\hline \multicolumn{3}{|c|}{ Transmissivity* } \\
\hline foot squared per day $\left(\mathrm{ft}^{2} / \mathrm{d}\right)$ & 0.09290 & meter squared per day $\left(\mathrm{m}^{2} / \mathrm{d}\right)$ \\
\hline \multicolumn{3}{|c|}{ Hydraulic gradient } \\
\hline foot per mile (ft/mi) & 0.1894 & meter per kilometer $(\mathrm{m} / \mathrm{km})$ \\
\hline
\end{tabular}

Temperature in degrees Fahrenheit $\left({ }^{\circ} \mathrm{F}\right)$ may be converted to degrees Celsius $\left({ }^{\circ} \mathrm{C}\right)$ as follows:

${ }^{\circ} \mathrm{C}=\left({ }^{\circ} \mathrm{F}-32\right) / 1.8$

Vertical coordinate information is referenced to the National Geodetic Vertical Datum of 1929 (NGVD 29).

Horizontal coordinate information is referenced to the North American Datum of 1983 (NAD 83).

Altitude, as used in this report, refers to distance above the vertical datum.

*Hydraulic conductivity: The standard unit for hydraulic conductivity is cubic foot per day per square foot $\left(\mathrm{ft}^{3} / \mathrm{d}\right) / \mathrm{ft}^{2}$. In this report, the mathematically reduced form, foot per day (ft/d) and similarly reduced form, inches per hour (in/h), are used for convenience.

*Transmissivity: The standard unit for transmissivity is cubic foot per day per square foot times foot of aquifer thickness [(ft $\left.\left.\mathrm{ft}^{3} \mathrm{~d}\right) / \mathrm{ft}^{2}\right] \mathrm{ft}$. In this report, the mathematically reduced form, foot squared per day $\left(\mathrm{ft}^{2} / \mathrm{d}\right)$, is used for convenience.

\section{Other abbreviations used in this report:}

$\mu \mathrm{S} / \mathrm{cm} \quad$ Microsiemens per centimeter

$\mathrm{dS} / \mathrm{m}$ Decisiemens per meter 


\section{Definition of Terms}

Bulk density—Mass of dry soil per unit volume.

Dielectric constant-A measure of the polarizability of a material in an electric field.

Electrical conductivity-The ability of a material to conduct electricity.

Gravimetric water content-Water content expressed as mass of water per mass of dry solid in a soil sample.

Specific yield-Ratio of the volume of water that drains from a saturated soil by gravity to the total volume of the soil.

Volumetric water content-Water content expressed as volume of water per total volume of soil sample. 



\title{
Estimates of Deep-Percolation Return Flow Beneath a Flood- and a Sprinkler-Irrigated Site in Weld County, Colorado, 2008-2009
}

\author{
By L.R. Arnold
}

\section{Abstract}

Water management and determination of consumptive water use in irrigated agricultural settings depend on understanding factors such as surface-water diversions, groundwater withdrawals, evapotranspiration, tail-water runoff, and deep-percolation return flow of water beneath irrigated fields. To improve understanding of deep-percolation return flow beneath irrigated fields, the U.S. Geological Survey, in cooperation with the Central Colorado Water Conservancy District, conducted a study to estimate deep-percolation return flow at two irrigated sites in the Central Colorado Water Conservancy District during the 2008 and 2009 irrigation seasons. At one site, deep percolation was monitored beneath an agricultural field irrigated by flood furrows, and at a second site, deep percolation was monitored beneath an agricultural field irrigated by a center-pivot sprinkler. Deep-percolation return flow at each site was estimated by using an unsaturated zone water-balance method and the water-table fluctuation method, and the ratio of deep percolation to applied water (irrigation and irrigation plus precipitation) was computed for each site. Cumulative deep percolation at the flood-irrigated site was estimated to range from 7.7 to 8.8 in. during the monitoring periods, which is about $40-52$ percent of irrigation water applied during the same periods and about 29-39 percent of irrigation water plus precipitation during those periods. Deep percolation at the sprinkler-irrigated site was estimated to range from 1.2 to $3.2 \mathrm{in}$. during the monitoring periods, which is about 5-14 percent of irrigation water applied during the periods and about 4-11 percent of irrigation water plus precipitation during the periods.

\section{Introduction}

Water management and determination of consumptive water use in irrigated agricultural settings depend on understanding factors such as surface-water diversions, groundwater withdrawals, evapotranspiration, tail-water runoff, and deep-percolation return flow of water beneath irrigated fields.
Of these components, deep-percolation return flow of water beneath irrigated fields can be the most difficult to measure and the least understood. To help improve understanding of deep-percolation return flow beneath irrigated fields, the U.S. Geological Survey (USGS), in cooperation with the Central Colorado Water Conservancy District (CCWCD), conducted a study to estimate deep-percolation return flow beneath two irrigated fields in the CCWCD (fig. 1) during the 2008 and 2009 irrigation seasons.

\section{Purpose and Scope}

This report presents the methods of investigation and results of a study to estimate deep-percolation return flow at two irrigated agricultural sites in the CCWCD during the 2008 and 2009 irrigation seasons. One site was irrigated by flood furrows, and the other site was irrigated by a centerpivot sprinkler. Deep-percolation return flow at each site was estimated by using two different methods. The first method estimated deep-percolation return flow by using an unsaturated zone water-balance (UZWB) approach (Delin and others, 2000), in which water that infiltrates below the root zone is assumed not to be substantially affected by evapotranspiration and ultimately reaches the water table as deep percolation return flow. Soil moisture was monitored at each site by using sensors installed in the unsaturated zone, and increases in soil-water storage between the bottom of the root zone and the top of the capillary fringe above the water table were assumed to equal deep-percolation return flow. The second method estimated deep-percolation return flow by using the water-table fluctuation (WTF) method (Healy and Cook, 2002), in which rises in groundwater levels are assumed to be caused by downward-moving water arriving at the water table as recharge. Results of each estimation method were used to compute the ratio of deep percolation to applied water (irrigation and irrigation plus precipitation) for each site. 


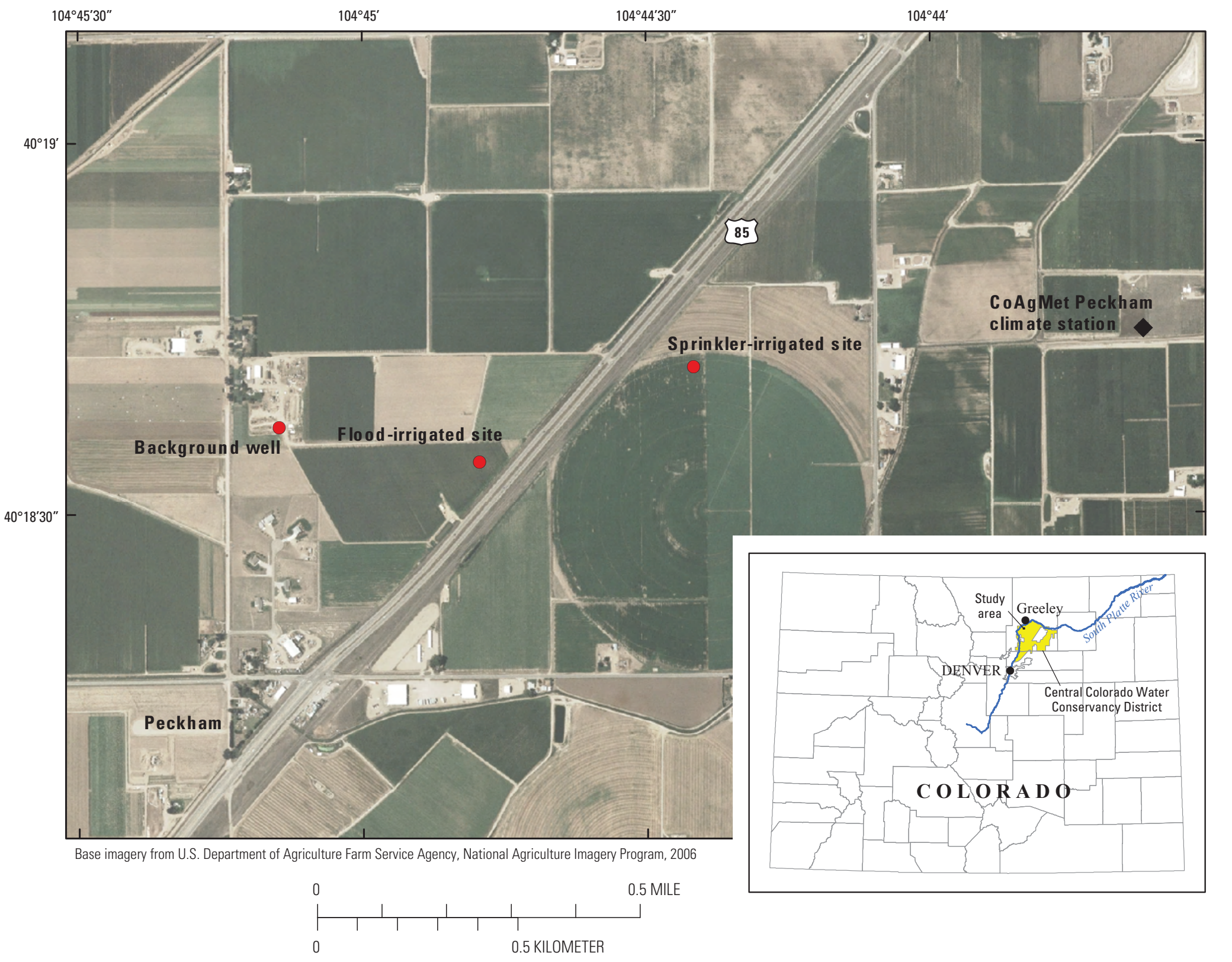

Figure 1. Location of study area, Weld County, Colorado. 


\section{Study Area Description}

The study area is in a gently sloping agricultural area of the South Platte River valley about 7 mi south of the City of Greeley in northeastern Colorado (fig. 1). The climate of the study area is characterized by semiarid conditions and large seasonal variations in temperature. During the period 1967-2005, the mean January minimum temperature at Greeley was $15.6^{\circ} \mathrm{F}$, and the mean July maximum temperature was $89.5^{\circ} \mathrm{F}$ (Western Regional Climate Center, 2010). Mean annual precipitation at Greeley for the same period was 14.0 in. with about 70 percent of the precipitation occurring during April through September.

Soil at the flood-irrigated site classifies as Julesburg sandy loam with 1 to 3 percent slopes and high saturated hydraulic conductivity (4-12 ft/d or 2.0-6.0 in/h) (U.S. Department of Agriculture, 2005). Soil at the sprinklerirrigated site classifies as Olney fine sandy loam with 1 to 3 percent slopes and moderately high to high saturated hydraulic conductivity (1.2-4 ft/d or 0.6-2.0 in/h) (U.S. Department of Agriculture, 2005).

Soils in the study area overlie predominantly unconsolidated sand and gravel alluvium of the South Platte River valley (Colton, 1978). Unconsolidated sediments in the study area are as much as about $100 \mathrm{ft}$ thick with about 50 to 90 $\mathrm{ft}$ of saturated thickness (Robson and others, 2000, sheets 1 and 4). Depth to water in the alluvial aquifer in the study area generally ranges from about 10 to $30 \mathrm{ft}$ below land surface, and the direction of groundwater flow in the alluvial aquifer is predominantly to the northeast with a hydraulic gradient of about $11 \mathrm{ft} / \mathrm{mi}$ (Robson and others, 2000, sheets 3 and 5). Transmissivity of the alluvial aquifer in the study area is about 27,000 ft²/d (Hurr and others, 1972, pl. 5).

Most land in the study area is used for irrigated agriculture, and irrigation water is supplied by both surface water and groundwater. Surface water is diverted to fields from the South Platte River through irrigation ditches, and groundwater is pumped from irrigation wells completed in the alluvial aquifer (Colorado Division of Water Resources, 2010). Corn was grown at the flood-irrigated site in 2008 and 2009. Corn was grown at the sprinkler-irrigated site in 2008, and sugar beets were grown in 2009.

\section{Site Selection and Instrumentation}

Sites were selected to represent typical soil and hydrogeologic conditions in the CCWCD and commonly used irrigation methods. Sites also were selected at locations with the potential for long-term monitoring and in close proximity to nonirrigated land for monitoring background water levels in the alluvial aquifer. Instrumentation at each site was installed as far as practicable inside the field to minimize potential influence on water levels from deep percolation beneath adjacent irrigated fields, seepage from nearby irrigation ponds or ditches, and well pumping. Instrumentation at the flood-irrigated site was installed about $175 \mathrm{ft}$ southeast (aligned with furrows) from the northern edge of the field (fig. 1). Instrumentation at the sprinkler-irrigated site was installed about $155 \mathrm{ft}$ south from the northern edge of the field in an area irrigated by drop nozzles rather than an area irrigated by the spray gun on the end of the pivot arm.

Each site was equipped with soil-moisture sensors (Decagon Devices, Inc., models EC-5, EC-TM, and $\mathrm{ECH}_{2} \mathrm{O}-\mathrm{TE}$ ) installed at multiple depths between land surface and the water table (fig. 2) to monitor soil-moisture content in the unsaturated zone throughout the irrigation season. Soil-moisture sensors at the flood-irrigated site were installed at depths of 2, $3,4,6,8,10,12,15$, and $18.5 \mathrm{ft}$ below ground surface. Soilmoisture sensors at the sprinkler-irrigated site were installed at depths of 2, 3, 4, 5, 7, 9, 13, and $16.5 \mathrm{ft}$ below ground surface. An additional soil-moisture sensor was added at a depth of $1 \mathrm{ft}$ at each site in 2009 to monitor the response of the shallow root zone to irrigation events and provide additional information concerning the timing and magnitude of irrigation events. The sensors are capacitance-type probes that measure the dielectric constant of soil in order to determine its volumetric water content (Decagon Devices, Inc., 2006, 2007), where volumetric water content is determined as the average value over the 5 - $\mathrm{cm}$ length of the probes. The sensors are reported by Decagon Devices, Inc. $(2006,2007)$ to be capable of measuring volumetric water content from 0 to 100 percent and have a measurement accuracy of about 3 percent for most soil types (including those at the study sites) having electrical conductivity up to about $8,000 \mu \mathrm{S} / \mathrm{cm}(8 \mathrm{dS} / \mathrm{m})$. Although not used to quantify deep percolation, temperature also was measured in the unsaturated zone at each site to help identify and interpret deep-percolation events. Temperature was measured by using multiple-parameter sensors (Decagon Devices, Inc., model EC-TM) capable of measuring both soil moisture and temperature at depths of $2,4,8,12$, and $15 \mathrm{ft}$ below ground surface at the flood-irrigated site and at depths of 2, 4, 7, and $13 \mathrm{ft}$ below ground surface at the sprinkler-irrigated site. The sensor (Decagon Devices, Inc., model $\mathrm{ECH}_{2} \mathrm{O}-\mathrm{TE}$ ) installed at a depth of $4 \mathrm{ft}$ at each site also measured the bulk electrical conductivity of the soil-water medium to verify that electrical conductivity did not exceed the range over which the soilmoisture sensors are accurate.

The sensors were installed by first excavating a 4-in.-wide trench about $2 \mathrm{ft}$ deep and $6 \mathrm{ft}$ long (fig. 2). A handdriven bucket auger was then used to make a 2.75 -in.-diameter hole at one end of the trench bottom, and soils were described (fig. 3) as they were removed from the hole. A small soil core was collected from the bottom of the hole by driving a length of 1-in.-diameter metal conduit into the bottom of the hole at the target sensor depth, cutting off the portion of the conduit containing the sample (about 3 in.), immediately placing the conduit containing the sample in a soil tin, and sealing the tin with plastic tape to preserve soil moisture. A soil-moisture sensor was then inserted vertically into undisturbed soil at the bottom of the hole by using a modified length of conduit designed for inserting the sensor, and the conduit was 
Top View

Backfilled trench

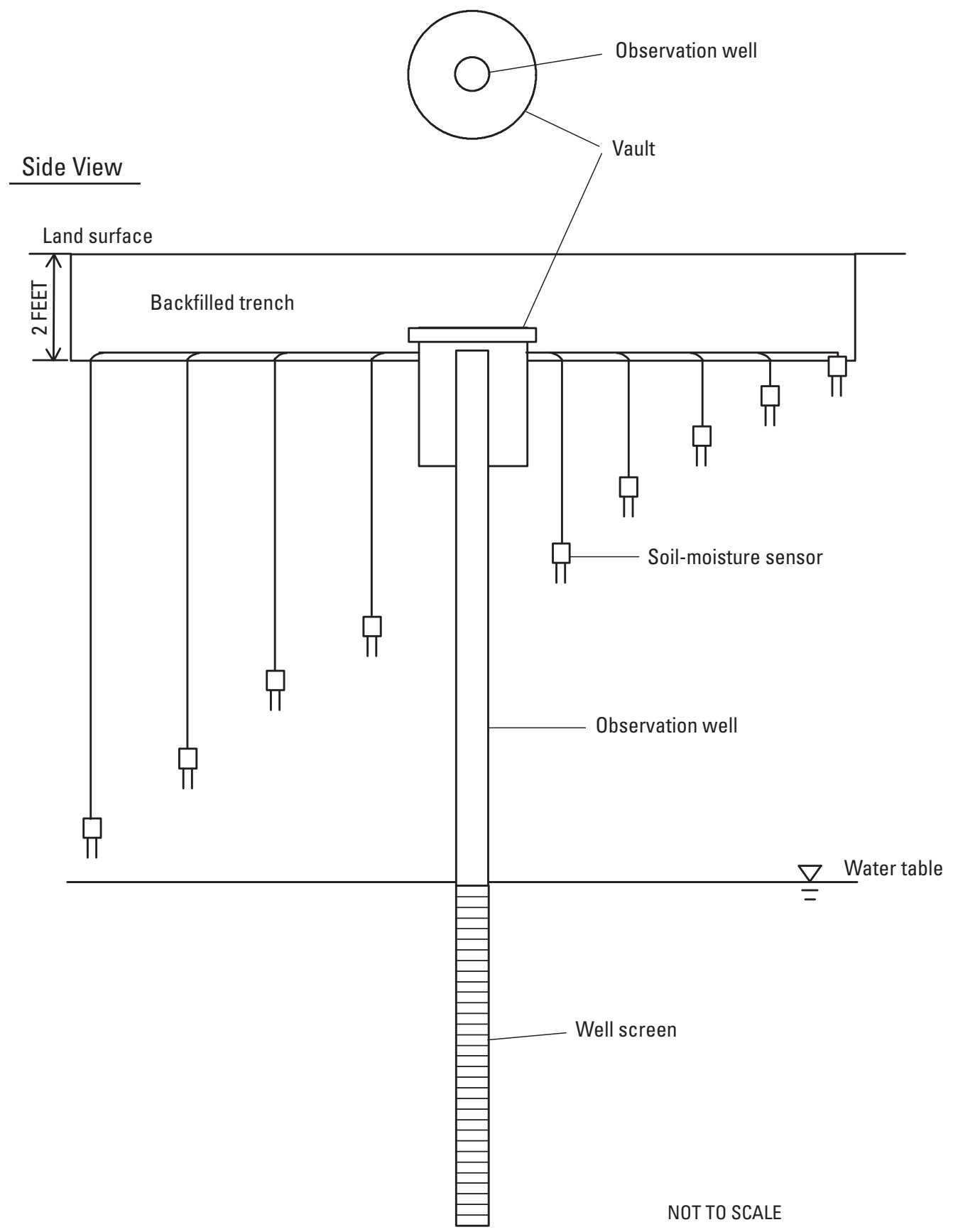

Figure 2. Installation design of soil-moisture sensors and observation wells used to monitor deep-percolation return flow at the flood- and sprinkler-irrigated sites, Weld County, Colorado. 


\begin{tabular}{|}
\begin{tabular}{|c|}
\hline $\begin{array}{l}\text { Flood-irrigated site } \\
\text { Latitude: } 40^{\circ} 18^{\prime} 34.2^{\prime \prime} \\
\text { Longitude: } 104^{\circ} 44^{\prime} 47.7^{\prime \prime}\end{array}$ \\
Land-surface altitude: 4,724 feet \\
\hline $\begin{array}{c}\text { Depth } \\
\text { interval } \\
\text { (feet) }\end{array}$
\end{tabular} \\
\hline $0-1.5$ & Silty sand, fine to medium grained, slightly clayey, brown, damp to moist \\
\hline $1.5-9$ & $\begin{array}{l}\text { Silty sand, fine to medium grained, slightly clayey to slightly gravelly in places, more clayey with } \\
\text { caliche from } 4 \text { to } 6.5 \text { feet, light brown with infrequent rust staining, damp }\end{array}$ \\
\hline $9-11$ & $\begin{array}{l}\text { Gravelly sand, medium to coarse grained with minor fine, 2-inch-thick clay layer at } 11 \text { feet, light } \\
\text { brown, damp }\end{array}$ \\
\hline $11-13$ & $\begin{array}{l}\text { Sand, fine to medium grained with minor coarse grains, light brown with rust streaks and staining } \\
\text { below } 12 \text { feet, damp }\end{array}$ \\
\hline $13-16$ & Silty sand, fine grained, slightly clayey, light brown to light gray with rust streaks, moist \\
\hline $16-18.5$ & $\begin{array}{l}\text { Sand, fine to coarse grained, slightly gravelly, gravelly in places, light brown with rust staining, } \\
\text { moist to wet }\end{array}$ \\
\hline $18.5-20$ & Not observed \\
\hline $20-25$ & Gravelly sand, medium to coarse grained, light brown, saturated \\
\hline
\end{tabular}

\begin{tabular}{|c|c|}
\hline \multicolumn{2}{|c|}{$\begin{array}{l}\text { Sprinkler-irrigated site } \\
\text { Latitude: } 40^{\circ} 18^{\prime} 41.8^{\prime \prime} \\
\text { Longitude: } 104^{\circ} 44^{\prime} 25.1^{\prime \prime} \\
\text { Land-surface altitude: } 4,720 \text { feet }\end{array}$} \\
\hline $\begin{array}{c}\text { Depth } \\
\text { interval } \\
\text { (feet) }\end{array}$ & Description $^{1}$ \\
\hline $0-1.5$ & Silty sand, fine to medium grained, slightly clayey to clayey, brown, damp to moist \\
\hline $1.5-10$ & $\begin{array}{l}\text { Silty sand, fine to medium grained, slightly clayey to slightly gravelly in places, infrequent caliche } \\
\text { nodules up to } 0.5 \text {-inch size, light brown, damp to moist }\end{array}$ \\
\hline $10-13.5$ & Gravelly sand, fine to coarse grained, light brown with rust at 12-13.5 feet, damp \\
\hline $13.5-16.5$ & $\begin{array}{l}\text { Silty sand, very fine to fine grained, infrequent thin clay layers, light brown to light gray with rust } \\
\text { streaks, moist }\end{array}$ \\
\hline $16.5-20$ & Not observed \\
\hline $20-25$ & Gravelly sand, medium to coarse grained, light brown, saturated \\
\hline
\end{tabular}

${ }^{1}$ Sediments from 0 to 18.5 feet at the flood-irrigated site and from 0 to 16.5 feet at the sprinkler-irrigated site were logged during hand augering of holes for soil-moisture sensors.

Sediments from 20 to 25 feet were logged from core samples collected during well installation.

Sediments in the interval between the bottom of the deepest hole augered for soil-moisture sensors and the top of core samples collected during well installation were not observed.
Figure 3. Lithologic descriptions of sediments at the flood- and sprinkler-irrigated sites, Weld County, Colorado. removed. Additional holes were augered to different depths at a spacing of about $8 \mathrm{in}$. along the trench bottom, soil cores were collected from each hole, and sensors were inserted as with the first hole. Holes were backfilled above the sensors with $0.5-3 \mathrm{ft}$ (depending on hole depth) of soil excavated from the hole, which was compacted in layers to restore soil to conditions similar to surrounding soils. A layer of bentonite crumbles (0.5-3.5 ft thick) was added above the compacted soil to preclude preferential flow of water down the hole, and additional soil excavated from the hole was added and compacted as needed to complete backfilling. The trench was then backfilled in compacted layers with soil excavated from the trench and any vegetation was replaced at ground surface. Prior to backfilling the trench, all sensor wires were extended to an observation well vault (fig. 2) located about 3-4 ft from the trench for protection while not in use. After tilling and planting were completed at each site, sensor wires were connected to an above-ground data logger (Decagon Devices, Inc., model Em50) for automated collection of soil-moisture, temperature, and electrical-conductivity data during each irrigation season. The data logger was removed prior to harvest at the end of each irrigation season, and sensor wires were returned to the vault for protection during the nonirrigation season. Laboratory measurements of moisture content and soil bulk density (table 1) of core samples were completed at the U.S. Geological Survey National Research Program laboratory in Lakewood, Colo., using methods described by Dane and Topp (2002).

An observation well (fig. 2) was installed at each site to monitor water levels during the irrigation season. Wells were installed April 17, 2008, by using a Geoprobe Model 6610DT direct-push machine. Because wells were installed 
Table 1. Unsaturated gravimetric water content, bulk density, saturated and unsaturated volumetric water content, and specific yield of sediments at the flood- and sprinkler-irrigated sites, Weld County, Colorado.

[Depth in feet below land surface; bulk density in grams per cubic centimeter; all other variables dimensionless; --, no data]

\begin{tabular}{|c|c|c|c|c|c|c|}
\hline Depth & $\begin{array}{l}\text { Sediment } \\
\text { type }^{1}\end{array}$ & $\begin{array}{c}\text { Unsaturated } \\
\text { gravimetric } \\
\text { water content }^{2}\end{array}$ & $\begin{array}{c}\text { Bulk } \\
\text { density }^{2}\end{array}$ & $\begin{array}{c}\text { Unsaturated } \\
\text { volumetric } \\
\text { water content }^{2}\end{array}$ & $\begin{array}{c}\text { Saturated } \\
\text { volumetric } \\
\text { water content }^{3}\end{array}$ & $\begin{array}{c}\text { Specific } \\
\text { yield }^{4}\end{array}$ \\
\hline 2 & Silty sand & 0.042 & 1.14 & 0.048 & -- & -- \\
\hline 3 & Silty sand & 0.084 & 1.12 & 0.093 & -- & -- \\
\hline 8 & Silty sand & 0.044 & 1.16 & 0.051 & -- & -- \\
\hline 10 & Gravelly sand & 0.014 & 1.22 & 0.017 & -- & -- \\
\hline 12 & Sand & 0.177 & 1.10 & 0.194 & 0.417 & 0.22 \\
\hline 15 & Silty sand & 0.205 & 1.11 & 0.227 & 0.410 & 0.18 \\
\hline 3 & Silty sand & 0.111 & 1.09 & 0.121 & -- & -- \\
\hline 4 & Silty sand & 0.136 & 1.08 & 0.147 & -- & -- \\
\hline 5 & Silty sand & 0.074 & 1.10 & 0.082 & -- & -- \\
\hline 7 & Silty sand & 0.108 & 1.17 & 0.127 & -- & -- \\
\hline 9 & Silty sand & 0.065 & 1.08 & 0.070 & -- & -- \\
\hline 10 & Gravelly sand & 0.013 & 1.22 & 0.016 & -- & -- \\
\hline 13 & Gravelly sand & 0.015 & 1.14 & 0.017 & 0.317 & 0.30 \\
\hline 16.5 & Silty sand & 0.194 & 1.20 & 0.233 & 0.434 & 0.20 \\
\hline
\end{tabular}

${ }^{1}$ Soils were collected at the flood-irrigated site on May 9, 2008. Soils were collected at the sprinkler-irrigated site on April 25, 2008.

${ }^{2}$ Unsaturated gravimetric water content, bulk density, and unsaturated volumetric water content were determined by laboratory analysis of soil core samples collected above the water table.

${ }^{3}$ Saturated volumetric water content was measured by soil-moisture sensors on the date sensor was submerged by water-table rise.

${ }^{4}$ Specific yield estimated as the difference between saturated and unsaturated volumetric water contents.

using direct-push methods, soils could not be logged during well installation. However, a 5-ft-long core sample was collected at a depth of 20-25 ft below land surface at each site for examination of aquifer sediments within the screened interval of the well. Wells were constructed of 2-in. nominal-diameter Schedule 40 polyvinyl chloride (PVC) casing with a screen length of 10 or $15 \mathrm{ft}$ located near the water table to facilitate monitoring of potential water-table fluctuations. The top of each well casing was located about $2 \mathrm{ft}$ below ground surface inside an 8-in.-diameter metal vault (fig. 2) to prevent interfering with farming practices, such as tilling and planting, and ensure that crop and soil conditions at the well location would be representative of the irrigated field. Well-completion details are summarized in table 2. Each well was equipped with a pressure transducer and data logger (Global Water Instrumentation, Inc., model WL16) during the irrigation season to monitor groundwater levels. Pressure transducers were vented to the surface to compensate for barometric effects on waterlevel measurements. Wells were developed after installation for about $0.5 \mathrm{hr}$ using a combination of pumping and mechanical surging to remove mud from the well and establish good hydraulic connection between the well and aquifer.

An additional observation well located on nonirrigated land (fig. 1) also was equipped with a pressure transducer and data logger to assist in distinguishing groundwater-level rises caused by deep-percolation return flow at each site from more regional groundwater-level trends. The observation well used to monitor background groundwater levels is a $41-\mathrm{ft}$ deep unused stock well with a 5-in. nominal-diameter steel casing. Other construction aspects of the background well are unknown. Water-level data for each well used by the study are available through the U.S. Geological Survey National Water 
Table 2. Locations, construction information, and depth to water of observation wells used to monitor groundwater levels at the flood- and sprinkler-irrigated sites, Weld County, Colorado.

[DDMMSS.S, degrees, minutes, decimal seconds; land-surface altitude in feet; all depths in feet below land surface; --, no data]

\begin{tabular}{|c|c|c|c|c|c|c|c|c|c|c|}
\hline $\begin{array}{c}\text { Site } \\
\text { ID }\end{array}$ & Site & $\begin{array}{c}\text { Latitude }^{1} \\
\text { (DDMMSS.S) }\end{array}$ & $\begin{array}{l}\text { Longitude }^{1} \\
\text { (DDMMSS.S) }\end{array}$ & $\begin{array}{l}\text { Land- } \\
\text { surface }^{2} \\
\text { altitude }\end{array}$ & $\begin{array}{c}\text { Depth } \\
\text { to } \\
\text { water }\end{array}$ & $\begin{array}{c}\text { Water- } \\
\text { level } \\
\text { date }\end{array}$ & $\begin{array}{l}\text { Depth } \\
\text { to top } \\
\text { of } \\
\text { casing }\end{array}$ & $\begin{array}{l}\text { Depth } \\
\text { to } \\
\text { top of } \\
\text { screen }\end{array}$ & $\begin{array}{l}\text { Depth to } \\
\text { bottom } \\
\text { of } \\
\text { screen }\end{array}$ & $\begin{array}{l}\text { Total } \\
\text { well } \\
\text { depth }\end{array}$ \\
\hline 401842104442501 & Sprinkler & 401841.8 & 1044425.1 & 4,720 & 17.4 & $04 / 17 / 2008$ & 1.9 & 18 & 28 & 33 \\
\hline 401837104450901 & Background & 401837.0 & 1044508.9 & 4,729 & 22.1 & $05 / 06 / 2008$ & 3.2 & -- & -- & 41 \\
\hline
\end{tabular}

${ }^{1}$ Latitude and longitude determined by Global Positioning System. North American Datum of 1983.

${ }^{2}$ Land-surface altitude estimated from U.S. Geological Survey 1:24,000-scale topgraphic map with a contour interval of 10 feet. National Geodetic Vertical Datum of 1929.

Information System at http://waterdata.usgs.gov/nwis. Site identification numbers for each observation well are listed in table 2.

Irrigation data (application dates and quantities) for the flood-irrigated site were provided by Troy Bauder (Colorado State University, written commun., 2009, 2010). Net irrigation (table 3 ) at the flood-irrigated site was estimated by using furrow flumes equipped with a pressure sensor and data logger to monitor water flow onto and off the field. Irrigation data (total quantity for season) for the sprinkler-irrigated site were provided by Frank Eckhardt (Eckhardt Farms, Inc., oral commun., 2010). Dates and quantities of individual irrigation applications at the sprinkler-irrigated site were estimated on the basis of peaks in volumetric water content measured by soil-moisture sensors at depths of 1 and $2 \mathrm{ft}$ and the typical magnitude and frequency of irrigation events reported by Eckhardt Farms, Inc. (oral commun., 2010). Precipitation measured at the Colorado Agricultural Meteorological Network (CoAgMet) Peckham climate station (fig. 1) located about $0.7 \mathrm{mi}$ east of the sprinkler-irrigated site was used to represent precipitation at each site.

\section{Estimates of Deep-Percolation Return Flow}

\section{Analytical Methods}

Two methods were used to estimate deep-percolation return flow at the study sites. Deep-percolation return flow was estimated by using an unsaturated zone water-balance (UZWB) method (Delin and others, 2000) and the water-table fluctuation (WTF) method (Healy and Cook, 2002).

\section{Unsaturated Zone Water-Balance Method}

The UZWB method equates deep-percolation return flow to increases in soil-water storage below a plane in the unsaturated zone (zero-flux plane) that separates upward movement of soil water in response to evapotranspiration from downward drainage of soil water toward the water table. For the purpose of this study, the depth of the zero-flux plane was assumed to occur at the bottom of the root zone, which was estimated to have a depth of $4 \mathrm{ft}$ below ground surface. Increases in soil moisture between the bottom of the root zone and the top of the capillary fringe above the water table (estimated as $1.5 \mathrm{ft}$ above the water table - see section "Deep Percolation Return Flow at the Flood-Irrigated Site") were used to estimate deeppercolation return flow. Changes in soil moisture shallower than $4 \mathrm{ft}$ were not used because soil water at these depths could be substantially affected by evapotranspiration and likely does not represent water that percolates to the water table. Changes in soil moisture within $1.5 \mathrm{ft}$ above the water table were not used because soil water in this zone could be substantially affected by the capillary fringe of the water table. Although evapotranspiration can occur at depths greater than $4 \mathrm{ft}$, the time interval between successive irrigation or precipitation events was sufficiently short that evapotranspiration at depths greater than $4 \mathrm{ft}$ likely was not substantial between events. If the depth of substantial evapotranspiration was deeper than $4 \mathrm{ft}$, deep-percolation return flow determined by using the UZWB method could be overestimated. Similarly, if the depth of substantial evapotranspiration was shallower than $4 \mathrm{ft}$, deep-percolation return flow determined by using the UZWB method could be underestimated.

The change in soil-water storage was determined as the difference between the maximum total soil water in storage following a deep-percolation event and the minimum total soil 
Table 3. Net irrigation, precipitation, and deep percolation estimated by using the unsaturated zone water balance and water-table fluctuation methods at the flood- and sprinkler-irrigated sites, Weld County, Colorado.

[UZWB, unsaturated zone water balance; WTF, water-table fluctuation; --, no data]

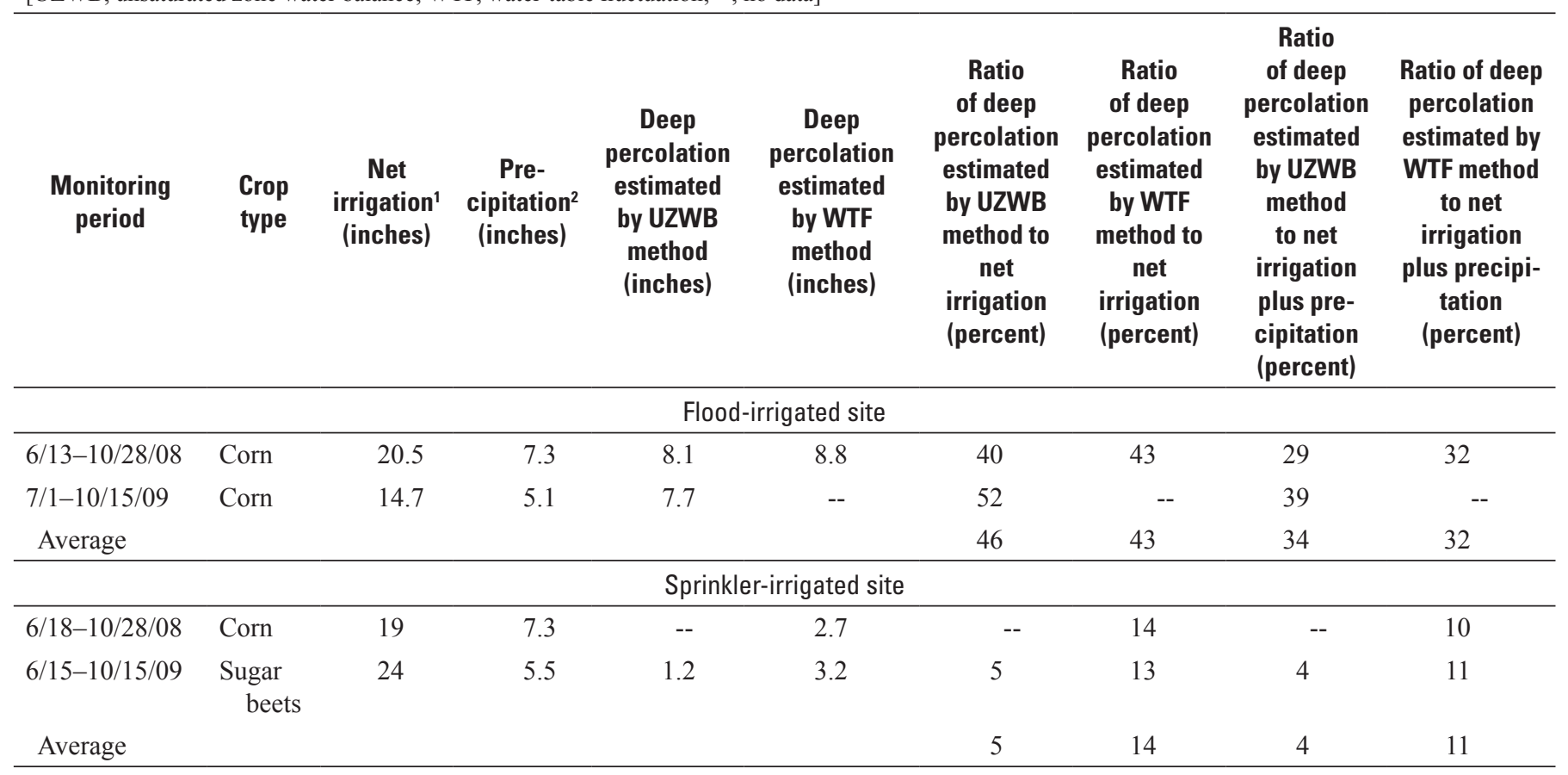

${ }^{1}$ Net irrigation of flood-irrigated site provided by Troy Bauder, Colorado State University Extension (written commun., 2009, 2010). Net irrigation of sprinkler-irrigated site provided by Frank Eckhardt, Eckhardt Farms, Inc. (oral commun., 2010).

${ }^{2}$ Precipitation measured at the Colorado Agricultural Meteorological Network Peckham Station (see figure 1 for location).

water in storage immediately prior to the event (fig. 4), calculated as (modified from Delin and others, 2000):

$$
\Delta S=\sum_{i=1}^{\mathrm{N}}\left[\left(\Delta \theta_{i}+\Delta \theta_{i+1}\right) / 2\right] \Delta z
$$

where

$\Delta S$ is the change in soil water stored in a column of unit surface area extending from the bottom of the root zone to the top of the capillary fringe, in feet,

$i \quad$ is an index to the soil-moisture sensors equal to 1 for the sensor at a depth of $4 \mathrm{ft}$ and increasing to a value of $\mathrm{N}$ for the deepest sensor in the interval of analysis,

$\Delta \theta_{i}$ is the change in volumetric water content between the times of minimum and maximum total soil water in storage for sensor $i$ (dimensionless), and

$\Delta z \quad$ is the vertical thickness of the unsaturated zone in the interval between sensors $i$ and $i+1$, in feet.

Deep-percolation return flow for the entire irrigation season was then calculated by summing all individual increases in soil-water storage related to deep-percolation events during the monitoring period. In cases where the capillary fringe was below the deepest sensor in the unsaturated zone, the volumetric water content at the top of the capillary fringe was estimated by interpolating between the deepest sensor in the unsaturated zone and the shallowest sensor below the water table, where the average saturated volumetric water content was used to represent the volumetric water content of the sensor below the water table.

\section{Water-Table Fluctuation Method}

The WTF method is based on the assumption that rises in groundwater levels in unconfined aquifers are caused by recharge from downward-percolating water and that no other sources or sinks affect groundwater levels during the recharge event. Recharge is calculated as (Healy and Cook, 2002):

$$
R=S_{y}(d h / d t)
$$

where

$R \quad$ is aquifer recharge, in length per time,

$S_{\mathrm{y}} \quad$ is specific yield (dimensionless), and

$d h / d t \quad$ is the change in water-table hydraulic head over time, in length per time.

For the purposes of this report, deep-percolation return flow and recharge are synonymous, and recharge calculated using equation 2 represents deep-percolation return flow at the study sites. Total deep-percolation return flow during 


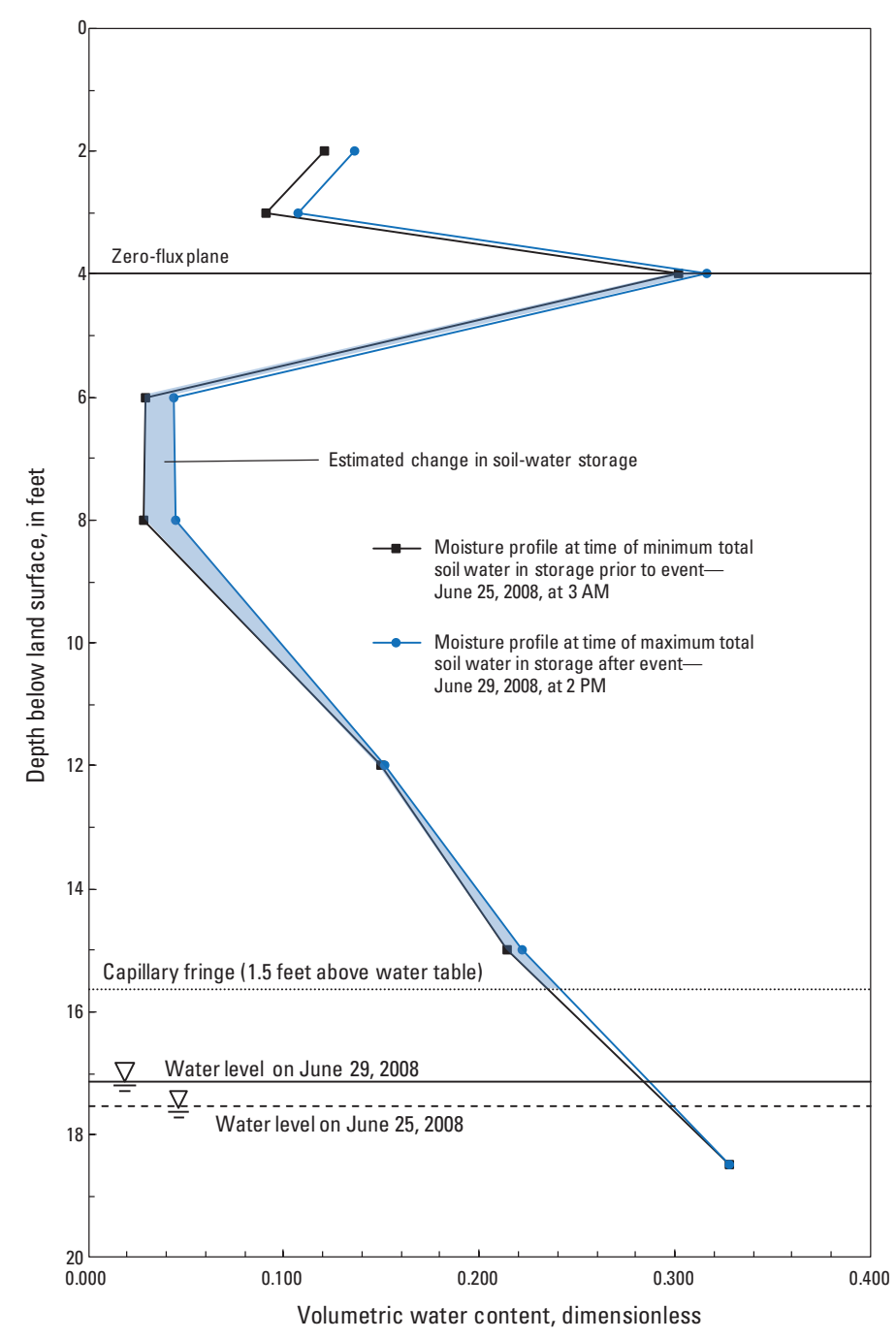

Figure 4. Estimated change in soil-water storage in response to the deep-percolation event on June 25, 2008, at the flood-irrigated site, Weld County, Colorado.

the irrigation season was determined by applying equation 2 to each individual period of water-table rise (in feet), and summing the results of all individual deep-percolation events during the season. The WTF method is best applied where the water table is shallow and groundwater levels have sharp fluctuations. Because rises in groundwater levels typically reflect water-table conditions over an area of at least a few tens of square feet, the method may provide a more general indication of deep-percolation return flow at the scale of the irrigated field than the UZWB method, which represents deeppercolation return flow at a specific location in the field.

Specific yield was estimated for use in equation 2 by calculating the difference between unsaturated and saturated volumetric water content (table 1) for each soil layer (fig. 3) in the interval of water-level rise. The volumetric water content determined in the laboratory for core samples collected above the water table was used to represent the volumetric water content of soils under unsaturated conditions. Because the core samples were collected prior to the start of irrigation at each site, when the water table was low, the samples were assumed to represent the volumetric water content of the soils under well-drained conditions. The volumetric water content measured by soil-moisture sensors as the water table rose above the sensors was used to represent the volumetric water content of each soil layer under saturated conditions. In cases where more than one soil layer was present in the interval of the water-level rise, the weighted-average specific yield of the soil layers in the interval of rise was used to estimate deeppercolation return flow using equation 2 .

\section{Deep-Percolation Return Flow at the Flood-Irrigated Site}

Soil-moisture measurements and deep-percolation return flow estimated by using the UZWB method. Soil moisture at the flood-irrigated site was monitored hourly from June 13 to October 28, 2008, and every 4 hours from July 1 to October 15, 2009. Graphs showing moisture content at the flood-irrigated site during the 2008 and 2009 monitoring periods are presented in figures 5 and 6 , respectively. Soilmoisture data collected at the site are presented in appendixes 1 and 2 and are available through the U.S. Geological Survey National Water Information System at http://waterdata.usgs. gov/nwis (Site ID 401834104444801). Soil-moisture data at a depth of $10 \mathrm{ft}$ are not displayed in figures 5 and 6 because the sensor provided erratic and inaccurate measurements, possibly because of inadequate insertion into the gravelly sand sediments (fig. 3) at that depth. Soil moisture at a depth of $18.5 \mathrm{ft}$ is not displayed in figure 5 because the water table had already risen above the sensor by the time data collection began on June 13, 2008.

Irrigation did not begin at the flood-irrigated site until July 2, 2009, because substantial precipitation and belowaverage temperatures during the month of June precluded the need for irrigation to meet the water requirements of the crops. Because data loggers could not be installed above ground at the site until irrigation furrows had been installed, data collection at the site also was delayed until July. Soil moisture at depths of 15 and $18.5 \mathrm{ft}$ are not displayed in figure 6 because the water table already had risen above the sensors by the time data collection began on July 1, 2009. Beginning on August 17, 2009, some sensors began measuring differently (perhaps because of electrical damage). The sensor at a depth of $4 \mathrm{ft}$ began displaying apparently anomalous spikes in soil moisture (above expected peak values) in response to irrigation or precipitation events beginning on August 17. To correct for the apparently anomalous spikes, changes in soil moisture at the 4-ft depth were computed using peak values without consideration of the spikes from August 17 through the end of the monitoring period. Soil moisture at a depth of $6 \mathrm{ft}$ is not displayed after August 17, 2009, because the sensor appeared 

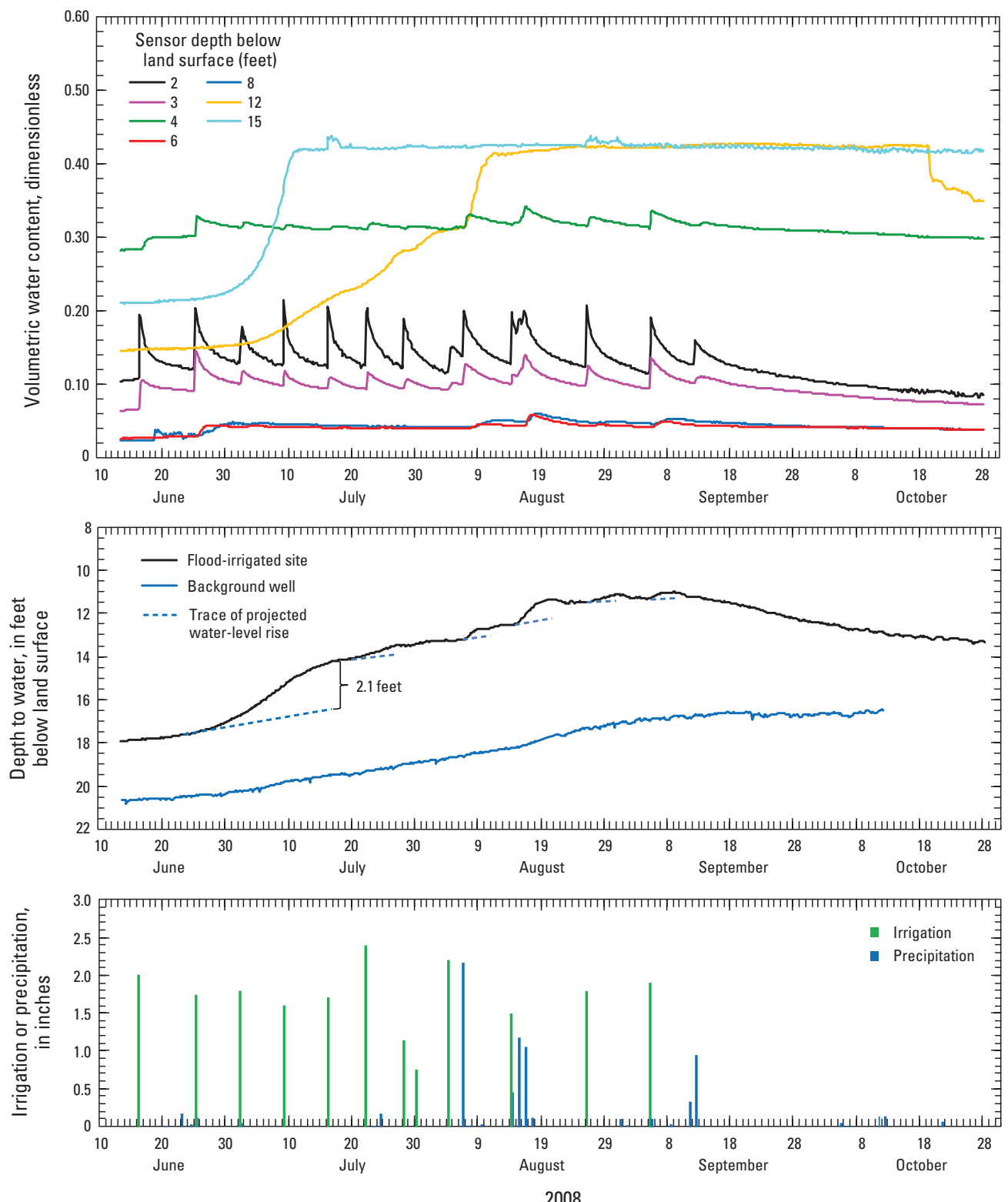

Figure 5. Volumetric water content, depth to water, irrigation, and precipitation at the flood-irrigated site during 2008, Weld County, Colorado.

to be malfunctioning. Deep-percolation return flow after the loss of the 6-ft sensor was calculated by using equation 1 without consideration of measurements by the 6 - $\mathrm{ft}$ sensor. Soil moisture at a depth of $12 \mathrm{ft}$ is not displayed after August 17,2009 , because the sensor at this depth ceased to operate. Because the 12-ft sensor was within the capillary fringe of the water table and the water table did not change substantially after the loss of the 12-ft sensor, deep-percolation return flow after the sensor loss was estimated using the average volumetric water content measured by the $12-\mathrm{ft}$ sensor in the capillary fringe prior to the sensor loss.
Soil moisture at the flood-irrigated site generally exhibits sharp increases at depths to as much as $3 \mathrm{ft}$ after irrigation or precipitation events that exceed about 1 in., and the increases generally become more attenuated with increasing depth below land surface (figs. 5 and 6). At depths greater than or equal to $6 \mathrm{ft}$, soil moisture commonly does not appear to increase substantially in response to individual irrigation or precipitation events. The time lag between an irrigation or precipitation event and a corresponding increase in soil moisture increases with increasing depth below ground surface. At a depth of $2 \mathrm{ft}$ below ground surface, soil moisture generally 

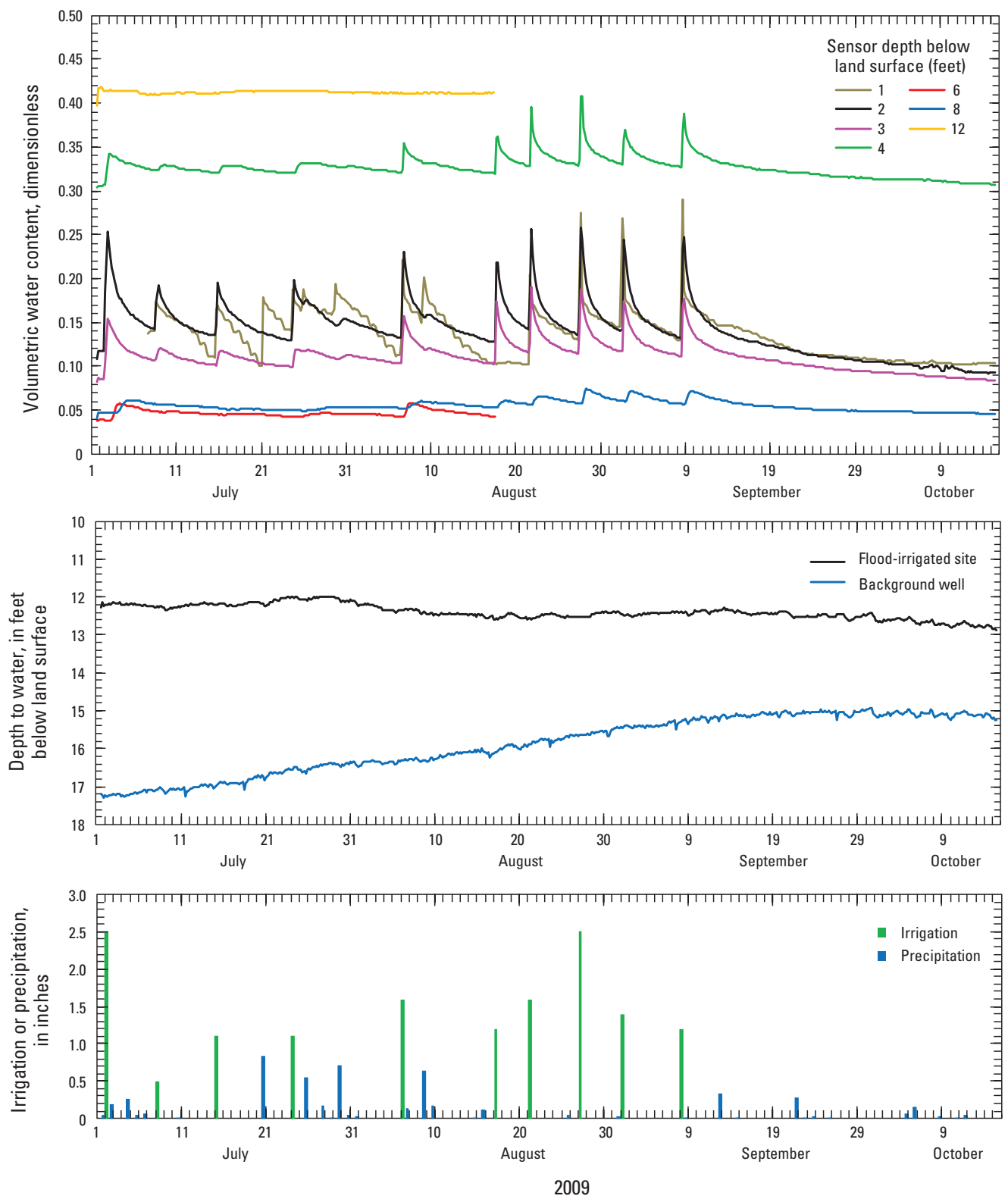

Figure 6. Volumetric water content, depth to water, irrigation, and precipitation at the flood-irrigated site during 2009, Weld County, Colorado.

increases to a maximum value about 1 to 8 hours after the event, whereas at a depth of $8 \mathrm{ft}$, soil moisture generally increases (when increases occur) to a maximum value after about 2 to 6 days. The exceptionally high soil-moisture content measured by the sensor at a depth of $4 \mathrm{ft}$ may be related to incipient perching caused by a zone of higher clay content and caliche present from 4 to $6.5 \mathrm{ft}$ below ground surface (fig. 3) and reflect the presence of a relatively stable zero-flux plane at the top of the zone.

The rapid, steady rises in volumetric water content beginning about July 3 at a depth of $15 \mathrm{ft}$ and August 7 at a depth of $12 \mathrm{ft}$ in 2008 (fig. 5) at the flood-irrigated site likely were caused by the infilling of pores by the capillary fringe of the rising water table. Once the water table reached the level of the soil-moisture sensors, volumetric water content remained at saturation until the water table again declined below the sensors. The water table rose to the level of the moisture sensor at a depth of about $15 \mathrm{ft}$ on July 10, 2008, and the water table rose to the level of the moisture sensor at a depth of about $12 \mathrm{ft}$ on about August 10,2008. By comparing the depth of the water table at the start of the rapid, steady rise in volumetric water content to its depth when the sensors 
become submerged, the thickness of the capillary fringe can be estimated. Using this method, the thickness of the capillary fringe is estimated as about $1.5 \mathrm{ft}$ on the basis of soil-moisture measurements in the silty sand at a depth of $15 \mathrm{ft}$. Similarly, the thickness of the capillary fringe is estimated as about $1.2 \mathrm{ft}$ on the basis of soil-moisture measurements in the fine- to medium-grained sand at a depth of $12 \mathrm{ft}$.

Using the UZWB method, increases in soil moisture between the bottom of the root zone ( $4 \mathrm{ft}$ below ground surface) and the top of the capillary fringe ( $1.5 \mathrm{ft}$ above the water table) during the 2008 monitoring period indicate that deep percolation was about $8.1 \mathrm{in}$. (table 3 ), which is about 40 percent of the $20.5 \mathrm{in}$. of net irrigation water (inflow minus outflow) applied and about 29 percent of irrigation water plus precipitation (27.8 in.) during the period. Similarly, increases in soil moisture between $4 \mathrm{ft}$ below ground surface and $1.5 \mathrm{ft}$ above the water table during the 2009 monitoring period indicate that deep percolation was about 7.7 in. (table 3), which is about 52 percent of the $14.7 \mathrm{in}$. of net irrigation water applied during the period and about 39 percent of irrigation water plus precipitation (19.8 in.) during the period.

Water-level measurements and deep-percolation return flow estimated by using the WTF method. Water levels at the flood-irrigated site were monitored every 4 hours from June 13 to October 28, 2008, and from July 1 to October 15, 2009. Because of the substantial precipitation during June 2009, and irrigation of crops in adjacent fields prior to the monitoring period (Troy Bauder, Colorado State University, written commun., 2010), the water table already was near its highest level (fig. 6) for the season at the time data collection began on July 1, 2009. Therefore, using the WTF method to estimate deep-percolation return flow proved problematic, and deep-percolation return flow at the flood-irrigated site could not be determined by the WTF method during 2009 .

Because background water levels generally rose throughout the 2008 irrigation season (fig. 5), local rises in the water table resulting from deep-percolation return flow at each site were obscured by the regional water-level trend. Rather than rising in response to deep-percolation events and then returning to pre-event levels, the rate of water-level rise commonly increased temporarily following a deep-percolation event and then returned to a slower rate of rise (fig. 5). Therefore, the water-level rise resulting from a deep-percolation event was estimated as the difference between the projected pre-event water level and the peak of the accelerated rise after the event, where the trace of the projected water level is the expected trend of the hydrograph had there been no deep percolation. Drawing the trace of the projected water level is a matter of interpretation and is somewhat subjective.

Groundwater levels at the flood-irrigated site began rising substantially relative to the rate of background waterlevel rise about 10 days after the start of irrigation on June 16, 2008. Rises in groundwater levels resulting from individual irrigation events are not evident during the early part of the irrigation season; however, the cumulative effects of irrigation are substantial (about $2.1 \mathrm{ft}$ from June 22 to July 10) (fig. 5).
Later in the season, beginning with the irrigation event on July 22, 2008, groundwater levels generally rose in response to individual or closely spaced irrigation and precipitation events that were greater than about 1.5 in., and the peak of the groundwater-level rises occurred about 3 to 6 days after the event or closely spaced events. Water levels in the background well were not recorded after October 12 during the 2008 monitoring period because of power failure to the data logger.

Rises in groundwater levels relative to the projected water-level trend (fig. 5) during the irrigation season in 2008 ranged from about 0.2 to $2.1 \mathrm{ft}$. Using equation 2 with specific-yield values (table 1) representing the sediment layer in each interval of water-table rise, deep-percolation return flow during the 2008 monitoring period is estimated as about $8.8 \mathrm{in}$. (table 3 ), which is about 43 percent of the $20.5 \mathrm{in}$. of net irrigation water applied during the period and about 32 percent of irrigation water plus precipitation (27.8 in.) during the period.

\section{Deep-Percolation Return Flow at the Sprinkler- Irrigated Site}

Soil-moisture measurements and deep-percolation return flow estimated by using the UZWB method. Soil moisture at the sprinkler-irrigated site was monitored hourly from June 18 to October 28, 2008, and every 4 hours from June 15 to October 15, 2009. Graphs showing moisture content at the sprinkler-irrigated site during the 2008 and 2009 monitoring periods are presented in figures 7 and 8 , respectively. Soil-moisture data collected at the site are presented in appendixes 3 and 4 and are available through the U.S. Geological Survey National Water Information System at http://waterdata.usgs.gov/nwis (Site ID 401842104442501).

Damage to sensor wires during the 2008 monitoring period prevented collection of soil-moisture data after July 14, 2008, at depths of $7 \mathrm{ft}$ and $16.5 \mathrm{ft}$ and after July 25, 2008, at depths of $9 \mathrm{ft}$ and $13 \mathrm{ft}$. Soil moisture at a depth of $16.5 \mathrm{ft}$ is not displayed in figure 7 because the water table had already risen above the sensor by the time data collection began on June 18, 2008. Soil moisture at a depths of 13 and $16.5 \mathrm{ft}$ are not displayed in figure 8 because the water table had already risen above the sensor by the time data collection began on June 15, 2009.

Similar to the flood-irrigated site, soil moisture at the sprinkler-irrigated site generally exhibits sharp increases at depths up to $3 \mathrm{ft}$ after irrigation or precipitation events that exceed about $1 \mathrm{in}$,, and the increases generally become more attenuated with increasing depth below land surface (figs. 7 and 8). The increases in soil moisture at the sprinkler-irrigated site in 2009 (fig. 8) generally are smaller and less abrupt than those in 2008 (fig. 7). At depths greater than or equal to $3 \mathrm{ft}$, soil moisture commonly does not appear to increase substantially in response to individual irrigation or precipitation events in 2009. Also, the time lag between an irrigation or pre- 

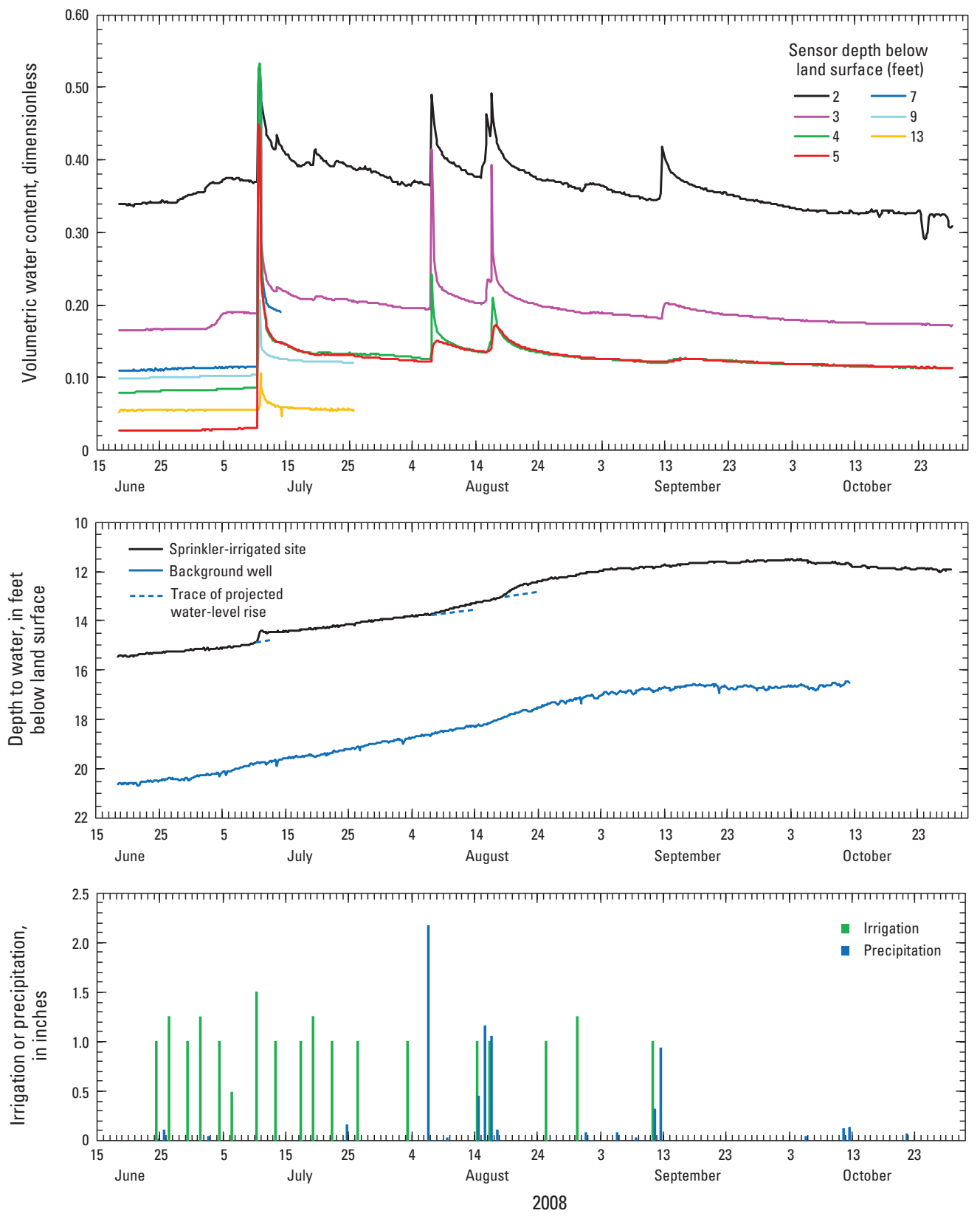

Figure 7. Volumetric water content, depth to water, irrigation, and precipitation at the sprinkler-irrigated site during 2008, Weld County, Colorado.

cipitation event and a corresponding increase in soil moisture increases with increasing depth below ground surface.

Because of damage to sensor wires and unreliable measurements, deep percolation at the sprinkler-irrigated site is not estimated using the UZWB method for 2008. For 2009, use of the UZWB method with increases in soil moisture between the bottom of the root zone ( $4 \mathrm{ft}$ below ground surface) and the top of the capillary fringe ( $1.5 \mathrm{ft}$ above the water table) during the 2009 monitoring period indicates that deep percolation was about $1.2 \mathrm{in}$. (table 3 ), which is about 5 percent of the $24 \mathrm{in}$. of irrigation water applied during the period and about 4 percent of irrigation water plus precipitation (29.5 in.) during the same period.

Water-level measurements and deep-percolation return flow estimated by using the WTF method. Water levels at the sprinkler-irrigated site were monitored every 4 hours from June 18 to October 28, 2008, and from June 15 to October 15, 2009. As with the flood-irrigated site, 

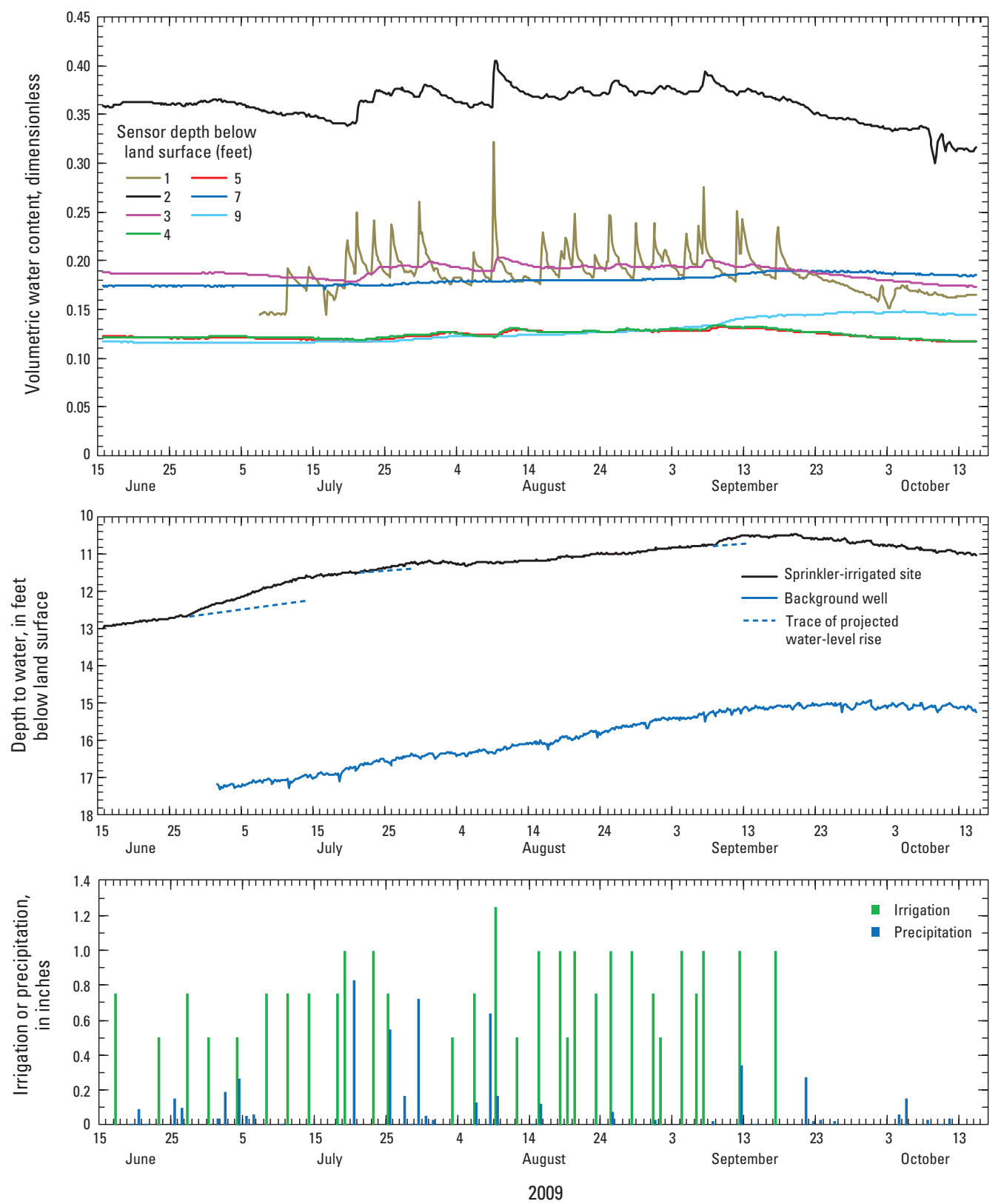

Figure 8. Volumetric water content, depth to water, irrigation, and precipitation at the sprinkler-irrigated site during 2009, Weld County, Colorado.

the water-level rise caused by deep-percolation events was estimated by comparing the difference between the projected pre-event water level and the peak of the accelerated rise after the event (figs. 7 and 8), where the trace of the projected water level is the expected trend of the hydrograph had there been no deep percolation. Rises in groundwater levels generally are smaller and less frequent at the sprinkler-irrigated site than at the flood-irrigated site in 2008, and rises in response to individual irrigation or precipitation events are not apparent. Unlike the flood-irrigated site in 2009 (fig. 6), although the water table had risen substantially by the time data collection began on June 15, 2009, rises in groundwater levels do occur several times at the sprinkler-irrigated site throughout the 2009 monitoring period (fig. 8).

Rises in groundwater levels relative to the projected water-level trend during the 2008 monitoring period ranged from about 0.2 to $0.4 \mathrm{ft}$ (fig. 7), and relative rises in groundwater levels during the 2009 monitoring period ranged from about 0.1 to $0.6 \mathrm{ft}$ (fig. 8). Using equation 2 with specificyield values (table 1 ) representing the sediment layer in each 
interval of water-table rise, deep-percolation return flow during the 2008 monitoring period is estimated as about $2.7 \mathrm{in}$. (table 3), which is about 14 percent of the 19 in. of irrigation water applied during the period and about 10 percent of irrigation water plus precipitation (26.3 in.) during the same period. Similarly, deep-percolation return flow during the 2009 monitoring period is estimated as about 3.2 in. (table 3), which is about 13 percent of the $24 \mathrm{in}$. of irrigation water applied during the period and about 11 percent of irrigation water plus precipitation (29.5 in.) during the same period.

\section{Summary}

To improve understanding of deep-percolation return flow beneath irrigated fields, the U.S. Geological Survey, in cooperation with Central Colorado Water Conservancy District, conducted a study to estimate deep-percolation return flow at two irrigated sites in the Central Colorado Water Conservancy District during the 2008 and 2009 irrigation seasons. One site was irrigated by flood furrows, and the other site was irrigated by a center-pivot sprinkler. Each site was equipped with sensors installed at multiple depths between land surface and the water table to monitor soil moisture in the unsaturated zone, and an observation well equipped with a pressure transducer and data logger was installed at each site to monitor groundwater levels throughout the irrigation season. An observation well located on adjacent nonirrigated land also was equipped with a pressure transducer and data logger to monitor background water-level trends.

Deep-percolation return flow was estimated at each site by using two different methods. The first method estimated deep-percolation return flow by using an unsaturated zone water-balance method, in which water that infiltrates below the root zone is assumed not to be substantially affected by evapotranspiration, and changes in soil-water storage between the bottom of the root zone and the top of the capillary fringe above the water table are assumed to equal deep-percolation return flow. The second method estimated deep-percolation return flow by using the water-table fluctuation method, in which rises in groundwater levels are assumed to be caused by downward-moving water arriving at the water table as recharge.

For the unsaturated zone water-balance method, the depth of substantial evapotranspiration was assumed to occur at the bottom of the root zone, which was estimated to have a depth of $4 \mathrm{ft}$ below ground surface. Increases in soil moisture between the bottom of the root zone and the top of the capillary fringe above the water table (estimated as $1.5 \mathrm{ft}$ above the water table) were used to estimate deep-percolation return flow. Although evapotranspiration can occur at depths greater than $4 \mathrm{ft}$, the time interval between successive irrigation or precipitation events was sufficiently short that evapotranspiration at depths greater than $4 \mathrm{ft}$ likely was not substantial between events. If the depth of substantial evapotranspiration was deeper than $4 \mathrm{ft}$, deep-percolation return flow determined by using the unsaturated zone water-balance method could be overestimated. Similarly, if the depth of substantial evapotranspiration was shallower than $4 \mathrm{ft}$, deep-percolation return flow determined by using the unsaturated zone water-balance method could be underestimated. Because rises in groundwater levels typically reflect water-table conditions over an area of at least a few tens of square feet, the water-table fluctuation method may provide a more general indication of deeppercolation return flow at the scale of the irrigated field than the unsaturated zone water-balance method, which represents deep-percolation return flow at a specific location in the field.

Deep percolation at the flood-irrigated site was estimated to range from 7.7 to $8.8 \mathrm{in}$. during the monitoring periods, which is about $40-52$ percent of irrigation water applied during each monitoring period and about 29-39 percent of irrigation water plus precipitation during each period. Deep percolation at the sprinkler-irrigated site was estimated to range from 1.2 to $3.2 \mathrm{in}$. during the monitoring periods, which is about 5-14 percent of irrigation water applied during each monitoring period and about 4-11 percent of irrigation water plus precipitation during each period.

\section{Acknowledgments}

Thanks are extended to Eckhardt Farms, Inc., and DSW Farms, Inc., for granting access to their properties for data collection. Thanks also are extended to Eckhardt Farms, Inc., and to Troy Bauder of the Department of Soil and Crop Sciences, Colorado State University, for providing data concerning irrigation at the study sites. Technical support provided by Rick Healy (USGS National Research Program) concerning the collection and analysis of data used to estimate recharge also is gratefully acknowledged.

\section{References Cited}

Colorado Division of Water Resources, 2010, Colorado's Decision Support Systems: Colorado Division of Water Resources structure diversion data available on the Web, accessed December 17, 2010, at http://cdss.state.co.us/ DNN/StructuresDiversions/tabid/75/Default.aspx.

Colton, R.B., 1978, Geological map of the Boulder-Fort Collins-Greeley area, Front Range Urban Corridor, Colorado: U.S. Geological Survey Miscellaneous Investigations Map I-855-G, scale 1:100,000.

Dane, J.H., and Topp, G.C., eds., 2002, Methods of soil analysis, part 4, Physical methods: Madison, Wis., Soil Science Society of America Book Series, v. 5, 1692 p. 
Decagon Devices, Inc., 2006, $\mathrm{ECH}_{2} \mathrm{O}$ soil moisture sensor operator's manual, version 5: Pullman, Wash., Decagon Devices, Inc., 23 p.

Decagon Devices, Inc., 2007, ECH ${ }_{2} \mathrm{O}-\mathrm{TE} / \mathrm{EC}-\mathrm{TM}$ water content, EC and temperature sensors operator's manual, version 5: Pullman, Wash., Decagon Devices, Inc., 23 p.

Delin, G.N., Healy, R.W., Landon, M.K., and Bohlke, J.K., 2000, Effects of topography and soil properties on recharge at two sites in an agricultural field: Journal of the American Water Resources Association, v. 36, no. 6, p. 1401-1416.

Healy, R.W., and Cook, P.G., 2002, Using groundwater levels to estimate recharge: Hydrogeology Journal, v. 10, no. 1, p. 91-109.

Hurr, R.T., Schneider, P.A., Jr., and others, 1972, Hydrogeologic characteristics of the valley-fill aquifer in the Greeley reach of the South Platte River valley, Colorado: U.S. Geological Survey Open-File Report 73-124, 2 p. 6 pls.

Robson, S.G., Heiny, J.S., and Arnold, L.R., 2000, Geohydrology of the shallow aquifers in the Fort Lupton-Gilcrest area, Colorado: U.S. Geological Survey Hydrologic Investigations Atlas HA-746-C, 5 sheets, scale 1:50,000.

U.S. Department of Agriculture, Natural Resources Conservation Service, 2005, Soil survey geographic (SSURGO) database for Weld County, Colorado, southern part: U.S. Department of Agriculture- Natural Resources Conservation Service tabular and spatial soil data available on the Web, accessed March 12, 2008, at http:/soildatamart.nrcs. usda.gov.

Western Regional Climate Center, 2010, Historical climate information: Western Regional Climate Center data available on the Web, accessed January 6, 2010, at $h t t p: / / w w w$. wrcc.dri.edu/summary/climsmco.html. 


\section{Appendixes}


Appendix 1. Volumetric water content, temperature, and electrical-conductivity data collected at the flood-irrigated site during 2008.

[Depth in feet below land surface; volumetric water content dimensionless; temperature in degrees Celsius; bulk electrical conductivity in decisiemens per meter; --, no data]

\begin{tabular}{|c|c|c|c|c|c|c|c|c|c|c|c|c|c|c|c|}
\hline \multirow[b]{3}{*}{$\begin{array}{l}\text { Measurement } \\
\text { date and time }\end{array}$} & \multicolumn{15}{|c|}{ Depth } \\
\hline & \multicolumn{2}{|c|}{2} & \multirow{2}{*}{$\begin{array}{c}3 \\
\begin{array}{c}\text { Volumetric } \\
\text { water } \\
\text { content }\end{array}\end{array}$} & \multicolumn{3}{|c|}{4} & \multirow{2}{*}{$\begin{array}{c}6 \\
\text { Volumetric } \\
\text { water } \\
\text { content }\end{array}$} & \multicolumn{2}{|c|}{8} & \multirow{2}{*}{$\begin{array}{c}10 \\
\text { Volumetric } \\
\text { water } \\
\text { content }\end{array}$} & \multicolumn{2}{|l|}{12} & \multicolumn{2}{|c|}{15} & \multirow{2}{*}{$\begin{array}{c}18.5 \\
\text { Volumetric } \\
\text { water } \\
\text { content }\end{array}$} \\
\hline & $\begin{array}{c}\text { Volumetric } \\
\text { water } \\
\text { content }\end{array}$ & $\begin{array}{l}\text { Temper- } \\
\text { ature }\end{array}$ & & $\begin{array}{c}\text { Volumetric } \\
\text { water } \\
\text { content }\end{array}$ & $\begin{array}{l}\text { Temper- } \\
\text { ature }\end{array}$ & $\begin{array}{c}\text { Bulk } \\
\text { electrical } \\
\text { conduct- } \\
\text { ivity } \\
\end{array}$ & & $\begin{array}{c}\text { Volumetric } \\
\text { water } \\
\text { content }\end{array}$ & $\begin{array}{l}\text { Temper- } \\
\text { ature }\end{array}$ & & $\begin{array}{c}\text { Volumetric } \\
\text { water } \\
\text { content }\end{array}$ & $\begin{array}{l}\text { Temper- } \\
\text { ature }\end{array}$ & $\begin{array}{c}\text { Volumetric } \\
\text { water } \\
\text { content }\end{array}$ & $\begin{array}{l}\text { Temper- } \\
\text { ature }\end{array}$ & \\
\hline 6/13/2008 3:00 PM & 0.107 & 18.5 & 0.064 & 0.281 & 16.6 & 0.38 & 0.026 & 0.024 & 13.2 & -- & 0.145 & 11.6 & 0.210 & 11.4 & 0.318 \\
\hline 6/13/2008 4:00 PM & 0.106 & 18.5 & 0.064 & 0.281 & 16.6 & 0.38 & 0.025 & 0.024 & 13.2 & -- & 0.146 & 11.7 & 0.210 & 11.4 & 0.310 \\
\hline 6/13/2008 5:00 PM & 0.104 & 18.5 & 0.063 & 0.282 & 16.6 & 0.38 & 0.026 & 0.023 & -- & -- & 0.145 & 11.7 & 0.210 & 11.4 & 0.311 \\
\hline 6/13/2008 6:00 PM & 0.104 & 18.5 & 0.063 & 0.282 & 16.6 & 0.38 & 0.025 & 0.023 & -- & -- & 0.145 & 11.7 & 0.210 & 11.4 & 0.311 \\
\hline 6/13/2008 7:00 PM & 0.104 & 18.5 & 0.063 & 0.282 & 16.7 & 0.38 & 0.025 & 0.023 & -- & -- & 0.144 & 11.7 & 0.211 & 11.4 & 0.310 \\
\hline 6/13/2008 8:00 PM & 0.104 & 18.5 & 0.063 & 0.282 & 16.7 & 0.38 & 0.026 & 0.023 & -- & -- & 0.145 & 11.7 & 0.210 & 11.4 & 0.309 \\
\hline 6/13/2008 9:00 PM & 0.105 & 18.4 & 0.063 & 0.282 & 16.7 & 0.38 & 0.025 & 0.024 & -- & -- & 0.146 & 11.7 & 0.210 & 11.4 & 0.308 \\
\hline 6/13/2008 10:00 PM & 0.104 & 18.5 & 0.064 & 0.283 & 16.7 & 0.38 & 0.025 & 0.023 & -- & -- & 0.145 & 11.7 & 0.210 & 11.4 & 0.309 \\
\hline 6/13/2008 11:00 PM & 0.105 & 18.5 & 0.064 & 0.282 & 16.7 & 0.38 & 0.026 & 0.023 & -- & -- & 0.146 & 11.7 & 0.210 & 11.4 & 0.312 \\
\hline 6/14/2008 12:00 AM & 0.105 & 18.5 & 0.064 & 0.282 & 16.7 & 0.38 & 0.026 & 0.023 & -- & -- & 0.145 & 11.7 & 0.210 & 11.4 & 0.313 \\
\hline 6/14/2008 1:00 AM & 0.105 & 18.5 & 0.064 & 0.282 & 16.7 & 0.38 & 0.026 & 0.023 & -- & -- & 0.145 & 11.7 & 0.210 & 11.4 & 0.323 \\
\hline 6/14/2008 2:00 AM & 0.105 & 18.6 & 0.064 & 0.282 & 16.7 & 0.38 & 0.026 & 0.023 & -- & -- & 0.145 & 11.7 & 0.210 & 11.4 & 0.341 \\
\hline 6/14/2008 3:00 AM & 0.105 & 18.6 & 0.064 & 0.282 & 16.7 & 0.38 & 0.026 & 0.023 & -- & -- & 0.145 & 11.7 & 0.210 & 11.5 & 0.341 \\
\hline 6/14/2008 4:00 AM & 0.105 & 18.6 & 0.064 & 0.282 & 16.7 & 0.38 & 0.026 & 0.024 & -- & -- & 0.145 & 11.7 & 0.210 & 11.5 & 0.313 \\
\hline 6/14/2008 5:00 AM & 0.105 & 18.6 & 0.064 & 0.282 & 16.7 & 0.38 & 0.026 & 0.029 & -- & -- & 0.145 & 11.7 & 0.209 & 11.4 & 0.311 \\
\hline 6/14/2008 6:00 AM & 0.105 & 18.6 & 0.064 & 0.282 & 16.7 & 0.38 & 0.026 & 0.024 & -- & -- & 0.145 & 11.7 & 0.209 & 11.5 & 0.313 \\
\hline 6/14/2008 7:00 AM & 0.105 & 18.7 & 0.064 & 0.282 & 16.7 & 0.38 & 0.026 & 0.024 & -- & -- & 0.145 & 11.7 & 0.210 & 11.4 & 0.319 \\
\hline 6/14/2008 8:00 AM & 0.105 & 18.7 & 0.064 & 0.282 & 16.7 & 0.38 & 0.025 & 0.024 & -- & -- & 0.145 & 11.7 & 0.210 & 11.5 & 0.315 \\
\hline 6/14/2008 9:00 AM & 0.105 & 18.8 & 0.064 & 0.283 & 16.7 & 0.38 & 0.025 & 0.024 & -- & -- & 0.145 & 11.7 & 0.209 & 11.5 & 0.325 \\
\hline 6/14/2008 10:00 AM & 0.106 & 18.7 & 0.064 & 0.283 & 16.7 & 0.38 & 0.025 & 0.024 & -- & -- & 0.145 & 11.7 & 0.210 & 11.4 & 0.352 \\
\hline 6/14/2008 11:00 AM & 0.104 & 18.7 & 0.064 & 0.283 & 16.7 & 0.38 & 0.025 & 0.024 & -- & -- & 0.145 & 11.8 & 0.211 & 11.4 & 0.353 \\
\hline 6/14/2008 12:00 PM & 0.104 & 18.7 & 0.064 & 0.283 & 16.7 & 0.38 & 0.026 & 0.024 & -- & -- & 0.145 & 11.7 & 0.210 & 11.4 & 0.321 \\
\hline 6/14/2008 1:00 PM & 0.105 & 18.7 & 0.064 & 0.283 & 16.7 & 0.38 & 0.026 & 0.024 & -- & -- & 0.145 & 11.8 & 0.210 & 11.5 & 0.315 \\
\hline 6/14/2008 2:00 PM & 0.106 & 18.6 & 0.064 & 0.283 & 16.7 & 0.38 & 0.026 & 0.024 & -- & -- & 0.145 & 11.8 & 0.210 & 11.5 & 0.313 \\
\hline 6/14/2008 3:00 PM & 0.105 & 18.6 & 0.064 & 0.284 & 16.7 & 0.38 & 0.026 & 0.024 & -- & -- & 0.145 & 11.8 & 0.210 & 11.5 & 0.313 \\
\hline 6/14/2008 4:00 PM & 0.105 & 18.6 & 0.064 & 0.284 & 16.7 & 0.38 & 0.026 & 0.024 & -- & -- & 0.145 & 11.8 & 0.210 & 11.5 & 0.345 \\
\hline 6/14/2008 5:00 PM & 0.105 & 18.6 & 0.064 & 0.283 & 16.7 & 0.38 & 0.026 & 0.024 & -- & -- & 0.145 & 11.8 & 0.210 & 11.5 & 0.364 \\
\hline 6/14/2008 6:00 PM & 0.105 & 18.5 & 0.064 & 0.283 & 16.7 & 0.38 & 0.026 & 0.024 & -- & -- & 0.145 & 11.7 & 0.210 & 11.5 & 0.353 \\
\hline 6/14/2008 7:00 PM & 0.105 & 18.5 & 0.064 & 0.283 & 16.8 & 0.38 & 0.026 & 0.024 & -- & -- & 0.146 & 11.8 & 0.210 & 11.5 & 0.424 \\
\hline 6/14/2008 8:00 PM & 0.105 & 18.5 & 0.064 & 0.284 & 16.8 & 0.38 & 0.026 & 0.024 & -- & -- & 0.147 & 11.8 & 0.210 & 11.5 & 0.360 \\
\hline 6/14/2008 9:00 PM & 0.105 & 18.6 & 0.064 & 0.283 & 16.8 & 0.38 & 0.025 & 0.024 & -- & -- & 0.146 & 11.8 & 0.210 & 11.5 & 0.336 \\
\hline 6/14/2008 10:00 PM & 0.105 & 18.6 & 0.064 & 0.283 & 16.8 & 0.38 & 0.025 & 0.024 & -- & -- & 0.146 & 11.8 & 0.210 & 11.5 & 0.324 \\
\hline 6/14/2008 11:00 PM & 0.106 & 18.6 & 0.064 & 0.283 & 16.8 & 0.38 & 0.025 & 0.024 & -- & -- & 0.147 & 11.7 & 0.210 & 11.5 & 0.349 \\
\hline 6/15/2008 12:00 AM & 0.105 & 18.6 & 0.064 & 0.283 & 16.8 & 0.38 & 0.025 & 0.024 & -- & -- & 0.147 & 11.8 & 0.210 & 11.5 & 0.352 \\
\hline 6/15/2008 1:00 AM & 0.105 & 18.7 & 0.064 & 0.283 & 16.8 & 0.38 & 0.025 & 0.024 & -- & -- & 0.147 & 11.8 & 0.210 & 11.5 & 0.314 \\
\hline 6/15/2008 2:00 AM & 0.105 & 18.8 & 0.064 & 0.283 & 16.8 & 0.38 & 0.025 & 0.024 & -- & -- & 0.146 & 11.7 & 0.210 & 11.5 & 0.314 \\
\hline 6/15/2008 3:00 AM & 0.106 & 18.8 & 0.064 & 0.283 & 16.8 & 0.38 & 0.026 & 0.024 & -- & -- & 0.146 & 11.8 & 0.210 & 11.5 & 0.314 \\
\hline 6/15/2008 4:00 AM & 0.106 & 18.9 & 0.064 & 0.283 & 16.8 & 0.38 & 0.026 & 0.024 & -- & -- & 0.145 & 11.8 & 0.210 & 11.5 & 0.318 \\
\hline 6/15/2008 5:00 AM & 0.106 & 19.0 & 0.064 & 0.282 & 16.8 & 0.38 & 0.026 & 0.024 & -- & -- & 0.145 & 11.8 & 0.210 & 11.5 & 0.324 \\
\hline 6/15/2008 6:00 AM & 0.106 & 19.0 & 0.064 & 0.283 & 16.8 & 0.38 & 0.026 & 0.024 & -- & -- & 0.145 & 11.8 & 0.210 & 11.5 & 0.352 \\
\hline
\end{tabular}


Appendix 1. Volumetric water content, temperature, and electrical-conductivity data collected at the flood-irrigated site during 2008.-Continued

[Depth in feet below land surface; volumetric water content dimensionless; temperature in degrees Celsius; bulk electrical conductivity in decisiemens per meter; --, no data]

\begin{tabular}{|c|c|c|c|c|c|c|c|c|c|c|c|c|c|c|c|}
\hline \multirow[b]{3}{*}{$\begin{array}{l}\text { Measurement } \\
\text { date and time }\end{array}$} & \multicolumn{15}{|c|}{ Depth } \\
\hline & \multicolumn{2}{|c|}{2} & \multirow{2}{*}{$\begin{array}{c}3 \\
\begin{array}{c}\text { Volumetric } \\
\text { water } \\
\text { content }\end{array}\end{array}$} & \multicolumn{3}{|c|}{4} & \multirow{2}{*}{$\begin{array}{c}6 \\
\begin{array}{c}\text { Volumetric } \\
\text { water } \\
\text { content }\end{array}\end{array}$} & \multicolumn{2}{|l|}{8} & \multirow{2}{*}{$\begin{array}{c}10 \\
\begin{array}{c}\text { Volumetric } \\
\text { water } \\
\text { content }\end{array}\end{array}$} & \multicolumn{2}{|c|}{12} & \multicolumn{2}{|c|}{15} & \multirow{2}{*}{$\begin{array}{c}18.5 \\
\text { Volumetric } \\
\text { water } \\
\text { content }\end{array}$} \\
\hline & $\begin{array}{l}\text { Volumetric } \\
\text { water } \\
\text { content }\end{array}$ & $\begin{array}{l}\text { Temper- } \\
\text { ature }\end{array}$ & & $\begin{array}{c}\text { Volumetric } \\
\text { water } \\
\text { content }\end{array}$ & $\begin{array}{l}\text { Temper- } \\
\text { ature }\end{array}$ & $\begin{array}{c}\text { Bulk } \\
\text { electrical } \\
\text { conduct- } \\
\text { ivity } \\
\end{array}$ & & $\begin{array}{l}\text { Volumetric } \\
\text { water } \\
\text { content }\end{array}$ & $\begin{array}{l}\text { Temper- } \\
\text { ature }\end{array}$ & & $\begin{array}{l}\text { Volumetric } \\
\text { water } \\
\text { content }\end{array}$ & $\begin{array}{l}\text { Temper- } \\
\text { ature }\end{array}$ & $\begin{array}{l}\text { Volumetric } \\
\text { water } \\
\text { content }\end{array}$ & $\begin{array}{l}\text { Temper- } \\
\text { ature }\end{array}$ & \\
\hline 6/15/2008 7:00 AM & 0.106 & 19.0 & 0.064 & 0.283 & 16.8 & 0.38 & 0.026 & 0.024 & -- & -- & 0.146 & 11.8 & 0.210 & 11.5 & 0.367 \\
\hline 6/15/2008 8:00 AM & 0.106 & 19.0 & 0.064 & 0.283 & 16.8 & 0.38 & 0.025 & 0.024 & -- & -- & 0.147 & 11.8 & 0.209 & 11.5 & 0.392 \\
\hline 6/15/2008 9:00 AM & 0.106 & 19.0 & 0.064 & 0.283 & 16.8 & 0.38 & 0.025 & 0.024 & -- & -- & 0.145 & 11.8 & 0.210 & 11.5 & 0.410 \\
\hline 6/15/2008 10:00 AM & 0.106 & 19.0 & 0.064 & 0.283 & 16.8 & 0.38 & 0.025 & 0.024 & -- & -- & 0.146 & 11.8 & 0.210 & 11.5 & 0.330 \\
\hline 6/15/2008 11:00 AM & 0.106 & 19.1 & 0.064 & 0.283 & 16.8 & 0.38 & 0.025 & 0.024 & -- & -- & 0.147 & 11.8 & 0.210 & 11.5 & 0.338 \\
\hline 6/15/2008 12:00 PM & 0.106 & 19.0 & 0.065 & 0.283 & 16.8 & 0.38 & 0.025 & 0.024 & -- & -- & 0.147 & 11.8 & 0.210 & 11.5 & 0.316 \\
\hline 6/15/2008 1:00 PM & 0.106 & 19.0 & 0.065 & 0.284 & 16.8 & 0.38 & 0.026 & 0.024 & -- & -- & 0.147 & 11.8 & 0.210 & 11.5 & 0.315 \\
\hline 6/15/2008 2:00 PM & 0.106 & 19.0 & 0.065 & 0.284 & 16.8 & 0.38 & 0.026 & 0.024 & -- & -- & 0.145 & 11.8 & 0.210 & 11.5 & 0.319 \\
\hline 6/15/2008 3:00 PM & 0.106 & 19.0 & 0.065 & 0.284 & 16.8 & 0.38 & 0.026 & 0.024 & -- & -- & 0.145 & 11.8 & 0.210 & 11.5 & 0.331 \\
\hline 6/15/2008 4:00 PM & 0.107 & 19.0 & 0.065 & 0.284 & 16.9 & 0.38 & 0.026 & 0.024 & -- & -- & 0.145 & 11.8 & 0.210 & 11.5 & 0.320 \\
\hline 6/15/2008 5:00 PM & 0.107 & 19.0 & 0.065 & 0.284 & 16.9 & 0.38 & 0.026 & 0.024 & -- & -- & 0.145 & 11.8 & 0.210 & 11.5 & 0.326 \\
\hline 6/15/2008 6:00 PM & 0.106 & 19.0 & 0.065 & 0.284 & 16.9 & 0.38 & 0.026 & 0.024 & -- & -- & 0.145 & 11.8 & 0.210 & 11.5 & 0.326 \\
\hline 6/15/2008 7:00 PM & 0.106 & 19.0 & 0.065 & 0.284 & 16.9 & 0.38 & 0.026 & 0.024 & -- & -- & 0.146 & 11.8 & 0.210 & 11.5 & 0.328 \\
\hline 6/15/2008 8:00 PM & 0.106 & 19.1 & 0.065 & 0.284 & 16.9 & 0.38 & 0.026 & 0.024 & -- & -- & 0.147 & 11.8 & 0.210 & 11.5 & 0.324 \\
\hline 6/15/2008 9:00 PM & 0.107 & 19.1 & 0.065 & 0.284 & 16.9 & 0.38 & 0.026 & 0.024 & -- & -- & 0.147 & 11.8 & 0.210 & 11.5 & 0.326 \\
\hline 6/15/2008 10:00 PM & 0.107 & 19.1 & 0.065 & 0.284 & 16.9 & 0.38 & 0.026 & 0.024 & -- & -- & 0.147 & 11.8 & 0.211 & 11.5 & 0.328 \\
\hline 6/15/2008 11:00 PM & 0.107 & 19.2 & 0.065 & 0.284 & 16.9 & 0.38 & 0.026 & 0.024 & -- & -- & 0.146 & 11.8 & 0.210 & 11.5 & 0.383 \\
\hline 6/16/2008 12:00 AM & 0.107 & 19.2 & 0.065 & 0.283 & 16.9 & 0.38 & 0.026 & 0.024 & -- & -- & 0.145 & 11.8 & 0.210 & 11.5 & 0.329 \\
\hline 6/16/2008 1:00 AM & 0.107 & 19.3 & 0.065 & 0.283 & 16.9 & 0.38 & 0.026 & 0.024 & -- & -- & 0.146 & 11.8 & 0.210 & 11.6 & 0.326 \\
\hline 6/16/2008 2:00 AM & 0.107 & 19.4 & 0.065 & 0.284 & 16.9 & 0.38 & 0.026 & 0.024 & -- & -- & 0.146 & 11.8 & 0.210 & 11.6 & 0.327 \\
\hline 6/16/2008 3:00 AM & 0.107 & 19.4 & 0.065 & 0.283 & 16.9 & 0.38 & 0.026 & 0.024 & -- & -- & 0.146 & 11.8 & 0.210 & 11.6 & 0.326 \\
\hline 6/16/2008 4:00 AM & 0.108 & 19.5 & 0.065 & 0.283 & 16.9 & 0.38 & 0.026 & 0.024 & -- & -- & 0.147 & 11.8 & 0.210 & 11.6 & 0.325 \\
\hline 6/16/2008 5:00 AM & 0.107 & 19.6 & 0.065 & 0.283 & 16.9 & 0.38 & 0.026 & 0.024 & -- & -- & 0.147 & 11.8 & 0.210 & 11.6 & 0.326 \\
\hline 6/16/2008 6:00 AM & 0.107 & 19.6 & 0.065 & 0.283 & 17.0 & 0.38 & 0.026 & 0.024 & -- & -- & 0.147 & 11.8 & 0.210 & 11.6 & 0.324 \\
\hline 6/16/2008 7:00 AM & 0.108 & 19.7 & 0.065 & 0.284 & 17.0 & 0.38 & 0.026 & 0.024 & -- & -- & 0.147 & 11.9 & 0.210 & 11.6 & 0.323 \\
\hline 6/16/2008 8:00 AM & 0.107 & 19.7 & 0.065 & 0.284 & 17.0 & 0.38 & 0.026 & 0.024 & -- & -- & 0.147 & 11.8 & 0.210 & 11.6 & 0.323 \\
\hline 6/16/2008 9:00 AM & 0.108 & 19.7 & 0.065 & 0.284 & 17.0 & 0.38 & 0.026 & 0.024 & -- & -- & 0.147 & 11.8 & 0.210 & 11.6 & 0.328 \\
\hline 6/16/2008 10:00 AM & 0.107 & 19.8 & 0.065 & 0.284 & 17.0 & 0.38 & 0.026 & 0.024 & -- & -- & 0.146 & 11.9 & 0.210 & 11.6 & 0.340 \\
\hline 6/16/2008 11:00 AM & 0.108 & 19.8 & 0.066 & 0.284 & 17.0 & 0.38 & 0.026 & 0.024 & -- & -- & 0.146 & 11.9 & 0.210 & 11.6 & 0.376 \\
\hline 6/16/2008 12:00 PM & 0.108 & 19.8 & 0.066 & 0.283 & 17.0 & 0.38 & 0.026 & 0.024 & -- & -- & 0.146 & 11.9 & 0.211 & 11.6 & 0.318 \\
\hline 6/16/2008 1:00 PM & 0.108 & 19.8 & 0.066 & 0.284 & 17.0 & 0.38 & 0.026 & 0.024 & -- & -- & 0.147 & 11.9 & 0.211 & 11.6 & 0.321 \\
\hline 6/16/2008 2:00 PM & 0.108 & 19.8 & 0.066 & 0.284 & 17.0 & 0.38 & 0.026 & 0.024 & -- & -- & 0.147 & 11.9 & 0.210 & 11.6 & 0.322 \\
\hline 6/16/2008 3:00 PM & 0.108 & 19.8 & 0.066 & 0.284 & 17.0 & 0.38 & 0.026 & 0.024 & -- & -- & 0.147 & 11.9 & 0.211 & 11.6 & 0.365 \\
\hline 6/16/2008 4:00 PM & 0.110 & 19.8 & 0.066 & 0.284 & 17.0 & 0.38 & 0.026 & 0.024 & -- & -- & 0.147 & 11.9 & 0.210 & 11.6 & 0.368 \\
\hline 6/16/2008 5:00 PM & 0.165 & 19.9 & 0.067 & 0.284 & 17.1 & 0.38 & 0.026 & 0.024 & -- & -- & 0.147 & 11.9 & 0.211 & 11.6 & 0.330 \\
\hline 6/16/2008 6:00 PM & 0.194 & 20.0 & 0.067 & 0.284 & 17.1 & 0.38 & 0.026 & 0.024 & -- & -- & 0.147 & 11.9 & 0.211 & 11.6 & 0.328 \\
\hline 6/16/2008 7:00 PM & 0.200 & 20.1 & 0.068 & 0.284 & 17.1 & 0.38 & 0.026 & 0.024 & -- & -- & 0.148 & 11.9 & 0.211 & 11.6 & 0.330 \\
\hline 6/16/2008 8:00 PM & 0.201 & 20.1 & 0.075 & 0.284 & 17.1 & 0.38 & 0.026 & 0.024 & -- & -- & 0.147 & 11.9 & 0.211 & 11.6 & 0.338 \\
\hline 6/16/2008 9:00 PM & 0.196 & 20.2 & 0.092 & 0.283 & 17.1 & 0.38 & 0.026 & 0.024 & -- & -- & 0.146 & 11.9 & 0.211 & 11.6 & 0.342 \\
\hline 6/16/2008 10:00 PM & 0.191 & 20.3 & 0.101 & 0.284 & 17.1 & 0.38 & 0.026 & 0.024 & -- & -- & 0.146 & 11.9 & 0.211 & 11.6 & 0.337 \\
\hline
\end{tabular}


Appendix 1. Volumetric water content, temperature, and electrical-conductivity data collected at the flood-irrigated site during 2008.-Continued

[Depth in feet below land surface; volumetric water content dimensionless; temperature in degrees Celsius; bulk electrical conductivity in decisiemens per meter; --, no data]

\begin{tabular}{|c|c|c|c|c|c|c|c|c|c|c|c|c|c|c|c|}
\hline \multirow[b]{3}{*}{$\begin{array}{l}\text { Measurement } \\
\text { date and time }\end{array}$} & \multicolumn{15}{|c|}{ Depth } \\
\hline & \multicolumn{2}{|c|}{2} & \multirow{2}{*}{$\begin{array}{c}\mathbf{3} \\
\text { Volumetric } \\
\text { water } \\
\text { content }\end{array}$} & \multicolumn{3}{|c|}{4} & \multirow{2}{*}{$\begin{array}{c}6 \\
\text { Volumetric } \\
\text { water } \\
\text { content }\end{array}$} & \multicolumn{2}{|l|}{8} & \multirow{2}{*}{$\begin{array}{c}10 \\
\begin{array}{c}\text { Volumetric } \\
\text { water } \\
\text { content }\end{array}\end{array}$} & \multicolumn{2}{|c|}{12} & \multicolumn{2}{|c|}{15} & \multirow{2}{*}{$\begin{array}{c}18.5 \\
\text { Volumetric } \\
\text { water } \\
\text { content }\end{array}$} \\
\hline & $\begin{array}{l}\text { Volumetric } \\
\text { water } \\
\text { content }\end{array}$ & $\begin{array}{l}\text { Temper- } \\
\text { ature }\end{array}$ & & $\begin{array}{c}\text { Volumetric } \\
\text { water } \\
\text { content }\end{array}$ & $\begin{array}{l}\text { Temper- } \\
\text { ature }\end{array}$ & $\begin{array}{c}\text { Bulk } \\
\text { electrical } \\
\text { conduct- } \\
\text { ivity }\end{array}$ & & $\begin{array}{l}\text { Volumetric } \\
\text { water } \\
\text { content }\end{array}$ & $\begin{array}{l}\text { Temper- } \\
\text { ature }\end{array}$ & & $\begin{array}{c}\text { Volumetric } \\
\text { water } \\
\text { content }\end{array}$ & $\begin{array}{l}\text { Temper- } \\
\text { ature }\end{array}$ & $\begin{array}{l}\text { Volumetric } \\
\text { water } \\
\text { content }\end{array}$ & $\begin{array}{l}\text { Temper- } \\
\text { ature }\end{array}$ & \\
\hline 6/16/2008 11:00 PM & 0.186 & 20.3 & 0.104 & 0.284 & 17.1 & 0.38 & 0.026 & 0.024 & -- & -- & 0.146 & 11.9 & 0.211 & 11.6 & 0.345 \\
\hline 6/17/2008 12:00 AM & 0.182 & 20.2 & 0.105 & 0.284 & 17.1 & 0.38 & 0.026 & 0.024 & -- & -- & 0.147 & 11.9 & 0.210 & 11.6 & 0.330 \\
\hline 6/17/2008 1:00 AM & 0.179 & 20.2 & 0.105 & 0.284 & 17.2 & 0.38 & 0.026 & 0.024 & -- & -- & 0.147 & 11.9 & 0.210 & 11.6 & 0.328 \\
\hline 6/17/2008 2:00 AM & 0.175 & 20.2 & 0.105 & 0.284 & 17.2 & 0.38 & 0.026 & 0.024 & -- & -- & 0.147 & 11.9 & 0.210 & 11.6 & 0.341 \\
\hline 6/17/2008 3:00 AM & 0.173 & 20.2 & 0.105 & 0.284 & 17.2 & 0.38 & 0.026 & 0.024 & -- & -- & 0.147 & 11.9 & 0.210 & 11.6 & 0.351 \\
\hline 6/17/2008 4:00 AM & 0.171 & 20.2 & 0.105 & 0.284 & 17.2 & 0.39 & 0.026 & 0.024 & -- & -- & 0.147 & 11.9 & 0.210 & 11.6 & 0.362 \\
\hline 6/17/2008 5:00 AM & 0.169 & 20.3 & 0.105 & 0.285 & 17.3 & 0.39 & 0.026 & 0.024 & -- & -- & 0.147 & 11.9 & 0.210 & 11.6 & 0.326 \\
\hline 6/17/2008 6:00 AM & 0.167 & 20.2 & 0.104 & 0.286 & 17.3 & 0.39 & 0.026 & 0.024 & -- & -- & 0.147 & 11.9 & 0.210 & 11.6 & 0.322 \\
\hline 6/17/2008 7:00 AM & 0.165 & 20.2 & 0.104 & 0.286 & 17.3 & 0.39 & 0.026 & 0.024 & -- & -- & 0.147 & 11.9 & 0.210 & 11.6 & 0.322 \\
\hline 6/17/2008 8:00 AM & 0.163 & 20.1 & 0.104 & 0.287 & 17.3 & 0.39 & 0.026 & 0.024 & -- & -- & 0.146 & 11.9 & 0.211 & 11.6 & 0.323 \\
\hline 6/17/2008 9:00 AM & 0.162 & 20.1 & 0.104 & 0.288 & 17.3 & 0.39 & 0.026 & 0.024 & -- & -- & 0.147 & 11.9 & 0.211 & 11.6 & 0.317 \\
\hline 6/17/2008 10:00 AM & 0.160 & 20.0 & 0.104 & 0.288 & 17.3 & 0.39 & 0.026 & 0.024 & -- & -- & 0.147 & 12.0 & 0.211 & 11.6 & 0.317 \\
\hline 6/17/2008 11:00 AM & 0.158 & 20.0 & 0.104 & 0.290 & 17.4 & 0.39 & 0.026 & 0.024 & -- & -- & 0.147 & 12.0 & 0.211 & 11.6 & 0.317 \\
\hline 6/17/2008 12:00 PM & 0.157 & 19.9 & 0.104 & 0.291 & 17.4 & 0.39 & 0.026 & 0.024 & -- & -- & 0.145 & 11.9 & 0.211 & 11.6 & 0.321 \\
\hline 6/17/2008 1:00 PM & 0.156 & 19.9 & 0.104 & 0.292 & 17.4 & 0.39 & 0.026 & 0.024 & -- & -- & 0.145 & 12.0 & 0.211 & 11.6 & 0.316 \\
\hline 6/17/2008 2:00 PM & 0.155 & 19.9 & 0.103 & 0.293 & 17.4 & 0.39 & 0.026 & 0.024 & -- & -- & 0.146 & 11.9 & 0.211 & 11.6 & 0.315 \\
\hline 6/17/2008 3:00 PM & 0.153 & 19.8 & 0.103 & 0.293 & 17.4 & 0.39 & 0.026 & 0.024 & -- & -- & 0.147 & 12.0 & 0.211 & 11.6 & 0.315 \\
\hline 6/17/2008 4:00 PM & 0.153 & 19.7 & 0.103 & 0.294 & 17.4 & 0.39 & 0.026 & 0.024 & -- & -- & 0.148 & 12.0 & 0.211 & 11.6 & 0.316 \\
\hline 6/17/2008 5:00 PM & 0.150 & 19.7 & 0.102 & 0.294 & 17.4 & 0.40 & 0.026 & 0.024 & -- & -- & 0.149 & 12.0 & 0.211 & 11.6 & 0.316 \\
\hline 6/17/2008 6:00 PM & 0.150 & 19.7 & 0.102 & 0.295 & 17.5 & 0.40 & 0.026 & 0.024 & -- & -- & 0.148 & 12.0 & 0.211 & 11.6 & 0.316 \\
\hline 6/17/2008 7:00 PM & 0.149 & 19.7 & 0.102 & 0.295 & 17.5 & 0.40 & 0.026 & 0.024 & -- & -- & 0.147 & 12.0 & 0.211 & 11.6 & 0.317 \\
\hline 6/17/2008 8:00 PM & 0.148 & 19.7 & 0.101 & 0.295 & 17.5 & 0.40 & 0.026 & 0.024 & -- & -- & 0.146 & 12.0 & 0.211 & 11.6 & 0.321 \\
\hline 6/17/2008 9:00 PM & 0.148 & 19.7 & 0.101 & 0.296 & 17.5 & 0.40 & 0.026 & 0.024 & -- & -- & 0.148 & 12.0 & 0.211 & 11.7 & 0.320 \\
\hline 6/17/2008 10:00 PM & 0.148 & 19.7 & 0.101 & 0.296 & 17.5 & 0.40 & 0.026 & 0.024 & -- & -- & 0.147 & 12.0 & 0.211 & 11.6 & 0.321 \\
\hline 6/17/2008 11:00 PM & 0.147 & 19.7 & 0.100 & 0.296 & 17.5 & 0.40 & 0.026 & 0.024 & -- & -- & 0.146 & 12.0 & 0.211 & 11.6 & 0.323 \\
\hline 6/18/2008 12:00 AM & 0.146 & 19.8 & 0.100 & 0.296 & 17.5 & 0.40 & 0.026 & 0.024 & -- & -- & 0.146 & 11.9 & 0.211 & 11.6 & 0.324 \\
\hline 6/18/2008 1:00 AM & 0.146 & 19.8 & 0.100 & 0.296 & 17.5 & 0.40 & 0.026 & 0.024 & -- & -- & 0.147 & 11.9 & 0.211 & 11.6 & 0.322 \\
\hline 6/18/2008 2:00 AM & 0.145 & 19.9 & 0.100 & 0.297 & 17.5 & 0.40 & 0.026 & 0.024 & -- & -- & 0.147 & 12.0 & 0.211 & 11.6 & 0.324 \\
\hline 6/18/2008 3:00 AM & 0.145 & 19.9 & 0.099 & 0.296 & 17.5 & 0.40 & 0.026 & 0.024 & -- & -- & 0.147 & 12.0 & 0.211 & 11.6 & 0.323 \\
\hline 6/18/2008 4:00 AM & 0.145 & 19.9 & 0.099 & 0.296 & 17.5 & 0.40 & 0.026 & 0.024 & -- & -- & 0.147 & 12.0 & 0.211 & 11.6 & 0.324 \\
\hline 6/18/2008 5:00 AM & 0.144 & 20.0 & 0.099 & 0.296 & 17.6 & 0.40 & 0.026 & 0.024 & -- & -- & 0.147 & 12.0 & 0.211 & 11.6 & 0.325 \\
\hline 6/18/2008 6:00 AM & 0.144 & 20.0 & 0.099 & 0.297 & 17.6 & 0.40 & 0.026 & 0.024 & -- & -- & 0.147 & 12.0 & 0.211 & 11.6 & 0.328 \\
\hline 6/18/2008 7:00 AM & 0.143 & 20.0 & 0.099 & 0.297 & 17.6 & 0.40 & 0.026 & 0.024 & -- & -- & 0.147 & 12.0 & 0.211 & 11.7 & 0.324 \\
\hline 6/18/2008 8:00 AM & 0.142 & 20.1 & 0.099 & 0.297 & 17.6 & 0.40 & 0.026 & 0.024 & -- & -- & 0.147 & 12.0 & 0.211 & 11.7 & 0.327 \\
\hline 6/18/2008 9:00 AM & 0.142 & 20.1 & 0.099 & 0.298 & 17.6 & 0.40 & 0.026 & 0.024 & -- & -- & 0.146 & 12.0 & 0.211 & 11.7 & 0.323 \\
\hline 6/18/2008 10:00 AM & 0.141 & 20.1 & 0.098 & 0.297 & 17.6 & 0.40 & 0.026 & 0.024 & -- & -- & 0.148 & 12.0 & 0.211 & 11.7 & 0.323 \\
\hline 6/18/2008 11:00 AM & 0.141 & 20.1 & 0.098 & 0.298 & 17.6 & 0.40 & 0.026 & 0.024 & -- & -- & 0.148 & 12.0 & 0.211 & 11.7 & 0.321 \\
\hline 6/18/2008 12:00 PM & 0.140 & 20.1 & 0.098 & 0.298 & 17.6 & 0.40 & 0.026 & 0.024 & -- & -- & 0.146 & 12.0 & 0.211 & 11.7 & 0.320 \\
\hline 6/18/2008 1:00 PM & 0.140 & 20.0 & 0.098 & 0.298 & 17.6 & 0.40 & 0.026 & 0.024 & -- & -- & 0.146 & 12.0 & 0.211 & 11.6 & 0.319 \\
\hline 6/18/2008 2:00 PM & 0.140 & 20.0 & 0.098 & 0.298 & 17.6 & 0.40 & 0.026 & 0.024 & -- & -- & 0.146 & 12.0 & 0.211 & 11.7 & 0.318 \\
\hline
\end{tabular}


Appendix 1. Volumetric water content, temperature, and electrical-conductivity data collected at the flood-irrigated site during 2008.-Continued

[Depth in feet below land surface; volumetric water content dimensionless; temperature in degrees Celsius; bulk electrical conductivity in decisiemens per meter; --, no data]

\begin{tabular}{|c|c|c|c|c|c|c|c|c|c|c|c|c|c|c|c|}
\hline \multirow[b]{3}{*}{$\begin{array}{l}\text { Measurement } \\
\text { date and time }\end{array}$} & \multicolumn{15}{|c|}{ Depth } \\
\hline & \multicolumn{2}{|c|}{2} & \multirow[b]{2}{*}{$\begin{array}{c}3 \\
\begin{array}{c}\text { Volumetric } \\
\text { water } \\
\text { content }\end{array}\end{array}$} & \multicolumn{3}{|c|}{4} & \multirow{2}{*}{$\begin{array}{c}6 \\
\text { Volumetric } \\
\text { water } \\
\text { content }\end{array}$} & \multicolumn{2}{|l|}{8} & \multirow{2}{*}{$\begin{array}{c}10 \\
\begin{array}{l}\text { Volumetric } \\
\text { water } \\
\text { content }\end{array}\end{array}$} & \multicolumn{2}{|c|}{12} & \multicolumn{2}{|l|}{15} & \multirow{2}{*}{$\begin{array}{c}18.5 \\
\text { Volumetric } \\
\text { water } \\
\text { content }\end{array}$} \\
\hline & $\begin{array}{c}\text { Volumetric } \\
\text { water } \\
\text { content }\end{array}$ & $\begin{array}{l}\text { Temper- } \\
\text { ature }\end{array}$ & & $\begin{array}{c}\text { Volumetric } \\
\text { water } \\
\text { content }\end{array}$ & $\begin{array}{l}\text { Temper- } \\
\text { ature }\end{array}$ & $\begin{array}{c}\text { Bulk } \\
\text { electrical } \\
\text { conduct- } \\
\text { ivity }\end{array}$ & & $\begin{array}{c}\text { Volumetric } \\
\text { water } \\
\text { content }\end{array}$ & $\begin{array}{l}\text { Temper- } \\
\text { ature }\end{array}$ & & $\begin{array}{c}\text { Volumetric } \\
\text { water } \\
\text { content }\end{array}$ & $\begin{array}{l}\text { Temper- } \\
\text { ature }\end{array}$ & $\begin{array}{c}\text { Volumetric } \\
\text { water } \\
\text { content }\end{array}$ & $\begin{array}{l}\text { Temper- } \\
\text { ature }\end{array}$ & \\
\hline 6/18/2008 3:00 PM & 0.138 & 20.0 & 0.098 & 0.298 & 17.6 & 0.40 & 0.026 & 0.024 & -- & -- & 0.147 & 12.0 & 0.211 & 11.7 & 0.318 \\
\hline 6/18/2008 4:00 PM & 0.138 & 20.0 & 0.098 & 0.298 & 17.6 & 0.40 & 0.026 & 0.024 & -- & -- & 0.146 & 12.0 & 0.211 & 11.7 & 0.318 \\
\hline 6/18/2008 5:00 PM & 0.138 & 19.9 & 0.098 & 0.299 & 17.6 & 0.40 & 0.026 & 0.024 & -- & -- & 0.147 & 12.0 & 0.211 & 11.7 & 0.318 \\
\hline 6/18/2008 6:00 PM & 0.138 & 19.9 & 0.098 & 0.299 & 17.6 & 0.40 & 0.026 & 0.024 & -- & -- & 0.147 & 12.0 & 0.211 & 11.7 & 0.319 \\
\hline 6/18/2008 7:00 PM & 0.137 & 19.9 & 0.098 & 0.299 & 17.7 & 0.40 & 0.026 & 0.024 & -- & -- & 0.147 & 12.0 & 0.211 & 11.7 & 0.319 \\
\hline 6/18/2008 8:00 PM & 0.137 & 19.9 & 0.098 & 0.299 & 17.7 & 0.40 & 0.026 & 0.024 & -- & -- & 0.148 & 12.0 & 0.211 & 11.7 & 0.319 \\
\hline 6/18/2008 9:00 PM & 0.136 & 20.0 & 0.098 & 0.298 & 17.7 & 0.40 & 0.026 & 0.024 & -- & -- & 0.148 & 12.1 & 0.211 & 11.7 & 0.319 \\
\hline 6/18/2008 10:00 PM & 0.136 & 19.9 & 0.098 & 0.299 & 17.7 & 0.40 & 0.026 & 0.024 & -- & -- & 0.146 & 12.0 & 0.211 & 11.7 & 0.318 \\
\hline 6/18/2008 11:00 PM & 0.136 & 20.0 & 0.097 & 0.299 & 17.7 & 0.40 & 0.026 & 0.024 & -- & -- & 0.147 & 12.0 & 0.212 & 11.7 & 0.318 \\
\hline 6/19/2008 12:00 AM & 0.136 & 20.0 & 0.097 & 0.299 & 17.7 & 0.40 & 0.026 & 0.024 & -- & -- & 0.146 & 12.0 & 0.211 & 11.7 & 0.318 \\
\hline 6/19/2008 1:00 AM & 0.135 & 20.0 & 0.097 & 0.299 & 17.7 & 0.40 & 0.026 & 0.024 & -- & -- & 0.146 & 12.0 & 0.212 & 11.7 & 0.318 \\
\hline 6/19/2008 2:00 AM & 0.135 & 20.0 & 0.097 & 0.299 & 17.7 & 0.40 & 0.026 & 0.024 & -- & -- & 0.146 & 12.0 & 0.211 & 11.7 & 0.318 \\
\hline 6/19/2008 3:00 AM & 0.135 & 20.0 & 0.097 & 0.299 & 17.7 & 0.40 & 0.026 & 0.034 & -- & -- & 0.147 & 12.0 & 0.212 & 11.7 & 0.318 \\
\hline 6/19/2008 4:00 AM & 0.135 & 20.0 & 0.097 & 0.299 & 17.7 & 0.40 & 0.026 & 0.042 & -- & -- & 0.147 & 12.0 & 0.212 & 11.7 & 0.318 \\
\hline 6/19/2008 5:00 AM & 0.135 & 20.1 & 0.097 & 0.299 & 17.7 & 0.40 & 0.026 & 0.037 & -- & -- & 0.148 & 12.0 & 0.212 & 11.7 & 0.318 \\
\hline 6/19/2008 6:00 AM & 0.134 & 20.1 & 0.097 & 0.299 & 17.7 & 0.40 & 0.026 & 0.038 & -- & -- & 0.148 & 12.0 & 0.212 & 11.7 & 0.318 \\
\hline 6/19/2008 7:00 AM & 0.134 & 20.1 & 0.096 & 0.299 & 17.7 & 0.40 & 0.026 & 0.037 & -- & -- & 0.148 & 12.0 & 0.212 & 11.7 & 0.318 \\
\hline 6/19/2008 8:00 AM & 0.134 & 20.1 & 0.096 & 0.299 & 17.7 & 0.40 & 0.026 & 0.037 & -- & -- & 0.148 & 12.0 & 0.212 & 11.7 & 0.318 \\
\hline 6/19/2008 9:00 AM & 0.133 & 20.0 & 0.096 & 0.299 & 17.8 & 0.40 & 0.026 & 0.031 & -- & -- & 0.147 & 12.1 & 0.211 & 11.7 & 0.318 \\
\hline 6/19/2008 10:00 AM & 0.133 & 20.0 & 0.096 & 0.299 & 17.8 & 0.40 & 0.026 & 0.035 & -- & -- & 0.148 & 12.1 & 0.211 & 11.7 & 0.319 \\
\hline 6/19/2008 11:00 AM & 0.133 & 20.0 & 0.096 & 0.299 & 17.8 & 0.40 & 0.026 & 0.036 & -- & -- & 0.148 & 12.1 & 0.211 & 11.7 & 0.319 \\
\hline 6/19/2008 12:00 PM & 0.133 & 20.0 & 0.096 & 0.299 & 17.8 & 0.40 & 0.026 & 0.034 & -- & -- & 0.148 & 12.1 & 0.211 & 11.7 & 0.319 \\
\hline 6/19/2008 1:00 PM & 0.132 & 20.0 & 0.096 & 0.299 & 17.8 & 0.40 & 0.027 & 0.034 & -- & -- & 0.147 & 12.1 & 0.212 & 11.7 & 0.319 \\
\hline 6/19/2008 2:00 PM & 0.132 & 19.9 & 0.096 & 0.299 & 17.8 & 0.40 & 0.027 & 0.038 & -- & -- & 0.146 & 12.1 & 0.212 & 11.7 & 0.320 \\
\hline 6/19/2008 3:00 PM & 0.132 & 19.9 & 0.096 & 0.299 & 17.8 & 0.40 & 0.027 & 0.038 & -- & -- & 0.145 & 12.1 & 0.212 & 11.7 & 0.320 \\
\hline 6/19/2008 4:00 PM & 0.131 & 19.9 & 0.096 & 0.299 & 17.8 & 0.40 & 0.027 & 0.038 & -- & -- & 0.146 & 12.1 & 0.212 & 11.7 & 0.321 \\
\hline 6/19/2008 5:00 PM & 0.131 & 19.9 & 0.096 & 0.299 & 17.8 & 0.40 & 0.027 & 0.034 & -- & -- & 0.146 & 12.1 & 0.212 & 11.7 & 0.321 \\
\hline 6/19/2008 6:00 PM & 0.131 & 19.9 & 0.096 & 0.299 & 17.8 & 0.40 & 0.027 & 0.036 & -- & -- & 0.146 & 12.1 & 0.213 & 11.7 & 0.322 \\
\hline 6/19/2008 7:00 PM & 0.131 & 19.9 & 0.096 & 0.299 & 17.8 & 0.40 & 0.027 & 0.037 & -- & -- & 0.146 & 12.1 & 0.212 & 11.7 & 0.324 \\
\hline 6/19/2008 8:00 PM & 0.130 & 19.9 & 0.096 & 0.300 & 17.8 & 0.40 & 0.027 & 0.036 & -- & -- & 0.148 & 12.1 & 0.212 & 11.7 & 0.323 \\
\hline 6/19/2008 9:00 PM & 0.130 & 20.0 & 0.095 & 0.300 & 17.8 & 0.40 & 0.027 & 0.036 & -- & -- & 0.146 & 12.1 & 0.211 & 11.7 & 0.327 \\
\hline 6/19/2008 10:00 PM & 0.131 & 20.1 & 0.095 & 0.299 & 17.8 & 0.40 & 0.027 & 0.035 & -- & -- & 0.147 & 12.1 & 0.211 & 11.7 & 0.336 \\
\hline 6/19/2008 11:00 PM & 0.130 & 20.1 & 0.095 & 0.300 & 17.8 & 0.40 & 0.027 & 0.036 & -- & -- & 0.148 & 12.1 & 0.212 & 11.7 & 0.331 \\
\hline 6/20/2008 12:00 AM & 0.130 & 20.1 & 0.095 & 0.299 & 17.8 & 0.40 & 0.027 & 0.036 & -- & -- & 0.148 & 12.1 & 0.212 & 11.7 & 0.325 \\
\hline 6/20/2008 1:00 AM & 0.130 & 20.2 & 0.095 & 0.299 & 17.8 & 0.40 & 0.027 & 0.035 & -- & -- & 0.148 & 12.1 & 0.212 & 11.7 & 0.327 \\
\hline 6/20/2008 2:00 AM & 0.130 & 20.3 & 0.095 & 0.299 & 17.8 & 0.40 & 0.027 & 0.035 & -- & -- & 0.148 & 12.1 & 0.212 & 11.7 & 0.327 \\
\hline 6/20/2008 3:00 AM & 0.130 & 20.3 & 0.095 & 0.299 & 17.8 & 0.40 & 0.027 & 0.035 & -- & -- & 0.148 & 12.1 & 0.212 & 11.7 & 0.326 \\
\hline 6/20/2008 4:00 AM & 0.130 & 20.4 & 0.095 & 0.299 & 17.9 & 0.40 & 0.027 & 0.034 & -- & -- & 0.147 & 12.1 & 0.212 & 11.7 & 0.325 \\
\hline 6/20/2008 5:00 AM & 0.130 & 20.4 & 0.095 & 0.299 & 17.9 & 0.40 & 0.027 & 0.032 & -- & -- & 0.147 & 12.1 & 0.212 & 11.7 & 0.326 \\
\hline 6/20/2008 6:00 AM & 0.130 & 20.5 & 0.094 & 0.299 & 17.9 & 0.40 & 0.027 & 0.032 & -- & -- & 0.147 & 12.1 & 0.212 & 11.7 & 0.330 \\
\hline
\end{tabular}


Appendix 1. Volumetric water content, temperature, and electrical-conductivity data collected at the flood-irrigated site during 2008.-Continued

[Depth in feet below land surface; volumetric water content dimensionless; temperature in degrees Celsius; bulk electrical conductivity in decisiemens per meter; --, no data]

\begin{tabular}{|c|c|c|c|c|c|c|c|c|c|c|c|c|c|c|c|}
\hline \multirow[b]{3}{*}{$\begin{array}{l}\text { Measurement } \\
\text { date and time }\end{array}$} & \multicolumn{15}{|c|}{ Depth } \\
\hline & \multicolumn{2}{|c|}{2} & \multirow{2}{*}{$\begin{array}{c}\mathbf{3} \\
\begin{array}{c}\text { Volumetric } \\
\text { water } \\
\text { content }\end{array}\end{array}$} & \multicolumn{3}{|c|}{4} & \multirow{2}{*}{$\begin{array}{c}6 \\
\text { Volumetric } \\
\text { water } \\
\text { content }\end{array}$} & \multicolumn{2}{|l|}{8} & \multirow{2}{*}{$\begin{array}{c}10 \\
\text { Volumetric } \\
\text { water } \\
\text { content }\end{array}$} & \multicolumn{2}{|c|}{12} & \multicolumn{2}{|l|}{15} & \multirow{2}{*}{$\begin{array}{c}18.5 \\
\text { Volumetric } \\
\text { water } \\
\text { content }\end{array}$} \\
\hline & $\begin{array}{c}\text { Volumetric } \\
\text { water } \\
\text { content }\end{array}$ & $\begin{array}{l}\text { Temper- } \\
\text { ature }\end{array}$ & & $\begin{array}{c}\text { Volumetric } \\
\text { water } \\
\text { content }\end{array}$ & $\begin{array}{l}\text { Temper- } \\
\text { ature }\end{array}$ & $\begin{array}{c}\text { Bulk } \\
\text { electrical } \\
\text { conduct- } \\
\text { ivity }\end{array}$ & & $\begin{array}{c}\text { Volumetric } \\
\text { water } \\
\text { content }\end{array}$ & $\begin{array}{l}\text { Temper- } \\
\text { ature }\end{array}$ & & $\begin{array}{c}\text { Volumetric } \\
\text { water } \\
\text { content }\end{array}$ & $\begin{array}{l}\text { Temper- } \\
\text { ature }\end{array}$ & $\begin{array}{c}\text { Volumetric } \\
\text { water } \\
\text { content }\end{array}$ & $\begin{array}{l}\text { Temper- } \\
\text { ature }\end{array}$ & \\
\hline 6/20/2008 7:00 AM & 0.130 & 20.5 & 0.094 & 0.299 & 17.9 & 0.40 & 0.027 & 0.032 & -- & -- & 0.147 & 12.1 & 0.212 & 11.7 & 0.333 \\
\hline 6/20/2008 8:00 AM & 0.130 & 20.6 & 0.094 & 0.300 & 17.9 & 0.40 & 0.027 & 0.032 & -- & -- & 0.148 & 12.1 & 0.212 & 11.7 & 0.336 \\
\hline 6/20/2008 9:00 AM & 0.130 & 20.6 & 0.095 & 0.300 & 17.9 & 0.40 & 0.027 & 0.030 & -- & -- & 0.147 & 12.1 & 0.212 & 11.8 & 0.339 \\
\hline 6/20/2008 10:00 AM & 0.129 & 20.6 & 0.095 & 0.299 & 17.9 & 0.40 & 0.027 & 0.031 & -- & -- & 0.148 & 12.2 & 0.212 & 11.7 & 0.345 \\
\hline 6/20/2008 11:00 AM & 0.129 & 20.6 & 0.094 & 0.299 & 17.9 & 0.40 & 0.027 & 0.031 & -- & -- & 0.148 & 12.2 & 0.212 & 11.7 & 0.363 \\
\hline 6/20/2008 12:00 PM & 0.129 & 20.6 & 0.094 & 0.299 & 17.9 & 0.40 & 0.028 & 0.030 & -- & -- & 0.148 & 12.2 & 0.213 & 11.7 & 0.344 \\
\hline 6/20/2008 1:00 PM & 0.129 & 20.5 & 0.094 & 0.299 & 17.9 & 0.40 & 0.028 & 0.032 & -- & -- & 0.148 & 12.2 & 0.213 & 11.7 & 0.325 \\
\hline 6/20/2008 2:00 PM & 0.129 & 20.5 & 0.094 & 0.299 & 17.9 & 0.40 & 0.028 & 0.032 & -- & -- & 0.147 & 12.2 & 0.213 & 11.7 & 0.324 \\
\hline 6/20/2008 3:00 PM & 0.129 & 20.5 & 0.094 & 0.299 & 17.9 & 0.40 & 0.028 & 0.034 & -- & -- & 0.146 & 12.1 & 0.213 & 11.7 & 0.321 \\
\hline 6/20/2008 4:00 PM & 0.129 & 20.5 & 0.094 & 0.299 & 17.9 & 0.40 & 0.028 & 0.034 & -- & -- & 0.146 & 12.2 & 0.213 & 11.7 & 0.324 \\
\hline 6/20/2008 5:00 PM & 0.129 & 20.5 & 0.094 & 0.300 & 17.9 & 0.40 & 0.028 & 0.034 & -- & -- & 0.145 & 12.2 & 0.213 & 11.7 & 0.324 \\
\hline 6/20/2008 6:00 PM & 0.129 & 20.5 & 0.094 & 0.300 & 17.9 & 0.40 & 0.028 & 0.032 & -- & -- & 0.146 & 12.2 & 0.213 & 11.7 & 0.323 \\
\hline 6/20/2008 7:00 PM & 0.128 & 20.5 & 0.094 & 0.299 & 17.9 & 0.40 & 0.028 & 0.034 & -- & -- & 0.148 & 12.2 & 0.213 & 11.8 & 0.322 \\
\hline 6/20/2008 8:00 PM & 0.128 & 20.6 & 0.094 & 0.299 & 18.0 & 0.40 & 0.027 & 0.034 & -- & -- & 0.147 & 12.2 & 0.212 & 11.8 & 0.320 \\
\hline 6/20/2008 9:00 PM & 0.128 & 20.6 & 0.094 & 0.299 & 18.0 & 0.40 & 0.027 & 0.033 & -- & -- & 0.148 & 12.1 & 0.212 & 11.8 & 0.320 \\
\hline 6/20/2008 10:00 PM & 0.128 & 20.6 & 0.094 & 0.299 & 18.0 & 0.40 & 0.027 & 0.032 & -- & -- & 0.148 & 12.1 & 0.212 & 11.8 & 0.324 \\
\hline 6/20/2008 11:00 PM & 0.128 & 20.7 & 0.094 & 0.299 & 18.0 & 0.40 & 0.027 & 0.032 & -- & -- & 0.148 & 12.1 & 0.212 & 11.8 & 0.328 \\
\hline 6/21/2008 12:00 AM & 0.126 & 20.7 & 0.094 & 0.299 & 18.0 & 0.40 & 0.028 & 0.031 & -- & -- & 0.148 & 12.1 & 0.212 & 11.8 & 0.328 \\
\hline 6/21/2008 1:00 AM & 0.126 & 20.8 & 0.093 & 0.300 & 18.0 & 0.40 & 0.028 & 0.032 & -- & -- & 0.147 & 12.2 & 0.212 & 11.8 & 0.320 \\
\hline 6/21/2008 2:00 AM & 0.126 & 20.8 & 0.093 & 0.299 & 18.0 & 0.40 & 0.028 & 0.027 & -- & -- & 0.147 & 12.1 & 0.212 & 11.8 & 0.328 \\
\hline 6/21/2008 3:00 AM & 0.126 & 20.9 & 0.093 & 0.299 & 18.0 & 0.40 & 0.028 & 0.030 & -- & -- & 0.147 & 12.2 & 0.212 & 11.8 & 0.336 \\
\hline 6/21/2008 4:00 AM & 0.126 & 20.8 & 0.093 & 0.299 & 18.0 & 0.40 & 0.028 & 0.033 & -- & -- & 0.147 & 12.1 & 0.213 & 11.8 & 0.325 \\
\hline 6/21/2008 5:00 AM & 0.126 & 20.9 & 0.093 & 0.299 & 18.0 & 0.40 & 0.028 & 0.031 & -- & -- & 0.147 & 12.1 & 0.212 & 11.8 & 0.325 \\
\hline 6/21/2008 6:00 AM & 0.126 & 20.9 & 0.093 & 0.300 & 18.0 & 0.40 & 0.028 & 0.030 & -- & -- & 0.147 & 12.2 & 0.212 & 11.8 & 0.329 \\
\hline 6/21/2008 7:00 AM & 0.126 & 21.0 & 0.093 & 0.299 & 18.0 & 0.40 & 0.028 & 0.034 & -- & -- & 0.147 & 12.2 & 0.212 & 11.8 & 0.322 \\
\hline 6/21/2008 8:00 AM & 0.126 & 21.0 & 0.093 & 0.300 & 18.0 & 0.40 & 0.028 & 0.033 & -- & -- & 0.148 & 12.2 & 0.212 & 11.8 & 0.321 \\
\hline 6/21/2008 9:00 AM & 0.126 & 21.1 & 0.093 & 0.300 & 18.1 & 0.40 & 0.028 & 0.028 & -- & -- & 0.147 & 12.2 & 0.212 & 11.8 & 0.324 \\
\hline 6/21/2008 10:00 AM & 0.126 & 21.1 & 0.093 & 0.299 & 18.1 & 0.40 & 0.028 & 0.028 & -- & -- & 0.148 & 12.2 & 0.213 & 11.8 & 0.325 \\
\hline 6/21/2008 11:00 AM & 0.126 & 21.1 & 0.093 & 0.299 & 18.1 & 0.40 & 0.028 & 0.029 & -- & -- & 0.149 & 12.2 & 0.213 & 11.8 & 0.329 \\
\hline 6/21/2008 12:00 PM & 0.126 & 20.9 & 0.093 & 0.300 & 18.1 & 0.40 & 0.028 & 0.032 & -- & -- & 0.148 & 12.2 & 0.213 & 11.8 & 0.328 \\
\hline 6/21/2008 1:00 PM & 0.126 & 20.9 & 0.093 & 0.300 & 18.1 & 0.40 & 0.028 & 0.025 & -- & -- & 0.148 & 12.2 & 0.213 & 11.8 & 0.334 \\
\hline 6/21/2008 2:00 PM & 0.126 & 20.9 & 0.093 & 0.300 & 18.1 & 0.40 & 0.028 & 0.032 & -- & -- & 0.148 & 12.2 & 0.213 & 11.8 & 0.343 \\
\hline 6/21/2008 3:00 PM & 0.126 & 21.0 & 0.093 & 0.300 & 18.1 & 0.40 & 0.028 & 0.031 & -- & -- & 0.147 & 12.2 & 0.213 & 11.8 & 0.323 \\
\hline 6/21/2008 4:00 PM & 0.126 & 21.0 & 0.093 & 0.299 & 18.1 & 0.40 & 0.028 & 0.029 & -- & -- & 0.146 & 12.3 & 0.213 & 11.8 & 0.338 \\
\hline 6/21/2008 5:00 PM & 0.125 & 20.9 & 0.093 & 0.300 & 18.1 & 0.40 & 0.028 & 0.033 & -- & -- & 0.146 & 12.2 & 0.213 & 11.8 & 0.322 \\
\hline 6/21/2008 6:00 PM & 0.125 & 20.9 & 0.093 & 0.300 & 18.1 & 0.40 & 0.028 & 0.033 & -- & -- & 0.146 & 12.3 & 0.213 & 11.8 & 0.322 \\
\hline 6/21/2008 7:00 PM & 0.125 & 20.9 & 0.093 & 0.300 & 18.1 & 0.40 & 0.028 & 0.033 & -- & -- & 0.146 & 12.2 & 0.213 & 11.8 & 0.338 \\
\hline 6/21/2008 8:00 PM & 0.125 & 20.8 & 0.093 & 0.299 & 18.1 & 0.40 & 0.028 & 0.029 & -- & -- & 0.148 & 12.3 & 0.213 & 11.8 & 0.327 \\
\hline 6/21/2008 9:00 PM & 0.125 & 20.9 & 0.093 & 0.300 & 18.2 & 0.40 & 0.028 & 0.030 & -- & -- & 0.147 & 12.2 & 0.213 & 11.8 & 0.362 \\
\hline 6/21/2008 10:00 PM & 0.125 & 20.9 & 0.092 & 0.300 & 18.2 & 0.40 & 0.028 & 0.030 & -- & -- & 0.148 & 12.2 & 0.213 & 11.8 & 0.351 \\
\hline
\end{tabular}


Appendix 1. Volumetric water content, temperature, and electrical-conductivity data collected at the flood-irrigated site during 2008.-Continued

[Depth in feet below land surface; volumetric water content dimensionless; temperature in degrees Celsius; bulk electrical conductivity in decisiemens per meter; --, no data]

\begin{tabular}{|c|c|c|c|c|c|c|c|c|c|c|c|c|c|c|c|}
\hline \multirow[b]{3}{*}{$\begin{array}{l}\text { Measurement } \\
\text { date and time }\end{array}$} & \multicolumn{15}{|c|}{ Depth } \\
\hline & \multicolumn{2}{|c|}{2} & \multirow[b]{2}{*}{$\begin{array}{c}3 \\
\begin{array}{c}\text { Volumetric } \\
\text { water } \\
\text { content }\end{array}\end{array}$} & \multicolumn{3}{|c|}{4} & \multirow{2}{*}{$\begin{array}{c}6 \\
\text { Volumetric } \\
\text { water } \\
\text { content }\end{array}$} & \multicolumn{2}{|l|}{8} & \multirow{2}{*}{$\begin{array}{c}10 \\
\begin{array}{l}\text { Volumetric } \\
\text { water } \\
\text { content }\end{array}\end{array}$} & \multicolumn{2}{|c|}{12} & \multicolumn{2}{|l|}{15} & \multirow{2}{*}{$\begin{array}{c}18.5 \\
\text { Volumetric } \\
\text { water } \\
\text { content }\end{array}$} \\
\hline & $\begin{array}{c}\text { Volumetric } \\
\text { water } \\
\text { content }\end{array}$ & $\begin{array}{l}\text { Temper- } \\
\text { ature }\end{array}$ & & $\begin{array}{c}\text { Volumetric } \\
\text { water } \\
\text { content }\end{array}$ & $\begin{array}{l}\text { Temper- } \\
\text { ature }\end{array}$ & $\begin{array}{c}\text { Bulk } \\
\text { electrical } \\
\text { conduct- } \\
\text { ivity }\end{array}$ & & $\begin{array}{c}\text { Volumetric } \\
\text { water } \\
\text { content }\end{array}$ & $\begin{array}{l}\text { Temper- } \\
\text { ature }\end{array}$ & & $\begin{array}{c}\text { Volumetric } \\
\text { water } \\
\text { content }\end{array}$ & $\begin{array}{l}\text { Temper- } \\
\text { ature }\end{array}$ & $\begin{array}{c}\text { Volumetric } \\
\text { water } \\
\text { content }\end{array}$ & $\begin{array}{l}\text { Temper- } \\
\text { ature }\end{array}$ & \\
\hline 6/21/2008 11:00 PM & 0.124 & 21.0 & 0.092 & 0.300 & 18.2 & 0.40 & 0.028 & 0.026 & -- & -- & 0.147 & 12.2 & 0.212 & 11.8 & 0.321 \\
\hline 6/22/2008 12:00 AM & 0.124 & 21.1 & 0.092 & 0.300 & 18.2 & 0.40 & 0.028 & 0.029 & -- & -- & 0.147 & 12.2 & 0.212 & 11.8 & 0.319 \\
\hline 6/22/2008 1:00 AM & 0.124 & 21.2 & 0.092 & 0.300 & 18.2 & 0.40 & 0.028 & 0.030 & -- & -- & 0.147 & 12.2 & 0.212 & 11.8 & 0.318 \\
\hline 6/22/2008 2:00 AM & 0.124 & 21.2 & 0.092 & 0.300 & 18.2 & 0.40 & 0.028 & 0.033 & -- & -- & 0.147 & 12.2 & 0.212 & 11.8 & 0.318 \\
\hline 6/22/2008 3:00 AM & 0.125 & 21.3 & 0.092 & 0.300 & 18.2 & 0.40 & 0.028 & 0.033 & -- & -- & 0.147 & 12.2 & 0.213 & 11.8 & 0.318 \\
\hline 6/22/2008 4:00 AM & 0.125 & 21.3 & 0.092 & 0.300 & 18.2 & 0.40 & 0.028 & 0.034 & -- & -- & 0.147 & 12.2 & 0.213 & 11.8 & 0.321 \\
\hline 6/22/2008 5:00 AM & 0.125 & 21.3 & 0.092 & 0.300 & 18.2 & 0.40 & 0.028 & 0.034 & -- & -- & 0.148 & 12.2 & 0.213 & 11.8 & 0.323 \\
\hline 6/22/2008 6:00 AM & 0.125 & 21.3 & 0.092 & 0.300 & 18.2 & 0.40 & 0.028 & 0.034 & -- & -- & 0.148 & 12.2 & 0.213 & 11.8 & 0.324 \\
\hline $6 / 22 / 2008$ 7:00 AM & 0.125 & 21.4 & 0.092 & 0.300 & 18.2 & 0.40 & 0.028 & 0.035 & -- & -- & 0.147 & 12.2 & 0.212 & 11.8 & 0.336 \\
\hline 6/22/2008 8:00 AM & 0.124 & 21.5 & 0.092 & 0.300 & 18.2 & 0.40 & 0.028 & 0.035 & -- & -- & 0.148 & 12.2 & 0.212 & 11.8 & 0.327 \\
\hline 6/22/2008 9:00 AM & 0.124 & 21.5 & 0.092 & 0.300 & 18.3 & 0.40 & 0.028 & 0.035 & -- & -- & 0.148 & 12.3 & 0.213 & 11.8 & 0.339 \\
\hline 6/22/2008 10:00 AM & 0.124 & 21.4 & 0.092 & 0.300 & 18.3 & 0.40 & 0.029 & 0.033 & -- & -- & 0.148 & 12.3 & 0.215 & 11.8 & 0.328 \\
\hline 6/22/2008 11:00 AM & 0.124 & 21.4 & 0.092 & 0.300 & 18.3 & 0.40 & 0.029 & 0.033 & -- & -- & 0.149 & 12.3 & 0.213 & 11.8 & 0.331 \\
\hline 6/22/2008 12:00 PM & 0.124 & 21.4 & 0.092 & 0.300 & 18.3 & 0.40 & 0.029 & 0.033 & -- & -- & 0.148 & 12.3 & 0.213 & 11.8 & 0.320 \\
\hline 6/22/2008 1:00 PM & 0.124 & 21.4 & 0.092 & 0.300 & 18.3 & 0.40 & 0.029 & 0.031 & -- & -- & 0.149 & 12.3 & 0.213 & 11.8 & 0.324 \\
\hline 6/22/2008 2:00 PM & 0.124 & 21.3 & 0.092 & 0.300 & 18.3 & 0.40 & 0.029 & 0.033 & -- & -- & 0.149 & 12.3 & 0.215 & 11.8 & 0.360 \\
\hline 6/22/2008 3:00 PM & 0.124 & 21.3 & 0.092 & 0.300 & 18.3 & 0.40 & 0.029 & 0.027 & -- & -- & 0.149 & 12.3 & 0.213 & 11.8 & 0.384 \\
\hline 6/22/2008 4:00 PM & 0.124 & 21.4 & 0.092 & 0.300 & 18.3 & 0.40 & 0.029 & 0.025 & -- & -- & 0.147 & 12.3 & 0.213 & 11.8 & 0.362 \\
\hline 6/22/2008 5:00 PM & 0.124 & 21.3 & 0.092 & 0.300 & 18.3 & 0.40 & 0.029 & 0.029 & -- & -- & 0.148 & 12.3 & 0.213 & 11.8 & 0.356 \\
\hline 6/22/2008 6:00 PM & 0.124 & 21.2 & 0.092 & 0.300 & 18.3 & 0.40 & 0.029 & 0.035 & -- & -- & 0.149 & 12.3 & 0.215 & 11.8 & 0.337 \\
\hline 6/22/2008 7:00 PM & 0.123 & 21.2 & 0.092 & 0.300 & 18.4 & 0.40 & 0.029 & 0.035 & -- & -- & 0.148 & 12.3 & 0.215 & 11.9 & 0.357 \\
\hline 6/22/2008 8:00 PM & 0.123 & 21.3 & 0.092 & 0.300 & 18.4 & 0.41 & 0.029 & 0.035 & -- & -- & 0.147 & 12.3 & 0.215 & 11.9 & 0.331 \\
\hline 6/22/2008 9:00 PM & 0.123 & 21.3 & 0.092 & 0.300 & 18.4 & 0.41 & 0.029 & 0.035 & -- & -- & 0.148 & 12.3 & 0.215 & 11.8 & 0.366 \\
\hline 6/22/2008 10:00 PM & 0.123 & 21.4 & 0.092 & 0.300 & 18.4 & 0.41 & 0.029 & 0.035 & -- & -- & 0.149 & 12.3 & 0.215 & 11.9 & 0.321 \\
\hline 6/22/2008 11:00 PM & 0.123 & 21.4 & 0.092 & 0.300 & 18.4 & 0.40 & 0.029 & 0.034 & -- & -- & 0.148 & 12.3 & 0.213 & 11.8 & 0.322 \\
\hline 6/23/2008 12:00 AM & 0.123 & 21.4 & 0.092 & 0.300 & 18.4 & 0.40 & 0.029 & 0.030 & -- & -- & 0.148 & 12.3 & 0.213 & 11.9 & 0.322 \\
\hline 6/23/2008 1:00 AM & 0.124 & 21.4 & 0.092 & 0.300 & 18.4 & 0.40 & 0.029 & 0.030 & -- & -- & 0.147 & 12.3 & 0.213 & 11.9 & 0.324 \\
\hline 6/23/2008 2:00 AM & 0.124 & 21.4 & 0.092 & 0.300 & 18.4 & 0.40 & 0.029 & 0.032 & -- & -- & 0.147 & 12.3 & 0.213 & 11.9 & 0.331 \\
\hline 6/23/2008 3:00 AM & 0.123 & 21.5 & 0.092 & 0.300 & 18.4 & 0.41 & 0.029 & 0.032 & -- & -- & 0.147 & 12.3 & 0.213 & 11.9 & 0.330 \\
\hline 6/23/2008 4:00 AM & 0.124 & 21.5 & 0.092 & 0.300 & 18.4 & 0.41 & 0.029 & 0.030 & -- & -- & 0.148 & 12.3 & 0.213 & 11.9 & 0.329 \\
\hline 6/23/2008 5:00 AM & 0.124 & 21.5 & 0.092 & 0.300 & 18.4 & 0.41 & 0.029 & 0.030 & -- & -- & 0.148 & 12.3 & 0.213 & 11.9 & 0.329 \\
\hline 6/23/2008 6:00 AM & 0.124 & 21.5 & 0.092 & 0.300 & 18.5 & 0.41 & 0.029 & 0.034 & -- & -- & 0.148 & 12.3 & 0.213 & 11.9 & 0.334 \\
\hline 6/23/2008 7:00 AM & 0.123 & 21.6 & 0.092 & 0.300 & 18.5 & 0.41 & 0.029 & 0.034 & -- & -- & 0.148 & 12.3 & 0.213 & 11.9 & 0.339 \\
\hline 6/23/2008 8:00 AM & 0.123 & 21.6 & 0.092 & 0.300 & 18.5 & 0.41 & 0.029 & 0.032 & -- & -- & 0.148 & 12.3 & 0.213 & 11.9 & 0.335 \\
\hline 6/23/2008 9:00 AM & 0.123 & 21.6 & 0.092 & 0.300 & 18.5 & 0.41 & 0.029 & 0.031 & -- & -- & 0.148 & 12.4 & 0.215 & 11.9 & 0.330 \\
\hline 6/23/2008 10:00 AM & 0.122 & 21.6 & 0.092 & 0.300 & 18.5 & 0.41 & 0.029 & 0.031 & -- & -- & 0.147 & 12.4 & 0.215 & 11.9 & 0.325 \\
\hline 6/23/2008 11:00 AM & 0.122 & 21.6 & 0.092 & 0.301 & 18.5 & 0.41 & 0.029 & 0.025 & -- & -- & 0.148 & 12.3 & 0.215 & 11.9 & 0.325 \\
\hline 6/23/2008 12:00 PM & 0.122 & 21.5 & 0.092 & 0.300 & 18.5 & 0.41 & 0.029 & 0.025 & -- & -- & 0.149 & 12.3 & 0.213 & 11.9 & 0.325 \\
\hline 6/23/2008 1:00 PM & 0.122 & 21.5 & 0.092 & 0.301 & 18.5 & 0.41 & 0.029 & 0.025 & -- & -- & 0.149 & 12.4 & 0.213 & 11.9 & 0.324 \\
\hline 6/23/2008 2:00 PM & 0.122 & 21.5 & 0.092 & 0.301 & 18.5 & 0.41 & 0.029 & 0.025 & -- & -- & 0.149 & 12.4 & 0.213 & 11.9 & 0.324 \\
\hline
\end{tabular}


Appendix 1. Volumetric water content, temperature, and electrical-conductivity data collected at the flood-irrigated site during 2008.-Continued

[Depth in feet below land surface; volumetric water content dimensionless; temperature in degrees Celsius; bulk electrical conductivity in decisiemens per meter; --, no data]

\begin{tabular}{|c|c|c|c|c|c|c|c|c|c|c|c|c|c|c|c|}
\hline \multirow[b]{3}{*}{$\begin{array}{l}\text { Measurement } \\
\text { date and time }\end{array}$} & \multicolumn{15}{|c|}{ Depth } \\
\hline & \multicolumn{2}{|c|}{2} & \multirow{2}{*}{$\begin{array}{c}\mathbf{3} \\
\begin{array}{c}\text { Volumetric } \\
\text { water } \\
\text { content }\end{array}\end{array}$} & \multicolumn{3}{|c|}{4} & \multirow{2}{*}{$\begin{array}{c}6 \\
\text { Volumetric } \\
\text { water } \\
\text { content }\end{array}$} & \multicolumn{2}{|l|}{8} & \multirow{2}{*}{$\begin{array}{c}10 \\
\text { Volumetric } \\
\text { water } \\
\text { content }\end{array}$} & \multicolumn{2}{|c|}{12} & \multicolumn{2}{|l|}{15} & \multirow{2}{*}{$\begin{array}{c}18.5 \\
\text { Volumetric } \\
\text { water } \\
\text { content }\end{array}$} \\
\hline & $\begin{array}{c}\text { Volumetric } \\
\text { water } \\
\text { content }\end{array}$ & $\begin{array}{l}\text { Temper- } \\
\text { ature }\end{array}$ & & $\begin{array}{c}\text { Volumetric } \\
\text { water } \\
\text { content }\end{array}$ & $\begin{array}{l}\text { Temper- } \\
\text { ature }\end{array}$ & $\begin{array}{c}\text { Bulk } \\
\text { electrical } \\
\text { conduct- } \\
\text { ivity }\end{array}$ & & $\begin{array}{c}\text { Volumetric } \\
\text { water } \\
\text { content }\end{array}$ & $\begin{array}{l}\text { Temper- } \\
\text { ature }\end{array}$ & & $\begin{array}{c}\text { Volumetric } \\
\text { water } \\
\text { content }\end{array}$ & $\begin{array}{l}\text { Temper- } \\
\text { ature }\end{array}$ & $\begin{array}{c}\text { Volumetric } \\
\text { water } \\
\text { content }\end{array}$ & $\begin{array}{l}\text { Temper- } \\
\text { ature }\end{array}$ & \\
\hline 6/23/2008 3:00 PM & 0.121 & 21.5 & 0.092 & 0.301 & 18.5 & 0.41 & 0.029 & 0.025 & -- & -- & 0.148 & 12.4 & 0.213 & 11.9 & 0.324 \\
\hline 6/23/2008 4:00 PM & 0.121 & 21.4 & 0.092 & 0.301 & 18.5 & 0.41 & 0.030 & 0.025 & -- & -- & 0.147 & 12.4 & 0.215 & 11.9 & 0.324 \\
\hline 6/23/2008 5:00 PM & 0.121 & 21.4 & 0.092 & 0.301 & 18.6 & 0.41 & 0.030 & 0.026 & -- & -- & 0.148 & 12.4 & 0.215 & 11.9 & 0.324 \\
\hline 6/23/2008 6:00 PM & 0.121 & 21.4 & 0.092 & 0.300 & 18.6 & 0.41 & 0.030 & 0.026 & -- & -- & 0.147 & 12.4 & 0.215 & 11.9 & 0.325 \\
\hline 6/23/2008 7:00 PM & 0.121 & 21.4 & 0.092 & 0.301 & 18.6 & 0.41 & 0.030 & 0.026 & -- & -- & 0.147 & 12.4 & 0.215 & 11.9 & 0.324 \\
\hline 6/23/2008 8:00 PM & 0.121 & 21.4 & 0.092 & 0.301 & 18.6 & 0.41 & 0.030 & 0.026 & -- & -- & 0.147 & 12.4 & 0.215 & 11.9 & 0.324 \\
\hline 6/23/2008 9:00 PM & 0.121 & 21.4 & 0.092 & 0.301 & 18.6 & 0.41 & 0.029 & 0.026 & -- & -- & 0.149 & 12.4 & 0.215 & 11.9 & 0.325 \\
\hline 6/23/2008 10:00 PM & 0.122 & 21.4 & 0.092 & 0.301 & 18.6 & 0.41 & 0.029 & 0.026 & -- & -- & 0.147 & 12.3 & 0.215 & 11.9 & 0.324 \\
\hline 6/23/2008 11:00 PM & 0.122 & 21.5 & 0.092 & 0.301 & 18.6 & 0.41 & 0.029 & 0.026 & -- & -- & 0.147 & 12.3 & 0.215 & 11.9 & 0.325 \\
\hline 6/24/2008 12:00 AM & 0.122 & 21.5 & 0.092 & 0.301 & 18.6 & 0.41 & 0.029 & 0.026 & -- & -- & 0.147 & 12.4 & 0.215 & 11.9 & 0.329 \\
\hline 6/24/2008 1:00 AM & 0.122 & 21.5 & 0.091 & 0.301 & 18.6 & 0.41 & 0.029 & 0.027 & -- & -- & 0.148 & 12.4 & 0.213 & 11.9 & 0.366 \\
\hline 6/24/2008 2:00 AM & 0.121 & 21.6 & 0.092 & 0.300 & 18.6 & 0.41 & 0.029 & 0.026 & -- & -- & 0.148 & 12.4 & 0.215 & 11.9 & 0.360 \\
\hline 6/24/2008 3:00 AM & 0.122 & 21.5 & 0.091 & 0.300 & 18.6 & 0.41 & 0.029 & 0.026 & -- & -- & 0.149 & 12.4 & 0.213 & 11.9 & 0.337 \\
\hline 6/24/2008 4:00 AM & 0.122 & 21.5 & 0.091 & 0.301 & 18.6 & 0.41 & 0.029 & 0.026 & -- & -- & 0.148 & 12.4 & 0.213 & 11.9 & 0.330 \\
\hline 6/24/2008 5:00 AM & 0.122 & 21.6 & 0.091 & 0.301 & 18.7 & 0.41 & 0.029 & 0.028 & -- & -- & 0.149 & 12.4 & 0.213 & 11.9 & 0.381 \\
\hline 6/24/2008 6:00 AM & 0.122 & 21.6 & 0.091 & 0.301 & 18.7 & 0.41 & 0.029 & 0.030 & -- & -- & 0.148 & 12.4 & 0.215 & 11.9 & 0.354 \\
\hline 6/24/2008 7:00 AM & 0.122 & 21.6 & 0.091 & 0.300 & 18.7 & 0.41 & 0.029 & 0.027 & -- & -- & 0.148 & 12.4 & 0.213 & 11.9 & 0.335 \\
\hline 6/24/2008 8:00 AM & 0.122 & 21.6 & 0.091 & 0.301 & 18.7 & 0.41 & 0.029 & 0.030 & -- & -- & 0.148 & 12.4 & 0.215 & 11.9 & 0.367 \\
\hline 6/24/2008 9:00 AM & 0.122 & 21.7 & 0.091 & 0.301 & 18.7 & 0.41 & 0.030 & 0.030 & -- & -- & 0.149 & 12.4 & 0.215 & 11.9 & 0.380 \\
\hline 6/24/2008 10:00 AM & 0.121 & 21.6 & 0.091 & 0.301 & 18.7 & 0.41 & 0.030 & 0.029 & -- & -- & 0.148 & 12.4 & 0.215 & 11.9 & 0.409 \\
\hline 6/24/2008 11:00 AM & 0.121 & 21.6 & 0.091 & 0.301 & 18.7 & 0.41 & 0.030 & 0.030 & -- & -- & 0.147 & 12.4 & 0.215 & 11.9 & 0.341 \\
\hline 6/24/2008 12:00 PM & 0.120 & 21.6 & 0.091 & 0.301 & 18.7 & 0.41 & 0.030 & 0.029 & -- & -- & 0.149 & 12.4 & 0.215 & 11.9 & 0.335 \\
\hline 6/24/2008 1:00 PM & 0.120 & 21.6 & 0.091 & 0.301 & 18.7 & 0.41 & 0.030 & 0.032 & -- & -- & 0.149 & 12.4 & 0.215 & 11.9 & 0.345 \\
\hline 6/24/2008 2:00 PM & 0.120 & 21.6 & 0.091 & 0.301 & 18.7 & 0.41 & 0.030 & 0.032 & -- & -- & 0.149 & 12.4 & 0.215 & 11.9 & 0.358 \\
\hline 6/24/2008 3:00 PM & 0.121 & 21.6 & 0.091 & 0.301 & 18.7 & 0.41 & 0.030 & 0.031 & -- & -- & 0.149 & 12.4 & 0.215 & 11.9 & 0.338 \\
\hline 6/24/2008 4:00 PM & 0.121 & 21.6 & 0.091 & 0.301 & 18.7 & 0.41 & 0.030 & 0.030 & -- & -- & 0.147 & 12.4 & 0.216 & 11.9 & 0.392 \\
\hline 6/24/2008 5:00 PM & 0.120 & 21.6 & 0.091 & 0.301 & 18.7 & 0.41 & 0.030 & 0.030 & -- & -- & 0.148 & 12.4 & 0.215 & 11.9 & 0.322 \\
\hline 6/24/2008 6:00 PM & 0.120 & 21.6 & 0.091 & 0.301 & 18.7 & 0.41 & 0.030 & 0.030 & -- & -- & 0.149 & 12.4 & 0.215 & 11.9 & 0.321 \\
\hline 6/24/2008 7:00 PM & 0.120 & 21.5 & 0.091 & 0.301 & 18.8 & 0.41 & 0.030 & 0.030 & -- & -- & 0.149 & 12.5 & 0.215 & 11.9 & 0.319 \\
\hline 6/24/2008 8:00 PM & 0.120 & 21.5 & 0.091 & 0.301 & 18.8 & 0.41 & 0.030 & 0.030 & -- & -- & 0.149 & 12.4 & 0.216 & 12.0 & 0.322 \\
\hline 6/24/2008 9:00 PM & 0.120 & 21.5 & 0.091 & 0.301 & 18.8 & 0.41 & 0.030 & 0.033 & -- & -- & 0.149 & 12.4 & 0.215 & 12.0 & 0.317 \\
\hline 6/24/2008 10:00 PM & 0.120 & 21.5 & 0.091 & 0.301 & 18.8 & 0.41 & 0.030 & 0.032 & -- & -- & 0.148 & 12.4 & 0.216 & 11.9 & 0.318 \\
\hline 6/24/2008 11:00 PM & 0.120 & 21.6 & 0.091 & 0.301 & 18.8 & 0.41 & 0.030 & 0.031 & -- & -- & 0.148 & 12.4 & 0.216 & 12.0 & 0.318 \\
\hline 6/25/2008 12:00 AM & 0.120 & 21.7 & 0.091 & 0.301 & 18.8 & 0.41 & 0.030 & 0.030 & -- & -- & 0.148 & 12.5 & 0.216 & 12.0 & 0.318 \\
\hline 6/25/2008 1:00 AM & 0.121 & 21.7 & 0.090 & 0.301 & 18.8 & 0.41 & 0.030 & 0.030 & -- & -- & 0.148 & 12.4 & 0.216 & 12.0 & 0.318 \\
\hline 6/25/2008 2:00 AM & 0.121 & 21.8 & 0.091 & 0.301 & 18.8 & 0.41 & 0.030 & 0.030 & -- & -- & 0.148 & 12.4 & 0.216 & 12.0 & 0.318 \\
\hline 6/25/2008 3:00 AM & 0.121 & 21.8 & 0.091 & 0.301 & 18.8 & 0.41 & 0.030 & 0.028 & -- & -- & 0.149 & 12.4 & 0.215 & 11.9 & 0.318 \\
\hline 6/25/2008 4:00 AM & 0.121 & 21.9 & 0.090 & 0.301 & 18.8 & 0.41 & 0.030 & 0.028 & -- & -- & 0.149 & 12.4 & 0.215 & 11.9 & 0.318 \\
\hline 6/25/2008 5:00 AM & 0.121 & 22.0 & 0.090 & 0.301 & 18.8 & 0.41 & 0.030 & 0.028 & -- & -- & 0.149 & 12.4 & 0.215 & 11.9 & 0.318 \\
\hline 6/25/2008 6:00 AM & 0.120 & 22.0 & 0.090 & 0.301 & 18.8 & 0.41 & 0.030 & 0.028 & -- & -- & 0.149 & 12.4 & 0.215 & 11.9 & 0.318 \\
\hline
\end{tabular}


Appendix 1. Volumetric water content, temperature, and electrical-conductivity data collected at the flood-irrigated site during 2008.-Continued

[Depth in feet below land surface; volumetric water content dimensionless; temperature in degrees Celsius; bulk electrical conductivity in decisiemens per meter; --, no data]

\begin{tabular}{|c|c|c|c|c|c|c|c|c|c|c|c|c|c|c|c|}
\hline \multirow[b]{3}{*}{$\begin{array}{l}\text { Measurement } \\
\text { date and time }\end{array}$} & \multicolumn{15}{|c|}{ Depth } \\
\hline & \multicolumn{2}{|c|}{2} & \multirow[b]{2}{*}{$\begin{array}{c}3 \\
\begin{array}{c}\text { Volumetric } \\
\text { water } \\
\text { content }\end{array}\end{array}$} & \multicolumn{3}{|c|}{4} & \multirow{2}{*}{$\begin{array}{c}6 \\
\begin{array}{c}\text { Volumetric } \\
\text { water } \\
\text { content }\end{array}\end{array}$} & \multicolumn{2}{|c|}{8} & \multirow{2}{*}{$\begin{array}{c}10 \\
\text { Volumetric } \\
\text { water } \\
\text { content }\end{array}$} & \multicolumn{2}{|c|}{12} & \multicolumn{2}{|c|}{15} & \multirow{2}{*}{$\begin{array}{c}18.5 \\
\text { Volumetric } \\
\text { water } \\
\text { content }\end{array}$} \\
\hline & $\begin{array}{c}\text { Volumetric } \\
\text { water } \\
\text { content }\end{array}$ & $\begin{array}{l}\text { Temper- } \\
\text { ature }\end{array}$ & & $\begin{array}{c}\text { Volumetric } \\
\text { water } \\
\text { content }\end{array}$ & $\begin{array}{l}\text { Temper- } \\
\text { ature }\end{array}$ & $\begin{array}{c}\text { Bulk } \\
\text { electrical } \\
\text { conduct- } \\
\text { ivity }\end{array}$ & & $\begin{array}{c}\text { Volumetric } \\
\text { water } \\
\text { content }\end{array}$ & $\begin{array}{l}\text { Temper- } \\
\text { ature }\end{array}$ & & $\begin{array}{c}\text { Volumetric } \\
\text { water } \\
\text { content }\end{array}$ & $\begin{array}{l}\text { Temper- } \\
\text { ature }\end{array}$ & $\begin{array}{c}\text { Volumetric } \\
\text { water } \\
\text { content }\end{array}$ & $\begin{array}{l}\text { Temper- } \\
\text { ature }\end{array}$ & \\
\hline$\overline{6 / 25 / 2008 ~ 7: 00 ~ A M ~}$ & 0.121 & 22.1 & 0.090 & 0.301 & 18.8 & 0.41 & 0.030 & 0.028 & -- & -- & 0.149 & 12.5 & 0.216 & 12.0 & 0.318 \\
\hline 6/25/2008 8:00 AM & 0.121 & 22.1 & 0.091 & 0.301 & 18.8 & 0.41 & 0.030 & 0.029 & -- & -- & 0.148 & 12.5 & 0.216 & 11.9 & 0.318 \\
\hline 6/25/2008 9:00 AM & 0.121 & 22.1 & 0.091 & 0.301 & 18.8 & 0.41 & 0.030 & 0.029 & -- & -- & 0.149 & 12.5 & 0.216 & 12.0 & 0.318 \\
\hline 6/25/2008 10:00 AM & 0.123 & 22.1 & 0.090 & 0.301 & 18.8 & 0.41 & 0.030 & 0.032 & -- & -- & 0.148 & 12.5 & 0.216 & 12.0 & 0.318 \\
\hline 6/25/2008 11:00 AM & 0.163 & 22.2 & 0.090 & 0.300 & 18.9 & 0.41 & 0.030 & 0.031 & -- & -- & 0.148 & 12.5 & 0.216 & 12.0 & 0.318 \\
\hline 6/25/2008 12:00 PM & 0.180 & 22.1 & 0.090 & 0.299 & 18.9 & 0.41 & 0.030 & 0.030 & -- & -- & 0.149 & 12.5 & 0.215 & 12.0 & 0.318 \\
\hline 6/25/2008 1:00 PM & 0.188 & 22.1 & 0.116 & 0.300 & 18.9 & 0.41 & 0.030 & 0.030 & -- & -- & 0.149 & 12.5 & 0.216 & 12.0 & 0.318 \\
\hline 6/25/2008 2:00 PM & 0.204 & 22.2 & 0.146 & 0.301 & 18.9 & 0.41 & 0.030 & 0.030 & -- & -- & 0.150 & 12.5 & 0.215 & 12.0 & 0.318 \\
\hline 6/25/2008 3:00 PM & 0.203 & 22.1 & 0.147 & 0.319 & 18.9 & 0.47 & 0.030 & 0.030 & -- & -- & 0.149 & 12.5 & 0.216 & 12.0 & 0.318 \\
\hline 6/25/2008 4:00 PM & 0.198 & 22.2 & 0.146 & 0.328 & 19.0 & 0.51 & 0.030 & 0.030 & -- & -- & 0.149 & 12.5 & 0.216 & 12.0 & 0.318 \\
\hline 6/25/2008 5:00 PM & 0.196 & 22.2 & 0.144 & 0.329 & 19.0 & 0.51 & 0.030 & 0.030 & -- & -- & 0.148 & 12.5 & 0.216 & 12.0 & 0.318 \\
\hline 6/25/2008 6:00 PM & 0.194 & 22.1 & 0.143 & 0.329 & 19.0 & 0.51 & 0.030 & 0.030 & -- & -- & 0.148 & 12.5 & 0.216 & 12.0 & 0.318 \\
\hline 6/25/2008 7:00 PM & 0.192 & 22.2 & 0.142 & 0.329 & 19.0 & 0.51 & 0.030 & 0.030 & -- & -- & 0.148 & 12.5 & 0.216 & 12.0 & 0.318 \\
\hline 6/25/2008 8:00 PM & 0.187 & 22.2 & 0.141 & 0.329 & 19.1 & 0.52 & 0.030 & 0.030 & -- & -- & 0.149 & 12.5 & 0.216 & 12.0 & 0.318 \\
\hline 6/25/2008 9:00 PM & 0.185 & 22.2 & 0.140 & 0.328 & 19.1 & 0.52 & 0.030 & 0.029 & -- & -- & 0.149 & 12.5 & 0.216 & 12.0 & 0.318 \\
\hline 6/25/2008 10:00 PM & 0.182 & 22.2 & 0.138 & 0.328 & 19.1 & 0.52 & 0.030 & 0.029 & -- & -- & 0.148 & 12.5 & 0.217 & 12.0 & 0.318 \\
\hline 6/25/2008 11:00 PM & 0.180 & 22.2 & 0.138 & 0.326 & 19.1 & 0.52 & 0.030 & 0.030 & -- & -- & 0.148 & 12.5 & 0.217 & 12.0 & 0.319 \\
\hline 6/26/2008 12:00 AM & 0.179 & 22.3 & 0.137 & 0.326 & 19.2 & 0.52 & 0.030 & 0.030 & -- & -- & 0.148 & 12.5 & 0.217 & 12.0 & 0.321 \\
\hline 6/26/2008 1:00 AM & 0.176 & 22.2 & 0.135 & 0.326 & 19.2 & 0.52 & 0.031 & 0.030 & -- & -- & 0.148 & 12.5 & 0.216 & 12.0 & 0.322 \\
\hline 6/26/2008 2:00 AM & 0.175 & 22.2 & 0.134 & 0.326 & 19.2 & 0.52 & 0.031 & 0.030 & -- & -- & 0.148 & 12.5 & 0.217 & 12.0 & 0.324 \\
\hline 6/26/2008 3:00 AM & 0.174 & 22.3 & 0.133 & 0.325 & 19.2 & 0.52 & 0.032 & 0.030 & -- & -- & 0.149 & 12.5 & 0.217 & 12.0 & 0.473 \\
\hline 6/26/2008 4:00 AM & 0.174 & 22.3 & 0.132 & 0.325 & 19.2 & 0.53 & 0.032 & 0.030 & -- & -- & 0.149 & 12.5 & 0.217 & 12.0 & 0.441 \\
\hline 6/26/2008 5:00 AM & 0.173 & 22.2 & 0.132 & 0.325 & 19.3 & 0.53 & 0.033 & 0.031 & -- & -- & 0.149 & 12.5 & 0.217 & 12.0 & 0.443 \\
\hline 6/26/2008 6:00 AM & 0.171 & 22.2 & 0.131 & 0.325 & 19.3 & 0.53 & 0.033 & 0.030 & -- & -- & 0.149 & 12.5 & 0.217 & 12.0 & 0.330 \\
\hline 6/26/2008 7:00 AM & 0.169 & 22.2 & 0.130 & 0.324 & 19.3 & 0.53 & 0.034 & 0.032 & -- & -- & 0.149 & 12.5 & 0.217 & 12.0 & 0.335 \\
\hline 6/26/2008 8:00 AM & 0.171 & 22.2 & 0.130 & 0.324 & 19.3 & 0.53 & 0.035 & 0.031 & -- & -- & 0.149 & 12.5 & 0.217 & 12.0 & 0.486 \\
\hline 6/26/2008 9:00 AM & 0.171 & 22.2 & 0.129 & 0.324 & 19.3 & 0.53 & 0.036 & 0.030 & -- & -- & 0.149 & 12.5 & 0.217 & 12.0 & 0.693 \\
\hline 6/26/2008 10:00 AM & 0.170 & 22.1 & 0.128 & 0.324 & 19.3 & 0.53 & 0.036 & 0.031 & -- & -- & 0.150 & 12.5 & 0.217 & 12.0 & -- \\
\hline 6/26/2008 11:00 AM & 0.169 & 22.0 & 0.127 & 0.324 & 19.3 & 0.52 & 0.036 & 0.030 & -- & -- & 0.148 & 12.6 & 0.217 & 12.0 & 0.652 \\
\hline 6/26/2008 12:00 PM & 0.168 & 21.9 & 0.127 & 0.324 & 19.3 & 0.52 & 0.037 & 0.030 & -- & -- & 0.148 & 12.5 & 0.217 & 12.0 & 0.677 \\
\hline 6/26/2008 1:00 PM & 0.167 & 22.0 & 0.126 & 0.324 & 19.4 & 0.52 & 0.037 & 0.030 & -- & -- & 0.149 & 12.5 & 0.217 & 12.0 & 0.704 \\
\hline 6/26/2008 2:00 PM & 0.166 & 21.9 & 0.126 & 0.323 & 19.4 & 0.51 & 0.038 & 0.030 & -- & -- & 0.150 & 12.5 & 0.217 & 12.0 & -- \\
\hline 6/26/2008 3:00 PM & 0.165 & 21.9 & 0.126 & 0.323 & 19.4 & 0.51 & 0.038 & 0.030 & -- & -- & 0.149 & 12.6 & 0.217 & 12.0 & -- \\
\hline 6/26/2008 4:00 PM & 0.163 & 21.8 & 0.125 & 0.323 & 19.4 & 0.51 & 0.039 & 0.031 & -- & -- & 0.149 & 12.6 & 0.217 & 12.0 & -- \\
\hline 6/26/2008 5:00 PM & 0.163 & 21.8 & 0.125 & 0.323 & 19.4 & 0.51 & 0.040 & 0.032 & -- & -- & 0.149 & 12.6 & 0.217 & 12.0 & -- \\
\hline 6/26/2008 6:00 PM & 0.161 & 21.8 & 0.124 & 0.323 & 19.4 & 0.50 & 0.040 & 0.032 & -- & -- & 0.150 & 12.6 & 0.217 & 12.0 & -- \\
\hline 6/26/2008 7:00 PM & 0.161 & 21.8 & 0.123 & 0.323 & 19.4 & 0.50 & 0.041 & 0.032 & -- & -- & 0.149 & 12.6 & 0.217 & 12.0 & -- \\
\hline 6/26/2008 8:00 PM & 0.161 & 21.7 & 0.123 & 0.323 & 19.4 & 0.50 & 0.041 & 0.033 & -- & -- & 0.150 & 12.6 & 0.217 & 12.1 & -- \\
\hline 6/26/2008 9:00 PM & 0.160 & 21.7 & 0.123 & 0.323 & 19.4 & 0.49 & 0.042 & 0.033 & -- & -- & 0.150 & 12.6 & 0.217 & 12.0 & -- \\
\hline 6/26/2008 10:00 PM & 0.160 & 21.7 & 0.122 & 0.323 & 19.4 & 0.49 & 0.042 & 0.034 & -- & -- & 0.149 & 12.6 & 0.217 & 12.1 & -- \\
\hline
\end{tabular}


Appendix 1. Volumetric water content, temperature, and electrical-conductivity data collected at the flood-irrigated site during 2008.-Continued

[Depth in feet below land surface; volumetric water content dimensionless; temperature in degrees Celsius; bulk electrical conductivity in decisiemens per meter; --, no data]

\begin{tabular}{|c|c|c|c|c|c|c|c|c|c|c|c|c|c|c|c|}
\hline \multirow[b]{3}{*}{$\begin{array}{l}\text { Measurement } \\
\text { date and time }\end{array}$} & \multicolumn{15}{|c|}{ Depth } \\
\hline & \multicolumn{2}{|l|}{2} & \multirow{2}{*}{$\begin{array}{c}3 \\
\text { Volumetric } \\
\text { water } \\
\text { content }\end{array}$} & \multicolumn{3}{|c|}{4} & \multirow{2}{*}{$\begin{array}{c}6 \\
\begin{array}{c}\text { Volumetric } \\
\text { water } \\
\text { content }\end{array}\end{array}$} & \multicolumn{2}{|l|}{8} & \multirow{2}{*}{$\begin{array}{c}10 \\
\text { Volumetric } \\
\text { water } \\
\text { content }\end{array}$} & \multicolumn{2}{|c|}{12} & \multicolumn{2}{|c|}{15} & \multirow{2}{*}{$\begin{array}{c}18.5 \\
\text { Volumetric } \\
\text { water } \\
\text { content }\end{array}$} \\
\hline & $\begin{array}{l}\text { Volumetric } \\
\text { water } \\
\text { content }\end{array}$ & $\begin{array}{l}\text { Temper- } \\
\text { ature }\end{array}$ & & $\begin{array}{c}\text { Volumetric } \\
\text { water } \\
\text { content }\end{array}$ & $\begin{array}{l}\text { Temper- } \\
\text { ature }\end{array}$ & $\begin{array}{c}\text { Bulk } \\
\text { electrical } \\
\text { conduct- } \\
\text { ivity } \\
\end{array}$ & & $\begin{array}{l}\text { Volumetric } \\
\text { water } \\
\text { content }\end{array}$ & $\begin{array}{l}\text { Temper- } \\
\text { ature }\end{array}$ & & $\begin{array}{l}\text { Volumetric } \\
\text { water } \\
\text { content }\end{array}$ & $\begin{array}{l}\text { Temper- } \\
\text { ature }\end{array}$ & $\begin{array}{l}\text { Volumetric } \\
\text { water } \\
\text { content }\end{array}$ & $\begin{array}{l}\text { Temper- } \\
\text { ature }\end{array}$ & \\
\hline 6/26/2008 11:00 PM & 0.159 & 21.7 & 0.121 & 0.322 & 19.4 & 0.49 & 0.042 & 0.035 & -- & -- & 0.148 & 12.6 & 0.217 & 12.0 & -- \\
\hline 6/27/2008 12:00 AM & 0.159 & 21.7 & 0.121 & 0.322 & 19.4 & 0.49 & 0.042 & 0.033 & -- & -- & 0.149 & 12.6 & 0.217 & 12.1 & -- \\
\hline 6/27/2008 1:00 AM & 0.159 & 21.7 & 0.121 & 0.322 & 19.4 & 0.48 & 0.042 & 0.034 & -- & -- & 0.149 & 12.6 & 0.217 & 12.1 & -- \\
\hline 6/27/2008 2:00 AM & 0.158 & 21.6 & 0.121 & 0.322 & 19.4 & 0.48 & 0.042 & 0.035 & -- & -- & 0.149 & 12.6 & 0.218 & 12.0 & -- \\
\hline 6/27/2008 3:00 AM & 0.157 & 21.6 & 0.120 & 0.322 & 19.4 & 0.48 & 0.042 & 0.035 & -- & -- & 0.149 & 12.6 & 0.218 & 12.0 & -- \\
\hline 6/27/2008 4:00 AM & 0.157 & 21.6 & 0.120 & 0.322 & 19.4 & 0.48 & 0.042 & 0.035 & -- & -- & 0.149 & 12.6 & 0.218 & 12.0 & -- \\
\hline 6/27/2008 5:00 AM & 0.156 & 21.6 & 0.120 & 0.321 & 19.4 & 0.47 & 0.042 & 0.035 & -- & -- & 0.149 & 12.6 & 0.218 & 12.0 & 0.706 \\
\hline 6/27/2008 6:00 AM & 0.156 & 21.6 & 0.119 & 0.321 & 19.5 & 0.47 & 0.043 & 0.035 & -- & -- & 0.149 & 12.6 & 0.218 & 12.0 & 0.705 \\
\hline 6/27/2008 7:00 AM & 0.155 & 21.6 & 0.119 & 0.322 & 19.5 & 0.47 & 0.043 & 0.035 & -- & -- & 0.149 & 12.6 & 0.218 & 12.0 & 0.674 \\
\hline 6/27/2008 8:00 AM & 0.154 & 21.6 & 0.119 & 0.322 & 19.5 & 0.47 & 0.043 & 0.036 & -- & -- & 0.149 & 12.6 & 0.218 & 12.0 & -- \\
\hline 6/27/2008 9:00 AM & 0.154 & 21.7 & 0.118 & 0.322 & 19.5 & 0.46 & 0.043 & 0.036 & -- & -- & 0.149 & 12.6 & 0.218 & 12.1 & -- \\
\hline 6/27/2008 10:00 AM & 0.153 & 21.7 & 0.118 & 0.322 & 19.5 & 0.46 & 0.044 & 0.036 & -- & -- & 0.150 & 12.6 & 0.218 & 12.1 & -- \\
\hline 6/27/2008 11:00 AM & 0.153 & 21.6 & 0.117 & 0.322 & 19.5 & 0.46 & 0.044 & 0.036 & -- & -- & 0.150 & 12.6 & 0.217 & 12.1 & -- \\
\hline 6/27/2008 12:00 PM & 0.151 & 21.6 & 0.117 & 0.321 & 19.5 & 0.46 & 0.044 & 0.036 & -- & -- & 0.150 & 12.6 & 0.218 & 12.1 & -- \\
\hline 6/27/2008 1:00 PM & 0.150 & 21.5 & 0.117 & 0.321 & 19.5 & 0.46 & 0.044 & 0.036 & -- & -- & 0.149 & 12.6 & 0.218 & 12.1 & -- \\
\hline 6/27/2008 2:00 PM & 0.150 & 21.5 & 0.116 & 0.321 & 19.5 & 0.46 & 0.044 & 0.036 & -- & -- & 0.149 & 12.6 & 0.218 & 12.1 & -- \\
\hline 6/27/2008 3:00 PM & 0.150 & 21.4 & 0.116 & 0.322 & 19.5 & 0.45 & 0.044 & 0.036 & -- & -- & 0.149 & 12.6 & 0.218 & 12.1 & -- \\
\hline 6/27/2008 4:00 PM & 0.150 & 21.3 & 0.116 & 0.321 & 19.5 & 0.45 & 0.044 & 0.036 & -- & -- & 0.150 & 12.6 & 0.218 & 12.1 & -- \\
\hline 6/27/2008 5:00 PM & 0.149 & 21.3 & 0.115 & 0.321 & 19.5 & 0.45 & 0.044 & 0.035 & -- & -- & 0.150 & 12.7 & 0.218 & 12.1 & -- \\
\hline 6/27/2008 6:00 PM & 0.149 & 21.3 & 0.115 & 0.321 & 19.5 & 0.45 & 0.044 & 0.035 & -- & -- & 0.150 & 12.6 & 0.219 & 12.1 & 0.714 \\
\hline 6/27/2008 7:00 PM & 0.148 & 21.3 & 0.115 & 0.321 & 19.5 & 0.45 & 0.044 & 0.036 & -- & -- & 0.149 & 12.7 & 0.218 & 12.1 & 0.693 \\
\hline 6/27/2008 8:00 PM & 0.148 & 21.3 & 0.115 & 0.321 & 19.5 & 0.45 & 0.044 & 0.037 & -- & -- & 0.150 & 12.6 & 0.218 & 12.1 & -- \\
\hline 6/27/2008 9:00 PM & 0.147 & 21.3 & 0.115 & 0.321 & 19.5 & 0.44 & 0.044 & 0.037 & -- & 0.000 & 0.149 & 12.6 & 0.218 & 12.1 & -- \\
\hline 6/27/2008 10:00 PM & 0.147 & 21.3 & 0.115 & 0.320 & 19.5 & 0.44 & 0.043 & 0.036 & -- & 0.000 & 0.149 & 12.6 & 0.218 & 12.1 & -- \\
\hline 6/27/2008 11:00 PM & 0.147 & 21.3 & 0.114 & 0.320 & 19.5 & 0.44 & 0.043 & 0.036 & -- & 0.000 & 0.149 & 12.6 & 0.218 & 12.1 & -- \\
\hline 6/28/2008 12:00 AM & 0.147 & 21.4 & 0.114 & 0.320 & 19.5 & 0.44 & 0.043 & 0.037 & -- & 0.000 & 0.150 & 12.6 & 0.219 & 12.1 & -- \\
\hline 6/28/2008 1:00 AM & 0.147 & 21.4 & 0.114 & 0.320 & 19.5 & 0.44 & 0.043 & 0.036 & -- & 0.000 & 0.149 & 12.6 & 0.218 & 12.1 & -- \\
\hline 6/28/2008 2:00 AM & 0.147 & 21.4 & 0.113 & 0.320 & 19.5 & 0.44 & 0.043 & 0.036 & -- & 0.000 & 0.150 & 12.7 & 0.219 & 12.1 & -- \\
\hline 6/28/2008 3:00 AM & 0.146 & 21.4 & 0.113 & 0.320 & 19.5 & 0.44 & 0.043 & 0.036 & -- & 0.000 & 0.150 & 12.6 & 0.219 & 12.1 & -- \\
\hline 6/28/2008 4:00 AM & 0.146 & 21.5 & 0.113 & 0.320 & 19.5 & 0.43 & 0.044 & 0.036 & -- & 0.000 & 0.150 & 12.6 & 0.219 & 12.1 & -- \\
\hline 6/28/2008 5:00 AM & 0.146 & 21.5 & 0.113 & 0.320 & 19.4 & 0.43 & 0.044 & 0.036 & -- & 0.000 & 0.150 & 12.6 & 0.219 & 12.1 & -- \\
\hline 6/28/2008 6:00 AM & 0.146 & 21.5 & 0.112 & 0.320 & 19.4 & 0.43 & 0.044 & 0.036 & -- & 0.000 & 0.149 & 12.6 & 0.219 & 12.1 & -- \\
\hline 6/28/2008 7:00 AM & 0.145 & 21.5 & 0.112 & 0.319 & 19.4 & 0.43 & 0.044 & 0.037 & -- & 0.000 & 0.150 & 12.7 & 0.219 & 12.1 & -- \\
\hline 6/28/2008 8:00 AM & 0.145 & 21.6 & 0.112 & 0.320 & 19.4 & 0.43 & 0.044 & 0.038 & -- & 0.000 & 0.150 & 12.7 & 0.219 & 12.1 & 0.684 \\
\hline 6/28/2008 9:00 AM & 0.145 & 21.6 & 0.112 & 0.319 & 19.4 & 0.43 & 0.044 & 0.038 & -- & 0.000 & 0.150 & 12.7 & 0.219 & 12.1 & -- \\
\hline 6/28/2008 10:00 AM & 0.145 & 21.6 & 0.111 & 0.320 & 19.4 & 0.43 & 0.044 & 0.038 & -- & 0.000 & 0.150 & 12.7 & 0.219 & 12.1 & -- \\
\hline 6/28/2008 11:00 AM & 0.144 & 21.6 & 0.111 & 0.319 & 19.4 & 0.43 & 0.044 & 0.038 & -- & 0.000 & 0.149 & 12.7 & 0.219 & 12.1 & -- \\
\hline 6/28/2008 12:00 PM & 0.143 & 21.6 & 0.111 & 0.319 & 19.4 & 0.43 & 0.044 & 0.039 & -- & 0.000 & 0.150 & 12.7 & 0.219 & 12.1 & -- \\
\hline 6/28/2008 1:00 PM & 0.143 & 21.6 & 0.111 & 0.319 & 19.4 & 0.42 & 0.044 & 0.039 & -- & 0.000 & 0.151 & 12.7 & 0.220 & 12.1 & -- \\
\hline 6/28/2008 2:00 PM & 0.142 & 21.5 & 0.110 & 0.319 & 19.4 & 0.42 & 0.044 & 0.039 & -- & 0.000 & 0.151 & 12.7 & 0.220 & 12.1 & -- \\
\hline
\end{tabular}


Appendix 1. Volumetric water content, temperature, and electrical-conductivity data collected at the flood-irrigated site during 2008.-Continued

[Depth in feet below land surface; volumetric water content dimensionless; temperature in degrees Celsius; bulk electrical conductivity in decisiemens per meter; --, no data]

\begin{tabular}{|c|c|c|c|c|c|c|c|c|c|c|c|c|c|c|c|}
\hline \multirow[b]{3}{*}{$\begin{array}{l}\text { Measurement } \\
\text { date and time }\end{array}$} & \multicolumn{15}{|c|}{ Depth } \\
\hline & \multicolumn{2}{|c|}{2} & \multirow{2}{*}{$\begin{array}{c}3 \\
\begin{array}{c}\text { Volumetric } \\
\text { water } \\
\text { content }\end{array}\end{array}$} & \multicolumn{3}{|c|}{4} & \multirow{2}{*}{$\begin{array}{c}6 \\
\text { Volumetric } \\
\text { water } \\
\text { content }\end{array}$} & \multicolumn{2}{|c|}{8} & \multirow{2}{*}{$\begin{array}{c}10 \\
\text { Volumetric } \\
\text { water } \\
\text { content }\end{array}$} & \multicolumn{2}{|l|}{12} & \multicolumn{2}{|c|}{15} & \multirow{2}{*}{$\begin{array}{c}18.5 \\
\text { Volumetric } \\
\text { water } \\
\text { content }\end{array}$} \\
\hline & $\begin{array}{c}\text { Volumetric } \\
\text { water } \\
\text { content }\end{array}$ & $\begin{array}{l}\text { Temper- } \\
\text { ature }\end{array}$ & & $\begin{array}{c}\text { Volumetric } \\
\text { water } \\
\text { content }\end{array}$ & $\begin{array}{l}\text { Temper- } \\
\text { ature }\end{array}$ & $\begin{array}{c}\text { Bulk } \\
\text { electrical } \\
\text { conduct- } \\
\text { ivity } \\
\end{array}$ & & $\begin{array}{c}\text { Volumetric } \\
\text { water } \\
\text { content }\end{array}$ & $\begin{array}{l}\text { Temper- } \\
\text { ature }\end{array}$ & & $\begin{array}{c}\text { Volumetric } \\
\text { water } \\
\text { content }\end{array}$ & $\begin{array}{l}\text { Temper- } \\
\text { ature }\end{array}$ & $\begin{array}{c}\text { Volumetric } \\
\text { water } \\
\text { content }\end{array}$ & $\begin{array}{l}\text { Temper- } \\
\text { ature }\end{array}$ & \\
\hline 6/28/2008 3:00 PM & 0.142 & 21.5 & 0.110 & 0.319 & 19.4 & 0.42 & 0.044 & 0.039 & -- & 0.000 & 0.150 & 12.7 & 0.220 & 12.1 & -- \\
\hline 6/28/2008 4:00 PM & 0.143 & 21.5 & 0.110 & 0.319 & 19.4 & 0.42 & 0.044 & 0.039 & -- & 0.000 & 0.150 & 12.7 & 0.220 & 12.1 & -- \\
\hline 6/28/2008 5:00 PM & 0.142 & 21.4 & 0.110 & 0.319 & 19.4 & 0.42 & 0.044 & 0.039 & -- & 0.000 & 0.150 & 12.7 & 0.220 & 12.1 & -- \\
\hline 6/28/2008 6:00 PM & 0.142 & 21.4 & 0.110 & 0.319 & 19.4 & 0.42 & 0.044 & 0.040 & -- & 0.000 & 0.150 & 12.7 & 0.220 & 12.1 & 0.713 \\
\hline 6/28/2008 7:00 PM & 0.141 & 21.4 & 0.109 & 0.319 & 19.4 & 0.42 & 0.044 & 0.040 & -- & 0.000 & 0.150 & 12.7 & 0.220 & 12.1 & 0.682 \\
\hline 6/28/2008 8:00 PM & 0.141 & 21.4 & 0.109 & 0.319 & 19.4 & 0.42 & 0.044 & 0.039 & -- & 0.000 & 0.149 & 12.7 & 0.220 & 12.1 & 0.709 \\
\hline 6/28/2008 9:00 PM & 0.141 & 21.4 & 0.109 & 0.318 & 19.4 & 0.42 & 0.044 & 0.040 & -- & 0.001 & 0.150 & 12.7 & 0.220 & 12.1 & 0.717 \\
\hline 6/28/2008 10:00 PM & 0.141 & 21.4 & 0.109 & 0.318 & 19.4 & 0.42 & 0.043 & 0.041 & -- & 0.001 & 0.150 & 12.7 & 0.220 & 12.1 & 0.708 \\
\hline 6/28/2008 11:00 PM & 0.141 & 21.4 & 0.109 & 0.318 & 19.4 & 0.42 & 0.043 & 0.042 & -- & 0.001 & 0.150 & 12.7 & 0.220 & 12.1 & 0.704 \\
\hline 6/29/2008 12:00 AM & 0.141 & 21.4 & 0.109 & 0.318 & 19.4 & 0.42 & 0.043 & 0.041 & -- & 0.001 & 0.150 & 12.7 & 0.220 & 12.1 & 0.713 \\
\hline 6/29/2008 1:00 AM & 0.141 & 21.5 & 0.109 & 0.318 & 19.4 & 0.42 & 0.043 & 0.041 & -- & 0.001 & 0.150 & 12.7 & 0.221 & 12.1 & -- \\
\hline 6/29/2008 2:00 AM & 0.141 & 21.5 & 0.109 & 0.318 & 19.4 & 0.42 & 0.043 & 0.042 & -- & 0.001 & 0.150 & 12.7 & 0.221 & 12.1 & -- \\
\hline 6/29/2008 3:00 AM & 0.141 & 21.5 & 0.108 & 0.318 & 19.4 & 0.42 & 0.043 & 0.042 & -- & 0.001 & 0.150 & 12.7 & 0.221 & 12.1 & -- \\
\hline 6/29/2008 4:00 AM & 0.141 & 21.6 & 0.108 & 0.318 & 19.4 & 0.42 & 0.043 & 0.042 & -- & 0.001 & 0.150 & 12.7 & 0.220 & 12.1 & -- \\
\hline 6/29/2008 5:00 AM & 0.140 & 21.6 & 0.108 & 0.318 & 19.4 & 0.41 & 0.043 & 0.042 & -- & 0.001 & 0.151 & 12.7 & 0.221 & 12.1 & 0.714 \\
\hline 6/29/2008 6:00 AM & 0.140 & 21.6 & 0.108 & 0.318 & 19.4 & 0.41 & 0.043 & 0.042 & -- & 0.001 & 0.151 & 12.7 & 0.221 & 12.1 & 0.682 \\
\hline 6/29/2008 7:00 AM & 0.140 & 21.6 & 0.108 & 0.318 & 19.4 & 0.41 & 0.043 & 0.042 & -- & 0.001 & 0.151 & 12.7 & 0.221 & 12.2 & 0.686 \\
\hline 6/29/2008 8:00 AM & 0.138 & 21.7 & 0.108 & 0.318 & 19.4 & 0.41 & 0.043 & 0.042 & -- & 0.001 & 0.150 & 12.7 & 0.221 & 12.1 & 0.681 \\
\hline 6/29/2008 9:00 AM & 0.138 & 21.7 & 0.108 & 0.318 & 19.4 & 0.41 & 0.044 & 0.043 & -- & 0.001 & 0.150 & 12.8 & 0.221 & 12.2 & 0.667 \\
\hline 6/29/2008 10:00 AM & 0.138 & 21.7 & 0.108 & 0.318 & 19.4 & 0.41 & 0.044 & 0.042 & -- & 0.001 & 0.150 & 12.8 & 0.221 & 12.2 & 0.663 \\
\hline 6/29/2008 11:00 AM & 0.137 & 21.7 & 0.108 & 0.318 & 19.5 & 0.41 & 0.044 & 0.043 & -- & 0.001 & 0.151 & 12.8 & 0.221 & 12.2 & 0.651 \\
\hline 6/29/2008 12:00 PM & 0.137 & 21.7 & 0.107 & 0.318 & 19.5 & 0.41 & 0.044 & 0.042 & -- & 0.001 & 0.151 & 12.8 & 0.222 & 12.2 & 0.651 \\
\hline 6/29/2008 1:00 PM & 0.136 & 21.6 & 0.107 & 0.318 & 19.5 & 0.41 & 0.044 & 0.042 & -- & 0.001 & 0.151 & 12.8 & 0.222 & 12.2 & 0.658 \\
\hline 6/29/2008 2:00 PM & 0.136 & 21.6 & 0.107 & 0.317 & 19.5 & 0.41 & 0.044 & 0.045 & -- & 0.001 & 0.151 & 12.8 & 0.222 & 12.2 & 0.702 \\
\hline 6/29/2008 3:00 PM & 0.136 & 21.6 & 0.107 & 0.317 & 19.5 & 0.41 & 0.043 & 0.045 & -- & 0.001 & 0.150 & 12.8 & 0.222 & 12.2 & 0.652 \\
\hline 6/29/2008 4:00 PM & 0.136 & 21.5 & 0.106 & 0.317 & 19.5 & 0.41 & 0.043 & 0.044 & -- & 0.001 & 0.150 & 12.8 & 0.222 & 12.2 & 0.653 \\
\hline 6/29/2008 5:00 PM & 0.136 & 21.5 & 0.106 & 0.317 & 19.5 & 0.41 & 0.043 & 0.044 & -- & 0.001 & 0.150 & 12.8 & 0.222 & 12.2 & 0.646 \\
\hline 6/29/2008 6:00 PM & 0.136 & 21.5 & 0.106 & 0.317 & 19.5 & 0.41 & 0.043 & 0.044 & -- & 0.001 & 0.150 & 12.8 & 0.223 & 12.2 & 0.661 \\
\hline 6/29/2008 7:00 PM & 0.136 & 21.5 & 0.106 & 0.317 & 19.5 & 0.41 & 0.043 & 0.044 & -- & 0.001 & 0.151 & 12.8 & 0.222 & 12.2 & 0.693 \\
\hline 6/29/2008 8:00 PM & 0.135 & 21.5 & 0.106 & 0.317 & 19.5 & 0.41 & 0.043 & 0.044 & -- & 0.001 & 0.150 & 12.8 & 0.222 & 12.2 & 0.713 \\
\hline 6/29/2008 9:00 PM & 0.135 & 21.5 & 0.106 & 0.317 & 19.5 & 0.41 & 0.043 & 0.044 & -- & 0.001 & 0.150 & 12.8 & 0.222 & 12.2 & -- \\
\hline 6/29/2008 10:00 PM & 0.136 & 21.5 & 0.106 & 0.317 & 19.5 & 0.41 & 0.043 & 0.045 & -- & 0.001 & 0.151 & 12.8 & 0.222 & 12.2 & -- \\
\hline 6/29/2008 11:00 PM & 0.136 & 21.5 & 0.105 & 0.317 & 19.5 & 0.41 & 0.043 & 0.045 & -- & 0.001 & 0.151 & 12.8 & 0.222 & 12.2 & -- \\
\hline 6/30/2008 12:00 AM & 0.135 & 21.6 & 0.105 & 0.316 & 19.5 & 0.41 & 0.043 & 0.045 & -- & 0.002 & 0.151 & 12.8 & 0.223 & 12.2 & -- \\
\hline 6/30/2008 1:00 AM & 0.135 & 21.6 & 0.105 & 0.316 & 19.5 & 0.41 & 0.043 & 0.045 & -- & 0.002 & 0.151 & 12.8 & 0.223 & 12.2 & -- \\
\hline 6/30/2008 2:00 AM & 0.135 & 21.7 & 0.105 & 0.316 & 19.5 & 0.41 & 0.043 & 0.045 & -- & 0.002 & 0.151 & 12.8 & 0.223 & 12.2 & -- \\
\hline 6/30/2008 3:00 AM & 0.136 & 21.7 & 0.105 & 0.316 & 19.5 & 0.41 & 0.043 & 0.045 & -- & 0.002 & 0.151 & 12.8 & 0.223 & 12.2 & -- \\
\hline 6/30/2008 4:00 AM & 0.135 & 21.7 & 0.105 & 0.316 & 19.5 & 0.41 & 0.043 & 0.045 & -- & 0.002 & 0.151 & 12.8 & 0.223 & 12.2 & -- \\
\hline 6/30/2008 5:00 AM & 0.135 & 21.8 & 0.104 & 0.316 & 19.5 & 0.41 & 0.043 & 0.045 & -- & 0.002 & 0.151 & 12.8 & 0.223 & 12.2 & -- \\
\hline 6/30/2008 6:00 AM & 0.135 & 21.8 & 0.104 & 0.316 & 19.5 & 0.41 & 0.043 & 0.045 & -- & 0.002 & 0.151 & 12.8 & 0.223 & 12.2 & -- \\
\hline
\end{tabular}


Appendix 1. Volumetric water content, temperature, and electrical-conductivity data collected at the flood-irrigated site during 2008.-Continued

[Depth in feet below land surface; volumetric water content dimensionless; temperature in degrees Celsius; bulk electrical conductivity in decisiemens per meter; --, no data]

\begin{tabular}{|c|c|c|c|c|c|c|c|c|c|c|c|c|c|c|c|}
\hline \multirow[b]{3}{*}{$\begin{array}{l}\text { Measurement } \\
\text { date and time }\end{array}$} & \multicolumn{15}{|c|}{ Depth } \\
\hline & \multicolumn{2}{|c|}{2} & \multirow{2}{*}{$\begin{array}{c}3 \\
\text { Volumetric } \\
\text { water } \\
\text { content }\end{array}$} & \multicolumn{3}{|c|}{4} & \multirow{2}{*}{$\begin{array}{c}6 \\
\begin{array}{c}\text { Volumetric } \\
\text { water } \\
\text { content }\end{array}\end{array}$} & \multicolumn{2}{|l|}{8} & \multirow{2}{*}{$\begin{array}{c}10 \\
\text { Volumetric } \\
\text { water } \\
\text { content }\end{array}$} & \multicolumn{2}{|c|}{12} & \multicolumn{2}{|c|}{15} & \multirow{2}{*}{$\begin{array}{c}18.5 \\
\text { Volumetric } \\
\text { water } \\
\text { content }\end{array}$} \\
\hline & $\begin{array}{l}\text { Volumetric } \\
\text { water } \\
\text { content }\end{array}$ & $\begin{array}{l}\text { Temper- } \\
\text { ature }\end{array}$ & & $\begin{array}{c}\text { Volumetric } \\
\text { water } \\
\text { content }\end{array}$ & $\begin{array}{l}\text { Temper- } \\
\text { ature }\end{array}$ & $\begin{array}{c}\text { Bulk } \\
\text { electrical } \\
\text { conduct- } \\
\text { ivity } \\
\end{array}$ & & $\begin{array}{l}\text { Volumetric } \\
\text { water } \\
\text { content }\end{array}$ & $\begin{array}{l}\text { Temper- } \\
\text { ature }\end{array}$ & & $\begin{array}{l}\text { Volumetric } \\
\text { water } \\
\text { content }\end{array}$ & $\begin{array}{l}\text { Temper- } \\
\text { ature }\end{array}$ & $\begin{array}{l}\text { Volumetric } \\
\text { water } \\
\text { content }\end{array}$ & $\begin{array}{l}\text { Temper- } \\
\text { ature }\end{array}$ & \\
\hline 6/30/2008 7:00 AM & 0.135 & 21.8 & 0.104 & 0.316 & 19.5 & 0.41 & 0.043 & 0.047 & -- & 0.002 & 0.151 & 12.8 & 0.223 & 12.2 & -- \\
\hline 6/30/2008 8:00 AM & 0.135 & 21.8 & 0.104 & 0.316 & 19.5 & 0.41 & 0.043 & 0.046 & -- & 0.002 & 0.151 & 12.8 & 0.223 & 12.2 & -- \\
\hline 6/30/2008 9:00 AM & 0.134 & 21.8 & 0.104 & 0.316 & 19.5 & 0.41 & 0.043 & 0.045 & -- & 0.002 & 0.151 & 12.9 & 0.224 & 12.2 & -- \\
\hline 6/30/2008 10:00 AM & 0.134 & 21.9 & 0.104 & 0.316 & 19.5 & 0.41 & 0.043 & 0.045 & -- & 0.002 & 0.151 & 12.9 & 0.225 & 12.2 & -- \\
\hline 6/30/2008 11:00 AM & 0.134 & 21.9 & 0.104 & 0.316 & 19.5 & 0.41 & 0.043 & 0.046 & -- & 0.002 & 0.153 & 12.9 & 0.224 & 12.2 & -- \\
\hline 6/30/2008 12:00 PM & 0.134 & 21.8 & 0.104 & 0.316 & 19.5 & 0.41 & 0.043 & 0.047 & -- & 0.002 & 0.153 & 12.9 & 0.224 & 12.2 & -- \\
\hline 6/30/2008 1:00 PM & 0.133 & 21.8 & 0.104 & 0.316 & 19.5 & 0.41 & 0.043 & 0.047 & -- & 0.002 & 0.153 & 12.9 & 0.224 & 12.2 & -- \\
\hline 6/30/2008 2:00 PM & 0.133 & 21.9 & 0.104 & 0.316 & 19.5 & 0.41 & 0.043 & 0.046 & -- & 0.002 & 0.151 & 12.9 & 0.225 & 12.2 & -- \\
\hline 6/30/2008 3:00 PM & 0.133 & 21.9 & 0.104 & 0.316 & 19.5 & 0.41 & 0.043 & 0.047 & -- & 0.002 & 0.151 & 12.9 & 0.225 & 12.2 & -- \\
\hline 6/30/2008 4:00 PM & 0.132 & 21.8 & 0.104 & 0.315 & 19.5 & 0.41 & 0.042 & 0.047 & -- & 0.002 & 0.151 & 12.9 & 0.225 & 12.2 & -- \\
\hline 6/30/2008 5:00 PM & 0.132 & 21.8 & 0.104 & 0.316 & 19.5 & 0.41 & 0.042 & 0.047 & -- & 0.002 & 0.151 & 12.9 & 0.225 & 12.2 & -- \\
\hline 6/30/2008 6:00 PM & 0.132 & 21.8 & 0.104 & 0.315 & 19.5 & 0.41 & 0.042 & 0.047 & -- & 0.002 & 0.151 & 12.9 & 0.225 & 12.2 & -- \\
\hline 6/30/2008 7:00 PM & 0.132 & 21.8 & 0.104 & 0.315 & 19.5 & 0.41 & 0.043 & 0.047 & -- & 0.002 & 0.153 & 12.9 & 0.225 & 12.2 & -- \\
\hline 6/30/2008 8:00 PM & 0.133 & 21.8 & 0.104 & 0.315 & 19.5 & 0.41 & 0.043 & 0.047 & -- & 0.002 & 0.151 & 12.9 & 0.226 & 12.2 & -- \\
\hline 6/30/2008 9:00 PM & 0.132 & 21.8 & 0.104 & 0.315 & 19.5 & 0.41 & 0.043 & 0.046 & -- & 0.002 & 0.151 & 12.9 & 0.226 & 12.2 & -- \\
\hline 6/30/2008 10:00 PM & 0.133 & 21.8 & 0.104 & 0.315 & 19.5 & 0.41 & 0.043 & 0.046 & -- & 0.002 & 0.153 & 12.9 & 0.226 & 12.2 & -- \\
\hline 6/30/2008 11:00 PM & 0.133 & 21.8 & 0.103 & 0.315 & 19.5 & 0.41 & 0.043 & 0.045 & -- & 0.002 & 0.153 & 12.9 & 0.226 & 12.2 & -- \\
\hline 7/1/2008 12:00 AM & 0.133 & 21.9 & 0.103 & 0.315 & 19.6 & 0.41 & 0.042 & 0.045 & -- & 0.003 & 0.151 & 12.9 & 0.226 & 12.2 & -- \\
\hline 7/1/2008 1:00 AM & 0.133 & 22.0 & 0.103 & 0.315 & 19.6 & 0.41 & 0.042 & 0.047 & -- & 0.003 & 0.151 & 12.9 & 0.226 & 12.2 & -- \\
\hline 7/1/2008 2:00 AM & 0.132 & 22.0 & 0.103 & 0.315 & 19.6 & 0.41 & 0.042 & 0.047 & -- & 0.003 & 0.153 & 12.9 & 0.226 & 12.2 & -- \\
\hline 7/1/2008 3:00 AM & 0.131 & 22.1 & 0.103 & 0.315 & 19.6 & 0.41 & 0.042 & 0.047 & -- & 0.003 & 0.153 & 12.9 & 0.226 & 12.3 & -- \\
\hline 7/1/2008 4:00 AM & 0.129 & 22.1 & 0.103 & 0.315 & 19.6 & 0.41 & 0.042 & 0.047 & -- & 0.003 & 0.153 & 12.9 & 0.228 & 12.2 & -- \\
\hline 7/1/2008 5:00 AM & 0.129 & 22.2 & 0.103 & 0.315 & 19.6 & 0.41 & 0.042 & 0.046 & -- & 0.003 & 0.153 & 12.9 & 0.226 & 12.2 & -- \\
\hline 7/1/2008 6:00 AM & 0.130 & 22.2 & 0.102 & 0.315 & 19.6 & 0.41 & 0.042 & 0.046 & -- & 0.004 & 0.153 & 12.9 & 0.226 & 12.2 & -- \\
\hline 7/1/2008 7:00 AM & 0.129 & 22.2 & 0.103 & 0.313 & 19.6 & 0.41 & 0.042 & 0.046 & -- & 0.004 & 0.153 & 12.9 & 0.228 & 12.2 & -- \\
\hline 7/1/2008 8:00 AM & 0.131 & 22.2 & 0.103 & 0.313 & 19.6 & 0.41 & 0.042 & 0.048 & -- & 0.004 & 0.153 & 12.9 & 0.228 & 12.2 & -- \\
\hline 7/1/2008 9:00 AM & 0.132 & 22.3 & 0.103 & 0.315 & 19.6 & 0.41 & 0.042 & 0.048 & -- & 0.004 & 0.153 & 12.9 & 0.229 & 12.3 & -- \\
\hline 7/1/2008 10:00 AM & 0.132 & 22.3 & 0.103 & 0.313 & 19.6 & 0.41 & 0.042 & 0.048 & -- & 0.004 & 0.153 & 13.0 & 0.229 & 12.3 & -- \\
\hline 7/1/2008 11:00 AM & 0.132 & 22.4 & 0.102 & 0.315 & 19.6 & 0.41 & 0.042 & 0.048 & -- & 0.004 & 0.154 & 13.0 & 0.229 & 12.3 & -- \\
\hline 7/1/2008 12:00 PM & 0.132 & 22.4 & 0.102 & 0.315 & 19.6 & 0.41 & 0.042 & 0.048 & -- & 0.004 & 0.154 & 13.0 & 0.229 & 12.3 & -- \\
\hline 7/1/2008 1:00 PM & 0.132 & 22.4 & 0.102 & 0.313 & 19.6 & 0.41 & 0.042 & 0.047 & -- & 0.005 & 0.154 & 13.0 & 0.230 & 12.3 & -- \\
\hline 7/1/2008 2:00 PM & 0.132 & 22.3 & 0.102 & 0.313 & 19.6 & 0.41 & 0.042 & 0.047 & -- & 0.005 & 0.153 & 13.0 & 0.230 & 12.2 & -- \\
\hline 7/1/2008 3:00 PM & 0.132 & 22.3 & 0.102 & 0.313 & 19.6 & 0.41 & 0.042 & 0.048 & -- & 0.005 & 0.153 & 13.0 & 0.230 & 12.3 & -- \\
\hline 7/1/2008 4:00 PM & 0.132 & 22.2 & 0.102 & 0.313 & 19.6 & 0.41 & 0.042 & 0.048 & -- & 0.006 & 0.151 & 13.0 & 0.230 & 12.3 & -- \\
\hline 7/1/2008 5:00 PM & 0.131 & 22.2 & 0.102 & 0.313 & 19.6 & 0.41 & 0.042 & 0.047 & -- & 0.006 & 0.153 & 13.0 & 0.231 & 12.3 & -- \\
\hline 7/1/2008 6:00 PM & 0.131 & 22.2 & 0.102 & 0.313 & 19.7 & 0.41 & 0.042 & 0.046 & -- & 0.006 & 0.153 & 13.0 & 0.231 & 12.3 & -- \\
\hline 7/1/2008 7:00 PM & 0.131 & 22.2 & 0.101 & 0.313 & 19.7 & 0.41 & 0.042 & 0.046 & -- & 0.006 & 0.153 & 13.0 & 0.231 & 12.3 & -- \\
\hline 7/1/2008 8:00 PM & 0.130 & 22.2 & 0.102 & 0.313 & 19.7 & 0.41 & 0.042 & 0.046 & -- & 0.007 & 0.154 & 13.0 & 0.231 & 12.3 & -- \\
\hline 7/1/2008 9:00 PM & 0.129 & 22.3 & 0.101 & 0.313 & 19.7 & 0.41 & 0.042 & 0.047 & -- & 0.007 & 0.154 & 13.0 & 0.231 & 12.3 & -- \\
\hline 7/1/2008 10:00 PM & 0.129 & 22.3 & 0.101 & 0.313 & 19.7 & 0.41 & 0.042 & 0.047 & -- & 0.008 & 0.154 & 13.0 & 0.231 & 12.3 & -- \\
\hline
\end{tabular}


Appendix 1. Volumetric water content, temperature, and electrical-conductivity data collected at the flood-irrigated site during 2008.-Continued

[Depth in feet below land surface; volumetric water content dimensionless; temperature in degrees Celsius; bulk electrical conductivity in decisiemens per meter; --, no data]

\begin{tabular}{|c|c|c|c|c|c|c|c|c|c|c|c|c|c|c|c|}
\hline \multirow[b]{3}{*}{$\begin{array}{l}\text { Measurement } \\
\text { date and time }\end{array}$} & \multicolumn{15}{|c|}{ Depth } \\
\hline & \multicolumn{2}{|c|}{2} & \multirow[b]{2}{*}{$\begin{array}{c}3 \\
\begin{array}{c}\text { Volumetric } \\
\text { water } \\
\text { content }\end{array}\end{array}$} & \multicolumn{3}{|c|}{4} & \multirow{2}{*}{$\begin{array}{c}6 \\
\text { Volumetric } \\
\text { water } \\
\text { content }\end{array}$} & \multicolumn{2}{|c|}{8} & \multirow{2}{*}{$\begin{array}{c}10 \\
\text { Volumetric } \\
\text { water } \\
\text { content }\end{array}$} & \multicolumn{2}{|c|}{12} & \multicolumn{2}{|l|}{15} & \multirow{2}{*}{$\begin{array}{c}18.5 \\
\text { Volumetric } \\
\text { water } \\
\text { content }\end{array}$} \\
\hline & $\begin{array}{c}\text { Volumetric } \\
\text { water } \\
\text { content }\end{array}$ & $\begin{array}{l}\text { Temper- } \\
\text { ature }\end{array}$ & & $\begin{array}{c}\text { Volumetric } \\
\text { water } \\
\text { content }\end{array}$ & $\begin{array}{l}\text { Temper- } \\
\text { ature }\end{array}$ & $\begin{array}{c}\text { Bulk } \\
\text { electrical } \\
\text { conduct- } \\
\text { ivity }\end{array}$ & & $\begin{array}{c}\text { Volumetric } \\
\text { water } \\
\text { content }\end{array}$ & $\begin{array}{l}\text { Temper- } \\
\text { ature }\end{array}$ & & $\begin{array}{c}\text { Volumetric } \\
\text { water } \\
\text { content }\end{array}$ & $\begin{array}{l}\text { Temper- } \\
\text { ature }\end{array}$ & $\begin{array}{c}\text { Volumetric } \\
\text { water } \\
\text { content }\end{array}$ & $\begin{array}{l}\text { Temper- } \\
\text { ature }\end{array}$ & \\
\hline 7/1/2008 11:00 PM & 0.128 & 22.3 & 0.101 & 0.313 & 19.7 & 0.41 & 0.042 & 0.046 & -- & 0.008 & 0.154 & 13.0 & 0.231 & 12.3 & -- \\
\hline 7/2/2008 12:00 AM & 0.126 & 22.3 & 0.101 & 0.313 & 19.7 & 0.41 & 0.042 & 0.047 & -- & 0.008 & 0.153 & 13.0 & 0.231 & 12.3 & -- \\
\hline 7/2/2008 1:00 AM & 0.126 & 22.3 & 0.101 & 0.313 & 19.7 & 0.41 & 0.042 & 0.047 & -- & 0.008 & 0.153 & 13.0 & 0.232 & 12.3 & -- \\
\hline 7/2/2008 2:00 AM & 0.126 & 22.3 & 0.101 & 0.313 & 19.7 & 0.41 & 0.042 & 0.047 & -- & 0.008 & 0.153 & 13.0 & 0.232 & 12.3 & -- \\
\hline 7/2/2008 3:00 AM & 0.126 & 22.3 & 0.101 & 0.313 & 19.7 & 0.41 & 0.042 & 0.047 & -- & 0.008 & 0.154 & 13.0 & 0.232 & 12.3 & -- \\
\hline 7/2/2008 4:00 AM & 0.128 & 22.4 & 0.101 & 0.312 & 19.7 & 0.41 & 0.042 & 0.047 & -- & 0.009 & 0.154 & 13.0 & 0.232 & 12.3 & -- \\
\hline 7/2/2008 5:00 AM & 0.129 & 22.4 & 0.101 & 0.313 & 19.7 & 0.41 & 0.042 & 0.047 & -- & 0.009 & 0.154 & 13.0 & 0.232 & 12.3 & -- \\
\hline 7/2/2008 6:00 AM & 0.129 & 22.4 & 0.101 & 0.313 & 19.7 & 0.41 & 0.042 & 0.047 & -- & 0.009 & 0.154 & 13.0 & 0.233 & 12.3 & -- \\
\hline 7/2/2008 7:00 AM & 0.126 & 22.4 & 0.101 & 0.312 & 19.8 & 0.41 & 0.042 & 0.047 & -- & 0.010 & 0.154 & 13.0 & 0.233 & 12.3 & -- \\
\hline 7/2/2008 8:00 AM & 0.126 & 22.4 & 0.101 & 0.313 & 19.8 & 0.41 & 0.042 & 0.046 & -- & 0.010 & 0.154 & 13.0 & 0.233 & 12.3 & -- \\
\hline 7/2/2008 9:00 AM & 0.128 & 22.4 & 0.100 & 0.313 & 19.8 & 0.41 & 0.042 & 0.046 & -- & 0.010 & 0.154 & 13.1 & 0.233 & 12.3 & -- \\
\hline 7/2/2008 10:00 AM & 0.130 & 22.4 & 0.100 & 0.312 & 19.8 & 0.41 & 0.042 & 0.045 & -- & 0.011 & 0.153 & 13.1 & 0.233 & 12.3 & -- \\
\hline 7/2/2008 11:00 AM & 0.130 & 22.4 & 0.100 & 0.312 & 19.8 & 0.41 & 0.042 & 0.045 & -- & 0.011 & 0.153 & 13.1 & 0.234 & 12.3 & -- \\
\hline 7/2/2008 12:00 PM & 0.130 & 22.4 & 0.100 & 0.312 & 19.8 & 0.41 & 0.042 & 0.047 & -- & 0.012 & 0.154 & 13.1 & 0.234 & 12.3 & -- \\
\hline 7/2/2008 1:00 PM & 0.130 & 22.4 & 0.100 & 0.312 & 19.8 & 0.41 & 0.042 & 0.047 & -- & 0.012 & 0.155 & 13.1 & 0.234 & 12.3 & -- \\
\hline 7/2/2008 2:00 PM & 0.129 & 22.4 & 0.100 & 0.312 & 19.8 & 0.41 & 0.042 & 0.047 & -- & 0.013 & 0.155 & 13.1 & 0.235 & 12.3 & -- \\
\hline 7/2/2008 3:00 PM & 0.130 & 22.3 & 0.100 & 0.312 & 19.8 & 0.41 & 0.042 & 0.047 & -- & 0.013 & 0.155 & 13.1 & 0.235 & 12.3 & -- \\
\hline 7/2/2008 4:00 PM & 0.145 & 22.4 & 0.099 & 0.312 & 19.8 & 0.41 & 0.042 & 0.047 & -- & 0.028 & 0.155 & 13.1 & 0.235 & 12.3 & -- \\
\hline 7/2/2008 5:00 PM & 0.159 & 22.3 & 0.099 & 0.311 & 19.8 & 0.41 & 0.042 & 0.047 & -- & 0.025 & 0.154 & 13.1 & 0.235 & 12.3 & -- \\
\hline 7/2/2008 6:00 PM & 0.160 & 22.3 & 0.102 & 0.312 & 19.8 & 0.41 & 0.042 & 0.047 & -- & 0.024 & 0.154 & 13.1 & 0.236 & 12.3 & -- \\
\hline 7/2/2008 7:00 PM & 0.162 & 22.3 & 0.106 & 0.312 & 19.8 & 0.41 & 0.042 & 0.047 & -- & 0.021 & 0.155 & 13.1 & 0.236 & 12.3 & -- \\
\hline 7/2/2008 8:00 PM & 0.171 & 22.4 & 0.111 & 0.312 & 19.9 & 0.41 & 0.042 & 0.046 & -- & 0.025 & 0.155 & 13.0 & 0.236 & 12.3 & -- \\
\hline 7/2/2008 9:00 PM & 0.178 & 22.5 & 0.115 & 0.312 & 19.9 & 0.41 & 0.042 & 0.046 & -- & 0.025 & 0.155 & 13.0 & 0.236 & 12.3 & -- \\
\hline 7/2/2008 10:00 PM & 0.179 & 22.5 & 0.116 & 0.313 & 19.9 & 0.41 & 0.042 & 0.046 & -- & 0.028 & 0.155 & 13.1 & 0.236 & 12.4 & -- \\
\hline 7/2/2008 11:00 PM & 0.178 & 22.6 & 0.117 & 0.313 & 19.9 & 0.41 & 0.042 & 0.045 & -- & 0.026 & 0.155 & 13.1 & 0.237 & 12.3 & -- \\
\hline 7/3/2008 12:00 AM & 0.175 & 22.6 & 0.118 & 0.315 & 19.9 & 0.41 & 0.042 & 0.047 & -- & 0.026 & 0.155 & 13.1 & 0.237 & 12.3 & -- \\
\hline 7/3/2008 1:00 AM & 0.173 & 22.6 & 0.118 & 0.316 & 19.9 & 0.42 & 0.042 & 0.047 & -- & 0.025 & 0.155 & 13.1 & 0.237 & 12.3 & -- \\
\hline 7/3/2008 2:00 AM & 0.170 & 22.7 & 0.118 & 0.317 & 19.9 & 0.42 & 0.042 & 0.047 & -- & 0.028 & 0.155 & 13.1 & 0.237 & 12.3 & -- \\
\hline 7/3/2008 3:00 AM & 0.170 & 22.7 & 0.117 & 0.318 & 19.9 & 0.42 & 0.042 & 0.047 & -- & 0.028 & 0.156 & 13.1 & 0.237 & 12.3 & -- \\
\hline 7/3/2008 4:00 AM & 0.168 & 22.6 & 0.117 & 0.318 & 19.9 & 0.42 & 0.042 & 0.046 & -- & 0.028 & 0.156 & 13.1 & 0.238 & 12.3 & -- \\
\hline 7/3/2008 5:00 AM & 0.165 & 22.7 & 0.117 & 0.319 & 19.9 & 0.42 & 0.042 & 0.046 & -- & 0.028 & 0.155 & 13.1 & 0.238 & 12.3 & -- \\
\hline 7/3/2008 6:00 AM & 0.162 & 22.7 & 0.117 & 0.319 & 20.0 & 0.42 & 0.042 & 0.046 & -- & 0.030 & 0.155 & 13.1 & 0.238 & 12.4 & -- \\
\hline 7/3/2008 7:00 AM & 0.162 & 22.7 & 0.116 & 0.319 & 20.0 & 0.42 & 0.042 & 0.046 & -- & 0.032 & 0.155 & 13.1 & 0.238 & 12.3 & -- \\
\hline 7/3/2008 8:00 AM & 0.165 & 22.6 & 0.116 & 0.319 & 20.0 & 0.42 & 0.042 & 0.046 & -- & 0.030 & 0.156 & 13.1 & 0.240 & 12.3 & -- \\
\hline 7/3/2008 9:00 AM & 0.166 & 22.6 & 0.116 & 0.319 & 20.0 & 0.43 & 0.042 & 0.046 & -- & 0.031 & 0.156 & 13.1 & 0.240 & 12.4 & -- \\
\hline 7/3/2008 10:00 AM & 0.165 & 22.6 & 0.115 & 0.319 & 20.0 & 0.43 & 0.042 & 0.046 & -- & 0.031 & 0.155 & 13.1 & 0.241 & 12.4 & -- \\
\hline 7/3/2008 11:00 AM & 0.161 & 22.5 & 0.115 & 0.319 & 20.0 & 0.43 & 0.042 & 0.046 & -- & 0.033 & 0.155 & 13.1 & 0.241 & 12.4 & -- \\
\hline 7/3/2008 12:00 PM & 0.158 & 22.5 & 0.115 & 0.319 & 20.0 & 0.43 & 0.042 & 0.046 & -- & 0.033 & 0.156 & 13.1 & 0.241 & 12.4 & -- \\
\hline 7/3/2008 1:00 PM & 0.158 & 22.4 & 0.115 & 0.320 & 20.0 & 0.43 & 0.042 & 0.046 & -- & 0.029 & 0.156 & 13.1 & 0.241 & 12.4 & -- \\
\hline 7/3/2008 2:00 PM & 0.158 & 22.4 & 0.115 & 0.320 & 20.0 & 0.43 & 0.042 & 0.046 & -- & 0.030 & 0.156 & 13.2 & 0.242 & 12.4 & -- \\
\hline
\end{tabular}


Appendix 1. Volumetric water content, temperature, and electrical-conductivity data collected at the flood-irrigated site during 2008.-Continued

[Depth in feet below land surface; volumetric water content dimensionless; temperature in degrees Celsius; bulk electrical conductivity in decisiemens per meter; --, no data]

\begin{tabular}{|c|c|c|c|c|c|c|c|c|c|c|c|c|c|c|c|}
\hline \multirow[b]{3}{*}{$\begin{array}{l}\text { Measurement } \\
\text { date and time }\end{array}$} & \multicolumn{15}{|c|}{ Depth } \\
\hline & \multirow{2}{*}{$\begin{array}{c}2 \\
\text { Volumetric } \\
\text { water } \\
\text { content }\end{array}$} & & \multirow{2}{*}{ 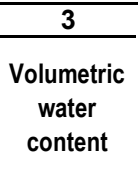 } & \multicolumn{3}{|c|}{4} & \multirow{2}{*}{$\begin{array}{c}6 \\
\begin{array}{c}\text { Volumetric } \\
\text { water } \\
\text { content }\end{array}\end{array}$} & \multicolumn{2}{|l|}{8} & \multirow{2}{*}{$\begin{array}{c}10 \\
\text { Volumetric } \\
\text { water } \\
\text { content }\end{array}$} & \multicolumn{2}{|c|}{12} & \multicolumn{2}{|c|}{15} & \multirow{2}{*}{$\begin{array}{c}18.5 \\
\text { Volumetric } \\
\text { water } \\
\text { content }\end{array}$} \\
\hline & & $\begin{array}{l}\text { Temper- } \\
\text { ature }\end{array}$ & & $\begin{array}{c}\text { Volumetric } \\
\text { water } \\
\text { content }\end{array}$ & $\begin{array}{l}\text { Temper- } \\
\text { ature }\end{array}$ & $\begin{array}{l}\text { Bulk } \\
\text { electrical } \\
\text { conduct- } \\
\text { ivity }\end{array}$ & & $\begin{array}{l}\text { Volumetric } \\
\text { water } \\
\text { content }\end{array}$ & $\begin{array}{l}\text { Temper- } \\
\text { ature }\end{array}$ & & $\begin{array}{c}\text { Volumetric } \\
\text { water } \\
\text { content }\end{array}$ & $\begin{array}{l}\text { Temper- } \\
\text { ature }\end{array}$ & $\begin{array}{l}\text { Volumetric } \\
\text { water } \\
\text { content }\end{array}$ & $\begin{array}{l}\text { Temper- } \\
\text { ature }\end{array}$ & \\
\hline 7/3/2008 3:00 PM & 0.158 & 22.3 & 0.114 & 0.319 & 20.1 & 0.43 & 0.042 & 0.046 & -- & 0.030 & 0.156 & 13.2 & 0.242 & 12.4 & -- \\
\hline 7/3/2008 4:00 PM & 0.158 & 22.3 & 0.114 & 0.319 & 20.1 & 0.43 & 0.042 & 0.046 & -- & 0.030 & 0.156 & 13.2 & 0.242 & 12.4 & -- \\
\hline 7/3/2008 5:00 PM & 0.158 & 22.3 & 0.114 & 0.319 & 20.1 & 0.43 & 0.042 & 0.046 & -- & 0.030 & 0.156 & 13.2 & 0.243 & 12.4 & -- \\
\hline 7/3/2008 6:00 PM & 0.157 & 22.2 & 0.113 & 0.319 & 20.1 & 0.43 & 0.042 & 0.046 & -- & 0.031 & 0.156 & 13.2 & 0.243 & 12.4 & -- \\
\hline 7/3/2008 7:00 PM & 0.156 & 22.1 & 0.113 & 0.320 & 20.1 & 0.43 & 0.042 & 0.046 & -- & 0.031 & 0.156 & 13.2 & 0.243 & 12.4 & -- \\
\hline 7/3/2008 8:00 PM & 0.155 & 22.1 & 0.113 & 0.319 & 20.1 & 0.43 & 0.043 & 0.045 & -- & 0.031 & 0.156 & 13.2 & 0.243 & 12.4 & -- \\
\hline 7/3/2008 9:00 PM & 0.154 & 22.1 & 0.113 & 0.319 & 20.1 & 0.43 & 0.043 & 0.047 & -- & 0.032 & 0.156 & 13.1 & 0.243 & 12.4 & -- \\
\hline 7/3/2008 10:00 PM & 0.153 & 22.0 & 0.112 & 0.319 & 20.1 & 0.43 & 0.043 & 0.046 & -- & 0.032 & 0.156 & 13.1 & 0.244 & 12.4 & -- \\
\hline 7/3/2008 11:00 PM & 0.151 & 22.0 & 0.112 & 0.319 & 20.1 & 0.43 & 0.043 & 0.045 & -- & 0.032 & 0.156 & 13.2 & 0.244 & 12.4 & -- \\
\hline 7/4/2008 12:00 AM & 0.150 & 22.0 & 0.112 & 0.319 & 20.1 & 0.43 & 0.043 & 0.046 & -- & 0.033 & 0.157 & 13.2 & 0.245 & 12.4 & -- \\
\hline 7/4/2008 1:00 AM & 0.150 & 22.0 & 0.111 & 0.319 & 20.1 & 0.43 & 0.043 & 0.046 & -- & 0.033 & 0.157 & 13.2 & 0.245 & 12.4 & -- \\
\hline 7/4/2008 2:00 AM & 0.150 & 22.1 & 0.111 & 0.319 & 20.1 & 0.43 & 0.043 & 0.046 & -- & 0.034 & 0.157 & 13.2 & 0.246 & 12.4 & -- \\
\hline 7/4/2008 3:00 AM & 0.149 & 22.1 & 0.111 & 0.319 & 20.1 & 0.43 & 0.043 & 0.046 & -- & 0.034 & 0.156 & 13.2 & 0.246 & 12.4 & -- \\
\hline 7/4/2008 4:00 AM & 0.148 & 22.1 & 0.111 & 0.319 & 20.2 & 0.43 & 0.043 & 0.046 & -- & 0.034 & 0.156 & 13.2 & 0.246 & 12.4 & -- \\
\hline 7/4/2008 5:00 AM & 0.147 & 22.1 & 0.110 & 0.319 & 20.2 & 0.43 & 0.043 & 0.046 & -- & 0.034 & 0.156 & 13.2 & 0.247 & 12.4 & -- \\
\hline 7/4/2008 6:00 AM & 0.147 & 22.1 & 0.110 & 0.318 & 20.2 & 0.43 & 0.043 & 0.046 & -- & 0.035 & 0.156 & 13.2 & 0.247 & 12.4 & -- \\
\hline 7/4/2008 7:00 AM & 0.147 & 22.1 & 0.110 & 0.318 & 20.2 & 0.43 & 0.043 & 0.046 & -- & 0.035 & 0.156 & 13.2 & 0.247 & 12.4 & -- \\
\hline 7/4/2008 8:00 AM & 0.148 & 22.1 & 0.110 & 0.319 & 20.2 & 0.43 & 0.043 & 0.046 & -- & 0.036 & 0.157 & 13.2 & 0.248 & 12.4 & -- \\
\hline 7/4/2008 9:00 AM & 0.149 & 22.1 & 0.110 & 0.319 & 20.2 & 0.43 & 0.043 & 0.046 & -- & 0.039 & 0.157 & 13.2 & 0.247 & 12.4 & -- \\
\hline 7/4/2008 10:00 AM & 0.148 & 22.0 & 0.109 & 0.318 & 20.2 & 0.43 & 0.043 & 0.046 & -- & 0.035 & 0.157 & 13.2 & 0.248 & 12.4 & -- \\
\hline 7/4/2008 11:00 AM & 0.147 & 22.0 & 0.109 & 0.319 & 20.2 & 0.43 & 0.043 & 0.046 & -- & 0.034 & 0.157 & 13.3 & 0.249 & 12.4 & -- \\
\hline 7/4/2008 12:00 PM & 0.147 & 22.0 & 0.109 & 0.318 & 20.2 & 0.43 & 0.043 & 0.046 & -- & 0.035 & 0.157 & 13.2 & 0.249 & 12.4 & -- \\
\hline 7/4/2008 1:00 PM & 0.147 & 22.0 & 0.109 & 0.318 & 20.2 & 0.43 & 0.043 & 0.047 & -- & 0.035 & 0.157 & 13.3 & 0.250 & 12.4 & -- \\
\hline 7/4/2008 2:00 PM & 0.146 & 21.9 & 0.109 & 0.318 & 20.2 & 0.43 & 0.043 & 0.047 & -- & 0.035 & 0.156 & 13.3 & 0.250 & 12.4 & -- \\
\hline 7/4/2008 3:00 PM & 0.145 & 21.9 & 0.109 & 0.318 & 20.2 & 0.43 & 0.043 & 0.046 & -- & 0.035 & 0.157 & 13.3 & 0.251 & 12.4 & -- \\
\hline 7/4/2008 4:00 PM & 0.145 & 21.9 & 0.109 & 0.318 & 20.2 & 0.43 & 0.043 & 0.046 & -- & 0.036 & 0.157 & 13.3 & 0.251 & 12.4 & -- \\
\hline 7/4/2008 5:00 PM & 0.145 & 21.9 & 0.109 & 0.318 & 20.2 & 0.43 & 0.043 & 0.046 & -- & 0.036 & 0.157 & 13.3 & 0.251 & 12.4 & -- \\
\hline 7/4/2008 6:00 PM & 0.144 & 21.8 & 0.109 & 0.318 & 20.2 & 0.43 & 0.043 & 0.046 & -- & 0.037 & 0.157 & 13.3 & 0.253 & 12.4 & -- \\
\hline 7/4/2008 7:00 PM & 0.144 & 21.8 & 0.108 & 0.318 & 20.2 & 0.43 & 0.043 & 0.046 & -- & 0.037 & 0.158 & 13.3 & 0.253 & 12.4 & -- \\
\hline 7/4/2008 8:00 PM & 0.144 & 21.8 & 0.108 & 0.318 & 20.2 & 0.43 & 0.044 & 0.046 & -- & 0.038 & 0.158 & 13.3 & 0.254 & 12.4 & -- \\
\hline 7/4/2008 9:00 PM & 0.144 & 21.8 & 0.108 & 0.318 & 20.2 & 0.43 & 0.044 & 0.046 & -- & 0.038 & 0.157 & 13.3 & 0.254 & 12.4 & -- \\
\hline 7/4/2008 10:00 PM & 0.144 & 21.8 & 0.108 & 0.317 & 20.2 & 0.43 & 0.044 & 0.046 & -- & 0.039 & 0.158 & 13.3 & 0.255 & 12.4 & -- \\
\hline 7/4/2008 11:00 PM & 0.144 & 21.8 & 0.108 & 0.317 & 20.2 & 0.43 & 0.043 & 0.046 & -- & 0.039 & 0.158 & 13.3 & 0.255 & 12.4 & -- \\
\hline 7/5/2008 12:00 AM & 0.144 & 21.8 & 0.108 & 0.317 & 20.2 & 0.43 & 0.043 & 0.047 & -- & 0.039 & 0.158 & 13.3 & 0.255 & 12.4 & -- \\
\hline 7/5/2008 1:00 AM & 0.144 & 21.9 & 0.107 & 0.317 & 20.2 & 0.43 & 0.043 & 0.047 & -- & 0.039 & 0.159 & 13.3 & 0.256 & 12.5 & -- \\
\hline 7/5/2008 2:00 AM & 0.143 & 21.9 & 0.107 & 0.317 & 20.2 & 0.43 & 0.043 & 0.047 & -- & 0.039 & 0.158 & 13.3 & 0.256 & 12.4 & -- \\
\hline 7/5/2008 3:00 AM & 0.142 & 22.0 & 0.107 & 0.317 & 20.2 & 0.43 & 0.043 & 0.046 & -- & 0.040 & 0.158 & 13.3 & 0.257 & 12.5 & -- \\
\hline 7/5/2008 4:00 AM & 0.141 & 22.0 & 0.107 & 0.317 & 20.2 & 0.43 & 0.043 & 0.046 & -- & 0.040 & 0.158 & 13.3 & 0.258 & 12.5 & -- \\
\hline 7/5/2008 5:00 AM & 0.141 & 22.0 & 0.107 & 0.317 & 20.2 & 0.43 & 0.043 & 0.046 & -- & 0.041 & 0.158 & 13.3 & 0.258 & 12.5 & -- \\
\hline 7/5/2008 6:00 AM & 0.140 & 22.0 & 0.107 & 0.317 & 20.2 & 0.43 & 0.043 & 0.046 & -- & 0.041 & 0.158 & 13.3 & 0.259 & 12.5 & -- \\
\hline
\end{tabular}


Appendix 1. Volumetric water content, temperature, and electrical-conductivity data collected at the flood-irrigated site during 2008.-Continued

[Depth in feet below land surface; volumetric water content dimensionless; temperature in degrees Celsius; bulk electrical conductivity in decisiemens per meter; --, no data]

\begin{tabular}{|c|c|c|c|c|c|c|c|c|c|c|c|c|c|c|c|}
\hline \multirow[b]{3}{*}{$\begin{array}{l}\text { Measurement } \\
\text { date and time }\end{array}$} & \multicolumn{15}{|c|}{ Depth } \\
\hline & \multicolumn{2}{|c|}{2} & \multirow[b]{2}{*}{$\begin{array}{c}3 \\
\begin{array}{c}\text { Volumetric } \\
\text { water } \\
\text { content }\end{array}\end{array}$} & \multicolumn{3}{|c|}{4} & \multirow{2}{*}{$\begin{array}{c}6 \\
\text { Volumetric } \\
\text { water } \\
\text { content }\end{array}$} & \multicolumn{2}{|l|}{8} & \multirow{2}{*}{$\begin{array}{c}10 \\
\begin{array}{c}\text { Volumetric } \\
\text { water } \\
\text { content }\end{array}\end{array}$} & \multicolumn{2}{|c|}{12} & \multicolumn{2}{|l|}{15} & \multirow{2}{*}{$\begin{array}{c}18.5 \\
\text { Volumetric } \\
\text { water } \\
\text { content }\end{array}$} \\
\hline & $\begin{array}{c}\text { Volumetric } \\
\text { water } \\
\text { content }\end{array}$ & $\begin{array}{l}\text { Temper- } \\
\text { ature }\end{array}$ & & $\begin{array}{c}\text { Volumetric } \\
\text { water } \\
\text { content }\end{array}$ & $\begin{array}{l}\text { Temper- } \\
\text { ature }\end{array}$ & $\begin{array}{c}\text { Bulk } \\
\text { electrical } \\
\text { conduct- } \\
\text { ivity }\end{array}$ & & $\begin{array}{c}\text { Volumetric } \\
\text { water } \\
\text { content }\end{array}$ & $\begin{array}{l}\text { Temper- } \\
\text { ature }\end{array}$ & & $\begin{array}{c}\text { Volumetric } \\
\text { water } \\
\text { content }\end{array}$ & $\begin{array}{l}\text { Temper- } \\
\text { ature }\end{array}$ & $\begin{array}{c}\text { Volumetric } \\
\text { water } \\
\text { content }\end{array}$ & $\begin{array}{l}\text { Temper- } \\
\text { ature }\end{array}$ & \\
\hline 7/5/2008 7:00 AM & 0.140 & 22.1 & 0.106 & 0.317 & 20.2 & 0.43 & 0.043 & 0.046 & -- & 0.041 & 0.158 & 13.3 & 0.259 & 12.5 & -- \\
\hline 7/5/2008 8:00 AM & 0.142 & 22.1 & 0.106 & 0.317 & 20.2 & 0.43 & 0.043 & 0.047 & -- & 0.041 & 0.159 & 13.3 & 0.260 & 12.4 & -- \\
\hline 7/5/2008 9:00 AM & 0.142 & 22.1 & 0.106 & 0.317 & 20.2 & 0.43 & 0.043 & 0.046 & -- & 0.042 & 0.159 & 13.3 & 0.260 & 12.5 & -- \\
\hline 7/5/2008 10:00 AM & 0.142 & 22.1 & 0.106 & 0.317 & 20.2 & 0.43 & 0.043 & 0.046 & -- & 0.042 & 0.159 & 13.3 & 0.261 & 12.5 & -- \\
\hline 7/5/2008 11:00 AM & 0.141 & 22.1 & 0.106 & 0.317 & 20.2 & 0.43 & 0.043 & 0.047 & -- & 0.042 & 0.160 & 13.4 & 0.262 & 12.5 & -- \\
\hline 7/5/2008 12:00 PM & 0.141 & 22.2 & 0.106 & 0.317 & 20.2 & 0.43 & 0.043 & 0.047 & -- & 0.042 & 0.160 & 13.4 & 0.263 & 12.5 & -- \\
\hline 7/5/2008 1:00 PM & 0.140 & 22.2 & 0.106 & 0.317 & 20.2 & 0.43 & 0.043 & 0.047 & -- & 0.042 & 0.159 & 13.4 & 0.265 & 12.5 & -- \\
\hline 7/5/2008 2:00 PM & 0.140 & 22.1 & 0.106 & 0.317 & 20.2 & 0.43 & 0.043 & 0.047 & -- & 0.042 & 0.159 & 13.4 & 0.265 & 12.5 & -- \\
\hline 7/5/2008 3:00 PM & 0.140 & 22.1 & 0.105 & 0.316 & 20.2 & 0.43 & 0.043 & 0.047 & -- & 0.042 & 0.160 & 13.4 & 0.266 & 12.5 & -- \\
\hline 7/5/2008 4:00 PM & 0.138 & 22.1 & 0.105 & 0.316 & 20.2 & 0.43 & 0.043 & 0.047 & -- & 0.042 & 0.160 & 13.4 & 0.266 & 12.5 & -- \\
\hline 7/5/2008 5:00 PM & 0.138 & 22.0 & 0.105 & 0.316 & 20.2 & 0.43 & 0.043 & 0.047 & -- & 0.042 & 0.160 & 13.4 & 0.267 & 12.5 & -- \\
\hline 7/5/2008 6:00 PM & 0.138 & 22.0 & 0.105 & 0.316 & 20.2 & 0.43 & 0.043 & 0.047 & -- & 0.043 & 0.160 & 13.4 & 0.267 & 12.5 & -- \\
\hline 7/5/2008 7:00 PM & 0.138 & 22.0 & 0.105 & 0.316 & 20.2 & 0.43 & 0.043 & 0.047 & -- & 0.043 & 0.160 & 13.4 & 0.268 & 12.5 & -- \\
\hline 7/5/2008 8:00 PM & 0.138 & 22.0 & 0.105 & 0.316 & 20.2 & 0.43 & 0.043 & 0.047 & -- & 0.043 & 0.160 & 13.3 & 0.268 & 12.5 & -- \\
\hline 7/5/2008 9:00 PM & 0.138 & 22.0 & 0.105 & 0.316 & 20.2 & 0.43 & 0.043 & 0.047 & -- & 0.044 & 0.160 & 13.3 & 0.269 & 12.5 & -- \\
\hline 7/5/2008 10:00 PM & 0.138 & 22.0 & 0.104 & 0.316 & 20.2 & 0.43 & 0.043 & 0.047 & -- & 0.046 & 0.161 & 13.4 & 0.269 & 12.5 & -- \\
\hline 7/5/2008 11:00 PM & 0.138 & 22.1 & 0.104 & 0.316 & 20.2 & 0.43 & 0.043 & 0.047 & -- & 0.042 & 0.161 & 13.4 & 0.270 & 12.5 & -- \\
\hline 7/6/2008 12:00 AM & 0.137 & 22.1 & 0.104 & 0.316 & 20.2 & 0.43 & 0.043 & 0.047 & -- & 0.042 & 0.161 & 13.4 & 0.270 & 12.5 & -- \\
\hline 7/6/2008 1:00 AM & 0.136 & 22.1 & 0.104 & 0.316 & 20.2 & 0.43 & 0.043 & 0.047 & -- & 0.042 & 0.161 & 13.4 & 0.271 & 12.5 & -- \\
\hline 7/6/2008 2:00 AM & 0.136 & 22.2 & 0.104 & 0.316 & 20.2 & 0.43 & 0.043 & 0.047 & -- & 0.042 & 0.161 & 13.4 & 0.272 & 12.5 & -- \\
\hline 7/6/2008 3:00 AM & 0.135 & 22.2 & 0.104 & 0.316 & 20.2 & 0.43 & 0.043 & 0.047 & -- & 0.042 & 0.161 & 13.4 & 0.273 & 12.5 & -- \\
\hline 7/6/2008 4:00 AM & 0.135 & 22.2 & 0.104 & 0.315 & 20.2 & 0.43 & 0.043 & 0.047 & -- & 0.042 & 0.161 & 13.4 & 0.274 & 12.5 & -- \\
\hline 7/6/2008 5:00 AM & 0.135 & 22.2 & 0.104 & 0.315 & 20.2 & 0.43 & 0.043 & 0.047 & -- & 0.042 & 0.161 & 13.4 & 0.274 & 12.5 & -- \\
\hline 7/6/2008 6:00 AM & 0.134 & 22.2 & 0.104 & 0.315 & 20.2 & 0.43 & 0.043 & 0.047 & -- & 0.042 & 0.161 & 13.4 & 0.275 & 12.5 & -- \\
\hline 7/6/2008 7:00 AM & 0.133 & 22.2 & 0.104 & 0.315 & 20.2 & 0.43 & 0.043 & 0.047 & -- & 0.042 & 0.161 & 13.4 & 0.276 & 12.5 & -- \\
\hline 7/6/2008 8:00 AM & 0.134 & 22.3 & 0.104 & 0.315 & 20.2 & 0.43 & 0.043 & 0.047 & -- & 0.042 & 0.161 & 13.4 & 0.276 & 12.5 & -- \\
\hline 7/6/2008 9:00 AM & 0.135 & 22.3 & 0.104 & 0.315 & 20.2 & 0.43 & 0.043 & 0.047 & -- & 0.043 & 0.162 & 13.4 & 0.278 & 12.5 & -- \\
\hline 7/6/2008 10:00 AM & 0.136 & 22.3 & 0.104 & 0.315 & 20.2 & 0.43 & 0.043 & 0.047 & -- & 0.043 & 0.162 & 13.4 & 0.279 & 12.5 & -- \\
\hline 7/6/2008 11:00 AM & 0.136 & 22.3 & 0.104 & 0.315 & 20.2 & 0.43 & 0.043 & 0.047 & -- & 0.043 & 0.162 & 13.4 & 0.280 & 12.5 & -- \\
\hline 7/6/2008 12:00 PM & 0.136 & 22.2 & 0.104 & 0.315 & 20.2 & 0.43 & 0.043 & 0.047 & -- & 0.045 & 0.162 & 13.4 & 0.281 & 12.5 & -- \\
\hline 7/6/2008 1:00 PM & 0.136 & 22.3 & 0.104 & 0.315 & 20.2 & 0.43 & 0.042 & 0.047 & -- & 0.045 & 0.163 & 13.4 & 0.282 & 12.5 & -- \\
\hline 7/6/2008 2:00 PM & 0.135 & 22.3 & 0.103 & 0.315 & 20.2 & 0.43 & 0.042 & 0.047 & -- & 0.047 & 0.163 & 13.4 & 0.283 & 12.5 & -- \\
\hline 7/6/2008 3:00 PM & 0.135 & 22.2 & 0.103 & 0.315 & 20.2 & 0.43 & 0.043 & 0.047 & -- & 0.046 & 0.162 & 13.5 & 0.283 & 12.5 & -- \\
\hline 7/6/2008 4:00 PM & 0.135 & 22.2 & 0.103 & 0.315 & 20.2 & 0.43 & 0.043 & 0.047 & -- & 0.047 & 0.162 & 13.4 & 0.284 & 12.5 & -- \\
\hline 7/6/2008 5:00 PM & 0.135 & 22.2 & 0.103 & 0.315 & 20.2 & 0.43 & 0.043 & 0.047 & -- & 0.047 & 0.163 & 13.4 & 0.285 & 12.6 & -- \\
\hline 7/6/2008 6:00 PM & 0.135 & 22.1 & 0.103 & 0.315 & 20.2 & 0.43 & 0.043 & 0.047 & -- & 0.047 & 0.163 & 13.5 & 0.286 & 12.5 & -- \\
\hline 7/6/2008 7:00 PM & 0.134 & 22.2 & 0.103 & 0.315 & 20.2 & 0.43 & 0.043 & 0.047 & -- & 0.047 & 0.165 & 13.4 & 0.287 & 12.5 & -- \\
\hline 7/6/2008 8:00 PM & 0.133 & 22.2 & 0.103 & 0.315 & 20.2 & 0.43 & 0.043 & 0.047 & -- & 0.046 & 0.165 & 13.5 & 0.287 & 12.6 & -- \\
\hline 7/6/2008 9:00 PM & 0.132 & 22.2 & 0.103 & 0.315 & 20.2 & 0.43 & 0.043 & 0.047 & -- & 0.046 & 0.163 & 13.4 & 0.288 & 12.6 & -- \\
\hline 7/6/2008 10:00 PM & 0.132 & 22.2 & 0.103 & 0.313 & 20.2 & 0.43 & 0.042 & 0.047 & -- & 0.045 & 0.163 & 13.4 & 0.290 & 12.6 & -- \\
\hline
\end{tabular}


Appendix 1. Volumetric water content, temperature, and electrical-conductivity data collected at the flood-irrigated site during 2008.-Continued

[Depth in feet below land surface; volumetric water content dimensionless; temperature in degrees Celsius; bulk electrical conductivity in decisiemens per meter; --, no data]

\begin{tabular}{|c|c|c|c|c|c|c|c|c|c|c|c|c|c|c|c|}
\hline \multirow[b]{3}{*}{$\begin{array}{l}\text { Measurement } \\
\text { date and time }\end{array}$} & \multicolumn{15}{|c|}{ Depth } \\
\hline & \multicolumn{2}{|l|}{2} & \multirow{2}{*}{$\begin{array}{c}3 \\
\begin{array}{c}\text { Volumetric } \\
\text { water } \\
\text { content }\end{array}\end{array}$} & \multicolumn{3}{|c|}{4} & \multirow{2}{*}{$\begin{array}{c}6 \\
\text { Volumetric } \\
\text { water } \\
\text { content }\end{array}$} & \multicolumn{2}{|l|}{8} & \multirow{2}{*}{$\begin{array}{c}10 \\
\text { Volumetric } \\
\text { water } \\
\text { content }\end{array}$} & \multicolumn{2}{|c|}{12} & \multicolumn{2}{|c|}{15} & \multirow{2}{*}{$\begin{array}{c}18.5 \\
\text { Volumetric } \\
\text { water } \\
\text { content }\end{array}$} \\
\hline & $\begin{array}{l}\text { Volumetric } \\
\text { water } \\
\text { content }\end{array}$ & $\begin{array}{l}\text { Temper- } \\
\text { ature }\end{array}$ & & $\begin{array}{c}\text { Volumetric } \\
\text { water } \\
\text { content }\end{array}$ & $\begin{array}{l}\text { Temper- } \\
\text { ature }\end{array}$ & $\begin{array}{c}\text { Bulk } \\
\text { electrical } \\
\text { conduct- } \\
\text { ivity } \\
\end{array}$ & & $\begin{array}{l}\text { Volumetric } \\
\text { water } \\
\text { content }\end{array}$ & $\begin{array}{l}\text { Temper- } \\
\text { ature }\end{array}$ & & $\begin{array}{l}\text { Volumetric } \\
\text { water } \\
\text { content }\end{array}$ & $\begin{array}{l}\text { Temper- } \\
\text { ature }\end{array}$ & $\begin{array}{l}\text { Volumetric } \\
\text { water } \\
\text { content }\end{array}$ & $\begin{array}{l}\text { Temper- } \\
\text { ature }\end{array}$ & \\
\hline 7/6/2008 11:00 PM & 0.131 & 22.2 & 0.103 & 0.313 & 20.3 & 0.43 & 0.042 & 0.047 & -- & 0.045 & 0.163 & 13.5 & 0.290 & 12.5 & -- \\
\hline 7/7/2008 12:00 AM & 0.130 & 22.2 & 0.102 & 0.313 & 20.3 & 0.43 & 0.042 & 0.047 & -- & 0.046 & 0.165 & 13.5 & 0.291 & 12.6 & -- \\
\hline 7/7/2008 1:00 AM & 0.130 & 22.3 & 0.102 & 0.313 & 20.3 & 0.43 & 0.042 & 0.047 & -- & 0.046 & 0.165 & 13.4 & 0.292 & 12.6 & -- \\
\hline 7/7/2008 2:00 AM & 0.130 & 22.3 & 0.102 & 0.313 & 20.3 & 0.43 & 0.042 & 0.047 & -- & 0.045 & 0.165 & 13.5 & 0.293 & 12.6 & -- \\
\hline 7/7/2008 3:00 AM & 0.131 & 22.3 & 0.102 & 0.313 & 20.3 & 0.43 & 0.042 & 0.047 & -- & 0.045 & 0.165 & 13.5 & 0.294 & 12.6 & -- \\
\hline 7/7/2008 4:00 AM & 0.132 & 22.3 & 0.102 & 0.313 & 20.3 & 0.43 & 0.042 & 0.046 & -- & 0.045 & 0.166 & 13.5 & 0.295 & 12.6 & -- \\
\hline 7/7/2008 5:00 AM & 0.131 & 22.3 & 0.102 & 0.313 & 20.3 & 0.43 & 0.042 & 0.047 & -- & 0.046 & 0.166 & 13.5 & 0.296 & 12.6 & -- \\
\hline 7/7/2008 6:00 AM & 0.131 & 22.4 & 0.102 & 0.313 & 20.3 & 0.43 & 0.042 & 0.047 & -- & 0.047 & 0.166 & 13.5 & 0.297 & 12.6 & -- \\
\hline 7/7/2008 7:00 AM & 0.130 & 22.4 & 0.102 & 0.313 & 20.3 & 0.43 & 0.042 & 0.047 & -- & 0.047 & 0.166 & 13.5 & 0.297 & 12.6 & -- \\
\hline 7/7/2008 8:00 AM & 0.129 & 22.4 & 0.102 & 0.313 & 20.3 & 0.43 & 0.042 & 0.047 & -- & 0.047 & 0.166 & 13.5 & 0.298 & 12.6 & -- \\
\hline 7/7/2008 9:00 AM & 0.129 & 22.3 & 0.102 & 0.313 & 20.3 & 0.43 & 0.042 & 0.047 & -- & 0.050 & 0.166 & 13.5 & 0.299 & 12.6 & -- \\
\hline 7/7/2008 10:00 AM & 0.130 & 22.3 & 0.101 & 0.313 & 20.3 & 0.43 & 0.042 & 0.046 & -- & 0.047 & 0.167 & 13.5 & 0.301 & 12.6 & -- \\
\hline 7/7/2008 11:00 AM & 0.132 & 22.3 & 0.101 & 0.313 & 20.3 & 0.43 & 0.042 & 0.046 & -- & 0.042 & 0.167 & 13.5 & 0.303 & 12.6 & -- \\
\hline 7/7/2008 12:00 PM & 0.132 & 22.3 & 0.101 & 0.313 & 20.3 & 0.43 & 0.042 & 0.046 & -- & 0.043 & 0.168 & 13.5 & 0.303 & 12.6 & -- \\
\hline 7/7/2008 1:00 PM & 0.132 & 22.3 & 0.101 & 0.313 & 20.3 & 0.43 & 0.042 & 0.046 & -- & 0.045 & 0.168 & 13.5 & 0.304 & 12.6 & -- \\
\hline 7/7/2008 2:00 PM & 0.132 & 22.3 & 0.101 & 0.313 & 20.3 & 0.43 & 0.042 & 0.046 & -- & 0.060 & 0.167 & 13.5 & 0.305 & 12.6 & -- \\
\hline 7/7/2008 3:00 PM & 0.131 & 22.3 & 0.101 & 0.313 & 20.3 & 0.43 & 0.042 & 0.046 & -- & 0.077 & 0.167 & 13.5 & 0.306 & 12.6 & -- \\
\hline 7/7/2008 4:00 PM & 0.131 & 22.3 & 0.101 & 0.313 & 20.3 & 0.43 & 0.042 & 0.047 & -- & 0.061 & 0.168 & 13.5 & 0.308 & 12.6 & -- \\
\hline 7/7/2008 5:00 PM & 0.131 & 22.2 & 0.101 & 0.313 & 20.3 & 0.43 & 0.042 & 0.046 & -- & 0.063 & 0.168 & 13.5 & 0.308 & 12.6 & -- \\
\hline 7/7/2008 6:00 PM & 0.129 & 22.2 & 0.101 & 0.313 & 20.3 & 0.43 & 0.042 & 0.047 & -- & 0.059 & 0.168 & 13.5 & 0.309 & 12.6 & -- \\
\hline 7/7/2008 7:00 PM & 0.129 & 22.2 & 0.100 & 0.313 & 20.3 & 0.43 & 0.042 & 0.047 & -- & 0.067 & 0.168 & 13.5 & 0.310 & 12.6 & -- \\
\hline 7/7/2008 8:00 PM & 0.130 & 22.2 & 0.100 & 0.313 & 20.3 & 0.43 & 0.042 & 0.046 & -- & 0.065 & 0.168 & 13.5 & 0.311 & 12.6 & -- \\
\hline 7/7/2008 9:00 PM & 0.129 & 22.1 & 0.100 & 0.313 & 20.3 & 0.43 & 0.042 & 0.047 & -- & 0.056 & 0.168 & 13.5 & 0.312 & 12.6 & -- \\
\hline 7/7/2008 10:00 PM & 0.128 & 22.1 & 0.100 & 0.313 & 20.3 & 0.43 & 0.042 & 0.047 & -- & 0.048 & 0.168 & 13.5 & 0.313 & 12.6 & -- \\
\hline 7/7/2008 11:00 PM & 0.128 & 22.1 & 0.100 & 0.312 & 20.3 & 0.43 & 0.042 & 0.047 & -- & 0.047 & 0.169 & 13.5 & 0.315 & 12.6 & -- \\
\hline 7/8/2008 12:00 AM & 0.128 & 22.1 & 0.100 & 0.312 & 20.3 & 0.43 & 0.042 & 0.046 & -- & 0.047 & 0.169 & 13.5 & 0.316 & 12.6 & -- \\
\hline 7/8/2008 1:00 AM & 0.126 & 22.1 & 0.100 & 0.312 & 20.3 & 0.43 & 0.042 & 0.046 & -- & 0.047 & 0.169 & 13.5 & 0.317 & 12.6 & -- \\
\hline 7/8/2008 2:00 AM & 0.126 & 22.1 & 0.100 & 0.312 & 20.3 & 0.43 & 0.042 & 0.046 & -- & 0.047 & 0.169 & 13.5 & 0.318 & 12.6 & -- \\
\hline 7/8/2008 3:00 AM & 0.126 & 22.1 & 0.100 & 0.312 & 20.3 & 0.43 & 0.042 & 0.046 & -- & 0.047 & 0.169 & 13.5 & 0.319 & 12.6 & -- \\
\hline 7/8/2008 4:00 AM & 0.126 & 22.1 & 0.100 & 0.312 & 20.3 & 0.43 & 0.042 & 0.046 & -- & 0.047 & 0.169 & 13.5 & 0.320 & 12.7 & -- \\
\hline 7/8/2008 5:00 AM & 0.125 & 22.1 & 0.099 & 0.312 & 20.3 & 0.43 & 0.042 & 0.046 & -- & 0.047 & 0.169 & 13.6 & 0.321 & 12.7 & -- \\
\hline 7/8/2008 6:00 AM & 0.125 & 22.1 & 0.099 & 0.312 & 20.3 & 0.43 & 0.042 & 0.046 & -- & 0.047 & 0.170 & 13.6 & 0.322 & 12.6 & -- \\
\hline 7/8/2008 7:00 AM & 0.125 & 22.1 & 0.099 & 0.312 & 20.3 & 0.43 & 0.042 & 0.046 & -- & 0.046 & 0.170 & 13.5 & 0.323 & 12.7 & -- \\
\hline 7/8/2008 8:00 AM & 0.128 & 22.1 & 0.099 & 0.312 & 20.3 & 0.43 & 0.042 & 0.046 & -- & 0.045 & 0.170 & 13.5 & 0.324 & 12.6 & -- \\
\hline 7/8/2008 9:00 AM & 0.129 & 22.1 & 0.099 & 0.312 & 20.3 & 0.43 & 0.042 & 0.046 & -- & 0.044 & 0.170 & 13.6 & 0.325 & 12.6 & -- \\
\hline 7/8/2008 10:00 AM & 0.128 & 22.0 & 0.099 & 0.312 & 20.3 & 0.43 & 0.042 & 0.046 & -- & 0.045 & 0.171 & 13.6 & 0.326 & 12.7 & -- \\
\hline 7/8/2008 11:00 AM & 0.128 & 22.0 & 0.099 & 0.312 & 20.3 & 0.43 & 0.042 & 0.046 & -- & 0.047 & 0.171 & 13.6 & 0.328 & 12.6 & -- \\
\hline 7/8/2008 12:00 PM & 0.128 & 22.0 & 0.099 & 0.312 & 20.3 & 0.43 & 0.042 & 0.046 & -- & 0.048 & 0.171 & 13.6 & 0.329 & 12.7 & -- \\
\hline 7/8/2008 1:00 PM & 0.126 & 22.0 & 0.099 & 0.312 & 20.3 & 0.43 & 0.042 & 0.046 & -- & 0.047 & 0.171 & 13.6 & 0.331 & 12.7 & -- \\
\hline 7/8/2008 2:00 PM & 0.126 & 22.0 & 0.099 & 0.312 & 20.4 & 0.43 & 0.042 & 0.046 & -- & 0.047 & 0.172 & 13.6 & 0.333 & 12.6 & -- \\
\hline
\end{tabular}


Appendix 1. Volumetric water content, temperature, and electrical-conductivity data collected at the flood-irrigated site during 2008.-Continued

[Depth in feet below land surface; volumetric water content dimensionless; temperature in degrees Celsius; bulk electrical conductivity in decisiemens per meter; --, no data]

\begin{tabular}{|c|c|c|c|c|c|c|c|c|c|c|c|c|c|c|c|}
\hline \multirow[b]{3}{*}{$\begin{array}{l}\text { Measurement } \\
\text { date and time }\end{array}$} & \multicolumn{15}{|c|}{ Depth } \\
\hline & \multicolumn{2}{|l|}{2} & \multirow{2}{*}{$\begin{array}{c}3 \\
\begin{array}{c}\text { Volumetric } \\
\text { water } \\
\text { content }\end{array}\end{array}$} & \multicolumn{3}{|c|}{4} & \multirow{2}{*}{$\begin{array}{c}6 \\
\begin{array}{c}\text { Volumetric } \\
\text { water } \\
\text { content }\end{array}\end{array}$} & \multicolumn{2}{|l|}{8} & \multirow{2}{*}{$\begin{array}{c}10 \\
\begin{array}{c}\text { Volumetric } \\
\text { water } \\
\text { content }\end{array}\end{array}$} & \multicolumn{2}{|c|}{12} & \multicolumn{2}{|c|}{15} & \multirow{2}{*}{$\begin{array}{c}18.5 \\
\text { Volumetric } \\
\text { water } \\
\text { content }\end{array}$} \\
\hline & $\begin{array}{l}\text { Volumetric } \\
\text { water } \\
\text { content }\end{array}$ & $\begin{array}{l}\text { Temper- } \\
\text { ature }\end{array}$ & & $\begin{array}{c}\text { Volumetric } \\
\text { water } \\
\text { content }\end{array}$ & $\begin{array}{l}\text { Temper- } \\
\text { ature }\end{array}$ & $\begin{array}{c}\text { Bulk } \\
\text { electrical } \\
\text { conduct- } \\
\text { ivity }\end{array}$ & & $\begin{array}{l}\text { Volumetric } \\
\text { water } \\
\text { content }\end{array}$ & $\begin{array}{l}\text { Temper- } \\
\text { ature }\end{array}$ & & $\begin{array}{c}\text { Volumetric } \\
\text { water } \\
\text { content }\end{array}$ & $\begin{array}{l}\text { Temper- } \\
\text { ature }\end{array}$ & $\begin{array}{l}\text { Volumetric } \\
\text { water } \\
\text { content }\end{array}$ & $\begin{array}{l}\text { Temper- } \\
\text { ature }\end{array}$ & \\
\hline 7/8/2008 3:00 PM & 0.126 & 21.9 & 0.099 & 0.312 & 20.4 & 0.43 & 0.042 & 0.046 & -- & 0.047 & 0.172 & 13.6 & 0.333 & 12.7 & -- \\
\hline 7/8/2008 4:00 PM & 0.126 & 21.9 & 0.099 & 0.312 & 20.4 & 0.43 & 0.042 & 0.046 & -- & 0.047 & 0.173 & 13.6 & 0.334 & 12.7 & -- \\
\hline 7/8/2008 5:00 PM & 0.126 & 21.8 & 0.099 & 0.312 & 20.4 & 0.43 & 0.042 & 0.046 & -- & 0.047 & 0.173 & 13.6 & 0.335 & 12.7 & -- \\
\hline 7/8/2008 6:00 PM & 0.126 & 21.8 & 0.099 & 0.312 & 20.4 & 0.43 & 0.042 & 0.046 & -- & 0.047 & 0.173 & 13.6 & 0.337 & 12.7 & -- \\
\hline 7/8/2008 7:00 PM & 0.126 & 21.8 & 0.099 & 0.312 & 20.4 & 0.43 & 0.042 & 0.046 & -- & 0.047 & 0.173 & 13.6 & 0.338 & 12.7 & -- \\
\hline 7/8/2008 8:00 PM & 0.126 & 21.8 & 0.098 & 0.312 & 20.4 & 0.43 & 0.042 & 0.046 & -- & 0.047 & 0.172 & 13.6 & 0.341 & 12.7 & -- \\
\hline 7/8/2008 9:00 PM & 0.125 & 21.8 & 0.098 & 0.312 & 20.4 & 0.43 & 0.042 & 0.046 & -- & 0.047 & 0.172 & 13.6 & 0.341 & 12.7 & -- \\
\hline 7/8/2008 10:00 PM & 0.126 & 21.8 & 0.098 & 0.312 & 20.4 & 0.43 & 0.042 & 0.046 & -- & 0.047 & 0.173 & 13.6 & 0.343 & 12.7 & -- \\
\hline 7/8/2008 11:00 PM & 0.126 & 21.7 & 0.098 & 0.312 & 20.4 & 0.43 & 0.042 & 0.046 & -- & 0.047 & 0.173 & 13.6 & 0.344 & 12.7 & -- \\
\hline 7/9/2008 12:00 AM & 0.126 & 21.8 & 0.098 & 0.312 & 20.4 & 0.43 & 0.042 & 0.046 & -- & 0.047 & 0.173 & 13.6 & 0.345 & 12.7 & -- \\
\hline 7/9/2008 1:00 AM & 0.126 & 21.8 & 0.098 & 0.312 & 20.4 & 0.43 & 0.042 & 0.046 & -- & 0.047 & 0.173 & 13.6 & 0.346 & 12.7 & -- \\
\hline 7/9/2008 2:00 AM & 0.126 & 21.8 & 0.098 & 0.311 & 20.4 & 0.43 & 0.042 & 0.046 & -- & 0.047 & 0.173 & 13.6 & 0.347 & 12.7 & -- \\
\hline 7/9/2008 3:00 AM & 0.125 & 21.8 & 0.098 & 0.311 & 20.4 & 0.43 & 0.042 & 0.046 & -- & 0.046 & 0.173 & 13.6 & 0.349 & 12.7 & -- \\
\hline 7/9/2008 4:00 AM & 0.125 & 21.8 & 0.098 & 0.311 & 20.4 & 0.43 & 0.042 & 0.046 & -- & 0.046 & 0.174 & 13.6 & 0.350 & 12.7 & -- \\
\hline 7/9/2008 5:00 AM & 0.125 & 21.9 & 0.098 & 0.311 & 20.4 & 0.43 & 0.042 & 0.046 & -- & 0.046 & 0.174 & 13.6 & 0.353 & 12.7 & -- \\
\hline 7/9/2008 6:00 AM & 0.125 & 21.9 & 0.098 & 0.311 & 20.4 & 0.43 & 0.042 & 0.046 & -- & 0.046 & 0.174 & 13.6 & 0.354 & 12.7 & -- \\
\hline 7/9/2008 7:00 AM & 0.125 & 21.8 & 0.098 & 0.311 & 20.4 & 0.43 & 0.042 & 0.046 & -- & 0.046 & 0.174 & 13.6 & 0.355 & 12.7 & -- \\
\hline 7/9/2008 8:00 AM & 0.125 & 21.8 & 0.098 & 0.311 & 20.4 & 0.43 & 0.042 & 0.046 & -- & 0.046 & 0.174 & 13.6 & 0.356 & 12.7 & -- \\
\hline 7/9/2008 9:00 AM & 0.125 & 21.8 & 0.098 & 0.311 & 20.4 & 0.43 & 0.042 & 0.046 & -- & 0.046 & 0.175 & 13.6 & 0.357 & 12.7 & -- \\
\hline 7/9/2008 10:00 AM & 0.129 & 21.9 & 0.098 & 0.311 & 20.4 & 0.43 & 0.042 & 0.046 & -- & 0.047 & 0.175 & 13.6 & 0.359 & 12.7 & -- \\
\hline 7/9/2008 11:00 AM & 0.173 & 21.9 & 0.098 & 0.311 & 20.4 & 0.43 & 0.042 & 0.046 & -- & 0.047 & 0.176 & 13.6 & 0.362 & 12.7 & -- \\
\hline 7/9/2008 12:00 PM & 0.200 & 21.9 & 0.098 & 0.311 & 20.4 & 0.43 & 0.042 & 0.046 & -- & 0.047 & 0.176 & 13.6 & 0.365 & 12.7 & -- \\
\hline 7/9/2008 1:00 PM & 0.209 & 21.9 & 0.102 & 0.311 & 20.4 & 0.43 & 0.042 & 0.046 & -- & 0.047 & 0.176 & 13.6 & 0.367 & 12.7 & -- \\
\hline 7/9/2008 2:00 PM & 0.213 & 21.9 & 0.109 & 0.311 & 20.4 & 0.43 & 0.042 & 0.046 & -- & 0.046 & 0.176 & 13.6 & 0.369 & 12.7 & -- \\
\hline 7/9/2008 3:00 PM & 0.211 & 21.9 & 0.115 & 0.311 & 20.4 & 0.43 & 0.042 & 0.046 & -- & 0.045 & 0.176 & 13.6 & 0.372 & 12.7 & -- \\
\hline 7/9/2008 4:00 PM & 0.205 & 21.8 & 0.117 & 0.311 & 20.4 & 0.43 & 0.042 & 0.046 & -- & 0.053 & 0.176 & 13.6 & 0.373 & 12.7 & -- \\
\hline 7/9/2008 5:00 PM & 0.198 & 21.8 & 0.117 & 0.312 & 20.4 & 0.43 & 0.042 & 0.046 & -- & 0.053 & 0.178 & 13.6 & 0.376 & 12.7 & -- \\
\hline 7/9/2008 6:00 PM & 0.194 & 21.7 & 0.117 & 0.312 & 20.4 & 0.43 & 0.042 & 0.046 & -- & 0.048 & 0.178 & 13.7 & 0.379 & 12.7 & -- \\
\hline 7/9/2008 7:00 PM & 0.190 & 21.7 & 0.117 & 0.313 & 20.4 & 0.43 & 0.042 & 0.046 & -- & 0.047 & 0.179 & 13.6 & 0.379 & 12.7 & -- \\
\hline 7/9/2008 8:00 PM & 0.186 & 21.7 & 0.116 & 0.315 & 20.4 & 0.43 & 0.042 & 0.046 & -- & 0.047 & 0.179 & 13.6 & 0.381 & 12.7 & -- \\
\hline 7/9/2008 9:00 PM & 0.183 & 21.7 & 0.115 & 0.315 & 20.4 & 0.44 & 0.042 & 0.046 & -- & 0.047 & 0.178 & 13.6 & 0.383 & 12.7 & -- \\
\hline 7/9/2008 10:00 PM & 0.180 & 21.7 & 0.115 & 0.316 & 20.4 & 0.44 & 0.042 & 0.046 & -- & 0.046 & 0.178 & 13.6 & 0.384 & 12.7 & -- \\
\hline 7/9/2008 11:00 PM & 0.178 & 21.7 & 0.115 & 0.316 & 20.4 & 0.44 & 0.042 & 0.046 & -- & 0.046 & 0.179 & 13.6 & 0.385 & 12.7 & -- \\
\hline 7/10/2008 12:00 AM & 0.175 & 21.7 & 0.114 & 0.316 & 20.4 & 0.44 & 0.042 & 0.045 & -- & 0.046 & 0.179 & 13.6 & 0.386 & 12.7 & -- \\
\hline 7/10/2008 1:00 AM & 0.173 & 21.7 & 0.113 & 0.316 & 20.4 & 0.44 & 0.042 & 0.045 & -- & 0.046 & 0.179 & 13.6 & 0.387 & 12.7 & -- \\
\hline 7/10/2008 2:00 AM & 0.172 & 21.7 & 0.113 & 0.316 & 20.4 & 0.44 & 0.042 & 0.045 & -- & 0.046 & 0.180 & 13.7 & 0.390 & 12.7 & -- \\
\hline 7/10/2008 3:00 AM & 0.170 & 21.7 & 0.112 & 0.316 & 20.4 & 0.44 & 0.042 & 0.045 & -- & 0.046 & 0.179 & 13.6 & 0.391 & 12.7 & -- \\
\hline 7/10/2008 4:00 AM & 0.169 & 21.8 & 0.112 & 0.316 & 20.4 & 0.44 & 0.042 & 0.046 & -- & 0.046 & 0.180 & 13.6 & 0.392 & 12.7 & -- \\
\hline 7/10/2008 5:00 AM & 0.167 & 21.8 & 0.111 & 0.316 & 20.4 & 0.44 & 0.042 & 0.046 & -- & 0.046 & 0.180 & 13.6 & 0.392 & 12.7 & -- \\
\hline 7/10/2008 6:00 AM & 0.166 & 21.8 & 0.111 & 0.316 & 20.4 & 0.44 & 0.042 & 0.046 & -- & 0.046 & 0.181 & 13.6 & 0.393 & 12.7 & -- \\
\hline
\end{tabular}


Appendix 1. Volumetric water content, temperature, and electrical-conductivity data collected at the flood-irrigated site during 2008.-Continued

[Depth in feet below land surface; volumetric water content dimensionless; temperature in degrees Celsius; bulk electrical conductivity in decisiemens per meter; --, no data]

\begin{tabular}{|c|c|c|c|c|c|c|c|c|c|c|c|c|c|c|c|}
\hline \multirow[b]{3}{*}{$\begin{array}{l}\text { Measurement } \\
\text { date and time }\end{array}$} & \multicolumn{15}{|c|}{ Depth } \\
\hline & \multicolumn{2}{|c|}{2} & \multirow{2}{*}{$\begin{array}{c}3 \\
\begin{array}{c}\text { Volumetric } \\
\text { water } \\
\text { content }\end{array}\end{array}$} & \multicolumn{3}{|c|}{4} & \multirow{2}{*}{$\begin{array}{c}6 \\
\begin{array}{c}\text { Volumetric } \\
\text { water } \\
\text { content }\end{array}\end{array}$} & \multicolumn{2}{|l|}{8} & \multirow{2}{*}{$\begin{array}{c}10 \\
\text { Volumetric } \\
\text { water } \\
\text { content }\end{array}$} & \multicolumn{2}{|c|}{12} & \multicolumn{2}{|c|}{15} & \multirow{2}{*}{$\begin{array}{c}18.5 \\
\text { Volumetric } \\
\text { water } \\
\text { content }\end{array}$} \\
\hline & $\begin{array}{l}\text { Volumetric } \\
\text { water } \\
\text { content }\end{array}$ & $\begin{array}{l}\text { Temper- } \\
\text { ature }\end{array}$ & & $\begin{array}{c}\text { Volumetric } \\
\text { water } \\
\text { content }\end{array}$ & $\begin{array}{l}\text { Temper- } \\
\text { ature }\end{array}$ & $\begin{array}{c}\text { Bulk } \\
\text { electrical } \\
\text { conduct- } \\
\text { ivity } \\
\end{array}$ & & $\begin{array}{l}\text { Volumetric } \\
\text { water } \\
\text { content }\end{array}$ & $\begin{array}{l}\text { Temper- } \\
\text { ature }\end{array}$ & & $\begin{array}{l}\text { Volumetric } \\
\text { water } \\
\text { content }\end{array}$ & $\begin{array}{l}\text { Temper- } \\
\text { ature }\end{array}$ & $\begin{array}{l}\text { Volumetric } \\
\text { water } \\
\text { content }\end{array}$ & $\begin{array}{l}\text { Temper- } \\
\text { ature }\end{array}$ & \\
\hline 7/10/2008 7:00 AM & 0.163 & 21.8 & 0.110 & 0.316 & 20.4 & 0.44 & 0.042 & 0.046 & -- & 0.046 & 0.181 & 13.7 & 0.395 & 12.7 & -- \\
\hline 7/10/2008 8:00 AM & 0.162 & 21.8 & 0.110 & 0.316 & 20.4 & 0.44 & 0.042 & 0.046 & -- & 0.045 & 0.181 & 13.6 & 0.395 & 12.7 & -- \\
\hline 7/10/2008 9:00 AM & 0.161 & 21.8 & 0.109 & 0.316 & 20.4 & 0.44 & 0.042 & 0.045 & -- & 0.045 & 0.181 & 13.7 & 0.397 & 12.7 & -- \\
\hline 7/10/2008 10:00 AM & 0.160 & 21.8 & 0.109 & 0.316 & 20.4 & 0.44 & 0.042 & 0.045 & -- & 0.045 & 0.182 & 13.6 & 0.399 & 12.7 & -- \\
\hline 7/10/2008 11:00 AM & 0.158 & 21.8 & 0.109 & 0.316 & 20.4 & 0.44 & 0.042 & 0.046 & -- & 0.045 & 0.183 & 13.6 & 0.400 & 12.7 & -- \\
\hline 7/10/2008 12:00 PM & 0.157 & 21.8 & 0.109 & 0.316 & 20.4 & 0.44 & 0.041 & 0.046 & -- & 0.045 & 0.183 & 13.7 & 0.401 & 12.8 & -- \\
\hline 7/10/2008 1:00 PM & 0.156 & 21.8 & 0.108 & 0.316 & 20.4 & 0.44 & 0.042 & 0.046 & -- & 0.045 & 0.183 & 13.7 & 0.403 & 12.7 & -- \\
\hline 7/10/2008 2:00 PM & 0.155 & 21.8 & 0.108 & 0.316 & 20.4 & 0.44 & 0.042 & 0.046 & -- & 0.045 & 0.182 & 13.7 & 0.405 & 12.8 & -- \\
\hline 7/10/2008 3:00 PM & 0.154 & 21.8 & 0.108 & 0.316 & 20.4 & 0.44 & 0.042 & 0.046 & -- & 0.045 & 0.183 & 13.7 & 0.405 & 12.8 & -- \\
\hline 7/10/2008 4:00 PM & 0.153 & 21.7 & 0.107 & 0.316 & 20.4 & 0.44 & 0.042 & 0.046 & -- & 0.045 & 0.183 & 13.7 & 0.406 & 12.7 & -- \\
\hline 7/10/2008 5:00 PM & 0.151 & 21.7 & 0.107 & 0.316 & 20.4 & 0.44 & 0.042 & 0.046 & -- & 0.045 & 0.184 & 13.7 & 0.407 & 12.7 & -- \\
\hline 7/10/2008 6:00 PM & 0.151 & 21.7 & 0.107 & 0.316 & 20.4 & 0.44 & 0.042 & 0.046 & -- & 0.045 & 0.184 & 13.7 & 0.408 & 12.8 & -- \\
\hline 7/10/2008 7:00 PM & 0.151 & 21.7 & 0.107 & 0.316 & 20.4 & 0.44 & 0.042 & 0.045 & -- & 0.045 & 0.185 & 13.7 & 0.409 & 12.8 & -- \\
\hline 7/10/2008 8:00 PM & 0.150 & 21.7 & 0.106 & 0.316 & 20.4 & 0.44 & 0.042 & 0.045 & -- & 0.045 & 0.184 & 13.7 & 0.409 & 12.8 & -- \\
\hline 7/10/2008 9:00 PM & 0.150 & 21.7 & 0.106 & 0.316 & 20.4 & 0.44 & 0.042 & 0.045 & -- & 0.045 & 0.184 & 13.7 & 0.409 & 12.8 & -- \\
\hline 7/10/2008 10:00 PM & 0.149 & 21.7 & 0.106 & 0.316 & 20.4 & 0.44 & 0.042 & 0.045 & -- & 0.045 & 0.185 & 13.7 & 0.410 & 12.8 & -- \\
\hline 7/10/2008 11:00 PM & 0.149 & 21.7 & 0.106 & 0.315 & 20.4 & 0.44 & 0.042 & 0.045 & -- & 0.045 & 0.185 & 13.7 & 0.410 & 12.8 & -- \\
\hline 7/11/2008 12:00 AM & 0.149 & 21.7 & 0.105 & 0.315 & 20.4 & 0.43 & 0.042 & 0.045 & -- & 0.045 & 0.185 & 13.7 & 0.411 & 12.8 & -- \\
\hline 7/11/2008 1:00 AM & 0.148 & 21.8 & 0.105 & 0.315 & 20.4 & 0.43 & 0.042 & 0.045 & -- & 0.045 & 0.185 & 13.7 & 0.412 & 12.8 & -- \\
\hline 7/11/2008 2:00 AM & 0.148 & 21.8 & 0.105 & 0.315 & 20.4 & 0.43 & 0.042 & 0.045 & -- & 0.045 & 0.185 & 13.7 & 0.413 & 12.8 & -- \\
\hline 7/11/2008 3:00 AM & 0.147 & 21.9 & 0.105 & 0.315 & 20.4 & 0.43 & 0.042 & 0.045 & -- & 0.045 & 0.185 & 13.7 & 0.415 & 12.8 & -- \\
\hline 7/11/2008 4:00 AM & 0.147 & 21.9 & 0.104 & 0.315 & 20.4 & 0.43 & 0.042 & 0.045 & -- & 0.045 & 0.186 & 13.7 & 0.416 & 12.8 & -- \\
\hline 7/11/2008 5:00 AM & 0.146 & 21.9 & 0.104 & 0.315 & 20.4 & 0.43 & 0.042 & 0.045 & -- & 0.045 & 0.186 & 13.7 & 0.417 & 12.8 & -- \\
\hline 7/11/2008 6:00 AM & 0.144 & 21.9 & 0.104 & 0.315 & 20.4 & 0.43 & 0.042 & 0.045 & -- & 0.046 & 0.187 & 13.7 & 0.417 & 12.8 & -- \\
\hline 7/11/2008 7:00 AM & 0.144 & 21.9 & 0.104 & 0.315 & 20.4 & 0.43 & 0.042 & 0.045 & -- & 0.049 & 0.187 & 13.7 & 0.417 & 12.8 & -- \\
\hline 7/11/2008 8:00 AM & 0.145 & 21.9 & 0.104 & 0.315 & 20.4 & 0.43 & 0.042 & 0.045 & -- & 0.051 & 0.187 & 13.7 & 0.416 & 12.8 & -- \\
\hline 7/11/2008 9:00 AM & 0.145 & 21.9 & 0.104 & 0.315 & 20.4 & 0.43 & 0.042 & 0.045 & -- & 0.053 & 0.187 & 13.7 & 0.416 & 12.8 & -- \\
\hline 7/11/2008 10:00 AM & 0.144 & 21.9 & 0.104 & 0.315 & 20.4 & 0.43 & 0.042 & 0.045 & -- & 0.070 & 0.187 & 13.7 & 0.416 & 12.8 & -- \\
\hline 7/11/2008 11:00 AM & 0.143 & 22.0 & 0.103 & 0.315 & 20.4 & 0.43 & 0.042 & 0.045 & -- & 0.070 & 0.188 & 13.8 & 0.417 & 12.8 & -- \\
\hline 7/11/2008 12:00 PM & 0.143 & 22.0 & 0.103 & 0.315 & 20.4 & 0.43 & 0.042 & 0.045 & -- & 0.092 & 0.190 & 13.8 & 0.416 & 12.8 & -- \\
\hline 7/11/2008 1:00 PM & 0.143 & 22.0 & 0.103 & 0.315 & 20.4 & 0.43 & 0.042 & 0.045 & -- & 0.110 & 0.190 & 13.8 & 0.417 & 12.8 & -- \\
\hline 7/11/2008 2:00 PM & 0.142 & 22.0 & 0.103 & 0.315 & 20.4 & 0.43 & 0.042 & 0.045 & -- & 0.076 & 0.188 & 13.8 & 0.418 & 12.8 & -- \\
\hline 7/11/2008 3:00 PM & 0.142 & 21.9 & 0.103 & 0.315 & 20.4 & 0.43 & 0.042 & 0.045 & -- & 0.081 & 0.190 & 13.8 & 0.418 & 12.8 & -- \\
\hline 7/11/2008 4:00 PM & 0.141 & 21.9 & 0.102 & 0.315 & 20.4 & 0.43 & 0.042 & 0.045 & -- & 0.061 & 0.190 & 13.8 & 0.417 & 12.8 & -- \\
\hline 7/11/2008 5:00 PM & 0.141 & 21.9 & 0.102 & 0.315 & 20.4 & 0.43 & 0.042 & 0.045 & -- & 0.063 & 0.190 & 13.8 & 0.417 & 12.8 & -- \\
\hline 7/11/2008 6:00 PM & 0.141 & 22.0 & 0.102 & 0.313 & 20.4 & 0.43 & 0.042 & 0.045 & -- & 0.058 & 0.191 & 13.8 & 0.416 & 12.8 & -- \\
\hline 7/11/2008 7:00 PM & 0.141 & 21.9 & 0.102 & 0.313 & 20.4 & 0.43 & 0.042 & 0.045 & -- & 0.058 & 0.191 & 13.8 & 0.417 & 12.8 & -- \\
\hline 7/11/2008 8:00 PM & 0.140 & 21.9 & 0.102 & 0.313 & 20.4 & 0.43 & 0.042 & 0.045 & -- & 0.056 & 0.191 & 13.8 & 0.417 & 12.8 & -- \\
\hline 7/11/2008 9:00 PM & 0.140 & 21.9 & 0.102 & 0.313 & 20.4 & 0.43 & 0.042 & 0.045 & -- & 0.056 & 0.191 & 13.8 & 0.418 & 12.8 & -- \\
\hline 7/11/2008 10:00 PM & 0.140 & 22.0 & 0.102 & 0.313 & 20.4 & 0.43 & 0.042 & 0.045 & -- & 0.109 & 0.191 & 13.8 & 0.418 & 12.8 & -- \\
\hline
\end{tabular}


Appendix 1. Volumetric water content, temperature, and electrical-conductivity data collected at the flood-irrigated site during 2008.-Continued

[Depth in feet below land surface; volumetric water content dimensionless; temperature in degrees Celsius; bulk electrical conductivity in decisiemens per meter; --, no data]

\begin{tabular}{|c|c|c|c|c|c|c|c|c|c|c|c|c|c|c|c|}
\hline \multirow[b]{3}{*}{$\begin{array}{l}\text { Measurement } \\
\text { date and time }\end{array}$} & \multicolumn{15}{|c|}{ Depth } \\
\hline & \multicolumn{2}{|c|}{2} & \multirow{2}{*}{$\begin{array}{c}3 \\
\begin{array}{c}\text { Volumetric } \\
\text { water } \\
\text { content }\end{array}\end{array}$} & \multicolumn{3}{|c|}{4} & \multirow{2}{*}{$\begin{array}{c}6 \\
\text { Volumetric } \\
\text { water } \\
\text { content }\end{array}$} & \multicolumn{2}{|l|}{8} & \multirow{2}{*}{$\begin{array}{c}10 \\
\begin{array}{c}\text { Volumetric } \\
\text { water } \\
\text { content }\end{array}\end{array}$} & \multicolumn{2}{|l|}{12} & \multicolumn{2}{|c|}{15} & \multirow{2}{*}{$\begin{array}{c}18.5 \\
\text { Volumetric } \\
\text { water } \\
\text { content }\end{array}$} \\
\hline & $\begin{array}{c}\text { Volumetric } \\
\text { water } \\
\text { content }\end{array}$ & $\begin{array}{l}\text { Temper- } \\
\text { ature }\end{array}$ & & $\begin{array}{l}\text { Volumetric } \\
\text { water } \\
\text { content }\end{array}$ & $\begin{array}{l}\text { Temper- } \\
\text { ature }\end{array}$ & $\begin{array}{c}\text { Bulk } \\
\text { electrical } \\
\text { conduct- } \\
\text { ivity }\end{array}$ & & $\begin{array}{c}\text { Volumetric } \\
\text { water } \\
\text { content }\end{array}$ & $\begin{array}{l}\text { Temper- } \\
\text { ature }\end{array}$ & & $\begin{array}{c}\text { Volumetric } \\
\text { water } \\
\text { content }\end{array}$ & $\begin{array}{l}\text { Temper- } \\
\text { ature }\end{array}$ & $\begin{array}{c}\text { Volumetric } \\
\text { water } \\
\text { content }\end{array}$ & $\begin{array}{l}\text { Temper- } \\
\text { ature }\end{array}$ & \\
\hline 7/11/2008 11:00 PM & 0.140 & 22.0 & 0.101 & 0.313 & 20.4 & 0.43 & 0.042 & 0.045 & - & 0.144 & 0.191 & 13.8 & 0.418 & 12.9 & -- \\
\hline 7/12/2008 12:00 AM & 0.140 & 22.0 & 0.101 & 0.313 & 20.4 & 0.43 & 0.042 & 0.045 & -- & 0.160 & 0.191 & 13.7 & 0.419 & 12.8 & -- \\
\hline 7/12/2008 1:00 AM & 0.137 & 22.0 & 0.101 & 0.313 & 20.4 & 0.43 & 0.042 & 0.045 & -- & 0.109 & 0.191 & 13.8 & 0.419 & 12.9 & -- \\
\hline 7/12/2008 2:00 AM & 0.136 & 22.1 & 0.101 & 0.313 & 20.4 & 0.43 & 0.042 & 0.045 & -- & 0.082 & 0.191 & 13.8 & 0.419 & 12.8 & -- \\
\hline 7/12/2008 3:00 AM & 0.135 & 22.1 & 0.101 & 0.313 & 20.4 & 0.43 & 0.042 & 0.045 & -- & 0.091 & 0.192 & 13.8 & 0.419 & 12.8 & -- \\
\hline 7/12/2008 4:00 AM & 0.134 & 22.1 & 0.101 & 0.313 & 20.4 & 0.43 & 0.042 & 0.045 & -- & 0.062 & 0.192 & 13.8 & 0.418 & 12.8 & -- \\
\hline 7/12/2008 5:00 AM & 0.136 & 22.2 & 0.100 & 0.312 & 20.4 & 0.43 & 0.042 & 0.045 & -- & 0.062 & 0.192 & 13.7 & 0.418 & 12.8 & -- \\
\hline 7/12/2008 6:00 AM & 0.136 & 22.2 & 0.100 & 0.312 & 20.4 & 0.43 & 0.042 & 0.045 & -- & 0.127 & 0.193 & 13.8 & 0.418 & 12.8 & -- \\
\hline 7/12/2008 7:00 AM & 0.136 & 22.3 & 0.100 & 0.312 & 20.4 & 0.43 & 0.042 & 0.045 & -- & 0.069 & 0.193 & 13.8 & 0.418 & 12.8 & -- \\
\hline 7/12/2008 8:00 AM & 0.134 & 22.2 & 0.100 & 0.312 & 20.4 & 0.43 & 0.042 & 0.045 & -- & 0.080 & 0.192 & 13.8 & 0.419 & 12.8 & -- \\
\hline 7/12/2008 9:00 AM & 0.134 & 22.2 & 0.100 & 0.312 & 20.4 & 0.43 & 0.042 & 0.045 & -- & 0.058 & 0.193 & 13.8 & 0.419 & 12.8 & -- \\
\hline 7/12/2008 10:00 AM & 0.136 & 22.2 & 0.100 & 0.313 & 20.4 & 0.43 & 0.042 & 0.045 & -- & 0.061 & 0.193 & 13.8 & 0.419 & 12.9 & -- \\
\hline 7/12/2008 11:00 AM & 0.136 & 22.2 & 0.100 & 0.312 & 20.4 & 0.43 & 0.042 & 0.045 & -- & 0.065 & 0.193 & 13.8 & 0.420 & 12.9 & -- \\
\hline 7/12/2008 12:00 PM & 0.136 & 22.2 & 0.099 & 0.312 & 20.4 & 0.43 & 0.042 & 0.045 & -- & 0.061 & 0.193 & 13.8 & 0.420 & 12.9 & -- \\
\hline 7/12/2008 1:00 PM & 0.136 & 22.2 & 0.099 & 0.312 & 20.4 & 0.43 & 0.042 & 0.045 & -- & 0.061 & 0.194 & 13.8 & 0.420 & 12.9 & -- \\
\hline 7/12/2008 2:00 PM & 0.136 & 22.2 & 0.099 & 0.312 & 20.4 & 0.43 & 0.041 & 0.045 & -- & 0.062 & 0.195 & 13.8 & 0.420 & 12.9 & -- \\
\hline 7/12/2008 3:00 PM & 0.136 & 22.2 & 0.099 & 0.312 & 20.4 & 0.43 & 0.041 & 0.045 & -- & 0.063 & 0.195 & 13.8 & 0.420 & 12.9 & -- \\
\hline 7/12/2008 4:00 PM & 0.135 & 22.2 & 0.099 & 0.312 & 20.4 & 0.43 & 0.041 & 0.045 & -- & 0.067 & 0.195 & 13.8 & 0.420 & 12.9 & -- \\
\hline 7/12/2008 5:00 PM & 0.135 & 22.2 & 0.099 & 0.312 & 20.4 & 0.43 & 0.041 & 0.045 & -- & 0.061 & 0.195 & 13.8 & 0.420 & 12.9 & -- \\
\hline 7/12/2008 6:00 PM & 0.135 & 22.2 & 0.099 & 0.312 & 20.5 & 0.43 & 0.041 & 0.045 & -- & 0.069 & 0.196 & 13.8 & 0.419 & 12.9 & -- \\
\hline 7/12/2008 7:00 PM & 0.134 & 22.1 & 0.099 & 0.312 & 20.5 & 0.43 & 0.042 & 0.045 & -- & 0.064 & 0.195 & 13.8 & 0.418 & 12.9 & -- \\
\hline 7/12/2008 8:00 PM & 0.135 & 22.1 & 0.098 & 0.312 & 20.5 & 0.43 & 0.042 & 0.045 & -- & 0.061 & 0.195 & 13.8 & 0.418 & 12.9 & -- \\
\hline 7/12/2008 9:00 PM & 0.133 & 22.1 & 0.099 & 0.312 & 20.5 & 0.43 & 0.042 & 0.045 & -- & 0.063 & 0.195 & 13.8 & 0.419 & 12.9 & -- \\
\hline 7/12/2008 10:00 PM & 0.132 & 22.2 & 0.098 & 0.312 & 20.5 & 0.43 & 0.042 & 0.045 & -- & 0.064 & 0.196 & 13.8 & 0.419 & 12.9 & -- \\
\hline 7/12/2008 11:00 PM & 0.132 & 22.1 & 0.098 & 0.312 & 20.5 & 0.43 & 0.041 & 0.045 & -- & 0.064 & 0.196 & 13.8 & 0.419 & 12.9 & -- \\
\hline 7/13/2008 12:00 AM & 0.130 & 22.2 & 0.098 & 0.312 & 20.5 & 0.43 & 0.041 & 0.045 & -- & 0.061 & 0.196 & 13.8 & 0.419 & 12.9 & -- \\
\hline 7/13/2008 1:00 AM & 0.130 & 22.2 & 0.098 & 0.312 & 20.5 & 0.43 & 0.041 & 0.045 & -- & 0.057 & 0.196 & 13.8 & 0.419 & 12.9 & -- \\
\hline 7/13/2008 2:00 AM & 0.131 & 22.3 & 0.098 & 0.312 & 20.5 & 0.43 & 0.041 & 0.045 & -- & 0.056 & 0.197 & 13.8 & 0.419 & 12.9 & -- \\
\hline 7/13/2008 3:00 AM & 0.132 & 22.4 & 0.098 & 0.312 & 20.5 & 0.43 & 0.041 & 0.045 & -- & 0.058 & 0.197 & 13.8 & 0.419 & 12.9 & -- \\
\hline 7/13/2008 4:00 AM & 0.132 & 22.4 & 0.098 & 0.312 & 20.5 & 0.43 & 0.041 & 0.045 & -- & 0.058 & 0.197 & 13.8 & 0.419 & 12.9 & -- \\
\hline 7/13/2008 5:00 AM & 0.133 & 22.4 & 0.098 & 0.312 & 20.5 & 0.43 & 0.041 & 0.045 & -- & 0.067 & 0.197 & 13.8 & 0.419 & 12.9 & -- \\
\hline 7/13/2008 6:00 AM & 0.134 & 22.4 & 0.098 & 0.312 & 20.5 & 0.43 & 0.041 & 0.045 & -- & 0.081 & 0.197 & 13.8 & 0.419 & 12.9 & -- \\
\hline 7/13/2008 7:00 AM & 0.133 & 22.4 & 0.098 & 0.312 & 20.5 & 0.43 & 0.041 & 0.045 & -- & 0.060 & 0.198 & 13.9 & 0.418 & 12.9 & -- \\
\hline 7/13/2008 8:00 AM & 0.131 & 22.4 & 0.098 & 0.311 & 20.5 & 0.43 & 0.041 & 0.045 & -- & 0.057 & 0.198 & 13.8 & 0.418 & 12.9 & -- \\
\hline 7/13/2008 9:00 AM & 0.129 & 22.3 & 0.098 & 0.312 & 20.5 & 0.43 & 0.041 & 0.045 & -- & 0.060 & 0.197 & 13.8 & 0.418 & 12.9 & -- \\
\hline 7/13/2008 10:00 AM & 0.131 & 22.3 & 0.098 & 0.312 & 20.5 & 0.43 & 0.041 & 0.045 & -- & 0.059 & 0.198 & 13.9 & 0.419 & 12.9 & -- \\
\hline 7/13/2008 11:00 AM & 0.132 & 22.3 & 0.098 & 0.312 & 20.5 & 0.43 & 0.041 & 0.045 & -- & 0.072 & 0.198 & 13.9 & 0.418 & 12.9 & -- \\
\hline 7/13/2008 12:00 PM & 0.132 & 22.3 & 0.098 & 0.312 & 20.5 & 0.43 & 0.041 & 0.045 & -- & 0.088 & 0.199 & 13.9 & 0.418 & 12.9 & -- \\
\hline 7/13/2008 1:00 PM & 0.132 & 22.3 & 0.098 & 0.312 & 20.5 & 0.43 & 0.041 & 0.045 & -- & 0.061 & 0.199 & 13.9 & 0.418 & 12.9 & -- \\
\hline 7/13/2008 2:00 PM & 0.131 & 22.3 & 0.098 & 0.312 & 20.5 & 0.43 & 0.041 & 0.045 & -- & 0.087 & 0.200 & 13.9 & 0.418 & 12.9 & -- \\
\hline
\end{tabular}


Appendix 1. Volumetric water content, temperature, and electrical-conductivity data collected at the flood-irrigated site during 2008.-Continued

[Depth in feet below land surface; volumetric water content dimensionless; temperature in degrees Celsius; bulk electrical conductivity in decisiemens per meter; --, no data]

\begin{tabular}{|c|c|c|c|c|c|c|c|c|c|c|c|c|c|c|c|}
\hline \multirow[b]{3}{*}{$\begin{array}{l}\text { Measurement } \\
\text { date and time }\end{array}$} & \multicolumn{15}{|c|}{ Depth } \\
\hline & \multicolumn{2}{|c|}{2} & \multirow{2}{*}{$\begin{array}{c}3 \\
\begin{array}{c}\text { Volumetric } \\
\text { water } \\
\text { content }\end{array}\end{array}$} & \multicolumn{3}{|c|}{4} & \multirow{2}{*}{$\begin{array}{c}6 \\
\text { Volumetric } \\
\text { water } \\
\text { content }\end{array}$} & \multicolumn{2}{|l|}{8} & \multirow{2}{*}{$\begin{array}{c}10 \\
\text { Volumetric } \\
\text { water } \\
\text { content }\end{array}$} & \multicolumn{2}{|c|}{12} & \multicolumn{2}{|c|}{15} & \multirow{2}{*}{$\begin{array}{c}18.5 \\
\text { Volumetric } \\
\text { water } \\
\text { content }\end{array}$} \\
\hline & $\begin{array}{l}\text { Volumetric } \\
\text { water } \\
\text { content }\end{array}$ & $\begin{array}{l}\text { Temper- } \\
\text { ature }\end{array}$ & & $\begin{array}{c}\text { Volumetric } \\
\text { water } \\
\text { content }\end{array}$ & $\begin{array}{l}\text { Temper- } \\
\text { ature }\end{array}$ & $\begin{array}{c}\text { Bulk } \\
\text { electrical } \\
\text { conduct- } \\
\text { ivity } \\
\end{array}$ & & $\begin{array}{l}\text { Volumetric } \\
\text { water } \\
\text { content }\end{array}$ & $\begin{array}{l}\text { Temper- } \\
\text { ature }\end{array}$ & & $\begin{array}{l}\text { Volumetric } \\
\text { water } \\
\text { content }\end{array}$ & $\begin{array}{l}\text { Temper- } \\
\text { ature }\end{array}$ & $\begin{array}{l}\text { Volumetric } \\
\text { water } \\
\text { content }\end{array}$ & $\begin{array}{l}\text { Temper- } \\
\text { ature }\end{array}$ & \\
\hline 7/13/2008 3:00 PM & 0.131 & 22.2 & 0.098 & 0.312 & 20.5 & 0.43 & 0.041 & 0.045 & -- & 0.243 & 0.200 & 13.9 & 0.419 & 12.9 & -- \\
\hline 7/13/2008 4:00 PM & 0.131 & 22.2 & 0.098 & 0.312 & 20.5 & 0.43 & 0.041 & 0.045 & -- & -- & 0.200 & 13.9 & 0.419 & 12.9 & -- \\
\hline 7/13/2008 5:00 PM & 0.130 & 22.2 & 0.098 & 0.312 & 20.5 & 0.43 & 0.041 & 0.045 & -- & 0.727 & 0.200 & 13.9 & 0.420 & 12.9 & -- \\
\hline 7/13/2008 6:00 PM & 0.130 & 22.2 & 0.097 & 0.312 & 20.5 & 0.43 & 0.041 & 0.045 & -- & -- & 0.200 & 13.9 & 0.419 & 12.9 & -- \\
\hline 7/13/2008 7:00 PM & 0.130 & 22.2 & 0.097 & 0.312 & 20.5 & 0.43 & 0.041 & 0.045 & -- & 0.463 & 0.200 & 13.9 & 0.419 & 12.9 & -- \\
\hline 7/13/2008 8:00 PM & 0.131 & 22.1 & 0.097 & 0.311 & 20.5 & 0.43 & 0.041 & 0.044 & -- & 0.712 & 0.201 & 13.9 & 0.419 & 12.9 & -- \\
\hline 7/13/2008 9:00 PM & 0.130 & 22.1 & 0.097 & 0.311 & 20.5 & 0.43 & 0.041 & 0.044 & -- & 0.684 & 0.201 & 13.9 & 0.418 & 12.9 & -- \\
\hline 7/13/2008 10:00 PM & 0.129 & 22.2 & 0.097 & 0.311 & 20.5 & 0.43 & 0.041 & 0.044 & -- & 0.497 & 0.201 & 13.9 & 0.418 & 12.9 & -- \\
\hline 7/13/2008 11:00 PM & 0.129 & 22.2 & 0.097 & 0.311 & 20.6 & 0.43 & 0.041 & 0.044 & -- & 0.456 & 0.201 & 13.9 & 0.418 & 12.9 & -- \\
\hline 7/14/2008 12:00 AM & 0.128 & 22.2 & 0.097 & 0.311 & 20.6 & 0.43 & 0.041 & 0.044 & -- & 0.460 & 0.201 & 13.9 & 0.418 & 12.9 & -- \\
\hline 7/14/2008 1:00 AM & 0.126 & 22.2 & 0.097 & 0.311 & 20.6 & 0.43 & 0.041 & 0.044 & -- & 0.567 & 0.201 & 13.9 & 0.418 & 12.9 & -- \\
\hline 7/14/2008 2:00 AM & 0.126 & 22.3 & 0.097 & 0.311 & 20.6 & 0.43 & 0.041 & 0.045 & -- & 0.652 & 0.203 & 13.9 & 0.418 & 12.9 & -- \\
\hline 7/14/2008 3:00 AM & 0.128 & 22.3 & 0.097 & 0.311 & 20.6 & 0.43 & 0.041 & 0.045 & -- & 0.645 & 0.203 & 13.9 & 0.418 & 13.0 & -- \\
\hline 7/14/2008 4:00 AM & 0.128 & 22.4 & 0.097 & 0.311 & 20.6 & 0.43 & 0.041 & 0.045 & -- & 0.643 & 0.203 & 13.9 & 0.418 & 13.0 & -- \\
\hline 7/14/2008 5:00 AM & 0.129 & 22.4 & 0.097 & 0.311 & 20.6 & 0.43 & 0.041 & 0.045 & -- & 0.652 & 0.203 & 13.9 & 0.418 & 13.0 & -- \\
\hline 7/14/2008 6:00 AM & 0.130 & 22.5 & 0.096 & 0.311 & 20.6 & 0.43 & 0.041 & 0.044 & -- & 0.704 & 0.204 & 13.9 & 0.418 & 13.0 & -- \\
\hline 7/14/2008 7:00 AM & 0.130 & 22.5 & 0.096 & 0.311 & 20.6 & 0.43 & 0.041 & 0.044 & -- & -- & 0.204 & 13.9 & 0.418 & 13.0 & -- \\
\hline 7/14/2008 8:00 AM & 0.128 & 22.5 & 0.096 & 0.311 & 20.6 & 0.43 & 0.041 & 0.045 & -- & -- & 0.204 & 13.9 & 0.418 & 13.0 & -- \\
\hline 7/14/2008 9:00 AM & 0.126 & 22.4 & 0.096 & 0.311 & 20.6 & 0.43 & 0.041 & 0.044 & -- & -- & 0.204 & 13.9 & 0.418 & 13.0 & -- \\
\hline 7/14/2008 10:00 AM & 0.128 & 22.5 & 0.096 & 0.311 & 20.6 & 0.43 & 0.041 & 0.044 & -- & -- & 0.204 & 13.9 & 0.418 & 13.0 & -- \\
\hline 7/14/2008 11:00 AM & 0.130 & 22.5 & 0.096 & 0.311 & 20.6 & 0.43 & 0.041 & 0.045 & -- & -- & 0.204 & 13.9 & 0.419 & 13.0 & -- \\
\hline 7/14/2008 12:00 PM & 0.129 & 22.4 & 0.096 & 0.311 & 20.6 & 0.43 & 0.041 & 0.045 & -- & -- & 0.205 & 14.0 & 0.420 & 13.0 & -- \\
\hline 7/14/2008 1:00 PM & 0.129 & 22.4 & 0.096 & 0.311 & 20.6 & 0.43 & 0.041 & 0.045 & -- & 0.584 & 0.206 & 14.0 & 0.420 & 13.0 & -- \\
\hline 7/14/2008 2:00 PM & 0.129 & 22.5 & 0.096 & 0.311 & 20.6 & 0.43 & 0.041 & 0.045 & -- & 0.619 & 0.206 & 14.0 & 0.420 & 13.0 & -- \\
\hline 7/14/2008 3:00 PM & 0.129 & 22.5 & 0.096 & 0.311 & 20.6 & 0.43 & 0.041 & 0.045 & -- & 0.712 & 0.206 & 14.0 & 0.420 & 13.0 & -- \\
\hline 7/14/2008 4:00 PM & 0.129 & 22.5 & 0.096 & 0.311 & 20.6 & 0.43 & 0.041 & 0.045 & -- & -- & 0.206 & 14.0 & 0.420 & 13.0 & -- \\
\hline 7/14/2008 5:00 PM & 0.129 & 22.5 & 0.096 & 0.311 & 20.6 & 0.43 & 0.041 & 0.045 & -- & 0.650 & 0.207 & 14.0 & 0.420 & 13.0 & -- \\
\hline 7/14/2008 6:00 PM & 0.129 & 22.4 & 0.096 & 0.311 & 20.6 & 0.43 & 0.041 & 0.045 & -- & 0.459 & 0.206 & 14.0 & 0.420 & 13.0 & -- \\
\hline 7/14/2008 7:00 PM & 0.129 & 22.4 & 0.096 & 0.311 & 20.6 & 0.43 & 0.041 & 0.045 & -- & 0.635 & 0.206 & 14.0 & 0.420 & 13.0 & -- \\
\hline 7/14/2008 8:00 PM & 0.126 & 22.4 & 0.096 & 0.311 & 20.6 & 0.43 & 0.041 & 0.044 & -- & 0.670 & 0.206 & 14.0 & 0.419 & 13.0 & -- \\
\hline 7/14/2008 9:00 PM & 0.125 & 22.4 & 0.096 & 0.311 & 20.6 & 0.43 & 0.041 & 0.044 & -- & 0.581 & 0.207 & 14.0 & 0.419 & 13.0 & -- \\
\hline 7/14/2008 10:00 PM & 0.124 & 22.4 & 0.096 & 0.311 & 20.6 & 0.43 & 0.041 & 0.044 & -- & 0.575 & 0.206 & 14.0 & 0.419 & 13.0 & -- \\
\hline 7/14/2008 11:00 PM & 0.124 & 22.5 & 0.096 & 0.311 & 20.6 & 0.43 & 0.041 & 0.044 & -- & 0.604 & 0.207 & 14.0 & 0.418 & 13.0 & -- \\
\hline 7/15/2008 12:00 AM & 0.124 & 22.5 & 0.096 & 0.311 & 20.6 & 0.43 & 0.041 & 0.044 & -- & 0.601 & 0.207 & 14.0 & 0.418 & 13.0 & -- \\
\hline 7/15/2008 1:00 AM & 0.124 & 22.5 & 0.095 & 0.311 & 20.6 & 0.43 & 0.041 & 0.044 & -- & 0.630 & 0.207 & 14.0 & 0.418 & 13.0 & -- \\
\hline 7/15/2008 2:00 AM & 0.125 & 22.6 & 0.095 & 0.311 & 20.6 & 0.43 & 0.041 & 0.044 & -- & -- & 0.207 & 14.0 & 0.418 & 13.0 & -- \\
\hline 7/15/2008 3:00 AM & 0.126 & 22.7 & 0.095 & 0.311 & 20.6 & 0.43 & 0.041 & 0.044 & -- & -- & 0.208 & 14.0 & 0.418 & 13.0 & -- \\
\hline 7/15/2008 4:00 AM & 0.128 & 22.7 & 0.095 & 0.311 & 20.6 & 0.43 & 0.041 & 0.044 & -- & -- & 0.208 & 14.0 & 0.418 & 13.0 & -- \\
\hline 7/15/2008 5:00 AM & 0.129 & 22.7 & 0.095 & 0.311 & 20.6 & 0.43 & 0.041 & 0.044 & -- & -- & 0.208 & 14.0 & 0.418 & 13.0 & -- \\
\hline 7/15/2008 6:00 AM & 0.124 & 22.8 & 0.095 & 0.310 & 20.6 & 0.43 & 0.041 & 0.044 & -- & -- & 0.208 & 14.0 & 0.418 & 13.0 & -- \\
\hline
\end{tabular}


Appendix 1. Volumetric water content, temperature, and electrical-conductivity data collected at the flood-irrigated site during 2008.-Continued

[Depth in feet below land surface; volumetric water content dimensionless; temperature in degrees Celsius; bulk electrical conductivity in decisiemens per meter; --, no data]

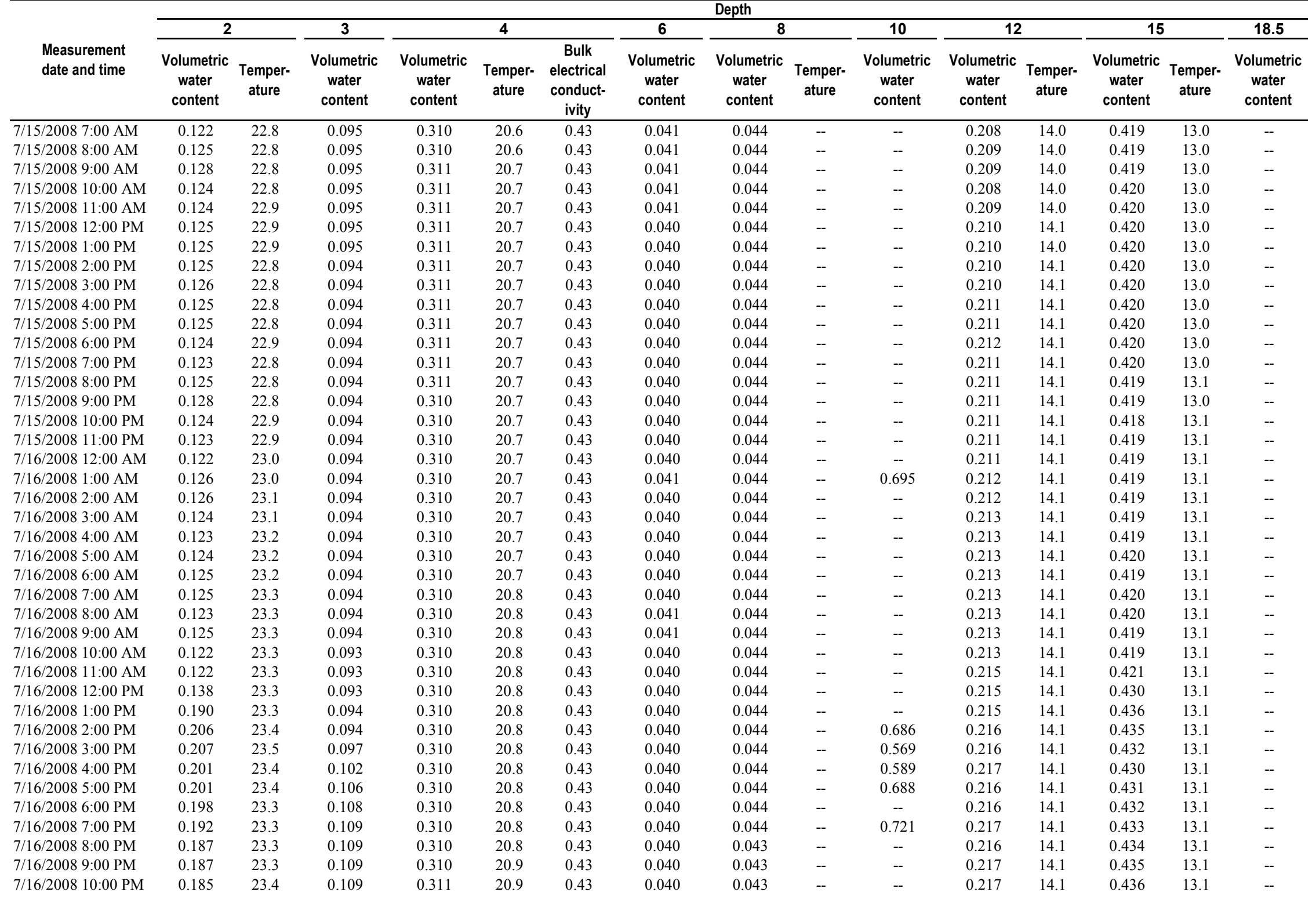


Appendix 1. Volumetric water content, temperature, and electrical-conductivity data collected at the flood-irrigated site during 2008.-Continued

[Depth in feet below land surface; volumetric water content dimensionless; temperature in degrees Celsius; bulk electrical conductivity in decisiemens per meter; --, no data]

\begin{tabular}{|c|c|c|c|c|c|c|c|c|c|c|c|c|c|c|c|}
\hline \multirow[b]{3}{*}{$\begin{array}{l}\text { Measurement } \\
\text { date and time }\end{array}$} & \multicolumn{15}{|c|}{ Depth } \\
\hline & \multicolumn{2}{|l|}{2} & \multirow{2}{*}{$\begin{array}{c}3 \\
\begin{array}{c}\text { Volumetric } \\
\text { water } \\
\text { content }\end{array}\end{array}$} & \multicolumn{3}{|c|}{4} & \multirow{2}{*}{$\begin{array}{c}6 \\
\begin{array}{c}\text { Volumetric } \\
\text { water } \\
\text { content }\end{array}\end{array}$} & \multicolumn{2}{|l|}{8} & \multirow{2}{*}{$\begin{array}{c}10 \\
\text { Volumetric } \\
\text { water } \\
\text { content }\end{array}$} & \multicolumn{2}{|c|}{12} & \multicolumn{2}{|c|}{15} & \multirow{2}{*}{$\begin{array}{c}18.5 \\
\text { Volumetric } \\
\text { water } \\
\text { content }\end{array}$} \\
\hline & $\begin{array}{l}\text { Volumetric } \\
\text { water } \\
\text { content }\end{array}$ & $\begin{array}{l}\text { Temper- } \\
\text { ature }\end{array}$ & & $\begin{array}{c}\text { Volumetric } \\
\text { water } \\
\text { content }\end{array}$ & $\begin{array}{l}\text { Temper- } \\
\text { ature }\end{array}$ & $\begin{array}{c}\text { Bulk } \\
\text { electrical } \\
\text { conduct- } \\
\text { ivity } \\
\end{array}$ & & $\begin{array}{l}\text { Volumetric } \\
\text { water } \\
\text { content }\end{array}$ & $\begin{array}{l}\text { Temper- } \\
\text { ature }\end{array}$ & & $\begin{array}{l}\text { Volumetric } \\
\text { water } \\
\text { content }\end{array}$ & $\begin{array}{l}\text { Temper- } \\
\text { ature }\end{array}$ & $\begin{array}{l}\text { Volumetric } \\
\text { water } \\
\text { content }\end{array}$ & $\begin{array}{l}\text { Temper- } \\
\text { ature }\end{array}$ & \\
\hline 7/16/2008 11:00 PM & 0.183 & 23.4 & 0.109 & 0.311 & 20.9 & 0.43 & 0.040 & 0.043 & -- & -- & 0.217 & 14.1 & 0.435 & 13.1 & -- \\
\hline 7/17/2008 12:00 AM & 0.181 & 23.4 & 0.109 & 0.311 & 20.9 & 0.43 & 0.040 & 0.043 & -- & -- & 0.217 & 14.1 & 0.436 & 13.2 & -- \\
\hline 7/17/2008 1:00 AM & 0.179 & 23.5 & 0.109 & 0.311 & 20.9 & 0.43 & 0.040 & 0.043 & -- & -- & 0.218 & 14.1 & 0.433 & 13.1 & -- \\
\hline 7/17/2008 2:00 AM & 0.176 & 23.5 & 0.109 & 0.312 & 20.9 & 0.43 & 0.040 & 0.043 & -- & 0.687 & 0.218 & 14.1 & 0.438 & 13.1 & -- \\
\hline 7/17/2008 3:00 AM & 0.174 & 23.5 & 0.109 & 0.312 & 20.9 & 0.43 & 0.040 & 0.043 & -- & 0.599 & 0.218 & 14.1 & 0.435 & 13.2 & -- \\
\hline 7/17/2008 4:00 AM & 0.173 & 23.5 & 0.109 & 0.312 & 20.9 & 0.43 & 0.040 & 0.043 & -- & 0.690 & 0.218 & 14.1 & 0.435 & 13.1 & -- \\
\hline 7/17/2008 5:00 AM & 0.171 & 23.5 & 0.109 & 0.313 & 20.9 & 0.43 & 0.040 & 0.043 & -- & -- & 0.219 & 14.1 & 0.435 & 13.2 & -- \\
\hline 7/17/2008 6:00 AM & 0.169 & 23.5 & 0.108 & 0.313 & 20.9 & 0.43 & 0.040 & 0.043 & -- & -- & 0.218 & 14.1 & 0.433 & 13.1 & -- \\
\hline 7/17/2008 7:00 AM & 0.168 & 23.4 & 0.108 & 0.313 & 21.0 & 0.43 & 0.040 & 0.043 & -- & 0.697 & 0.219 & 14.1 & 0.431 & 13.2 & -- \\
\hline 7/17/2008 8:00 AM & 0.167 & 23.5 & 0.108 & 0.313 & 21.0 & 0.43 & 0.040 & 0.043 & -- & 0.699 & 0.219 & 14.1 & 0.431 & 13.1 & -- \\
\hline 7/17/2008 9:00 AM & 0.167 & 23.5 & 0.108 & 0.313 & 21.0 & 0.43 & 0.040 & 0.043 & -- & -- & 0.219 & 14.1 & 0.432 & 13.2 & -- \\
\hline 7/17/2008 10:00 AM & 0.165 & 23.5 & 0.107 & 0.313 & 21.0 & 0.43 & 0.040 & 0.043 & -- & -- & 0.219 & 14.2 & 0.431 & 13.1 & -- \\
\hline 7/17/2008 11:00 AM & 0.165 & 23.4 & 0.107 & 0.313 & 21.0 & 0.43 & 0.040 & 0.044 & -- & -- & 0.219 & 14.2 & 0.434 & 13.2 & -- \\
\hline 7/17/2008 12:00 PM & 0.165 & 23.3 & 0.107 & 0.315 & 21.0 & 0.43 & 0.040 & 0.044 & -- & -- & 0.219 & 14.2 & 0.433 & 13.2 & -- \\
\hline 7/17/2008 1:00 PM & 0.161 & 23.3 & 0.107 & 0.315 & 21.0 & 0.44 & 0.040 & 0.044 & -- & -- & 0.220 & 14.2 & 0.431 & 13.2 & -- \\
\hline 7/17/2008 2:00 PM & 0.157 & 23.3 & 0.106 & 0.315 & 21.0 & 0.44 & 0.040 & 0.044 & -- & -- & 0.220 & 14.2 & 0.433 & 13.2 & -- \\
\hline 7/17/2008 3:00 PM & 0.159 & 23.3 & 0.106 & 0.315 & 21.0 & 0.44 & 0.040 & 0.044 & -- & -- & 0.220 & 14.2 & 0.433 & 13.2 & -- \\
\hline 7/17/2008 4:00 PM & 0.158 & 23.3 & 0.106 & 0.315 & 21.0 & 0.44 & 0.040 & 0.044 & -- & -- & 0.220 & 14.2 & 0.433 & 13.2 & -- \\
\hline 7/17/2008 5:00 PM & 0.158 & 23.2 & 0.106 & 0.315 & 21.0 & 0.44 & 0.040 & 0.044 & -- & -- & 0.220 & 14.2 & 0.434 & 13.2 & -- \\
\hline 7/17/2008 6:00 PM & 0.158 & 23.3 & 0.105 & 0.315 & 21.0 & 0.43 & 0.040 & 0.044 & -- & -- & 0.221 & 14.2 & 0.434 & 13.2 & -- \\
\hline 7/17/2008 7:00 PM & 0.158 & 23.2 & 0.105 & 0.315 & 21.1 & 0.43 & 0.040 & 0.043 & -- & -- & 0.220 & 14.2 & 0.434 & 13.2 & -- \\
\hline 7/17/2008 8:00 PM & 0.157 & 23.3 & 0.105 & 0.315 & 21.1 & 0.43 & 0.040 & 0.043 & -- & -- & 0.220 & 14.2 & 0.434 & 13.2 & -- \\
\hline 7/17/2008 9:00 PM & 0.154 & 23.2 & 0.104 & 0.315 & 21.1 & 0.43 & 0.040 & 0.043 & -- & -- & 0.221 & 14.2 & 0.432 & 13.2 & -- \\
\hline 7/17/2008 10:00 PM & 0.153 & 23.2 & 0.104 & 0.315 & 21.1 & 0.44 & 0.040 & 0.043 & -- & -- & 0.221 & 14.2 & 0.432 & 13.2 & -- \\
\hline 7/17/2008 11:00 PM & 0.151 & 23.2 & 0.104 & 0.315 & 21.1 & 0.44 & 0.040 & 0.043 & -- & -- & 0.221 & 14.2 & 0.429 & 13.2 & -- \\
\hline 7/18/2008 12:00 AM & 0.151 & 23.2 & 0.104 & 0.315 & 21.1 & 0.44 & 0.040 & 0.043 & -- & -- & 0.221 & 14.2 & 0.432 & 13.2 & -- \\
\hline 7/18/2008 1:00 AM & 0.150 & 23.2 & 0.104 & 0.315 & 21.1 & 0.44 & 0.040 & 0.043 & -- & -- & 0.222 & 14.2 & 0.429 & 13.2 & -- \\
\hline 7/18/2008 2:00 AM & 0.151 & 23.2 & 0.104 & 0.315 & 21.1 & 0.44 & 0.040 & 0.043 & -- & -- & 0.222 & 14.2 & 0.430 & 13.2 & -- \\
\hline 7/18/2008 3:00 AM & 0.151 & 23.2 & 0.104 & 0.313 & 21.1 & 0.44 & 0.040 & 0.043 & -- & -- & 0.222 & 14.2 & 0.430 & 13.2 & -- \\
\hline 7/18/2008 4:00 AM & 0.151 & 23.2 & 0.104 & 0.313 & 21.1 & 0.44 & 0.040 & 0.043 & -- & -- & 0.222 & 14.2 & 0.429 & 13.2 & -- \\
\hline 7/18/2008 5:00 AM & 0.150 & 23.2 & 0.104 & 0.313 & 21.1 & 0.44 & 0.040 & 0.043 & -- & -- & 0.222 & 14.2 & 0.428 & 13.2 & -- \\
\hline 7/18/2008 6:00 AM & 0.150 & 23.3 & 0.103 & 0.313 & 21.1 & 0.44 & 0.040 & 0.043 & -- & -- & 0.222 & 14.2 & 0.429 & 13.2 & -- \\
\hline 7/18/2008 7:00 AM & 0.149 & 23.3 & 0.103 & 0.313 & 21.1 & 0.44 & 0.040 & 0.043 & -- & -- & 0.223 & 14.2 & 0.428 & 13.2 & -- \\
\hline 7/18/2008 8:00 AM & 0.148 & 23.2 & 0.103 & 0.313 & 21.1 & 0.44 & 0.040 & 0.043 & -- & -- & 0.223 & 14.2 & 0.425 & 13.2 & -- \\
\hline 7/18/2008 9:00 AM & 0.146 & 23.2 & 0.103 & 0.313 & 21.1 & 0.44 & 0.041 & 0.043 & -- & -- & 0.223 & 14.2 & 0.431 & 13.2 & -- \\
\hline 7/18/2008 10:00 AM & 0.147 & 23.2 & 0.103 & 0.315 & 21.1 & 0.44 & 0.041 & 0.043 & -- & -- & 0.223 & 14.2 & 0.426 & 13.2 & -- \\
\hline 7/18/2008 11:00 AM & 0.143 & 23.2 & 0.102 & 0.315 & 21.1 & 0.44 & 0.040 & 0.043 & -- & -- & 0.223 & 14.2 & 0.424 & 13.2 & -- \\
\hline 7/18/2008 12:00 PM & 0.146 & 23.2 & 0.102 & 0.315 & 21.1 & 0.44 & 0.040 & 0.043 & -- & -- & 0.223 & 14.2 & 0.424 & 13.2 & -- \\
\hline 7/18/2008 1:00 PM & 0.147 & 23.2 & 0.102 & 0.315 & 21.1 & 0.44 & 0.040 & 0.043 & -- & -- & 0.223 & 14.2 & 0.424 & 13.2 & -- \\
\hline 7/18/2008 2:00 PM & 0.147 & 23.2 & 0.102 & 0.313 & 21.1 & 0.44 & 0.040 & 0.043 & -- & -- & 0.224 & 14.3 & 0.421 & 13.2 & -- \\
\hline
\end{tabular}


Appendix 1. Volumetric water content, temperature, and electrical-conductivity data collected at the flood-irrigated site during 2008.-Continued

[Depth in feet below land surface; volumetric water content dimensionless; temperature in degrees Celsius; bulk electrical conductivity in decisiemens per meter; --, no data]

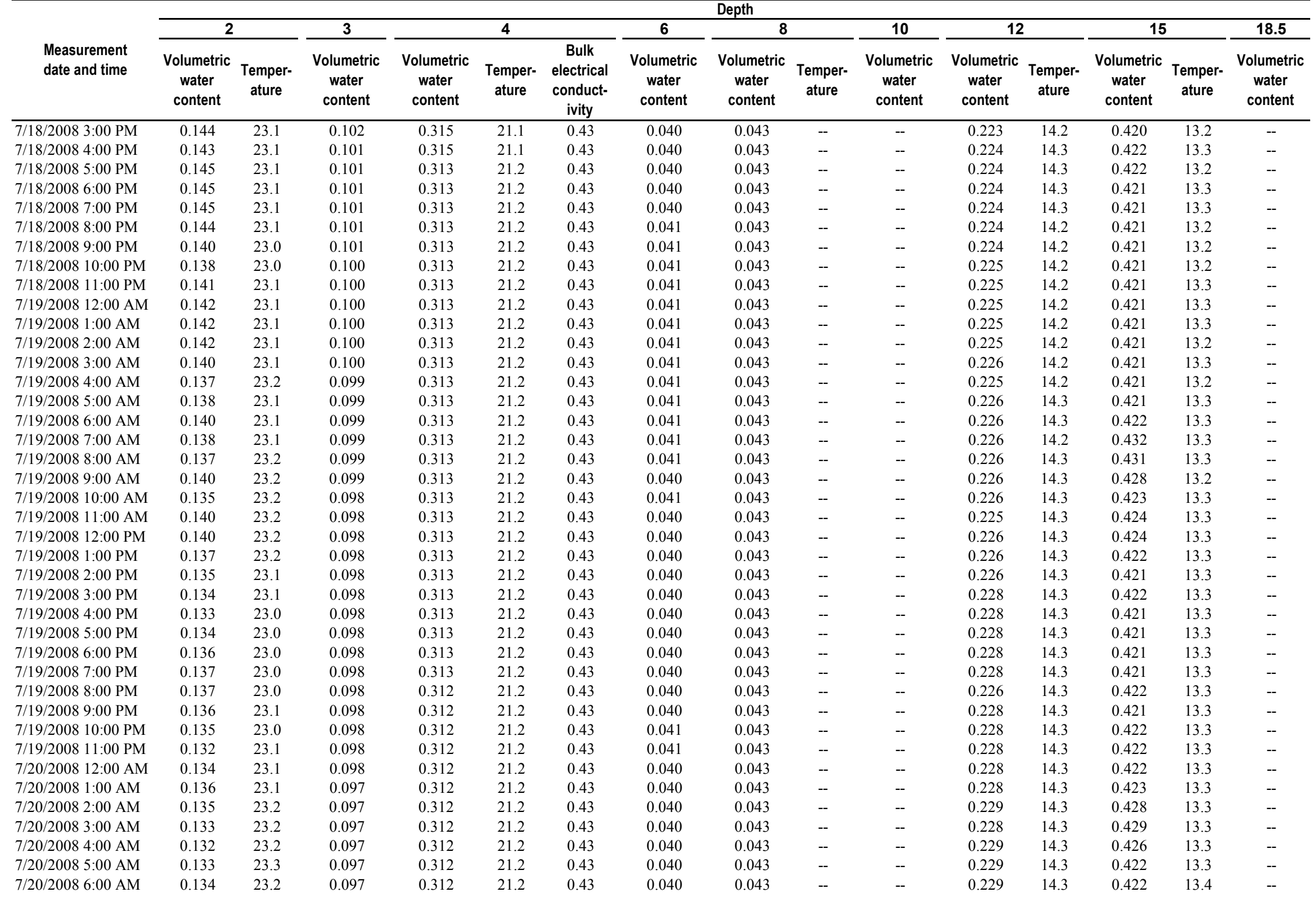


Appendix 1. Volumetric water content, temperature, and electrical-conductivity data collected at the flood-irrigated site during 2008.-Continued

[Depth in feet below land surface; volumetric water content dimensionless; temperature in degrees Celsius; bulk electrical conductivity in decisiemens per meter; --, no data]

\begin{tabular}{|c|c|c|c|c|c|c|c|c|c|c|c|c|c|c|c|}
\hline \multirow[b]{3}{*}{$\begin{array}{l}\text { Measurement } \\
\text { date and time }\end{array}$} & \multicolumn{15}{|c|}{ Depth } \\
\hline & \multicolumn{2}{|l|}{2} & \multirow{2}{*}{$\begin{array}{c}3 \\
\begin{array}{c}\text { Volumetric } \\
\text { water } \\
\text { content }\end{array}\end{array}$} & \multicolumn{3}{|c|}{4} & \multirow{2}{*}{$\begin{array}{c}6 \\
\begin{array}{c}\text { Volumetric } \\
\text { water } \\
\text { content }\end{array}\end{array}$} & \multicolumn{2}{|l|}{8} & \multirow{2}{*}{$\begin{array}{c}10 \\
\text { Volumetric } \\
\text { water } \\
\text { content }\end{array}$} & \multicolumn{2}{|c|}{12} & \multicolumn{2}{|c|}{15} & \multirow{2}{*}{$\begin{array}{c}18.5 \\
\text { Volumetric } \\
\text { water } \\
\text { content }\end{array}$} \\
\hline & $\begin{array}{l}\text { Volumetric } \\
\text { water } \\
\text { content }\end{array}$ & $\begin{array}{l}\text { Temper- } \\
\text { ature }\end{array}$ & & $\begin{array}{c}\text { Volumetric } \\
\text { water } \\
\text { content }\end{array}$ & $\begin{array}{l}\text { Temper- } \\
\text { ature }\end{array}$ & $\begin{array}{c}\text { Bulk } \\
\text { electrical } \\
\text { conduct- } \\
\text { ivity } \\
\end{array}$ & & $\begin{array}{l}\text { Volumetric } \\
\text { water } \\
\text { content }\end{array}$ & $\begin{array}{l}\text { Temper- } \\
\text { ature }\end{array}$ & & $\begin{array}{l}\text { Volumetric } \\
\text { water } \\
\text { content }\end{array}$ & $\begin{array}{l}\text { Temper- } \\
\text { ature }\end{array}$ & $\begin{array}{l}\text { Volumetric } \\
\text { water } \\
\text { content }\end{array}$ & $\begin{array}{l}\text { Temper- } \\
\text { ature }\end{array}$ & \\
\hline 7/20/2008 7:00 AM & 0.134 & 23.2 & 0.097 & 0.312 & 21.2 & 0.43 & 0.040 & 0.043 & -- & -- & 0.229 & 14.3 & 0.422 & 13.3 & -- \\
\hline 7/20/2008 8:00 AM & 0.133 & 23.3 & 0.097 & 0.312 & 21.2 & 0.43 & 0.040 & 0.043 & -- & -- & 0.229 & 14.3 & 0.422 & 13.4 & -- \\
\hline 7/20/2008 9:00 AM & 0.133 & 23.3 & 0.096 & 0.312 & 21.2 & 0.43 & 0.040 & 0.043 & -- & -- & 0.229 & 14.3 & 0.422 & 13.3 & -- \\
\hline 7/20/2008 10:00 AM & 0.133 & 23.3 & 0.096 & 0.312 & 21.2 & 0.43 & 0.040 & 0.043 & -- & -- & 0.229 & 14.3 & 0.422 & 13.3 & -- \\
\hline 7/20/2008 11:00 AM & 0.131 & 23.3 & 0.096 & 0.312 & 21.2 & 0.43 & 0.040 & 0.043 & -- & -- & 0.228 & 14.3 & 0.421 & 13.3 & -- \\
\hline 7/20/2008 12:00 PM & 0.134 & 23.3 & 0.096 & 0.312 & 21.2 & 0.43 & 0.040 & 0.043 & -- & -- & 0.229 & 14.3 & 0.422 & 13.4 & -- \\
\hline 7/20/2008 1:00 PM & 0.134 & 23.3 & 0.096 & 0.312 & 21.2 & 0.43 & 0.040 & 0.043 & -- & -- & 0.229 & 14.3 & 0.422 & 13.3 & -- \\
\hline 7/20/2008 2:00 PM & 0.132 & 23.3 & 0.096 & 0.312 & 21.2 & 0.43 & 0.040 & 0.043 & -- & 0.677 & 0.229 & 14.4 & 0.421 & 13.3 & -- \\
\hline 7/20/2008 3:00 PM & 0.131 & 23.3 & 0.096 & 0.312 & 21.2 & 0.43 & 0.040 & 0.043 & -- & -- & 0.230 & 14.3 & 0.421 & 13.4 & -- \\
\hline 7/20/2008 4:00 PM & 0.133 & 23.2 & 0.096 & 0.312 & 21.2 & 0.43 & 0.040 & 0.043 & -- & -- & 0.229 & 14.3 & 0.421 & 13.3 & -- \\
\hline 7/20/2008 5:00 PM & 0.133 & 23.3 & 0.096 & 0.312 & 21.2 & 0.43 & 0.040 & 0.043 & -- & -- & 0.230 & 14.3 & 0.421 & 13.3 & -- \\
\hline 7/20/2008 6:00 PM & 0.133 & 23.3 & 0.096 & 0.312 & 21.2 & 0.43 & 0.040 & 0.043 & -- & -- & 0.229 & 14.4 & 0.421 & 13.4 & -- \\
\hline 7/20/2008 7:00 PM & 0.130 & 23.2 & 0.096 & 0.312 & 21.3 & 0.43 & 0.040 & 0.043 & -- & -- & 0.229 & 14.3 & 0.421 & 13.3 & -- \\
\hline 7/20/2008 8:00 PM & 0.126 & 23.2 & 0.095 & 0.312 & 21.3 & 0.43 & 0.040 & 0.043 & -- & -- & 0.230 & 14.3 & 0.421 & 13.4 & -- \\
\hline 7/20/2008 9:00 PM & 0.129 & 23.2 & 0.095 & 0.312 & 21.3 & 0.43 & 0.040 & 0.043 & -- & -- & 0.230 & 14.3 & 0.422 & 13.4 & -- \\
\hline 7/20/2008 10:00 PM & 0.131 & 23.2 & 0.095 & 0.312 & 21.3 & 0.43 & 0.040 & 0.043 & -- & -- & 0.230 & 14.4 & 0.422 & 13.3 & -- \\
\hline 7/20/2008 11:00 PM & 0.131 & 23.3 & 0.095 & 0.312 & 21.3 & 0.43 & 0.040 & 0.043 & -- & -- & 0.231 & 14.3 & 0.422 & 13.4 & -- \\
\hline 7/21/2008 12:00 AM & 0.128 & 23.3 & 0.095 & 0.312 & 21.3 & 0.43 & 0.040 & 0.043 & -- & -- & 0.230 & 14.3 & 0.421 & 13.4 & -- \\
\hline 7/21/2008 1:00 AM & 0.129 & 23.3 & 0.095 & 0.311 & 21.3 & 0.43 & 0.040 & 0.043 & -- & -- & 0.231 & 14.3 & 0.422 & 13.4 & -- \\
\hline 7/21/2008 2:00 AM & 0.130 & 23.4 & 0.095 & 0.311 & 21.3 & 0.43 & 0.040 & 0.043 & -- & -- & 0.231 & 14.3 & 0.421 & 13.4 & -- \\
\hline 7/21/2008 3:00 AM & 0.130 & 23.4 & 0.095 & 0.312 & 21.3 & 0.43 & 0.040 & 0.043 & -- & -- & 0.231 & 14.4 & 0.421 & 13.4 & -- \\
\hline 7/21/2008 4:00 AM & 0.130 & 23.4 & 0.095 & 0.311 & 21.3 & 0.43 & 0.040 & 0.043 & -- & -- & 0.231 & 14.3 & 0.421 & 13.4 & -- \\
\hline 7/21/2008 5:00 AM & 0.129 & 23.4 & 0.095 & 0.311 & 21.3 & 0.43 & 0.040 & 0.043 & -- & -- & 0.231 & 14.3 & 0.421 & 13.4 & -- \\
\hline 7/21/2008 6:00 AM & 0.129 & 23.5 & 0.095 & 0.311 & 21.3 & 0.43 & 0.040 & 0.043 & -- & -- & 0.231 & 14.4 & 0.421 & 13.4 & -- \\
\hline 7/21/2008 7:00 AM & 0.129 & 23.5 & 0.094 & 0.311 & 21.3 & 0.43 & 0.040 & 0.043 & -- & -- & 0.232 & 14.3 & 0.420 & 13.4 & -- \\
\hline 7/21/2008 8:00 AM & 0.129 & 23.5 & 0.094 & 0.312 & 21.3 & 0.43 & 0.040 & 0.043 & -- & -- & 0.231 & 14.3 & 0.421 & 13.4 & -- \\
\hline 7/21/2008 9:00 AM & 0.128 & 23.5 & 0.094 & 0.311 & 21.3 & 0.43 & 0.040 & 0.043 & -- & -- & 0.232 & 14.3 & 0.421 & 13.4 & -- \\
\hline 7/21/2008 10:00 AM & 0.129 & 23.5 & 0.094 & 0.312 & 21.3 & 0.43 & 0.040 & 0.043 & -- & -- & 0.232 & 14.4 & 0.421 & 13.4 & -- \\
\hline 7/21/2008 11:00 AM & 0.126 & 23.5 & 0.094 & 0.312 & 21.3 & 0.43 & 0.040 & 0.043 & -- & 0.726 & 0.231 & 14.4 & 0.422 & 13.4 & -- \\
\hline 7/21/2008 12:00 PM & 0.123 & 23.5 & 0.094 & 0.312 & 21.3 & 0.43 & 0.040 & 0.043 & -- & 0.725 & 0.232 & 14.4 & 0.422 & 13.4 & -- \\
\hline 7/21/2008 1:00 PM & 0.125 & 23.4 & 0.094 & 0.312 & 21.3 & 0.43 & 0.040 & 0.043 & -- & -- & 0.232 & 14.4 & 0.422 & 13.4 & -- \\
\hline 7/21/2008 2:00 PM & 0.129 & 23.5 & 0.094 & 0.312 & 21.3 & 0.43 & 0.040 & 0.043 & -- & -- & 0.232 & 14.4 & 0.422 & 13.4 & -- \\
\hline 7/21/2008 3:00 PM & 0.130 & 23.5 & 0.094 & 0.312 & 21.3 & 0.43 & 0.040 & 0.043 & -- & -- & 0.233 & 14.4 & 0.421 & 13.4 & -- \\
\hline 7/21/2008 4:00 PM & 0.130 & 23.4 & 0.094 & 0.312 & 21.3 & 0.43 & 0.040 & 0.043 & -- & -- & 0.232 & 14.4 & 0.421 & 13.4 & -- \\
\hline 7/21/2008 5:00 PM & 0.125 & 23.4 & 0.094 & 0.312 & 21.3 & 0.43 & 0.040 & 0.043 & -- & -- & 0.233 & 14.4 & 0.422 & 13.4 & -- \\
\hline 7/21/2008 6:00 PM & 0.122 & 23.4 & 0.094 & 0.312 & 21.3 & 0.43 & 0.040 & 0.043 & -- & -- & 0.233 & 14.4 & 0.421 & 13.4 & -- \\
\hline 7/21/2008 7:00 PM & 0.123 & 23.3 & 0.094 & 0.312 & 21.3 & 0.43 & 0.040 & 0.043 & -- & -- & 0.233 & 14.4 & 0.421 & 13.4 & -- \\
\hline 7/21/2008 8:00 PM & 0.126 & 23.3 & 0.093 & 0.311 & 21.3 & 0.43 & 0.040 & 0.043 & -- & -- & 0.233 & 14.4 & 0.421 & 13.4 & -- \\
\hline 7/21/2008 9:00 PM & 0.125 & 23.4 & 0.093 & 0.311 & 21.3 & 0.43 & 0.040 & 0.043 & -- & -- & 0.233 & 14.4 & 0.421 & 13.4 & -- \\
\hline 7/21/2008 10:00 PM & 0.124 & 23.4 & 0.093 & 0.311 & 21.3 & 0.43 & 0.040 & 0.043 & -- & -- & 0.234 & 14.4 & 0.421 & 13.4 & -- \\
\hline
\end{tabular}


Appendix 1. Volumetric water content, temperature, and electrical-conductivity data collected at the flood-irrigated site during 2008.-Continued

[Depth in feet below land surface; volumetric water content dimensionless; temperature in degrees Celsius; bulk electrical conductivity in decisiemens per meter; --, no data]

\begin{tabular}{|c|c|c|c|c|c|c|c|c|c|c|c|c|c|c|c|}
\hline \multirow[b]{3}{*}{$\begin{array}{l}\text { Measurement } \\
\text { date and time }\end{array}$} & \multicolumn{15}{|c|}{ Depth } \\
\hline & \multicolumn{2}{|c|}{2} & \multirow{2}{*}{$\begin{array}{c}3 \\
\text { Volumetric } \\
\text { water } \\
\text { content }\end{array}$} & \multicolumn{3}{|c|}{4} & \multirow{2}{*}{$\begin{array}{c}6 \\
\text { Volumetric } \\
\text { water } \\
\text { content }\end{array}$} & \multicolumn{2}{|l|}{8} & \multirow{2}{*}{$\begin{array}{c}10 \\
\begin{array}{c}\text { Volumetric } \\
\text { water } \\
\text { content }\end{array}\end{array}$} & \multicolumn{2}{|c|}{12} & \multicolumn{2}{|c|}{15} & \multirow{2}{*}{$\begin{array}{c}18.5 \\
\text { Volumetric } \\
\text { water } \\
\text { content }\end{array}$} \\
\hline & $\begin{array}{c}\text { Volumetric } \\
\text { water } \\
\text { content }\end{array}$ & $\begin{array}{l}\text { Temper- } \\
\text { ature }\end{array}$ & & $\begin{array}{l}\text { Volumetric } \\
\text { water } \\
\text { content }\end{array}$ & $\begin{array}{l}\text { Temper- } \\
\text { ature }\end{array}$ & $\begin{array}{c}\text { Bulk } \\
\text { electrical } \\
\text { conduct- } \\
\text { ivity }\end{array}$ & & $\begin{array}{c}\text { Volumetric } \\
\text { water } \\
\text { content }\end{array}$ & $\begin{array}{l}\text { Temper- } \\
\text { ature }\end{array}$ & & $\begin{array}{c}\text { Volumetric } \\
\text { water } \\
\text { content }\end{array}$ & $\begin{array}{l}\text { Temper- } \\
\text { ature }\end{array}$ & $\begin{array}{c}\text { Volumetric } \\
\text { water } \\
\text { content }\end{array}$ & $\begin{array}{l}\text { Temper- } \\
\text { ature }\end{array}$ & \\
\hline 7/21/2008 11:00 PM & 0.125 & 23.5 & 0.093 & 0.311 & 21.3 & 0.43 & 0.040 & 0.043 & - & -- & 0.234 & 14.4 & 0.422 & 13.4 & -- \\
\hline 7/22/2008 12:00 AM & 0.126 & 23.4 & 0.093 & 0.311 & 21.3 & 0.43 & 0.040 & 0.042 & -- & -- & 0.234 & 14.4 & 0.422 & 13.4 & -- \\
\hline 7/22/2008 1:00 AM & 0.126 & 23.5 & 0.093 & 0.311 & 21.3 & 0.43 & 0.040 & 0.042 & -- & -- & 0.234 & 14.4 & 0.422 & 13.4 & -- \\
\hline 7/22/2008 2:00 AM & 0.125 & 23.6 & 0.094 & 0.311 & 21.3 & 0.43 & 0.040 & 0.043 & -- & -- & 0.235 & 14.4 & 0.422 & 13.4 & -- \\
\hline 7/22/2008 3:00 AM & 0.126 & 23.6 & 0.093 & 0.311 & 21.4 & 0.43 & 0.040 & 0.043 & -- & -- & 0.235 & 14.4 & 0.422 & 13.4 & -- \\
\hline 7/22/2008 4:00 AM & 0.126 & 23.6 & 0.093 & 0.311 & 21.4 & 0.43 & 0.040 & 0.043 & -- & -- & 0.235 & 14.4 & 0.422 & 13.4 & -- \\
\hline 7/22/2008 5:00 AM & 0.126 & 23.6 & 0.093 & 0.311 & 21.4 & 0.43 & 0.040 & 0.043 & -- & -- & 0.235 & 14.4 & 0.422 & 13.4 & -- \\
\hline 7/22/2008 6:00 AM & 0.126 & 23.6 & 0.093 & 0.311 & 21.4 & 0.43 & 0.040 & 0.043 & -- & -- & 0.235 & 14.4 & 0.422 & 13.4 & -- \\
\hline 7/22/2008 7:00 AM & 0.125 & 23.7 & 0.093 & 0.311 & 21.4 & 0.43 & 0.040 & 0.043 & -- & -- & 0.235 & 14.4 & 0.422 & 13.4 & -- \\
\hline 7/22/2008 8:00 AM & 0.126 & 23.7 & 0.093 & 0.311 & 21.4 & 0.43 & 0.040 & 0.043 & -- & -- & 0.235 & 14.4 & 0.422 & 13.4 & -- \\
\hline 7/22/2008 9:00 AM & 0.125 & 23.7 & 0.093 & 0.311 & 21.4 & 0.43 & 0.040 & 0.043 & -- & -- & 0.236 & 14.4 & 0.422 & 13.4 & -- \\
\hline 7/22/2008 10:00 AM & 0.125 & 23.7 & 0.093 & 0.311 & 21.4 & 0.43 & 0.040 & 0.043 & -- & -- & 0.236 & 14.4 & 0.422 & 13.5 & -- \\
\hline 7/22/2008 11:00 AM & 0.123 & 23.7 & 0.093 & 0.311 & 21.4 & 0.43 & 0.040 & 0.043 & -- & -- & 0.235 & 14.4 & 0.421 & 13.5 & -- \\
\hline 7/22/2008 12:00 PM & 0.129 & 23.7 & 0.093 & 0.311 & 21.4 & 0.43 & 0.040 & 0.043 & -- & -- & 0.236 & 14.4 & 0.423 & 13.5 & -- \\
\hline 7/22/2008 1:00 PM & 0.169 & 23.7 & 0.093 & 0.311 & 21.4 & 0.43 & 0.040 & 0.043 & -- & -- & 0.236 & 14.4 & 0.422 & 13.5 & -- \\
\hline 7/22/2008 2:00 PM & 0.197 & 23.7 & 0.092 & 0.310 & 21.4 & 0.43 & 0.039 & 0.043 & -- & -- & 0.237 & 14.4 & 0.421 & 13.5 & -- \\
\hline 7/22/2008 3:00 PM & 0.203 & 23.7 & 0.093 & 0.310 & 21.4 & 0.43 & 0.039 & 0.043 & -- & -- & 0.237 & 14.4 & 0.421 & 13.5 & -- \\
\hline 7/22/2008 4:00 PM & 0.203 & 23.7 & 0.098 & 0.311 & 21.4 & 0.43 & 0.040 & 0.043 & -- & -- & 0.237 & 14.4 & 0.421 & 13.5 & -- \\
\hline 7/22/2008 5:00 PM & 0.207 & 23.7 & 0.106 & 0.311 & 21.4 & 0.43 & 0.040 & 0.043 & -- & -- & 0.237 & 14.5 & 0.421 & 13.5 & -- \\
\hline 7/22/2008 6:00 PM & 0.204 & 23.7 & 0.112 & 0.311 & 21.4 & 0.43 & 0.040 & 0.043 & -- & -- & 0.237 & 14.5 & 0.420 & 13.5 & -- \\
\hline 7/22/2008 7:00 PM & 0.199 & 23.7 & 0.115 & 0.311 & 21.4 & 0.43 & 0.040 & 0.043 & -- & -- & 0.237 & 14.5 & 0.420 & 13.5 & -- \\
\hline 7/22/2008 8:00 PM & 0.196 & 23.7 & 0.115 & 0.311 & 21.4 & 0.43 & 0.040 & 0.042 & -- & -- & 0.238 & 14.5 & 0.420 & 13.5 & -- \\
\hline 7/22/2008 9:00 PM & 0.194 & 23.7 & 0.115 & 0.311 & 21.4 & 0.44 & 0.040 & 0.042 & -- & -- & 0.238 & 14.5 & 0.420 & 13.5 & -- \\
\hline 7/22/2008 10:00 PM & 0.192 & 23.7 & 0.115 & 0.312 & 21.5 & 0.44 & 0.040 & 0.042 & -- & -- & 0.240 & 14.4 & 0.420 & 13.5 & -- \\
\hline 7/22/2008 11:00 PM & 0.188 & 23.8 & 0.115 & 0.312 & 21.5 & 0.44 & 0.040 & 0.042 & -- & -- & 0.240 & 14.5 & 0.420 & 13.5 & -- \\
\hline 7/23/2008 12:00 AM & 0.187 & 23.8 & 0.115 & 0.313 & 21.5 & 0.44 & 0.040 & 0.042 & -- & -- & 0.240 & 14.5 & 0.420 & 13.5 & -- \\
\hline 7/23/2008 1:00 AM & 0.185 & 23.8 & 0.115 & 0.315 & 21.5 & 0.44 & 0.040 & 0.042 & -- & -- & 0.241 & 14.5 & 0.420 & 13.5 & -- \\
\hline 7/23/2008 2:00 AM & 0.182 & 23.8 & 0.115 & 0.316 & 21.5 & 0.44 & 0.040 & 0.042 & -- & -- & 0.241 & 14.5 & 0.420 & 13.5 & -- \\
\hline 7/23/2008 3:00 AM & 0.180 & 23.8 & 0.115 & 0.316 & 21.5 & 0.44 & 0.040 & 0.042 & -- & -- & 0.241 & 14.5 & 0.420 & 13.5 & -- \\
\hline 7/23/2008 4:00 AM & 0.179 & 23.8 & 0.114 & 0.317 & 21.5 & 0.44 & 0.040 & 0.042 & -- & -- & 0.241 & 14.5 & 0.419 & 13.5 & -- \\
\hline 7/23/2008 5:00 AM & 0.178 & 23.8 & 0.114 & 0.317 & 21.5 & 0.45 & 0.040 & 0.042 & -- & -- & 0.241 & 14.5 & 0.419 & 13.5 & -- \\
\hline 7/23/2008 6:00 AM & 0.176 & 23.8 & 0.113 & 0.318 & 21.5 & 0.45 & 0.040 & 0.042 & -- & -- & 0.241 & 14.5 & 0.419 & 13.5 & -- \\
\hline 7/23/2008 7:00 AM & 0.174 & 23.8 & 0.113 & 0.318 & 21.5 & 0.45 & 0.040 & 0.042 & -- & -- & 0.242 & 14.5 & 0.419 & 13.5 & -- \\
\hline 7/23/2008 8:00 AM & 0.173 & 23.8 & 0.113 & 0.318 & 21.5 & 0.45 & 0.040 & 0.042 & -- & -- & 0.242 & 14.5 & 0.420 & 13.5 & -- \\
\hline 7/23/2008 9:00 AM & 0.173 & 23.8 & 0.112 & 0.318 & 21.6 & 0.45 & 0.040 & 0.042 & -- & -- & 0.242 & 14.5 & 0.420 & 13.5 & -- \\
\hline 7/23/2008 10:00 AM & 0.171 & 23.8 & 0.112 & 0.318 & 21.6 & 0.45 & 0.040 & 0.042 & -- & -- & 0.243 & 14.5 & 0.420 & 13.5 & -- \\
\hline 7/23/2008 11:00 AM & 0.170 & 23.7 & 0.112 & 0.318 & 21.6 & 0.45 & 0.040 & 0.043 & -- & -- & 0.243 & 14.5 & 0.420 & 13.5 & -- \\
\hline 7/23/2008 12:00 PM & 0.169 & 23.7 & 0.111 & 0.319 & 21.6 & 0.45 & 0.040 & 0.043 & -- & -- & 0.242 & 14.5 & 0.421 & 13.5 & -- \\
\hline 7/23/2008 1:00 PM & 0.167 & 23.7 & 0.111 & 0.319 & 21.6 & 0.45 & 0.040 & 0.043 & -- & -- & 0.243 & 14.5 & 0.421 & 13.5 & -- \\
\hline 7/23/2008 2:00 PM & 0.165 & 23.7 & 0.111 & 0.319 & 21.6 & 0.45 & 0.040 & 0.043 & -- & -- & 0.243 & 14.5 & 0.421 & 13.5 & -- \\
\hline
\end{tabular}


Appendix 1. Volumetric water content, temperature, and electrical-conductivity data collected at the flood-irrigated site during 2008.-Continued

Depth in feet below land surface; volumetric water content dimensionless; temperature in degrees Celsius; bulk electrical conductivity in decisiemens per meter; --, no data]

\begin{tabular}{|c|c|c|c|c|c|c|c|c|c|c|c|c|c|c|c|}
\hline \multirow[b]{3}{*}{$\begin{array}{l}\text { Measurement } \\
\text { date and time }\end{array}$} & \multicolumn{15}{|c|}{ Depth } \\
\hline & \multicolumn{2}{|l|}{2} & \multirow{2}{*}{$\begin{array}{c}3 \\
\begin{array}{c}\text { Volumetric } \\
\text { water } \\
\text { content }\end{array}\end{array}$} & \multicolumn{3}{|c|}{4} & \multirow{2}{*}{$\begin{array}{c}6 \\
\text { Volumetric } \\
\text { water } \\
\text { content }\end{array}$} & \multicolumn{2}{|l|}{8} & \multirow{2}{*}{$\begin{array}{c}10 \\
\text { Volumetric } \\
\text { water } \\
\text { content }\end{array}$} & \multicolumn{2}{|c|}{12} & \multicolumn{2}{|c|}{15} & \multirow{2}{*}{$\begin{array}{c}18.5 \\
\text { Volumetric } \\
\text { water } \\
\text { content }\end{array}$} \\
\hline & $\begin{array}{l}\text { Volumetric } \\
\text { water } \\
\text { content }\end{array}$ & $\begin{array}{l}\text { Temper- } \\
\text { ature }\end{array}$ & & $\begin{array}{c}\text { Volumetric } \\
\text { water } \\
\text { content }\end{array}$ & $\begin{array}{l}\text { Temper- } \\
\text { ature }\end{array}$ & $\begin{array}{c}\text { Bulk } \\
\text { electrical } \\
\text { conduct- } \\
\text { ivity } \\
\end{array}$ & & $\begin{array}{l}\text { Volumetric } \\
\text { water } \\
\text { content }\end{array}$ & $\begin{array}{l}\text { Temper- } \\
\text { ature }\end{array}$ & & $\begin{array}{l}\text { Volumetric } \\
\text { water } \\
\text { content }\end{array}$ & $\begin{array}{l}\text { Temper- } \\
\text { ature }\end{array}$ & $\begin{array}{l}\text { Volumetric } \\
\text { water } \\
\text { content }\end{array}$ & $\begin{array}{l}\text { Temper- } \\
\text { ature }\end{array}$ & \\
\hline 7/23/2008 3:00 PM & 0.166 & 23.6 & 0.110 & 0.319 & 21.6 & 0.45 & 0.040 & 0.043 & -- & -- & 0.243 & 14.5 & 0.420 & 13.5 & -- \\
\hline 7/23/2008 4:00 PM & 0.165 & 23.6 & 0.110 & 0.319 & 21.6 & 0.45 & 0.040 & 0.042 & -- & -- & 0.244 & 14.5 & 0.420 & 13.5 & -- \\
\hline 7/23/2008 5:00 PM & 0.163 & 23.6 & 0.109 & 0.319 & 21.6 & 0.45 & 0.040 & 0.042 & -- & -- & 0.244 & 14.5 & 0.420 & 13.5 & -- \\
\hline 7/23/2008 6:00 PM & 0.163 & 23.5 & 0.109 & 0.318 & 21.6 & 0.45 & 0.040 & 0.042 & -- & -- & 0.244 & 14.5 & 0.420 & 13.5 & -- \\
\hline 7/23/2008 7:00 PM & 0.162 & 23.5 & 0.109 & 0.319 & 21.6 & 0.45 & 0.040 & 0.042 & -- & -- & 0.245 & 14.5 & 0.420 & 13.5 & -- \\
\hline 7/23/2008 8:00 PM & 0.162 & 23.5 & 0.109 & 0.318 & 21.6 & 0.45 & 0.040 & 0.042 & -- & -- & 0.245 & 14.5 & 0.420 & 13.5 & -- \\
\hline 7/23/2008 9:00 PM & 0.161 & 23.5 & 0.109 & 0.318 & 21.6 & 0.45 & 0.040 & 0.042 & -- & -- & 0.245 & 14.5 & 0.420 & 13.5 & -- \\
\hline 7/23/2008 10:00 PM & 0.160 & 23.5 & 0.109 & 0.318 & 21.6 & 0.45 & 0.040 & 0.042 & -- & -- & 0.245 & 14.5 & 0.420 & 13.5 & -- \\
\hline 7/23/2008 11:00 PM & 0.159 & 23.5 & 0.108 & 0.318 & 21.6 & 0.45 & 0.040 & 0.042 & -- & -- & 0.246 & 14.5 & 0.420 & 13.5 & -- \\
\hline 7/24/2008 12:00 AM & 0.158 & 23.5 & 0.108 & 0.318 & 21.6 & 0.45 & 0.040 & 0.042 & -- & -- & 0.246 & 14.5 & 0.419 & 13.5 & -- \\
\hline 7/24/2008 1:00 AM & 0.157 & 23.5 & 0.108 & 0.318 & 21.6 & 0.45 & 0.040 & 0.042 & -- & -- & 0.246 & 14.5 & 0.420 & 13.5 & -- \\
\hline 7/24/2008 2:00 AM & 0.156 & 23.4 & 0.108 & 0.318 & 21.6 & 0.45 & 0.040 & 0.042 & -- & -- & 0.246 & 14.5 & 0.419 & 13.5 & -- \\
\hline 7/24/2008 3:00 AM & 0.155 & 23.4 & 0.107 & 0.318 & 21.6 & 0.45 & 0.040 & 0.042 & -- & -- & 0.247 & 14.5 & 0.420 & 13.6 & -- \\
\hline 7/24/2008 4:00 AM & 0.155 & 23.5 & 0.107 & 0.318 & 21.7 & 0.45 & 0.040 & 0.043 & -- & -- & 0.247 & 14.5 & 0.419 & 13.5 & -- \\
\hline 7/24/2008 5:00 AM & 0.154 & 23.4 & 0.107 & 0.318 & 21.7 & 0.45 & 0.040 & 0.043 & -- & -- & 0.248 & 14.5 & 0.419 & 13.6 & -- \\
\hline 7/24/2008 6:00 AM & 0.154 & 23.4 & 0.106 & 0.318 & 21.7 & 0.45 & 0.040 & 0.043 & -- & -- & 0.248 & 14.5 & 0.419 & 13.5 & -- \\
\hline 7/24/2008 7:00 AM & 0.153 & 23.4 & 0.106 & 0.318 & 21.7 & 0.45 & 0.040 & 0.043 & -- & -- & 0.248 & 14.5 & 0.419 & 13.6 & -- \\
\hline 7/24/2008 8:00 AM & 0.153 & 23.4 & 0.106 & 0.318 & 21.7 & 0.45 & 0.040 & 0.042 & -- & -- & 0.248 & 14.5 & 0.420 & 13.6 & -- \\
\hline 7/24/2008 9:00 AM & 0.151 & 23.3 & 0.106 & 0.318 & 21.7 & 0.45 & 0.040 & 0.042 & -- & -- & 0.248 & 14.5 & 0.420 & 13.6 & -- \\
\hline 7/24/2008 10:00 AM & 0.151 & 23.3 & 0.106 & 0.318 & 21.7 & 0.45 & 0.040 & 0.042 & -- & -- & 0.248 & 14.5 & 0.420 & 13.6 & -- \\
\hline 7/24/2008 11:00 AM & 0.148 & 23.3 & 0.105 & 0.318 & 21.7 & 0.45 & 0.040 & 0.043 & -- & -- & 0.249 & 14.5 & 0.421 & 13.6 & -- \\
\hline 7/24/2008 12:00 PM & 0.147 & 23.3 & 0.105 & 0.318 & 21.7 & 0.45 & 0.040 & 0.043 & -- & -- & 0.249 & 14.6 & 0.421 & 13.6 & -- \\
\hline 7/24/2008 1:00 PM & 0.150 & 23.2 & 0.105 & 0.318 & 21.7 & 0.45 & 0.040 & 0.043 & -- & -- & 0.249 & 14.6 & 0.421 & 13.6 & -- \\
\hline 7/24/2008 2:00 PM & 0.150 & 23.2 & 0.104 & 0.318 & 21.7 & 0.45 & 0.040 & 0.043 & -- & -- & 0.249 & 14.5 & 0.421 & 13.6 & -- \\
\hline 7/24/2008 3:00 PM & 0.149 & 23.1 & 0.104 & 0.318 & 21.7 & 0.45 & 0.040 & 0.043 & -- & -- & 0.249 & 14.6 & 0.421 & 13.6 & -- \\
\hline 7/24/2008 4:00 PM & 0.149 & 23.1 & 0.104 & 0.318 & 21.7 & 0.45 & 0.040 & 0.043 & -- & -- & 0.250 & 14.5 & 0.421 & 13.6 & -- \\
\hline 7/24/2008 5:00 PM & 0.149 & 23.1 & 0.104 & 0.317 & 21.7 & 0.45 & 0.040 & 0.043 & -- & -- & 0.250 & 14.5 & 0.420 & 13.6 & -- \\
\hline 7/24/2008 6:00 PM & 0.149 & 23.1 & 0.104 & 0.318 & 21.7 & 0.45 & 0.040 & 0.043 & -- & -- & 0.250 & 14.5 & 0.420 & 13.6 & -- \\
\hline 7/24/2008 7:00 PM & 0.148 & 23.1 & 0.104 & 0.317 & 21.7 & 0.45 & 0.040 & 0.043 & -- & -- & 0.251 & 14.6 & 0.420 & 13.6 & -- \\
\hline 7/24/2008 8:00 PM & 0.146 & 23.0 & 0.104 & 0.317 & 21.7 & 0.45 & 0.040 & 0.042 & -- & -- & 0.251 & 14.6 & 0.420 & 13.6 & -- \\
\hline 7/24/2008 9:00 PM & 0.145 & 23.0 & 0.104 & 0.317 & 21.7 & 0.45 & 0.041 & 0.042 & -- & -- & 0.251 & 14.5 & 0.420 & 13.6 & -- \\
\hline 7/24/2008 10:00 PM & 0.146 & 23.0 & 0.104 & 0.317 & 21.7 & 0.45 & 0.041 & 0.042 & -- & -- & 0.251 & 14.5 & 0.420 & 13.6 & -- \\
\hline 7/24/2008 11:00 PM & 0.146 & 23.0 & 0.104 & 0.317 & 21.7 & 0.45 & 0.041 & 0.042 & -- & -- & 0.253 & 14.5 & 0.420 & 13.6 & -- \\
\hline 7/25/2008 12:00 AM & 0.146 & 23.0 & 0.104 & 0.317 & 21.7 & 0.45 & 0.041 & 0.042 & -- & -- & 0.253 & 14.6 & 0.420 & 13.6 & -- \\
\hline 7/25/2008 1:00 AM & 0.146 & 23.0 & 0.104 & 0.317 & 21.7 & 0.45 & 0.041 & 0.042 & -- & -- & 0.254 & 14.5 & 0.420 & 13.6 & -- \\
\hline 7/25/2008 2:00 AM & 0.145 & 23.0 & 0.103 & 0.317 & 21.7 & 0.45 & 0.041 & 0.042 & -- & -- & 0.254 & 14.5 & 0.420 & 13.6 & -- \\
\hline 7/25/2008 3:00 AM & 0.145 & 23.0 & 0.103 & 0.317 & 21.7 & 0.45 & 0.041 & 0.042 & -- & -- & 0.254 & 14.5 & 0.420 & 13.6 & -- \\
\hline 7/25/2008 4:00 AM & 0.144 & 23.0 & 0.103 & 0.317 & 21.7 & 0.45 & 0.041 & 0.042 & -- & -- & 0.254 & 14.6 & 0.420 & 13.6 & -- \\
\hline 7/25/2008 5:00 AM & 0.144 & 23.0 & 0.103 & 0.317 & 21.7 & 0.45 & 0.041 & 0.042 & -- & -- & 0.254 & 14.5 & 0.420 & 13.6 & -- \\
\hline 7/25/2008 6:00 AM & 0.143 & 23.0 & 0.103 & 0.316 & 21.7 & 0.45 & 0.041 & 0.042 & -- & -- & 0.255 & 14.5 & 0.420 & 13.6 & -- \\
\hline
\end{tabular}


Appendix 1. Volumetric water content, temperature, and electrical-conductivity data collected at the flood-irrigated site during 2008.-Continued

[Depth in feet below land surface; volumetric water content dimensionless; temperature in degrees Celsius; bulk electrical conductivity in decisiemens per meter; --, no data]

\begin{tabular}{|c|c|c|c|c|c|c|c|c|c|c|c|c|c|c|c|}
\hline \multirow[b]{3}{*}{$\begin{array}{l}\text { Measurement } \\
\text { date and time }\end{array}$} & \multicolumn{15}{|c|}{ Depth } \\
\hline & \multicolumn{2}{|c|}{2} & \multirow{2}{*}{$\begin{array}{c}3 \\
\text { Volumetric } \\
\text { water } \\
\text { content }\end{array}$} & \multicolumn{3}{|c|}{4} & \multirow{2}{*}{$\begin{array}{c}6 \\
\text { Volumetric } \\
\text { water } \\
\text { content }\end{array}$} & \multicolumn{2}{|l|}{8} & \multirow{2}{*}{$\begin{array}{c}10 \\
\begin{array}{c}\text { Volumetric } \\
\text { water } \\
\text { content }\end{array}\end{array}$} & \multicolumn{2}{|c|}{12} & \multicolumn{2}{|c|}{15} & \multirow{2}{*}{$\begin{array}{c}18.5 \\
\text { Volumetric } \\
\text { water } \\
\text { content }\end{array}$} \\
\hline & $\begin{array}{c}\text { Volumetric } \\
\text { water } \\
\text { content }\end{array}$ & $\begin{array}{l}\text { Temper- } \\
\text { ature }\end{array}$ & & $\begin{array}{l}\text { Volumetric } \\
\text { water } \\
\text { content }\end{array}$ & $\begin{array}{l}\text { Temper- } \\
\text { ature }\end{array}$ & $\begin{array}{c}\text { Bulk } \\
\text { electrical } \\
\text { conduct- } \\
\text { ivity }\end{array}$ & & $\begin{array}{c}\text { Volumetric } \\
\text { water } \\
\text { content }\end{array}$ & $\begin{array}{l}\text { Temper- } \\
\text { ature }\end{array}$ & & $\begin{array}{c}\text { Volumetric } \\
\text { water } \\
\text { content }\end{array}$ & $\begin{array}{l}\text { Temper- } \\
\text { ature }\end{array}$ & $\begin{array}{c}\text { Volumetric } \\
\text { water } \\
\text { content }\end{array}$ & $\begin{array}{l}\text { Temper- } \\
\text { ature }\end{array}$ & \\
\hline 7/25/2008 7:00 AM & 0.143 & 23.0 & 0.102 & 0.316 & 21.7 & 0.45 & 0.041 & 0.042 & - & -- & 0.255 & 14.5 & 0.420 & 13.6 & -- \\
\hline 7/25/2008 8:00 AM & 0.143 & 23.0 & 0.102 & 0.316 & 21.7 & 0.45 & 0.041 & 0.042 & -- & -- & 0.255 & 14.5 & 0.420 & 13.6 & -- \\
\hline 7/25/2008 9:00 AM & 0.137 & 23.0 & 0.102 & 0.316 & 21.7 & 0.45 & 0.041 & 0.042 & -- & -- & 0.255 & 14.6 & 0.420 & 13.6 & -- \\
\hline $7 / 25 / 2008$ 10:00 AM & 0.140 & 23.0 & 0.102 & 0.316 & 21.7 & 0.45 & 0.041 & 0.042 & -- & -- & 0.255 & 14.6 & 0.420 & 13.6 & -- \\
\hline 7/25/2008 11:00 AM & 0.142 & 22.9 & 0.101 & 0.316 & 21.6 & 0.45 & 0.040 & 0.042 & -- & -- & 0.256 & 14.6 & 0.421 & 13.6 & -- \\
\hline 7/25/2008 12:00 PM & 0.140 & 22.9 & 0.101 & 0.316 & 21.6 & 0.45 & 0.040 & 0.043 & -- & -- & 0.256 & 14.6 & 0.421 & 13.6 & -- \\
\hline 7/25/2008 1:00 PM & 0.138 & 22.9 & 0.101 & 0.316 & 21.6 & 0.45 & 0.040 & 0.043 & -- & -- & 0.256 & 14.6 & 0.421 & 13.6 & -- \\
\hline 7/25/2008 2:00 PM & 0.136 & 22.8 & 0.101 & 0.316 & 21.6 & 0.45 & 0.040 & 0.043 & -- & -- & 0.256 & 14.6 & 0.421 & 13.6 & -- \\
\hline 7/25/2008 3:00 PM & 0.136 & 22.8 & 0.101 & 0.316 & 21.6 & 0.45 & 0.040 & 0.043 & -- & -- & 0.257 & 14.6 & 0.421 & 13.6 & -- \\
\hline 7/25/2008 4:00 PM & 0.136 & 22.8 & 0.101 & 0.316 & 21.6 & 0.45 & 0.040 & 0.043 & -- & -- & 0.258 & 14.6 & 0.421 & 13.7 & -- \\
\hline 7/25/2008 5:00 PM & 0.137 & 22.8 & 0.101 & 0.316 & 21.6 & 0.45 & 0.040 & 0.043 & -- & -- & 0.258 & 14.6 & 0.421 & 13.6 & -- \\
\hline 7/25/2008 6:00 PM & 0.137 & 22.7 & 0.100 & 0.316 & 21.6 & 0.45 & 0.040 & 0.042 & -- & -- & 0.259 & 14.6 & 0.421 & 13.6 & -- \\
\hline 7/25/2008 7:00 PM & 0.136 & 22.7 & 0.100 & 0.316 & 21.6 & 0.45 & 0.040 & 0.042 & -- & -- & 0.259 & 14.6 & 0.421 & 13.6 & -- \\
\hline 7/25/2008 8:00 PM & 0.136 & 22.8 & 0.100 & 0.316 & 21.6 & 0.45 & 0.041 & 0.042 & -- & -- & 0.259 & 14.6 & 0.420 & 13.6 & -- \\
\hline 7/25/2008 9:00 PM & 0.136 & 22.8 & 0.100 & 0.316 & 21.6 & 0.45 & 0.041 & 0.042 & -- & -- & 0.260 & 14.6 & 0.420 & 13.7 & -- \\
\hline 7/25/2008 10:00 PM & 0.136 & 22.7 & 0.100 & 0.316 & 21.6 & 0.45 & 0.041 & 0.042 & -- & -- & 0.260 & 14.6 & 0.420 & 13.7 & -- \\
\hline 7/25/2008 11:00 PM & 0.136 & 22.7 & 0.100 & 0.316 & 21.6 & 0.45 & 0.041 & 0.042 & -- & -- & 0.260 & 14.6 & 0.420 & 13.6 & -- \\
\hline 7/26/2008 12:00 AM & 0.136 & 22.7 & 0.100 & 0.315 & 21.6 & 0.45 & 0.041 & 0.042 & -- & 0.414 & 0.260 & 14.6 & 0.420 & 13.7 & -- \\
\hline 7/26/2008 1:00 AM & 0.137 & 22.8 & 0.099 & 0.315 & 21.6 & 0.45 & 0.041 & 0.042 & -- & 0.054 & 0.261 & 14.6 & 0.420 & 13.7 & -- \\
\hline 7/26/2008 2:00 AM & 0.137 & 22.7 & 0.099 & 0.315 & 21.6 & 0.45 & 0.040 & 0.042 & -- & 0.053 & 0.261 & 14.6 & 0.420 & 13.7 & -- \\
\hline 7/26/2008 3:00 AM & 0.137 & 22.8 & 0.099 & 0.315 & 21.6 & 0.45 & 0.040 & 0.042 & -- & 0.053 & 0.262 & 14.6 & 0.420 & 13.7 & -- \\
\hline 7/26/2008 4:00 AM & 0.137 & 22.8 & 0.099 & 0.315 & 21.6 & 0.45 & 0.040 & 0.042 & -- & 0.052 & 0.262 & 14.6 & 0.420 & 13.7 & -- \\
\hline 7/26/2008 5:00 AM & 0.137 & 22.7 & 0.099 & 0.315 & 21.6 & 0.45 & 0.040 & 0.042 & -- & 0.052 & 0.263 & 14.6 & 0.420 & 13.7 & -- \\
\hline 7/26/2008 6:00 AM & 0.136 & 22.7 & 0.099 & 0.315 & 21.6 & 0.45 & 0.041 & 0.042 & -- & 0.052 & 0.263 & 14.6 & 0.420 & 13.7 & -- \\
\hline 7/26/2008 7:00 AM & 0.136 & 22.7 & 0.099 & 0.315 & 21.6 & 0.45 & 0.041 & 0.042 & -- & 0.051 & 0.263 & 14.6 & 0.420 & 13.7 & -- \\
\hline 7/26/2008 8:00 AM & 0.135 & 22.7 & 0.099 & 0.315 & 21.6 & 0.45 & 0.041 & 0.042 & -- & 0.051 & 0.265 & 14.6 & 0.420 & 13.7 & -- \\
\hline 7/26/2008 9:00 AM & 0.133 & 22.7 & 0.098 & 0.315 & 21.6 & 0.45 & 0.041 & 0.042 & -- & 0.050 & 0.265 & 14.6 & 0.420 & 13.7 & -- \\
\hline 7/26/2008 10:00 AM & 0.132 & 22.7 & 0.098 & 0.315 & 21.6 & 0.46 & 0.041 & 0.042 & -- & 0.050 & 0.265 & 14.6 & 0.420 & 13.7 & -- \\
\hline 7/26/2008 11:00 AM & 0.131 & 22.7 & 0.098 & 0.315 & 21.6 & 0.45 & 0.040 & 0.042 & -- & 0.050 & 0.266 & 14.6 & 0.421 & 13.7 & -- \\
\hline 7/26/2008 12:00 PM & 0.132 & 22.7 & 0.098 & 0.315 & 21.6 & 0.45 & 0.040 & 0.043 & -- & 0.050 & 0.266 & 14.6 & 0.421 & 13.7 & -- \\
\hline 7/26/2008 1:00 PM & 0.132 & 22.7 & 0.098 & 0.315 & 21.6 & 0.45 & 0.040 & 0.043 & -- & 0.049 & 0.266 & 14.6 & 0.421 & 13.7 & -- \\
\hline 7/26/2008 2:00 PM & 0.133 & 22.5 & 0.098 & 0.315 & 21.6 & 0.45 & 0.040 & 0.043 & -- & 0.049 & 0.266 & 14.6 & 0.421 & 13.7 & -- \\
\hline 7/26/2008 3:00 PM & 0.133 & 22.5 & 0.098 & 0.315 & 21.6 & 0.45 & 0.040 & 0.043 & -- & 0.049 & 0.267 & 14.6 & 0.421 & 13.7 & -- \\
\hline 7/26/2008 4:00 PM & 0.132 & 22.5 & 0.098 & 0.315 & 21.6 & 0.45 & 0.040 & 0.043 & -- & 0.049 & 0.268 & 14.6 & 0.421 & 13.7 & -- \\
\hline 7/26/2008 5:00 PM & 0.132 & 22.5 & 0.098 & 0.315 & 21.6 & 0.46 & 0.040 & 0.043 & -- & 0.048 & 0.268 & 14.6 & 0.421 & 13.7 & -- \\
\hline 7/26/2008 6:00 PM & 0.132 & 22.5 & 0.098 & 0.315 & 21.6 & 0.46 & 0.040 & 0.043 & -- & 0.048 & 0.269 & 14.6 & 0.421 & 13.7 & -- \\
\hline 7/26/2008 7:00 PM & 0.131 & 22.5 & 0.098 & 0.315 & 21.6 & 0.46 & 0.040 & 0.043 & -- & 0.048 & 0.270 & 14.6 & 0.421 & 13.7 & -- \\
\hline 7/26/2008 8:00 PM & 0.130 & 22.4 & 0.098 & 0.315 & 21.6 & 0.46 & 0.040 & 0.042 & -- & 0.047 & 0.270 & 14.6 & 0.421 & 13.7 & -- \\
\hline 7/26/2008 9:00 PM & 0.129 & 22.5 & 0.098 & 0.315 & 21.6 & 0.46 & 0.040 & 0.042 & -- & 0.047 & 0.270 & 14.6 & 0.421 & 13.7 & -- \\
\hline 7/26/2008 10:00 PM & 0.128 & 22.5 & 0.098 & 0.313 & 21.5 & 0.46 & 0.040 & 0.042 & -- & 0.047 & 0.271 & 14.6 & 0.421 & 13.7 & -- \\
\hline
\end{tabular}


Appendix 1. Volumetric water content, temperature, and electrical-conductivity data collected at the flood-irrigated site during 2008.-Continued

Depth in feet below land surface; volumetric water content dimensionless; temperature in degrees Celsius; bulk electrical conductivity in decisiemens per meter; --, no data]

\begin{tabular}{|c|c|c|c|c|c|c|c|c|c|c|c|c|c|c|c|}
\hline \multirow[b]{3}{*}{$\begin{array}{l}\text { Measurement } \\
\text { date and time }\end{array}$} & \multicolumn{15}{|c|}{ Depth } \\
\hline & \multicolumn{2}{|l|}{2} & \multirow{2}{*}{$\begin{array}{c}3 \\
\begin{array}{c}\text { Volumetric } \\
\text { water } \\
\text { content }\end{array}\end{array}$} & \multicolumn{3}{|c|}{4} & \multirow{2}{*}{$\begin{array}{c}6 \\
\text { Volumetric } \\
\text { water } \\
\text { content }\end{array}$} & \multicolumn{2}{|l|}{8} & \multirow{2}{*}{$\begin{array}{c}10 \\
\text { Volumetric } \\
\text { water } \\
\text { content }\end{array}$} & \multicolumn{2}{|c|}{12} & \multicolumn{2}{|c|}{15} & \multirow{2}{*}{$\begin{array}{c}18.5 \\
\text { Volumetric } \\
\text { water } \\
\text { content }\end{array}$} \\
\hline & $\begin{array}{l}\text { Volumetric } \\
\text { water } \\
\text { content }\end{array}$ & $\begin{array}{l}\text { Temper- } \\
\text { ature }\end{array}$ & & $\begin{array}{l}\text { Volumetric } \\
\text { water } \\
\text { content }\end{array}$ & $\begin{array}{l}\text { Temper- } \\
\text { ature }\end{array}$ & $\begin{array}{c}\text { Bulk } \\
\text { electrical } \\
\text { conduct- } \\
\text { ivity } \\
\end{array}$ & & $\begin{array}{l}\text { Volumetric } \\
\text { water } \\
\text { content }\end{array}$ & $\begin{array}{l}\text { Temper- } \\
\text { ature }\end{array}$ & & $\begin{array}{l}\text { Volumetric } \\
\text { water } \\
\text { content }\end{array}$ & $\begin{array}{l}\text { Temper- } \\
\text { ature }\end{array}$ & $\begin{array}{l}\text { Volumetric } \\
\text { water } \\
\text { content }\end{array}$ & $\begin{array}{l}\text { Temper- } \\
\text { ature }\end{array}$ & \\
\hline 7/26/2008 11:00 PM & 0.128 & 22.4 & 0.098 & 0.313 & 21.5 & 0.46 & 0.040 & 0.042 & -- & 0.047 & 0.271 & 14.6 & 0.420 & 13.7 & -- \\
\hline 7/27/2008 12:00 AM & 0.128 & 22.4 & 0.098 & 0.313 & 21.5 & 0.46 & 0.040 & 0.042 & -- & 0.047 & 0.272 & 14.6 & 0.420 & 13.7 & -- \\
\hline 7/27/2008 1:00 AM & 0.128 & 22.5 & 0.097 & 0.313 & 21.5 & 0.46 & 0.040 & 0.042 & -- & 0.047 & 0.272 & 14.6 & 0.420 & 13.7 & -- \\
\hline 7/27/2008 2:00 AM & 0.128 & 22.5 & 0.097 & 0.313 & 21.5 & 0.46 & 0.040 & 0.042 & -- & 0.047 & 0.273 & 14.6 & 0.420 & 13.7 & -- \\
\hline 7/27/2008 3:00 AM & 0.129 & 22.5 & 0.097 & 0.313 & 21.5 & 0.46 & 0.040 & 0.042 & -- & 0.046 & 0.273 & 14.7 & 0.420 & 13.7 & -- \\
\hline 7/27/2008 4:00 AM & 0.129 & 22.5 & 0.097 & 0.313 & 21.5 & 0.46 & 0.040 & 0.042 & -- & 0.046 & 0.273 & 14.6 & 0.420 & 13.7 & -- \\
\hline 7/27/2008 5:00 AM & 0.129 & 22.5 & 0.097 & 0.313 & 21.5 & 0.46 & 0.040 & 0.042 & -- & 0.045 & 0.274 & 14.6 & 0.420 & 13.7 & -- \\
\hline 7/27/2008 6:00 AM & 0.129 & 22.5 & 0.096 & 0.313 & 21.5 & 0.46 & 0.040 & 0.042 & -- & 0.045 & 0.274 & 14.6 & 0.420 & 13.7 & -- \\
\hline 7/27/2008 7:00 AM & 0.129 & 22.5 & 0.096 & 0.313 & 21.5 & 0.46 & 0.040 & 0.042 & -- & 0.045 & 0.274 & 14.6 & 0.420 & 13.7 & -- \\
\hline 7/27/2008 8:00 AM & 0.128 & 22.5 & 0.096 & 0.313 & 21.5 & 0.46 & 0.040 & 0.042 & -- & 0.044 & 0.275 & 14.6 & 0.420 & 13.7 & -- \\
\hline 7/27/2008 9:00 AM & 0.126 & 22.5 & 0.096 & 0.313 & 21.5 & 0.46 & 0.040 & 0.042 & -- & 0.044 & 0.275 & 14.6 & 0.421 & 13.7 & -- \\
\hline 7/27/2008 10:00 AM & 0.128 & 22.5 & 0.096 & 0.313 & 21.5 & 0.46 & 0.040 & 0.042 & -- & 0.043 & 0.275 & 14.6 & 0.421 & 13.7 & -- \\
\hline 7/27/2008 11:00 AM & 0.129 & 22.5 & 0.096 & 0.313 & 21.5 & 0.46 & 0.040 & 0.043 & -- & 0.043 & 0.275 & 14.7 & 0.422 & 13.7 & -- \\
\hline 7/27/2008 12:00 PM & 0.129 & 22.5 & 0.096 & 0.313 & 21.5 & 0.46 & 0.040 & 0.043 & -- & 0.042 & 0.276 & 14.7 & 0.422 & 13.8 & -- \\
\hline 7/27/2008 1:00 PM & 0.129 & 22.5 & 0.096 & 0.313 & 21.5 & 0.46 & 0.040 & 0.043 & -- & 0.042 & 0.275 & 14.7 & 0.422 & 13.8 & -- \\
\hline 7/27/2008 2:00 PM & 0.129 & 22.4 & 0.096 & 0.313 & 21.5 & 0.46 & 0.040 & 0.043 & -- & 0.042 & 0.276 & 14.7 & 0.421 & 13.7 & -- \\
\hline 7/27/2008 3:00 PM & 0.128 & 22.4 & 0.096 & 0.313 & 21.5 & 0.46 & 0.040 & 0.043 & -- & 0.042 & 0.276 & 14.7 & 0.421 & 13.8 & -- \\
\hline 7/27/2008 4:00 PM & 0.128 & 22.4 & 0.096 & 0.313 & 21.5 & 0.46 & 0.040 & 0.042 & -- & 0.042 & 0.278 & 14.7 & 0.421 & 13.8 & -- \\
\hline 7/27/2008 5:00 PM & 0.128 & 22.4 & 0.095 & 0.313 & 21.5 & 0.46 & 0.040 & 0.043 & -- & 0.042 & 0.278 & 14.7 & 0.421 & 13.8 & -- \\
\hline 7/27/2008 6:00 PM & 0.128 & 22.4 & 0.095 & 0.313 & 21.5 & 0.46 & 0.040 & 0.043 & -- & 0.042 & 0.278 & 14.7 & 0.422 & 13.8 & -- \\
\hline 7/27/2008 7:00 PM & 0.126 & 22.3 & 0.095 & 0.313 & 21.5 & 0.46 & 0.040 & 0.043 & -- & 0.042 & 0.279 & 14.7 & 0.422 & 13.8 & -- \\
\hline 7/27/2008 8:00 PM & 0.126 & 22.4 & 0.095 & 0.313 & 21.5 & 0.46 & 0.040 & 0.043 & -- & 0.042 & 0.279 & 14.7 & 0.422 & 13.8 & -- \\
\hline 7/27/2008 9:00 PM & 0.125 & 22.4 & 0.095 & 0.313 & 21.5 & 0.46 & 0.040 & 0.042 & -- & 0.042 & 0.279 & 14.7 & 0.422 & 13.8 & -- \\
\hline 7/27/2008 10:00 PM & 0.124 & 22.4 & 0.095 & 0.313 & 21.5 & 0.46 & 0.040 & 0.042 & -- & 0.042 & 0.279 & 14.7 & 0.422 & 13.8 & -- \\
\hline 7/27/2008 11:00 PM & 0.124 & 22.4 & 0.095 & 0.312 & 21.5 & 0.46 & 0.040 & 0.042 & -- & 0.042 & 0.279 & 14.7 & 0.422 & 13.8 & -- \\
\hline 7/28/2008 12:00 AM & 0.123 & 22.4 & 0.095 & 0.312 & 21.5 & 0.46 & 0.040 & 0.042 & -- & 0.042 & 0.279 & 14.7 & 0.422 & 13.8 & -- \\
\hline 7/28/2008 1:00 AM & 0.123 & 22.5 & 0.095 & 0.312 & 21.5 & 0.46 & 0.040 & 0.042 & -- & 0.042 & 0.279 & 14.7 & 0.422 & 13.8 & -- \\
\hline 7/28/2008 2:00 AM & 0.122 & 22.5 & 0.095 & 0.312 & 21.5 & 0.46 & 0.040 & 0.042 & -- & 0.041 & 0.280 & 14.7 & 0.422 & 13.8 & -- \\
\hline 7/28/2008 3:00 AM & 0.123 & 22.5 & 0.095 & 0.312 & 21.5 & 0.46 & 0.040 & 0.042 & -- & 0.041 & 0.280 & 14.7 & 0.422 & 13.8 & -- \\
\hline 7/28/2008 4:00 AM & 0.124 & 22.6 & 0.095 & 0.312 & 21.4 & 0.46 & 0.040 & 0.042 & -- & 0.041 & 0.281 & 14.7 & 0.422 & 13.8 & -- \\
\hline 7/28/2008 5:00 AM & 0.124 & 22.6 & 0.094 & 0.312 & 21.4 & 0.46 & 0.040 & 0.042 & -- & 0.041 & 0.280 & 14.7 & 0.422 & 13.8 & -- \\
\hline 7/28/2008 6:00 AM & 0.125 & 22.5 & 0.094 & 0.312 & 21.4 & 0.46 & 0.040 & 0.042 & -- & 0.041 & 0.280 & 14.7 & 0.421 & 13.8 & -- \\
\hline 7/28/2008 7:00 AM & 0.125 & 22.6 & 0.094 & 0.312 & 21.4 & 0.46 & 0.040 & 0.042 & -- & 0.041 & 0.281 & 14.7 & 0.421 & 13.8 & -- \\
\hline 7/28/2008 8:00 AM & 0.124 & 22.6 & 0.094 & 0.312 & 21.4 & 0.46 & 0.040 & 0.042 & -- & 0.042 & 0.281 & 14.7 & 0.422 & 13.8 & -- \\
\hline 7/28/2008 9:00 AM & 0.123 & 22.7 & 0.094 & 0.312 & 21.4 & 0.46 & 0.040 & 0.042 & -- & 0.042 & 0.281 & 14.7 & 0.422 & 13.8 & -- \\
\hline 7/28/2008 10:00 AM & 0.122 & 22.7 & 0.094 & 0.312 & 21.4 & 0.46 & 0.040 & 0.042 & -- & 0.040 & 0.281 & 14.7 & 0.422 & 13.8 & -- \\
\hline 7/28/2008 11:00 AM & 0.123 & 22.6 & 0.094 & 0.312 & 21.4 & 0.46 & 0.040 & 0.043 & -- & 0.041 & 0.281 & 14.7 & 0.422 & 13.8 & -- \\
\hline 7/28/2008 12:00 PM & 0.133 & 22.6 & 0.094 & 0.312 & 21.4 & 0.46 & 0.040 & 0.043 & -- & 0.041 & 0.281 & 14.7 & 0.422 & 13.8 & -- \\
\hline 7/28/2008 1:00 PM & 0.178 & 22.6 & 0.093 & 0.312 & 21.4 & 0.46 & 0.040 & 0.043 & -- & 0.039 & 0.281 & 14.7 & 0.422 & 13.8 & -- \\
\hline 7/28/2008 2:00 PM & 0.190 & 22.6 & 0.094 & 0.312 & 21.4 & 0.46 & 0.039 & 0.043 & -- & 0.040 & 0.281 & 14.7 & 0.423 & 13.8 & -- \\
\hline
\end{tabular}


Appendix 1. Volumetric water content, temperature, and electrical-conductivity data collected at the flood-irrigated site during 2008.-Continued

[Depth in feet below land surface; volumetric water content dimensionless; temperature in degrees Celsius; bulk electrical conductivity in decisiemens per meter; --, no data]

\begin{tabular}{|c|c|c|c|c|c|c|c|c|c|c|c|c|c|c|c|}
\hline \multirow[b]{3}{*}{$\begin{array}{l}\text { Measurement } \\
\text { date and time }\end{array}$} & \multicolumn{15}{|c|}{ Depth } \\
\hline & \multicolumn{2}{|c|}{2} & \multirow{2}{*}{$\begin{array}{c}3 \\
\text { Volumetric } \\
\text { water } \\
\text { content }\end{array}$} & \multicolumn{3}{|c|}{4} & \multirow{2}{*}{$\begin{array}{c}6 \\
\text { Volumetric } \\
\text { water } \\
\text { content }\end{array}$} & \multicolumn{2}{|l|}{8} & \multirow{2}{*}{$\begin{array}{c}10 \\
\begin{array}{c}\text { Volumetric } \\
\text { water } \\
\text { content }\end{array}\end{array}$} & \multicolumn{2}{|l|}{12} & \multicolumn{2}{|c|}{15} & \multirow{2}{*}{$\begin{array}{c}18.5 \\
\text { Volumetric } \\
\text { water } \\
\text { content }\end{array}$} \\
\hline & $\begin{array}{c}\text { Volumetric } \\
\text { water } \\
\text { content }\end{array}$ & $\begin{array}{l}\text { Temper- } \\
\text { ature }\end{array}$ & & $\begin{array}{l}\text { Volumetric } \\
\text { water } \\
\text { content }\end{array}$ & $\begin{array}{l}\text { Temper- } \\
\text { ature }\end{array}$ & $\begin{array}{c}\text { Bulk } \\
\text { electrical } \\
\text { conduct- } \\
\text { ivity }\end{array}$ & & $\begin{array}{c}\text { Volumetric } \\
\text { water } \\
\text { content }\end{array}$ & $\begin{array}{l}\text { Temper- } \\
\text { ature }\end{array}$ & & $\begin{array}{c}\text { Volumetric } \\
\text { water } \\
\text { content }\end{array}$ & $\begin{array}{l}\text { Temper- } \\
\text { ature }\end{array}$ & $\begin{array}{c}\text { Volumetric } \\
\text { water } \\
\text { content }\end{array}$ & $\begin{array}{l}\text { Temper- } \\
\text { ature }\end{array}$ & \\
\hline 7/28/2008 3:00 PM & 0.187 & 22.6 & 0.095 & 0.312 & 21.4 & 0.46 & 0.040 & 0.043 & - & 0.041 & 0.281 & 14.7 & 0.423 & 13.8 & -- \\
\hline 7/28/2008 4:00 PM & 0.184 & 22.6 & 0.098 & 0.311 & 21.4 & 0.46 & 0.040 & 0.043 & -- & 0.043 & 0.282 & 14.7 & 0.422 & 13.8 & -- \\
\hline 7/28/2008 5:00 PM & 0.181 & 22.6 & 0.099 & 0.311 & 21.4 & 0.46 & 0.040 & 0.043 & -- & 0.043 & 0.282 & 14.8 & 0.423 & 13.8 & -- \\
\hline 7/28/2008 6:00 PM & 0.178 & 22.5 & 0.101 & 0.311 & 21.4 & 0.46 & 0.040 & 0.043 & -- & 0.043 & 0.282 & 14.7 & 0.422 & 13.8 & -- \\
\hline 7/28/2008 7:00 PM & 0.176 & 22.7 & 0.102 & 0.312 & 21.4 & 0.46 & 0.040 & 0.043 & -- & 0.043 & 0.282 & 14.7 & 0.422 & 13.8 & -- \\
\hline 7/28/2008 8:00 PM & 0.176 & 22.7 & 0.104 & 0.311 & 21.4 & 0.46 & 0.039 & 0.042 & -- & 0.043 & 0.282 & 14.7 & 0.423 & 13.8 & -- \\
\hline 7/28/2008 9:00 PM & 0.175 & 22.7 & 0.104 & 0.312 & 21.4 & 0.46 & 0.039 & 0.042 & -- & 0.043 & 0.282 & 14.7 & 0.423 & 13.8 & -- \\
\hline 7/28/2008 10:00 PM & 0.173 & 22.7 & 0.105 & 0.312 & 21.4 & 0.46 & 0.040 & 0.042 & -- & 0.042 & 0.282 & 14.7 & 0.423 & 13.9 & -- \\
\hline 7/28/2008 11:00 PM & 0.173 & 22.7 & 0.105 & 0.312 & 21.4 & 0.46 & 0.040 & 0.042 & -- & 0.042 & 0.282 & 14.7 & 0.423 & 13.9 & -- \\
\hline 7/29/2008 12:00 AM & 0.173 & 22.6 & 0.106 & 0.312 & 21.4 & 0.46 & 0.040 & 0.042 & -- & 0.042 & 0.282 & 14.7 & 0.423 & 13.8 & -- \\
\hline 7/29/2008 1:00 AM & 0.172 & 22.7 & 0.106 & 0.312 & 21.4 & 0.46 & 0.040 & 0.042 & -- & 0.042 & 0.282 & 14.7 & 0.424 & 13.9 & -- \\
\hline 7/29/2008 2:00 AM & 0.171 & 22.7 & 0.106 & 0.312 & 21.4 & 0.46 & 0.040 & 0.042 & -- & 0.042 & 0.282 & 14.8 & 0.424 & 13.8 & -- \\
\hline 7/29/2008 3:00 AM & 0.169 & 22.6 & 0.106 & 0.312 & 21.4 & 0.46 & 0.040 & 0.042 & -- & 0.042 & 0.282 & 14.8 & 0.423 & 13.9 & -- \\
\hline 7/29/2008 4:00 AM & 0.166 & 22.6 & 0.106 & 0.312 & 21.4 & 0.46 & 0.040 & 0.042 & -- & 0.042 & 0.282 & 14.8 & 0.423 & 13.8 & -- \\
\hline 7/29/2008 5:00 AM & 0.162 & 22.7 & 0.106 & 0.312 & 21.4 & 0.46 & 0.040 & 0.042 & -- & 0.042 & 0.282 & 14.8 & 0.423 & 13.9 & -- \\
\hline 7/29/2008 6:00 AM & 0.161 & 22.7 & 0.106 & 0.313 & 21.4 & 0.46 & 0.040 & 0.042 & -- & 0.042 & 0.282 & 14.8 & 0.423 & 13.9 & -- \\
\hline 7/29/2008 7:00 AM & 0.160 & 22.7 & 0.105 & 0.313 & 21.4 & 0.46 & 0.040 & 0.042 & -- & 0.042 & 0.282 & 14.8 & 0.423 & 13.9 & -- \\
\hline 7/29/2008 8:00 AM & 0.163 & 22.6 & 0.105 & 0.313 & 21.4 & 0.46 & 0.040 & 0.042 & -- & 0.042 & 0.282 & 14.8 & 0.424 & 13.9 & -- \\
\hline 7/29/2008 9:00 AM & 0.163 & 22.7 & 0.105 & 0.313 & 21.4 & 0.46 & 0.040 & 0.042 & -- & 0.042 & 0.282 & 14.8 & 0.424 & 13.9 & -- \\
\hline 7/29/2008 10:00 AM & 0.159 & 22.7 & 0.105 & 0.313 & 21.4 & 0.46 & 0.040 & 0.042 & -- & 0.041 & 0.282 & 14.7 & 0.423 & 13.9 & -- \\
\hline 7/29/2008 11:00 AM & 0.158 & 22.6 & 0.105 & 0.313 & 21.4 & 0.46 & 0.039 & 0.042 & -- & 0.041 & 0.282 & 14.8 & 0.423 & 13.9 & -- \\
\hline 7/29/2008 12:00 PM & 0.158 & 22.5 & 0.104 & 0.313 & 21.4 & 0.46 & 0.039 & 0.042 & -- & 0.041 & 0.282 & 14.8 & 0.423 & 13.9 & -- \\
\hline 7/29/2008 1:00 PM & 0.158 & 22.5 & 0.104 & 0.315 & 21.4 & 0.46 & 0.039 & 0.042 & -- & 0.041 & 0.282 & 14.8 & 0.423 & 13.9 & -- \\
\hline 7/29/2008 2:00 PM & 0.158 & 22.6 & 0.104 & 0.315 & 21.4 & 0.46 & 0.039 & 0.042 & -- & 0.041 & 0.282 & 14.8 & 0.424 & 13.9 & -- \\
\hline 7/29/2008 3:00 PM & 0.158 & 22.5 & 0.104 & 0.315 & 21.4 & 0.46 & 0.039 & 0.042 & -- & 0.041 & 0.282 & 14.8 & 0.424 & 13.9 & -- \\
\hline 7/29/2008 4:00 PM & 0.157 & 22.5 & 0.104 & 0.315 & 21.4 & 0.46 & 0.039 & 0.042 & -- & 0.041 & 0.282 & 14.8 & 0.424 & 13.9 & -- \\
\hline 7/29/2008 5:00 PM & 0.155 & 22.5 & 0.104 & 0.315 & 21.4 & 0.46 & 0.039 & 0.042 & -- & 0.041 & 0.283 & 14.8 & 0.423 & 13.9 & -- \\
\hline 7/29/2008 6:00 PM & 0.154 & 22.5 & 0.104 & 0.315 & 21.4 & 0.46 & 0.039 & 0.042 & -- & 0.041 & 0.283 & 14.8 & 0.423 & 13.9 & -- \\
\hline 7/29/2008 7:00 PM & 0.153 & 22.4 & 0.104 & 0.315 & 21.4 & 0.46 & 0.039 & 0.042 & -- & 0.041 & 0.282 & 14.8 & 0.423 & 13.9 & -- \\
\hline 7/29/2008 8:00 PM & 0.151 & 22.4 & 0.104 & 0.315 & 21.4 & 0.46 & 0.039 & 0.042 & -- & 0.041 & 0.283 & 14.8 & 0.423 & 13.9 & -- \\
\hline 7/29/2008 9:00 PM & 0.149 & 22.4 & 0.104 & 0.315 & 21.4 & 0.47 & 0.040 & 0.042 & -- & 0.041 & 0.283 & 14.8 & 0.423 & 13.9 & -- \\
\hline 7/29/2008 10:00 PM & 0.149 & 22.4 & 0.104 & 0.315 & 21.4 & 0.47 & 0.040 & 0.042 & -- & 0.041 & 0.283 & 14.8 & 0.423 & 13.9 & -- \\
\hline 7/29/2008 11:00 PM & 0.148 & 22.4 & 0.104 & 0.315 & 21.4 & 0.47 & 0.040 & 0.042 & -- & 0.041 & 0.283 & 14.8 & 0.424 & 13.9 & -- \\
\hline 7/30/2008 12:00 AM & 0.148 & 22.4 & 0.103 & 0.315 & 21.4 & 0.47 & 0.040 & 0.042 & -- & 0.041 & 0.283 & 14.8 & 0.423 & 13.9 & -- \\
\hline 7/30/2008 1:00 AM & 0.147 & 22.4 & 0.103 & 0.315 & 21.4 & 0.47 & 0.040 & 0.042 & -- & 0.041 & 0.283 & 14.8 & 0.423 & 13.9 & -- \\
\hline 7/30/2008 2:00 AM & 0.147 & 22.3 & 0.103 & 0.315 & 21.4 & 0.47 & 0.040 & 0.042 & -- & 0.041 & 0.284 & 14.8 & 0.423 & 13.9 & -- \\
\hline 7/30/2008 3:00 AM & 0.147 & 22.4 & 0.103 & 0.315 & 21.4 & 0.47 & 0.040 & 0.042 & -- & 0.041 & 0.284 & 14.8 & 0.424 & 13.9 & -- \\
\hline 7/30/2008 4:00 AM & 0.147 & 22.4 & 0.103 & 0.315 & 21.4 & 0.47 & 0.040 & 0.042 & -- & 0.041 & 0.284 & 14.8 & 0.423 & 13.9 & -- \\
\hline 7/30/2008 5:00 AM & 0.147 & 22.4 & 0.102 & 0.315 & 21.4 & 0.47 & 0.040 & 0.042 & -- & 0.041 & 0.284 & 14.8 & 0.424 & 13.9 & -- \\
\hline 7/30/2008 6:00 AM & 0.147 & 22.4 & 0.102 & 0.315 & 21.4 & 0.47 & 0.040 & 0.042 & -- & 0.041 & 0.284 & 14.8 & 0.423 & 13.9 & -- \\
\hline
\end{tabular}


Appendix 1. Volumetric water content, temperature, and electrical-conductivity data collected at the flood-irrigated site during 2008.-Continued

[Depth in feet below land surface; volumetric water content dimensionless; temperature in degrees Celsius; bulk electrical conductivity in decisiemens per meter; --, no data]

\begin{tabular}{|c|c|c|c|c|c|c|c|c|c|c|c|c|c|c|c|}
\hline \multirow[b]{3}{*}{$\begin{array}{l}\text { Measurement } \\
\text { date and time }\end{array}$} & \multicolumn{15}{|c|}{ Depth } \\
\hline & \multicolumn{2}{|l|}{2} & \multirow{2}{*}{$\begin{array}{c}3 \\
\begin{array}{c}\text { Volumetric } \\
\text { water } \\
\text { content }\end{array}\end{array}$} & \multicolumn{3}{|c|}{4} & \multirow{2}{*}{$\begin{array}{c}6 \\
\text { Volumetric } \\
\text { water } \\
\text { content }\end{array}$} & \multicolumn{2}{|l|}{8} & \multirow{2}{*}{$\begin{array}{c}10 \\
\text { Volumetric } \\
\text { water } \\
\text { content }\end{array}$} & \multicolumn{2}{|c|}{12} & \multicolumn{2}{|c|}{15} & \multirow{2}{*}{$\begin{array}{c}18.5 \\
\text { Volumetric } \\
\text { water } \\
\text { content }\end{array}$} \\
\hline & $\begin{array}{l}\text { Volumetric } \\
\text { water } \\
\text { content }\end{array}$ & $\begin{array}{l}\text { Temper- } \\
\text { ature }\end{array}$ & & $\begin{array}{c}\text { Volumetric } \\
\text { water } \\
\text { content }\end{array}$ & $\begin{array}{l}\text { Temper- } \\
\text { ature }\end{array}$ & $\begin{array}{c}\text { Bulk } \\
\text { electrical } \\
\text { conduct- } \\
\text { ivity } \\
\end{array}$ & & $\begin{array}{l}\text { Volumetric } \\
\text { water } \\
\text { content }\end{array}$ & $\begin{array}{l}\text { Temper- } \\
\text { ature }\end{array}$ & & $\begin{array}{l}\text { Volumetric } \\
\text { water } \\
\text { content }\end{array}$ & $\begin{array}{l}\text { Temper- } \\
\text { ature }\end{array}$ & $\begin{array}{l}\text { Volumetric } \\
\text { water } \\
\text { content }\end{array}$ & $\begin{array}{l}\text { Temper- } \\
\text { ature }\end{array}$ & \\
\hline 7/30/2008 7:00 AM & 0.147 & 22.4 & 0.102 & 0.315 & 21.4 & 0.47 & 0.040 & 0.042 & -- & 0.041 & 0.284 & 14.8 & 0.423 & 13.9 & -- \\
\hline 7/30/2008 8:00 AM & 0.145 & 22.4 & 0.102 & 0.315 & 21.4 & 0.47 & 0.040 & 0.042 & -- & 0.041 & 0.284 & 14.8 & 0.423 & 13.9 & -- \\
\hline 7/30/2008 9:00 AM & 0.144 & 22.3 & 0.102 & 0.315 & 21.4 & 0.47 & 0.040 & 0.042 & -- & 0.041 & 0.284 & 14.8 & 0.423 & 13.9 & -- \\
\hline 7/30/2008 10:00 AM & 0.144 & 22.3 & 0.102 & 0.315 & 21.4 & 0.47 & 0.040 & 0.042 & -- & 0.041 & 0.285 & 14.8 & 0.423 & 13.9 & -- \\
\hline 7/30/2008 11:00 AM & 0.146 & 22.3 & 0.101 & 0.315 & 21.4 & 0.47 & 0.039 & 0.042 & -- & 0.041 & 0.285 & 14.9 & 0.423 & 13.9 & -- \\
\hline 7/30/2008 12:00 PM & 0.145 & 22.3 & 0.101 & 0.315 & 21.4 & 0.47 & 0.039 & 0.042 & -- & 0.041 & 0.285 & 14.8 & 0.423 & 13.9 & -- \\
\hline 7/30/2008 1:00 PM & 0.145 & 22.3 & 0.101 & 0.315 & 21.4 & 0.47 & 0.039 & 0.042 & -- & 0.041 & 0.285 & 14.9 & 0.423 & 13.9 & -- \\
\hline 7/30/2008 2:00 PM & 0.144 & 22.3 & 0.101 & 0.315 & 21.4 & 0.47 & 0.039 & 0.042 & -- & 0.041 & 0.285 & 14.8 & 0.423 & 13.9 & -- \\
\hline 7/30/2008 3:00 PM & 0.143 & 22.2 & 0.101 & 0.315 & 21.4 & 0.47 & 0.040 & 0.042 & -- & 0.041 & 0.286 & 14.9 & 0.423 & 13.9 & -- \\
\hline 7/30/2008 4:00 PM & 0.142 & 22.2 & 0.100 & 0.315 & 21.4 & 0.47 & 0.040 & 0.042 & -- & 0.041 & 0.286 & 14.8 & 0.423 & 13.9 & -- \\
\hline 7/30/2008 5:00 PM & 0.142 & 22.2 & 0.100 & 0.315 & 21.4 & 0.47 & 0.039 & 0.042 & -- & 0.041 & 0.287 & 14.9 & 0.423 & 13.9 & -- \\
\hline 7/30/2008 6:00 PM & 0.142 & 22.1 & 0.100 & 0.315 & 21.4 & 0.47 & 0.039 & 0.042 & -- & 0.041 & 0.288 & 14.9 & 0.423 & 13.9 & -- \\
\hline 7/30/2008 7:00 PM & 0.142 & 22.1 & 0.100 & 0.315 & 21.4 & 0.47 & 0.039 & 0.042 & -- & 0.041 & 0.288 & 14.9 & 0.423 & 13.9 & -- \\
\hline 7/30/2008 8:00 PM & 0.141 & 22.1 & 0.100 & 0.315 & 21.4 & 0.47 & 0.039 & 0.042 & -- & 0.041 & 0.288 & 14.9 & 0.423 & 14.0 & -- \\
\hline 7/30/2008 9:00 PM & 0.141 & 22.1 & 0.100 & 0.313 & 21.4 & 0.47 & 0.039 & 0.042 & -- & 0.041 & 0.290 & 14.8 & 0.422 & 14.0 & -- \\
\hline 7/30/2008 10:00 PM & 0.140 & 22.1 & 0.100 & 0.313 & 21.4 & 0.47 & 0.039 & 0.042 & -- & 0.041 & 0.290 & 14.8 & 0.423 & 14.0 & -- \\
\hline 7/30/2008 11:00 PM & 0.140 & 22.1 & 0.099 & 0.313 & 21.4 & 0.47 & 0.039 & 0.042 & -- & 0.041 & 0.290 & 14.9 & 0.423 & 14.0 & -- \\
\hline 7/31/2008 12:00 AM & 0.138 & 22.1 & 0.099 & 0.313 & 21.4 & 0.47 & 0.040 & 0.042 & -- & 0.041 & 0.290 & 14.8 & 0.423 & 14.0 & -- \\
\hline 7/31/2008 1:00 AM & 0.137 & 22.1 & 0.099 & 0.313 & 21.4 & 0.47 & 0.040 & 0.042 & -- & 0.041 & 0.291 & 14.9 & 0.423 & 14.0 & -- \\
\hline 7/31/2008 2:00 AM & 0.137 & 22.1 & 0.099 & 0.313 & 21.4 & 0.47 & 0.040 & 0.042 & -- & 0.041 & 0.291 & 14.9 & 0.423 & 14.0 & -- \\
\hline 7/31/2008 3:00 AM & 0.136 & 22.1 & 0.099 & 0.313 & 21.4 & 0.47 & 0.040 & 0.042 & -- & 0.040 & 0.291 & 14.9 & 0.423 & 14.0 & -- \\
\hline 7/31/2008 4:00 AM & 0.136 & 22.1 & 0.099 & 0.313 & 21.4 & 0.47 & 0.040 & 0.042 & -- & 0.040 & 0.291 & 14.9 & 0.423 & 14.0 & -- \\
\hline 7/31/2008 5:00 AM & 0.135 & 22.1 & 0.099 & 0.313 & 21.4 & 0.47 & 0.040 & 0.042 & -- & 0.040 & 0.291 & 14.9 & 0.423 & 14.0 & -- \\
\hline 7/31/2008 6:00 AM & 0.135 & 22.1 & 0.098 & 0.313 & 21.4 & 0.47 & 0.040 & 0.042 & -- & 0.040 & 0.291 & 14.9 & 0.423 & 14.0 & -- \\
\hline 7/31/2008 7:00 AM & 0.134 & 22.1 & 0.098 & 0.313 & 21.4 & 0.47 & 0.040 & 0.042 & -- & 0.040 & 0.292 & 14.8 & 0.423 & 14.0 & -- \\
\hline 7/31/2008 8:00 AM & 0.135 & 22.1 & 0.098 & 0.313 & 21.4 & 0.47 & 0.040 & 0.042 & -- & 0.040 & 0.292 & 14.9 & 0.423 & 14.0 & -- \\
\hline 7/31/2008 9:00 AM & 0.136 & 22.1 & 0.098 & 0.313 & 21.4 & 0.47 & 0.040 & 0.042 & -- & 0.040 & 0.292 & 14.9 & 0.423 & 14.0 & -- \\
\hline 7/31/2008 10:00 AM & 0.136 & 22.0 & 0.098 & 0.313 & 21.4 & 0.47 & 0.039 & 0.042 & -- & 0.040 & 0.292 & 14.9 & 0.423 & 14.0 & -- \\
\hline 7/31/2008 11:00 AM & 0.135 & 22.0 & 0.098 & 0.313 & 21.4 & 0.47 & 0.039 & 0.042 & -- & 0.040 & 0.292 & 14.9 & 0.423 & 14.0 & -- \\
\hline 7/31/2008 12:00 PM & 0.135 & 22.1 & 0.098 & 0.313 & 21.4 & 0.47 & 0.039 & 0.042 & -- & 0.040 & 0.293 & 14.9 & 0.424 & 14.0 & -- \\
\hline 7/31/2008 1:00 PM & 0.134 & 22.1 & 0.098 & 0.313 & 21.4 & 0.47 & 0.039 & 0.042 & -- & 0.040 & 0.293 & 14.9 & 0.424 & 14.0 & -- \\
\hline 7/31/2008 2:00 PM & 0.134 & 22.1 & 0.098 & 0.313 & 21.4 & 0.47 & 0.039 & 0.042 & -- & 0.040 & 0.293 & 14.9 & 0.424 & 14.0 & -- \\
\hline 7/31/2008 3:00 PM & 0.133 & 22.1 & 0.098 & 0.313 & 21.4 & 0.47 & 0.039 & 0.042 & -- & 0.040 & 0.293 & 14.9 & 0.423 & 14.0 & -- \\
\hline 7/31/2008 4:00 PM & 0.133 & 22.0 & 0.098 & 0.313 & 21.4 & 0.47 & 0.040 & 0.042 & -- & 0.040 & 0.293 & 14.9 & 0.424 & 14.0 & -- \\
\hline 7/31/2008 5:00 PM & 0.133 & 22.0 & 0.098 & 0.313 & 21.4 & 0.47 & 0.039 & 0.042 & -- & 0.040 & 0.294 & 14.9 & 0.423 & 14.0 & -- \\
\hline 7/31/2008 6:00 PM & 0.133 & 22.0 & 0.098 & 0.313 & 21.4 & 0.47 & 0.039 & 0.042 & -- & 0.040 & 0.295 & 14.9 & 0.423 & 14.0 & -- \\
\hline 7/31/2008 7:00 PM & 0.132 & 22.0 & 0.098 & 0.313 & 21.4 & 0.47 & 0.039 & 0.042 & -- & 0.039 & 0.295 & 14.9 & 0.423 & 14.0 & -- \\
\hline 7/31/2008 8:00 PM & 0.132 & 21.9 & 0.097 & 0.313 & 21.3 & 0.47 & 0.039 & 0.042 & -- & 0.039 & 0.295 & 14.9 & 0.423 & 14.0 & -- \\
\hline 7/31/2008 9:00 PM & 0.132 & 21.9 & 0.098 & 0.313 & 21.3 & 0.47 & 0.039 & 0.042 & -- & 0.039 & 0.296 & 14.9 & 0.423 & 14.0 & -- \\
\hline 7/31/2008 10:00 PM & 0.132 & 21.9 & 0.097 & 0.313 & 21.3 & 0.47 & 0.039 & 0.042 & -- & 0.040 & 0.296 & 14.9 & 0.423 & 14.0 & -- \\
\hline
\end{tabular}


Appendix 1. Volumetric water content, temperature, and electrical-conductivity data collected at the flood-irrigated site during 2008.-Continued

[Depth in feet below land surface; volumetric water content dimensionless; temperature in degrees Celsius; bulk electrical conductivity in decisiemens per meter; --, no data]

\begin{tabular}{|c|c|c|c|c|c|c|c|c|c|c|c|c|c|c|c|}
\hline \multirow[b]{3}{*}{$\begin{array}{l}\text { Measurement } \\
\text { date and time }\end{array}$} & \multicolumn{15}{|c|}{ Depth } \\
\hline & \multicolumn{2}{|c|}{2} & \multirow[b]{2}{*}{$\begin{array}{c}3 \\
\begin{array}{c}\text { Volumetric } \\
\text { water } \\
\text { content }\end{array}\end{array}$} & \multicolumn{3}{|c|}{4} & \multirow{2}{*}{$\begin{array}{c}6 \\
\begin{array}{c}\text { Volumetric } \\
\text { water } \\
\text { content }\end{array}\end{array}$} & \multicolumn{2}{|c|}{8} & \multirow{2}{*}{$\begin{array}{c}10 \\
\text { Volumetric } \\
\text { water } \\
\text { content }\end{array}$} & \multicolumn{2}{|c|}{12} & \multicolumn{2}{|c|}{15} & \multirow{2}{*}{$\begin{array}{c}18.5 \\
\text { Volumetric } \\
\text { water } \\
\text { content }\end{array}$} \\
\hline & $\begin{array}{c}\text { Volumetric } \\
\text { water } \\
\text { content }\end{array}$ & $\begin{array}{l}\text { Temper- } \\
\text { ature }\end{array}$ & & $\begin{array}{c}\text { Volumetric } \\
\text { water } \\
\text { content }\end{array}$ & $\begin{array}{l}\text { Temper- } \\
\text { ature }\end{array}$ & $\begin{array}{c}\text { Bulk } \\
\text { electrical } \\
\text { conduct- } \\
\text { ivity }\end{array}$ & & $\begin{array}{c}\text { Volumetric } \\
\text { water } \\
\text { content }\end{array}$ & $\begin{array}{l}\text { Temper- } \\
\text { ature }\end{array}$ & & $\begin{array}{c}\text { Volumetric } \\
\text { water } \\
\text { content }\end{array}$ & $\begin{array}{l}\text { Temper- } \\
\text { ature }\end{array}$ & $\begin{array}{c}\text { Volumetric } \\
\text { water } \\
\text { content }\end{array}$ & $\begin{array}{l}\text { Temper- } \\
\text { ature }\end{array}$ & \\
\hline $7 / 31 / 2008$ 11:00 PM & 0.132 & 21.9 & 0.097 & 0.313 & 21.3 & 0.47 & 0.040 & 0.042 & -- & 0.039 & 0.296 & 14.9 & 0.423 & 14.0 & $\overline{--}$ \\
\hline 8/1/2008 12:00 AM & 0.131 & 21.9 & 0.097 & 0.313 & 21.3 & 0.47 & 0.040 & 0.042 & -- & 0.039 & 0.296 & 14.9 & 0.423 & 14.0 & -- \\
\hline 8/1/2008 1:00 AM & 0.131 & 21.9 & 0.097 & 0.313 & 21.3 & 0.47 & 0.040 & 0.042 & -- & 0.039 & 0.297 & 14.9 & 0.423 & 14.0 & -- \\
\hline 8/1/2008 2:00 AM & 0.131 & 21.9 & 0.097 & 0.313 & 21.3 & 0.47 & 0.040 & 0.042 & -- & 0.039 & 0.297 & 14.9 & 0.423 & 14.0 & -- \\
\hline 8/1/2008 3:00 AM & 0.131 & 22.0 & 0.097 & 0.313 & 21.3 & 0.47 & 0.040 & 0.042 & -- & 0.039 & 0.297 & 14.9 & 0.423 & 14.0 & -- \\
\hline 8/1/2008 4:00 AM & 0.131 & 22.0 & 0.097 & 0.313 & 21.3 & 0.47 & 0.040 & 0.042 & -- & 0.039 & 0.297 & 14.9 & 0.423 & 14.0 & -- \\
\hline 8/1/2008 5:00 AM & 0.131 & 22.0 & 0.097 & 0.312 & 21.3 & 0.47 & 0.040 & 0.042 & -- & 0.039 & 0.298 & 14.9 & 0.423 & 14.0 & -- \\
\hline 8/1/2008 6:00 AM & 0.130 & 22.0 & 0.096 & 0.312 & 21.3 & 0.47 & 0.040 & 0.042 & -- & 0.039 & 0.298 & 14.9 & 0.423 & 14.1 & -- \\
\hline 8/1/2008 7:00 AM & 0.130 & 22.0 & 0.096 & 0.312 & 21.3 & 0.47 & 0.040 & 0.042 & -- & 0.039 & 0.298 & 14.9 & 0.424 & 14.0 & -- \\
\hline 8/1/2008 8:00 AM & 0.130 & 22.0 & 0.096 & 0.312 & 21.3 & 0.47 & 0.040 & 0.042 & -- & 0.039 & 0.298 & 15.0 & 0.423 & 14.0 & -- \\
\hline 8/1/2008 9:00 AM & 0.130 & 22.0 & 0.096 & 0.312 & 21.3 & 0.47 & 0.039 & 0.042 & -- & 0.039 & 0.298 & 14.9 & 0.422 & 14.1 & -- \\
\hline 8/1/2008 10:00 AM & 0.129 & 22.1 & 0.096 & 0.312 & 21.3 & 0.47 & 0.039 & 0.042 & -- & 0.038 & 0.299 & 14.9 & 0.422 & 14.1 & -- \\
\hline 8/1/2008 11:00 AM & 0.129 & 22.1 & 0.096 & 0.313 & 21.3 & 0.47 & 0.039 & 0.042 & -- & 0.038 & 0.299 & 15.0 & 0.422 & 14.0 & -- \\
\hline 8/1/2008 12:00 PM & 0.128 & 22.1 & 0.096 & 0.313 & 21.3 & 0.47 & 0.039 & 0.042 & -- & 0.038 & 0.299 & 15.0 & 0.422 & 14.0 & -- \\
\hline 8/1/2008 1:00 PM & 0.128 & 22.1 & 0.096 & 0.312 & 21.3 & 0.47 & 0.039 & 0.042 & -- & 0.038 & 0.300 & 15.0 & 0.422 & 14.0 & -- \\
\hline 8/1/2008 2:00 PM & 0.126 & 22.0 & 0.095 & 0.313 & 21.3 & 0.47 & 0.039 & 0.042 & -- & 0.038 & 0.299 & 15.0 & 0.422 & 14.1 & -- \\
\hline 8/1/2008 3:00 PM & 0.126 & 22.0 & 0.095 & 0.313 & 21.3 & 0.47 & 0.039 & 0.042 & -- & 0.038 & 0.299 & 15.0 & 0.422 & 14.1 & -- \\
\hline 8/1/2008 4:00 PM & 0.125 & 22.0 & 0.095 & 0.313 & 21.3 & 0.47 & 0.039 & 0.042 & -- & 0.038 & 0.300 & 15.0 & 0.422 & 14.1 & -- \\
\hline 8/1/2008 5:00 PM & 0.125 & 22.0 & 0.095 & 0.312 & 21.3 & 0.47 & 0.040 & 0.042 & -- & 0.039 & 0.301 & 15.0 & 0.422 & 14.1 & -- \\
\hline 8/1/2008 6:00 PM & 0.125 & 22.0 & 0.095 & 0.312 & 21.3 & 0.47 & 0.039 & 0.042 & -- & 0.042 & 0.301 & 15.0 & 0.422 & 14.1 & -- \\
\hline 8/1/2008 7:00 PM & 0.125 & 22.0 & 0.095 & 0.312 & 21.3 & 0.47 & 0.039 & 0.042 & -- & 0.041 & 0.303 & 15.0 & 0.422 & 14.0 & -- \\
\hline 8/1/2008 8:00 PM & 0.125 & 22.0 & 0.095 & 0.312 & 21.3 & 0.47 & 0.039 & 0.042 & -- & 0.041 & 0.303 & 15.0 & 0.422 & 14.1 & -- \\
\hline 8/1/2008 9:00 PM & 0.125 & 22.1 & 0.095 & 0.312 & 21.3 & 0.47 & 0.039 & 0.042 & -- & 0.041 & 0.303 & 15.0 & 0.422 & 14.1 & -- \\
\hline 8/1/2008 10:00 PM & 0.125 & 22.0 & 0.095 & 0.312 & 21.3 & 0.47 & 0.039 & 0.042 & -- & 0.041 & 0.303 & 15.0 & 0.422 & 14.1 & -- \\
\hline 8/1/2008 11:00 PM & 0.125 & 22.0 & 0.095 & 0.312 & 21.3 & 0.47 & 0.039 & 0.042 & -- & 0.041 & 0.304 & 15.0 & 0.422 & 14.1 & -- \\
\hline 8/2/2008 12:00 AM & 0.125 & 22.1 & 0.095 & 0.312 & 21.3 & 0.47 & 0.039 & 0.042 & -- & 0.040 & 0.304 & 15.0 & 0.422 & 14.1 & -- \\
\hline 8/2/2008 1:00 AM & 0.125 & 22.1 & 0.095 & 0.312 & 21.3 & 0.47 & 0.039 & 0.042 & -- & 0.040 & 0.304 & 15.0 & 0.422 & 14.1 & -- \\
\hline 8/2/2008 2:00 AM & 0.124 & 22.1 & 0.094 & 0.312 & 21.3 & 0.47 & 0.039 & 0.042 & -- & 0.040 & 0.304 & 15.0 & 0.422 & 14.1 & -- \\
\hline 8/2/2008 3:00 AM & 0.124 & 22.1 & 0.094 & 0.312 & 21.3 & 0.47 & 0.039 & 0.042 & -- & 0.040 & 0.305 & 15.0 & 0.422 & 14.1 & -- \\
\hline 8/2/2008 4:00 AM & 0.123 & 22.1 & 0.094 & 0.312 & 21.3 & 0.47 & 0.039 & 0.042 & -- & 0.040 & 0.305 & 15.0 & 0.422 & 14.1 & -- \\
\hline 8/2/2008 5:00 AM & 0.123 & 22.2 & 0.094 & 0.312 & 21.3 & 0.47 & 0.039 & 0.042 & -- & 0.040 & 0.305 & 15.0 & 0.422 & 14.1 & -- \\
\hline 8/2/2008 6:00 AM & 0.122 & 22.2 & 0.094 & 0.312 & 21.2 & 0.47 & 0.039 & 0.042 & -- & 0.041 & 0.305 & 15.0 & 0.422 & 14.1 & -- \\
\hline 8/2/2008 7:00 AM & 0.121 & 22.2 & 0.094 & 0.312 & 21.2 & 0.47 & 0.039 & 0.042 & -- & 0.040 & 0.305 & 15.0 & 0.422 & 14.1 & -- \\
\hline 8/2/2008 8:00 AM & 0.122 & 22.2 & 0.094 & 0.312 & 21.2 & 0.47 & 0.039 & 0.042 & -- & 0.040 & 0.305 & 15.0 & 0.422 & 14.1 & -- \\
\hline 8/2/2008 9:00 AM & 0.123 & 22.2 & 0.094 & 0.312 & 21.2 & 0.47 & 0.039 & 0.042 & -- & 0.041 & 0.305 & 15.0 & 0.422 & 14.1 & -- \\
\hline 8/2/2008 10:00 AM & 0.124 & 22.3 & 0.094 & 0.312 & 21.2 & 0.47 & 0.039 & 0.042 & -- & 0.043 & 0.305 & 15.0 & 0.422 & 14.1 & -- \\
\hline 8/2/2008 11:00 AM & 0.124 & 22.4 & 0.094 & 0.312 & 21.2 & 0.47 & 0.039 & 0.042 & -- & 0.094 & 0.305 & 15.0 & 0.422 & 14.1 & -- \\
\hline 8/2/2008 12:00 PM & 0.123 & 22.3 & 0.094 & 0.312 & 21.2 & 0.47 & 0.039 & 0.042 & -- & 0.152 & 0.306 & 15.0 & 0.422 & 14.1 & -- \\
\hline 8/2/2008 1:00 PM & 0.122 & 22.3 & 0.094 & 0.312 & 21.2 & 0.47 & 0.039 & 0.042 & -- & 0.392 & 0.305 & 15.0 & 0.422 & 14.1 & -- \\
\hline 8/2/2008 2:00 PM & 0.122 & 22.2 & 0.093 & 0.312 & 21.2 & 0.47 & 0.039 & 0.042 & -- & 0.523 & 0.305 & 15.0 & 0.422 & 14.1 & -- \\
\hline
\end{tabular}


Appendix 1. Volumetric water content, temperature, and electrical-conductivity data collected at the flood-irrigated site during 2008.-Continued

[Depth in feet below land surface; volumetric water content dimensionless; temperature in degrees Celsius; bulk electrical conductivity in decisiemens per meter; --, no data]

\begin{tabular}{|c|c|c|c|c|c|c|c|c|c|c|c|c|c|c|c|}
\hline \multirow[b]{3}{*}{$\begin{array}{l}\text { Measurement } \\
\text { date and time }\end{array}$} & \multicolumn{15}{|c|}{ Depth } \\
\hline & \multicolumn{2}{|l|}{2} & \multirow{2}{*}{$\begin{array}{c}3 \\
\begin{array}{c}\text { Volumetric } \\
\text { water } \\
\text { content }\end{array}\end{array}$} & \multicolumn{3}{|c|}{4} & \multirow{2}{*}{$\begin{array}{c}6 \\
\begin{array}{c}\text { Volumetric } \\
\text { water } \\
\text { content }\end{array}\end{array}$} & \multicolumn{2}{|l|}{8} & \multirow{2}{*}{$\begin{array}{c}10 \\
\text { Volumetric } \\
\text { water } \\
\text { content }\end{array}$} & \multicolumn{2}{|c|}{12} & \multicolumn{2}{|c|}{15} & \multirow{2}{*}{$\begin{array}{c}18.5 \\
\text { Volumetric } \\
\text { water } \\
\text { content }\end{array}$} \\
\hline & $\begin{array}{l}\text { Volumetric } \\
\text { water } \\
\text { content }\end{array}$ & $\begin{array}{l}\text { Temper- } \\
\text { ature }\end{array}$ & & $\begin{array}{c}\text { Volumetric } \\
\text { water } \\
\text { content }\end{array}$ & $\begin{array}{l}\text { Temper- } \\
\text { ature }\end{array}$ & $\begin{array}{c}\text { Bulk } \\
\text { electrical } \\
\text { conduct- } \\
\text { ivity } \\
\end{array}$ & & $\begin{array}{l}\text { Volumetric } \\
\text { water } \\
\text { content }\end{array}$ & $\begin{array}{l}\text { Temper- } \\
\text { ature }\end{array}$ & & $\begin{array}{l}\text { Volumetric } \\
\text { water } \\
\text { content }\end{array}$ & $\begin{array}{l}\text { Temper- } \\
\text { ature }\end{array}$ & $\begin{array}{l}\text { Volumetric } \\
\text { water } \\
\text { content }\end{array}$ & $\begin{array}{l}\text { Temper- } \\
\text { ature }\end{array}$ & \\
\hline 8/2/2008 3:00 PM & 0.122 & 22.2 & 0.093 & 0.312 & 21.2 & 0.47 & 0.039 & 0.042 & -- & -- & 0.305 & 15.1 & 0.422 & 14.1 & -- \\
\hline 8/2/2008 4:00 PM & 0.122 & 22.2 & 0.093 & 0.312 & 21.2 & 0.47 & 0.039 & 0.042 & -- & -- & 0.306 & 15.1 & 0.422 & 14.1 & -- \\
\hline 8/2/2008 5:00 PM & 0.122 & 22.3 & 0.093 & 0.312 & 21.2 & 0.47 & 0.039 & 0.042 & -- & -- & 0.307 & 15.1 & 0.422 & 14.1 & -- \\
\hline 8/2/2008 6:00 PM & 0.122 & 22.3 & 0.093 & 0.312 & 21.2 & 0.47 & 0.039 & 0.042 & -- & -- & 0.307 & 15.1 & 0.422 & 14.1 & -- \\
\hline 8/2/2008 7:00 PM & 0.121 & 22.3 & 0.093 & 0.312 & 21.2 & 0.47 & 0.039 & 0.042 & -- & -- & 0.307 & 15.1 & 0.423 & 14.1 & -- \\
\hline 8/2/2008 8:00 PM & 0.121 & 22.3 & 0.093 & 0.312 & 21.2 & 0.47 & 0.039 & 0.042 & -- & -- & 0.307 & 15.1 & 0.422 & 14.1 & -- \\
\hline 8/2/2008 9:00 PM & 0.121 & 22.3 & 0.093 & 0.312 & 21.2 & 0.47 & 0.039 & 0.042 & -- & -- & 0.307 & 15.1 & 0.422 & 14.1 & -- \\
\hline 8/2/2008 10:00 PM & 0.121 & 22.3 & 0.093 & 0.312 & 21.2 & 0.47 & 0.039 & 0.042 & -- & -- & 0.307 & 15.1 & 0.422 & 14.1 & -- \\
\hline 8/2/2008 11:00 PM & 0.121 & 22.3 & 0.093 & 0.312 & 21.2 & 0.47 & 0.039 & 0.042 & -- & -- & 0.307 & 15.1 & 0.422 & 14.1 & -- \\
\hline 8/3/2008 12:00 AM & 0.121 & 22.3 & 0.093 & 0.312 & 21.2 & 0.47 & 0.039 & 0.042 & -- & -- & 0.307 & 15.1 & 0.422 & 14.1 & -- \\
\hline 8/3/2008 1:00 AM & 0.120 & 22.3 & 0.093 & 0.312 & 21.2 & 0.47 & 0.039 & 0.042 & -- & -- & 0.307 & 15.0 & 0.422 & 14.1 & -- \\
\hline 8/3/2008 2:00 AM & 0.120 & 22.3 & 0.093 & 0.311 & 21.2 & 0.47 & 0.039 & 0.042 & -- & -- & 0.307 & 15.1 & 0.422 & 14.1 & -- \\
\hline 8/3/2008 3:00 AM & 0.119 & 22.3 & 0.093 & 0.311 & 21.2 & 0.47 & 0.039 & 0.042 & -- & -- & 0.307 & 15.0 & 0.422 & 14.1 & -- \\
\hline 8/3/2008 4:00 AM & 0.119 & 22.4 & 0.093 & 0.312 & 21.2 & 0.47 & 0.039 & 0.042 & -- & -- & 0.308 & 15.1 & 0.422 & 14.1 & -- \\
\hline 8/3/2008 5:00 AM & 0.118 & 22.4 & 0.093 & 0.311 & 21.2 & 0.47 & 0.039 & 0.042 & -- & -- & 0.308 & 15.0 & 0.422 & 14.1 & -- \\
\hline 8/3/2008 6:00 AM & 0.118 & 22.4 & 0.093 & 0.311 & 21.2 & 0.47 & 0.039 & 0.042 & -- & -- & 0.308 & 15.0 & 0.423 & 14.1 & -- \\
\hline 8/3/2008 7:00 AM & 0.117 & 22.4 & 0.093 & 0.311 & 21.2 & 0.47 & 0.039 & 0.042 & -- & -- & 0.308 & 15.1 & 0.423 & 14.2 & -- \\
\hline 8/3/2008 8:00 AM & 0.118 & 22.4 & 0.093 & 0.311 & 21.2 & 0.47 & 0.039 & 0.042 & -- & -- & 0.308 & 15.0 & 0.422 & 14.1 & -- \\
\hline 8/3/2008 9:00 AM & 0.119 & 22.4 & 0.092 & 0.311 & 21.2 & 0.47 & 0.039 & 0.042 & -- & -- & 0.308 & 15.1 & 0.422 & 14.1 & -- \\
\hline 8/3/2008 10:00 AM & 0.120 & 22.5 & 0.092 & 0.311 & 21.2 & 0.47 & 0.039 & 0.042 & -- & -- & 0.307 & 15.1 & 0.422 & 14.1 & -- \\
\hline 8/3/2008 11:00 AM & 0.120 & 22.4 & 0.092 & 0.312 & 21.2 & 0.47 & 0.039 & 0.042 & -- & -- & 0.308 & 15.1 & 0.422 & 14.1 & -- \\
\hline 8/3/2008 12:00 PM & 0.120 & 22.4 & 0.092 & 0.312 & 21.2 & 0.47 & 0.039 & 0.042 & -- & -- & 0.308 & 15.1 & 0.422 & 14.2 & -- \\
\hline 8/3/2008 1:00 PM & 0.119 & 22.3 & 0.092 & 0.312 & 21.2 & 0.47 & 0.039 & 0.042 & -- & -- & 0.308 & 15.1 & 0.422 & 14.1 & -- \\
\hline 8/3/2008 2:00 PM & 0.119 & 22.3 & 0.092 & 0.312 & 21.2 & 0.47 & 0.039 & 0.042 & -- & -- & 0.307 & 15.1 & 0.423 & 14.2 & -- \\
\hline 8/3/2008 3:00 PM & 0.119 & 22.4 & 0.092 & 0.312 & 21.2 & 0.47 & 0.039 & 0.042 & -- & -- & 0.308 & 15.1 & 0.422 & 14.2 & -- \\
\hline 8/3/2008 4:00 PM & 0.119 & 22.4 & 0.092 & 0.311 & 21.2 & 0.47 & 0.039 & 0.042 & -- & -- & 0.309 & 15.1 & 0.422 & 14.2 & -- \\
\hline 8/3/2008 5:00 PM & 0.118 & 22.4 & 0.092 & 0.311 & 21.2 & 0.47 & 0.039 & 0.042 & -- & -- & 0.308 & 15.1 & 0.422 & 14.2 & -- \\
\hline 8/3/2008 6:00 PM & 0.118 & 22.4 & 0.092 & 0.311 & 21.2 & 0.47 & 0.039 & 0.042 & -- & -- & 0.308 & 15.1 & 0.423 & 14.2 & -- \\
\hline 8/3/2008 7:00 PM & 0.118 & 22.4 & 0.092 & 0.311 & 21.2 & 0.47 & 0.039 & 0.042 & -- & -- & 0.308 & 15.1 & 0.423 & 14.2 & -- \\
\hline 8/3/2008 8:00 PM & 0.118 & 22.4 & 0.092 & 0.311 & 21.2 & 0.47 & 0.039 & 0.042 & -- & -- & 0.308 & 15.1 & 0.423 & 14.2 & -- \\
\hline 8/3/2008 9:00 PM & 0.117 & 22.4 & 0.092 & 0.311 & 21.2 & 0.47 & 0.039 & 0.042 & -- & -- & 0.308 & 15.1 & 0.423 & 14.2 & -- \\
\hline 8/3/2008 10:00 PM & 0.116 & 22.3 & 0.092 & 0.311 & 21.2 & 0.47 & 0.039 & 0.042 & -- & -- & 0.308 & 15.1 & 0.423 & 14.2 & -- \\
\hline 8/3/2008 11:00 PM & 0.115 & 22.3 & 0.092 & 0.311 & 21.2 & 0.47 & 0.039 & 0.042 & -- & -- & 0.308 & 15.1 & 0.423 & 14.2 & -- \\
\hline 8/4/2008 12:00 AM & 0.115 & 22.4 & 0.092 & 0.311 & 21.2 & 0.47 & 0.039 & 0.042 & -- & -- & 0.308 & 15.1 & 0.423 & 14.2 & -- \\
\hline 8/4/2008 1:00 AM & 0.113 & 22.4 & 0.092 & 0.311 & 21.2 & 0.47 & 0.039 & 0.042 & -- & -- & 0.308 & 15.1 & 0.422 & 14.2 & -- \\
\hline 8/4/2008 2:00 AM & 0.115 & 22.4 & 0.092 & 0.311 & 21.2 & 0.47 & 0.039 & 0.042 & -- & -- & 0.308 & 15.1 & 0.422 & 14.2 & -- \\
\hline 8/4/2008 3:00 AM & 0.115 & 22.5 & 0.092 & 0.311 & 21.3 & 0.47 & 0.039 & 0.042 & -- & 0.714 & 0.308 & 15.1 & 0.422 & 14.2 & -- \\
\hline 8/4/2008 4:00 AM & 0.115 & 22.4 & 0.092 & 0.311 & 21.2 & 0.47 & 0.039 & 0.042 & -- & -- & 0.309 & 15.1 & 0.423 & 14.2 & -- \\
\hline 8/4/2008 5:00 AM & 0.115 & 22.4 & 0.092 & 0.311 & 21.2 & 0.47 & 0.039 & 0.042 & -- & -- & 0.309 & 15.1 & 0.422 & 14.2 & -- \\
\hline 8/4/2008 6:00 AM & 0.116 & 22.4 & 0.092 & 0.311 & 21.3 & 0.47 & 0.039 & 0.042 & -- & 0.702 & 0.309 & 15.1 & 0.422 & 14.2 & -- \\
\hline
\end{tabular}


Appendix 1. Volumetric water content, temperature, and electrical-conductivity data collected at the flood-irrigated site during 2008.-Continued

[Depth in feet below land surface; volumetric water content dimensionless; temperature in degrees Celsius; bulk electrical conductivity in decisiemens per meter; --, no data]

\begin{tabular}{|c|c|c|c|c|c|c|c|c|c|c|c|c|c|c|c|}
\hline \multirow[b]{3}{*}{$\begin{array}{l}\text { Measurement } \\
\text { date and time }\end{array}$} & \multicolumn{15}{|c|}{ Depth } \\
\hline & \multicolumn{2}{|c|}{2} & \multirow[b]{2}{*}{$\begin{array}{c}3 \\
\begin{array}{c}\text { Volumetric } \\
\text { water } \\
\text { content }\end{array}\end{array}$} & \multicolumn{3}{|c|}{4} & \multirow{2}{*}{$\begin{array}{c}6 \\
\text { Volumetric } \\
\text { water } \\
\text { content }\end{array}$} & \multicolumn{2}{|c|}{8} & \multirow{2}{*}{$\begin{array}{c}10 \\
\begin{array}{c}\text { Volumetric } \\
\text { water } \\
\text { content }\end{array}\end{array}$} & \multicolumn{2}{|c|}{12} & \multicolumn{2}{|l|}{15} & \multirow{2}{*}{$\begin{array}{c}18.5 \\
\text { Volumetric } \\
\text { water } \\
\text { content }\end{array}$} \\
\hline & $\begin{array}{c}\text { Volumetric } \\
\text { water } \\
\text { content }\end{array}$ & $\begin{array}{l}\text { Temper- } \\
\text { ature }\end{array}$ & & $\begin{array}{c}\text { Volumetric } \\
\text { water } \\
\text { content }\end{array}$ & $\begin{array}{l}\text { Temper- } \\
\text { ature }\end{array}$ & $\begin{array}{c}\text { Bulk } \\
\text { electrical } \\
\text { conduct- } \\
\text { ivity }\end{array}$ & & $\begin{array}{c}\text { Volumetric } \\
\text { water } \\
\text { content }\end{array}$ & $\begin{array}{l}\text { Temper- } \\
\text { ature }\end{array}$ & & $\begin{array}{c}\text { Volumetric } \\
\text { water } \\
\text { content }\end{array}$ & $\begin{array}{l}\text { Temper- } \\
\text { ature }\end{array}$ & $\begin{array}{c}\text { Volumetric } \\
\text { water } \\
\text { content }\end{array}$ & $\begin{array}{l}\text { Temper- } \\
\text { ature }\end{array}$ & \\
\hline 8/4/2008 7:00 AM & 0.116 & 22.4 & 0.092 & 0.311 & 21.3 & 0.47 & 0.039 & 0.042 & -- & -- & 0.309 & 15.1 & 0.422 & 14.2 & -- \\
\hline 8/4/2008 8:00 AM & 0.115 & 22.4 & 0.092 & 0.311 & 21.3 & 0.47 & 0.039 & 0.042 & -- & -- & 0.309 & 15.1 & 0.423 & 14.2 & -- \\
\hline 8/4/2008 9:00 AM & 0.115 & 22.5 & 0.092 & 0.311 & 21.3 & 0.47 & 0.039 & 0.042 & -- & -- & 0.309 & 15.1 & 0.422 & 14.2 & -- \\
\hline 8/4/2008 10:00 AM & 0.117 & 22.5 & 0.092 & 0.310 & 21.3 & 0.47 & 0.039 & 0.042 & -- & -- & 0.309 & 15.1 & 0.422 & 14.2 & -- \\
\hline 8/4/2008 11:00 AM & 0.118 & 22.5 & 0.091 & 0.310 & 21.3 & 0.47 & 0.039 & 0.042 & -- & -- & 0.309 & 15.2 & 0.422 & 14.2 & -- \\
\hline 8/4/2008 12:00 PM & 0.118 & 22.4 & 0.091 & 0.310 & 21.3 & 0.47 & 0.039 & 0.042 & -- & -- & 0.309 & 15.1 & 0.422 & 14.2 & -- \\
\hline 8/4/2008 1:00 PM & 0.117 & 22.5 & 0.091 & 0.311 & 21.3 & 0.47 & 0.039 & 0.042 & -- & 0.482 & 0.309 & 15.2 & 0.422 & 14.2 & -- \\
\hline 8/4/2008 2:00 PM & 0.117 & 22.4 & 0.092 & 0.311 & 21.3 & 0.47 & 0.039 & 0.042 & -- & 0.108 & 0.310 & 15.2 & 0.423 & 14.2 & -- \\
\hline 8/4/2008 3:00 PM & 0.117 & 22.3 & 0.092 & 0.311 & 21.3 & 0.47 & 0.039 & 0.042 & -- & 0.101 & 0.310 & 15.1 & 0.423 & 14.2 & -- \\
\hline 8/4/2008 4:00 PM & 0.117 & 22.4 & 0.092 & 0.311 & 21.3 & 0.47 & 0.039 & 0.042 & -- & 0.102 & 0.310 & 15.1 & 0.422 & 14.2 & -- \\
\hline 8/4/2008 5:00 PM & 0.118 & 22.3 & 0.094 & 0.311 & 21.3 & 0.47 & 0.039 & 0.042 & -- & 0.114 & 0.310 & 15.2 & 0.422 & 14.2 & -- \\
\hline 8/4/2008 6:00 PM & 0.126 & 22.3 & 0.095 & 0.311 & 21.3 & 0.47 & 0.039 & 0.042 & -- & 0.121 & 0.310 & 15.2 & 0.422 & 14.2 & -- \\
\hline 8/4/2008 7:00 PM & 0.137 & 22.3 & 0.096 & 0.311 & 21.3 & 0.47 & 0.039 & 0.042 & -- & 0.088 & 0.310 & 15.2 & 0.422 & 14.2 & -- \\
\hline 8/4/2008 8:00 PM & 0.144 & 22.3 & 0.098 & 0.311 & 21.3 & 0.47 & 0.039 & 0.042 & -- & 0.105 & 0.310 & 15.1 & 0.422 & 14.2 & -- \\
\hline 8/4/2008 9:00 PM & 0.147 & 22.2 & 0.098 & 0.311 & 21.3 & 0.47 & 0.039 & 0.042 & -- & 0.086 & 0.310 & 15.2 & 0.422 & 14.2 & -- \\
\hline 8/4/2008 10:00 PM & 0.148 & 22.2 & 0.098 & 0.311 & 21.3 & 0.47 & 0.039 & 0.042 & -- & 0.072 & 0.310 & 15.1 & 0.422 & 14.2 & -- \\
\hline 8/4/2008 11:00 PM & 0.149 & 22.1 & 0.099 & 0.311 & 21.3 & 0.47 & 0.039 & 0.042 & -- & 0.083 & 0.310 & 15.1 & 0.423 & 14.2 & -- \\
\hline 8/5/2008 12:00 AM & 0.149 & 22.1 & 0.099 & 0.311 & 21.3 & 0.47 & 0.039 & 0.042 & -- & 0.064 & 0.310 & 15.1 & 0.423 & 14.2 & -- \\
\hline 8/5/2008 1:00 AM & 0.150 & 22.2 & 0.100 & 0.311 & 21.3 & 0.47 & 0.039 & 0.042 & -- & 0.063 & 0.310 & 15.1 & 0.423 & 14.2 & -- \\
\hline 8/5/2008 2:00 AM & 0.150 & 22.1 & 0.100 & 0.311 & 21.3 & 0.47 & 0.039 & 0.042 & -- & 0.089 & 0.310 & 15.1 & 0.423 & 14.2 & -- \\
\hline 8/5/2008 3:00 AM & 0.149 & 22.1 & 0.100 & 0.311 & 21.3 & 0.47 & 0.039 & 0.042 & -- & 0.099 & 0.310 & 15.1 & 0.423 & 14.2 & -- \\
\hline 8/5/2008 4:00 AM & 0.149 & 22.1 & 0.101 & 0.311 & 21.3 & 0.47 & 0.039 & 0.042 & -- & 0.086 & 0.310 & 15.1 & 0.423 & 14.2 & -- \\
\hline 8/5/2008 5:00 AM & 0.149 & 22.1 & 0.101 & 0.311 & 21.3 & 0.47 & 0.039 & 0.042 & -- & 0.077 & 0.310 & 15.1 & 0.423 & 14.2 & -- \\
\hline 8/5/2008 6:00 AM & 0.148 & 22.1 & 0.101 & 0.311 & 21.3 & 0.47 & 0.039 & 0.042 & -- & 0.073 & 0.310 & 15.1 & 0.423 & 14.2 & -- \\
\hline 8/5/2008 7:00 AM & 0.148 & 22.1 & 0.101 & 0.311 & 21.3 & 0.47 & 0.039 & 0.042 & -- & 0.070 & 0.310 & 15.1 & 0.423 & 14.2 & -- \\
\hline 8/5/2008 8:00 AM & 0.148 & 22.1 & 0.101 & 0.311 & 21.3 & 0.47 & 0.039 & 0.042 & -- & 0.070 & 0.310 & 15.2 & 0.423 & 14.2 & -- \\
\hline 8/5/2008 9:00 AM & 0.149 & 22.1 & 0.101 & 0.312 & 21.3 & 0.47 & 0.039 & 0.042 & -- & 0.065 & 0.310 & 15.2 & 0.423 & 14.2 & -- \\
\hline 8/5/2008 10:00 AM & 0.150 & 22.1 & 0.101 & 0.312 & 21.3 & 0.47 & 0.039 & 0.042 & -- & 0.062 & 0.310 & 15.2 & 0.423 & 14.2 & -- \\
\hline 8/5/2008 11:00 AM & 0.150 & 22.1 & 0.101 & 0.312 & 21.3 & 0.47 & 0.039 & 0.042 & -- & 0.078 & 0.310 & 15.2 & 0.423 & 14.2 & -- \\
\hline 8/5/2008 12:00 PM & 0.149 & 22.1 & 0.101 & 0.312 & 21.3 & 0.47 & 0.039 & 0.042 & -- & 0.155 & 0.309 & 15.2 & 0.423 & 14.3 & -- \\
\hline 8/5/2008 1:00 PM & 0.148 & 22.1 & 0.101 & 0.312 & 21.3 & 0.47 & 0.039 & 0.042 & -- & 0.123 & 0.310 & 15.2 & 0.423 & 14.3 & -- \\
\hline 8/5/2008 2:00 PM & 0.148 & 22.0 & 0.101 & 0.312 & 21.3 & 0.47 & 0.039 & 0.042 & -- & 0.121 & 0.310 & 15.2 & 0.423 & 14.2 & -- \\
\hline 8/5/2008 3:00 PM & 0.147 & 22.0 & 0.101 & 0.312 & 21.3 & 0.47 & 0.039 & 0.042 & -- & 0.068 & 0.310 & 15.2 & 0.423 & 14.3 & -- \\
\hline 8/5/2008 4:00 PM & 0.147 & 21.9 & 0.101 & 0.312 & 21.3 & 0.47 & 0.039 & 0.042 & -- & 0.070 & 0.311 & 15.2 & 0.423 & 14.2 & -- \\
\hline 8/5/2008 5:00 PM & 0.146 & 21.9 & 0.101 & 0.312 & 21.3 & 0.47 & 0.039 & 0.042 & -- & 0.072 & 0.311 & 15.2 & 0.422 & 14.3 & -- \\
\hline 8/5/2008 6:00 PM & 0.146 & 21.9 & 0.101 & 0.313 & 21.3 & 0.47 & 0.039 & 0.042 & -- & 0.075 & 0.311 & 15.2 & 0.422 & 14.3 & -- \\
\hline 8/5/2008 7:00 PM & 0.146 & 21.9 & 0.101 & 0.313 & 21.3 & 0.47 & 0.039 & 0.042 & -- & 0.086 & 0.311 & 15.2 & 0.422 & 14.3 & -- \\
\hline 8/5/2008 8:00 PM & 0.145 & 21.8 & 0.101 & 0.313 & 21.3 & 0.47 & 0.039 & 0.042 & -- & 0.065 & 0.311 & 15.2 & 0.422 & 14.3 & -- \\
\hline 8/5/2008 9:00 PM & 0.145 & 21.8 & 0.101 & 0.313 & 21.3 & 0.47 & 0.039 & 0.042 & -- & 0.066 & 0.311 & 15.2 & 0.422 & 14.3 & -- \\
\hline 8/5/2008 10:00 PM & 0.145 & 21.8 & 0.101 & 0.313 & 21.3 & 0.47 & 0.039 & 0.042 & -- & 0.062 & 0.311 & 15.2 & 0.423 & 14.2 & -- \\
\hline
\end{tabular}


Appendix 1. Volumetric water content, temperature, and electrical-conductivity data collected at the flood-irrigated site during 2008.-Continued

[Depth in feet below land surface; volumetric water content dimensionless; temperature in degrees Celsius; bulk electrical conductivity in decisiemens per meter; --, no data]

\begin{tabular}{|c|c|c|c|c|c|c|c|c|c|c|c|c|c|c|c|}
\hline \multirow[b]{3}{*}{$\begin{array}{l}\text { Measurement } \\
\text { date and time }\end{array}$} & \multicolumn{15}{|c|}{ Depth } \\
\hline & \multicolumn{2}{|c|}{2} & \multirow{2}{*}{$\begin{array}{c}3 \\
\text { Volumetric } \\
\text { water } \\
\text { content }\end{array}$} & \multicolumn{3}{|c|}{4} & \multirow{2}{*}{$\begin{array}{c}6 \\
\begin{array}{c}\text { Volumetric } \\
\text { water } \\
\text { content }\end{array}\end{array}$} & \multicolumn{2}{|l|}{8} & \multirow{2}{*}{$\begin{array}{c}10 \\
\text { Volumetric } \\
\text { water } \\
\text { content }\end{array}$} & \multicolumn{2}{|c|}{12} & \multicolumn{2}{|c|}{15} & \multirow{2}{*}{$\begin{array}{c}18.5 \\
\text { Volumetric } \\
\text { water } \\
\text { content }\end{array}$} \\
\hline & $\begin{array}{l}\text { Volumetric } \\
\text { water } \\
\text { content }\end{array}$ & $\begin{array}{l}\text { Temper- } \\
\text { ature }\end{array}$ & & $\begin{array}{c}\text { Volumetric } \\
\text { water } \\
\text { content }\end{array}$ & $\begin{array}{l}\text { Temper- } \\
\text { ature }\end{array}$ & $\begin{array}{c}\text { Bulk } \\
\text { electrical } \\
\text { conduct- } \\
\text { ivity } \\
\end{array}$ & & $\begin{array}{l}\text { Volumetric } \\
\text { water } \\
\text { content }\end{array}$ & $\begin{array}{l}\text { Temper- } \\
\text { ature }\end{array}$ & & $\begin{array}{l}\text { Volumetric } \\
\text { water } \\
\text { content }\end{array}$ & $\begin{array}{l}\text { Temper- } \\
\text { ature }\end{array}$ & $\begin{array}{l}\text { Volumetric } \\
\text { water } \\
\text { content }\end{array}$ & $\begin{array}{l}\text { Temper- } \\
\text { ature }\end{array}$ & \\
\hline 8/5/2008 11:00 PM & 0.145 & 21.8 & 0.101 & 0.313 & 21.3 & 0.47 & 0.039 & 0.042 & -- & 0.064 & 0.311 & 15.2 & 0.423 & 14.3 & -- \\
\hline 8/6/2008 12:00 AM & 0.144 & 21.7 & 0.101 & 0.313 & 21.3 & 0.47 & 0.039 & 0.042 & -- & 0.064 & 0.311 & 15.2 & 0.423 & 14.2 & -- \\
\hline 8/6/2008 1:00 AM & 0.144 & 21.7 & 0.101 & 0.313 & 21.3 & 0.47 & 0.039 & 0.042 & -- & 0.058 & 0.311 & 15.2 & 0.423 & 14.2 & -- \\
\hline 8/6/2008 2:00 AM & 0.144 & 21.8 & 0.101 & 0.312 & 21.3 & 0.47 & 0.039 & 0.042 & -- & 0.060 & 0.311 & 15.2 & 0.423 & 14.2 & -- \\
\hline 8/6/2008 3:00 AM & 0.144 & 21.7 & 0.101 & 0.313 & 21.3 & 0.47 & 0.039 & 0.042 & -- & 0.064 & 0.311 & 15.2 & 0.423 & 14.2 & -- \\
\hline 8/6/2008 4:00 AM & 0.143 & 21.8 & 0.101 & 0.313 & 21.3 & 0.47 & 0.039 & 0.042 & -- & 0.071 & 0.311 & 15.2 & 0.423 & 14.2 & -- \\
\hline 8/6/2008 5:00 AM & 0.143 & 21.8 & 0.100 & 0.313 & 21.3 & 0.47 & 0.039 & 0.042 & -- & 0.087 & 0.312 & 15.2 & 0.423 & 14.2 & -- \\
\hline 8/6/2008 6:00 AM & 0.143 & 21.8 & 0.100 & 0.312 & 21.3 & 0.47 & 0.039 & 0.042 & -- & 0.101 & 0.312 & 15.2 & 0.423 & 14.2 & -- \\
\hline 8/6/2008 7:00 AM & 0.142 & 21.8 & 0.100 & 0.313 & 21.3 & 0.47 & 0.039 & 0.042 & -- & 0.095 & 0.312 & 15.2 & 0.423 & 14.2 & -- \\
\hline 8/6/2008 8:00 AM & 0.142 & 21.7 & 0.100 & 0.313 & 21.3 & 0.47 & 0.039 & 0.042 & -- & 0.092 & 0.312 & 15.2 & 0.423 & 14.2 & -- \\
\hline 8/6/2008 9:00 AM & 0.142 & 21.8 & 0.100 & 0.313 & 21.3 & 0.47 & 0.039 & 0.042 & -- & 0.087 & 0.312 & 15.2 & 0.422 & 14.2 & -- \\
\hline 8/6/2008 10:00 AM & 0.141 & 21.8 & 0.100 & 0.313 & 21.2 & 0.47 & 0.039 & 0.042 & -- & 0.064 & 0.312 & 15.2 & 0.422 & 14.2 & -- \\
\hline 8/6/2008 11:00 AM & 0.141 & 21.8 & 0.100 & 0.313 & 21.2 & 0.47 & 0.039 & 0.042 & -- & 0.058 & 0.312 & 15.2 & 0.423 & 14.2 & -- \\
\hline 8/6/2008 12:00 PM & 0.140 & 21.8 & 0.100 & 0.313 & 21.2 & 0.47 & 0.039 & 0.042 & -- & 0.058 & 0.312 & 15.3 & 0.423 & 14.2 & -- \\
\hline 8/6/2008 1:00 PM & 0.140 & 21.9 & 0.099 & 0.313 & 21.2 & 0.47 & 0.039 & 0.042 & -- & 0.057 & 0.313 & 15.2 & 0.423 & 14.2 & -- \\
\hline 8/6/2008 2:00 PM & 0.138 & 21.8 & 0.099 & 0.313 & 21.2 & 0.47 & 0.039 & 0.042 & -- & 0.060 & 0.313 & 15.3 & 0.423 & 14.2 & -- \\
\hline 8/6/2008 3:00 PM & 0.137 & 21.8 & 0.099 & 0.313 & 21.2 & 0.47 & 0.039 & 0.042 & -- & 0.061 & 0.313 & 15.3 & 0.423 & 14.2 & -- \\
\hline 8/6/2008 4:00 PM & 0.138 & 21.7 & 0.099 & 0.313 & 21.2 & 0.47 & 0.039 & 0.042 & -- & 0.064 & 0.313 & 15.3 & 0.423 & 14.2 & -- \\
\hline 8/6/2008 5:00 PM & 0.137 & 21.7 & 0.099 & 0.313 & 21.2 & 0.47 & 0.039 & 0.042 & -- & 0.064 & 0.313 & 15.3 & 0.422 & 14.2 & -- \\
\hline 8/6/2008 6:00 PM & 0.137 & 21.7 & 0.099 & 0.313 & 21.2 & 0.47 & 0.039 & 0.042 & -- & 0.060 & 0.313 & 15.3 & 0.422 & 14.2 & -- \\
\hline 8/6/2008 7:00 PM & 0.137 & 21.7 & 0.099 & 0.312 & 21.2 & 0.47 & 0.039 & 0.042 & -- & 0.065 & 0.315 & 15.2 & 0.423 & 14.2 & -- \\
\hline 8/6/2008 8:00 PM & 0.137 & 21.7 & 0.099 & 0.312 & 21.2 & 0.47 & 0.040 & 0.042 & -- & 0.070 & 0.313 & 15.2 & 0.423 & 14.2 & -- \\
\hline 8/6/2008 9:00 PM & 0.137 & 21.7 & 0.099 & 0.312 & 21.2 & 0.47 & 0.040 & 0.042 & -- & 0.068 & 0.313 & 15.3 & 0.423 & 14.2 & -- \\
\hline 8/6/2008 10:00 PM & 0.137 & 21.6 & 0.099 & 0.312 & 21.2 & 0.47 & 0.040 & 0.042 & -- & 0.063 & 0.313 & 15.2 & 0.423 & 14.2 & -- \\
\hline 8/6/2008 11:00 PM & 0.146 & 21.6 & 0.099 & 0.312 & 21.2 & 0.47 & 0.040 & 0.042 & -- & 0.066 & 0.313 & 15.2 & 0.423 & 14.2 & -- \\
\hline 8/7/2008 12:00 AM & 0.184 & 21.7 & 0.100 & 0.312 & 21.2 & 0.47 & 0.040 & 0.042 & -- & 0.059 & 0.313 & 15.2 & 0.423 & 14.3 & -- \\
\hline 8/7/2008 1:00 AM & 0.199 & 21.7 & 0.105 & 0.312 & 21.2 & 0.47 & 0.040 & 0.042 & -- & 0.063 & 0.313 & 15.2 & 0.423 & 14.2 & -- \\
\hline 8/7/2008 2:00 AM & 0.200 & 21.7 & 0.114 & 0.312 & 21.2 & 0.47 & 0.040 & 0.042 & -- & 0.061 & 0.313 & 15.3 & 0.423 & 14.2 & -- \\
\hline 8/7/2008 3:00 AM & 0.199 & 21.8 & 0.121 & 0.312 & 21.2 & 0.47 & 0.040 & 0.042 & -- & 0.065 & 0.313 & 15.2 & 0.424 & 14.2 & -- \\
\hline 8/7/2008 4:00 AM & 0.196 & 21.7 & 0.124 & 0.312 & 21.2 & 0.47 & 0.039 & 0.042 & -- & 0.066 & 0.315 & 15.3 & 0.426 & 14.3 & -- \\
\hline 8/7/2008 5:00 AM & 0.194 & 21.7 & 0.126 & 0.313 & 21.2 & 0.48 & 0.039 & 0.042 & -- & 0.059 & 0.315 & 15.3 & 0.425 & 14.3 & -- \\
\hline 8/7/2008 6:00 АМ & 0.192 & 21.7 & 0.126 & 0.315 & 21.2 & 0.48 & 0.040 & 0.042 & -- & 0.089 & 0.315 & 15.2 & 0.424 & 14.3 & -- \\
\hline 8/7/2008 7:00 AM & 0.190 & 21.6 & 0.126 & 0.317 & 21.2 & 0.48 & 0.040 & 0.042 & -- & 0.080 & 0.316 & 15.2 & 0.423 & 14.3 & -- \\
\hline 8/7/2008 8:00 AM & 0.187 & 21.6 & 0.126 & 0.319 & 21.2 & 0.49 & 0.039 & 0.042 & -- & 0.058 & 0.316 & 15.3 & 0.422 & 14.2 & -- \\
\hline 8/7/2008 9:00 AM & 0.186 & 21.6 & 0.126 & 0.322 & 21.2 & 0.49 & 0.039 & 0.042 & -- & 0.058 & 0.316 & 15.3 & 0.423 & 14.3 & -- \\
\hline 8/7/2008 10:00 AM & 0.185 & 21.6 & 0.126 & 0.323 & 21.2 & 0.50 & 0.039 & 0.042 & -- & 0.058 & 0.317 & 15.3 & 0.423 & 14.2 & -- \\
\hline 8/7/2008 11:00 AM & 0.183 & 21.5 & 0.126 & 0.325 & 21.2 & 0.50 & 0.039 & 0.042 & -- & 0.058 & 0.318 & 15.3 & 0.423 & 14.3 & -- \\
\hline 8/7/2008 12:00 PM & 0.182 & 21.5 & 0.126 & 0.326 & 21.2 & 0.50 & 0.039 & 0.042 & -- & 0.057 & 0.318 & 15.3 & 0.422 & 14.3 & -- \\
\hline 8/7/2008 1:00 PM & 0.181 & 21.5 & 0.126 & 0.328 & 21.2 & 0.50 & 0.039 & 0.042 & -- & 0.054 & 0.318 & 15.3 & 0.423 & 14.2 & -- \\
\hline 8/7/2008 2:00 PM & 0.180 & 21.5 & 0.126 & 0.328 & 21.2 & 0.50 & 0.039 & 0.042 & -- & 0.053 & 0.319 & 15.3 & 0.422 & 14.3 & -- \\
\hline
\end{tabular}


Appendix 1. Volumetric water content, temperature, and electrical-conductivity data collected at the flood-irrigated site during 2008.-Continued

[Depth in feet below land surface; volumetric water content dimensionless; temperature in degrees Celsius; bulk electrical conductivity in decisiemens per meter; --, no data]

\begin{tabular}{|c|c|c|c|c|c|c|c|c|c|c|c|c|c|c|c|}
\hline \multirow[b]{3}{*}{$\begin{array}{l}\text { Measurement } \\
\text { date and time }\end{array}$} & \multicolumn{15}{|c|}{ Depth } \\
\hline & \multicolumn{2}{|c|}{2} & \multirow[b]{2}{*}{$\begin{array}{c}3 \\
\begin{array}{c}\text { Volumetric } \\
\text { water } \\
\text { content }\end{array}\end{array}$} & \multicolumn{3}{|c|}{4} & \multirow{2}{*}{$\begin{array}{c}6 \\
\text { Volumetric } \\
\text { water } \\
\text { content }\end{array}$} & \multicolumn{2}{|l|}{8} & \multirow{2}{*}{$\begin{array}{c}10 \\
\begin{array}{c}\text { Volumetric } \\
\text { water } \\
\text { content }\end{array}\end{array}$} & \multicolumn{2}{|c|}{12} & \multicolumn{2}{|l|}{15} & \multirow{2}{*}{$\begin{array}{c}18.5 \\
\text { Volumetric } \\
\text { water } \\
\text { content }\end{array}$} \\
\hline & $\begin{array}{c}\text { Volumetric } \\
\text { water } \\
\text { content }\end{array}$ & $\begin{array}{l}\text { Temper- } \\
\text { ature }\end{array}$ & & $\begin{array}{c}\text { Volumetric } \\
\text { water } \\
\text { content }\end{array}$ & $\begin{array}{l}\text { Temper- } \\
\text { ature }\end{array}$ & $\begin{array}{c}\text { Bulk } \\
\text { electrical } \\
\text { conduct- } \\
\text { ivity }\end{array}$ & & $\begin{array}{c}\text { Volumetric } \\
\text { water } \\
\text { content }\end{array}$ & $\begin{array}{l}\text { Temper- } \\
\text { ature }\end{array}$ & & $\begin{array}{c}\text { Volumetric } \\
\text { water } \\
\text { content }\end{array}$ & $\begin{array}{l}\text { Temper- } \\
\text { ature }\end{array}$ & $\begin{array}{c}\text { Volumetric } \\
\text { water } \\
\text { content }\end{array}$ & $\begin{array}{l}\text { Temper- } \\
\text { ature }\end{array}$ & \\
\hline 8/7/2008 3:00 PM & 0.179 & 21.4 & 0.126 & 0.329 & 21.2 & 0.50 & 0.039 & 0.042 & -- & 0.052 & 0.320 & 15.3 & 0.422 & 14.3 & -- \\
\hline 8/7/2008 4:00 PM & 0.178 & 21.4 & 0.126 & 0.329 & 21.2 & 0.50 & 0.039 & 0.042 & -- & 0.049 & 0.321 & 15.3 & 0.422 & 14.3 & -- \\
\hline 8/7/2008 5:00 PM & 0.178 & 21.3 & 0.126 & 0.329 & 21.2 & 0.50 & 0.039 & 0.042 & -- & 0.048 & 0.322 & 15.3 & 0.422 & 14.3 & -- \\
\hline 8/7/2008 6:00 PM & 0.175 & 21.3 & 0.126 & 0.329 & 21.2 & 0.50 & 0.039 & 0.042 & -- & 0.048 & 0.323 & 15.3 & 0.422 & 14.3 & -- \\
\hline 8/7/2008 7:00 PM & 0.175 & 21.3 & 0.125 & 0.329 & 21.2 & 0.51 & 0.039 & 0.042 & -- & 0.048 & 0.324 & 15.3 & 0.422 & 14.3 & -- \\
\hline 8/7/2008 8:00 PM & 0.174 & 21.3 & 0.125 & 0.330 & 21.2 & 0.51 & 0.039 & 0.042 & -- & 0.048 & 0.325 & 15.3 & 0.422 & 14.3 & -- \\
\hline 8/7/2008 9:00 PM & 0.173 & 21.3 & 0.125 & 0.330 & 21.2 & 0.51 & 0.040 & 0.042 & -- & 0.047 & 0.326 & 15.3 & 0.422 & 14.3 & -- \\
\hline 8/7/2008 10:00 PM & 0.173 & 21.3 & 0.124 & 0.330 & 21.2 & 0.51 & 0.040 & 0.042 & -- & 0.047 & 0.328 & 15.3 & 0.422 & 14.3 & -- \\
\hline 8/7/2008 11:00 PM & 0.172 & 21.3 & 0.124 & 0.330 & 21.2 & 0.51 & 0.040 & 0.042 & -- & 0.047 & 0.329 & 15.3 & 0.422 & 14.3 & -- \\
\hline 8/8/2008 12:00 AM & 0.171 & 21.3 & 0.124 & 0.330 & 21.2 & 0.51 & 0.040 & 0.042 & -- & 0.047 & 0.331 & 15.3 & 0.422 & 14.3 & -- \\
\hline 8/8/2008 1:00 AM & 0.171 & 21.3 & 0.123 & 0.330 & 21.2 & 0.50 & 0.040 & 0.042 & -- & 0.047 & 0.332 & 15.3 & 0.422 & 14.3 & -- \\
\hline 8/8/2008 2:00 AM & 0.170 & 21.3 & 0.123 & 0.330 & 21.2 & 0.50 & 0.040 & 0.042 & -- & 0.047 & 0.333 & 15.3 & 0.422 & 14.3 & -- \\
\hline 8/8/2008 3:00 AM & 0.170 & 21.3 & 0.123 & 0.330 & 21.2 & 0.50 & 0.040 & 0.042 & -- & 0.047 & 0.335 & 15.3 & 0.422 & 14.3 & -- \\
\hline 8/8/2008 4:00 AM & 0.169 & 21.3 & 0.123 & 0.330 & 21.2 & 0.50 & 0.041 & 0.042 & -- & 0.047 & 0.336 & 15.3 & 0.422 & 14.3 & -- \\
\hline 8/8/2008 5:00 AM & 0.168 & 21.3 & 0.122 & 0.330 & 21.2 & 0.50 & 0.041 & 0.042 & -- & 0.047 & 0.338 & 15.3 & 0.422 & 14.3 & -- \\
\hline 8/8/2008 6:00 AM & 0.168 & 21.3 & 0.122 & 0.330 & 21.2 & 0.50 & 0.041 & 0.042 & -- & 0.047 & 0.340 & 15.3 & 0.422 & 14.3 & -- \\
\hline 8/8/2008 7:00 AM & 0.167 & 21.3 & 0.121 & 0.330 & 21.2 & 0.50 & 0.041 & 0.042 & -- & 0.047 & 0.341 & 15.3 & 0.422 & 14.3 & -- \\
\hline 8/8/2008 8:00 AM & 0.166 & 21.3 & 0.121 & 0.329 & 21.2 & 0.50 & 0.041 & 0.042 & -- & 0.047 & 0.342 & 15.3 & 0.422 & 14.3 & -- \\
\hline 8/8/2008 9:00 AM & 0.166 & 21.3 & 0.121 & 0.329 & 21.1 & 0.50 & 0.041 & 0.042 & -- & 0.047 & 0.344 & 15.3 & 0.422 & 14.4 & -- \\
\hline 8/8/2008 10:00 AM & 0.166 & 21.3 & 0.121 & 0.329 & 21.1 & 0.50 & 0.042 & 0.042 & -- & 0.047 & 0.345 & 15.3 & 0.423 & 14.4 & -- \\
\hline 8/8/2008 11:00 AM & 0.165 & 21.2 & 0.121 & 0.330 & 21.1 & 0.50 & 0.042 & 0.042 & -- & 0.047 & 0.346 & 15.3 & 0.423 & 14.3 & -- \\
\hline 8/8/2008 12:00 PM & 0.165 & 21.2 & 0.121 & 0.330 & 21.1 & 0.50 & 0.042 & 0.042 & -- & 0.048 & 0.347 & 15.3 & 0.423 & 14.4 & -- \\
\hline 8/8/2008 1:00 PM & 0.163 & 21.2 & 0.120 & 0.329 & 21.1 & 0.50 & 0.042 & 0.042 & -- & 0.049 & 0.348 & 15.4 & 0.423 & 14.3 & -- \\
\hline 8/8/2008 2:00 PM & 0.163 & 21.1 & 0.120 & 0.329 & 21.1 & 0.50 & 0.042 & 0.042 & -- & 0.048 & 0.350 & 15.3 & 0.421 & 14.3 & -- \\
\hline 8/8/2008 3:00 PM & 0.163 & 21.1 & 0.120 & 0.329 & 21.1 & 0.50 & 0.042 & 0.042 & -- & 0.047 & 0.353 & 15.3 & 0.421 & 14.3 & -- \\
\hline 8/8/2008 4:00 PM & 0.162 & 21.1 & 0.119 & 0.329 & 21.1 & 0.50 & 0.042 & 0.042 & -- & 0.048 & 0.354 & 15.3 & 0.422 & 14.3 & -- \\
\hline 8/8/2008 5:00 PM & 0.161 & 21.1 & 0.119 & 0.329 & 21.1 & 0.50 & 0.042 & 0.042 & -- & 0.049 & 0.356 & 15.4 & 0.421 & 14.3 & -- \\
\hline 8/8/2008 6:00 PM & 0.161 & 21.0 & 0.119 & 0.329 & 21.1 & 0.50 & 0.042 & 0.042 & -- & 0.051 & 0.357 & 15.4 & 0.423 & 14.4 & -- \\
\hline 8/8/2008 7:00 PM & 0.160 & 21.1 & 0.118 & 0.329 & 21.1 & 0.50 & 0.042 & 0.042 & -- & 0.051 & 0.358 & 15.4 & 0.422 & 14.4 & -- \\
\hline 8/8/2008 8:00 PM & 0.160 & 21.1 & 0.118 & 0.329 & 21.1 & 0.49 & 0.043 & 0.042 & -- & 0.052 & 0.359 & 15.4 & 0.423 & 14.4 & -- \\
\hline 8/8/2008 9:00 PM & 0.159 & 21.1 & 0.118 & 0.329 & 21.1 & 0.49 & 0.043 & 0.042 & -- & 0.052 & 0.360 & 15.4 & 0.423 & 14.4 & -- \\
\hline 8/8/2008 10:00 PM & 0.159 & 21.1 & 0.118 & 0.328 & 21.1 & 0.49 & 0.043 & 0.042 & -- & 0.051 & 0.361 & 15.4 & 0.423 & 14.4 & -- \\
\hline 8/8/2008 11:00 PM & 0.158 & 21.1 & 0.117 & 0.328 & 21.1 & 0.49 & 0.043 & 0.042 & -- & 0.051 & 0.362 & 15.4 & 0.423 & 14.4 & -- \\
\hline 8/9/2008 12:00 AM & 0.158 & 21.1 & 0.117 & 0.328 & 21.1 & 0.49 & 0.043 & 0.043 & -- & 0.051 & 0.365 & 15.4 & 0.423 & 14.4 & -- \\
\hline 8/9/2008 1:00 AM & 0.157 & 21.1 & 0.117 & 0.328 & 21.1 & 0.49 & 0.044 & 0.043 & -- & 0.051 & 0.366 & 15.4 & 0.423 & 14.4 & -- \\
\hline 8/9/2008 2:00 AM & 0.157 & 21.1 & 0.116 & 0.328 & 21.1 & 0.49 & 0.044 & 0.043 & -- & 0.051 & 0.367 & 15.4 & 0.423 & 14.4 & -- \\
\hline 8/9/2008 3:00 AM & 0.156 & 21.1 & 0.116 & 0.328 & 21.1 & 0.49 & 0.044 & 0.043 & -- & 0.051 & 0.369 & 15.4 & 0.423 & 14.4 & -- \\
\hline 8/9/2008 4:00 AM & 0.156 & 21.1 & 0.116 & 0.328 & 21.1 & 0.49 & 0.044 & 0.043 & -- & 0.051 & 0.371 & 15.4 & 0.423 & 14.4 & -- \\
\hline 8/9/2008 5:00 AM & 0.155 & 21.1 & 0.116 & 0.328 & 21.1 & 0.49 & 0.044 & 0.043 & -- & 0.051 & 0.372 & 15.4 & 0.423 & 14.4 & -- \\
\hline 8/9/2008 6:00 AM & 0.155 & 21.2 & 0.115 & 0.328 & 21.1 & 0.49 & 0.045 & 0.043 & -- & 0.051 & 0.374 & 15.4 & 0.423 & 14.4 & -- \\
\hline
\end{tabular}


Appendix 1. Volumetric water content, temperature, and electrical-conductivity data collected at the flood-irrigated site during 2008.-Continued

[Depth in feet below land surface; volumetric water content dimensionless; temperature in degrees Celsius; bulk electrical conductivity in decisiemens per meter; --, no data]

\begin{tabular}{|c|c|c|c|c|c|c|c|c|c|c|c|c|c|c|c|}
\hline \multirow[b]{3}{*}{$\begin{array}{l}\text { Measurement } \\
\text { date and time }\end{array}$} & \multicolumn{15}{|c|}{ Depth } \\
\hline & \multicolumn{2}{|l|}{$\begin{array}{r}2 \\
\end{array}$} & \multirow{2}{*}{ 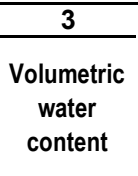 } & \multicolumn{3}{|c|}{4} & \multirow{2}{*}{$\begin{array}{c}6 \\
\begin{array}{c}\text { Volumetric } \\
\text { water } \\
\text { content }\end{array}\end{array}$} & \multicolumn{2}{|l|}{8} & \multirow{2}{*}{$\begin{array}{c}10 \\
\text { Volumetric } \\
\text { water } \\
\text { content }\end{array}$} & \multicolumn{2}{|c|}{12} & \multicolumn{2}{|c|}{15} & \multirow{2}{*}{$\begin{array}{c}18.5 \\
\text { Volumetric } \\
\text { water } \\
\text { content }\end{array}$} \\
\hline & $\begin{array}{c}\text { Volumetric } \\
\text { water } \\
\text { content }\end{array}$ & $\begin{array}{l}\text { Temper- } \\
\text { ature }\end{array}$ & & $\begin{array}{c}\text { Volumetric } \\
\text { water } \\
\text { content }\end{array}$ & $\begin{array}{l}\text { Temper- } \\
\text { ature }\end{array}$ & $\begin{array}{l}\text { Bulk } \\
\text { electrical } \\
\text { conduct- } \\
\text { ivity }\end{array}$ & & $\begin{array}{l}\text { Volumetric } \\
\text { water } \\
\text { content }\end{array}$ & $\begin{array}{l}\text { Temper- } \\
\text { ature }\end{array}$ & & $\begin{array}{c}\text { Volumetric } \\
\text { water } \\
\text { content }\end{array}$ & $\begin{array}{l}\text { Temper- } \\
\text { ature }\end{array}$ & $\begin{array}{l}\text { Volumetric } \\
\text { water } \\
\text { content }\end{array}$ & $\begin{array}{l}\text { Temper- } \\
\text { ature }\end{array}$ & \\
\hline 8/9/2008 7:00 AM & 0.155 & 21.1 & 0.115 & 0.326 & 21.0 & 0.49 & 0.045 & 0.044 & -- & 0.051 & 0.376 & 15.4 & 0.423 & 14.4 & -- \\
\hline 8/9/2008 8:00 AM & 0.154 & 21.2 & 0.115 & 0.326 & 21.0 & 0.49 & 0.045 & 0.044 & -- & 0.051 & 0.378 & 15.4 & 0.423 & 14.4 & -- \\
\hline 8/9/2008 9:00 AM & 0.154 & 21.2 & 0.115 & 0.326 & 21.0 & 0.49 & 0.045 & 0.044 & -- & 0.051 & 0.379 & 15.4 & 0.423 & 14.4 & -- \\
\hline 8/9/2008 10:00 AM & 0.154 & 21.1 & 0.115 & 0.326 & 21.0 & 0.49 & 0.045 & 0.044 & -- & 0.051 & 0.380 & 15.4 & 0.423 & 14.4 & -- \\
\hline 8/9/2008 11:00 AM & 0.153 & 21.1 & 0.115 & 0.326 & 21.0 & 0.49 & 0.045 & 0.045 & -- & 0.051 & 0.382 & 15.4 & 0.424 & 14.4 & -- \\
\hline 8/9/2008 12:00 PM & 0.153 & 21.1 & 0.115 & 0.326 & 21.0 & 0.49 & 0.045 & 0.045 & -- & 0.051 & 0.383 & 15.4 & 0.424 & 14.4 & -- \\
\hline 8/9/2008 1:00 PM & 0.153 & 21.0 & 0.114 & 0.326 & 21.0 & 0.49 & 0.045 & 0.045 & -- & 0.050 & 0.384 & 15.4 & 0.424 & 14.4 & -- \\
\hline 8/9/2008 2:00 PM & 0.153 & 21.0 & 0.114 & 0.326 & 21.0 & 0.49 & 0.045 & 0.045 & -- & 0.050 & 0.384 & 15.4 & 0.423 & 14.4 & -- \\
\hline 8/9/2008 3:00 PM & 0.151 & 21.0 & 0.114 & 0.326 & 21.0 & 0.49 & 0.045 & 0.045 & -- & 0.050 & 0.386 & 15.4 & 0.423 & 14.4 & -- \\
\hline 8/9/2008 4:00 PM & 0.151 & 21.0 & 0.114 & 0.326 & 21.0 & 0.49 & 0.045 & 0.046 & -- & 0.051 & 0.387 & 15.4 & 0.424 & 14.4 & -- \\
\hline 8/9/2008 5:00 PM & 0.151 & 21.0 & 0.114 & 0.326 & 21.0 & 0.49 & 0.045 & 0.046 & -- & 0.052 & 0.388 & 15.4 & 0.424 & 14.4 & -- \\
\hline 8/9/2008 6:00 PM & 0.150 & 21.0 & 0.113 & 0.326 & 21.0 & 0.49 & 0.045 & 0.046 & -- & 0.052 & 0.390 & 15.4 & 0.424 & 14.4 & -- \\
\hline 8/9/2008 7:00 PM & 0.149 & 21.0 & 0.113 & 0.326 & 21.0 & 0.49 & 0.045 & 0.046 & -- & 0.051 & 0.392 & 15.4 & 0.424 & 14.4 & -- \\
\hline 8/9/2008 8:00 PM & 0.149 & 21.0 & 0.113 & 0.326 & 21.0 & 0.49 & 0.045 & 0.046 & -- & 0.051 & 0.393 & 15.4 & 0.423 & 14.4 & -- \\
\hline 8/9/2008 9:00 PM & 0.148 & 21.1 & 0.113 & 0.325 & 21.0 & 0.49 & 0.046 & 0.046 & -- & 0.051 & 0.394 & 15.4 & 0.423 & 14.4 & -- \\
\hline 8/9/2008 10:00 PM & 0.148 & 21.1 & 0.113 & 0.325 & 21.0 & 0.49 & 0.046 & 0.046 & -- & 0.051 & 0.394 & 15.4 & 0.423 & 14.4 & -- \\
\hline 8/9/2008 11:00 PM & 0.148 & 21.1 & 0.112 & 0.325 & 21.0 & 0.49 & 0.046 & 0.046 & -- & 0.051 & 0.394 & 15.4 & 0.424 & 14.4 & -- \\
\hline 8/10/2008 12:00 AM & 0.148 & 21.1 & 0.112 & 0.325 & 21.0 & 0.49 & 0.046 & 0.047 & -- & 0.051 & 0.394 & 15.4 & 0.424 & 14.4 & -- \\
\hline 8/10/2008 1:00 AM & 0.148 & 21.1 & 0.112 & 0.325 & 21.0 & 0.49 & 0.046 & 0.047 & -- & 0.051 & 0.395 & 15.4 & 0.424 & 14.4 & -- \\
\hline 8/10/2008 2:00 AM & 0.147 & 21.1 & 0.112 & 0.325 & 21.0 & 0.49 & 0.046 & 0.047 & -- & 0.051 & 0.396 & 15.4 & 0.424 & 14.4 & -- \\
\hline 8/10/2008 3:00 AM & 0.147 & 21.2 & 0.111 & 0.325 & 21.0 & 0.49 & 0.046 & 0.047 & -- & 0.051 & 0.396 & 15.4 & 0.424 & 14.4 & -- \\
\hline 8/10/2008 4:00 AM & 0.147 & 21.1 & 0.111 & 0.324 & 21.0 & 0.49 & 0.046 & 0.047 & -- & 0.051 & 0.397 & 15.4 & 0.424 & 14.4 & -- \\
\hline 8/10/2008 5:00 AM & 0.146 & 21.1 & 0.111 & 0.324 & 21.0 & 0.49 & 0.046 & 0.047 & -- & 0.051 & 0.397 & 15.4 & 0.424 & 14.4 & -- \\
\hline 8/10/2008 6:00 AM & 0.146 & 21.1 & 0.111 & 0.324 & 21.0 & 0.49 & 0.046 & 0.047 & -- & 0.051 & 0.398 & 15.4 & 0.424 & 14.4 & -- \\
\hline 8/10/2008 7:00 AM & 0.146 & 21.1 & 0.111 & 0.324 & 21.0 & 0.49 & 0.046 & 0.047 & -- & 0.051 & 0.398 & 15.5 & 0.424 & 14.4 & -- \\
\hline 8/10/2008 8:00 AM & 0.146 & 21.1 & 0.111 & 0.324 & 21.0 & 0.48 & 0.046 & 0.047 & -- & 0.051 & 0.398 & 15.4 & 0.424 & 14.4 & -- \\
\hline 8/10/2008 9:00 AM & 0.145 & 21.2 & 0.110 & 0.324 & 21.0 & 0.49 & 0.046 & 0.047 & -- & 0.051 & 0.398 & 15.5 & 0.424 & 14.5 & -- \\
\hline 8/10/2008 10:00 AM & 0.145 & 21.2 & 0.110 & 0.324 & 20.9 & 0.49 & 0.046 & 0.047 & -- & 0.052 & 0.398 & 15.5 & 0.424 & 14.5 & -- \\
\hline 8/10/2008 11:00 AM & 0.145 & 21.2 & 0.110 & 0.324 & 20.9 & 0.49 & 0.046 & 0.047 & -- & 0.052 & 0.398 & 15.5 & 0.424 & 14.4 & -- \\
\hline 8/10/2008 12:00 PM & 0.145 & 21.1 & 0.110 & 0.324 & 20.9 & 0.48 & 0.046 & 0.048 & -- & 0.052 & 0.398 & 15.4 & 0.424 & 14.5 & -- \\
\hline 8/10/2008 1:00 PM & 0.145 & 21.1 & 0.109 & 0.324 & 20.9 & 0.48 & 0.046 & 0.048 & -- & 0.052 & 0.398 & 15.4 & 0.424 & 14.5 & -- \\
\hline 8/10/2008 2:00 PM & 0.145 & 21.0 & 0.109 & 0.324 & 20.9 & 0.48 & 0.046 & 0.048 & -- & 0.052 & 0.399 & 15.4 & 0.424 & 14.5 & -- \\
\hline 8/10/2008 3:00 PM & 0.144 & 21.0 & 0.109 & 0.324 & 20.9 & 0.48 & 0.046 & 0.048 & -- & 0.052 & 0.400 & 15.4 & 0.424 & 14.5 & -- \\
\hline 8/10/2008 4:00 PM & 0.144 & 21.0 & 0.109 & 0.324 & 20.9 & 0.48 & 0.046 & 0.048 & -- & 0.052 & 0.403 & 15.5 & 0.424 & 14.5 & -- \\
\hline 8/10/2008 5:00 PM & 0.143 & 21.0 & 0.109 & 0.324 & 20.9 & 0.48 & 0.046 & 0.049 & -- & 0.052 & 0.404 & 15.4 & 0.424 & 14.5 & -- \\
\hline 8/10/2008 6:00 PM & 0.143 & 21.1 & 0.109 & 0.324 & 20.9 & 0.48 & 0.046 & 0.049 & -- & 0.052 & 0.405 & 15.4 & 0.424 & 14.5 & -- \\
\hline 8/10/2008 7:00 PM & 0.143 & 21.1 & 0.109 & 0.323 & 20.9 & 0.48 & 0.046 & 0.048 & -- & 0.052 & 0.405 & 15.5 & 0.424 & 14.5 & -- \\
\hline 8/10/2008 8:00 PM & 0.143 & 21.1 & 0.109 & 0.323 & 20.9 & 0.48 & 0.046 & 0.048 & -- & 0.052 & 0.406 & 15.5 & 0.424 & 14.5 & -- \\
\hline 8/10/2008 9:00 PM & 0.142 & 21.0 & 0.109 & 0.323 & 20.9 & 0.48 & 0.046 & 0.049 & -- & 0.052 & 0.407 & 15.5 & 0.424 & 14.5 & -- \\
\hline 8/10/2008 10:00 PM & 0.142 & 21.0 & 0.109 & 0.323 & 20.9 & 0.48 & 0.046 & 0.049 & -- & 0.052 & 0.407 & 15.5 & 0.424 & 14.5 & -- \\
\hline
\end{tabular}


Appendix 1. Volumetric water content, temperature, and electrical-conductivity data collected at the flood-irrigated site during 2008.-Continued

[Depth in feet below land surface; volumetric water content dimensionless; temperature in degrees Celsius; bulk electrical conductivity in decisiemens per meter; --, no data]

\begin{tabular}{|c|c|c|c|c|c|c|c|c|c|c|c|c|c|c|c|}
\hline \multirow[b]{3}{*}{$\begin{array}{l}\text { Measurement } \\
\text { date and time }\end{array}$} & \multicolumn{15}{|c|}{ Depth } \\
\hline & \multicolumn{2}{|c|}{2} & \multirow[b]{2}{*}{$\begin{array}{c}3 \\
\begin{array}{c}\text { Volumetric } \\
\text { water } \\
\text { content }\end{array}\end{array}$} & \multicolumn{3}{|c|}{4} & \multirow{2}{*}{$\begin{array}{c}6 \\
\begin{array}{c}\text { Volumetric } \\
\text { water } \\
\text { content }\end{array}\end{array}$} & \multicolumn{2}{|c|}{8} & \multirow{2}{*}{$\begin{array}{c}10 \\
\text { Volumetric } \\
\text { water } \\
\text { content }\end{array}$} & \multicolumn{2}{|c|}{12} & \multicolumn{2}{|c|}{15} & \multirow{2}{*}{$\begin{array}{c}18.5 \\
\text { Volumetric } \\
\text { water } \\
\text { content }\end{array}$} \\
\hline & $\begin{array}{c}\text { Volumetric } \\
\text { water } \\
\text { content }\end{array}$ & $\begin{array}{l}\text { Temper- } \\
\text { ature }\end{array}$ & & $\begin{array}{c}\text { Volumetric } \\
\text { water } \\
\text { content }\end{array}$ & $\begin{array}{l}\text { Temper- } \\
\text { ature }\end{array}$ & $\begin{array}{c}\text { Bulk } \\
\text { electrical } \\
\text { conduct- } \\
\text { ivity }\end{array}$ & & $\begin{array}{c}\text { Volumetric } \\
\text { water } \\
\text { content }\end{array}$ & $\begin{array}{l}\text { Temper- } \\
\text { ature }\end{array}$ & & $\begin{array}{c}\text { Volumetric } \\
\text { water } \\
\text { content }\end{array}$ & $\begin{array}{l}\text { Temper- } \\
\text { ature }\end{array}$ & $\begin{array}{c}\text { Volumetric } \\
\text { water } \\
\text { content }\end{array}$ & $\begin{array}{l}\text { Temper- } \\
\text { ature }\end{array}$ & \\
\hline$\overline{8 / 10 / 2008 ~ 11: 00 ~ P M}$ & 0.142 & 21.0 & 0.109 & 0.323 & 20.9 & 0.48 & 0.046 & 0.049 & -- & 0.052 & 0.407 & 15.5 & 0.424 & 14.5 & $\overline{--}$ \\
\hline 8/11/2008 12:00 AM & 0.142 & 21.0 & 0.109 & 0.323 & 20.9 & 0.48 & 0.046 & 0.049 & -- & 0.052 & 0.407 & 15.5 & 0.424 & 14.5 & -- \\
\hline 8/11/2008 1:00 AM & 0.142 & 21.0 & 0.109 & 0.323 & 20.9 & 0.48 & 0.046 & 0.049 & -- & 0.052 & 0.407 & 15.5 & 0.424 & 14.5 & -- \\
\hline 8/11/2008 2:00 AM & 0.141 & 21.0 & 0.108 & 0.323 & 20.9 & 0.48 & 0.046 & 0.049 & -- & 0.052 & 0.409 & 15.5 & 0.424 & 14.5 & -- \\
\hline 8/11/2008 3:00 AM & 0.141 & 21.0 & 0.108 & 0.323 & 20.9 & 0.48 & 0.046 & 0.049 & -- & 0.052 & 0.409 & 15.5 & 0.424 & 14.5 & -- \\
\hline 8/11/2008 4:00 AM & 0.141 & 21.0 & 0.108 & 0.323 & 20.9 & 0.48 & 0.046 & 0.049 & -- & 0.052 & 0.410 & 15.5 & 0.424 & 14.5 & -- \\
\hline 8/11/2008 5:00 AM & 0.141 & 21.0 & 0.108 & 0.322 & 20.9 & 0.48 & 0.046 & 0.049 & -- & 0.052 & 0.410 & 15.5 & 0.424 & 14.5 & -- \\
\hline 8/11/2008 6:00 AM & 0.140 & 21.0 & 0.108 & 0.322 & 20.9 & 0.48 & 0.046 & 0.049 & -- & 0.053 & 0.410 & 15.5 & 0.424 & 14.5 & -- \\
\hline 8/11/2008 7:00 AM & 0.140 & 21.0 & 0.108 & 0.322 & 20.9 & 0.48 & 0.046 & 0.049 & -- & 0.053 & 0.410 & 15.5 & 0.424 & 14.5 & -- \\
\hline 8/11/2008 8:00 AM & 0.140 & 21.0 & 0.107 & 0.322 & 20.9 & 0.48 & 0.046 & 0.050 & -- & 0.054 & 0.411 & 15.5 & 0.424 & 14.5 & -- \\
\hline 8/11/2008 9:00 AM & 0.140 & 21.0 & 0.107 & 0.322 & 20.9 & 0.48 & 0.046 & 0.050 & -- & 0.054 & 0.411 & 15.5 & 0.424 & 14.5 & -- \\
\hline 8/11/2008 10:00 AM & 0.140 & 21.0 & 0.107 & 0.322 & 20.9 & 0.48 & 0.046 & 0.050 & -- & 0.054 & 0.411 & 15.5 & 0.424 & 14.4 & -- \\
\hline 8/11/2008 11:00 AM & 0.140 & 20.9 & 0.107 & 0.322 & 20.9 & 0.48 & 0.045 & 0.050 & -- & 0.054 & 0.411 & 15.5 & 0.424 & 14.5 & -- \\
\hline 8/11/2008 12:00 PM & 0.140 & 20.9 & 0.107 & 0.322 & 20.9 & 0.48 & 0.045 & 0.050 & -- & 0.054 & 0.411 & 15.5 & 0.424 & 14.4 & -- \\
\hline 8/11/2008 1:00 PM & 0.138 & 20.9 & 0.107 & 0.322 & 20.9 & 0.48 & 0.045 & 0.050 & -- & 0.054 & 0.411 & 15.5 & 0.424 & 14.4 & -- \\
\hline 8/11/2008 2:00 PM & 0.138 & 20.9 & 0.107 & 0.322 & 20.9 & 0.48 & 0.045 & 0.050 & -- & 0.054 & 0.411 & 15.5 & 0.424 & 14.5 & -- \\
\hline 8/11/2008 3:00 PM & 0.138 & 20.9 & 0.106 & 0.322 & 20.9 & 0.48 & 0.045 & 0.050 & -- & 0.055 & 0.411 & 15.5 & 0.424 & 14.4 & -- \\
\hline 8/11/2008 4:00 PM & 0.138 & 20.8 & 0.106 & 0.322 & 20.9 & 0.48 & 0.045 & 0.050 & -- & 0.055 & 0.412 & 15.5 & 0.424 & 14.4 & -- \\
\hline 8/11/2008 5:00 PM & 0.138 & 20.8 & 0.106 & 0.322 & 20.9 & 0.48 & 0.045 & 0.051 & -- & 0.054 & 0.413 & 15.5 & 0.424 & 14.5 & -- \\
\hline 8/11/2008 6:00 PM & 0.137 & 20.8 & 0.106 & 0.322 & 20.9 & 0.48 & 0.045 & 0.051 & -- & 0.054 & 0.413 & 15.5 & 0.424 & 14.5 & -- \\
\hline 8/11/2008 7:00 PM & 0.137 & 20.8 & 0.106 & 0.322 & 20.9 & 0.48 & 0.045 & 0.051 & -- & 0.055 & 0.413 & 15.5 & 0.424 & 14.4 & -- \\
\hline 8/11/2008 8:00 PM & 0.137 & 20.8 & 0.106 & 0.321 & 20.9 & 0.48 & 0.045 & 0.050 & -- & 0.055 & 0.413 & 15.5 & 0.424 & 14.5 & -- \\
\hline 8/11/2008 9:00 PM & 0.137 & 20.8 & 0.106 & 0.321 & 20.9 & 0.48 & 0.045 & 0.050 & -- & 0.055 & 0.413 & 15.5 & 0.424 & 14.5 & -- \\
\hline 8/11/2008 10:00 PM & 0.136 & 20.8 & 0.106 & 0.321 & 20.9 & 0.48 & 0.045 & 0.050 & -- & 0.055 & 0.413 & 15.5 & 0.424 & 14.5 & -- \\
\hline 8/11/2008 11:00 PM & 0.136 & 20.8 & 0.105 & 0.321 & 20.9 & 0.48 & 0.045 & 0.050 & -- & 0.056 & 0.413 & 15.5 & 0.424 & 14.5 & -- \\
\hline 8/12/2008 12:00 AM & 0.136 & 20.8 & 0.105 & 0.321 & 20.8 & 0.48 & 0.045 & 0.050 & -- & 0.056 & 0.413 & 15.5 & 0.424 & 14.5 & -- \\
\hline 8/12/2008 1:00 AM & 0.136 & 20.8 & 0.105 & 0.321 & 20.8 & 0.48 & 0.045 & 0.050 & -- & 0.056 & 0.413 & 15.5 & 0.424 & 14.5 & -- \\
\hline 8/12/2008 2:00 AM & 0.136 & 20.8 & 0.105 & 0.321 & 20.8 & 0.48 & 0.045 & 0.050 & -- & 0.056 & 0.413 & 15.5 & 0.424 & 14.5 & -- \\
\hline 8/12/2008 3:00 AM & 0.135 & 20.8 & 0.105 & 0.321 & 20.8 & 0.48 & 0.045 & 0.050 & -- & 0.056 & 0.413 & 15.5 & 0.424 & 14.5 & -- \\
\hline 8/12/2008 4:00 AM & 0.135 & 20.8 & 0.104 & 0.321 & 20.8 & 0.48 & 0.045 & 0.050 & -- & 0.057 & 0.413 & 15.5 & 0.424 & 14.5 & -- \\
\hline 8/12/2008 5:00 AM & 0.135 & 20.8 & 0.104 & 0.321 & 20.8 & 0.48 & 0.045 & 0.050 & -- & 0.058 & 0.413 & 15.5 & 0.424 & 14.5 & -- \\
\hline 8/12/2008 6:00 AM & 0.135 & 20.8 & 0.104 & 0.321 & 20.8 & 0.48 & 0.045 & 0.050 & -- & 0.058 & 0.413 & 15.5 & 0.424 & 14.5 & -- \\
\hline 8/12/2008 7:00 AM & 0.135 & 20.8 & 0.104 & 0.320 & 20.8 & 0.48 & 0.045 & 0.051 & -- & 0.058 & 0.413 & 15.5 & 0.424 & 14.5 & -- \\
\hline 8/12/2008 8:00 AM & 0.135 & 20.8 & 0.104 & 0.320 & 20.8 & 0.48 & 0.045 & 0.051 & -- & 0.056 & 0.413 & 15.5 & 0.424 & 14.5 & -- \\
\hline 8/12/2008 9:00 AM & 0.135 & 20.8 & 0.104 & 0.320 & 20.8 & 0.48 & 0.045 & 0.051 & -- & 0.056 & 0.413 & 15.5 & 0.424 & 14.5 & -- \\
\hline 8/12/2008 10:00 AM & 0.135 & 20.7 & 0.104 & 0.320 & 20.8 & 0.48 & 0.045 & 0.051 & -- & 0.056 & 0.413 & 15.5 & 0.424 & 14.5 & -- \\
\hline 8/12/2008 11:00 AM & 0.135 & 20.7 & 0.104 & 0.320 & 20.8 & 0.48 & 0.045 & 0.051 & -- & 0.056 & 0.413 & 15.5 & 0.424 & 14.5 & -- \\
\hline 8/12/2008 12:00 PM & 0.134 & 20.7 & 0.104 & 0.321 & 20.8 & 0.48 & 0.045 & 0.051 & -- & 0.056 & 0.412 & 15.5 & 0.425 & 14.5 & -- \\
\hline 8/12/2008 1:00 PM & 0.134 & 20.7 & 0.104 & 0.320 & 20.8 & 0.48 & 0.045 & 0.051 & -- & 0.056 & 0.412 & 15.5 & 0.424 & 14.5 & -- \\
\hline 8/12/2008 2:00 PM & 0.134 & 20.6 & 0.104 & 0.320 & 20.8 & 0.48 & 0.045 & 0.051 & -- & 0.056 & 0.411 & 15.5 & 0.423 & 14.5 & -- \\
\hline
\end{tabular}


Appendix 1. Volumetric water content, temperature, and electrical-conductivity data collected at the flood-irrigated site during 2008.-Continued

[Depth in feet below land surface; volumetric water content dimensionless; temperature in degrees Celsius; bulk electrical conductivity in decisiemens per meter; --, no data]

\begin{tabular}{|c|c|c|c|c|c|c|c|c|c|c|c|c|c|c|c|}
\hline \multirow[b]{3}{*}{$\begin{array}{l}\text { Measurement } \\
\text { date and time }\end{array}$} & \multicolumn{15}{|c|}{ Depth } \\
\hline & \multicolumn{2}{|c|}{2} & \multirow{2}{*}{$\begin{array}{c}3 \\
\begin{array}{c}\text { Volumetric } \\
\text { water } \\
\text { content }\end{array}\end{array}$} & \multicolumn{3}{|c|}{4} & \multirow{2}{*}{$\begin{array}{c}6 \\
\begin{array}{c}\text { Volumetric } \\
\text { water } \\
\text { content }\end{array}\end{array}$} & \multicolumn{2}{|l|}{8} & \multirow{2}{*}{$\begin{array}{c}10 \\
\text { Volumetric } \\
\text { water } \\
\text { content }\end{array}$} & \multicolumn{2}{|c|}{12} & \multicolumn{2}{|c|}{15} & \multirow{2}{*}{$\begin{array}{c}18.5 \\
\text { Volumetric } \\
\text { water } \\
\text { content }\end{array}$} \\
\hline & $\begin{array}{l}\text { Volumetric } \\
\text { water } \\
\text { content }\end{array}$ & $\begin{array}{l}\text { Temper- } \\
\text { ature }\end{array}$ & & $\begin{array}{c}\text { Volumetric } \\
\text { water } \\
\text { content }\end{array}$ & $\begin{array}{l}\text { Temper- } \\
\text { ature }\end{array}$ & $\begin{array}{c}\text { Bulk } \\
\text { electrical } \\
\text { conduct- } \\
\text { ivity } \\
\end{array}$ & & $\begin{array}{l}\text { Volumetric } \\
\text { water } \\
\text { content }\end{array}$ & $\begin{array}{l}\text { Temper- } \\
\text { ature }\end{array}$ & & $\begin{array}{l}\text { Volumetric } \\
\text { water } \\
\text { content }\end{array}$ & $\begin{array}{l}\text { Temper- } \\
\text { ature }\end{array}$ & $\begin{array}{l}\text { Volumetric } \\
\text { water } \\
\text { content }\end{array}$ & $\begin{array}{l}\text { Temper- } \\
\text { ature }\end{array}$ & \\
\hline 8/12/2008 3:00 PM & 0.134 & 20.6 & 0.104 & 0.320 & 20.8 & 0.48 & 0.045 & 0.051 & -- & 0.056 & 0.412 & 15.5 & 0.425 & 14.5 & -- \\
\hline 8/12/2008 4:00 PM & 0.134 & 20.6 & 0.104 & 0.320 & 20.8 & 0.48 & 0.045 & 0.051 & -- & 0.057 & 0.412 & 15.5 & 0.425 & 14.5 & -- \\
\hline 8/12/2008 5:00 PM & 0.133 & 20.5 & 0.104 & 0.320 & 20.8 & 0.48 & 0.045 & 0.051 & -- & 0.057 & 0.412 & 15.5 & 0.425 & 14.5 & -- \\
\hline 8/12/2008 6:00 PM & 0.133 & 20.5 & 0.104 & 0.320 & 20.8 & 0.48 & 0.045 & 0.051 & -- & 0.058 & 0.412 & 15.5 & 0.425 & 14.5 & -- \\
\hline 8/12/2008 7:00 PM & 0.133 & 20.6 & 0.104 & 0.320 & 20.8 & 0.47 & 0.044 & 0.051 & -- & 0.058 & 0.412 & 15.5 & 0.425 & 14.5 & -- \\
\hline 8/12/2008 8:00 PM & 0.133 & 20.5 & 0.104 & 0.320 & 20.8 & 0.47 & 0.044 & 0.051 & -- & 0.063 & 0.413 & 15.5 & 0.425 & 14.5 & -- \\
\hline 8/12/2008 9:00 PM & 0.132 & 20.5 & 0.104 & 0.320 & 20.8 & 0.47 & 0.044 & 0.051 & -- & 0.061 & 0.413 & 15.5 & 0.425 & 14.5 & -- \\
\hline 8/12/2008 10:00 PM & 0.132 & 20.5 & 0.104 & 0.320 & 20.8 & 0.47 & 0.045 & 0.050 & -- & 0.060 & 0.412 & 15.6 & 0.425 & 14.5 & -- \\
\hline 8/12/2008 11:00 PM & 0.132 & 20.5 & 0.104 & 0.320 & 20.8 & 0.47 & 0.045 & 0.050 & -- & 0.060 & 0.412 & 15.5 & 0.423 & 14.5 & -- \\
\hline 8/13/2008 12:00 AM & 0.132 & 20.5 & 0.103 & 0.319 & 20.8 & 0.47 & 0.045 & 0.050 & -- & 0.060 & 0.413 & 15.5 & 0.423 & 14.5 & -- \\
\hline 8/13/2008 1:00 AM & 0.132 & 20.6 & 0.103 & 0.319 & 20.8 & 0.47 & 0.045 & 0.050 & -- & 0.060 & 0.412 & 15.6 & 0.424 & 14.5 & -- \\
\hline 8/13/2008 2:00 AM & 0.132 & 20.6 & 0.103 & 0.319 & 20.8 & 0.47 & 0.045 & 0.050 & -- & 0.061 & 0.412 & 15.6 & 0.425 & 14.5 & -- \\
\hline 8/13/2008 3:00 AM & 0.132 & 20.5 & 0.103 & 0.319 & 20.8 & 0.47 & 0.045 & 0.050 & -- & 0.061 & 0.413 & 15.5 & 0.424 & 14.5 & -- \\
\hline 8/13/2008 4:00 AM & 0.132 & 20.6 & 0.103 & 0.319 & 20.8 & 0.47 & 0.045 & 0.050 & -- & 0.060 & 0.413 & 15.6 & 0.424 & 14.5 & -- \\
\hline 8/13/2008 5:00 AM & 0.132 & 20.6 & 0.103 & 0.319 & 20.7 & 0.47 & 0.045 & 0.050 & -- & 0.058 & 0.413 & 15.6 & 0.425 & 14.5 & -- \\
\hline 8/13/2008 6:00 AM & 0.132 & 20.6 & 0.102 & 0.319 & 20.7 & 0.47 & 0.044 & 0.050 & -- & 0.058 & 0.413 & 15.6 & 0.423 & 14.5 & -- \\
\hline 8/13/2008 7:00 AM & 0.132 & 20.6 & 0.103 & 0.319 & 20.7 & 0.47 & 0.044 & 0.050 & -- & 0.058 & 0.413 & 15.5 & 0.424 & 14.5 & -- \\
\hline 8/13/2008 8:00 AM & 0.132 & 20.6 & 0.103 & 0.319 & 20.7 & 0.47 & 0.044 & 0.050 & -- & 0.058 & 0.413 & 15.6 & 0.424 & 14.5 & -- \\
\hline 8/13/2008 9:00 AM & 0.131 & 20.6 & 0.102 & 0.319 & 20.7 & 0.47 & 0.044 & 0.050 & -- & 0.058 & 0.413 & 15.6 & 0.424 & 14.5 & -- \\
\hline 8/13/2008 10:00 AM & 0.131 & 20.6 & 0.102 & 0.319 & 20.7 & 0.47 & 0.044 & 0.050 & -- & 0.058 & 0.412 & 15.6 & 0.424 & 14.5 & -- \\
\hline 8/13/2008 11:00 AM & 0.131 & 20.5 & 0.102 & 0.319 & 20.7 & 0.47 & 0.044 & 0.051 & -- & 0.058 & 0.412 & 15.6 & 0.423 & 14.6 & -- \\
\hline 8/13/2008 12:00 PM & 0.131 & 20.5 & 0.102 & 0.319 & 20.7 & 0.47 & 0.044 & 0.051 & -- & 0.058 & 0.412 & 15.6 & 0.424 & 14.5 & -- \\
\hline 8/13/2008 1:00 PM & 0.131 & 20.5 & 0.102 & 0.319 & 20.7 & 0.47 & 0.044 & 0.051 & -- & 0.058 & 0.412 & 15.6 & 0.425 & 14.6 & -- \\
\hline 8/13/2008 2:00 PM & 0.130 & 20.5 & 0.102 & 0.319 & 20.7 & 0.47 & 0.044 & 0.051 & -- & 0.058 & 0.411 & 15.6 & 0.425 & 14.6 & -- \\
\hline 8/13/2008 3:00 PM & 0.130 & 20.5 & 0.102 & 0.319 & 20.7 & 0.47 & 0.044 & 0.050 & -- & 0.058 & 0.412 & 15.6 & 0.425 & 14.6 & -- \\
\hline 8/13/2008 4:00 PM & 0.129 & 20.4 & 0.102 & 0.319 & 20.7 & 0.47 & 0.044 & 0.051 & -- & 0.058 & 0.411 & 15.6 & 0.425 & 14.6 & -- \\
\hline 8/13/2008 5:00 PM & 0.129 & 20.4 & 0.102 & 0.319 & 20.7 & 0.47 & 0.044 & 0.051 & -- & 0.058 & 0.411 & 15.6 & 0.425 & 14.6 & -- \\
\hline 8/13/2008 6:00 PM & 0.130 & 20.4 & 0.101 & 0.319 & 20.7 & 0.47 & 0.044 & 0.051 & -- & 0.059 & 0.412 & 15.6 & 0.424 & 14.6 & -- \\
\hline 8/13/2008 7:00 PM & 0.129 & 20.4 & 0.101 & 0.319 & 20.7 & 0.47 & 0.044 & 0.051 & -- & 0.059 & 0.412 & 15.6 & 0.423 & 14.6 & -- \\
\hline 8/13/2008 8:00 PM & 0.129 & 20.4 & 0.101 & 0.318 & 20.7 & 0.47 & 0.043 & 0.051 & -- & 0.059 & 0.412 & 15.6 & 0.423 & 14.6 & -- \\
\hline 8/13/2008 9:00 PM & 0.129 & 20.4 & 0.101 & 0.318 & 20.7 & 0.47 & 0.043 & 0.050 & -- & 0.059 & 0.412 & 15.6 & 0.423 & 14.6 & -- \\
\hline 8/13/2008 10:00 PM & 0.129 & 20.4 & 0.101 & 0.318 & 20.7 & 0.47 & 0.044 & 0.050 & -- & 0.059 & 0.412 & 15.6 & 0.424 & 14.6 & -- \\
\hline 8/13/2008 11:00 PM & 0.129 & 20.4 & 0.101 & 0.318 & 20.7 & 0.47 & 0.044 & 0.050 & -- & 0.059 & 0.413 & 15.6 & 0.424 & 14.6 & -- \\
\hline 8/14/2008 12:00 AM & 0.129 & 20.4 & 0.101 & 0.318 & 20.7 & 0.47 & 0.044 & 0.050 & -- & 0.059 & 0.412 & 15.6 & 0.424 & 14.6 & -- \\
\hline 8/14/2008 1:00 AM & 0.129 & 20.4 & 0.101 & 0.318 & 20.7 & 0.47 & 0.044 & 0.050 & -- & 0.060 & 0.412 & 15.6 & 0.424 & 14.6 & -- \\
\hline 8/14/2008 2:00 AM & 0.129 & 20.5 & 0.101 & 0.318 & 20.7 & 0.47 & 0.044 & 0.050 & -- & 0.060 & 0.413 & 15.6 & 0.424 & 14.6 & -- \\
\hline 8/14/2008 3:00 AM & 0.129 & 20.5 & 0.101 & 0.318 & 20.7 & 0.47 & 0.044 & 0.050 & -- & 0.062 & 0.413 & 15.6 & 0.424 & 14.6 & -- \\
\hline 8/14/2008 4:00 AM & 0.129 & 20.5 & 0.100 & 0.318 & 20.7 & 0.47 & 0.044 & 0.050 & -- & 0.064 & 0.412 & 15.6 & 0.424 & 14.6 & -- \\
\hline 8/14/2008 5:00 AM & 0.129 & 20.5 & 0.100 & 0.318 & 20.7 & 0.47 & 0.044 & 0.050 & -- & 0.064 & 0.413 & 15.6 & 0.424 & 14.6 & -- \\
\hline 8/14/2008 6:00 AM & 0.129 & 20.5 & 0.100 & 0.318 & 20.6 & 0.47 & 0.044 & 0.050 & -- & 0.064 & 0.413 & 15.6 & 0.424 & 14.6 & -- \\
\hline
\end{tabular}


Appendix 1. Volumetric water content, temperature, and electrical-conductivity data collected at the flood-irrigated site during 2008.-Continued

[Depth in feet below land surface; volumetric water content dimensionless; temperature in degrees Celsius; bulk electrical conductivity in decisiemens per meter; --, no data]

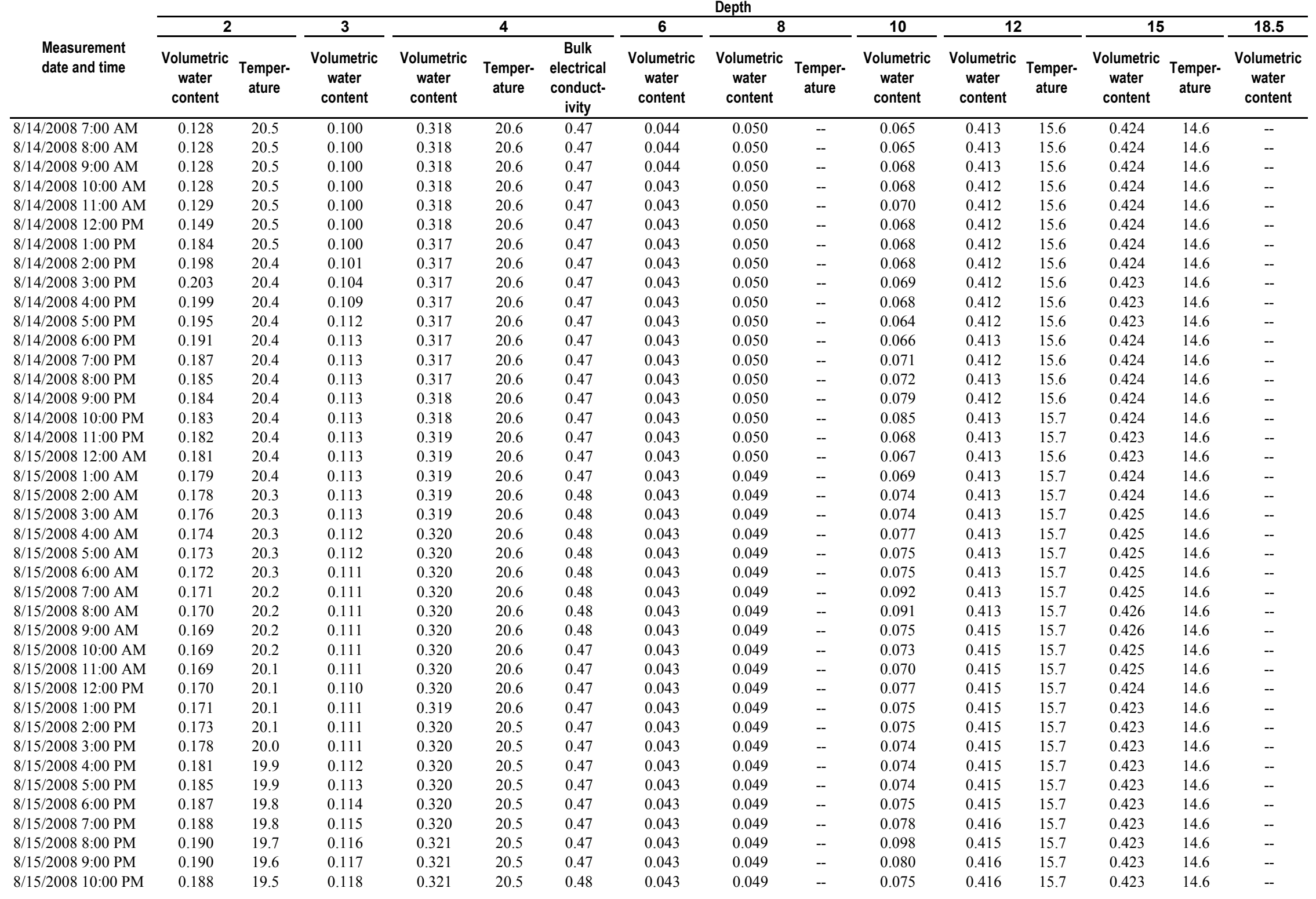


Appendix 1. Volumetric water content, temperature, and electrical-conductivity data collected at the flood-irrigated site during 2008.-Continued

[Depth in feet below land surface; volumetric water content dimensionless; temperature in degrees Celsius; bulk electrical conductivity in decisiemens per meter; --, no data]

\begin{tabular}{|c|c|c|c|c|c|c|c|c|c|c|c|c|c|c|c|}
\hline \multirow[b]{3}{*}{$\begin{array}{l}\text { Measurement } \\
\text { date and time }\end{array}$} & \multicolumn{15}{|c|}{ Depth } \\
\hline & \multicolumn{2}{|c|}{2} & \multirow{2}{*}{$\begin{array}{c}3 \\
\text { Volumetric } \\
\text { water } \\
\text { content }\end{array}$} & \multicolumn{3}{|c|}{4} & \multirow{2}{*}{$\begin{array}{c}6 \\
\begin{array}{c}\text { Volumetric } \\
\text { water } \\
\text { content }\end{array}\end{array}$} & \multicolumn{2}{|l|}{8} & \multirow{2}{*}{$\begin{array}{c}10 \\
\text { Volumetric } \\
\text { water } \\
\text { content }\end{array}$} & \multicolumn{2}{|c|}{12} & \multicolumn{2}{|c|}{15} & \multirow{2}{*}{$\begin{array}{c}18.5 \\
\text { Volumetric } \\
\text { water } \\
\text { content }\end{array}$} \\
\hline & $\begin{array}{l}\text { Volumetric } \\
\text { water } \\
\text { content }\end{array}$ & $\begin{array}{l}\text { Temper- } \\
\text { ature }\end{array}$ & & $\begin{array}{c}\text { Volumetric } \\
\text { water } \\
\text { content }\end{array}$ & $\begin{array}{l}\text { Temper- } \\
\text { ature }\end{array}$ & $\begin{array}{c}\text { Bulk } \\
\text { electrical } \\
\text { conduct- } \\
\text { ivity } \\
\end{array}$ & & $\begin{array}{l}\text { Volumetric } \\
\text { water } \\
\text { content }\end{array}$ & $\begin{array}{l}\text { Temper- } \\
\text { ature }\end{array}$ & & $\begin{array}{l}\text { Volumetric } \\
\text { water } \\
\text { content }\end{array}$ & $\begin{array}{l}\text { Temper- } \\
\text { ature }\end{array}$ & $\begin{array}{l}\text { Volumetric } \\
\text { water } \\
\text { content }\end{array}$ & $\begin{array}{l}\text { Temper- } \\
\text { ature }\end{array}$ & \\
\hline 8/15/2008 11:00 PM & 0.187 & 19.5 & 0.119 & 0.322 & 20.5 & 0.48 & 0.043 & 0.049 & -- & 0.075 & 0.416 & 15.7 & 0.423 & 14.6 & -- \\
\hline 8/16/2008 12:00 AM & 0.186 & 19.4 & 0.120 & 0.323 & 20.5 & 0.48 & 0.043 & 0.049 & -- & 0.077 & 0.416 & 15.7 & 0.423 & 14.6 & -- \\
\hline 8/16/2008 1:00 AM & 0.185 & 19.3 & 0.120 & 0.323 & 20.5 & 0.48 & 0.043 & 0.049 & -- & 0.073 & 0.416 & 15.7 & 0.423 & 14.6 & -- \\
\hline 8/16/2008 2:00 AM & 0.185 & 19.3 & 0.121 & 0.324 & 20.5 & 0.48 & 0.043 & 0.049 & -- & 0.087 & 0.416 & 15.7 & 0.423 & 14.6 & -- \\
\hline 8/16/2008 3:00 AM & 0.184 & 19.3 & 0.121 & 0.325 & 20.5 & 0.48 & 0.043 & 0.049 & -- & 0.089 & 0.416 & 15.7 & 0.423 & 14.6 & -- \\
\hline 8/16/2008 4:00 AM & 0.183 & 19.3 & 0.121 & 0.325 & 20.5 & 0.48 & 0.043 & 0.049 & -- & 0.087 & 0.416 & 15.7 & 0.423 & 14.6 & -- \\
\hline 8/16/2008 5:00 AM & 0.182 & 19.3 & 0.121 & 0.326 & 20.5 & 0.48 & 0.043 & 0.049 & -- & 0.080 & 0.416 & 15.7 & 0.424 & 14.7 & -- \\
\hline 8/16/2008 6:00 AM & 0.181 & 19.2 & 0.121 & 0.326 & 20.5 & 0.48 & 0.043 & 0.049 & -- & 0.075 & 0.416 & 15.7 & 0.424 & 14.7 & -- \\
\hline 8/16/2008 7:00 AM & 0.180 & 19.1 & 0.121 & 0.326 & 20.5 & 0.48 & 0.043 & 0.049 & -- & 0.075 & 0.416 & 15.7 & 0.423 & 14.6 & -- \\
\hline 8/16/2008 8:00 AM & 0.181 & 19.1 & 0.121 & 0.328 & 20.5 & 0.47 & 0.043 & 0.049 & -- & 0.078 & 0.416 & 15.7 & 0.423 & 14.6 & -- \\
\hline 8/16/2008 9:00 AM & 0.184 & 19.1 & 0.121 & 0.328 & 20.5 & 0.47 & 0.043 & 0.049 & -- & 0.080 & 0.416 & 15.7 & 0.423 & 14.7 & -- \\
\hline 8/16/2008 10:00 AM & 0.187 & 19.0 & 0.121 & 0.328 & 20.4 & 0.47 & 0.043 & 0.049 & -- & 0.081 & 0.417 & 15.7 & 0.426 & 14.7 & -- \\
\hline 8/16/2008 11:00 AM & 0.192 & 19.0 & 0.125 & 0.328 & 20.4 & 0.47 & 0.043 & 0.049 & -- & 0.085 & 0.417 & 15.7 & 0.430 & 14.7 & -- \\
\hline 8/16/2008 12:00 PM & 0.196 & 18.9 & 0.131 & 0.329 & 20.4 & 0.47 & 0.043 & 0.049 & -- & 0.082 & 0.417 & 15.7 & 0.431 & 14.7 & -- \\
\hline 8/16/2008 1:00 PM & 0.197 & 18.8 & 0.134 & 0.332 & 20.4 & 0.48 & 0.043 & 0.049 & -- & 0.091 & 0.417 & 15.7 & 0.432 & 14.7 & -- \\
\hline 8/16/2008 2:00 PM & 0.199 & 18.9 & 0.137 & 0.334 & 20.4 & 0.48 & 0.043 & 0.049 & -- & 0.109 & 0.417 & 15.7 & 0.425 & 14.7 & -- \\
\hline 8/16/2008 3:00 PM & 0.199 & 18.8 & 0.138 & 0.337 & 20.4 & 0.49 & 0.043 & 0.049 & -- & 0.109 & 0.417 & 15.7 & 0.425 & 14.7 & -- \\
\hline 8/16/2008 4:00 PM & 0.198 & 18.6 & 0.140 & 0.338 & 20.4 & 0.49 & 0.043 & 0.049 & -- & 0.110 & 0.417 & 15.7 & 0.425 & 14.7 & -- \\
\hline 8/16/2008 5:00 PM & 0.197 & 18.6 & 0.140 & 0.340 & 20.4 & 0.50 & 0.043 & 0.049 & -- & 0.109 & 0.417 & 15.7 & 0.426 & 14.7 & -- \\
\hline 8/16/2008 6:00 PM & 0.196 & 18.5 & 0.140 & 0.341 & 20.4 & 0.50 & 0.044 & 0.049 & -- & 0.115 & 0.417 & 15.7 & 0.424 & 14.7 & -- \\
\hline 8/16/2008 7:00 PM & 0.194 & 18.4 & 0.140 & 0.341 & 20.4 & 0.50 & 0.044 & 0.049 & -- & 0.142 & 0.417 & 15.7 & 0.424 & 14.7 & -- \\
\hline 8/16/2008 8:00 PM & 0.192 & 18.4 & 0.139 & 0.341 & 20.3 & 0.50 & 0.044 & 0.049 & -- & 0.155 & 0.417 & 15.7 & 0.424 & 14.7 & -- \\
\hline 8/16/2008 9:00 PM & 0.191 & 18.3 & 0.138 & 0.341 & 20.3 & 0.50 & 0.044 & 0.049 & -- & 0.217 & 0.417 & 15.8 & 0.424 & 14.7 & -- \\
\hline 8/16/2008 10:00 PM & 0.188 & 18.3 & 0.138 & 0.341 & 20.3 & 0.50 & 0.045 & 0.049 & -- & 0.239 & 0.417 & 15.7 & 0.424 & 14.7 & -- \\
\hline 8/16/2008 11:00 PM & 0.187 & 18.3 & 0.137 & 0.341 & 20.3 & 0.50 & 0.045 & 0.049 & -- & 0.209 & 0.417 & 15.7 & 0.425 & 14.7 & -- \\
\hline 8/17/2008 12:00 AM & 0.186 & 18.3 & 0.137 & 0.341 & 20.3 & 0.50 & 0.046 & 0.049 & -- & 0.217 & 0.417 & 15.7 & 0.425 & 14.7 & -- \\
\hline 8/17/2008 1:00 AM & 0.185 & 18.3 & 0.136 & 0.341 & 20.3 & 0.49 & 0.046 & 0.049 & -- & 0.245 & 0.417 & 15.7 & 0.425 & 14.7 & -- \\
\hline 8/17/2008 2:00 AM & 0.184 & 18.2 & 0.135 & 0.340 & 20.3 & 0.49 & 0.047 & 0.049 & -- & 0.275 & 0.417 & 15.7 & 0.425 & 14.7 & -- \\
\hline 8/17/2008 3:00 AM & 0.182 & 18.2 & 0.134 & 0.340 & 20.2 & 0.49 & 0.047 & 0.049 & -- & 0.289 & 0.417 & 15.7 & 0.425 & 14.7 & -- \\
\hline 8/17/2008 4:00 AM & 0.182 & 18.1 & 0.133 & 0.338 & 20.2 & 0.49 & 0.048 & 0.049 & -- & 0.233 & 0.417 & 15.7 & 0.425 & 14.7 & -- \\
\hline 8/17/2008 5:00 AM & 0.180 & 18.1 & 0.132 & 0.338 & 20.2 & 0.49 & 0.050 & 0.049 & -- & 0.269 & 0.417 & 15.8 & 0.425 & 14.7 & -- \\
\hline 8/17/2008 6:00 AM & 0.179 & 18.1 & 0.132 & 0.338 & 20.2 & 0.48 & 0.052 & 0.050 & -- & 0.234 & 0.417 & 15.7 & 0.425 & 14.7 & -- \\
\hline 8/17/2008 7:00 AM & 0.178 & 18.0 & 0.132 & 0.338 & 20.2 & 0.48 & 0.053 & 0.050 & -- & 0.211 & 0.417 & 15.7 & 0.425 & 14.7 & -- \\
\hline 8/17/2008 8:00 AM & 0.176 & 18.0 & 0.131 & 0.337 & 20.2 & 0.48 & 0.054 & 0.050 & -- & 0.275 & 0.417 & 15.8 & 0.425 & 14.7 & -- \\
\hline 8/17/2008 9:00 AM & 0.175 & 18.0 & 0.130 & 0.337 & 20.1 & 0.48 & 0.055 & 0.050 & -- & 0.277 & 0.417 & 15.8 & 0.425 & 14.7 & -- \\
\hline 8/17/2008 10:00 AM & 0.175 & 18.0 & 0.130 & 0.337 & 20.1 & 0.47 & 0.056 & 0.050 & -- & 0.244 & 0.418 & 15.8 & 0.425 & 14.7 & -- \\
\hline 8/17/2008 11:00 AM & 0.174 & 17.9 & 0.129 & 0.337 & 20.1 & 0.47 & 0.057 & 0.051 & -- & 0.213 & 0.417 & 15.7 & 0.425 & 14.7 & -- \\
\hline 8/17/2008 12:00 PM & 0.174 & 17.9 & 0.129 & 0.337 & 20.1 & 0.47 & 0.057 & 0.051 & -- & 0.234 & 0.417 & 15.7 & 0.425 & 14.7 & -- \\
\hline 8/17/2008 1:00 PM & 0.173 & 17.9 & 0.128 & 0.336 & 20.1 & 0.47 & 0.058 & 0.052 & -- & 0.118 & 0.417 & 15.7 & 0.425 & 14.7 & -- \\
\hline 8/17/2008 2:00 PM & 0.172 & 17.9 & 0.128 & 0.336 & 20.1 & 0.46 & 0.058 & 0.052 & -- & 0.119 & 0.418 & 15.7 & 0.425 & 14.7 & -- \\
\hline
\end{tabular}


Appendix 1. Volumetric water content, temperature, and electrical-conductivity data collected at the flood-irrigated site during 2008.-Continued

[Depth in feet below land surface; volumetric water content dimensionless; temperature in degrees Celsius; bulk electrical conductivity in decisiemens per meter; --, no data]

\begin{tabular}{|c|c|c|c|c|c|c|c|c|c|c|c|c|c|c|c|}
\hline \multirow{3}{*}{$\begin{array}{l}\text { Measurement } \\
\text { date and time }\end{array}$} & \multicolumn{15}{|c|}{ Depth } \\
\hline & \multicolumn{2}{|c|}{2} & \multirow{2}{*}{$\begin{array}{c}3 \\
\begin{array}{c}\text { Volumetric } \\
\text { water } \\
\text { content }\end{array}\end{array}$} & \multicolumn{3}{|c|}{4} & \multirow{2}{*}{$\begin{array}{c}6 \\
\text { Volumetric } \\
\text { water } \\
\text { content }\end{array}$} & \multicolumn{2}{|l|}{ DepuI } & \multirow{2}{*}{$\begin{array}{c}10 \\
\text { Volumetric } \\
\text { water } \\
\text { content }\end{array}$} & \multicolumn{2}{|c|}{12} & \multicolumn{2}{|c|}{15} & \multirow{2}{*}{$\begin{array}{c}18.5 \\
\text { Volumetric } \\
\text { water } \\
\text { content }\end{array}$} \\
\hline & $\begin{array}{c}\text { Volumetric } \\
\text { water } \\
\text { content }\end{array}$ & $\begin{array}{l}\text { Temper- } \\
\text { ature }\end{array}$ & & $\begin{array}{c}\text { Volumetric } \\
\text { water } \\
\text { content }\end{array}$ & $\begin{array}{l}\text { Temper- } \\
\text { ature }\end{array}$ & $\begin{array}{c}\text { Bulk } \\
\text { electrical } \\
\text { conduct- } \\
\text { ivity }\end{array}$ & & $\begin{array}{c}\text { Volumetric } \\
\text { water } \\
\text { content }\end{array}$ & $\begin{array}{l}\text { Temper- } \\
\text { ature }\end{array}$ & & $\begin{array}{c}\text { Volumetric } \\
\text { water } \\
\text { content }\end{array}$ & $\begin{array}{l}\text { Temper- } \\
\text { ature }\end{array}$ & $\begin{array}{c}\text { Volumetric } \\
\text { water } \\
\text { content }\end{array}$ & $\begin{array}{l}\text { Temper- } \\
\text { ature }\end{array}$ & \\
\hline 8/17/2008 3:00 PM & 0.172 & 17.9 & 0.127 & 0.336 & 20.1 & 0.46 & 0.058 & 0.053 & -- & 0.093 & 0.418 & 15.7 & 0.425 & 14.7 & $\overline{--}$ \\
\hline 8/17/2008 4:00 PM & 0.171 & 17.8 & 0.127 & 0.336 & 20.0 & 0.46 & 0.058 & 0.053 & -- & 0.062 & 0.417 & 15.7 & 0.425 & 14.7 & -- \\
\hline 8/17/2008 5:00 PM & 0.170 & 17.8 & 0.126 & 0.336 & 20.0 & 0.46 & 0.058 & 0.053 & -- & 0.063 & 0.418 & 15.7 & 0.425 & 14.7 & -- \\
\hline 8/17/2008 6:00 PM & 0.169 & 17.8 & 0.126 & 0.335 & 20.0 & 0.45 & 0.058 & 0.054 & -- & 0.082 & 0.417 & 15.7 & 0.425 & 14.7 & -- \\
\hline 8/17/2008 7:00 PM & 0.168 & 17.8 & 0.126 & 0.335 & 20.0 & 0.45 & 0.058 & 0.054 & -- & 0.081 & 0.417 & 15.7 & 0.425 & 14.7 & -- \\
\hline 8/17/2008 8:00 PM & 0.168 & 17.8 & 0.125 & 0.335 & 20.0 & 0.45 & 0.058 & 0.055 & -- & 0.076 & 0.417 & 15.7 & 0.425 & 14.7 & -- \\
\hline 8/17/2008 9:00 PM & 0.167 & 17.8 & 0.125 & 0.335 & 20.0 & 0.45 & 0.058 & 0.055 & -- & 0.071 & 0.418 & 15.8 & 0.425 & 14.7 & -- \\
\hline 8/17/2008 10:00 PM & 0.166 & 17.8 & 0.124 & 0.334 & 19.9 & 0.45 & 0.058 & 0.056 & -- & 0.072 & 0.417 & 15.7 & 0.425 & 14.7 & -- \\
\hline 8/17/2008 11:00 PM & 0.166 & 17.8 & 0.124 & 0.334 & 19.9 & 0.44 & 0.058 & 0.057 & -- & 0.075 & 0.418 & 15.8 & 0.425 & 14.7 & -- \\
\hline 8/18/2008 12:00 AM & 0.165 & 17.8 & 0.123 & 0.334 & 19.9 & 0.44 & 0.058 & 0.057 & -- & 0.073 & 0.418 & 15.8 & 0.425 & 14.7 & -- \\
\hline 8/18/2008 1:00 AM & 0.165 & 17.9 & 0.123 & 0.334 & 19.9 & 0.44 & 0.058 & 0.058 & -- & 0.075 & 0.418 & 15.8 & 0.425 & 14.7 & -- \\
\hline 8/18/2008 2:00 AM & 0.163 & 17.8 & 0.122 & 0.333 & 19.9 & 0.44 & 0.058 & 0.058 & -- & 0.075 & 0.418 & 15.8 & 0.425 & 14.7 & -- \\
\hline 8/18/2008 3:00 AM & 0.162 & 17.8 & 0.122 & 0.333 & 19.9 & 0.44 & 0.058 & 0.058 & -- & 0.075 & 0.418 & 15.8 & 0.425 & 14.7 & -- \\
\hline 8/18/2008 4:00 AM & 0.162 & 17.8 & 0.121 & 0.333 & 19.9 & 0.43 & 0.058 & 0.058 & -- & 0.073 & 0.418 & 15.8 & 0.425 & 14.7 & -- \\
\hline 8/18/2008 5:00 AM & 0.161 & 17.8 & 0.121 & 0.333 & 19.8 & 0.43 & 0.058 & 0.058 & -- & 0.073 & 0.418 & 15.8 & 0.425 & 14.7 & -- \\
\hline 8/18/2008 6:00 AM & 0.160 & 17.8 & 0.121 & 0.332 & 19.8 & 0.43 & 0.058 & 0.059 & -- & 0.073 & 0.418 & 15.8 & 0.425 & 14.7 & -- \\
\hline 8/18/2008 7:00 AM & 0.160 & 17.9 & 0.121 & 0.332 & 19.8 & 0.43 & 0.058 & 0.059 & -- & 0.075 & 0.418 & 15.8 & 0.425 & 14.7 & -- \\
\hline 8/18/2008 8:00 AM & 0.159 & 17.9 & 0.120 & 0.332 & 19.8 & 0.43 & 0.058 & 0.059 & -- & 0.072 & 0.418 & 15.8 & 0.425 & 14.8 & -- \\
\hline 8/18/2008 9:00 AM & 0.159 & 17.9 & 0.120 & 0.332 & 19.8 & 0.42 & 0.058 & 0.059 & -- & 0.070 & 0.418 & 15.8 & 0.425 & 14.8 & -- \\
\hline 8/18/2008 10:00 AM & 0.159 & 17.9 & 0.120 & 0.332 & 19.8 & 0.42 & 0.058 & 0.059 & -- & 0.070 & 0.418 & 15.8 & 0.425 & 14.7 & -- \\
\hline 8/18/2008 11:00 AM & 0.158 & 17.8 & 0.120 & 0.332 & 19.8 & 0.42 & 0.057 & 0.060 & -- & 0.072 & 0.418 & 15.8 & 0.426 & 14.7 & -- \\
\hline 8/18/2008 12:00 PM & 0.158 & 17.8 & 0.119 & 0.332 & 19.8 & 0.42 & 0.057 & 0.060 & -- & 0.074 & 0.418 & 15.8 & 0.426 & 14.8 & -- \\
\hline 8/18/2008 1:00 PM & 0.157 & 17.8 & 0.119 & 0.332 & 19.7 & 0.42 & 0.057 & 0.060 & -- & 0.070 & 0.418 & 15.8 & 0.426 & 14.8 & -- \\
\hline 8/18/2008 2:00 PM & 0.157 & 17.9 & 0.118 & 0.332 & 19.7 & 0.42 & 0.057 & 0.060 & -- & 0.080 & 0.418 & 15.8 & 0.426 & 14.7 & -- \\
\hline 8/18/2008 3:00 PM & 0.157 & 17.8 & 0.118 & 0.332 & 19.7 & 0.42 & 0.056 & 0.060 & -- & 0.067 & 0.418 & 15.8 & 0.426 & 14.8 & -- \\
\hline 8/18/2008 4:00 PM & 0.156 & 17.8 & 0.118 & 0.331 & 19.7 & 0.41 & 0.056 & 0.060 & -- & 0.063 & 0.418 & 15.8 & 0.426 & 14.8 & -- \\
\hline 8/18/2008 5:00 PM & 0.155 & 17.9 & 0.117 & 0.331 & 19.7 & 0.41 & 0.056 & 0.060 & -- & 0.067 & 0.418 & 15.8 & 0.426 & 14.8 & -- \\
\hline 8/18/2008 6:00 PM & 0.155 & 17.8 & 0.117 & 0.331 & 19.7 & 0.41 & 0.056 & 0.060 & -- & 0.067 & 0.418 & 15.8 & 0.426 & 14.8 & -- \\
\hline 8/18/2008 7:00 PM & 0.154 & 17.8 & 0.117 & 0.331 & 19.7 & 0.41 & 0.056 & 0.060 & -- & 0.068 & 0.418 & 15.8 & 0.426 & 14.8 & -- \\
\hline 8/18/2008 8:00 PM & 0.154 & 17.8 & 0.116 & 0.330 & 19.7 & 0.41 & 0.056 & 0.059 & -- & 0.067 & 0.418 & 15.8 & 0.426 & 14.8 & -- \\
\hline 8/18/2008 9:00 PM & 0.154 & 17.8 & 0.116 & 0.330 & 19.6 & 0.41 & 0.056 & 0.059 & -- & 0.068 & 0.418 & 15.8 & 0.426 & 14.8 & -- \\
\hline 8/18/2008 10:00 PM & 0.153 & 17.8 & 0.115 & 0.330 & 19.6 & 0.41 & 0.056 & 0.059 & -- & 0.065 & 0.418 & 15.8 & 0.425 & 14.8 & -- \\
\hline 8/18/2008 11:00 PM & 0.153 & 17.9 & 0.115 & 0.330 & 19.6 & 0.41 & 0.055 & 0.059 & -- & 0.065 & 0.418 & 15.8 & 0.426 & 14.8 & -- \\
\hline 8/19/2008 12:00 AM & 0.151 & 17.9 & 0.115 & 0.330 & 19.6 & 0.41 & 0.055 & 0.059 & -- & 0.065 & 0.418 & 15.8 & 0.425 & 14.8 & -- \\
\hline 8/19/2008 1:00 AM & 0.151 & 17.9 & 0.115 & 0.329 & 19.6 & 0.41 & 0.055 & 0.059 & -- & 0.065 & 0.418 & 15.8 & 0.425 & 14.8 & -- \\
\hline 8/19/2008 2:00 AM & 0.150 & 17.9 & 0.115 & 0.329 & 19.6 & 0.41 & 0.055 & 0.059 & -- & 0.065 & 0.418 & 15.8 & 0.425 & 14.8 & -- \\
\hline 8/19/2008 3:00 AM & 0.150 & 17.9 & 0.115 & 0.329 & 19.6 & 0.41 & 0.055 & 0.059 & -- & 0.065 & 0.418 & 15.8 & 0.425 & 14.8 & -- \\
\hline 8/19/2008 4:00 AM & 0.150 & 17.9 & 0.115 & 0.329 & 19.6 & 0.40 & 0.055 & 0.059 & -- & 0.065 & 0.418 & 15.8 & 0.425 & 14.8 & -- \\
\hline 8/19/2008 5:00 AM & 0.149 & 18.0 & 0.114 & 0.329 & 19.6 & 0.40 & 0.055 & 0.059 & -- & 0.066 & 0.418 & 15.8 & 0.425 & 14.8 & -- \\
\hline 8/19/2008 6:00 AM & 0.149 & 18.0 & 0.114 & 0.329 & 19.6 & 0.40 & 0.055 & 0.059 & -- & 0.066 & 0.418 & 15.8 & 0.425 & 14.8 & -- \\
\hline
\end{tabular}


Appendix 1. Volumetric water content, temperature, and electrical-conductivity data collected at the flood-irrigated site during 2008.-Continued

[Depth in feet below land surface; volumetric water content dimensionless; temperature in degrees Celsius; bulk electrical conductivity in decisiemens per meter; --, no data]

\begin{tabular}{|c|c|c|c|c|c|c|c|c|c|c|c|c|c|c|c|}
\hline \multirow[b]{3}{*}{$\begin{array}{l}\text { Measurement } \\
\text { date and time }\end{array}$} & \multicolumn{15}{|c|}{ Depth } \\
\hline & \multirow{2}{*}{$\begin{array}{c}2 \\
\begin{array}{c}\text { Volumetric } \\
\text { water } \\
\text { content }\end{array}\end{array}$} & & \multirow{2}{*}{ 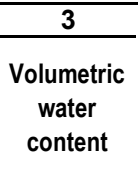 } & \multicolumn{3}{|c|}{4} & \multirow{2}{*}{$\begin{array}{c}6 \\
\begin{array}{c}\text { Volumetric } \\
\text { water } \\
\text { content }\end{array}\end{array}$} & \multicolumn{2}{|l|}{8} & \multirow{2}{*}{$\begin{array}{c}10 \\
\text { Volumetric } \\
\text { water } \\
\text { content }\end{array}$} & \multicolumn{2}{|c|}{12} & \multicolumn{2}{|c|}{15} & \multirow{2}{*}{$\begin{array}{c}18.5 \\
\text { Volumetric } \\
\text { water } \\
\text { content }\end{array}$} \\
\hline & & $\begin{array}{l}\text { Temper- } \\
\text { ature }\end{array}$ & & $\begin{array}{c}\text { Volumetric } \\
\text { water } \\
\text { content }\end{array}$ & $\begin{array}{l}\text { Temper- } \\
\text { ature }\end{array}$ & $\begin{array}{l}\text { Bulk } \\
\text { electrical } \\
\text { conduct- } \\
\text { ivity }\end{array}$ & & $\begin{array}{c}\text { Volumetric } \\
\text { water } \\
\text { content }\end{array}$ & $\begin{array}{l}\text { Temper- } \\
\text { ature }\end{array}$ & & $\begin{array}{c}\text { Volumetric } \\
\text { water } \\
\text { content }\end{array}$ & $\begin{array}{l}\text { Temper- } \\
\text { ature }\end{array}$ & $\begin{array}{l}\text { Volumetric } \\
\text { water } \\
\text { content }\end{array}$ & $\begin{array}{l}\text { Temper- } \\
\text { ature }\end{array}$ & \\
\hline $8 / 19 / 2008$ 7:00 AM & 0.149 & 18.0 & 0.114 & 0.329 & 19.6 & 0.40 & 0.054 & 0.058 & -- & 0.066 & 0.418 & 15.9 & 0.425 & 14.8 & -- \\
\hline 8/19/2008 8:00 AM & 0.148 & 18.0 & 0.113 & 0.328 & 19.5 & 0.40 & 0.054 & 0.058 & -- & 0.066 & 0.418 & 15.8 & 0.425 & 14.8 & -- \\
\hline 8/19/2008 9:00 AM & 0.148 & 18.0 & 0.113 & 0.328 & 19.5 & 0.40 & 0.054 & 0.058 & -- & 0.066 & 0.418 & 15.9 & 0.425 & 14.8 & -- \\
\hline 8/19/2008 10:00 AM & 0.148 & 18.0 & 0.113 & 0.328 & 19.5 & 0.40 & 0.054 & 0.058 & -- & 0.066 & 0.418 & 15.9 & 0.425 & 14.8 & -- \\
\hline 8/19/2008 11:00 AM & 0.148 & 18.0 & 0.113 & 0.328 & 19.5 & 0.40 & 0.054 & 0.058 & -- & 0.066 & 0.418 & 15.9 & 0.426 & 14.8 & -- \\
\hline 8/19/2008 12:00 PM & 0.148 & 18.0 & 0.113 & 0.328 & 19.5 & 0.40 & 0.053 & 0.058 & -- & 0.066 & 0.418 & 15.8 & 0.426 & 14.8 & -- \\
\hline 8/19/2008 1:00 PM & 0.148 & 18.0 & 0.113 & 0.328 & 19.5 & 0.40 & 0.053 & 0.058 & -- & 0.066 & 0.418 & 15.8 & 0.425 & 14.8 & -- \\
\hline 8/19/2008 2:00 PM & 0.147 & 18.0 & 0.112 & 0.328 & 19.5 & 0.40 & 0.053 & 0.058 & -- & 0.066 & 0.418 & 15.9 & 0.425 & 14.8 & -- \\
\hline 8/19/2008 3:00 PM & 0.147 & 18.0 & 0.112 & 0.328 & 19.5 & 0.40 & 0.053 & 0.058 & -- & 0.067 & 0.418 & 15.9 & 0.425 & 14.8 & -- \\
\hline 8/19/2008 4:00 PM & 0.147 & 18.0 & 0.112 & 0.328 & 19.5 & 0.40 & 0.053 & 0.058 & -- & 0.067 & 0.417 & 15.8 & 0.425 & 14.8 & -- \\
\hline 8/19/2008 5:00 PM & 0.146 & 18.0 & 0.112 & 0.328 & 19.5 & 0.40 & 0.053 & 0.058 & -- & 0.069 & 0.418 & 15.8 & 0.425 & 14.8 & -- \\
\hline 8/19/2008 6:00 PM & 0.146 & 17.9 & 0.112 & 0.326 & 19.5 & 0.40 & 0.053 & 0.058 & -- & 0.070 & 0.418 & 15.9 & 0.425 & 14.8 & -- \\
\hline 8/19/2008 7:00 PM & 0.146 & 18.0 & 0.111 & 0.326 & 19.5 & 0.40 & 0.053 & 0.058 & -- & 0.069 & 0.418 & 15.8 & 0.425 & 14.8 & -- \\
\hline 8/19/2008 8:00 PM & 0.145 & 17.9 & 0.111 & 0.326 & 19.5 & 0.40 & 0.053 & 0.058 & -- & 0.070 & 0.418 & 15.9 & 0.425 & 14.8 & -- \\
\hline 8/19/2008 9:00 PM & 0.145 & 17.9 & 0.111 & 0.326 & 19.4 & 0.40 & 0.053 & 0.058 & -- & 0.071 & 0.418 & 15.9 & 0.425 & 14.8 & -- \\
\hline 8/19/2008 10:00 PM & 0.145 & 17.9 & 0.110 & 0.326 & 19.4 & 0.40 & 0.053 & 0.058 & -- & 0.075 & 0.418 & 15.9 & 0.425 & 14.8 & -- \\
\hline 8/19/2008 11:00 PM & 0.145 & 18.0 & 0.110 & 0.325 & 19.4 & 0.40 & 0.053 & 0.058 & -- & 0.087 & 0.418 & 15.9 & 0.425 & 14.9 & -- \\
\hline 8/20/2008 12:00 AM & 0.144 & 17.9 & 0.110 & 0.326 & 19.4 & 0.40 & 0.053 & 0.058 & -- & 0.068 & 0.418 & 15.9 & 0.425 & 14.8 & -- \\
\hline 8/20/2008 1:00 AM & 0.144 & 18.0 & 0.110 & 0.325 & 19.4 & 0.40 & 0.053 & 0.058 & -- & 0.068 & 0.418 & 15.9 & 0.425 & 14.8 & -- \\
\hline 8/20/2008 2:00 AM & 0.143 & 18.0 & 0.110 & 0.325 & 19.4 & 0.39 & 0.053 & 0.058 & -- & 0.068 & 0.418 & 15.9 & 0.425 & 14.8 & -- \\
\hline 8/20/2008 3:00 AM & 0.143 & 18.1 & 0.109 & 0.325 & 19.4 & 0.39 & 0.053 & 0.057 & -- & 0.068 & 0.418 & 15.9 & 0.425 & 14.8 & -- \\
\hline 8/20/2008 4:00 AM & 0.144 & 18.1 & 0.109 & 0.325 & 19.4 & 0.39 & 0.052 & 0.057 & -- & 0.068 & 0.418 & 15.9 & 0.425 & 14.8 & -- \\
\hline 8/20/2008 5:00 AM & 0.144 & 18.1 & 0.109 & 0.325 & 19.4 & 0.39 & 0.052 & 0.057 & -- & 0.068 & 0.418 & 15.9 & 0.425 & 14.9 & -- \\
\hline 8/20/2008 6:00 AM & 0.143 & 18.2 & 0.109 & 0.325 & 19.4 & 0.39 & 0.052 & 0.057 & -- & 0.068 & 0.418 & 15.9 & 0.425 & 14.9 & -- \\
\hline 8/20/2008 7:00 AM & 0.143 & 18.2 & 0.109 & 0.325 & 19.4 & 0.39 & 0.052 & 0.057 & -- & 0.068 & 0.418 & 15.9 & 0.425 & 14.9 & -- \\
\hline 8/20/2008 8:00 AM & 0.143 & 18.2 & 0.109 & 0.324 & 19.4 & 0.39 & 0.052 & 0.057 & -- & 0.068 & 0.418 & 15.9 & 0.425 & 14.9 & -- \\
\hline 8/20/2008 9:00 AM & 0.142 & 18.2 & 0.109 & 0.324 & 19.4 & 0.39 & 0.052 & 0.057 & -- & 0.068 & 0.418 & 15.9 & 0.425 & 14.9 & -- \\
\hline 8/20/2008 10:00 AM & 0.142 & 18.2 & 0.109 & 0.324 & 19.4 & 0.39 & 0.052 & 0.057 & -- & 0.068 & 0.418 & 15.9 & 0.425 & 14.9 & -- \\
\hline 8/20/2008 11:00 AM & 0.142 & 18.2 & 0.109 & 0.325 & 19.4 & 0.39 & 0.051 & 0.057 & -- & 0.068 & 0.418 & 15.9 & 0.425 & 14.9 & -- \\
\hline 8/20/2008 12:00 PM & 0.142 & 18.2 & 0.109 & 0.324 & 19.4 & 0.39 & 0.051 & 0.057 & -- & 0.068 & 0.419 & 15.9 & 0.425 & 14.9 & -- \\
\hline 8/20/2008 1:00 PM & 0.142 & 18.2 & 0.109 & 0.324 & 19.4 & 0.39 & 0.051 & 0.057 & -- & 0.068 & 0.418 & 15.9 & 0.425 & 14.9 & -- \\
\hline 8/20/2008 2:00 PM & 0.142 & 18.2 & 0.109 & 0.324 & 19.4 & 0.39 & 0.051 & 0.057 & -- & 0.068 & 0.418 & 15.9 & 0.425 & 14.9 & -- \\
\hline 8/20/2008 3:00 PM & 0.141 & 18.2 & 0.109 & 0.324 & 19.3 & 0.39 & 0.051 & 0.057 & -- & 0.069 & 0.418 & 15.9 & 0.425 & 14.9 & -- \\
\hline 8/20/2008 4:00 PM & 0.141 & 18.2 & 0.109 & 0.324 & 19.3 & 0.39 & 0.051 & 0.057 & -- & 0.070 & 0.418 & 15.9 & 0.425 & 14.9 & -- \\
\hline 8/20/2008 5:00 PM & 0.141 & 18.2 & 0.108 & 0.324 & 19.3 & 0.39 & 0.050 & 0.057 & -- & 0.068 & 0.418 & 15.9 & 0.425 & 14.9 & -- \\
\hline 8/20/2008 6:00 PM & 0.141 & 18.2 & 0.108 & 0.323 & 19.3 & 0.39 & 0.050 & 0.056 & -- & 0.067 & 0.419 & 15.9 & 0.425 & 14.9 & -- \\
\hline 8/20/2008 7:00 PM & 0.140 & 18.2 & 0.108 & 0.324 & 19.3 & 0.39 & 0.050 & 0.056 & -- & 0.070 & 0.419 & 15.9 & 0.425 & 14.9 & -- \\
\hline 8/20/2008 8:00 PM & 0.140 & 18.2 & 0.108 & 0.323 & 19.3 & 0.39 & 0.050 & 0.056 & -- & 0.070 & 0.419 & 15.9 & 0.425 & 14.9 & -- \\
\hline 8/20/2008 9:00 PM & 0.140 & 18.2 & 0.108 & 0.323 & 19.3 & 0.39 & 0.050 & 0.056 & -- & 0.069 & 0.419 & 15.9 & 0.425 & 14.9 & -- \\
\hline 8/20/2008 10:00 PM & 0.140 & 18.2 & 0.107 & 0.323 & 19.3 & 0.39 & 0.050 & 0.056 & -- & 0.069 & 0.419 & 15.9 & 0.425 & 14.9 & -- \\
\hline
\end{tabular}


Appendix 1. Volumetric water content, temperature, and electrical-conductivity data collected at the flood-irrigated site during 2008.-Continued

[Depth in feet below land surface; volumetric water content dimensionless; temperature in degrees Celsius; bulk electrical conductivity in decisiemens per meter; --, no data]

\begin{tabular}{|c|c|c|c|c|c|c|c|c|c|c|c|c|c|c|c|}
\hline \multirow{3}{*}{$\begin{array}{l}\text { Measurement } \\
\text { date and time }\end{array}$} & \multicolumn{15}{|c|}{ Depth } \\
\hline & \multicolumn{2}{|c|}{2} & \multirow{2}{*}{$\begin{array}{c}3 \\
\begin{array}{c}\text { Volumetric } \\
\text { water } \\
\text { content }\end{array}\end{array}$} & \multicolumn{3}{|c|}{4} & \multirow{2}{*}{$\begin{array}{c}6 \\
\text { Volumetric } \\
\text { water } \\
\text { content }\end{array}$} & \multicolumn{2}{|l|}{ DepuI } & \multirow{2}{*}{$\begin{array}{c}10 \\
\text { Volumetric } \\
\text { water } \\
\text { content }\end{array}$} & \multicolumn{2}{|c|}{12} & \multicolumn{2}{|c|}{15} & \multirow{2}{*}{$\begin{array}{c}18.5 \\
\text { Volumetric } \\
\text { water } \\
\text { content }\end{array}$} \\
\hline & $\begin{array}{c}\text { Volumetric } \\
\text { water } \\
\text { content }\end{array}$ & $\begin{array}{l}\text { Temper- } \\
\text { ature }\end{array}$ & & $\begin{array}{c}\text { Volumetric } \\
\text { water } \\
\text { content }\end{array}$ & $\begin{array}{l}\text { Temper- } \\
\text { ature }\end{array}$ & $\begin{array}{c}\text { Bulk } \\
\text { electrical } \\
\text { conduct- } \\
\text { ivity }\end{array}$ & & $\begin{array}{c}\text { Volumetric } \\
\text { water } \\
\text { content }\end{array}$ & $\begin{array}{l}\text { Temper- } \\
\text { ature }\end{array}$ & & $\begin{array}{c}\text { Volumetric } \\
\text { water } \\
\text { content }\end{array}$ & $\begin{array}{l}\text { Temper- } \\
\text { ature }\end{array}$ & $\begin{array}{c}\text { Volumetric } \\
\text { water } \\
\text { content }\end{array}$ & $\begin{array}{l}\text { Temper- } \\
\text { ature }\end{array}$ & \\
\hline$\overline{8 / 20 / 2008 ~ 11: 00 ~ P M}$ & 0.140 & 18.2 & 0.107 & 0.323 & 19.3 & 0.39 & 0.050 & 0.055 & -- & 0.070 & 0.419 & 15.9 & 0.425 & 14.9 & $\overline{--}$ \\
\hline 8/21/2008 12:00 AM & 0.140 & 18.2 & 0.107 & 0.323 & 19.3 & 0.39 & 0.050 & 0.055 & -- & 0.070 & 0.419 & 15.9 & 0.425 & 14.9 & -- \\
\hline 8/21/2008 1:00 AM & 0.140 & 18.3 & 0.107 & 0.323 & 19.3 & 0.39 & 0.050 & 0.055 & -- & 0.069 & 0.419 & 15.9 & 0.425 & 14.9 & -- \\
\hline 8/21/2008 2:00 AM & 0.138 & 18.3 & 0.107 & 0.323 & 19.3 & 0.39 & 0.050 & 0.055 & -- & 0.067 & 0.419 & 16.0 & 0.425 & 14.9 & -- \\
\hline 8/21/2008 3:00 AM & 0.138 & 18.3 & 0.107 & 0.323 & 19.3 & 0.39 & 0.050 & 0.055 & -- & 0.067 & 0.419 & 16.0 & 0.425 & 14.9 & -- \\
\hline 8/21/2008 4:00 AM & 0.138 & 18.2 & 0.106 & 0.322 & 19.3 & 0.39 & 0.050 & 0.055 & -- & 0.068 & 0.419 & 15.9 & 0.425 & 14.9 & -- \\
\hline 8/21/2008 5:00 AM & 0.138 & 18.2 & 0.106 & 0.322 & 19.3 & 0.39 & 0.050 & 0.055 & -- & 0.067 & 0.419 & 16.0 & 0.425 & 14.9 & -- \\
\hline 8/21/2008 6:00 AM & 0.138 & 18.3 & 0.106 & 0.322 & 19.3 & 0.39 & 0.049 & 0.055 & -- & 0.068 & 0.419 & 16.0 & 0.425 & 14.9 & -- \\
\hline 8/21/2008 7:00 AM & 0.137 & 18.3 & 0.106 & 0.322 & 19.3 & 0.39 & 0.049 & 0.055 & -- & 0.070 & 0.419 & 16.0 & 0.425 & 14.9 & -- \\
\hline 8/21/2008 8:00 AM & 0.137 & 18.3 & 0.106 & 0.322 & 19.3 & 0.39 & 0.049 & 0.055 & -- & 0.069 & 0.419 & 16.0 & 0.425 & 14.9 & -- \\
\hline 8/21/2008 9:00 AM & 0.137 & 18.3 & 0.106 & 0.322 & 19.3 & 0.39 & 0.049 & 0.055 & -- & 0.067 & 0.420 & 15.9 & 0.425 & 14.9 & -- \\
\hline 8/21/2008 10:00 AM & 0.137 & 18.3 & 0.106 & 0.322 & 19.3 & 0.39 & 0.049 & 0.055 & -- & 0.066 & 0.420 & 16.0 & 0.424 & 14.9 & -- \\
\hline 8/21/2008 11:00 AM & 0.137 & 18.3 & 0.106 & 0.322 & 19.3 & 0.39 & 0.049 & 0.055 & -- & 0.066 & 0.420 & 15.9 & 0.424 & 14.9 & -- \\
\hline 8/21/2008 12:00 PM & 0.137 & 18.4 & 0.106 & 0.322 & 19.3 & 0.39 & 0.049 & 0.055 & -- & 0.066 & 0.420 & 15.9 & 0.424 & 14.9 & -- \\
\hline 8/21/2008 1:00 PM & 0.137 & 18.4 & 0.106 & 0.322 & 19.3 & 0.39 & 0.049 & 0.055 & -- & 0.066 & 0.420 & 15.9 & 0.424 & 14.9 & -- \\
\hline 8/21/2008 2:00 PM & 0.136 & 18.4 & 0.106 & 0.322 & 19.3 & 0.39 & 0.048 & 0.055 & -- & 0.066 & 0.420 & 16.0 & 0.425 & 14.9 & -- \\
\hline 8/21/2008 3:00 PM & 0.136 & 18.4 & 0.105 & 0.322 & 19.3 & 0.39 & 0.048 & 0.054 & -- & 0.066 & 0.419 & 16.0 & 0.425 & 14.9 & -- \\
\hline 8/21/2008 4:00 PM & 0.136 & 18.4 & 0.105 & 0.322 & 19.3 & 0.39 & 0.048 & 0.054 & -- & 0.066 & 0.420 & 16.0 & 0.425 & 15.0 & -- \\
\hline 8/21/2008 5:00 PM & 0.136 & 18.3 & 0.105 & 0.322 & 19.3 & 0.39 & 0.048 & 0.054 & -- & 0.067 & 0.420 & 16.0 & 0.424 & 15.0 & -- \\
\hline 8/21/2008 6:00 PM & 0.136 & 18.3 & 0.105 & 0.322 & 19.3 & 0.39 & 0.048 & 0.054 & -- & 0.070 & 0.420 & 16.0 & 0.424 & 14.9 & -- \\
\hline 8/21/2008 7:00 PM & 0.135 & 18.3 & 0.105 & 0.322 & 19.3 & 0.39 & 0.048 & 0.054 & -- & 0.080 & 0.420 & 16.0 & 0.424 & 14.9 & -- \\
\hline 8/21/2008 8:00 PM & 0.136 & 18.3 & 0.105 & 0.321 & 19.3 & 0.39 & 0.048 & 0.054 & -- & 0.107 & 0.420 & 16.0 & 0.424 & 15.0 & -- \\
\hline 8/21/2008 9:00 PM & 0.136 & 18.3 & 0.105 & 0.321 & 19.3 & 0.39 & 0.048 & 0.053 & -- & 0.104 & 0.420 & 16.0 & 0.424 & 15.0 & -- \\
\hline 8/21/2008 10:00 PM & 0.136 & 18.3 & 0.104 & 0.321 & 19.3 & 0.39 & 0.048 & 0.054 & -- & 0.081 & 0.420 & 16.0 & 0.424 & 14.9 & -- \\
\hline 8/21/2008 11:00 PM & 0.135 & 18.3 & 0.104 & 0.321 & 19.3 & 0.39 & 0.048 & 0.053 & -- & 0.118 & 0.420 & 16.0 & 0.424 & 15.0 & -- \\
\hline 8/22/2008 12:00 AM & 0.135 & 18.4 & 0.104 & 0.321 & 19.3 & 0.39 & 0.048 & 0.053 & -- & 0.083 & 0.420 & 16.0 & 0.424 & 15.0 & -- \\
\hline 8/22/2008 1:00 AM & 0.134 & 18.4 & 0.104 & 0.321 & 19.3 & 0.39 & 0.048 & 0.053 & -- & 0.081 & 0.421 & 16.0 & 0.424 & 15.0 & -- \\
\hline 8/22/2008 2:00 AM & 0.135 & 18.5 & 0.104 & 0.321 & 19.3 & 0.39 & 0.048 & 0.053 & -- & 0.074 & 0.420 & 16.0 & 0.424 & 15.0 & -- \\
\hline 8/22/2008 3:00 AM & 0.135 & 18.6 & 0.104 & 0.321 & 19.3 & 0.39 & 0.048 & 0.053 & -- & 0.077 & 0.421 & 16.0 & 0.424 & 15.0 & -- \\
\hline 8/22/2008 4:00 AM & 0.135 & 18.6 & 0.104 & 0.321 & 19.3 & 0.39 & 0.048 & 0.053 & -- & 0.078 & 0.420 & 16.0 & 0.424 & 15.0 & -- \\
\hline 8/22/2008 5:00 AM & 0.134 & 18.6 & 0.104 & 0.320 & 19.3 & 0.39 & 0.048 & 0.053 & -- & 0.087 & 0.421 & 16.0 & 0.424 & 15.0 & -- \\
\hline 8/22/2008 6:00 AM & 0.133 & 18.6 & 0.104 & 0.320 & 19.3 & 0.39 & 0.048 & 0.053 & -- & 0.082 & 0.421 & 16.0 & 0.424 & 15.0 & -- \\
\hline 8/22/2008 7:00 AM & 0.133 & 18.6 & 0.104 & 0.320 & 19.3 & 0.39 & 0.047 & 0.053 & -- & 0.086 & 0.422 & 16.0 & 0.424 & 15.0 & -- \\
\hline 8/22/2008 8:00 AM & 0.134 & 18.7 & 0.104 & 0.320 & 19.3 & 0.39 & 0.047 & 0.053 & -- & 0.091 & 0.422 & 16.0 & 0.424 & 15.0 & -- \\
\hline 8/22/2008 9:00 AM & 0.134 & 18.7 & 0.104 & 0.320 & 19.2 & 0.39 & 0.047 & 0.053 & -- & 0.078 & 0.422 & 16.0 & 0.424 & 15.0 & -- \\
\hline 8/22/2008 10:00 AM & 0.133 & 18.7 & 0.104 & 0.320 & 19.2 & 0.39 & 0.047 & 0.053 & -- & 0.075 & 0.422 & 16.0 & 0.424 & 15.0 & -- \\
\hline 8/22/2008 11:00 AM & 0.134 & 18.7 & 0.104 & 0.320 & 19.2 & 0.39 & 0.047 & 0.053 & -- & 0.071 & 0.422 & 16.0 & 0.424 & 15.0 & -- \\
\hline 8/22/2008 12:00 PM & 0.134 & 18.6 & 0.104 & 0.320 & 19.2 & 0.39 & 0.047 & 0.053 & -- & 0.078 & 0.422 & 16.0 & 0.424 & 15.0 & -- \\
\hline 8/22/2008 1:00 PM & 0.134 & 18.6 & 0.104 & 0.320 & 19.2 & 0.39 & 0.047 & 0.053 & -- & 0.078 & 0.422 & 16.0 & 0.425 & 15.0 & -- \\
\hline 8/22/2008 2:00 PM & 0.133 & 18.6 & 0.104 & 0.320 & 19.2 & 0.39 & 0.047 & 0.053 & -- & 0.079 & 0.422 & 16.0 & 0.425 & 15.0 & -- \\
\hline
\end{tabular}


Appendix 1. Volumetric water content, temperature, and electrical-conductivity data collected at the flood-irrigated site during 2008.-Continued

[Depth in feet below land surface; volumetric water content dimensionless; temperature in degrees Celsius; bulk electrical conductivity in decisiemens per meter; --, no data]

\begin{tabular}{|c|c|c|c|c|c|c|c|c|c|c|c|c|c|c|c|}
\hline \multirow[b]{3}{*}{$\begin{array}{l}\text { Measurement } \\
\text { date and time }\end{array}$} & \multicolumn{15}{|c|}{ Depth } \\
\hline & \multicolumn{2}{|c|}{2} & \multirow{2}{*}{$\begin{array}{c}3 \\
\begin{array}{c}\text { Volumetric } \\
\text { water } \\
\text { content }\end{array}\end{array}$} & \multicolumn{3}{|c|}{4} & \multirow{2}{*}{$\begin{array}{c}6 \\
\begin{array}{c}\text { Volumetric } \\
\text { water } \\
\text { content }\end{array}\end{array}$} & \multicolumn{2}{|l|}{8} & \multirow{2}{*}{$\begin{array}{c}10 \\
\text { Volumetric } \\
\text { water } \\
\text { content }\end{array}$} & \multicolumn{2}{|c|}{12} & \multicolumn{2}{|c|}{15} & \multirow{2}{*}{$\begin{array}{c}18.5 \\
\text { Volumetric } \\
\text { water } \\
\text { content }\end{array}$} \\
\hline & $\begin{array}{l}\text { Volumetric } \\
\text { water } \\
\text { content }\end{array}$ & $\begin{array}{l}\text { Temper- } \\
\text { ature }\end{array}$ & & $\begin{array}{c}\text { Volumetric } \\
\text { water } \\
\text { content }\end{array}$ & $\begin{array}{l}\text { Temper- } \\
\text { ature }\end{array}$ & $\begin{array}{c}\text { Bulk } \\
\text { electrical } \\
\text { conduct- } \\
\text { ivity } \\
\end{array}$ & & $\begin{array}{l}\text { Volumetric } \\
\text { water } \\
\text { content }\end{array}$ & $\begin{array}{l}\text { Temper- } \\
\text { ature }\end{array}$ & & $\begin{array}{l}\text { Volumetric } \\
\text { water } \\
\text { content }\end{array}$ & $\begin{array}{l}\text { Temper- } \\
\text { ature }\end{array}$ & $\begin{array}{l}\text { Volumetric } \\
\text { water } \\
\text { content }\end{array}$ & $\begin{array}{l}\text { Temper- } \\
\text { ature }\end{array}$ & \\
\hline 8/22/2008 3:00 PM & 0.133 & 18.6 & 0.104 & 0.320 & 19.2 & 0.39 & 0.047 & 0.053 & -- & 0.087 & 0.422 & 16.0 & 0.425 & 15.0 & -- \\
\hline 8/22/2008 4:00 PM & 0.133 & 18.6 & 0.104 & 0.320 & 19.2 & 0.39 & 0.047 & 0.053 & -- & 0.077 & 0.422 & 16.0 & 0.425 & 15.0 & -- \\
\hline 8/22/2008 5:00 PM & 0.133 & 18.6 & 0.104 & 0.320 & 19.2 & 0.39 & 0.047 & 0.053 & -- & 0.076 & 0.422 & 16.0 & 0.425 & 15.0 & -- \\
\hline 8/22/2008 6:00 PM & 0.133 & 18.6 & 0.103 & 0.320 & 19.2 & 0.39 & 0.047 & 0.053 & -- & 0.078 & 0.422 & 16.0 & 0.425 & 15.0 & -- \\
\hline 8/22/2008 7:00 PM & 0.132 & 18.6 & 0.103 & 0.320 & 19.2 & 0.39 & 0.047 & 0.053 & -- & 0.073 & 0.422 & 16.0 & 0.425 & 15.0 & -- \\
\hline 8/22/2008 8:00 PM & 0.132 & 18.6 & 0.103 & 0.320 & 19.2 & 0.39 & 0.047 & 0.052 & -- & 0.081 & 0.422 & 16.0 & 0.424 & 15.0 & -- \\
\hline 8/22/2008 9:00 PM & 0.132 & 18.6 & 0.103 & 0.320 & 19.2 & 0.39 & 0.047 & 0.052 & -- & 0.079 & 0.422 & 16.0 & 0.424 & 15.0 & -- \\
\hline 8/22/2008 10:00 PM & 0.132 & 18.6 & 0.103 & 0.320 & 19.2 & 0.39 & 0.047 & 0.052 & -- & 0.081 & 0.422 & 16.0 & 0.424 & 15.0 & -- \\
\hline 8/22/2008 11:00 PM & 0.132 & 18.6 & 0.103 & 0.320 & 19.2 & 0.39 & 0.047 & 0.052 & -- & 0.081 & 0.422 & 16.0 & 0.424 & 15.0 & -- \\
\hline 8/23/2008 12:00 AM & 0.132 & 18.6 & 0.103 & 0.319 & 19.2 & 0.39 & 0.047 & 0.052 & -- & 0.081 & 0.422 & 16.0 & 0.424 & 15.0 & -- \\
\hline 8/23/2008 1:00 AM & 0.132 & 18.7 & 0.102 & 0.319 & 19.2 & 0.39 & 0.047 & 0.052 & -- & 0.080 & 0.422 & 16.0 & 0.424 & 15.0 & -- \\
\hline 8/23/2008 2:00 AM & 0.132 & 18.7 & 0.102 & 0.319 & 19.2 & 0.39 & 0.047 & 0.052 & -- & 0.081 & 0.422 & 16.0 & 0.425 & 15.0 & -- \\
\hline 8/23/2008 3:00 AM & 0.131 & 18.7 & 0.102 & 0.319 & 19.2 & 0.39 & 0.047 & 0.052 & -- & 0.071 & 0.422 & 16.1 & 0.425 & 15.0 & -- \\
\hline 8/23/2008 4:00 AM & 0.130 & 18.7 & 0.102 & 0.319 & 19.2 & 0.39 & 0.047 & 0.052 & -- & 0.078 & 0.422 & 16.0 & 0.425 & 15.0 & -- \\
\hline 8/23/2008 5:00 AM & 0.130 & 18.7 & 0.102 & 0.319 & 19.2 & 0.39 & 0.047 & 0.052 & -- & 0.083 & 0.422 & 16.0 & 0.425 & 15.0 & -- \\
\hline 8/23/2008 6:00 AM & 0.130 & 18.8 & 0.102 & 0.319 & 19.2 & 0.39 & 0.047 & 0.052 & -- & 0.087 & 0.423 & 16.0 & 0.425 & 15.0 & -- \\
\hline 8/23/2008 7:00 AM & 0.130 & 18.7 & 0.102 & 0.319 & 19.2 & 0.39 & 0.047 & 0.052 & -- & 0.079 & 0.423 & 16.0 & 0.425 & 15.0 & -- \\
\hline 8/23/2008 8:00 AM & 0.131 & 18.8 & 0.102 & 0.319 & 19.2 & 0.39 & 0.047 & 0.052 & -- & 0.083 & 0.422 & 16.1 & 0.425 & 15.0 & -- \\
\hline 8/23/2008 9:00 AM & 0.132 & 18.8 & 0.102 & 0.319 & 19.2 & 0.39 & 0.047 & 0.052 & -- & 0.084 & 0.422 & 16.1 & 0.425 & 15.0 & -- \\
\hline 8/23/2008 10:00 AM & 0.131 & 18.8 & 0.102 & 0.319 & 19.2 & 0.39 & 0.047 & 0.052 & -- & 0.088 & 0.422 & 16.1 & 0.425 & 15.0 & -- \\
\hline 8/23/2008 11:00 AM & 0.131 & 18.7 & 0.102 & 0.319 & 19.2 & 0.39 & 0.046 & 0.052 & -- & 0.089 & 0.423 & 16.0 & 0.425 & 15.0 & -- \\
\hline 8/23/2008 12:00 PM & 0.131 & 18.7 & 0.102 & 0.319 & 19.2 & 0.39 & 0.046 & 0.052 & -- & 0.088 & 0.422 & 16.1 & 0.426 & 15.0 & -- \\
\hline 8/23/2008 1:00 PM & 0.131 & 18.7 & 0.102 & 0.319 & 19.2 & 0.39 & 0.046 & 0.052 & -- & 0.092 & 0.422 & 16.1 & 0.425 & 15.1 & -- \\
\hline 8/23/2008 2:00 PM & 0.130 & 18.7 & 0.101 & 0.319 & 19.2 & 0.39 & 0.046 & 0.052 & -- & 0.094 & 0.423 & 16.0 & 0.425 & 15.1 & -- \\
\hline 8/23/2008 3:00 PM & 0.130 & 18.6 & 0.102 & 0.319 & 19.2 & 0.39 & 0.046 & 0.052 & -- & 0.096 & 0.422 & 16.1 & 0.425 & 15.1 & -- \\
\hline 8/23/2008 4:00 PM & 0.130 & 18.6 & 0.101 & 0.319 & 19.2 & 0.39 & 0.046 & 0.051 & -- & 0.098 & 0.422 & 16.0 & 0.425 & 15.0 & -- \\
\hline 8/23/2008 5:00 PM & 0.130 & 18.6 & 0.101 & 0.319 & 19.2 & 0.39 & 0.046 & 0.051 & -- & 0.098 & 0.423 & 16.1 & 0.425 & 15.1 & -- \\
\hline 8/23/2008 6:00 PM & 0.129 & 18.6 & 0.101 & 0.319 & 19.2 & 0.39 & 0.046 & 0.051 & -- & 0.098 & 0.423 & 16.1 & 0.425 & 15.1 & -- \\
\hline 8/23/2008 7:00 PM & 0.129 & 18.6 & 0.101 & 0.319 & 19.2 & 0.39 & 0.046 & 0.051 & -- & 0.097 & 0.423 & 16.0 & 0.425 & 15.1 & -- \\
\hline 8/23/2008 8:00 PM & 0.129 & 18.7 & 0.101 & 0.318 & 19.2 & 0.39 & 0.046 & 0.051 & -- & 0.104 & 0.423 & 16.1 & 0.425 & 15.1 & -- \\
\hline 8/23/2008 9:00 PM & 0.129 & 18.7 & 0.101 & 0.318 & 19.2 & 0.39 & 0.046 & 0.051 & -- & 0.101 & 0.422 & 16.1 & 0.425 & 15.1 & -- \\
\hline 8/23/2008 10:00 PM & 0.130 & 18.7 & 0.101 & 0.318 & 19.2 & 0.39 & 0.046 & 0.051 & -- & 0.092 & 0.422 & 16.1 & 0.425 & 15.1 & -- \\
\hline 8/23/2008 11:00 PM & 0.130 & 18.7 & 0.101 & 0.318 & 19.2 & 0.39 & 0.046 & 0.051 & -- & 0.087 & 0.422 & 16.1 & 0.425 & 15.1 & -- \\
\hline 8/24/2008 12:00 AM & 0.130 & 18.7 & 0.100 & 0.318 & 19.2 & 0.39 & 0.046 & 0.051 & -- & 0.088 & 0.423 & 16.1 & 0.425 & 15.1 & -- \\
\hline 8/24/2008 1:00 AM & 0.129 & 18.7 & 0.100 & 0.318 & 19.2 & 0.39 & 0.046 & 0.050 & -- & 0.073 & 0.423 & 16.1 & 0.425 & 15.1 & -- \\
\hline 8/24/2008 2:00 AM & 0.128 & 18.7 & 0.100 & 0.318 & 19.2 & 0.39 & 0.046 & 0.050 & -- & 0.071 & 0.423 & 16.1 & 0.424 & 15.1 & -- \\
\hline 8/24/2008 3:00 AM & 0.128 & 18.7 & 0.100 & 0.318 & 19.2 & 0.39 & 0.046 & 0.050 & -- & 0.070 & 0.423 & 16.1 & 0.424 & 15.1 & -- \\
\hline 8/24/2008 4:00 AM & 0.129 & 18.7 & 0.100 & 0.318 & 19.2 & 0.39 & 0.046 & 0.050 & -- & 0.068 & 0.423 & 16.1 & 0.424 & 15.1 & -- \\
\hline 8/24/2008 5:00 AM & 0.129 & 18.7 & 0.100 & 0.318 & 19.2 & 0.39 & 0.046 & 0.050 & -- & 0.066 & 0.423 & 16.1 & 0.425 & 15.1 & -- \\
\hline 8/24/2008 6:00 AM & 0.129 & 18.7 & 0.100 & 0.318 & 19.2 & 0.39 & 0.046 & 0.050 & -- & 0.066 & 0.423 & 16.1 & 0.425 & 15.1 & -- \\
\hline
\end{tabular}


Appendix 1. Volumetric water content, temperature, and electrical-conductivity data collected at the flood-irrigated site during 2008.-Continued

[Depth in feet below land surface; volumetric water content dimensionless; temperature in degrees Celsius; bulk electrical conductivity in decisiemens per meter; --, no data]

\begin{tabular}{|c|c|c|c|c|c|c|c|c|c|c|c|c|c|c|c|}
\hline \multirow[b]{3}{*}{$\begin{array}{l}\text { Measurement } \\
\text { date and time }\end{array}$} & \multicolumn{15}{|c|}{ Depth } \\
\hline & \multicolumn{2}{|c|}{2} & \multirow{2}{*}{$\begin{array}{c}3 \\
\begin{array}{c}\text { Volumetric } \\
\text { water } \\
\text { content }\end{array}\end{array}$} & \multicolumn{3}{|c|}{4} & \multirow{2}{*}{$\begin{array}{c}6 \\
\begin{array}{c}\text { Volumetric } \\
\text { water } \\
\text { content }\end{array}\end{array}$} & \multicolumn{2}{|l|}{8} & \multirow{2}{*}{$\begin{array}{c}10 \\
\begin{array}{c}\text { Volumetric } \\
\text { water } \\
\text { content }\end{array}\end{array}$} & \multicolumn{2}{|c|}{12} & \multicolumn{2}{|c|}{15} & \multirow{2}{*}{$\begin{array}{c}18.5 \\
\text { Volumetric } \\
\text { water } \\
\text { content }\end{array}$} \\
\hline & $\begin{array}{l}\text { Volumetric } \\
\text { water } \\
\text { content }\end{array}$ & $\begin{array}{l}\text { Temper- } \\
\text { ature }\end{array}$ & & $\begin{array}{c}\text { Volumetric } \\
\text { water } \\
\text { content }\end{array}$ & $\begin{array}{l}\text { Temper- } \\
\text { ature }\end{array}$ & $\begin{array}{c}\text { Bulk } \\
\text { electrical } \\
\text { conduct- } \\
\text { ivity } \\
\end{array}$ & & $\begin{array}{l}\text { Volumetric } \\
\text { water } \\
\text { content }\end{array}$ & $\begin{array}{l}\text { Temper- } \\
\text { ature }\end{array}$ & & $\begin{array}{l}\text { Volumetric } \\
\text { water } \\
\text { content }\end{array}$ & $\begin{array}{l}\text { Temper- } \\
\text { ature }\end{array}$ & $\begin{array}{l}\text { Volumetric } \\
\text { water } \\
\text { content }\end{array}$ & $\begin{array}{l}\text { Temper- } \\
\text { ature }\end{array}$ & \\
\hline $8 / 24 / 2008$ 7:00 AM & 0.129 & 18.7 & 0.100 & 0.318 & 19.2 & 0.39 & 0.045 & 0.050 & -- & 0.067 & 0.423 & 16.1 & 0.424 & 15.1 & -- \\
\hline 8/24/2008 8:00 AM & 0.129 & 18.8 & 0.100 & 0.318 & 19.2 & 0.39 & 0.045 & 0.050 & -- & 0.069 & 0.423 & 16.1 & 0.424 & 15.1 & -- \\
\hline 8/24/2008 9:00 AM & 0.126 & 18.8 & 0.100 & 0.318 & 19.2 & 0.39 & 0.045 & 0.050 & -- & 0.070 & 0.423 & 16.1 & 0.425 & 15.1 & -- \\
\hline 8/24/2008 10:00 AM & 0.128 & 18.8 & 0.100 & 0.318 & 19.2 & 0.39 & 0.045 & 0.050 & -- & 0.071 & 0.422 & 16.1 & 0.425 & 15.1 & -- \\
\hline 8/24/2008 11:00 AM & 0.128 & 18.8 & 0.100 & 0.318 & 19.2 & 0.39 & 0.045 & 0.050 & -- & 0.072 & 0.423 & 16.1 & 0.425 & 15.1 & -- \\
\hline 8/24/2008 12:00 PM & 0.128 & 18.8 & 0.100 & 0.318 & 19.2 & 0.39 & 0.045 & 0.050 & -- & 0.081 & 0.423 & 16.1 & 0.425 & 15.1 & -- \\
\hline 8/24/2008 1:00 PM & 0.128 & 18.8 & 0.100 & 0.318 & 19.2 & 0.39 & 0.045 & 0.050 & -- & 0.078 & 0.423 & 16.1 & 0.425 & 15.1 & -- \\
\hline 8/24/2008 2:00 PM & 0.128 & 18.8 & 0.100 & 0.318 & 19.2 & 0.39 & 0.045 & 0.050 & -- & 0.080 & 0.423 & 16.1 & 0.425 & 15.1 & -- \\
\hline 8/24/2008 3:00 PM & 0.128 & 18.8 & 0.100 & 0.318 & 19.2 & 0.39 & 0.045 & 0.050 & -- & 0.081 & 0.423 & 16.1 & 0.425 & 15.1 & -- \\
\hline 8/24/2008 4:00 PM & 0.126 & 18.8 & 0.100 & 0.318 & 19.2 & 0.39 & 0.045 & 0.050 & -- & 0.104 & 0.423 & 16.1 & 0.425 & 15.1 & -- \\
\hline 8/24/2008 5:00 PM & 0.126 & 18.8 & 0.100 & 0.318 & 19.2 & 0.39 & 0.045 & 0.050 & -- & 0.084 & 0.423 & 16.1 & 0.425 & 15.1 & -- \\
\hline 8/24/2008 6:00 PM & 0.126 & 18.8 & 0.099 & 0.318 & 19.2 & 0.39 & 0.045 & 0.050 & -- & 0.087 & 0.423 & 16.1 & 0.425 & 15.1 & -- \\
\hline 8/24/2008 7:00 PM & 0.126 & 18.8 & 0.099 & 0.318 & 19.2 & 0.39 & 0.045 & 0.050 & -- & 0.099 & 0.423 & 16.1 & 0.425 & 15.1 & -- \\
\hline 8/24/2008 8:00 PM & 0.126 & 18.8 & 0.099 & 0.317 & 19.2 & 0.39 & 0.045 & 0.049 & -- & 0.095 & 0.423 & 16.1 & 0.425 & 15.1 & -- \\
\hline 8/24/2008 9:00 PM & 0.126 & 18.7 & 0.099 & 0.317 & 19.2 & 0.39 & 0.045 & 0.049 & -- & 0.098 & 0.423 & 16.1 & 0.425 & 15.1 & -- \\
\hline 8/24/2008 10:00 PM & 0.126 & 18.8 & 0.099 & 0.317 & 19.2 & 0.39 & 0.045 & 0.049 & -- & 0.104 & 0.423 & 16.1 & 0.425 & 15.1 & -- \\
\hline 8/24/2008 11:00 PM & 0.126 & 18.8 & 0.099 & 0.317 & 19.2 & 0.39 & 0.045 & 0.049 & -- & 0.106 & 0.423 & 16.1 & 0.425 & 15.1 & -- \\
\hline 8/25/2008 12:00 AM & 0.125 & 18.8 & 0.099 & 0.317 & 19.2 & 0.39 & 0.045 & 0.049 & -- & 0.093 & 0.423 & 16.1 & 0.425 & 15.1 & -- \\
\hline 8/25/2008 1:00 AM & 0.125 & 18.8 & 0.099 & 0.317 & 19.2 & 0.39 & 0.045 & 0.049 & -- & 0.089 & 0.423 & 16.1 & 0.425 & 15.1 & -- \\
\hline 8/25/2008 2:00 AM & 0.126 & 18.8 & 0.098 & 0.317 & 19.2 & 0.39 & 0.045 & 0.049 & -- & 0.088 & 0.423 & 16.1 & 0.425 & 15.1 & -- \\
\hline 8/25/2008 3:00 AM & 0.126 & 18.8 & 0.098 & 0.317 & 19.2 & 0.39 & 0.045 & 0.049 & -- & 0.093 & 0.423 & 16.1 & 0.425 & 15.1 & -- \\
\hline 8/25/2008 4:00 AM & 0.126 & 18.9 & 0.098 & 0.317 & 19.2 & 0.39 & 0.045 & 0.049 & -- & 0.090 & 0.423 & 16.1 & 0.425 & 15.1 & -- \\
\hline 8/25/2008 5:00 AM & 0.125 & 18.9 & 0.098 & 0.317 & 19.2 & 0.39 & 0.044 & 0.049 & -- & 0.110 & 0.424 & 16.1 & 0.425 & 15.1 & -- \\
\hline 8/25/2008 6:00 AM & 0.125 & 18.9 & 0.098 & 0.317 & 19.2 & 0.39 & 0.044 & 0.049 & -- & 0.112 & 0.424 & 16.1 & 0.425 & 15.1 & -- \\
\hline 8/25/2008 7:00 AM & 0.126 & 18.9 & 0.098 & 0.317 & 19.2 & 0.39 & 0.044 & 0.049 & -- & 0.104 & 0.424 & 16.1 & 0.424 & 15.1 & -- \\
\hline 8/25/2008 8:00 AM & 0.125 & 18.9 & 0.098 & 0.317 & 19.2 & 0.39 & 0.044 & 0.049 & -- & 0.093 & 0.424 & 16.1 & 0.425 & 15.1 & -- \\
\hline 8/25/2008 9:00 AM & 0.125 & 18.9 & 0.098 & 0.317 & 19.2 & 0.39 & 0.044 & 0.049 & -- & 0.099 & 0.423 & 16.1 & 0.425 & 15.1 & -- \\
\hline 8/25/2008 10:00 AM & 0.125 & 18.9 & 0.098 & 0.317 & 19.2 & 0.39 & 0.044 & 0.049 & -- & 0.099 & 0.423 & 16.1 & 0.425 & 15.1 & -- \\
\hline 8/25/2008 11:00 AM & 0.125 & 18.9 & 0.098 & 0.317 & 19.2 & 0.39 & 0.044 & 0.049 & -- & 0.087 & 0.423 & 16.1 & 0.425 & 15.2 & -- \\
\hline 8/25/2008 12:00 PM & 0.125 & 18.8 & 0.098 & 0.317 & 19.2 & 0.39 & 0.044 & 0.049 & -- & 0.115 & 0.423 & 16.1 & 0.425 & 15.2 & -- \\
\hline 8/25/2008 1:00 PM & 0.124 & 18.8 & 0.098 & 0.317 & 19.2 & 0.39 & 0.044 & 0.049 & -- & 0.095 & 0.423 & 16.1 & 0.424 & 15.1 & -- \\
\hline 8/25/2008 2:00 PM & 0.124 & 18.8 & 0.098 & 0.317 & 19.2 & 0.39 & 0.044 & 0.049 & -- & 0.091 & 0.423 & 16.1 & 0.424 & 15.2 & -- \\
\hline 8/25/2008 3:00 PM & 0.124 & 18.8 & 0.098 & 0.317 & 19.2 & 0.39 & 0.044 & 0.049 & -- & $* * *$ & 0.423 & 16.1 & 0.424 & 15.2 & -- \\
\hline 8/25/2008 4:00 PM & 0.124 & 18.8 & 0.098 & 0.317 & 19.2 & 0.39 & 0.044 & 0.049 & -- & 0.221 & 0.423 & 16.1 & 0.424 & 15.2 & -- \\
\hline 8/25/2008 5:00 PM & 0.124 & 18.9 & 0.098 & 0.317 & 19.2 & 0.39 & 0.044 & 0.049 & -- & 0.064 & 0.423 & 16.1 & 0.423 & 15.1 & -- \\
\hline 8/25/2008 6:00 PM & 0.124 & 18.9 & 0.098 & 0.317 & 19.2 & 0.39 & 0.044 & 0.049 & -- & 0.082 & 0.423 & 16.1 & 0.423 & 15.2 & -- \\
\hline 8/25/2008 7:00 PM & 0.124 & 18.9 & 0.098 & 0.317 & 19.2 & 0.39 & 0.044 & 0.049 & -- & 0.086 & 0.423 & 16.1 & 0.423 & 15.1 & -- \\
\hline 8/25/2008 8:00 PM & 0.123 & 18.9 & 0.098 & 0.317 & 19.2 & 0.39 & 0.044 & 0.049 & -- & 0.098 & 0.423 & 16.1 & 0.423 & 15.2 & -- \\
\hline 8/25/2008 9:00 PM & 0.123 & 18.9 & 0.098 & 0.317 & 19.2 & 0.39 & 0.044 & 0.048 & -- & 0.108 & 0.423 & 16.1 & 0.424 & 15.2 & -- \\
\hline 8/25/2008 10:00 PM & 0.123 & 18.9 & 0.098 & 0.317 & 19.2 & 0.39 & 0.044 & 0.048 & -- & 0.121 & 0.423 & 16.1 & 0.423 & 15.2 & -- \\
\hline
\end{tabular}


Appendix 1. Volumetric water content, temperature, and electrical-conductivity data collected at the flood-irrigated site during 2008.-Continued

[Depth in feet below land surface; volumetric water content dimensionless; temperature in degrees Celsius; bulk electrical conductivity in decisiemens per meter; --, no data]

\begin{tabular}{|c|c|c|c|c|c|c|c|c|c|c|c|c|c|c|c|}
\hline \multirow[b]{3}{*}{$\begin{array}{l}\text { Measurement } \\
\text { date and time }\end{array}$} & \multicolumn{15}{|c|}{ Depth } \\
\hline & \multicolumn{2}{|l|}{$\begin{array}{r}2 \\
\end{array}$} & \multirow{2}{*}{ 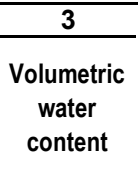 } & \multicolumn{3}{|c|}{4} & \multirow{2}{*}{$\begin{array}{c}6 \\
\begin{array}{c}\text { Volumetric } \\
\text { water } \\
\text { content }\end{array}\end{array}$} & \multicolumn{2}{|l|}{8} & \multirow{2}{*}{$\begin{array}{c}10 \\
\text { Volumetric } \\
\text { water } \\
\text { content }\end{array}$} & \multicolumn{2}{|c|}{12} & \multicolumn{2}{|c|}{15} & \multirow{2}{*}{$\begin{array}{c}18.5 \\
\text { Volumetric } \\
\text { water } \\
\text { content }\end{array}$} \\
\hline & $\begin{array}{c}\text { Volumetric } \\
\text { water } \\
\text { content }\end{array}$ & $\begin{array}{l}\text { Temper- } \\
\text { ature }\end{array}$ & & $\begin{array}{c}\text { Volumetric } \\
\text { water } \\
\text { content }\end{array}$ & $\begin{array}{l}\text { Temper- } \\
\text { ature }\end{array}$ & $\begin{array}{l}\text { Bulk } \\
\text { electrical } \\
\text { conduct- } \\
\text { ivity }\end{array}$ & & $\begin{array}{l}\text { Volumetric } \\
\text { water } \\
\text { content }\end{array}$ & $\begin{array}{l}\text { Temper- } \\
\text { ature }\end{array}$ & & $\begin{array}{c}\text { Volumetric } \\
\text { water } \\
\text { content }\end{array}$ & $\begin{array}{l}\text { Temper- } \\
\text { ature }\end{array}$ & $\begin{array}{l}\text { Volumetric } \\
\text { water } \\
\text { content }\end{array}$ & $\begin{array}{l}\text { Temper- } \\
\text { ature }\end{array}$ & \\
\hline 8/25/2008 11:00 PM & 0.124 & 18.9 & 0.098 & 0.316 & 19.2 & 0.39 & 0.044 & 0.048 & -- & 0.113 & 0.423 & 16.1 & 0.424 & 15.2 & -- \\
\hline 8/26/2008 12:00 AM & 0.124 & 18.9 & 0.098 & 0.316 & 19.2 & 0.39 & 0.044 & 0.048 & -- & 0.104 & 0.423 & 16.1 & 0.423 & 15.2 & -- \\
\hline 8/26/2008 1:00 AM & 0.123 & 18.9 & 0.098 & 0.316 & 19.2 & 0.39 & 0.044 & 0.048 & -- & 0.109 & 0.423 & 16.1 & 0.423 & 15.2 & -- \\
\hline 8/26/2008 2:00 AM & 0.123 & 18.9 & 0.098 & 0.316 & 19.2 & 0.39 & 0.044 & 0.048 & -- & 0.093 & 0.423 & 16.1 & 0.423 & 15.2 & -- \\
\hline 8/26/2008 3:00 AM & 0.123 & 18.9 & 0.098 & 0.316 & 19.3 & 0.39 & 0.044 & 0.048 & -- & 0.104 & 0.423 & 16.1 & 0.423 & 15.2 & -- \\
\hline 8/26/2008 4:00 AM & 0.123 & 18.9 & 0.098 & 0.316 & 19.2 & 0.39 & 0.044 & 0.048 & -- & 0.073 & 0.423 & 16.2 & 0.423 & 15.2 & -- \\
\hline 8/26/2008 5:00 AM & 0.123 & 18.9 & 0.098 & 0.316 & 19.3 & 0.39 & 0.043 & 0.048 & -- & 0.081 & 0.423 & 16.1 & 0.423 & 15.2 & -- \\
\hline 8/26/2008 6:00 AM & 0.123 & 18.9 & 0.098 & 0.316 & 19.3 & 0.39 & 0.043 & 0.048 & -- & 0.093 & 0.424 & 16.1 & 0.423 & 15.2 & -- \\
\hline 8/26/2008 7:00 AM & 0.123 & 18.9 & 0.098 & 0.316 & 19.3 & 0.39 & 0.043 & 0.048 & -- & 0.093 & 0.424 & 16.1 & 0.423 & 15.2 & -- \\
\hline 8/26/2008 8:00 AM & 0.123 & 18.9 & 0.098 & 0.316 & 19.3 & 0.39 & 0.043 & 0.048 & -- & 0.096 & 0.424 & 16.2 & 0.423 & 15.2 & -- \\
\hline 8/26/2008 9:00 AM & 0.123 & 18.9 & 0.098 & 0.316 & 19.3 & 0.39 & 0.043 & 0.048 & -- & 0.089 & 0.423 & 16.1 & 0.423 & 15.2 & -- \\
\hline 8/26/2008 10:00 AM & 0.123 & 19.0 & 0.098 & 0.316 & 19.3 & 0.39 & 0.043 & 0.048 & -- & 0.127 & 0.422 & 16.2 & 0.424 & 15.2 & -- \\
\hline 8/26/2008 11:00 AM & 0.142 & 19.0 & 0.098 & 0.316 & 19.3 & 0.39 & 0.043 & 0.048 & -- & 0.182 & 0.423 & 16.1 & 0.425 & 15.2 & -- \\
\hline 8/26/2008 12:00 PM & 0.190 & 19.0 & 0.098 & 0.316 & 19.3 & 0.39 & 0.043 & 0.048 & -- & 0.177 & 0.423 & 16.1 & 0.424 & 15.2 & -- \\
\hline 8/26/2008 1:00 PM & 0.206 & 19.0 & 0.099 & 0.315 & 19.3 & 0.39 & 0.043 & 0.048 & -- & 0.200 & 0.422 & 16.1 & 0.429 & 15.2 & -- \\
\hline 8/26/2008 2:00 PM & 0.207 & 19.0 & 0.106 & 0.315 & 19.3 & 0.39 & 0.043 & 0.048 & -- & 0.100 & 0.423 & 16.1 & 0.430 & 15.2 & -- \\
\hline 8/26/2008 3:00 PM & 0.201 & 18.9 & 0.112 & 0.315 & 19.3 & 0.38 & 0.043 & 0.048 & -- & 0.139 & 0.422 & 16.1 & 0.431 & 15.2 & -- \\
\hline 8/26/2008 4:00 PM & 0.195 & 18.9 & 0.119 & 0.316 & 19.3 & 0.38 & 0.043 & 0.048 & -- & 0.062 & 0.422 & 16.1 & 0.432 & 15.2 & -- \\
\hline 8/26/2008 5:00 PM & 0.191 & 18.9 & 0.124 & 0.316 & 19.3 & 0.39 & 0.043 & 0.048 & -- & 0.266 & 0.423 & 16.1 & 0.431 & 15.2 & -- \\
\hline 8/26/2008 6:00 PM & 0.188 & 18.9 & 0.125 & 0.317 & 19.3 & 0.39 & 0.043 & 0.048 & -- & 0.284 & 0.422 & 16.1 & 0.432 & 15.1 & -- \\
\hline 8/26/2008 7:00 PM & 0.187 & 18.9 & 0.125 & 0.319 & 19.3 & 0.39 & 0.043 & 0.048 & -- & -- & 0.422 & 16.1 & 0.432 & 15.2 & -- \\
\hline 8/26/2008 8:00 PM & 0.184 & 18.9 & 0.124 & 0.321 & 19.3 & 0.39 & 0.043 & 0.048 & -- & -- & 0.422 & 16.2 & 0.431 & 15.2 & -- \\
\hline 8/26/2008 9:00 PM & 0.181 & 18.9 & 0.124 & 0.323 & 19.3 & 0.40 & 0.043 & 0.047 & -- & -- & 0.423 & 16.2 & 0.430 & 15.2 & -- \\
\hline 8/26/2008 10:00 PM & 0.179 & 18.9 & 0.123 & 0.325 & 19.3 & 0.40 & 0.043 & 0.047 & -- & -- & 0.423 & 16.2 & 0.431 & 15.2 & -- \\
\hline 8/26/2008 11:00 PM & 0.179 & 18.9 & 0.122 & 0.326 & 19.3 & 0.40 & 0.043 & 0.047 & -- & -- & 0.423 & 16.2 & 0.434 & 15.2 & -- \\
\hline 8/27/2008 12:00 AM & 0.176 & 18.9 & 0.122 & 0.326 & 19.3 & 0.40 & 0.043 & 0.047 & -- & -- & 0.423 & 16.2 & 0.435 & 15.2 & -- \\
\hline 8/27/2008 1:00 AM & 0.175 & 19.0 & 0.121 & 0.326 & 19.3 & 0.40 & 0.043 & 0.047 & -- & -- & 0.423 & 16.2 & 0.435 & 15.2 & -- \\
\hline 8/27/2008 2:00 AM & 0.173 & 19.0 & 0.121 & 0.328 & 19.3 & 0.40 & 0.043 & 0.047 & -- & -- & 0.423 & 16.2 & 0.437 & 15.2 & -- \\
\hline 8/27/2008 3:00 AM & 0.172 & 19.0 & 0.121 & 0.328 & 19.3 & 0.40 & 0.043 & 0.047 & -- & -- & 0.423 & 16.2 & 0.434 & 15.2 & -- \\
\hline 8/27/2008 4:00 AM & 0.170 & 19.1 & 0.120 & 0.328 & 19.3 & 0.40 & 0.043 & 0.047 & -- & -- & 0.423 & 16.2 & 0.432 & 15.1 & -- \\
\hline 8/27/2008 5:00 AM & 0.169 & 19.1 & 0.119 & 0.328 & 19.3 & 0.40 & 0.043 & 0.047 & -- & -- & 0.422 & 16.2 & 0.436 & 15.2 & -- \\
\hline 8/27/2008 6:00 AM & 0.169 & 19.1 & 0.119 & 0.326 & 19.3 & 0.40 & 0.043 & 0.047 & -- & -- & 0.422 & 16.2 & 0.434 & 15.2 & -- \\
\hline 8/27/2008 7:00 AM & 0.167 & 19.1 & 0.118 & 0.326 & 19.3 & 0.40 & 0.043 & 0.047 & -- & -- & 0.422 & 16.2 & 0.433 & 15.2 & -- \\
\hline 8/27/2008 8:00 AM & 0.167 & 19.2 & 0.118 & 0.326 & 19.3 & 0.40 & 0.043 & 0.047 & -- & -- & 0.422 & 16.2 & 0.430 & 15.2 & -- \\
\hline 8/27/2008 9:00 AM & 0.165 & 19.2 & 0.118 & 0.326 & 19.3 & 0.40 & 0.043 & 0.047 & -- & -- & 0.422 & 16.2 & 0.433 & 15.2 & -- \\
\hline 8/27/2008 10:00 AM & 0.163 & 19.2 & 0.117 & 0.326 & 19.3 & 0.40 & 0.043 & 0.047 & -- & -- & 0.423 & 16.2 & 0.431 & 15.2 & -- \\
\hline 8/27/2008 11:00 AM & 0.162 & 19.3 & 0.117 & 0.328 & 19.3 & 0.39 & 0.043 & 0.047 & -- & -- & 0.423 & 16.2 & 0.433 & 15.2 & -- \\
\hline 8/27/2008 12:00 PM & 0.161 & 19.3 & 0.117 & 0.326 & 19.3 & 0.39 & 0.043 & 0.047 & -- & -- & 0.423 & 16.2 & 0.433 & 15.2 & -- \\
\hline 8/27/2008 1:00 PM & 0.160 & 19.2 & 0.116 & 0.326 & 19.3 & 0.39 & 0.043 & 0.047 & -- & -- & 0.423 & 16.2 & 0.432 & 15.2 & -- \\
\hline 8/27/2008 2:00 PM & 0.159 & 19.2 & 0.116 & 0.326 & 19.3 & 0.39 & 0.043 & 0.047 & -- & -- & 0.423 & 16.2 & 0.433 & 15.2 & -- \\
\hline
\end{tabular}


Appendix 1. Volumetric water content, temperature, and electrical-conductivity data collected at the flood-irrigated site during 2008.-Continued

[Depth in feet below land surface; volumetric water content dimensionless; temperature in degrees Celsius; bulk electrical conductivity in decisiemens per meter; --, no data]

\begin{tabular}{|c|c|c|c|c|c|c|c|c|c|c|c|c|c|c|c|}
\hline \multirow[b]{3}{*}{$\begin{array}{l}\text { Measurement } \\
\text { date and time }\end{array}$} & \multicolumn{15}{|c|}{ Depth } \\
\hline & \multicolumn{2}{|c|}{2} & \multirow[b]{2}{*}{$\begin{array}{c}3 \\
\begin{array}{c}\text { Volumetric } \\
\text { water } \\
\text { content }\end{array}\end{array}$} & \multicolumn{3}{|c|}{4} & \multirow{2}{*}{$\begin{array}{c}6 \\
\begin{array}{c}\text { Volumetric } \\
\text { water } \\
\text { content }\end{array}\end{array}$} & \multicolumn{2}{|c|}{8} & \multirow{2}{*}{$\begin{array}{c}10 \\
\text { Volumetric } \\
\text { water } \\
\text { content }\end{array}$} & \multicolumn{2}{|c|}{12} & \multicolumn{2}{|c|}{15} & \multirow{2}{*}{$\begin{array}{c}18.5 \\
\text { Volumetric } \\
\text { water } \\
\text { content }\end{array}$} \\
\hline & $\begin{array}{c}\text { Volumetric } \\
\text { water } \\
\text { content }\end{array}$ & $\begin{array}{l}\text { Temper- } \\
\text { ature }\end{array}$ & & $\begin{array}{c}\text { Volumetric } \\
\text { water } \\
\text { content }\end{array}$ & $\begin{array}{l}\text { Temper- } \\
\text { ature }\end{array}$ & $\begin{array}{c}\text { Bulk } \\
\text { electrical } \\
\text { conduct- } \\
\text { ivity }\end{array}$ & & $\begin{array}{c}\text { Volumetric } \\
\text { water } \\
\text { content }\end{array}$ & $\begin{array}{l}\text { Temper- } \\
\text { ature }\end{array}$ & & $\begin{array}{c}\text { Volumetric } \\
\text { water } \\
\text { content }\end{array}$ & $\begin{array}{l}\text { Temper- } \\
\text { ature }\end{array}$ & $\begin{array}{c}\text { Volumetric } \\
\text { water } \\
\text { content }\end{array}$ & $\begin{array}{l}\text { Temper- } \\
\text { ature }\end{array}$ & \\
\hline 8/27/2008 3:00 PM & 0.159 & 19.2 & 0.115 & 0.326 & 19.3 & 0.39 & 0.043 & 0.047 & -- & -- & 0.423 & 16.2 & 0.434 & 15.2 & $\overline{--}$ \\
\hline 8/27/2008 4:00 PM & 0.158 & 19.2 & 0.115 & 0.326 & 19.3 & 0.39 & 0.044 & 0.047 & -- & -- & 0.422 & 16.2 & 0.437 & 15.2 & -- \\
\hline 8/27/2008 5:00 PM & 0.157 & 19.2 & 0.115 & 0.326 & 19.3 & 0.39 & 0.044 & 0.047 & -- & -- & 0.422 & 16.2 & 0.435 & 15.1 & -- \\
\hline 8/27/2008 6:00 PM & 0.157 & 19.2 & 0.115 & 0.326 & 19.3 & 0.39 & 0.044 & 0.047 & -- & -- & 0.422 & 16.2 & 0.434 & 15.2 & -- \\
\hline 8/27/2008 7:00 PM & 0.156 & 19.2 & 0.115 & 0.326 & 19.3 & 0.39 & 0.044 & 0.047 & -- & -- & 0.423 & 16.2 & 0.432 & 15.2 & -- \\
\hline 8/27/2008 8:00 PM & 0.155 & 19.2 & 0.115 & 0.326 & 19.3 & 0.39 & 0.044 & 0.047 & -- & -- & 0.423 & 16.2 & 0.431 & 15.2 & -- \\
\hline 8/27/2008 9:00 PM & 0.155 & 19.1 & 0.114 & 0.326 & 19.3 & 0.39 & 0.044 & 0.047 & -- & -- & 0.423 & 16.2 & 0.433 & 15.2 & -- \\
\hline 8/27/2008 10:00 PM & 0.154 & 19.2 & 0.114 & 0.325 & 19.3 & 0.39 & 0.044 & 0.047 & -- & -- & 0.422 & 16.2 & 0.432 & 15.2 & -- \\
\hline 8/27/2008 11:00 PM & 0.154 & 19.1 & 0.114 & 0.325 & 19.3 & 0.39 & 0.044 & 0.047 & -- & -- & 0.422 & 16.2 & 0.438 & 15.2 & -- \\
\hline 8/28/2008 12:00 AM & 0.153 & 19.1 & 0.113 & 0.325 & 19.3 & 0.39 & 0.044 & 0.047 & -- & -- & 0.422 & 16.2 & 0.431 & 15.2 & -- \\
\hline 8/28/2008 1:00 AM & 0.153 & 19.2 & 0.113 & 0.325 & 19.3 & 0.39 & 0.044 & 0.047 & -- & -- & 0.422 & 16.2 & 0.432 & 15.2 & -- \\
\hline 8/28/2008 2:00 AM & 0.153 & 19.2 & 0.113 & 0.325 & 19.3 & 0.39 & 0.044 & 0.047 & -- & -- & 0.422 & 16.2 & 0.435 & 15.2 & -- \\
\hline 8/28/2008 3:00 AM & 0.151 & 19.1 & 0.113 & 0.325 & 19.3 & 0.39 & 0.045 & 0.047 & -- & -- & 0.422 & 16.2 & 0.437 & 15.2 & -- \\
\hline 8/28/2008 4:00 AM & 0.150 & 19.1 & 0.113 & 0.325 & 19.3 & 0.39 & 0.045 & 0.047 & -- & -- & 0.423 & 16.2 & 0.434 & 15.2 & -- \\
\hline 8/28/2008 5:00 AM & 0.150 & 19.1 & 0.112 & 0.324 & 19.3 & 0.39 & 0.045 & 0.047 & -- & -- & 0.423 & 16.2 & 0.434 & 15.2 & -- \\
\hline 8/28/2008 6:00 AM & 0.150 & 19.2 & 0.112 & 0.324 & 19.3 & 0.39 & 0.045 & 0.047 & -- & -- & 0.423 & 16.2 & 0.429 & 15.2 & -- \\
\hline 8/28/2008 7:00 AM & 0.149 & 19.1 & 0.112 & 0.325 & 19.3 & 0.39 & 0.045 & 0.047 & -- & -- & 0.423 & 16.2 & 0.429 & 15.2 & -- \\
\hline 8/28/2008 8:00 AM & 0.149 & 19.1 & 0.111 & 0.324 & 19.4 & 0.39 & 0.045 & 0.047 & -- & -- & 0.423 & 16.2 & 0.428 & 15.2 & -- \\
\hline 8/28/2008 9:00 AM & 0.149 & 19.1 & 0.111 & 0.324 & 19.4 & 0.39 & 0.045 & 0.047 & -- & 0.330 & 0.423 & 16.2 & 0.429 & 15.2 & -- \\
\hline 8/28/2008 10:00 AM & 0.148 & 19.2 & 0.111 & 0.324 & 19.4 & 0.39 & 0.045 & 0.047 & -- & 0.166 & 0.423 & 16.1 & 0.430 & 15.2 & -- \\
\hline 8/28/2008 11:00 AM & 0.148 & 19.2 & 0.111 & 0.324 & 19.4 & 0.39 & 0.045 & 0.047 & -- & 0.169 & 0.423 & 16.1 & 0.430 & 15.2 & -- \\
\hline 8/28/2008 12:00 PM & 0.147 & 19.2 & 0.111 & 0.324 & 19.4 & 0.39 & 0.044 & 0.048 & -- & 0.188 & 0.423 & 16.1 & 0.429 & 15.2 & -- \\
\hline 8/28/2008 1:00 PM & 0.147 & 19.1 & 0.111 & 0.324 & 19.4 & 0.39 & 0.044 & 0.048 & -- & 0.178 & 0.423 & 16.1 & 0.430 & 15.2 & -- \\
\hline 8/28/2008 2:00 PM & 0.147 & 19.2 & 0.111 & 0.324 & 19.4 & 0.39 & 0.045 & 0.048 & -- & 0.166 & 0.423 & 16.1 & 0.429 & 15.2 & -- \\
\hline 8/28/2008 3:00 PM & 0.146 & 19.1 & 0.111 & 0.324 & 19.4 & 0.39 & 0.045 & 0.048 & -- & 0.236 & 0.423 & 16.2 & 0.429 & 15.2 & -- \\
\hline 8/28/2008 4:00 PM & 0.146 & 19.0 & 0.111 & 0.324 & 19.4 & 0.39 & 0.045 & 0.048 & -- & 0.256 & 0.423 & 16.2 & 0.430 & 15.2 & -- \\
\hline 8/28/2008 5:00 PM & 0.146 & 19.0 & 0.110 & 0.324 & 19.4 & 0.39 & 0.045 & 0.048 & -- & 0.188 & 0.423 & 16.2 & 0.429 & 15.2 & -- \\
\hline 8/28/2008 6:00 PM & 0.145 & 19.0 & 0.110 & 0.324 & 19.4 & 0.39 & 0.045 & 0.048 & -- & 0.179 & 0.423 & 16.1 & 0.428 & 15.2 & -- \\
\hline 8/28/2008 7:00 PM & 0.144 & 18.9 & 0.110 & 0.324 & 19.4 & 0.39 & 0.045 & 0.048 & -- & 0.183 & 0.423 & 16.2 & 0.428 & 15.2 & -- \\
\hline 8/28/2008 8:00 PM & 0.144 & 19.0 & 0.109 & 0.324 & 19.4 & 0.39 & 0.045 & 0.047 & -- & 0.104 & 0.423 & 16.2 & 0.429 & 15.2 & -- \\
\hline 8/28/2008 9:00 PM & 0.144 & 19.0 & 0.109 & 0.323 & 19.4 & 0.39 & 0.045 & 0.047 & -- & 0.061 & 0.423 & 16.2 & 0.429 & 15.2 & -- \\
\hline 8/28/2008 10:00 PM & 0.143 & 19.0 & 0.109 & 0.323 & 19.4 & 0.39 & 0.045 & 0.047 & -- & 0.054 & 0.423 & 16.2 & 0.429 & 15.2 & -- \\
\hline 8/28/2008 11:00 PM & 0.143 & 19.0 & 0.109 & 0.323 & 19.4 & 0.39 & 0.045 & 0.047 & -- & 0.053 & 0.423 & 16.2 & 0.429 & 15.2 & -- \\
\hline 8/29/2008 12:00 AM & 0.143 & 19.0 & 0.109 & 0.323 & 19.4 & 0.39 & 0.045 & 0.047 & -- & 0.053 & 0.423 & 16.2 & 0.429 & 15.2 & -- \\
\hline 8/29/2008 1:00 AM & 0.142 & 18.9 & 0.109 & 0.323 & 19.4 & 0.39 & 0.045 & 0.047 & -- & 0.053 & 0.423 & 16.2 & 0.429 & 15.3 & -- \\
\hline 8/29/2008 2:00 AM & 0.142 & 19.0 & 0.109 & 0.323 & 19.4 & 0.39 & 0.045 & 0.047 & -- & 0.053 & 0.423 & 16.2 & 0.430 & 15.2 & -- \\
\hline 8/29/2008 3:00 AM & 0.142 & 19.0 & 0.109 & 0.323 & 19.4 & 0.39 & 0.045 & 0.047 & -- & 0.054 & 0.423 & 16.2 & 0.429 & 15.2 & -- \\
\hline 8/29/2008 4:00 AM & 0.142 & 19.0 & 0.109 & 0.323 & 19.4 & 0.39 & 0.045 & 0.047 & -- & 0.054 & 0.423 & 16.2 & 0.429 & 15.3 & -- \\
\hline 8/29/2008 5:00 AM & 0.142 & 19.0 & 0.109 & 0.323 & 19.4 & 0.39 & 0.045 & 0.047 & -- & 0.054 & 0.423 & 16.2 & 0.429 & 15.3 & -- \\
\hline 8/29/2008 6:00 AM & 0.141 & 19.0 & 0.108 & 0.322 & 19.4 & 0.39 & 0.045 & 0.047 & -- & 0.054 & 0.424 & 16.2 & 0.429 & 15.2 & -- \\
\hline
\end{tabular}


Appendix 1. Volumetric water content, temperature, and electrical-conductivity data collected at the flood-irrigated site during 2008.-Continued

[Depth in feet below land surface; volumetric water content dimensionless; temperature in degrees Celsius; bulk electrical conductivity in decisiemens per meter; --, no data]

\begin{tabular}{|c|c|c|c|c|c|c|c|c|c|c|c|c|c|c|c|}
\hline \multirow[b]{3}{*}{$\begin{array}{l}\text { Measurement } \\
\text { date and time }\end{array}$} & \multicolumn{15}{|c|}{ Depth } \\
\hline & \multirow{2}{*}{$\begin{array}{c}2 \\
\begin{array}{c}\text { Volumetric } \\
\text { water } \\
\text { content }\end{array}\end{array}$} & & \multirow{2}{*}{ 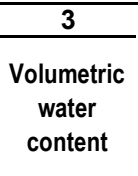 } & \multicolumn{3}{|c|}{4} & \multirow{2}{*}{$\begin{array}{c}6 \\
\begin{array}{c}\text { Volumetric } \\
\text { water } \\
\text { content }\end{array}\end{array}$} & \multicolumn{2}{|l|}{8} & \multirow{2}{*}{$\begin{array}{c}10 \\
\text { Volumetric } \\
\text { water } \\
\text { content }\end{array}$} & \multicolumn{2}{|c|}{12} & \multicolumn{2}{|c|}{15} & \multirow{2}{*}{$\begin{array}{c}18.5 \\
\text { Volumetric } \\
\text { water } \\
\text { content }\end{array}$} \\
\hline & & $\begin{array}{l}\text { Temper- } \\
\text { ature }\end{array}$ & & $\begin{array}{c}\text { Volumetric } \\
\text { water } \\
\text { content }\end{array}$ & $\begin{array}{l}\text { Temper- } \\
\text { ature }\end{array}$ & $\begin{array}{l}\text { Bulk } \\
\text { electrical } \\
\text { conduct- } \\
\text { ivity }\end{array}$ & & $\begin{array}{l}\text { Volumetric } \\
\text { water } \\
\text { content }\end{array}$ & $\begin{array}{l}\text { Temper- } \\
\text { ature }\end{array}$ & & $\begin{array}{c}\text { Volumetric } \\
\text { water } \\
\text { content }\end{array}$ & $\begin{array}{l}\text { Temper- } \\
\text { ature }\end{array}$ & $\begin{array}{l}\text { Volumetric } \\
\text { water } \\
\text { content }\end{array}$ & $\begin{array}{l}\text { Temper- } \\
\text { ature }\end{array}$ & \\
\hline $8 / 29 / 2008$ 7:00 AM & 0.141 & 18.9 & 0.108 & 0.322 & 19.4 & 0.39 & 0.045 & 0.047 & -- & 0.054 & 0.424 & 16.2 & 0.429 & 15.2 & -- \\
\hline 8/29/2008 8:00 AM & 0.141 & 18.9 & 0.108 & 0.323 & 19.4 & 0.39 & 0.045 & 0.047 & -- & 0.054 & 0.424 & 16.2 & 0.429 & 15.2 & -- \\
\hline 8/29/2008 9:00 AM & 0.140 & 18.9 & 0.108 & 0.322 & 19.4 & 0.39 & 0.045 & 0.047 & -- & 0.054 & 0.423 & 16.2 & 0.429 & 15.2 & -- \\
\hline 8/29/2008 10:00 AM & 0.140 & 18.9 & 0.108 & 0.323 & 19.4 & 0.39 & 0.045 & 0.047 & -- & 0.054 & 0.423 & 16.2 & 0.429 & 15.2 & -- \\
\hline 8/29/2008 11:00 AM & 0.140 & 19.0 & 0.108 & 0.323 & 19.4 & 0.39 & 0.044 & 0.048 & -- & 0.055 & 0.423 & 16.2 & 0.429 & 15.3 & -- \\
\hline 8/29/2008 12:00 PM & 0.140 & 18.9 & 0.108 & 0.323 & 19.4 & 0.39 & 0.044 & 0.048 & -- & 0.054 & 0.423 & 16.2 & 0.429 & 15.3 & -- \\
\hline 8/29/2008 1:00 PM & 0.138 & 18.9 & 0.108 & 0.323 & 19.4 & 0.39 & 0.044 & 0.048 & -- & 0.054 & 0.423 & 16.1 & 0.429 & 15.3 & -- \\
\hline 8/29/2008 2:00 PM & 0.138 & 18.8 & 0.107 & 0.322 & 19.4 & 0.39 & 0.044 & 0.048 & -- & 0.054 & 0.423 & 16.2 & 0.429 & 15.2 & -- \\
\hline 8/29/2008 3:00 PM & 0.138 & 18.8 & 0.107 & 0.322 & 19.4 & 0.39 & 0.044 & 0.048 & -- & 0.055 & 0.423 & 16.2 & 0.428 & 15.2 & -- \\
\hline 8/29/2008 4:00 PM & 0.137 & 18.8 & 0.107 & 0.322 & 19.4 & 0.39 & 0.044 & 0.048 & -- & 0.054 & 0.423 & 16.2 & 0.428 & 15.2 & -- \\
\hline 8/29/2008 5:00 PM & 0.137 & 18.8 & 0.107 & 0.322 & 19.4 & 0.39 & 0.044 & 0.048 & -- & 0.053 & 0.423 & 16.2 & 0.429 & 15.2 & -- \\
\hline 8/29/2008 6:00 PM & 0.137 & 18.8 & 0.107 & 0.322 & 19.4 & 0.39 & 0.044 & 0.048 & -- & 0.052 & 0.422 & 16.2 & 0.429 & 15.3 & -- \\
\hline 8/29/2008 7:00 PM & 0.137 & 18.8 & 0.107 & 0.322 & 19.4 & 0.39 & 0.044 & 0.048 & -- & 0.053 & 0.423 & 16.2 & 0.429 & 15.3 & -- \\
\hline 8/29/2008 8:00 PM & 0.136 & 18.7 & 0.107 & 0.322 & 19.4 & 0.39 & 0.044 & 0.048 & -- & 0.053 & 0.423 & 16.1 & 0.429 & 15.2 & -- \\
\hline 8/29/2008 9:00 PM & 0.136 & 18.7 & 0.106 & 0.322 & 19.4 & 0.39 & 0.045 & 0.048 & -- & 0.053 & 0.422 & 16.1 & 0.429 & 15.3 & -- \\
\hline 8/29/2008 10:00 PM & 0.136 & 18.7 & 0.106 & 0.322 & 19.4 & 0.39 & 0.045 & 0.048 & -- & 0.053 & 0.422 & 16.2 & 0.428 & 15.3 & -- \\
\hline 8/29/2008 11:00 PM & 0.136 & 18.7 & 0.106 & 0.322 & 19.4 & 0.39 & 0.044 & 0.048 & -- & 0.053 & 0.422 & 16.2 & 0.429 & 15.3 & -- \\
\hline 8/30/2008 12:00 AM & 0.136 & 18.7 & 0.106 & 0.322 & 19.4 & 0.39 & 0.044 & 0.048 & -- & 0.053 & 0.423 & 16.2 & 0.429 & 15.3 & -- \\
\hline 8/30/2008 1:00 AM & 0.136 & 18.7 & 0.106 & 0.321 & 19.3 & 0.39 & 0.044 & 0.048 & -- & 0.053 & 0.422 & 16.2 & 0.429 & 15.3 & -- \\
\hline 8/30/2008 2:00 AM & 0.136 & 18.7 & 0.105 & 0.321 & 19.3 & 0.39 & 0.044 & 0.048 & -- & 0.053 & 0.422 & 16.2 & 0.429 & 15.3 & -- \\
\hline 8/30/2008 3:00 AM & 0.135 & 18.7 & 0.105 & 0.321 & 19.3 & 0.39 & 0.044 & 0.048 & -- & 0.053 & 0.423 & 16.2 & 0.429 & 15.3 & -- \\
\hline 8/30/2008 4:00 AM & 0.134 & 18.7 & 0.105 & 0.321 & 19.3 & 0.39 & 0.044 & 0.048 & -- & 0.052 & 0.422 & 16.2 & 0.430 & 15.3 & -- \\
\hline 8/30/2008 5:00 AM & 0.134 & 18.7 & 0.105 & 0.321 & 19.3 & 0.39 & 0.044 & 0.048 & -- & 0.052 & 0.423 & 16.2 & 0.430 & 15.3 & -- \\
\hline 8/30/2008 6:00 AM & 0.134 & 18.8 & 0.105 & 0.321 & 19.3 & 0.39 & 0.044 & 0.048 & -- & 0.052 & 0.422 & 16.2 & 0.430 & 15.3 & -- \\
\hline 8/30/2008 7:00 AM & 0.134 & 18.8 & 0.105 & 0.321 & 19.3 & 0.39 & 0.044 & 0.048 & -- & 0.052 & 0.423 & 16.2 & 0.429 & 15.3 & -- \\
\hline 8/30/2008 8:00 AM & 0.133 & 18.8 & 0.105 & 0.321 & 19.3 & 0.39 & 0.044 & 0.048 & -- & 0.052 & 0.423 & 16.2 & 0.430 & 15.3 & -- \\
\hline 8/30/2008 9:00 AM & 0.134 & 18.8 & 0.105 & 0.321 & 19.3 & 0.39 & 0.044 & 0.048 & -- & 0.052 & 0.422 & 16.2 & 0.429 & 15.3 & -- \\
\hline 8/30/2008 10:00 AM & 0.135 & 18.8 & 0.105 & 0.321 & 19.3 & 0.39 & 0.044 & 0.048 & -- & 0.051 & 0.422 & 16.2 & 0.430 & 15.3 & -- \\
\hline 8/30/2008 11:00 AM & 0.134 & 18.8 & 0.105 & 0.321 & 19.3 & 0.39 & 0.044 & 0.048 & -- & 0.051 & 0.423 & 16.2 & 0.430 & 15.3 & -- \\
\hline 8/30/2008 12:00 PM & 0.133 & 18.8 & 0.104 & 0.321 & 19.3 & 0.39 & 0.044 & 0.048 & -- & 0.051 & 0.422 & 16.2 & 0.430 & 15.3 & -- \\
\hline 8/30/2008 1:00 PM & 0.133 & 18.8 & 0.104 & 0.321 & 19.3 & 0.39 & 0.044 & 0.048 & -- & 0.051 & 0.422 & 16.2 & 0.430 & 15.3 & -- \\
\hline 8/30/2008 2:00 PM & 0.134 & 18.8 & 0.104 & 0.321 & 19.3 & 0.39 & 0.044 & 0.048 & -- & 0.051 & 0.423 & 16.2 & 0.430 & 15.3 & -- \\
\hline 8/30/2008 3:00 PM & 0.134 & 18.8 & 0.104 & 0.321 & 19.3 & 0.39 & 0.044 & 0.048 & -- & 0.051 & 0.422 & 16.2 & 0.430 & 15.3 & -- \\
\hline 8/30/2008 4:00 PM & 0.133 & 18.8 & 0.104 & 0.321 & 19.3 & 0.39 & 0.044 & 0.048 & -- & 0.051 & 0.422 & 16.2 & 0.430 & 15.3 & -- \\
\hline 8/30/2008 5:00 PM & 0.133 & 18.8 & 0.104 & 0.320 & 19.3 & 0.39 & 0.044 & 0.048 & -- & 0.051 & 0.422 & 16.2 & 0.430 & 15.3 & -- \\
\hline 8/30/2008 6:00 PM & 0.132 & 18.8 & 0.104 & 0.320 & 19.3 & 0.39 & 0.044 & 0.048 & -- & 0.051 & 0.422 & 16.2 & 0.430 & 15.3 & -- \\
\hline 8/30/2008 7:00 PM & 0.132 & 18.8 & 0.104 & 0.320 & 19.3 & 0.39 & 0.044 & 0.048 & -- & 0.052 & 0.422 & 16.2 & 0.430 & 15.3 & -- \\
\hline 8/30/2008 8:00 PM & 0.132 & 18.8 & 0.104 & 0.320 & 19.3 & 0.39 & 0.044 & 0.048 & -- & 0.052 & 0.423 & 16.2 & 0.430 & 15.3 & -- \\
\hline 8/30/2008 9:00 PM & 0.132 & 18.8 & 0.104 & 0.320 & 19.3 & 0.39 & 0.044 & 0.048 & -- & 0.052 & 0.422 & 16.2 & 0.430 & 15.3 & -- \\
\hline 8/30/2008 10:00 PM & 0.133 & 18.8 & 0.104 & 0.320 & 19.3 & 0.39 & 0.044 & 0.048 & -- & 0.052 & 0.422 & 16.2 & 0.430 & 15.3 & -- \\
\hline
\end{tabular}


Appendix 1. Volumetric water content, temperature, and electrical-conductivity data collected at the flood-irrigated site during 2008.-Continued

[Depth in feet below land surface; volumetric water content dimensionless; temperature in degrees Celsius; bulk electrical conductivity in decisiemens per meter; --, no data]

\begin{tabular}{|c|c|c|c|c|c|c|c|c|c|c|c|c|c|c|c|}
\hline \multirow[b]{3}{*}{$\begin{array}{l}\text { Measurement } \\
\text { date and time }\end{array}$} & \multicolumn{15}{|c|}{ Depth } \\
\hline & \multicolumn{2}{|l|}{2} & \multirow{2}{*}{$\begin{array}{c}3 \\
\begin{array}{c}\text { Volumetric } \\
\text { water } \\
\text { content }\end{array}\end{array}$} & \multicolumn{3}{|c|}{4} & \multirow{2}{*}{$\begin{array}{c}6 \\
\begin{array}{c}\text { Volumetric } \\
\text { water } \\
\text { content }\end{array}\end{array}$} & \multicolumn{2}{|l|}{8} & \multirow{2}{*}{$\begin{array}{c}10 \\
\begin{array}{c}\text { Volumetric } \\
\text { water } \\
\text { content }\end{array}\end{array}$} & \multicolumn{2}{|c|}{12} & \multicolumn{2}{|c|}{15} & \multirow{2}{*}{$\begin{array}{c}18.5 \\
\text { Volumetric } \\
\text { water } \\
\text { content }\end{array}$} \\
\hline & $\begin{array}{l}\text { Volumetric } \\
\text { water } \\
\text { content }\end{array}$ & $\begin{array}{l}\text { Temper- } \\
\text { ature }\end{array}$ & & $\begin{array}{c}\text { Volumetric } \\
\text { water } \\
\text { content }\end{array}$ & $\begin{array}{l}\text { Temper- } \\
\text { ature }\end{array}$ & $\begin{array}{c}\text { Bulk } \\
\text { electrical } \\
\text { conduct- } \\
\text { ivity } \\
\end{array}$ & & $\begin{array}{l}\text { Volumetric } \\
\text { water } \\
\text { content }\end{array}$ & $\begin{array}{l}\text { Temper- } \\
\text { ature }\end{array}$ & & $\begin{array}{l}\text { Volumetric } \\
\text { water } \\
\text { content }\end{array}$ & $\begin{array}{l}\text { Temper- } \\
\text { ature }\end{array}$ & $\begin{array}{l}\text { Volumetric } \\
\text { water } \\
\text { content }\end{array}$ & $\begin{array}{l}\text { Temper- } \\
\text { ature }\end{array}$ & \\
\hline 8/30/2008 11:00 PM & 0.132 & 18.8 & 0.104 & 0.320 & 19.3 & 0.39 & 0.044 & 0.048 & -- & 0.052 & 0.422 & 16.2 & 0.429 & 15.3 & -- \\
\hline 8/31/2008 12:00 AM & 0.131 & 18.8 & 0.104 & 0.320 & 19.3 & 0.39 & 0.044 & 0.048 & -- & 0.052 & 0.422 & 16.2 & 0.429 & 15.3 & -- \\
\hline 8/31/2008 1:00 AM & 0.131 & 18.8 & 0.103 & 0.319 & 19.3 & 0.39 & 0.044 & 0.048 & -- & 0.052 & 0.422 & 16.2 & 0.428 & 15.3 & -- \\
\hline 8/31/2008 2:00 AM & 0.132 & 18.9 & 0.103 & 0.320 & 19.3 & 0.39 & 0.044 & 0.048 & -- & 0.052 & 0.422 & 16.2 & 0.430 & 15.3 & -- \\
\hline 8/31/2008 3:00 AM & 0.132 & 18.9 & 0.103 & 0.319 & 19.3 & 0.39 & 0.044 & 0.048 & -- & 0.052 & 0.422 & 16.2 & 0.430 & 15.3 & -- \\
\hline 8/31/2008 4:00 AM & 0.131 & 18.9 & 0.103 & 0.319 & 19.3 & 0.39 & 0.044 & 0.048 & -- & 0.052 & 0.422 & 16.2 & 0.430 & 15.3 & -- \\
\hline 8/31/2008 5:00 AM & 0.131 & 18.9 & 0.103 & 0.319 & 19.3 & 0.39 & 0.044 & 0.048 & -- & 0.052 & 0.422 & 16.2 & 0.430 & 15.3 & -- \\
\hline 8/31/2008 6:00 AM & 0.130 & 18.9 & 0.103 & 0.319 & 19.3 & 0.39 & 0.044 & 0.048 & -- & 0.052 & 0.422 & 16.2 & 0.430 & 15.3 & -- \\
\hline 8/31/2008 7:00 AM & 0.130 & 18.9 & 0.103 & 0.319 & 19.3 & 0.39 & 0.044 & 0.048 & -- & 0.052 & 0.422 & 16.2 & 0.430 & 15.3 & -- \\
\hline 8/31/2008 8:00 AM & 0.130 & 18.9 & 0.103 & 0.319 & 19.3 & 0.39 & 0.044 & 0.048 & -- & 0.052 & 0.422 & 16.2 & 0.430 & 15.3 & -- \\
\hline 8/31/2008 9:00 AM & 0.131 & 18.9 & 0.103 & 0.319 & 19.3 & 0.39 & 0.044 & 0.048 & -- & 0.052 & 0.422 & 16.2 & 0.431 & 15.3 & -- \\
\hline 8/31/2008 10:00 AM & 0.130 & 19.0 & 0.103 & 0.319 & 19.3 & 0.39 & 0.044 & 0.048 & -- & 0.052 & 0.422 & 16.2 & 0.435 & 15.3 & -- \\
\hline 8/31/2008 11:00 AM & 0.131 & 18.9 & 0.103 & 0.319 & 19.3 & 0.39 & 0.043 & 0.048 & -- & 0.052 & 0.422 & 16.2 & 0.432 & 15.3 & -- \\
\hline 8/31/2008 12:00 PM & 0.130 & 18.9 & 0.103 & 0.319 & 19.3 & 0.39 & 0.043 & 0.048 & -- & 0.053 & 0.422 & 16.2 & 0.433 & 15.3 & -- \\
\hline 8/31/2008 1:00 PM & 0.129 & 18.9 & 0.102 & 0.319 & 19.3 & 0.39 & 0.043 & 0.048 & -- & 0.053 & 0.422 & 16.2 & 0.429 & 15.3 & -- \\
\hline 8/31/2008 2:00 PM & 0.129 & 18.8 & 0.102 & 0.319 & 19.3 & 0.39 & 0.043 & 0.048 & -- & 0.053 & 0.422 & 16.2 & 0.429 & 15.3 & -- \\
\hline 8/31/2008 3:00 PM & 0.129 & 18.8 & 0.102 & 0.319 & 19.3 & 0.39 & 0.043 & 0.048 & -- & 0.053 & 0.422 & 16.2 & 0.428 & 15.3 & -- \\
\hline 8/31/2008 4:00 PM & 0.129 & 18.8 & 0.102 & 0.319 & 19.2 & 0.39 & 0.043 & 0.048 & -- & 0.053 & 0.422 & 16.2 & 0.429 & 15.3 & -- \\
\hline 8/31/2008 5:00 PM & 0.129 & 18.8 & 0.102 & 0.319 & 19.2 & 0.39 & 0.043 & 0.048 & -- & 0.052 & 0.422 & 16.2 & 0.430 & 15.3 & -- \\
\hline 8/31/2008 6:00 PM & 0.129 & 18.9 & 0.102 & 0.319 & 19.2 & 0.39 & 0.043 & 0.048 & -- & 0.052 & 0.422 & 16.2 & 0.430 & 15.3 & -- \\
\hline 8/31/2008 7:00 PM & 0.128 & 18.9 & 0.102 & 0.318 & 19.2 & 0.39 & 0.043 & 0.048 & -- & 0.052 & 0.422 & 16.2 & 0.430 & 15.3 & -- \\
\hline 8/31/2008 8:00 PM & 0.126 & 18.9 & 0.102 & 0.318 & 19.2 & 0.39 & 0.043 & 0.048 & -- & 0.052 & 0.422 & 16.2 & 0.432 & 15.3 & -- \\
\hline 8/31/2008 9:00 PM & 0.126 & 18.9 & 0.101 & 0.318 & 19.2 & 0.39 & 0.043 & 0.048 & -- & 0.052 & 0.421 & 16.2 & 0.438 & 15.3 & -- \\
\hline 8/31/2008 10:00 PM & 0.128 & 18.9 & 0.101 & 0.318 & 19.2 & 0.39 & 0.043 & 0.048 & -- & 0.053 & 0.422 & 16.2 & 0.430 & 15.3 & -- \\
\hline 8/31/2008 11:00 PM & 0.129 & 18.9 & 0.101 & 0.318 & 19.2 & 0.39 & 0.043 & 0.048 & -- & 0.052 & 0.422 & 16.2 & 0.425 & 15.3 & -- \\
\hline 9/1/2008 12:00 AM & 0.129 & 18.9 & 0.101 & 0.318 & 19.2 & 0.39 & 0.043 & 0.048 & -- & 0.052 & 0.422 & 16.2 & 0.424 & 15.3 & -- \\
\hline 9/1/2008 1:00 AM & 0.129 & 19.0 & 0.101 & 0.318 & 19.2 & 0.39 & 0.043 & 0.048 & -- & 0.053 & 0.421 & 16.2 & 0.426 & 15.4 & -- \\
\hline 9/1/2008 2:00 AM & 0.129 & 19.0 & 0.101 & 0.318 & 19.2 & 0.39 & 0.043 & 0.048 & -- & 0.053 & 0.422 & 16.2 & 0.428 & 15.4 & -- \\
\hline 9/1/2008 3:00 AM & 0.128 & 18.9 & 0.101 & 0.318 & 19.2 & 0.39 & 0.043 & 0.048 & -- & 0.052 & 0.422 & 16.2 & 0.426 & 15.4 & -- \\
\hline 9/1/2008 4:00 AM & 0.128 & 19.0 & 0.101 & 0.318 & 19.2 & 0.39 & 0.043 & 0.048 & -- & 0.052 & 0.421 & 16.2 & 0.426 & 15.4 & -- \\
\hline 9/1/2008 5:00 AM & 0.126 & 18.9 & 0.101 & 0.318 & 19.2 & 0.39 & 0.043 & 0.048 & -- & 0.053 & 0.421 & 16.2 & 0.426 & 15.4 & -- \\
\hline 9/1/2008 6:00 AM & 0.126 & 19.0 & 0.100 & 0.318 & 19.2 & 0.39 & 0.043 & 0.048 & -- & 0.053 & 0.421 & 16.2 & 0.425 & 15.4 & -- \\
\hline 9/1/2008 7:00 AM & 0.126 & 19.0 & 0.100 & 0.317 & 19.2 & 0.39 & 0.043 & 0.048 & -- & 0.053 & 0.422 & 16.2 & 0.426 & 15.4 & -- \\
\hline 9/1/2008 8:00 AM & 0.126 & 18.9 & 0.100 & 0.317 & 19.2 & 0.39 & 0.043 & 0.048 & -- & 0.053 & 0.421 & 16.2 & 0.428 & 15.4 & -- \\
\hline 9/1/2008 9:00 AM & 0.128 & 19.0 & 0.100 & 0.318 & 19.2 & 0.39 & 0.043 & 0.048 & -- & 0.053 & 0.421 & 16.2 & 0.428 & 15.4 & -- \\
\hline 9/1/2008 10:00 AM & 0.126 & 18.9 & 0.100 & 0.318 & 19.2 & 0.39 & 0.043 & 0.048 & -- & 0.053 & 0.421 & 16.2 & 0.426 & 15.4 & -- \\
\hline 9/1/2008 11:00 AM & 0.125 & 18.9 & 0.100 & 0.318 & 19.2 & 0.39 & 0.043 & 0.048 & -- & 0.053 & 0.422 & 16.2 & 0.425 & 15.4 & -- \\
\hline 9/1/2008 12:00 PM & 0.126 & 18.9 & 0.100 & 0.318 & 19.2 & 0.39 & 0.043 & 0.048 & -- & 0.053 & 0.422 & 16.2 & 0.426 & 15.4 & -- \\
\hline 9/1/2008 1:00 PM & 0.126 & 18.9 & 0.100 & 0.318 & 19.2 & 0.39 & 0.043 & 0.048 & -- & 0.053 & 0.421 & 16.2 & 0.425 & 15.4 & -- \\
\hline 9/1/2008 2:00 PM & 0.126 & 18.9 & 0.100 & 0.318 & 19.2 & 0.39 & 0.043 & 0.048 & -- & 0.053 & 0.422 & 16.2 & 0.423 & 15.4 & -- \\
\hline
\end{tabular}


Appendix 1. Volumetric water content, temperature, and electrical-conductivity data collected at the flood-irrigated site during 2008.-Continued

[Depth in feet below land surface; volumetric water content dimensionless; temperature in degrees Celsius; bulk electrical conductivity in decisiemens per meter; --, no data]

\begin{tabular}{|c|c|c|c|c|c|c|c|c|c|c|c|c|c|c|c|}
\hline \multirow[b]{3}{*}{$\begin{array}{l}\text { Measurement } \\
\text { date and time }\end{array}$} & \multicolumn{15}{|c|}{ Depth } \\
\hline & \multirow{2}{*}{$\begin{array}{c}2 \\
\text { Volumetric } \\
\text { water } \\
\text { content }\end{array}$} & & \multirow{2}{*}{ 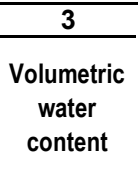 } & \multicolumn{3}{|c|}{4} & \multirow{2}{*}{$\begin{array}{c}6 \\
\begin{array}{c}\text { Volumetric } \\
\text { water } \\
\text { content }\end{array}\end{array}$} & \multicolumn{2}{|l|}{8} & \multirow{2}{*}{$\begin{array}{c}10 \\
\text { Volumetric } \\
\text { water } \\
\text { content }\end{array}$} & \multicolumn{2}{|c|}{12} & \multicolumn{2}{|c|}{15} & \multirow{2}{*}{$\begin{array}{c}18.5 \\
\text { Volumetric } \\
\text { water } \\
\text { content }\end{array}$} \\
\hline & & $\begin{array}{l}\text { Temper- } \\
\text { ature }\end{array}$ & & $\begin{array}{c}\text { Volumetric } \\
\text { water } \\
\text { content }\end{array}$ & $\begin{array}{l}\text { Temper- } \\
\text { ature }\end{array}$ & $\begin{array}{l}\text { Bulk } \\
\text { electrical } \\
\text { conduct- } \\
\text { ivity }\end{array}$ & & $\begin{array}{c}\text { Volumetric } \\
\text { water } \\
\text { content }\end{array}$ & $\begin{array}{l}\text { Temper- } \\
\text { ature }\end{array}$ & & $\begin{array}{c}\text { Volumetric } \\
\text { water } \\
\text { content }\end{array}$ & $\begin{array}{l}\text { Temper- } \\
\text { ature }\end{array}$ & $\begin{array}{l}\text { Volumetric } \\
\text { water } \\
\text { content }\end{array}$ & $\begin{array}{l}\text { Temper- } \\
\text { ature }\end{array}$ & \\
\hline 9/1/2008 3:00 PM & 0.125 & 18.9 & 0.100 & 0.317 & 19.2 & 0.39 & 0.043 & 0.048 & -- & 0.053 & 0.422 & 16.2 & 0.422 & 15.4 & -- \\
\hline 9/1/2008 4:00 PM & 0.125 & 18.9 & 0.100 & 0.317 & 19.2 & 0.39 & 0.043 & 0.048 & -- & 0.053 & 0.422 & 16.2 & 0.422 & 15.4 & -- \\
\hline 9/1/2008 5:00 PM & 0.125 & 18.9 & 0.100 & 0.317 & 19.2 & 0.39 & 0.043 & 0.048 & -- & 0.053 & 0.422 & 16.2 & 0.422 & 15.4 & -- \\
\hline 9/1/2008 6:00 PM & 0.125 & 18.9 & 0.100 & 0.317 & 19.2 & 0.39 & 0.043 & 0.048 & -- & 0.053 & 0.422 & 16.2 & 0.424 & 15.4 & -- \\
\hline 9/1/2008 7:00 PM & 0.124 & 18.9 & 0.099 & 0.317 & 19.2 & 0.39 & 0.043 & 0.048 & -- & 0.053 & 0.422 & 16.2 & 0.425 & 15.4 & -- \\
\hline 9/1/2008 8:00 PM & 0.125 & 18.9 & 0.099 & 0.317 & 19.2 & 0.39 & 0.043 & 0.047 & -- & 0.053 & 0.422 & 16.2 & 0.425 & 15.4 & -- \\
\hline 9/1/2008 9:00 PM & 0.125 & 18.9 & 0.099 & 0.317 & 19.2 & 0.39 & 0.043 & 0.047 & -- & 0.053 & 0.422 & 16.2 & 0.426 & 15.4 & -- \\
\hline 9/1/2008 10:00 PM & 0.124 & 18.9 & 0.099 & 0.317 & 19.2 & 0.39 & 0.043 & 0.047 & -- & 0.053 & 0.422 & 16.2 & 0.425 & 15.4 & -- \\
\hline 9/1/2008 11:00 PM & 0.124 & 19.0 & 0.099 & 0.317 & 19.2 & 0.39 & 0.043 & 0.047 & -- & 0.053 & 0.422 & 16.2 & 0.425 & 15.4 & -- \\
\hline 9/2/2008 12:00 AM & 0.124 & 19.0 & 0.099 & 0.316 & 19.2 & 0.39 & 0.043 & 0.047 & -- & 0.053 & 0.422 & 16.2 & 0.425 & 15.4 & -- \\
\hline 9/2/2008 1:00 AM & 0.124 & 19.0 & 0.099 & 0.317 & 19.2 & 0.39 & 0.043 & 0.047 & -- & 0.053 & 0.422 & 16.2 & 0.425 & 15.4 & -- \\
\hline 9/2/2008 2:00 AM & 0.124 & 19.0 & 0.099 & 0.317 & 19.2 & 0.39 & 0.043 & 0.047 & -- & 0.053 & 0.422 & 16.2 & 0.425 & 15.4 & -- \\
\hline 9/2/2008 3:00 AM & 0.124 & 19.0 & 0.098 & 0.317 & 19.2 & 0.39 & 0.043 & 0.047 & -- & 0.053 & 0.422 & 16.2 & 0.424 & 15.4 & -- \\
\hline 9/2/2008 4:00 AM & 0.124 & 19.0 & 0.098 & 0.317 & 19.2 & 0.39 & 0.043 & 0.047 & -- & 0.053 & 0.422 & 16.2 & 0.423 & 15.4 & -- \\
\hline 9/2/2008 5:00 AM & 0.124 & 19.0 & 0.098 & 0.317 & 19.2 & 0.39 & 0.043 & 0.047 & -- & 0.053 & 0.422 & 16.2 & 0.423 & 15.4 & -- \\
\hline 9/2/2008 6:00 AM & 0.124 & 19.0 & 0.098 & 0.316 & 19.2 & 0.39 & 0.043 & 0.047 & -- & 0.052 & 0.422 & 16.3 & 0.423 & 15.4 & -- \\
\hline 9/2/2008 7:00 AM & 0.124 & 19.0 & 0.098 & 0.316 & 19.2 & 0.39 & 0.043 & 0.047 & -- & 0.052 & 0.422 & 16.2 & 0.423 & 15.4 & -- \\
\hline 9/2/2008 8:00 AM & 0.123 & 19.0 & 0.098 & 0.316 & 19.2 & 0.39 & 0.043 & 0.047 & -- & 0.052 & 0.422 & 16.2 & 0.423 & 15.4 & -- \\
\hline 9/2/2008 9:00 AM & 0.123 & 19.0 & 0.098 & 0.316 & 19.2 & 0.39 & 0.043 & 0.047 & -- & 0.051 & 0.422 & 16.2 & 0.423 & 15.4 & -- \\
\hline 9/2/2008 10:00 AM & 0.124 & 19.0 & 0.098 & 0.316 & 19.2 & 0.39 & 0.043 & 0.047 & -- & 0.051 & 0.422 & 16.2 & 0.423 & 15.4 & -- \\
\hline 9/2/2008 11:00 AM & 0.123 & 19.0 & 0.098 & 0.316 & 19.2 & 0.39 & 0.043 & 0.047 & -- & 0.051 & 0.422 & 16.2 & 0.423 & 15.4 & -- \\
\hline 9/2/2008 12:00 PM & 0.123 & 19.0 & 0.098 & 0.316 & 19.2 & 0.39 & 0.043 & 0.047 & -- & 0.051 & 0.422 & 16.2 & 0.424 & 15.4 & -- \\
\hline 9/2/2008 1:00 PM & 0.123 & 19.0 & 0.098 & 0.316 & 19.2 & 0.39 & 0.043 & 0.047 & -- & 0.051 & 0.422 & 16.2 & 0.425 & 15.4 & -- \\
\hline 9/2/2008 2:00 PM & 0.122 & 19.0 & 0.098 & 0.316 & 19.2 & 0.39 & 0.043 & 0.047 & -- & 0.051 & 0.422 & 16.2 & 0.426 & 15.4 & -- \\
\hline 9/2/2008 3:00 PM & 0.121 & 19.0 & 0.098 & 0.316 & 19.2 & 0.39 & 0.043 & 0.047 & -- & 0.051 & 0.422 & 16.2 & 0.426 & 15.4 & -- \\
\hline 9/2/2008 4:00 PM & 0.121 & 19.0 & 0.098 & 0.316 & 19.2 & 0.39 & 0.043 & 0.047 & -- & 0.051 & 0.422 & 16.2 & 0.425 & 15.4 & -- \\
\hline 9/2/2008 5:00 PM & 0.121 & 18.9 & 0.098 & 0.316 & 19.2 & 0.39 & 0.043 & 0.047 & -- & 0.051 & 0.422 & 16.2 & 0.425 & 15.4 & -- \\
\hline 9/2/2008 6:00 PM & 0.121 & 18.9 & 0.098 & 0.316 & 19.2 & 0.39 & 0.043 & 0.047 & -- & 0.051 & 0.422 & 16.2 & 0.425 & 15.4 & -- \\
\hline 9/2/2008 7:00 PM & 0.120 & 18.9 & 0.098 & 0.316 & 19.2 & 0.39 & 0.042 & 0.047 & -- & 0.051 & 0.422 & 16.2 & 0.425 & 15.4 & -- \\
\hline 9/2/2008 8:00 PM & 0.120 & 18.8 & 0.098 & 0.316 & 19.2 & 0.39 & 0.042 & 0.047 & -- & 0.051 & 0.422 & 16.2 & 0.424 & 15.4 & -- \\
\hline 9/2/2008 9:00 PM & 0.121 & 18.8 & 0.098 & 0.316 & 19.2 & 0.39 & 0.042 & 0.047 & -- & 0.051 & 0.422 & 16.3 & 0.424 & 15.4 & -- \\
\hline 9/2/2008 10:00 PM & 0.121 & 18.8 & 0.098 & 0.316 & 19.2 & 0.39 & 0.042 & 0.047 & -- & 0.051 & 0.422 & 16.3 & 0.424 & 15.4 & -- \\
\hline 9/2/2008 11:00 PM & 0.120 & 18.7 & 0.098 & 0.316 & 19.2 & 0.39 & 0.042 & 0.047 & -- & 0.051 & 0.422 & 16.3 & 0.424 & 15.4 & -- \\
\hline 9/3/2008 12:00 AM & 0.120 & 18.7 & 0.098 & 0.316 & 19.2 & 0.39 & 0.042 & 0.047 & -- & 0.051 & 0.422 & 16.2 & 0.424 & 15.4 & -- \\
\hline 9/3/2008 1:00 AM & 0.120 & 18.6 & 0.098 & 0.316 & 19.2 & 0.39 & 0.042 & 0.047 & -- & 0.051 & 0.422 & 16.3 & 0.424 & 15.4 & -- \\
\hline 9/3/2008 2:00 AM & 0.121 & 18.6 & 0.097 & 0.316 & 19.2 & 0.39 & 0.042 & 0.047 & -- & 0.051 & 0.423 & 16.3 & 0.423 & 15.4 & -- \\
\hline 9/3/2008 3:00 AM & 0.121 & 18.6 & 0.097 & 0.316 & 19.2 & 0.39 & 0.042 & 0.047 & -- & 0.051 & 0.423 & 16.3 & 0.423 & 15.4 & -- \\
\hline 9/3/2008 4:00 АМ & 0.121 & 18.6 & 0.097 & 0.316 & 19.2 & 0.39 & 0.042 & 0.047 & -- & 0.051 & 0.423 & 16.3 & 0.422 & 15.4 & -- \\
\hline 9/3/2008 5:00 AM & 0.121 & 18.5 & 0.097 & 0.316 & 19.2 & 0.39 & 0.042 & 0.047 & -- & 0.052 & 0.423 & 16.3 & 0.422 & 15.4 & -- \\
\hline 9/3/2008 6:00 AM & 0.120 & 18.5 & 0.097 & 0.316 & 19.2 & 0.39 & 0.042 & 0.047 & -- & 0.052 & 0.423 & 16.3 & 0.422 & 15.4 & -- \\
\hline
\end{tabular}


Appendix 1. Volumetric water content, temperature, and electrical-conductivity data collected at the flood-irrigated site during 2008.-Continued

[Depth in feet below land surface; volumetric water content dimensionless; temperature in degrees Celsius; bulk electrical conductivity in decisiemens per meter; --, no data]

\begin{tabular}{|c|c|c|c|c|c|c|c|c|c|c|c|c|c|c|c|}
\hline \multirow{3}{*}{$\begin{array}{l}\text { Measurement } \\
\text { date and time }\end{array}$} & \multicolumn{15}{|c|}{ Depth } \\
\hline & \multicolumn{2}{|c|}{2} & \multirow{2}{*}{$\begin{array}{c}3 \\
\begin{array}{c}\text { Volumetric } \\
\text { water } \\
\text { content }\end{array}\end{array}$} & \multicolumn{3}{|c|}{4} & \multirow{2}{*}{$\begin{array}{c}6 \\
\text { Volumetric } \\
\text { water } \\
\text { content }\end{array}$} & \multicolumn{2}{|l|}{ DepuI } & \multirow{2}{*}{$\begin{array}{c}10 \\
\text { Volumetric } \\
\text { water } \\
\text { content }\end{array}$} & \multicolumn{2}{|c|}{12} & \multicolumn{2}{|c|}{15} & \multirow{2}{*}{$\begin{array}{c}18.5 \\
\text { Volumetric } \\
\text { water } \\
\text { content }\end{array}$} \\
\hline & $\begin{array}{c}\text { Volumetric } \\
\text { water } \\
\text { content }\end{array}$ & $\begin{array}{l}\text { Temper- } \\
\text { ature }\end{array}$ & & $\begin{array}{c}\text { Volumetric } \\
\text { water } \\
\text { content }\end{array}$ & $\begin{array}{l}\text { Temper- } \\
\text { ature }\end{array}$ & $\begin{array}{c}\text { Bulk } \\
\text { electrical } \\
\text { conduct- } \\
\text { ivity }\end{array}$ & & $\begin{array}{c}\text { Volumetric } \\
\text { water } \\
\text { content }\end{array}$ & $\begin{array}{l}\text { Temper- } \\
\text { ature }\end{array}$ & & $\begin{array}{c}\text { Volumetric } \\
\text { water } \\
\text { content }\end{array}$ & $\begin{array}{l}\text { Temper- } \\
\text { ature }\end{array}$ & $\begin{array}{c}\text { Volumetric } \\
\text { water } \\
\text { content }\end{array}$ & $\begin{array}{l}\text { Temper- } \\
\text { ature }\end{array}$ & \\
\hline 9/3/2008 7:00 AM & 0.120 & 18.5 & 0.097 & 0.316 & 19.2 & 0.39 & 0.042 & 0.047 & -- & 0.053 & 0.424 & 16.2 & 0.422 & 15.4 & -- \\
\hline 9/3/2008 8:00 AM & 0.119 & 18.5 & 0.097 & 0.316 & 19.2 & 0.39 & 0.042 & 0.047 & -- & 0.053 & 0.423 & 16.3 & 0.422 & 15.4 & -- \\
\hline 9/3/2008 9:00 AM & 0.120 & 18.5 & 0.097 & 0.316 & 19.2 & 0.39 & 0.042 & 0.047 & -- & 0.054 & 0.423 & 16.3 & 0.423 & 15.4 & -- \\
\hline 9/3/2008 10:00 AM & 0.120 & 18.5 & 0.097 & 0.316 & 19.2 & 0.39 & 0.042 & 0.047 & -- & 0.055 & 0.423 & 16.3 & 0.424 & 15.4 & -- \\
\hline 9/3/2008 11:00 AM & 0.120 & 18.4 & 0.097 & 0.316 & 19.2 & 0.39 & 0.042 & 0.047 & -- & 0.057 & 0.422 & 16.3 & 0.426 & 15.4 & -- \\
\hline 9/3/2008 12:00 PM & 0.120 & 18.4 & 0.097 & 0.316 & 19.2 & 0.39 & 0.042 & 0.047 & -- & 0.062 & 0.422 & 16.2 & 0.426 & 15.5 & -- \\
\hline 9/3/2008 1:00 PM & 0.119 & 18.3 & 0.097 & 0.316 & 19.2 & 0.39 & 0.042 & 0.047 & -- & 0.078 & 0.422 & 16.2 & 0.426 & 15.4 & -- \\
\hline 9/3/2008 2:00 PM & 0.119 & 18.3 & 0.097 & 0.316 & 19.2 & 0.39 & 0.042 & 0.047 & -- & 0.153 & 0.422 & 16.2 & 0.425 & 15.5 & -- \\
\hline 9/3/2008 3:00 PM & 0.119 & 18.3 & 0.097 & 0.316 & 19.2 & 0.39 & 0.042 & 0.047 & -- & 0.177 & 0.422 & 16.2 & 0.425 & 15.5 & -- \\
\hline 9/3/2008 4:00 PM & 0.119 & 18.2 & 0.097 & 0.316 & 19.2 & 0.39 & 0.042 & 0.047 & -- & 0.261 & 0.422 & 16.2 & 0.424 & 15.4 & -- \\
\hline 9/3/2008 5:00 PM & 0.119 & 18.1 & 0.097 & 0.316 & 19.2 & 0.39 & 0.042 & 0.047 & -- & 0.312 & 0.422 & 16.2 & 0.425 & 15.4 & -- \\
\hline 9/3/2008 6:00 PM & 0.118 & 18.1 & 0.097 & 0.316 & 19.1 & 0.39 & 0.042 & 0.047 & -- & 0.329 & 0.422 & 16.2 & 0.425 & 15.5 & -- \\
\hline 9/3/2008 7:00 PM & 0.118 & 18.1 & 0.096 & 0.316 & 19.1 & 0.39 & 0.042 & 0.047 & -- & 0.323 & 0.422 & 16.2 & 0.426 & 15.5 & -- \\
\hline 9/3/2008 8:00 PM & 0.118 & 18.1 & 0.096 & 0.315 & 19.1 & 0.39 & 0.042 & 0.047 & -- & 0.222 & 0.422 & 16.2 & 0.426 & 15.5 & -- \\
\hline 9/3/2008 9:00 PM & 0.118 & 18.1 & 0.096 & 0.315 & 19.1 & 0.38 & 0.042 & 0.047 & -- & 0.194 & 0.422 & 16.2 & 0.425 & 15.4 & -- \\
\hline 9/3/2008 10:00 PM & 0.118 & 18.1 & 0.096 & 0.315 & 19.1 & 0.39 & 0.042 & 0.047 & -- & 0.219 & 0.422 & 16.3 & 0.425 & 15.4 & -- \\
\hline 9/3/2008 11:00 PM & 0.118 & 18.2 & 0.096 & 0.315 & 19.1 & 0.39 & 0.042 & 0.047 & -- & 0.206 & 0.422 & 16.3 & 0.425 & 15.5 & -- \\
\hline 9/4/2008 12:00 AM & 0.118 & 18.1 & 0.096 & 0.315 & 19.1 & 0.39 & 0.042 & 0.047 & -- & 0.122 & 0.422 & 16.3 & 0.424 & 15.4 & -- \\
\hline 9/4/2008 1:00 AM & 0.118 & 18.1 & 0.096 & 0.315 & 19.1 & 0.39 & 0.042 & 0.047 & -- & 0.147 & 0.422 & 16.3 & 0.423 & 15.5 & -- \\
\hline 9/4/2008 2:00 AM & 0.118 & 18.1 & 0.096 & 0.315 & 19.1 & 0.39 & 0.042 & 0.047 & -- & 0.162 & 0.422 & 16.3 & 0.423 & 15.5 & -- \\
\hline 9/4/2008 3:00 AM & 0.118 & 18.1 & 0.096 & 0.315 & 19.1 & 0.39 & 0.042 & 0.047 & -- & 0.092 & 0.422 & 16.3 & 0.423 & 15.5 & -- \\
\hline 9/4/2008 4:00 AM & 0.118 & 18.1 & 0.095 & 0.315 & 19.1 & 0.39 & 0.042 & 0.047 & -- & 0.101 & 0.423 & 16.3 & 0.423 & 15.5 & -- \\
\hline 9/4/2008 5:00 AM & 0.118 & 18.1 & 0.095 & 0.315 & 19.1 & 0.39 & 0.042 & 0.047 & -- & 0.082 & 0.423 & 16.3 & 0.423 & 15.4 & -- \\
\hline 9/4/2008 6:00 AM & 0.118 & 18.0 & 0.095 & 0.315 & 19.1 & 0.39 & 0.042 & 0.047 & -- & 0.094 & 0.423 & 16.3 & 0.423 & 15.5 & -- \\
\hline 9/4/2008 7:00 AM & 0.118 & 18.0 & 0.095 & 0.315 & 19.1 & 0.39 & 0.042 & 0.047 & -- & 0.167 & 0.423 & 16.3 & 0.423 & 15.5 & -- \\
\hline 9/4/2008 8:00 AM & 0.118 & 18.1 & 0.095 & 0.315 & 19.1 & 0.38 & 0.042 & 0.047 & -- & 0.148 & 0.423 & 16.3 & 0.423 & 15.4 & -- \\
\hline 9/4/2008 9:00 AM & 0.117 & 18.1 & 0.095 & 0.315 & 19.1 & 0.38 & 0.042 & 0.047 & -- & 0.100 & 0.423 & 16.3 & 0.424 & 15.5 & -- \\
\hline 9/4/2008 10:00 AM & 0.117 & 18.1 & 0.095 & 0.315 & 19.1 & 0.38 & 0.042 & 0.047 & -- & 0.109 & 0.422 & 16.3 & 0.425 & 15.5 & -- \\
\hline 9/4/2008 11:00 AM & 0.117 & 18.0 & 0.095 & 0.315 & 19.1 & 0.38 & 0.042 & 0.047 & -- & 0.058 & 0.421 & 16.3 & 0.426 & 15.5 & -- \\
\hline 9/4/2008 12:00 PM & 0.117 & 18.0 & 0.095 & 0.315 & 19.1 & 0.38 & 0.042 & 0.047 & -- & 0.053 & 0.422 & 16.3 & 0.426 & 15.5 & -- \\
\hline 9/4/2008 1:00 PM & 0.116 & 18.0 & 0.095 & 0.315 & 19.1 & 0.38 & 0.042 & 0.047 & -- & 0.049 & 0.422 & 16.2 & 0.426 & 15.5 & -- \\
\hline 9/4/2008 2:00 PM & 0.116 & 17.9 & 0.095 & 0.315 & 19.0 & 0.38 & 0.042 & 0.047 & -- & 0.048 & 0.422 & 16.2 & 0.426 & 15.5 & -- \\
\hline 9/4/2008 3:00 PM & 0.116 & 17.9 & 0.095 & 0.315 & 19.0 & 0.38 & 0.042 & 0.047 & -- & 0.048 & 0.421 & 16.2 & 0.426 & 15.5 & -- \\
\hline 9/4/2008 4:00 PM & 0.116 & 17.9 & 0.095 & 0.315 & 19.0 & 0.38 & 0.042 & 0.047 & -- & 0.063 & 0.422 & 16.3 & 0.425 & 15.5 & -- \\
\hline 9/4/2008 5:00 PM & 0.116 & 17.9 & 0.095 & 0.315 & 19.0 & 0.38 & 0.042 & 0.047 & -- & 0.143 & 0.421 & 16.3 & 0.425 & 15.5 & -- \\
\hline 9/4/2008 6:00 PM & 0.116 & 17.8 & 0.095 & 0.315 & 19.0 & 0.38 & 0.042 & 0.047 & -- & 0.174 & 0.422 & 16.2 & 0.423 & 15.5 & -- \\
\hline 9/4/2008 7:00 PM & 0.115 & 17.8 & 0.095 & 0.315 & 19.0 & 0.38 & 0.042 & 0.047 & -- & 0.225 & 0.422 & 16.3 & 0.422 & 15.5 & -- \\
\hline 9/4/2008 8:00 PM & 0.115 & 17.8 & 0.094 & 0.313 & 19.0 & 0.38 & 0.042 & 0.047 & -- & 0.143 & 0.422 & 16.3 & 0.422 & 15.5 & -- \\
\hline 9/4/2008 9:00 PM & 0.115 & 17.8 & 0.094 & 0.313 & 19.0 & 0.38 & 0.042 & 0.047 & -- & 0.101 & 0.421 & 16.2 & 0.423 & 15.5 & -- \\
\hline 9/4/2008 10:00 PM & 0.115 & 17.9 & 0.094 & 0.315 & 19.0 & 0.38 & 0.042 & 0.047 & -- & 0.100 & 0.422 & 16.3 & 0.423 & 15.5 & -- \\
\hline
\end{tabular}


Appendix 1. Volumetric water content, temperature, and electrical-conductivity data collected at the flood-irrigated site during 2008.-Continued

[Depth in feet below land surface; volumetric water content dimensionless; temperature in degrees Celsius; bulk electrical conductivity in decisiemens per meter; --, no data]

\begin{tabular}{|c|c|c|c|c|c|c|c|c|c|c|c|c|c|c|c|}
\hline \multirow[b]{3}{*}{$\begin{array}{l}\text { Measurement } \\
\text { date and time }\end{array}$} & \multicolumn{15}{|c|}{ Depth } \\
\hline & \multirow{2}{*}{$\begin{array}{c}2 \\
\text { Volumetric } \\
\text { water } \\
\text { content }\end{array}$} & & \multirow{2}{*}{ 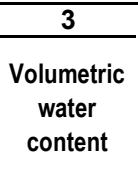 } & \multicolumn{3}{|c|}{4} & \multirow{2}{*}{$\begin{array}{c}6 \\
\begin{array}{c}\text { Volumetric } \\
\text { water } \\
\text { content }\end{array}\end{array}$} & \multicolumn{2}{|l|}{8} & \multirow{2}{*}{$\begin{array}{c}10 \\
\text { Volumetric } \\
\text { water } \\
\text { content }\end{array}$} & \multicolumn{2}{|c|}{12} & \multicolumn{2}{|c|}{15} & \multirow{2}{*}{$\begin{array}{c}18.5 \\
\text { Volumetric } \\
\text { water } \\
\text { content }\end{array}$} \\
\hline & & $\begin{array}{l}\text { Temper- } \\
\text { ature }\end{array}$ & & $\begin{array}{c}\text { Volumetric } \\
\text { water } \\
\text { content }\end{array}$ & $\begin{array}{l}\text { Temper- } \\
\text { ature }\end{array}$ & $\begin{array}{l}\text { Bulk } \\
\text { electrical } \\
\text { conduct- } \\
\text { ivity }\end{array}$ & & $\begin{array}{l}\text { Volumetric } \\
\text { water } \\
\text { content }\end{array}$ & $\begin{array}{l}\text { Temper- } \\
\text { ature }\end{array}$ & & $\begin{array}{c}\text { Volumetric } \\
\text { water } \\
\text { content }\end{array}$ & $\begin{array}{l}\text { Temper- } \\
\text { ature }\end{array}$ & $\begin{array}{l}\text { Volumetric } \\
\text { water } \\
\text { content }\end{array}$ & $\begin{array}{l}\text { Temper- } \\
\text { ature }\end{array}$ & \\
\hline 9/4/2008 11:00 PM & 0.115 & 17.9 & 0.094 & 0.313 & 19.0 & 0.38 & 0.042 & 0.047 & -- & 0.101 & 0.422 & 16.3 & 0.424 & 15.5 & -- \\
\hline 9/5/2008 12:00 AM & 0.115 & 17.9 & 0.094 & 0.313 & 19.0 & 0.38 & 0.042 & 0.047 & -- & 0.114 & 0.422 & 16.3 & 0.425 & 15.5 & -- \\
\hline 9/5/2008 1:00 AM & 0.115 & 17.9 & 0.094 & 0.313 & 19.0 & 0.38 & 0.042 & 0.047 & -- & 0.078 & 0.422 & 16.3 & 0.425 & 15.5 & -- \\
\hline 9/5/2008 2:00 AM & 0.115 & 17.9 & 0.094 & 0.313 & 19.0 & 0.38 & 0.042 & 0.047 & -- & 0.059 & 0.422 & 16.3 & 0.425 & 15.5 & -- \\
\hline 9/5/2008 3:00 AM & 0.116 & 17.9 & 0.094 & 0.313 & 19.0 & 0.38 & 0.042 & 0.047 & -- & 0.056 & 0.422 & 16.3 & 0.425 & 15.5 & -- \\
\hline 9/5/2008 4:00 AM & 0.115 & 17.9 & 0.094 & 0.313 & 19.0 & 0.38 & 0.042 & 0.047 & -- & 0.056 & 0.422 & 16.3 & 0.425 & 15.5 & -- \\
\hline 9/5/2008 5:00 AM & 0.116 & 17.9 & 0.094 & 0.313 & 19.0 & 0.38 & 0.042 & 0.047 & -- & 0.056 & 0.422 & 16.3 & 0.425 & 15.5 & -- \\
\hline 9/5/2008 6:00 AM & 0.115 & 17.9 & 0.094 & 0.313 & 18.9 & 0.38 & 0.042 & 0.047 & -- & 0.056 & 0.422 & 16.3 & 0.425 & 15.5 & -- \\
\hline 9/5/2008 7:00 AM & 0.115 & 17.9 & 0.094 & 0.313 & 18.9 & 0.38 & 0.042 & 0.047 & -- & 0.053 & 0.422 & 16.3 & 0.425 & 15.5 & -- \\
\hline 9/5/2008 8:00 AM & 0.116 & 17.9 & 0.093 & 0.312 & 18.9 & 0.38 & 0.042 & 0.047 & -- & 0.045 & 0.422 & 16.3 & 0.425 & 15.5 & -- \\
\hline 9/5/2008 9:00 АM & 0.118 & 17.9 & 0.092 & 0.312 & 18.9 & 0.38 & 0.042 & 0.047 & -- & -- & 0.422 & 16.3 & 0.425 & 15.5 & -- \\
\hline 9/5/2008 10:00 AM & 0.118 & 17.9 & 0.093 & 0.311 & 18.9 & 0.38 & 0.042 & 0.047 & -- & -- & 0.422 & 16.3 & 0.425 & 15.5 & -- \\
\hline 9/5/2008 11:00 AM & 0.118 & 17.9 & 0.114 & 0.311 & 18.9 & 0.38 & 0.042 & 0.047 & -- & 0.103 & 0.422 & 16.3 & 0.425 & 15.5 & -- \\
\hline 9/5/2008 12:00 PM & 0.135 & 17.9 & 0.132 & 0.312 & 18.9 & 0.38 & 0.042 & 0.047 & -- & 0.111 & 0.422 & 16.3 & 0.425 & 15.5 & -- \\
\hline 9/5/2008 1:00 PM & 0.148 & 17.9 & 0.132 & 0.315 & 18.9 & 0.38 & 0.042 & 0.047 & -- & 0.439 & 0.422 & 16.3 & 0.425 & 15.5 & -- \\
\hline 9/5/2008 2:00 PM & 0.156 & 17.9 & 0.134 & 0.322 & 18.9 & 0.40 & 0.042 & 0.047 & -- & 0.088 & 0.422 & 16.3 & 0.426 & 15.5 & -- \\
\hline 9/5/2008 3:00 PM & 0.181 & 17.8 & 0.135 & 0.328 & 18.9 & 0.41 & 0.042 & 0.046 & -- & 0.138 & 0.422 & 16.3 & 0.426 & 15.5 & -- \\
\hline 9/5/2008 4:00 PM & 0.195 & 17.8 & 0.137 & 0.331 & 18.9 & 0.41 & 0.042 & 0.046 & -- & 0.158 & 0.422 & 16.3 & 0.426 & 15.5 & -- \\
\hline 9/5/2008 5:00 PM & 0.194 & 17.7 & 0.137 & 0.333 & 18.9 & 0.41 & 0.042 & 0.046 & -- & 0.175 & 0.422 & 16.3 & 0.426 & 15.5 & -- \\
\hline 9/5/2008 6:00 PM & 0.191 & 17.7 & 0.137 & 0.334 & 18.9 & 0.41 & 0.042 & 0.046 & -- & 0.128 & 0.422 & 16.3 & 0.425 & 15.5 & -- \\
\hline 9/5/2008 7:00 PM & 0.187 & 17.6 & 0.136 & 0.335 & 18.9 & 0.42 & 0.042 & 0.046 & -- & 0.129 & 0.422 & 16.3 & 0.425 & 15.5 & -- \\
\hline 9/5/2008 8:00 PM & 0.185 & 17.6 & 0.135 & 0.335 & 18.9 & 0.42 & 0.042 & 0.046 & -- & 0.131 & 0.422 & 16.3 & 0.425 & 15.5 & -- \\
\hline 9/5/2008 9:00 PM & 0.183 & 17.6 & 0.134 & 0.335 & 18.8 & 0.42 & 0.042 & 0.046 & -- & 0.124 & 0.422 & 16.3 & 0.425 & 15.5 & -- \\
\hline 9/5/2008 10:00 PM & 0.181 & 17.6 & 0.133 & 0.335 & 18.8 & 0.42 & 0.042 & 0.046 & -- & 0.101 & 0.422 & 16.3 & 0.425 & 15.5 & -- \\
\hline 9/5/2008 11:00 PM & 0.180 & 17.6 & 0.132 & 0.335 & 18.8 & 0.42 & 0.042 & 0.046 & -- & 0.098 & 0.423 & 16.3 & 0.425 & 15.5 & -- \\
\hline 9/6/2008 12:00 AM & 0.178 & 17.5 & 0.132 & 0.334 & 18.8 & 0.42 & 0.042 & 0.046 & -- & 0.092 & 0.422 & 16.3 & 0.425 & 15.5 & -- \\
\hline 9/6/2008 1:00 AM & 0.176 & 17.4 & 0.132 & 0.334 & 18.8 & 0.42 & 0.042 & 0.046 & -- & 0.079 & 0.422 & 16.3 & 0.425 & 15.6 & -- \\
\hline 9/6/2008 2:00 AM & 0.175 & 17.4 & 0.132 & 0.334 & 18.8 & 0.42 & 0.042 & 0.046 & -- & 0.069 & 0.422 & 16.3 & 0.425 & 15.5 & -- \\
\hline 9/6/2008 3:00 AM & 0.174 & 17.4 & 0.131 & 0.334 & 18.8 & 0.42 & 0.042 & 0.046 & -- & 0.140 & 0.422 & 16.3 & 0.425 & 15.5 & -- \\
\hline 9/6/2008 4:00 AM & 0.173 & 17.4 & 0.130 & 0.334 & 18.8 & 0.42 & 0.042 & 0.046 & -- & 0.223 & 0.422 & 16.3 & 0.425 & 15.5 & -- \\
\hline 9/6/2008 5:00 AM & 0.172 & 17.4 & 0.130 & 0.334 & 18.8 & 0.42 & 0.043 & 0.046 & -- & 0.327 & 0.422 & 16.3 & 0.425 & 15.5 & -- \\
\hline 9/6/2008 6:00 AM & 0.172 & 17.4 & 0.129 & 0.334 & 18.8 & 0.42 & 0.043 & 0.046 & -- & 0.407 & 0.423 & 16.3 & 0.425 & 15.5 & -- \\
\hline 9/6/2008 7:00 AM & 0.171 & 17.3 & 0.129 & 0.333 & 18.8 & 0.42 & 0.043 & 0.046 & -- & 0.224 & 0.423 & 16.3 & 0.425 & 15.5 & -- \\
\hline 9/6/2008 8:00 AM & 0.170 & 17.3 & 0.128 & 0.333 & 18.8 & 0.42 & 0.044 & 0.046 & -- & 0.310 & 0.423 & 16.3 & 0.425 & 15.5 & -- \\
\hline 9/6/2008 9:00 AM & 0.169 & 17.3 & 0.128 & 0.333 & 18.7 & 0.42 & 0.044 & 0.046 & -- & 0.240 & 0.422 & 16.3 & 0.425 & 15.5 & -- \\
\hline 9/6/2008 10:00 AM & 0.168 & 17.3 & 0.127 & 0.333 & 18.7 & 0.42 & 0.044 & 0.046 & -- & 0.144 & 0.422 & 16.3 & 0.426 & 15.5 & -- \\
\hline 9/6/2008 11:00 AM & 0.168 & 17.3 & 0.127 & 0.333 & 18.7 & 0.42 & 0.045 & 0.047 & -- & 0.090 & 0.422 & 16.3 & 0.425 & 15.5 & -- \\
\hline 9/6/2008 12:00 PM & 0.167 & 17.3 & 0.126 & 0.333 & 18.7 & 0.42 & 0.045 & 0.047 & -- & 0.095 & 0.422 & 16.3 & 0.425 & 15.5 & -- \\
\hline 9/6/2008 1:00 PM & 0.166 & 17.3 & 0.126 & 0.333 & 18.7 & 0.42 & 0.045 & 0.047 & -- & 0.100 & 0.422 & 16.3 & 0.424 & 15.5 & -- \\
\hline 9/6/2008 2:00 PM & 0.166 & 17.2 & 0.126 & 0.333 & 18.7 & 0.41 & 0.045 & 0.047 & -- & 0.168 & 0.422 & 16.3 & 0.423 & 15.5 & -- \\
\hline
\end{tabular}


Appendix 1. Volumetric water content, temperature, and electrical-conductivity data collected at the flood-irrigated site during 2008.-Continued

[Depth in feet below land surface; volumetric water content dimensionless; temperature in degrees Celsius; bulk electrical conductivity in decisiemens per meter; --, no data]

\begin{tabular}{|c|c|c|c|c|c|c|c|c|c|c|c|c|c|c|c|}
\hline \multirow[b]{3}{*}{$\begin{array}{l}\text { Measurement } \\
\text { date and time }\end{array}$} & \multicolumn{15}{|c|}{ Depth } \\
\hline & \multicolumn{2}{|c|}{2} & \multirow[b]{2}{*}{$\begin{array}{c}3 \\
\begin{array}{c}\text { Volumetric } \\
\text { water } \\
\text { content }\end{array}\end{array}$} & \multicolumn{3}{|c|}{4} & \multirow{2}{*}{$\begin{array}{c}6 \\
\begin{array}{c}\text { Volumetric } \\
\text { water } \\
\text { content }\end{array}\end{array}$} & \multicolumn{2}{|c|}{8} & \multirow{2}{*}{$\begin{array}{c}10 \\
\text { Volumetric } \\
\text { water } \\
\text { content }\end{array}$} & \multicolumn{2}{|c|}{12} & \multicolumn{2}{|c|}{15} & \multirow{2}{*}{$\begin{array}{c}18.5 \\
\text { Volumetric } \\
\text { water } \\
\text { content }\end{array}$} \\
\hline & $\begin{array}{c}\text { Volumetric } \\
\text { water } \\
\text { content }\end{array}$ & $\begin{array}{l}\text { Temper- } \\
\text { ature }\end{array}$ & & $\begin{array}{c}\text { Volumetric } \\
\text { water } \\
\text { content }\end{array}$ & $\begin{array}{l}\text { Temper- } \\
\text { ature }\end{array}$ & $\begin{array}{c}\text { Bulk } \\
\text { electrical } \\
\text { conduct- } \\
\text { ivity }\end{array}$ & & $\begin{array}{c}\text { Volumetric } \\
\text { water } \\
\text { content }\end{array}$ & $\begin{array}{l}\text { Temper- } \\
\text { ature }\end{array}$ & & $\begin{array}{c}\text { Volumetric } \\
\text { water } \\
\text { content }\end{array}$ & $\begin{array}{l}\text { Temper- } \\
\text { ature }\end{array}$ & $\begin{array}{c}\text { Volumetric } \\
\text { water } \\
\text { content }\end{array}$ & $\begin{array}{l}\text { Temper- } \\
\text { ature }\end{array}$ & \\
\hline 9/6/2008 3:00 PM & 0.165 & 17.2 & 0.126 & 0.333 & 18.7 & 0.41 & 0.045 & 0.047 & -- & 0.101 & 0.422 & 16.3 & 0.423 & 15.5 & $\overline{--}$ \\
\hline 9/6/2008 4:00 PM & 0.163 & 17.2 & 0.126 & 0.332 & 18.7 & 0.41 & 0.046 & 0.047 & -- & 0.218 & 0.422 & 16.3 & 0.423 & 15.5 & -- \\
\hline 9/6/2008 5:00 PM & 0.163 & 17.2 & 0.125 & 0.332 & 18.7 & 0.41 & 0.046 & 0.047 & -- & 0.163 & 0.422 & 16.3 & 0.423 & 15.6 & -- \\
\hline 9/6/2008 6:00 PM & 0.162 & 17.2 & 0.125 & 0.332 & 18.7 & 0.41 & 0.046 & 0.047 & -- & 0.092 & 0.422 & 16.3 & 0.424 & 15.5 & -- \\
\hline 9/6/2008 7:00 PM & 0.161 & 17.2 & 0.124 & 0.332 & 18.7 & 0.41 & 0.047 & 0.047 & -- & 0.095 & 0.422 & 16.3 & 0.425 & 15.5 & -- \\
\hline 9/6/2008 8:00 PM & 0.161 & 17.1 & 0.124 & 0.332 & 18.7 & 0.41 & 0.047 & 0.047 & -- & 0.101 & 0.422 & 16.3 & 0.426 & 15.5 & -- \\
\hline 9/6/2008 9:00 PM & 0.160 & 17.1 & 0.123 & 0.331 & 18.7 & 0.41 & 0.047 & 0.047 & -- & 0.101 & 0.422 & 16.3 & 0.426 & 15.5 & -- \\
\hline 9/6/2008 10:00 PM & 0.160 & 17.1 & 0.123 & 0.331 & 18.7 & 0.41 & 0.047 & 0.047 & -- & 0.157 & 0.423 & 16.3 & 0.426 & 15.5 & -- \\
\hline 9/6/2008 11:00 PM & 0.159 & 17.1 & 0.122 & 0.331 & 18.6 & 0.41 & 0.047 & 0.047 & -- & 0.194 & 0.422 & 16.3 & 0.426 & 15.6 & -- \\
\hline 9/7/2008 12:00 AM & 0.159 & 17.1 & 0.122 & 0.331 & 18.6 & 0.41 & 0.047 & 0.047 & -- & 0.189 & 0.422 & 16.3 & 0.426 & 15.5 & -- \\
\hline 9/7/2008 1:00 AM & 0.158 & 17.1 & 0.122 & 0.331 & 18.6 & 0.41 & 0.047 & 0.047 & -- & 0.125 & 0.422 & 16.3 & 0.426 & 15.5 & -- \\
\hline 9/7/2008 2:00 AM & 0.157 & 17.0 & 0.121 & 0.331 & 18.6 & 0.41 & 0.047 & 0.047 & -- & 0.134 & 0.422 & 16.3 & 0.426 & 15.5 & -- \\
\hline 9/7/2008 3:00 AM & 0.157 & 17.1 & 0.121 & 0.331 & 18.6 & 0.41 & 0.047 & 0.047 & -- & 0.154 & 0.422 & 16.3 & 0.426 & 15.6 & -- \\
\hline 9/7/2008 4:00 AM & 0.157 & 17.1 & 0.121 & 0.331 & 18.6 & 0.41 & 0.047 & 0.048 & -- & 0.158 & 0.423 & 16.3 & 0.426 & 15.6 & -- \\
\hline 9/7/2008 5:00 AM & 0.156 & 17.1 & 0.121 & 0.330 & 18.6 & 0.41 & 0.048 & 0.048 & -- & 0.142 & 0.423 & 16.3 & 0.426 & 15.5 & -- \\
\hline 9/7/2008 6:00 AM & 0.156 & 17.0 & 0.121 & 0.330 & 18.6 & 0.41 & 0.048 & 0.048 & -- & 0.152 & 0.423 & 16.3 & 0.426 & 15.5 & -- \\
\hline 9/7/2008 7:00 AM & 0.155 & 17.0 & 0.120 & 0.330 & 18.6 & 0.41 & 0.048 & 0.048 & -- & 0.146 & 0.423 & 16.3 & 0.426 & 15.5 & -- \\
\hline 9/7/2008 8:00 AM & 0.155 & 17.1 & 0.120 & 0.330 & 18.6 & 0.41 & 0.048 & 0.048 & -- & 0.151 & 0.423 & 16.3 & 0.426 & 15.5 & -- \\
\hline 9/7/2008 9:00 AM & 0.154 & 17.0 & 0.120 & 0.330 & 18.6 & 0.41 & 0.048 & 0.048 & -- & 0.152 & 0.422 & 16.3 & 0.426 & 15.6 & -- \\
\hline 9/7/2008 10:00 AM & 0.154 & 17.1 & 0.119 & 0.330 & 18.6 & 0.41 & 0.048 & 0.048 & -- & 0.114 & 0.422 & 16.3 & 0.425 & 15.6 & -- \\
\hline 9/7/2008 11:00 AM & 0.153 & 17.0 & 0.119 & 0.330 & 18.6 & 0.41 & 0.048 & 0.049 & -- & 0.121 & 0.422 & 16.3 & 0.424 & 15.6 & -- \\
\hline 9/7/2008 12:00 PM & 0.153 & 17.0 & 0.119 & 0.330 & 18.5 & 0.41 & 0.048 & 0.049 & -- & 0.118 & 0.422 & 16.3 & 0.423 & 15.6 & -- \\
\hline 9/7/2008 1:00 PM & 0.151 & 17.1 & 0.119 & 0.329 & 18.5 & 0.41 & 0.048 & 0.049 & -- & 0.111 & 0.422 & 16.3 & 0.422 & 15.6 & -- \\
\hline 9/7/2008 2:00 PM & 0.151 & 17.1 & 0.118 & 0.329 & 18.5 & 0.41 & 0.048 & 0.049 & -- & 0.112 & 0.422 & 16.3 & 0.422 & 15.6 & -- \\
\hline 9/7/2008 3:00 PM & 0.150 & 17.0 & 0.118 & 0.329 & 18.5 & 0.41 & 0.048 & 0.050 & -- & 0.109 & 0.422 & 16.3 & 0.423 & 15.6 & -- \\
\hline 9/7/2008 4:00 PM & 0.150 & 17.0 & 0.118 & 0.329 & 18.5 & 0.41 & 0.048 & 0.050 & -- & 0.107 & 0.422 & 16.3 & 0.423 & 15.6 & -- \\
\hline 9/7/2008 5:00 PM & 0.150 & 17.0 & 0.117 & 0.329 & 18.5 & 0.41 & 0.048 & 0.050 & -- & 0.204 & 0.422 & 16.3 & 0.422 & 15.6 & -- \\
\hline 9/7/2008 6:00 PM & 0.149 & 16.9 & 0.117 & 0.329 & 18.5 & 0.41 & 0.048 & 0.050 & -- & 0.185 & 0.422 & 16.2 & 0.422 & 15.6 & -- \\
\hline 9/7/2008 7:00 PM & 0.149 & 16.9 & 0.116 & 0.329 & 18.5 & 0.41 & 0.048 & 0.050 & -- & 0.118 & 0.422 & 16.3 & 0.422 & 15.6 & -- \\
\hline 9/7/2008 8:00 PM & 0.149 & 16.9 & 0.116 & 0.328 & 18.5 & 0.41 & 0.048 & 0.050 & -- & 0.148 & 0.422 & 16.3 & 0.423 & 15.6 & -- \\
\hline 9/7/2008 9:00 PM & 0.148 & 16.9 & 0.116 & 0.328 & 18.5 & 0.41 & 0.048 & 0.050 & -- & 0.216 & 0.421 & 16.3 & 0.424 & 15.6 & -- \\
\hline 9/7/2008 10:00 PM & 0.148 & 16.9 & 0.115 & 0.328 & 18.5 & 0.41 & 0.048 & 0.050 & -- & 0.190 & 0.422 & 16.3 & 0.425 & 15.6 & -- \\
\hline 9/7/2008 11:00 PM & 0.148 & 16.9 & 0.115 & 0.328 & 18.5 & 0.41 & 0.048 & 0.050 & -- & 0.164 & 0.422 & 16.3 & 0.425 & 15.6 & -- \\
\hline 9/8/2008 12:00 AM & 0.147 & 17.0 & 0.115 & 0.328 & 18.5 & 0.41 & 0.048 & 0.051 & -- & 0.186 & 0.423 & 16.3 & 0.426 & 15.6 & -- \\
\hline 9/8/2008 1:00 AM & 0.147 & 17.0 & 0.115 & 0.326 & 18.5 & 0.41 & 0.048 & 0.051 & -- & 0.113 & 0.423 & 16.3 & 0.426 & 15.6 & -- \\
\hline 9/8/2008 2:00 AM & 0.146 & 17.0 & 0.115 & 0.326 & 18.4 & 0.41 & 0.048 & 0.051 & -- & 0.245 & 0.423 & 16.3 & 0.426 & 15.6 & -- \\
\hline 9/8/2008 3:00 AM & 0.146 & 17.0 & 0.115 & 0.326 & 18.4 & 0.41 & 0.048 & 0.051 & -- & 0.262 & 0.423 & 16.3 & 0.426 & 15.6 & -- \\
\hline 9/8/2008 4:00 AM & 0.146 & 17.0 & 0.115 & 0.326 & 18.4 & 0.41 & 0.048 & 0.051 & -- & 0.176 & 0.423 & 16.3 & 0.426 & 15.6 & -- \\
\hline 9/8/2008 5:00 AM & 0.146 & 17.0 & 0.115 & 0.326 & 18.4 & 0.41 & 0.048 & 0.052 & -- & 0.132 & 0.423 & 16.3 & 0.426 & 15.6 & -- \\
\hline 9/8/2008 6:00 AM & 0.145 & 17.0 & 0.114 & 0.326 & 18.4 & 0.41 & 0.048 & 0.052 & -- & 0.140 & 0.423 & 16.3 & 0.426 & 15.6 & -- \\
\hline
\end{tabular}


Appendix 1. Volumetric water content, temperature, and electrical-conductivity data collected at the flood-irrigated site during 2008.-Continued

[Depth in feet below land surface; volumetric water content dimensionless; temperature in degrees Celsius; bulk electrical conductivity in decisiemens per meter; --, no data]

\begin{tabular}{|c|c|c|c|c|c|c|c|c|c|c|c|c|c|c|c|}
\hline \multirow[b]{3}{*}{$\begin{array}{l}\text { Measurement } \\
\text { date and time }\end{array}$} & \multicolumn{15}{|c|}{ Depth } \\
\hline & \multicolumn{2}{|c|}{2} & \multirow{2}{*}{$\begin{array}{c}3 \\
\text { Volumetric } \\
\text { water } \\
\text { content }\end{array}$} & \multicolumn{3}{|c|}{4} & \multirow{2}{*}{$\begin{array}{c}6 \\
\begin{array}{c}\text { Volumetric } \\
\text { water } \\
\text { content }\end{array}\end{array}$} & \multicolumn{2}{|l|}{8} & \multirow{2}{*}{$\begin{array}{c}10 \\
\text { Volumetric } \\
\text { water } \\
\text { content }\end{array}$} & \multicolumn{2}{|c|}{12} & \multicolumn{2}{|c|}{15} & \multirow{2}{*}{$\begin{array}{c}18.5 \\
\text { Volumetric } \\
\text { water } \\
\text { content }\end{array}$} \\
\hline & $\begin{array}{l}\text { Volumetric } \\
\text { water } \\
\text { content }\end{array}$ & $\begin{array}{l}\text { Temper- } \\
\text { ature }\end{array}$ & & $\begin{array}{c}\text { Volumetric } \\
\text { water } \\
\text { content }\end{array}$ & $\begin{array}{l}\text { Temper- } \\
\text { ature }\end{array}$ & $\begin{array}{c}\text { Bulk } \\
\text { electrical } \\
\text { conduct- } \\
\text { ivity } \\
\end{array}$ & & $\begin{array}{l}\text { Volumetric } \\
\text { water } \\
\text { content }\end{array}$ & $\begin{array}{l}\text { Temper- } \\
\text { ature }\end{array}$ & & $\begin{array}{l}\text { Volumetric } \\
\text { water } \\
\text { content }\end{array}$ & $\begin{array}{l}\text { Temper- } \\
\text { ature }\end{array}$ & $\begin{array}{l}\text { Volumetric } \\
\text { water } \\
\text { content }\end{array}$ & $\begin{array}{l}\text { Temper- } \\
\text { ature }\end{array}$ & \\
\hline 9/8/2008 7:00 AM & 0.145 & 17.0 & 0.114 & 0.325 & 18.4 & 0.41 & 0.048 & 0.052 & -- & 0.125 & 0.423 & 16.3 & 0.426 & 15.6 & -- \\
\hline 9/8/2008 8:00 AM & 0.145 & 17.0 & 0.114 & 0.325 & 18.4 & 0.41 & 0.048 & 0.052 & -- & 0.117 & 0.423 & 16.3 & 0.426 & 15.6 & -- \\
\hline 9/8/2008 9:00 AM & 0.145 & 17.0 & 0.114 & 0.325 & 18.4 & 0.41 & 0.048 & 0.052 & -- & 0.203 & 0.423 & 16.3 & 0.426 & 15.6 & -- \\
\hline 9/8/2008 10:00 AM & 0.144 & 17.0 & 0.113 & 0.325 & 18.4 & 0.41 & 0.048 & 0.052 & -- & 0.254 & 0.423 & 16.3 & 0.426 & 15.6 & -- \\
\hline 9/8/2008 11:00 AM & 0.144 & 17.0 & 0.113 & 0.325 & 18.4 & 0.41 & 0.048 & 0.052 & -- & 0.259 & 0.423 & 16.3 & 0.425 & 15.6 & -- \\
\hline 9/8/2008 12:00 PM & 0.144 & 16.9 & 0.113 & 0.325 & 18.4 & 0.41 & 0.048 & 0.052 & -- & 0.476 & 0.423 & 16.3 & 0.425 & 15.6 & -- \\
\hline 9/8/2008 1:00 PM & 0.143 & 17.0 & 0.113 & 0.325 & 18.4 & 0.41 & 0.048 & 0.052 & -- & 0.327 & 0.423 & 16.3 & 0.425 & 15.6 & -- \\
\hline 9/8/2008 2:00 PM & 0.143 & 16.9 & 0.112 & 0.325 & 18.4 & 0.41 & 0.048 & 0.052 & -- & 0.276 & 0.423 & 16.3 & 0.425 & 15.6 & -- \\
\hline 9/8/2008 3:00 PM & 0.143 & 16.9 & 0.112 & 0.325 & 18.4 & 0.41 & 0.048 & 0.052 & -- & 0.233 & 0.422 & 16.3 & 0.424 & 15.6 & -- \\
\hline 9/8/2008 4:00 PM & 0.142 & 16.9 & 0.112 & 0.325 & 18.4 & 0.41 & 0.048 & 0.052 & -- & 0.267 & 0.422 & 16.3 & 0.424 & 15.6 & -- \\
\hline 9/8/2008 5:00 PM & 0.142 & 16.8 & 0.112 & 0.325 & 18.4 & 0.40 & 0.048 & 0.052 & -- & 0.268 & 0.422 & 16.3 & 0.424 & 15.6 & -- \\
\hline 9/8/2008 6:00 PM & 0.141 & 16.8 & 0.111 & 0.324 & 18.3 & 0.41 & 0.048 & 0.052 & -- & 0.198 & 0.422 & 16.3 & 0.424 & 15.6 & -- \\
\hline 9/8/2008 7:00 PM & 0.141 & 16.8 & 0.111 & 0.324 & 18.3 & 0.40 & 0.048 & 0.052 & -- & 0.122 & 0.422 & 16.3 & 0.424 & 15.6 & -- \\
\hline 9/8/2008 8:00 PM & 0.141 & 16.8 & 0.111 & 0.324 & 18.3 & 0.40 & 0.048 & 0.052 & -- & 0.108 & 0.423 & 16.3 & 0.424 & 15.6 & -- \\
\hline 9/8/2008 9:00 PM & 0.141 & 16.7 & 0.111 & 0.324 & 18.3 & 0.40 & 0.048 & 0.052 & -- & 0.091 & 0.423 & 16.3 & 0.424 & 15.6 & -- \\
\hline 9/8/2008 10:00 PM & 0.140 & 16.7 & 0.111 & 0.324 & 18.3 & 0.40 & 0.048 & 0.052 & -- & 0.106 & 0.423 & 16.3 & 0.424 & 15.6 & -- \\
\hline 9/8/2008 11:00 PM & 0.140 & 16.6 & 0.110 & 0.324 & 18.3 & 0.40 & 0.048 & 0.052 & -- & 0.132 & 0.423 & 16.3 & 0.423 & 15.6 & -- \\
\hline 9/9/2008 12:00 AM & 0.140 & 16.7 & 0.110 & 0.324 & 18.3 & 0.40 & 0.048 & 0.052 & -- & 0.148 & 0.423 & 16.3 & 0.423 & 15.6 & -- \\
\hline 9/9/2008 1:00 AM & 0.140 & 16.6 & 0.110 & 0.323 & 18.3 & 0.40 & 0.048 & 0.052 & -- & 0.152 & 0.423 & 16.3 & 0.423 & 15.6 & -- \\
\hline 9/9/2008 2:00 AM & 0.138 & 16.6 & 0.110 & 0.323 & 18.3 & 0.40 & 0.048 & 0.052 & -- & 0.141 & 0.423 & 16.3 & 0.423 & 15.6 & -- \\
\hline 9/9/2008 3:00 AM & 0.138 & 16.6 & 0.110 & 0.323 & 18.3 & 0.40 & 0.048 & 0.052 & -- & 0.174 & 0.423 & 16.3 & 0.422 & 15.6 & -- \\
\hline 9/9/2008 4:00 AM & 0.138 & 16.6 & 0.109 & 0.323 & 18.3 & 0.40 & 0.047 & 0.052 & -- & 0.171 & 0.423 & 16.3 & 0.422 & 15.6 & -- \\
\hline 9/9/2008 5:00 AM & 0.137 & 16.6 & 0.109 & 0.323 & 18.3 & 0.40 & 0.047 & 0.053 & -- & 0.151 & 0.423 & 16.3 & 0.422 & 15.6 & -- \\
\hline 9/9/2008 6:00 AM & 0.137 & 16.6 & 0.109 & 0.323 & 18.3 & 0.40 & 0.047 & 0.053 & -- & 0.142 & 0.423 & 16.3 & 0.422 & 15.6 & -- \\
\hline 9/9/2008 7:00 AM & 0.137 & 16.6 & 0.109 & 0.323 & 18.3 & 0.40 & 0.047 & 0.053 & -- & 0.120 & 0.423 & 16.3 & 0.423 & 15.6 & -- \\
\hline 9/9/2008 8:00 AM & 0.137 & 16.6 & 0.109 & 0.323 & 18.3 & 0.40 & 0.047 & 0.053 & -- & 0.132 & 0.423 & 16.3 & 0.423 & 15.6 & -- \\
\hline 9/9/2008 9:00 AM & 0.137 & 16.5 & 0.109 & 0.323 & 18.3 & 0.40 & 0.047 & 0.053 & -- & 0.116 & 0.423 & 16.3 & 0.423 & 15.6 & -- \\
\hline 9/9/2008 10:00 AM & 0.136 & 16.5 & 0.109 & 0.323 & 18.3 & 0.40 & 0.047 & 0.053 & -- & 0.115 & 0.422 & 16.3 & 0.422 & 15.6 & -- \\
\hline 9/9/2008 11:00 AM & 0.136 & 16.5 & 0.109 & 0.322 & 18.2 & 0.40 & 0.047 & 0.053 & -- & 0.132 & 0.422 & 16.3 & 0.423 & 15.6 & -- \\
\hline 9/9/2008 12:00 PM & 0.135 & 16.4 & 0.109 & 0.322 & 18.2 & 0.40 & 0.047 & 0.053 & -- & 0.183 & 0.422 & 16.3 & 0.424 & 15.6 & -- \\
\hline 9/9/2008 1:00 PM & 0.135 & 16.4 & 0.109 & 0.322 & 18.2 & 0.40 & 0.047 & 0.053 & -- & 0.138 & 0.421 & 16.3 & 0.425 & 15.6 & -- \\
\hline 9/9/2008 2:00 PM & 0.135 & 16.4 & 0.109 & 0.322 & 18.2 & 0.40 & 0.047 & 0.053 & -- & 0.120 & 0.422 & 16.3 & 0.425 & 15.6 & -- \\
\hline 9/9/2008 3:00 PM & 0.135 & 16.4 & 0.109 & 0.322 & 18.2 & 0.40 & 0.047 & 0.053 & -- & 0.131 & 0.422 & 16.3 & 0.426 & 15.6 & -- \\
\hline 9/9/2008 4:00 PM & 0.135 & 16.4 & 0.108 & 0.322 & 18.2 & 0.40 & 0.047 & 0.053 & -- & 0.121 & 0.421 & 16.3 & 0.425 & 15.6 & -- \\
\hline 9/9/2008 5:00 PM & 0.134 & 16.3 & 0.108 & 0.322 & 18.2 & 0.40 & 0.047 & 0.053 & -- & 0.145 & 0.422 & 16.3 & 0.425 & 15.6 & -- \\
\hline 9/9/2008 6:00 PM & 0.134 & 16.3 & 0.108 & 0.322 & 18.2 & 0.40 & 0.047 & 0.053 & -- & 0.152 & 0.421 & 16.3 & 0.424 & 15.6 & -- \\
\hline 9/9/2008 7:00 PM & 0.134 & 16.3 & 0.108 & 0.322 & 18.2 & 0.40 & 0.047 & 0.052 & -- & 0.186 & 0.421 & 16.3 & 0.423 & 15.6 & -- \\
\hline 9/9/2008 8:00 PM & 0.134 & 16.3 & 0.107 & 0.322 & 18.2 & 0.40 & 0.047 & 0.052 & -- & 0.121 & 0.421 & 16.3 & 0.422 & 15.6 & -- \\
\hline 9/9/2008 9:00 PM & 0.133 & 16.3 & 0.107 & 0.321 & 18.2 & 0.40 & 0.047 & 0.052 & -- & 0.103 & 0.421 & 16.3 & 0.423 & 15.6 & -- \\
\hline 9/9/2008 10:00 PM & 0.133 & 16.3 & 0.107 & 0.321 & 18.2 & 0.40 & 0.047 & 0.052 & -- & 0.096 & 0.422 & 16.3 & 0.422 & 15.6 & -- \\
\hline
\end{tabular}


Appendix 1. Volumetric water content, temperature, and electrical-conductivity data collected at the flood-irrigated site during 2008.-Continued

[Depth in feet below land surface; volumetric water content dimensionless; temperature in degrees Celsius; bulk electrical conductivity in decisiemens per meter; --, no data]

\begin{tabular}{|c|c|c|c|c|c|c|c|c|c|c|c|c|c|c|c|}
\hline \multirow[b]{3}{*}{$\begin{array}{l}\text { Measurement } \\
\text { date and time }\end{array}$} & \multicolumn{15}{|c|}{ Depth } \\
\hline & \multicolumn{2}{|c|}{2} & \multirow[b]{2}{*}{$\begin{array}{c}3 \\
\begin{array}{c}\text { Volumetric } \\
\text { water } \\
\text { content }\end{array}\end{array}$} & \multicolumn{3}{|c|}{4} & \multirow{2}{*}{$\begin{array}{c}6 \\
\begin{array}{c}\text { Volumetric } \\
\text { water } \\
\text { content }\end{array}\end{array}$} & \multicolumn{2}{|c|}{8} & \multirow{2}{*}{$\begin{array}{c}10 \\
\text { Volumetric } \\
\text { water } \\
\text { content }\end{array}$} & \multicolumn{2}{|c|}{12} & \multicolumn{2}{|c|}{15} & \multirow{2}{*}{$\begin{array}{c}18.5 \\
\text { Volumetric } \\
\text { water } \\
\text { content }\end{array}$} \\
\hline & $\begin{array}{c}\text { Volumetric } \\
\text { water } \\
\text { content }\end{array}$ & $\begin{array}{l}\text { Temper- } \\
\text { ature }\end{array}$ & & $\begin{array}{c}\text { Volumetric } \\
\text { water } \\
\text { content }\end{array}$ & $\begin{array}{l}\text { Temper- } \\
\text { ature }\end{array}$ & $\begin{array}{c}\text { Bulk } \\
\text { electrical } \\
\text { conduct- } \\
\text { ivity }\end{array}$ & & $\begin{array}{c}\text { Volumetric } \\
\text { water } \\
\text { content }\end{array}$ & $\begin{array}{l}\text { Temper- } \\
\text { ature }\end{array}$ & & $\begin{array}{c}\text { Volumetric } \\
\text { water } \\
\text { content }\end{array}$ & $\begin{array}{l}\text { Temper- } \\
\text { ature }\end{array}$ & $\begin{array}{c}\text { Volumetric } \\
\text { water } \\
\text { content }\end{array}$ & $\begin{array}{l}\text { Temper- } \\
\text { ature }\end{array}$ & \\
\hline 9/9/2008 11:00 PM & 0.133 & 16.3 & 0.107 & 0.321 & 18.2 & 0.40 & 0.047 & 0.052 & -- & 0.086 & 0.422 & 16.3 & 0.422 & 15.6 & $\overline{--}$ \\
\hline 9/10/2008 12:00 AM & 0.133 & 16.3 & 0.107 & 0.321 & 18.2 & 0.40 & 0.047 & 0.052 & -- & 0.075 & 0.422 & 16.3 & 0.422 & 15.6 & -- \\
\hline 9/10/2008 1:00 AM & 0.133 & 16.3 & 0.107 & 0.321 & 18.1 & 0.40 & 0.047 & 0.052 & -- & 0.070 & 0.422 & 16.3 & 0.422 & 15.5 & -- \\
\hline 9/10/2008 2:00 AM & 0.133 & 16.3 & 0.107 & 0.321 & 18.1 & 0.40 & 0.047 & 0.052 & -- & 0.068 & 0.422 & 16.3 & 0.422 & 15.5 & -- \\
\hline 9/10/2008 3:00 AM & 0.133 & 16.3 & 0.106 & 0.320 & 18.1 & 0.40 & 0.047 & 0.052 & -- & 0.068 & 0.422 & 16.3 & 0.422 & 15.5 & -- \\
\hline 9/10/2008 4:00 AM & 0.133 & 16.3 & 0.106 & 0.321 & 18.1 & 0.40 & 0.047 & 0.053 & -- & 0.067 & 0.423 & 16.3 & 0.423 & 15.6 & -- \\
\hline 9/10/2008 5:00 AM & 0.132 & 16.3 & 0.106 & 0.321 & 18.1 & 0.40 & 0.047 & 0.053 & -- & 0.067 & 0.423 & 16.3 & 0.423 & 15.5 & -- \\
\hline 9/10/2008 6:00 AM & 0.132 & 16.4 & 0.106 & 0.321 & 18.1 & 0.40 & 0.047 & 0.053 & -- & 0.066 & 0.423 & 16.3 & 0.423 & 15.5 & -- \\
\hline 9/10/2008 7:00 AM & 0.132 & 16.4 & 0.106 & 0.320 & 18.1 & 0.40 & 0.047 & 0.053 & -- & 0.066 & 0.423 & 16.3 & 0.423 & 15.5 & -- \\
\hline 9/10/2008 8:00 AM & 0.132 & 16.4 & 0.106 & 0.320 & 18.1 & 0.40 & 0.047 & 0.052 & -- & 0.065 & 0.423 & 16.3 & 0.423 & 15.5 & -- \\
\hline 9/10/2008 9:00 AM & 0.132 & 16.4 & 0.105 & 0.320 & 18.1 & 0.40 & 0.047 & 0.052 & -- & 0.065 & 0.423 & 16.3 & 0.422 & 15.5 & -- \\
\hline 9/10/2008 10:00 AM & 0.131 & 16.4 & 0.105 & 0.320 & 18.1 & 0.40 & 0.047 & 0.052 & -- & 0.065 & 0.422 & 16.3 & 0.422 & 15.5 & -- \\
\hline 9/10/2008 11:00 AM & 0.131 & 16.4 & 0.105 & 0.320 & 18.1 & 0.40 & 0.047 & 0.052 & -- & 0.064 & 0.422 & 16.3 & 0.423 & 15.5 & -- \\
\hline 9/10/2008 12:00 PM & 0.131 & 16.3 & 0.105 & 0.320 & 18.1 & 0.40 & 0.046 & 0.052 & -- & 0.064 & 0.422 & 16.3 & 0.425 & 15.5 & -- \\
\hline 9/10/2008 1:00 PM & 0.131 & 16.3 & 0.105 & 0.320 & 18.1 & 0.40 & 0.046 & 0.052 & -- & 0.064 & 0.422 & 16.3 & 0.425 & 15.5 & -- \\
\hline 9/10/2008 2:00 PM & 0.131 & 16.3 & 0.105 & 0.320 & 18.0 & 0.40 & 0.046 & 0.052 & -- & 0.064 & 0.422 & 16.3 & 0.425 & 15.6 & -- \\
\hline 9/10/2008 3:00 PM & 0.130 & 16.3 & 0.105 & 0.320 & 18.0 & 0.40 & 0.046 & 0.052 & -- & 0.064 & 0.422 & 16.3 & 0.425 & 15.6 & -- \\
\hline 9/10/2008 4:00 PM & 0.130 & 16.3 & 0.105 & 0.320 & 18.0 & 0.40 & 0.046 & 0.052 & -- & 0.064 & 0.422 & 16.3 & 0.425 & 15.5 & -- \\
\hline 9/10/2008 5:00 PM & 0.130 & 16.3 & 0.105 & 0.320 & 18.0 & 0.40 & 0.046 & 0.052 & -- & 0.064 & 0.422 & 16.3 & 0.425 & 15.5 & -- \\
\hline 9/10/2008 6:00 PM & 0.130 & 16.3 & 0.104 & 0.320 & 18.0 & 0.40 & 0.046 & 0.052 & -- & 0.065 & 0.422 & 16.3 & 0.425 & 15.6 & -- \\
\hline 9/10/2008 7:00 PM & 0.130 & 16.3 & 0.104 & 0.320 & 18.0 & 0.40 & 0.046 & 0.052 & -- & 0.066 & 0.422 & 16.3 & 0.424 & 15.6 & -- \\
\hline 9/10/2008 8:00 PM & 0.129 & 16.4 & 0.104 & 0.319 & 18.0 & 0.40 & 0.046 & 0.052 & -- & 0.068 & 0.422 & 16.3 & 0.423 & 15.6 & -- \\
\hline 9/10/2008 9:00 PM & 0.130 & 16.3 & 0.104 & 0.319 & 18.0 & 0.40 & 0.046 & 0.052 & -- & 0.068 & 0.422 & 16.3 & 0.422 & 15.5 & -- \\
\hline 9/10/2008 10:00 PM & 0.130 & 16.3 & 0.104 & 0.319 & 18.0 & 0.40 & 0.046 & 0.052 & -- & 0.068 & 0.422 & 16.4 & 0.422 & 15.5 & -- \\
\hline 9/10/2008 11:00 PM & 0.130 & 16.3 & 0.104 & 0.319 & 18.0 & 0.40 & 0.046 & 0.052 & -- & 0.068 & 0.423 & 16.3 & 0.421 & 15.5 & -- \\
\hline 9/11/2008 12:00 AM & 0.130 & 16.3 & 0.104 & 0.319 & 18.0 & 0.40 & 0.046 & 0.052 & -- & 0.068 & 0.423 & 16.3 & 0.422 & 15.6 & -- \\
\hline 9/11/2008 1:00 AM & 0.129 & 16.4 & 0.104 & 0.319 & 18.0 & 0.40 & 0.046 & 0.051 & -- & 0.068 & 0.424 & 16.4 & 0.422 & 15.5 & -- \\
\hline 9/11/2008 2:00 AM & 0.129 & 16.4 & 0.104 & 0.319 & 18.0 & 0.40 & 0.046 & 0.051 & -- & 0.065 & 0.424 & 16.3 & 0.422 & 15.6 & -- \\
\hline 9/11/2008 3:00 AM & 0.129 & 16.4 & 0.104 & 0.319 & 18.0 & 0.40 & 0.046 & 0.052 & -- & 0.065 & 0.424 & 16.3 & 0.422 & 15.5 & -- \\
\hline 9/11/2008 4:00 AM & 0.129 & 16.5 & 0.104 & 0.318 & 18.0 & 0.40 & 0.046 & 0.051 & -- & 0.064 & 0.424 & 16.3 & 0.422 & 15.5 & -- \\
\hline 9/11/2008 5:00 AM & 0.129 & 16.5 & 0.104 & 0.318 & 18.0 & 0.40 & 0.046 & 0.051 & -- & 0.064 & 0.424 & 16.3 & 0.422 & 15.5 & -- \\
\hline 9/11/2008 6:00 AM & 0.129 & 16.5 & 0.104 & 0.318 & 17.9 & 0.40 & 0.046 & 0.052 & -- & 0.064 & 0.424 & 16.3 & 0.422 & 15.6 & -- \\
\hline 9/11/2008 7:00 AM & 0.129 & 16.5 & 0.104 & 0.318 & 17.9 & 0.40 & 0.046 & 0.052 & -- & 0.064 & 0.424 & 16.3 & 0.422 & 15.6 & -- \\
\hline 9/11/2008 8:00 AM & 0.128 & 16.5 & 0.104 & 0.318 & 17.9 & 0.40 & 0.046 & 0.051 & -- & 0.064 & 0.424 & 16.3 & 0.422 & 15.6 & -- \\
\hline 9/11/2008 9:00 AM & 0.129 & 16.5 & 0.103 & 0.319 & 17.9 & 0.40 & 0.046 & 0.051 & -- & 0.064 & 0.424 & 16.3 & 0.422 & 15.6 & -- \\
\hline 9/11/2008 10:00 AM & 0.129 & 16.5 & 0.103 & 0.318 & 17.9 & 0.40 & 0.046 & 0.051 & -- & 0.063 & 0.424 & 16.4 & 0.422 & 15.6 & -- \\
\hline 9/11/2008 11:00 AM & 0.129 & 16.5 & 0.103 & 0.318 & 17.9 & 0.40 & 0.045 & 0.051 & -- & 0.064 & 0.424 & 16.3 & 0.422 & 15.6 & -- \\
\hline 9/11/2008 12:00 PM & 0.128 & 16.5 & 0.103 & 0.318 & 17.9 & 0.40 & 0.045 & 0.051 & -- & 0.063 & 0.424 & 16.3 & 0.423 & 15.6 & -- \\
\hline 9/11/2008 1:00 PM & 0.128 & 16.5 & 0.103 & 0.318 & 17.9 & 0.40 & 0.045 & 0.051 & -- & 0.064 & 0.423 & 16.4 & 0.423 & 15.6 & -- \\
\hline 9/11/2008 2:00 PM & 0.128 & 16.4 & 0.103 & 0.318 & 17.9 & 0.40 & 0.045 & 0.051 & -- & 0.064 & 0.423 & 16.3 & 0.424 & 15.6 & -- \\
\hline
\end{tabular}


Appendix 1. Volumetric water content, temperature, and electrical-conductivity data collected at the flood-irrigated site during 2008.-Continued

[Depth in feet below land surface; volumetric water content dimensionless; temperature in degrees Celsius; bulk electrical conductivity in decisiemens per meter; --, no data]

\begin{tabular}{|c|c|c|c|c|c|c|c|c|c|c|c|c|c|c|c|}
\hline \multirow[b]{3}{*}{$\begin{array}{l}\text { Measurement } \\
\text { date and time }\end{array}$} & \multicolumn{15}{|c|}{ Depth } \\
\hline & \multicolumn{2}{|c|}{2} & \multirow{2}{*}{$\begin{array}{c}3 \\
\text { Volumetric } \\
\text { water } \\
\text { content }\end{array}$} & \multicolumn{3}{|c|}{4} & \multirow{2}{*}{$\begin{array}{c}6 \\
\begin{array}{c}\text { Volumetric } \\
\text { water } \\
\text { content }\end{array}\end{array}$} & \multicolumn{2}{|l|}{8} & \multirow{2}{*}{$\begin{array}{c}10 \\
\text { Volumetric } \\
\text { water } \\
\text { content }\end{array}$} & \multicolumn{2}{|c|}{12} & \multicolumn{2}{|c|}{15} & \multirow{2}{*}{$\begin{array}{c}18.5 \\
\text { Volumetric } \\
\text { water } \\
\text { content }\end{array}$} \\
\hline & $\begin{array}{l}\text { Volumetric } \\
\text { water } \\
\text { content }\end{array}$ & $\begin{array}{l}\text { Temper- } \\
\text { ature }\end{array}$ & & $\begin{array}{c}\text { Volumetric } \\
\text { water } \\
\text { content }\end{array}$ & $\begin{array}{l}\text { Temper- } \\
\text { ature }\end{array}$ & $\begin{array}{c}\text { Bulk } \\
\text { electrical } \\
\text { conduct- } \\
\text { ivity } \\
\end{array}$ & & $\begin{array}{l}\text { Volumetric } \\
\text { water } \\
\text { content }\end{array}$ & $\begin{array}{l}\text { Temper- } \\
\text { ature }\end{array}$ & & $\begin{array}{l}\text { Volumetric } \\
\text { water } \\
\text { content }\end{array}$ & $\begin{array}{l}\text { Temper- } \\
\text { ature }\end{array}$ & $\begin{array}{l}\text { Volumetric } \\
\text { water } \\
\text { content }\end{array}$ & $\begin{array}{l}\text { Temper- } \\
\text { ature }\end{array}$ & \\
\hline 9/11/2008 3:00 PM & 0.126 & 16.4 & 0.103 & 0.318 & 17.9 & 0.40 & 0.045 & 0.051 & -- & 0.064 & 0.423 & 16.3 & 0.425 & 15.5 & -- \\
\hline 9/11/2008 4:00 PM & 0.126 & 16.4 & 0.103 & 0.318 & 17.9 & 0.40 & 0.045 & 0.051 & -- & 0.063 & 0.423 & 16.3 & 0.425 & 15.6 & -- \\
\hline 9/11/2008 5:00 PM & 0.126 & 16.5 & 0.103 & 0.318 & 17.9 & 0.40 & 0.045 & 0.050 & -- & 0.063 & 0.423 & 16.3 & 0.424 & 15.6 & -- \\
\hline 9/11/2008 6:00 PM & 0.126 & 16.4 & 0.103 & 0.318 & 17.9 & 0.40 & 0.045 & 0.050 & -- & 0.063 & 0.424 & 16.3 & 0.423 & 15.6 & -- \\
\hline 9/11/2008 7:00 PM & 0.126 & 16.4 & 0.103 & 0.317 & 17.9 & 0.40 & 0.045 & 0.050 & -- & 0.063 & 0.424 & 16.3 & 0.423 & 15.6 & -- \\
\hline 9/11/2008 8:00 PM & 0.126 & 16.4 & 0.102 & 0.317 & 17.9 & 0.40 & 0.045 & 0.050 & -- & 0.063 & 0.424 & 16.3 & 0.423 & 15.6 & -- \\
\hline 9/11/2008 9:00 PM & 0.126 & 16.4 & 0.102 & 0.317 & 17.9 & 0.40 & 0.045 & 0.050 & -- & 0.062 & 0.425 & 16.3 & 0.422 & 15.6 & -- \\
\hline 9/11/2008 10:00 PM & 0.128 & 16.4 & 0.102 & 0.317 & 17.9 & 0.40 & 0.045 & 0.050 & -- & 0.062 & 0.425 & 16.3 & 0.422 & 15.6 & -- \\
\hline 9/11/2008 11:00 PM & 0.126 & 16.4 & 0.102 & 0.317 & 17.9 & 0.40 & 0.045 & 0.050 & -- & 0.062 & 0.425 & 16.4 & 0.422 & 15.6 & -- \\
\hline 9/12/2008 12:00 AM & 0.126 & 16.4 & 0.102 & 0.317 & 17.9 & 0.40 & 0.045 & 0.050 & -- & 0.062 & 0.425 & 16.4 & 0.422 & 15.6 & -- \\
\hline 9/12/2008 1:00 AM & 0.126 & 16.4 & 0.102 & 0.317 & 17.9 & 0.40 & 0.045 & 0.050 & -- & 0.062 & 0.425 & 16.4 & 0.422 & 15.6 & -- \\
\hline 9/12/2008 2:00 AM & 0.126 & 16.4 & 0.102 & 0.317 & 17.9 & 0.40 & 0.045 & 0.050 & -- & 0.062 & 0.425 & 16.3 & 0.422 & 15.6 & -- \\
\hline 9/12/2008 3:00 AM & 0.126 & 16.4 & 0.102 & 0.317 & 17.9 & 0.40 & 0.045 & 0.050 & -- & 0.062 & 0.425 & 16.3 & 0.422 & 15.6 & -- \\
\hline 9/12/2008 4:00 AM & 0.126 & 16.4 & 0.102 & 0.316 & 17.9 & 0.40 & 0.045 & 0.050 & -- & 0.061 & 0.425 & 16.3 & 0.422 & 15.6 & -- \\
\hline 9/12/2008 5:00 AM & 0.126 & 16.4 & 0.102 & 0.316 & 17.9 & 0.40 & 0.045 & 0.050 & -- & 0.060 & 0.425 & 16.4 & 0.422 & 15.6 & -- \\
\hline 9/12/2008 6:00 AM & 0.126 & 16.4 & 0.101 & 0.316 & 17.8 & 0.40 & 0.045 & 0.050 & -- & 0.060 & 0.425 & 16.4 & 0.422 & 15.6 & -- \\
\hline 9/12/2008 7:00 AM & 0.126 & 16.3 & 0.101 & 0.316 & 17.8 & 0.40 & 0.045 & 0.050 & -- & 0.060 & 0.425 & 16.3 & 0.422 & 15.6 & -- \\
\hline 9/12/2008 8:00 AM & 0.126 & 16.3 & 0.101 & 0.316 & 17.8 & 0.40 & 0.045 & 0.050 & -- & 0.060 & 0.425 & 16.3 & 0.423 & 15.6 & -- \\
\hline 9/12/2008 9:00 AM & 0.125 & 16.3 & 0.101 & 0.316 & 17.8 & 0.40 & 0.045 & 0.050 & -- & 0.059 & 0.425 & 16.4 & 0.423 & 15.6 & -- \\
\hline 9/12/2008 10:00 AM & 0.126 & 16.3 & 0.101 & 0.316 & 17.8 & 0.40 & 0.045 & 0.050 & -- & 0.059 & 0.425 & 16.3 & 0.423 & 15.6 & -- \\
\hline 9/12/2008 11:00 AM & 0.131 & 16.2 & 0.101 & 0.316 & 17.8 & 0.40 & 0.045 & 0.050 & -- & 0.059 & 0.425 & 16.3 & 0.423 & 15.6 & -- \\
\hline 9/12/2008 12:00 PM & 0.135 & 16.2 & 0.101 & 0.316 & 17.8 & 0.40 & 0.045 & 0.050 & -- & 0.059 & 0.425 & 16.3 & 0.423 & 15.6 & -- \\
\hline 9/12/2008 1:00 PM & 0.142 & 16.2 & 0.101 & 0.316 & 17.8 & 0.40 & 0.045 & 0.049 & -- & 0.059 & 0.425 & 16.4 & 0.423 & 15.6 & -- \\
\hline 9/12/2008 2:00 PM & 0.149 & 16.2 & 0.101 & 0.316 & 17.8 & 0.40 & 0.044 & 0.049 & -- & 0.058 & 0.425 & 16.4 & 0.423 & 15.6 & -- \\
\hline 9/12/2008 3:00 PM & 0.155 & 16.2 & 0.101 & 0.316 & 17.8 & 0.40 & 0.044 & 0.049 & -- & 0.058 & 0.424 & 16.3 & 0.423 & 15.6 & -- \\
\hline 9/12/2008 4:00 PM & 0.158 & 16.2 & 0.102 & 0.316 & 17.8 & 0.40 & 0.044 & 0.049 & -- & 0.059 & 0.424 & 16.3 & 0.424 & 15.6 & -- \\
\hline 9/12/2008 5:00 PM & 0.159 & 16.1 & 0.103 & 0.316 & 17.8 & 0.40 & 0.044 & 0.049 & -- & 0.058 & 0.424 & 16.4 & 0.424 & 15.6 & -- \\
\hline 9/12/2008 6:00 PM & 0.159 & 16.1 & 0.104 & 0.316 & 17.8 & 0.40 & 0.044 & 0.049 & -- & 0.058 & 0.424 & 16.3 & 0.423 & 15.6 & -- \\
\hline 9/12/2008 7:00 PM & 0.159 & 16.0 & 0.104 & 0.316 & 17.8 & 0.40 & 0.044 & 0.049 & -- & 0.058 & 0.425 & 16.3 & 0.422 & 15.6 & -- \\
\hline 9/12/2008 8:00 PM & 0.158 & 16.0 & 0.105 & 0.316 & 17.8 & 0.40 & 0.044 & 0.049 & -- & 0.058 & 0.425 & 16.3 & 0.422 & 15.6 & -- \\
\hline 9/12/2008 9:00 PM & 0.158 & 16.0 & 0.106 & 0.316 & 17.8 & 0.40 & 0.044 & 0.049 & -- & 0.058 & 0.425 & 16.4 & 0.421 & 15.6 & -- \\
\hline 9/12/2008 10:00 PM & 0.157 & 16.0 & 0.106 & 0.316 & 17.8 & 0.40 & 0.044 & 0.049 & -- & 0.058 & 0.425 & 16.4 & 0.421 & 15.6 & -- \\
\hline 9/12/2008 11:00 PM & 0.156 & 16.0 & 0.107 & 0.316 & 17.8 & 0.40 & 0.044 & 0.049 & -- & 0.058 & 0.425 & 16.3 & 0.421 & 15.6 & -- \\
\hline 9/13/2008 12:00 AM & 0.156 & 15.9 & 0.108 & 0.316 & 17.8 & 0.40 & 0.044 & 0.049 & -- & 0.058 & 0.425 & 16.3 & 0.422 & 15.6 & -- \\
\hline 9/13/2008 1:00 AM & 0.155 & 16.0 & 0.108 & 0.316 & 17.8 & 0.40 & 0.044 & 0.049 & -- & 0.058 & 0.425 & 16.3 & 0.422 & 15.6 & -- \\
\hline 9/13/2008 2:00 AM & 0.155 & 16.0 & 0.109 & 0.317 & 17.8 & 0.40 & 0.044 & 0.049 & -- & 0.058 & 0.425 & 16.3 & 0.422 & 15.6 & -- \\
\hline 9/13/2008 3:00 AM & 0.154 & 15.9 & 0.109 & 0.317 & 17.8 & 0.40 & 0.044 & 0.049 & -- & 0.058 & 0.424 & 16.3 & 0.422 & 15.6 & -- \\
\hline 9/13/2008 4:00 AM & 0.154 & 15.9 & 0.109 & 0.316 & 17.8 & 0.40 & 0.044 & 0.049 & -- & 0.058 & 0.424 & 16.3 & 0.422 & 15.6 & -- \\
\hline 9/13/2008 5:00 AM & 0.153 & 15.9 & 0.109 & 0.317 & 17.8 & 0.40 & 0.044 & 0.049 & -- & 0.058 & 0.424 & 16.3 & 0.422 & 15.6 & -- \\
\hline 9/13/2008 6:00 AM & 0.153 & 15.9 & 0.109 & 0.317 & 17.7 & 0.40 & 0.044 & 0.049 & -- & 0.058 & 0.424 & 16.3 & 0.422 & 15.6 & -- \\
\hline
\end{tabular}


Appendix 1. Volumetric water content, temperature, and electrical-conductivity data collected at the flood-irrigated site during 2008.-Continued

[Depth in feet below land surface; volumetric water content dimensionless; temperature in degrees Celsius; bulk electrical conductivity in decisiemens per meter; --, no data]

\begin{tabular}{|c|c|c|c|c|c|c|c|c|c|c|c|c|c|c|c|}
\hline \multirow[b]{3}{*}{$\begin{array}{l}\text { Measurement } \\
\text { date and time }\end{array}$} & \multicolumn{15}{|c|}{ Depth } \\
\hline & \multicolumn{2}{|c|}{2} & \multirow[b]{2}{*}{$\begin{array}{c}3 \\
\begin{array}{c}\text { Volumetric } \\
\text { water } \\
\text { content }\end{array}\end{array}$} & \multicolumn{3}{|c|}{4} & \multirow{2}{*}{$\begin{array}{c}6 \\
\text { Volumetric } \\
\text { water } \\
\text { content }\end{array}$} & \multicolumn{2}{|c|}{8} & \multirow{2}{*}{$\begin{array}{c}10 \\
\text { Volumetric } \\
\text { water } \\
\text { content }\end{array}$} & \multicolumn{2}{|c|}{12} & \multicolumn{2}{|c|}{15} & \multirow{2}{*}{$\begin{array}{c}18.5 \\
\text { Volumetric } \\
\text { water } \\
\text { content }\end{array}$} \\
\hline & $\begin{array}{c}\text { Volumetric } \\
\text { water } \\
\text { content }\end{array}$ & $\begin{array}{l}\text { Temper- } \\
\text { ature }\end{array}$ & & $\begin{array}{c}\text { Volumetric } \\
\text { water } \\
\text { content }\end{array}$ & $\begin{array}{l}\text { Temper- } \\
\text { ature }\end{array}$ & $\begin{array}{c}\text { Bulk } \\
\text { electrical } \\
\text { conduct- } \\
\text { ivity }\end{array}$ & & $\begin{array}{c}\text { Volumetric } \\
\text { water } \\
\text { content }\end{array}$ & $\begin{array}{l}\text { Temper- } \\
\text { ature }\end{array}$ & & $\begin{array}{c}\text { Volumetric } \\
\text { water } \\
\text { content }\end{array}$ & $\begin{array}{l}\text { Temper- } \\
\text { ature }\end{array}$ & $\begin{array}{c}\text { Volumetric } \\
\text { water } \\
\text { content }\end{array}$ & $\begin{array}{l}\text { Temper- } \\
\text { ature }\end{array}$ & \\
\hline 9/13/2008 7:00 AM & 0.151 & 15.9 & 0.109 & 0.317 & 17.7 & 0.40 & 0.044 & 0.049 & -- & 0.058 & 0.424 & 16.3 & 0.422 & 15.6 & $\overline{--}$ \\
\hline 9/13/2008 8:00 AM & 0.151 & 15.8 & 0.109 & 0.317 & 17.7 & 0.40 & 0.044 & 0.049 & -- & 0.059 & 0.424 & 16.3 & 0.422 & 15.6 & -- \\
\hline 9/13/2008 9:00 AM & 0.151 & 15.8 & 0.110 & 0.318 & 17.7 & 0.40 & 0.044 & 0.049 & -- & 0.059 & 0.425 & 16.3 & 0.422 & 15.6 & -- \\
\hline 9/13/2008 10:00 AM & 0.150 & 15.9 & 0.109 & 0.317 & 17.7 & 0.40 & 0.044 & 0.049 & -- & 0.059 & 0.425 & 16.3 & 0.421 & 15.6 & -- \\
\hline 9/13/2008 11:00 AM & 0.149 & 15.8 & 0.110 & 0.318 & 17.7 & 0.40 & 0.044 & 0.048 & -- & 0.059 & 0.424 & 16.3 & 0.422 & 15.6 & -- \\
\hline 9/13/2008 12:00 PM & 0.149 & 15.8 & 0.110 & 0.318 & 17.7 & 0.40 & 0.044 & 0.048 & -- & 0.059 & 0.423 & 16.4 & 0.424 & 15.6 & -- \\
\hline 9/13/2008 1:00 PM & 0.149 & 15.8 & 0.110 & 0.318 & 17.7 & 0.40 & 0.043 & 0.049 & -- & 0.059 & 0.424 & 16.4 & 0.425 & 15.6 & -- \\
\hline 9/13/2008 2:00 PM & 0.148 & 15.8 & 0.110 & 0.318 & 17.7 & 0.40 & 0.043 & 0.049 & -- & 0.059 & 0.424 & 16.3 & 0.425 & 15.6 & -- \\
\hline 9/13/2008 3:00 PM & 0.148 & 15.7 & 0.110 & 0.318 & 17.7 & 0.40 & 0.043 & 0.049 & -- & 0.059 & 0.423 & 16.4 & 0.425 & 15.6 & -- \\
\hline 9/13/2008 4:00 PM & 0.147 & 15.7 & 0.110 & 0.318 & 17.7 & 0.40 & 0.043 & 0.049 & -- & 0.059 & 0.424 & 16.3 & 0.425 & 15.6 & -- \\
\hline 9/13/2008 5:00 PM & 0.147 & 15.7 & 0.110 & 0.319 & 17.7 & 0.40 & 0.043 & 0.049 & -- & 0.059 & 0.424 & 16.3 & 0.425 & 15.6 & -- \\
\hline 9/13/2008 6:00 PM & 0.147 & 15.7 & 0.110 & 0.318 & 17.7 & 0.40 & 0.044 & 0.048 & -- & 0.059 & 0.423 & 16.3 & 0.425 & 15.6 & -- \\
\hline 9/13/2008 7:00 PM & 0.146 & 15.7 & 0.110 & 0.319 & 17.7 & 0.40 & 0.044 & 0.048 & -- & 0.060 & 0.424 & 16.3 & 0.424 & 15.6 & -- \\
\hline 9/13/2008 8:00 PM & 0.146 & 15.6 & 0.110 & 0.318 & 17.7 & 0.40 & 0.044 & 0.048 & -- & 0.060 & 0.424 & 16.4 & 0.424 & 15.6 & -- \\
\hline 9/13/2008 9:00 PM & 0.146 & 15.6 & 0.110 & 0.318 & 17.7 & 0.40 & 0.044 & 0.048 & -- & 0.060 & 0.424 & 16.4 & 0.423 & 15.6 & -- \\
\hline 9/13/2008 10:00 PM & 0.145 & 15.6 & 0.109 & 0.319 & 17.7 & 0.40 & 0.044 & 0.048 & -- & 0.059 & 0.425 & 16.4 & 0.423 & 15.6 & -- \\
\hline 9/13/2008 11:00 PM & 0.145 & 15.6 & 0.109 & 0.319 & 17.6 & 0.40 & 0.044 & 0.048 & -- & 0.059 & 0.426 & 16.3 & 0.423 & 15.6 & -- \\
\hline 9/14/2008 12:00 AM & 0.145 & 15.6 & 0.109 & 0.319 & 17.6 & 0.40 & 0.044 & 0.048 & -- & 0.059 & 0.426 & 16.3 & 0.423 & 15.6 & -- \\
\hline 9/14/2008 1:00 AM & 0.144 & 15.6 & 0.109 & 0.319 & 17.6 & 0.40 & 0.044 & 0.048 & -- & 0.059 & 0.426 & 16.3 & 0.422 & 15.6 & -- \\
\hline 9/14/2008 2:00 AM & 0.145 & 15.6 & 0.110 & 0.319 & 17.6 & 0.40 & 0.044 & 0.048 & -- & 0.059 & 0.426 & 16.4 & 0.422 & 15.6 & -- \\
\hline 9/14/2008 3:00 AM & 0.144 & 15.6 & 0.109 & 0.319 & 17.6 & 0.40 & 0.044 & 0.048 & -- & 0.059 & 0.426 & 16.3 & 0.422 & 15.6 & -- \\
\hline 9/14/2008 4:00 AM & 0.144 & 15.6 & 0.109 & 0.319 & 17.6 & 0.40 & 0.044 & 0.048 & -- & 0.059 & 0.426 & 16.3 & 0.422 & 15.6 & -- \\
\hline 9/14/2008 5:00 AM & 0.144 & 15.6 & 0.109 & 0.319 & 17.6 & 0.40 & 0.044 & 0.048 & -- & 0.059 & 0.426 & 16.4 & 0.422 & 15.6 & -- \\
\hline 9/14/2008 6:00 AM & 0.144 & 15.6 & 0.109 & 0.319 & 17.6 & 0.40 & 0.044 & 0.048 & -- & 0.059 & 0.426 & 16.3 & 0.422 & 15.6 & -- \\
\hline 9/14/2008 7:00 AM & 0.144 & 15.6 & 0.109 & 0.319 & 17.6 & 0.40 & 0.044 & 0.048 & -- & 0.058 & 0.426 & 16.4 & 0.422 & 15.6 & -- \\
\hline 9/14/2008 8:00 AM & 0.143 & 15.7 & 0.109 & 0.319 & 17.6 & 0.40 & 0.044 & 0.048 & -- & 0.059 & 0.426 & 16.3 & 0.422 & 15.6 & -- \\
\hline 9/14/2008 9:00 AM & 0.143 & 15.7 & 0.109 & 0.319 & 17.6 & 0.40 & 0.044 & 0.048 & -- & 0.059 & 0.426 & 16.3 & 0.422 & 15.6 & -- \\
\hline 9/14/2008 10:00 AM & 0.143 & 15.7 & 0.109 & 0.319 & 17.6 & 0.40 & 0.044 & 0.048 & -- & 0.059 & 0.426 & 16.3 & 0.423 & 15.6 & -- \\
\hline 9/14/2008 11:00 AM & 0.142 & 15.7 & 0.109 & 0.319 & 17.6 & 0.40 & 0.044 & 0.048 & -- & 0.059 & 0.426 & 16.4 & 0.423 & 15.6 & -- \\
\hline 9/14/2008 12:00 PM & 0.142 & 15.7 & 0.109 & 0.319 & 17.6 & 0.40 & 0.044 & 0.048 & -- & 0.059 & 0.426 & 16.3 & 0.424 & 15.6 & -- \\
\hline 9/14/2008 1:00 PM & 0.142 & 15.7 & 0.109 & 0.319 & 17.5 & 0.40 & 0.044 & 0.048 & -- & 0.059 & 0.425 & 16.3 & 0.425 & 15.6 & -- \\
\hline 9/14/2008 2:00 PM & 0.141 & 15.7 & 0.109 & 0.319 & 17.5 & 0.40 & 0.044 & 0.048 & -- & 0.059 & 0.425 & 16.3 & 0.425 & 15.7 & -- \\
\hline 9/14/2008 3:00 PM & 0.141 & 15.7 & 0.109 & 0.319 & 17.5 & 0.40 & 0.044 & 0.048 & -- & 0.059 & 0.425 & 16.3 & 0.425 & 15.7 & -- \\
\hline 9/14/2008 4:00 PM & 0.141 & 15.7 & 0.109 & 0.319 & 17.5 & 0.40 & 0.044 & 0.048 & -- & 0.060 & 0.425 & 16.4 & 0.425 & 15.6 & -- \\
\hline 9/14/2008 5:00 PM & 0.141 & 15.7 & 0.109 & 0.319 & 17.5 & 0.40 & 0.044 & 0.048 & -- & 0.060 & 0.425 & 16.4 & 0.425 & 15.6 & -- \\
\hline 9/14/2008 6:00 PM & 0.140 & 15.7 & 0.109 & 0.319 & 17.5 & 0.40 & 0.044 & 0.048 & -- & 0.060 & 0.425 & 16.4 & 0.425 & 15.7 & -- \\
\hline 9/14/2008 7:00 PM & 0.140 & 15.6 & 0.109 & 0.319 & 17.5 & 0.40 & 0.044 & 0.048 & -- & 0.060 & 0.426 & 16.4 & 0.424 & 15.7 & -- \\
\hline 9/14/2008 8:00 PM & 0.141 & 15.6 & 0.109 & 0.319 & 17.5 & 0.40 & 0.044 & 0.048 & -- & 0.060 & 0.426 & 16.4 & 0.423 & 15.6 & -- \\
\hline 9/14/2008 9:00 PM & 0.140 & 15.5 & 0.109 & 0.319 & 17.5 & 0.40 & 0.044 & 0.048 & -- & 0.060 & 0.426 & 16.4 & 0.423 & 15.6 & -- \\
\hline 9/14/2008 10:00 PM & 0.140 & 15.6 & 0.109 & 0.318 & 17.5 & 0.40 & 0.044 & 0.048 & -- & 0.060 & 0.426 & 16.3 & 0.422 & 15.6 & -- \\
\hline
\end{tabular}


Appendix 1. Volumetric water content, temperature, and electrical-conductivity data collected at the flood-irrigated site during 2008.-Continued

[Depth in feet below land surface; volumetric water content dimensionless; temperature in degrees Celsius; bulk electrical conductivity in decisiemens per meter; --, no data]

\begin{tabular}{|c|c|c|c|c|c|c|c|c|c|c|c|c|c|c|c|}
\hline \multirow[b]{3}{*}{$\begin{array}{l}\text { Measurement } \\
\text { date and time }\end{array}$} & \multicolumn{15}{|c|}{ Depth } \\
\hline & \multicolumn{2}{|c|}{2} & \multirow{2}{*}{$\begin{array}{c}3 \\
\text { Volumetric } \\
\text { water } \\
\text { content }\end{array}$} & \multicolumn{3}{|c|}{4} & \multirow{2}{*}{$\begin{array}{c}6 \\
\begin{array}{c}\text { Volumetric } \\
\text { water } \\
\text { content }\end{array}\end{array}$} & \multicolumn{2}{|l|}{8} & \multirow{2}{*}{$\begin{array}{c}10 \\
\text { Volumetric } \\
\text { water } \\
\text { content }\end{array}$} & \multicolumn{2}{|c|}{12} & \multicolumn{2}{|c|}{15} & \multirow{2}{*}{$\begin{array}{c}18.5 \\
\text { Volumetric } \\
\text { water } \\
\text { content }\end{array}$} \\
\hline & $\begin{array}{l}\text { Volumetric } \\
\text { water } \\
\text { content }\end{array}$ & $\begin{array}{l}\text { Temper- } \\
\text { ature }\end{array}$ & & $\begin{array}{c}\text { Volumetric } \\
\text { water } \\
\text { content }\end{array}$ & $\begin{array}{l}\text { Temper- } \\
\text { ature }\end{array}$ & $\begin{array}{c}\text { Bulk } \\
\text { electrical } \\
\text { conduct- } \\
\text { ivity } \\
\end{array}$ & & $\begin{array}{l}\text { Volumetric } \\
\text { water } \\
\text { content }\end{array}$ & $\begin{array}{l}\text { Temper- } \\
\text { ature }\end{array}$ & & $\begin{array}{l}\text { Volumetric } \\
\text { water } \\
\text { content }\end{array}$ & $\begin{array}{l}\text { Temper- } \\
\text { ature }\end{array}$ & $\begin{array}{l}\text { Volumetric } \\
\text { water } \\
\text { content }\end{array}$ & $\begin{array}{l}\text { Temper- } \\
\text { ature }\end{array}$ & \\
\hline 9/14/2008 11:00 PM & 0.140 & 15.6 & 0.109 & 0.318 & 17.5 & 0.40 & 0.044 & 0.048 & -- & 0.060 & 0.426 & 16.3 & 0.422 & 15.7 & -- \\
\hline 9/15/2008 12:00 AM & 0.140 & 15.6 & 0.109 & 0.318 & 17.5 & 0.40 & 0.044 & 0.047 & -- & 0.061 & 0.426 & 16.3 & 0.422 & 15.7 & -- \\
\hline 9/15/2008 1:00 AM & 0.138 & 15.6 & 0.108 & 0.318 & 17.5 & 0.40 & 0.044 & 0.048 & -- & 0.061 & 0.426 & 16.3 & 0.422 & 15.6 & -- \\
\hline 9/15/2008 2:00 AM & 0.138 & 15.6 & 0.108 & 0.318 & 17.5 & 0.40 & 0.044 & 0.048 & -- & 0.060 & 0.425 & 16.3 & 0.422 & 15.6 & -- \\
\hline 9/15/2008 3:00 AM & 0.138 & 15.6 & 0.108 & 0.318 & 17.5 & 0.40 & 0.044 & 0.048 & -- & 0.060 & 0.425 & 16.3 & 0.422 & 15.6 & -- \\
\hline 9/15/2008 4:00 AM & 0.138 & 15.6 & 0.108 & 0.318 & 17.5 & 0.40 & 0.044 & 0.048 & -- & 0.059 & 0.425 & 16.3 & 0.422 & 15.6 & -- \\
\hline 9/15/2008 5:00 AM & 0.138 & 15.6 & 0.108 & 0.318 & 17.5 & 0.40 & 0.044 & 0.048 & -- & 0.059 & 0.425 & 16.4 & 0.422 & 15.6 & -- \\
\hline 9/15/2008 6:00 AM & 0.138 & 15.6 & 0.108 & 0.318 & 17.5 & 0.40 & 0.044 & 0.048 & -- & 0.059 & 0.425 & 16.3 & 0.422 & 15.7 & -- \\
\hline 9/15/2008 7:00 AM & 0.138 & 15.6 & 0.108 & 0.318 & 17.4 & 0.40 & 0.044 & 0.048 & -- & 0.059 & 0.425 & 16.3 & 0.422 & 15.6 & -- \\
\hline 9/15/2008 8:00 AM & 0.137 & 15.6 & 0.108 & 0.318 & 17.4 & 0.40 & 0.044 & 0.048 & -- & 0.060 & 0.425 & 16.3 & 0.422 & 15.6 & -- \\
\hline 9/15/2008 9:00 AM & 0.137 & 15.6 & 0.107 & 0.318 & 17.4 & 0.40 & 0.044 & 0.047 & -- & 0.061 & 0.426 & 16.3 & 0.422 & 15.7 & -- \\
\hline 9/15/2008 10:00 AM & 0.137 & 15.5 & 0.107 & 0.318 & 17.4 & 0.40 & 0.044 & 0.047 & -- & 0.062 & 0.426 & 16.3 & 0.423 & 15.7 & -- \\
\hline 9/15/2008 11:00 AM & 0.136 & 15.6 & 0.107 & 0.318 & 17.4 & 0.40 & 0.044 & 0.047 & -- & 0.062 & 0.426 & 16.3 & 0.425 & 15.7 & -- \\
\hline 9/15/2008 12:00 PM & 0.136 & 15.6 & 0.107 & 0.318 & 17.4 & 0.40 & 0.044 & 0.047 & -- & 0.062 & 0.425 & 16.4 & 0.426 & 15.7 & -- \\
\hline 9/15/2008 1:00 PM & 0.136 & 15.6 & 0.107 & 0.318 & 17.4 & 0.40 & 0.044 & 0.048 & -- & 0.063 & 0.425 & 16.3 & 0.426 & 15.7 & -- \\
\hline 9/15/2008 2:00 PM & 0.136 & 15.5 & 0.107 & 0.318 & 17.4 & 0.40 & 0.044 & 0.048 & -- & 0.064 & 0.425 & 16.4 & 0.426 & 15.7 & -- \\
\hline 9/15/2008 3:00 PM & 0.135 & 15.5 & 0.107 & 0.319 & 17.4 & 0.40 & 0.044 & 0.048 & -- & 0.066 & 0.425 & 16.3 & 0.426 & 15.7 & -- \\
\hline 9/15/2008 4:00 PM & 0.135 & 15.5 & 0.107 & 0.319 & 17.4 & 0.40 & 0.044 & 0.048 & -- & 0.065 & 0.425 & 16.3 & 0.426 & 15.7 & -- \\
\hline 9/15/2008 5:00 PM & 0.135 & 15.5 & 0.107 & 0.318 & 17.4 & 0.40 & 0.044 & 0.048 & -- & 0.065 & 0.425 & 16.4 & 0.426 & 15.7 & -- \\
\hline 9/15/2008 6:00 PM & 0.135 & 15.5 & 0.106 & 0.318 & 17.4 & 0.40 & 0.044 & 0.048 & -- & 0.067 & 0.425 & 16.4 & 0.425 & 15.7 & -- \\
\hline 9/15/2008 7:00 PM & 0.135 & 15.5 & 0.106 & 0.318 & 17.4 & 0.40 & 0.044 & 0.047 & -- & 0.062 & 0.425 & 16.4 & 0.424 & 15.7 & -- \\
\hline 9/15/2008 8:00 PM & 0.134 & 15.4 & 0.106 & 0.318 & 17.4 & 0.40 & 0.044 & 0.047 & -- & 0.064 & 0.426 & 16.4 & 0.423 & 15.7 & -- \\
\hline 9/15/2008 9:00 PM & 0.134 & 15.4 & 0.106 & 0.318 & 17.4 & 0.40 & 0.044 & 0.047 & -- & 0.066 & 0.426 & 16.4 & 0.422 & 15.7 & -- \\
\hline 9/15/2008 10:00 PM & 0.134 & 15.4 & 0.106 & 0.317 & 17.4 & 0.40 & 0.044 & 0.047 & -- & 0.065 & 0.426 & 16.4 & 0.422 & 15.7 & -- \\
\hline 9/15/2008 11:00 PM & 0.134 & 15.4 & 0.106 & 0.317 & 17.4 & 0.40 & 0.044 & 0.047 & -- & 0.064 & 0.426 & 16.4 & 0.422 & 15.7 & -- \\
\hline 9/16/2008 12:00 AM & 0.134 & 15.4 & 0.106 & 0.317 & 17.4 & 0.40 & 0.044 & 0.047 & -- & 0.064 & 0.426 & 16.4 & 0.422 & 15.7 & -- \\
\hline 9/16/2008 1:00 AM & 0.134 & 15.4 & 0.106 & 0.317 & 17.4 & 0.40 & 0.044 & 0.047 & -- & 0.064 & 0.426 & 16.3 & 0.422 & 15.7 & -- \\
\hline 9/16/2008 2:00 AM & 0.134 & 15.4 & 0.105 & 0.317 & 17.3 & 0.40 & 0.044 & 0.048 & -- & 0.064 & 0.426 & 16.3 & 0.422 & 15.7 & -- \\
\hline 9/16/2008 3:00 AM & 0.133 & 15.4 & 0.105 & 0.317 & 17.3 & 0.40 & 0.044 & 0.048 & -- & 0.064 & 0.425 & 16.3 & 0.422 & 15.7 & -- \\
\hline 9/16/2008 4:00 AM & 0.133 & 15.4 & 0.105 & 0.317 & 17.3 & 0.40 & 0.044 & 0.048 & -- & 0.064 & 0.425 & 16.4 & 0.422 & 15.7 & -- \\
\hline 9/16/2008 5:00 AM & 0.133 & 15.4 & 0.105 & 0.317 & 17.3 & 0.40 & 0.044 & 0.048 & -- & 0.064 & 0.426 & 16.4 & 0.422 & 15.7 & -- \\
\hline 9/16/2008 6:00 AM & 0.133 & 15.4 & 0.105 & 0.317 & 17.3 & 0.40 & 0.044 & 0.048 & -- & 0.063 & 0.426 & 16.3 & 0.422 & 15.7 & -- \\
\hline 9/16/2008 7:00 AM & 0.133 & 15.4 & 0.105 & 0.317 & 17.3 & 0.40 & 0.044 & 0.048 & -- & 0.064 & 0.426 & 16.4 & 0.422 & 15.7 & -- \\
\hline 9/16/2008 8:00 AM & 0.133 & 15.4 & 0.105 & 0.317 & 17.3 & 0.40 & 0.044 & 0.048 & -- & 0.066 & 0.426 & 16.3 & 0.422 & 15.7 & -- \\
\hline 9/16/2008 9:00 AM & 0.133 & 15.4 & 0.104 & 0.317 & 17.3 & 0.40 & 0.044 & 0.047 & -- & 0.067 & 0.426 & 16.3 & 0.422 & 15.7 & -- \\
\hline 9/16/2008 10:00 AM & 0.133 & 15.5 & 0.104 & 0.317 & 17.3 & 0.40 & 0.044 & 0.047 & -- & 0.066 & 0.426 & 16.4 & 0.422 & 15.7 & -- \\
\hline 9/16/2008 11:00 AM & 0.132 & 15.4 & 0.104 & 0.317 & 17.3 & 0.40 & 0.044 & 0.047 & -- & 0.066 & 0.426 & 16.4 & 0.424 & 15.7 & -- \\
\hline 9/16/2008 12:00 PM & 0.132 & 15.5 & 0.104 & 0.317 & 17.3 & 0.40 & 0.044 & 0.047 & -- & 0.066 & 0.425 & 16.4 & 0.425 & 15.7 & -- \\
\hline 9/16/2008 1:00 PM & 0.132 & 15.4 & 0.104 & 0.317 & 17.3 & 0.40 & 0.044 & 0.048 & -- & 0.066 & 0.425 & 16.4 & 0.425 & 15.7 & -- \\
\hline 9/16/2008 2:00 PM & 0.132 & 15.4 & 0.104 & 0.317 & 17.3 & 0.40 & 0.044 & 0.048 & -- & 0.067 & 0.425 & 16.3 & 0.426 & 15.7 & -- \\
\hline
\end{tabular}


Appendix 1. Volumetric water content, temperature, and electrical-conductivity data collected at the flood-irrigated site during 2008.-Continued

[Depth in feet below land surface; volumetric water content dimensionless; temperature in degrees Celsius; bulk electrical conductivity in decisiemens per meter; --, no data]

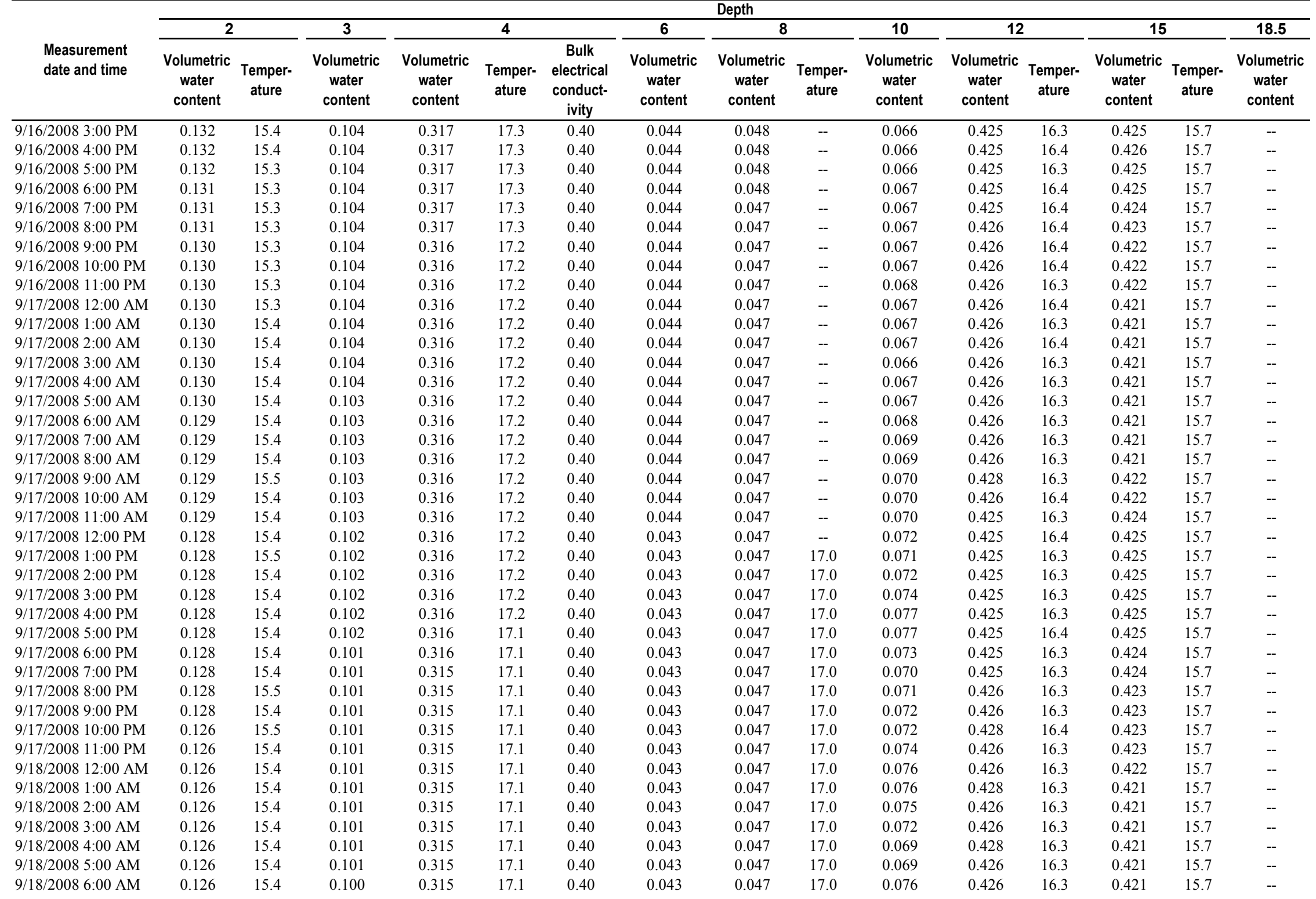


Appendix 1. Volumetric water content, temperature, and electrical-conductivity data collected at the flood-irrigated site during 2008.-Continued

[Depth in feet below land surface; volumetric water content dimensionless; temperature in degrees Celsius; bulk electrical conductivity in decisiemens per meter; --, no data]

\begin{tabular}{|c|c|c|c|c|c|c|c|c|c|c|c|c|c|c|c|}
\hline \multirow[b]{3}{*}{$\begin{array}{l}\text { Measurement } \\
\text { date and time }\end{array}$} & \multicolumn{15}{|c|}{ Depth } \\
\hline & \multirow{2}{*}{$\begin{array}{c}2 \\
\text { Volumetric } \\
\text { water } \\
\text { content }\end{array}$} & & \multirow{2}{*}{$\begin{array}{c}3 \\
\text { Volumetric } \\
\text { water } \\
\text { content }\end{array}$} & \multicolumn{3}{|c|}{4} & \multirow{2}{*}{$\begin{array}{c}\mathbf{6} \\
\text { Volumetric } \\
\text { water } \\
\text { content }\end{array}$} & \multicolumn{2}{|c|}{8} & \multirow{2}{*}{$\begin{array}{c}10 \\
\text { Volumetric } \\
\text { water } \\
\text { content }\end{array}$} & \multicolumn{2}{|c|}{12} & \multicolumn{2}{|c|}{15} & \multirow{2}{*}{$\begin{array}{c}18.5 \\
\text { Volumetric } \\
\text { water } \\
\text { content }\end{array}$} \\
\hline & & $\begin{array}{l}\text { Temper- } \\
\text { ature }\end{array}$ & & $\begin{array}{l}\text { Volumetric } \\
\text { water } \\
\text { content }\end{array}$ & $\begin{array}{l}\text { Temper- } \\
\text { ature }\end{array}$ & $\begin{array}{c}\text { Bulk } \\
\text { electrical } \\
\text { conduct- } \\
\text { ivity }\end{array}$ & & $\begin{array}{c}\text { Volumetric } \\
\text { water } \\
\text { content }\end{array}$ & $\begin{array}{l}\text { Temper- } \\
\text { ature }\end{array}$ & & $\begin{array}{l}\text { Volumetric } \\
\text { water } \\
\text { content }\end{array}$ & $\begin{array}{l}\text { Temper- } \\
\text { ature }\end{array}$ & $\begin{array}{l}\text { Volumetric } \\
\text { water } \\
\text { content }\end{array}$ & $\begin{array}{l}\text { Temper- } \\
\text { ature }\end{array}$ & \\
\hline 9/18/2008 7:00 AM & 0.126 & 15.5 & 0.100 & 0.315 & 17.1 & 0.40 & 0.043 & 0.047 & 17.0 & 0.076 & 0.426 & 16.4 & 0.421 & 15.7 & -- \\
\hline 9/18/2008 8:00 AM & 0.126 & 15.5 & 0.100 & 0.315 & 17.1 & 0.40 & 0.043 & 0.047 & 17.0 & 0.077 & 0.426 & 16.3 & 0.421 & 15.7 & -- \\
\hline 9/18/2008 9:00 AM & 0.125 & 15.5 & 0.100 & 0.315 & 17.1 & 0.40 & 0.043 & 0.047 & 17.0 & 0.077 & 0.426 & 16.3 & 0.421 & 15.7 & -- \\
\hline 9/18/2008 10:00 AM & 0.125 & 15.5 & 0.100 & 0.315 & 17.1 & 0.40 & 0.043 & 0.047 & 17.0 & 0.077 & 0.428 & 16.3 & 0.422 & 15.7 & -- \\
\hline 9/18/2008 11:00 AM & 0.125 & 15.6 & 0.100 & 0.315 & 17.1 & 0.40 & 0.043 & 0.047 & 17.0 & 0.075 & 0.426 & 16.4 & 0.424 & 15.7 & -- \\
\hline 9/18/2008 12:00 PM & 0.125 & 15.6 & 0.100 & 0.315 & 17.1 & 0.40 & 0.043 & 0.047 & 17.0 & 0.073 & 0.425 & 16.3 & 0.424 & 15.8 & -- \\
\hline 9/18/2008 1:00 PM & 0.125 & 15.6 & 0.100 & 0.315 & 17.1 & 0.40 & 0.042 & 0.047 & 17.0 & 0.073 & 0.425 & 16.3 & 0.425 & 15.7 & -- \\
\hline 9/18/2008 2:00 PM & 0.125 & 15.5 & 0.100 & 0.315 & 17.1 & 0.40 & 0.042 & 0.047 & 17.0 & 0.073 & 0.425 & 16.3 & 0.425 & 15.7 & -- \\
\hline 9/18/2008 3:00 PM & 0.124 & 15.5 & 0.100 & 0.315 & 17.1 & 0.40 & 0.042 & 0.047 & 16.9 & 0.072 & 0.425 & 16.3 & 0.425 & 15.7 & -- \\
\hline 9/18/2008 4:00 PM & 0.124 & 15.6 & 0.100 & 0.315 & 17.1 & 0.40 & 0.042 & 0.047 & 16.9 & 0.075 & 0.425 & 16.3 & 0.425 & 15.8 & -- \\
\hline 9/18/2008 5:00 PM & 0.124 & 15.6 & 0.099 & 0.315 & 17.1 & 0.40 & 0.042 & 0.047 & 16.9 & 0.076 & 0.425 & 16.3 & 0.425 & 15.8 & -- \\
\hline 9/18/2008 6:00 PM & 0.124 & 15.5 & 0.099 & 0.315 & 17.1 & 0.40 & 0.043 & 0.047 & 16.9 & 0.075 & 0.425 & 16.3 & 0.424 & 15.7 & -- \\
\hline 9/18/2008 7:00 PM & 0.124 & 15.5 & 0.099 & 0.313 & 17.1 & 0.40 & 0.043 & 0.047 & 16.9 & 0.075 & 0.426 & 16.3 & 0.424 & 15.7 & -- \\
\hline 9/18/2008 8:00 PM & 0.124 & 15.5 & 0.099 & 0.315 & 17.1 & 0.40 & 0.043 & 0.047 & 16.9 & 0.075 & 0.428 & 16.3 & 0.424 & 15.8 & -- \\
\hline 9/18/2008 9:00 PM & 0.124 & 15.5 & 0.099 & 0.315 & 17.1 & 0.40 & 0.043 & 0.047 & 16.9 & 0.072 & 0.428 & 16.3 & 0.423 & 15.7 & -- \\
\hline 9/18/2008 10:00 PM & 0.124 & 15.5 & 0.099 & 0.313 & 17.1 & 0.40 & 0.043 & 0.047 & 16.9 & 0.071 & 0.428 & 16.3 & 0.423 & 15.7 & -- \\
\hline 9/18/2008 11:00 PM & 0.124 & 15.5 & 0.099 & 0.313 & 17.1 & 0.40 & 0.043 & 0.047 & 16.9 & 0.071 & 0.428 & 16.3 & 0.422 & 15.8 & -- \\
\hline 9/19/2008 12:00 AM & 0.124 & 15.6 & 0.099 & 0.313 & 17.1 & 0.40 & 0.043 & 0.047 & 16.9 & 0.070 & 0.428 & 16.3 & 0.421 & 15.7 & -- \\
\hline 9/19/2008 1:00 AM & 0.124 & 15.6 & 0.099 & 0.313 & 17.1 & 0.40 & 0.043 & 0.047 & 16.9 & 0.067 & 0.428 & 16.3 & 0.421 & 15.7 & -- \\
\hline 9/19/2008 2:00 AM & 0.124 & 15.6 & 0.099 & 0.313 & 17.1 & 0.40 & 0.043 & 0.047 & 16.9 & 0.065 & 0.428 & 16.3 & 0.421 & 15.7 & -- \\
\hline 9/19/2008 3:00 AM & 0.124 & 15.6 & 0.099 & 0.313 & 17.1 & 0.40 & 0.043 & 0.047 & 16.9 & 0.064 & 0.426 & 16.3 & 0.421 & 15.7 & -- \\
\hline 9/19/2008 4:00 AM & 0.124 & 15.6 & 0.099 & 0.313 & 17.1 & 0.40 & 0.043 & 0.047 & 16.9 & 0.063 & 0.426 & 16.3 & 0.421 & 15.8 & -- \\
\hline 9/19/2008 5:00 AM & 0.124 & 15.6 & 0.099 & 0.313 & 17.1 & 0.40 & 0.043 & 0.047 & 16.9 & 0.063 & 0.428 & 16.3 & 0.421 & 15.8 & -- \\
\hline 9/19/2008 6:00 AM & 0.124 & 15.6 & 0.099 & 0.313 & 17.0 & 0.40 & 0.043 & 0.047 & 16.9 & 0.062 & 0.426 & 16.4 & 0.421 & 15.8 & -- \\
\hline 9/19/2008 7:00 AM & 0.124 & 15.6 & 0.099 & 0.313 & 17.0 & 0.40 & 0.043 & 0.047 & 16.9 & 0.062 & 0.426 & 16.3 & 0.421 & 15.8 & -- \\
\hline 9/19/2008 8:00 AM & 0.124 & 15.7 & 0.099 & 0.313 & 17.0 & 0.40 & 0.043 & 0.047 & 16.9 & 0.061 & 0.428 & 16.3 & 0.421 & 15.8 & -- \\
\hline 9/19/2008 9:00 AM & 0.123 & 15.7 & 0.098 & 0.313 & 17.0 & 0.40 & 0.043 & 0.047 & 16.9 & 0.060 & 0.428 & 16.3 & 0.422 & 15.8 & -- \\
\hline 9/19/2008 10:00 AM & 0.123 & 15.7 & 0.098 & 0.313 & 17.0 & 0.40 & 0.043 & 0.047 & 16.9 & 0.063 & 0.428 & 16.3 & 0.424 & 15.8 & -- \\
\hline 9/19/2008 11:00 AM & 0.123 & 15.7 & 0.098 & 0.313 & 17.0 & 0.40 & 0.043 & 0.047 & 16.9 & 0.062 & 0.426 & 16.3 & 0.425 & 15.8 & -- \\
\hline 9/19/2008 12:00 PM & 0.123 & 15.7 & 0.098 & 0.313 & 17.0 & 0.40 & 0.043 & 0.047 & 16.9 & 0.063 & 0.426 & 16.3 & 0.425 & 15.8 & -- \\
\hline 9/19/2008 1:00 PM & 0.123 & 15.7 & 0.098 & 0.313 & 17.0 & 0.40 & 0.042 & 0.047 & 16.9 & 0.062 & 0.426 & 16.3 & 0.425 & 15.8 & -- \\
\hline 9/19/2008 2:00 PM & 0.123 & 15.7 & 0.098 & 0.313 & 17.0 & 0.40 & 0.042 & 0.047 & 16.9 & 0.061 & 0.426 & 16.3 & 0.425 & 15.7 & -- \\
\hline 9/19/2008 3:00 PM & 0.122 & 15.7 & 0.098 & 0.313 & 17.0 & 0.40 & 0.042 & 0.047 & 16.9 & 0.060 & 0.426 & 16.3 & 0.425 & 15.8 & -- \\
\hline 9/19/2008 4:00 PM & 0.122 & 15.7 & 0.098 & 0.313 & 17.0 & 0.40 & 0.042 & 0.047 & 16.9 & 0.060 & 0.426 & 16.3 & 0.425 & 15.8 & -- \\
\hline 9/19/2008 5:00 PM & 0.122 & 15.7 & 0.098 & 0.313 & 17.0 & 0.40 & 0.042 & 0.047 & 16.9 & 0.062 & 0.426 & 16.3 & 0.425 & 15.8 & -- \\
\hline 9/19/2008 6:00 PM & 0.122 & 15.7 & 0.098 & 0.313 & 17.0 & 0.40 & 0.042 & 0.047 & 16.9 & 0.061 & 0.426 & 16.3 & 0.425 & 15.8 & -- \\
\hline 9/19/2008 7:00 PM & 0.122 & 15.7 & 0.098 & 0.313 & 17.0 & 0.40 & 0.042 & 0.047 & 16.9 & 0.058 & 0.426 & 16.3 & 0.425 & 15.8 & -- \\
\hline 9/19/2008 8:00 PM & 0.122 & 15.7 & 0.098 & 0.313 & 17.0 & 0.40 & 0.042 & 0.047 & 16.9 & 0.058 & 0.428 & 16.4 & 0.424 & 15.8 & -- \\
\hline 9/19/2008 9:00 PM & 0.122 & 15.6 & 0.098 & 0.313 & 17.0 & 0.40 & 0.042 & 0.047 & 16.9 & 0.057 & 0.428 & 16.3 & 0.423 & 15.8 & -- \\
\hline 9/19/2008 10:00 PM & 0.122 & 15.7 & 0.098 & 0.313 & 17.0 & 0.40 & 0.042 & 0.047 & 16.9 & 0.055 & 0.428 & 16.3 & 0.423 & 15.8 & -- \\
\hline
\end{tabular}


Appendix 1. Volumetric water content, temperature, and electrical-conductivity data collected at the flood-irrigated site during 2008.-Continued

[Depth in feet below land surface; volumetric water content dimensionless; temperature in degrees Celsius; bulk electrical conductivity in decisiemens per meter; --, no data]

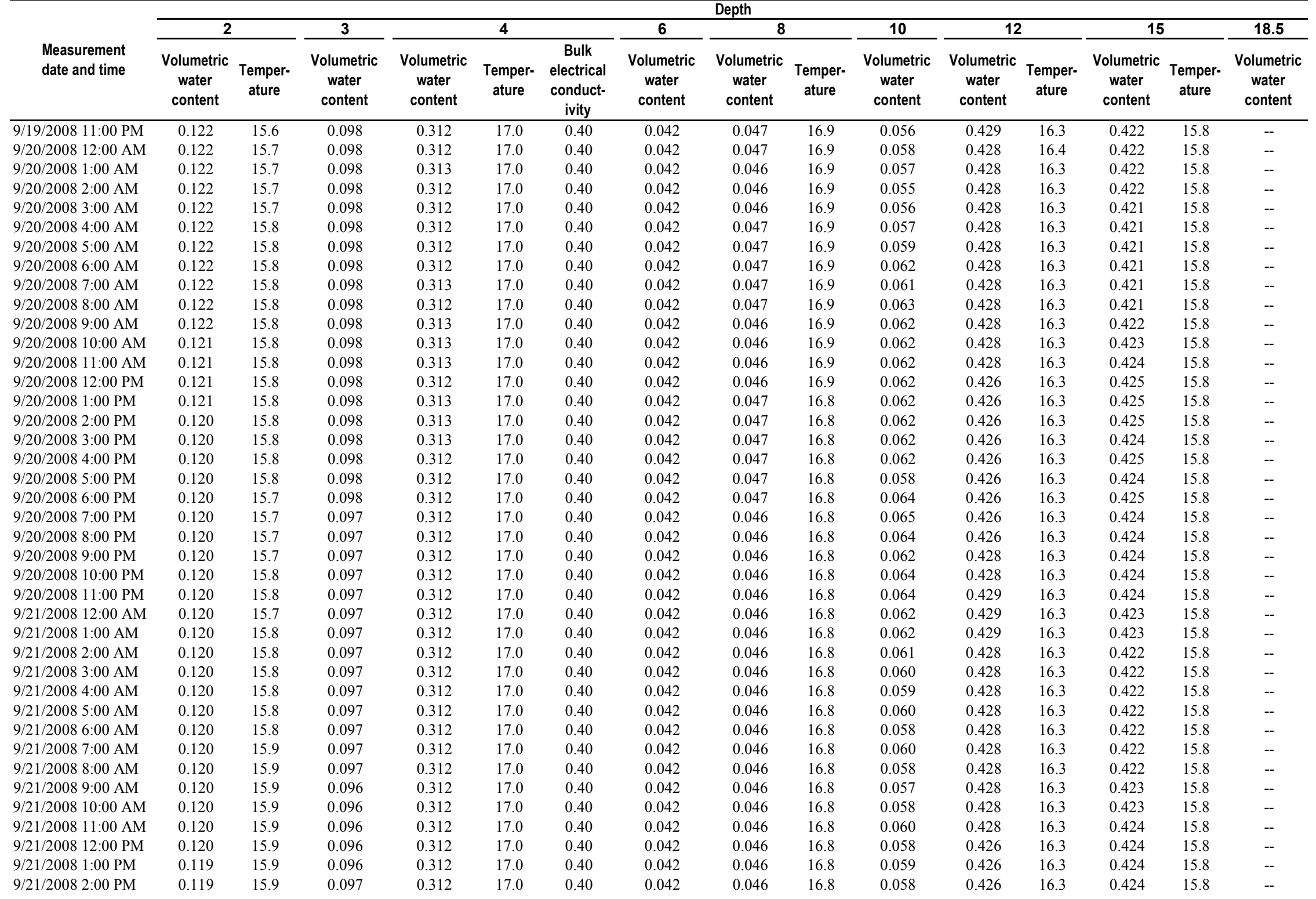


Appendix 1. Volumetric water content, temperature, and electrical-conductivity data collected at the flood-irrigated site during 2008.-Continued

[Depth in feet below land surface; volumetric water content dimensionless; temperature in degrees Celsius; bulk electrical conductivity in decisiemens per meter; --, no data]

\begin{tabular}{|c|c|c|c|c|c|c|c|c|c|c|c|c|c|c|c|}
\hline \multirow[b]{3}{*}{$\begin{array}{l}\text { Measurement } \\
\text { date and time }\end{array}$} & \multicolumn{15}{|c|}{ Depth } \\
\hline & \multicolumn{2}{|c|}{2} & \multirow{2}{*}{$\begin{array}{c}3 \\
\text { Volumetric } \\
\text { water } \\
\text { content }\end{array}$} & \multicolumn{3}{|c|}{4} & \multirow{2}{*}{$\begin{array}{c}6 \\
\begin{array}{c}\text { Volumetric } \\
\text { water } \\
\text { content }\end{array}\end{array}$} & \multicolumn{2}{|l|}{8} & \multirow{2}{*}{$\begin{array}{c}10 \\
\text { Volumetric } \\
\text { water } \\
\text { content }\end{array}$} & \multicolumn{2}{|c|}{12} & \multicolumn{2}{|c|}{15} & \multirow{2}{*}{$\begin{array}{c}18.5 \\
\text { Volumetric } \\
\text { water } \\
\text { content }\end{array}$} \\
\hline & $\begin{array}{l}\text { Volumetric } \\
\text { water } \\
\text { content }\end{array}$ & $\begin{array}{l}\text { Temper- } \\
\text { ature }\end{array}$ & & $\begin{array}{c}\text { Volumetric } \\
\text { water } \\
\text { content }\end{array}$ & $\begin{array}{l}\text { Temper- } \\
\text { ature }\end{array}$ & $\begin{array}{c}\text { Bulk } \\
\text { electrical } \\
\text { conduct- } \\
\text { ivity } \\
\end{array}$ & & $\begin{array}{l}\text { Volumetric } \\
\text { water } \\
\text { content }\end{array}$ & $\begin{array}{l}\text { Temper- } \\
\text { ature }\end{array}$ & & $\begin{array}{l}\text { Volumetric } \\
\text { water } \\
\text { content }\end{array}$ & $\begin{array}{l}\text { Temper- } \\
\text { ature }\end{array}$ & $\begin{array}{l}\text { Volumetric } \\
\text { water } \\
\text { content }\end{array}$ & $\begin{array}{l}\text { Temper- } \\
\text { ature }\end{array}$ & \\
\hline 9/21/2008 3:00 PM & 0.118 & 15.9 & 0.097 & 0.312 & 17.0 & 0.40 & 0.042 & 0.046 & 16.8 & 0.058 & 0.426 & 16.3 & 0.424 & 15.8 & -- \\
\hline 9/21/2008 4:00 PM & 0.119 & 15.9 & 0.097 & 0.312 & 17.0 & 0.40 & 0.042 & 0.046 & 16.8 & 0.060 & 0.426 & 16.3 & 0.423 & 15.8 & -- \\
\hline 9/21/2008 5:00 PM & 0.118 & 15.9 & 0.096 & 0.312 & 17.0 & 0.40 & 0.042 & 0.046 & 16.8 & 0.061 & 0.426 & 16.3 & 0.423 & 15.8 & -- \\
\hline 9/21/2008 6:00 PM & 0.118 & 16.0 & 0.096 & 0.312 & 17.0 & 0.40 & 0.042 & 0.046 & 16.8 & 0.064 & 0.426 & 16.3 & 0.424 & 15.8 & -- \\
\hline 9/21/2008 7:00 PM & 0.119 & 16.0 & 0.096 & 0.312 & 17.0 & 0.40 & 0.042 & 0.046 & 16.8 & 0.062 & 0.426 & 16.3 & 0.423 & 15.8 & -- \\
\hline 9/21/2008 8:00 PM & 0.119 & 16.0 & 0.096 & 0.312 & 17.0 & 0.40 & 0.042 & 0.046 & 16.8 & 0.061 & 0.428 & 16.3 & 0.423 & 15.8 & -- \\
\hline 9/21/2008 9:00 PM & 0.119 & 16.0 & 0.096 & 0.312 & 17.0 & 0.40 & 0.042 & 0.046 & 16.8 & 0.061 & 0.428 & 16.3 & 0.422 & 15.8 & -- \\
\hline 9/21/2008 10:00 PM & 0.119 & 16.0 & 0.096 & 0.311 & 17.0 & 0.40 & 0.042 & 0.046 & 16.8 & 0.059 & 0.428 & 16.3 & 0.422 & 15.8 & -- \\
\hline 9/21/2008 11:00 PM & 0.119 & 16.0 & 0.096 & 0.311 & 17.0 & 0.40 & 0.042 & 0.045 & 16.8 & 0.059 & 0.428 & 16.3 & 0.422 & 15.8 & -- \\
\hline 9/22/2008 12:00 AM & 0.119 & 16.0 & 0.096 & 0.311 & 17.0 & 0.40 & 0.042 & 0.045 & 16.8 & 0.058 & 0.428 & 16.3 & 0.421 & 15.8 & -- \\
\hline 9/22/2008 1:00 AM & 0.119 & 16.1 & 0.096 & 0.311 & 17.0 & 0.40 & 0.042 & 0.045 & 16.8 & 0.059 & 0.428 & 16.3 & 0.421 & 15.8 & -- \\
\hline 9/22/2008 2:00 AM & 0.119 & 16.1 & 0.096 & 0.311 & 17.0 & 0.40 & 0.042 & 0.045 & 16.8 & 0.060 & 0.428 & 16.3 & 0.421 & 15.8 & -- \\
\hline 9/22/2008 3:00 AM & 0.119 & 16.1 & 0.096 & 0.312 & 17.0 & 0.39 & 0.042 & 0.045 & 16.8 & 0.064 & 0.428 & 16.3 & 0.421 & 15.8 & -- \\
\hline 9/22/2008 4:00 AM & 0.119 & 16.1 & 0.096 & 0.311 & 17.0 & 0.39 & 0.042 & 0.045 & 16.8 & 0.065 & 0.428 & 16.3 & 0.421 & 15.8 & -- \\
\hline 9/22/2008 5:00 AM & 0.119 & 16.1 & 0.096 & 0.311 & 17.0 & 0.39 & 0.042 & 0.045 & 16.8 & 0.065 & 0.428 & 16.3 & 0.421 & 15.8 & -- \\
\hline 9/22/2008 6:00 AM & 0.119 & 16.1 & 0.096 & 0.311 & 17.0 & 0.40 & 0.042 & 0.046 & 16.8 & 0.063 & 0.428 & 16.3 & 0.420 & 15.8 & -- \\
\hline 9/22/2008 7:00 AM & 0.119 & 16.1 & 0.096 & 0.311 & 17.0 & 0.40 & 0.042 & 0.046 & 16.7 & 0.063 & 0.428 & 16.3 & 0.420 & 15.8 & -- \\
\hline 9/22/2008 8:00 AM & 0.119 & 16.1 & 0.096 & 0.311 & 17.0 & 0.40 & 0.042 & 0.045 & 16.7 & 0.065 & 0.428 & 16.3 & 0.420 & 15.8 & -- \\
\hline 9/22/2008 9:00 AM & 0.118 & 16.2 & 0.096 & 0.311 & 17.0 & 0.40 & 0.042 & 0.045 & 16.7 & 0.065 & 0.428 & 16.3 & 0.421 & 15.8 & -- \\
\hline 9/22/2008 10:00 AM & 0.119 & 16.1 & 0.095 & 0.311 & 17.0 & 0.40 & 0.042 & 0.045 & 16.8 & 0.065 & 0.428 & 16.3 & 0.422 & 15.8 & -- \\
\hline 9/22/2008 11:00 AM & 0.118 & 16.1 & 0.095 & 0.311 & 17.0 & 0.40 & 0.042 & 0.045 & 16.7 & 0.064 & 0.426 & 16.3 & 0.423 & 15.8 & -- \\
\hline 9/22/2008 12:00 PM & 0.118 & 16.1 & 0.095 & 0.311 & 17.0 & 0.40 & 0.042 & 0.046 & 16.7 & 0.063 & 0.425 & 16.3 & 0.423 & 15.8 & -- \\
\hline 9/22/2008 1:00 PM & 0.117 & 16.1 & 0.096 & 0.312 & 17.0 & 0.40 & 0.042 & 0.046 & 16.7 & 0.060 & 0.425 & 16.3 & 0.423 & 15.8 & -- \\
\hline 9/22/2008 2:00 PM & 0.117 & 16.1 & 0.095 & 0.312 & 17.0 & 0.40 & 0.042 & 0.046 & 16.7 & 0.064 & 0.424 & 16.3 & 0.422 & 15.8 & -- \\
\hline 9/22/2008 3:00 PM & 0.117 & 16.1 & 0.095 & 0.311 & 17.0 & 0.40 & 0.042 & 0.046 & 16.7 & 0.067 & 0.425 & 16.3 & 0.422 & 15.8 & -- \\
\hline 9/22/2008 4:00 PM & 0.117 & 16.1 & 0.095 & 0.312 & 17.0 & 0.40 & 0.042 & 0.046 & 16.7 & 0.064 & 0.425 & 16.3 & 0.423 & 15.8 & -- \\
\hline 9/22/2008 5:00 PM & 0.117 & 16.1 & 0.095 & 0.311 & 17.0 & 0.40 & 0.042 & 0.046 & 16.7 & 0.066 & 0.425 & 16.3 & 0.423 & 15.8 & -- \\
\hline 9/22/2008 6:00 PM & 0.117 & 16.1 & 0.095 & 0.311 & 17.0 & 0.40 & 0.042 & 0.045 & 16.7 & 0.066 & 0.425 & 16.3 & 0.423 & 15.8 & -- \\
\hline 9/22/2008 7:00 PM & 0.117 & 16.1 & 0.095 & 0.311 & 17.0 & 0.40 & 0.042 & 0.045 & 16.7 & 0.066 & 0.425 & 16.3 & 0.423 & 15.8 & -- \\
\hline 9/22/2008 8:00 PM & 0.117 & 16.0 & 0.095 & 0.311 & 17.0 & 0.40 & 0.042 & 0.045 & 16.7 & 0.064 & 0.425 & 16.3 & 0.423 & 15.8 & -- \\
\hline 9/22/2008 9:00 PM & 0.117 & 16.1 & 0.095 & 0.311 & 17.0 & 0.40 & 0.042 & 0.045 & 16.7 & 0.066 & 0.426 & 16.3 & 0.422 & 15.8 & -- \\
\hline 9/22/2008 10:00 PM & 0.117 & 16.1 & 0.095 & 0.311 & 17.0 & 0.40 & 0.042 & 0.045 & 16.7 & 0.067 & 0.426 & 16.3 & 0.422 & 15.8 & -- \\
\hline 9/22/2008 11:00 PM & 0.117 & 16.1 & 0.095 & 0.311 & 17.0 & 0.40 & 0.042 & 0.045 & 16.7 & 0.064 & 0.428 & 16.3 & 0.422 & 15.8 & -- \\
\hline 9/23/2008 12:00 AM & 0.117 & 16.2 & 0.095 & 0.311 & 17.0 & 0.40 & 0.042 & 0.045 & 16.7 & 0.065 & 0.428 & 16.3 & 0.421 & 15.8 & -- \\
\hline 9/23/2008 1:00 AM & 0.117 & 16.2 & 0.095 & 0.311 & 17.0 & 0.40 & 0.042 & 0.045 & 16.7 & 0.063 & 0.428 & 16.3 & 0.421 & 15.8 & -- \\
\hline 9/23/2008 2:00 AM & 0.117 & 16.2 & 0.095 & 0.311 & 17.0 & 0.40 & 0.042 & 0.045 & 16.7 & 0.061 & 0.428 & 16.3 & 0.421 & 15.8 & -- \\
\hline 9/23/2008 3:00 AM & 0.117 & 16.2 & 0.095 & 0.311 & 17.0 & 0.40 & 0.042 & 0.045 & 16.7 & 0.063 & 0.428 & 16.3 & 0.421 & 15.8 & -- \\
\hline 9/23/2008 4:00 AM & 0.117 & 16.2 & 0.095 & 0.310 & 17.0 & 0.40 & 0.042 & 0.045 & 16.7 & 0.061 & 0.426 & 16.3 & 0.421 & 15.8 & -- \\
\hline 9/23/2008 5:00 AM & 0.118 & 16.3 & 0.094 & 0.311 & 17.0 & 0.40 & 0.042 & 0.045 & 16.7 & 0.062 & 0.426 & 16.3 & 0.420 & 15.8 & -- \\
\hline 9/23/2008 6:00 AM & 0.118 & 16.3 & 0.094 & 0.311 & 17.0 & 0.40 & 0.042 & 0.045 & 16.7 & 0.061 & 0.426 & 16.3 & 0.420 & 15.8 & -- \\
\hline
\end{tabular}


Appendix 1. Volumetric water content, temperature, and electrical-conductivity data collected at the flood-irrigated site during 2008.-Continued

[Depth in feet below land surface; volumetric water content dimensionless; temperature in degrees Celsius; bulk electrical conductivity in decisiemens per meter; --, no data]

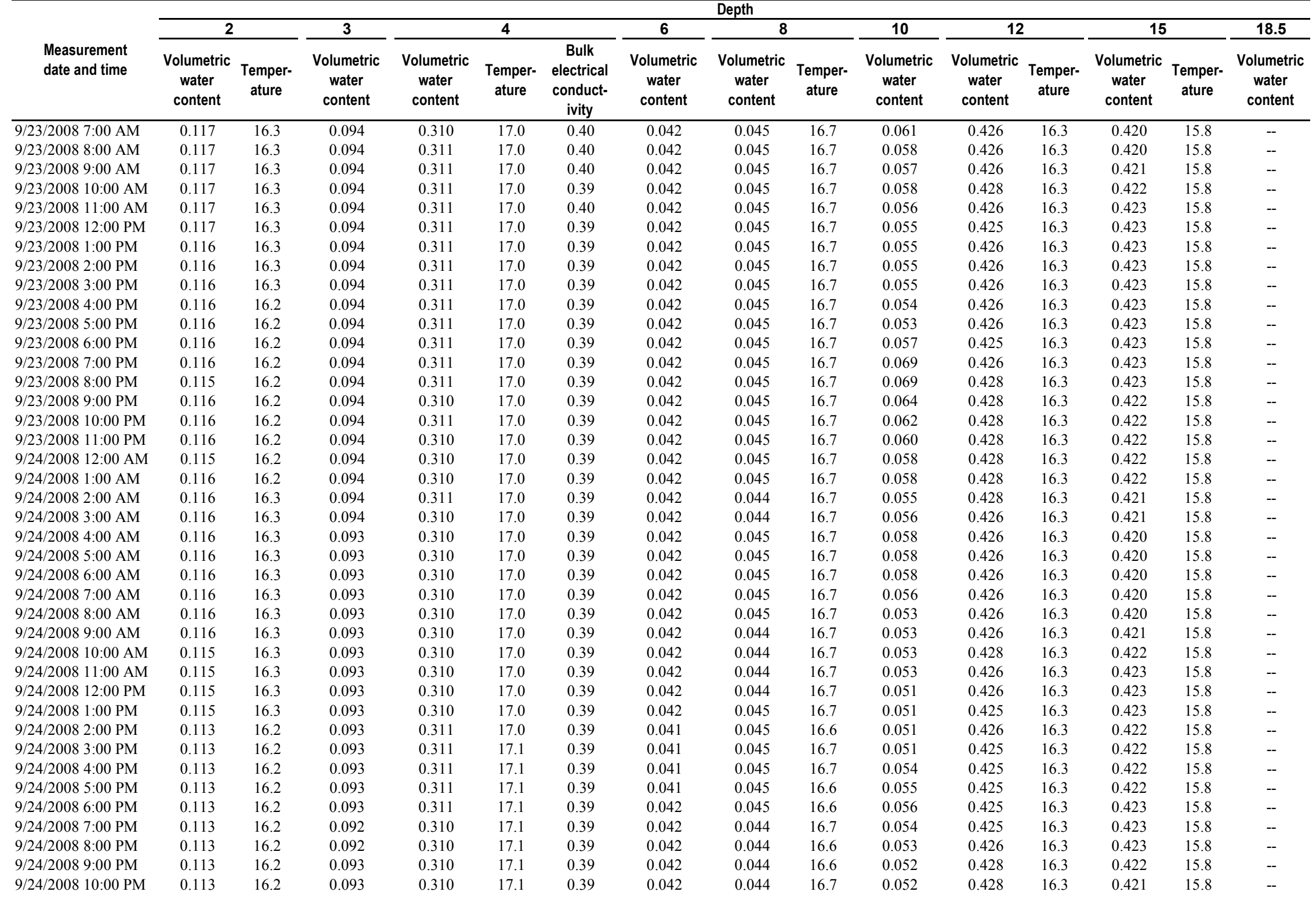


Appendix 1. Volumetric water content, temperature, and electrical-conductivity data collected at the flood-irrigated site during 2008.-Continued

[Depth in feet below land surface; volumetric water content dimensionless; temperature in degrees Celsius; bulk electrical conductivity in decisiemens per meter; --, no data]

\begin{tabular}{|c|c|c|c|c|c|c|c|c|c|c|c|c|c|c|c|}
\hline \multirow[b]{3}{*}{$\begin{array}{l}\text { Measurement } \\
\text { date and time }\end{array}$} & \multicolumn{15}{|c|}{ Depth } \\
\hline & \multicolumn{2}{|l|}{2} & \multirow{2}{*}{$\begin{array}{c}3 \\
\text { Volumetric } \\
\text { water } \\
\text { content }\end{array}$} & \multicolumn{3}{|c|}{4} & \multirow{2}{*}{$\begin{array}{c}6 \\
\begin{array}{c}\text { Volumetric } \\
\text { water } \\
\text { content }\end{array}\end{array}$} & \multicolumn{2}{|l|}{8} & \multirow{2}{*}{$\begin{array}{c}10 \\
\text { Volumetric } \\
\text { water } \\
\text { content }\end{array}$} & \multicolumn{2}{|c|}{12} & \multicolumn{2}{|c|}{15} & \multirow{2}{*}{$\begin{array}{c}18.5 \\
\text { Volumetric } \\
\text { water } \\
\text { content }\end{array}$} \\
\hline & $\begin{array}{l}\text { Volumetric } \\
\text { water } \\
\text { content }\end{array}$ & $\begin{array}{l}\text { Temper- } \\
\text { ature }\end{array}$ & & $\begin{array}{c}\text { Volumetric } \\
\text { water } \\
\text { content }\end{array}$ & $\begin{array}{l}\text { Temper- } \\
\text { ature }\end{array}$ & $\begin{array}{c}\text { Bulk } \\
\text { electrical } \\
\text { conduct- } \\
\text { ivity } \\
\end{array}$ & & $\begin{array}{l}\text { Volumetric } \\
\text { water } \\
\text { content }\end{array}$ & $\begin{array}{l}\text { Temper- } \\
\text { ature }\end{array}$ & & $\begin{array}{l}\text { Volumetric } \\
\text { water } \\
\text { content }\end{array}$ & $\begin{array}{l}\text { Temper- } \\
\text { ature }\end{array}$ & $\begin{array}{l}\text { Volumetric } \\
\text { water } \\
\text { content }\end{array}$ & $\begin{array}{l}\text { Temper- } \\
\text { ature }\end{array}$ & \\
\hline 9/24/2008 11:00 PM & 0.113 & 16.2 & 0.093 & 0.310 & 17.1 & 0.39 & 0.042 & 0.044 & 16.7 & 0.050 & 0.428 & 16.3 & 0.421 & 15.8 & -- \\
\hline 9/25/2008 12:00 AM & 0.113 & 16.2 & 0.092 & 0.310 & 17.1 & 0.39 & 0.042 & 0.044 & 16.6 & 0.050 & 0.428 & 16.3 & 0.421 & 15.8 & -- \\
\hline 9/25/2008 1:00 AM & 0.113 & 16.2 & 0.092 & 0.310 & 17.1 & 0.39 & 0.042 & 0.044 & 16.6 & 0.049 & 0.428 & 16.3 & 0.421 & 15.8 & -- \\
\hline 9/25/2008 2:00 AM & 0.113 & 16.2 & 0.092 & 0.310 & 17.1 & 0.39 & 0.042 & 0.044 & 16.6 & 0.049 & 0.426 & 16.3 & 0.421 & 15.8 & -- \\
\hline 9/25/2008 3:00 AM & 0.113 & 16.2 & 0.092 & 0.310 & 17.1 & 0.39 & 0.042 & 0.044 & 16.6 & 0.048 & 0.426 & 16.3 & 0.421 & 15.9 & -- \\
\hline 9/25/2008 4:00 AM & 0.113 & 16.2 & 0.092 & 0.310 & 17.1 & 0.39 & 0.042 & 0.044 & 16.6 & 0.048 & 0.426 & 16.3 & 0.421 & 15.9 & -- \\
\hline 9/25/2008 5:00 AM & 0.113 & 16.2 & 0.092 & 0.310 & 17.1 & 0.39 & 0.042 & 0.044 & 16.6 & 0.048 & 0.426 & 16.3 & 0.421 & 15.8 & -- \\
\hline 9/25/2008 6:00 AM & 0.113 & 16.3 & 0.092 & 0.310 & 17.1 & 0.39 & 0.042 & 0.044 & 16.6 & 0.048 & 0.426 & 16.3 & 0.420 & 15.8 & -- \\
\hline 9/25/2008 7:00 AM & 0.113 & 16.3 & 0.092 & 0.309 & 17.1 & 0.39 & 0.042 & 0.044 & 16.6 & 0.048 & 0.426 & 16.3 & 0.420 & 15.8 & -- \\
\hline 9/25/2008 8:00 AM & 0.115 & 16.3 & 0.092 & 0.310 & 17.1 & 0.39 & 0.042 & 0.044 & 16.6 & 0.048 & 0.426 & 16.3 & 0.420 & 15.8 & -- \\
\hline 9/25/2008 9:00 AM & 0.113 & 16.2 & 0.092 & 0.310 & 17.1 & 0.39 & 0.042 & 0.044 & 16.6 & 0.048 & 0.426 & 16.3 & 0.421 & 15.9 & -- \\
\hline 9/25/2008 10:00 AM & 0.113 & 16.3 & 0.092 & 0.310 & 17.1 & 0.39 & 0.042 & 0.044 & 16.6 & 0.048 & 0.428 & 16.2 & 0.422 & 15.8 & -- \\
\hline 9/25/2008 11:00 AM & 0.113 & 16.2 & 0.092 & 0.310 & 17.1 & 0.39 & 0.042 & 0.044 & 16.6 & 0.047 & 0.425 & 16.3 & 0.423 & 15.9 & -- \\
\hline 9/25/2008 12:00 PM & 0.113 & 16.2 & 0.092 & 0.310 & 17.1 & 0.39 & 0.042 & 0.044 & 16.6 & 0.047 & 0.425 & 16.3 & 0.423 & 15.8 & -- \\
\hline 9/25/2008 1:00 PM & 0.112 & 16.2 & 0.092 & 0.310 & 17.1 & 0.39 & 0.041 & 0.044 & 16.6 & 0.047 & 0.425 & 16.3 & 0.422 & 15.8 & -- \\
\hline 9/25/2008 2:00 PM & 0.111 & 16.1 & 0.092 & 0.310 & 17.1 & 0.39 & 0.041 & 0.044 & 16.6 & 0.047 & 0.425 & 16.3 & 0.421 & 15.8 & -- \\
\hline 9/25/2008 3:00 PM & 0.111 & 16.1 & 0.092 & 0.310 & 17.1 & 0.39 & 0.041 & 0.044 & 16.6 & 0.047 & 0.425 & 16.3 & 0.421 & 15.8 & -- \\
\hline 9/25/2008 4:00 PM & 0.111 & 16.1 & 0.092 & 0.310 & 17.1 & 0.39 & 0.041 & 0.044 & 16.6 & 0.047 & 0.425 & 16.3 & 0.421 & 15.8 & -- \\
\hline 9/25/2008 5:00 PM & 0.111 & 16.1 & 0.092 & 0.310 & 17.1 & 0.39 & 0.041 & 0.044 & 16.6 & 0.049 & 0.425 & 16.3 & 0.421 & 15.8 & -- \\
\hline 9/25/2008 6:00 PM & 0.111 & 16.2 & 0.092 & 0.310 & 17.1 & 0.39 & 0.041 & 0.044 & 16.6 & 0.050 & 0.425 & 16.3 & 0.422 & 15.9 & -- \\
\hline 9/25/2008 7:00 PM & 0.111 & 16.1 & 0.092 & 0.310 & 17.1 & 0.39 & 0.042 & 0.044 & 16.6 & 0.049 & 0.425 & 16.2 & 0.423 & 15.8 & -- \\
\hline 9/25/2008 8:00 PM & 0.111 & 16.2 & 0.092 & 0.310 & 17.1 & 0.39 & 0.042 & 0.044 & 16.6 & 0.049 & 0.425 & 16.3 & 0.422 & 15.8 & -- \\
\hline 9/25/2008 9:00 PM & 0.111 & 16.2 & 0.092 & 0.310 & 17.1 & 0.39 & 0.042 & 0.044 & 16.6 & 0.049 & 0.426 & 16.3 & 0.422 & 15.8 & -- \\
\hline 9/25/2008 10:00 PM & 0.111 & 16.1 & 0.092 & 0.310 & 17.1 & 0.39 & 0.042 & 0.044 & 16.6 & 0.048 & 0.426 & 16.3 & 0.421 & 15.8 & -- \\
\hline 9/25/2008 11:00 PM & 0.111 & 16.2 & 0.092 & 0.309 & 17.1 & 0.39 & 0.042 & 0.044 & 16.6 & 0.048 & 0.426 & 16.3 & 0.421 & 15.9 & -- \\
\hline 9/26/2008 12:00 AM & 0.111 & 16.2 & 0.092 & 0.310 & 17.1 & 0.39 & 0.042 & 0.044 & 16.6 & 0.048 & 0.426 & 16.3 & 0.421 & 15.9 & -- \\
\hline 9/26/2008 1:00 AM & 0.112 & 16.2 & 0.092 & 0.310 & 17.1 & 0.39 & 0.041 & 0.044 & 16.6 & 0.048 & 0.426 & 16.3 & 0.421 & 15.9 & -- \\
\hline 9/26/2008 2:00 AM & 0.112 & 16.3 & 0.092 & 0.310 & 17.1 & 0.39 & 0.041 & 0.044 & 16.6 & 0.048 & 0.426 & 16.2 & 0.421 & 15.8 & -- \\
\hline 9/26/2008 3:00 AM & 0.112 & 16.3 & 0.092 & 0.310 & 17.1 & 0.39 & 0.042 & 0.044 & 16.6 & 0.048 & 0.426 & 16.3 & 0.420 & 15.9 & -- \\
\hline 9/26/2008 4:00 AM & 0.112 & 16.3 & 0.092 & 0.310 & 17.1 & 0.39 & 0.042 & 0.044 & 16.6 & 0.048 & 0.425 & 16.3 & 0.420 & 15.9 & -- \\
\hline 9/26/2008 5:00 AM & 0.112 & 16.3 & 0.092 & 0.310 & 17.1 & 0.39 & 0.042 & 0.044 & 16.6 & 0.048 & 0.425 & 16.3 & 0.420 & 15.9 & -- \\
\hline 9/26/2008 6:00 AM & 0.112 & 16.3 & 0.092 & 0.310 & 17.1 & 0.39 & 0.041 & 0.044 & 16.6 & 0.047 & 0.425 & 16.2 & 0.420 & 15.9 & -- \\
\hline 9/26/2008 7:00 AM & 0.112 & 16.4 & 0.092 & 0.309 & 17.1 & 0.39 & 0.042 & 0.044 & 16.6 & 0.047 & 0.425 & 16.3 & 0.420 & 15.9 & -- \\
\hline 9/26/2008 8:00 AM & 0.112 & 16.3 & 0.092 & 0.310 & 17.1 & 0.39 & 0.042 & 0.044 & 16.6 & 0.047 & 0.426 & 16.3 & 0.420 & 15.9 & -- \\
\hline 9/26/2008 9:00 AM & 0.112 & 16.3 & 0.092 & 0.309 & 17.1 & 0.39 & 0.042 & 0.043 & 16.6 & 0.047 & 0.426 & 16.3 & 0.421 & 15.9 & -- \\
\hline 9/26/2008 10:00 AM & 0.111 & 16.3 & 0.092 & 0.310 & 17.0 & 0.39 & 0.041 & 0.043 & 16.6 & 0.047 & 0.425 & 16.3 & 0.422 & 15.9 & -- \\
\hline 9/26/2008 11:00 AM & 0.111 & 16.3 & 0.092 & 0.309 & 17.0 & 0.39 & 0.041 & 0.044 & 16.6 & 0.047 & 0.425 & 16.3 & 0.423 & 15.8 & -- \\
\hline 9/26/2008 12:00 PM & 0.111 & 16.3 & 0.092 & 0.309 & 17.0 & 0.39 & 0.041 & 0.044 & 16.6 & 0.047 & 0.425 & 16.2 & 0.422 & 15.8 & -- \\
\hline 9/26/2008 1:00 PM & 0.110 & 16.2 & 0.092 & 0.310 & 17.1 & 0.39 & 0.041 & 0.044 & 16.6 & 0.047 & 0.424 & 16.2 & 0.421 & 15.9 & -- \\
\hline 9/26/2008 2:00 PM & 0.110 & 16.2 & 0.092 & 0.310 & 17.0 & 0.39 & 0.041 & 0.044 & 16.6 & 0.047 & 0.425 & 16.2 & 0.420 & 15.8 & -- \\
\hline
\end{tabular}


Appendix 1. Volumetric water content, temperature, and electrical-conductivity data collected at the flood-irrigated site during 2008.-Continued

[Depth in feet below land surface; volumetric water content dimensionless; temperature in degrees Celsius; bulk electrical conductivity in decisiemens per meter; --, no data]

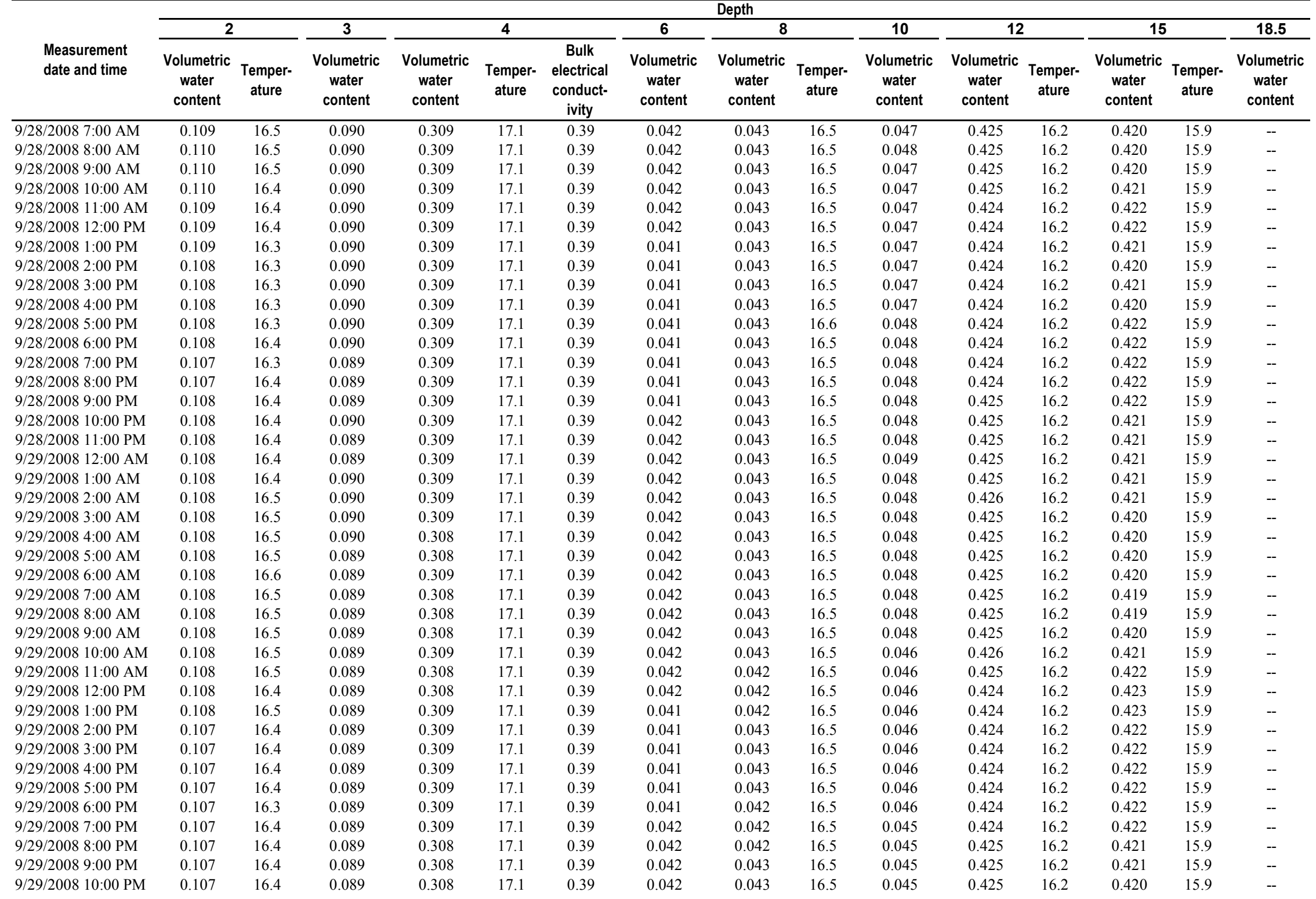


Appendix 1. Volumetric water content, temperature, and electrical-conductivity data collected at the flood-irrigated site during 2008.-Continued

[Depth in feet below land surface; volumetric water content dimensionless; temperature in degrees Celsius; bulk electrical conductivity in decisiemens per meter; --, no data]

\begin{tabular}{|c|c|c|c|c|c|c|c|c|c|c|c|c|c|c|c|}
\hline \multirow[b]{3}{*}{$\begin{array}{l}\text { Measurement } \\
\text { date and time }\end{array}$} & \multicolumn{15}{|c|}{ Depth } \\
\hline & \multicolumn{2}{|l|}{2} & \multirow{2}{*}{$\begin{array}{c}3 \\
\text { Volumetric } \\
\text { water } \\
\text { content }\end{array}$} & \multicolumn{3}{|c|}{4} & \multirow{2}{*}{$\begin{array}{c}6 \\
\begin{array}{c}\text { Volumetric } \\
\text { water } \\
\text { content }\end{array}\end{array}$} & \multicolumn{2}{|l|}{8} & \multirow{2}{*}{$\begin{array}{c}10 \\
\text { Volumetric } \\
\text { water } \\
\text { content }\end{array}$} & \multicolumn{2}{|c|}{12} & \multicolumn{2}{|c|}{15} & \multirow{2}{*}{$\begin{array}{c}18.5 \\
\text { Volumetric } \\
\text { water } \\
\text { content }\end{array}$} \\
\hline & $\begin{array}{l}\text { Volumetric } \\
\text { water } \\
\text { content }\end{array}$ & $\begin{array}{l}\text { Temper- } \\
\text { ature }\end{array}$ & & $\begin{array}{c}\text { Volumetric } \\
\text { water } \\
\text { content }\end{array}$ & $\begin{array}{l}\text { Temper- } \\
\text { ature }\end{array}$ & $\begin{array}{c}\text { Bulk } \\
\text { electrical } \\
\text { conduct- } \\
\text { ivity } \\
\end{array}$ & & $\begin{array}{l}\text { Volumetric } \\
\text { water } \\
\text { content }\end{array}$ & $\begin{array}{l}\text { Temper- } \\
\text { ature }\end{array}$ & & $\begin{array}{l}\text { Volumetric } \\
\text { water } \\
\text { content }\end{array}$ & $\begin{array}{l}\text { Temper- } \\
\text { ature }\end{array}$ & $\begin{array}{l}\text { Volumetric } \\
\text { water } \\
\text { content }\end{array}$ & $\begin{array}{l}\text { Temper- } \\
\text { ature }\end{array}$ & \\
\hline 9/29/2008 11:00 PM & 0.107 & 16.4 & 0.089 & 0.309 & 17.1 & 0.39 & 0.042 & 0.043 & 16.5 & 0.045 & 0.425 & 16.2 & 0.420 & 15.9 & -- \\
\hline 9/30/2008 12:00 AM & 0.107 & 16.4 & 0.089 & 0.308 & 17.1 & 0.39 & 0.042 & 0.043 & 16.5 & 0.045 & 0.425 & 16.2 & 0.420 & 15.9 & -- \\
\hline 9/30/2008 1:00 AM & 0.107 & 16.5 & 0.089 & 0.308 & 17.1 & 0.39 & 0.042 & 0.043 & 16.5 & 0.045 & 0.425 & 16.2 & 0.420 & 15.9 & -- \\
\hline 9/30/2008 2:00 AM & 0.107 & 16.4 & 0.089 & 0.308 & 17.1 & 0.39 & 0.042 & 0.043 & 16.5 & 0.045 & 0.424 & 16.2 & 0.420 & 15.9 & -- \\
\hline 9/30/2008 3:00 AM & 0.107 & 16.4 & 0.089 & 0.308 & 17.1 & 0.39 & 0.042 & 0.043 & 16.5 & 0.045 & 0.424 & 16.2 & 0.419 & 15.9 & -- \\
\hline 9/30/2008 4:00 AM & 0.107 & 16.4 & 0.088 & 0.308 & 17.1 & 0.39 & 0.042 & 0.043 & 16.5 & 0.045 & 0.424 & 16.2 & 0.419 & 15.9 & -- \\
\hline 9/30/2008 5:00 AM & 0.107 & 16.4 & 0.088 & 0.308 & 17.1 & 0.39 & 0.042 & 0.043 & 16.5 & 0.045 & 0.424 & 16.2 & 0.419 & 15.9 & -- \\
\hline 9/30/2008 6:00 AM & 0.107 & 16.4 & 0.088 & 0.308 & 17.1 & 0.39 & 0.042 & 0.042 & 16.5 & 0.047 & 0.424 & 16.2 & 0.419 & 15.9 & -- \\
\hline 9/30/2008 7:00 AM & 0.107 & 16.4 & 0.088 & 0.308 & 17.1 & 0.39 & 0.042 & 0.042 & 16.5 & 0.047 & 0.424 & 16.2 & 0.419 & 15.9 & -- \\
\hline 9/30/2008 8:00 AM & 0.107 & 16.4 & 0.088 & 0.308 & 17.1 & 0.39 & 0.042 & 0.043 & 16.5 & 0.048 & 0.424 & 16.2 & 0.419 & 15.9 & -- \\
\hline 9/30/2008 9:00 AM & 0.107 & 16.4 & 0.088 & 0.308 & 17.1 & 0.39 & 0.042 & 0.043 & 16.5 & 0.048 & 0.425 & 16.2 & 0.420 & 15.9 & -- \\
\hline 9/30/2008 10:00 AM & 0.107 & 16.4 & 0.088 & 0.308 & 17.1 & 0.39 & 0.042 & 0.042 & 16.5 & 0.047 & 0.425 & 16.2 & 0.421 & 15.9 & -- \\
\hline 9/30/2008 11:00 AM & 0.107 & 16.4 & 0.088 & 0.308 & 17.1 & 0.39 & 0.042 & 0.042 & 16.5 & 0.047 & 0.424 & 16.2 & 0.422 & 15.9 & -- \\
\hline 9/30/2008 12:00 PM & 0.107 & 16.3 & 0.088 & 0.309 & 17.1 & 0.39 & 0.042 & 0.042 & 16.5 & 0.047 & 0.424 & 16.2 & 0.423 & 15.9 & -- \\
\hline 9/30/2008 1:00 PM & 0.106 & 16.3 & 0.088 & 0.309 & 17.1 & 0.39 & 0.041 & 0.043 & 16.5 & 0.047 & 0.424 & 16.2 & 0.422 & 15.9 & -- \\
\hline 9/30/2008 2:00 PM & 0.106 & 16.2 & 0.088 & 0.309 & 17.1 & 0.39 & 0.041 & 0.043 & 16.5 & 0.047 & 0.424 & 16.2 & 0.421 & 15.9 & -- \\
\hline 9/30/2008 3:00 PM & 0.105 & 16.2 & 0.088 & 0.309 & 17.1 & 0.39 & 0.041 & 0.043 & 16.5 & 0.047 & 0.423 & 16.2 & 0.421 & 15.9 & -- \\
\hline 9/30/2008 4:00 PM & 0.105 & 16.2 & 0.088 & 0.309 & 17.1 & 0.39 & 0.041 & 0.043 & 16.5 & 0.047 & 0.423 & 16.2 & 0.421 & 15.9 & -- \\
\hline 9/30/2008 5:00 PM & 0.105 & 16.2 & 0.088 & 0.308 & 17.1 & 0.39 & 0.041 & 0.043 & 16.5 & 0.047 & 0.423 & 16.2 & 0.421 & 15.8 & -- \\
\hline 9/30/2008 6:00 PM & 0.105 & 16.2 & 0.088 & 0.308 & 17.1 & 0.39 & 0.041 & 0.042 & 16.5 & 0.050 & 0.424 & 16.2 & 0.422 & 15.9 & -- \\
\hline 9/30/2008 7:00 PM & 0.105 & 16.1 & 0.088 & 0.308 & 17.1 & 0.39 & 0.042 & 0.042 & 16.5 & 0.048 & 0.423 & 16.2 & 0.422 & 15.9 & -- \\
\hline 9/30/2008 8:00 PM & 0.105 & 16.1 & 0.088 & 0.308 & 17.1 & 0.39 & 0.042 & 0.042 & 16.5 & 0.049 & 0.424 & 16.2 & 0.421 & 15.9 & -- \\
\hline 9/30/2008 9:00 PM & 0.105 & 16.1 & 0.088 & 0.308 & 17.1 & 0.39 & 0.042 & 0.042 & 16.5 & 0.048 & 0.425 & 16.2 & 0.420 & 15.9 & -- \\
\hline 9/30/2008 10:00 PM & 0.105 & 16.1 & 0.088 & 0.308 & 17.1 & 0.39 & 0.042 & 0.042 & 16.5 & 0.048 & 0.425 & 16.2 & 0.420 & 15.9 & -- \\
\hline 9/30/2008 11:00 PM & 0.105 & 16.1 & 0.088 & 0.308 & 17.1 & 0.39 & 0.042 & 0.042 & 16.5 & 0.048 & 0.425 & 16.2 & 0.420 & 15.9 & -- \\
\hline 10/1/2008 12:00 AM & 0.105 & 16.1 & 0.088 & 0.308 & 17.1 & 0.39 & 0.042 & 0.042 & 16.5 & 0.047 & 0.425 & 16.2 & 0.420 & 15.9 & -- \\
\hline 10/1/2008 1:00 AM & 0.105 & 16.2 & 0.088 & 0.308 & 17.1 & 0.39 & 0.042 & 0.042 & 16.5 & 0.047 & 0.424 & 16.2 & 0.420 & 15.9 & -- \\
\hline 10/1/2008 2:00 AM & 0.105 & 16.2 & 0.088 & 0.308 & 17.1 & 0.39 & 0.042 & 0.042 & 16.5 & 0.047 & 0.424 & 16.2 & 0.419 & 15.9 & -- \\
\hline 10/1/2008 3:00 AM & 0.106 & 16.2 & 0.088 & 0.308 & 17.1 & 0.39 & 0.042 & 0.042 & 16.5 & 0.047 & 0.424 & 16.2 & 0.419 & 15.9 & -- \\
\hline 10/1/2008 4:00 AM & 0.106 & 16.2 & 0.088 & 0.308 & 17.1 & 0.39 & 0.042 & 0.042 & 16.5 & 0.046 & 0.424 & 16.2 & 0.418 & 15.9 & -- \\
\hline 10/1/2008 5:00 AM & 0.106 & 16.2 & 0.087 & 0.308 & 17.1 & 0.39 & 0.042 & 0.042 & 16.5 & 0.045 & 0.424 & 16.2 & 0.418 & 15.9 & -- \\
\hline 10/1/2008 6:00 AM & 0.106 & 16.2 & 0.087 & 0.308 & 17.1 & 0.39 & 0.042 & 0.042 & 16.5 & 0.045 & 0.424 & 16.2 & 0.418 & 15.9 & -- \\
\hline 10/1/2008 7:00 AM & 0.106 & 16.2 & 0.087 & 0.308 & 17.1 & 0.39 & 0.042 & 0.042 & 16.5 & 0.047 & 0.424 & 16.2 & 0.418 & 15.9 & -- \\
\hline 10/1/2008 8:00 AM & 0.106 & 16.2 & 0.087 & 0.308 & 17.1 & 0.39 & 0.042 & 0.042 & 16.5 & 0.045 & 0.424 & 16.2 & 0.418 & 15.9 & -- \\
\hline 10/1/2008 9:00 AM & 0.105 & 16.2 & 0.087 & 0.308 & 17.1 & 0.39 & 0.042 & 0.042 & 16.5 & 0.045 & 0.424 & 16.2 & 0.419 & 15.9 & -- \\
\hline 10/1/2008 10:00 AM & 0.105 & 16.2 & 0.087 & 0.308 & 17.1 & 0.39 & 0.042 & 0.042 & 16.5 & 0.045 & 0.425 & 16.2 & 0.420 & 15.9 & -- \\
\hline 10/1/2008 11:00 AM & 0.105 & 16.2 & 0.087 & 0.308 & 17.1 & 0.39 & 0.042 & 0.042 & 16.5 & 0.047 & 0.423 & 16.2 & 0.422 & 15.9 & -- \\
\hline 10/1/2008 12:00 PM & 0.105 & 16.2 & 0.087 & 0.308 & 17.1 & 0.39 & 0.042 & 0.042 & 16.5 & 0.047 & 0.423 & 16.2 & 0.422 & 15.9 & -- \\
\hline 10/1/2008 1:00 PM & 0.104 & 16.1 & 0.087 & 0.308 & 17.1 & 0.39 & 0.041 & 0.042 & 16.5 & 0.047 & 0.423 & 16.2 & 0.422 & 15.9 & -- \\
\hline 10/1/2008 2:00 PM & 0.104 & 16.1 & 0.087 & 0.308 & 17.1 & 0.39 & 0.041 & 0.042 & 16.5 & 0.047 & 0.423 & 16.2 & 0.423 & 15.9 & -- \\
\hline
\end{tabular}


Appendix 1. Volumetric water content, temperature, and electrical-conductivity data collected at the flood-irrigated site during 2008.-Continued

[Depth in feet below land surface; volumetric water content dimensionless; temperature in degrees Celsius; bulk electrical conductivity in decisiemens per meter; --, no data]

\begin{tabular}{|c|c|c|c|c|c|c|c|c|c|c|c|c|c|c|c|}
\hline \multirow[b]{3}{*}{$\begin{array}{l}\text { Measurement } \\
\text { date and time }\end{array}$} & \multicolumn{15}{|c|}{ Depth } \\
\hline & \multicolumn{2}{|c|}{2} & \multirow{2}{*}{$\begin{array}{c}3 \\
\begin{array}{c}\text { Volumetric } \\
\text { water } \\
\text { content }\end{array}\end{array}$} & \multicolumn{3}{|c|}{4} & \multirow{2}{*}{$\begin{array}{c}6 \\
\begin{array}{c}\text { Volumetric } \\
\text { water } \\
\text { content }\end{array}\end{array}$} & \multicolumn{2}{|l|}{8} & \multirow{2}{*}{$\begin{array}{c}10 \\
\begin{array}{c}\text { Volumetric } \\
\text { water } \\
\text { content }\end{array}\end{array}$} & \multicolumn{2}{|c|}{12} & \multicolumn{2}{|c|}{15} & \multirow{2}{*}{$\begin{array}{c}18.5 \\
\text { Volumetric } \\
\text { water } \\
\text { content }\end{array}$} \\
\hline & $\begin{array}{c}\text { Volumetric } \\
\text { water } \\
\text { content }\end{array}$ & $\begin{array}{l}\text { Temper- } \\
\text { ature }\end{array}$ & & $\begin{array}{c}\text { Volumetric } \\
\text { water } \\
\text { content }\end{array}$ & $\begin{array}{l}\text { Temper- } \\
\text { ature }\end{array}$ & $\begin{array}{c}\text { Bulk } \\
\text { electrical } \\
\text { conduct- } \\
\text { ivity } \\
\end{array}$ & & $\begin{array}{l}\text { Volumetric } \\
\text { water } \\
\text { content }\end{array}$ & $\begin{array}{l}\text { Temper- } \\
\text { ature }\end{array}$ & & $\begin{array}{l}\text { Volumetric } \\
\text { water } \\
\text { content }\end{array}$ & $\begin{array}{l}\text { Temper- } \\
\text { ature }\end{array}$ & $\begin{array}{l}\text { Volumetric } \\
\text { water } \\
\text { content }\end{array}$ & $\begin{array}{l}\text { Temper- } \\
\text { ature }\end{array}$ & \\
\hline 10/1/2008 3:00 PM & 0.104 & 16.1 & 0.087 & 0.308 & 17.1 & 0.39 & 0.041 & 0.042 & 16.5 & 0.047 & 0.423 & 16.2 & 0.422 & 15.9 & -- \\
\hline 10/1/2008 4:00 PM & 0.104 & 16.1 & 0.087 & 0.308 & 17.1 & 0.39 & 0.041 & 0.042 & 16.5 & 0.047 & 0.423 & 16.2 & 0.422 & 15.9 & -- \\
\hline 10/1/2008 5:00 PM & 0.104 & 16.1 & 0.087 & 0.308 & 17.1 & 0.39 & 0.041 & 0.042 & 16.5 & 0.045 & 0.423 & 16.2 & 0.422 & 15.9 & -- \\
\hline 10/1/2008 6:00 PM & 0.104 & 16.0 & 0.087 & 0.308 & 17.1 & 0.39 & 0.041 & 0.042 & 16.5 & 0.044 & 0.423 & 16.2 & 0.423 & 15.9 & -- \\
\hline 10/1/2008 7:00 PM & 0.104 & 16.0 & 0.087 & 0.308 & 17.1 & 0.39 & 0.041 & 0.042 & 16.5 & 0.043 & 0.423 & 16.2 & 0.423 & 15.9 & -- \\
\hline 10/1/2008 8:00 PM & 0.104 & 16.0 & 0.087 & 0.308 & 17.1 & 0.39 & 0.042 & 0.042 & 16.5 & 0.043 & 0.423 & 16.2 & 0.422 & 15.9 & -- \\
\hline 10/1/2008 9:00 PM & 0.104 & 16.0 & 0.087 & 0.308 & 17.1 & 0.39 & 0.041 & 0.042 & 16.5 & 0.043 & 0.424 & 16.2 & 0.422 & 15.9 & -- \\
\hline 10/1/2008 10:00 PM & 0.104 & 16.0 & 0.087 & 0.308 & 17.1 & 0.39 & 0.042 & 0.042 & 16.5 & 0.043 & 0.424 & 16.2 & 0.423 & 15.9 & -- \\
\hline 10/1/2008 11:00 PM & 0.104 & 16.0 & 0.087 & 0.308 & 17.1 & 0.39 & 0.042 & 0.042 & 16.5 & 0.043 & 0.424 & 16.2 & 0.423 & 15.9 & -- \\
\hline 10/2/2008 12:00 AM & 0.104 & 16.0 & 0.087 & 0.308 & 17.0 & 0.39 & 0.042 & 0.042 & 16.5 & 0.043 & 0.424 & 16.2 & 0.423 & 15.9 & -- \\
\hline 10/2/2008 1:00 AM & 0.104 & 16.0 & 0.087 & 0.308 & 17.0 & 0.39 & 0.042 & 0.042 & 16.5 & 0.043 & 0.424 & 16.2 & 0.423 & 15.9 & -- \\
\hline 10/2/2008 2:00 AM & 0.104 & 16.0 & 0.087 & 0.308 & 17.0 & 0.39 & 0.041 & 0.042 & 16.5 & 0.043 & 0.424 & 16.2 & 0.422 & 15.9 & -- \\
\hline 10/2/2008 3:00 AM & 0.104 & 16.0 & 0.087 & 0.308 & 17.0 & 0.39 & 0.042 & 0.042 & 16.5 & 0.042 & 0.424 & 16.2 & 0.421 & 15.9 & -- \\
\hline 10/2/2008 4:00 AM & 0.104 & 16.1 & 0.087 & 0.308 & 17.0 & 0.39 & 0.042 & 0.042 & 16.5 & 0.042 & 0.424 & 16.2 & 0.420 & 15.9 & -- \\
\hline 10/2/2008 5:00 AM & 0.104 & 16.1 & 0.087 & 0.308 & 17.0 & 0.39 & 0.042 & 0.042 & 16.5 & 0.042 & 0.424 & 16.2 & 0.419 & 15.9 & -- \\
\hline 10/2/2008 6:00 AM & 0.104 & 16.1 & 0.087 & 0.307 & 17.0 & 0.39 & 0.042 & 0.042 & 16.5 & 0.042 & 0.424 & 16.2 & 0.419 & 15.9 & -- \\
\hline 10/2/2008 7:00 AM & 0.104 & 16.1 & 0.087 & 0.308 & 17.0 & 0.39 & 0.042 & 0.042 & 16.5 & 0.042 & 0.424 & 16.2 & 0.419 & 15.9 & -- \\
\hline 10/2/2008 8:00 AM & 0.104 & 16.1 & 0.087 & 0.308 & 17.0 & 0.39 & 0.042 & 0.042 & 16.5 & 0.042 & 0.424 & 16.2 & 0.419 & 15.9 & -- \\
\hline 10/2/2008 9:00 AM & 0.104 & 16.1 & 0.087 & 0.308 & 17.0 & 0.39 & 0.042 & 0.042 & 16.5 & 0.042 & 0.424 & 16.2 & 0.419 & 15.9 & -- \\
\hline 10/2/2008 10:00 AM & 0.104 & 16.1 & 0.087 & 0.308 & 17.0 & 0.39 & 0.042 & 0.042 & 16.5 & 0.043 & 0.424 & 16.2 & 0.421 & 15.9 & -- \\
\hline 10/2/2008 11:00 AM & 0.104 & 16.1 & 0.087 & 0.308 & 17.0 & 0.39 & 0.042 & 0.042 & 16.5 & 0.045 & 0.422 & 16.2 & 0.422 & 15.9 & -- \\
\hline 10/2/2008 12:00 PM & 0.103 & 16.1 & 0.087 & 0.308 & 17.0 & 0.39 & 0.041 & 0.042 & 16.5 & 0.046 & 0.423 & 16.2 & 0.423 & 15.9 & -- \\
\hline 10/2/2008 1:00 PM & 0.103 & 16.0 & 0.087 & 0.308 & 17.0 & 0.39 & 0.041 & 0.042 & 16.5 & 0.047 & 0.423 & 16.2 & 0.422 & 15.9 & -- \\
\hline 10/2/2008 2:00 PM & 0.103 & 16.1 & 0.087 & 0.308 & 17.0 & 0.39 & 0.041 & 0.042 & 16.5 & 0.047 & 0.423 & 16.2 & 0.423 & 15.9 & -- \\
\hline 10/2/2008 3:00 PM & 0.103 & 16.1 & 0.087 & 0.308 & 17.0 & 0.39 & 0.041 & 0.042 & 16.5 & 0.047 & 0.423 & 16.2 & 0.422 & 15.9 & -- \\
\hline 10/2/2008 4:00 PM & 0.103 & 16.0 & 0.087 & 0.308 & 17.0 & 0.38 & 0.041 & 0.042 & 16.5 & 0.047 & 0.423 & 16.2 & 0.423 & 15.9 & -- \\
\hline 10/2/2008 5:00 PM & 0.103 & 16.0 & 0.087 & 0.308 & 17.0 & 0.38 & 0.041 & 0.042 & 16.5 & 0.047 & 0.422 & 16.2 & 0.422 & 15.9 & -- \\
\hline 10/2/2008 6:00 PM & 0.103 & 16.0 & 0.087 & 0.308 & 17.0 & 0.38 & 0.041 & 0.042 & 16.5 & 0.046 & 0.422 & 16.2 & 0.421 & 15.8 & -- \\
\hline 10/2/2008 7:00 PM & 0.103 & 16.0 & 0.087 & 0.307 & 17.0 & 0.38 & 0.041 & 0.042 & 16.5 & 0.046 & 0.422 & 16.2 & 0.419 & 15.9 & -- \\
\hline 10/2/2008 8:00 PM & 0.103 & 16.0 & 0.087 & 0.308 & 17.0 & 0.38 & 0.041 & 0.042 & 16.5 & 0.046 & 0.422 & 16.2 & 0.418 & 15.9 & -- \\
\hline 10/2/2008 9:00 PM & 0.103 & 16.0 & 0.087 & 0.308 & 17.0 & 0.38 & 0.041 & 0.042 & 16.5 & 0.046 & 0.423 & 16.2 & 0.419 & 15.9 & -- \\
\hline 10/2/2008 10:00 PM & 0.103 & 16.0 & 0.087 & 0.308 & 17.0 & 0.38 & 0.041 & 0.042 & 16.5 & 0.046 & 0.424 & 16.2 & 0.419 & 15.9 & -- \\
\hline 10/2/2008 11:00 PM & 0.103 & 16.0 & 0.087 & 0.307 & 17.0 & 0.38 & 0.041 & 0.042 & 16.5 & 0.045 & 0.424 & 16.2 & 0.420 & 15.9 & -- \\
\hline 10/3/2008 12:00 AM & 0.103 & 16.0 & 0.087 & 0.307 & 17.0 & 0.38 & 0.041 & 0.042 & 16.5 & 0.045 & 0.424 & 16.2 & 0.420 & 15.9 & -- \\
\hline 10/3/2008 1:00 AM & 0.103 & 16.0 & 0.087 & 0.307 & 17.0 & 0.38 & 0.041 & 0.042 & 16.5 & 0.045 & 0.424 & 16.2 & 0.420 & 15.9 & -- \\
\hline 10/3/2008 2:00 AM & 0.103 & 16.0 & 0.087 & 0.308 & 17.0 & 0.38 & 0.041 & 0.042 & 16.5 & 0.045 & 0.424 & 16.1 & 0.420 & 15.9 & -- \\
\hline 10/3/2008 3:00 AM & 0.103 & 16.0 & 0.087 & 0.307 & 17.0 & 0.38 & 0.041 & 0.042 & 16.5 & 0.045 & 0.423 & 16.2 & 0.420 & 15.9 & -- \\
\hline 10/3/2008 4:00 AM & 0.103 & 16.0 & 0.087 & 0.307 & 17.0 & 0.38 & 0.041 & 0.042 & 16.5 & 0.045 & 0.423 & 16.2 & 0.420 & 15.9 & -- \\
\hline 10/3/2008 5:00 AM & 0.103 & 16.0 & 0.087 & 0.307 & 17.0 & 0.39 & 0.041 & 0.042 & 16.5 & 0.045 & 0.423 & 16.2 & 0.420 & 15.9 & -- \\
\hline 10/3/2008 6:00 AM & 0.103 & 16.1 & 0.087 & 0.307 & 17.0 & 0.39 & 0.041 & 0.042 & 16.5 & 0.047 & 0.423 & 16.1 & 0.421 & 15.9 & -- \\
\hline
\end{tabular}


Appendix 1. Volumetric water content, temperature, and electrical-conductivity data collected at the flood-irrigated site during 2008.-Continued

[Depth in feet below land surface; volumetric water content dimensionless; temperature in degrees Celsius; bulk electrical conductivity in decisiemens per meter; --, no data]

\begin{tabular}{|c|c|c|c|c|c|c|c|c|c|c|c|c|c|c|c|}
\hline \multirow[b]{3}{*}{$\begin{array}{l}\text { Measurement } \\
\text { date and time }\end{array}$} & \multicolumn{15}{|c|}{ Depth } \\
\hline & \multicolumn{2}{|l|}{$\begin{array}{r}2 \\
\end{array}$} & \multirow{2}{*}{ 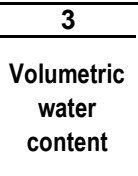 } & \multicolumn{3}{|c|}{4} & \multirow{2}{*}{$\begin{array}{c}6 \\
\begin{array}{c}\text { Volumetric } \\
\text { water } \\
\text { content }\end{array}\end{array}$} & \multicolumn{2}{|l|}{8} & \multirow{2}{*}{$\begin{array}{c}10 \\
\text { Volumetric } \\
\text { water } \\
\text { content }\end{array}$} & \multicolumn{2}{|c|}{12} & \multicolumn{2}{|c|}{15} & \multirow{2}{*}{$\begin{array}{c}18.5 \\
\text { Volumetric } \\
\text { water } \\
\text { content }\end{array}$} \\
\hline & $\begin{array}{c}\text { Volumetric } \\
\text { water } \\
\text { content }\end{array}$ & $\begin{array}{l}\text { Temper- } \\
\text { ature }\end{array}$ & & $\begin{array}{c}\text { Volumetric } \\
\text { water } \\
\text { content }\end{array}$ & $\begin{array}{l}\text { Temper- } \\
\text { ature }\end{array}$ & $\begin{array}{l}\text { Bulk } \\
\text { electrical } \\
\text { conduct- } \\
\text { ivity }\end{array}$ & & $\begin{array}{l}\text { Volumetric } \\
\text { water } \\
\text { content }\end{array}$ & $\begin{array}{l}\text { Temper- } \\
\text { ature }\end{array}$ & & $\begin{array}{c}\text { Volumetric } \\
\text { water } \\
\text { content }\end{array}$ & $\begin{array}{l}\text { Temper- } \\
\text { ature }\end{array}$ & $\begin{array}{l}\text { Volumetric } \\
\text { water } \\
\text { content }\end{array}$ & $\begin{array}{l}\text { Temper- } \\
\text { ature }\end{array}$ & \\
\hline 10/3/2008 7:00 AM & 0.103 & 16.0 & 0.087 & 0.307 & 17.0 & 0.39 & 0.041 & 0.042 & 16.5 & 0.045 & 0.423 & 16.1 & 0.421 & 15.9 & -- \\
\hline 10/3/2008 8:00 AM & 0.103 & 16.1 & 0.087 & 0.307 & 17.0 & 0.39 & 0.041 & 0.042 & 16.5 & 0.043 & 0.423 & 16.2 & 0.421 & 15.9 & -- \\
\hline 10/3/2008 9:00 AM & 0.103 & 16.1 & 0.087 & 0.307 & 17.0 & 0.39 & 0.042 & 0.042 & 16.5 & 0.045 & 0.424 & 16.2 & 0.420 & 15.9 & -- \\
\hline 10/3/2008 10:00 AM & 0.103 & 16.1 & 0.086 & 0.307 & 17.0 & 0.39 & 0.041 & 0.042 & 16.5 & 0.045 & 0.423 & 16.2 & 0.419 & 15.9 & -- \\
\hline 10/3/2008 11:00 AM & 0.103 & 16.0 & 0.086 & 0.307 & 17.0 & 0.39 & 0.041 & 0.042 & 16.5 & 0.044 & 0.422 & 16.2 & 0.418 & 15.9 & -- \\
\hline 10/3/2008 12:00 PM & 0.103 & 16.1 & 0.086 & 0.307 & 17.0 & 0.39 & 0.041 & 0.042 & 16.5 & 0.043 & 0.422 & 16.2 & 0.419 & 15.9 & -- \\
\hline 10/3/2008 1:00 PM & 0.101 & 16.1 & 0.087 & 0.307 & 17.0 & 0.39 & 0.041 & 0.042 & 16.5 & 0.043 & 0.422 & 16.2 & 0.421 & 15.9 & -- \\
\hline 10/3/2008 2:00 PM & 0.101 & 16.0 & 0.087 & 0.308 & 17.0 & 0.39 & 0.041 & 0.042 & 16.5 & 0.043 & 0.422 & 16.1 & 0.422 & 15.9 & -- \\
\hline 10/3/2008 3:00 PM & 0.101 & 16.0 & 0.087 & 0.308 & 17.0 & 0.39 & 0.041 & 0.042 & 16.5 & 0.043 & 0.422 & 16.2 & 0.422 & 15.8 & -- \\
\hline 10/3/2008 4:00 PM & 0.101 & 16.0 & 0.087 & 0.308 & 17.0 & 0.38 & 0.041 & 0.042 & 16.5 & 0.043 & 0.422 & 16.2 & 0.422 & 15.9 & -- \\
\hline 10/3/2008 5:00 PM & 0.100 & 16.0 & 0.086 & 0.307 & 17.0 & 0.38 & 0.041 & 0.042 & 16.5 & 0.043 & 0.422 & 16.2 & 0.421 & 15.8 & -- \\
\hline 10/3/2008 6:00 PM & 0.101 & 15.9 & 0.086 & 0.308 & 17.0 & 0.38 & 0.041 & 0.042 & 16.5 & 0.044 & 0.422 & 16.2 & 0.420 & 15.8 & -- \\
\hline 10/3/2008 7:00 PM & 0.101 & 15.9 & 0.086 & 0.307 & 17.0 & 0.38 & 0.041 & 0.042 & 16.5 & 0.045 & 0.422 & 16.2 & 0.421 & 15.8 & -- \\
\hline 10/3/2008 8:00 PM & 0.101 & 15.9 & 0.086 & 0.307 & 17.0 & 0.38 & 0.041 & 0.042 & 16.5 & 0.045 & 0.422 & 16.2 & 0.421 & 15.8 & -- \\
\hline 10/3/2008 9:00 PM & 0.101 & 15.9 & 0.086 & 0.307 & 17.0 & 0.38 & 0.041 & 0.042 & 16.5 & 0.045 & 0.423 & 16.2 & 0.421 & 15.8 & -- \\
\hline 10/3/2008 10:00 PM & 0.101 & 15.8 & 0.086 & 0.307 & 17.0 & 0.38 & 0.041 & 0.042 & 16.5 & 0.045 & 0.423 & 16.2 & 0.422 & 15.8 & -- \\
\hline 10/3/2008 11:00 PM & 0.101 & 15.9 & 0.086 & 0.307 & 17.0 & 0.38 & 0.041 & 0.042 & 16.5 & 0.045 & 0.424 & 16.1 & 0.421 & 15.8 & -- \\
\hline 10/4/2008 12:00 AM & 0.101 & 15.9 & 0.086 & 0.307 & 17.0 & 0.38 & 0.041 & 0.042 & 16.5 & 0.045 & 0.424 & 16.1 & 0.420 & 15.8 & -- \\
\hline 10/4/2008 1:00 AM & 0.101 & 15.9 & 0.086 & 0.307 & 17.0 & 0.38 & 0.041 & 0.042 & 16.5 & 0.044 & 0.424 & 16.2 & 0.421 & 15.8 & -- \\
\hline 10/4/2008 2:00 AM & 0.101 & 15.9 & 0.086 & 0.307 & 17.0 & 0.38 & 0.041 & 0.042 & 16.5 & 0.044 & 0.424 & 16.2 & 0.420 & 15.8 & -- \\
\hline 10/4/2008 3:00 AM & 0.101 & 15.9 & 0.086 & 0.307 & 17.0 & 0.38 & 0.041 & 0.042 & 16.5 & 0.044 & 0.424 & 16.2 & 0.419 & 15.8 & -- \\
\hline 10/4/2008 4:00 AM & 0.101 & 16.0 & 0.086 & 0.307 & 17.0 & 0.38 & 0.041 & 0.042 & 16.5 & 0.044 & 0.423 & 16.2 & 0.419 & 15.8 & -- \\
\hline 10/4/2008 5:00 AM & 0.101 & 15.9 & 0.086 & 0.307 & 17.0 & 0.38 & 0.041 & 0.042 & 16.5 & 0.044 & 0.423 & 16.1 & 0.419 & 15.8 & -- \\
\hline 10/4/2008 6:00 AM & 0.101 & 16.0 & 0.086 & 0.307 & 17.0 & 0.38 & 0.041 & 0.042 & 16.5 & 0.043 & 0.423 & 16.1 & 0.419 & 15.8 & -- \\
\hline 10/4/2008 7:00 AM & 0.101 & 16.0 & 0.086 & 0.307 & 17.0 & 0.38 & 0.041 & 0.042 & 16.5 & 0.043 & 0.423 & 16.1 & 0.419 & 15.8 & -- \\
\hline 10/4/2008 8:00 AM & 0.101 & 16.0 & 0.086 & 0.307 & 17.0 & 0.38 & 0.041 & 0.042 & 16.5 & 0.042 & 0.423 & 16.2 & 0.419 & 15.8 & -- \\
\hline 10/4/2008 9:00 AM & 0.101 & 16.0 & 0.086 & 0.307 & 17.0 & 0.38 & 0.041 & 0.042 & 16.5 & 0.042 & 0.423 & 16.2 & 0.420 & 15.8 & -- \\
\hline 10/4/2008 10:00 AM & 0.101 & 16.0 & 0.086 & 0.307 & 17.0 & 0.38 & 0.041 & 0.042 & 16.4 & 0.042 & 0.422 & 16.2 & 0.422 & 15.8 & -- \\
\hline 10/4/2008 11:00 AM & 0.101 & 16.0 & 0.086 & 0.307 & 17.0 & 0.38 & 0.041 & 0.042 & 16.4 & 0.042 & 0.422 & 16.2 & 0.422 & 15.8 & -- \\
\hline 10/4/2008 12:00 PM & 0.101 & 16.0 & 0.086 & 0.307 & 17.0 & 0.38 & 0.041 & 0.042 & 16.4 & 0.042 & 0.422 & 16.2 & 0.421 & 15.8 & -- \\
\hline 10/4/2008 1:00 PM & 0.100 & 16.1 & 0.086 & 0.307 & 17.0 & 0.38 & 0.041 & 0.042 & 16.5 & 0.042 & 0.422 & 16.2 & 0.421 & 15.8 & -- \\
\hline 10/4/2008 2:00 PM & 0.100 & 16.0 & 0.086 & 0.307 & 17.0 & 0.38 & 0.041 & 0.042 & 16.5 & 0.042 & 0.422 & 16.1 & 0.420 & 15.8 & -- \\
\hline 10/4/2008 3:00 PM & 0.100 & 16.0 & 0.086 & 0.307 & 17.0 & 0.38 & 0.041 & 0.042 & 16.4 & 0.042 & 0.422 & 16.1 & 0.420 & 15.8 & -- \\
\hline 10/4/2008 4:00 PM & 0.100 & 16.0 & 0.086 & 0.307 & 17.0 & 0.38 & 0.041 & 0.042 & 16.5 & 0.042 & 0.422 & 16.2 & 0.420 & 15.8 & -- \\
\hline 10/4/2008 5:00 PM & 0.100 & 16.0 & 0.085 & 0.307 & 17.0 & 0.38 & 0.041 & 0.042 & 16.5 & 0.042 & 0.422 & 16.2 & 0.421 & 15.8 & -- \\
\hline 10/4/2008 6:00 PM & 0.100 & 15.9 & 0.085 & 0.307 & 17.0 & 0.38 & 0.041 & 0.042 & 16.4 & 0.042 & 0.422 & 16.1 & 0.421 & 15.8 & -- \\
\hline 10/4/2008 7:00 PM & 0.100 & 15.9 & 0.085 & 0.307 & 16.9 & 0.38 & 0.041 & 0.042 & 16.4 & 0.042 & 0.422 & 16.2 & 0.421 & 15.8 & -- \\
\hline 10/4/2008 8:00 PM & 0.100 & 15.9 & 0.085 & 0.307 & 16.9 & 0.38 & 0.041 & 0.042 & 16.5 & 0.042 & 0.421 & 16.2 & 0.421 & 15.8 & -- \\
\hline 10/4/2008 9:00 PM & 0.100 & 15.9 & 0.085 & 0.307 & 16.9 & 0.38 & 0.041 & 0.042 & 16.5 & 0.042 & 0.422 & 16.1 & 0.421 & 15.8 & -- \\
\hline 10/4/2008 10:00 PM & 0.100 & 15.9 & 0.085 & 0.307 & 16.9 & 0.38 & 0.041 & 0.042 & 16.5 & 0.042 & 0.422 & 16.2 & 0.421 & 15.8 & -- \\
\hline
\end{tabular}


Appendix 1. Volumetric water content, temperature, and electrical-conductivity data collected at the flood-irrigated site during 2008.-Continued

[Depth in feet below land surface; volumetric water content dimensionless; temperature in degrees Celsius; bulk electrical conductivity in decisiemens per meter; --, no data]

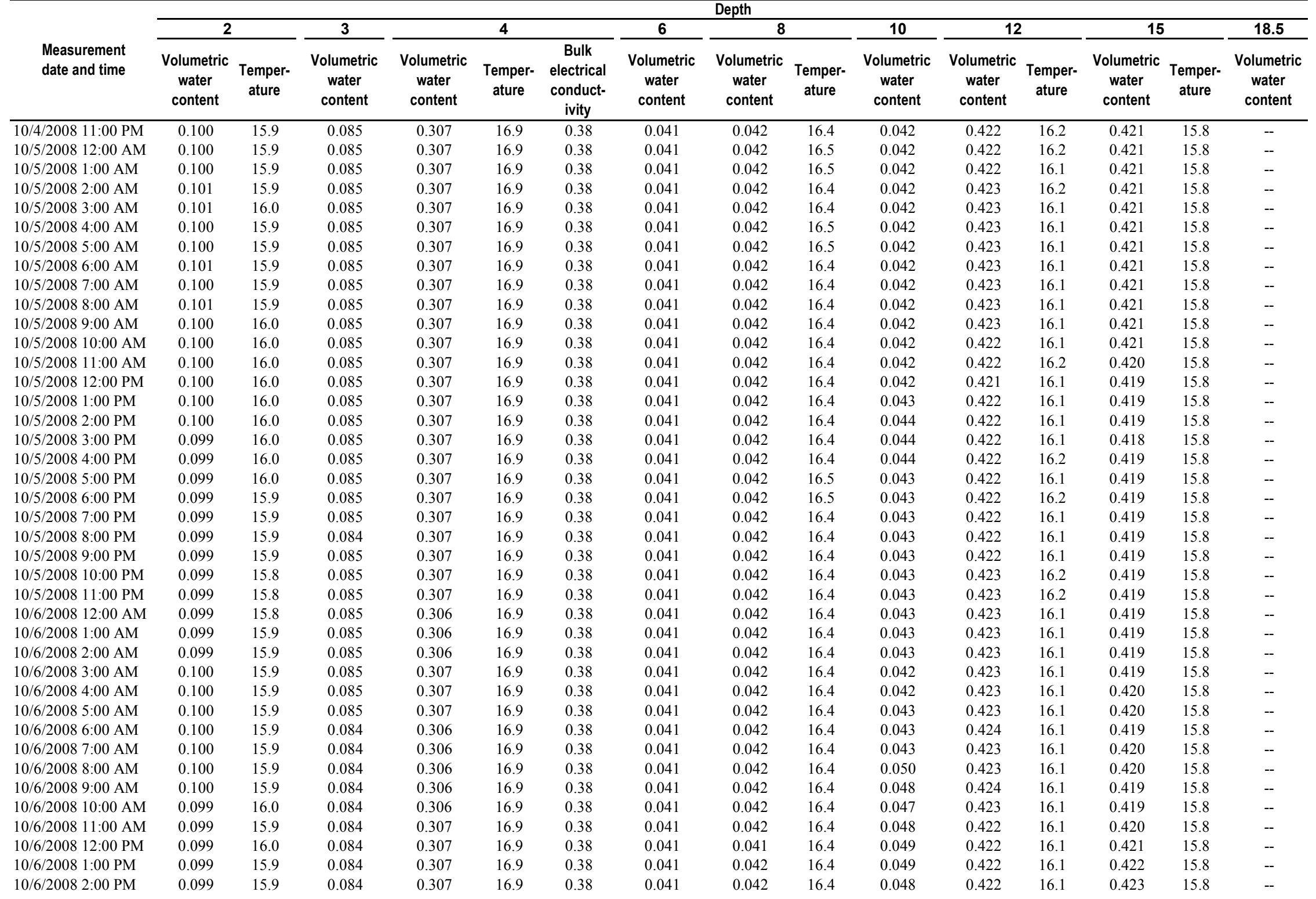


Appendix 1. Volumetric water content, temperature, and electrical-conductivity data collected at the flood-irrigated site during 2008.-Continued

[Depth in feet below land surface; volumetric water content dimensionless; temperature in degrees Celsius; bulk electrical conductivity in decisiemens per meter; --, no data]

\begin{tabular}{|c|c|c|c|c|c|c|c|c|c|c|c|c|c|c|c|}
\hline \multirow[b]{3}{*}{$\begin{array}{l}\text { Measurement } \\
\text { date and time }\end{array}$} & \multicolumn{15}{|c|}{ Depth } \\
\hline & \multicolumn{2}{|l|}{2} & \multirow{2}{*}{$\begin{array}{c}3 \\
\begin{array}{c}\text { Volumetric } \\
\text { water } \\
\text { content }\end{array}\end{array}$} & \multicolumn{3}{|c|}{4} & \multirow{2}{*}{$\begin{array}{c}6 \\
\begin{array}{c}\text { Volumetric } \\
\text { water } \\
\text { content }\end{array}\end{array}$} & \multicolumn{2}{|l|}{8} & \multirow{2}{*}{$\begin{array}{c}10 \\
\text { Volumetric } \\
\text { water } \\
\text { content }\end{array}$} & \multicolumn{2}{|c|}{12} & \multicolumn{2}{|c|}{15} & \multirow{2}{*}{$\begin{array}{c}18.5 \\
\text { Volumetric } \\
\text { water } \\
\text { content }\end{array}$} \\
\hline & $\begin{array}{l}\text { Volumetric } \\
\text { water } \\
\text { content }\end{array}$ & $\begin{array}{l}\text { Temper- } \\
\text { ature }\end{array}$ & & $\begin{array}{c}\text { Volumetric } \\
\text { water } \\
\text { content }\end{array}$ & $\begin{array}{l}\text { Temper- } \\
\text { ature }\end{array}$ & $\begin{array}{c}\text { Bulk } \\
\text { electrical } \\
\text { conduct- } \\
\text { ivity } \\
\end{array}$ & & $\begin{array}{l}\text { Volumetric } \\
\text { water } \\
\text { content }\end{array}$ & $\begin{array}{l}\text { Temper- } \\
\text { ature }\end{array}$ & & $\begin{array}{l}\text { Volumetric } \\
\text { water } \\
\text { content }\end{array}$ & $\begin{array}{l}\text { Temper- } \\
\text { ature }\end{array}$ & $\begin{array}{l}\text { Volumetric } \\
\text { water } \\
\text { content }\end{array}$ & $\begin{array}{l}\text { Temper- } \\
\text { ature }\end{array}$ & \\
\hline 10/6/2008 3:00 PM & 0.098 & 16.0 & 0.084 & 0.307 & 16.9 & 0.38 & 0.041 & 0.042 & 16.4 & 0.048 & 0.422 & 16.1 & 0.422 & 15.8 & -- \\
\hline 10/6/2008 4:00 PM & 0.098 & 15.9 & 0.084 & 0.307 & 16.9 & 0.38 & 0.041 & 0.042 & 16.4 & 0.048 & 0.422 & 16.2 & 0.421 & 15.8 & -- \\
\hline 10/6/2008 5:00 PM & 0.098 & 15.9 & 0.084 & 0.307 & 16.9 & 0.38 & 0.041 & 0.041 & 16.4 & 0.048 & 0.422 & 16.1 & 0.421 & 15.8 & -- \\
\hline 10/6/2008 6:00 PM & 0.098 & 15.8 & 0.084 & 0.307 & 16.9 & 0.38 & 0.040 & 0.041 & 16.4 & 0.048 & 0.422 & 16.1 & 0.421 & 15.8 & -- \\
\hline 10/6/2008 7:00 PM & 0.098 & 15.8 & 0.084 & 0.306 & 16.9 & 0.38 & 0.040 & 0.041 & 16.4 & 0.047 & 0.423 & 16.1 & 0.422 & 15.8 & -- \\
\hline 10/6/2008 8:00 PM & 0.098 & 15.8 & 0.084 & 0.307 & 16.9 & 0.38 & 0.040 & 0.041 & 16.4 & 0.047 & 0.424 & 16.1 & 0.422 & 15.8 & -- \\
\hline 10/6/2008 9:00 PM & 0.098 & 15.8 & 0.084 & 0.306 & 16.9 & 0.38 & 0.040 & 0.042 & 16.4 & 0.047 & 0.424 & 16.1 & 0.422 & 15.8 & -- \\
\hline 10/6/2008 10:00 PM & 0.098 & 15.8 & 0.084 & 0.306 & 16.9 & 0.38 & 0.040 & 0.042 & 16.4 & 0.047 & 0.424 & 16.1 & 0.421 & 15.8 & -- \\
\hline 10/6/2008 11:00 PM & 0.098 & 15.8 & 0.084 & 0.306 & 16.9 & 0.38 & 0.040 & 0.042 & 16.4 & 0.047 & 0.423 & 16.1 & 0.422 & 15.8 & -- \\
\hline 10/7/2008 12:00 AM & 0.098 & 15.8 & 0.084 & 0.306 & 16.9 & 0.38 & 0.040 & 0.042 & 16.4 & 0.047 & 0.423 & 16.1 & 0.423 & 15.8 & -- \\
\hline 10/7/2008 1:00 AM & 0.098 & 15.8 & 0.084 & 0.306 & 16.9 & 0.38 & 0.040 & 0.042 & 16.4 & 0.047 & 0.423 & 16.1 & 0.422 & 15.8 & -- \\
\hline 10/7/2008 2:00 AM & 0.098 & 15.8 & 0.084 & 0.306 & 16.9 & 0.38 & 0.040 & 0.042 & 16.4 & 0.047 & 0.423 & 16.1 & 0.422 & 15.8 & -- \\
\hline 10/7/2008 3:00 AM & 0.098 & 15.8 & 0.084 & 0.306 & 16.9 & 0.38 & 0.040 & 0.042 & 16.4 & 0.047 & 0.423 & 16.1 & 0.419 & 15.8 & -- \\
\hline 10/7/2008 4:00 AM & 0.098 & 15.8 & 0.084 & 0.306 & 16.9 & 0.38 & 0.040 & 0.042 & 16.4 & 0.047 & 0.423 & 16.1 & 0.419 & 15.8 & -- \\
\hline 10/7/2008 5:00 AM & 0.099 & 15.8 & 0.084 & 0.306 & 16.9 & 0.38 & 0.040 & 0.042 & 16.4 & 0.047 & 0.422 & 16.1 & 0.419 & 15.8 & -- \\
\hline 10/7/2008 6:00 AM & 0.098 & 15.8 & 0.084 & 0.306 & 16.9 & 0.38 & 0.040 & 0.041 & 16.4 & 0.047 & 0.422 & 16.1 & 0.419 & 15.8 & -- \\
\hline 10/7/2008 7:00 AM & 0.098 & 15.7 & 0.084 & 0.306 & 16.9 & 0.38 & 0.040 & 0.042 & 16.4 & 0.047 & 0.422 & 16.1 & 0.419 & 15.8 & -- \\
\hline 10/7/2008 8:00 AM & 0.099 & 15.7 & 0.083 & 0.306 & 16.9 & 0.38 & 0.040 & 0.041 & 16.4 & 0.047 & 0.423 & 16.1 & 0.420 & 15.8 & -- \\
\hline 10/7/2008 9:00 AM & 0.098 & 15.7 & 0.084 & 0.306 & 16.9 & 0.38 & 0.040 & 0.042 & 16.4 & 0.046 & 0.423 & 16.1 & 0.421 & 15.8 & -- \\
\hline 10/7/2008 10:00 AM & 0.098 & 15.7 & 0.083 & 0.306 & 16.9 & 0.38 & 0.041 & 0.041 & 16.4 & 0.046 & 0.424 & 16.1 & 0.422 & 15.8 & -- \\
\hline 10/7/2008 11:00 AM & 0.098 & 15.7 & 0.083 & 0.306 & 16.9 & 0.38 & 0.040 & 0.041 & 16.4 & 0.046 & 0.423 & 16.1 & 0.422 & 15.8 & -- \\
\hline 10/7/2008 12:00 PM & 0.098 & 15.7 & 0.083 & 0.306 & 16.9 & 0.38 & 0.041 & 0.041 & 16.4 & 0.046 & 0.422 & 16.1 & 0.420 & 15.8 & -- \\
\hline 10/7/2008 1:00 PM & 0.098 & 15.7 & 0.083 & 0.306 & 16.9 & 0.38 & 0.040 & 0.041 & 16.4 & 0.045 & 0.422 & 16.1 & 0.420 & 15.8 & -- \\
\hline 10/7/2008 2:00 PM & 0.098 & 15.6 & 0.084 & 0.306 & 16.9 & 0.38 & 0.040 & 0.042 & 16.4 & 0.045 & 0.423 & 16.1 & 0.421 & 15.8 & -- \\
\hline 10/7/2008 3:00 PM & 0.098 & 15.6 & 0.084 & 0.307 & 16.9 & 0.38 & 0.040 & 0.042 & 16.4 & 0.045 & 0.422 & 16.1 & 0.421 & 15.8 & -- \\
\hline 10/7/2008 4:00 PM & 0.098 & 15.6 & 0.084 & 0.307 & 16.9 & 0.38 & 0.040 & 0.042 & 16.4 & 0.045 & 0.422 & 16.1 & 0.421 & 15.8 & -- \\
\hline 10/7/2008 5:00 PM & 0.098 & 15.6 & 0.083 & 0.306 & 16.9 & 0.38 & 0.040 & 0.042 & 16.4 & 0.044 & 0.422 & 16.1 & 0.421 & 15.8 & -- \\
\hline 10/7/2008 6:00 PM & 0.097 & 15.5 & 0.083 & 0.306 & 16.9 & 0.38 & 0.041 & 0.041 & 16.4 & 0.044 & 0.422 & 16.1 & 0.420 & 15.8 & -- \\
\hline 10/7/2008 7:00 PM & 0.097 & 15.5 & 0.083 & 0.306 & 16.9 & 0.38 & 0.041 & 0.041 & 16.4 & 0.044 & 0.422 & 16.1 & 0.422 & 15.8 & -- \\
\hline 10/7/2008 8:00 PM & 0.098 & 15.4 & 0.083 & 0.306 & 16.9 & 0.38 & 0.040 & 0.041 & 16.4 & 0.044 & 0.423 & 16.1 & 0.422 & 15.8 & -- \\
\hline 10/7/2008 9:00 PM & 0.097 & 15.3 & 0.083 & 0.306 & 16.9 & 0.38 & 0.040 & 0.042 & 16.4 & 0.044 & 0.423 & 16.1 & 0.424 & 15.8 & -- \\
\hline 10/7/2008 10:00 PM & 0.098 & 15.3 & 0.083 & 0.306 & 16.9 & 0.38 & 0.040 & 0.042 & 16.4 & 0.044 & 0.423 & 16.1 & 0.424 & 15.9 & -- \\
\hline 10/7/2008 11:00 PM & 0.098 & 15.3 & 0.083 & 0.306 & 16.9 & 0.38 & 0.040 & 0.042 & 16.4 & 0.045 & 0.423 & 16.1 & 0.423 & 15.8 & -- \\
\hline 10/8/2008 12:00 AM & 0.098 & 15.4 & 0.083 & 0.306 & 16.9 & 0.38 & 0.040 & 0.042 & 16.4 & 0.044 & 0.423 & 16.1 & 0.420 & 15.8 & -- \\
\hline 10/8/2008 1:00 AM & 0.098 & 15.4 & 0.083 & 0.306 & 16.9 & 0.38 & 0.040 & 0.042 & 16.4 & 0.045 & 0.423 & 16.1 & 0.421 & 15.8 & -- \\
\hline 10/8/2008 2:00 AM & 0.098 & 15.4 & 0.083 & 0.306 & 16.9 & 0.38 & 0.040 & 0.042 & 16.4 & 0.045 & 0.422 & 16.1 & 0.422 & 15.8 & -- \\
\hline 10/8/2008 3:00 AM & 0.098 & 15.3 & 0.083 & 0.306 & 16.9 & 0.38 & 0.040 & 0.042 & 16.4 & 0.045 & 0.423 & 16.1 & 0.419 & 15.8 & -- \\
\hline 10/8/2008 4:00 AM & 0.098 & 15.3 & 0.083 & 0.306 & 16.9 & 0.38 & 0.040 & 0.041 & 16.4 & 0.045 & 0.422 & 16.1 & 0.420 & 15.8 & -- \\
\hline 10/8/2008 5:00 AM & 0.097 & 15.3 & 0.083 & 0.306 & 16.9 & 0.38 & 0.040 & 0.042 & 16.4 & 0.045 & 0.422 & 16.1 & 0.418 & 15.8 & -- \\
\hline 10/8/2008 6:00 AM & 0.098 & 15.3 & 0.083 & 0.306 & 16.9 & 0.38 & 0.040 & 0.042 & 16.4 & 0.046 & 0.423 & 16.1 & 0.418 & 15.8 & -- \\
\hline
\end{tabular}


Appendix 1. Volumetric water content, temperature, and electrical-conductivity data collected at the flood-irrigated site during 2008.-Continued

[Depth in feet below land surface; volumetric water content dimensionless; temperature in degrees Celsius; bulk electrical conductivity in decisiemens per meter; --, no data]

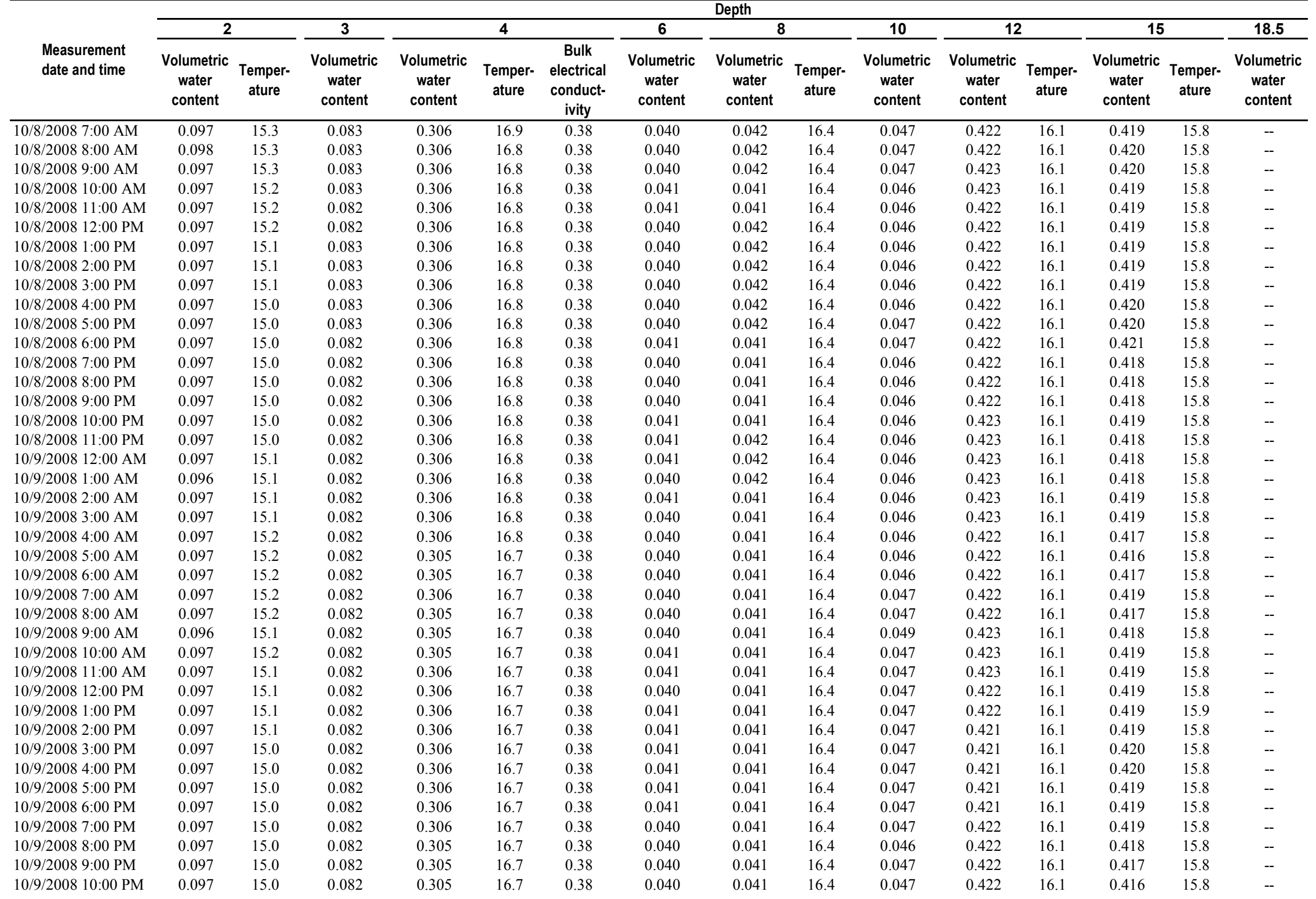


Appendix 1. Volumetric water content, temperature, and electrical-conductivity data collected at the flood-irrigated site during 2008.-Continued

[Depth in feet below land surface; volumetric water content dimensionless; temperature in degrees Celsius; bulk electrical conductivity in decisiemens per meter; --, no data]

\begin{tabular}{|c|c|c|c|c|c|c|c|c|c|c|c|c|c|c|c|}
\hline \multirow[b]{3}{*}{$\begin{array}{l}\text { Measurement } \\
\text { date and time }\end{array}$} & \multicolumn{15}{|c|}{ Depth } \\
\hline & \multicolumn{2}{|l|}{2} & \multirow{2}{*}{$\begin{array}{c}3 \\
\begin{array}{c}\text { Volumetric } \\
\text { water } \\
\text { content }\end{array}\end{array}$} & \multicolumn{3}{|c|}{4} & \multirow{2}{*}{$\begin{array}{c}6 \\
\begin{array}{c}\text { Volumetric } \\
\text { water } \\
\text { content }\end{array}\end{array}$} & \multicolumn{2}{|l|}{8} & \multirow{2}{*}{$\begin{array}{c}10 \\
\text { Volumetric } \\
\text { water } \\
\text { content }\end{array}$} & \multicolumn{2}{|c|}{12} & \multicolumn{2}{|c|}{15} & \multirow{2}{*}{$\begin{array}{c}18.5 \\
\text { Volumetric } \\
\text { water } \\
\text { content }\end{array}$} \\
\hline & $\begin{array}{l}\text { Volumetric } \\
\text { water } \\
\text { content }\end{array}$ & $\begin{array}{l}\text { Temper- } \\
\text { ature }\end{array}$ & & $\begin{array}{c}\text { Volumetric } \\
\text { water } \\
\text { content }\end{array}$ & $\begin{array}{l}\text { Temper- } \\
\text { ature }\end{array}$ & $\begin{array}{c}\text { Bulk } \\
\text { electrical } \\
\text { conduct- } \\
\text { ivity } \\
\end{array}$ & & $\begin{array}{l}\text { Volumetric } \\
\text { water } \\
\text { content }\end{array}$ & $\begin{array}{l}\text { Temper- } \\
\text { ature }\end{array}$ & & $\begin{array}{l}\text { Volumetric } \\
\text { water } \\
\text { content }\end{array}$ & $\begin{array}{l}\text { Temper- } \\
\text { ature }\end{array}$ & $\begin{array}{l}\text { Volumetric } \\
\text { water } \\
\text { content }\end{array}$ & $\begin{array}{l}\text { Temper- } \\
\text { ature }\end{array}$ & \\
\hline 10/9/2008 11:00 PM & 0.097 & 15.0 & 0.082 & 0.305 & 16.7 & 0.38 & 0.040 & 0.041 & 16.4 & 0.047 & 0.422 & 16.1 & 0.417 & 15.8 & -- \\
\hline 10/10/2008 12:00 AM & 0.097 & 15.0 & 0.082 & 0.305 & 16.7 & 0.38 & 0.040 & 0.041 & 16.4 & 0.047 & 0.422 & 16.1 & 0.417 & 15.8 & -- \\
\hline 10/10/2008 1:00 AM & 0.097 & 14.9 & 0.082 & 0.305 & 16.7 & 0.38 & 0.040 & 0.041 & 16.4 & 0.047 & 0.422 & 16.1 & 0.416 & 15.8 & -- \\
\hline 10/10/2008 2:00 AM & 0.096 & 14.9 & 0.081 & 0.305 & 16.7 & 0.38 & 0.040 & 0.041 & 16.4 & 0.047 & 0.421 & 16.1 & 0.416 & 15.8 & -- \\
\hline 10/10/2008 3:00 AM & 0.096 & 14.9 & 0.081 & 0.305 & 16.6 & 0.38 & 0.040 & 0.041 & 16.4 & 0.047 & 0.422 & 16.1 & 0.415 & 15.8 & -- \\
\hline 10/10/2008 4:00 AM & 0.096 & 14.9 & 0.081 & 0.305 & 16.6 & 0.38 & 0.040 & 0.041 & 16.4 & 0.047 & 0.422 & 16.1 & 0.415 & 15.8 & -- \\
\hline 10/10/2008 5:00 AM & 0.096 & 14.9 & 0.081 & 0.305 & 16.6 & 0.38 & 0.040 & 0.041 & 16.4 & 0.047 & 0.421 & 16.1 & 0.416 & 15.8 & -- \\
\hline 10/10/2008 6:00 AM & 0.096 & 14.9 & 0.081 & 0.305 & 16.6 & 0.38 & 0.040 & 0.041 & 16.4 & 0.048 & 0.421 & 16.1 & 0.418 & 15.8 & -- \\
\hline 10/10/2008 7:00 AM & 0.096 & 14.9 & 0.081 & 0.305 & 16.6 & 0.38 & 0.040 & 0.041 & 16.4 & 0.048 & 0.422 & 16.1 & 0.418 & 15.8 & -- \\
\hline 10/10/2008 8:00 AM & 0.096 & 14.9 & 0.081 & 0.305 & 16.6 & 0.38 & 0.040 & 0.041 & 16.4 & 0.048 & 0.422 & 16.1 & 0.418 & 15.8 & -- \\
\hline 10/10/2008 9:00 AM & 0.096 & 14.8 & 0.081 & 0.305 & 16.6 & 0.38 & 0.040 & 0.041 & 16.4 & 0.048 & 0.422 & 16.1 & 0.418 & 15.8 & -- \\
\hline 10/10/2008 10:00 AM & 0.096 & 14.8 & 0.081 & 0.305 & 16.6 & 0.38 & 0.040 & 0.041 & 16.4 & 0.048 & 0.423 & 16.1 & 0.416 & 15.8 & -- \\
\hline 10/10/2008 11:00 AM & 0.096 & 14.8 & 0.081 & 0.305 & 16.6 & 0.38 & 0.040 & 0.041 & 16.4 & 0.048 & 0.422 & 16.1 & 0.415 & 15.8 & -- \\
\hline 10/10/2008 12:00 PM & 0.096 & 14.8 & 0.081 & 0.306 & 16.6 & 0.38 & 0.040 & 0.041 & 16.4 & 0.048 & 0.422 & 16.1 & 0.415 & 15.8 & -- \\
\hline 10/10/2008 1:00 PM & 0.096 & 14.8 & 0.081 & 0.305 & 16.6 & 0.38 & 0.040 & 0.041 & 16.4 & 0.048 & 0.421 & 16.1 & 0.416 & 15.8 & -- \\
\hline 10/10/2008 2:00 PM & 0.096 & 14.8 & 0.081 & 0.305 & 16.6 & 0.38 & 0.040 & 0.041 & 16.4 & 0.049 & 0.421 & 16.1 & 0.418 & 15.8 & -- \\
\hline 10/10/2008 3:00 PM & 0.096 & 14.7 & 0.081 & 0.305 & 16.6 & 0.38 & 0.040 & 0.041 & 16.4 & 0.050 & 0.421 & 16.1 & 0.418 & 15.8 & -- \\
\hline 10/10/2008 4:00 PM & 0.096 & 14.6 & 0.081 & 0.305 & 16.6 & 0.38 & 0.040 & 0.041 & 16.4 & 0.050 & 0.421 & 16.1 & 0.419 & 15.8 & -- \\
\hline 10/10/2008 5:00 PM & 0.096 & 14.7 & 0.081 & 0.305 & 16.6 & 0.38 & 0.040 & 0.041 & 16.4 & 0.050 & 0.421 & 16.1 & 0.419 & 15.8 & -- \\
\hline 10/10/2008 6:00 PM & 0.096 & 14.6 & 0.081 & 0.305 & 16.6 & 0.38 & 0.040 & 0.041 & 16.4 & 0.050 & 0.422 & 16.1 & 0.420 & 15.8 & -- \\
\hline 10/10/2008 7:00 PM & 0.096 & 14.5 & 0.081 & 0.305 & 16.6 & 0.38 & 0.040 & 0.041 & 16.4 & 0.050 & 0.423 & 16.1 & 0.420 & 15.8 & -- \\
\hline 10/10/2008 8:00 PM & 0.096 & 14.5 & 0.081 & 0.305 & 16.6 & 0.38 & 0.040 & 0.041 & 16.3 & 0.050 & 0.423 & 16.1 & 0.420 & 15.8 & -- \\
\hline 10/10/2008 9:00 PM & 0.096 & 14.5 & 0.081 & 0.305 & 16.6 & 0.38 & 0.040 & 0.041 & 16.3 & 0.050 & 0.423 & 16.1 & 0.418 & 15.8 & -- \\
\hline 10/10/2008 10:00 PM & 0.096 & 14.5 & 0.081 & 0.305 & 16.6 & 0.38 & 0.040 & 0.041 & 16.4 & 0.050 & 0.423 & 16.1 & 0.415 & 15.8 & -- \\
\hline 10/10/2008 11:00 PM & 0.096 & 14.5 & 0.081 & 0.305 & 16.6 & 0.38 & 0.040 & 0.041 & 16.4 & 0.050 & 0.423 & 16.1 & 0.416 & 15.8 & -- \\
\hline 10/11/2008 12:00 AM & 0.096 & 14.5 & 0.081 & 0.305 & 16.6 & 0.38 & 0.040 & 0.041 & 16.4 & 0.049 & 0.423 & 16.1 & 0.416 & 15.8 & -- \\
\hline 10/11/2008 1:00 AM & 0.096 & 14.5 & 0.081 & 0.305 & 16.5 & 0.38 & 0.040 & 0.041 & 16.4 & 0.050 & 0.423 & 16.1 & 0.416 & 15.8 & -- \\
\hline 10/11/2008 2:00 AM & 0.096 & 14.5 & 0.081 & 0.305 & 16.5 & 0.38 & 0.040 & 0.041 & 16.3 & 0.051 & 0.423 & 16.1 & 0.416 & 15.8 & -- \\
\hline 10/11/2008 3:00 AM & 0.096 & 14.5 & 0.081 & 0.305 & 16.5 & 0.38 & 0.040 & 0.041 & 16.4 & 0.051 & 0.423 & 16.1 & 0.417 & 15.8 & -- \\
\hline 10/11/2008 4:00 AM & 0.096 & 14.5 & 0.081 & 0.305 & 16.5 & 0.38 & 0.040 & 0.041 & 16.3 & 0.051 & 0.423 & 16.1 & 0.417 & 15.8 & -- \\
\hline 10/11/2008 5:00 AM & 0.096 & 14.4 & 0.081 & 0.305 & 16.5 & 0.38 & 0.040 & 0.041 & 16.4 & 0.051 & 0.423 & 16.1 & 0.417 & 15.8 & -- \\
\hline 10/11/2008 6:00 AM & 0.096 & 14.4 & 0.081 & 0.305 & 16.5 & 0.38 & 0.040 & 0.041 & 16.3 & 0.051 & 0.423 & 16.1 & 0.417 & 15.8 & -- \\
\hline 10/11/2008 7:00 AM & 0.096 & 14.4 & 0.081 & 0.305 & 16.5 & 0.38 & 0.040 & 0.041 & 16.3 & 0.049 & 0.423 & 16.1 & 0.418 & 15.8 & -- \\
\hline 10/11/2008 8:00 AM & 0.096 & 14.4 & 0.081 & 0.305 & 16.5 & 0.38 & 0.040 & 0.041 & 16.3 & 0.050 & 0.422 & 16.1 & 0.418 & 15.8 & -- \\
\hline 10/11/2008 9:00 AM & 0.096 & 14.4 & 0.081 & 0.305 & 16.5 & 0.38 & 0.040 & 0.041 & 16.3 & 0.049 & 0.422 & 16.1 & 0.417 & 15.8 & -- \\
\hline 10/11/2008 10:00 AM & 0.096 & 14.4 & 0.081 & 0.305 & 16.5 & 0.38 & 0.040 & 0.041 & 16.3 & 0.050 & 0.423 & 16.1 & 0.417 & 15.8 & -- \\
\hline 10/11/2008 11:00 AM & 0.096 & 14.4 & 0.081 & 0.305 & 16.5 & 0.38 & 0.040 & 0.041 & 16.3 & 0.050 & 0.423 & 16.1 & 0.416 & 15.8 & -- \\
\hline 10/11/2008 12:00 PM & 0.096 & 14.3 & 0.081 & 0.305 & 16.5 & 0.38 & 0.040 & 0.041 & 16.3 & 0.050 & 0.423 & 16.1 & 0.416 & 15.8 & -- \\
\hline 10/11/2008 1:00 PM & 0.095 & 14.3 & 0.081 & 0.305 & 16.5 & 0.38 & 0.040 & 0.041 & 16.3 & 0.051 & 0.423 & 16.1 & 0.418 & 15.8 & -- \\
\hline 10/11/2008 2:00 PM & 0.095 & 14.3 & 0.081 & 0.305 & 16.5 & 0.38 & 0.040 & 0.041 & 16.3 & 0.051 & 0.423 & 16.1 & 0.419 & 15.8 & -- \\
\hline
\end{tabular}


Appendix 1. Volumetric water content, temperature, and electrical-conductivity data collected at the flood-irrigated site during 2008.-Continued

[Depth in feet below land surface; volumetric water content dimensionless; temperature in degrees Celsius; bulk electrical conductivity in decisiemens per meter; --, no data]

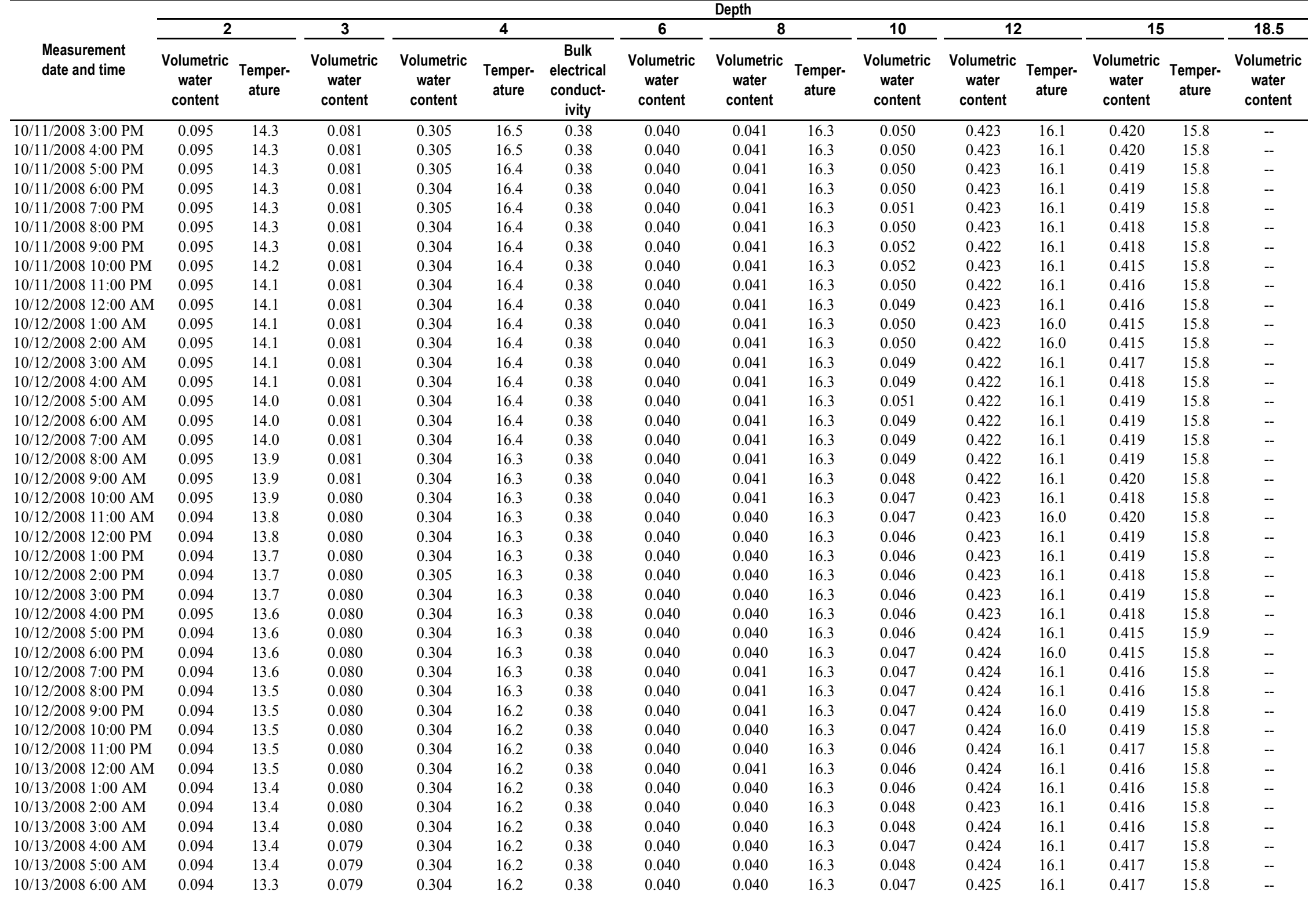


Appendix 1. Volumetric water content, temperature, and electrical-conductivity data collected at the flood-irrigated site during 2008.-Continued

[Depth in feet below land surface; volumetric water content dimensionless; temperature in degrees Celsius; bulk electrical conductivity in decisiemens per meter; --, no data]

\begin{tabular}{|c|c|c|c|c|c|c|c|c|c|c|c|c|c|c|c|}
\hline \multirow[b]{3}{*}{$\begin{array}{l}\text { Measurement } \\
\text { date and time }\end{array}$} & \multicolumn{15}{|c|}{ Depth } \\
\hline & \multirow{2}{*}{$\begin{array}{c}\mathbf{2} \\
\begin{array}{c}\text { Volumetric } \\
\text { water } \\
\text { content }\end{array}\end{array}$} & & \multirow{2}{*}{ 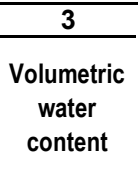 } & \multicolumn{3}{|c|}{4} & \multirow{2}{*}{$\begin{array}{c}6 \\
\begin{array}{c}\text { Volumetric } \\
\text { water } \\
\text { content }\end{array}\end{array}$} & \multicolumn{2}{|l|}{8} & \multirow{2}{*}{$\begin{array}{c}10 \\
\text { Volumetric } \\
\text { water } \\
\text { content }\end{array}$} & \multicolumn{2}{|c|}{12} & \multicolumn{2}{|c|}{15} & \multirow{2}{*}{$\begin{array}{c}18.5 \\
\text { Volumetric } \\
\text { water } \\
\text { content }\end{array}$} \\
\hline & & $\begin{array}{l}\text { Temper- } \\
\text { ature }\end{array}$ & & $\begin{array}{c}\text { Volumetric } \\
\text { water } \\
\text { content }\end{array}$ & $\begin{array}{l}\text { Temper- } \\
\text { ature }\end{array}$ & $\begin{array}{l}\text { Bulk } \\
\text { electrical } \\
\text { conduct- } \\
\text { ivity }\end{array}$ & & $\begin{array}{l}\text { Volumetric } \\
\text { water } \\
\text { content }\end{array}$ & $\begin{array}{l}\text { Temper- } \\
\text { ature }\end{array}$ & & $\begin{array}{c}\text { Volumetric } \\
\text { water } \\
\text { content }\end{array}$ & $\begin{array}{l}\text { Temper- } \\
\text { ature }\end{array}$ & $\begin{array}{l}\text { Volumetric } \\
\text { water } \\
\text { content }\end{array}$ & $\begin{array}{l}\text { Temper- } \\
\text { ature }\end{array}$ & \\
\hline 10/13/2008 7:00 AM & 0.094 & 13.3 & 0.079 & 0.304 & 16.1 & 0.38 & 0.040 & 0.040 & 16.3 & 0.047 & 0.425 & 16.1 & 0.417 & 15.8 & -- \\
\hline 10/13/2008 8:00 AM & 0.094 & 13.3 & 0.079 & 0.304 & 16.1 & 0.38 & 0.040 & 0.040 & 16.3 & 0.046 & 0.425 & 16.1 & 0.416 & 15.8 & -- \\
\hline 10/13/2008 9:00 AM & 0.094 & 13.3 & 0.079 & 0.304 & 16.1 & 0.38 & 0.040 & 0.040 & 16.3 & 0.044 & 0.424 & 16.1 & 0.416 & 15.8 & -- \\
\hline 10/13/2008 10:00 AM & 0.093 & 13.3 & 0.079 & 0.304 & 16.1 & 0.38 & 0.040 & 0.040 & 16.3 & 0.047 & 0.424 & 16.0 & 0.417 & 15.8 & -- \\
\hline 10/13/2008 11:00 AM & 0.093 & 13.2 & 0.079 & 0.304 & 16.1 & 0.38 & 0.040 & 0.041 & 16.3 & 0.046 & 0.425 & 16.1 & 0.419 & 15.8 & -- \\
\hline 10/13/2008 12:00 PM & 0.093 & 13.2 & 0.079 & 0.304 & 16.1 & 0.38 & 0.040 & 0.040 & 16.3 & 0.046 & 0.425 & 16.1 & 0.421 & 15.8 & -- \\
\hline 10/13/2008 1:00 PM & 0.093 & 13.1 & 0.079 & 0.304 & 16.1 & 0.38 & 0.040 & 0.040 & 16.3 & 0.047 & 0.424 & 16.1 & 0.419 & 15.8 & -- \\
\hline 10/13/2008 2:00 PM & 0.093 & 13.1 & 0.079 & 0.304 & 16.1 & 0.38 & 0.040 & 0.040 & 16.3 & 0.046 & 0.424 & 16.1 & 0.419 & 15.8 & -- \\
\hline 10/13/2008 3:00 PM & 0.093 & 13.1 & 0.079 & 0.304 & 16.1 & 0.38 & 0.040 & 0.040 & 16.3 & 0.046 & 0.424 & 16.1 & 0.418 & 15.8 & -- \\
\hline 10/13/2008 4:00 PM & 0.093 & 13.0 & 0.079 & 0.304 & 16.1 & 0.38 & 0.040 & 0.040 & 16.3 & 0.046 & 0.424 & 16.1 & 0.419 & 15.8 & -- \\
\hline 10/13/2008 5:00 PM & 0.093 & 13.0 & 0.079 & 0.304 & 16.0 & 0.38 & 0.040 & 0.040 & 16.3 & 0.046 & 0.424 & 16.1 & 0.418 & 15.8 & -- \\
\hline 10/13/2008 6:00 PM & 0.092 & 13.0 & 0.079 & 0.304 & 16.0 & 0.38 & 0.040 & 0.040 & 16.3 & 0.046 & 0.425 & 16.0 & 0.418 & 15.8 & -- \\
\hline 10/13/2008 7:00 PM & 0.093 & 12.9 & 0.079 & 0.304 & 16.0 & 0.38 & 0.040 & 0.040 & 16.3 & 0.046 & 0.424 & 16.1 & 0.418 & 15.8 & -- \\
\hline 10/13/2008 8:00 PM & 0.093 & 12.9 & 0.079 & 0.304 & 16.0 & 0.38 & 0.040 & 0.040 & 16.3 & 0.046 & 0.424 & 16.1 & 0.420 & 15.8 & -- \\
\hline 10/13/2008 9:00 PM & 0.093 & 12.9 & 0.079 & 0.304 & 16.0 & 0.38 & 0.040 & 0.040 & 16.3 & 0.046 & 0.424 & 16.1 & 0.420 & 15.8 & -- \\
\hline 10/13/2008 10:00 PM & 0.093 & 12.8 & 0.079 & 0.303 & 16.0 & 0.38 & 0.040 & 0.040 & 16.3 & 0.046 & 0.424 & 16.1 & 0.420 & 15.8 & -- \\
\hline 10/13/2008 11:00 PM & 0.093 & 12.9 & 0.079 & 0.303 & 16.0 & 0.38 & 0.040 & 0.040 & 16.3 & 0.046 & 0.424 & 16.1 & 0.421 & 15.8 & -- \\
\hline 10/14/2008 12:00 AM & 0.093 & 12.8 & 0.079 & 0.303 & 16.0 & 0.38 & 0.040 & 0.040 & 16.3 & 0.045 & 0.424 & 16.1 & 0.421 & 15.8 & -- \\
\hline 10/14/2008 1:00 AM & 0.093 & 12.8 & 0.079 & 0.303 & 16.0 & 0.38 & 0.040 & 0.040 & 16.3 & 0.045 & 0.424 & 16.1 & 0.421 & 15.8 & -- \\
\hline 10/14/2008 2:00 AM & 0.093 & 12.8 & 0.079 & 0.303 & 16.0 & 0.38 & 0.040 & 0.040 & 16.3 & 0.045 & 0.424 & 16.1 & 0.421 & 15.8 & -- \\
\hline 10/14/2008 3:00 AM & 0.093 & 12.8 & 0.078 & 0.303 & 15.9 & 0.38 & 0.040 & 0.040 & 16.3 & 0.046 & 0.425 & 16.1 & 0.421 & 15.8 & -- \\
\hline 10/14/2008 4:00 AM & 0.093 & 12.8 & 0.078 & 0.303 & 15.9 & 0.37 & 0.040 & 0.040 & 16.3 & 0.047 & 0.425 & 16.1 & 0.421 & 15.8 & -- \\
\hline 10/14/2008 5:00 AM & 0.093 & 12.8 & 0.078 & 0.303 & 15.9 & 0.38 & 0.039 & 0.040 & 16.3 & 0.047 & 0.425 & 16.1 & 0.421 & 15.8 & -- \\
\hline 10/14/2008 6:00 AM & 0.093 & 12.7 & 0.078 & 0.303 & 15.9 & 0.38 & 0.039 & 0.040 & 16.3 & 0.044 & 0.425 & 16.1 & 0.421 & 15.8 & -- \\
\hline 10/14/2008 7:00 AM & 0.093 & 12.7 & 0.078 & 0.303 & 15.9 & 0.38 & 0.039 & 0.040 & 16.3 & 0.047 & 0.425 & 16.1 & 0.417 & 15.8 & -- \\
\hline 10/14/2008 8:00 AM & 0.092 & 12.7 & 0.078 & 0.303 & 15.9 & 0.38 & 0.039 & 0.040 & 16.2 & 0.047 & 0.424 & 16.1 & 0.417 & 15.8 & -- \\
\hline 10/14/2008 9:00 AM & 0.093 & 12.7 & 0.078 & 0.303 & 15.9 & 0.38 & 0.040 & 0.040 & 16.3 & 0.047 & 0.424 & 16.1 & 0.420 & 15.8 & -- \\
\hline 10/14/2008 10:00 AM & 0.092 & 12.7 & 0.078 & 0.303 & 15.9 & 0.38 & 0.040 & 0.040 & 16.3 & 0.046 & 0.424 & 16.1 & 0.419 & 15.8 & -- \\
\hline 10/14/2008 11:00 AM & 0.092 & 12.6 & 0.079 & 0.303 & 15.9 & 0.38 & 0.040 & 0.040 & 16.3 & 0.044 & 0.424 & 16.1 & 0.421 & 15.8 & -- \\
\hline 10/14/2008 12:00 PM & 0.093 & 12.6 & 0.079 & 0.303 & 15.8 & 0.37 & 0.040 & 0.040 & 16.2 & 0.042 & 0.425 & 16.1 & 0.421 & 15.8 & -- \\
\hline 10/14/2008 1:00 PM & 0.093 & 12.5 & 0.079 & 0.303 & 15.8 & 0.37 & 0.040 & 0.040 & 16.2 & 0.042 & 0.425 & 16.0 & 0.420 & 15.8 & -- \\
\hline 10/14/2008 2:00 PM & 0.092 & 12.5 & 0.079 & 0.303 & 15.8 & 0.37 & 0.040 & 0.040 & 16.2 & 0.042 & 0.425 & 16.0 & 0.419 & 15.8 & -- \\
\hline 10/14/2008 3:00 PM & 0.092 & 12.4 & 0.079 & 0.303 & 15.8 & 0.37 & 0.040 & 0.040 & 16.2 & 0.042 & 0.424 & 16.1 & 0.420 & 15.9 & -- \\
\hline 10/14/2008 4:00 PM & 0.092 & 12.4 & 0.079 & 0.303 & 15.8 & 0.37 & 0.040 & 0.040 & 16.2 & 0.042 & 0.424 & 16.0 & 0.420 & 15.8 & -- \\
\hline 10/14/2008 5:00 PM & 0.093 & 12.3 & 0.079 & 0.303 & 15.8 & 0.38 & 0.040 & 0.040 & 16.2 & 0.042 & 0.424 & 16.0 & 0.420 & 15.8 & -- \\
\hline 10/14/2008 6:00 PM & 0.092 & 12.3 & 0.078 & 0.303 & 15.8 & 0.38 & 0.040 & 0.040 & 16.2 & 0.042 & 0.425 & 16.1 & 0.417 & 15.8 & -- \\
\hline 10/14/2008 7:00 PM & 0.092 & 12.3 & 0.078 & 0.303 & 15.8 & 0.37 & 0.040 & 0.040 & 16.2 & 0.042 & 0.424 & 16.0 & 0.416 & 15.8 & -- \\
\hline 10/14/2008 8:00 PM & 0.092 & 12.3 & 0.078 & 0.303 & 15.7 & 0.37 & 0.040 & 0.040 & 16.2 & 0.042 & 0.424 & 16.0 & 0.416 & 15.8 & -- \\
\hline 10/14/2008 9:00 PM & 0.092 & 12.3 & 0.078 & 0.303 & 15.7 & 0.37 & 0.040 & 0.040 & 16.2 & 0.042 & 0.424 & 16.1 & 0.417 & 15.8 & -- \\
\hline 10/14/2008 10:00 PM & 0.091 & 12.3 & 0.078 & 0.303 & 15.7 & 0.37 & 0.040 & 0.040 & 16.2 & 0.042 & 0.424 & 16.0 & 0.419 & 15.8 & -- \\
\hline
\end{tabular}


Appendix 1. Volumetric water content, temperature, and electrical-conductivity data collected at the flood-irrigated site during 2008.-Continued

[Depth in feet below land surface; volumetric water content dimensionless; temperature in degrees Celsius; bulk electrical conductivity in decisiemens per meter; --, no data]

\begin{tabular}{|c|c|c|c|c|c|c|c|c|c|c|c|c|c|c|c|}
\hline \multirow[b]{3}{*}{$\begin{array}{l}\text { Measurement } \\
\text { date and time }\end{array}$} & \multicolumn{15}{|c|}{ Depth } \\
\hline & \multicolumn{2}{|c|}{2} & \multirow{2}{*}{$\begin{array}{c}3 \\
\text { Volumetric } \\
\text { water } \\
\text { content }\end{array}$} & \multicolumn{3}{|c|}{4} & \multirow{2}{*}{$\begin{array}{c}6 \\
\text { Volumetric } \\
\text { water } \\
\text { content }\end{array}$} & \multicolumn{2}{|l|}{ DeptiI } & \multirow{2}{*}{$\begin{array}{c}10 \\
\begin{array}{c}\text { Volumetric } \\
\text { water } \\
\text { content }\end{array}\end{array}$} & \multicolumn{2}{|c|}{12} & \multicolumn{2}{|c|}{15} & \multirow{2}{*}{$\begin{array}{c}18.5 \\
\text { Volumetric } \\
\text { water } \\
\text { content }\end{array}$} \\
\hline & $\begin{array}{c}\text { Volumetric } \\
\text { water } \\
\text { content }\end{array}$ & $\begin{array}{l}\text { Temper- } \\
\text { ature }\end{array}$ & & $\begin{array}{c}\text { Volumetric } \\
\text { water } \\
\text { content }\end{array}$ & $\begin{array}{l}\text { Temper- } \\
\text { ature }\end{array}$ & $\begin{array}{c}\text { Bulk } \\
\text { electrical } \\
\text { conduct- } \\
\text { ivity }\end{array}$ & & $\begin{array}{c}\text { Volumetric } \\
\text { water } \\
\text { content }\end{array}$ & $\begin{array}{l}\text { Temper- } \\
\text { ature }\end{array}$ & & $\begin{array}{c}\text { Volumetric } \\
\text { water } \\
\text { content }\end{array}$ & $\begin{array}{l}\text { Temper- } \\
\text { ature }\end{array}$ & $\begin{array}{c}\text { Volumetric } \\
\text { water } \\
\text { content }\end{array}$ & $\begin{array}{l}\text { Temper- } \\
\text { ature }\end{array}$ & \\
\hline $\begin{array}{l}\text { 10/14/2008 11:00 PM } \\
\end{array}$ & 0.092 & 12.3 & 0.078 & 0.303 & 15.7 & 0.37 & 0.040 & 0.040 & 16.2 & 0.042 & 0.424 & 16.0 & 0.419 & 15.8 & -- \\
\hline $10 / 15 / 2008$ 12:00 AM & 0.092 & 12.3 & 0.078 & 0.303 & 15.7 & 0.37 & 0.040 & 0.040 & 16.2 & 0.042 & 0.424 & 16.0 & 0.419 & 15.8 & -- \\
\hline 10/15/2008 1:00 AM & 0.091 & 12.2 & 0.078 & 0.303 & 15.7 & 0.37 & 0.040 & 0.040 & 16.2 & 0.042 & 0.424 & 16.0 & 0.418 & 15.8 & -- \\
\hline $10 / 15 / 20082: 00 \mathrm{AM}$ & 0.091 & 12.2 & 0.078 & 0.303 & 15.7 & 0.37 & 0.040 & 0.040 & 16.2 & 0.042 & 0.424 & 16.0 & 0.419 & 15.8 & -- \\
\hline 10/15/2008 3:00 AM & 0.091 & 12.2 & 0.078 & 0.303 & 15.7 & 0.37 & 0.040 & 0.040 & 16.2 & 0.042 & 0.424 & 16.0 & 0.419 & 15.8 & -- \\
\hline 10/15/2008 4:00 AM & 0.090 & 12.2 & 0.078 & 0.303 & 15.7 & 0.37 & 0.040 & 0.040 & 16.2 & 0.042 & 0.424 & 16.0 & 0.420 & 15.8 & -- \\
\hline 10/15/2008 5:00 AM & 0.091 & 12.2 & 0.077 & 0.303 & 15.6 & 0.37 & 0.039 & 0.040 & 16.2 & 0.042 & 0.424 & 16.1 & 0.420 & 15.8 & -- \\
\hline 10/15/2008 6:00 AM & 0.092 & 12.2 & 0.077 & 0.303 & 15.6 & 0.37 & 0.039 & 0.040 & 16.2 & 0.042 & 0.424 & 16.1 & 0.420 & 15.8 & -- \\
\hline 10/15/2008 7:00 AM & 0.091 & 12.2 & 0.077 & 0.303 & 15.6 & 0.37 & 0.039 & 0.040 & 16.2 & 0.042 & 0.425 & 16.1 & 0.420 & 15.8 & -- \\
\hline 10/15/2008 8:00 AM & 0.090 & 12.2 & 0.077 & 0.303 & 15.6 & 0.37 & 0.039 & 0.040 & 16.2 & 0.042 & 0.425 & 16.1 & 0.420 & 15.8 & -- \\
\hline 10/15/2008 9:00 AM & 0.091 & 12.2 & 0.077 & 0.303 & 15.6 & 0.37 & 0.040 & 0.040 & 16.2 & 0.042 & 0.424 & 16.1 & 0.420 & 15.8 & -- \\
\hline $10 / 15 / 2008$ 10:00 AM & 0.090 & 12.1 & 0.078 & 0.303 & 15.6 & 0.37 & 0.040 & 0.040 & 16.2 & 0.042 & 0.424 & 16.0 & 0.420 & 15.8 & -- \\
\hline 10/15/2008 11:00 AM & 0.091 & 12.1 & 0.078 & 0.303 & 15.6 & 0.37 & 0.040 & 0.040 & 16.2 & 0.041 & 0.425 & 16.0 & 0.417 & 15.8 & -- \\
\hline 10/15/2008 12:00 PM & 0.090 & 12.1 & 0.078 & 0.303 & 15.6 & 0.37 & 0.040 & 0.040 & 16.2 & 0.041 & 0.424 & 16.0 & 0.417 & 15.8 & -- \\
\hline 10/15/2008 1:00 PM & 0.092 & 12.1 & 0.078 & 0.303 & 15.5 & 0.37 & 0.040 & 0.040 & 16.2 & 0.041 & 0.423 & 16.0 & 0.420 & 15.8 & -- \\
\hline 10/15/2008 2:00 PM & 0.091 & 12.1 & 0.078 & 0.303 & 15.5 & 0.37 & 0.040 & 0.040 & 16.2 & 0.041 & 0.423 & 16.0 & 0.420 & 15.8 & -- \\
\hline 10/15/2008 3:00 PM & 0.092 & 12.0 & 0.078 & 0.303 & 15.5 & 0.37 & 0.040 & 0.040 & 16.2 & 0.041 & 0.423 & 16.1 & 0.417 & 15.8 & -- \\
\hline 10/15/2008 4:00 PM & 0.091 & 12.0 & 0.078 & 0.303 & 15.5 & 0.37 & 0.040 & 0.040 & 16.2 & 0.041 & 0.423 & 16.0 & 0.417 & 15.8 & -- \\
\hline 10/15/2008 5:00 PM & 0.092 & 12.0 & 0.078 & 0.303 & 15.5 & 0.37 & 0.040 & 0.040 & 16.2 & 0.041 & 0.423 & 16.0 & 0.416 & 15.8 & -- \\
\hline 10/15/2008 6:00 PM & 0.092 & 12.0 & 0.077 & 0.303 & 15.5 & 0.37 & 0.040 & 0.040 & 16.2 & 0.041 & 0.424 & 16.1 & 0.418 & 15.8 & -- \\
\hline 10/15/2008 7:00 PM & 0.090 & 12.0 & 0.077 & 0.303 & 15.5 & 0.37 & 0.040 & 0.040 & 16.2 & 0.041 & 0.425 & 16.0 & 0.420 & 15.8 & -- \\
\hline 10/15/2008 8:00 PM & 0.091 & 11.9 & 0.077 & 0.303 & 15.5 & 0.37 & 0.040 & 0.040 & 16.2 & 0.041 & 0.424 & 16.0 & 0.420 & 15.9 & -- \\
\hline 10/15/2008 9:00 PM & 0.091 & 11.8 & 0.077 & 0.303 & 15.4 & 0.37 & 0.040 & 0.040 & 16.2 & 0.041 & 0.424 & 16.0 & 0.419 & 15.8 & -- \\
\hline 10/15/2008 10:00 PM & 0.092 & 11.8 & 0.077 & 0.303 & 15.4 & 0.37 & 0.040 & 0.040 & 16.2 & 0.041 & 0.423 & 16.0 & 0.419 & 15.9 & -- \\
\hline 10/15/2008 11:00 PM & 0.091 & 11.9 & 0.077 & 0.301 & 15.4 & 0.37 & 0.039 & 0.040 & 16.2 & 0.041 & 0.423 & 16.0 & 0.418 & 15.9 & -- \\
\hline 10/16/2008 12:00 AM & 0.091 & 11.8 & 0.077 & 0.301 & 15.4 & 0.37 & 0.039 & 0.040 & 16.2 & 0.041 & 0.423 & 16.0 & 0.418 & 15.8 & -- \\
\hline 10/16/2008 1:00 AM & 0.091 & 11.8 & 0.077 & 0.301 & 15.4 & 0.37 & 0.039 & 0.040 & 16.2 & 0.041 & 0.424 & 16.0 & 0.418 & 15.8 & -- \\
\hline 10/16/2008 2:00 AM & 0.092 & 11.9 & 0.077 & 0.301 & 15.4 & 0.37 & 0.039 & 0.040 & 16.2 & 0.041 & 0.424 & 16.1 & 0.418 & 15.8 & -- \\
\hline 10/16/2008 3:00 AM & 0.091 & 11.9 & 0.077 & 0.301 & 15.4 & 0.37 & 0.039 & 0.040 & 16.1 & 0.041 & 0.424 & 16.1 & 0.418 & 15.8 & -- \\
\hline 10/16/2008 4:00 AM & 0.092 & 11.8 & 0.077 & 0.301 & 15.4 & 0.37 & 0.039 & 0.040 & 16.2 & 0.041 & 0.424 & 16.0 & 0.418 & 15.8 & -- \\
\hline 10/16/2008 5:00 AM & 0.092 & 11.9 & 0.077 & 0.301 & 15.4 & 0.37 & 0.039 & 0.040 & 16.2 & 0.041 & 0.424 & 16.1 & 0.417 & 15.8 & -- \\
\hline 10/16/2008 6:00 AM & 0.090 & 11.9 & 0.077 & 0.301 & 15.3 & 0.37 & 0.039 & 0.040 & 16.1 & 0.041 & 0.424 & 16.1 & 0.417 & 15.8 & -- \\
\hline 10/16/2008 7:00 AM & 0.088 & 11.9 & 0.077 & 0.301 & 15.3 & 0.37 & 0.039 & 0.040 & 16.2 & 0.041 & 0.424 & 16.1 & 0.417 & 15.8 & -- \\
\hline 10/16/2008 8:00 AM & 0.090 & 11.9 & 0.077 & 0.301 & 15.3 & 0.37 & 0.039 & 0.040 & 16.1 & 0.042 & 0.424 & 16.1 & 0.417 & 15.8 & -- \\
\hline 10/16/2008 9:00 AM & 0.091 & 11.9 & 0.077 & 0.301 & 15.3 & 0.37 & 0.039 & 0.040 & 16.2 & 0.042 & 0.423 & 16.0 & 0.417 & 15.8 & -- \\
\hline 10/16/2008 10:00 AM & 0.093 & 11.9 & 0.077 & 0.301 & 15.3 & 0.37 & 0.040 & 0.040 & 16.2 & 0.042 & 0.424 & 16.0 & 0.419 & 15.8 & -- \\
\hline 10/16/2008 11:00 AM & 0.093 & 11.9 & 0.077 & 0.301 & 15.3 & 0.37 & 0.040 & 0.040 & 16.2 & 0.042 & 0.424 & 16.0 & 0.419 & 15.8 & -- \\
\hline 10/16/2008 12:00 PM & 0.092 & 11.9 & 0.077 & 0.301 & 15.3 & 0.37 & 0.040 & 0.040 & 16.1 & 0.043 & 0.423 & 16.0 & 0.419 & 15.8 & -- \\
\hline 10/16/2008 1:00 PM & 0.091 & 11.8 & 0.077 & 0.303 & 15.3 & 0.37 & 0.039 & 0.040 & 16.1 & 0.044 & 0.423 & 16.0 & 0.418 & 15.8 & -- \\
\hline 10/16/2008 2:00 PM & 0.090 & 11.9 & 0.077 & 0.303 & 15.3 & 0.37 & 0.039 & 0.040 & 16.1 & 0.044 & 0.423 & 16.0 & 0.419 & 15.8 & -- \\
\hline
\end{tabular}


Appendix 1. Volumetric water content, temperature, and electrical-conductivity data collected at the flood-irrigated site during 2008.-Continued

[Depth in feet below land surface; volumetric water content dimensionless; temperature in degrees Celsius; bulk electrical conductivity in decisiemens per meter; --, no data]

\begin{tabular}{|c|c|c|c|c|c|c|c|c|c|c|c|c|c|c|c|}
\hline \multirow[b]{3}{*}{$\begin{array}{l}\text { Measurement } \\
\text { date and time }\end{array}$} & \multicolumn{15}{|c|}{ Depth } \\
\hline & \multicolumn{2}{|c|}{2} & \multirow{2}{*}{$\begin{array}{c}3 \\
\text { Volumetric } \\
\text { water } \\
\text { content }\end{array}$} & \multicolumn{3}{|c|}{4} & \multirow{2}{*}{$\begin{array}{c}6 \\
\begin{array}{c}\text { Volumetric } \\
\text { water } \\
\text { content }\end{array}\end{array}$} & \multicolumn{2}{|l|}{8} & \multirow{2}{*}{$\begin{array}{c}10 \\
\text { Volumetric } \\
\text { water } \\
\text { content }\end{array}$} & \multicolumn{2}{|c|}{12} & \multicolumn{2}{|c|}{15} & \multirow{2}{*}{$\begin{array}{c}18.5 \\
\text { Volumetric } \\
\text { water } \\
\text { content }\end{array}$} \\
\hline & $\begin{array}{c}\text { Volumetric } \\
\text { water } \\
\text { content }\end{array}$ & $\begin{array}{l}\text { Temper- } \\
\text { ature }\end{array}$ & & $\begin{array}{c}\text { Volumetric } \\
\text { water } \\
\text { content }\end{array}$ & $\begin{array}{l}\text { Temper- } \\
\text { ature }\end{array}$ & $\begin{array}{c}\text { Bulk } \\
\text { electrical } \\
\text { conduct- } \\
\text { ivity } \\
\end{array}$ & & $\begin{array}{l}\text { Volumetric } \\
\text { water } \\
\text { content }\end{array}$ & $\begin{array}{l}\text { Temper- } \\
\text { ature }\end{array}$ & & $\begin{array}{l}\text { Volumetric } \\
\text { water } \\
\text { content }\end{array}$ & $\begin{array}{l}\text { Temper- } \\
\text { ature }\end{array}$ & $\begin{array}{l}\text { Volumetric } \\
\text { water } \\
\text { content }\end{array}$ & $\begin{array}{l}\text { Temper- } \\
\text { ature }\end{array}$ & \\
\hline 10/16/2008 3:00 PM & 0.091 & 11.8 & 0.077 & 0.303 & 15.2 & 0.37 & 0.039 & 0.040 & 16.1 & 0.044 & 0.423 & 16.0 & 0.419 & 15.8 & -- \\
\hline 10/16/2008 4:00 PM & 0.091 & 11.8 & 0.077 & 0.303 & 15.2 & 0.37 & 0.039 & 0.040 & 16.1 & 0.045 & 0.423 & 16.0 & 0.419 & 15.8 & -- \\
\hline 10/16/2008 5:00 PM & 0.091 & 11.8 & 0.077 & 0.301 & 15.2 & 0.37 & 0.040 & 0.040 & 16.1 & 0.047 & 0.423 & 16.0 & 0.419 & 15.8 & -- \\
\hline 10/16/2008 6:00 PM & 0.091 & 11.7 & 0.077 & 0.301 & 15.2 & 0.37 & 0.040 & 0.039 & 16.1 & 0.047 & 0.423 & 16.0 & 0.418 & 15.8 & -- \\
\hline 10/16/2008 7:00 PM & 0.090 & 11.7 & 0.077 & 0.301 & 15.2 & 0.37 & 0.040 & 0.039 & 16.1 & 0.044 & 0.423 & 16.0 & 0.418 & 15.8 & -- \\
\hline 10/16/2008 8:00 PM & 0.092 & 11.7 & 0.077 & 0.301 & 15.2 & 0.37 & 0.040 & 0.039 & 16.1 & 0.043 & 0.424 & 16.0 & 0.417 & 15.8 & -- \\
\hline 10/16/2008 9:00 PM & 0.093 & 11.7 & 0.077 & 0.301 & 15.2 & 0.37 & 0.040 & 0.039 & 16.1 & 0.042 & 0.424 & 16.0 & 0.416 & 15.8 & -- \\
\hline 10/16/2008 10:00 PM & 0.094 & 11.8 & 0.077 & 0.301 & 15.2 & 0.37 & 0.039 & 0.040 & 16.1 & 0.042 & 0.424 & 16.0 & 0.416 & 15.8 & -- \\
\hline 10/16/2008 11:00 PM & 0.092 & 11.7 & 0.077 & 0.301 & 15.2 & 0.37 & 0.040 & 0.040 & 16.1 & 0.042 & 0.424 & 16.0 & 0.415 & 15.8 & -- \\
\hline 10/17/2008 12:00 AM & 0.093 & 11.8 & 0.077 & 0.301 & 15.2 & 0.37 & 0.039 & 0.040 & 16.1 & 0.042 & 0.424 & 16.0 & 0.415 & 15.8 & -- \\
\hline 10/17/2008 1:00 AM & 0.094 & 11.8 & 0.077 & 0.301 & 15.1 & 0.37 & 0.039 & 0.039 & 16.1 & 0.042 & 0.425 & 16.0 & 0.415 & 15.8 & -- \\
\hline 10/17/2008 2:00 AM & 0.092 & 11.8 & 0.077 & 0.301 & 15.1 & 0.37 & 0.040 & 0.040 & 16.1 & 0.042 & 0.425 & 16.0 & 0.415 & 15.8 & -- \\
\hline 10/17/2008 3:00 AM & 0.093 & 11.8 & 0.076 & 0.301 & 15.1 & 0.37 & 0.040 & 0.040 & 16.1 & 0.042 & 0.424 & 16.0 & 0.416 & 15.8 & -- \\
\hline 10/17/2008 4:00 AM & 0.092 & 11.8 & 0.076 & 0.301 & 15.1 & 0.37 & 0.039 & 0.040 & 16.1 & 0.042 & 0.424 & 16.0 & 0.417 & 15.8 & -- \\
\hline 10/17/2008 5:00 AM & 0.093 & 11.8 & 0.076 & 0.301 & 15.1 & 0.37 & 0.039 & 0.039 & 16.1 & 0.042 & 0.423 & 16.0 & 0.417 & 15.8 & -- \\
\hline 10/17/2008 6:00 AM & 0.091 & 11.8 & 0.076 & 0.301 & 15.1 & 0.37 & 0.039 & 0.039 & 16.1 & 0.042 & 0.424 & 16.1 & 0.417 & 15.8 & -- \\
\hline 10/17/2008 7:00 AM & 0.092 & 11.8 & 0.076 & 0.301 & 15.1 & 0.37 & 0.039 & 0.039 & 16.1 & 0.042 & 0.424 & 16.0 & 0.417 & 15.8 & -- \\
\hline 10/17/2008 8:00 AM & 0.092 & 11.8 & 0.076 & 0.301 & 15.1 & 0.37 & 0.039 & 0.039 & 16.1 & 0.042 & 0.424 & 16.0 & 0.417 & 15.8 & -- \\
\hline 10/17/2008 9:00 AM & 0.091 & 11.9 & 0.076 & 0.301 & 15.1 & 0.37 & 0.039 & 0.039 & 16.1 & 0.042 & 0.424 & 16.0 & 0.417 & 15.8 & -- \\
\hline 10/17/2008 10:00 AM & 0.092 & 11.9 & 0.077 & 0.301 & 15.1 & 0.37 & 0.040 & 0.040 & 16.1 & 0.042 & 0.424 & 16.0 & 0.416 & 15.8 & -- \\
\hline 10/17/2008 11:00 AM & 0.093 & 11.9 & 0.077 & 0.301 & 15.0 & 0.37 & 0.040 & 0.039 & 16.1 & 0.042 & 0.424 & 16.0 & 0.417 & 15.8 & -- \\
\hline 10/17/2008 12:00 PM & 0.092 & 11.9 & 0.077 & 0.301 & 15.0 & 0.37 & 0.040 & 0.039 & 16.1 & 0.042 & 0.423 & 16.0 & 0.418 & 15.8 & -- \\
\hline 10/17/2008 1:00 PM & 0.090 & 11.9 & 0.076 & 0.301 & 15.0 & 0.37 & 0.039 & 0.040 & 16.1 & 0.042 & 0.424 & 16.0 & 0.419 & 15.8 & -- \\
\hline 10/17/2008 2:00 PM & 0.088 & 11.9 & 0.076 & 0.301 & 15.0 & 0.37 & 0.039 & 0.040 & 16.1 & 0.042 & 0.424 & 16.0 & 0.419 & 15.8 & -- \\
\hline 10/17/2008 3:00 PM & 0.091 & 11.9 & 0.076 & 0.301 & 15.0 & 0.37 & 0.039 & 0.040 & 16.1 & 0.042 & 0.423 & 16.0 & 0.419 & 15.8 & -- \\
\hline 10/17/2008 4:00 PM & 0.091 & 11.9 & 0.076 & 0.301 & 15.0 & 0.37 & 0.039 & 0.040 & 16.1 & 0.042 & 0.424 & 16.0 & 0.419 & 15.8 & -- \\
\hline 10/17/2008 5:00 PM & 0.091 & 11.9 & 0.076 & 0.301 & 15.0 & 0.37 & 0.039 & 0.040 & 16.1 & 0.042 & 0.423 & 16.0 & 0.419 & 15.8 & -- \\
\hline 10/17/2008 6:00 PM & 0.092 & 11.9 & 0.076 & 0.301 & 15.0 & 0.37 & 0.040 & 0.039 & 16.1 & 0.042 & 0.423 & 16.0 & 0.418 & 15.8 & -- \\
\hline 10/17/2008 7:00 PM & 0.092 & 11.9 & 0.076 & 0.301 & 15.0 & 0.37 & 0.039 & 0.039 & 16.1 & 0.042 & 0.424 & 16.0 & 0.416 & 15.8 & -- \\
\hline 10/17/2008 8:00 PM & 0.091 & 11.9 & 0.076 & 0.301 & 15.0 & 0.37 & 0.039 & 0.040 & 16.1 & 0.042 & 0.424 & 16.0 & 0.415 & 15.8 & -- \\
\hline 10/17/2008 9:00 PM & 0.091 & 11.9 & 0.076 & 0.301 & 15.0 & 0.37 & 0.039 & 0.040 & 16.1 & 0.042 & 0.424 & 16.0 & 0.416 & 15.8 & -- \\
\hline 10/17/2008 10:00 PM & 0.091 & 11.9 & 0.076 & 0.301 & 15.0 & 0.37 & 0.039 & 0.040 & 16.1 & 0.042 & 0.424 & 16.0 & 0.416 & 15.8 & -- \\
\hline 10/17/2008 11:00 PM & 0.092 & 11.9 & 0.076 & 0.300 & 14.9 & 0.37 & 0.039 & 0.040 & 16.0 & 0.042 & 0.424 & 16.0 & 0.417 & 15.8 & -- \\
\hline 10/18/2008 12:00 AM & 0.090 & 11.9 & 0.076 & 0.300 & 14.9 & 0.37 & 0.039 & 0.040 & 16.0 & 0.042 & 0.423 & 16.0 & 0.417 & 15.8 & -- \\
\hline 10/18/2008 1:00 AM & 0.090 & 11.9 & 0.076 & 0.300 & 14.9 & 0.37 & 0.039 & 0.039 & 16.0 & 0.042 & 0.423 & 16.0 & 0.418 & 15.8 & -- \\
\hline 10/18/2008 2:00 AM & 0.091 & 12.0 & 0.076 & 0.300 & 14.9 & 0.37 & 0.039 & 0.039 & 16.0 & 0.042 & 0.423 & 16.0 & 0.418 & 15.8 & -- \\
\hline 10/18/2008 3:00 AM & 0.088 & 12.0 & 0.076 & 0.300 & 14.9 & 0.37 & 0.039 & 0.039 & 16.0 & 0.042 & 0.423 & 16.0 & 0.418 & 15.8 & -- \\
\hline 10/18/2008 4:00 AM & 0.091 & 12.0 & 0.076 & 0.300 & 14.9 & 0.37 & 0.039 & 0.039 & 16.0 & 0.042 & 0.423 & 16.0 & 0.418 & 15.8 & -- \\
\hline 10/18/2008 5:00 AM & 0.087 & 12.0 & 0.076 & 0.300 & 14.9 & 0.37 & 0.039 & 0.039 & 16.0 & 0.042 & 0.423 & 16.0 & 0.418 & 15.8 & -- \\
\hline 10/18/2008 6:00 AM & 0.087 & 12.0 & 0.076 & 0.300 & 14.9 & 0.37 & 0.039 & 0.039 & 16.0 & 0.042 & 0.423 & 16.0 & 0.418 & 15.8 & -- \\
\hline
\end{tabular}


Appendix 1. Volumetric water content, temperature, and electrical-conductivity data collected at the flood-irrigated site during 2008.-Continued

[Depth in feet below land surface; volumetric water content dimensionless; temperature in degrees Celsius; bulk electrical conductivity in decisiemens per meter; --, no data]

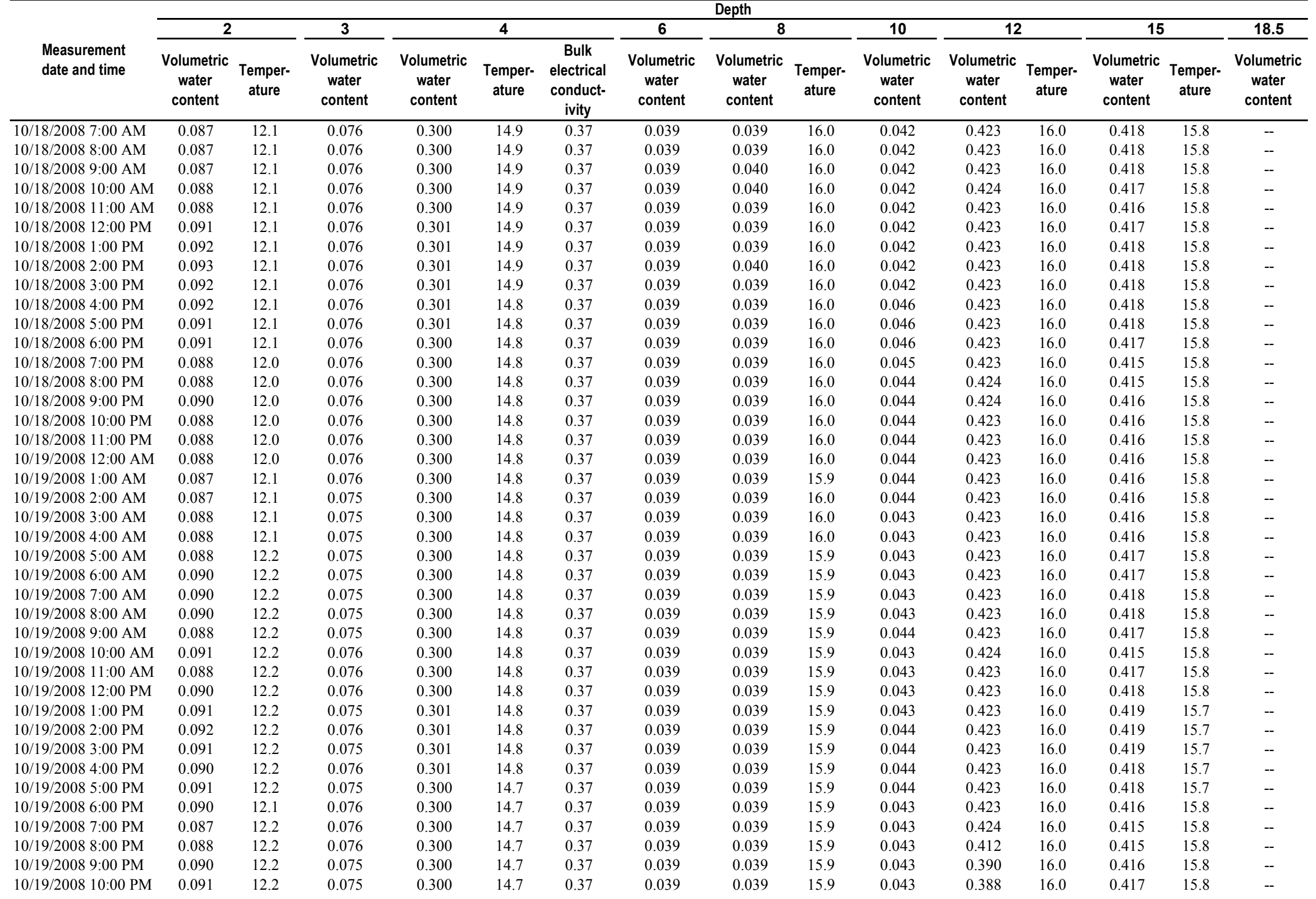


Appendix 1. Volumetric water content, temperature, and electrical-conductivity data collected at the flood-irrigated site during 2008.-Continued

[Depth in feet below land surface; volumetric water content dimensionless; temperature in degrees Celsius; bulk electrical conductivity in decisiemens per meter; --, no data]

\begin{tabular}{|c|c|c|c|c|c|c|c|c|c|c|c|c|c|c|c|}
\hline \multirow[b]{3}{*}{$\begin{array}{l}\text { Measurement } \\
\text { date and time }\end{array}$} & \multicolumn{15}{|c|}{ Depth } \\
\hline & \multicolumn{2}{|l|}{2} & \multirow{2}{*}{$\begin{array}{c}3 \\
\text { Volumetric } \\
\text { water } \\
\text { content }\end{array}$} & \multicolumn{3}{|c|}{4} & \multirow{2}{*}{$\begin{array}{c}6 \\
\begin{array}{c}\text { Volumetric } \\
\text { water } \\
\text { content }\end{array}\end{array}$} & \multicolumn{2}{|l|}{8} & \multirow{2}{*}{$\begin{array}{c}10 \\
\text { Volumetric } \\
\text { water } \\
\text { content }\end{array}$} & \multicolumn{2}{|c|}{12} & \multicolumn{2}{|c|}{15} & \multirow{2}{*}{$\begin{array}{c}18.5 \\
\text { Volumetric } \\
\text { water } \\
\text { content }\end{array}$} \\
\hline & $\begin{array}{l}\text { Volumetric } \\
\text { water } \\
\text { content }\end{array}$ & $\begin{array}{l}\text { Temper- } \\
\text { ature }\end{array}$ & & $\begin{array}{c}\text { Volumetric } \\
\text { water } \\
\text { content }\end{array}$ & $\begin{array}{l}\text { Temper- } \\
\text { ature }\end{array}$ & $\begin{array}{c}\text { Bulk } \\
\text { electrical } \\
\text { conduct- } \\
\text { ivity } \\
\end{array}$ & & $\begin{array}{l}\text { Volumetric } \\
\text { water } \\
\text { content }\end{array}$ & $\begin{array}{l}\text { Temper- } \\
\text { ature }\end{array}$ & & $\begin{array}{l}\text { Volumetric } \\
\text { water } \\
\text { content }\end{array}$ & $\begin{array}{l}\text { Temper- } \\
\text { ature }\end{array}$ & $\begin{array}{l}\text { Volumetric } \\
\text { water } \\
\text { content }\end{array}$ & $\begin{array}{l}\text { Temper- } \\
\text { ature }\end{array}$ & \\
\hline 10/19/2008 11:00 PM & 0.090 & 12.2 & 0.076 & 0.300 & 14.7 & 0.37 & 0.039 & 0.039 & 15.9 & 0.044 & 0.386 & 16.0 & 0.416 & 15.8 & -- \\
\hline 10/20/2008 12:00 AM & 0.088 & 12.2 & 0.075 & 0.300 & 14.7 & 0.37 & 0.039 & 0.039 & 15.9 & 0.044 & 0.384 & 16.0 & 0.416 & 15.7 & -- \\
\hline 10/20/2008 1:00 AM & 0.091 & 12.3 & 0.075 & 0.300 & 14.7 & 0.37 & 0.039 & 0.039 & 15.9 & 0.043 & 0.383 & 16.0 & 0.416 & 15.8 & -- \\
\hline 10/20/2008 2:00 AM & 0.090 & 12.3 & 0.075 & 0.300 & 14.7 & 0.37 & 0.039 & 0.039 & 15.9 & 0.043 & 0.382 & 15.9 & 0.416 & 15.7 & -- \\
\hline 10/20/2008 3:00 AM & 0.091 & 12.3 & 0.075 & 0.300 & 14.7 & 0.37 & 0.039 & 0.039 & 15.9 & 0.043 & 0.381 & 16.0 & 0.416 & 15.8 & -- \\
\hline 10/20/2008 4:00 AM & 0.091 & 12.3 & 0.075 & 0.300 & 14.7 & 0.37 & 0.039 & 0.039 & 15.9 & 0.043 & 0.381 & 16.0 & 0.416 & 15.8 & -- \\
\hline 10/20/2008 5:00 AM & 0.090 & 12.3 & 0.075 & 0.300 & 14.7 & 0.37 & 0.039 & 0.039 & 15.9 & 0.043 & 0.380 & 16.0 & 0.416 & 15.8 & -- \\
\hline 10/20/2008 6:00 AM & 0.091 & 12.4 & 0.075 & 0.300 & 14.7 & 0.37 & 0.039 & 0.039 & 15.9 & 0.043 & 0.380 & 16.0 & 0.416 & 15.7 & -- \\
\hline 10/20/2008 7:00 AM & 0.091 & 12.4 & 0.075 & 0.300 & 14.7 & 0.37 & 0.039 & 0.039 & 15.8 & 0.043 & 0.379 & 16.0 & 0.416 & 15.8 & -- \\
\hline 10/20/2008 8:00 AM & 0.092 & 12.5 & 0.075 & 0.300 & 14.7 & 0.37 & 0.039 & 0.039 & 15.8 & 0.043 & 0.378 & 16.0 & 0.416 & 15.7 & -- \\
\hline 10/20/2008 9:00 AM & 0.091 & 12.5 & 0.075 & 0.300 & 14.7 & 0.37 & 0.039 & 0.039 & 15.8 & 0.043 & 0.378 & 16.0 & 0.416 & 15.8 & -- \\
\hline 10/20/2008 10:00 AM & 0.091 & 12.4 & 0.075 & 0.300 & 14.7 & 0.37 & 0.039 & 0.039 & 15.8 & 0.043 & 0.376 & 16.0 & 0.416 & 15.8 & -- \\
\hline 10/20/2008 11:00 AM & 0.092 & 12.5 & 0.075 & 0.300 & 14.7 & 0.37 & 0.039 & 0.039 & 15.8 & 0.043 & 0.376 & 16.0 & 0.416 & 15.8 & -- \\
\hline 10/20/2008 12:00 PM & 0.091 & 12.4 & 0.075 & 0.300 & 14.7 & 0.37 & 0.039 & 0.039 & 15.8 & 0.043 & 0.378 & 16.0 & 0.415 & 15.8 & -- \\
\hline 10/20/2008 1:00 PM & 0.091 & 12.5 & 0.075 & 0.300 & 14.7 & 0.37 & 0.039 & 0.039 & 15.8 & 0.043 & 0.376 & 16.0 & 0.415 & 15.8 & -- \\
\hline 10/20/2008 2:00 PM & 0.090 & 12.4 & 0.075 & 0.300 & 14.7 & 0.37 & 0.039 & 0.039 & 15.8 & 0.043 & 0.376 & 16.0 & 0.416 & 15.8 & -- \\
\hline 10/20/2008 3:00 PM & 0.091 & 12.4 & 0.075 & 0.300 & 14.7 & 0.37 & 0.039 & 0.039 & 15.8 & 0.043 & 0.376 & 16.0 & 0.416 & 15.8 & -- \\
\hline 10/20/2008 4:00 PM & 0.091 & 12.4 & 0.075 & 0.300 & 14.7 & 0.37 & 0.039 & 0.039 & 15.8 & 0.043 & 0.376 & 16.0 & 0.416 & 15.8 & -- \\
\hline 10/20/2008 5:00 PM & 0.090 & 12.4 & 0.075 & 0.300 & 14.7 & 0.37 & 0.039 & 0.039 & 15.8 & 0.043 & 0.378 & 16.0 & 0.416 & 15.8 & -- \\
\hline 10/20/2008 6:00 PM & 0.091 & 12.5 & 0.075 & 0.300 & 14.7 & 0.37 & 0.039 & 0.039 & 15.8 & 0.043 & 0.378 & 16.0 & 0.415 & 15.8 & -- \\
\hline 10/20/2008 7:00 PM & 0.091 & 12.5 & 0.075 & 0.300 & 14.7 & 0.37 & 0.039 & 0.039 & 15.8 & 0.043 & 0.378 & 16.0 & 0.415 & 15.8 & -- \\
\hline 10/20/2008 8:00 PM & 0.090 & 12.5 & 0.075 & 0.300 & 14.7 & 0.37 & 0.039 & 0.039 & 15.8 & 0.043 & 0.378 & 16.0 & 0.415 & 15.8 & -- \\
\hline 10/20/2008 9:00 PM & 0.090 & 12.5 & 0.075 & 0.300 & 14.7 & 0.37 & 0.039 & 0.039 & 15.8 & 0.043 & 0.378 & 16.0 & 0.415 & 15.8 & -- \\
\hline 10/20/2008 10:00 PM & 0.091 & 12.5 & 0.075 & 0.300 & 14.7 & 0.37 & 0.039 & 0.039 & 15.8 & 0.043 & 0.378 & 16.0 & 0.416 & 15.7 & -- \\
\hline 10/20/2008 11:00 PM & 0.090 & 12.6 & 0.075 & 0.300 & 14.7 & 0.37 & 0.039 & 0.039 & 15.8 & 0.043 & 0.376 & 15.9 & 0.416 & 15.7 & -- \\
\hline 10/21/2008 12:00 AM & 0.090 & 12.6 & 0.075 & 0.300 & 14.7 & 0.37 & 0.039 & 0.039 & 15.8 & 0.043 & 0.376 & 16.0 & 0.416 & 15.7 & -- \\
\hline 10/21/2008 1:00 AM & 0.091 & 12.6 & 0.075 & 0.300 & 14.7 & 0.37 & 0.039 & 0.039 & 15.8 & 0.043 & 0.376 & 15.9 & 0.417 & 15.8 & -- \\
\hline 10/21/2008 2:00 AM & 0.091 & 12.6 & 0.075 & 0.300 & 14.7 & 0.37 & 0.039 & 0.039 & 15.8 & 0.043 & 0.376 & 16.0 & 0.418 & 15.8 & -- \\
\hline 10/21/2008 3:00 AM & 0.091 & 12.6 & 0.075 & 0.300 & 14.7 & 0.37 & 0.039 & 0.039 & 15.8 & 0.043 & 0.376 & 16.0 & 0.418 & 15.8 & -- \\
\hline 10/21/2008 4:00 AM & 0.091 & 12.6 & 0.075 & 0.300 & 14.7 & 0.37 & 0.039 & 0.039 & 15.8 & 0.043 & 0.376 & 15.9 & 0.418 & 15.8 & -- \\
\hline 10/21/2008 5:00 AM & 0.091 & 12.7 & 0.075 & 0.300 & 14.7 & 0.37 & 0.039 & 0.039 & 15.8 & 0.043 & 0.376 & 15.9 & 0.418 & 15.8 & -- \\
\hline 10/21/2008 6:00 AM & 0.091 & 12.7 & 0.075 & 0.300 & 14.7 & 0.37 & 0.039 & 0.039 & 15.8 & 0.043 & 0.376 & 16.0 & 0.418 & 15.8 & -- \\
\hline 10/21/2008 7:00 AM & 0.091 & 12.7 & 0.075 & 0.300 & 14.7 & 0.37 & 0.039 & 0.039 & 15.8 & 0.043 & 0.375 & 15.9 & 0.418 & 15.8 & -- \\
\hline 10/21/2008 8:00 AM & 0.090 & 12.7 & 0.075 & 0.300 & 14.7 & 0.37 & 0.039 & 0.039 & 15.8 & 0.042 & 0.375 & 15.9 & 0.417 & 15.8 & -- \\
\hline 10/21/2008 9:00 AM & 0.091 & 12.7 & 0.075 & 0.300 & 14.7 & 0.37 & 0.039 & 0.039 & 15.7 & 0.043 & 0.375 & 15.9 & 0.417 & 15.7 & -- \\
\hline 10/21/2008 10:00 AM & 0.091 & 12.7 & 0.075 & 0.300 & 14.7 & 0.37 & 0.039 & 0.039 & 15.8 & 0.043 & 0.376 & 15.9 & 0.417 & 15.8 & -- \\
\hline 10/21/2008 11:00 AM & 0.091 & 12.7 & 0.075 & 0.300 & 14.7 & 0.37 & 0.039 & 0.039 & 15.8 & 0.043 & 0.376 & 15.9 & 0.416 & 15.8 & -- \\
\hline 10/21/2008 12:00 PM & 0.092 & 12.6 & 0.075 & 0.300 & 14.7 & 0.37 & 0.039 & 0.039 & 15.7 & 0.043 & 0.376 & 15.9 & 0.415 & 15.8 & -- \\
\hline 10/21/2008 1:00 PM & 0.091 & 12.6 & 0.075 & 0.300 & 14.7 & 0.37 & 0.039 & 0.038 & 15.7 & 0.043 & 0.376 & 16.0 & 0.415 & 15.8 & -- \\
\hline 10/21/2008 2:00 PM & 0.091 & 12.6 & 0.075 & 0.300 & 14.7 & 0.37 & 0.039 & 0.038 & 15.7 & 0.043 & 0.376 & 15.9 & 0.415 & 15.8 & -- \\
\hline
\end{tabular}


Appendix 1. Volumetric water content, temperature, and electrical-conductivity data collected at the flood-irrigated site during 2008.-Continued

[Depth in feet below land surface; volumetric water content dimensionless; temperature in degrees Celsius; bulk electrical conductivity in decisiemens per meter; --, no data]

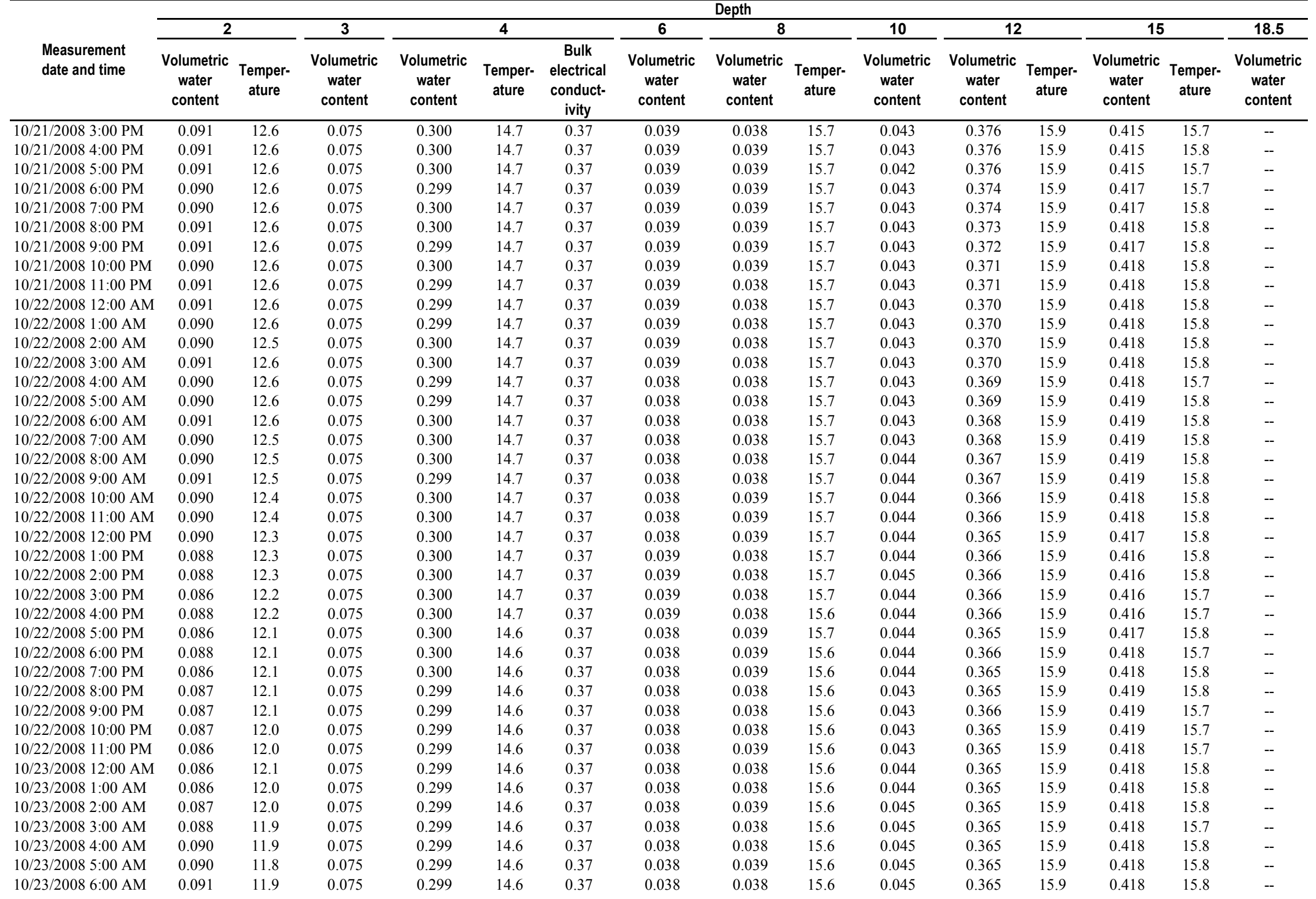


Appendix 1. Volumetric water content, temperature, and electrical-conductivity data collected at the flood-irrigated site during 2008.-Continued

[Depth in feet below land surface; volumetric water content dimensionless; temperature in degrees Celsius; bulk electrical conductivity in decisiemens per meter; --, no data]

\begin{tabular}{|c|c|c|c|c|c|c|c|c|c|c|c|c|c|c|c|}
\hline \multirow[b]{3}{*}{$\begin{array}{l}\text { Measurement } \\
\text { date and time }\end{array}$} & \multicolumn{15}{|c|}{ Depth } \\
\hline & \multicolumn{2}{|l|}{2} & \multirow{2}{*}{$\begin{array}{c}3 \\
\begin{array}{c}\text { Volumetric } \\
\text { water } \\
\text { content }\end{array}\end{array}$} & \multicolumn{3}{|c|}{4} & \multirow{2}{*}{$\begin{array}{c}6 \\
\begin{array}{c}\text { Volumetric } \\
\text { water } \\
\text { content }\end{array}\end{array}$} & \multicolumn{2}{|l|}{8} & \multirow{2}{*}{$\begin{array}{c}10 \\
\text { Volumetric } \\
\text { water } \\
\text { content }\end{array}$} & \multicolumn{2}{|c|}{12} & \multicolumn{2}{|c|}{15} & \multirow{2}{*}{$\begin{array}{c}18.5 \\
\text { Volumetric } \\
\text { water } \\
\text { content }\end{array}$} \\
\hline & $\begin{array}{l}\text { Volumetric } \\
\text { water } \\
\text { content }\end{array}$ & $\begin{array}{l}\text { Temper- } \\
\text { ature }\end{array}$ & & $\begin{array}{c}\text { Volumetric } \\
\text { water } \\
\text { content }\end{array}$ & $\begin{array}{l}\text { Temper- } \\
\text { ature }\end{array}$ & $\begin{array}{c}\text { Bulk } \\
\text { electrical } \\
\text { conduct- } \\
\text { ivity } \\
\end{array}$ & & $\begin{array}{l}\text { Volumetric } \\
\text { water } \\
\text { content }\end{array}$ & $\begin{array}{l}\text { Temper- } \\
\text { ature }\end{array}$ & & $\begin{array}{l}\text { Volumetric } \\
\text { water } \\
\text { content }\end{array}$ & $\begin{array}{l}\text { Temper- } \\
\text { ature }\end{array}$ & $\begin{array}{l}\text { Volumetric } \\
\text { water } \\
\text { content }\end{array}$ & $\begin{array}{l}\text { Temper- } \\
\text { ature }\end{array}$ & \\
\hline 10/23/2008 7:00 AM & 0.091 & 11.8 & 0.075 & 0.299 & 14.6 & 0.37 & 0.038 & 0.038 & 15.6 & 0.046 & 0.365 & 15.9 & 0.418 & 15.8 & -- \\
\hline 10/23/2008 8:00 AM & 0.091 & 11.8 & 0.075 & 0.299 & 14.6 & 0.37 & 0.038 & 0.038 & 15.6 & 0.046 & 0.366 & 15.9 & 0.418 & 15.7 & -- \\
\hline 10/23/2008 9:00 AM & 0.088 & 11.7 & 0.075 & 0.299 & 14.6 & 0.37 & 0.038 & 0.038 & 15.6 & 0.046 & 0.366 & 15.9 & 0.418 & 15.8 & -- \\
\hline 10/23/2008 10:00 AM & 0.087 & 11.8 & 0.075 & 0.299 & 14.6 & 0.37 & 0.038 & 0.038 & 15.6 & 0.051 & 0.366 & 15.9 & 0.418 & 15.8 & -- \\
\hline 10/23/2008 11:00 AM & 0.090 & 11.8 & 0.075 & 0.300 & 14.6 & 0.37 & 0.038 & 0.039 & 15.6 & 0.054 & 0.366 & 15.9 & 0.417 & 15.7 & -- \\
\hline 10/23/2008 12:00 PM & 0.086 & 11.7 & 0.075 & 0.300 & 14.6 & 0.37 & 0.039 & 0.038 & 15.6 & 0.049 & 0.367 & 15.9 & 0.415 & 15.8 & -- \\
\hline 10/23/2008 1:00 PM & 0.087 & 11.6 & 0.075 & 0.300 & 14.6 & 0.37 & 0.039 & 0.038 & 15.6 & 0.048 & 0.367 & 15.9 & 0.415 & 15.8 & -- \\
\hline 10/23/2008 2:00 PM & 0.087 & 11.6 & 0.075 & 0.300 & 14.6 & 0.37 & 0.039 & 0.038 & 15.6 & 0.047 & 0.367 & 15.9 & 0.416 & 15.8 & -- \\
\hline 10/23/2008 3:00 PM & 0.087 & 11.5 & 0.075 & 0.300 & 14.6 & 0.37 & 0.039 & 0.038 & 15.6 & 0.047 & 0.367 & 15.9 & 0.415 & 15.7 & -- \\
\hline 10/23/2008 4:00 PM & 0.087 & 11.5 & 0.075 & 0.300 & 14.6 & 0.37 & 0.039 & 0.038 & 15.6 & 0.047 & 0.368 & 15.9 & 0.415 & 15.8 & -- \\
\hline 10/23/2008 5:00 PM & 0.086 & 11.5 & 0.075 & 0.300 & 14.6 & 0.37 & 0.039 & 0.038 & 15.6 & 0.047 & 0.368 & 15.9 & 0.415 & 15.8 & -- \\
\hline 10/23/2008 6:00 PM & 0.088 & 11.5 & 0.075 & 0.300 & 14.5 & 0.37 & 0.038 & 0.038 & 15.6 & 0.047 & 0.367 & 15.9 & 0.416 & 15.7 & -- \\
\hline 10/23/2008 7:00 PM & 0.088 & 11.4 & 0.075 & 0.299 & 14.5 & 0.37 & 0.038 & 0.038 & 15.6 & 0.047 & 0.366 & 15.9 & 0.418 & 15.8 & -- \\
\hline 10/23/2008 8:00 PM & 0.087 & 11.4 & 0.074 & 0.299 & 14.5 & 0.37 & 0.038 & 0.038 & 15.6 & 0.047 & 0.366 & 15.9 & 0.418 & 15.7 & -- \\
\hline 10/23/2008 9:00 PM & 0.087 & 11.4 & 0.074 & 0.299 & 14.5 & 0.37 & 0.038 & 0.038 & 15.6 & 0.047 & 0.366 & 15.9 & 0.418 & 15.8 & -- \\
\hline 10/23/2008 10:00 PM & 0.084 & 11.3 & 0.074 & 0.299 & 14.5 & 0.37 & 0.038 & 0.038 & 15.6 & 0.047 & 0.366 & 15.9 & 0.417 & 15.8 & -- \\
\hline 10/23/2008 11:00 PM & 0.084 & 11.3 & 0.074 & 0.299 & 14.5 & 0.37 & 0.038 & 0.038 & 15.6 & 0.047 & 0.365 & 15.9 & 0.417 & 15.7 & -- \\
\hline 10/24/2008 12:00 AM & 0.085 & 11.3 & 0.074 & 0.299 & 14.5 & 0.37 & 0.038 & 0.038 & 15.6 & 0.048 & 0.365 & 15.9 & 0.417 & 15.7 & -- \\
\hline 10/24/2008 1:00 AM & 0.085 & 11.3 & 0.074 & 0.299 & 14.5 & 0.37 & 0.038 & 0.038 & 15.6 & 0.048 & 0.365 & 15.9 & 0.417 & 15.7 & -- \\
\hline 10/24/2008 2:00 AM & 0.084 & 11.3 & 0.074 & 0.299 & 14.5 & 0.37 & 0.038 & 0.038 & 15.6 & 0.049 & 0.365 & 15.9 & 0.417 & 15.7 & -- \\
\hline 10/24/2008 3:00 AM & 0.084 & 11.3 & 0.074 & 0.299 & 14.5 & 0.37 & 0.038 & 0.038 & 15.5 & 0.048 & 0.365 & 15.9 & 0.417 & 15.7 & -- \\
\hline 10/24/2008 4:00 AM & 0.084 & 11.3 & 0.074 & 0.299 & 14.5 & 0.37 & 0.038 & 0.038 & 15.5 & 0.049 & 0.365 & 15.9 & 0.417 & 15.8 & -- \\
\hline 10/24/2008 5:00 AM & 0.084 & 11.3 & 0.074 & 0.299 & 14.5 & 0.37 & 0.038 & 0.038 & 15.5 & 0.050 & 0.365 & 15.9 & 0.417 & 15.8 & -- \\
\hline 10/24/2008 6:00 AM & 0.084 & 11.3 & 0.074 & 0.299 & 14.5 & 0.37 & 0.038 & 0.038 & 15.5 & 0.050 & 0.365 & 15.9 & 0.417 & 15.7 & -- \\
\hline 10/24/2008 7:00 AM & 0.086 & 11.3 & 0.074 & 0.299 & 14.4 & 0.37 & 0.038 & 0.038 & 15.5 & 0.051 & 0.365 & 15.9 & 0.417 & 15.8 & -- \\
\hline 10/24/2008 8:00 AM & 0.085 & 11.2 & 0.074 & 0.299 & 14.4 & 0.37 & 0.038 & 0.038 & 15.5 & 0.051 & 0.363 & 15.9 & 0.417 & 15.7 & -- \\
\hline 10/24/2008 9:00 AM & 0.088 & 11.2 & 0.074 & 0.299 & 14.4 & 0.37 & 0.038 & 0.038 & 15.5 & 0.047 & 0.363 & 15.9 & 0.418 & 15.7 & -- \\
\hline 10/24/2008 10:00 AM & 0.088 & 11.3 & 0.074 & 0.299 & 14.4 & 0.37 & 0.038 & 0.038 & 15.5 & 0.049 & 0.363 & 15.9 & 0.418 & 15.8 & -- \\
\hline 10/24/2008 11:00 AM & 0.088 & 11.2 & 0.074 & 0.299 & 14.4 & 0.37 & 0.038 & 0.038 & 15.5 & 0.049 & 0.363 & 15.9 & 0.415 & 15.7 & -- \\
\hline 10/24/2008 12:00 PM & 0.087 & 11.2 & 0.074 & 0.299 & 14.4 & 0.37 & 0.039 & 0.038 & 15.5 & 0.050 & 0.363 & 15.9 & 0.415 & 15.7 & -- \\
\hline 10/24/2008 1:00 PM & 0.085 & 11.1 & 0.074 & 0.300 & 14.4 & 0.37 & 0.039 & 0.038 & 15.5 & 0.050 & 0.362 & 15.8 & 0.417 & 15.7 & -- \\
\hline 10/24/2008 2:00 PM & 0.085 & 11.1 & 0.074 & 0.300 & 14.4 & 0.37 & 0.039 & 0.038 & 15.5 & 0.049 & 0.362 & 15.9 & 0.418 & 15.7 & -- \\
\hline 10/24/2008 3:00 PM & 0.085 & 11.1 & 0.074 & 0.300 & 14.4 & 0.37 & 0.039 & 0.038 & 15.5 & 0.048 & 0.363 & 15.9 & 0.418 & 15.7 & -- \\
\hline 10/24/2008 4:00 PM & 0.084 & 11.0 & 0.074 & 0.300 & 14.4 & 0.37 & 0.039 & 0.038 & 15.5 & 0.047 & 0.363 & 15.9 & 0.418 & 15.7 & -- \\
\hline 10/24/2008 5:00 PM & 0.084 & 11.0 & 0.074 & 0.300 & 14.4 & 0.37 & 0.039 & 0.038 & 15.5 & 0.050 & 0.363 & 15.9 & 0.418 & 15.8 & -- \\
\hline 10/24/2008 6:00 PM & 0.085 & 11.0 & 0.074 & 0.299 & 14.3 & 0.37 & 0.038 & 0.038 & 15.5 & 0.049 & 0.363 & 15.9 & 0.418 & 15.7 & -- \\
\hline 10/24/2008 7:00 PM & 0.085 & 11.0 & 0.074 & 0.299 & 14.3 & 0.37 & 0.038 & 0.038 & 15.5 & 0.051 & 0.362 & 15.8 & 0.417 & 15.8 & -- \\
\hline 10/24/2008 8:00 PM & 0.084 & 11.0 & 0.074 & 0.299 & 14.3 & 0.37 & 0.038 & 0.038 & 15.5 & 0.050 & 0.362 & 15.8 & 0.416 & 15.8 & -- \\
\hline 10/24/2008 9:00 PM & 0.085 & 11.0 & 0.074 & 0.299 & 14.3 & 0.37 & 0.038 & 0.038 & 15.5 & 0.050 & 0.362 & 15.9 & 0.415 & 15.7 & -- \\
\hline 10/24/2008 10:00 PM & 0.084 & 11.0 & 0.074 & 0.299 & 14.3 & 0.37 & 0.038 & 0.038 & 15.5 & 0.049 & 0.361 & 15.9 & 0.415 & 15.7 & -- \\
\hline
\end{tabular}


Appendix 1. Volumetric water content, temperature, and electrical-conductivity data collected at the flood-irrigated site during 2008.-Continued

[Depth in feet below land surface; volumetric water content dimensionless; temperature in degrees Celsius; bulk electrical conductivity in decisiemens per meter; --, no data]

\begin{tabular}{|c|c|c|c|c|c|c|c|c|c|c|c|c|c|c|c|}
\hline \multirow[b]{3}{*}{$\begin{array}{l}\text { Measurement } \\
\text { date and time }\end{array}$} & \multicolumn{15}{|c|}{ Depth } \\
\hline & \multicolumn{2}{|c|}{2} & \multirow[b]{2}{*}{$\begin{array}{c}3 \\
\begin{array}{c}\text { Volumetric } \\
\text { water } \\
\text { content }\end{array}\end{array}$} & \multicolumn{3}{|c|}{4} & \multirow{2}{*}{$\begin{array}{c}6 \\
\begin{array}{c}\text { Volumetric } \\
\text { water } \\
\text { content }\end{array}\end{array}$} & \multicolumn{2}{|c|}{8} & \multirow{2}{*}{$\begin{array}{c}10 \\
\text { Volumetric } \\
\text { water } \\
\text { content }\end{array}$} & \multicolumn{2}{|c|}{12} & \multicolumn{2}{|c|}{15} & \multirow{2}{*}{$\begin{array}{c}18.5 \\
\text { Volumetric } \\
\text { water } \\
\text { content }\end{array}$} \\
\hline & $\begin{array}{c}\text { Volumetric } \\
\text { water } \\
\text { content }\end{array}$ & $\begin{array}{l}\text { Temper- } \\
\text { ature }\end{array}$ & & $\begin{array}{c}\text { Volumetric } \\
\text { water } \\
\text { content }\end{array}$ & $\begin{array}{l}\text { Temper- } \\
\text { ature }\end{array}$ & $\begin{array}{c}\text { Bulk } \\
\text { electrical } \\
\text { conduct- } \\
\text { ivity }\end{array}$ & & $\begin{array}{c}\text { Volumetric } \\
\text { water } \\
\text { content }\end{array}$ & $\begin{array}{l}\text { Temper- } \\
\text { ature }\end{array}$ & & $\begin{array}{c}\text { Volumetric } \\
\text { water } \\
\text { content }\end{array}$ & $\begin{array}{l}\text { Temper- } \\
\text { ature }\end{array}$ & $\begin{array}{c}\text { Volumetric } \\
\text { water } \\
\text { content }\end{array}$ & $\begin{array}{l}\text { Temper- } \\
\text { ature }\end{array}$ & \\
\hline 10/24/2008 11:00 PM & 0.086 & 11.0 & 0.074 & 0.299 & 14.3 & 0.37 & 0.038 & 0.038 & 15.5 & 0.051 & 0.361 & 15.8 & 0.415 & 15.7 & $\overline{--}$ \\
\hline 10/25/2008 12:00 AM & 0.085 & 11.0 & 0.074 & 0.299 & 14.3 & 0.37 & 0.038 & 0.038 & 15.5 & 0.049 & 0.361 & 15.8 & 0.415 & 15.7 & -- \\
\hline 10/25/2008 1:00 AM & 0.086 & 11.0 & 0.074 & 0.299 & 14.3 & 0.37 & 0.038 & 0.038 & 15.5 & 0.047 & 0.360 & 15.8 & 0.415 & 15.7 & -- \\
\hline 10/25/2008 2:00 AM & 0.086 & 11.0 & 0.074 & 0.299 & 14.3 & 0.37 & 0.038 & 0.038 & 15.5 & 0.047 & 0.360 & 15.8 & 0.415 & 15.7 & -- \\
\hline 10/25/2008 3:00 AM & 0.086 & 11.0 & 0.074 & 0.299 & 14.3 & 0.37 & 0.038 & 0.038 & 15.5 & 0.047 & 0.361 & 15.8 & 0.415 & 15.8 & -- \\
\hline 10/25/2008 4:00 AM & 0.086 & 11.1 & 0.074 & 0.299 & 14.3 & 0.37 & 0.038 & 0.038 & 15.5 & 0.050 & 0.361 & 15.9 & 0.415 & 15.7 & -- \\
\hline 10/25/2008 5:00 AM & 0.087 & 11.1 & 0.074 & 0.299 & 14.2 & 0.37 & 0.038 & 0.038 & 15.5 & 0.050 & 0.361 & 15.9 & 0.415 & 15.7 & -- \\
\hline 10/25/2008 6:00 AM & 0.087 & 11.1 & 0.074 & 0.299 & 14.2 & 0.37 & 0.038 & 0.038 & 15.5 & 0.058 & 0.361 & 15.8 & 0.415 & 15.8 & -- \\
\hline 10/25/2008 7:00 AM & 0.086 & 11.1 & 0.073 & 0.299 & 14.2 & 0.37 & 0.038 & 0.038 & 15.5 & 0.062 & 0.360 & 15.9 & 0.415 & 15.7 & -- \\
\hline 10/25/2008 8:00 AM & 0.087 & 11.1 & 0.073 & 0.299 & 14.2 & 0.37 & 0.038 & 0.038 & 15.5 & 0.065 & 0.360 & 15.8 & 0.415 & 15.7 & -- \\
\hline 10/25/2008 9:00 AM & 0.085 & 11.0 & 0.074 & 0.299 & 14.2 & 0.37 & 0.038 & 0.038 & 15.5 & 0.062 & 0.360 & 15.8 & 0.415 & 15.8 & -- \\
\hline 10/25/2008 10:00 AM & 0.084 & 11.0 & 0.073 & 0.299 & 14.2 & 0.37 & 0.038 & 0.038 & 15.5 & 0.058 & 0.360 & 15.8 & 0.415 & 15.7 & -- \\
\hline 10/25/2008 11:00 AM & 0.084 & 11.0 & 0.074 & 0.299 & 14.2 & 0.37 & 0.038 & 0.038 & 15.4 & 0.060 & 0.361 & 15.8 & 0.417 & 15.7 & -- \\
\hline 10/25/2008 12:00 PM & 0.083 & 11.0 & 0.074 & 0.299 & 14.2 & 0.37 & 0.038 & 0.038 & 15.5 & 0.058 & 0.361 & 15.8 & 0.418 & 15.7 & -- \\
\hline 10/25/2008 1:00 PM & 0.084 & 10.9 & 0.074 & 0.299 & 14.2 & 0.37 & 0.038 & 0.038 & 15.5 & 0.057 & 0.360 & 15.8 & 0.418 & 15.7 & -- \\
\hline 10/25/2008 2:00 PM & 0.084 & 10.9 & 0.074 & 0.299 & 14.2 & 0.37 & 0.038 & 0.038 & 15.5 & 0.060 & 0.360 & 15.8 & 0.418 & 15.7 & -- \\
\hline 10/25/2008 3:00 PM & 0.084 & 10.9 & 0.073 & 0.299 & 14.2 & 0.37 & 0.038 & 0.038 & 15.4 & 0.056 & 0.360 & 15.8 & 0.418 & 15.7 & -- \\
\hline 10/25/2008 4:00 PM & 0.084 & 10.9 & 0.073 & 0.299 & 14.1 & 0.37 & 0.038 & 0.038 & 15.5 & 0.049 & 0.360 & 15.8 & 0.418 & 15.7 & -- \\
\hline 10/25/2008 5:00 PM & 0.084 & 10.9 & 0.073 & 0.299 & 14.1 & 0.37 & 0.038 & 0.038 & 15.5 & 0.047 & 0.360 & 15.8 & 0.418 & 15.7 & -- \\
\hline 10/25/2008 6:00 PM & 0.083 & 10.8 & 0.074 & 0.299 & 14.1 & 0.37 & 0.038 & 0.038 & 15.4 & 0.047 & 0.361 & 15.8 & 0.418 & 15.7 & -- \\
\hline 10/25/2008 7:00 PM & 0.083 & 10.9 & 0.074 & 0.299 & 14.1 & 0.37 & 0.038 & 0.038 & 15.4 & 0.047 & 0.360 & 15.8 & 0.417 & 15.7 & -- \\
\hline 10/25/2008 8:00 PM & 0.084 & 10.9 & 0.073 & 0.299 & 14.1 & 0.37 & 0.038 & 0.038 & 15.4 & 0.047 & 0.360 & 15.8 & 0.415 & 15.7 & -- \\
\hline 10/25/2008 9:00 PM & 0.084 & 10.8 & 0.073 & 0.299 & 14.1 & 0.37 & 0.038 & 0.038 & 15.4 & 0.047 & 0.360 & 15.8 & 0.415 & 15.7 & -- \\
\hline 10/25/2008 10:00 PM & 0.083 & 10.8 & 0.073 & 0.299 & 14.1 & 0.37 & 0.038 & 0.038 & 15.4 & 0.047 & 0.359 & 15.8 & 0.413 & 15.8 & -- \\
\hline 10/25/2008 11:00 PM & 0.083 & 10.8 & 0.073 & 0.299 & 14.1 & 0.37 & 0.038 & 0.038 & 15.4 & 0.047 & 0.359 & 15.8 & 0.413 & 15.7 & -- \\
\hline 10/26/2008 12:00 AM & 0.084 & 10.9 & 0.073 & 0.299 & 14.1 & 0.37 & 0.038 & 0.038 & 15.4 & 0.048 & 0.359 & 15.8 & 0.413 & 15.7 & -- \\
\hline 10/26/2008 1:00 AM & 0.083 & 10.8 & 0.073 & 0.299 & 14.1 & 0.37 & 0.038 & 0.038 & 15.4 & 0.049 & 0.359 & 15.8 & 0.413 & 15.7 & -- \\
\hline $10 / 26 / 20082: 00 \mathrm{AM}$ & 0.083 & 10.9 & 0.073 & 0.299 & 14.1 & 0.37 & 0.038 & 0.038 & 15.4 & 0.051 & 0.358 & 15.8 & 0.413 & 15.7 & -- \\
\hline 10/26/2008 3:00 AM & 0.083 & 10.9 & 0.073 & 0.299 & 14.1 & 0.37 & 0.038 & 0.038 & 15.4 & 0.053 & 0.358 & 15.8 & 0.415 & 15.7 & -- \\
\hline 10/26/2008 4:00 AM & 0.083 & 10.9 & 0.073 & 0.299 & 14.1 & 0.37 & 0.038 & 0.038 & 15.4 & 0.064 & 0.358 & 15.8 & 0.415 & 15.8 & -- \\
\hline 10/26/2008 5:00 AM & 0.084 & 10.9 & 0.073 & 0.298 & 14.0 & 0.37 & 0.038 & 0.038 & 15.4 & 0.062 & 0.357 & 15.8 & 0.415 & 15.8 & -- \\
\hline 10/26/2008 6:00 AM & 0.084 & 10.9 & 0.073 & 0.298 & 14.0 & 0.37 & 0.038 & 0.038 & 15.4 & 0.059 & 0.357 & 15.8 & 0.415 & 15.8 & -- \\
\hline 10/26/2008 7:00 AM & 0.085 & 11.0 & 0.073 & 0.298 & 14.0 & 0.37 & 0.038 & 0.038 & 15.4 & 0.058 & 0.356 & 15.8 & 0.416 & 15.8 & -- \\
\hline 10/26/2008 8:00 AM & 0.084 & 11.0 & 0.073 & 0.299 & 14.0 & 0.37 & 0.038 & 0.038 & 15.4 & 0.057 & 0.356 & 15.8 & 0.415 & 15.7 & -- \\
\hline 10/26/2008 9:00 AM & 0.083 & 11.0 & 0.073 & 0.299 & 14.0 & 0.37 & 0.038 & 0.038 & 15.4 & 0.057 & 0.355 & 15.8 & 0.415 & 15.7 & -- \\
\hline 10/26/2008 10:00 AM & 0.083 & 11.0 & 0.073 & 0.299 & 14.0 & 0.37 & 0.038 & 0.038 & 15.4 & 0.057 & 0.354 & 15.8 & 0.415 & 15.7 & -- \\
\hline 10/26/2008 11:00 AM & 0.083 & 11.0 & 0.073 & 0.299 & 14.0 & 0.37 & 0.038 & 0.038 & 15.4 & 0.058 & 0.353 & 15.8 & 0.416 & 15.7 & -- \\
\hline 10/26/2008 12:00 PM & 0.084 & 11.0 & 0.073 & 0.299 & 14.0 & 0.37 & 0.038 & 0.038 & 15.4 & 0.059 & 0.353 & 15.8 & 0.416 & 15.7 & -- \\
\hline 10/26/2008 1:00 PM & 0.084 & 11.0 & 0.073 & 0.299 & 14.0 & 0.37 & 0.038 & 0.038 & 15.4 & 0.062 & 0.353 & 15.8 & 0.418 & 15.7 & -- \\
\hline 10/26/2008 2:00 PM & 0.083 & 11.0 & 0.073 & 0.299 & 14.0 & 0.37 & 0.038 & 0.038 & 15.4 & 0.061 & 0.353 & 15.8 & 0.417 & 15.7 & -- \\
\hline
\end{tabular}


Appendix 1. Volumetric water content, temperature, and electrical-conductivity data collected at the flood-irrigated site during 2008.-Continued

[Depth in feet below land surface; volumetric water content dimensionless; temperature in degrees Celsius; bulk electrical conductivity in decisiemens per meter; --, no data]

\begin{tabular}{|c|c|c|c|c|c|c|c|c|c|c|c|c|c|c|c|}
\hline \multirow[b]{3}{*}{$\begin{array}{l}\text { Measurement } \\
\text { date and time }\end{array}$} & \multicolumn{15}{|c|}{ Depth } \\
\hline & \multicolumn{2}{|l|}{2} & \multirow{2}{*}{ 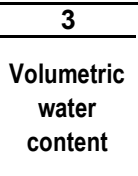 } & \multicolumn{3}{|c|}{4} & \multirow{2}{*}{$\begin{array}{c}6 \\
\begin{array}{c}\text { Volumetric } \\
\text { water } \\
\text { content }\end{array}\end{array}$} & \multicolumn{2}{|l|}{8} & \multirow{2}{*}{$\begin{array}{c}10 \\
\text { Volumetric } \\
\text { water } \\
\text { content }\end{array}$} & \multicolumn{2}{|c|}{12} & \multicolumn{2}{|c|}{15} & \multirow{2}{*}{$\begin{array}{c}18.5 \\
\text { Volumetric } \\
\text { water } \\
\text { content }\end{array}$} \\
\hline & $\begin{array}{l}\text { Volumetric } \\
\text { water } \\
\text { content }\end{array}$ & $\begin{array}{l}\text { Temper- } \\
\text { ature }\end{array}$ & & $\begin{array}{c}\text { Volumetric } \\
\text { water } \\
\text { content }\end{array}$ & $\begin{array}{l}\text { Temper- } \\
\text { ature }\end{array}$ & $\begin{array}{l}\text { Bulk } \\
\text { electrical } \\
\text { conduct- } \\
\text { ivity }\end{array}$ & & $\begin{array}{l}\text { Volumetric } \\
\text { water } \\
\text { content }\end{array}$ & $\begin{array}{l}\text { Temper- } \\
\text { ature }\end{array}$ & & $\begin{array}{c}\text { Volumetric } \\
\text { water } \\
\text { content }\end{array}$ & $\begin{array}{l}\text { Temper- } \\
\text { ature }\end{array}$ & $\begin{array}{l}\text { Volumetric } \\
\text { water } \\
\text { content }\end{array}$ & $\begin{array}{l}\text { Temper- } \\
\text { ature }\end{array}$ & \\
\hline 10/26/2008 3:00 PM & 0.083 & 11.0 & 0.073 & 0.299 & 14.0 & 0.37 & 0.038 & 0.038 & 15.4 & 0.058 & 0.353 & 15.8 & 0.417 & 15.7 & -- \\
\hline 10/26/2008 4:00 PM & 0.083 & 11.0 & 0.073 & 0.299 & 14.0 & 0.37 & 0.038 & 0.038 & 15.4 & 0.058 & 0.353 & 15.8 & 0.417 & 15.7 & -- \\
\hline 10/26/2008 5:00 PM & 0.083 & 11.0 & 0.073 & 0.299 & 14.0 & 0.37 & 0.038 & 0.038 & 15.4 & 0.057 & 0.351 & 15.8 & 0.417 & 15.7 & -- \\
\hline 10/26/2008 6:00 PM & 0.084 & 11.0 & 0.073 & 0.299 & 14.0 & 0.37 & 0.038 & 0.038 & 15.4 & 0.058 & 0.351 & 15.8 & 0.416 & 15.7 & -- \\
\hline 10/26/2008 7:00 PM & 0.084 & 10.9 & 0.073 & 0.298 & 13.9 & 0.37 & 0.038 & 0.038 & 15.4 & 0.059 & 0.351 & 15.8 & 0.416 & 15.7 & -- \\
\hline 10/26/2008 8:00 PM & 0.084 & 10.9 & 0.073 & 0.298 & 13.9 & 0.37 & 0.038 & 0.038 & 15.4 & 0.061 & 0.350 & 15.8 & 0.416 & 15.7 & -- \\
\hline 10/26/2008 9:00 PM & 0.085 & 10.9 & 0.073 & 0.298 & 13.9 & 0.37 & 0.038 & 0.038 & 15.4 & 0.062 & 0.349 & 15.8 & 0.417 & 15.7 & -- \\
\hline 10/26/2008 10:00 PM & 0.085 & 11.0 & 0.073 & 0.298 & 13.9 & 0.37 & 0.038 & 0.038 & 15.4 & 0.062 & 0.349 & 15.8 & 0.418 & 15.7 & -- \\
\hline 10/26/2008 11:00 PM & 0.085 & 11.0 & 0.073 & 0.298 & 13.9 & 0.37 & 0.038 & 0.038 & 15.4 & 0.061 & 0.349 & 15.8 & 0.418 & 15.7 & -- \\
\hline 10/27/2008 12:00 AM & 0.086 & 11.0 & 0.073 & 0.298 & 13.9 & 0.37 & 0.038 & 0.037 & 15.4 & 0.059 & 0.349 & 15.8 & 0.418 & 15.7 & -- \\
\hline 10/27/2008 1:00 AM & 0.086 & 11.0 & 0.073 & 0.298 & 13.9 & 0.37 & 0.038 & 0.037 & 15.4 & 0.064 & 0.349 & 15.8 & 0.418 & 15.7 & -- \\
\hline 10/27/2008 2:00 AM & 0.086 & 11.0 & 0.073 & 0.298 & 13.9 & 0.37 & 0.038 & 0.037 & 15.3 & 0.064 & 0.349 & 15.8 & 0.419 & 15.7 & -- \\
\hline 10/27/2008 3:00 AM & 0.085 & 11.0 & 0.073 & 0.298 & 13.9 & 0.37 & 0.038 & 0.037 & 15.3 & 0.063 & 0.349 & 15.8 & 0.418 & 15.7 & -- \\
\hline 10/27/2008 4:00 AM & 0.085 & 11.0 & 0.073 & 0.298 & 13.9 & 0.37 & 0.038 & 0.037 & 15.4 & 0.060 & 0.349 & 15.8 & 0.419 & 15.7 & -- \\
\hline 10/27/2008 5:00 AM & 0.085 & 10.9 & 0.073 & 0.298 & 13.9 & 0.37 & 0.038 & 0.037 & 15.3 & 0.061 & 0.349 & 15.8 & 0.419 & 15.7 & -- \\
\hline 10/27/2008 6:00 AM & 0.086 & 10.9 & 0.073 & 0.298 & 13.9 & 0.37 & 0.038 & 0.037 & 15.3 & 0.062 & 0.349 & 15.8 & 0.419 & 15.7 & -- \\
\hline 10/27/2008 7:00 AM & 0.086 & 10.9 & 0.073 & 0.298 & 13.9 & 0.37 & 0.038 & 0.037 & 15.3 & 0.062 & 0.349 & 15.8 & 0.419 & 15.7 & -- \\
\hline 10/27/2008 8:00 AM & 0.085 & 10.9 & 0.073 & 0.298 & 13.9 & 0.37 & 0.038 & 0.037 & 15.3 & 0.061 & 0.349 & 15.8 & 0.419 & 15.7 & -- \\
\hline 10/27/2008 9:00 AM & 0.085 & 10.9 & 0.072 & 0.298 & 13.9 & 0.37 & 0.038 & 0.037 & 15.3 & 0.058 & 0.349 & 15.8 & 0.419 & 15.7 & -- \\
\hline 10/27/2008 10:00 AM & 0.083 & 10.9 & 0.073 & 0.298 & 13.9 & 0.37 & 0.038 & 0.038 & 15.3 & 0.058 & 0.348 & 15.8 & 0.418 & 15.7 & -- \\
\hline 10/27/2008 11:00 AM & 0.083 & 10.8 & 0.073 & 0.298 & 13.9 & 0.37 & 0.038 & 0.037 & 15.3 & 0.064 & 0.349 & 15.7 & 0.415 & 15.7 & -- \\
\hline 10/27/2008 12:00 PM & 0.084 & 10.7 & 0.073 & 0.298 & 13.9 & 0.37 & 0.038 & 0.038 & 15.3 & 0.062 & 0.349 & 15.8 & 0.416 & 15.7 & -- \\
\hline 10/27/2008 1:00 PM & 0.082 & 10.7 & 0.073 & 0.298 & 13.9 & 0.37 & 0.038 & 0.038 & 15.3 & 0.059 & 0.349 & 15.8 & 0.419 & 15.7 & -- \\
\hline 10/27/2008 2:00 PM & 0.082 & 10.8 & 0.073 & 0.299 & 13.8 & 0.37 & 0.038 & 0.037 & 15.3 & 0.060 & 0.350 & 15.8 & 0.419 & 15.7 & -- \\
\hline 10/27/2008 3:00 PM & 0.082 & 10.7 & 0.073 & 0.299 & 13.8 & 0.37 & 0.038 & 0.037 & 15.3 & 0.063 & 0.350 & 15.8 & 0.417 & 15.7 & -- \\
\hline 10/27/2008 4:00 PM & 0.082 & 10.7 & 0.073 & 0.298 & 13.8 & 0.37 & 0.038 & 0.037 & 15.3 & 0.066 & 0.351 & 15.8 & 0.419 & 15.7 & -- \\
\hline 10/27/2008 5:00 PM & 0.082 & 10.6 & 0.073 & 0.298 & 13.8 & 0.37 & 0.038 & 0.037 & 15.3 & 0.064 & 0.351 & 15.8 & 0.419 & 15.7 & -- \\
\hline 10/27/2008 6:00 PM & 0.082 & 10.6 & 0.073 & 0.298 & 13.8 & 0.37 & 0.038 & 0.037 & 15.3 & 0.063 & 0.350 & 15.8 & 0.416 & 15.7 & -- \\
\hline 10/27/2008 7:00 PM & 0.086 & 10.5 & 0.072 & 0.298 & 13.8 & 0.37 & 0.038 & 0.037 & 15.3 & 0.061 & 0.350 & 15.8 & 0.418 & 15.7 & -- \\
\hline 10/27/2008 8:00 PM & 0.085 & 10.5 & 0.073 & 0.298 & 13.8 & 0.37 & 0.038 & 0.037 & 15.3 & 0.058 & 0.350 & 15.7 & 0.419 & 15.7 & -- \\
\hline 10/27/2008 9:00 PM & 0.086 & 10.5 & 0.073 & 0.298 & 13.8 & 0.37 & 0.038 & 0.037 & 15.3 & 0.058 & 0.349 & 15.7 & 0.419 & 15.7 & -- \\
\hline 10/27/2008 10:00 PM & 0.086 & 10.5 & 0.073 & 0.298 & 13.8 & 0.37 & 0.038 & 0.037 & 15.3 & 0.058 & 0.349 & 15.8 & 0.419 & 15.7 & -- \\
\hline 10/27/2008 11:00 PM & 0.086 & 10.5 & 0.072 & 0.298 & 13.8 & 0.37 & 0.038 & 0.037 & 15.3 & 0.057 & 0.349 & 15.8 & 0.418 & 15.7 & -- \\
\hline 10/28/2008 12:00 AM & 0.086 & 10.5 & 0.072 & 0.298 & 13.8 & 0.37 & 0.038 & 0.037 & 15.3 & 0.056 & 0.349 & 15.7 & 0.418 & 15.7 & -- \\
\hline 10/28/2008 1:00 AM & 0.085 & 10.4 & 0.072 & 0.298 & 13.8 & 0.37 & 0.038 & 0.037 & 15.3 & 0.056 & 0.349 & 15.7 & 0.418 & 15.7 & -- \\
\hline 10/28/2008 2:00 AM & 0.085 & 10.5 & 0.072 & 0.298 & 13.8 & 0.37 & 0.038 & 0.037 & 15.3 & 0.055 & 0.349 & 15.8 & 0.417 & 15.7 & -- \\
\hline 10/28/2008 3:00 AM & 0.086 & 10.5 & 0.072 & 0.298 & 13.8 & 0.37 & 0.038 & 0.037 & 15.3 & 0.056 & 0.349 & 15.8 & 0.417 & 15.7 & -- \\
\hline 10/28/2008 4:00 AM & 0.086 & 10.4 & 0.072 & 0.298 & 13.8 & 0.37 & 0.038 & 0.037 & 15.3 & 0.054 & 0.349 & 15.8 & 0.417 & 15.7 & -- \\
\hline 10/28/2008 5:00 AM & 0.086 & 10.4 & 0.072 & 0.298 & 13.8 & 0.37 & 0.038 & 0.037 & 15.3 & 0.056 & 0.349 & 15.8 & 0.417 & 15.7 & -- \\
\hline 10/28/2008 6:00 AM & 0.086 & 10.4 & 0.072 & 0.298 & 13.8 & 0.37 & 0.038 & 0.037 & 15.3 & 0.053 & 0.349 & 15.7 & 0.416 & 15.7 & -- \\
\hline
\end{tabular}


Appendix 1. Volumetric water content, temperature, and electrical-conductivity data collected at the flood-irrigated site during 2008.-Continued

[Depth in feet below land surface; volumetric water content dimensionless; temperature in degrees Celsius; bulk electrical conductivity in decisiemens per meter; --, no data]

\begin{tabular}{|c|c|c|c|c|c|c|c|c|c|c|c|c|c|c|c|}
\hline \multirow[b]{3}{*}{$\begin{array}{l}\text { Measurement } \\
\text { date and time }\end{array}$} & \multicolumn{15}{|c|}{ Depth } \\
\hline & \multicolumn{2}{|c|}{2} & \multirow{2}{*}{$\begin{array}{c}3 \\
\begin{array}{c}\text { Volumetric } \\
\text { water } \\
\text { content }\end{array}\end{array}$} & \multicolumn{3}{|c|}{4} & \multirow{2}{*}{$\begin{array}{c}6 \\
\text { Volumetric } \\
\text { water } \\
\text { content }\end{array}$} & \multicolumn{2}{|l|}{8} & \multirow{2}{*}{$\begin{array}{c}10 \\
\text { Volumetric } \\
\text { water } \\
\text { content }\end{array}$} & \multicolumn{2}{|l|}{12} & \multicolumn{2}{|l|}{15} & \multirow{2}{*}{$\begin{array}{c}18.5 \\
\text { Volumetric } \\
\text { water } \\
\text { content }\end{array}$} \\
\hline & $\begin{array}{c}\text { Volumetric } \\
\text { water } \\
\text { content }\end{array}$ & $\begin{array}{l}\text { Temper- } \\
\text { ature }\end{array}$ & & $\begin{array}{c}\text { Volumetric } \\
\text { water } \\
\text { content }\end{array}$ & $\begin{array}{l}\text { Temper- } \\
\text { ature }\end{array}$ & $\begin{array}{c}\text { Bulk } \\
\text { electrical } \\
\text { conduct- } \\
\text { ivity } \\
\end{array}$ & & $\begin{array}{c}\text { Volumetric } \\
\text { water } \\
\text { content }\end{array}$ & $\begin{array}{c}\text { Temper- } \\
\text { ature }\end{array}$ & & $\begin{array}{c}\text { Volumetric } \\
\text { water } \\
\text { content }\end{array}$ & $\begin{array}{l}\text { Temper- } \\
\text { ature }\end{array}$ & $\begin{array}{c}\text { Volumetric } \\
\text { water } \\
\text { content }\end{array}$ & $\begin{array}{c}\text { Temper- } \\
\text { ature }\end{array}$ & \\
\hline 10/28/2008 7:00 AM & 0.087 & 10.4 & 0.072 & 0.298 & 13.7 & 0.37 & 0.038 & 0.038 & 15.3 & 0.053 & 0.349 & 15.8 & 0.416 & 15.7 & -- \\
\hline 10/28/2008 8:00 AM & 0.087 & 10.4 & 0.072 & 0.298 & 13.7 & 0.37 & 0.038 & 0.037 & 15.3 & 0.054 & 0.349 & 15.8 & 0.416 & 15.7 & -- \\
\hline 10/28/2008 9:00 AM & 0.085 & 10.4 & 0.072 & 0.298 & 13.7 & 0.37 & 0.038 & 0.037 & 15.3 & 0.056 & 0.348 & 15.7 & 0.416 & 15.7 & -- \\
\hline 10/28/2008 10:00 AM & 0.085 & 10.3 & 0.072 & 0.298 & 13.7 & 0.37 & 0.038 & 0.037 & 15.3 & 0.057 & 0.349 & 15.7 & 0.418 & 15.7 & -- \\
\hline 10/28/2008 11:00 AM & 0.085 & 10.3 & 0.072 & 0.298 & 13.7 & 0.37 & 0.038 & 0.037 & 15.2 & 0.056 & 0.349 & 15.7 & 0.419 & 15.7 & -- \\
\hline 10/28/2008 12:00 PM & 0.085 & 10.3 & 0.072 & 0.298 & 13.7 & 0.37 & 0.038 & 0.037 & 15.2 & 0.055 & 0.349 & 15.7 & 0.419 & 15.7 & -- \\
\hline 10/28/2008 1:00 PM & 0.086 & 10.3 & 0.072 & 0.298 & 13.7 & 0.37 & 0.038 & 0.038 & 15.2 & 0.056 & 0.349 & 15.8 & 0.417 & 15.7 & -- \\
\hline
\end{tabular}


Appendix 2. Volumetric water content, temperature, and electrical-conductivity data collected at the flood-irrigated site during 2009.

[Depth in feet below land surface; volumetric water content dimensionless; temperature in degrees Celsius; bulk electrical conductivity in decisiemens per meter; --, no data]

\begin{tabular}{|c|c|c|c|c|c|c|c|c|c|c|c|c|c|c|c|c|}
\hline \multirow[b]{3}{*}{$\begin{array}{l}\text { Measurement } \\
\text { date and time }\end{array}$} & \multicolumn{16}{|c|}{ Depth } \\
\hline & \multirow{2}{*}{$\begin{array}{c}1 \\
\text { Volumetric } \\
\text { water } \\
\text { content }\end{array}$} & \multicolumn{2}{|c|}{2} & \multirow{2}{*}{$\begin{array}{c}3 \\
\text { Volumetric } \\
\text { water } \\
\text { content }\end{array}$} & \multicolumn{3}{|c|}{4} & \multirow{2}{*}{$\begin{array}{c}6 \\
\text { Volumetric } \\
\text { water } \\
\text { content }\end{array}$} & \multicolumn{2}{|c|}{8} & \multirow{2}{*}{$\begin{array}{c}10 \\
\text { Volumetric } \\
\text { water } \\
\text { content }\end{array}$} & \multicolumn{2}{|c|}{12} & \multicolumn{2}{|c|}{15} & \multirow{2}{*}{$\begin{array}{c}18.5 \\
\text { Volumetric } \\
\text { water } \\
\text { content }\end{array}$} \\
\hline & & $\begin{array}{c}\text { Volumetric } \\
\text { water } \\
\text { content }\end{array}$ & $\begin{array}{l}\text { Temper- } \\
\text { ature }\end{array}$ & & $\begin{array}{c}\text { Volumetric } \\
\text { water } \\
\text { content }\end{array}$ & $\begin{array}{l}\text { Temper- } \\
\text { ature }\end{array}$ & $\begin{array}{l}\text { Bulk } \\
\text { electrical } \\
\text { conduct- } \\
\text { ivity }\end{array}$ & & $\begin{array}{c}\text { Volumetric } \\
\text { water } \\
\text { content }\end{array}$ & $\begin{array}{l}\text { Temper- } \\
\text { ature }\end{array}$ & & $\begin{array}{c}\text { Volumetric } \\
\text { water } \\
\text { content }\end{array}$ & $\begin{array}{l}\text { Temper- } \\
\text { ature }\end{array}$ & $\begin{array}{c}\text { Volumetric } \\
\text { water } \\
\text { content }\end{array}$ & $\begin{array}{l}\text { Temper- } \\
\text { ature }\end{array}$ & \\
\hline 7/1/2009 4:00 PM & -- & 0.108 & 18.5 & 0.082 & 0.304 & 17.6 & 0.43 & 0.038 & 0.042 & 15.0 & 0.042 & 0.397 & 13.7 & 0.415 & 13.3 & -- \\
\hline 7/1/2009 8:00 PM & -- & 0.117 & 21.7 & 0.087 & 0.306 & 19.1 & 0.45 & 0.038 & 0.047 & 14.9 & 0.041 & 0.417 & 13.0 & 0.413 & 12.3 & -- \\
\hline 7/2/2009 12:00 AM & -- & 0.117 & 21.8 & 0.086 & 0.306 & 19.1 & 0.45 & 0.039 & 0.047 & 14.9 & 0.046 & 0.417 & 13.0 & 0.412 & 12.3 & -- \\
\hline 7/2/2009 4:00 AM & -- & 0.117 & 21.9 & 0.086 & 0.306 & 19.2 & 0.45 & 0.039 & 0.047 & 14.9 & 0.049 & 0.418 & 13.0 & 0.412 & 12.3 & -- \\
\hline 7/2/2009 8:00 AM & -- & 0.117 & 21.9 & 0.086 & 0.306 & 19.2 & 0.45 & 0.039 & 0.047 & 15.0 & 0.043 & 0.417 & 13.0 & 0.412 & 12.4 & -- \\
\hline 7/2/2009 12:00 PM & -- & 0.117 & 21.9 & 0.086 & 0.307 & 19.2 & 0.45 & 0.039 & 0.047 & 15.0 & 0.042 & 0.415 & 13.0 & 0.413 & 12.4 & -- \\
\hline 7/2/2009 4:00 PM & -- & 0.185 & 22.0 & 0.102 & 0.307 & 19.3 & 0.45 & 0.038 & 0.047 & 15.0 & 0.042 & 0.415 & 13.0 & 0.413 & 12.4 & -- \\
\hline 7/2/2009 8:00 PM & -- & 0.254 & 22.7 & 0.154 & 0.332 & 19.4 & 0.51 & 0.038 & 0.047 & 15.0 & 0.043 & 0.415 & 13.0 & 0.413 & 12.4 & -- \\
\hline 7/3/2009 12:00 AM & -- & 0.238 & 23.1 & 0.151 & 0.342 & 19.6 & 0.52 & 0.038 & 0.047 & 15.0 & 0.043 & 0.415 & 13.0 & 0.417 & 12.3 & -- \\
\hline 7/3/2009 4:00 AM & -- & 0.224 & 23.2 & 0.147 & 0.342 & 19.7 & 0.49 & 0.038 & 0.047 & 15.1 & 0.044 & 0.416 & 13.0 & 0.413 & 12.4 & -- \\
\hline 7/3/2009 8:00 AM & -- & 0.212 & 23.2 & 0.143 & 0.341 & 19.8 & 0.48 & 0.040 & 0.047 & 15.1 & 0.044 & 0.415 & 13.0 & 0.413 & 12.4 & -- \\
\hline 7/3/2009 12:00 PM & -- & 0.206 & 23.1 & 0.140 & 0.340 & 19.9 & 0.46 & 0.042 & 0.047 & 15.1 & 0.055 & 0.415 & 13.0 & 0.415 & 12.3 & -- \\
\hline 7/3/2009 4:00 PM & -- & 0.198 & 22.9 & 0.138 & 0.340 & 19.9 & 0.45 & 0.047 & 0.047 & 15.1 & 0.071 & 0.413 & 13.0 & 0.415 & 12.3 & -- \\
\hline 7/3/2009 8:00 PM & -- & 0.193 & 22.8 & 0.134 & 0.338 & 20.0 & 0.43 & 0.053 & 0.047 & 15.1 & 0.064 & 0.413 & 13.0 & 0.415 & 12.3 & -- \\
\hline 7/4/2009 12:00 AM & -- & 0.185 & 22.7 & 0.132 & 0.337 & 20.1 & 0.42 & 0.057 & 0.047 & 15.1 & 0.049 & 0.415 & 13.1 & 0.413 & 12.3 & -- \\
\hline 7/4/2009 4:00 AM & -- & 0.181 & 22.7 & 0.129 & 0.336 & 20.1 & 0.41 & 0.057 & 0.049 & 15.2 & 0.058 & 0.415 & 13.1 & 0.413 & 12.3 & -- \\
\hline 7/4/2009 8:00 AM & -- & 0.178 & 22.7 & 0.127 & 0.335 & 20.1 & 0.41 & 0.058 & 0.051 & 15.2 & 0.054 & 0.415 & 13.1 & 0.415 & 12.3 & -- \\
\hline 7/4/2009 12:00 PM & -- & 0.176 & 22.5 & 0.126 & 0.334 & 20.2 & 0.40 & 0.057 & 0.053 & 15.2 & 0.044 & 0.413 & 13.1 & 0.415 & 12.4 & -- \\
\hline 7/4/2009 4:00 PM & -- & 0.173 & 22.4 & 0.124 & 0.334 & 20.2 & 0.40 & 0.057 & 0.056 & 15.2 & 0.374 & 0.413 & 13.1 & 0.415 & 12.4 & -- \\
\hline 7/4/2009 8:00 PM & -- & 0.171 & 22.3 & 0.122 & 0.333 & 20.2 & 0.39 & 0.056 & 0.058 & 15.3 & 0.669 & 0.415 & 13.1 & 0.415 & 12.3 & -- \\
\hline 7/5/2009 12:00 AM & -- & 0.168 & 22.2 & 0.121 & 0.332 & 20.2 & 0.39 & 0.056 & 0.059 & 15.3 & 0.063 & 0.415 & 13.1 & 0.415 & 12.3 & -- \\
\hline 7/5/2009 4:00 AM & -- & 0.166 & 22.2 & 0.120 & 0.332 & 20.2 & 0.39 & 0.055 & 0.060 & 15.3 & 0.085 & 0.415 & 13.2 & 0.413 & 12.4 & -- \\
\hline 7/5/2009 8:00 AM & -- & 0.163 & 22.1 & 0.118 & 0.331 & 20.2 & 0.38 & 0.055 & 0.060 & 15.4 & 0.055 & 0.415 & 13.2 & 0.413 & 12.4 & -- \\
\hline 7/5/2009 12:00 PM & -- & 0.161 & 22.0 & 0.117 & 0.331 & 20.2 & 0.38 & 0.054 & 0.061 & 15.4 & 0.055 & 0.413 & 13.2 & 0.415 & 12.5 & -- \\
\hline 7/5/2009 4:00 PM & -- & 0.159 & 21.7 & 0.116 & 0.331 & 20.2 & 0.38 & 0.054 & 0.061 & 15.4 & 0.050 & 0.413 & 13.2 & 0.415 & 12.5 & -- \\
\hline 7/5/2009 8:00 PM & -- & 0.158 & 21.5 & 0.115 & 0.330 & 20.2 & 0.38 & 0.054 & 0.061 & 15.4 & 0.056 & 0.413 & 13.2 & 0.413 & 12.5 & -- \\
\hline 7/6/2009 12:00 AM & -- & 0.156 & 21.5 & 0.115 & 0.330 & 20.2 & 0.38 & 0.053 & 0.060 & 15.5 & 0.085 & 0.413 & 13.2 & 0.412 & 12.4 & -- \\
\hline 7/6/2009 4:00 AM & -- & 0.155 & 21.5 & 0.114 & 0.329 & 20.2 & 0.38 & 0.053 & 0.060 & 15.5 & 0.112 & 0.413 & 13.2 & 0.413 & 12.4 & -- \\
\hline 7/6/2009 8:00 AM & -- & 0.154 & 21.4 & 0.113 & 0.328 & 20.2 & 0.38 & 0.053 & 0.060 & 15.5 & 0.075 & 0.413 & 13.2 & 0.413 & 12.5 & -- \\
\hline 7/6/2009 12:00 PM & -- & 0.153 & 21.4 & 0.113 & 0.328 & 20.2 & 0.38 & 0.053 & 0.060 & 15.6 & 0.060 & 0.412 & 13.2 & 0.415 & 12.5 & -- \\
\hline 7/6/2009 4:00 PM & -- & 0.151 & 21.3 & 0.112 & 0.328 & 20.2 & 0.38 & 0.052 & 0.060 & 15.6 & 0.085 & 0.411 & 13.2 & 0.413 & 12.5 & -- \\
\hline 7/6/2009 8:00 PM & -- & 0.150 & 21.2 & 0.111 & 0.326 & 20.2 & 0.38 & 0.052 & 0.059 & 15.6 & 0.352 & 0.411 & 13.2 & 0.413 & 12.5 & -- \\
\hline 7/7/2009 12:00 AM & -- & 0.149 & 21.1 & 0.110 & 0.326 & 20.2 & 0.38 & 0.051 & 0.059 & 15.6 & 0.070 & 0.411 & 13.3 & 0.412 & 12.6 & -- \\
\hline 7/7/2009 4:00 AM & -- & 0.148 & 21.2 & 0.110 & 0.326 & 20.2 & 0.38 & 0.051 & 0.059 & 15.7 & -- & 0.411 & 13.3 & 0.412 & 12.6 & -- \\
\hline 7/7/2009 8:00 AM & -- & 0.147 & 21.1 & 0.109 & 0.325 & 20.1 & 0.38 & 0.051 & 0.058 & 15.7 & 0.089 & 0.410 & 13.3 & 0.412 & 12.6 & -- \\
\hline 7/7/2009 12:00 PM & -- & 0.147 & 21.1 & 0.109 & 0.325 & 20.1 & 0.37 & 0.050 & 0.058 & 15.7 & 0.064 & 0.409 & 13.3 & 0.413 & 12.5 & -- \\
\hline 7/7/2009 4:00 PM & 0.137 & 0.145 & 21.0 & 0.109 & 0.325 & 20.1 & 0.37 & 0.050 & 0.058 & 15.7 & 0.059 & 0.409 & 13.3 & 0.413 & 12.5 & -- \\
\hline 7/7/2009 8:00 PM & 0.138 & 0.145 & 20.9 & 0.108 & 0.325 & 20.1 & 0.37 & 0.050 & 0.058 & 15.8 & 0.059 & 0.409 & 13.3 & 0.415 & 12.5 & -- \\
\hline 7/8/2009 12:00 AM & 0.140 & 0.144 & 20.9 & 0.108 & 0.324 & 20.1 & 0.37 & 0.049 & 0.058 & 15.8 & 0.077 & 0.410 & 13.3 & 0.412 & 12.6 & -- \\
\hline 7/8/2009 4:00 AM & 0.140 & 0.144 & 21.0 & 0.107 & 0.324 & 20.1 & 0.37 & 0.049 & 0.058 & 15.8 & 0.068 & 0.409 & 13.4 & 0.412 & 12.6 & -- \\
\hline 7/8/2009 8:00 AM & 0.140 & 0.143 & 21.0 & 0.107 & 0.324 & 20.0 & 0.37 & 0.049 & 0.057 & 15.8 & 0.076 & 0.409 & 13.4 & 0.412 & 12.6 & -- \\
\hline 7/8/2009 12:00 PM & 0.140 & 0.142 & 21.0 & 0.106 & 0.324 & 20.0 & 0.37 & 0.048 & 0.058 & 15.9 & 0.065 & 0.410 & 13.4 & 0.415 & 12.6 & -- \\
\hline 7/8/2009 4:00 PM & 0.174 & 0.173 & 21.0 & 0.109 & 0.323 & 20.0 & 0.37 & 0.048 & 0.057 & 15.9 & 0.059 & 0.409 & 13.4 & 0.413 & 12.5 & -- \\
\hline 7/8/2009 8:00 PM & 0.168 & 0.192 & 21.1 & 0.120 & 0.324 & 20.0 & 0.38 & 0.048 & 0.057 & 15.9 & 0.062 & 0.409 & 13.4 & 0.413 & 12.5 & -- \\
\hline
\end{tabular}


Appendix 2. Volumetric water content, temperature, and electrical-conductivity data collected at the flood-irrigated site during 2009.-Continued

[Depth in feet below land surface; volumetric water content dimensionless; temperature in degrees Celsius; bulk electrical conductivity in decisiemens per meter; --, no data]

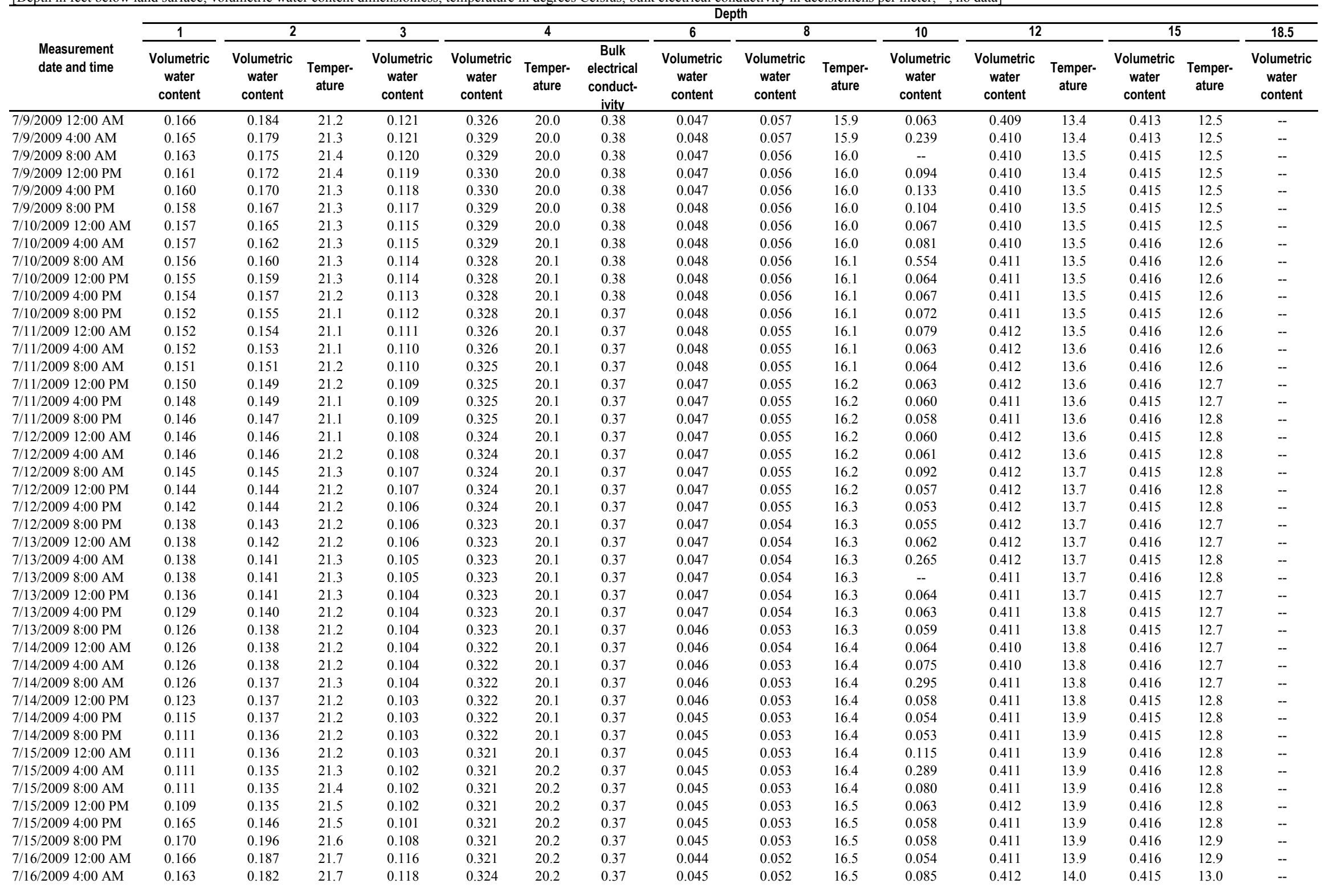


Appendix 2. Volumetric water content, temperature, and electrical-conductivity data collected at the flood-irrigated site during 2009.-Continued

[Depth in feet below land surface; volumetric water content dimensionless; temperature in degrees Celsius; bulk electrical conductivity in decisiemens per meter; --, no data]

\begin{tabular}{|c|c|c|c|c|c|c|c|c|c|c|c|c|c|c|c|c|}
\hline \multirow[b]{3}{*}{$\begin{array}{l}\text { Measurement } \\
\text { date and time }\end{array}$} & \multicolumn{16}{|c|}{ Depth } \\
\hline & \multirow{2}{*}{$\begin{array}{c}1 \\
\text { Volumetric } \\
\text { water } \\
\text { content }\end{array}$} & \multicolumn{2}{|c|}{2} & \multirow[b]{2}{*}{$\begin{array}{c}3 \\
\text { Volumetric } \\
\text { water } \\
\text { content }\end{array}$} & \multicolumn{3}{|c|}{4} & \multirow{2}{*}{$\begin{array}{c}6 \\
\text { Volumetric } \\
\text { water } \\
\text { content }\end{array}$} & \multicolumn{2}{|c|}{8} & \multirow{2}{*}{$\begin{array}{c}10 \\
\text { Volumetric } \\
\text { water } \\
\text { content }\end{array}$} & \multicolumn{2}{|c|}{12} & \multicolumn{2}{|c|}{15} & \multirow{2}{*}{$\begin{array}{c}18.5 \\
\text { Volumetric } \\
\text { water } \\
\text { content }\end{array}$} \\
\hline & & $\begin{array}{c}\text { Volumetric } \\
\text { water } \\
\text { content }\end{array}$ & $\begin{array}{l}\text { Temper- } \\
\text { ature }\end{array}$ & & $\begin{array}{c}\text { Volumetric } \\
\text { water } \\
\text { content }\end{array}$ & $\begin{array}{l}\text { Temper- } \\
\text { ature }\end{array}$ & $\begin{array}{c}\text { Bulk } \\
\text { electrical } \\
\text { conduct- } \\
\text { ivity } \\
\end{array}$ & & $\begin{array}{c}\text { Volumetric } \\
\text { water } \\
\text { content }\end{array}$ & $\begin{array}{l}\text { Temper- } \\
\text { ature }\end{array}$ & & $\begin{array}{c}\text { Volumetric } \\
\text { water } \\
\text { content }\end{array}$ & $\begin{array}{l}\text { Temper- } \\
\text { ature }\end{array}$ & $\begin{array}{c}\text { Volumetric } \\
\text { water } \\
\text { content }\end{array}$ & $\begin{array}{l}\text { Temper- } \\
\text { ature }\end{array}$ & \\
\hline 7/16/2009 8:00 AM & 0.162 & 0.178 & 21.8 & 0.118 & 0.326 & 20.3 & 0.38 & 0.045 & 0.052 & 16.5 & 0.215 & 0.412 & 14.0 & 0.415 & 13.0 & -- \\
\hline 7/16/2009 12:00 PM & 0.159 & 0.175 & 21.7 & 0.117 & 0.328 & 20.3 & 0.38 & 0.044 & 0.052 & 16.5 & 0.052 & 0.412 & 14.0 & 0.416 & 13.0 & -- \\
\hline 7/16/2009 4:00 PM & 0.154 & 0.172 & 21.6 & 0.116 & 0.329 & 20.3 & 0.38 & 0.044 & 0.052 & 16.5 & 0.052 & 0.412 & 14.0 & 0.416 & 12.9 & -- \\
\hline 7/16/2009 8:00 PM & 0.152 & 0.169 & 21.5 & 0.116 & 0.329 & 20.3 & 0.38 & 0.044 & 0.052 & 16.5 & 0.054 & 0.412 & 14.0 & 0.416 & 12.9 & -- \\
\hline 7/17/2009 12:00 AM & 0.152 & 0.167 & 21.5 & 0.115 & 0.329 & 20.3 & 0.38 & 0.045 & 0.051 & 16.5 & 0.055 & 0.412 & 14.0 & 0.415 & 12.9 & -- \\
\hline 7/17/2009 4:00 AM & 0.152 & 0.163 & 21.5 & 0.115 & 0.329 & 20.3 & 0.38 & 0.045 & 0.051 & 16.5 & 0.053 & 0.412 & 14.0 & 0.416 & 12.9 & -- \\
\hline 7/17/2009 8:00 AM & 0.151 & 0.162 & 21.5 & 0.114 & 0.329 & 20.4 & 0.38 & 0.045 & 0.051 & 16.6 & 0.053 & 0.412 & 14.0 & 0.416 & 12.9 & -- \\
\hline 7/17/2009 12:00 PM & 0.149 & 0.161 & 21.4 & 0.113 & 0.329 & 20.4 & 0.37 & 0.045 & 0.052 & 16.6 & 0.050 & 0.412 & 14.0 & 0.416 & 12.9 & -- \\
\hline 7/17/2009 4:00 PM & 0.143 & 0.159 & 21.4 & 0.113 & 0.329 & 20.4 & 0.37 & 0.045 & 0.052 & 16.6 & 0.050 & 0.412 & 14.0 & 0.415 & 12.9 & -- \\
\hline 7/17/2009 8:00 PM & 0.139 & 0.158 & 21.3 & 0.112 & 0.329 & 20.4 & 0.37 & 0.046 & 0.052 & 16.6 & 0.052 & 0.412 & 14.1 & 0.416 & 12.9 & -- \\
\hline 7/18/2009 12:00 AM & 0.139 & 0.156 & 21.3 & 0.111 & 0.328 & 20.4 & 0.37 & 0.046 & 0.051 & 16.6 & 0.055 & 0.413 & 14.1 & 0.416 & 13.0 & -- \\
\hline 7/18/2009 4:00 AM & 0.140 & 0.155 & 21.3 & 0.111 & 0.328 & 20.4 & 0.37 & 0.046 & 0.051 & 16.6 & 0.057 & 0.413 & 14.1 & 0.416 & 13.0 & -- \\
\hline 7/18/2009 8:00 AM & 0.140 & 0.153 & 21.3 & 0.110 & 0.328 & 20.4 & 0.37 & 0.046 & 0.051 & 16.6 & 0.064 & 0.413 & 14.1 & 0.415 & 13.0 & -- \\
\hline 7/18/2009 12:00 PM & 0.138 & 0.153 & 21.3 & 0.109 & 0.328 & 20.4 & 0.37 & 0.046 & 0.052 & 16.6 & 0.048 & 0.413 & 14.1 & 0.416 & 13.0 & -- \\
\hline 7/18/2009 4:00 PM & 0.132 & 0.150 & 21.2 & 0.109 & 0.328 & 20.4 & 0.37 & 0.046 & 0.052 & 16.6 & 0.051 & 0.413 & 14.1 & 0.416 & 13.0 & -- \\
\hline 7/18/2009 8:00 PM & 0.126 & 0.149 & 21.1 & 0.109 & 0.326 & 20.4 & 0.37 & 0.046 & 0.052 & 16.6 & 0.052 & 0.413 & 14.1 & 0.416 & 13.0 & -- \\
\hline 7/19/2009 12:00 AM & 0.126 & 0.148 & 21.1 & 0.109 & 0.326 & 20.4 & 0.37 & 0.046 & 0.052 & 16.7 & 0.053 & 0.413 & 14.1 & 0.416 & 13.0 & -- \\
\hline 7/19/2009 4:00 AM & 0.127 & 0.147 & 21.2 & 0.108 & 0.326 & 20.4 & 0.37 & 0.046 & 0.052 & 16.7 & 0.061 & 0.413 & 14.1 & 0.415 & 13.0 & -- \\
\hline 7/19/2009 8:00 AM & 0.128 & 0.146 & 21.2 & 0.108 & 0.325 & 20.4 & 0.37 & 0.046 & 0.052 & 16.7 & 0.064 & 0.413 & 14.1 & 0.415 & 13.1 & -- \\
\hline 7/19/2009 12:00 PM & 0.125 & 0.146 & 21.2 & 0.107 & 0.325 & 20.4 & 0.37 & 0.045 & 0.052 & 16.7 & 0.049 & 0.413 & 14.2 & 0.415 & 13.0 & -- \\
\hline 7/19/2009 4:00 PM & 0.119 & 0.145 & 21.1 & 0.107 & 0.325 & 20.4 & 0.37 & 0.045 & 0.052 & 16.7 & 0.053 & 0.413 & 14.2 & 0.415 & 13.1 & -- \\
\hline 7/19/2009 8:00 PM & 0.113 & 0.144 & 21.1 & 0.107 & 0.325 & 20.4 & 0.37 & 0.045 & 0.052 & 16.7 & 0.053 & 0.413 & 14.2 & 0.415 & 13.1 & -- \\
\hline 7/20/2009 12:00 AM & 0.113 & 0.143 & 21.1 & 0.106 & 0.325 & 20.4 & 0.37 & 0.045 & 0.052 & 16.7 & 0.051 & 0.413 & 14.2 & 0.413 & 13.1 & -- \\
\hline 7/20/2009 4:00 AM & 0.114 & 0.142 & 21.1 & 0.106 & 0.324 & 20.4 & 0.37 & 0.045 & 0.051 & 16.7 & 0.051 & 0.412 & 14.2 & 0.413 & 13.1 & -- \\
\hline 7/20/2009 8:00 AM & 0.115 & 0.142 & 21.2 & 0.105 & 0.324 & 20.4 & 0.37 & 0.045 & 0.052 & 16.7 & 0.050 & 0.413 & 14.2 & 0.413 & 13.1 & -- \\
\hline 7/20/2009 12:00 PM & 0.111 & 0.141 & 21.2 & 0.105 & 0.324 & 20.3 & 0.37 & 0.045 & 0.052 & 16.8 & 0.043 & 0.413 & 14.2 & 0.415 & 13.1 & -- \\
\hline 7/20/2009 4:00 PM & 0.105 & 0.140 & 21.2 & 0.104 & 0.324 & 20.3 & 0.37 & 0.045 & 0.052 & 16.8 & 0.051 & 0.413 & 14.2 & 0.415 & 13.1 & -- \\
\hline 7/20/2009 8:00 PM & 0.101 & 0.140 & 21.2 & 0.104 & 0.324 & 20.3 & 0.37 & 0.045 & 0.052 & 16.8 & 0.045 & 0.413 & 14.2 & 0.415 & 13.1 & -- \\
\hline 7/21/2009 12:00 AM & 0.101 & 0.138 & 21.2 & 0.104 & 0.323 & 20.3 & 0.37 & 0.045 & 0.051 & 16.8 & 0.048 & 0.413 & 14.2 & 0.413 & 13.1 & -- \\
\hline 7/21/2009 4:00 AM & 0.179 & 0.138 & 21.3 & 0.104 & 0.323 & 20.4 & 0.37 & 0.045 & 0.051 & 16.8 & 0.095 & 0.413 & 14.2 & 0.413 & 13.1 & -- \\
\hline 7/21/2009 8:00 AM & 0.173 & 0.137 & 21.3 & 0.103 & 0.322 & 20.4 & 0.37 & 0.045 & 0.051 & 16.8 & 0.106 & 0.413 & 14.3 & 0.413 & 13.2 & -- \\
\hline 7/21/2009 12:00 PM & 0.171 & 0.137 & 21.3 & 0.103 & 0.322 & 20.4 & 0.37 & 0.044 & 0.051 & 16.8 & 0.049 & 0.413 & 14.3 & 0.413 & 13.2 & -- \\
\hline 7/21/2009 4:00 PM & 0.169 & 0.136 & 21.2 & 0.103 & 0.322 & 20.4 & 0.37 & 0.044 & 0.051 & 16.8 & 0.042 & 0.413 & 14.3 & 0.415 & 13.2 & -- \\
\hline 7/21/2009 8:00 PM & 0.166 & 0.136 & 21.1 & 0.103 & 0.322 & 20.4 & 0.37 & 0.044 & 0.051 & 16.8 & 0.042 & 0.415 & 14.3 & 0.415 & 13.2 & -- \\
\hline $7 / 22 / 200912: 00 \mathrm{AM}$ & 0.165 & 0.136 & 21.0 & 0.103 & 0.322 & 20.4 & 0.37 & 0.044 & 0.051 & 16.8 & 0.049 & 0.415 & 14.3 & 0.413 & 13.2 & -- \\
\hline 7/22/2009 4:00 AM & 0.164 & 0.135 & 21.0 & 0.102 & 0.322 & 20.4 & 0.37 & 0.044 & 0.051 & 16.9 & 0.058 & 0.415 & 14.3 & 0.413 & 13.2 & -- \\
\hline 7/22/2009 8:00 AM & 0.163 & 0.135 & 21.0 & 0.102 & 0.322 & 20.4 & 0.36 & 0.044 & 0.051 & 16.9 & 0.056 & 0.415 & 14.3 & 0.413 & 13.2 & -- \\
\hline 7/22/2009 12:00 PM & 0.161 & 0.134 & 20.9 & 0.102 & 0.322 & 20.4 & 0.36 & 0.044 & 0.051 & 16.9 & 0.046 & 0.415 & 14.3 & 0.415 & 13.2 & -- \\
\hline 7/22/2009 4:00 PM & 0.155 & 0.134 & 20.8 & 0.101 & 0.322 & 20.4 & 0.36 & 0.044 & 0.051 & 16.9 & 0.045 & 0.415 & 14.3 & 0.415 & 13.2 & -- \\
\hline 7/22/2009 8:00 PM & 0.155 & 0.133 & 20.7 & 0.101 & 0.321 & 20.4 & 0.36 & 0.044 & 0.051 & 16.9 & 0.051 & 0.415 & 14.4 & 0.415 & 13.2 & -- \\
\hline 7/23/2009 12:00 AM & 0.154 & 0.133 & 20.7 & 0.101 & 0.321 & 20.4 & 0.36 & 0.044 & 0.050 & 16.9 & 0.047 & 0.415 & 14.4 & 0.413 & 13.2 & -- \\
\hline 7/23/2009 4:00 AM & 0.154 & 0.133 & 20.7 & 0.101 & 0.321 & 20.3 & 0.36 & 0.044 & 0.050 & 16.9 & 0.052 & 0.413 & 14.4 & 0.413 & 13.2 & -- \\
\hline 7/23/2009 8:00 AM & 0.154 & 0.132 & 20.7 & 0.101 & 0.321 & 20.3 & 0.36 & 0.044 & 0.051 & 16.9 & 0.062 & 0.413 & 14.4 & 0.413 & 13.3 & -- \\
\hline 7/23/2009 12:00 PM & 0.151 & 0.132 & 20.7 & 0.100 & 0.321 & 20.3 & 0.36 & 0.043 & 0.051 & 16.9 & 0.029 & 0.415 & 14.4 & 0.415 & 13.3 & -- \\
\hline
\end{tabular}


Appendix 2. Volumetric water content, temperature, and electrical-conductivity data collected at the flood-irrigated site during 2009.-Continued

[Depth in feet below land surface; volumetric water content dimensionless; temperature in degrees Celsius; bulk electrical conductivity in decisiemens per meter; --, no data]

\begin{tabular}{|c|c|c|c|c|c|c|c|c|c|c|c|c|c|c|c|c|}
\hline \multirow[b]{3}{*}{$\begin{array}{l}\text { Measurement } \\
\text { date and time }\end{array}$} & \multicolumn{16}{|c|}{ Depth } \\
\hline & \multirow{2}{*}{$\begin{array}{c}1 \\
\text { Volumetric } \\
\text { water } \\
\text { content } \\
\end{array}$} & \multicolumn{2}{|l|}{2} & \multirow{2}{*}{$\begin{array}{c}3 \\
\text { Volumetric } \\
\text { water } \\
\text { content }\end{array}$} & \multicolumn{3}{|c|}{4} & \multirow{2}{*}{$\begin{array}{c}6 \\
\text { Volumetric } \\
\text { water } \\
\text { content }\end{array}$} & \multicolumn{2}{|c|}{8} & \multirow{2}{*}{$\begin{array}{c}10 \\
\text { Volumetric } \\
\text { water } \\
\text { content }\end{array}$} & \multicolumn{2}{|c|}{12} & \multicolumn{2}{|c|}{15} & \multirow{2}{*}{$\begin{array}{c}18.5 \\
\text { Volumetric } \\
\text { water } \\
\text { content }\end{array}$} \\
\hline & & $\begin{array}{c}\text { Volumetric } \\
\text { water } \\
\text { content }\end{array}$ & $\begin{array}{l}\text { Temper- } \\
\text { ature }\end{array}$ & & $\begin{array}{l}\text { Volumetric } \\
\text { water } \\
\text { content }\end{array}$ & $\begin{array}{l}\text { Temper- } \\
\text { ature }\end{array}$ & $\begin{array}{c}\text { Bulk } \\
\text { electrical } \\
\text { conduct- } \\
\text { ivity } \\
\end{array}$ & & $\begin{array}{l}\text { Volumetric } \\
\text { water } \\
\text { content }\end{array}$ & $\begin{array}{l}\text { Temper- } \\
\text { ature }\end{array}$ & & $\begin{array}{l}\text { Volumetric } \\
\text { water } \\
\text { content }\end{array}$ & $\begin{array}{l}\text { Temper- } \\
\text { ature }\end{array}$ & $\begin{array}{l}\text { Volumetric } \\
\text { water } \\
\text { content }\end{array}$ & $\begin{array}{l}\text { Temper- } \\
\text { ature }\end{array}$ & \\
\hline 7/23/2009 4:00 PM & 0.147 & 0.131 & 20.6 & 0.100 & 0.321 & 20.3 & 0.36 & 0.043 & 0.051 & 16.9 & 0.048 & 0.415 & 14.4 & 0.416 & 13.3 & -- \\
\hline 7/23/2009 8:00 PM & 0.143 & 0.131 & 20.5 & 0.100 & 0.321 & 20.3 & 0.36 & 0.043 & 0.051 & 16.9 & 0.049 & 0.415 & 14.4 & 0.416 & 13.3 & -- \\
\hline 7/24/2009 12:00 AM & 0.143 & 0.130 & 20.5 & 0.100 & 0.321 & 20.3 & 0.36 & 0.043 & 0.050 & 16.9 & 0.025 & 0.413 & 14.4 & 0.415 & 13.3 & -- \\
\hline 7/24/2009 4:00 AM & 0.143 & 0.130 & 20.5 & 0.100 & 0.321 & 20.3 & 0.36 & 0.043 & 0.050 & 16.9 & 0.044 & 0.413 & 14.4 & 0.413 & 13.3 & -- \\
\hline 7/24/2009 8:00 AM & 0.142 & 0.130 & 20.6 & 0.099 & 0.320 & 20.3 & 0.36 & 0.043 & 0.050 & 16.9 & 0.047 & 0.413 & 14.4 & 0.413 & 13.3 & -- \\
\hline 7/24/2009 12:00 PM & 0.140 & 0.130 & 20.5 & 0.099 & 0.320 & 20.3 & 0.36 & 0.043 & 0.050 & 16.9 & 0.031 & 0.413 & 14.5 & 0.416 & 13.3 & -- \\
\hline 7/24/2009 4:00 PM & 0.188 & 0.150 & 20.5 & 0.099 & 0.320 & 20.3 & 0.36 & 0.043 & 0.050 & 16.9 & 0.051 & 0.415 & 14.5 & 0.416 & 13.4 & -- \\
\hline 7/24/2009 8:00 PM & 0.176 & 0.198 & 20.5 & 0.111 & 0.320 & 20.2 & 0.36 & 0.043 & 0.050 & 17.0 & 0.045 & 0.415 & 14.5 & 0.415 & 13.4 & -- \\
\hline 7/25/2009 12:00 AM & 0.172 & 0.190 & 20.7 & 0.119 & 0.321 & 20.2 & 0.36 & 0.043 & 0.050 & 17.0 & 0.042 & 0.413 & 14.5 & 0.416 & 13.4 & -- \\
\hline 7/25/2009 4:00 AM & 0.171 & 0.184 & 20.8 & 0.120 & 0.325 & 20.2 & 0.37 & 0.043 & 0.050 & 17.0 & 0.041 & 0.413 & 14.5 & 0.416 & 13.4 & -- \\
\hline 7/25/2009 8:00 AM & 0.169 & 0.181 & 20.9 & 0.120 & 0.328 & 20.2 & 0.37 & 0.043 & 0.050 & 17.0 & 0.041 & 0.415 & 14.5 & 0.417 & 13.4 & -- \\
\hline 7/25/2009 12:00 PM & 0.167 & 0.178 & 20.9 & 0.119 & 0.330 & 20.2 & 0.38 & 0.042 & 0.050 & 17.0 & 0.039 & 0.413 & 14.5 & 0.416 & 13.4 & -- \\
\hline 7/25/2009 4:00 PM & 0.163 & 0.175 & 20.9 & 0.118 & 0.331 & 20.2 & 0.38 & 0.043 & 0.050 & 17.0 & 0.040 & 0.413 & 14.5 & 0.416 & 13.3 & -- \\
\hline 7/25/2009 8:00 PM & 0.168 & 0.172 & 20.9 & 0.118 & 0.331 & 20.2 & 0.38 & 0.043 & 0.049 & 17.0 & 0.040 & 0.413 & 14.5 & 0.416 & 13.3 & -- \\
\hline 7/26/2009 12:00 AM & 0.188 & 0.171 & 20.8 & 0.117 & 0.331 & 20.3 & 0.38 & 0.043 & 0.049 & 17.0 & 0.039 & 0.415 & 14.5 & 0.416 & 13.3 & -- \\
\hline 7/26/2009 4:00 AM & 0.181 & 0.174 & 20.8 & 0.117 & 0.331 & 20.3 & 0.38 & 0.044 & 0.049 & 17.0 & 0.039 & 0.413 & 14.5 & 0.416 & 13.3 & -- \\
\hline 7/26/2009 8:00 AM & 0.177 & 0.175 & 20.8 & 0.118 & 0.331 & 20.3 & 0.38 & 0.044 & 0.049 & 17.0 & 0.039 & 0.413 & 14.5 & 0.416 & 13.4 & -- \\
\hline 7/26/2009 12:00 PM & 0.174 & 0.175 & 20.8 & 0.119 & 0.332 & 20.3 & 0.38 & 0.044 & 0.050 & 17.0 & 0.032 & 0.413 & 14.6 & 0.416 & 13.5 & -- \\
\hline 7/26/2009 4:00 PM & 0.171 & 0.173 & 20.7 & 0.119 & 0.332 & 20.3 & 0.38 & 0.044 & 0.050 & 17.0 & 0.038 & 0.413 & 14.6 & 0.416 & 13.4 & -- \\
\hline 7/26/2009 8:00 PM & 0.169 & 0.171 & 20.6 & 0.118 & 0.332 & 20.3 & 0.38 & 0.045 & 0.050 & 17.0 & 0.039 & 0.413 & 14.6 & 0.416 & 13.4 & -- \\
\hline 7/27/2009 12:00 AM & 0.169 & 0.170 & 20.6 & 0.118 & 0.332 & 20.3 & 0.38 & 0.045 & 0.050 & 17.0 & 0.038 & 0.413 & 14.6 & 0.417 & 13.4 & -- \\
\hline 7/27/2009 4:00 AM & 0.169 & 0.169 & 20.6 & 0.117 & 0.332 & 20.3 & 0.38 & 0.046 & 0.050 & 17.0 & 0.038 & 0.415 & 14.6 & 0.416 & 13.4 & -- \\
\hline 7/27/2009 8:00 AM & 0.168 & 0.167 & 20.7 & 0.116 & 0.332 & 20.3 & 0.38 & 0.046 & 0.051 & 17.1 & 0.039 & 0.415 & 14.6 & 0.416 & 13.4 & -- \\
\hline 7/27/2009 12:00 PM & 0.166 & 0.166 & 20.7 & 0.116 & 0.331 & 20.3 & 0.38 & 0.046 & 0.051 & 17.1 & -- & 0.413 & 14.6 & 0.416 & 13.5 & -- \\
\hline 7/27/2009 4:00 PM & 0.162 & 0.163 & 20.6 & 0.115 & 0.331 & 20.3 & 0.38 & 0.046 & 0.052 & 17.1 & -- & 0.413 & 14.6 & 0.416 & 13.4 & -- \\
\hline 7/27/2009 8:00 PM & 0.160 & 0.162 & 20.6 & 0.115 & 0.331 & 20.3 & 0.38 & 0.046 & 0.051 & 17.1 & 0.039 & 0.413 & 14.6 & 0.416 & 13.5 & -- \\
\hline 7/28/2009 12:00 AM & 0.161 & 0.161 & 20.6 & 0.114 & 0.331 & 20.3 & 0.38 & 0.047 & 0.052 & 17.1 & 0.040 & 0.413 & 14.6 & 0.416 & 13.4 & -- \\
\hline 7/28/2009 4:00 AM & 0.163 & 0.160 & 20.6 & 0.114 & 0.330 & 20.3 & 0.38 & 0.047 & 0.052 & 17.1 & 0.045 & 0.413 & 14.6 & 0.417 & 13.5 & -- \\
\hline 7/28/2009 8:00 AM & 0.165 & 0.158 & 20.5 & 0.113 & 0.330 & 20.3 & 0.38 & 0.047 & 0.052 & 17.1 & 0.045 & 0.413 & 14.6 & 0.417 & 13.5 & -- \\
\hline 7/28/2009 12:00 PM & 0.165 & 0.156 & 20.5 & 0.113 & 0.330 & 20.3 & 0.38 & 0.047 & 0.052 & 17.1 & 0.040 & 0.413 & 14.7 & 0.416 & 13.5 & -- \\
\hline 7/28/2009 4:00 PM & 0.162 & 0.155 & 20.4 & 0.112 & 0.330 & 20.3 & 0.37 & 0.047 & 0.053 & 17.1 & 0.039 & 0.413 & 14.7 & 0.416 & 13.5 & -- \\
\hline 7/28/2009 8:00 PM & 0.160 & 0.154 & 20.4 & 0.111 & 0.329 & 20.3 & 0.37 & 0.047 & 0.053 & 17.1 & 0.040 & 0.413 & 14.7 & 0.417 & 13.6 & -- \\
\hline 7/29/2009 12:00 AM & 0.160 & 0.153 & 20.3 & 0.111 & 0.329 & 20.3 & 0.37 & 0.047 & 0.053 & 17.1 & 0.042 & 0.412 & 14.7 & 0.416 & 13.6 & -- \\
\hline 7/29/2009 4:00 AM & 0.160 & 0.151 & 20.3 & 0.110 & 0.329 & 20.2 & 0.37 & 0.047 & 0.053 & 17.1 & 0.052 & 0.412 & 14.7 & 0.415 & 13.6 & -- \\
\hline 7/29/2009 8:00 AM & 0.159 & 0.150 & 20.3 & 0.109 & 0.328 & 20.2 & 0.37 & 0.046 & 0.053 & 17.1 & 0.053 & 0.412 & 14.7 & 0.415 & 13.6 & -- \\
\hline 7/29/2009 12:00 PM & 0.161 & 0.149 & 20.2 & 0.109 & 0.328 & 20.2 & 0.37 & 0.046 & 0.053 & 17.1 & 0.043 & 0.412 & 14.7 & 0.416 & 13.6 & -- \\
\hline 7/29/2009 4:00 PM & 0.166 & 0.148 & 20.2 & 0.109 & 0.328 & 20.2 & 0.37 & 0.046 & 0.053 & 17.1 & 0.043 & 0.412 & 14.7 & 0.417 & 13.6 & -- \\
\hline 7/29/2009 8:00 PM & 0.194 & 0.147 & 20.1 & 0.109 & 0.328 & 20.2 & 0.37 & 0.046 & 0.053 & 17.1 & 0.056 & 0.412 & 14.7 & 0.417 & 13.6 & -- \\
\hline 7/30/2009 12:00 AM & 0.183 & 0.147 & 20.0 & 0.108 & 0.326 & 20.2 & 0.37 & 0.046 & 0.053 & 17.1 & 0.053 & 0.412 & 14.7 & 0.416 & 13.6 & -- \\
\hline 7/30/2009 4:00 AM & 0.182 & 0.148 & 19.9 & 0.109 & 0.326 & 20.2 & 0.37 & 0.046 & 0.053 & 17.2 & 0.052 & 0.412 & 14.8 & 0.417 & 13.6 & -- \\
\hline 7/30/2009 8:00 AM & 0.178 & 0.151 & 19.8 & 0.109 & 0.326 & 20.2 & 0.37 & 0.046 & 0.053 & 17.2 & 0.052 & 0.412 & 14.7 & 0.416 & 13.6 & -- \\
\hline 7/30/2009 12:00 PM & 0.177 & 0.153 & 19.7 & 0.110 & 0.326 & 20.1 & 0.37 & 0.046 & 0.053 & 17.2 & 0.052 & 0.412 & 14.7 & 0.416 & 13.7 & -- \\
\hline 7/30/2009 4:00 PM & 0.177 & 0.154 & 19.6 & 0.111 & 0.326 & 20.1 & 0.38 & 0.045 & 0.053 & 17.2 & 0.045 & 0.412 & 14.8 & 0.417 & 13.7 & -- \\
\hline 7/30/2009 8:00 PM & 0.175 & 0.154 & 19.4 & 0.112 & 0.326 & 20.1 & 0.38 & 0.045 & 0.053 & 17.2 & 0.048 & 0.412 & 14.8 & 0.416 & 13.7 & -- \\
\hline
\end{tabular}


Appendix 2. Volumetric water content, temperature, and electrical-conductivity data collected at the flood-irrigated site during 2009.-Continued

[Depth in feet below land surface; volumetric water content dimensionless; temperature in degrees Celsius; bulk electrical conductivity in decisiemens per meter; --, no data]

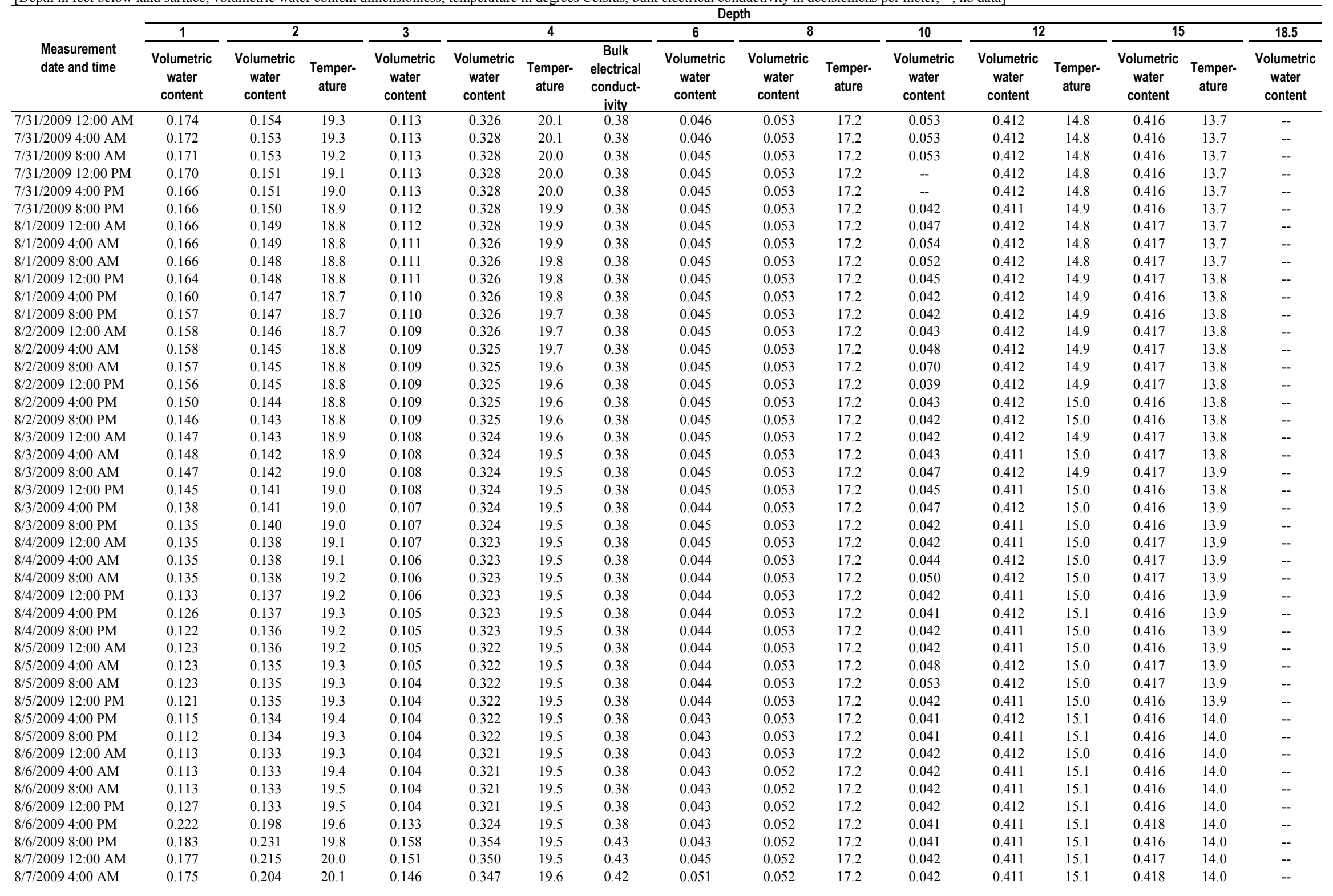


Appendix 2. Volumetric water content, temperature, and electrical-conductivity data collected at the flood-irrigated site during 2009.-Continued

[Depth in feet below land surface; volumetric water content dimensionless; temperature in degrees Celsius; bulk electrical conductivity in decisiemens per meter; --, no data]

\begin{tabular}{|c|c|c|c|c|c|c|c|c|c|c|c|c|c|c|c|c|}
\hline \multirow[b]{3}{*}{$\begin{array}{l}\text { Measurement } \\
\text { date and time }\end{array}$} & \multicolumn{16}{|c|}{ Depth } \\
\hline & \multirow{2}{*}{$\begin{array}{c}1 \\
\text { Volumetric } \\
\text { water } \\
\text { content }\end{array}$} & \multicolumn{2}{|c|}{2} & \multirow{2}{*}{$\begin{array}{c}3 \\
\text { Volumetric } \\
\text { water } \\
\text { content }\end{array}$} & \multicolumn{3}{|c|}{4} & \multirow{2}{*}{$\begin{array}{c}6 \\
\text { Volumetric } \\
\text { water } \\
\text { content }\end{array}$} & \multicolumn{2}{|c|}{8} & \multirow{2}{*}{$\begin{array}{c}10 \\
\text { Volumetric } \\
\text { water } \\
\text { content }\end{array}$} & \multicolumn{2}{|c|}{12} & \multicolumn{2}{|c|}{15} & \multirow{2}{*}{$\begin{array}{c}18.5 \\
\text { Volumetric } \\
\text { water } \\
\text { content }\end{array}$} \\
\hline & & $\begin{array}{c}\text { Volumetric } \\
\text { water } \\
\text { content }\end{array}$ & $\begin{array}{l}\text { Temper- } \\
\text { ature }\end{array}$ & & $\begin{array}{c}\text { Volumetric } \\
\text { water } \\
\text { content }\end{array}$ & $\begin{array}{l}\text { Temper- } \\
\text { ature }\end{array}$ & $\begin{array}{l}\text { Bulk } \\
\text { electrical } \\
\text { conduct- } \\
\text { ivity } \\
\end{array}$ & & $\begin{array}{l}\text { Volumetric } \\
\text { water } \\
\text { content }\end{array}$ & $\begin{array}{l}\text { Temper- } \\
\text { ature }\end{array}$ & & $\begin{array}{l}\text { Volumetric } \\
\text { water } \\
\text { content }\end{array}$ & $\begin{array}{l}\text { Temper- } \\
\text { ature }\end{array}$ & $\begin{array}{c}\text { Volumetric } \\
\text { water } \\
\text { content }\end{array}$ & $\begin{array}{l}\text { Temper- } \\
\text { ature }\end{array}$ & \\
\hline 8/7/2009 8:00 AM & 0.172 & 0.195 & 20.1 & 0.142 & 0.344 & 19.6 & 0.41 & 0.057 & 0.053 & 17.2 & 0.042 & 0.411 & 15.1 & 0.417 & 14.0 & -- \\
\hline 8/7/2009 12:00 PM & 0.171 & 0.187 & 20.1 & 0.138 & 0.343 & 19.6 & 0.41 & 0.058 & 0.053 & 17.2 & 0.042 & 0.411 & 15.1 & 0.416 & 14.0 & -- \\
\hline 8/7/2009 4:00 PM & 0.166 & 0.182 & 20.1 & 0.135 & 0.341 & 19.7 & 0.41 & 0.058 & 0.054 & 17.2 & 0.041 & 0.411 & 15.1 & 0.416 & 14.0 & -- \\
\hline 8/7/2009 8:00 PM & 0.162 & 0.178 & 20.0 & 0.132 & 0.340 & 19.7 & 0.41 & 0.058 & 0.056 & 17.2 & 0.040 & 0.411 & 15.2 & 0.416 & 14.0 & -- \\
\hline 8/8/2009 12:00 AM & 0.162 & 0.173 & 20.1 & 0.131 & 0.338 & 19.7 & 0.40 & 0.058 & 0.057 & 17.2 & 0.042 & 0.411 & 15.1 & 0.416 & 14.1 & -- \\
\hline 8/8/2009 4:00 AM & 0.162 & 0.170 & 20.1 & 0.128 & 0.336 & 19.7 & 0.40 & 0.057 & 0.058 & 17.2 & 0.043 & 0.411 & 15.1 & 0.417 & 14.1 & -- \\
\hline 8/8/2009 8:00 AM & 0.161 & 0.167 & 20.1 & 0.126 & 0.336 & 19.7 & 0.40 & 0.057 & 0.058 & 17.2 & 0.047 & 0.412 & 15.1 & 0.417 & 14.0 & -- \\
\hline 8/8/2009 12:00 PM & 0.160 & 0.165 & 20.1 & 0.125 & 0.335 & 19.8 & 0.40 & 0.056 & 0.059 & 17.2 & 0.044 & 0.411 & 15.2 & 0.418 & 14.1 & -- \\
\hline 8/8/2009 4:00 PM & 0.154 & 0.162 & 20.1 & 0.123 & 0.334 & 19.8 & 0.40 & 0.055 & 0.059 & 17.2 & 0.045 & 0.411 & 15.2 & 0.416 & 14.1 & -- \\
\hline 8/8/2009 8:00 PM & 0.150 & 0.159 & 20.0 & 0.122 & 0.334 & 19.8 & 0.40 & 0.055 & 0.060 & 17.2 & 0.047 & 0.411 & 15.2 & 0.416 & 14.1 & -- \\
\hline 8/9/2009 12:00 AM & 0.179 & 0.158 & 20.0 & 0.121 & 0.332 & 19.8 & 0.40 & 0.054 & 0.059 & 17.2 & 0.056 & 0.411 & 15.2 & 0.416 & 14.2 & _- \\
\hline 8/9/2009 4:00 AM & 0.202 & 0.156 & 20.0 & 0.120 & 0.332 & 19.8 & 0.40 & 0.053 & 0.059 & 17.2 & 0.059 & 0.412 & 15.2 & 0.416 & 14.2 & -- \\
\hline 8/9/2009 8:00 AM & 0.192 & 0.156 & 20.0 & 0.119 & 0.331 & 19.8 & 0.40 & 0.053 & 0.059 & 17.2 & 0.059 & 0.412 & 15.2 & 0.416 & 14.2 & -- \\
\hline 8/9/2009 12:00 PM & 0.183 & 0.158 & 19.9 & 0.120 & 0.331 & 19.8 & 0.40 & 0.053 & 0.059 & 17.2 & 0.054 & 0.412 & 15.2 & 0.416 & 14.2 & -- \\
\hline 8/9/2009 4:00 PM & 0.176 & 0.159 & 19.8 & 0.121 & 0.331 & 19.8 & 0.40 & 0.052 & 0.059 & 17.2 & 0.050 & 0.411 & 15.2 & 0.416 & 14.1 & -- \\
\hline 8/9/2009 8:00 PM & 0.172 & 0.159 & 19.7 & 0.121 & 0.331 & 19.8 & 0.40 & 0.052 & 0.059 & 17.2 & 0.050 & 0.411 & 15.2 & 0.416 & 14.1 & -- \\
\hline 8/10/2009 12:00 AM & 0.171 & 0.158 & 19.6 & 0.121 & 0.331 & 19.8 & 0.40 & 0.052 & 0.059 & 17.2 & 0.051 & 0.412 & 15.2 & 0.416 & 14.2 & -- \\
\hline 8/10/2009 4:00 AM & 0.171 & 0.157 & 19.7 & 0.120 & 0.331 & 19.8 & 0.40 & 0.051 & 0.058 & 17.2 & 0.054 & 0.412 & 15.2 & 0.417 & 14.2 & -- \\
\hline 8/10/2009 8:00 AM & 0.169 & 0.156 & 19.6 & 0.120 & 0.331 & 19.8 & 0.40 & 0.051 & 0.058 & 17.3 & 0.060 & 0.412 & 15.2 & 0.417 & 14.2 & -- \\
\hline 8/10/2009 12:00 PM & 0.167 & 0.155 & 19.6 & 0.119 & 0.331 & 19.8 & 0.40 & 0.051 & 0.059 & 17.3 & 0.052 & 0.412 & 15.2 & 0.417 & 14.2 & -- \\
\hline 8/10/2009 4:00 PM & 0.162 & 0.154 & 19.6 & 0.118 & 0.331 & 19.8 & 0.40 & 0.050 & 0.059 & 17.3 & 0.050 & 0.411 & 15.3 & 0.417 & 14.2 & -- \\
\hline 8/10/2009 8:00 PM & 0.159 & 0.153 & 19.5 & 0.118 & 0.330 & 19.8 & 0.40 & 0.050 & 0.058 & 17.3 & 0.050 & 0.411 & 15.2 & 0.416 & 14.2 & -- \\
\hline 8/11/2009 12:00 AM & 0.160 & 0.151 & 19.5 & 0.117 & 0.330 & 19.8 & 0.39 & 0.050 & 0.058 & 17.3 & 0.051 & 0.412 & 15.3 & 0.417 & 14.2 & -- \\
\hline 8/11/2009 4:00 AM & 0.159 & 0.150 & 19.5 & 0.116 & 0.329 & 19.8 & 0.39 & 0.050 & 0.058 & 17.3 & 0.058 & 0.412 & 15.3 & 0.417 & 14.2 & -- \\
\hline 8/11/2009 8:00 AM & 0.159 & 0.149 & 19.6 & 0.116 & 0.329 & 19.8 & 0.39 & 0.050 & 0.058 & 17.3 & 0.060 & 0.412 & 15.3 & 0.417 & 14.3 & -- \\
\hline 8/11/2009 12:00 PM & 0.157 & 0.148 & 19.6 & 0.115 & 0.329 & 19.8 & 0.39 & 0.050 & 0.058 & 17.3 & 0.051 & 0.411 & 15.3 & 0.417 & 14.2 & -- \\
\hline 8/11/2009 4:00 PM & 0.151 & 0.147 & 19.5 & 0.115 & 0.329 & 19.8 & 0.39 & 0.049 & 0.058 & 17.3 & 0.048 & 0.411 & 15.3 & 0.417 & 14.2 & -- \\
\hline 8/11/2009 8:00 PM & 0.147 & 0.147 & 19.5 & 0.115 & 0.328 & 19.7 & 0.39 & 0.049 & 0.058 & 17.3 & 0.048 & 0.411 & 15.3 & 0.417 & 14.2 & -- \\
\hline 8/12/2009 12:00 AM & 0.147 & 0.145 & 19.5 & 0.114 & 0.328 & 19.7 & 0.39 & 0.049 & 0.058 & 17.3 & 0.049 & 0.412 & 15.3 & 0.417 & 14.2 & -- \\
\hline 8/12/2009 4:00 AM & 0.147 & 0.145 & 19.5 & 0.113 & 0.326 & 19.7 & 0.39 & 0.048 & 0.058 & 17.3 & 0.052 & 0.412 & 15.3 & 0.417 & 14.2 & -- \\
\hline 8/12/2009 8:00 AM & 0.147 & 0.144 & 19.6 & 0.113 & 0.326 & 19.7 & 0.39 & 0.048 & 0.058 & 17.3 & 0.060 & 0.411 & 15.3 & 0.417 & 14.3 & -- \\
\hline 8/12/2009 12:00 PM & 0.145 & 0.144 & 19.6 & 0.112 & 0.326 & 19.7 & 0.39 & 0.048 & 0.058 & 17.3 & 0.050 & 0.411 & 15.3 & 0.417 & 14.3 & -- \\
\hline 8/12/2009 4:00 PM & 0.138 & 0.143 & 19.5 & 0.111 & 0.326 & 19.7 & 0.39 & 0.048 & 0.058 & 17.3 & 0.049 & 0.411 & 15.3 & 0.417 & 14.3 & -- \\
\hline 8/12/2009 8:00 PM & 0.132 & 0.142 & 19.5 & 0.111 & 0.325 & 19.7 & 0.39 & 0.047 & 0.057 & 17.3 & 0.048 & 0.411 & 15.3 & 0.417 & 14.3 & -- \\
\hline 8/13/2009 12:00 AM & 0.133 & 0.141 & 19.5 & 0.111 & 0.325 & 19.7 & 0.39 & 0.047 & 0.057 & 17.3 & 0.048 & 0.411 & 15.3 & 0.417 & 14.3 & -- \\
\hline 8/13/2009 4:00 AM & 0.133 & 0.141 & 19.6 & 0.110 & 0.325 & 19.7 & 0.39 & 0.047 & 0.056 & 17.3 & 0.051 & 0.411 & 15.3 & 0.417 & 14.3 & -- \\
\hline 8/13/2009 8:00 AM & 0.133 & 0.141 & 19.6 & 0.110 & 0.324 & 19.7 & 0.39 & 0.047 & 0.056 & 17.3 & 0.058 & 0.411 & 15.3 & 0.417 & 14.3 & -- \\
\hline 8/13/2009 12:00 PM & 0.132 & 0.140 & 19.6 & 0.110 & 0.324 & 19.7 & 0.39 & 0.047 & 0.056 & 17.3 & 0.047 & 0.411 & 15.3 & 0.417 & 14.3 & -- \\
\hline 8/13/2009 4:00 PM & 0.126 & 0.140 & 19.6 & 0.109 & 0.324 & 19.7 & 0.39 & 0.047 & 0.057 & 17.3 & 0.047 & 0.410 & 15.4 & 0.417 & 14.3 & -- \\
\hline 8/13/2009 8:00 PM & 0.122 & 0.138 & 19.5 & 0.109 & 0.324 & 19.7 & 0.39 & 0.047 & 0.056 & 17.3 & 0.047 & 0.410 & 15.4 & 0.417 & 14.3 & -- \\
\hline 8/14/2009 12:00 AM & 0.123 & 0.137 & 19.6 & 0.109 & 0.324 & 19.7 & 0.39 & 0.047 & 0.056 & 17.3 & 0.047 & 0.411 & 15.4 & 0.417 & 14.3 & -- \\
\hline 8/14/2009 4:00 AM & 0.123 & 0.137 & 19.6 & 0.109 & 0.323 & 19.7 & 0.39 & 0.047 & 0.055 & 17.3 & 0.047 & 0.411 & 15.4 & 0.417 & 14.4 & -- \\
\hline 8/14/2009 8:00 AM & 0.123 & 0.137 & 19.6 & 0.108 & 0.323 & 19.7 & 0.39 & 0.046 & 0.055 & 17.3 & 0.047 & 0.411 & 15.4 & 0.417 & 14.4 & -- \\
\hline 8/14/2009 12:00 PM & 0.121 & 0.136 & 19.6 & 0.108 & 0.323 & 19.7 & 0.39 & 0.046 & 0.055 & 17.3 & 0.046 & 0.411 & 15.4 & 0.417 & 14.3 & -- \\
\hline
\end{tabular}


Appendix 2. Volumetric water content, temperature, and electrical-conductivity data collected at the flood-irrigated site during 2009.-Continued

[Depth in feet below land surface; volumetric water content dimensionless; temperature in degrees Celsius; bulk electrical conductivity in decisiemens per meter; --, no data]

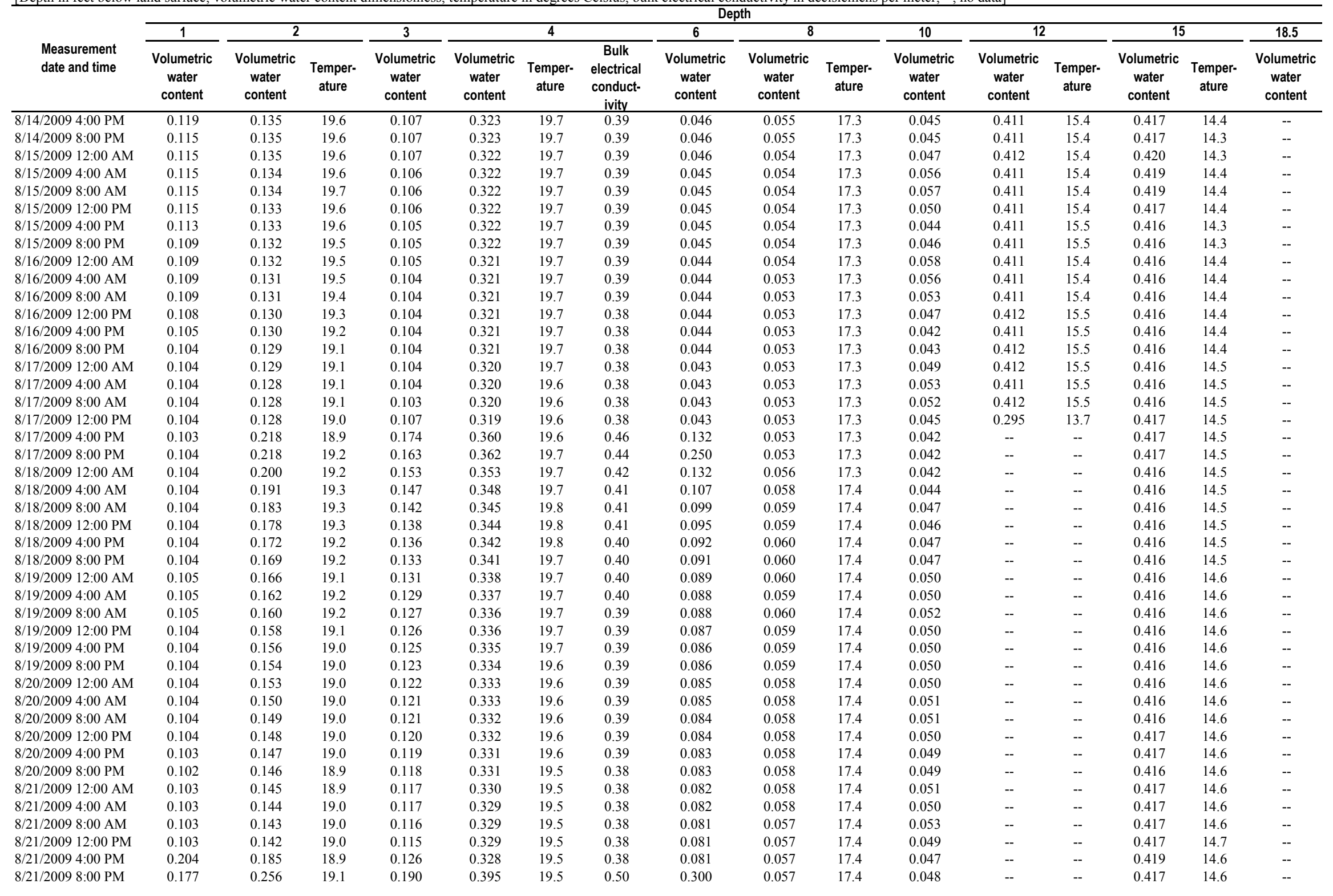


Appendix 2. Volumetric water content, temperature, and electrical-conductivity data collected at the flood-irrigated site during 2009.-Continued

[Depth in feet below land surface; volumetric water content dimensionless; temperature in degrees Celsius; bulk electrical conductivity in decisiemens per meter; --, no data]

\begin{tabular}{|c|c|c|c|c|c|c|c|c|c|c|c|c|c|c|c|c|}
\hline \multirow[b]{3}{*}{$\begin{array}{l}\text { Measurement } \\
\text { date and time }\end{array}$} & \multicolumn{16}{|c|}{ Depth } \\
\hline & \multirow{2}{*}{$\begin{array}{c}1 \\
\text { Volumetric } \\
\text { water } \\
\text { content }\end{array}$} & \multicolumn{2}{|c|}{2} & \multirow{2}{*}{$\begin{array}{c}3 \\
\text { Volumetric } \\
\text { water } \\
\text { content }\end{array}$} & \multicolumn{3}{|c|}{4} & \multirow{2}{*}{$\begin{array}{c}6 \\
\text { Volumetric } \\
\text { water } \\
\text { content }\end{array}$} & \multicolumn{2}{|c|}{8} & \multirow{2}{*}{$\begin{array}{c}10 \\
\text { Volumetric } \\
\text { water } \\
\text { content }\end{array}$} & \multicolumn{2}{|c|}{12} & \multicolumn{2}{|c|}{15} & \multirow{2}{*}{$\begin{array}{c}18.5 \\
\text { Volumetric } \\
\text { water } \\
\text { content }\end{array}$} \\
\hline & & $\begin{array}{c}\text { Volumetric } \\
\text { water } \\
\text { content }\end{array}$ & $\begin{array}{l}\text { Temper- } \\
\text { ature }\end{array}$ & & $\begin{array}{c}\text { Volumetric } \\
\text { water } \\
\text { content }\end{array}$ & $\begin{array}{l}\text { Temper- } \\
\text { ature }\end{array}$ & $\begin{array}{c}\text { Bulk } \\
\text { electrical } \\
\text { conduct- } \\
\text { ivity }\end{array}$ & & $\begin{array}{c}\text { Volumetric } \\
\text { water } \\
\text { content }\end{array}$ & $\begin{array}{l}\text { Temper- } \\
\text { ature }\end{array}$ & & $\begin{array}{c}\text { Volumetric } \\
\text { water } \\
\text { content }\end{array}$ & $\begin{array}{l}\text { Temper- } \\
\text { ature }\end{array}$ & $\begin{array}{c}\text { Volumetric } \\
\text { water } \\
\text { content }\end{array}$ & $\begin{array}{l}\text { Temper- } \\
\text { ature }\end{array}$ & \\
\hline 8/22/2009 12:00 AM & 0.171 & 0.229 & 19.6 & 0.170 & 0.371 & 19.6 & 0.44 & 0.392 & 0.057 & 17.4 & 0.048 & -- & -- & 0.417 & 14.6 & -- \\
\hline 8/22/2009 4:00 AM & 0.169 & 0.211 & 19.8 & 0.160 & 0.362 & 19.6 & 0.42 & 0.269 & 0.060 & 17.4 & 0.048 & -- & -- & 0.417 & 14.7 & -- \\
\hline 8/22/2009 8:00 AM & 0.166 & 0.198 & 19.8 & 0.153 & 0.358 & 19.7 & 0.41 & 0.185 & 0.063 & 17.4 & 0.050 & -- & -- & 0.417 & 14.6 & -- \\
\hline 8/22/2009 12:00 PM & 0.164 & 0.190 & 19.8 & 0.149 & 0.354 & 19.7 & 0.40 & 0.161 & 0.064 & 17.5 & 0.051 & -- & -- & 0.417 & 14.7 & -- \\
\hline 8/22/2009 4:00 PM & 0.160 & 0.183 & 19.7 & 0.144 & 0.351 & 19.7 & 0.40 & 0.151 & 0.066 & 17.5 & 0.053 & -- & -- & 0.417 & 14.7 & -- \\
\hline 8/22/2009 8:00 PM & 0.157 & 0.178 & 19.7 & 0.141 & 0.349 & 19.7 & 0.40 & 0.145 & 0.066 & 17.5 & 0.056 & -- & -- & 0.417 & 14.6 & -- \\
\hline 8/23/2009 12:00 AM & 0.157 & 0.172 & 19.6 & 0.138 & 0.347 & 19.7 & 0.39 & 0.140 & 0.065 & 17.5 & 0.056 & -- & -- & 0.417 & 14.7 & -- \\
\hline 8/23/2009 4:00 AM & 0.156 & 0.169 & 19.7 & 0.136 & 0.345 & 19.7 & 0.39 & 0.137 & 0.065 & 17.5 & 0.057 & -- & -- & 0.417 & 14.7 & -- \\
\hline 8/23/2009 8:00 AM & 0.155 & 0.166 & 19.7 & 0.134 & 0.344 & 19.7 & 0.39 & 0.134 & 0.065 & 17.5 & 0.057 & -- & -- & 0.417 & 14.7 & -- \\
\hline 8/23/2009 12:00 PM & 0.155 & 0.162 & 19.7 & 0.132 & 0.343 & 19.7 & 0.39 & 0.132 & 0.065 & 17.5 & 0.057 & -- & -- & 0.417 & 14.7 & -- \\
\hline 8/23/2009 4:00 PM & 0.151 & 0.160 & 19.7 & 0.130 & 0.342 & 19.7 & 0.39 & 0.131 & 0.065 & 17.5 & 0.057 & -- & -- & 0.417 & 14.8 & -- \\
\hline 8/23/2009 8:00 PM & 0.149 & 0.158 & 19.7 & 0.129 & 0.341 & 19.7 & 0.39 & 0.129 & 0.064 & 17.5 & 0.057 & -- & -- & 0.417 & 14.8 & -- \\
\hline 8/24/2009 12:00 AM & 0.149 & 0.156 & 19.7 & 0.127 & 0.340 & 19.7 & 0.38 & 0.127 & 0.064 & 17.5 & 0.056 & -- & -- & 0.418 & 14.8 & -- \\
\hline 8/24/2009 4:00 AM & 0.149 & 0.155 & 19.8 & 0.126 & 0.338 & 19.7 & 0.38 & 0.126 & 0.064 & 17.5 & 0.056 & -- & -- & 0.418 & 14.8 & -- \\
\hline 8/24/2009 8:00 AM & 0.149 & 0.153 & 19.9 & 0.126 & 0.337 & 19.7 & 0.38 & 0.125 & 0.064 & 17.5 & 0.058 & -- & -- & 0.418 & 14.8 & -- \\
\hline 8/24/2009 12:00 PM & 0.148 & 0.151 & 19.9 & 0.125 & 0.337 & 19.7 & 0.38 & 0.124 & 0.063 & 17.5 & 0.055 & -- & -- & 0.418 & 14.8 & -- \\
\hline 8/24/2009 4:00 PM & 0.144 & 0.150 & 19.8 & 0.123 & 0.336 & 19.7 & 0.38 & 0.123 & 0.063 & 17.5 & 0.054 & -- & -- & 0.418 & 14.8 & -- \\
\hline 8/24/2009 8:00 PM & 0.143 & 0.149 & 19.8 & 0.123 & 0.335 & 19.7 & 0.38 & 0.121 & 0.062 & 17.5 & 0.053 & -- & -- & 0.418 & 14.8 & -- \\
\hline 8/25/2009 12:00 AM & 0.143 & 0.148 & 19.8 & 0.122 & 0.335 & 19.7 & 0.38 & 0.121 & 0.062 & 17.5 & 0.053 & -- & -- & 0.418 & 14.8 & -- \\
\hline 8/25/2009 4:00 AM & 0.143 & 0.147 & 19.8 & 0.121 & 0.334 & 19.7 & 0.38 & 0.120 & 0.062 & 17.5 & 0.054 & -- & -- & 0.419 & 14.8 & -- \\
\hline 8/25/2009 8:00 AM & 0.143 & 0.146 & 19.8 & 0.121 & 0.334 & 19.7 & 0.38 & 0.120 & 0.061 & 17.5 & 0.053 & -- & -- & 0.419 & 14.9 & -- \\
\hline 8/25/2009 12:00 PM & 0.142 & 0.145 & 19.8 & 0.120 & 0.333 & 19.7 & 0.38 & 0.119 & 0.061 & 17.5 & 0.052 & -- & -- & 0.419 & 14.8 & -- \\
\hline 8/25/2009 4:00 PM & 0.138 & 0.144 & 19.7 & 0.120 & 0.333 & 19.7 & 0.38 & 0.118 & 0.061 & 17.5 & 0.051 & -- & -- & 0.418 & 14.9 & -- \\
\hline 8/25/2009 8:00 PM & 0.138 & 0.143 & 19.6 & 0.119 & 0.333 & 19.7 & 0.38 & 0.117 & 0.060 & 17.5 & 0.051 & -- & -- & 0.418 & 14.9 & -- \\
\hline 8/26/2009 12:00 AM & 0.138 & 0.142 & 19.6 & 0.118 & 0.332 & 19.7 & 0.38 & 0.116 & 0.060 & 17.5 & 0.052 & -- & -- & 0.419 & 14.9 & -- \\
\hline 8/26/2009 4:00 AM & 0.138 & 0.142 & 19.7 & 0.118 & 0.332 & 19.7 & 0.38 & 0.116 & 0.059 & 17.5 & 0.053 & -- & -- & 0.418 & 14.9 & -- \\
\hline 8/26/2009 8:00 AM & 0.138 & 0.141 & 19.7 & 0.117 & 0.331 & 19.7 & 0.38 & 0.115 & 0.059 & 17.5 & 0.052 & -- & -- & 0.418 & 14.9 & -- \\
\hline 8/26/2009 12:00 PM & 0.137 & 0.140 & 19.6 & 0.117 & 0.331 & 19.7 & 0.38 & 0.115 & 0.059 & 17.5 & 0.050 & -- & -- & 0.419 & 14.9 & -- \\
\hline 8/26/2009 4:00 PM & 0.133 & 0.140 & 19.5 & 0.116 & 0.331 & 19.7 & 0.38 & 0.115 & 0.059 & 17.5 & 0.048 & -- & -- & 0.418 & 14.9 & -- \\
\hline 8/26/2009 8:00 PM & 0.131 & 0.138 & 19.5 & 0.115 & 0.331 & 19.7 & 0.38 & 0.115 & 0.059 & 17.5 & 0.048 & -- & -- & 0.418 & 14.9 & -- \\
\hline 8/27/2009 12:00 AM & 0.131 & 0.137 & 19.5 & 0.115 & 0.330 & 19.7 & 0.38 & 0.114 & 0.058 & 17.5 & 0.050 & -- & -- & 0.418 & 14.9 & -- \\
\hline 8/27/2009 4:00 AM & 0.131 & 0.136 & 19.5 & 0.115 & 0.330 & 19.7 & 0.38 & 0.113 & 0.058 & 17.5 & 0.048 & -- & -- & 0.418 & 14.9 & -- \\
\hline 8/27/2009 8:00 AM & 0.130 & 0.136 & 19.5 & 0.115 & 0.329 & 19.7 & 0.38 & 0.113 & 0.058 & 17.5 & 0.049 & -- & -- & 0.418 & 14.9 & -- \\
\hline 8/27/2009 12:00 PM & 0.203 & 0.167 & 19.4 & 0.143 & 0.335 & 19.7 & 0.39 & 0.112 & 0.058 & 17.5 & 0.048 & -- & -- & 0.418 & 14.9 & -- \\
\hline 8/27/2009 4:00 PM & 0.275 & 0.258 & 19.4 & 0.188 & 0.408 & 19.7 & 0.51 & 0.204 & 0.058 & 17.5 & 0.048 & -- & -- & 0.418 & 14.8 & -- \\
\hline 8/27/2009 8:00 PM & 0.182 & 0.244 & 19.3 & 0.179 & 0.408 & 19.8 & 0.49 & 0.386 & 0.058 & 17.5 & 0.047 & -- & -- & 0.417 & 14.9 & -- \\
\hline 8/28/2009 12:00 AM & 0.173 & 0.222 & 19.5 & 0.166 & 0.371 & 19.8 & 0.43 & 0.384 & 0.064 & 17.5 & 0.048 & -- & -- & 0.417 & 14.9 & -- \\
\hline 8/28/2009 4:00 AM & 0.171 & 0.207 & 19.5 & 0.157 & 0.365 & 19.8 & 0.41 & 0.266 & 0.072 & 17.5 & 0.049 & -- & -- & 0.418 & 14.9 & -- \\
\hline 8/28/2009 8:00 AM & 0.169 & 0.196 & 19.6 & 0.151 & 0.358 & 19.8 & 0.40 & 0.194 & 0.075 & 17.5 & 0.057 & -- & -- & 0.418 & 14.9 & -- \\
\hline 8/28/2009 12:00 PM & 0.166 & 0.188 & 19.5 & 0.147 & 0.355 & 19.8 & 0.40 & 0.173 & 0.074 & 17.6 & 0.059 & -- & -- & 0.418 & 14.9 & -- \\
\hline 8/28/2009 4:00 PM & 0.162 & 0.182 & 19.5 & 0.143 & 0.351 & 19.8 & 0.39 & 0.163 & 0.074 & 17.6 & 0.060 & -- & -- & 0.417 & 14.9 & -- \\
\hline 8/28/2009 8:00 PM & 0.160 & 0.176 & 19.4 & 0.140 & 0.349 & 19.8 & 0.39 & 0.157 & 0.073 & 17.6 & 0.060 & -- & -- & 0.417 & 14.9 & -- \\
\hline 8/29/2009 12:00 AM & 0.159 & 0.172 & 19.4 & 0.138 & 0.347 & 19.8 & 0.39 & 0.152 & 0.071 & 17.6 & 0.062 & -- & -- & 0.417 & 14.9 & -- \\
\hline 8/29/2009 4:00 AM & 0.159 & 0.169 & 19.4 & 0.135 & 0.346 & 19.8 & 0.39 & 0.149 & 0.070 & 17.6 & 0.063 & -- & -- & 0.417 & 14.9 & -- \\
\hline
\end{tabular}


Appendix 2. Volumetric water content, temperature, and electrical-conductivity data collected at the flood-irrigated site during 2009.-Continued

[Depth in feet below land surface; volumetric water content dimensionless; temperature in degrees Celsius; bulk electrical conductivity in decisiemens per meter; --, no data]

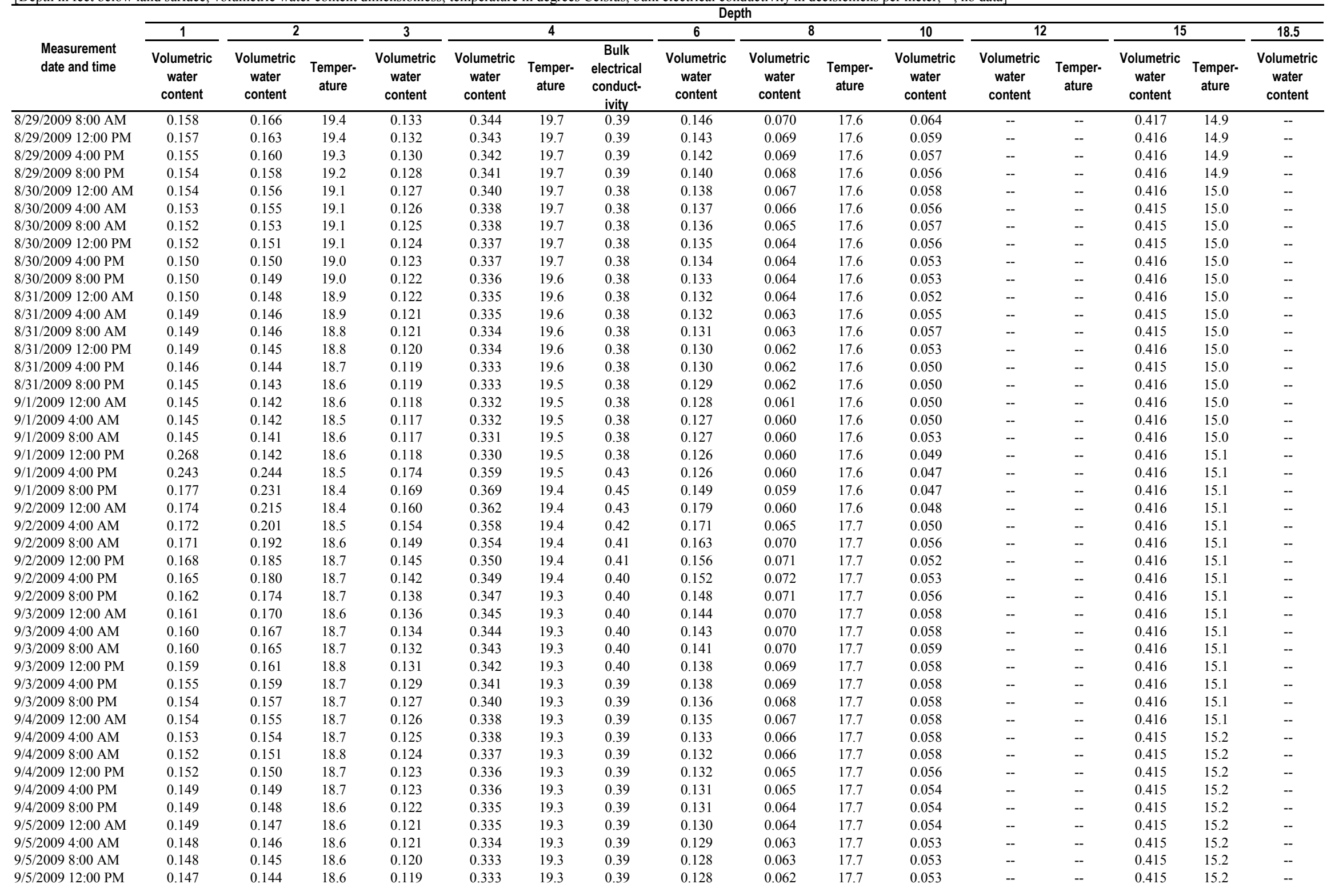


Appendix 2. Volumetric water content, temperature, and electrical-conductivity data collected at the flood-irrigated site during 2009.-Continued

[Depth in feet below land surface; volumetric water content dimensionless; temperature in degrees Celsius; bulk electrical conductivity in decisiemens per meter; --, no data]

\begin{tabular}{|c|c|c|c|c|c|c|c|c|c|c|c|c|c|c|c|c|}
\hline \multirow{3}{*}{$\begin{array}{l}\text { Measurement } \\
\text { date and time }\end{array}$} & \multicolumn{16}{|c|}{ Depth } \\
\hline & \multirow{2}{*}{$\begin{array}{c}1 \\
\text { Volumetric } \\
\text { water } \\
\text { content }\end{array}$} & \multicolumn{2}{|c|}{2} & \multirow{2}{*}{$\begin{array}{c}3 \\
\text { Volumetric } \\
\text { water } \\
\text { content }\end{array}$} & \multicolumn{3}{|c|}{4} & \multirow{2}{*}{$\begin{array}{c}6 \\
\text { Volumetric } \\
\text { water } \\
\text { content }\end{array}$} & \multicolumn{2}{|c|}{8} & \multirow{2}{*}{$\begin{array}{c}10 \\
\text { Volumetric } \\
\text { water } \\
\text { content }\end{array}$} & \multicolumn{2}{|c|}{12} & \multicolumn{2}{|c|}{15} & \multirow{2}{*}{$\begin{array}{c}18.5 \\
\text { Volumetric } \\
\text { water } \\
\text { content }\end{array}$} \\
\hline & & $\begin{array}{c}\text { Volumetric } \\
\text { water } \\
\text { content }\end{array}$ & $\begin{array}{l}\text { Temper- } \\
\text { ature }\end{array}$ & & $\begin{array}{c}\text { Volumetric } \\
\text { water } \\
\text { content }\end{array}$ & $\begin{array}{l}\text { Temper- } \\
\text { ature }\end{array}$ & $\begin{array}{c}\text { Bulk } \\
\text { electrical } \\
\text { conduct- } \\
\text { ivity } \\
\end{array}$ & & $\begin{array}{c}\text { Volumetric } \\
\text { water } \\
\text { content }\end{array}$ & $\begin{array}{l}\text { Temper- } \\
\text { ature }\end{array}$ & & $\begin{array}{l}\text { Volumetric } \\
\text { water } \\
\text { content }\end{array}$ & $\begin{array}{l}\text { Temper- } \\
\text { ature }\end{array}$ & $\begin{array}{c}\text { Volumetric } \\
\text { water } \\
\text { content }\end{array}$ & $\begin{array}{l}\text { Temper- } \\
\text { ature }\end{array}$ & \\
\hline 9/5/2009 4:00 PM & 0.145 & 0.143 & 18.6 & 0.119 & 0.333 & 19.2 & 0.39 & 0.127 & 0.062 & 17.7 & 0.051 & -- & -- & 0.415 & 15.2 & -- \\
\hline 9/5/2009 8:00 PM & 0.143 & 0.142 & 18.5 & 0.118 & 0.332 & 19.2 & 0.39 & 0.127 & 0.062 & 17.7 & 0.051 & -- & -- & 0.415 & 15.3 & -- \\
\hline 9/6/2009 12:00 AM & 0.143 & 0.142 & 18.5 & 0.118 & 0.332 & 19.2 & 0.39 & 0.126 & 0.061 & 17.7 & 0.053 & -- & -- & 0.416 & 15.2 & -- \\
\hline 9/6/2009 4:00 AM & 0.143 & 0.141 & 18.5 & 0.117 & 0.331 & 19.2 & 0.39 & 0.126 & 0.061 & 17.7 & 0.051 & -- & -- & 0.416 & 15.3 & -- \\
\hline 9/6/2009 8:00 AM & 0.143 & 0.140 & 18.6 & 0.116 & 0.331 & 19.2 & 0.39 & 0.126 & 0.060 & 17.7 & 0.052 & -- & -- & 0.416 & 15.3 & -- \\
\hline 9/6/2009 12:00 PM & 0.142 & 0.140 & 18.6 & 0.116 & 0.331 & 19.2 & 0.39 & 0.126 & 0.060 & 17.7 & 0.050 & -- & -- & 0.416 & 15.3 & -- \\
\hline 9/6/2009 4:00 PM & 0.140 & 0.138 & 18.5 & 0.115 & 0.330 & 19.2 & 0.38 & 0.125 & 0.060 & 17.7 & 0.048 & -- & -- & 0.416 & 15.3 & -- \\
\hline 9/6/2009 8:00 PM & 0.138 & 0.137 & 18.5 & 0.115 & 0.330 & 19.2 & 0.38 & 0.125 & 0.060 & 17.7 & 0.048 & -- & -- & 0.416 & 15.3 & -- \\
\hline 9/7/2009 12:00 AM & 0.138 & 0.137 & 18.4 & 0.115 & 0.330 & 19.2 & 0.38 & 0.124 & 0.059 & 17.7 & 0.049 & -- & -- & 0.415 & 15.3 & -- \\
\hline 9/7/2009 4:00 AM & 0.138 & 0.136 & 18.5 & 0.115 & 0.330 & 19.2 & 0.38 & 0.124 & 0.058 & 17.7 & 0.050 & -- & -- & 0.415 & 15.3 & -- \\
\hline 9/7/2009 8:00 AM & 0.138 & 0.136 & 18.5 & 0.114 & 0.329 & 19.2 & 0.38 & 0.124 & 0.058 & 17.7 & 0.051 & -- & -- & 0.415 & 15.3 & -- \\
\hline 9/7/2009 12:00 PM & 0.137 & 0.135 & 18.5 & 0.114 & 0.329 & 19.2 & 0.38 & 0.124 & 0.058 & 17.7 & 0.048 & -- & -- & 0.416 & 15.3 & -- \\
\hline 9/7/2009 4:00 PM & 0.133 & 0.135 & 18.5 & 0.114 & 0.329 & 19.2 & 0.38 & 0.123 & 0.058 & 17.7 & 0.049 & -- & -- & 0.416 & 15.3 & -- \\
\hline 9/7/2009 8:00 PM & 0.131 & 0.134 & 18.5 & 0.113 & 0.329 & 19.2 & 0.38 & 0.123 & 0.058 & 17.6 & 0.048 & -- & -- & 0.416 & 15.3 & -- \\
\hline 9/8/2009 12:00 AM & 0.130 & 0.133 & 18.5 & 0.113 & 0.328 & 19.1 & 0.38 & 0.123 & 0.058 & 17.6 & 0.048 & -- & -- & 0.416 & 15.3 & -- \\
\hline 9/8/2009 4:00 AM & 0.130 & 0.133 & 18.5 & 0.112 & 0.328 & 19.1 & 0.38 & 0.122 & 0.058 & 17.6 & 0.049 & -- & -- & 0.416 & 15.3 & -- \\
\hline 9/8/2009 8:00 AM & 0.130 & 0.133 & 18.6 & 0.112 & 0.326 & 19.1 & 0.38 & 0.122 & 0.058 & 17.6 & 0.048 & -- & -- & 0.416 & 15.3 & -- \\
\hline 9/8/2009 12:00 PM & 0.201 & 0.132 & 18.7 & 0.112 & 0.326 & 19.1 & 0.38 & 0.122 & 0.058 & 17.6 & 0.047 & -- & -- & 0.416 & 15.3 & -- \\
\hline 9/8/2009 4:00 PM & 0.290 & 0.233 & 18.7 & 0.167 & 0.369 & 19.1 & 0.46 & 0.121 & 0.057 & 17.6 & 0.047 & -- & -- & 0.416 & 15.4 & -- \\
\hline 9/8/2009 8:00 PM & 0.187 & 0.247 & 18.8 & 0.177 & 0.388 & 19.2 & 0.51 & 0.312 & 0.057 & 17.6 & 0.046 & -- & -- & 0.416 & 15.4 & -- \\
\hline 9/9/2009 12:00 AM & 0.180 & 0.223 & 19.0 & 0.166 & 0.369 & 19.2 & 0.46 & 0.335 & 0.058 & 17.6 & 0.046 & -- & -- & 0.417 & 15.4 & -- \\
\hline 9/9/2009 4:00 AM & 0.177 & 0.208 & 19.1 & 0.158 & 0.362 & 19.2 & 0.43 & 0.228 & 0.062 & 17.6 & 0.050 & -- & -- & 0.416 & 15.4 & -- \\
\hline 9/9/2009 8:00 AM & 0.175 & 0.197 & 19.2 & 0.151 & 0.358 & 19.2 & 0.42 & 0.190 & 0.067 & 17.7 & 0.049 & -- & -- & 0.416 & 15.4 & -- \\
\hline 9/9/2009 12:00 PM & 0.173 & 0.188 & 19.3 & 0.147 & 0.355 & 19.3 & 0.41 & 0.175 & 0.070 & 17.7 & 0.048 & -- & -- & 0.417 & 15.4 & -- \\
\hline 9/9/2009 4:00 PM & 0.170 & 0.183 & 19.3 & 0.143 & 0.351 & 19.3 & 0.41 & 0.167 & 0.071 & 17.7 & 0.050 & -- & -- & 0.417 & 15.4 & -- \\
\hline 9/9/2009 8:00 PM & 0.167 & 0.178 & 19.2 & 0.140 & 0.349 & 19.3 & 0.40 & 0.162 & 0.072 & 17.7 & 0.053 & -- & -- & 0.417 & 15.4 & -- \\
\hline 9/10/2009 12:00 AM & 0.167 & 0.173 & 19.2 & 0.138 & 0.348 & 19.3 & 0.40 & 0.158 & 0.071 & 17.7 & 0.060 & -- & -- & 0.416 & 15.4 & -- \\
\hline 9/10/2009 4:00 AM & 0.166 & 0.169 & 19.2 & 0.136 & 0.346 & 19.3 & 0.40 & 0.155 & 0.070 & 17.7 & 0.061 & -- & -- & 0.416 & 15.4 & -- \\
\hline 9/10/2009 8:00 AM & 0.166 & 0.166 & 19.2 & 0.133 & 0.345 & 19.3 & 0.39 & 0.153 & 0.070 & 17.7 & 0.062 & -- & -- & 0.416 & 15.4 & -- \\
\hline 9/10/2009 12:00 PM & 0.165 & 0.163 & 19.2 & 0.132 & 0.344 & 19.3 & 0.39 & 0.151 & 0.070 & 17.7 & 0.060 & -- & -- & 0.417 & 15.4 & -- \\
\hline 9/10/2009 4:00 PM & 0.162 & 0.161 & 19.1 & 0.130 & 0.343 & 19.3 & 0.39 & 0.149 & 0.069 & 17.7 & 0.062 & -- & -- & 0.416 & 15.4 & -- \\
\hline 9/10/2009 8:00 PM & 0.160 & 0.159 & 19.1 & 0.129 & 0.342 & 19.4 & 0.39 & 0.148 & 0.069 & 17.7 & 0.061 & -- & -- & 0.416 & 15.4 & -- \\
\hline 9/11/2009 12:00 AM & 0.160 & 0.157 & 19.1 & 0.127 & 0.341 & 19.4 & 0.39 & 0.146 & 0.067 & 17.7 & 0.062 & -- & -- & 0.417 & 15.4 & -- \\
\hline 9/11/2009 4:00 AM & 0.159 & 0.155 & 19.1 & 0.126 & 0.340 & 19.4 & 0.39 & 0.144 & 0.067 & 17.7 & 0.060 & -- & -- & 0.417 & 15.4 & -- \\
\hline 9/11/2009 8:00 AM & 0.159 & 0.153 & 19.1 & 0.126 & 0.340 & 19.4 & 0.38 & 0.143 & 0.066 & 17.7 & 0.060 & -- & -- & 0.417 & 15.5 & -- \\
\hline 9/11/2009 12:00 PM & 0.157 & 0.151 & 19.1 & 0.125 & 0.338 & 19.4 & 0.38 & 0.142 & 0.065 & 17.7 & 0.058 & -- & -- & 0.417 & 15.5 & -- \\
\hline 9/11/2009 4:00 PM & 0.155 & 0.150 & 19.0 & 0.123 & 0.337 & 19.4 & 0.38 & 0.142 & 0.065 & 17.7 & 0.056 & -- & -- & 0.417 & 15.5 & -- \\
\hline 9/11/2009 8:00 PM & 0.153 & 0.149 & 18.9 & 0.123 & 0.337 & 19.3 & 0.38 & 0.141 & 0.064 & 17.7 & 0.056 & -- & -- & 0.417 & 15.5 & -- \\
\hline 9/12/2009 12:00 AM & 0.153 & 0.147 & 18.8 & 0.121 & 0.336 & 19.3 & 0.38 & 0.139 & 0.064 & 17.7 & 0.058 & -- & -- & 0.417 & 15.5 & -- \\
\hline 9/12/2009 4:00 AM & 0.152 & 0.146 & 18.8 & 0.121 & 0.335 & 19.3 & 0.38 & 0.138 & 0.064 & 17.7 & 0.058 & -- & -- & 0.417 & 15.5 & -- \\
\hline 9/12/2009 8:00 AM & 0.152 & 0.145 & 18.7 & 0.121 & 0.335 & 19.3 & 0.38 & 0.138 & 0.064 & 17.7 & 0.058 & -- & -- & 0.418 & 15.5 & -- \\
\hline 9/12/2009 12:00 PM & 0.150 & 0.144 & 18.7 & 0.120 & 0.335 & 19.3 & 0.38 & 0.137 & 0.063 & 17.7 & 0.057 & -- & -- & 0.417 & 15.5 & -- \\
\hline 9/12/2009 4:00 PM & 0.148 & 0.143 & 18.6 & 0.119 & 0.334 & 19.3 & 0.38 & 0.136 & 0.063 & 17.7 & 0.056 & -- & -- & 0.417 & 15.5 & -- \\
\hline 9/12/2009 8:00 PM & 0.147 & 0.142 & 18.5 & 0.119 & 0.333 & 19.3 & 0.38 & 0.135 & 0.062 & 17.7 & 0.055 & -- & -- & 0.417 & 15.5 & -- \\
\hline
\end{tabular}


Appendix 2. Volumetric water content, temperature, and electrical-conductivity data collected at the flood-irrigated site during 2009.-Continued

[Depth in feet below land surface; volumetric water content dimensionless; temperature in degrees Celsius; bulk electrical conductivity in decisiemens per meter; --, no data]

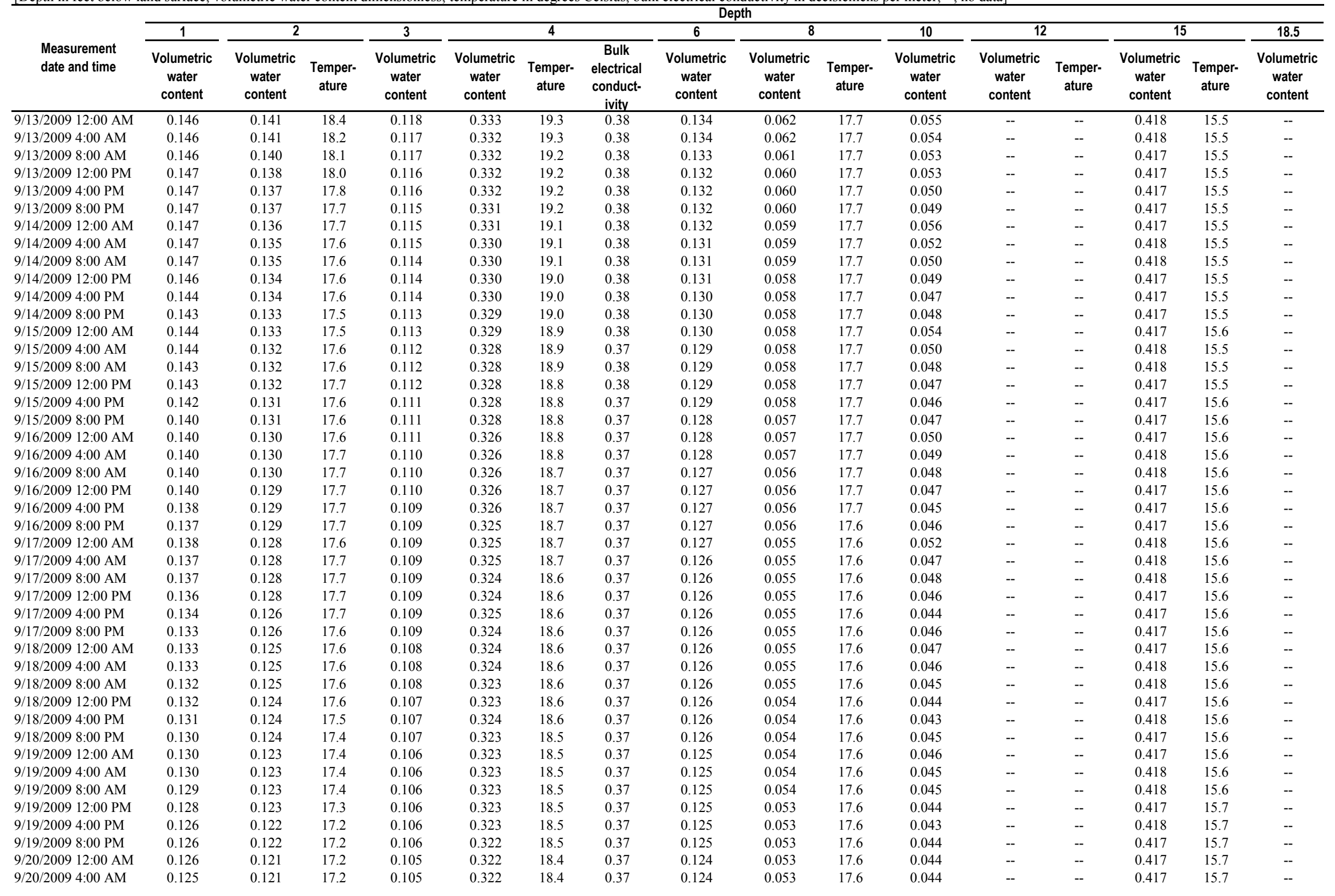


Appendix 2. Volumetric water content, temperature, and electrical-conductivity data collected at the flood-irrigated site during 2009.-Continued

[Depth in feet below land surface; volumetric water content dimensionless; temperature in degrees Celsius; bulk electrical conductivity in decisiemens per meter; --, no data]

\begin{tabular}{|c|c|c|c|c|c|c|c|c|c|c|c|c|c|c|c|c|}
\hline \multirow[b]{3}{*}{$\begin{array}{l}\text { Measurement } \\
\text { date and time }\end{array}$} & \multicolumn{16}{|c|}{ Depth } \\
\hline & \multirow{2}{*}{$\begin{array}{c}1 \\
\text { Volumetric } \\
\text { water } \\
\text { content } \\
\end{array}$} & \multicolumn{2}{|c|}{2} & \multirow{2}{*}{$\begin{array}{c}3 \\
\text { Volumetric } \\
\text { water } \\
\text { content }\end{array}$} & \multicolumn{3}{|c|}{4} & \multirow{2}{*}{$\begin{array}{c}6 \\
\text { Volumetric } \\
\text { water } \\
\text { content } \\
\end{array}$} & \multicolumn{2}{|c|}{8} & \multirow{2}{*}{$\begin{array}{c}10 \\
\text { Volumetric } \\
\text { water } \\
\text { content } \\
\end{array}$} & \multicolumn{2}{|c|}{12} & \multicolumn{2}{|c|}{15} & \multirow{2}{*}{$\begin{array}{c}18.5 \\
\text { Volumetric } \\
\text { water } \\
\text { content } \\
\end{array}$} \\
\hline & & $\begin{array}{c}\text { Volumetric } \\
\text { water } \\
\text { content }\end{array}$ & $\begin{array}{l}\text { Temper- } \\
\text { ature }\end{array}$ & & $\begin{array}{c}\text { Volumetric } \\
\text { water } \\
\text { content } \\
\end{array}$ & $\begin{array}{l}\text { Temper- } \\
\text { ature }\end{array}$ & $\begin{array}{c}\text { Bulk } \\
\text { electrical } \\
\text { conduct- } \\
\text { ivity }\end{array}$ & & $\begin{array}{c}\text { Volumetric } \\
\text { water } \\
\text { content }\end{array}$ & $\begin{array}{l}\text { Temper- } \\
\text { ature }\end{array}$ & & $\begin{array}{c}\text { Volumetric } \\
\text { water } \\
\text { content }\end{array}$ & $\begin{array}{l}\text { Temper- } \\
\text { ature }\end{array}$ & $\begin{array}{c}\text { Volumetric } \\
\text { water } \\
\text { content }\end{array}$ & $\begin{array}{l}\text { Temper- } \\
\text { ature }\end{array}$ & \\
\hline 9/20/2009 8:00 AM & 0.125 & 0.121 & 17.2 & 0.104 & 0.322 & 18.4 & 0.37 & 0.124 & 0.053 & 17.6 & 0.044 & -- & -- & 0.417 & 15.7 & -- \\
\hline 9/20/2009 12:00 PM & 0.124 & 0.121 & 17.2 & 0.104 & 0.322 & 18.4 & 0.37 & 0.124 & 0.053 & 17.6 & 0.043 & -- & -- & 0.417 & 15.7 & -- \\
\hline 9/20/2009 4:00 PM & 0.122 & 0.120 & 17.0 & 0.104 & 0.322 & 18.4 & 0.37 & 0.124 & 0.053 & 17.6 & 0.048 & -- & -- & 0.418 & 15.7 & -- \\
\hline 9/20/2009 8:00 PM & 0.121 & 0.120 & 17.0 & 0.104 & 0.322 & 18.4 & 0.37 & 0.124 & 0.053 & 17.6 & 0.046 & -- & -- & 0.417 & 15.7 & -- \\
\hline 9/21/2009 12:00 AM & 0.121 & 0.120 & 17.0 & 0.104 & 0.321 & 18.3 & 0.37 & 0.123 & 0.053 & 17.6 & 0.043 & -- & -- & 0.416 & 15.7 & -- \\
\hline 9/21/2009 4:00 AM & 0.121 & 0.119 & 17.1 & 0.104 & 0.321 & 18.3 & 0.37 & 0.123 & 0.053 & 17.6 & 0.042 & -- & -- & 0.417 & 15.7 & -- \\
\hline 9/21/2009 8:00 AM & 0.121 & 0.119 & 17.1 & 0.104 & 0.321 & 18.3 & 0.37 & 0.123 & 0.053 & 17.6 & 0.042 & -- & -- & 0.417 & 15.7 & -- \\
\hline 9/21/2009 12:00 PM & 0.118 & 0.119 & 17.1 & 0.104 & 0.321 & 18.3 & 0.37 & 0.123 & 0.053 & 17.6 & 0.042 & -- & -- & 0.417 & 15.7 & -- \\
\hline 9/21/2009 4:00 PM & 0.120 & 0.119 & 17.0 & 0.103 & 0.321 & 18.3 & 0.37 & 0.123 & 0.053 & 17.5 & 0.042 & -- & -- & 0.417 & 15.7 & -- \\
\hline 9/21/2009 8:00 PM & 0.118 & 0.118 & 16.9 & 0.103 & 0.321 & 18.2 & 0.37 & 0.123 & 0.053 & 17.5 & 0.042 & -- & -- & 0.417 & 15.7 & -- \\
\hline 9/22/2009 12:00 AM & 0.118 & 0.118 & 16.8 & 0.103 & 0.321 & 18.2 & 0.37 & 0.123 & 0.052 & 17.5 & 0.042 & -- & -- & 0.418 & 15.7 & -- \\
\hline 9/22/2009 4:00 AM & 0.118 & 0.118 & 16.7 & 0.103 & 0.320 & 18.2 & 0.37 & 0.122 & 0.052 & 17.5 & 0.042 & -- & -- & 0.418 & 15.7 & -- \\
\hline 9/22/2009 8:00 AM & 0.117 & 0.117 & 16.5 & 0.103 & 0.320 & 18.2 & 0.37 & 0.122 & 0.052 & 17.5 & 0.042 & -- & -- & 0.417 & 15.7 & -- \\
\hline 9/22/2009 12:00 PM & 0.116 & 0.117 & 16.4 & 0.102 & 0.320 & 18.2 & 0.37 & 0.123 & 0.052 & 17.5 & 0.042 & -- & -- & 0.417 & 15.7 & -- \\
\hline 9/22/2009 4:00 PM & 0.115 & 0.116 & 16.2 & 0.102 & 0.320 & 18.1 & 0.37 & 0.123 & 0.052 & 17.5 & 0.041 & -- & -- & 0.418 & 15.7 & -- \\
\hline 9/22/2009 8:00 PM & 0.115 & 0.115 & 16.0 & 0.102 & 0.320 & 18.1 & 0.37 & 0.122 & 0.052 & 17.5 & 0.041 & -- & -- & 0.417 & 15.7 & -- \\
\hline 9/23/2009 12:00 AM & 0.114 & 0.115 & 15.9 & 0.101 & 0.320 & 18.1 & 0.37 & 0.122 & 0.052 & 17.5 & 0.041 & -- & -- & 0.417 & 15.7 & -- \\
\hline 9/23/2009 4:00 AM & 0.114 & 0.115 & 15.7 & 0.101 & 0.320 & 18.1 & 0.37 & 0.122 & 0.052 & 17.5 & 0.041 & -- & -- & 0.417 & 15.7 & -- \\
\hline 9/23/2009 8:00 AM & 0.115 & 0.113 & 15.6 & 0.101 & 0.320 & 18.0 & 0.37 & 0.122 & 0.052 & 17.5 & 0.041 & -- & -- & 0.418 & 15.7 & -- \\
\hline 9/23/2009 12:00 PM & 0.115 & 0.113 & 15.5 & 0.101 & 0.320 & 18.0 & 0.37 & 0.122 & 0.052 & 17.5 & 0.041 & -- & -- & 0.417 & 15.7 & -- \\
\hline 9/23/2009 4:00 PM & 0.113 & 0.113 & 15.4 & 0.100 & 0.319 & 17.9 & 0.37 & 0.122 & 0.052 & 17.5 & 0.041 & -- & -- & 0.417 & 15.7 & -- \\
\hline 9/23/2009 8:00 PM & 0.114 & 0.113 & 15.3 & 0.100 & 0.319 & 17.9 & 0.37 & 0.122 & 0.052 & 17.5 & 0.041 & -- & -- & 0.417 & 15.7 & -- \\
\hline 9/24/2009 12:00 AM & 0.114 & 0.113 & 15.2 & 0.100 & 0.319 & 17.8 & 0.37 & 0.121 & 0.051 & 17.5 & 0.041 & -- & -- & 0.417 & 15.7 & -- \\
\hline 9/24/2009 4:00 AM & 0.113 & 0.112 & 15.0 & 0.100 & 0.319 & 17.8 & 0.37 & 0.121 & 0.051 & 17.5 & 0.041 & -- & -- & 0.417 & 15.7 & -- \\
\hline 9/24/2009 8:00 AM & 0.113 & 0.112 & 15.0 & 0.099 & 0.319 & 17.7 & 0.37 & 0.121 & 0.051 & 17.4 & 0.041 & -- & -- & 0.416 & 15.7 & -- \\
\hline 9/24/2009 12:00 PM & 0.113 & 0.112 & 14.9 & 0.099 & 0.319 & 17.7 & 0.37 & 0.121 & 0.051 & 17.4 & 0.040 & -- & -- & 0.417 & 15.7 & -- \\
\hline 9/24/2009 4:00 PM & 0.113 & 0.111 & 14.7 & 0.099 & 0.319 & 17.6 & 0.37 & 0.121 & 0.051 & 17.4 & 0.042 & -- & -- & 0.418 & 15.7 & -- \\
\hline 9/24/2009 8:00 PM & 0.113 & 0.111 & 14.6 & 0.098 & 0.319 & 17.6 & 0.37 & 0.121 & 0.051 & 17.4 & 0.042 & -- & -- & 0.417 & 15.7 & -- \\
\hline 9/25/2009 12:00 AM & 0.113 & 0.111 & 14.6 & 0.098 & 0.318 & 17.5 & 0.37 & 0.121 & 0.051 & 17.4 & 0.040 & -- & -- & 0.416 & 15.7 & -- \\
\hline 9/25/2009 4:00 AM & 0.113 & 0.111 & 14.5 & 0.098 & 0.318 & 17.5 & 0.37 & 0.121 & 0.051 & 17.4 & 0.040 & -- & -- & 0.416 & 15.7 & -- \\
\hline 9/25/2009 8:00 AM & 0.113 & 0.111 & 14.6 & 0.098 & 0.318 & 17.4 & 0.37 & 0.121 & 0.051 & 17.4 & 0.040 & -- & -- & 0.416 & 15.7 & -- \\
\hline 9/25/2009 12:00 PM & 0.113 & 0.111 & 14.6 & 0.098 & 0.318 & 17.4 & 0.37 & 0.121 & 0.051 & 17.4 & 0.040 & -- & -- & 0.417 & 15.7 & -- \\
\hline 9/25/2009 4:00 PM & 0.113 & 0.111 & 14.5 & 0.098 & 0.318 & 17.3 & 0.37 & 0.121 & 0.051 & 17.4 & 0.039 & -- & -- & 0.418 & 15.8 & -- \\
\hline 9/25/2009 8:00 PM & 0.113 & 0.110 & 14.5 & 0.098 & 0.318 & 17.3 & 0.37 & 0.121 & 0.050 & 17.4 & 0.041 & -- & -- & 0.417 & 15.7 & -- \\
\hline 9/26/2009 12:00 AM & 0.113 & 0.110 & 14.5 & 0.098 & 0.317 & 17.2 & 0.37 & 0.121 & 0.051 & 17.4 & 0.040 & -- & -- & 0.416 & 15.7 & -- \\
\hline 9/26/2009 4:00 AM & 0.113 & 0.110 & 14.6 & 0.098 & 0.317 & 17.2 & 0.37 & 0.121 & 0.050 & 17.4 & 0.040 & -- & -- & 0.417 & 15.7 & -- \\
\hline 9/26/2009 8:00 AM & 0.113 & 0.110 & 14.6 & 0.098 & 0.317 & 17.1 & 0.37 & 0.121 & 0.050 & 17.4 & 0.040 & -- & -- & 0.417 & 15.7 & -- \\
\hline 9/26/2009 12:00 PM & 0.112 & 0.110 & 14.6 & 0.097 & 0.317 & 17.1 & 0.37 & 0.121 & 0.051 & 17.3 & 0.040 & -- & -- & 0.417 & 15.7 & -- \\
\hline 9/26/2009 4:00 PM & 0.111 & 0.109 & 14.7 & 0.097 & 0.317 & 17.1 & 0.36 & 0.121 & 0.051 & 17.3 & 0.051 & -- & -- & 0.418 & 15.8 & -- \\
\hline 9/26/2009 8:00 PM & 0.111 & 0.109 & 14.6 & 0.097 & 0.317 & 17.0 & 0.37 & 0.121 & 0.050 & 17.3 & 0.047 & -- & -- & 0.418 & 15.8 & -- \\
\hline 9/27/2009 12:00 AM & 0.111 & 0.109 & 14.6 & 0.097 & 0.316 & 17.0 & 0.37 & 0.121 & 0.050 & 17.3 & 0.040 & -- & -- & 0.416 & 15.8 & -- \\
\hline 9/27/2009 4:00 AM & 0.111 & 0.109 & 14.7 & 0.097 & 0.316 & 17.0 & 0.36 & 0.121 & 0.050 & 17.3 & 0.040 & -- & -- & 0.416 & 15.8 & -- \\
\hline 9/27/2009 8:00 AM & 0.111 & 0.109 & 14.8 & 0.097 & 0.316 & 16.9 & 0.36 & 0.120 & 0.050 & 17.3 & 0.040 & -- & -- & 0.416 & 15.8 & -- \\
\hline 9/27/2009 12:00 PM & 0.111 & 0.109 & 14.8 & 0.096 & 0.316 & 16.9 & 0.36 & 0.120 & 0.050 & 17.3 & 0.042 & -- & -- & 0.418 & 15.8 & -- \\
\hline
\end{tabular}


Appendix 2. Volumetric water content, temperature, and electrical-conductivity data collected at the flood-irrigated site during 2009.-Continued

[Depth in feet below land surface; volumetric water content dimensionless; temperature in degrees Celsius; bulk electrical conductivity in decisiemens per meter; --, no data]

\begin{tabular}{|c|c|c|c|c|c|c|c|c|c|c|c|c|c|c|c|c|}
\hline \multirow[b]{3}{*}{$\begin{array}{l}\text { Measurement } \\
\text { date and time }\end{array}$} & \multirow{3}{*}{$\begin{array}{c}1 \\
\begin{array}{c}\text { Volumetric } \\
\text { water } \\
\text { content }\end{array}\end{array}$} & \multirow{3}{*}{\multicolumn{3}{|c|}{ 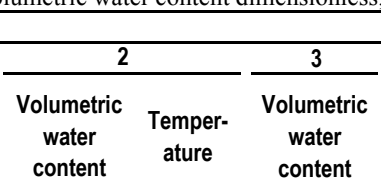 }} & \multirow{2}{*}{\multicolumn{7}{|c|}{ Depth }} & \multirow{2}{*}{\multicolumn{2}{|c|}{12}} & \multirow{2}{*}{\multicolumn{2}{|c|}{15}} & \multirow{3}{*}{$\begin{array}{c}18.5 \\
\text { Volumetric } \\
\text { water } \\
\text { content }\end{array}$} \\
\hline & & & & & & & & & & & & & & & & \\
\hline & & & & & $\begin{array}{c}\text { Volumetric } \\
\text { water } \\
\text { content }\end{array}$ & $\begin{array}{l}\text { Temper- } \\
\text { ature }\end{array}$ & $\begin{array}{c}\text { Bulk } \\
\text { electrical } \\
\text { conduct- } \\
\text { ivity }\end{array}$ & $\begin{array}{c}6 \\
\text { Volumetric } \\
\text { water } \\
\text { content }\end{array}$ & $\begin{array}{c}\text { Volumetric } \\
\text { water } \\
\text { content }\end{array}$ & $\begin{array}{l}\text { Temper- } \\
\text { ature }\end{array}$ & $\begin{array}{c}10 \\
\begin{array}{l}\text { Volumetric } \\
\text { water } \\
\text { content }\end{array}\end{array}$ & $\begin{array}{l}\text { Volumetric } \\
\text { water } \\
\text { content }\end{array}$ & $\begin{array}{l}\text { Temper- } \\
\text { ature }\end{array}$ & $\begin{array}{c}\text { Volumetric } \\
\text { water } \\
\text { content }\end{array}$ & $\begin{array}{l}\text { Temper- } \\
\text { ature }\end{array}$ & \\
\hline 9/27/2009 4:00 PM & 0.110 & 0.109 & 14.9 & 0.096 & 0.316 & 16.9 & 0.36 & 0.121 & 0.050 & 17.3 & 0.043 & -- & -- & 0.419 & 15.8 & -- \\
\hline 9/27/2009 8:00 PM & 0.111 & 0.108 & 14.8 & 0.096 & 0.316 & 16.9 & 0.36 & 0.120 & 0.050 & 17.2 & 0.042 & -- & -- & 0.418 & 15.8 & -- \\
\hline 9/28/2009 12:00 AM & 0.111 & 0.108 & 14.9 & 0.096 & 0.316 & 16.9 & 0.36 & 0.120 & 0.050 & 17.2 & 0.039 & -- & -- & 0.417 & 15.8 & -- \\
\hline 9/28/2009 4:00 AM & 0.110 & 0.108 & 14.9 & 0.096 & 0.316 & 16.8 & 0.36 & 0.120 & 0.049 & 17.2 & 0.040 & -- & -- & 0.417 & 15.8 & -- \\
\hline 9/28/2009 8:00 AM & 0.109 & 0.108 & 15.0 & 0.096 & 0.315 & 16.8 & 0.36 & 0.120 & 0.049 & 17.2 & 0.040 & -- & -- & 0.417 & 15.7 & -- \\
\hline 9/28/2009 12:00 PM & 0.109 & 0.108 & 15.0 & 0.096 & 0.316 & 16.8 & 0.36 & 0.120 & 0.049 & 17.2 & 0.040 & -- & -- & 0.418 & 15.8 & -- \\
\hline 9/28/2009 4:00 PM & 0.109 & 0.108 & 15.0 & 0.095 & 0.316 & 16.8 & 0.36 & 0.120 & 0.050 & 17.2 & 0.039 & -- & -- & 0.419 & 15.8 & -- \\
\hline 9/28/2009 8:00 PM & 0.109 & 0.107 & 14.9 & 0.095 & 0.316 & 16.8 & 0.36 & 0.120 & 0.049 & 17.2 & 0.040 & -- & -- & 0.418 & 15.8 & -- \\
\hline 9/29/2009 12:00 AM & 0.109 & 0.107 & 14.9 & 0.095 & 0.315 & 16.8 & 0.36 & 0.120 & 0.050 & 17.2 & 0.040 & -- & -- & 0.416 & 15.8 & -- \\
\hline 9/29/2009 4:00 AM & 0.109 & 0.107 & 14.9 & 0.095 & 0.315 & 16.8 & 0.36 & 0.119 & 0.049 & 17.2 & 0.040 & -- & -- & 0.417 & 15.8 & -- \\
\hline 9/29/2009 8:00 AM & 0.109 & 0.107 & 14.9 & 0.095 & 0.315 & 16.7 & 0.36 & 0.119 & 0.049 & 17.1 & 0.040 & -- & -- & 0.417 & 15.8 & -- \\
\hline 9/29/2009 12:00 PM & 0.109 & 0.107 & 14.9 & 0.095 & 0.315 & 16.7 & 0.36 & 0.120 & 0.049 & 17.1 & 0.039 & -- & -- & 0.417 & 15.8 & -- \\
\hline 9/29/2009 4:00 PM & 0.108 & 0.107 & 14.9 & 0.095 & 0.316 & 16.7 & 0.36 & 0.120 & 0.049 & 17.1 & 0.043 & -- & -- & 0.418 & 15.8 & -- \\
\hline 9/29/2009 8:00 PM & 0.108 & 0.106 & 14.8 & 0.094 & 0.315 & 16.7 & 0.36 & 0.119 & 0.049 & 17.1 & 0.043 & -- & -- & 0.418 & 15.8 & -- \\
\hline 9/30/2009 12:00 AM & 0.109 & 0.106 & 14.8 & 0.094 & 0.315 & 16.7 & 0.36 & 0.119 & 0.049 & 17.1 & 0.042 & -- & -- & 0.417 & 15.8 & -- \\
\hline 9/30/2009 4:00 AM & 0.109 & 0.106 & 14.8 & 0.094 & 0.315 & 16.7 & 0.36 & 0.119 & 0.049 & 17.1 & 0.039 & -- & -- & 0.416 & 15.8 & -- \\
\hline 9/30/2009 8:00 AM & 0.109 & 0.106 & 14.9 & 0.094 & 0.315 & 16.7 & 0.36 & 0.119 & 0.049 & 17.1 & 0.039 & -- & -- & 0.416 & 15.8 & -- \\
\hline 9/30/2009 12:00 PM & 0.108 & 0.106 & 15.0 & 0.094 & 0.315 & 16.7 & 0.36 & 0.119 & 0.049 & 17.1 & 0.042 & -- & -- & 0.417 & 15.8 & -- \\
\hline 9/30/2009 4:00 PM & 0.108 & 0.106 & 15.0 & 0.094 & 0.315 & 16.6 & 0.36 & 0.119 & 0.049 & 17.0 & 0.045 & -- & -- & 0.417 & 15.8 & -- \\
\hline 9/30/2009 8:00 PM & 0.108 & 0.106 & 14.9 & 0.094 & 0.315 & 16.6 & 0.36 & 0.119 & 0.049 & 17.0 & 0.046 & -- & -- & 0.417 & 15.8 & -- \\
\hline 10/1/2009 12:00 AM & 0.109 & 0.106 & 15.0 & 0.093 & 0.315 & 16.6 & 0.36 & 0.119 & 0.049 & 17.0 & 0.040 & -- & -- & 0.416 & 15.8 & -- \\
\hline 10/1/2009 4:00 AM & 0.108 & 0.106 & 15.1 & 0.093 & 0.313 & 16.6 & 0.36 & 0.118 & 0.048 & 17.0 & 0.039 & -- & -- & 0.416 & 15.8 & -- \\
\hline 10/1/2009 8:00 AM & 0.108 & 0.106 & 15.2 & 0.093 & 0.313 & 16.6 & 0.36 & 0.118 & 0.048 & 17.0 & 0.039 & -- & -- & 0.416 & 15.8 & -- \\
\hline 10/1/2009 12:00 PM & 0.108 & 0.105 & 15.2 & 0.093 & 0.313 & 16.6 & 0.36 & 0.118 & 0.049 & 17.0 & 0.038 & -- & -- & 0.416 & 15.8 & -- \\
\hline 10/1/2009 4:00 PM & 0.107 & 0.106 & 15.2 & 0.093 & 0.313 & 16.6 & 0.36 & 0.118 & 0.048 & 16.9 & 0.038 & -- & -- & 0.418 & 15.8 & -- \\
\hline 10/1/2009 8:00 PM & 0.107 & 0.105 & 15.1 & 0.093 & 0.313 & 16.6 & 0.36 & 0.118 & 0.049 & 16.9 & 0.038 & -- & -- & 0.416 & 15.8 & -- \\
\hline 10/2/2009 12:00 AM & 0.107 & 0.105 & 15.1 & 0.093 & 0.313 & 16.6 & 0.36 & 0.118 & 0.048 & 16.9 & 0.038 & -- & -- & 0.417 & 15.8 & -- \\
\hline 10/2/2009 4:00 AM & 0.106 & 0.105 & 15.1 & 0.092 & 0.313 & 16.6 & 0.36 & 0.118 & 0.048 & 16.9 & 0.038 & -- & -- & 0.417 & 15.8 & -- \\
\hline 10/2/2009 8:00 AM & 0.106 & 0.105 & 15.0 & 0.092 & 0.313 & 16.6 & 0.36 & 0.118 & 0.048 & 16.9 & 0.038 & -- & -- & 0.417 & 15.8 & -- \\
\hline 10/2/2009 12:00 PM & 0.105 & 0.104 & 14.9 & 0.092 & 0.313 & 16.6 & 0.36 & 0.118 & 0.048 & 16.9 & 0.039 & -- & -- & 0.417 & 15.8 & -- \\
\hline 10/2/2009 4:00 PM & 0.105 & 0.104 & 14.8 & 0.092 & 0.313 & 16.6 & 0.36 & 0.118 & 0.048 & 16.9 & 0.042 & -- & -- & 0.418 & 15.8 & -- \\
\hline 10/2/2009 8:00 PM & 0.106 & 0.104 & 14.7 & 0.092 & 0.313 & 16.6 & 0.36 & 0.118 & 0.048 & 16.9 & 0.041 & -- & -- & 0.417 & 15.8 & -- \\
\hline 10/3/2009 12:00 AM & 0.106 & 0.103 & 14.5 & 0.092 & 0.313 & 16.6 & 0.36 & 0.118 & 0.048 & 16.9 & 0.037 & -- & -- & 0.417 & 15.8 & -- \\
\hline 10/3/2009 4:00 AM & 0.106 & 0.104 & 14.4 & 0.092 & 0.313 & 16.5 & 0.36 & 0.117 & 0.048 & 16.9 & 0.037 & -- & -- & 0.417 & 15.8 & -- \\
\hline 10/3/2009 8:00 AM & 0.105 & 0.103 & 14.4 & 0.092 & 0.313 & 16.5 & 0.36 & 0.117 & 0.048 & 16.8 & 0.037 & -- & -- & 0.417 & 15.8 & -- \\
\hline 10/3/2009 12:00 PM & 0.105 & 0.103 & 14.4 & 0.092 & 0.313 & 16.5 & 0.36 & 0.118 & 0.048 & 16.8 & 0.036 & -- & -- & 0.417 & 15.8 & -- \\
\hline 10/3/2009 4:00 PM & 0.105 & 0.103 & 14.3 & 0.092 & 0.313 & 16.5 & 0.36 & 0.118 & 0.048 & 16.8 & 0.036 & -- & -- & 0.418 & 15.8 & -- \\
\hline 10/3/2009 8:00 PM & 0.106 & 0.103 & 14.1 & 0.092 & 0.313 & 16.5 & 0.36 & 0.118 & 0.048 & 16.8 & 0.036 & -- & -- & 0.417 & 15.8 & -- \\
\hline 10/4/2009 12:00 AM & 0.106 & 0.103 & 14.1 & 0.092 & 0.313 & 16.4 & 0.36 & 0.118 & 0.048 & 16.8 & 0.036 & -- & -- & 0.416 & 15.8 & -- \\
\hline 10/4/2009 4:00 AM & 0.106 & 0.103 & 14.1 & 0.092 & 0.313 & 16.4 & 0.36 & 0.117 & 0.048 & 16.8 & 0.036 & -- & -- & 0.416 & 15.8 & -- \\
\hline 10/4/2009 8:00 AM & 0.106 & 0.103 & 14.2 & 0.092 & 0.313 & 16.4 & 0.36 & 0.117 & 0.048 & 16.8 & 0.036 & -- & -- & 0.416 & 15.8 & -- \\
\hline 10/4/2009 12:00 PM & 0.106 & 0.101 & 14.1 & 0.092 & 0.313 & 16.4 & 0.36 & 0.117 & 0.048 & 16.8 & 0.036 & -- & -- & 0.416 & 15.8 & -- \\
\hline 10/4/2009 4:00 PM & 0.106 & 0.103 & 14.0 & 0.092 & 0.313 & 16.3 & 0.36 & 0.118 & 0.048 & 16.8 & 0.036 & -- & -- & 0.416 & 15.8 & -- \\
\hline 10/4/2009 8:00 PM & 0.106 & 0.103 & 13.9 & 0.092 & 0.313 & 16.3 & 0.36 & 0.117 & 0.048 & 16.7 & 0.036 & -- & -- & 0.416 & 15.8 & -- \\
\hline
\end{tabular}


Appendix 2. Volumetric water content, temperature, and electrical-conductivity data collected at the flood-irrigated site during 2009.-Continued

[Depth in feet below land surface; volumetric water content dimensionless; temperature in degrees Celsius; bulk electrical conductivity in decisiemens per meter; --, no data]

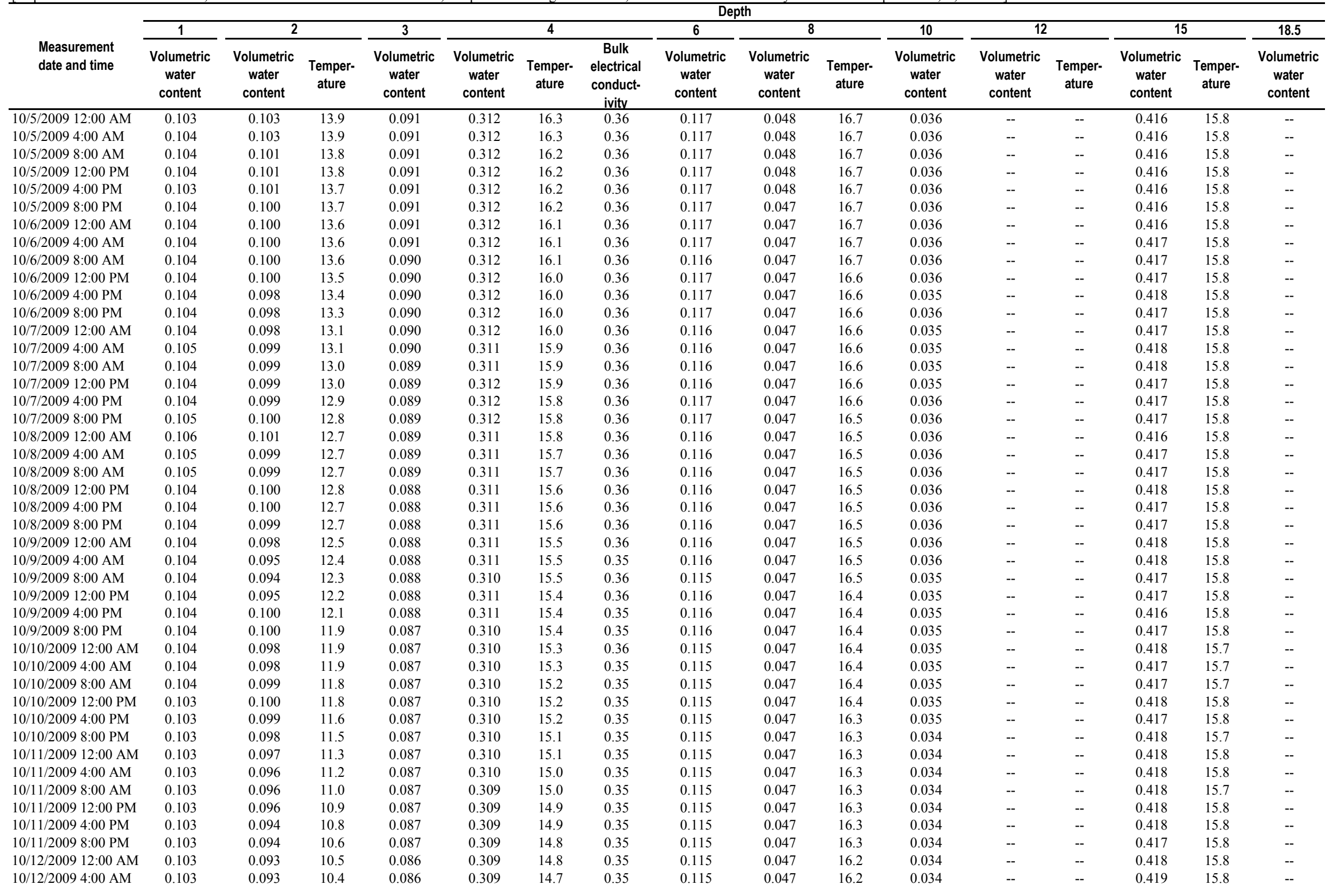


Appendix 2. Volumetric water content, temperature, and electrical-conductivity data collected at the flood-irrigated site during 2009.-Continued

[Depth in feet below land surface; volumetric water content dimensionless; temperature in degrees Celsius; bulk electrical conductivity in decisiemens per meter; --, no data]

\begin{tabular}{|c|c|c|c|c|c|c|c|c|c|c|c|c|c|c|c|c|}
\hline \multirow[b]{3}{*}{$\begin{array}{l}\text { Measurement } \\
\text { date and time }\end{array}$} & \multicolumn{16}{|c|}{ Depth } \\
\hline & \multirow{2}{*}{$\begin{array}{c}1 \\
\text { Volumetric } \\
\text { water } \\
\text { content }\end{array}$} & \multicolumn{2}{|c|}{2} & \multirow{2}{*}{$\begin{array}{c}3 \\
\text { Volumetric } \\
\text { water } \\
\text { content }\end{array}$} & \multicolumn{3}{|c|}{4} & \multirow{2}{*}{$\begin{array}{c}6 \\
\text { Volumetric } \\
\text { water } \\
\text { content }\end{array}$} & \multicolumn{2}{|c|}{8} & \multirow{2}{*}{$\begin{array}{c}10 \\
\text { Volumetric } \\
\text { water } \\
\text { content }\end{array}$} & \multicolumn{2}{|c|}{12} & \multicolumn{2}{|c|}{15} & \multirow{2}{*}{$\begin{array}{c}18.5 \\
\text { Volumetric } \\
\text { water } \\
\text { content }\end{array}$} \\
\hline & & $\begin{array}{c}\text { Volumetric } \\
\text { water } \\
\text { content }\end{array}$ & $\begin{array}{l}\text { Temper- } \\
\text { ature }\end{array}$ & & $\begin{array}{c}\text { Volumetric } \\
\text { water } \\
\text { content }\end{array}$ & $\begin{array}{l}\text { Temper- } \\
\text { ature }\end{array}$ & $\begin{array}{c}\text { Bulk } \\
\text { electrical } \\
\text { conduct- } \\
\text { ivity } \\
\end{array}$ & & $\begin{array}{c}\text { Volumetric } \\
\text { water } \\
\text { content }\end{array}$ & $\begin{array}{l}\text { Temper- } \\
\text { ature }\end{array}$ & & $\begin{array}{l}\text { Volumetric } \\
\text { water } \\
\text { content }\end{array}$ & $\begin{array}{l}\text { Temper- } \\
\text { ature }\end{array}$ & $\begin{array}{c}\text { Volumetric } \\
\text { water } \\
\text { content }\end{array}$ & $\begin{array}{l}\text { Temper- } \\
\text { ature }\end{array}$ & \\
\hline 10/12/2009 8:00 AM & 0.103 & 0.094 & 10.4 & 0.086 & 0.309 & 14.7 & 0.35 & 0.115 & 0.047 & 16.2 & 0.034 & -- & -- & 0.419 & 15.7 & -- \\
\hline 10/12/2009 12:00 PM & 0.103 & 0.093 & 10.3 & 0.086 & 0.309 & 14.6 & 0.35 & 0.115 & 0.047 & 16.2 & 0.034 & -- & -- & 0.419 & 15.7 & -- \\
\hline 10/12/2009 4:00 PM & 0.103 & 0.094 & 10.1 & 0.086 & 0.309 & 14.5 & 0.35 & 0.115 & 0.047 & 16.2 & 0.034 & -- & -- & 0.416 & 15.8 & -- \\
\hline 10/12/2009 8:00 PM & 0.103 & 0.093 & 10.1 & 0.086 & 0.309 & 14.5 & 0.35 & 0.115 & 0.047 & 16.2 & 0.034 & -- & -- & 0.418 & 15.7 & -- \\
\hline 10/13/2009 12:00 AM & 0.104 & 0.093 & 10.0 & 0.085 & 0.309 & 14.4 & 0.35 & 0.115 & 0.047 & 16.2 & 0.034 & -- & -- & 0.419 & 15.7 & -- \\
\hline 10/13/2009 4:00 AM & 0.103 & 0.093 & 10.1 & 0.085 & 0.308 & 14.3 & 0.35 & 0.115 & 0.047 & 16.1 & 0.034 & -- & -- & 0.419 & 15.7 & -- \\
\hline 10/13/2009 8:00 AM & 0.103 & 0.093 & 10.0 & 0.085 & 0.308 & 14.3 & 0.35 & 0.115 & 0.046 & 16.1 & 0.034 & -- & -- & 0.419 & 15.7 & -- \\
\hline 10/13/2009 12:00 PM & 0.104 & 0.093 & 10.0 & 0.085 & 0.308 & 14.2 & 0.35 & 0.115 & 0.047 & 16.1 & 0.034 & -- & -- & 0.419 & 15.7 & -- \\
\hline 10/13/2009 4:00 PM & 0.104 & 0.093 & 10.0 & 0.085 & 0.308 & 14.2 & 0.35 & 0.115 & 0.046 & 16.1 & 0.034 & -- & -- & 0.417 & 15.8 & -- \\
\hline 10/13/2009 8:00 PM & 0.104 & 0.093 & 9.9 & 0.085 & 0.308 & 14.1 & 0.35 & 0.115 & 0.046 & 16.1 & 0.034 & -- & -- & 0.418 & 15.7 & -- \\
\hline 10/14/2009 12:00 AM & 0.104 & 0.093 & 9.9 & 0.085 & 0.308 & 14.1 & 0.35 & 0.115 & 0.046 & 16.1 & 0.033 & -- & -- & 0.419 & 15.7 & -- \\
\hline 10/14/2009 4:00 AM & 0.104 & 0.093 & 10.0 & 0.085 & 0.308 & 14.0 & 0.35 & 0.115 & 0.046 & 16.0 & 0.033 & -- & -- & 0.419 & 15.7 & -- \\
\hline 10/14/2009 8:00 AM & 0.104 & 0.093 & 10.0 & 0.085 & 0.308 & 14.0 & 0.35 & 0.115 & 0.046 & 16.0 & 0.033 & -- & -- & 0.419 & 15.7 & -- \\
\hline 10/14/2009 12:00 PM & 0.104 & 0.093 & 10.0 & 0.084 & 0.308 & 13.9 & 0.35 & 0.115 & 0.046 & 16.0 & 0.033 & -- & -- & 0.419 & 15.7 & -- \\
\hline 10/14/2009 4:00 PM & 0.104 & 0.092 & 10.0 & 0.084 & 0.308 & 13.9 & 0.35 & 0.115 & 0.046 & 16.0 & 0.033 & -- & -- & 0.416 & 15.7 & -- \\
\hline 10/14/2009 8:00 PM & 0.104 & 0.093 & 10.0 & 0.084 & 0.308 & 13.9 & 0.35 & 0.115 & 0.046 & 16.0 & 0.033 & -- & -- & 0.417 & 15.7 & -- \\
\hline 10/15/2009 12:00 AM & 0.104 & 0.093 & 10.1 & 0.084 & 0.307 & 13.8 & 0.35 & 0.115 & 0.046 & 16.0 & 0.033 & -- & -- & 0.419 & 15.7 & -- \\
\hline 10/15/2009 4:00 AM & 0.104 & 0.093 & 10.2 & 0.084 & 0.307 & 13.8 & 0.35 & 0.115 & 0.046 & 15.9 & 0.033 & -- & -- & 0.419 & 15.7 & -- \\
\hline 10/15/2009 8:00 AM & 0.104 & 0.093 & 10.2 & 0.084 & 0.307 & 13.8 & 0.35 & 0.115 & 0.046 & 15.9 & 0.034 & -- & -- & 0.419 & 15.7 & -- \\
\hline 10/15/2009 12:00 PM & 0.104 & 0.093 & 10.3 & 0.084 & 0.307 & 13.7 & 0.35 & 0.115 & 0.046 & 15.9 & 0.034 & -- & -- & 0.418 & 15.7 & - \\
\hline
\end{tabular}


Appendix 3. Volumetric water content, temperature, and electrical-conductivity data collected at the sprinkler-irrigated site during 2008.

[Depth in feet below land surface; volumetric water content dimensionless; temperature in degrees Celsius; bulk electrical conductivity in decisiemens per meter; --, no data]

\begin{tabular}{|c|c|c|c|c|c|c|c|c|c|c|c|c|c|}
\hline \multirow[b]{3}{*}{$\begin{array}{l}\text { Measurement } \\
\text { date and time }\end{array}$} & \multicolumn{13}{|c|}{ Depth } \\
\hline & \multicolumn{2}{|l|}{2} & \multirow{2}{*}{$\begin{array}{c}3 \\
\begin{array}{c}\text { Volumetric } \\
\text { water } \\
\text { content }\end{array}\end{array}$} & \multicolumn{3}{|c|}{4} & \multirow{2}{*}{$\begin{array}{c}5 \\
\text { Volumetric } \\
\text { water } \\
\text { content }\end{array}$} & \multicolumn{2}{|c|}{7} & \multirow{2}{*}{$\begin{array}{c}9 \\
\text { Volumetric } \\
\text { water } \\
\text { content }\end{array}$} & \multicolumn{2}{|c|}{13} & \multirow{2}{*}{$\begin{array}{c}16.5 \\
\text { Volumetric } \\
\text { water } \\
\text { content }\end{array}$} \\
\hline & $\begin{array}{c}\text { Volumetric } \\
\text { water } \\
\text { content }\end{array}$ & $\begin{array}{c}\text { Temper- } \\
\text { ature }\end{array}$ & & $\begin{array}{c}\text { Volumetric } \\
\text { water } \\
\text { content }\end{array}$ & $\begin{array}{l}\text { Temper- } \\
\text { ature }\end{array}$ & $\begin{array}{c}\text { Bulk } \\
\text { electrical } \\
\text { conduct- } \\
\text { ivity }\end{array}$ & & $\begin{array}{c}\text { Volumetric } \\
\text { water } \\
\text { content }\end{array}$ & $\begin{array}{l}\text { Temper- } \\
\text { ature }\end{array}$ & & $\begin{array}{c}\text { Volumetric } \\
\text { water } \\
\text { content }\end{array}$ & $\begin{array}{l}\text { Temper- } \\
\text { ature }\end{array}$ & \\
\hline 6/18/2008 2:00 PM & 0.338 & 18.8 & 0.166 & 0.080 & 16.0 & 0.06 & 0.026 & 0.109 & 13.0 & 0.098 & 0.051 & 8.2 & 0.434 \\
\hline 6/18/2008 3:00 PM & 0.340 & 18.7 & 0.166 & 0.080 & 16.0 & 0.06 & 0.026 & 0.110 & 13.1 & 0.099 & 0.056 & 10.9 & 0.455 \\
\hline 6/18/2008 4:00 PM & 0.340 & 18.7 & 0.166 & 0.080 & 16.0 & 0.06 & 0.027 & 0.110 & 13.1 & 0.099 & 0.056 & 10.9 & 0.455 \\
\hline 6/18/2008 5:00 PM & 0.340 & 18.7 & 0.166 & 0.080 & 16.0 & 0.06 & 0.027 & 0.110 & 13.1 & 0.099 & 0.056 & 10.8 & 0.455 \\
\hline 6/18/2008 6:00 PM & 0.338 & 18.6 & 0.166 & 0.080 & 16.0 & 0.06 & 0.027 & 0.110 & 13.1 & 0.099 & 0.056 & 10.9 & 0.455 \\
\hline 6/18/2008 7:00 PM & 0.338 & 18.6 & 0.166 & 0.080 & 16.1 & 0.06 & 0.026 & 0.110 & 13.1 & 0.099 & 0.056 & 10.9 & 0.455 \\
\hline 6/18/2008 8:00 PM & 0.338 & 18.6 & 0.166 & 0.080 & 16.1 & 0.06 & 0.026 & 0.110 & 13.1 & 0.099 & 0.056 & 10.9 & 0.455 \\
\hline 6/18/2008 9:00 PM & 0.338 & 18.6 & 0.166 & 0.080 & 16.1 & 0.06 & 0.026 & 0.109 & 13.1 & 0.099 & 0.055 & 10.9 & 0.454 \\
\hline 6/18/2008 10:00 PM & 0.338 & 18.6 & 0.166 & 0.080 & 16.1 & 0.06 & 0.026 & 0.109 & 13.1 & 0.098 & 0.056 & 10.9 & 0.455 \\
\hline 6/18/2008 11:00 PM & 0.338 & 18.6 & 0.166 & 0.080 & 16.1 & 0.06 & 0.026 & 0.109 & 13.1 & 0.098 & 0.055 & 10.8 & 0.455 \\
\hline 6/19/2008 12:00 AM & 0.338 & 18.6 & 0.166 & 0.080 & 16.1 & 0.06 & 0.026 & 0.109 & 13.1 & 0.098 & 0.056 & 10.8 & 0.454 \\
\hline 6/19/2008 1:00 AM & 0.338 & 18.6 & 0.166 & 0.080 & 16.1 & 0.06 & 0.026 & 0.109 & 13.1 & 0.098 & 0.055 & 10.9 & 0.455 \\
\hline 6/19/2008 2:00 AM & 0.338 & 18.6 & 0.166 & 0.080 & 16.1 & 0.06 & 0.026 & 0.109 & 13.1 & 0.098 & 0.055 & 10.8 & 0.455 \\
\hline 6/19/2008 3:00 AM & 0.338 & 18.6 & 0.166 & 0.080 & 16.1 & 0.06 & 0.026 & 0.109 & 13.1 & 0.098 & 0.055 & 10.8 & 0.455 \\
\hline 6/19/2008 4:00 AM & 0.338 & 18.6 & 0.166 & 0.080 & 16.1 & 0.06 & 0.026 & 0.109 & 13.1 & 0.098 & 0.055 & 10.8 & 0.455 \\
\hline 6/19/2008 5:00 AM & 0.340 & 18.6 & 0.166 & 0.080 & 16.1 & 0.06 & 0.026 & 0.109 & 13.1 & 0.098 & 0.055 & 10.8 & 0.455 \\
\hline 6/19/2008 6:00 AM & 0.340 & 18.6 & 0.166 & 0.080 & 16.1 & 0.06 & 0.026 & 0.109 & 13.1 & 0.098 & 0.056 & 10.9 & 0.454 \\
\hline 6/19/2008 7:00 AM & 0.340 & 18.5 & 0.166 & 0.080 & 16.1 & 0.06 & 0.027 & 0.109 & 13.1 & 0.098 & 0.055 & 10.9 & 0.455 \\
\hline 6/19/2008 8:00 AM & 0.338 & 18.5 & 0.166 & 0.080 & 16.2 & 0.06 & 0.027 & 0.109 & 13.2 & 0.098 & 0.055 & 10.9 & 0.455 \\
\hline 6/19/2008 9:00 AM & 0.340 & 18.5 & 0.166 & 0.080 & 16.1 & 0.06 & 0.027 & 0.110 & 13.1 & 0.099 & 0.056 & 10.9 & 0.455 \\
\hline 6/19/2008 10:00 AM & 0.340 & 18.5 & 0.166 & 0.080 & 16.1 & 0.06 & 0.026 & 0.109 & 13.1 & 0.099 & 0.056 & 10.9 & 0.455 \\
\hline 6/19/2008 11:00 AM & 0.338 & 18.5 & 0.166 & 0.080 & 16.1 & 0.06 & 0.027 & 0.109 & 13.1 & 0.099 & 0.056 & 10.9 & 0.455 \\
\hline 6/19/2008 12:00 PM & 0.338 & 18.4 & 0.166 & 0.080 & 16.2 & 0.06 & 0.027 & 0.109 & 13.1 & 0.099 & 0.056 & 10.9 & 0.455 \\
\hline 6/19/2008 1:00 PM & 0.338 & 18.4 & 0.166 & 0.080 & 16.2 & 0.06 & 0.027 & 0.110 & 13.2 & 0.099 & 0.056 & 10.9 & 0.455 \\
\hline 6/19/2008 2:00 PM & 0.338 & 18.4 & 0.166 & 0.080 & 16.2 & 0.06 & 0.027 & 0.110 & 13.2 & 0.099 & 0.056 & 10.9 & 0.455 \\
\hline 6/19/2008 3:00 PM & 0.337 & 18.3 & 0.166 & 0.080 & 16.2 & 0.06 & 0.027 & 0.110 & 13.2 & 0.099 & 0.055 & 10.9 & 0.455 \\
\hline 6/19/2008 4:00 PM & 0.338 & 18.4 & 0.166 & 0.080 & 16.2 & 0.06 & 0.027 & 0.110 & 13.2 & 0.099 & 0.055 & 10.9 & 0.455 \\
\hline 6/19/2008 5:00 PM & 0.337 & 18.3 & 0.166 & 0.080 & 16.2 & 0.06 & 0.027 & 0.110 & 13.2 & 0.099 & 0.055 & 10.9 & 0.455 \\
\hline 6/19/2008 6:00 PM & 0.337 & 18.3 & 0.166 & 0.080 & 16.2 & 0.06 & 0.026 & 0.110 & 13.2 & 0.099 & 0.055 & 10.9 & 0.455 \\
\hline 6/19/2008 7:00 PM & 0.338 & 18.3 & 0.166 & 0.080 & 16.2 & 0.06 & 0.027 & 0.110 & 13.2 & 0.099 & 0.056 & 10.9 & 0.455 \\
\hline 6/19/2008 8:00 PM & 0.338 & 18.3 & 0.166 & 0.080 & 16.2 & 0.06 & 0.026 & 0.109 & 13.2 & 0.099 & 0.056 & 10.9 & 0.455 \\
\hline 6/19/2008 9:00 PM & 0.338 & 18.3 & 0.166 & 0.081 & 16.2 & 0.06 & 0.026 & 0.110 & 13.2 & 0.099 & 0.056 & 10.9 & 0.455 \\
\hline 6/19/2008 10:00 PM & 0.338 & 18.3 & 0.166 & 0.080 & 16.2 & 0.06 & 0.026 & 0.110 & 13.2 & 0.099 & 0.056 & 10.9 & 0.455 \\
\hline 6/19/2008 11:00 PM & 0.338 & 18.3 & 0.166 & 0.080 & 16.2 & 0.06 & 0.026 & 0.110 & 13.2 & 0.099 & 0.055 & 10.9 & 0.455 \\
\hline 6/20/2008 12:00 AM & 0.337 & 18.4 & 0.166 & 0.080 & 16.2 & 0.06 & 0.026 & 0.109 & 13.2 & 0.099 & 0.055 & 10.9 & 0.455 \\
\hline
\end{tabular}


Appendix 3. Volumetric water content, temperature, and electrical-conductivity data collected at the sprinkler-irrigated site during 2008.-Continued

[Depth in feet below land surface; volumetric water content dimensionless; temperature in degrees Celsius; bulk electrical conductivity in decisiemens per meter; --, no data]

\begin{tabular}{|c|c|c|c|c|c|c|c|c|c|c|c|c|c|}
\hline \multirow[b]{3}{*}{$\begin{array}{l}\text { Measurement } \\
\text { date and time }\end{array}$} & \multicolumn{13}{|c|}{ Depth } \\
\hline & \multicolumn{2}{|l|}{2} & \multirow{2}{*}{$\begin{array}{c}3 \\
\begin{array}{c}\text { Volumetric } \\
\text { water } \\
\text { content }\end{array}\end{array}$} & \multicolumn{3}{|c|}{4} & \multirow{2}{*}{$\begin{array}{c}5 \\
\begin{array}{c}\text { Volumetric } \\
\text { water } \\
\text { content }\end{array}\end{array}$} & \multicolumn{2}{|l|}{7} & \multirow{2}{*}{$\begin{array}{c}9 \\
\text { Volumetric } \\
\text { water } \\
\text { content }\end{array}$} & \multicolumn{2}{|c|}{13} & \multirow{2}{*}{$\begin{array}{c}16.5 \\
\text { Volumetric } \\
\text { water } \\
\text { content }\end{array}$} \\
\hline & $\begin{array}{c}\text { Volumetric } \\
\text { water } \\
\text { content }\end{array}$ & $\begin{array}{l}\text { Temper- } \\
\text { ature }\end{array}$ & & $\begin{array}{c}\text { Volumetric } \\
\text { water } \\
\text { content }\end{array}$ & $\begin{array}{l}\text { Temper- } \\
\text { ature }\end{array}$ & $\begin{array}{c}\text { Bulk } \\
\text { electrical } \\
\text { conduct- } \\
\text { ivity }\end{array}$ & & $\begin{array}{c}\text { Volumetric } \\
\text { water } \\
\text { content }\end{array}$ & $\begin{array}{l}\text { Temper- } \\
\text { ature }\end{array}$ & & $\begin{array}{c}\text { Volumetric } \\
\text { water } \\
\text { content }\end{array}$ & $\begin{array}{l}\text { Temper- } \\
\text { ature }\end{array}$ & \\
\hline 6/20/2008 1:00 AM & 0.337 & 18.4 & 0.166 & 0.080 & 16.2 & 0.06 & 0.026 & 0.109 & 13.2 & 0.099 & 0.055 & 10.9 & 0.455 \\
\hline 6/20/2008 2:00 AM & 0.337 & 18.5 & 0.166 & 0.080 & 16.2 & 0.06 & 0.026 & 0.109 & 13.2 & 0.099 & 0.055 & 10.9 & 0.455 \\
\hline 6/20/2008 3:00 AM & 0.337 & 18.5 & 0.166 & 0.080 & 16.2 & 0.06 & 0.026 & 0.109 & 13.2 & 0.099 & 0.055 & 10.9 & 0.455 \\
\hline 6/20/2008 4:00 AM & 0.338 & 18.6 & 0.166 & 0.080 & 16.2 & 0.06 & 0.026 & 0.109 & 13.2 & 0.099 & 0.055 & 10.9 & 0.455 \\
\hline 6/20/2008 5:00 AM & 0.338 & 18.6 & 0.166 & 0.080 & 16.2 & 0.06 & 0.026 & 0.109 & 13.2 & 0.099 & 0.055 & 10.9 & 0.455 \\
\hline 6/20/2008 6:00 AM & 0.338 & 18.6 & 0.166 & 0.080 & 16.2 & 0.06 & 0.026 & 0.110 & 13.2 & 0.099 & 0.055 & 10.9 & 0.455 \\
\hline 6/20/2008 7:00 AM & 0.337 & 18.6 & 0.166 & 0.080 & 16.2 & 0.06 & 0.026 & 0.110 & 13.2 & 0.099 & 0.055 & 10.9 & 0.455 \\
\hline 6/20/2008 8:00 AM & 0.337 & 18.6 & 0.166 & 0.080 & 16.2 & 0.06 & 0.026 & 0.110 & 13.2 & 0.099 & 0.056 & 10.9 & 0.455 \\
\hline 6/20/2008 9:00 AM & 0.337 & 18.7 & 0.166 & 0.080 & 16.2 & 0.06 & 0.027 & 0.110 & 13.2 & 0.099 & 0.056 & 10.9 & 0.455 \\
\hline 6/20/2008 10:00 AM & 0.337 & 18.7 & 0.166 & 0.080 & 16.2 & 0.06 & 0.026 & 0.109 & 13.2 & 0.099 & 0.056 & 10.9 & 0.455 \\
\hline 6/20/2008 11:00 AM & 0.337 & 18.7 & 0.166 & 0.080 & 16.2 & 0.06 & 0.026 & 0.110 & 13.2 & 0.099 & 0.056 & 10.9 & 0.455 \\
\hline 6/20/2008 12:00 PM & 0.337 & 18.6 & 0.166 & 0.080 & 16.2 & 0.06 & 0.027 & 0.110 & 13.2 & 0.099 & 0.056 & 10.9 & 0.455 \\
\hline 6/20/2008 1:00 PM & 0.337 & 18.7 & 0.166 & 0.080 & 16.3 & 0.06 & 0.026 & 0.111 & 13.2 & 0.099 & 0.056 & 11.0 & 0.455 \\
\hline 6/20/2008 2:00 PM & 0.337 & 18.6 & 0.166 & 0.080 & 16.2 & 0.06 & 0.027 & 0.110 & 13.2 & 0.099 & 0.056 & 11.0 & 0.455 \\
\hline 6/20/2008 3:00 PM & 0.337 & 18.6 & 0.166 & 0.080 & 16.2 & 0.06 & 0.027 & 0.110 & 13.2 & 0.099 & 0.056 & 10.9 & 0.455 \\
\hline 6/20/2008 4:00 PM & 0.337 & 18.6 & 0.166 & 0.081 & 16.3 & 0.06 & 0.027 & 0.110 & 13.3 & 0.099 & 0.056 & 10.9 & 0.455 \\
\hline 6/20/2008 5:00 PM & 0.337 & 18.6 & 0.166 & 0.081 & 16.3 & 0.06 & 0.027 & 0.110 & 13.3 & 0.099 & 0.056 & 10.9 & 0.455 \\
\hline 6/20/2008 6:00 PM & 0.337 & 18.6 & 0.166 & 0.080 & 16.2 & 0.06 & 0.027 & 0.110 & 13.3 & 0.099 & 0.056 & 10.9 & 0.455 \\
\hline 6/20/2008 7:00 PM & 0.337 & 18.6 & 0.166 & 0.080 & 16.3 & 0.06 & 0.026 & 0.110 & 13.3 & 0.099 & 0.056 & 11.0 & 0.455 \\
\hline 6/20/2008 8:00 PM & 0.337 & 18.6 & 0.166 & 0.080 & 16.3 & 0.06 & 0.027 & 0.109 & 13.3 & 0.099 & 0.056 & 10.9 & 0.455 \\
\hline 6/20/2008 9:00 PM & 0.337 & 18.6 & 0.166 & 0.080 & 16.3 & 0.06 & 0.027 & 0.111 & 13.3 & 0.099 & 0.056 & 11.0 & 0.456 \\
\hline 6/20/2008 10:00 PM & 0.336 & 18.7 & 0.166 & 0.080 & 16.3 & 0.06 & 0.027 & 0.111 & 13.3 & 0.099 & 0.055 & 11.0 & 0.456 \\
\hline 6/20/2008 11:00 PM & 0.337 & 18.7 & 0.166 & 0.080 & 16.3 & 0.06 & 0.026 & 0.110 & 13.3 & 0.099 & 0.055 & 11.0 & 0.456 \\
\hline 6/21/2008 12:00 AM & 0.337 & 18.8 & 0.166 & 0.081 & 16.3 & 0.06 & 0.026 & 0.111 & 13.3 & 0.099 & 0.056 & 10.9 & 0.456 \\
\hline 6/21/2008 1:00 AM & 0.338 & 18.8 & 0.166 & 0.080 & 16.3 & 0.06 & 0.026 & 0.110 & 13.3 & 0.099 & 0.055 & 10.9 & 0.455 \\
\hline 6/21/2008 2:00 AM & 0.338 & 18.8 & 0.166 & 0.080 & 16.3 & 0.06 & 0.026 & 0.110 & 13.3 & 0.099 & 0.055 & 10.9 & 0.456 \\
\hline 6/21/2008 3:00 AM & 0.338 & 18.9 & 0.166 & 0.080 & 16.3 & 0.06 & 0.026 & 0.110 & 13.3 & 0.099 & 0.055 & 11.0 & 0.456 \\
\hline 6/21/2008 4:00 AM & 0.340 & 18.9 & 0.166 & 0.081 & 16.3 & 0.06 & 0.026 & 0.110 & 13.3 & 0.099 & 0.055 & 10.9 & 0.456 \\
\hline 6/21/2008 5:00 AM & 0.340 & 19.0 & 0.166 & 0.081 & 16.3 & 0.06 & 0.026 & 0.110 & 13.3 & 0.099 & 0.055 & 11.0 & 0.456 \\
\hline 6/21/2008 6:00 AM & 0.340 & 19.0 & 0.166 & 0.081 & 16.3 & 0.06 & 0.026 & 0.110 & 13.3 & 0.099 & 0.055 & 11.0 & 0.456 \\
\hline 6/21/2008 7:00 AM & 0.340 & 19.0 & 0.166 & 0.081 & 16.3 & 0.06 & 0.026 & 0.110 & 13.3 & 0.099 & 0.055 & 11.0 & 0.456 \\
\hline 6/21/2008 8:00 AM & 0.340 & 19.0 & 0.166 & 0.080 & 16.3 & 0.06 & 0.027 & 0.111 & 13.3 & 0.099 & 0.056 & 11.0 & 0.456 \\
\hline 6/21/2008 9:00 AM & 0.340 & 19.1 & 0.166 & 0.080 & 16.3 & 0.06 & 0.027 & 0.109 & 13.3 & 0.099 & 0.056 & 11.0 & 0.456 \\
\hline 6/21/2008 10:00 AM & 0.340 & 19.1 & 0.166 & 0.081 & 16.3 & 0.06 & 0.027 & 0.110 & 13.3 & 0.099 & 0.056 & 11.0 & 0.456 \\
\hline 6/21/2008 11:00 AM & 0.340 & 19.1 & 0.166 & 0.081 & 16.3 & 0.06 & 0.027 & 0.111 & 13.3 & 0.099 & 0.056 & 11.0 & 0.456 \\
\hline
\end{tabular}


Appendix 3. Volumetric water content, temperature, and electrical-conductivity data collected at the sprinkler-irrigated site during 2008.-Continued

[Depth in feet below land surface; volumetric water content dimensionless; temperature in degrees Celsius; bulk electrical conductivity in decisiemens per meter; --, no data]

\begin{tabular}{|c|c|c|c|c|c|c|c|c|c|c|c|c|c|}
\hline \multirow[b]{3}{*}{$\begin{array}{l}\text { Measurement } \\
\text { date and time }\end{array}$} & \multicolumn{13}{|c|}{ Depth } \\
\hline & \multicolumn{2}{|l|}{2} & \multirow{2}{*}{$\begin{array}{c}3 \\
\text { Volumetric } \\
\text { water } \\
\text { content }\end{array}$} & \multicolumn{3}{|c|}{4} & \multirow{2}{*}{$\begin{array}{c}5 \\
\text { Volumetric } \\
\text { water } \\
\text { content }\end{array}$} & \multicolumn{2}{|l|}{7} & \multirow{2}{*}{$\begin{array}{c}9 \\
\text { Volumetric } \\
\text { water } \\
\text { content }\end{array}$} & \multicolumn{2}{|c|}{13} & \multirow{2}{*}{$\begin{array}{c}16.5 \\
\text { Volumetric } \\
\text { water } \\
\text { content }\end{array}$} \\
\hline & $\begin{array}{c}\text { Volumetric } \\
\text { water } \\
\text { content }\end{array}$ & $\begin{array}{l}\text { Temper- } \\
\text { ature }\end{array}$ & & $\begin{array}{c}\text { Volumetric } \\
\text { water } \\
\text { content }\end{array}$ & $\begin{array}{l}\text { Temper- } \\
\text { ature }\end{array}$ & $\begin{array}{c}\text { Bulk } \\
\text { electrical } \\
\text { conduct- } \\
\text { ivity }\end{array}$ & & $\begin{array}{l}\text { Volumetric } \\
\text { water } \\
\text { content }\end{array}$ & $\begin{array}{l}\text { Temper- } \\
\text { ature }\end{array}$ & & $\begin{array}{l}\text { Volumetric } \\
\text { water } \\
\text { content }\end{array}$ & $\begin{array}{l}\text { Temper- } \\
\text { ature }\end{array}$ & \\
\hline 6/21/2008 12:00 PM & 0.340 & 19.1 & 0.166 & 0.081 & 16.3 & 0.06 & 0.027 & 0.110 & 13.3 & 0.099 & 0.056 & 11.0 & 0.456 \\
\hline 6/21/2008 1:00 PM & 0.338 & 19.1 & 0.166 & 0.081 & 16.3 & 0.06 & 0.027 & 0.110 & 13.3 & 0.099 & 0.056 & 11.0 & 0.456 \\
\hline 6/21/2008 2:00 PM & 0.340 & 19.0 & 0.166 & 0.081 & 16.3 & 0.06 & 0.027 & 0.110 & 13.4 & 0.099 & 0.056 & 11.0 & 0.456 \\
\hline 6/21/2008 3:00 PM & 0.340 & 19.0 & 0.166 & 0.081 & 16.3 & 0.06 & 0.027 & 0.110 & 13.4 & 0.099 & 0.056 & 11.0 & 0.456 \\
\hline 6/21/2008 4:00 PM & 0.340 & 19.0 & 0.166 & 0.081 & 16.4 & 0.06 & 0.027 & 0.110 & 13.4 & 0.099 & 0.056 & 11.0 & 0.456 \\
\hline 6/21/2008 5:00 PM & 0.340 & 19.0 & 0.166 & 0.081 & 16.4 & 0.06 & 0.027 & 0.110 & 13.4 & 0.099 & 0.056 & 11.0 & 0.456 \\
\hline 6/21/2008 6:00 PM & 0.340 & 19.0 & 0.166 & 0.081 & 16.4 & 0.06 & 0.027 & 0.110 & 13.4 & 0.099 & 0.056 & 11.0 & 0.456 \\
\hline 6/21/2008 7:00 PM & 0.338 & 19.0 & 0.166 & 0.081 & 16.4 & 0.06 & 0.027 & 0.110 & 13.3 & 0.099 & 0.056 & 11.0 & 0.456 \\
\hline 6/21/2008 8:00 PM & 0.340 & 19.0 & 0.166 & 0.081 & 16.4 & 0.06 & 0.027 & 0.111 & 13.4 & 0.099 & 0.056 & 11.0 & 0.456 \\
\hline 6/21/2008 9:00 PM & 0.340 & 19.0 & 0.166 & 0.081 & 16.4 & 0.06 & 0.027 & 0.110 & 13.4 & 0.099 & 0.056 & 11.0 & 0.456 \\
\hline 6/21/2008 10:00 PM & 0.340 & 19.0 & 0.166 & 0.081 & 16.4 & 0.06 & 0.026 & 0.110 & 13.4 & 0.099 & 0.056 & 11.0 & 0.456 \\
\hline 6/21/2008 11:00 PM & 0.340 & 19.1 & 0.166 & 0.081 & 16.4 & 0.06 & 0.026 & 0.111 & 13.4 & 0.099 & 0.056 & 11.0 & 0.455 \\
\hline 6/22/2008 12:00 AM & 0.340 & 19.2 & 0.166 & 0.081 & 16.4 & 0.06 & 0.026 & 0.111 & 13.4 & 0.099 & 0.056 & 11.0 & 0.456 \\
\hline 6/22/2008 1:00 AM & 0.338 & 19.2 & 0.166 & 0.081 & 16.4 & 0.06 & 0.026 & 0.111 & 13.4 & 0.099 & 0.055 & 11.0 & 0.456 \\
\hline 6/22/2008 2:00 AM & 0.340 & 19.3 & 0.166 & 0.081 & 16.4 & 0.06 & 0.026 & 0.111 & 13.4 & 0.099 & 0.056 & 11.0 & 0.456 \\
\hline 6/22/2008 3:00 AM & 0.340 & 19.3 & 0.166 & 0.081 & 16.4 & 0.06 & 0.026 & 0.110 & 13.4 & 0.099 & 0.055 & 11.0 & 0.456 \\
\hline 6/22/2008 4:00 AM & 0.340 & 19.4 & 0.166 & 0.081 & 16.4 & 0.06 & 0.026 & 0.110 & 13.4 & 0.099 & 0.056 & 11.0 & 0.456 \\
\hline 6/22/2008 5:00 AM & 0.341 & 19.4 & 0.166 & 0.081 & 16.4 & 0.06 & 0.026 & 0.110 & 13.4 & 0.099 & 0.055 & 11.0 & 0.456 \\
\hline 6/22/2008 6:00 AM & 0.341 & 19.5 & 0.166 & 0.081 & 16.4 & 0.06 & 0.027 & 0.110 & 13.4 & 0.099 & 0.055 & 11.0 & 0.455 \\
\hline 6/22/2008 7:00 AM & 0.341 & 19.5 & 0.166 & 0.081 & 16.4 & 0.06 & 0.026 & 0.111 & 13.4 & 0.099 & 0.055 & 11.0 & 0.456 \\
\hline 6/22/2008 8:00 AM & 0.340 & 19.5 & 0.166 & 0.080 & 16.4 & 0.06 & 0.027 & 0.110 & 13.4 & 0.099 & 0.056 & 11.0 & 0.456 \\
\hline 6/22/2008 9:00 AM & 0.341 & 19.5 & 0.166 & 0.081 & 16.5 & 0.06 & 0.027 & 0.111 & 13.4 & 0.099 & 0.056 & 11.0 & 0.456 \\
\hline 6/22/2008 10:00 AM & 0.341 & 19.6 & 0.166 & 0.081 & 16.4 & 0.06 & 0.027 & 0.111 & 13.4 & 0.099 & 0.056 & 11.1 & 0.456 \\
\hline 6/22/2008 11:00 AM & 0.341 & 19.6 & 0.166 & 0.081 & 16.5 & 0.06 & 0.027 & 0.110 & 13.4 & 0.099 & 0.056 & 11.1 & 0.456 \\
\hline 6/22/2008 12:00 PM & 0.341 & 19.6 & 0.166 & 0.081 & 16.5 & 0.06 & 0.027 & 0.110 & 13.4 & 0.099 & 0.056 & 11.0 & 0.456 \\
\hline 6/22/2008 1:00 PM & 0.341 & 19.6 & 0.166 & 0.081 & 16.5 & 0.06 & 0.027 & 0.110 & 13.4 & 0.099 & 0.056 & 11.1 & 0.456 \\
\hline 6/22/2008 2:00 PM & 0.341 & 19.5 & 0.166 & 0.081 & 16.5 & 0.06 & 0.027 & 0.110 & 13.4 & 0.099 & 0.056 & 11.1 & 0.456 \\
\hline 6/22/2008 3:00 PM & 0.341 & 19.6 & 0.166 & 0.081 & 16.5 & 0.06 & 0.027 & 0.110 & 13.4 & 0.099 & 0.056 & 11.0 & 0.456 \\
\hline 6/22/2008 4:00 PM & 0.341 & 19.5 & 0.166 & 0.081 & 16.5 & 0.06 & 0.027 & 0.110 & 13.4 & 0.099 & 0.056 & 11.0 & 0.455 \\
\hline 6/22/2008 5:00 PM & 0.341 & 19.5 & 0.166 & 0.081 & 16.5 & 0.06 & 0.027 & 0.110 & 13.5 & 0.099 & 0.056 & 11.1 & 0.456 \\
\hline 6/22/2008 6:00 PM & 0.341 & 19.5 & 0.166 & 0.081 & 16.5 & 0.06 & 0.027 & 0.110 & 13.5 & 0.099 & 0.056 & 11.1 & 0.456 \\
\hline 6/22/2008 7:00 PM & 0.340 & 19.5 & 0.166 & 0.081 & 16.5 & 0.06 & 0.027 & 0.111 & 13.5 & 0.099 & 0.056 & 11.0 & 0.456 \\
\hline 6/22/2008 8:00 PM & 0.340 & 19.5 & 0.166 & 0.081 & 16.5 & 0.06 & 0.027 & 0.111 & 13.5 & 0.099 & 0.056 & 11.1 & 0.456 \\
\hline 6/22/2008 9:00 PM & 0.340 & 19.5 & 0.166 & 0.081 & 16.5 & 0.06 & 0.027 & 0.111 & 13.5 & 0.099 & 0.056 & 11.0 & 0.456 \\
\hline 6/22/2008 10:00 PM & 0.340 & 19.6 & 0.166 & 0.081 & 16.6 & 0.06 & 0.027 & 0.110 & 13.5 & 0.099 & 0.056 & 11.1 & 0.456 \\
\hline
\end{tabular}


Appendix 3. Volumetric water content, temperature, and electrical-conductivity data collected at the sprinkler-irrigated site during 2008.-Continued

[Depth in feet below land surface; volumetric water content dimensionless; temperature in degrees Celsius; bulk electrical conductivity in decisiemens per meter; --, no data]

\begin{tabular}{|c|c|c|c|c|c|c|c|c|c|c|c|c|c|}
\hline \multirow[b]{3}{*}{$\begin{array}{l}\text { Measurement } \\
\text { date and time }\end{array}$} & \multicolumn{13}{|c|}{ Depth } \\
\hline & \multicolumn{2}{|l|}{2} & \multirow{2}{*}{$\begin{array}{c}3 \\
\text { Volumetric } \\
\text { water } \\
\text { content }\end{array}$} & \multicolumn{3}{|c|}{4} & \multirow{2}{*}{$\begin{array}{c}5 \\
\text { Volumetric } \\
\text { water } \\
\text { content }\end{array}$} & \multicolumn{2}{|l|}{7} & \multirow{2}{*}{$\begin{array}{c}9 \\
\text { Volumetric } \\
\text { water } \\
\text { content }\end{array}$} & \multicolumn{2}{|c|}{13} & \multirow{2}{*}{$\begin{array}{c}16.5 \\
\text { Volumetric } \\
\text { water } \\
\text { content }\end{array}$} \\
\hline & $\begin{array}{c}\text { Volumetric } \\
\text { water } \\
\text { content }\end{array}$ & $\begin{array}{l}\text { Temper- } \\
\text { ature }\end{array}$ & & $\begin{array}{c}\text { Volumetric } \\
\text { water } \\
\text { content }\end{array}$ & $\begin{array}{l}\text { Temper- } \\
\text { ature }\end{array}$ & $\begin{array}{c}\text { Bulk } \\
\text { electrical } \\
\text { conduct- } \\
\text { ivity }\end{array}$ & & $\begin{array}{l}\text { Volumetric } \\
\text { water } \\
\text { content }\end{array}$ & $\begin{array}{l}\text { Temper- } \\
\text { ature }\end{array}$ & & $\begin{array}{l}\text { Volumetric } \\
\text { water } \\
\text { content }\end{array}$ & $\begin{array}{l}\text { Temper- } \\
\text { ature }\end{array}$ & \\
\hline 6/22/2008 11:00 PM & 0.341 & 19.6 & 0.166 & 0.081 & 16.5 & 0.06 & 0.027 & 0.110 & 13.5 & 0.099 & 0.056 & 11.0 & 0.455 \\
\hline 6/23/2008 12:00 AM & 0.341 & 19.6 & 0.166 & 0.081 & 16.6 & 0.06 & 0.027 & 0.110 & 13.5 & 0.099 & 0.056 & 11.0 & 0.456 \\
\hline 6/23/2008 1:00 AM & 0.341 & 19.6 & 0.166 & 0.081 & 16.6 & 0.06 & 0.026 & 0.110 & 13.5 & 0.099 & 0.056 & 11.1 & 0.456 \\
\hline 6/23/2008 2:00 AM & 0.341 & 19.7 & 0.166 & 0.081 & 16.6 & 0.06 & 0.026 & 0.111 & 13.5 & 0.099 & 0.056 & 11.1 & 0.456 \\
\hline 6/23/2008 3:00 AM & 0.341 & 19.7 & 0.166 & 0.081 & 16.6 & 0.06 & 0.026 & 0.111 & 13.5 & 0.099 & 0.055 & 11.1 & 0.456 \\
\hline 6/23/2008 4:00 AM & 0.341 & 19.7 & 0.166 & 0.081 & 16.6 & 0.06 & 0.026 & 0.111 & 13.5 & 0.099 & 0.055 & 11.1 & 0.455 \\
\hline 6/23/2008 5:00 AM & 0.341 & 19.8 & 0.166 & 0.081 & 16.6 & 0.06 & 0.026 & 0.111 & 13.5 & 0.099 & 0.056 & 11.1 & 0.455 \\
\hline 6/23/2008 6:00 AM & 0.341 & 19.8 & 0.166 & 0.081 & 16.6 & 0.06 & 0.026 & 0.111 & 13.5 & 0.099 & 0.056 & 11.1 & 0.455 \\
\hline 6/23/2008 7:00 AM & 0.341 & 19.8 & 0.166 & 0.081 & 16.6 & 0.06 & 0.026 & 0.111 & 13.5 & 0.100 & 0.056 & 11.1 & 0.456 \\
\hline 6/23/2008 8:00 AM & 0.341 & 19.8 & 0.166 & 0.081 & 16.6 & 0.06 & 0.027 & 0.110 & 13.5 & 0.100 & 0.056 & 11.1 & 0.456 \\
\hline 6/23/2008 9:00 AM & 0.342 & 19.8 & 0.166 & 0.081 & 16.6 & 0.06 & 0.027 & 0.111 & 13.5 & 0.100 & 0.056 & 11.1 & 0.456 \\
\hline 6/23/2008 10:00 AM & 0.343 & 19.8 & 0.166 & 0.081 & 16.6 & 0.06 & 0.027 & 0.111 & 13.5 & 0.100 & 0.056 & 11.1 & 0.456 \\
\hline 6/23/2008 11:00 AM & 0.342 & 19.8 & 0.166 & 0.081 & 16.6 & 0.06 & 0.027 & 0.111 & 13.5 & 0.100 & 0.056 & 11.1 & 0.456 \\
\hline 6/23/2008 12:00 PM & 0.341 & 19.8 & 0.166 & 0.081 & 16.7 & 0.06 & 0.027 & 0.110 & 13.5 & 0.100 & 0.056 & 11.1 & 0.456 \\
\hline 6/23/2008 1:00 PM & 0.341 & 19.8 & 0.166 & 0.081 & 16.7 & 0.06 & 0.027 & 0.110 & 13.5 & 0.100 & 0.056 & 11.1 & 0.456 \\
\hline 6/23/2008 2:00 PM & 0.341 & 19.8 & 0.166 & 0.081 & 16.7 & 0.06 & 0.027 & 0.110 & 13.5 & 0.100 & 0.056 & 11.1 & 0.456 \\
\hline 6/23/2008 3:00 PM & 0.342 & 19.8 & 0.166 & 0.081 & 16.7 & 0.06 & 0.027 & 0.111 & 13.5 & 0.100 & 0.056 & 11.1 & 0.456 \\
\hline 6/23/2008 4:00 PM & 0.342 & 19.8 & 0.166 & 0.081 & 16.7 & 0.06 & 0.027 & 0.111 & 13.5 & 0.100 & 0.056 & 11.1 & 0.456 \\
\hline 6/23/2008 5:00 PM & 0.342 & 19.7 & 0.166 & 0.081 & 16.7 & 0.06 & 0.027 & 0.110 & 13.5 & 0.100 & 0.056 & 11.1 & 0.456 \\
\hline 6/23/2008 6:00 PM & 0.342 & 19.8 & 0.166 & 0.081 & 16.7 & 0.06 & 0.027 & 0.111 & 13.5 & 0.100 & 0.056 & 11.1 & 0.456 \\
\hline 6/23/2008 7:00 PM & 0.342 & 19.7 & 0.166 & 0.081 & 16.7 & 0.06 & 0.027 & 0.111 & 13.5 & 0.100 & 0.056 & 11.1 & 0.456 \\
\hline 6/23/2008 8:00 PM & 0.341 & 19.8 & 0.166 & 0.081 & 16.7 & 0.06 & 0.027 & 0.111 & 13.6 & 0.100 & 0.056 & 11.1 & 0.456 \\
\hline 6/23/2008 9:00 PM & 0.341 & 19.7 & 0.166 & 0.081 & 16.7 & 0.06 & 0.027 & 0.111 & 13.6 & 0.100 & 0.056 & 11.1 & 0.456 \\
\hline 6/23/2008 10:00 PM & 0.341 & 19.8 & 0.166 & 0.081 & 16.7 & 0.06 & 0.027 & 0.110 & 13.6 & 0.100 & 0.056 & 11.1 & 0.456 \\
\hline 6/23/2008 11:00 PM & 0.341 & 19.8 & 0.166 & 0.081 & 16.8 & 0.06 & 0.027 & 0.110 & 13.6 & 0.100 & 0.056 & 11.1 & 0.456 \\
\hline 6/24/2008 12:00 AM & 0.340 & 19.8 & 0.166 & 0.081 & 16.8 & 0.06 & 0.027 & 0.110 & 13.6 & 0.100 & 0.056 & 11.1 & 0.456 \\
\hline 6/24/2008 1:00 AM & 0.341 & 19.8 & 0.166 & 0.081 & 16.8 & 0.06 & 0.027 & 0.110 & 13.6 & 0.100 & 0.056 & 11.1 & 0.456 \\
\hline 6/24/2008 2:00 AM & 0.341 & 19.9 & 0.166 & 0.081 & 16.8 & 0.06 & 0.027 & 0.110 & 13.6 & 0.100 & 0.056 & 11.1 & 0.456 \\
\hline 6/24/2008 3:00 AM & 0.342 & 19.9 & 0.166 & 0.081 & 16.8 & 0.06 & 0.027 & 0.111 & 13.6 & 0.100 & 0.056 & 11.1 & 0.456 \\
\hline 6/24/2008 4:00 AM & 0.342 & 19.9 & 0.166 & 0.081 & 16.8 & 0.06 & 0.027 & 0.111 & 13.6 & 0.100 & 0.056 & 11.1 & 0.456 \\
\hline 6/24/2008 5:00 AM & 0.343 & 19.9 & 0.166 & 0.081 & 16.8 & 0.06 & 0.027 & 0.112 & 13.6 & 0.100 & 0.055 & 11.1 & 0.456 \\
\hline 6/24/2008 6:00 AM & 0.342 & 20.0 & 0.166 & 0.081 & 16.8 & 0.06 & 0.027 & 0.112 & 13.6 & 0.100 & 0.056 & 11.1 & 0.456 \\
\hline $6 / 24 / 2008$ 7:00 AM & 0.342 & 20.0 & 0.166 & 0.081 & 16.8 & 0.06 & 0.027 & 0.111 & 13.6 & 0.100 & 0.056 & 11.1 & 0.456 \\
\hline 6/24/2008 8:00 AM & 0.341 & 20.0 & 0.166 & 0.081 & 16.8 & 0.06 & 0.027 & 0.111 & 13.6 & 0.100 & 0.056 & 11.1 & 0.456 \\
\hline 6/24/2008 9:00 AM & 0.341 & 20.0 & 0.166 & 0.081 & 16.8 & 0.06 & 0.027 & 0.112 & 13.6 & 0.100 & 0.056 & 11.1 & 0.456 \\
\hline
\end{tabular}


Appendix 3. Volumetric water content, temperature, and electrical-conductivity data collected at the sprinkler-irrigated site during 2008.-Continued

[Depth in feet below land surface; volumetric water content dimensionless; temperature in degrees Celsius; bulk electrical conductivity in decisiemens per meter; --, no data]

\begin{tabular}{|c|c|c|c|c|c|c|c|c|c|c|c|c|c|}
\hline \multirow[b]{3}{*}{$\begin{array}{l}\text { Measurement } \\
\text { date and time }\end{array}$} & \multicolumn{13}{|c|}{ Depth } \\
\hline & \multicolumn{2}{|l|}{2} & \multirow{2}{*}{$\begin{array}{c}3 \\
\begin{array}{c}\text { Volumetric } \\
\text { water } \\
\text { content }\end{array}\end{array}$} & \multicolumn{3}{|c|}{4} & \multirow{2}{*}{$\begin{array}{c}5 \\
\text { Volumetric } \\
\text { water } \\
\text { content }\end{array}$} & \multicolumn{2}{|c|}{7} & \multirow{2}{*}{$\begin{array}{c}9 \\
\text { Volumetric } \\
\text { water } \\
\text { content }\end{array}$} & \multicolumn{2}{|c|}{13} & \multirow{2}{*}{$\begin{array}{c}16.5 \\
\text { Volumetric } \\
\text { water } \\
\text { content }\end{array}$} \\
\hline & $\begin{array}{c}\text { Volumetric } \\
\text { water } \\
\text { content }\end{array}$ & $\begin{array}{l}\text { Temper- } \\
\text { ature }\end{array}$ & & $\begin{array}{c}\text { Volumetric } \\
\text { water } \\
\text { content }\end{array}$ & $\begin{array}{l}\text { Temper- } \\
\text { ature }\end{array}$ & $\begin{array}{c}\text { Bulk } \\
\text { electrical } \\
\text { conduct- } \\
\text { ivity }\end{array}$ & & $\begin{array}{c}\text { Volumetric } \\
\text { water } \\
\text { content }\end{array}$ & $\begin{array}{l}\text { Temper- } \\
\text { ature }\end{array}$ & & $\begin{array}{c}\text { Volumetric } \\
\text { water } \\
\text { content }\end{array}$ & $\begin{array}{l}\text { Temper- } \\
\text { ature }\end{array}$ & \\
\hline 6/24/2008 10:00 AM & 0.341 & 20.0 & 0.166 & 0.081 & 16.8 & 0.06 & 0.027 & 0.111 & 13.6 & 0.100 & 0.056 & 11.1 & 0.456 \\
\hline 6/24/2008 11:00 AM & 0.341 & 20.0 & 0.166 & 0.081 & 16.8 & 0.06 & 0.027 & 0.111 & 13.6 & 0.100 & 0.056 & 11.2 & 0.456 \\
\hline 6/24/2008 12:00 PM & 0.342 & 20.0 & 0.166 & 0.081 & 16.8 & 0.06 & 0.027 & 0.110 & 13.6 & 0.100 & 0.056 & 11.2 & 0.456 \\
\hline 6/24/2008 1:00 PM & 0.342 & 19.9 & 0.166 & 0.081 & 16.8 & 0.06 & 0.027 & 0.110 & 13.6 & 0.100 & 0.056 & 11.2 & 0.456 \\
\hline 6/24/2008 2:00 PM & 0.342 & 19.9 & 0.166 & 0.081 & 16.9 & 0.06 & 0.027 & 0.111 & 13.6 & 0.100 & 0.056 & 11.1 & 0.456 \\
\hline 6/24/2008 3:00 PM & 0.342 & 19.9 & 0.166 & 0.081 & 16.9 & 0.06 & 0.027 & 0.111 & 13.6 & 0.100 & 0.056 & 11.2 & 0.456 \\
\hline 6/24/2008 4:00 PM & 0.341 & 19.9 & 0.166 & 0.081 & 16.9 & 0.06 & 0.027 & 0.111 & 13.7 & 0.100 & 0.056 & 11.1 & 0.456 \\
\hline 6/24/2008 5:00 PM & 0.342 & 19.9 & 0.166 & 0.081 & 16.9 & 0.06 & 0.027 & 0.111 & 13.6 & 0.100 & 0.056 & 11.2 & 0.456 \\
\hline 6/24/2008 6:00 PM & 0.341 & 19.9 & 0.166 & 0.081 & 16.9 & 0.06 & 0.027 & 0.110 & 13.6 & 0.100 & 0.056 & 11.2 & 0.456 \\
\hline 6/24/2008 7:00 PM & 0.340 & 19.9 & 0.166 & 0.082 & 16.9 & 0.06 & 0.027 & 0.111 & 13.6 & 0.100 & 0.056 & 11.2 & 0.456 \\
\hline 6/24/2008 8:00 PM & 0.341 & 19.9 & 0.166 & 0.081 & 16.9 & 0.06 & 0.027 & 0.111 & 13.7 & 0.100 & 0.056 & 11.2 & 0.456 \\
\hline 6/24/2008 9:00 PM & 0.342 & 19.9 & 0.166 & 0.081 & 16.9 & 0.06 & 0.027 & 0.112 & 13.7 & 0.100 & 0.056 & 11.1 & 0.456 \\
\hline 6/24/2008 10:00 PM & 0.342 & 20.0 & 0.166 & 0.081 & 16.9 & 0.06 & 0.027 & 0.111 & 13.7 & 0.100 & 0.056 & 11.2 & 0.456 \\
\hline 6/24/2008 11:00 PM & 0.341 & 20.0 & 0.166 & 0.082 & 16.9 & 0.06 & 0.027 & 0.110 & 13.6 & 0.100 & 0.056 & 11.1 & 0.456 \\
\hline 6/25/2008 12:00 AM & 0.341 & 20.0 & 0.166 & 0.082 & 16.9 & 0.06 & 0.027 & 0.110 & 13.7 & 0.100 & 0.056 & 11.1 & 0.456 \\
\hline 6/25/2008 1:00 AM & 0.342 & 20.1 & 0.166 & 0.082 & 16.9 & 0.06 & 0.027 & 0.110 & 13.7 & 0.100 & 0.056 & 11.1 & 0.456 \\
\hline 6/25/2008 2:00 AM & 0.343 & 20.1 & 0.166 & 0.081 & 17.0 & 0.06 & 0.027 & 0.110 & 13.7 & 0.100 & 0.056 & 11.1 & 0.456 \\
\hline 6/25/2008 3:00 AM & 0.342 & 20.2 & 0.166 & 0.081 & 17.0 & 0.06 & 0.027 & 0.110 & 13.7 & 0.100 & 0.055 & 11.2 & 0.456 \\
\hline 6/25/2008 4:00 AM & 0.341 & 20.3 & 0.166 & 0.081 & 17.0 & 0.06 & 0.027 & 0.110 & 13.7 & 0.100 & 0.055 & 11.1 & 0.456 \\
\hline 6/25/2008 5:00 AM & 0.341 & 20.3 & 0.166 & 0.081 & 17.0 & 0.06 & 0.027 & 0.111 & 13.7 & 0.100 & 0.056 & 11.1 & 0.456 \\
\hline 6/25/2008 6:00 AM & 0.342 & 20.3 & 0.166 & 0.082 & 17.0 & 0.06 & 0.027 & 0.111 & 13.7 & 0.100 & 0.056 & 11.1 & 0.456 \\
\hline 6/25/2008 7:00 AM & 0.343 & 20.3 & 0.166 & 0.082 & 17.0 & 0.06 & 0.027 & 0.110 & 13.7 & 0.100 & 0.056 & 11.2 & 0.456 \\
\hline 6/25/2008 8:00 AM & 0.344 & 20.3 & 0.166 & 0.082 & 17.0 & 0.06 & 0.027 & 0.111 & 13.7 & 0.100 & 0.056 & 11.1 & 0.456 \\
\hline 6/25/2008 9:00 AM & 0.344 & 20.3 & 0.166 & 0.082 & 17.0 & 0.06 & 0.027 & 0.112 & 13.7 & 0.100 & 0.056 & 11.2 & 0.456 \\
\hline 6/25/2008 10:00 AM & 0.344 & 20.3 & 0.166 & 0.082 & 17.0 & 0.06 & 0.027 & 0.111 & 13.7 & 0.100 & 0.056 & 11.2 & 0.456 \\
\hline 6/25/2008 11:00 AM & 0.343 & 20.3 & 0.166 & 0.082 & 17.0 & 0.06 & 0.027 & 0.111 & 13.7 & 0.100 & 0.056 & 11.2 & 0.456 \\
\hline 6/25/2008 12:00 PM & 0.343 & 20.3 & 0.166 & 0.082 & 17.0 & 0.06 & 0.027 & 0.111 & 13.7 & 0.100 & 0.056 & 11.2 & 0.456 \\
\hline 6/25/2008 1:00 PM & 0.343 & 20.3 & 0.166 & 0.082 & 17.0 & 0.06 & 0.028 & 0.110 & 13.7 & 0.100 & 0.056 & 11.2 & 0.456 \\
\hline 6/25/2008 2:00 PM & 0.343 & 20.2 & 0.166 & 0.082 & 17.1 & 0.06 & 0.028 & 0.110 & 13.7 & 0.100 & 0.056 & 11.2 & 0.456 \\
\hline 6/25/2008 3:00 PM & 0.343 & 20.3 & 0.166 & 0.082 & 17.1 & 0.06 & 0.028 & 0.111 & 13.7 & 0.100 & 0.056 & 11.2 & 0.456 \\
\hline 6/25/2008 4:00 PM & 0.341 & 20.2 & 0.166 & 0.082 & 17.1 & 0.06 & 0.028 & 0.112 & 13.7 & 0.100 & 0.056 & 11.2 & 0.456 \\
\hline 6/25/2008 5:00 PM & 0.341 & 20.2 & 0.166 & 0.082 & 17.1 & 0.06 & 0.028 & 0.111 & 13.7 & 0.100 & 0.056 & 11.2 & 0.456 \\
\hline 6/25/2008 6:00 PM & 0.341 & 20.2 & 0.166 & 0.082 & 17.1 & 0.06 & 0.028 & 0.111 & 13.8 & 0.100 & 0.056 & 11.2 & 0.456 \\
\hline 6/25/2008 7:00 PM & 0.341 & 20.2 & 0.166 & 0.082 & 17.1 & 0.06 & 0.028 & 0.111 & 13.8 & 0.100 & 0.056 & 11.2 & 0.456 \\
\hline 6/25/2008 8:00 PM & 0.342 & 20.1 & 0.166 & 0.082 & 17.1 & 0.06 & 0.027 & 0.112 & 13.7 & 0.100 & 0.056 & 11.2 & 0.456 \\
\hline
\end{tabular}


Appendix 3. Volumetric water content, temperature, and electrical-conductivity data collected at the sprinkler-irrigated site during 2008.-Continued

[Depth in feet below land surface; volumetric water content dimensionless; temperature in degrees Celsius; bulk electrical conductivity in decisiemens per meter; --, no data]

\begin{tabular}{|c|c|c|c|c|c|c|c|c|c|c|c|c|c|}
\hline \multirow[b]{3}{*}{$\begin{array}{l}\text { Measurement } \\
\text { date and time }\end{array}$} & \multicolumn{13}{|c|}{ Depth } \\
\hline & \multicolumn{2}{|l|}{2} & \multirow{2}{*}{$\begin{array}{c}3 \\
\text { Volumetric } \\
\text { water } \\
\text { content }\end{array}$} & \multicolumn{3}{|c|}{4} & \multirow{2}{*}{$\begin{array}{c}5 \\
\text { Volumetric } \\
\text { water } \\
\text { content }\end{array}$} & \multicolumn{2}{|l|}{7} & \multirow{2}{*}{$\begin{array}{c}9 \\
\text { Volumetric } \\
\text { water } \\
\text { content }\end{array}$} & \multicolumn{2}{|c|}{13} & \multirow{2}{*}{$\begin{array}{c}16.5 \\
\text { Volumetric } \\
\text { water } \\
\text { content }\end{array}$} \\
\hline & $\begin{array}{c}\text { Volumetric } \\
\text { water } \\
\text { content }\end{array}$ & $\begin{array}{l}\text { Temper- } \\
\text { ature }\end{array}$ & & $\begin{array}{c}\text { Volumetric } \\
\text { water } \\
\text { content }\end{array}$ & $\begin{array}{l}\text { Temper- } \\
\text { ature }\end{array}$ & $\begin{array}{c}\text { Bulk } \\
\text { electrical } \\
\text { conduct- } \\
\text { ivity }\end{array}$ & & $\begin{array}{l}\text { Volumetric } \\
\text { water } \\
\text { content }\end{array}$ & $\begin{array}{l}\text { Temper- } \\
\text { ature }\end{array}$ & & $\begin{array}{l}\text { Volumetric } \\
\text { water } \\
\text { content }\end{array}$ & $\begin{array}{l}\text { Temper- } \\
\text { ature }\end{array}$ & \\
\hline 6/25/2008 9:00 PM & 0.343 & 20.1 & 0.166 & 0.082 & 17.1 & 0.06 & 0.027 & 0.112 & 13.7 & 0.100 & 0.056 & 11.2 & 0.457 \\
\hline 6/25/2008 10:00 PM & 0.343 & 20.1 & 0.166 & 0.082 & 17.1 & 0.06 & 0.027 & 0.112 & 13.8 & 0.100 & 0.056 & 11.2 & 0.457 \\
\hline 6/25/2008 11:00 PM & 0.343 & 20.1 & 0.166 & 0.082 & 17.1 & 0.06 & 0.027 & 0.112 & 13.8 & 0.100 & 0.056 & 11.2 & 0.457 \\
\hline 6/26/2008 12:00 AM & 0.342 & 20.1 & 0.166 & 0.082 & 17.1 & 0.06 & 0.027 & 0.112 & 13.8 & 0.100 & 0.056 & 11.2 & 0.457 \\
\hline 6/26/2008 1:00 AM & 0.342 & 20.1 & 0.166 & 0.082 & 17.1 & 0.06 & 0.027 & 0.111 & 13.8 & 0.100 & 0.056 & 11.1 & 0.457 \\
\hline 6/26/2008 2:00 AM & 0.343 & 20.1 & 0.166 & 0.082 & 17.1 & 0.06 & 0.027 & 0.111 & 13.8 & 0.100 & 0.056 & 11.2 & 0.457 \\
\hline 6/26/2008 3:00 AM & 0.343 & 20.2 & 0.166 & 0.082 & 17.1 & 0.06 & 0.027 & 0.111 & 13.8 & 0.100 & 0.056 & 11.2 & 0.457 \\
\hline 6/26/2008 4:00 AM & 0.342 & 20.1 & 0.166 & 0.082 & 17.2 & 0.06 & 0.027 & 0.111 & 13.8 & 0.100 & 0.056 & 11.2 & 0.457 \\
\hline 6/26/2008 5:00 AM & 0.342 & 20.1 & 0.166 & 0.082 & 17.2 & 0.06 & 0.027 & 0.111 & 13.8 & 0.100 & 0.056 & 11.2 & 0.457 \\
\hline 6/26/2008 6:00 AM & 0.341 & 20.1 & 0.166 & 0.082 & 17.2 & 0.06 & 0.027 & 0.111 & 13.8 & 0.100 & 0.055 & 11.2 & 0.457 \\
\hline 6/26/2008 7:00 AM & 0.342 & 20.1 & 0.166 & 0.082 & 17.2 & 0.06 & 0.027 & 0.111 & 13.8 & 0.100 & 0.056 & 11.2 & 0.457 \\
\hline 6/26/2008 8:00 AM & 0.342 & 20.1 & 0.166 & 0.082 & 17.2 & 0.06 & 0.027 & 0.112 & 13.8 & 0.100 & 0.056 & 11.2 & 0.457 \\
\hline 6/26/2008 9:00 AM & 0.342 & 20.1 & 0.166 & 0.082 & 17.2 & 0.06 & 0.027 & 0.111 & 13.8 & 0.100 & 0.056 & 11.2 & 0.457 \\
\hline 6/26/2008 10:00 AM & 0.342 & 20.1 & 0.166 & 0.082 & 17.2 & 0.06 & 0.028 & 0.111 & 13.8 & 0.100 & 0.056 & 11.3 & 0.457 \\
\hline 6/26/2008 11:00 AM & 0.342 & 20.0 & 0.166 & 0.082 & 17.2 & 0.06 & 0.028 & 0.111 & 13.9 & 0.100 & 0.056 & 11.2 & 0.457 \\
\hline 6/26/2008 12:00 PM & 0.342 & 20.0 & 0.166 & 0.082 & 17.2 & 0.06 & 0.028 & 0.111 & 13.8 & 0.101 & 0.056 & 11.3 & 0.457 \\
\hline 6/26/2008 1:00 PM & 0.341 & 19.9 & 0.166 & 0.082 & 17.2 & 0.06 & 0.028 & 0.111 & 13.8 & 0.101 & 0.056 & 11.3 & 0.456 \\
\hline 6/26/2008 2:00 PM & 0.341 & 19.9 & 0.166 & 0.082 & 17.2 & 0.06 & 0.028 & 0.111 & 13.8 & 0.101 & 0.056 & 11.3 & 0.456 \\
\hline 6/26/2008 3:00 PM & 0.341 & 19.9 & 0.166 & 0.082 & 17.3 & 0.06 & 0.028 & 0.111 & 13.8 & 0.101 & 0.056 & 11.3 & 0.456 \\
\hline 6/26/2008 4:00 PM & 0.342 & 19.9 & 0.166 & 0.082 & 17.3 & 0.06 & 0.028 & 0.112 & 13.9 & 0.100 & 0.056 & 11.3 & 0.457 \\
\hline 6/26/2008 5:00 PM & 0.343 & 19.8 & 0.166 & 0.082 & 17.3 & 0.06 & 0.028 & 0.111 & 13.9 & 0.101 & 0.056 & 11.2 & 0.456 \\
\hline 6/26/2008 6:00 PM & 0.342 & 19.8 & 0.166 & 0.082 & 17.3 & 0.06 & 0.028 & 0.111 & 13.9 & 0.101 & 0.056 & 11.2 & 0.456 \\
\hline 6/26/2008 7:00 PM & 0.342 & 19.8 & 0.166 & 0.082 & 17.3 & 0.06 & 0.028 & 0.111 & 13.8 & 0.101 & 0.056 & 11.3 & 0.456 \\
\hline 6/26/2008 8:00 PM & 0.342 & 19.8 & 0.166 & 0.082 & 17.3 & 0.06 & 0.028 & 0.112 & 13.9 & 0.101 & 0.056 & 11.3 & 0.457 \\
\hline 6/26/2008 9:00 PM & 0.341 & 19.8 & 0.166 & 0.082 & 17.3 & 0.06 & 0.028 & 0.111 & 13.9 & 0.101 & 0.056 & 11.3 & 0.457 \\
\hline 6/26/2008 10:00 PM & 0.341 & 19.8 & 0.166 & 0.082 & 17.3 & 0.06 & 0.027 & 0.112 & 13.9 & 0.101 & 0.056 & 11.2 & 0.457 \\
\hline 6/26/2008 11:00 PM & 0.342 & 19.8 & 0.166 & 0.082 & 17.3 & 0.06 & 0.027 & 0.112 & 13.9 & 0.101 & 0.056 & 11.2 & 0.457 \\
\hline 6/27/2008 12:00 AM & 0.342 & 19.8 & 0.166 & 0.082 & 17.3 & 0.06 & 0.028 & 0.112 & 13.9 & 0.101 & 0.056 & 11.2 & 0.457 \\
\hline 6/27/2008 1:00 AM & 0.342 & 19.8 & 0.166 & 0.082 & 17.3 & 0.06 & 0.027 & 0.112 & 13.9 & 0.101 & 0.056 & 11.2 & 0.457 \\
\hline 6/27/2008 2:00 AM & 0.342 & 19.8 & 0.166 & 0.082 & 17.3 & 0.06 & 0.027 & 0.112 & 13.9 & 0.101 & 0.056 & 11.2 & 0.457 \\
\hline 6/27/2008 3:00 AM & 0.342 & 19.8 & 0.166 & 0.082 & 17.3 & 0.06 & 0.027 & 0.112 & 13.9 & 0.101 & 0.056 & 11.2 & 0.457 \\
\hline 6/27/2008 4:00 AM & 0.342 & 19.8 & 0.166 & 0.082 & 17.3 & 0.06 & 0.027 & 0.111 & 13.9 & 0.101 & 0.055 & 11.2 & 0.457 \\
\hline 6/27/2008 5:00 AM & 0.342 & 19.8 & 0.166 & 0.082 & 17.3 & 0.06 & 0.027 & 0.111 & 13.9 & 0.101 & 0.056 & 11.2 & 0.457 \\
\hline $6 / 27 / 2008$ 6:00 AM & 0.342 & 19.8 & 0.166 & 0.082 & 17.3 & 0.06 & 0.027 & 0.111 & 13.9 & 0.101 & 0.055 & 11.3 & 0.456 \\
\hline 6/27/2008 7:00 AM & 0.342 & 19.8 & 0.166 & 0.082 & 17.3 & 0.06 & 0.027 & 0.112 & 13.9 & 0.101 & 0.056 & 11.3 & 0.457 \\
\hline
\end{tabular}


Appendix 3. Volumetric water content, temperature, and electrical-conductivity data collected at the sprinkler-irrigated site during 2008.-Continued

[Depth in feet below land surface; volumetric water content dimensionless; temperature in degrees Celsius; bulk electrical conductivity in decisiemens per meter; --, no data]

\begin{tabular}{|c|c|c|c|c|c|c|c|c|c|c|c|c|c|}
\hline \multirow[b]{3}{*}{$\begin{array}{l}\text { Measurement } \\
\text { date and time }\end{array}$} & \multicolumn{13}{|c|}{ Depth } \\
\hline & \multicolumn{2}{|l|}{2} & \multirow{2}{*}{$\begin{array}{c}3 \\
\text { Volumetric } \\
\text { water } \\
\text { content }\end{array}$} & \multicolumn{3}{|c|}{4} & \multirow{2}{*}{$\begin{array}{c}5 \\
\text { Volumetric } \\
\text { water } \\
\text { content }\end{array}$} & \multicolumn{2}{|c|}{7} & \multirow[b]{2}{*}{$\begin{array}{c}9 \\
\text { Volumetric } \\
\text { water } \\
\text { content }\end{array}$} & \multicolumn{2}{|c|}{13} & \multirow{2}{*}{$\begin{array}{c}16.5 \\
\text { Volumetric } \\
\text { water } \\
\text { content }\end{array}$} \\
\hline & $\begin{array}{c}\text { Volumetric } \\
\text { water } \\
\text { content }\end{array}$ & $\begin{array}{l}\text { Temper- } \\
\text { ature }\end{array}$ & & $\begin{array}{c}\text { Volumetric } \\
\text { water } \\
\text { content }\end{array}$ & $\begin{array}{l}\text { Temper- } \\
\text { ature }\end{array}$ & $\begin{array}{c}\text { Bulk } \\
\text { electrical } \\
\text { conduct- } \\
\text { ivity }\end{array}$ & & $\begin{array}{c}\text { Volumetric } \\
\text { water } \\
\text { content }\end{array}$ & $\begin{array}{l}\text { Temper- } \\
\text { ature }\end{array}$ & & $\begin{array}{c}\text { Volumetric } \\
\text { water } \\
\text { content }\end{array}$ & $\begin{array}{l}\text { Temper- } \\
\text { ature }\end{array}$ & \\
\hline 6/27/2008 8:00 AM & 0.342 & 19.8 & 0.166 & 0.082 & 17.4 & 0.06 & 0.028 & 0.112 & 13.9 & 0.101 & 0.056 & 11.2 & 0.457 \\
\hline 6/27/2008 9:00 AM & 0.342 & 19.8 & 0.166 & 0.082 & 17.3 & 0.06 & 0.028 & 0.112 & 13.9 & 0.101 & 0.056 & 11.3 & 0.457 \\
\hline 6/27/2008 10:00 AM & 0.342 & 19.8 & 0.166 & 0.082 & 17.3 & 0.06 & 0.028 & 0.112 & 13.9 & 0.101 & 0.056 & 11.3 & 0.457 \\
\hline 6/27/2008 11:00 AM & 0.342 & 19.7 & 0.166 & 0.082 & 17.4 & 0.06 & 0.028 & 0.111 & 13.9 & 0.101 & 0.056 & 11.3 & 0.457 \\
\hline 6/27/2008 12:00 PM & 0.342 & 19.7 & 0.166 & 0.082 & 17.4 & 0.06 & 0.028 & 0.111 & 14.0 & 0.101 & 0.056 & 11.3 & 0.457 \\
\hline 6/27/2008 1:00 PM & 0.342 & 19.7 & 0.166 & 0.082 & 17.3 & 0.06 & 0.028 & 0.111 & 14.0 & 0.101 & 0.056 & 11.3 & 0.457 \\
\hline 6/27/2008 2:00 PM & 0.342 & 19.6 & 0.166 & 0.082 & 17.4 & 0.06 & 0.028 & 0.111 & 13.9 & 0.101 & 0.056 & 11.3 & 0.457 \\
\hline 6/27/2008 3:00 PM & 0.343 & 19.6 & 0.166 & 0.082 & 17.4 & 0.06 & 0.028 & 0.111 & 14.0 & 0.101 & 0.056 & 11.3 & 0.457 \\
\hline 6/27/2008 4:00 PM & 0.343 & 19.5 & 0.166 & 0.082 & 17.4 & 0.06 & 0.028 & 0.112 & 14.0 & 0.101 & 0.056 & 11.3 & 0.457 \\
\hline 6/27/2008 5:00 PM & 0.343 & 19.6 & 0.166 & 0.082 & 17.4 & 0.06 & 0.028 & 0.111 & 14.0 & 0.101 & 0.056 & 11.3 & 0.457 \\
\hline 6/27/2008 6:00 PM & 0.343 & 19.5 & 0.166 & 0.082 & 17.4 & 0.06 & 0.028 & 0.111 & 14.0 & 0.101 & 0.056 & 11.3 & 0.457 \\
\hline 6/27/2008 7:00 PM & 0.343 & 19.5 & 0.166 & 0.082 & 17.4 & 0.06 & 0.028 & 0.111 & 14.0 & 0.101 & 0.056 & 11.3 & 0.457 \\
\hline 6/27/2008 8:00 PM & 0.343 & 19.5 & 0.166 & 0.082 & 17.4 & 0.06 & 0.028 & 0.112 & 14.0 & 0.101 & 0.056 & 11.3 & 0.457 \\
\hline 6/27/2008 9:00 PM & 0.343 & 19.4 & 0.166 & 0.082 & 17.4 & 0.06 & 0.028 & 0.112 & 14.0 & 0.101 & 0.056 & 11.3 & 0.457 \\
\hline 6/27/2008 10:00 PM & 0.344 & 19.5 & 0.166 & 0.082 & 17.4 & 0.06 & 0.027 & 0.113 & 14.0 & 0.101 & 0.056 & 11.3 & 0.457 \\
\hline 6/27/2008 11:00 PM & 0.345 & 19.5 & 0.166 & 0.082 & 17.4 & 0.06 & 0.027 & 0.113 & 14.0 & 0.101 & 0.056 & 11.3 & 0.457 \\
\hline 6/28/2008 12:00 AM & 0.345 & 19.5 & 0.166 & 0.082 & 17.4 & 0.06 & 0.028 & 0.112 & 14.0 & 0.101 & 0.056 & 11.2 & 0.457 \\
\hline 6/28/2008 1:00 AM & 0.345 & 19.4 & 0.166 & 0.082 & 17.4 & 0.06 & 0.027 & 0.113 & 14.0 & 0.101 & 0.056 & 11.3 & 0.457 \\
\hline 6/28/2008 2:00 AM & 0.345 & 19.5 & 0.166 & 0.082 & 17.4 & 0.06 & 0.028 & 0.113 & 14.0 & 0.101 & 0.056 & 11.3 & 0.457 \\
\hline 6/28/2008 3:00 AM & 0.346 & 19.5 & 0.166 & 0.082 & 17.4 & 0.06 & 0.028 & 0.113 & 14.0 & 0.101 & 0.056 & 11.3 & 0.457 \\
\hline 6/28/2008 4:00 AM & 0.346 & 19.5 & 0.166 & 0.082 & 17.4 & 0.06 & 0.028 & 0.113 & 14.0 & 0.101 & 0.056 & 11.3 & 0.457 \\
\hline 6/28/2008 5:00 AM & 0.346 & 19.5 & 0.166 & 0.082 & 17.4 & 0.06 & 0.028 & 0.113 & 14.0 & 0.101 & 0.056 & 11.3 & 0.457 \\
\hline 6/28/2008 6:00 AM & 0.346 & 19.5 & 0.166 & 0.082 & 17.4 & 0.06 & 0.028 & 0.113 & 14.0 & 0.101 & 0.056 & 11.3 & 0.457 \\
\hline 6/28/2008 7:00 AM & 0.346 & 19.5 & 0.166 & 0.082 & 17.4 & 0.06 & 0.028 & 0.113 & 14.0 & 0.101 & 0.056 & 11.3 & 0.458 \\
\hline 6/28/2008 8:00 AM & 0.346 & 19.5 & 0.167 & 0.082 & 17.4 & 0.06 & 0.028 & 0.112 & 14.1 & 0.101 & 0.056 & 11.3 & 0.458 \\
\hline 6/28/2008 9:00 AM & 0.347 & 19.5 & 0.167 & 0.082 & 17.4 & 0.06 & 0.028 & 0.112 & 14.1 & 0.101 & 0.056 & 11.3 & 0.458 \\
\hline 6/28/2008 10:00 AM & 0.347 & 19.5 & 0.167 & 0.082 & 17.4 & 0.06 & 0.028 & 0.112 & 14.0 & 0.101 & 0.056 & 11.3 & 0.458 \\
\hline 6/28/2008 11:00 AM & 0.347 & 19.5 & 0.166 & 0.082 & 17.4 & 0.06 & 0.028 & 0.112 & 14.0 & 0.101 & 0.056 & 11.3 & 0.458 \\
\hline 6/28/2008 12:00 PM & 0.347 & 19.5 & 0.166 & 0.082 & 17.4 & 0.06 & 0.028 & 0.112 & 14.0 & 0.101 & 0.056 & 11.4 & 0.458 \\
\hline 6/28/2008 1:00 PM & 0.347 & 19.5 & 0.166 & 0.082 & 17.4 & 0.06 & 0.028 & 0.111 & 14.1 & 0.101 & 0.056 & 11.4 & 0.458 \\
\hline 6/28/2008 2:00 PM & 0.347 & 19.4 & 0.166 & 0.082 & 17.4 & 0.06 & 0.028 & 0.111 & 14.1 & 0.101 & 0.056 & 11.4 & 0.458 \\
\hline 6/28/2008 3:00 PM & 0.347 & 19.4 & 0.166 & 0.082 & 17.4 & 0.06 & 0.028 & 0.112 & 14.1 & 0.101 & 0.056 & 11.3 & 0.458 \\
\hline 6/28/2008 4:00 PM & 0.347 & 19.4 & 0.167 & 0.082 & 17.4 & 0.06 & 0.028 & 0.112 & 14.1 & 0.101 & 0.056 & 11.4 & 0.458 \\
\hline 6/28/2008 5:00 PM & 0.347 & 19.3 & 0.167 & 0.082 & 17.4 & 0.06 & 0.028 & 0.112 & 14.1 & 0.101 & 0.056 & 11.3 & 0.458 \\
\hline 6/28/2008 6:00 PM & 0.348 & 19.3 & 0.167 & 0.082 & 17.4 & 0.06 & 0.028 & 0.111 & 14.1 & 0.101 & 0.056 & 11.4 & 0.458 \\
\hline
\end{tabular}


Appendix 3. Volumetric water content, temperature, and electrical-conductivity data collected at the sprinkler-irrigated site during 2008.-Continued

[Depth in feet below land surface; volumetric water content dimensionless; temperature in degrees Celsius; bulk electrical conductivity in decisiemens per meter; --, no data]

\begin{tabular}{|c|c|c|c|c|c|c|c|c|c|c|c|c|c|}
\hline \multirow[b]{3}{*}{$\begin{array}{l}\text { Measurement } \\
\text { date and time }\end{array}$} & \multicolumn{13}{|c|}{ Depth } \\
\hline & \multicolumn{2}{|l|}{2} & \multirow{2}{*}{$\begin{array}{c}3 \\
\begin{array}{c}\text { Volumetric } \\
\text { water } \\
\text { content }\end{array}\end{array}$} & \multicolumn{3}{|c|}{4} & \multirow{2}{*}{$\begin{array}{c}5 \\
\begin{array}{c}\text { Volumetric } \\
\text { water } \\
\text { content }\end{array}\end{array}$} & \multicolumn{2}{|l|}{7} & \multirow{2}{*}{$\begin{array}{c}9 \\
\begin{array}{c}\text { Volumetric } \\
\text { water } \\
\text { content }\end{array} \\
\end{array}$} & \multicolumn{2}{|c|}{13} & \multirow{2}{*}{$\begin{array}{c}16.5 \\
\text { Volumetric } \\
\text { water } \\
\text { content }\end{array}$} \\
\hline & $\begin{array}{c}\text { Volumetric } \\
\text { water } \\
\text { content }\end{array}$ & $\begin{array}{l}\text { Temper- } \\
\text { ature }\end{array}$ & & $\begin{array}{c}\text { Volumetric } \\
\text { water } \\
\text { content }\end{array}$ & $\begin{array}{l}\text { Temper- } \\
\text { ature }\end{array}$ & $\begin{array}{c}\text { Bulk } \\
\text { electrical } \\
\text { conduct- } \\
\text { ivity }\end{array}$ & & $\begin{array}{c}\text { Volumetric } \\
\text { water } \\
\text { content }\end{array}$ & $\begin{array}{l}\text { Temper- } \\
\text { ature }\end{array}$ & & $\begin{array}{c}\text { Volumetric } \\
\text { water } \\
\text { content }\end{array}$ & $\begin{array}{l}\text { Temper- } \\
\text { ature }\end{array}$ & \\
\hline 6/28/2008 7:00 PM & 0.347 & 19.3 & 0.166 & 0.082 & 17.4 & 0.06 & 0.028 & 0.112 & 14.1 & 0.101 & 0.056 & 11.4 & 0.458 \\
\hline 6/28/2008 8:00 PM & 0.347 & 19.3 & 0.166 & 0.082 & 17.4 & 0.06 & 0.028 & 0.112 & 14.1 & 0.101 & 0.056 & 11.4 & 0.458 \\
\hline 6/28/2008 9:00 PM & 0.348 & 19.3 & 0.167 & 0.082 & 17.4 & 0.06 & 0.028 & 0.112 & 14.1 & 0.101 & 0.056 & 11.3 & 0.458 \\
\hline 6/28/2008 10:00 PM & 0.348 & 19.3 & 0.167 & 0.082 & 17.4 & 0.06 & 0.028 & 0.113 & 14.1 & 0.101 & 0.056 & 11.3 & 0.458 \\
\hline 6/28/2008 11:00 PM & 0.348 & 19.3 & 0.167 & 0.082 & 17.4 & 0.06 & 0.028 & 0.113 & 14.1 & 0.101 & 0.056 & 11.3 & 0.457 \\
\hline 6/29/2008 12:00 AM & 0.349 & 19.3 & 0.167 & 0.082 & 17.4 & 0.06 & 0.028 & 0.113 & 14.1 & 0.101 & 0.056 & 11.3 & 0.457 \\
\hline 6/29/2008 1:00 AM & 0.349 & 19.3 & 0.167 & 0.082 & 17.4 & 0.06 & 0.028 & 0.113 & 14.1 & 0.101 & 0.055 & 11.3 & 0.457 \\
\hline 6/29/2008 2:00 AM & 0.349 & 19.3 & 0.167 & 0.082 & 17.4 & 0.06 & 0.028 & 0.113 & 14.1 & 0.101 & 0.055 & 11.3 & 0.458 \\
\hline 6/29/2008 3:00 AM & 0.350 & 19.3 & 0.167 & 0.082 & 17.4 & 0.06 & 0.028 & 0.113 & 14.1 & 0.101 & 0.055 & 11.4 & 0.458 \\
\hline 6/29/2008 4:00 AM & 0.350 & 19.4 & 0.167 & 0.082 & 17.4 & 0.06 & 0.027 & 0.112 & 14.1 & 0.101 & 0.055 & 11.4 & 0.458 \\
\hline 6/29/2008 5:00 AM & 0.350 & 19.4 & 0.167 & 0.082 & 17.4 & 0.06 & 0.027 & 0.112 & 14.1 & 0.101 & 0.055 & 11.4 & 0.458 \\
\hline 6/29/2008 6:00 AM & 0.350 & 19.4 & 0.167 & 0.082 & 17.4 & 0.06 & 0.028 & 0.112 & 14.1 & 0.101 & 0.055 & 11.3 & 0.458 \\
\hline 6/29/2008 7:00 AM & 0.350 & 19.4 & 0.167 & 0.082 & 17.4 & 0.06 & 0.028 & 0.113 & 14.1 & 0.101 & 0.055 & 11.3 & 0.458 \\
\hline 6/29/2008 8:00 AM & 0.351 & 19.4 & 0.167 & 0.082 & 17.4 & 0.06 & 0.028 & 0.113 & 14.1 & 0.101 & 0.056 & 11.3 & 0.458 \\
\hline 6/29/2008 9:00 AM & 0.351 & 19.4 & 0.167 & 0.082 & 17.4 & 0.06 & 0.028 & 0.112 & 14.1 & 0.101 & 0.056 & 11.4 & 0.458 \\
\hline 6/29/2008 10:00 AM & 0.351 & 19.4 & 0.167 & 0.082 & 17.4 & 0.06 & 0.028 & 0.112 & 14.2 & 0.101 & 0.056 & 11.4 & 0.458 \\
\hline 6/29/2008 11:00 AM & 0.351 & 19.3 & 0.167 & 0.083 & 17.4 & 0.06 & 0.028 & 0.112 & 14.1 & 0.101 & 0.056 & 11.4 & 0.458 \\
\hline 6/29/2008 12:00 PM & 0.351 & 19.3 & 0.167 & 0.083 & 17.4 & 0.06 & 0.028 & 0.112 & 14.1 & 0.101 & 0.056 & 11.4 & 0.458 \\
\hline 6/29/2008 1:00 PM & 0.351 & 19.3 & 0.167 & 0.083 & 17.5 & 0.06 & 0.028 & 0.112 & 14.2 & 0.101 & 0.056 & 11.4 & 0.458 \\
\hline 6/29/2008 2:00 PM & 0.351 & 19.3 & 0.167 & 0.083 & 17.4 & 0.06 & 0.028 & 0.112 & 14.2 & 0.101 & 0.056 & 11.4 & 0.458 \\
\hline 6/29/2008 3:00 PM & 0.350 & 19.2 & 0.167 & 0.083 & 17.4 & 0.06 & 0.028 & 0.112 & 14.2 & 0.101 & 0.056 & 11.4 & 0.458 \\
\hline 6/29/2008 4:00 PM & 0.350 & 19.2 & 0.167 & 0.083 & 17.4 & 0.06 & 0.028 & 0.112 & 14.2 & 0.101 & 0.056 & 11.4 & 0.458 \\
\hline 6/29/2008 5:00 PM & 0.350 & 19.2 & 0.166 & 0.083 & 17.4 & 0.06 & 0.028 & 0.112 & 14.2 & 0.101 & 0.056 & 11.4 & 0.458 \\
\hline 6/29/2008 6:00 PM & 0.351 & 19.2 & 0.167 & 0.083 & 17.4 & 0.06 & 0.028 & 0.112 & 14.2 & 0.101 & 0.056 & 11.4 & 0.458 \\
\hline 6/29/2008 7:00 PM & 0.351 & 19.1 & 0.167 & 0.083 & 17.5 & 0.06 & 0.028 & 0.112 & 14.2 & 0.101 & 0.056 & 11.4 & 0.458 \\
\hline 6/29/2008 8:00 PM & 0.351 & 19.1 & 0.167 & 0.082 & 17.4 & 0.06 & 0.028 & 0.112 & 14.2 & 0.101 & 0.056 & 11.4 & 0.458 \\
\hline 6/29/2008 9:00 PM & 0.351 & 19.1 & 0.167 & 0.083 & 17.4 & 0.06 & 0.028 & 0.113 & 14.2 & 0.101 & 0.056 & 11.4 & 0.458 \\
\hline 6/29/2008 10:00 PM & 0.351 & 19.2 & 0.167 & 0.083 & 17.5 & 0.06 & 0.028 & 0.112 & 14.2 & 0.101 & 0.056 & 11.4 & 0.458 \\
\hline 6/29/2008 11:00 PM & 0.351 & 19.2 & 0.167 & 0.083 & 17.5 & 0.06 & 0.028 & 0.113 & 14.2 & 0.101 & 0.056 & 11.4 & 0.458 \\
\hline $6 / 30 / 2008$ 12:00 AM & 0.351 & 19.2 & 0.167 & 0.082 & 17.5 & 0.06 & 0.028 & 0.113 & 14.2 & 0.101 & 0.056 & 11.4 & 0.458 \\
\hline 6/30/2008 1:00 AM & 0.353 & 19.2 & 0.167 & 0.083 & 17.4 & 0.06 & 0.028 & 0.113 & 14.2 & 0.101 & 0.056 & 11.4 & 0.458 \\
\hline 6/30/2008 2:00 AM & 0.353 & 19.2 & 0.167 & 0.083 & 17.4 & 0.06 & 0.028 & 0.113 & 14.2 & 0.101 & 0.056 & 11.4 & 0.458 \\
\hline 6/30/2008 3:00 AM & 0.354 & 19.3 & 0.167 & 0.083 & 17.5 & 0.06 & 0.028 & 0.113 & 14.2 & 0.101 & 0.056 & 11.4 & 0.458 \\
\hline 6/30/2008 4:00 AM & 0.354 & 19.3 & 0.167 & 0.083 & 17.4 & 0.06 & 0.028 & 0.115 & 14.2 & 0.101 & 0.056 & 11.4 & 0.458 \\
\hline 6/30/2008 5:00 AM & 0.354 & 19.3 & 0.167 & 0.083 & 17.5 & 0.06 & 0.028 & 0.113 & 14.2 & 0.101 & 0.055 & 11.4 & 0.458 \\
\hline
\end{tabular}


Appendix 3. Volumetric water content, temperature, and electrical-conductivity data collected at the sprinkler-irrigated site during 2008.-Continued

[Depth in feet below land surface; volumetric water content dimensionless; temperature in degrees Celsius; bulk electrical conductivity in decisiemens per meter; --, no data]

\begin{tabular}{|c|c|c|c|c|c|c|c|c|c|c|c|c|c|}
\hline \multirow[b]{3}{*}{$\begin{array}{l}\text { Measurement } \\
\text { date and time }\end{array}$} & \multirow{2}{*}{\multicolumn{2}{|c|}{2}} & \multirow{3}{*}{$\begin{array}{c}3 \\
\text { Volumetric } \\
\text { water } \\
\text { content }\end{array}$} & \multicolumn{9}{|c|}{ Depth } & \multirow{3}{*}{$\begin{array}{c}16.5 \\
\text { Volumetric } \\
\text { water } \\
\text { content }\end{array}$} \\
\hline & & & & \multicolumn{3}{|c|}{4} & \multirow[b]{2}{*}{$\begin{array}{c}\mathbf{5} \\
\text { Volumetric } \\
\text { water } \\
\text { content }\end{array}$} & \multicolumn{2}{|c|}{7} & \multirow{2}{*}{$\begin{array}{c}9 \\
\text { Volumetric } \\
\text { water } \\
\text { content }\end{array}$} & \multicolumn{2}{|c|}{13} & \\
\hline & $\begin{array}{c}\text { Volumetric } \\
\text { water } \\
\text { content }\end{array}$ & $\begin{array}{l}\text { Temper- } \\
\text { ature }\end{array}$ & & $\begin{array}{c}\text { Volumetric } \\
\text { water } \\
\text { content }\end{array}$ & $\begin{array}{l}\text { Temper- } \\
\text { ature }\end{array}$ & $\begin{array}{c}\text { Bulk } \\
\text { electrical } \\
\text { conduct- } \\
\text { ivity }\end{array}$ & & $\begin{array}{c}\text { Volumetric } \\
\text { water } \\
\text { content }\end{array}$ & $\begin{array}{l}\text { Temper- } \\
\text { ature }\end{array}$ & & $\begin{array}{l}\text { Volumetric } \\
\text { water } \\
\text { content }\end{array}$ & $\begin{array}{l}\text { Temper- } \\
\text { ature }\end{array}$ & \\
\hline 6/30/2008 6:00 AM & 0.354 & 19.3 & 0.167 & 0.083 & 17.5 & 0.06 & 0.028 & 0.115 & 14.2 & 0.101 & 0.055 & 11.4 & 0.458 \\
\hline 6/30/2008 7:00 AM & 0.354 & 19.3 & 0.167 & 0.083 & 17.5 & 0.06 & 0.028 & 0.115 & 14.2 & 0.101 & 0.056 & 11.4 & 0.458 \\
\hline 6/30/2008 8:00 AM & 0.354 & 19.4 & 0.167 & 0.083 & 17.4 & 0.06 & 0.028 & 0.113 & 14.3 & 0.101 & 0.056 & 11.4 & 0.458 \\
\hline 6/30/2008 9:00 AM & 0.354 & 19.4 & 0.167 & 0.083 & 17.5 & 0.06 & 0.028 & 0.112 & 14.3 & 0.101 & 0.056 & 11.4 & 0.458 \\
\hline 6/30/2008 10:00 AM & 0.354 & 19.4 & 0.167 & 0.083 & 17.4 & 0.06 & 0.028 & 0.112 & 14.2 & 0.101 & 0.056 & 11.5 & 0.458 \\
\hline 6/30/2008 11:00 AM & 0.354 & 19.4 & 0.167 & 0.083 & 17.5 & 0.06 & 0.028 & 0.112 & 14.3 & 0.101 & 0.056 & 11.5 & 0.458 \\
\hline 6/30/2008 12:00 PM & 0.354 & 19.3 & 0.167 & 0.083 & 17.5 & 0.06 & 0.028 & 0.113 & 14.2 & 0.101 & 0.056 & 11.5 & 0.458 \\
\hline 6/30/2008 1:00 PM & 0.354 & 19.3 & 0.167 & 0.083 & 17.4 & 0.06 & 0.028 & 0.113 & 14.3 & 0.101 & 0.056 & 11.5 & 0.458 \\
\hline 6/30/2008 2:00 PM & 0.354 & 19.3 & 0.167 & 0.083 & 17.5 & 0.06 & 0.028 & 0.113 & 14.2 & 0.101 & 0.056 & 11.5 & 0.458 \\
\hline 6/30/2008 3:00 PM & 0.354 & 19.2 & 0.167 & 0.083 & 17.5 & 0.06 & 0.028 & 0.112 & 14.3 & 0.101 & 0.056 & 11.5 & 0.458 \\
\hline 6/30/2008 4:00 PM & 0.354 & 19.2 & 0.167 & 0.083 & 17.4 & 0.06 & 0.028 & 0.113 & 14.3 & 0.101 & 0.056 & 11.5 & 0.458 \\
\hline 6/30/2008 5:00 PM & 0.353 & 19.2 & 0.167 & 0.083 & 17.5 & 0.06 & 0.028 & 0.113 & 14.3 & 0.101 & 0.056 & 11.5 & 0.458 \\
\hline 6/30/2008 6:00 PM & 0.354 & 19.2 & 0.167 & 0.083 & 17.5 & 0.06 & 0.028 & 0.113 & 14.3 & 0.101 & 0.056 & 11.5 & 0.458 \\
\hline 6/30/2008 7:00 PM & 0.354 & 19.2 & 0.167 & 0.083 & 17.5 & 0.06 & 0.028 & 0.112 & 14.3 & 0.101 & 0.056 & 11.5 & 0.458 \\
\hline 6/30/2008 8:00 PM & 0.354 & 19.2 & 0.167 & 0.083 & 17.5 & 0.06 & 0.028 & 0.112 & 14.3 & 0.101 & 0.056 & 11.5 & 0.458 \\
\hline 6/30/2008 9:00 PM & 0.355 & 19.2 & 0.167 & 0.083 & 17.5 & 0.06 & 0.028 & 0.112 & 14.3 & 0.101 & 0.056 & 11.5 & 0.458 \\
\hline 6/30/2008 10:00 PM & 0.355 & 19.2 & 0.167 & 0.083 & 17.5 & 0.06 & 0.028 & 0.113 & 14.3 & 0.101 & 0.056 & 11.4 & 0.458 \\
\hline 6/30/2008 11:00 PM & 0.355 & 19.2 & 0.167 & 0.083 & 17.5 & 0.06 & 0.028 & 0.112 & 14.3 & 0.101 & 0.056 & 11.5 & 0.458 \\
\hline 7/1/2008 12:00 AM & 0.355 & 19.2 & 0.167 & 0.083 & 17.5 & 0.06 & 0.028 & 0.113 & 14.3 & 0.101 & 0.056 & 11.5 & 0.458 \\
\hline 7/1/2008 1:00 AM & 0.355 & 19.2 & 0.167 & 0.083 & 17.5 & 0.06 & 0.028 & 0.113 & 14.3 & 0.101 & 0.056 & 11.4 & 0.458 \\
\hline 7/1/2008 2:00 AM & 0.355 & 19.3 & 0.167 & 0.083 & 17.5 & 0.06 & 0.028 & 0.113 & 14.3 & 0.101 & 0.056 & 11.4 & 0.458 \\
\hline 7/1/2008 3:00 AM & 0.356 & 19.3 & 0.167 & 0.083 & 17.5 & 0.06 & 0.028 & 0.113 & 14.3 & 0.101 & 0.056 & 11.5 & 0.458 \\
\hline 7/1/2008 4:00 AM & 0.356 & 19.4 & 0.167 & 0.083 & 17.5 & 0.06 & 0.028 & 0.113 & 14.3 & 0.101 & 0.056 & 11.4 & 0.458 \\
\hline 7/1/2008 5:00 AM & 0.356 & 19.4 & 0.167 & 0.083 & 17.5 & 0.06 & 0.028 & 0.113 & 14.3 & 0.101 & 0.056 & 11.4 & 0.458 \\
\hline 7/1/2008 6:00 AM & 0.356 & 19.4 & 0.167 & 0.083 & 17.5 & 0.06 & 0.028 & 0.113 & 14.3 & 0.101 & 0.056 & 11.4 & 0.458 \\
\hline 7/1/2008 7:00 AM & 0.356 & 19.4 & 0.167 & 0.083 & 17.5 & 0.06 & 0.028 & 0.113 & 14.3 & 0.101 & 0.056 & 11.5 & 0.458 \\
\hline 7/1/2008 8:00 AM & 0.356 & 19.4 & 0.167 & 0.083 & 17.5 & 0.06 & 0.028 & 0.112 & 14.3 & 0.101 & 0.056 & 11.5 & 0.458 \\
\hline 7/1/2008 9:00 AM & 0.356 & 19.4 & 0.167 & 0.083 & 17.5 & 0.06 & 0.028 & 0.112 & 14.3 & 0.101 & 0.056 & 11.5 & 0.458 \\
\hline 7/1/2008 10:00 AM & 0.355 & 19.4 & 0.167 & 0.083 & 17.5 & 0.06 & 0.028 & 0.113 & 14.3 & 0.102 & 0.056 & 11.5 & 0.458 \\
\hline 7/1/2008 11:00 AM & 0.355 & 19.4 & 0.167 & 0.083 & 17.5 & 0.06 & 0.028 & 0.113 & 14.3 & 0.102 & 0.056 & 11.5 & 0.458 \\
\hline 7/1/2008 12:00 PM & 0.355 & 19.4 & 0.167 & 0.083 & 17.5 & 0.06 & 0.028 & 0.113 & 14.3 & 0.102 & 0.056 & 11.5 & 0.458 \\
\hline 7/1/2008 1:00 PM & 0.355 & 19.4 & 0.167 & 0.083 & 17.5 & 0.06 & 0.028 & 0.113 & 14.3 & 0.102 & 0.056 & 11.5 & 0.458 \\
\hline 7/1/2008 2:00 PM & 0.355 & 19.4 & 0.167 & 0.083 & 17.5 & 0.06 & 0.028 & 0.113 & 14.3 & 0.102 & 0.056 & 11.6 & 0.458 \\
\hline 7/1/2008 3:00 PM & 0.355 & 19.3 & 0.167 & 0.083 & 17.5 & 0.06 & 0.028 & 0.113 & 14.4 & 0.102 & 0.056 & 11.5 & 0.458 \\
\hline 7/1/2008 4:00 PM & 0.356 & 19.4 & 0.167 & 0.083 & 17.5 & 0.06 & 0.028 & 0.113 & 14.4 & 0.102 & 0.056 & 11.5 & 0.458 \\
\hline
\end{tabular}


Appendix 3. Volumetric water content, temperature, and electrical-conductivity data collected at the sprinkler-irrigated site during 2008.-Continued

[Depth in feet below land surface; volumetric water content dimensionless; temperature in degrees Celsius; bulk electrical conductivity in decisiemens per meter; --, no data]

\begin{tabular}{|c|c|c|c|c|c|c|c|c|c|c|c|c|c|}
\hline \multirow[b]{3}{*}{$\begin{array}{l}\text { Measurement } \\
\text { date and time }\end{array}$} & \multicolumn{13}{|c|}{ Depth } \\
\hline & \multicolumn{2}{|c|}{2} & \multirow{2}{*}{$\begin{array}{c}3 \\
\begin{array}{c}\text { Volumetric } \\
\text { water } \\
\text { content }\end{array}\end{array}$} & \multicolumn{3}{|c|}{4} & \multirow{2}{*}{$\begin{array}{c}5 \\
\text { Volumetric } \\
\text { water } \\
\text { content }\end{array}$} & \multicolumn{2}{|l|}{7} & \multirow{2}{*}{$\begin{array}{c}9 \\
\text { Volumetric } \\
\text { water } \\
\text { content }\end{array}$} & \multicolumn{2}{|c|}{13} & \multirow{2}{*}{$\begin{array}{c}16.5 \\
\text { Volumetric } \\
\text { water } \\
\text { content }\end{array}$} \\
\hline & $\begin{array}{c}\text { Volumetric } \\
\text { water } \\
\text { content }\end{array}$ & $\begin{array}{l}\text { Temper- } \\
\text { ature }\end{array}$ & & $\begin{array}{c}\text { Volumetric } \\
\text { water } \\
\text { content }\end{array}$ & $\begin{array}{l}\text { Temper- } \\
\text { ature }\end{array}$ & $\begin{array}{c}\text { Bulk } \\
\text { electrical } \\
\text { conduct- } \\
\text { ivity }\end{array}$ & & $\begin{array}{c}\text { Volumetric } \\
\text { water } \\
\text { content }\end{array}$ & $\begin{array}{l}\text { Temper- } \\
\text { ature }\end{array}$ & & $\begin{array}{c}\text { Volumetric } \\
\text { water } \\
\text { content }\end{array}$ & $\begin{array}{l}\text { Temper- } \\
\text { ature }\end{array}$ & \\
\hline 7/1/2008 5:00 PM & 0.356 & 19.3 & 0.167 & 0.083 & 17.5 & 0.06 & 0.029 & 0.112 & 14.4 & 0.102 & 0.056 & 11.5 & 0.458 \\
\hline 7/1/2008 6:00 PM & 0.356 & 19.3 & 0.167 & 0.083 & 17.5 & 0.06 & 0.029 & 0.112 & 14.4 & 0.102 & 0.056 & 11.5 & 0.458 \\
\hline 7/1/2008 7:00 PM & 0.356 & 19.3 & 0.167 & 0.083 & 17.5 & 0.06 & 0.029 & 0.113 & 14.4 & 0.102 & 0.056 & 11.5 & 0.458 \\
\hline 7/1/2008 8:00 PM & 0.356 & 19.3 & 0.167 & 0.083 & 17.5 & 0.06 & 0.029 & 0.113 & 14.4 & 0.102 & 0.056 & 11.5 & 0.458 \\
\hline 7/1/2008 9:00 PM & 0.356 & 19.3 & 0.167 & 0.083 & 17.5 & 0.06 & 0.028 & 0.112 & 14.4 & 0.102 & 0.056 & 11.5 & 0.458 \\
\hline 7/1/2008 10:00 PM & 0.356 & 19.3 & 0.167 & 0.083 & 17.5 & 0.06 & 0.029 & 0.112 & 14.4 & 0.102 & 0.056 & 11.5 & 0.458 \\
\hline 7/1/2008 11:00 PM & 0.357 & 19.3 & 0.167 & 0.083 & 17.5 & 0.06 & 0.028 & 0.112 & 14.4 & 0.102 & 0.056 & 11.5 & 0.458 \\
\hline 7/2/2008 12:00 AM & 0.357 & 19.3 & 0.167 & 0.083 & 17.5 & 0.06 & 0.028 & 0.113 & 14.4 & 0.102 & 0.056 & 11.5 & 0.458 \\
\hline 7/2/2008 1:00 AM & 0.358 & 19.3 & 0.167 & 0.083 & 17.5 & 0.06 & 0.028 & 0.112 & 14.4 & 0.102 & 0.056 & 11.5 & 0.458 \\
\hline 7/2/2008 2:00 AM & 0.358 & 19.4 & 0.167 & 0.083 & 17.5 & 0.06 & 0.028 & 0.113 & 14.4 & 0.102 & 0.055 & 11.5 & 0.458 \\
\hline 7/2/2008 3:00 AM & 0.359 & 19.3 & 0.167 & 0.083 & 17.5 & 0.06 & 0.028 & 0.113 & 14.4 & 0.102 & 0.056 & 11.5 & 0.458 \\
\hline 7/2/2008 4:00 AM & 0.360 & 19.4 & 0.167 & 0.083 & 17.5 & 0.06 & 0.029 & 0.113 & 14.4 & 0.102 & 0.056 & 11.5 & 0.458 \\
\hline 7/2/2008 5:00 AM & 0.360 & 19.4 & 0.167 & 0.083 & 17.5 & 0.06 & 0.029 & 0.112 & 14.4 & 0.102 & 0.056 & 11.5 & 0.458 \\
\hline 7/2/2008 6:00 AM & 0.361 & 19.4 & 0.167 & 0.083 & 17.5 & 0.06 & 0.029 & 0.113 & 14.4 & 0.102 & 0.056 & 11.5 & 0.458 \\
\hline 7/2/2008 7:00 AM & 0.362 & 19.4 & 0.167 & 0.083 & 17.5 & 0.06 & 0.028 & 0.113 & 14.4 & 0.102 & 0.056 & 11.5 & 0.458 \\
\hline 7/2/2008 8:00 AM & 0.362 & 19.4 & 0.167 & 0.083 & 17.5 & 0.06 & 0.029 & 0.112 & 14.4 & 0.102 & 0.056 & 11.5 & 0.458 \\
\hline 7/2/2008 9:00 AM & 0.362 & 19.4 & 0.167 & 0.083 & 17.5 & 0.06 & 0.029 & 0.113 & 14.4 & 0.102 & 0.056 & 11.6 & 0.458 \\
\hline 7/2/2008 10:00 AM & 0.363 & 19.4 & 0.167 & 0.083 & 17.5 & 0.06 & 0.029 & 0.113 & 14.4 & 0.102 & 0.056 & 11.6 & 0.458 \\
\hline 7/2/2008 11:00 AM & 0.363 & 19.3 & 0.167 & 0.083 & 17.5 & 0.06 & 0.029 & 0.113 & 14.4 & 0.102 & 0.056 & 11.6 & 0.458 \\
\hline 7/2/2008 12:00 PM & 0.365 & 19.3 & 0.168 & 0.083 & 17.5 & 0.06 & 0.029 & 0.113 & 14.4 & 0.102 & 0.056 & 11.6 & 0.458 \\
\hline 7/2/2008 1:00 PM & 0.365 & 19.3 & 0.168 & 0.083 & 17.5 & 0.06 & 0.029 & 0.113 & 14.4 & 0.102 & 0.056 & 11.6 & 0.458 \\
\hline 7/2/2008 2:00 PM & 0.365 & 19.3 & 0.168 & 0.083 & 17.5 & 0.06 & 0.029 & 0.113 & 14.4 & 0.102 & 0.056 & 11.6 & 0.458 \\
\hline 7/2/2008 3:00 PM & 0.366 & 19.3 & 0.168 & 0.083 & 17.5 & 0.06 & 0.029 & 0.113 & 14.4 & 0.102 & 0.056 & 11.6 & 0.458 \\
\hline 7/2/2008 4:00 PM & 0.366 & 19.3 & 0.168 & 0.083 & 17.5 & 0.06 & 0.029 & 0.113 & 14.5 & 0.102 & 0.056 & 11.6 & 0.458 \\
\hline 7/2/2008 5:00 PM & 0.366 & 19.3 & 0.168 & 0.083 & 17.5 & 0.06 & 0.029 & 0.113 & 14.4 & 0.102 & 0.056 & 11.6 & 0.458 \\
\hline 7/2/2008 6:00 PM & 0.367 & 19.3 & 0.168 & 0.083 & 17.5 & 0.06 & 0.029 & 0.113 & 14.4 & 0.102 & 0.056 & 11.6 & 0.458 \\
\hline 7/2/2008 7:00 PM & 0.367 & 19.3 & 0.168 & 0.083 & 17.5 & 0.06 & 0.029 & 0.113 & 14.5 & 0.102 & 0.056 & 11.6 & 0.458 \\
\hline 7/2/2008 8:00 PM & 0.367 & 19.3 & 0.169 & 0.083 & 17.5 & 0.06 & 0.029 & 0.113 & 14.4 & 0.102 & 0.056 & 11.6 & 0.458 \\
\hline 7/2/2008 9:00 PM & 0.367 & 19.3 & 0.169 & 0.083 & 17.6 & 0.06 & 0.028 & 0.113 & 14.5 & 0.102 & 0.056 & 11.6 & 0.458 \\
\hline 7/2/2008 10:00 PM & 0.368 & 19.3 & 0.170 & 0.083 & 17.5 & 0.06 & 0.028 & 0.113 & 14.5 & 0.102 & 0.056 & 11.5 & 0.459 \\
\hline 7/2/2008 11:00 PM & 0.368 & 19.3 & 0.171 & 0.083 & 17.5 & 0.06 & 0.028 & 0.113 & 14.5 & 0.102 & 0.056 & 11.6 & 0.459 \\
\hline 7/3/2008 12:00 AM & 0.368 & 19.3 & 0.171 & 0.083 & 17.6 & 0.06 & 0.028 & 0.113 & 14.5 & 0.102 & 0.056 & 11.6 & 0.459 \\
\hline 7/3/2008 1:00 AM & 0.368 & 19.3 & 0.171 & 0.083 & 17.6 & 0.06 & 0.028 & 0.113 & 14.5 & 0.102 & 0.056 & 11.6 & 0.459 \\
\hline 7/3/2008 2:00 AM & 0.368 & 19.3 & 0.171 & 0.083 & 17.5 & 0.06 & 0.028 & 0.113 & 14.5 & 0.102 & 0.056 & 11.6 & 0.459 \\
\hline 7/3/2008 3:00 AM & 0.368 & 19.4 & 0.171 & 0.083 & 17.6 & 0.06 & 0.028 & 0.113 & 14.5 & 0.102 & 0.056 & 11.6 & 0.459 \\
\hline
\end{tabular}


Appendix 3. Volumetric water content, temperature, and electrical-conductivity data collected at the sprinkler-irrigated site during 2008.-Continued

[Depth in feet below land surface; volumetric water content dimensionless; temperature in degrees Celsius; bulk electrical conductivity in decisiemens per meter; --, no data]

\begin{tabular}{|c|c|c|c|c|c|c|c|c|c|c|c|c|c|}
\hline \multirow[b]{3}{*}{$\begin{array}{l}\text { Measurement } \\
\text { date and time }\end{array}$} & \multicolumn{13}{|c|}{ Depth } \\
\hline & \multicolumn{2}{|l|}{2} & \multirow{2}{*}{$\begin{array}{c}3 \\
\begin{array}{c}\text { Volumetric } \\
\text { water } \\
\text { content }\end{array}\end{array}$} & \multicolumn{3}{|c|}{4} & \multirow{2}{*}{$\begin{array}{c}5 \\
\begin{array}{c}\text { Volumetric } \\
\text { water } \\
\text { content }\end{array}\end{array}$} & \multicolumn{2}{|l|}{7} & \multirow{2}{*}{$\begin{array}{c}9 \\
\text { Volumetric } \\
\text { water } \\
\text { content }\end{array}$} & \multicolumn{2}{|c|}{13} & \multirow{2}{*}{$\begin{array}{c}16.5 \\
\text { Volumetric } \\
\text { water } \\
\text { content }\end{array}$} \\
\hline & $\begin{array}{c}\text { Volumetric } \\
\text { water } \\
\text { content }\end{array}$ & $\begin{array}{c}\text { Temper- } \\
\text { ature }\end{array}$ & & $\begin{array}{c}\text { Volumetric } \\
\text { water } \\
\text { content }\end{array}$ & $\begin{array}{l}\text { Temper- } \\
\text { ature }\end{array}$ & $\begin{array}{c}\text { Bulk } \\
\text { electrical } \\
\text { conduct- } \\
\text { ivity }\end{array}$ & & $\begin{array}{l}\text { Volumetric } \\
\text { water } \\
\text { content }\end{array}$ & $\begin{array}{l}\text { Temper- } \\
\text { ature }\end{array}$ & & $\begin{array}{c}\text { Volumetric } \\
\text { water } \\
\text { content }\end{array}$ & $\begin{array}{l}\text { Temper- } \\
\text { ature }\end{array}$ & \\
\hline 7/3/2008 4:00 AM & 0.368 & 19.4 & 0.171 & 0.083 & 17.6 & 0.06 & 0.028 & 0.113 & 14.5 & 0.102 & 0.056 & 11.6 & 0.459 \\
\hline 7/3/2008 5:00 AM & 0.368 & 19.4 & 0.172 & 0.083 & 17.5 & 0.06 & 0.029 & 0.113 & 14.5 & 0.102 & 0.056 & 11.6 & 0.459 \\
\hline 7/3/2008 6:00 AM & 0.368 & 19.4 & 0.172 & 0.083 & 17.6 & 0.06 & 0.029 & 0.113 & 14.5 & 0.102 & 0.056 & 11.6 & 0.459 \\
\hline 7/3/2008 7:00 AM & 0.368 & 19.4 & 0.172 & 0.083 & 17.6 & 0.06 & 0.029 & 0.113 & 14.5 & 0.102 & 0.056 & 11.6 & 0.459 \\
\hline 7/3/2008 8:00 AM & 0.369 & 19.4 & 0.172 & 0.083 & 17.5 & 0.06 & 0.029 & 0.113 & 14.5 & 0.102 & 0.056 & 11.6 & 0.459 \\
\hline 7/3/2008 9:00 AM & 0.369 & 19.4 & 0.172 & 0.083 & 17.6 & 0.06 & 0.029 & 0.113 & 14.5 & 0.102 & 0.056 & 11.6 & 0.459 \\
\hline 7/3/2008 10:00 AM & 0.369 & 19.4 & 0.172 & 0.083 & 17.6 & 0.06 & 0.029 & 0.113 & 14.5 & 0.102 & 0.056 & 11.6 & 0.459 \\
\hline 7/3/2008 11:00 AM & 0.369 & 19.4 & 0.172 & 0.083 & 17.6 & 0.06 & 0.029 & 0.113 & 14.5 & 0.102 & 0.056 & 11.6 & 0.459 \\
\hline 7/3/2008 12:00 PM & 0.368 & 19.3 & 0.172 & 0.083 & 17.6 & 0.06 & 0.029 & 0.113 & 14.5 & 0.102 & 0.056 & 11.6 & 0.459 \\
\hline 7/3/2008 1:00 PM & 0.368 & 19.3 & 0.173 & 0.083 & 17.6 & 0.06 & 0.029 & 0.113 & 14.5 & 0.102 & 0.056 & 11.6 & 0.459 \\
\hline 7/3/2008 2:00 PM & 0.368 & 19.3 & 0.173 & 0.083 & 17.6 & 0.06 & 0.029 & 0.113 & 14.5 & 0.102 & 0.056 & 11.6 & 0.459 \\
\hline 7/3/2008 3:00 PM & 0.368 & 19.3 & 0.173 & 0.083 & 17.6 & 0.06 & 0.029 & 0.113 & 14.5 & 0.102 & 0.056 & 11.7 & 0.459 \\
\hline 7/3/2008 4:00 PM & 0.368 & 19.3 & 0.173 & 0.084 & 17.6 & 0.06 & 0.029 & 0.113 & 14.5 & 0.102 & 0.056 & 11.7 & 0.459 \\
\hline 7/3/2008 5:00 PM & 0.368 & 19.2 & 0.174 & 0.084 & 17.6 & 0.06 & 0.029 & 0.113 & 14.5 & 0.102 & 0.056 & 11.6 & 0.459 \\
\hline 7/3/2008 6:00 PM & 0.368 & 19.2 & 0.174 & 0.083 & 17.6 & 0.06 & 0.029 & 0.115 & 14.5 & 0.102 & 0.056 & 11.6 & 0.459 \\
\hline 7/3/2008 7:00 PM & 0.368 & 19.2 & 0.174 & 0.083 & 17.6 & 0.06 & 0.029 & 0.113 & 14.5 & 0.102 & 0.056 & 11.6 & 0.459 \\
\hline 7/3/2008 8:00 PM & 0.368 & 19.2 & 0.175 & 0.084 & 17.6 & 0.06 & 0.029 & 0.113 & 14.5 & 0.102 & 0.056 & 11.7 & 0.459 \\
\hline 7/3/2008 9:00 PM & 0.368 & 19.2 & 0.175 & 0.084 & 17.6 & 0.06 & 0.029 & 0.113 & 14.5 & 0.102 & 0.056 & 11.7 & 0.459 \\
\hline 7/3/2008 10:00 PM & 0.368 & 19.2 & 0.176 & 0.083 & 17.6 & 0.06 & 0.029 & 0.113 & 14.6 & 0.102 & 0.056 & 11.6 & 0.459 \\
\hline 7/3/2008 11:00 PM & 0.368 & 19.2 & 0.177 & 0.084 & 17.6 & 0.06 & 0.029 & 0.113 & 14.5 & 0.102 & 0.056 & 11.6 & 0.459 \\
\hline 7/4/2008 12:00 AM & 0.368 & 19.2 & 0.177 & 0.084 & 17.6 & 0.06 & 0.029 & 0.113 & 14.5 & 0.102 & 0.056 & 11.7 & 0.459 \\
\hline 7/4/2008 1:00 AM & 0.368 & 19.2 & 0.177 & 0.084 & 17.6 & 0.06 & 0.029 & 0.113 & 14.5 & 0.102 & 0.056 & 11.6 & 0.459 \\
\hline 7/4/2008 2:00 AM & 0.368 & 19.2 & 0.177 & 0.084 & 17.6 & 0.06 & 0.029 & 0.113 & 14.5 & 0.102 & 0.056 & 11.6 & 0.459 \\
\hline 7/4/2008 3:00 AM & 0.369 & 19.2 & 0.178 & 0.084 & 17.6 & 0.06 & 0.029 & 0.113 & 14.6 & 0.102 & 0.056 & 11.6 & 0.459 \\
\hline 7/4/2008 4:00 AM & 0.369 & 19.3 & 0.178 & 0.084 & 17.6 & 0.06 & 0.029 & 0.113 & 14.6 & 0.102 & 0.056 & 11.7 & 0.459 \\
\hline 7/4/2008 5:00 AM & 0.369 & 19.3 & 0.179 & 0.084 & 17.6 & 0.06 & 0.029 & 0.113 & 14.6 & 0.102 & 0.056 & 11.7 & 0.459 \\
\hline 7/4/2008 6:00 AM & 0.368 & 19.3 & 0.180 & 0.084 & 17.6 & 0.06 & 0.029 & 0.113 & 14.6 & 0.102 & 0.056 & 11.7 & 0.459 \\
\hline 7/4/2008 7:00 AM & 0.369 & 19.3 & 0.180 & 0.084 & 17.6 & 0.06 & 0.029 & 0.113 & 14.5 & 0.102 & 0.056 & 11.6 & 0.459 \\
\hline 7/4/2008 8:00 AM & 0.369 & 19.3 & 0.180 & 0.084 & 17.6 & 0.06 & 0.029 & 0.113 & 14.5 & 0.102 & 0.056 & 11.7 & 0.459 \\
\hline 7/4/2008 9:00 AM & 0.369 & 19.3 & 0.181 & 0.084 & 17.6 & 0.06 & 0.029 & 0.115 & 14.6 & 0.102 & 0.056 & 11.7 & 0.459 \\
\hline 7/4/2008 10:00 AM & 0.368 & 19.3 & 0.182 & 0.084 & 17.6 & 0.06 & 0.029 & 0.115 & 14.6 & 0.102 & 0.056 & 11.7 & 0.459 \\
\hline 7/4/2008 11:00 AM & 0.368 & 19.3 & 0.183 & 0.084 & 17.6 & 0.06 & 0.029 & 0.113 & 14.6 & 0.102 & 0.056 & 11.7 & 0.459 \\
\hline 7/4/2008 12:00 PM & 0.368 & 19.3 & 0.183 & 0.084 & 17.6 & 0.06 & 0.029 & 0.113 & 14.6 & 0.102 & 0.056 & 11.7 & 0.459 \\
\hline 7/4/2008 1:00 PM & 0.368 & 19.3 & 0.184 & 0.084 & 17.6 & 0.06 & 0.029 & 0.112 & 14.6 & 0.102 & 0.056 & 11.7 & 0.459 \\
\hline 7/4/2008 2:00 PM & 0.369 & 19.2 & 0.185 & 0.084 & 17.6 & 0.06 & 0.029 & 0.112 & 14.6 & 0.102 & 0.056 & 11.8 & 0.459 \\
\hline
\end{tabular}


Appendix 3. Volumetric water content, temperature, and electrical-conductivity data collected at the sprinkler-irrigated site during 2008.-Continued

[Depth in feet below land surface; volumetric water content dimensionless; temperature in degrees Celsius; bulk electrical conductivity in decisiemens per meter; --, no data]

\begin{tabular}{|c|c|c|c|c|c|c|c|c|c|c|c|c|c|}
\hline \multirow[b]{3}{*}{$\begin{array}{l}\text { Measurement } \\
\text { date and time }\end{array}$} & \multicolumn{13}{|c|}{ Depth } \\
\hline & \multicolumn{2}{|l|}{2} & \multirow{2}{*}{$\begin{array}{c}3 \\
\text { Volumetric } \\
\text { water } \\
\text { content }\end{array}$} & \multicolumn{3}{|c|}{4} & \multirow{2}{*}{$\begin{array}{c}5 \\
\begin{array}{c}\text { Volumetric } \\
\text { water } \\
\text { content }\end{array}\end{array}$} & \multicolumn{2}{|c|}{7} & \multirow{2}{*}{$\begin{array}{c}9 \\
\text { Volumetric } \\
\text { water } \\
\text { content }\end{array}$} & \multicolumn{2}{|c|}{13} & \multirow{2}{*}{$\begin{array}{c}16.5 \\
\text { Volumetric } \\
\text { water } \\
\text { content }\end{array}$} \\
\hline & $\begin{array}{c}\text { Volumetric } \\
\text { water } \\
\text { content }\end{array}$ & $\begin{array}{l}\text { Temper- } \\
\text { ature }\end{array}$ & & $\begin{array}{c}\text { Volumetric } \\
\text { water } \\
\text { content }\end{array}$ & $\begin{array}{l}\text { Temper- } \\
\text { ature }\end{array}$ & $\begin{array}{c}\text { Bulk } \\
\text { electrical } \\
\text { conduct- } \\
\text { ivity }\end{array}$ & & $\begin{array}{c}\text { Volumetric } \\
\text { water } \\
\text { content }\end{array}$ & $\begin{array}{l}\text { Temper- } \\
\text { ature }\end{array}$ & & $\begin{array}{c}\text { Volumetric } \\
\text { water } \\
\text { content }\end{array}$ & $\begin{array}{l}\text { Temper- } \\
\text { ature }\end{array}$ & \\
\hline 7/4/2008 3:00 PM & 0.368 & 19.2 & 0.186 & 0.084 & 17.6 & 0.06 & 0.029 & 0.113 & 14.6 & 0.102 & 0.056 & 11.7 & 0.459 \\
\hline 7/4/2008 4:00 PM & 0.369 & 19.2 & 0.186 & 0.084 & 17.6 & 0.06 & 0.029 & 0.113 & 14.6 & 0.102 & 0.056 & 11.7 & 0.459 \\
\hline 7/4/2008 5:00 PM & 0.370 & 19.2 & 0.187 & 0.084 & 17.6 & 0.06 & 0.029 & 0.113 & 14.6 & 0.102 & 0.056 & 11.8 & 0.459 \\
\hline 7/4/2008 6:00 PM & 0.370 & 19.2 & 0.187 & 0.084 & 17.6 & 0.06 & 0.029 & 0.113 & 14.6 & 0.102 & 0.056 & 11.7 & 0.459 \\
\hline 7/4/2008 7:00 PM & 0.371 & 19.2 & 0.188 & 0.084 & 17.6 & 0.06 & 0.029 & 0.113 & 14.6 & 0.102 & 0.056 & 11.7 & 0.459 \\
\hline 7/4/2008 8:00 PM & 0.371 & 19.2 & 0.188 & 0.084 & 17.6 & 0.06 & 0.029 & 0.115 & 14.6 & 0.102 & 0.056 & 11.7 & 0.459 \\
\hline 7/4/2008 9:00 PM & 0.371 & 19.2 & 0.188 & 0.084 & 17.6 & 0.06 & 0.029 & 0.115 & 14.6 & 0.102 & 0.056 & 11.7 & 0.459 \\
\hline 7/4/2008 10:00 PM & 0.372 & 19.2 & 0.188 & 0.084 & 17.6 & 0.06 & 0.029 & 0.115 & 14.6 & 0.102 & 0.056 & 11.7 & 0.459 \\
\hline 7/4/2008 11:00 PM & 0.372 & 19.3 & 0.188 & 0.084 & 17.6 & 0.06 & 0.029 & 0.115 & 14.6 & 0.102 & 0.056 & 11.7 & 0.459 \\
\hline 7/5/2008 12:00 AM & 0.372 & 19.3 & 0.188 & 0.084 & 17.6 & 0.06 & 0.029 & 0.115 & 14.6 & 0.102 & 0.056 & 11.7 & 0.459 \\
\hline 7/5/2008 1:00 AM & 0.373 & 19.3 & 0.188 & 0.084 & 17.6 & 0.06 & 0.029 & 0.115 & 14.6 & 0.102 & 0.055 & 11.7 & 0.459 \\
\hline 7/5/2008 2:00 AM & 0.373 & 19.3 & 0.188 & 0.084 & 17.6 & 0.06 & 0.029 & 0.113 & 14.6 & 0.102 & 0.056 & 11.7 & 0.459 \\
\hline 7/5/2008 3:00 AM & 0.373 & 19.4 & 0.188 & 0.084 & 17.6 & 0.06 & 0.029 & 0.113 & 14.6 & 0.102 & 0.056 & 11.7 & 0.459 \\
\hline 7/5/2008 4:00 AM & 0.373 & 19.4 & 0.189 & 0.084 & 17.6 & 0.06 & 0.029 & 0.113 & 14.6 & 0.102 & 0.056 & 11.7 & 0.459 \\
\hline 7/5/2008 5:00 AM & 0.373 & 19.4 & 0.189 & 0.084 & 17.6 & 0.06 & 0.029 & 0.113 & 14.6 & 0.102 & 0.056 & 11.7 & 0.459 \\
\hline 7/5/2008 6:00 AM & 0.373 & 19.5 & 0.189 & 0.084 & 17.6 & 0.06 & 0.029 & 0.113 & 14.6 & 0.102 & 0.056 & 11.7 & 0.459 \\
\hline 7/5/2008 7:00 AM & 0.373 & 19.5 & 0.189 & 0.084 & 17.6 & 0.06 & 0.029 & 0.113 & 14.6 & 0.102 & 0.056 & 11.7 & 0.459 \\
\hline 7/5/2008 8:00 AM & 0.373 & 19.5 & 0.189 & 0.084 & 17.6 & 0.06 & 0.029 & 0.113 & 14.6 & 0.102 & 0.056 & 11.7 & 0.459 \\
\hline 7/5/2008 9:00 AM & 0.374 & 19.5 & 0.189 & 0.084 & 17.6 & 0.06 & 0.030 & 0.115 & 14.7 & 0.102 & 0.056 & 11.7 & 0.459 \\
\hline 7/5/2008 10:00 AM & 0.375 & 19.5 & 0.189 & 0.084 & 17.6 & 0.06 & 0.030 & 0.115 & 14.7 & 0.103 & 0.056 & 11.8 & 0.459 \\
\hline 7/5/2008 11:00 AM & 0.374 & 19.5 & 0.189 & 0.084 & 17.6 & 0.06 & 0.030 & 0.113 & 14.6 & 0.103 & 0.056 & 11.7 & 0.459 \\
\hline 7/5/2008 12:00 PM & 0.374 & 19.5 & 0.189 & 0.084 & 17.6 & 0.06 & 0.030 & 0.113 & 14.6 & 0.103 & 0.056 & 11.8 & 0.459 \\
\hline 7/5/2008 1:00 PM & 0.373 & 19.5 & 0.190 & 0.084 & 17.6 & 0.06 & 0.030 & 0.112 & 14.7 & 0.103 & 0.056 & 11.8 & 0.459 \\
\hline 7/5/2008 2:00 PM & 0.374 & 19.5 & 0.190 & 0.084 & 17.6 & 0.06 & 0.030 & 0.112 & 14.7 & 0.103 & 0.056 & 11.8 & 0.459 \\
\hline 7/5/2008 3:00 PM & 0.374 & 19.5 & 0.190 & 0.084 & 17.6 & 0.06 & 0.030 & 0.113 & 14.7 & 0.103 & 0.056 & 11.8 & 0.459 \\
\hline 7/5/2008 4:00 PM & 0.374 & 19.5 & 0.190 & 0.085 & 17.6 & 0.06 & 0.030 & 0.113 & 14.7 & 0.103 & 0.056 & 11.8 & 0.459 \\
\hline 7/5/2008 5:00 PM & 0.374 & 19.5 & 0.190 & 0.084 & 17.6 & 0.06 & 0.030 & 0.113 & 14.7 & 0.103 & 0.056 & 11.8 & 0.459 \\
\hline 7/5/2008 6:00 PM & 0.374 & 19.5 & 0.190 & 0.084 & 17.6 & 0.06 & 0.030 & 0.113 & 14.7 & 0.103 & 0.056 & 11.8 & 0.459 \\
\hline 7/5/2008 7:00 PM & 0.374 & 19.5 & 0.190 & 0.084 & 17.6 & 0.06 & 0.030 & 0.115 & 14.7 & 0.103 & 0.056 & 11.8 & 0.460 \\
\hline 7/5/2008 8:00 PM & 0.374 & 19.5 & 0.190 & 0.085 & 17.7 & 0.06 & 0.030 & 0.115 & 14.7 & 0.103 & 0.056 & 11.8 & 0.460 \\
\hline 7/5/2008 9:00 PM & 0.374 & 19.5 & 0.190 & 0.085 & 17.7 & 0.06 & 0.030 & 0.115 & 14.7 & 0.103 & 0.056 & 11.8 & 0.460 \\
\hline 7/5/2008 10:00 PM & 0.374 & 19.5 & 0.190 & 0.085 & 17.7 & 0.06 & 0.030 & 0.115 & 14.7 & 0.103 & 0.056 & 11.8 & 0.460 \\
\hline 7/5/2008 11:00 PM & 0.374 & 19.5 & 0.190 & 0.084 & 17.7 & 0.06 & 0.030 & 0.115 & 14.7 & 0.103 & 0.055 & 11.8 & 0.460 \\
\hline 7/6/2008 12:00 AM & 0.374 & 19.5 & 0.190 & 0.084 & 17.7 & 0.06 & 0.030 & 0.115 & 14.7 & 0.103 & 0.055 & 11.8 & 0.460 \\
\hline 7/6/2008 1:00 AM & 0.374 & 19.6 & 0.190 & 0.085 & 17.7 & 0.06 & 0.030 & 0.115 & 14.7 & 0.103 & 0.055 & 11.8 & 0.460 \\
\hline
\end{tabular}


Appendix 3. Volumetric water content, temperature, and electrical-conductivity data collected at the sprinkler-irrigated site during 2008.-Continued

[Depth in feet below land surface; volumetric water content dimensionless; temperature in degrees Celsius; bulk electrical conductivity in decisiemens per meter; --, no data]

\begin{tabular}{|c|c|c|c|c|c|c|c|c|c|c|c|c|c|}
\hline \multirow[b]{3}{*}{$\begin{array}{l}\text { Measurement } \\
\text { date and time }\end{array}$} & \multicolumn{13}{|c|}{ Depth } \\
\hline & \multicolumn{2}{|l|}{2} & \multirow{2}{*}{$\begin{array}{c}3 \\
\text { Volumetric } \\
\text { water } \\
\text { content }\end{array}$} & \multicolumn{3}{|c|}{4} & \multirow{2}{*}{$\begin{array}{c}5 \\
\text { Volumetric } \\
\text { water } \\
\text { content }\end{array}$} & \multicolumn{2}{|c|}{7} & \multirow{2}{*}{$\begin{array}{c}9 \\
\text { Volumetric } \\
\text { water } \\
\text { content }\end{array}$} & \multicolumn{2}{|c|}{13} & \multirow{2}{*}{$\begin{array}{c}16.5 \\
\text { Volumetric } \\
\text { water } \\
\text { content }\end{array}$} \\
\hline & $\begin{array}{c}\text { Volumetric } \\
\text { water } \\
\text { content }\end{array}$ & $\begin{array}{l}\text { Temper- } \\
\text { ature }\end{array}$ & & $\begin{array}{c}\text { Volumetric } \\
\text { water } \\
\text { content }\end{array}$ & $\begin{array}{l}\text { Temper- } \\
\text { ature }\end{array}$ & $\begin{array}{c}\text { Bulk } \\
\text { electrical } \\
\text { conduct- } \\
\text { ivity }\end{array}$ & & $\begin{array}{c}\text { Volumetric } \\
\text { water } \\
\text { content }\end{array}$ & $\begin{array}{l}\text { Temper- } \\
\text { ature }\end{array}$ & & $\begin{array}{c}\text { Volumetric } \\
\text { water } \\
\text { content }\end{array}$ & $\begin{array}{l}\text { Temper- } \\
\text { ature }\end{array}$ & \\
\hline 7/6/2008 2:00 AM & 0.374 & 19.6 & 0.190 & 0.085 & 17.7 & 0.06 & 0.030 & 0.115 & 14.7 & 0.103 & 0.056 & 11.8 & 0.460 \\
\hline 7/6/2008 3:00 AM & 0.374 & 19.6 & 0.190 & 0.085 & 17.7 & 0.06 & 0.030 & 0.115 & 14.7 & 0.103 & 0.056 & 11.8 & 0.460 \\
\hline 7/6/2008 4:00 AM & 0.374 & 19.6 & 0.190 & 0.085 & 17.7 & 0.06 & 0.030 & 0.115 & 14.7 & 0.103 & 0.056 & 11.8 & 0.459 \\
\hline 7/6/2008 5:00 AM & 0.374 & 19.6 & 0.190 & 0.085 & 17.7 & 0.06 & 0.030 & 0.115 & 14.7 & 0.103 & 0.056 & 11.8 & 0.460 \\
\hline 7/6/2008 6:00 AM & 0.374 & 19.6 & 0.190 & 0.085 & 17.7 & 0.06 & 0.030 & 0.115 & 14.7 & 0.103 & 0.056 & 11.8 & 0.460 \\
\hline 7/6/2008 7:00 AM & 0.374 & 19.6 & 0.190 & 0.085 & 17.7 & 0.06 & 0.030 & 0.115 & 14.7 & 0.103 & 0.056 & 11.8 & 0.460 \\
\hline 7/6/2008 8:00 AM & 0.374 & 19.6 & 0.190 & 0.085 & 17.7 & 0.06 & 0.030 & 0.115 & 14.7 & 0.103 & 0.056 & 11.8 & 0.460 \\
\hline 7/6/2008 9:00 AM & 0.374 & 19.6 & 0.190 & 0.085 & 17.7 & 0.06 & 0.030 & 0.115 & 14.7 & 0.103 & 0.055 & 11.8 & 0.460 \\
\hline 7/6/2008 10:00 AM & 0.374 & 19.6 & 0.189 & 0.085 & 17.7 & 0.06 & 0.030 & 0.115 & 14.7 & 0.103 & 0.056 & 11.8 & 0.460 \\
\hline 7/6/2008 11:00 AM & 0.373 & 19.6 & 0.190 & 0.085 & 17.7 & 0.06 & 0.030 & 0.115 & 14.7 & 0.103 & 0.056 & 11.8 & 0.460 \\
\hline 7/6/2008 12:00 PM & 0.373 & 19.6 & 0.190 & 0.085 & 17.7 & 0.06 & 0.030 & 0.113 & 14.7 & 0.103 & 0.056 & 11.8 & 0.460 \\
\hline 7/6/2008 1:00 PM & 0.373 & 19.6 & 0.190 & 0.085 & 17.7 & 0.06 & 0.030 & 0.113 & 14.7 & 0.103 & 0.056 & 11.8 & 0.460 \\
\hline 7/6/2008 2:00 PM & 0.373 & 19.6 & 0.190 & 0.085 & 17.7 & 0.06 & 0.030 & 0.113 & 14.7 & 0.103 & 0.056 & 11.8 & 0.460 \\
\hline 7/6/2008 3:00 PM & 0.373 & 19.6 & 0.190 & 0.085 & 17.7 & 0.06 & 0.030 & 0.113 & 14.7 & 0.103 & 0.056 & 11.8 & 0.460 \\
\hline 7/6/2008 4:00 PM & 0.373 & 19.6 & 0.190 & 0.085 & 17.7 & 0.06 & 0.030 & 0.115 & 14.7 & 0.103 & 0.056 & 11.8 & 0.460 \\
\hline 7/6/2008 5:00 PM & 0.373 & 19.5 & 0.190 & 0.085 & 17.7 & 0.06 & 0.030 & 0.115 & 14.7 & 0.103 & 0.056 & 11.9 & 0.460 \\
\hline 7/6/2008 6:00 PM & 0.373 & 19.6 & 0.190 & 0.085 & 17.7 & 0.06 & 0.030 & 0.115 & 14.7 & 0.103 & 0.056 & 11.8 & 0.460 \\
\hline 7/6/2008 7:00 PM & 0.373 & 19.5 & 0.190 & 0.085 & 17.7 & 0.06 & 0.030 & 0.115 & 14.7 & 0.103 & 0.056 & 11.8 & 0.460 \\
\hline 7/6/2008 8:00 PM & 0.373 & 19.5 & 0.190 & 0.085 & 17.7 & 0.06 & 0.030 & 0.115 & 14.7 & 0.103 & 0.056 & 11.8 & 0.460 \\
\hline 7/6/2008 9:00 PM & 0.373 & 19.6 & 0.190 & 0.085 & 17.7 & 0.06 & 0.030 & 0.115 & 14.7 & 0.103 & 0.056 & 11.8 & 0.460 \\
\hline 7/6/2008 10:00 PM & 0.373 & 19.6 & 0.190 & 0.085 & 17.7 & 0.06 & 0.030 & 0.115 & 14.7 & 0.103 & 0.056 & 11.8 & 0.460 \\
\hline 7/6/2008 11:00 PM & 0.373 & 19.5 & 0.190 & 0.085 & 17.7 & 0.06 & 0.030 & 0.115 & 14.7 & 0.103 & 0.056 & 11.8 & 0.460 \\
\hline 7/7/2008 12:00 AM & 0.373 & 19.6 & 0.190 & 0.085 & 17.7 & 0.06 & 0.030 & 0.116 & 14.8 & 0.103 & 0.056 & 11.8 & 0.460 \\
\hline 7/7/2008 1:00 AM & 0.373 & 19.6 & 0.190 & 0.085 & 17.7 & 0.06 & 0.030 & 0.115 & 14.8 & 0.103 & 0.056 & 11.8 & 0.460 \\
\hline 7/7/2008 2:00 AM & 0.374 & 19.6 & 0.190 & 0.085 & 17.7 & 0.06 & 0.030 & 0.115 & 14.8 & 0.103 & 0.056 & 11.8 & 0.460 \\
\hline 7/7/2008 3:00 AM & 0.374 & 19.6 & 0.190 & 0.085 & 17.7 & 0.06 & 0.030 & 0.115 & 14.8 & 0.103 & 0.056 & 11.8 & 0.460 \\
\hline 7/7/2008 4:00 AM & 0.374 & 19.6 & 0.190 & 0.085 & 17.8 & 0.06 & 0.030 & 0.115 & 14.8 & 0.103 & 0.056 & 11.8 & 0.460 \\
\hline 7/7/2008 5:00 AM & 0.374 & 19.6 & 0.190 & 0.085 & 17.8 & 0.06 & 0.030 & 0.115 & 14.8 & 0.103 & 0.056 & 11.8 & 0.460 \\
\hline 7/7/2008 6:00 AM & 0.374 & 19.6 & 0.190 & 0.085 & 17.7 & 0.06 & 0.030 & 0.116 & 14.8 & 0.103 & 0.056 & 11.8 & 0.460 \\
\hline 7/7/2008 7:00 AM & 0.374 & 19.6 & 0.190 & 0.085 & 17.8 & 0.06 & 0.030 & 0.115 & 14.8 & 0.103 & 0.056 & 11.8 & 0.460 \\
\hline 7/7/2008 8:00 AM & 0.374 & 19.6 & 0.190 & 0.085 & 17.8 & 0.06 & 0.030 & 0.115 & 14.8 & 0.103 & 0.056 & 11.8 & 0.460 \\
\hline 7/7/2008 9:00 AM & 0.374 & 19.6 & 0.190 & 0.085 & 17.8 & 0.06 & 0.030 & 0.115 & 14.8 & 0.103 & 0.056 & 11.8 & 0.460 \\
\hline 7/7/2008 10:00 AM & 0.374 & 19.6 & 0.190 & 0.085 & 17.8 & 0.06 & 0.030 & 0.115 & 14.8 & 0.103 & 0.056 & 11.9 & 0.460 \\
\hline 7/7/2008 11:00 AM & 0.374 & 19.6 & 0.190 & 0.085 & 17.8 & 0.06 & 0.030 & 0.115 & 14.8 & 0.103 & 0.056 & 11.9 & 0.460 \\
\hline 7/7/2008 12:00 PM & 0.374 & 19.6 & 0.190 & 0.085 & 17.8 & 0.06 & 0.030 & 0.115 & 14.8 & 0.103 & 0.056 & 11.9 & 0.460 \\
\hline
\end{tabular}


Appendix 3. Volumetric water content, temperature, and electrical-conductivity data collected at the sprinkler-irrigated site during 2008.-Continued

[Depth in feet below land surface; volumetric water content dimensionless; temperature in degrees Celsius; bulk electrical conductivity in decisiemens per meter; --, no data]

\begin{tabular}{|c|c|c|c|c|c|c|c|c|c|c|c|c|c|}
\hline \multirow[b]{3}{*}{$\begin{array}{l}\text { Measurement } \\
\text { date and time }\end{array}$} & \multicolumn{13}{|c|}{ Depth } \\
\hline & \multicolumn{2}{|c|}{2} & \multirow{2}{*}{$\begin{array}{c}3 \\
\begin{array}{c}\text { Volumetric } \\
\text { water } \\
\text { content }\end{array}\end{array}$} & \multicolumn{3}{|c|}{4} & \multirow{2}{*}{$\begin{array}{c}5 \\
\text { Volumetric } \\
\text { water } \\
\text { content }\end{array}$} & \multicolumn{2}{|l|}{7} & \multirow{2}{*}{$\begin{array}{c}9 \\
\text { Volumetric } \\
\text { water } \\
\text { content }\end{array}$} & \multicolumn{2}{|c|}{13} & \multirow{2}{*}{$\begin{array}{c}16.5 \\
\text { Volumetric } \\
\text { water } \\
\text { content }\end{array}$} \\
\hline & $\begin{array}{c}\text { Volumetric } \\
\text { water } \\
\text { content }\end{array}$ & $\begin{array}{l}\text { Temper- } \\
\text { ature }\end{array}$ & & $\begin{array}{c}\text { Volumetric } \\
\text { water } \\
\text { content }\end{array}$ & $\begin{array}{l}\text { Temper- } \\
\text { ature }\end{array}$ & $\begin{array}{c}\text { Bulk } \\
\text { electrical } \\
\text { conduct- } \\
\text { ivity }\end{array}$ & & $\begin{array}{c}\text { Volumetric } \\
\text { water } \\
\text { content }\end{array}$ & $\begin{array}{l}\text { Temper- } \\
\text { ature }\end{array}$ & & $\begin{array}{c}\text { Volumetric } \\
\text { water } \\
\text { content }\end{array}$ & $\begin{array}{l}\text { Temper- } \\
\text { ature }\end{array}$ & \\
\hline 7/7/2008 1:00 PM & 0.373 & 19.6 & 0.190 & 0.085 & 17.8 & 0.06 & 0.030 & 0.115 & 14.8 & 0.103 & 0.056 & 11.8 & 0.460 \\
\hline 7/7/2008 2:00 PM & 0.374 & 19.5 & 0.190 & 0.085 & 17.8 & 0.06 & 0.030 & 0.113 & 14.8 & 0.103 & 0.056 & 11.9 & 0.460 \\
\hline 7/7/2008 3:00 PM & 0.374 & 19.5 & 0.190 & 0.085 & 17.8 & 0.06 & 0.030 & 0.113 & 14.8 & 0.103 & 0.057 & 11.9 & 0.460 \\
\hline 7/7/2008 4:00 PM & 0.373 & 19.5 & 0.190 & 0.085 & 17.8 & 0.06 & 0.030 & 0.113 & 14.8 & 0.103 & 0.056 & 11.9 & 0.460 \\
\hline 7/7/2008 5:00 PM & 0.373 & 19.5 & 0.190 & 0.085 & 17.8 & 0.06 & 0.030 & 0.115 & 14.8 & 0.103 & 0.056 & 11.9 & 0.460 \\
\hline 7/7/2008 6:00 PM & 0.372 & 19.4 & 0.190 & 0.085 & 17.8 & 0.06 & 0.030 & 0.115 & 14.8 & 0.103 & 0.056 & 11.8 & 0.460 \\
\hline 7/7/2008 7:00 PM & 0.372 & 19.4 & 0.190 & 0.085 & 17.8 & 0.06 & 0.030 & 0.115 & 14.8 & 0.103 & 0.056 & 11.9 & 0.460 \\
\hline 7/7/2008 8:00 PM & 0.373 & 19.4 & 0.190 & 0.085 & 17.8 & 0.06 & 0.030 & 0.115 & 14.8 & 0.103 & 0.056 & 11.9 & 0.460 \\
\hline 7/7/2008 9:00 PM & 0.373 & 19.4 & 0.190 & 0.085 & 17.8 & 0.06 & 0.030 & 0.115 & 14.8 & 0.103 & 0.056 & 11.9 & 0.460 \\
\hline 7/7/2008 10:00 PM & 0.373 & 19.4 & 0.190 & 0.085 & 17.8 & 0.06 & 0.030 & 0.116 & 14.8 & 0.103 & 0.056 & 11.8 & 0.460 \\
\hline 7/7/2008 11:00 PM & 0.373 & 19.4 & 0.190 & 0.085 & 17.8 & 0.06 & 0.030 & 0.115 & 14.8 & 0.103 & 0.056 & 11.9 & 0.460 \\
\hline 7/8/2008 12:00 AM & 0.373 & 19.4 & 0.190 & 0.086 & 17.8 & 0.06 & 0.030 & 0.115 & 14.8 & 0.103 & 0.056 & 11.9 & 0.460 \\
\hline 7/8/2008 1:00 AM & 0.373 & 19.4 & 0.190 & 0.086 & 17.8 & 0.06 & 0.030 & 0.116 & 14.8 & 0.103 & 0.056 & 11.8 & 0.460 \\
\hline 7/8/2008 2:00 AM & 0.372 & 19.4 & 0.190 & 0.085 & 17.8 & 0.06 & 0.030 & 0.116 & 14.8 & 0.103 & 0.056 & 11.8 & 0.460 \\
\hline 7/8/2008 3:00 AM & 0.373 & 19.4 & 0.190 & 0.085 & 17.8 & 0.06 & 0.030 & 0.116 & 14.8 & 0.103 & 0.056 & 11.9 & 0.460 \\
\hline 7/8/2008 4:00 AM & 0.373 & 19.4 & 0.190 & 0.086 & 17.8 & 0.06 & 0.030 & 0.115 & 14.8 & 0.103 & 0.056 & 11.9 & 0.460 \\
\hline 7/8/2008 5:00 AM & 0.372 & 19.4 & 0.190 & 0.085 & 17.8 & 0.06 & 0.030 & 0.115 & 14.8 & 0.103 & 0.056 & 11.9 & 0.460 \\
\hline 7/8/2008 6:00 AM & 0.372 & 19.4 & 0.190 & 0.086 & 17.8 & 0.06 & 0.030 & 0.115 & 14.8 & 0.103 & 0.056 & 11.9 & 0.460 \\
\hline 7/8/2008 7:00 AM & 0.373 & 19.4 & 0.190 & 0.086 & 17.8 & 0.06 & 0.030 & 0.116 & 14.8 & 0.103 & 0.056 & 11.9 & 0.460 \\
\hline 7/8/2008 8:00 AM & 0.373 & 19.4 & 0.190 & 0.085 & 17.8 & 0.06 & 0.030 & 0.116 & 14.8 & 0.103 & 0.056 & 11.9 & 0.460 \\
\hline 7/8/2008 9:00 AM & 0.373 & 19.4 & 0.189 & 0.086 & 17.8 & 0.06 & 0.030 & 0.115 & 14.8 & 0.103 & 0.056 & 11.9 & 0.460 \\
\hline 7/8/2008 10:00 AM & 0.372 & 19.3 & 0.189 & 0.086 & 17.8 & 0.06 & 0.030 & 0.115 & 14.8 & 0.103 & 0.056 & 11.9 & 0.460 \\
\hline 7/8/2008 11:00 AM & 0.372 & 19.3 & 0.189 & 0.086 & 17.8 & 0.06 & 0.030 & 0.115 & 14.9 & 0.103 & 0.056 & 11.9 & 0.460 \\
\hline 7/8/2008 12:00 PM & 0.372 & 19.3 & 0.189 & 0.086 & 17.8 & 0.06 & 0.030 & 0.113 & 14.9 & 0.103 & 0.056 & 12.0 & 0.460 \\
\hline 7/8/2008 1:00 PM & 0.372 & 19.3 & 0.190 & 0.086 & 17.8 & 0.06 & 0.030 & 0.113 & 14.8 & 0.103 & 0.056 & 12.0 & 0.460 \\
\hline 7/8/2008 2:00 PM & 0.371 & 19.3 & 0.190 & 0.086 & 17.8 & 0.06 & 0.030 & 0.113 & 14.8 & 0.103 & 0.056 & 12.0 & 0.460 \\
\hline 7/8/2008 3:00 PM & 0.371 & 19.2 & 0.189 & 0.086 & 17.8 & 0.06 & 0.030 & 0.113 & 14.9 & 0.103 & 0.056 & 11.9 & 0.460 \\
\hline 7/8/2008 4:00 PM & 0.371 & 19.2 & 0.189 & 0.086 & 17.8 & 0.06 & 0.030 & 0.115 & 14.8 & 0.103 & 0.056 & 11.9 & 0.460 \\
\hline 7/8/2008 5:00 PM & 0.372 & 19.2 & 0.189 & 0.086 & 17.8 & 0.06 & 0.030 & 0.115 & 14.8 & 0.103 & 0.056 & 11.9 & 0.460 \\
\hline 7/8/2008 6:00 PM & 0.372 & 19.2 & 0.189 & 0.086 & 17.8 & 0.06 & 0.030 & 0.115 & 14.9 & 0.103 & 0.056 & 12.0 & 0.460 \\
\hline 7/8/2008 7:00 PM & 0.371 & 19.1 & 0.189 & 0.086 & 17.8 & 0.06 & 0.030 & 0.115 & 14.9 & 0.103 & 0.056 & 11.9 & 0.460 \\
\hline 7/8/2008 8:00 PM & 0.372 & 19.1 & 0.189 & 0.086 & 17.8 & 0.06 & 0.030 & 0.115 & 14.9 & 0.103 & 0.056 & 11.9 & 0.460 \\
\hline 7/8/2008 9:00 PM & 0.371 & 19.1 & 0.189 & 0.086 & 17.8 & 0.06 & 0.030 & 0.116 & 14.9 & 0.103 & 0.056 & 11.9 & 0.460 \\
\hline 7/8/2008 10:00 PM & 0.371 & 19.1 & 0.190 & 0.086 & 17.8 & 0.06 & 0.030 & 0.116 & 14.9 & 0.103 & 0.056 & 11.9 & 0.460 \\
\hline 7/8/2008 11:00 PM & 0.371 & 19.1 & 0.190 & 0.086 & 17.9 & 0.06 & 0.030 & 0.115 & 14.9 & 0.103 & 0.056 & 11.9 & 0.460 \\
\hline
\end{tabular}


Appendix 3. Volumetric water content, temperature, and electrical-conductivity data collected at the sprinkler-irrigated site during 2008.-Continued

[Depth in feet below land surface; volumetric water content dimensionless; temperature in degrees Celsius; bulk electrical conductivity in decisiemens per meter; --, no data]

\begin{tabular}{|c|c|c|c|c|c|c|c|c|c|c|c|c|c|}
\hline \multirow[b]{3}{*}{$\begin{array}{l}\text { Measurement } \\
\text { date and time }\end{array}$} & \multicolumn{13}{|c|}{ Depth } \\
\hline & \multicolumn{2}{|l|}{2} & \multirow{2}{*}{$\begin{array}{c}3 \\
\begin{array}{c}\text { Volumetric } \\
\text { water } \\
\text { content }\end{array}\end{array}$} & \multicolumn{3}{|c|}{4} & \multirow{2}{*}{$\begin{array}{c}5 \\
\text { Volumetric } \\
\text { water } \\
\text { content }\end{array}$} & \multicolumn{2}{|l|}{7} & \multirow{2}{*}{$\begin{array}{c}9 \\
\text { Volumetric } \\
\text { water } \\
\text { content }\end{array}$} & \multicolumn{2}{|c|}{13} & \multirow{2}{*}{$\begin{array}{c}16.5 \\
\text { Volumetric } \\
\text { water } \\
\text { content }\end{array}$} \\
\hline & $\begin{array}{l}\text { Volumetric } \\
\text { water } \\
\text { content }\end{array}$ & $\begin{array}{l}\text { Temper- } \\
\text { ature }\end{array}$ & & $\begin{array}{c}\text { Volumetric } \\
\text { water } \\
\text { content }\end{array}$ & $\begin{array}{l}\text { Temper- } \\
\text { ature }\end{array}$ & $\begin{array}{c}\text { Bulk } \\
\text { electrical } \\
\text { conduct- } \\
\text { ivity }\end{array}$ & & $\begin{array}{l}\text { Volumetric } \\
\text { water } \\
\text { content }\end{array}$ & $\begin{array}{l}\text { Temper- } \\
\text { ature }\end{array}$ & & $\begin{array}{c}\text { Volumetric } \\
\text { water } \\
\text { content }\end{array}$ & $\begin{array}{l}\text { Temper- } \\
\text { ature }\end{array}$ & \\
\hline 7/9/2008 12:00 AM & 0.371 & 19.1 & 0.189 & 0.086 & 17.8 & 0.06 & 0.030 & 0.116 & 14.9 & 0.103 & 0.056 & 12.0 & 0.460 \\
\hline 7/9/2008 1:00 AM & 0.371 & 19.1 & 0.189 & 0.086 & 17.8 & 0.06 & 0.030 & 0.116 & 14.9 & 0.103 & 0.056 & 11.9 & 0.460 \\
\hline 7/9/2008 2:00 AM & 0.371 & 19.2 & 0.189 & 0.086 & 17.9 & 0.06 & 0.030 & 0.116 & 14.9 & 0.103 & 0.056 & 11.9 & 0.460 \\
\hline 7/9/2008 3:00 AM & 0.371 & 19.2 & 0.189 & 0.086 & 17.8 & 0.06 & 0.030 & 0.116 & 14.9 & 0.103 & 0.056 & 11.9 & 0.460 \\
\hline 7/9/2008 4:00 AM & 0.371 & 19.2 & 0.189 & 0.086 & 17.8 & 0.06 & 0.030 & 0.116 & 14.9 & 0.103 & 0.056 & 11.9 & 0.460 \\
\hline 7/9/2008 5:00 AM & 0.371 & 19.2 & 0.189 & 0.086 & 17.8 & 0.06 & 0.030 & 0.116 & 14.9 & 0.103 & 0.056 & 12.0 & 0.460 \\
\hline 7/9/2008 6:00 AM & 0.370 & 19.2 & 0.189 & 0.086 & 17.9 & 0.06 & 0.030 & 0.116 & 14.9 & 0.103 & 0.056 & 11.9 & 0.460 \\
\hline 7/9/2008 7:00 AM & 0.371 & 19.2 & 0.189 & 0.086 & 17.8 & 0.06 & 0.030 & 0.116 & 14.9 & 0.103 & 0.056 & 11.9 & 0.460 \\
\hline 7/9/2008 8:00 AM & 0.371 & 19.2 & 0.189 & 0.086 & 17.8 & 0.06 & 0.030 & 0.116 & 14.9 & 0.103 & 0.056 & 11.9 & 0.460 \\
\hline 7/9/2008 9:00 AM & 0.371 & 19.2 & 0.189 & 0.086 & 17.8 & 0.06 & 0.030 & 0.116 & 14.9 & 0.103 & 0.056 & 12.0 & 0.460 \\
\hline 7/9/2008 10:00 AM & 0.371 & 19.1 & 0.189 & 0.086 & 17.9 & 0.06 & 0.030 & 0.115 & 14.9 & 0.103 & 0.056 & 12.0 & 0.460 \\
\hline 7/9/2008 11:00 AM & 0.370 & 19.1 & 0.189 & 0.086 & 17.8 & 0.06 & 0.030 & 0.115 & 14.9 & 0.103 & 0.056 & 12.0 & 0.460 \\
\hline 7/9/2008 12:00 PM & 0.371 & 19.1 & 0.189 & 0.086 & 17.8 & 0.06 & 0.030 & 0.113 & 14.9 & 0.104 & 0.056 & 12.0 & 0.460 \\
\hline 7/9/2008 1:00 PM & 0.370 & 19.1 & 0.189 & 0.086 & 17.8 & 0.06 & 0.030 & 0.115 & 14.9 & 0.103 & 0.057 & 12.0 & 0.460 \\
\hline 7/9/2008 2:00 PM & 0.370 & 19.1 & 0.189 & 0.086 & 17.8 & 0.06 & 0.030 & 0.115 & 14.9 & 0.104 & 0.056 & 12.0 & 0.460 \\
\hline 7/9/2008 3:00 PM & 0.369 & 19.0 & 0.189 & 0.086 & 17.8 & 0.06 & 0.030 & 0.115 & 14.9 & 0.104 & 0.056 & 12.0 & 0.460 \\
\hline 7/9/2008 4:00 PM & 0.369 & 19.0 & 0.189 & 0.086 & 17.8 & 0.06 & 0.030 & 0.115 & 14.9 & 0.104 & 0.056 & 12.0 & 0.460 \\
\hline 7/9/2008 5:00 PM & 0.368 & 19.0 & 0.189 & 0.086 & 17.8 & 0.06 & 0.030 & 0.115 & 14.9 & 0.104 & 0.056 & 12.0 & 0.460 \\
\hline 7/9/2008 6:00 PM & 0.368 & 19.0 & 0.189 & 0.086 & 17.8 & 0.06 & 0.030 & 0.115 & 14.9 & 0.104 & 0.056 & 12.0 & 0.460 \\
\hline 7/9/2008 7:00 PM & 0.369 & 19.0 & 0.189 & 0.086 & 17.8 & 0.06 & 0.030 & 0.113 & 14.9 & 0.104 & 0.056 & 12.0 & 0.460 \\
\hline 7/9/2008 8:00 PM & 0.369 & 19.0 & 0.189 & 0.086 & 17.8 & 0.06 & 0.030 & 0.115 & 14.9 & 0.104 & 0.056 & 12.0 & 0.460 \\
\hline 7/9/2008 9:00 PM & 0.369 & 18.9 & 0.189 & 0.086 & 17.8 & 0.06 & 0.030 & 0.115 & 14.9 & 0.104 & 0.056 & 12.0 & 0.460 \\
\hline 7/9/2008 10:00 PM & 0.369 & 19.0 & 0.189 & 0.086 & 17.8 & 0.06 & 0.030 & 0.115 & 14.9 & 0.104 & 0.056 & 12.0 & 0.460 \\
\hline 7/9/2008 11:00 PM & 0.369 & 19.0 & 0.189 & 0.086 & 17.8 & 0.06 & 0.030 & 0.115 & 14.9 & 0.104 & 0.056 & 12.0 & 0.460 \\
\hline 7/10/2008 12:00 AM & 0.369 & 19.0 & 0.189 & 0.086 & 17.8 & 0.06 & 0.030 & 0.115 & 15.0 & 0.104 & 0.056 & 12.0 & 0.460 \\
\hline 7/10/2008 1:00 AM & 0.369 & 19.0 & 0.189 & 0.086 & 17.8 & 0.06 & 0.030 & 0.115 & 15.0 & 0.104 & 0.056 & 12.0 & 0.460 \\
\hline 7/10/2008 2:00 AM & 0.369 & 19.0 & 0.189 & 0.086 & 17.8 & 0.06 & 0.030 & 0.116 & 15.0 & 0.104 & 0.056 & 12.0 & 0.460 \\
\hline 7/10/2008 3:00 AM & 0.369 & 19.1 & 0.189 & 0.086 & 17.8 & 0.06 & 0.030 & 0.116 & 14.9 & 0.104 & 0.056 & 12.0 & 0.460 \\
\hline 7/10/2008 4:00 AM & 0.369 & 19.1 & 0.189 & 0.086 & 17.8 & 0.06 & 0.030 & 0.116 & 15.0 & 0.104 & 0.056 & 12.0 & 0.460 \\
\hline 7/10/2008 5:00 AM & 0.370 & 19.1 & 0.189 & 0.086 & 17.8 & 0.06 & 0.030 & 0.116 & 15.0 & 0.104 & 0.056 & 12.0 & 0.460 \\
\hline 7/10/2008 6:00 AM & 0.369 & 19.1 & 0.189 & 0.086 & 17.8 & 0.06 & 0.030 & 0.116 & 14.9 & 0.104 & 0.056 & 12.0 & 0.460 \\
\hline 7/10/2008 7:00 AM & 0.369 & 19.1 & 0.189 & 0.086 & 17.8 & 0.06 & 0.030 & 0.116 & 15.0 & 0.104 & 0.056 & 12.0 & 0.460 \\
\hline 7/10/2008 8:00 AM & 0.369 & 19.2 & 0.189 & 0.086 & 17.8 & 0.06 & 0.030 & 0.116 & 15.0 & 0.104 & 0.056 & 12.0 & 0.460 \\
\hline 7/10/2008 9:00 AM & 0.369 & 19.1 & 0.189 & 0.086 & 17.8 & 0.06 & 0.030 & 0.115 & 15.0 & 0.104 & 0.056 & 12.0 & 0.460 \\
\hline 7/10/2008 10:00 AM & 0.370 & 19.2 & 0.188 & 0.086 & 17.8 & 0.06 & 0.030 & 0.115 & 15.0 & 0.104 & 0.056 & 12.0 & 0.460 \\
\hline
\end{tabular}


Appendix 3. Volumetric water content, temperature, and electrical-conductivity data collected at the sprinkler-irrigated site during 2008.-Continued

[Depth in feet below land surface; volumetric water content dimensionless; temperature in degrees Celsius; bulk electrical conductivity in decisiemens per meter; --, no data]

\begin{tabular}{|c|c|c|c|c|c|c|c|c|c|c|c|c|c|}
\hline \multirow[b]{3}{*}{$\begin{array}{l}\text { Measurement } \\
\text { date and time }\end{array}$} & \multicolumn{13}{|c|}{ Depth } \\
\hline & \multicolumn{2}{|l|}{2} & \multirow{2}{*}{$\begin{array}{c}3 \\
\text { Volumetric } \\
\text { water } \\
\text { content }\end{array}$} & \multicolumn{3}{|c|}{4} & \multirow{2}{*}{$\begin{array}{c}5 \\
\text { Volumetric } \\
\text { water } \\
\text { content }\end{array}$} & \multicolumn{2}{|l|}{7} & \multirow{2}{*}{$\begin{array}{c}9 \\
\text { Volumetric } \\
\text { water } \\
\text { content }\end{array}$} & \multicolumn{2}{|c|}{13} & \multirow{2}{*}{$\begin{array}{c}16.5 \\
\text { Volumetric } \\
\text { water } \\
\text { content }\end{array}$} \\
\hline & $\begin{array}{c}\text { Volumetric } \\
\text { water } \\
\text { content }\end{array}$ & $\begin{array}{l}\text { Temper- } \\
\text { ature }\end{array}$ & & $\begin{array}{c}\text { Volumetric } \\
\text { water } \\
\text { content }\end{array}$ & $\begin{array}{l}\text { Temper- } \\
\text { ature }\end{array}$ & $\begin{array}{c}\text { Bulk } \\
\text { electrical } \\
\text { conduct- } \\
\text { ivity }\end{array}$ & & $\begin{array}{l}\text { Volumetric } \\
\text { water } \\
\text { content }\end{array}$ & $\begin{array}{l}\text { Temper- } \\
\text { ature }\end{array}$ & & $\begin{array}{c}\text { Volumetric } \\
\text { water } \\
\text { content }\end{array}$ & $\begin{array}{l}\text { Temper- } \\
\text { ature }\end{array}$ & \\
\hline 7/10/2008 11:00 AM & 0.406 & 19.2 & 0.216 & 0.086 & 17.8 & 0.06 & 0.031 & 0.115 & 15.0 & 0.104 & 0.056 & 12.0 & 0.460 \\
\hline 7/10/2008 12:00 PM & 0.505 & 19.7 & 0.450 & 0.345 & 18.0 & 1.35 & 0.228 & 0.117 & 15.0 & 0.104 & 0.056 & 12.0 & 0.460 \\
\hline 7/10/2008 1:00 PM & 0.506 & 19.9 & 0.457 & 0.444 & 18.1 & 1.88 & 0.451 & 0.499 & 15.3 & 0.104 & 0.055 & 12.0 & 0.460 \\
\hline 7/10/2008 2:00 PM & 0.510 & 20.5 & 0.454 & 0.526 & 18.3 & 2.31 & 0.448 & 0.507 & 15.4 & 0.132 & 0.056 & 12.0 & 0.460 \\
\hline 7/10/2008 3:00 PM & 0.516 & 21.2 & 0.454 & 0.538 & 18.5 & 2.38 & 0.444 & 0.506 & 15.5 & 0.204 & 0.056 & 12.1 & 0.460 \\
\hline 7/10/2008 4:00 PM & 0.520 & 22.1 & 0.454 & 0.535 & 18.7 & 2.23 & 0.442 & 0.506 & 15.7 & 0.208 & 0.055 & 12.0 & 0.460 \\
\hline 7/10/2008 5:00 PM & 0.525 & 22.9 & 0.454 & 0.532 & 18.9 & 2.13 & 0.442 & 0.506 & 15.8 & 0.207 & 0.055 & 12.0 & 0.460 \\
\hline 7/10/2008 6:00 PM & 0.526 & 23.3 & 0.454 & 0.533 & 19.0 & 2.18 & 0.439 & 0.505 & 15.9 & 0.207 & 0.061 & 12.1 & 0.461 \\
\hline 7/10/2008 7:00 PM & 0.522 & 23.4 & 0.454 & 0.540 & 19.1 & 2.50 & 0.435 & 0.504 & 16.0 & 0.177 & 0.115 & 12.1 & 0.461 \\
\hline 7/10/2008 8:00 PM & 0.505 & 23.3 & 0.456 & 0.544 & 19.2 & 2.64 & 0.434 & 0.501 & 16.0 & 0.163 & 0.113 & 12.1 & 0.462 \\
\hline 7/10/2008 9:00 PM & 0.500 & 23.2 & 0.456 & 0.542 & 19.3 & 2.56 & 0.360 & 0.501 & 16.0 & 0.156 & 0.110 & 12.2 & 0.462 \\
\hline 7/10/2008 10:00 PM & 0.495 & 23.1 & 0.410 & 0.492 & 19.3 & 2.20 & 0.300 & 0.480 & 16.0 & 0.152 & 0.106 & 12.2 & 0.462 \\
\hline 7/10/2008 11:00 PM & 0.490 & 23.0 & 0.357 & 0.382 & 19.4 & 1.46 & 0.279 & 0.340 & 16.0 & 0.149 & 0.100 & 12.3 & 0.462 \\
\hline 7/11/2008 12:00 AM & 0.486 & 22.9 & 0.304 & 0.328 & 19.4 & 1.05 & 0.264 & 0.297 & 16.0 & 0.147 & 0.095 & 12.3 & 0.462 \\
\hline 7/11/2008 1:00 AM & 0.483 & 22.8 & 0.295 & 0.303 & 19.5 & 0.91 & 0.248 & 0.282 & 16.0 & 0.145 & 0.092 & 12.4 & 0.462 \\
\hline 7/11/2008 2:00 AM & 0.481 & 22.8 & 0.289 & 0.282 & 19.5 & 0.81 & 0.237 & 0.271 & 16.0 & 0.143 & 0.088 & 12.4 & 0.462 \\
\hline 7/11/2008 3:00 AM & 0.478 & 22.7 & 0.284 & 0.263 & 19.5 & 0.73 & 0.231 & 0.261 & 16.0 & 0.143 & 0.085 & 12.4 & 0.463 \\
\hline 7/11/2008 4:00 AM & 0.474 & 22.6 & 0.279 & 0.248 & 19.5 & 0.66 & 0.225 & 0.254 & 16.0 & 0.141 & 0.083 & 12.4 & 0.463 \\
\hline 7/11/2008 5:00 AM & 0.472 & 22.6 & 0.274 & 0.236 & 19.5 & 0.60 & 0.220 & 0.246 & 16.0 & 0.140 & 0.081 & 12.4 & 0.462 \\
\hline 7/11/2008 6:00 AM & 0.470 & 22.5 & 0.269 & 0.225 & 19.6 & 0.56 & 0.215 & 0.241 & 16.0 & 0.139 & 0.079 & 12.4 & 0.463 \\
\hline 7/11/2008 7:00 AM & 0.467 & 22.5 & 0.265 & 0.217 & 19.6 & 0.52 & 0.211 & 0.235 & 16.0 & 0.138 & 0.078 & 12.4 & 0.463 \\
\hline 7/11/2008 8:00 AM & 0.465 & 22.5 & 0.261 & 0.209 & 19.6 & 0.49 & 0.207 & 0.231 & 16.0 & 0.138 & 0.076 & 12.5 & 0.463 \\
\hline 7/11/2008 9:00 AM & 0.462 & 22.4 & 0.257 & 0.204 & 19.6 & 0.46 & 0.203 & 0.226 & 16.0 & 0.138 & 0.075 & 12.5 & 0.463 \\
\hline 7/11/2008 10:00 AM & 0.460 & 22.4 & 0.255 & 0.199 & 19.6 & 0.44 & 0.200 & 0.223 & 16.0 & 0.137 & 0.075 & 12.5 & 0.463 \\
\hline 7/11/2008 11:00 AM & 0.458 & 22.3 & 0.252 & 0.195 & 19.6 & 0.43 & 0.197 & 0.220 & 16.0 & 0.137 & 0.073 & 12.5 & 0.463 \\
\hline 7/11/2008 12:00 PM & 0.456 & 22.3 & 0.250 & 0.191 & 19.6 & 0.41 & 0.194 & 0.218 & 16.0 & 0.136 & 0.072 & 12.5 & 0.463 \\
\hline 7/11/2008 1:00 PM & 0.454 & 22.2 & 0.247 & 0.187 & 19.6 & 0.40 & 0.191 & 0.215 & 16.0 & 0.136 & 0.072 & 12.6 & 0.463 \\
\hline 7/11/2008 2:00 PM & 0.450 & 22.2 & 0.245 & 0.184 & 19.7 & 0.39 & 0.188 & 0.212 & 16.0 & 0.135 & 0.071 & 12.6 & 0.463 \\
\hline 7/11/2008 3:00 PM & 0.448 & 22.1 & 0.244 & 0.182 & 19.7 & 0.38 & 0.186 & 0.211 & 16.0 & 0.135 & 0.071 & 12.6 & 0.463 \\
\hline 7/11/2008 4:00 PM & 0.446 & 22.1 & 0.242 & 0.180 & 19.7 & 0.37 & 0.183 & 0.209 & 16.1 & 0.134 & 0.070 & 12.6 & 0.463 \\
\hline 7/11/2008 5:00 PM & 0.444 & 22.0 & 0.240 & 0.178 & 19.7 & 0.36 & 0.182 & 0.208 & 16.1 & 0.134 & 0.069 & 12.6 & 0.463 \\
\hline 7/11/2008 6:00 PM & 0.442 & 22.0 & 0.239 & 0.175 & 19.7 & 0.35 & 0.180 & 0.207 & 16.1 & 0.133 & 0.069 & 12.6 & 0.463 \\
\hline 7/11/2008 7:00 PM & 0.440 & 21.9 & 0.238 & 0.172 & 19.7 & 0.34 & 0.177 & 0.206 & 16.1 & 0.133 & 0.069 & 12.6 & 0.463 \\
\hline 7/11/2008 8:00 PM & 0.438 & 21.9 & 0.236 & 0.171 & 19.7 & 0.34 & 0.176 & 0.205 & 16.1 & 0.133 & 0.069 & 12.6 & 0.463 \\
\hline 7/11/2008 9:00 PM & 0.437 & 21.9 & 0.235 & 0.169 & 19.7 & 0.33 & 0.173 & 0.204 & 16.1 & 0.132 & 0.069 & 12.6 & 0.463 \\
\hline
\end{tabular}


Appendix 3. Volumetric water content, temperature, and electrical-conductivity data collected at the sprinkler-irrigated site during 2008.-Continued

[Depth in feet below land surface; volumetric water content dimensionless; temperature in degrees Celsius; bulk electrical conductivity in decisiemens per meter; --, no data]

\begin{tabular}{|c|c|c|c|c|c|c|c|c|c|c|c|c|c|}
\hline \multirow[b]{3}{*}{$\begin{array}{l}\text { Measurement } \\
\text { date and time }\end{array}$} & \multicolumn{13}{|c|}{ Depth } \\
\hline & \multicolumn{2}{|l|}{2} & \multirow{2}{*}{$\begin{array}{c}3 \\
\text { Volumetric } \\
\text { water } \\
\text { content }\end{array}$} & \multicolumn{3}{|c|}{4} & \multirow{2}{*}{$\begin{array}{c}5 \\
\text { Volumetric } \\
\text { water } \\
\text { content }\end{array}$} & \multicolumn{2}{|c|}{7} & \multirow{2}{*}{$\begin{array}{c}9 \\
\text { Volumetric } \\
\text { water } \\
\text { content }\end{array}$} & \multicolumn{2}{|c|}{13} & \multirow{2}{*}{$\begin{array}{c}16.5 \\
\text { Volumetric } \\
\text { water } \\
\text { content }\end{array}$} \\
\hline & $\begin{array}{c}\text { Volumetric } \\
\text { water } \\
\text { content }\end{array}$ & $\begin{array}{l}\text { Temper- } \\
\text { ature }\end{array}$ & & $\begin{array}{c}\text { Volumetric } \\
\text { water } \\
\text { content }\end{array}$ & $\begin{array}{l}\text { Temper- } \\
\text { ature }\end{array}$ & $\begin{array}{c}\text { Bulk } \\
\text { electrical } \\
\text { conduct- } \\
\text { ivity }\end{array}$ & & $\begin{array}{c}\text { Volumetric } \\
\text { water } \\
\text { content }\end{array}$ & $\begin{array}{l}\text { Temper- } \\
\text { ature }\end{array}$ & & $\begin{array}{l}\text { Volumetric } \\
\text { water } \\
\text { content }\end{array}$ & $\begin{array}{l}\text { Temper- } \\
\text { ature }\end{array}$ & \\
\hline 7/11/2008 10:00 PM & 0.435 & 21.9 & 0.234 & 0.167 & 19.7 & 0.33 & 0.171 & 0.203 & 16.1 & 0.132 & 0.069 & 12.6 & 0.463 \\
\hline 7/11/2008 11:00 PM & 0.435 & 21.8 & 0.234 & 0.167 & 19.7 & 0.32 & 0.171 & 0.201 & 16.1 & 0.132 & 0.068 & 12.6 & 0.463 \\
\hline 7/12/2008 12:00 AM & 0.434 & 21.8 & 0.233 & 0.166 & 19.7 & 0.32 & 0.169 & 0.201 & 16.1 & 0.132 & 0.067 & 12.6 & 0.464 \\
\hline 7/12/2008 1:00 AM & 0.433 & 21.8 & 0.232 & 0.165 & 19.7 & 0.31 & 0.168 & 0.200 & 16.1 & 0.132 & 0.067 & 12.6 & 0.464 \\
\hline 7/12/2008 2:00 AM & 0.432 & 21.8 & 0.231 & 0.163 & 19.7 & 0.31 & 0.167 & 0.200 & 16.1 & 0.132 & 0.066 & 12.6 & 0.464 \\
\hline 7/12/2008 3:00 AM & 0.431 & 21.8 & 0.230 & 0.162 & 19.7 & 0.31 & 0.166 & 0.199 & 16.1 & 0.132 & 0.066 & 12.6 & 0.464 \\
\hline 7/12/2008 4:00 AM & 0.431 & 21.8 & 0.230 & 0.161 & 19.7 & 0.31 & 0.165 & 0.199 & 16.1 & 0.131 & 0.066 & 12.6 & 0.464 \\
\hline 7/12/2008 5:00 AM & 0.430 & 21.7 & 0.229 & 0.160 & 19.7 & 0.30 & 0.164 & 0.199 & 16.1 & 0.131 & 0.066 & 12.6 & 0.464 \\
\hline 7/12/2008 6:00 AM & 0.429 & 21.7 & 0.228 & 0.160 & 19.7 & 0.30 & 0.163 & 0.198 & 16.1 & 0.131 & 0.067 & 12.6 & 0.464 \\
\hline 7/12/2008 7:00 AM & 0.428 & 21.7 & 0.228 & 0.159 & 19.7 & 0.30 & 0.162 & 0.198 & 16.1 & 0.131 & 0.066 & 12.6 & 0.464 \\
\hline 7/12/2008 8:00 AM & 0.428 & 21.7 & 0.228 & 0.158 & 19.7 & 0.30 & 0.161 & 0.198 & 16.2 & 0.131 & 0.066 & 12.6 & 0.464 \\
\hline 7/12/2008 9:00 AM & 0.426 & 21.7 & 0.227 & 0.158 & 19.7 & 0.30 & 0.160 & 0.198 & 16.2 & 0.131 & 0.065 & 12.6 & 0.464 \\
\hline 7/12/2008 10:00 AM & 0.426 & 21.7 & 0.227 & 0.157 & 19.7 & 0.29 & 0.160 & 0.197 & 16.2 & 0.130 & 0.065 & 12.7 & 0.464 \\
\hline 7/12/2008 11:00 AM & 0.426 & 21.7 & 0.226 & 0.157 & 19.7 & 0.29 & 0.160 & 0.197 & 16.2 & 0.130 & 0.065 & 12.7 & 0.464 \\
\hline 7/12/2008 12:00 PM & 0.425 & 21.6 & 0.226 & 0.156 & 19.7 & 0.29 & 0.159 & 0.196 & 16.2 & 0.130 & 0.065 & 12.6 & 0.464 \\
\hline 7/12/2008 1:00 PM & 0.425 & 21.6 & 0.226 & 0.156 & 19.7 & 0.29 & 0.159 & 0.196 & 16.2 & 0.130 & 0.065 & 12.7 & 0.464 \\
\hline 7/12/2008 2:00 PM & 0.424 & 21.5 & 0.225 & 0.156 & 19.7 & 0.29 & 0.158 & 0.196 & 16.2 & 0.130 & 0.065 & 12.7 & 0.464 \\
\hline 7/12/2008 3:00 PM & 0.423 & 21.5 & 0.225 & 0.156 & 19.7 & 0.29 & 0.158 & 0.196 & 16.2 & 0.130 & 0.063 & 12.7 & 0.464 \\
\hline 7/12/2008 4:00 PM & 0.422 & 21.5 & 0.224 & 0.155 & 19.7 & 0.29 & 0.157 & 0.196 & 16.2 & 0.130 & 0.065 & 12.7 & 0.464 \\
\hline 7/12/2008 5:00 PM & 0.421 & 21.4 & 0.224 & 0.154 & 19.7 & 0.28 & 0.156 & 0.195 & 16.2 & 0.130 & 0.063 & 12.7 & 0.464 \\
\hline 7/12/2008 6:00 PM & 0.420 & 21.4 & 0.223 & 0.154 & 19.7 & 0.28 & 0.156 & 0.195 & 16.2 & 0.129 & 0.063 & 12.7 & 0.464 \\
\hline 7/12/2008 7:00 PM & 0.419 & 21.4 & 0.223 & 0.154 & 19.7 & 0.28 & 0.155 & 0.195 & 16.2 & 0.129 & 0.063 & 12.7 & 0.464 \\
\hline 7/12/2008 8:00 PM & 0.418 & 21.3 & 0.222 & 0.153 & 19.7 & 0.28 & 0.155 & 0.195 & 16.2 & 0.129 & 0.063 & 12.7 & 0.465 \\
\hline 7/12/2008 9:00 PM & 0.417 & 21.3 & 0.222 & 0.153 & 19.7 & 0.28 & 0.155 & 0.195 & 16.2 & 0.129 & 0.062 & 12.7 & 0.465 \\
\hline 7/12/2008 10:00 PM & 0.417 & 21.3 & 0.222 & 0.151 & 19.7 & 0.28 & 0.154 & 0.195 & 16.2 & 0.129 & 0.062 & 12.7 & 0.465 \\
\hline 7/12/2008 11:00 PM & 0.416 & 21.2 & 0.222 & 0.151 & 19.7 & 0.28 & 0.154 & 0.195 & 16.3 & 0.129 & 0.062 & 12.7 & 0.464 \\
\hline 7/13/2008 12:00 AM & 0.416 & 21.2 & 0.221 & 0.150 & 19.7 & 0.28 & 0.153 & 0.194 & 16.2 & 0.129 & 0.063 & 12.7 & 0.465 \\
\hline 7/13/2008 1:00 AM & 0.415 & 21.2 & 0.221 & 0.150 & 19.7 & 0.28 & 0.152 & 0.194 & 16.3 & 0.128 & 0.063 & 12.7 & 0.465 \\
\hline 7/13/2008 2:00 AM & 0.415 & 21.2 & 0.220 & 0.150 & 19.7 & 0.27 & 0.152 & 0.194 & 16.3 & 0.128 & 0.062 & 12.7 & 0.465 \\
\hline 7/13/2008 3:00 AM & 0.413 & 21.1 & 0.220 & 0.149 & 19.7 & 0.27 & 0.151 & 0.194 & 16.3 & 0.128 & 0.061 & 12.7 & 0.465 \\
\hline 7/13/2008 4:00 AM & 0.415 & 21.1 & 0.220 & 0.150 & 19.7 & 0.27 & 0.151 & 0.194 & 16.3 & 0.128 & 0.061 & 12.7 & 0.465 \\
\hline 7/13/2008 5:00 AM & 0.413 & 21.1 & 0.220 & 0.149 & 19.7 & 0.27 & 0.150 & 0.194 & 16.3 & 0.128 & 0.060 & 12.7 & 0.465 \\
\hline 7/13/2008 6:00 AM & 0.415 & 21.1 & 0.220 & 0.149 & 19.7 & 0.27 & 0.150 & 0.194 & 16.3 & 0.128 & 0.060 & 12.8 & 0.465 \\
\hline 7/13/2008 7:00 AM & 0.416 & 21.1 & 0.220 & 0.148 & 19.7 & 0.27 & 0.150 & 0.194 & 16.3 & 0.128 & 0.060 & 12.7 & 0.465 \\
\hline 7/13/2008 8:00 AM & 0.416 & 21.1 & 0.219 & 0.148 & 19.7 & 0.27 & 0.149 & 0.193 & 16.3 & 0.128 & 0.062 & 12.7 & 0.465 \\
\hline
\end{tabular}


Appendix 3. Volumetric water content, temperature, and electrical-conductivity data collected at the sprinkler-irrigated site during 2008.-Continued

[Depth in feet below land surface; volumetric water content dimensionless; temperature in degrees Celsius; bulk electrical conductivity in decisiemens per meter; --, no data]

\begin{tabular}{|c|c|c|c|c|c|c|c|c|c|c|c|c|c|}
\hline \multirow[b]{3}{*}{$\begin{array}{l}\text { Measurement } \\
\text { date and time }\end{array}$} & \multicolumn{13}{|c|}{ Depth } \\
\hline & \multicolumn{2}{|l|}{2} & \multirow{2}{*}{$\begin{array}{c}3 \\
\text { Volumetric } \\
\text { water } \\
\text { content }\end{array}$} & \multicolumn{3}{|c|}{4} & \multirow{2}{*}{$\begin{array}{c}5 \\
\text { Volumetric } \\
\text { water } \\
\text { content }\end{array}$} & \multicolumn{2}{|l|}{7} & \multirow{2}{*}{$\begin{array}{c}9 \\
\text { Volumetric } \\
\text { water } \\
\text { content }\end{array}$} & \multicolumn{2}{|c|}{13} & \multirow{2}{*}{$\begin{array}{c}16.5 \\
\text { Volumetric } \\
\text { water } \\
\text { content }\end{array}$} \\
\hline & $\begin{array}{c}\text { Volumetric } \\
\text { water } \\
\text { content }\end{array}$ & $\begin{array}{l}\text { Temper- } \\
\text { ature }\end{array}$ & & $\begin{array}{c}\text { Volumetric } \\
\text { water } \\
\text { content }\end{array}$ & $\begin{array}{l}\text { Temper- } \\
\text { ature }\end{array}$ & $\begin{array}{c}\text { Bulk } \\
\text { electrical } \\
\text { conduct- } \\
\text { ivity }\end{array}$ & & $\begin{array}{l}\text { Volumetric } \\
\text { water } \\
\text { content }\end{array}$ & $\begin{array}{l}\text { Temper- } \\
\text { ature }\end{array}$ & & $\begin{array}{c}\text { Volumetric } \\
\text { water } \\
\text { content }\end{array}$ & $\begin{array}{l}\text { Temper- } \\
\text { ature }\end{array}$ & \\
\hline 7/13/2008 9:00 AM & 0.418 & 21.0 & 0.219 & 0.148 & 19.7 & 0.27 & 0.149 & 0.193 & 16.3 & 0.128 & 0.062 & 12.7 & 0.465 \\
\hline 7/13/2008 10:00 AM & 0.428 & 21.0 & 0.219 & 0.148 & 19.7 & 0.27 & 0.149 & 0.193 & 16.3 & 0.127 & 0.061 & 12.8 & 0.465 \\
\hline 7/13/2008 11:00 AM & 0.434 & 21.0 & 0.221 & 0.148 & 19.7 & 0.27 & 0.149 & 0.193 & 16.3 & 0.127 & 0.061 & 12.7 & 0.465 \\
\hline 7/13/2008 12:00 PM & 0.434 & 20.9 & 0.222 & 0.148 & 19.7 & 0.27 & 0.149 & 0.193 & 16.3 & 0.127 & 0.062 & 12.8 & 0.465 \\
\hline 7/13/2008 1:00 PM & 0.434 & 20.9 & 0.224 & 0.148 & 19.7 & 0.27 & 0.149 & 0.193 & 16.3 & 0.127 & 0.061 & 12.8 & 0.465 \\
\hline 7/13/2008 2:00 PM & 0.434 & 20.8 & 0.224 & 0.149 & 19.7 & 0.27 & 0.149 & 0.193 & 16.3 & 0.127 & 0.061 & 12.8 & 0.465 \\
\hline 7/13/2008 3:00 PM & 0.434 & 20.8 & 0.225 & 0.149 & 19.7 & 0.27 & 0.149 & 0.193 & 16.3 & 0.127 & 0.061 & 12.8 & 0.465 \\
\hline 7/13/2008 4:00 PM & 0.433 & 20.8 & 0.225 & 0.148 & 19.7 & 0.27 & 0.149 & 0.193 & 16.4 & 0.127 & 0.061 & 12.8 & 0.465 \\
\hline 7/13/2008 5:00 PM & 0.431 & 20.7 & 0.224 & 0.148 & 19.7 & 0.27 & 0.149 & 0.193 & 16.3 & 0.127 & 0.061 & 12.8 & 0.465 \\
\hline 7/13/2008 6:00 PM & 0.429 & 20.6 & 0.224 & 0.148 & 19.7 & 0.27 & 0.149 & 0.193 & 16.4 & 0.127 & 0.061 & 12.8 & 0.465 \\
\hline 7/13/2008 7:00 PM & 0.426 & 20.5 & 0.224 & 0.148 & 19.6 & 0.27 & 0.149 & 0.193 & 16.4 & 0.127 & 0.061 & 12.8 & 0.465 \\
\hline 7/13/2008 8:00 PM & 0.424 & 20.5 & 0.223 & 0.148 & 19.6 & 0.27 & 0.149 & 0.192 & 16.3 & 0.127 & 0.060 & 12.8 & 0.465 \\
\hline 7/13/2008 9:00 PM & 0.423 & 20.5 & 0.223 & 0.149 & 19.6 & 0.27 & 0.149 & 0.192 & 16.4 & 0.126 & 0.060 & 12.8 & 0.465 \\
\hline 7/13/2008 10:00 PM & 0.422 & 20.4 & 0.223 & 0.148 & 19.6 & 0.27 & 0.149 & 0.192 & 16.3 & 0.126 & 0.060 & 12.8 & 0.465 \\
\hline 7/13/2008 11:00 PM & 0.422 & 20.5 & 0.222 & 0.148 & 19.6 & 0.27 & 0.148 & 0.191 & 16.4 & 0.126 & 0.061 & 12.8 & 0.465 \\
\hline 7/14/2008 12:00 AM & 0.421 & 20.4 & 0.222 & 0.148 & 19.6 & 0.27 & 0.148 & 0.191 & 16.4 & 0.126 & 0.061 & 12.8 & 0.465 \\
\hline 7/14/2008 1:00 AM & 0.421 & 20.4 & 0.222 & 0.148 & 19.7 & 0.27 & 0.148 & 0.191 & 16.4 & 0.126 & 0.061 & 12.8 & 0.465 \\
\hline 7/14/2008 2:00 AM & 0.420 & 20.5 & 0.222 & 0.148 & 19.7 & 0.27 & 0.148 & 0.191 & 16.4 & 0.126 & 0.060 & 12.8 & 0.465 \\
\hline 7/14/2008 3:00 AM & 0.420 & 20.4 & 0.222 & 0.148 & 19.6 & 0.27 & 0.148 & 0.191 & 16.4 & 0.126 & 0.060 & 12.8 & 0.465 \\
\hline 7/14/2008 4:00 AM & 0.419 & 20.4 & 0.222 & 0.148 & 19.6 & 0.27 & 0.148 & 0.191 & 16.4 & 0.126 & 0.059 & 12.8 & 0.465 \\
\hline 7/14/2008 5:00 AM & 0.419 & 20.5 & 0.222 & 0.148 & 19.6 & 0.27 & 0.148 & 0.191 & 16.4 & 0.126 & 0.059 & 12.8 & 0.465 \\
\hline 7/14/2008 6:00 AM & 0.419 & 20.5 & 0.222 & 0.148 & 19.6 & 0.27 & 0.148 & -- & 0.4 & 0.126 & 0.047 & 12.8 & 0.197 \\
\hline 7/14/2008 7:00 AM & 0.418 & 20.5 & 0.222 & 0.148 & 19.6 & 0.26 & 0.148 & -- & -- & 0.126 & 0.059 & 12.8 & -- \\
\hline 7/14/2008 8:00 AM & 0.418 & 20.5 & 0.222 & 0.147 & 19.6 & 0.26 & 0.148 & -- & -- & 0.126 & 0.060 & 12.8 & -- \\
\hline 7/14/2008 9:00 AM & 0.417 & 20.5 & 0.221 & 0.147 & 19.6 & 0.26 & 0.148 & -- & -- & 0.126 & 0.060 & 12.8 & -- \\
\hline 7/14/2008 10:00 AM & 0.417 & 20.4 & 0.221 & 0.147 & 19.6 & 0.26 & 0.148 & -- & -- & 0.126 & 0.060 & 12.8 & -- \\
\hline 7/14/2008 11:00 AM & 0.416 & 20.4 & 0.221 & 0.147 & 19.6 & 0.26 & 0.148 & -- & -- & 0.126 & 0.060 & 12.8 & -- \\
\hline 7/14/2008 12:00 PM & 0.416 & 20.4 & 0.221 & 0.147 & 19.6 & 0.26 & 0.148 & -- & -- & 0.126 & 0.060 & 12.8 & -- \\
\hline 7/14/2008 1:00 PM & 0.415 & 20.4 & 0.221 & 0.147 & 19.6 & 0.26 & 0.148 & -- & -- & 0.126 & 0.060 & 12.8 & -- \\
\hline 7/14/2008 2:00 PM & 0.413 & 20.4 & 0.220 & 0.147 & 19.6 & 0.26 & 0.147 & -- & -- & 0.126 & 0.060 & 12.8 & -- \\
\hline 7/14/2008 3:00 PM & 0.411 & 20.4 & 0.220 & 0.147 & 19.6 & 0.26 & 0.147 & -- & -- & 0.126 & 0.060 & 12.9 & -- \\
\hline 7/14/2008 4:00 PM & 0.411 & 20.3 & 0.220 & 0.147 & 19.6 & 0.26 & 0.147 & -- & -- & 0.126 & 0.060 & 12.8 & -- \\
\hline 7/14/2008 5:00 PM & 0.411 & 20.3 & 0.219 & 0.147 & 19.6 & 0.26 & 0.147 & -- & -- & 0.126 & 0.060 & 12.8 & -- \\
\hline 7/14/2008 6:00 PM & 0.411 & 20.3 & 0.219 & 0.146 & 19.6 & 0.26 & 0.147 & -- & -- & 0.126 & 0.060 & 12.8 & -- \\
\hline 7/14/2008 7:00 PM & 0.411 & 20.3 & 0.219 & 0.146 & 19.6 & 0.26 & 0.147 & -- & -- & 0.126 & 0.059 & 12.8 & -- \\
\hline
\end{tabular}


Appendix 3. Volumetric water content, temperature, and electrical-conductivity data collected at the sprinkler-irrigated site during 2008.-Continued

[Depth in feet below land surface; volumetric water content dimensionless; temperature in degrees Celsius; bulk electrical conductivity in decisiemens per meter; --, no data]

\begin{tabular}{|c|c|c|c|c|c|c|c|c|c|c|c|c|c|}
\hline \multirow[b]{3}{*}{$\begin{array}{l}\text { Measurement } \\
\text { date and time }\end{array}$} & \multicolumn{13}{|c|}{ Depth } \\
\hline & \multicolumn{2}{|l|}{2} & \multirow{2}{*}{$\begin{array}{c}3 \\
\begin{array}{c}\text { Volumetric } \\
\text { water } \\
\text { content }\end{array}\end{array}$} & \multicolumn{3}{|c|}{4} & \multirow{2}{*}{$\begin{array}{c}\mathbf{5} \\
\text { Volumetric } \\
\text { water } \\
\text { content }\end{array}$} & \multicolumn{2}{|l|}{7} & \multirow{2}{*}{$\begin{array}{c}9 \\
\text { Volumetric } \\
\text { water } \\
\text { content }\end{array}$} & \multicolumn{2}{|c|}{13} & \multirow{2}{*}{$\begin{array}{c}16.5 \\
\text { Volumetric } \\
\text { water } \\
\text { content }\end{array}$} \\
\hline & $\begin{array}{c}\text { Volumetric } \\
\text { water } \\
\text { content }\end{array}$ & $\begin{array}{l}\text { Temper- } \\
\text { ature }\end{array}$ & & $\begin{array}{c}\text { Volumetric } \\
\text { water } \\
\text { content }\end{array}$ & $\begin{array}{l}\text { Temper- } \\
\text { ature }\end{array}$ & $\begin{array}{c}\text { Bulk } \\
\text { electrical } \\
\text { conduct- } \\
\text { ivity }\end{array}$ & & $\begin{array}{c}\text { Volumetric } \\
\text { water } \\
\text { content }\end{array}$ & $\begin{array}{l}\text { Temper- } \\
\text { ature }\end{array}$ & & $\begin{array}{c}\text { Volumetric } \\
\text { water } \\
\text { content }\end{array}$ & $\begin{array}{l}\text { Temper- } \\
\text { ature }\end{array}$ & \\
\hline 7/14/2008 8:00 PM & 0.410 & 20.2 & 0.218 & 0.146 & 19.6 & 0.26 & 0.146 & -- & -- & 0.126 & 0.059 & 12.8 & -- \\
\hline 7/14/2008 9:00 PM & 0.409 & 20.2 & 0.218 & 0.146 & 19.5 & 0.26 & 0.146 & -- & -- & 0.126 & 0.059 & 12.9 & -- \\
\hline 7/14/2008 10:00 PM & 0.408 & 20.2 & 0.218 & 0.145 & 19.5 & 0.26 & 0.146 & -- & -- & 0.126 & 0.059 & 12.9 & -- \\
\hline 7/14/2008 11:00 PM & 0.408 & 20.2 & 0.217 & 0.146 & 19.5 & 0.26 & 0.146 & -- & -- & 0.126 & 0.059 & 12.9 & -- \\
\hline 7/15/2008 12:00 AM & 0.408 & 20.2 & 0.217 & 0.145 & 19.5 & 0.26 & 0.145 & -- & -- & 0.126 & 0.059 & 12.9 & -- \\
\hline 7/15/2008 1:00 AM & 0.408 & 20.2 & 0.217 & 0.145 & 19.5 & 0.26 & 0.145 & -- & -- & 0.126 & 0.059 & 12.9 & -- \\
\hline 7/15/2008 2:00 AM & 0.408 & 20.2 & 0.217 & 0.145 & 19.5 & 0.25 & 0.145 & -- & -- & 0.126 & 0.059 & 12.9 & -- \\
\hline 7/15/2008 3:00 AM & 0.408 & 20.2 & 0.217 & 0.144 & 19.5 & 0.25 & 0.145 & -- & -- & 0.126 & 0.059 & 12.9 & -- \\
\hline 7/15/2008 4:00 AM & 0.407 & 20.2 & 0.217 & 0.145 & 19.5 & 0.25 & 0.144 & -- & -- & 0.126 & 0.059 & 12.9 & -- \\
\hline 7/15/2008 5:00 AM & 0.408 & 20.3 & 0.217 & 0.144 & 19.5 & 0.25 & 0.144 & -- & -- & 0.126 & 0.059 & 12.9 & -- \\
\hline 7/15/2008 6:00 AM & 0.407 & 20.3 & 0.217 & 0.144 & 19.5 & 0.25 & 0.144 & -- & -- & 0.125 & 0.058 & 12.9 & -- \\
\hline 7/15/2008 7:00 AM & 0.407 & 20.3 & 0.216 & 0.144 & 19.5 & 0.25 & 0.144 & -- & -- & 0.125 & 0.058 & 12.9 & -- \\
\hline 7/15/2008 8:00 AM & 0.407 & 20.3 & 0.216 & 0.144 & 19.5 & 0.25 & 0.144 & -- & -- & 0.125 & 0.059 & 12.9 & -- \\
\hline 7/15/2008 9:00 AM & 0.407 & 20.3 & 0.216 & 0.144 & 19.5 & 0.25 & 0.143 & -- & -- & 0.125 & 0.059 & 12.9 & -- \\
\hline 7/15/2008 10:00 AM & 0.406 & 20.3 & 0.216 & 0.143 & 19.5 & 0.25 & 0.143 & -- & -- & 0.125 & 0.058 & 12.9 & -- \\
\hline 7/15/2008 11:00 AM & 0.405 & 20.4 & 0.216 & 0.143 & 19.5 & 0.25 & 0.143 & -- & -- & 0.125 & 0.059 & 12.9 & -- \\
\hline 7/15/2008 12:00 PM & 0.404 & 20.3 & 0.216 & 0.143 & 19.5 & 0.25 & 0.143 & -- & -- & 0.125 & 0.059 & 12.9 & -- \\
\hline 7/15/2008 1:00 PM & 0.403 & 20.3 & 0.216 & 0.143 & 19.5 & 0.25 & 0.143 & -- & -- & 0.125 & 0.059 & 12.9 & -- \\
\hline 7/15/2008 2:00 PM & 0.403 & 20.3 & 0.215 & 0.143 & 19.5 & 0.25 & 0.143 & -- & -- & 0.125 & 0.059 & 12.9 & -- \\
\hline 7/15/2008 3:00 PM & 0.403 & 20.3 & 0.215 & 0.142 & 19.5 & 0.25 & 0.143 & -- & -- & 0.125 & 0.059 & 12.9 & -- \\
\hline 7/15/2008 4:00 PM & 0.401 & 20.3 & 0.215 & 0.143 & 19.4 & 0.25 & 0.143 & -- & -- & 0.125 & 0.059 & 12.9 & -- \\
\hline 7/15/2008 5:00 PM & 0.401 & 20.3 & 0.215 & 0.142 & 19.5 & 0.25 & 0.143 & -- & -- & 0.125 & 0.059 & 12.9 & -- \\
\hline 7/15/2008 6:00 PM & 0.400 & 20.3 & 0.214 & 0.142 & 19.4 & 0.25 & 0.143 & -- & -- & 0.125 & 0.058 & 12.9 & -- \\
\hline 7/15/2008 7:00 PM & 0.400 & 20.3 & 0.214 & 0.142 & 19.4 & 0.25 & 0.143 & -- & -- & 0.125 & 0.058 & 12.9 & -- \\
\hline 7/15/2008 8:00 PM & 0.400 & 20.2 & 0.214 & 0.142 & 19.4 & 0.24 & 0.142 & -- & -- & 0.125 & 0.058 & 12.9 & -- \\
\hline 7/15/2008 9:00 PM & 0.399 & 20.2 & 0.213 & 0.141 & 19.4 & 0.24 & 0.142 & -- & -- & 0.125 & 0.058 & 12.9 & -- \\
\hline 7/15/2008 10:00 PM & 0.399 & 20.2 & 0.213 & 0.141 & 19.4 & 0.24 & 0.142 & -- & -- & 0.125 & 0.059 & 13.0 & -- \\
\hline 7/15/2008 11:00 PM & 0.399 & 20.2 & 0.213 & 0.141 & 19.4 & 0.24 & 0.142 & -- & -- & 0.125 & 0.059 & 12.9 & -- \\
\hline 7/16/2008 12:00 AM & 0.399 & 20.2 & 0.213 & 0.141 & 19.4 & 0.24 & 0.141 & -- & -- & 0.125 & 0.058 & 13.0 & -- \\
\hline 7/16/2008 1:00 AM & 0.399 & 20.3 & 0.213 & 0.141 & 19.4 & 0.24 & 0.141 & -- & -- & 0.125 & 0.058 & 13.0 & -- \\
\hline 7/16/2008 2:00 AM & 0.399 & 20.3 & 0.212 & 0.141 & 19.4 & 0.24 & 0.141 & -- & -- & 0.125 & 0.057 & 12.9 & -- \\
\hline 7/16/2008 3:00 AM & 0.399 & 20.3 & 0.212 & 0.140 & 19.4 & 0.24 & 0.141 & -- & -- & 0.125 & 0.057 & 13.0 & -- \\
\hline 7/16/2008 4:00 AM & 0.399 & 20.3 & 0.212 & 0.140 & 19.4 & 0.24 & 0.141 & -- & -- & 0.125 & 0.057 & 13.0 & -- \\
\hline 7/16/2008 5:00 AM & 0.399 & 20.3 & 0.212 & 0.140 & 19.4 & 0.24 & 0.140 & -- & -- & 0.125 & 0.057 & 13.0 & -- \\
\hline 7/16/2008 6:00 AM & 0.399 & 20.4 & 0.212 & 0.140 & 19.4 & 0.24 & 0.140 & -- & -- & 0.125 & 0.057 & 13.0 & -- \\
\hline
\end{tabular}


Appendix 3. Volumetric water content, temperature, and electrical-conductivity data collected at the sprinkler-irrigated site during 2008.-Continued

[Depth in feet below land surface; volumetric water content dimensionless; temperature in degrees Celsius; bulk electrical conductivity in decisiemens per meter; --, no data]

\begin{tabular}{|c|c|c|c|c|c|c|c|c|c|c|c|c|c|}
\hline \multirow[b]{3}{*}{$\begin{array}{l}\text { Measurement } \\
\text { date and time }\end{array}$} & \multicolumn{13}{|c|}{ Depth } \\
\hline & \multicolumn{2}{|c|}{2} & \multirow{2}{*}{$\begin{array}{c}3 \\
\text { Volumetric } \\
\text { water } \\
\text { content }\end{array}$} & \multicolumn{3}{|c|}{4} & \multirow{2}{*}{$\begin{array}{c}5 \\
\text { Volumetric } \\
\text { water } \\
\text { content }\end{array}$} & \multicolumn{2}{|l|}{7} & \multirow[b]{2}{*}{$\begin{array}{c}9 \\
\text { Volumetric } \\
\text { water } \\
\text { content }\end{array}$} & \multicolumn{2}{|c|}{13} & \multirow{2}{*}{$\begin{array}{c}16.5 \\
\text { Volumetric } \\
\text { water } \\
\text { content }\end{array}$} \\
\hline & $\begin{array}{c}\text { Volumetric } \\
\text { water } \\
\text { content }\end{array}$ & $\begin{array}{l}\text { Temper- } \\
\text { ature }\end{array}$ & & $\begin{array}{c}\text { Volumetric } \\
\text { water } \\
\text { content }\end{array}$ & $\begin{array}{l}\text { Temper- } \\
\text { ature }\end{array}$ & $\begin{array}{c}\text { Bulk } \\
\text { electrical } \\
\text { conduct- } \\
\text { ivity }\end{array}$ & & $\begin{array}{c}\text { Volumetric } \\
\text { water } \\
\text { content }\end{array}$ & $\begin{array}{l}\text { Temper- } \\
\text { ature }\end{array}$ & & $\begin{array}{c}\text { Volumetric } \\
\text { water } \\
\text { content }\end{array}$ & $\begin{array}{l}\text { Temper- } \\
\text { ature }\end{array}$ & \\
\hline 7/16/2008 7:00 AM & 0.399 & 20.4 & 0.211 & 0.140 & 19.4 & 0.24 & 0.140 & -- & -- & 0.125 & 0.057 & 13.0 & -- \\
\hline 7/16/2008 8:00 AM & 0.398 & 20.4 & 0.211 & 0.140 & 19.4 & 0.24 & 0.140 & -- & -- & 0.125 & 0.057 & 13.0 & -- \\
\hline 7/16/2008 9:00 AM & 0.397 & 20.3 & 0.211 & 0.140 & 19.4 & 0.24 & 0.140 & -- & -- & 0.124 & 0.058 & 13.0 & -- \\
\hline 7/16/2008 10:00 AM & 0.397 & 20.3 & 0.211 & 0.138 & 19.4 & 0.24 & 0.140 & -- & -- & 0.124 & 0.058 & 13.0 & -- \\
\hline 7/16/2008 11:00 AM & 0.396 & 20.3 & 0.211 & 0.140 & 19.4 & 0.24 & 0.139 & -- & -- & 0.124 & 0.057 & 13.0 & -- \\
\hline 7/16/2008 12:00 PM & 0.396 & 20.4 & 0.211 & 0.138 & 19.4 & 0.24 & 0.139 & -- & -- & 0.124 & 0.058 & 13.0 & -- \\
\hline 7/16/2008 1:00 PM & 0.396 & 20.4 & 0.211 & 0.138 & 19.4 & 0.24 & 0.139 & -- & -- & 0.124 & 0.058 & 13.0 & -- \\
\hline 7/16/2008 2:00 PM & 0.395 & 20.4 & 0.211 & 0.138 & 19.4 & 0.24 & 0.139 & -- & -- & 0.124 & 0.058 & 13.0 & -- \\
\hline 7/16/2008 3:00 PM & 0.394 & 20.3 & 0.211 & 0.138 & 19.4 & 0.24 & 0.139 & -- & -- & 0.124 & 0.058 & 13.0 & -- \\
\hline 7/16/2008 4:00 PM & 0.393 & 20.3 & 0.211 & 0.138 & 19.4 & 0.23 & 0.139 & -- & -- & 0.124 & 0.058 & 13.0 & -- \\
\hline 7/16/2008 5:00 PM & 0.392 & 20.3 & 0.211 & 0.138 & 19.4 & 0.23 & 0.139 & -- & -- & 0.124 & 0.058 & 13.0 & -- \\
\hline 7/16/2008 6:00 PM & 0.392 & 20.3 & 0.211 & 0.137 & 19.4 & 0.23 & 0.138 & -- & -- & 0.124 & 0.058 & 13.0 & -- \\
\hline 7/16/2008 7:00 PM & 0.393 & 20.3 & 0.211 & 0.137 & 19.4 & 0.23 & 0.138 & -- & -- & 0.124 & 0.057 & 13.0 & -- \\
\hline 7/16/2008 8:00 PM & 0.393 & 20.3 & 0.210 & 0.137 & 19.4 & 0.23 & 0.138 & -- & -- & 0.124 & 0.057 & 13.0 & -- \\
\hline 7/16/2008 9:00 PM & 0.393 & 20.3 & 0.210 & 0.137 & 19.4 & 0.23 & 0.138 & -- & -- & 0.124 & 0.058 & 13.0 & -- \\
\hline 7/16/2008 10:00 PM & 0.393 & 20.3 & 0.210 & 0.137 & 19.4 & 0.23 & 0.138 & -- & -- & 0.124 & 0.058 & 13.0 & -- \\
\hline 7/16/2008 11:00 PM & 0.393 & 20.3 & 0.210 & 0.137 & 19.4 & 0.23 & 0.138 & -- & -- & 0.124 & 0.058 & 13.0 & -- \\
\hline 7/17/2008 12:00 AM & 0.393 & 20.3 & 0.210 & 0.137 & 19.4 & 0.23 & 0.138 & -- & -- & 0.124 & 0.057 & 13.0 & -- \\
\hline 7/17/2008 1:00 AM & 0.393 & 20.2 & 0.210 & 0.137 & 19.3 & 0.23 & 0.138 & -- & -- & 0.124 & 0.058 & 13.0 & -- \\
\hline 7/17/2008 2:00 AM & 0.392 & 20.3 & 0.210 & 0.136 & 19.4 & 0.23 & 0.138 & -- & -- & 0.124 & 0.057 & 13.0 & -- \\
\hline 7/17/2008 3:00 AM & 0.392 & 20.3 & 0.209 & 0.137 & 19.4 & 0.23 & 0.138 & -- & -- & 0.124 & 0.058 & 13.0 & -- \\
\hline 7/17/2008 4:00 AM & 0.391 & 20.3 & 0.209 & 0.137 & 19.4 & 0.23 & 0.137 & -- & -- & 0.124 & 0.058 & 13.0 & -- \\
\hline 7/17/2008 5:00 AM & 0.392 & 20.3 & 0.209 & 0.136 & 19.4 & 0.23 & 0.137 & -- & -- & 0.124 & 0.058 & 13.0 & -- \\
\hline 7/17/2008 6:00 AM & 0.391 & 20.4 & 0.208 & 0.136 & 19.4 & 0.23 & 0.137 & -- & -- & 0.124 & 0.057 & 13.0 & -- \\
\hline 7/17/2008 7:00 AM & 0.391 & 20.4 & 0.208 & 0.136 & 19.4 & 0.23 & 0.137 & -- & -- & 0.124 & 0.057 & 13.0 & -- \\
\hline 7/17/2008 8:00 AM & 0.391 & 20.4 & 0.208 & 0.136 & 19.3 & 0.23 & 0.137 & -- & -- & 0.124 & 0.057 & 13.0 & -- \\
\hline 7/17/2008 9:00 AM & 0.392 & 20.4 & 0.208 & 0.136 & 19.3 & 0.23 & 0.137 & -- & -- & 0.124 & 0.058 & 13.1 & -- \\
\hline 7/17/2008 10:00 AM & 0.392 & 20.4 & 0.208 & 0.136 & 19.3 & 0.23 & 0.137 & -- & -- & 0.124 & 0.057 & 13.1 & -- \\
\hline 7/17/2008 11:00 AM & 0.393 & 20.4 & 0.208 & 0.135 & 19.4 & 0.23 & 0.136 & -- & -- & 0.123 & 0.057 & 13.0 & -- \\
\hline 7/17/2008 12:00 PM & 0.393 & 20.4 & 0.209 & 0.135 & 19.4 & 0.23 & 0.136 & -- & -- & 0.123 & 0.057 & 13.0 & -- \\
\hline 7/17/2008 1:00 PM & 0.394 & 20.4 & 0.209 & 0.135 & 19.3 & 0.23 & 0.136 & -- & -- & 0.123 & 0.058 & 13.0 & -- \\
\hline 7/17/2008 2:00 PM & 0.394 & 20.4 & 0.209 & 0.135 & 19.3 & 0.23 & 0.136 & -- & -- & 0.123 & 0.057 & 13.0 & -- \\
\hline 7/17/2008 3:00 PM & 0.394 & 20.4 & 0.209 & 0.135 & 19.3 & 0.23 & 0.136 & -- & -- & 0.123 & 0.057 & 13.1 & -- \\
\hline 7/17/2008 4:00 PM & 0.394 & 20.4 & 0.209 & 0.135 & 19.3 & 0.23 & 0.136 & -- & -- & 0.123 & 0.057 & 13.0 & -- \\
\hline 7/17/2008 5:00 PM & 0.394 & 20.4 & 0.209 & 0.135 & 19.3 & 0.22 & 0.136 & -- & -- & 0.123 & 0.056 & 13.0 & -- \\
\hline
\end{tabular}


Appendix 3. Volumetric water content, temperature, and electrical-conductivity data collected at the sprinkler-irrigated site during 2008.-Continued

[Depth in feet below land surface; volumetric water content dimensionless; temperature in degrees Celsius; bulk electrical conductivity in decisiemens per meter; --, no data]

\begin{tabular}{|c|c|c|c|c|c|c|c|c|c|c|c|c|c|}
\hline \multirow[b]{3}{*}{$\begin{array}{l}\text { Measurement } \\
\text { date and time }\end{array}$} & \multicolumn{13}{|c|}{ Depth } \\
\hline & \multicolumn{2}{|l|}{2} & \multirow{2}{*}{$\begin{array}{c}3 \\
\begin{array}{c}\text { Volumetric } \\
\text { water } \\
\text { content }\end{array}\end{array}$} & \multicolumn{3}{|c|}{4} & \multirow{2}{*}{$\begin{array}{c}\mathbf{5} \\
\text { Volumetric } \\
\text { water } \\
\text { content }\end{array}$} & \multicolumn{2}{|l|}{7} & \multirow{2}{*}{$\begin{array}{c}9 \\
\text { Volumetric } \\
\text { water } \\
\text { content }\end{array}$} & \multicolumn{2}{|c|}{13} & \multirow{2}{*}{$\begin{array}{c}16.5 \\
\text { Volumetric } \\
\text { water } \\
\text { content }\end{array}$} \\
\hline & $\begin{array}{c}\text { Volumetric } \\
\text { water } \\
\text { content }\end{array}$ & $\begin{array}{l}\text { Temper- } \\
\text { ature }\end{array}$ & & $\begin{array}{c}\text { Volumetric } \\
\text { water } \\
\text { content }\end{array}$ & $\begin{array}{l}\text { Temper- } \\
\text { ature }\end{array}$ & $\begin{array}{c}\text { Bulk } \\
\text { electrical } \\
\text { conduct- } \\
\text { ivity }\end{array}$ & & $\begin{array}{c}\text { Volumetric } \\
\text { water } \\
\text { content }\end{array}$ & $\begin{array}{l}\text { Temper- } \\
\text { ature }\end{array}$ & & $\begin{array}{c}\text { Volumetric } \\
\text { water } \\
\text { content }\end{array}$ & $\begin{array}{l}\text { Temper- } \\
\text { ature }\end{array}$ & \\
\hline 7/17/2008 6:00 PM & 0.394 & 20.4 & 0.209 & 0.135 & 19.3 & 0.22 & 0.135 & -- & -- & 0.123 & 0.057 & 13.0 & -- \\
\hline 7/17/2008 7:00 PM & 0.394 & 20.3 & 0.209 & 0.135 & 19.4 & 0.22 & 0.135 & -- & -- & 0.123 & 0.057 & 13.1 & -- \\
\hline 7/17/2008 8:00 PM & 0.394 & 20.3 & 0.209 & 0.135 & 19.3 & 0.22 & 0.135 & -- & -- & 0.123 & 0.057 & 13.1 & -- \\
\hline 7/17/2008 9:00 PM & 0.394 & 20.3 & 0.209 & 0.135 & 19.3 & 0.22 & 0.135 & -- & -- & 0.123 & 0.057 & 13.1 & -- \\
\hline 7/17/2008 10:00 PM & 0.394 & 20.4 & 0.209 & 0.134 & 19.3 & 0.22 & 0.135 & -- & -- & 0.123 & 0.057 & 13.1 & -- \\
\hline 7/17/2008 11:00 PM & 0.394 & 20.4 & 0.209 & 0.134 & 19.3 & 0.22 & 0.135 & -- & -- & 0.123 & 0.057 & 13.1 & -- \\
\hline 7/18/2008 12:00 AM & 0.395 & 20.4 & 0.209 & 0.134 & 19.3 & 0.22 & 0.135 & -- & -- & 0.123 & 0.057 & 13.1 & -- \\
\hline 7/18/2008 1:00 AM & 0.395 & 20.4 & 0.209 & 0.134 & 19.3 & 0.22 & 0.134 & -- & -- & 0.123 & 0.057 & 13.1 & -- \\
\hline 7/18/2008 2:00 AM & 0.395 & 20.4 & 0.209 & 0.134 & 19.3 & 0.22 & 0.134 & -- & -- & 0.123 & 0.057 & 13.1 & -- \\
\hline 7/18/2008 3:00 AM & 0.395 & 20.4 & 0.209 & 0.134 & 19.3 & 0.22 & 0.134 & -- & -- & 0.123 & 0.056 & 13.1 & -- \\
\hline 7/18/2008 4:00 AM & 0.396 & 20.5 & 0.209 & 0.134 & 19.3 & 0.22 & 0.134 & -- & -- & 0.123 & 0.056 & 13.1 & -- \\
\hline 7/18/2008 5:00 AM & 0.396 & 20.5 & 0.209 & 0.134 & 19.3 & 0.22 & 0.134 & -- & -- & 0.123 & 0.056 & 13.1 & -- \\
\hline 7/18/2008 6:00 AM & 0.396 & 20.5 & 0.209 & 0.134 & 19.3 & 0.22 & 0.134 & -- & -- & 0.123 & 0.057 & 13.1 & -- \\
\hline 7/18/2008 7:00 AM & 0.396 & 20.5 & 0.209 & 0.134 & 19.3 & 0.22 & 0.134 & -- & -- & 0.123 & 0.057 & 13.1 & -- \\
\hline 7/18/2008 8:00 AM & 0.396 & 20.5 & 0.209 & 0.134 & 19.3 & 0.22 & 0.134 & -- & -- & 0.123 & 0.056 & 13.1 & -- \\
\hline 7/18/2008 9:00 AM & 0.397 & 20.5 & 0.209 & 0.134 & 19.3 & 0.22 & 0.134 & -- & -- & 0.123 & 0.057 & 13.1 & -- \\
\hline 7/18/2008 10:00 AM & 0.397 & 20.5 & 0.209 & 0.134 & 19.3 & 0.22 & 0.134 & -- & -- & 0.123 & 0.057 & 13.1 & -- \\
\hline 7/18/2008 11:00 AM & 0.396 & 20.5 & 0.209 & 0.134 & 19.4 & 0.22 & 0.134 & -- & -- & 0.123 & 0.057 & 13.1 & -- \\
\hline 7/18/2008 12:00 PM & 0.396 & 20.5 & 0.209 & 0.134 & 19.3 & 0.22 & 0.134 & -- & -- & 0.123 & 0.057 & 13.1 & -- \\
\hline 7/18/2008 1:00 PM & 0.396 & 20.5 & 0.209 & 0.134 & 19.4 & 0.22 & 0.133 & -- & -- & 0.123 & 0.057 & 13.1 & -- \\
\hline 7/18/2008 2:00 PM & 0.396 & 20.5 & 0.209 & 0.134 & 19.3 & 0.22 & 0.133 & -- & -- & 0.123 & 0.057 & 13.1 & -- \\
\hline 7/18/2008 3:00 PM & 0.396 & 20.5 & 0.209 & 0.134 & 19.3 & 0.22 & 0.133 & -- & -- & 0.122 & 0.057 & 13.1 & -- \\
\hline 7/18/2008 4:00 PM & 0.395 & 20.4 & 0.209 & 0.134 & 19.3 & 0.22 & 0.133 & -- & -- & 0.122 & 0.057 & 13.1 & -- \\
\hline 7/18/2008 5:00 PM & 0.394 & 20.4 & 0.209 & 0.134 & 19.3 & 0.22 & 0.133 & -- & -- & 0.122 & 0.056 & 13.1 & -- \\
\hline 7/18/2008 6:00 PM & 0.394 & 20.4 & 0.209 & 0.134 & 19.3 & 0.22 & 0.133 & -- & -- & 0.122 & 0.056 & 13.1 & -- \\
\hline 7/18/2008 7:00 PM & 0.393 & 20.4 & 0.209 & 0.133 & 19.3 & 0.22 & 0.133 & -- & -- & 0.122 & 0.057 & 13.1 & -- \\
\hline 7/18/2008 8:00 PM & 0.393 & 20.4 & 0.208 & 0.134 & 19.3 & 0.22 & 0.133 & -- & -- & 0.122 & 0.057 & 13.1 & -- \\
\hline 7/18/2008 9:00 PM & 0.393 & 20.4 & 0.208 & 0.133 & 19.3 & 0.22 & 0.133 & -- & -- & 0.122 & 0.057 & 13.1 & -- \\
\hline 7/18/2008 10:00 PM & 0.393 & 20.4 & 0.208 & 0.133 & 19.3 & 0.22 & 0.132 & -- & -- & 0.122 & 0.057 & 13.1 & -- \\
\hline 7/18/2008 11:00 PM & 0.393 & 20.4 & 0.208 & 0.133 & 19.3 & 0.22 & 0.132 & -- & -- & 0.122 & 0.057 & 13.1 & -- \\
\hline 7/19/2008 12:00 AM & 0.393 & 20.4 & 0.208 & 0.133 & 19.3 & 0.22 & 0.132 & -- & -- & 0.122 & 0.057 & 13.1 & -- \\
\hline 7/19/2008 1:00 AM & 0.393 & 20.4 & 0.208 & 0.133 & 19.3 & 0.22 & 0.132 & -- & -- & 0.122 & 0.057 & 13.1 & -- \\
\hline 7/19/2008 2:00 AM & 0.393 & 20.4 & 0.208 & 0.133 & 19.3 & 0.22 & 0.132 & -- & -- & 0.122 & 0.056 & 13.2 & -- \\
\hline 7/19/2008 3:00 AM & 0.393 & 20.5 & 0.207 & 0.133 & 19.3 & 0.22 & 0.132 & -- & -- & 0.122 & 0.057 & 13.2 & -- \\
\hline 7/19/2008 4:00 AM & 0.393 & 20.5 & 0.207 & 0.133 & 19.3 & 0.22 & 0.132 & -- & -- & 0.122 & 0.057 & 13.2 & -- \\
\hline
\end{tabular}


Appendix 3. Volumetric water content, temperature, and electrical-conductivity data collected at the sprinkler-irrigated site during 2008.-Continued

[Depth in feet below land surface; volumetric water content dimensionless; temperature in degrees Celsius; bulk electrical conductivity in decisiemens per meter; --, no data]

\begin{tabular}{|c|c|c|c|c|c|c|c|c|c|c|c|c|c|}
\hline \multirow[b]{3}{*}{$\begin{array}{l}\text { Measurement } \\
\text { date and time }\end{array}$} & \multicolumn{13}{|c|}{ Depth } \\
\hline & \multicolumn{2}{|c|}{2} & \multirow{2}{*}{$\begin{array}{c}3 \\
\text { Volumetric } \\
\text { water } \\
\text { content }\end{array}$} & \multicolumn{3}{|c|}{4} & \multirow{2}{*}{$\begin{array}{c}5 \\
\text { Volumetric } \\
\text { water } \\
\text { content }\end{array}$} & \multicolumn{2}{|l|}{7} & \multirow[b]{2}{*}{$\begin{array}{c}9 \\
\text { Volumetric } \\
\text { water } \\
\text { content }\end{array}$} & \multicolumn{2}{|c|}{13} & \multirow{2}{*}{$\begin{array}{c}16.5 \\
\text { Volumetric } \\
\text { water } \\
\text { content }\end{array}$} \\
\hline & $\begin{array}{c}\text { Volumetric } \\
\text { water } \\
\text { content }\end{array}$ & $\begin{array}{l}\text { Temper- } \\
\text { ature }\end{array}$ & & $\begin{array}{c}\text { Volumetric } \\
\text { water } \\
\text { content }\end{array}$ & $\begin{array}{l}\text { Temper- } \\
\text { ature }\end{array}$ & $\begin{array}{c}\text { Bulk } \\
\text { electrical } \\
\text { conduct- } \\
\text { ivity }\end{array}$ & & $\begin{array}{c}\text { Volumetric } \\
\text { water } \\
\text { content }\end{array}$ & $\begin{array}{l}\text { Temper- } \\
\text { ature }\end{array}$ & & $\begin{array}{c}\text { Volumetric } \\
\text { water } \\
\text { content }\end{array}$ & $\begin{array}{l}\text { Temper- } \\
\text { ature }\end{array}$ & \\
\hline 7/19/2008 5:00 AM & 0.393 & 20.5 & 0.207 & 0.133 & 19.3 & 0.22 & 0.132 & -- & -- & 0.122 & 0.057 & 13.2 & -- \\
\hline 7/19/2008 6:00 AM & 0.394 & 20.5 & 0.207 & 0.133 & 19.3 & 0.22 & 0.132 & -- & -- & 0.122 & 0.056 & 13.2 & -- \\
\hline 7/19/2008 7:00 AM & 0.397 & 20.5 & 0.207 & 0.133 & 19.3 & 0.22 & 0.132 & -- & -- & 0.122 & 0.057 & 13.2 & -- \\
\hline 7/19/2008 8:00 AM & 0.404 & 20.5 & 0.207 & 0.133 & 19.4 & 0.22 & 0.132 & -- & -- & 0.122 & 0.057 & 13.2 & -- \\
\hline 7/19/2008 9:00 AM & 0.410 & 20.5 & 0.208 & 0.133 & 19.4 & 0.22 & 0.132 & -- & -- & 0.122 & 0.056 & 13.2 & -- \\
\hline 7/19/2008 10:00 AM & 0.413 & 20.5 & 0.209 & 0.133 & 19.3 & 0.22 & 0.132 & -- & -- & 0.122 & 0.056 & 13.2 & -- \\
\hline 7/19/2008 11:00 AM & 0.416 & 20.5 & 0.210 & 0.133 & 19.3 & 0.21 & 0.132 & -- & -- & 0.122 & 0.057 & 13.2 & -- \\
\hline 7/19/2008 12:00 PM & 0.415 & 20.5 & 0.211 & 0.133 & 19.3 & 0.21 & 0.132 & -- & -- & 0.122 & 0.056 & 13.1 & -- \\
\hline 7/19/2008 1:00 PM & 0.415 & 20.5 & 0.211 & 0.133 & 19.3 & 0.21 & 0.132 & -- & -- & 0.122 & 0.056 & 13.2 & -- \\
\hline 7/19/2008 2:00 PM & 0.415 & 20.5 & 0.211 & 0.133 & 19.4 & 0.21 & 0.132 & -- & -- & 0.122 & 0.057 & 13.2 & -- \\
\hline 7/19/2008 3:00 PM & 0.413 & 20.5 & 0.211 & 0.133 & 19.4 & 0.21 & 0.132 & -- & -- & 0.122 & 0.056 & 13.1 & -- \\
\hline 7/19/2008 4:00 PM & 0.412 & 20.4 & 0.212 & 0.134 & 19.4 & 0.21 & 0.132 & -- & -- & 0.122 & 0.056 & 13.2 & -- \\
\hline 7/19/2008 5:00 PM & 0.411 & 20.4 & 0.212 & 0.133 & 19.3 & 0.22 & 0.132 & -- & -- & 0.122 & 0.056 & 13.2 & -- \\
\hline 7/19/2008 6:00 PM & 0.410 & 20.4 & 0.212 & 0.133 & 19.3 & 0.22 & 0.132 & -- & -- & 0.122 & 0.056 & 13.2 & -- \\
\hline 7/19/2008 7:00 PM & 0.408 & 20.3 & 0.212 & 0.133 & 19.3 & 0.22 & 0.132 & -- & -- & 0.121 & 0.056 & 13.2 & -- \\
\hline 7/19/2008 8:00 PM & 0.408 & 20.3 & 0.212 & 0.133 & 19.4 & 0.22 & 0.132 & -- & -- & 0.121 & 0.057 & 13.2 & -- \\
\hline 7/19/2008 9:00 PM & 0.408 & 20.3 & 0.212 & 0.134 & 19.4 & 0.22 & 0.132 & -- & -- & 0.121 & 0.057 & 13.2 & -- \\
\hline 7/19/2008 10:00 PM & 0.408 & 20.3 & 0.212 & 0.133 & 19.3 & 0.22 & 0.132 & -- & -- & 0.121 & 0.057 & 13.2 & -- \\
\hline 7/19/2008 11:00 PM & 0.408 & 20.3 & 0.212 & 0.133 & 19.4 & 0.22 & 0.132 & -- & -- & 0.121 & 0.057 & 13.2 & -- \\
\hline $7 / 20 / 2008$ 12:00 AM & 0.408 & 20.3 & 0.212 & 0.134 & 19.4 & 0.22 & 0.132 & & -- & 0.121 & 0.056 & 13.2 & \\
\hline 7/20/2008 1:00 AM & 0.408 & 20.3 & 0.211 & 0.134 & 19.3 & 0.22 & 0.132 & -- & -- & 0.121 & 0.056 & 13.2 & -- \\
\hline 7/20/2008 2:00 AM & 0.407 & 20.3 & 0.211 & 0.134 & 19.4 & 0.22 & 0.132 & -- & -- & 0.121 & 0.056 & 13.2 & -- \\
\hline 7/20/2008 3:00 AM & 0.407 & 20.3 & 0.211 & 0.134 & 19.4 & 0.22 & 0.132 & -- & -- & 0.121 & 0.057 & 13.2 & -- \\
\hline 7/20/2008 4:00 AM & 0.406 & 20.4 & 0.211 & 0.134 & 19.4 & 0.22 & 0.132 & -- & -- & 0.121 & 0.057 & 13.2 & -- \\
\hline 7/20/2008 5:00 AM & 0.406 & 20.3 & 0.211 & 0.134 & 19.4 & 0.22 & 0.132 & -- & -- & 0.121 & 0.057 & 13.2 & -- \\
\hline 7/20/2008 6:00 AM & 0.406 & 20.4 & 0.211 & 0.134 & 19.4 & 0.22 & 0.132 & -- & -- & 0.121 & 0.056 & 13.2 & -- \\
\hline 7/20/2008 7:00 AM & 0.406 & 20.4 & 0.211 & 0.134 & 19.4 & 0.21 & 0.132 & -- & -- & 0.121 & 0.056 & 13.2 & -- \\
\hline 7/20/2008 8:00 AM & 0.405 & 20.4 & 0.211 & 0.134 & 19.4 & 0.21 & 0.132 & -- & -- & 0.121 & 0.057 & 13.2 & -- \\
\hline 7/20/2008 9:00 AM & 0.405 & 20.4 & 0.211 & 0.134 & 19.4 & 0.21 & 0.132 & -- & -- & 0.121 & 0.056 & 13.2 & -- \\
\hline 7/20/2008 10:00 AM & 0.404 & 20.4 & 0.211 & 0.134 & 19.4 & 0.21 & 0.132 & -- & -- & 0.121 & 0.057 & 13.2 & -- \\
\hline 7/20/2008 11:00 AM & 0.404 & 20.4 & 0.211 & 0.134 & 19.4 & 0.21 & 0.132 & -- & -- & 0.121 & 0.057 & 13.2 & -- \\
\hline 7/20/2008 12:00 PM & 0.404 & 20.4 & 0.211 & 0.134 & 19.4 & 0.21 & 0.132 & -- & -- & 0.121 & 0.056 & 13.2 & -- \\
\hline 7/20/2008 1:00 PM & 0.403 & 20.4 & 0.211 & 0.134 & 19.4 & 0.21 & 0.132 & -- & -- & 0.121 & 0.056 & 13.2 & -- \\
\hline 7/20/2008 2:00 PM & 0.401 & 20.4 & 0.211 & 0.134 & 19.3 & 0.21 & 0.132 & -- & -- & 0.121 & 0.056 & 13.2 & -- \\
\hline 7/20/2008 3:00 PM & 0.401 & 20.4 & 0.211 & 0.134 & 19.4 & 0.21 & 0.132 & -- & -- & 0.121 & 0.056 & 13.2 & -- \\
\hline
\end{tabular}


Appendix 3. Volumetric water content, temperature, and electrical-conductivity data collected at the sprinkler-irrigated site during 2008.-Continued

[Depth in feet below land surface; volumetric water content dimensionless; temperature in degrees Celsius; bulk electrical conductivity in decisiemens per meter; --, no data]

\begin{tabular}{|c|c|c|c|c|c|c|c|c|c|c|c|c|c|}
\hline \multirow[b]{3}{*}{$\begin{array}{l}\text { Measurement } \\
\text { date and time }\end{array}$} & \multicolumn{13}{|c|}{ Depth } \\
\hline & \multicolumn{2}{|l|}{2} & \multirow{2}{*}{$\begin{array}{c}3 \\
\begin{array}{c}\text { Volumetric } \\
\text { water } \\
\text { content }\end{array}\end{array}$} & \multicolumn{3}{|c|}{4} & \multirow{2}{*}{$\begin{array}{c}\mathbf{5} \\
\text { Volumetric } \\
\text { water } \\
\text { content }\end{array}$} & \multicolumn{2}{|l|}{7} & \multirow{2}{*}{$\begin{array}{c}9 \\
\text { Volumetric } \\
\text { water } \\
\text { content }\end{array}$} & \multicolumn{2}{|c|}{13} & \multirow{2}{*}{$\begin{array}{c}16.5 \\
\text { Volumetric } \\
\text { water } \\
\text { content }\end{array}$} \\
\hline & $\begin{array}{c}\text { Volumetric } \\
\text { water } \\
\text { content }\end{array}$ & $\begin{array}{l}\text { Temper- } \\
\text { ature }\end{array}$ & & $\begin{array}{c}\text { Volumetric } \\
\text { water } \\
\text { content }\end{array}$ & $\begin{array}{l}\text { Temper- } \\
\text { ature }\end{array}$ & $\begin{array}{c}\text { Bulk } \\
\text { electrical } \\
\text { conduct- } \\
\text { ivity }\end{array}$ & & $\begin{array}{c}\text { Volumetric } \\
\text { water } \\
\text { content }\end{array}$ & $\begin{array}{l}\text { Temper- } \\
\text { ature }\end{array}$ & & $\begin{array}{c}\text { Volumetric } \\
\text { water } \\
\text { content }\end{array}$ & $\begin{array}{l}\text { Temper- } \\
\text { ature }\end{array}$ & \\
\hline 7/20/2008 4:00 PM & 0.401 & 20.3 & 0.211 & 0.134 & 19.4 & 0.21 & 0.132 & -- & -- & 0.121 & 0.056 & 13.3 & -- \\
\hline 7/20/2008 5:00 PM & 0.401 & 20.3 & 0.211 & 0.134 & 19.4 & 0.21 & 0.132 & -- & -- & 0.121 & 0.056 & 13.2 & -- \\
\hline 7/20/2008 6:00 PM & 0.401 & 20.3 & 0.211 & 0.134 & 19.4 & 0.21 & 0.132 & -- & -- & 0.121 & 0.056 & 13.2 & -- \\
\hline 7/20/2008 7:00 PM & 0.400 & 20.3 & 0.211 & 0.134 & 19.4 & 0.21 & 0.132 & -- & -- & 0.121 & 0.056 & 13.3 & -- \\
\hline 7/20/2008 8:00 PM & 0.400 & 20.3 & 0.211 & 0.134 & 19.4 & 0.21 & 0.132 & -- & -- & 0.121 & 0.057 & 13.2 & -- \\
\hline 7/20/2008 9:00 PM & 0.399 & 20.3 & 0.211 & 0.134 & 19.4 & 0.21 & 0.132 & -- & -- & 0.121 & 0.056 & 13.3 & -- \\
\hline 7/20/2008 10:00 PM & 0.399 & 20.2 & 0.211 & 0.134 & 19.4 & 0.21 & 0.132 & -- & -- & 0.121 & 0.056 & 13.2 & -- \\
\hline 7/20/2008 11:00 PM & 0.399 & 20.3 & 0.211 & 0.134 & 19.4 & 0.21 & 0.132 & -- & -- & 0.121 & 0.055 & 13.3 & -- \\
\hline 7/21/2008 12:00 AM & 0.399 & 20.3 & 0.210 & 0.134 & 19.4 & 0.21 & 0.132 & -- & -- & 0.121 & 0.056 & 13.3 & -- \\
\hline 7/21/2008 1:00 AM & 0.399 & 20.3 & 0.210 & 0.134 & 19.4 & 0.21 & 0.132 & -- & -- & 0.121 & 0.057 & 13.3 & -- \\
\hline 7/21/2008 2:00 AM & 0.399 & 20.3 & 0.210 & 0.134 & 19.4 & 0.21 & 0.132 & -- & -- & 0.121 & 0.057 & 13.3 & -- \\
\hline 7/21/2008 3:00 AM & 0.399 & 20.3 & 0.210 & 0.134 & 19.4 & 0.21 & 0.132 & -- & -- & 0.121 & 0.057 & 13.2 & -- \\
\hline 7/21/2008 4:00 AM & 0.399 & 20.3 & 0.210 & 0.134 & 19.4 & 0.21 & 0.132 & -- & -- & 0.121 & 0.057 & 13.3 & -- \\
\hline 7/21/2008 5:00 AM & 0.399 & 20.3 & 0.210 & 0.134 & 19.4 & 0.21 & 0.132 & -- & -- & 0.121 & 0.057 & 13.3 & -- \\
\hline 7/21/2008 6:00 AM & 0.399 & 20.4 & 0.210 & 0.134 & 19.4 & 0.21 & 0.132 & -- & -- & 0.121 & 0.056 & 13.3 & -- \\
\hline 7/21/2008 7:00 AM & 0.398 & 20.4 & 0.210 & 0.134 & 19.4 & 0.21 & 0.132 & -- & -- & 0.121 & 0.056 & 13.3 & -- \\
\hline 7/21/2008 8:00 AM & 0.398 & 20.3 & 0.210 & 0.134 & 19.4 & 0.21 & 0.132 & -- & -- & 0.121 & 0.057 & 13.3 & -- \\
\hline 7/21/2008 9:00 AM & 0.398 & 20.3 & 0.210 & 0.134 & 19.4 & 0.21 & 0.132 & -- & -- & 0.121 & 0.056 & 13.3 & -- \\
\hline 7/21/2008 10:00 AM & 0.397 & 20.3 & 0.209 & 0.134 & 19.4 & 0.21 & 0.132 & -- & -- & 0.121 & 0.056 & 13.3 & -- \\
\hline 7/21/2008 11:00 AM & 0.396 & 20.4 & 0.209 & 0.134 & 19.4 & 0.21 & 0.132 & -- & -- & 0.121 & 0.056 & 13.3 & -- \\
\hline 7/21/2008 12:00 PM & 0.395 & 20.4 & 0.210 & 0.134 & 19.4 & 0.21 & 0.132 & -- & -- & 0.121 & 0.056 & 13.3 & -- \\
\hline 7/21/2008 1:00 PM & 0.395 & 20.4 & 0.210 & 0.134 & 19.4 & 0.21 & 0.132 & -- & -- & 0.121 & 0.056 & 13.3 & -- \\
\hline 7/21/2008 2:00 PM & 0.395 & 20.4 & 0.209 & 0.134 & 19.4 & 0.21 & 0.132 & -- & -- & 0.121 & 0.056 & 13.3 & -- \\
\hline 7/21/2008 3:00 PM & 0.394 & 20.3 & 0.209 & 0.134 & 19.4 & 0.21 & 0.132 & -- & -- & 0.121 & 0.056 & 13.3 & -- \\
\hline 7/21/2008 4:00 PM & 0.394 & 20.3 & 0.209 & 0.134 & 19.4 & 0.21 & 0.132 & -- & -- & 0.121 & 0.056 & 13.3 & -- \\
\hline 7/21/2008 5:00 PM & 0.394 & 20.3 & 0.209 & 0.134 & 19.4 & 0.21 & 0.132 & -- & -- & 0.121 & 0.056 & 13.3 & -- \\
\hline 7/21/2008 6:00 PM & 0.394 & 20.3 & 0.208 & 0.134 & 19.4 & 0.21 & 0.132 & -- & -- & 0.121 & 0.056 & 13.3 & -- \\
\hline 7/21/2008 7:00 PM & 0.393 & 20.3 & 0.208 & 0.134 & 19.3 & 0.21 & 0.132 & -- & -- & 0.121 & 0.056 & 13.3 & -- \\
\hline 7/21/2008 8:00 PM & 0.393 & 20.3 & 0.208 & 0.134 & 19.4 & 0.21 & 0.132 & -- & -- & 0.121 & 0.057 & 13.3 & -- \\
\hline 7/21/2008 9:00 PM & 0.393 & 20.2 & 0.208 & 0.134 & 19.4 & 0.21 & 0.132 & -- & -- & 0.121 & 0.056 & 13.3 & -- \\
\hline 7/21/2008 10:00 PM & 0.392 & 20.3 & 0.208 & 0.134 & 19.4 & 0.21 & 0.132 & -- & -- & 0.121 & 0.055 & 13.3 & -- \\
\hline 7/21/2008 11:00 PM & 0.392 & 20.2 & 0.208 & 0.134 & 19.4 & 0.21 & 0.132 & -- & -- & 0.121 & 0.055 & 13.3 & -- \\
\hline 7/22/2008 12:00 AM & 0.393 & 20.3 & 0.208 & 0.133 & 19.3 & 0.21 & 0.132 & -- & -- & 0.121 & 0.056 & 13.3 & -- \\
\hline 7/22/2008 1:00 AM & 0.393 & 20.3 & 0.208 & 0.133 & 19.4 & 0.21 & 0.132 & -- & -- & 0.121 & 0.056 & 13.3 & -- \\
\hline 7/22/2008 2:00 AM & 0.392 & 20.3 & 0.208 & 0.133 & 19.4 & 0.21 & 0.132 & -- & -- & 0.121 & 0.057 & 13.3 & -- \\
\hline
\end{tabular}


Appendix 3. Volumetric water content, temperature, and electrical-conductivity data collected at the sprinkler-irrigated site during 2008.-Continued

[Depth in feet below land surface; volumetric water content dimensionless; temperature in degrees Celsius; bulk electrical conductivity in decisiemens per meter; --, no data]

\begin{tabular}{|c|c|c|c|c|c|c|c|c|c|c|c|c|c|}
\hline \multirow[b]{3}{*}{$\begin{array}{l}\text { Measurement } \\
\text { date and time }\end{array}$} & \multicolumn{13}{|c|}{ Depth } \\
\hline & \multicolumn{2}{|c|}{2} & \multirow{2}{*}{$\begin{array}{c}3 \\
\begin{array}{c}\text { Volumetric } \\
\text { water } \\
\text { content }\end{array}\end{array}$} & \multicolumn{3}{|c|}{4} & \multirow{2}{*}{$\begin{array}{c}5 \\
\text { Volumetric } \\
\text { water } \\
\text { content }\end{array}$} & \multicolumn{2}{|c|}{7} & \multirow[b]{2}{*}{$\begin{array}{c}9 \\
\text { Volumetric } \\
\text { water } \\
\text { content }\end{array}$} & \multicolumn{2}{|c|}{13} & \multirow{2}{*}{$\begin{array}{c}16.5 \\
\text { Volumetric } \\
\text { water } \\
\text { content }\end{array}$} \\
\hline & $\begin{array}{c}\text { Volumetric } \\
\text { water } \\
\text { content }\end{array}$ & $\begin{array}{l}\text { Temper- } \\
\text { ature }\end{array}$ & & $\begin{array}{c}\text { Volumetric } \\
\text { water } \\
\text { content }\end{array}$ & $\begin{array}{l}\text { Temper- } \\
\text { ature }\end{array}$ & $\begin{array}{c}\text { Bulk } \\
\text { electrical } \\
\text { conduct- } \\
\text { ivity }\end{array}$ & & $\begin{array}{c}\text { Volumetric } \\
\text { water } \\
\text { content }\end{array}$ & $\begin{array}{l}\text { Temper- } \\
\text { ature }\end{array}$ & & $\begin{array}{c}\text { Volumetric } \\
\text { water } \\
\text { content }\end{array}$ & $\begin{array}{l}\text { Temper- } \\
\text { ature }\end{array}$ & \\
\hline 7/22/2008 3:00 AM & 0.393 & 20.3 & 0.207 & 0.133 & 19.3 & 0.21 & 0.132 & -- & -- & 0.121 & 0.057 & 13.3 & -- \\
\hline 7/22/2008 4:00 AM & 0.392 & 20.3 & 0.207 & 0.133 & 19.3 & 0.21 & 0.132 & -- & -- & 0.121 & 0.056 & 13.4 & -- \\
\hline 7/22/2008 5:00 AM & 0.393 & 20.3 & 0.207 & 0.133 & 19.4 & 0.21 & 0.132 & -- & -- & 0.121 & 0.056 & 13.4 & -- \\
\hline 7/22/2008 6:00 AM & 0.392 & 20.3 & 0.207 & 0.133 & 19.3 & 0.21 & 0.132 & -- & -- & 0.121 & 0.056 & 13.4 & -- \\
\hline 7/22/2008 7:00 AM & 0.393 & 20.3 & 0.207 & 0.133 & 19.3 & 0.21 & 0.132 & -- & -- & 0.121 & 0.056 & 13.4 & -- \\
\hline 7/22/2008 8:00 AM & 0.392 & 20.4 & 0.206 & 0.133 & 19.3 & 0.21 & 0.132 & -- & -- & 0.121 & 0.057 & 13.4 & -- \\
\hline 7/22/2008 9:00 AM & 0.392 & 20.3 & 0.206 & 0.133 & 19.3 & 0.21 & 0.132 & -- & -- & 0.121 & 0.056 & 13.3 & -- \\
\hline 7/22/2008 10:00 AM & 0.392 & 20.3 & 0.206 & 0.133 & 19.4 & 0.21 & 0.132 & -- & -- & 0.121 & 0.055 & 13.3 & -- \\
\hline 7/22/2008 11:00 AM & 0.392 & 20.3 & 0.206 & 0.133 & 19.3 & 0.21 & 0.132 & -- & -- & 0.121 & 0.056 & 13.3 & -- \\
\hline 7/22/2008 12:00 PM & 0.391 & 20.4 & 0.206 & 0.133 & 19.3 & 0.21 & 0.132 & -- & -- & 0.121 & 0.056 & 13.4 & -- \\
\hline 7/22/2008 1:00 PM & 0.391 & 20.4 & 0.207 & 0.133 & 19.3 & 0.21 & 0.132 & -- & -- & 0.121 & 0.056 & 13.3 & -- \\
\hline 7/22/2008 2:00 PM & 0.392 & 20.4 & 0.207 & 0.133 & 19.3 & 0.21 & 0.132 & -- & -- & 0.121 & 0.056 & 13.4 & -- \\
\hline 7/22/2008 3:00 PM & 0.394 & 20.4 & 0.207 & 0.133 & 19.4 & 0.21 & 0.132 & -- & -- & 0.121 & 0.056 & 13.4 & -- \\
\hline 7/22/2008 4:00 PM & 0.395 & 20.3 & 0.208 & 0.133 & 19.3 & 0.21 & 0.132 & -- & -- & 0.121 & 0.056 & 13.4 & -- \\
\hline 7/22/2008 5:00 PM & 0.396 & 20.3 & 0.208 & 0.133 & 19.3 & 0.21 & 0.132 & -- & -- & 0.121 & 0.056 & 13.4 & -- \\
\hline 7/22/2008 6:00 PM & 0.397 & 20.3 & 0.208 & 0.133 & 19.3 & 0.21 & 0.132 & -- & -- & 0.121 & 0.056 & 13.4 & -- \\
\hline 7/22/2008 7:00 PM & 0.398 & 20.3 & 0.208 & 0.133 & 19.4 & 0.21 & 0.131 & -- & -- & 0.121 & 0.056 & 13.4 & -- \\
\hline 7/22/2008 8:00 PM & 0.397 & 20.3 & 0.208 & 0.133 & 19.4 & 0.21 & 0.131 & -- & -- & 0.121 & 0.056 & 13.4 & -- \\
\hline 7/22/2008 9:00 PM & 0.397 & 20.3 & 0.208 & 0.133 & 19.4 & 0.21 & 0.131 & -- & -- & 0.121 & 0.056 & 13.3 & -- \\
\hline 7/22/2008 10:00 PM & 0.398 & 20.2 & 0.208 & 0.133 & 19.3 & 0.21 & 0.132 & -- & -- & 0.121 & 0.055 & 13.4 & -- \\
\hline 7/22/2008 11:00 PM & 0.398 & 20.3 & 0.208 & 0.133 & 19.3 & 0.21 & 0.132 & -- & -- & 0.121 & 0.055 & 13.4 & -- \\
\hline 7/23/2008 12:00 AM & 0.398 & 20.3 & 0.209 & 0.133 & 19.4 & 0.21 & 0.132 & -- & -- & 0.121 & 0.055 & 13.4 & -- \\
\hline 7/23/2008 1:00 AM & 0.398 & 20.3 & 0.209 & 0.133 & 19.3 & 0.21 & 0.131 & -- & -- & 0.121 & 0.055 & 13.4 & -- \\
\hline 7/23/2008 2:00 AM & 0.399 & 20.3 & 0.209 & 0.133 & 19.4 & 0.21 & 0.131 & -- & -- & 0.121 & 0.056 & 13.4 & -- \\
\hline 7/23/2008 3:00 AM & 0.399 & 20.3 & 0.209 & 0.133 & 19.4 & 0.21 & 0.131 & -- & -- & 0.121 & 0.056 & 13.4 & -- \\
\hline 7/23/2008 4:00 AM & 0.399 & 20.3 & 0.209 & 0.134 & 19.3 & 0.21 & 0.131 & -- & -- & 0.121 & 0.057 & 13.4 & -- \\
\hline 7/23/2008 5:00 AM & 0.399 & 20.3 & 0.209 & 0.133 & 19.4 & 0.21 & 0.131 & -- & -- & 0.121 & 0.057 & 13.4 & -- \\
\hline 7/23/2008 6:00 AM & 0.399 & 20.3 & 0.209 & 0.133 & 19.3 & 0.21 & 0.131 & -- & -- & 0.121 & 0.057 & 13.4 & -- \\
\hline 7/23/2008 7:00 AM & 0.398 & 20.3 & 0.209 & 0.133 & 19.4 & 0.21 & 0.131 & -- & -- & 0.121 & 0.057 & 13.4 & -- \\
\hline 7/23/2008 8:00 AM & 0.399 & 20.3 & 0.209 & 0.133 & 19.3 & 0.21 & 0.131 & -- & -- & 0.121 & 0.057 & 13.4 & -- \\
\hline 7/23/2008 9:00 AM & 0.398 & 20.3 & 0.209 & 0.133 & 19.3 & 0.21 & 0.131 & -- & -- & 0.121 & 0.055 & 13.4 & -- \\
\hline 7/23/2008 10:00 AM & 0.397 & 20.3 & 0.209 & 0.133 & 19.4 & 0.21 & 0.131 & -- & -- & 0.121 & 0.056 & 13.4 & -- \\
\hline 7/23/2008 11:00 AM & 0.397 & 20.4 & 0.209 & 0.133 & 19.4 & 0.21 & 0.132 & -- & -- & 0.121 & 0.056 & 13.4 & -- \\
\hline 7/23/2008 12:00 PM & 0.397 & 20.4 & 0.209 & 0.133 & 19.3 & 0.21 & 0.132 & -- & -- & 0.121 & 0.056 & 13.4 & -- \\
\hline 7/23/2008 1:00 PM & 0.396 & 20.4 & 0.209 & 0.134 & 19.4 & 0.21 & 0.132 & -- & -- & 0.121 & 0.056 & 13.4 & -- \\
\hline
\end{tabular}


Appendix 3. Volumetric water content, temperature, and electrical-conductivity data collected at the sprinkler-irrigated site during 2008.-Continued

[Depth in feet below land surface; volumetric water content dimensionless; temperature in degrees Celsius; bulk electrical conductivity in decisiemens per meter; --, no data]

\begin{tabular}{|c|c|c|c|c|c|c|c|c|c|c|c|c|c|}
\hline \multirow[b]{3}{*}{$\begin{array}{l}\text { Measurement } \\
\text { date and time }\end{array}$} & \multicolumn{13}{|c|}{ Depth } \\
\hline & \multicolumn{2}{|l|}{2} & \multirow{2}{*}{$\begin{array}{c}3 \\
\text { Volumetric } \\
\text { water } \\
\text { content }\end{array}$} & \multicolumn{3}{|c|}{4} & \multirow{2}{*}{$\begin{array}{c}5 \\
\text { Volumetric } \\
\text { water } \\
\text { content }\end{array}$} & \multicolumn{2}{|l|}{7} & \multirow{2}{*}{$\begin{array}{c}9 \\
\text { Volumetric } \\
\text { water } \\
\text { content }\end{array}$} & \multicolumn{2}{|c|}{13} & \multirow{2}{*}{$\begin{array}{c}16.5 \\
\text { Volumetric } \\
\text { water } \\
\text { content }\end{array}$} \\
\hline & $\begin{array}{c}\text { Volumetric } \\
\text { water } \\
\text { content }\end{array}$ & $\begin{array}{l}\text { Temper- } \\
\text { ature }\end{array}$ & & $\begin{array}{c}\text { Volumetric } \\
\text { water } \\
\text { content }\end{array}$ & $\begin{array}{l}\text { Temper- } \\
\text { ature }\end{array}$ & $\begin{array}{c}\text { Bulk } \\
\text { electrical } \\
\text { conduct- } \\
\text { ivity }\end{array}$ & & $\begin{array}{c}\text { Volumetric } \\
\text { water } \\
\text { content }\end{array}$ & $\begin{array}{l}\text { Temper- } \\
\text { ature }\end{array}$ & & $\begin{array}{l}\text { Volumetric } \\
\text { water } \\
\text { content }\end{array}$ & $\begin{array}{l}\text { Temper- } \\
\text { ature }\end{array}$ & \\
\hline 7/23/2008 2:00 PM & 0.396 & 20.4 & 0.209 & 0.133 & 19.4 & 0.21 & 0.132 & -- & -- & 0.121 & 0.056 & 13.4 & -- \\
\hline 7/23/2008 3:00 PM & 0.396 & 20.4 & 0.209 & 0.134 & 19.4 & 0.21 & 0.132 & -- & -- & 0.121 & 0.056 & 13.4 & -- \\
\hline 7/23/2008 4:00 PM & 0.397 & 20.3 & 0.209 & 0.133 & 19.3 & 0.21 & 0.131 & -- & -- & 0.121 & 0.055 & 13.4 & -- \\
\hline 7/23/2008 5:00 PM & 0.397 & 20.3 & 0.209 & 0.133 & 19.4 & 0.21 & 0.132 & -- & -- & 0.121 & 0.055 & 13.4 & -- \\
\hline 7/23/2008 6:00 PM & 0.396 & 20.3 & 0.208 & 0.133 & 19.4 & 0.21 & 0.132 & -- & -- & 0.121 & 0.056 & 13.4 & -- \\
\hline 7/23/2008 7:00 PM & 0.396 & 20.3 & 0.208 & 0.133 & 19.4 & 0.20 & 0.132 & -- & -- & 0.121 & 0.056 & 13.4 & -- \\
\hline 7/23/2008 8:00 PM & 0.396 & 20.3 & 0.208 & 0.134 & 19.3 & 0.20 & 0.132 & -- & -- & 0.121 & 0.055 & 13.4 & -- \\
\hline 7/23/2008 9:00 PM & 0.396 & 20.3 & 0.209 & 0.133 & 19.4 & 0.20 & 0.132 & -- & -- & 0.121 & 0.055 & 13.4 & -- \\
\hline 7/23/2008 10:00 PM & 0.396 & 20.3 & 0.209 & 0.133 & 19.3 & 0.20 & 0.132 & -- & -- & 0.121 & 0.056 & 13.5 & -- \\
\hline 7/23/2008 11:00 PM & 0.396 & 20.4 & 0.208 & 0.133 & 19.3 & 0.20 & 0.132 & -- & -- & 0.121 & 0.056 & 13.4 & -- \\
\hline 7/24/2008 12:00 AM & 0.395 & 20.4 & 0.208 & 0.133 & 19.4 & 0.20 & 0.132 & -- & -- & 0.121 & 0.056 & 13.4 & -- \\
\hline 7/24/2008 1:00 AM & 0.396 & 20.4 & 0.208 & 0.133 & 19.3 & 0.20 & 0.132 & -- & -- & 0.121 & 0.057 & 13.4 & -- \\
\hline 7/24/2008 2:00 AM & 0.395 & 20.4 & 0.208 & 0.133 & 19.4 & 0.20 & 0.132 & -- & -- & 0.121 & 0.057 & 13.4 & -- \\
\hline 7/24/2008 3:00 AM & 0.396 & 20.4 & 0.208 & 0.133 & 19.3 & 0.20 & 0.132 & -- & -- & 0.121 & 0.057 & 13.4 & -- \\
\hline 7/24/2008 4:00 AM & 0.395 & 20.4 & 0.208 & 0.133 & 19.3 & 0.20 & 0.132 & -- & -- & 0.121 & 0.056 & 13.5 & -- \\
\hline 7/24/2008 5:00 AM & 0.395 & 20.4 & 0.208 & 0.133 & 19.3 & 0.20 & 0.132 & -- & -- & 0.121 & 0.056 & 13.5 & -- \\
\hline 7/24/2008 6:00 AM & 0.395 & 20.4 & 0.208 & 0.133 & 19.4 & 0.20 & 0.132 & -- & -- & 0.121 & 0.056 & 13.5 & -- \\
\hline 7/24/2008 7:00 AM & 0.395 & 20.4 & 0.208 & 0.133 & 19.4 & 0.20 & 0.132 & -- & -- & 0.121 & 0.056 & 13.5 & -- \\
\hline 7/24/2008 8:00 AM & 0.395 & 20.4 & 0.208 & 0.133 & 19.4 & 0.20 & 0.132 & -- & -- & 0.121 & 0.056 & 13.5 & -- \\
\hline 7/24/2008 9:00 AM & 0.394 & 20.4 & 0.208 & 0.133 & 19.4 & 0.20 & 0.132 & -- & -- & 0.121 & 0.056 & 13.5 & -- \\
\hline 7/24/2008 10:00 AM & 0.394 & 20.4 & 0.208 & 0.133 & 19.4 & 0.20 & 0.132 & -- & -- & 0.121 & 0.056 & 13.4 & -- \\
\hline 7/24/2008 11:00 AM & 0.394 & 20.4 & 0.208 & 0.133 & 19.4 & 0.20 & 0.132 & -- & -- & 0.121 & 0.056 & 13.5 & -- \\
\hline 7/24/2008 12:00 PM & 0.393 & 20.4 & 0.208 & 0.133 & 19.4 & 0.20 & 0.132 & -- & -- & 0.121 & 0.056 & 13.4 & -- \\
\hline 7/24/2008 1:00 PM & 0.392 & 20.4 & 0.208 & 0.133 & 19.4 & 0.20 & 0.132 & -- & -- & 0.121 & 0.056 & 13.4 & -- \\
\hline 7/24/2008 2:00 PM & 0.392 & 20.4 & 0.208 & 0.133 & 19.4 & 0.20 & 0.132 & -- & -- & 0.121 & 0.056 & 13.4 & -- \\
\hline 7/24/2008 3:00 PM & 0.391 & 20.4 & 0.208 & 0.133 & 19.4 & 0.20 & 0.132 & -- & -- & 0.121 & 0.056 & 13.4 & -- \\
\hline 7/24/2008 4:00 PM & 0.392 & 20.4 & 0.208 & 0.134 & 19.4 & 0.20 & 0.132 & -- & -- & 0.121 & 0.056 & 13.4 & -- \\
\hline 7/24/2008 5:00 PM & 0.392 & 20.4 & 0.207 & 0.133 & 19.4 & 0.20 & 0.132 & -- & -- & 0.121 & 0.056 & 13.5 & -- \\
\hline 7/24/2008 6:00 PM & 0.392 & 20.3 & 0.207 & 0.133 & 19.4 & 0.20 & 0.131 & -- & -- & 0.121 & 0.056 & 13.4 & -- \\
\hline 7/24/2008 7:00 PM & 0.392 & 20.3 & 0.207 & 0.133 & 19.4 & 0.20 & 0.131 & -- & -- & 0.121 & 0.056 & 13.4 & -- \\
\hline 7/24/2008 8:00 PM & 0.391 & 20.3 & 0.206 & 0.133 & 19.4 & 0.20 & 0.131 & -- & -- & 0.121 & 0.056 & 13.5 & -- \\
\hline 7/24/2008 9:00 PM & 0.392 & 20.3 & 0.206 & 0.133 & 19.4 & 0.20 & 0.131 & -- & -- & 0.121 & 0.056 & 13.5 & -- \\
\hline 7/24/2008 10:00 PM & 0.391 & 20.3 & 0.206 & 0.133 & 19.4 & 0.20 & 0.131 & -- & -- & 0.121 & 0.057 & 13.4 & -- \\
\hline 7/24/2008 11:00 PM & 0.391 & 20.3 & 0.206 & 0.133 & 19.4 & 0.20 & 0.131 & -- & -- & 0.121 & 0.057 & 13.4 & -- \\
\hline 7/25/2008 12:00 AM & 0.391 & 20.3 & 0.206 & 0.133 & 19.4 & 0.20 & 0.131 & -- & -- & 0.121 & 0.056 & 13.5 & -- \\
\hline
\end{tabular}


Appendix 3. Volumetric water content, temperature, and electrical-conductivity data collected at the sprinkler-irrigated site during 2008.-Continued

[Depth in feet below land surface; volumetric water content dimensionless; temperature in degrees Celsius; bulk electrical conductivity in decisiemens per meter; --, no data]

\begin{tabular}{|c|c|c|c|c|c|c|c|c|c|c|c|c|c|}
\hline \multirow[b]{3}{*}{$\begin{array}{l}\text { Measurement } \\
\text { date and time }\end{array}$} & \multicolumn{13}{|c|}{ Depth } \\
\hline & \multicolumn{2}{|c|}{2} & \multirow{2}{*}{$\begin{array}{c}3 \\
\text { Volumetric } \\
\text { water } \\
\text { content }\end{array}$} & \multicolumn{3}{|c|}{4} & \multirow{2}{*}{$\begin{array}{c}5 \\
\text { Volumetric } \\
\text { water } \\
\text { content }\end{array}$} & \multicolumn{2}{|l|}{7} & \multirow{2}{*}{$\begin{array}{c}9 \\
\text { Volumetric } \\
\text { water } \\
\text { content }\end{array}$} & \multicolumn{2}{|c|}{13} & \multirow{2}{*}{$\begin{array}{c}16.5 \\
\text { Volumetric } \\
\text { water } \\
\text { content }\end{array}$} \\
\hline & $\begin{array}{c}\text { Volumetric } \\
\text { water } \\
\text { content }\end{array}$ & $\begin{array}{l}\text { Temper- } \\
\text { ature }\end{array}$ & & $\begin{array}{c}\text { Volumetric } \\
\text { water } \\
\text { content }\end{array}$ & $\begin{array}{l}\text { Temper- } \\
\text { ature }\end{array}$ & $\begin{array}{c}\text { Bulk } \\
\text { electrical } \\
\text { conduct- } \\
\text { ivity }\end{array}$ & & $\begin{array}{c}\text { Volumetric } \\
\text { water } \\
\text { content }\end{array}$ & $\begin{array}{l}\text { Temper- } \\
\text { ature }\end{array}$ & & $\begin{array}{c}\text { Volumetric } \\
\text { water } \\
\text { content }\end{array}$ & $\begin{array}{l}\text { Temper- } \\
\text { ature }\end{array}$ & \\
\hline 7/25/2008 1:00 AM & 0.392 & 20.3 & 0.206 & 0.133 & 19.3 & 0.20 & 0.131 & -- & -- & 0.121 & 0.056 & 13.5 & -- \\
\hline 7/25/2008 2:00 AM & 0.392 & 20.3 & 0.206 & 0.134 & 19.4 & 0.20 & 0.131 & -- & -- & 0.121 & 0.056 & 13.5 & -- \\
\hline 7/25/2008 3:00 AM & 0.391 & 20.4 & 0.206 & 0.133 & 19.4 & 0.20 & 0.131 & -- & -- & 0.121 & 0.056 & 13.5 & -- \\
\hline 7/25/2008 4:00 AM & 0.391 & 20.4 & 0.206 & 0.133 & 19.4 & 0.20 & 0.131 & -- & -- & 0.121 & 0.055 & 13.5 & -- \\
\hline 7/25/2008 5:00 AM & 0.391 & 20.4 & 0.206 & 0.133 & 19.4 & 0.20 & 0.131 & -- & -- & 0.121 & 0.056 & 13.5 & -- \\
\hline 7/25/2008 6:00 AM & 0.391 & 20.4 & 0.206 & 0.133 & 19.4 & 0.20 & 0.131 & -- & -- & 0.121 & 0.056 & 13.5 & -- \\
\hline 7/25/2008 7:00 AM & 0.391 & 20.4 & 0.206 & 0.133 & 19.4 & 0.20 & 0.131 & -- & -- & 0.121 & 0.056 & 13.5 & -- \\
\hline 7/25/2008 8:00 AM & 0.391 & 20.4 & 0.206 & 0.133 & 19.4 & 0.20 & 0.131 & -- & -- & 0.121 & 0.056 & 13.5 & -- \\
\hline 7/25/2008 9:00 AM & 0.391 & 20.4 & 0.206 & 0.133 & 19.4 & 0.20 & 0.131 & -- & -- & 0.121 & 0.057 & 13.5 & -- \\
\hline 7/25/2008 10:00 AM & 0.390 & 20.4 & 0.205 & 0.133 & 19.4 & 0.20 & 0.131 & -- & -- & 0.121 & 0.056 & 13.5 & -- \\
\hline 7/25/2008 11:00 AM & 0.390 & 20.4 & 0.205 & 0.133 & 19.4 & 0.20 & 0.131 & -- & -- & 0.121 & 0.056 & 13.5 & -- \\
\hline 7/25/2008 12:00 PM & 0.388 & 20.4 & 0.206 & 0.133 & 19.4 & 0.20 & 0.131 & -- & -- & 0.121 & 0.056 & 13.5 & -- \\
\hline 7/25/2008 1:00 PM & 0.388 & 20.4 & 0.206 & 0.133 & 19.4 & 0.20 & 0.131 & -- & -- & 0.121 & 0.056 & 13.5 & -- \\
\hline 7/25/2008 2:00 PM & 0.387 & 20.4 & 0.206 & 0.133 & 19.4 & 0.20 & 0.131 & -- & -- & 0.121 & 0.055 & 13.5 & -- \\
\hline 7/25/2008 3:00 PM & 0.387 & 20.4 & 0.205 & 0.133 & 19.4 & 0.20 & 0.131 & -- & -- & 0.121 & 0.056 & 13.5 & -- \\
\hline 7/25/2008 4:00 PM & 0.387 & 20.4 & 0.205 & 0.133 & 19.4 & 0.20 & 0.131 & -- & -- & 0.121 & 0.057 & 13.5 & -- \\
\hline 7/25/2008 5:00 PM & 0.388 & 20.3 & 0.205 & 0.133 & 19.4 & 0.20 & 0.131 & -- & -- & 0.121 & 0.056 & 13.5 & -- \\
\hline 7/25/2008 6:00 PM & 0.388 & 20.3 & 0.205 & 0.133 & 19.4 & 0.20 & 0.131 & -- & -- & 0.121 & 0.056 & 13.5 & -- \\
\hline 7/25/2008 7:00 PM & 0.387 & 20.3 & 0.205 & 0.133 & 19.4 & 0.20 & 0.130 & -- & -- & 0.121 & 0.056 & 13.5 & -- \\
\hline 7/25/2008 8:00 PM & 0.387 & 20.3 & 0.205 & 0.133 & 19.4 & 0.20 & 0.130 & -- & -- & 0.012 & -- & -- & -- \\
\hline 7/25/2008 9:00 PM & 0.387 & 20.3 & 0.205 & 0.133 & 19.4 & 0.20 & 0.130 & -- & -- & -- & -- & -- & -- \\
\hline 7/25/2008 10:00 PM & 0.387 & 20.3 & 0.205 & 0.133 & 19.4 & 0.20 & 0.130 & -- & -- & -- & -- & -- & -- \\
\hline 7/25/2008 11:00 PM & 0.386 & 20.3 & 0.205 & 0.133 & 19.4 & 0.20 & 0.129 & -- & -- & -- & -- & -- & -- \\
\hline 7/26/2008 12:00 AM & 0.386 & 20.3 & 0.205 & 0.133 & 19.4 & 0.20 & 0.129 & -- & -- & -- & -- & -- & -- \\
\hline 7/26/2008 1:00 AM & 0.386 & 20.3 & 0.205 & 0.133 & 19.4 & 0.20 & 0.129 & -- & -- & -- & -- & -- & -- \\
\hline 7/26/2008 2:00 AM & 0.386 & 20.3 & 0.205 & 0.133 & 19.4 & 0.20 & 0.129 & -- & -- & -- & -- & -- & -- \\
\hline 7/26/2008 3:00 AM & 0.386 & 20.3 & 0.205 & 0.133 & 19.4 & 0.20 & 0.129 & -- & -- & -- & -- & -- & -- \\
\hline 7/26/2008 4:00 AM & 0.386 & 20.3 & 0.205 & 0.133 & 19.4 & 0.20 & 0.129 & -- & -- & -- & -- & -- & -- \\
\hline 7/26/2008 5:00 AM & 0.386 & 20.3 & 0.204 & 0.133 & 19.4 & 0.20 & 0.129 & -- & -- & -- & -- & -- & -- \\
\hline 7/26/2008 6:00 AM & 0.386 & 20.3 & 0.204 & 0.133 & 19.4 & 0.20 & 0.129 & -- & -- & -- & -- & -- & -- \\
\hline 7/26/2008 7:00 AM & 0.387 & 20.3 & 0.204 & 0.133 & 19.4 & 0.20 & 0.129 & -- & -- & -- & -- & -- & -- \\
\hline 7/26/2008 8:00 AM & 0.387 & 20.3 & 0.204 & 0.133 & 19.4 & 0.20 & 0.129 & -- & -- & -- & -- & -- & -- \\
\hline 7/26/2008 9:00 AM & 0.387 & 20.3 & 0.205 & 0.133 & 19.4 & 0.20 & 0.129 & -- & -- & -- & -- & -- & -- \\
\hline 7/26/2008 10:00 AM & 0.388 & 20.3 & 0.205 & 0.132 & 19.4 & 0.20 & 0.129 & -- & -- & -- & -- & -- & -- \\
\hline 7/26/2008 11:00 AM & 0.388 & 20.3 & 0.205 & 0.133 & 19.4 & 0.20 & 0.129 & -- & -- & -- & -- & -- & -- \\
\hline
\end{tabular}


Appendix 3. Volumetric water content, temperature, and electrical-conductivity data collected at the sprinkler-irrigated site during 2008.-Continued

[Depth in feet below land surface; volumetric water content dimensionless; temperature in degrees Celsius; bulk electrical conductivity in decisiemens per meter; --, no data]

\begin{tabular}{|c|c|c|c|c|c|c|c|c|c|c|c|c|c|}
\hline \multirow[b]{3}{*}{$\begin{array}{l}\text { Measurement } \\
\text { date and time }\end{array}$} & \multicolumn{13}{|c|}{ Depth } \\
\hline & \multicolumn{2}{|c|}{2} & \multirow[b]{2}{*}{$\begin{array}{c}3 \\
\text { Volumetric } \\
\text { water } \\
\text { content }\end{array}$} & \multicolumn{3}{|c|}{4} & \multirow{2}{*}{$\begin{array}{c}5 \\
\text { Volumetric } \\
\text { water } \\
\text { content }\end{array}$} & \multicolumn{2}{|c|}{7} & \multirow{2}{*}{$\begin{array}{c}9 \\
\text { Volumetric } \\
\text { water } \\
\text { content }\end{array}$} & \multicolumn{2}{|c|}{13} & \multirow{2}{*}{$\begin{array}{c}16.5 \\
\text { Volumetric } \\
\text { water } \\
\text { content }\end{array}$} \\
\hline & $\begin{array}{c}\text { Volumetric } \\
\text { water } \\
\text { content }\end{array}$ & $\begin{array}{l}\text { Temper- } \\
\text { ature }\end{array}$ & & $\begin{array}{c}\text { Volumetric } \\
\text { water } \\
\text { content }\end{array}$ & $\begin{array}{l}\text { Temper- } \\
\text { ature }\end{array}$ & $\begin{array}{c}\text { Bulk } \\
\text { electrical } \\
\text { conduct- } \\
\text { ivity }\end{array}$ & & $\begin{array}{c}\text { Volumetric } \\
\text { water } \\
\text { content }\end{array}$ & $\begin{array}{l}\text { Temper- } \\
\text { ature }\end{array}$ & & $\begin{array}{c}\text { Volumetric } \\
\text { water } \\
\text { content }\end{array}$ & $\begin{array}{l}\text { Temper- } \\
\text { ature }\end{array}$ & \\
\hline 7/26/2008 12:00 PM & 0.388 & 20.3 & 0.205 & 0.133 & 19.4 & 0.20 & 0.129 & -- & -- & -- & -- & -- & -- \\
\hline 7/26/2008 1:00 PM & 0.388 & 20.3 & 0.205 & 0.133 & 19.4 & 0.20 & 0.129 & -- & -- & -- & -- & -- & -- \\
\hline 7/26/2008 2:00 PM & 0.390 & 20.3 & 0.205 & 0.133 & 19.4 & 0.20 & 0.129 & -- & -- & -- & -- & -- & -- \\
\hline 7/26/2008 3:00 PM & 0.388 & 20.3 & 0.205 & 0.132 & 19.4 & 0.20 & 0.129 & -- & -- & -- & -- & -- & -- \\
\hline 7/26/2008 4:00 PM & 0.388 & 20.3 & 0.205 & 0.132 & 19.4 & 0.20 & 0.129 & -- & -- & -- & -- & -- & -- \\
\hline 7/26/2008 5:00 PM & 0.388 & 20.3 & 0.205 & 0.132 & 19.4 & 0.20 & 0.129 & -- & -- & -- & -- & -- & -- \\
\hline 7/26/2008 6:00 PM & 0.387 & 20.3 & 0.205 & 0.133 & 19.4 & 0.20 & 0.129 & -- & -- & -- & -- & -- & -- \\
\hline 7/26/2008 7:00 PM & 0.387 & 20.3 & 0.205 & 0.132 & 19.4 & 0.20 & 0.129 & -- & -- & -- & -- & -- & -- \\
\hline 7/26/2008 8:00 PM & 0.387 & 20.3 & 0.205 & 0.132 & 19.4 & 0.20 & 0.129 & -- & -- & -- & -- & -- & -- \\
\hline 7/26/2008 9:00 PM & 0.386 & 20.3 & 0.205 & 0.133 & 19.4 & 0.20 & 0.129 & -- & -- & -- & -- & -- & -- \\
\hline 7/26/2008 10:00 PM & 0.387 & 20.2 & 0.205 & 0.133 & 19.4 & 0.20 & 0.129 & -- & -- & -- & -- & -- & -- \\
\hline 7/26/2008 11:00 PM & 0.387 & 20.2 & 0.205 & 0.133 & 19.4 & 0.20 & 0.128 & -- & -- & -- & -- & -- & -- \\
\hline 7/27/2008 12:00 AM & 0.387 & 20.2 & 0.205 & 0.132 & 19.4 & 0.20 & 0.128 & -- & -- & -- & -- & -- & -- \\
\hline 7/27/2008 1:00 AM & 0.387 & 20.3 & 0.205 & 0.133 & 19.4 & 0.20 & 0.128 & -- & -- & -- & -- & -- & -- \\
\hline 7/27/2008 2:00 AM & 0.387 & 20.2 & 0.205 & 0.132 & 19.4 & 0.20 & 0.128 & -- & -- & -- & -- & -- & -- \\
\hline 7/27/2008 3:00 AM & 0.387 & 20.3 & 0.205 & 0.133 & 19.4 & 0.20 & 0.128 & -- & -- & -- & -- & -- & -- \\
\hline 7/27/2008 4:00 AM & 0.387 & 20.3 & 0.205 & 0.133 & 19.4 & 0.20 & 0.128 & -- & -- & -- & -- & -- & -- \\
\hline 7/27/2008 5:00 AM & 0.388 & 20.3 & 0.205 & 0.133 & 19.4 & 0.20 & 0.128 & -- & -- & -- & -- & -- & -- \\
\hline 7/27/2008 6:00 AM & 0.388 & 20.3 & 0.205 & 0.133 & 19.4 & 0.20 & 0.128 & -- & -- & -- & -- & -- & -- \\
\hline 7/27/2008 7:00 AM & 0.390 & 20.3 & 0.205 & 0.132 & 19.4 & 0.20 & 0.128 & -- & -- & -- & -- & -- & -- \\
\hline 7/27/2008 8:00 AM & 0.390 & 20.3 & 0.205 & 0.132 & 19.4 & 0.20 & 0.128 & -- & -- & -- & -- & -- & -- \\
\hline 7/27/2008 9:00 AM & 0.390 & 20.3 & 0.205 & 0.132 & 19.4 & 0.20 & 0.128 & -- & -- & -- & -- & -- & -- \\
\hline 7/27/2008 10:00 AM & 0.391 & 20.3 & 0.205 & 0.133 & 19.4 & 0.20 & 0.128 & -- & -- & -- & -- & -- & -- \\
\hline 7/27/2008 11:00 AM & 0.390 & 20.3 & 0.205 & 0.133 & 19.4 & 0.20 & 0.128 & -- & -- & -- & -- & -- & -- \\
\hline 7/27/2008 12:00 PM & 0.390 & 20.4 & 0.205 & 0.133 & 19.4 & 0.20 & 0.128 & -- & -- & -- & -- & -- & -- \\
\hline 7/27/2008 1:00 PM & 0.388 & 20.4 & 0.205 & 0.133 & 19.4 & 0.20 & 0.128 & -- & -- & -- & -- & -- & -- \\
\hline 7/27/2008 2:00 PM & 0.388 & 20.3 & 0.205 & 0.132 & 19.4 & 0.20 & 0.128 & -- & -- & -- & -- & -- & -- \\
\hline 7/27/2008 3:00 PM & 0.388 & 20.3 & 0.205 & 0.132 & 19.4 & 0.19 & 0.128 & -- & -- & -- & -- & -- & -- \\
\hline 7/27/2008 4:00 PM & 0.388 & 20.3 & 0.205 & 0.132 & 19.4 & 0.19 & 0.128 & -- & -- & -- & -- & -- & -- \\
\hline 7/27/2008 5:00 PM & 0.387 & 20.3 & 0.205 & 0.133 & 19.4 & 0.19 & 0.128 & -- & -- & -- & -- & -- & -- \\
\hline 7/27/2008 6:00 PM & 0.387 & 20.3 & 0.205 & 0.132 & 19.4 & 0.19 & 0.128 & -- & -- & -- & -- & -- & -- \\
\hline 7/27/2008 7:00 PM & 0.386 & 20.3 & 0.205 & 0.133 & 19.4 & 0.19 & 0.128 & -- & -- & -- & -- & -- & -- \\
\hline 7/27/2008 8:00 PM & 0.386 & 20.2 & 0.204 & 0.133 & 19.4 & 0.19 & 0.128 & -- & -- & -- & -- & -- & -- \\
\hline 7/27/2008 9:00 PM & 0.386 & 20.3 & 0.204 & 0.133 & 19.4 & 0.19 & 0.128 & -- & -- & -- & -- & -- & -- \\
\hline 7/27/2008 10:00 PM & 0.385 & 20.3 & 0.204 & 0.132 & 19.4 & 0.19 & 0.128 & -- & -- & -- & -- & -- & -- \\
\hline
\end{tabular}


Appendix 3. Volumetric water content, temperature, and electrical-conductivity data collected at the sprinkler-irrigated site during 2008.-Continued

[Depth in feet below land surface; volumetric water content dimensionless; temperature in degrees Celsius; bulk electrical conductivity in decisiemens per meter; --, no data]

\begin{tabular}{|c|c|c|c|c|c|c|c|c|c|c|c|c|c|}
\hline \multirow[b]{3}{*}{$\begin{array}{l}\text { Measurement } \\
\text { date and time }\end{array}$} & \multicolumn{13}{|c|}{ Depth } \\
\hline & \multicolumn{2}{|c|}{2} & \multirow{2}{*}{$\begin{array}{c}3 \\
\text { Volumetric } \\
\text { water } \\
\text { content }\end{array}$} & \multicolumn{3}{|c|}{4} & \multirow{2}{*}{$\begin{array}{c}5 \\
\text { Volumetric } \\
\text { water } \\
\text { content }\end{array}$} & \multicolumn{2}{|l|}{7} & \multirow{2}{*}{$\begin{array}{c}9 \\
\text { Volumetric } \\
\text { water } \\
\text { content }\end{array}$} & \multicolumn{2}{|c|}{13} & \multirow{2}{*}{$\begin{array}{c}16.5 \\
\text { Volumetric } \\
\text { water } \\
\text { content }\end{array}$} \\
\hline & $\begin{array}{c}\text { Volumetric } \\
\text { water } \\
\text { content }\end{array}$ & $\begin{array}{l}\text { Temper- } \\
\text { ature }\end{array}$ & & $\begin{array}{c}\text { Volumetric } \\
\text { water } \\
\text { content }\end{array}$ & $\begin{array}{l}\text { Temper- } \\
\text { ature }\end{array}$ & $\begin{array}{c}\text { Bulk } \\
\text { electrical } \\
\text { conduct- } \\
\text { ivity }\end{array}$ & & $\begin{array}{c}\text { Volumetric } \\
\text { water } \\
\text { content }\end{array}$ & $\begin{array}{l}\text { Temper- } \\
\text { ature }\end{array}$ & & $\begin{array}{c}\text { Volumetric } \\
\text { water } \\
\text { content }\end{array}$ & $\begin{array}{l}\text { Temper- } \\
\text { ature }\end{array}$ & \\
\hline 7/27/2008 11:00 PM & 0.386 & 20.3 & 0.204 & 0.133 & 19.4 & 0.19 & 0.128 & -- & -- & -- & -- & -- & -- \\
\hline 7/28/2008 12:00 AM & 0.386 & 20.3 & 0.204 & 0.133 & 19.4 & 0.19 & 0.128 & -- & -- & -- & -- & -- & -- \\
\hline 7/28/2008 1:00 AM & 0.387 & 20.3 & 0.204 & 0.133 & 19.4 & 0.19 & 0.128 & -- & -- & -- & -- & -- & -- \\
\hline 7/28/2008 2:00 AM & 0.387 & 20.3 & 0.204 & 0.132 & 19.4 & 0.19 & 0.128 & -- & -- & -- & -- & -- & -- \\
\hline 7/28/2008 3:00 AM & 0.387 & 20.3 & 0.204 & 0.133 & 19.4 & 0.19 & 0.128 & -- & -- & -- & -- & -- & -- \\
\hline 7/28/2008 4:00 AM & 0.387 & 20.3 & 0.204 & 0.133 & 19.4 & 0.19 & 0.128 & -- & -- & -- & -- & -- & -- \\
\hline 7/28/2008 5:00 AM & 0.388 & 20.4 & 0.204 & 0.133 & 19.4 & 0.19 & 0.128 & -- & -- & -- & -- & -- & -- \\
\hline 7/28/2008 6:00 AM & 0.387 & 20.4 & 0.204 & 0.132 & 19.4 & 0.19 & 0.128 & -- & -- & -- & -- & -- & -- \\
\hline 7/28/2008 7:00 AM & 0.387 & 20.4 & 0.204 & 0.132 & 19.4 & 0.19 & 0.127 & -- & -- & -- & -- & -- & -- \\
\hline 7/28/2008 8:00 AM & 0.387 & 20.4 & 0.204 & 0.132 & 19.4 & 0.19 & 0.128 & -- & -- & -- & -- & -- & -- \\
\hline 7/28/2008 9:00 AM & 0.387 & 20.4 & 0.204 & 0.132 & 19.4 & 0.19 & 0.128 & -- & -- & -- & -- & -- & -- \\
\hline 7/28/2008 10:00 AM & 0.386 & 20.4 & 0.204 & 0.133 & 19.4 & 0.19 & 0.128 & -- & -- & -- & -- & -- & -- \\
\hline 7/28/2008 11:00 AM & 0.385 & 20.4 & 0.204 & 0.132 & 19.4 & 0.19 & 0.128 & -- & -- & -- & -- & -- & -- \\
\hline 7/28/2008 12:00 PM & 0.385 & 20.4 & 0.204 & 0.133 & 19.4 & 0.19 & 0.128 & -- & -- & -- & -- & -- & -- \\
\hline 7/28/2008 1:00 PM & 0.384 & 20.4 & 0.204 & 0.133 & 19.4 & 0.19 & 0.128 & -- & -- & -- & -- & -- & -- \\
\hline 7/28/2008 2:00 PM & 0.383 & 20.4 & 0.204 & 0.133 & 19.4 & 0.19 & 0.128 & -- & -- & -- & -- & -- & -- \\
\hline 7/28/2008 3:00 PM & 0.384 & 20.3 & 0.203 & 0.133 & 19.4 & 0.19 & 0.128 & -- & -- & -- & -- & -- & -- \\
\hline 7/28/2008 4:00 PM & 0.384 & 20.4 & 0.203 & 0.133 & 19.4 & 0.19 & 0.128 & -- & -- & -- & -- & -- & -- \\
\hline 7/28/2008 5:00 PM & 0.384 & 20.4 & 0.203 & 0.133 & 19.4 & 0.19 & 0.127 & -- & -- & -- & -- & -- & -- \\
\hline 7/28/2008 6:00 PM & 0.384 & 20.3 & 0.203 & 0.133 & 19.4 & 0.19 & 0.127 & -- & -- & -- & -- & -- & -- \\
\hline 7/28/2008 7:00 PM & 0.384 & 20.3 & 0.203 & 0.133 & 19.4 & 0.19 & 0.127 & -- & -- & -- & -- & -- & -- \\
\hline 7/28/2008 8:00 PM & 0.384 & 20.3 & 0.203 & 0.133 & 19.4 & 0.19 & 0.127 & -- & -- & -- & -- & -- & -- \\
\hline 7/28/2008 9:00 PM & 0.384 & 20.3 & 0.203 & 0.133 & 19.4 & 0.19 & 0.127 & -- & -- & -- & -- & -- & -- \\
\hline 7/28/2008 10:00 PM & 0.384 & 20.3 & 0.203 & 0.133 & 19.4 & 0.19 & 0.127 & -- & -- & -- & -- & -- & -- \\
\hline 7/28/2008 11:00 PM & 0.384 & 20.3 & 0.203 & 0.133 & 19.4 & 0.19 & 0.127 & -- & -- & -- & -- & -- & -- \\
\hline 7/29/2008 12:00 AM & 0.384 & 20.4 & 0.203 & 0.133 & 19.4 & 0.19 & 0.127 & -- & -- & -- & -- & -- & -- \\
\hline 7/29/2008 1:00 AM & 0.384 & 20.4 & 0.203 & 0.133 & 19.4 & 0.19 & 0.127 & -- & -- & -- & -- & -- & -- \\
\hline 7/29/2008 2:00 AM & 0.384 & 20.4 & 0.202 & 0.133 & 19.4 & 0.19 & 0.127 & -- & -- & -- & -- & -- & -- \\
\hline 7/29/2008 3:00 AM & 0.384 & 20.4 & 0.202 & 0.133 & 19.4 & 0.19 & 0.127 & -- & -- & -- & -- & -- & -- \\
\hline 7/29/2008 4:00 AM & 0.384 & 20.4 & 0.202 & 0.133 & 19.4 & 0.19 & 0.127 & -- & -- & -- & -- & -- & -- \\
\hline 7/29/2008 5:00 AM & 0.384 & 20.4 & 0.202 & 0.133 & 19.4 & 0.19 & 0.127 & -- & -- & -- & -- & -- & -- \\
\hline 7/29/2008 6:00 AM & 0.384 & 20.4 & 0.202 & 0.132 & 19.4 & 0.19 & 0.127 & -- & -- & -- & -- & -- & -- \\
\hline 7/29/2008 7:00 AM & 0.384 & 20.4 & 0.202 & 0.133 & 19.4 & 0.19 & 0.127 & -- & -- & -- & -- & -- & -- \\
\hline 7/29/2008 8:00 AM & 0.384 & 20.4 & 0.202 & 0.133 & 19.4 & 0.19 & 0.127 & -- & -- & -- & -- & -- & -- \\
\hline 7/29/2008 9:00 AM & 0.384 & 20.4 & 0.202 & 0.132 & 19.4 & 0.19 & 0.127 & -- & -- & -- & -- & -- & -- \\
\hline
\end{tabular}


Appendix 3. Volumetric water content, temperature, and electrical-conductivity data collected at the sprinkler-irrigated site during 2008.-Continued

[Depth in feet below land surface; volumetric water content dimensionless; temperature in degrees Celsius; bulk electrical conductivity in decisiemens per meter; --, no data]

\begin{tabular}{|c|c|c|c|c|c|c|c|c|c|c|c|c|c|}
\hline \multirow[b]{3}{*}{$\begin{array}{l}\text { Measurement } \\
\text { date and time }\end{array}$} & \multicolumn{13}{|c|}{ Depth } \\
\hline & \multicolumn{2}{|c|}{2} & \multirow{2}{*}{$\begin{array}{c}3 \\
\text { Volumetric } \\
\text { water } \\
\text { content }\end{array}$} & \multicolumn{3}{|c|}{4} & \multirow{2}{*}{$\begin{array}{c}\mathbf{5} \\
\text { Volumetric } \\
\text { water } \\
\text { content }\end{array}$} & \multicolumn{2}{|l|}{7} & \multirow{2}{*}{$\begin{array}{c}9 \\
\text { Volumetric } \\
\text { water } \\
\text { content }\end{array}$} & \multicolumn{2}{|c|}{13} & \multirow{2}{*}{$\begin{array}{c}16.5 \\
\text { Volumetric } \\
\text { water } \\
\text { content }\end{array}$} \\
\hline & $\begin{array}{c}\text { Volumetric } \\
\text { water } \\
\text { content }\end{array}$ & $\begin{array}{l}\text { Temper- } \\
\text { ature }\end{array}$ & & $\begin{array}{c}\text { Volumetric } \\
\text { water } \\
\text { content }\end{array}$ & $\begin{array}{l}\text { Temper- } \\
\text { ature }\end{array}$ & $\begin{array}{c}\text { Bulk } \\
\text { electrical } \\
\text { conduct- } \\
\text { ivity }\end{array}$ & & $\begin{array}{c}\text { Volumetric } \\
\text { water } \\
\text { content }\end{array}$ & $\begin{array}{l}\text { Temper- } \\
\text { ature }\end{array}$ & & $\begin{array}{c}\text { Volumetric } \\
\text { water } \\
\text { content }\end{array}$ & $\begin{array}{l}\text { Temper- } \\
\text { ature }\end{array}$ & \\
\hline$\overline{7 / 29 / 2008 \text { 10:00 AM }}$ & 0.383 & 20.4 & 0.202 & 0.133 & 19.4 & 0.19 & 0.127 & $\overline{--}$ & -- & $\overline{--}$ & -- & $\overline{--}$ & $\overline{--}$ \\
\hline 7/29/2008 11:00 AM & 0.383 & 20.4 & 0.202 & 0.133 & 19.4 & 0.19 & 0.127 & -- & -- & -- & -- & -- & -- \\
\hline 7/29/2008 12:00 PM & 0.382 & 20.4 & 0.202 & 0.133 & 19.4 & 0.19 & 0.127 & -- & -- & -- & -- & -- & -- \\
\hline 7/29/2008 1:00 PM & 0.382 & 20.4 & 0.202 & 0.133 & 19.4 & 0.19 & 0.127 & -- & -- & -- & -- & -- & -- \\
\hline 7/29/2008 2:00 PM & 0.382 & 20.4 & 0.202 & 0.133 & 19.4 & 0.19 & 0.127 & -- & -- & -- & -- & -- & -- \\
\hline 7/29/2008 3:00 PM & 0.382 & 20.4 & 0.202 & 0.132 & 19.4 & 0.19 & 0.127 & -- & -- & -- & -- & -- & -- \\
\hline 7/29/2008 4:00 PM & 0.382 & 20.4 & 0.202 & 0.132 & 19.4 & 0.19 & 0.127 & -- & -- & -- & -- & -- & -- \\
\hline 7/29/2008 5:00 PM & 0.382 & 20.3 & 0.202 & 0.133 & 19.4 & 0.19 & 0.127 & -- & -- & -- & -- & -- & -- \\
\hline 7/29/2008 6:00 PM & 0.381 & 20.3 & 0.201 & 0.132 & 19.4 & 0.19 & 0.127 & -- & -- & -- & -- & -- & -- \\
\hline 7/29/2008 7:00 PM & 0.380 & 20.3 & 0.201 & 0.132 & 19.4 & 0.19 & 0.126 & -- & -- & -- & -- & -- & -- \\
\hline 7/29/2008 8:00 PM & 0.379 & 20.3 & 0.201 & 0.132 & 19.4 & 0.19 & 0.126 & -- & -- & -- & -- & -- & -- \\
\hline 7/29/2008 9:00 PM & 0.379 & 20.3 & 0.201 & 0.132 & 19.4 & 0.19 & 0.126 & -- & -- & -- & -- & -- & -- \\
\hline 7/29/2008 10:00 PM & 0.379 & 20.3 & 0.201 & 0.132 & 19.4 & 0.19 & 0.126 & -- & -- & -- & -- & -- & -- \\
\hline 7/29/2008 11:00 PM & 0.379 & 20.3 & 0.201 & 0.132 & 19.4 & 0.19 & 0.126 & -- & -- & -- & -- & -- & -- \\
\hline 7/30/2008 12:00 AM & 0.379 & 20.3 & 0.201 & 0.132 & 19.4 & 0.19 & 0.126 & -- & -- & -- & -- & -- & -- \\
\hline 7/30/2008 1:00 AM & 0.379 & 20.3 & 0.201 & 0.132 & 19.4 & 0.19 & 0.126 & -- & -- & -- & -- & -- & -- \\
\hline 7/30/2008 2:00 AM & 0.379 & 20.3 & 0.201 & 0.132 & 19.4 & 0.19 & 0.126 & -- & -- & -- & -- & -- & -- \\
\hline 7/30/2008 3:00 AM & 0.379 & 20.3 & 0.201 & 0.132 & 19.4 & 0.19 & 0.126 & -- & -- & -- & -- & -- & -- \\
\hline 7/30/2008 4:00 AM & 0.379 & 20.3 & 0.201 & 0.132 & 19.4 & 0.19 & 0.126 & -- & -- & -- & -- & -- & -- \\
\hline 7/30/2008 5:00 AM & 0.379 & 20.3 & 0.201 & 0.132 & 19.4 & 0.19 & 0.126 & -- & -- & -- & -- & -- & -- \\
\hline 7/30/2008 6:00 AM & 0.379 & 20.3 & 0.201 & 0.132 & 19.4 & 0.19 & 0.126 & -- & -- & -- & -- & -- & -- \\
\hline 7/30/2008 7:00 AM & 0.379 & 20.3 & 0.201 & 0.132 & 19.4 & 0.19 & 0.126 & -- & -- & -- & -- & -- & -- \\
\hline 7/30/2008 8:00 AM & 0.379 & 20.3 & 0.201 & 0.132 & 19.4 & 0.19 & 0.126 & -- & -- & -- & -- & -- & -- \\
\hline 7/30/2008 9:00 AM & 0.379 & 20.3 & 0.201 & 0.132 & 19.4 & 0.19 & 0.126 & -- & -- & -- & -- & -- & -- \\
\hline 7/30/2008 10:00 AM & 0.379 & 20.3 & 0.201 & 0.132 & 19.4 & 0.19 & 0.126 & -- & -- & -- & -- & -- & -- \\
\hline 7/30/2008 11:00 AM & 0.379 & 20.3 & 0.201 & 0.132 & 19.4 & 0.19 & 0.126 & -- & -- & -- & -- & -- & -- \\
\hline 7/30/2008 12:00 PM & 0.380 & 20.3 & 0.201 & 0.132 & 19.4 & 0.19 & 0.126 & -- & -- & -- & -- & -- & -- \\
\hline 7/30/2008 1:00 PM & 0.379 & 20.3 & 0.201 & 0.132 & 19.4 & 0.19 & 0.126 & -- & -- & -- & -- & -- & -- \\
\hline 7/30/2008 2:00 PM & 0.378 & 20.3 & 0.201 & 0.132 & 19.4 & 0.19 & 0.126 & -- & -- & -- & -- & -- & -- \\
\hline 7/30/2008 3:00 PM & 0.376 & 20.3 & 0.201 & 0.132 & 19.4 & 0.19 & 0.126 & -- & -- & -- & -- & -- & -- \\
\hline 7/30/2008 4:00 PM & 0.375 & 20.2 & 0.200 & 0.132 & 19.4 & 0.19 & 0.126 & -- & -- & -- & -- & -- & -- \\
\hline 7/30/2008 5:00 PM & 0.376 & 20.2 & 0.200 & 0.132 & 19.4 & 0.19 & 0.126 & -- & -- & -- & -- & -- & -- \\
\hline 7/30/2008 6:00 PM & 0.376 & 20.2 & 0.200 & 0.132 & 19.4 & 0.19 & 0.126 & -- & -- & -- & -- & -- & -- \\
\hline 7/30/2008 7:00 PM & 0.376 & 20.2 & 0.200 & 0.132 & 19.4 & 0.19 & 0.126 & -- & -- & -- & -- & -- & -- \\
\hline 7/30/2008 8:00 PM & 0.375 & 20.2 & 0.200 & 0.132 & 19.4 & 0.19 & 0.126 & -- & -- & -- & -- & -- & -- \\
\hline
\end{tabular}


Appendix 3. Volumetric water content, temperature, and electrical-conductivity data collected at the sprinkler-irrigated site during 2008.-Continued

[Depth in feet below land surface; volumetric water content dimensionless; temperature in degrees Celsius; bulk electrical conductivity in decisiemens per meter; --, no data]

\begin{tabular}{|c|c|c|c|c|c|c|c|c|c|c|c|c|c|}
\hline \multirow[b]{3}{*}{$\begin{array}{l}\text { Measurement } \\
\text { date and time }\end{array}$} & \multicolumn{13}{|c|}{ Depth } \\
\hline & \multicolumn{2}{|c|}{2} & \multirow{2}{*}{$\begin{array}{c}3 \\
\begin{array}{c}\text { Volumetric } \\
\text { water } \\
\text { content }\end{array}\end{array}$} & \multicolumn{3}{|c|}{4} & \multirow{2}{*}{$\begin{array}{c}5 \\
\text { Volumetric } \\
\text { water } \\
\text { content }\end{array}$} & \multicolumn{2}{|l|}{7} & \multirow{2}{*}{$\begin{array}{c}9 \\
\text { Volumetric } \\
\text { water } \\
\text { content }\end{array}$} & \multicolumn{2}{|c|}{13} & \multirow{2}{*}{$\begin{array}{c}16.5 \\
\text { Volumetric } \\
\text { water } \\
\text { content }\end{array}$} \\
\hline & $\begin{array}{c}\text { Volumetric } \\
\text { water } \\
\text { content }\end{array}$ & $\begin{array}{l}\text { Temper- } \\
\text { ature }\end{array}$ & & $\begin{array}{c}\text { Volumetric } \\
\text { water } \\
\text { content }\end{array}$ & $\begin{array}{l}\text { Temper- } \\
\text { ature }\end{array}$ & $\begin{array}{c}\text { Bulk } \\
\text { electrical } \\
\text { conduct- } \\
\text { ivity }\end{array}$ & & $\begin{array}{c}\text { Volumetric } \\
\text { water } \\
\text { content }\end{array}$ & $\begin{array}{l}\text { Temper- } \\
\text { ature }\end{array}$ & & $\begin{array}{c}\text { Volumetric } \\
\text { water } \\
\text { content }\end{array}$ & $\begin{array}{l}\text { Temper- } \\
\text { ature }\end{array}$ & \\
\hline 7/30/2008 9:00 PM & 0.376 & 20.1 & 0.200 & 0.132 & 19.4 & 0.19 & 0.126 & -- & -- & -- & -- & -- & -- \\
\hline 7/30/2008 10:00 PM & 0.375 & 20.1 & 0.200 & 0.132 & 19.4 & 0.19 & 0.126 & -- & -- & -- & -- & -- & -- \\
\hline 7/30/2008 11:00 PM & 0.375 & 20.1 & 0.200 & 0.132 & 19.4 & 0.19 & 0.126 & -- & -- & -- & -- & -- & -- \\
\hline 7/31/2008 12:00 AM & 0.375 & 20.2 & 0.200 & 0.132 & 19.4 & 0.19 & 0.126 & -- & -- & -- & -- & -- & -- \\
\hline 7/31/2008 1:00 AM & 0.375 & 20.2 & 0.200 & 0.131 & 19.4 & 0.19 & 0.126 & -- & -- & -- & -- & -- & -- \\
\hline 7/31/2008 2:00 AM & 0.375 & 20.2 & 0.200 & 0.131 & 19.4 & 0.19 & 0.126 & -- & -- & -- & -- & -- & -- \\
\hline 7/31/2008 3:00 AM & 0.376 & 20.2 & 0.200 & 0.131 & 19.4 & 0.19 & 0.126 & -- & -- & -- & -- & -- & -- \\
\hline 7/31/2008 4:00 AM & 0.375 & 20.2 & 0.200 & 0.131 & 19.4 & 0.19 & 0.126 & -- & -- & -- & -- & -- & -- \\
\hline 7/31/2008 5:00 AM & 0.376 & 20.2 & 0.200 & 0.131 & 19.4 & 0.19 & 0.126 & -- & -- & -- & -- & -- & -- \\
\hline 7/31/2008 6:00 AM & 0.375 & 20.2 & 0.200 & 0.131 & 19.4 & 0.19 & 0.126 & -- & -- & -- & -- & -- & -- \\
\hline 7/31/2008 7:00 AM & 0.376 & 20.2 & 0.200 & 0.131 & 19.4 & 0.19 & 0.126 & -- & -- & -- & -- & -- & -- \\
\hline 7/31/2008 8:00 AM & 0.376 & 20.2 & 0.200 & 0.131 & 19.4 & 0.18 & 0.126 & -- & -- & -- & -- & -- & -- \\
\hline 7/31/2008 9:00 AM & 0.376 & 20.2 & 0.200 & 0.131 & 19.4 & 0.19 & 0.126 & -- & -- & -- & -- & -- & -- \\
\hline 7/31/2008 10:00 AM & 0.376 & 20.2 & 0.200 & 0.131 & 19.4 & 0.18 & 0.126 & -- & -- & -- & -- & -- & -- \\
\hline 7/31/2008 11:00 AM & 0.376 & 20.2 & 0.200 & 0.131 & 19.4 & 0.18 & 0.126 & -- & -- & -- & -- & -- & -- \\
\hline 7/31/2008 12:00 PM & 0.375 & 20.2 & 0.200 & 0.131 & 19.4 & 0.19 & 0.126 & -- & -- & -- & -- & -- & -- \\
\hline 7/31/2008 1:00 PM & 0.374 & 20.2 & 0.200 & 0.131 & 19.4 & 0.19 & 0.126 & -- & -- & -- & -- & -- & -- \\
\hline 7/31/2008 2:00 PM & 0.373 & 20.2 & 0.200 & 0.131 & 19.4 & 0.19 & 0.126 & -- & -- & -- & -- & -- & -- \\
\hline 7/31/2008 3:00 PM & 0.372 & 20.2 & 0.199 & 0.131 & 19.4 & 0.19 & 0.126 & -- & -- & -- & -- & -- & -- \\
\hline 7/31/2008 4:00 PM & 0.371 & 20.1 & 0.199 & 0.131 & 19.4 & 0.19 & 0.126 & -- & -- & -- & -- & -- & -- \\
\hline 7/31/2008 5:00 PM & 0.370 & 20.1 & 0.199 & 0.131 & 19.4 & 0.18 & 0.126 & -- & -- & -- & -- & -- & -- \\
\hline 7/31/2008 6:00 PM & 0.369 & 20.1 & 0.199 & 0.131 & 19.4 & 0.18 & 0.126 & -- & -- & -- & -- & -- & -- \\
\hline 7/31/2008 7:00 PM & 0.368 & 20.1 & 0.198 & 0.131 & 19.4 & 0.18 & 0.126 & -- & -- & -- & -- & -- & -- \\
\hline 7/31/2008 8:00 PM & 0.369 & 20.0 & 0.198 & 0.130 & 19.4 & 0.18 & 0.126 & -- & -- & -- & -- & -- & -- \\
\hline 7/31/2008 9:00 PM & 0.370 & 20.0 & 0.198 & 0.131 & 19.4 & 0.18 & 0.126 & -- & -- & -- & -- & -- & -- \\
\hline 7/31/2008 10:00 PM & 0.370 & 20.0 & 0.198 & 0.131 & 19.4 & 0.18 & 0.126 & -- & -- & -- & -- & -- & -- \\
\hline 7/31/2008 11:00 PM & 0.370 & 20.0 & 0.198 & 0.131 & 19.4 & 0.18 & 0.126 & -- & -- & -- & -- & -- & -- \\
\hline 8/1/2008 12:00 AM & 0.370 & 20.0 & 0.198 & 0.131 & 19.4 & 0.18 & 0.126 & -- & -- & -- & -- & -- & -- \\
\hline 8/1/2008 1:00 AM & 0.370 & 20.1 & 0.198 & 0.131 & 19.4 & 0.18 & 0.126 & -- & -- & -- & -- & -- & -- \\
\hline 8/1/2008 2:00 AM & 0.369 & 20.1 & 0.198 & 0.131 & 19.4 & 0.18 & 0.126 & -- & -- & -- & -- & -- & -- \\
\hline 8/1/2008 3:00 AM & 0.369 & 20.1 & 0.198 & 0.131 & 19.4 & 0.18 & 0.126 & -- & -- & -- & -- & -- & -- \\
\hline 8/1/2008 4:00 AM & 0.369 & 20.1 & 0.198 & 0.131 & 19.4 & 0.18 & 0.126 & -- & -- & -- & -- & -- & -- \\
\hline 8/1/2008 5:00 AM & 0.369 & 20.1 & 0.198 & 0.130 & 19.4 & 0.18 & 0.126 & -- & -- & -- & -- & -- & -- \\
\hline 8/1/2008 6:00 AM & 0.369 & 20.1 & 0.198 & 0.130 & 19.4 & 0.18 & 0.126 & -- & -- & -- & -- & -- & -- \\
\hline 8/1/2008 7:00 AM & 0.369 & 20.1 & 0.198 & 0.130 & 19.4 & 0.18 & 0.126 & -- & -- & -- & -- & -- & -- \\
\hline
\end{tabular}


Appendix 3. Volumetric water content, temperature, and electrical-conductivity data collected at the sprinkler-irrigated site during 2008.-Continued

[Depth in feet below land surface; volumetric water content dimensionless; temperature in degrees Celsius; bulk electrical conductivity in decisiemens per meter; --, no data]

\begin{tabular}{|c|c|c|c|c|c|c|c|c|c|c|c|c|c|}
\hline \multirow[b]{3}{*}{$\begin{array}{l}\text { Measurement } \\
\text { date and time }\end{array}$} & \multirow{2}{*}{\multicolumn{2}{|c|}{2}} & \multicolumn{10}{|c|}{ Depth } & \multirow{3}{*}{$\begin{array}{c}16.5 \\
\text { Volumetric } \\
\text { water } \\
\text { content }\end{array}$} \\
\hline & & & \multirow[b]{2}{*}{$\begin{array}{c}3 \\
\begin{array}{c}\text { Volumetric } \\
\text { water } \\
\text { content }\end{array}\end{array}$} & \multicolumn{3}{|c|}{4} & \multirow[b]{2}{*}{$\begin{array}{c}\mathbf{5} \\
\text { Volumetric } \\
\text { water } \\
\text { content }\end{array}$} & \multicolumn{2}{|c|}{7} & \multirow{2}{*}{$\begin{array}{c}9 \\
\text { Volumetric } \\
\text { water } \\
\text { content }\end{array}$} & \multicolumn{2}{|c|}{13} & \\
\hline & $\begin{array}{c}\text { Volumetric } \\
\text { water } \\
\text { content }\end{array}$ & $\begin{array}{l}\text { Temper- } \\
\text { ature }\end{array}$ & & $\begin{array}{c}\text { Volumetric } \\
\text { water } \\
\text { content }\end{array}$ & $\begin{array}{l}\text { Temper- } \\
\text { ature }\end{array}$ & $\begin{array}{c}\text { Bulk } \\
\text { electrical } \\
\text { conduct- } \\
\text { ivity }\end{array}$ & & $\begin{array}{c}\text { Volumetric } \\
\text { water } \\
\text { content }\end{array}$ & $\begin{array}{l}\text { Temper- } \\
\text { ature }\end{array}$ & & $\begin{array}{c}\text { Volumetric } \\
\text { water } \\
\text { content }\end{array}$ & $\begin{array}{l}\text { Temper- } \\
\text { ature }\end{array}$ & \\
\hline 8/1/2008 8:00 AM & 0.369 & 20.1 & 0.198 & 0.130 & 19.4 & 0.18 & 0.126 & -- & -- & -- & -- & -- & -- \\
\hline 8/1/2008 9:00 AM & 0.369 & 20.1 & 0.198 & 0.130 & 19.4 & 0.18 & 0.126 & -- & -- & -- & -- & -- & -- \\
\hline 8/1/2008 10:00 AM & 0.370 & 20.1 & 0.198 & 0.130 & 19.4 & 0.18 & 0.126 & -- & -- & -- & -- & -- & -- \\
\hline 8/1/2008 11:00 AM & 0.370 & 20.2 & 0.198 & 0.130 & 19.4 & 0.18 & 0.126 & -- & -- & -- & -- & -- & -- \\
\hline 8/1/2008 12:00 PM & 0.370 & 20.2 & 0.198 & 0.130 & 19.4 & 0.18 & 0.126 & -- & -- & -- & -- & -- & -- \\
\hline 8/1/2008 1:00 PM & 0.370 & 20.1 & 0.198 & 0.130 & 19.4 & 0.18 & 0.126 & -- & -- & -- & -- & -- & -- \\
\hline 8/1/2008 2:00 PM & 0.370 & 20.1 & 0.198 & 0.130 & 19.4 & 0.18 & 0.126 & -- & -- & -- & -- & -- & -- \\
\hline 8/1/2008 3:00 PM & 0.369 & 20.1 & 0.198 & 0.130 & 19.4 & 0.18 & 0.126 & -- & -- & -- & -- & -- & -- \\
\hline 8/1/2008 4:00 PM & 0.368 & 20.1 & 0.198 & 0.130 & 19.4 & 0.18 & 0.126 & -- & -- & -- & -- & -- & -- \\
\hline 8/1/2008 5:00 PM & 0.367 & 20.1 & 0.198 & 0.130 & 19.3 & 0.18 & 0.126 & -- & -- & -- & -- & -- & -- \\
\hline 8/1/2008 6:00 PM & 0.367 & 20.1 & 0.197 & 0.130 & 19.4 & 0.18 & 0.126 & -- & -- & -- & -- & -- & -- \\
\hline 8/1/2008 7:00 PM & 0.367 & 20.1 & 0.197 & 0.130 & 19.3 & 0.18 & 0.126 & -- & -- & -- & -- & -- & -- \\
\hline 8/1/2008 8:00 PM & 0.366 & 20.1 & 0.197 & 0.130 & 19.3 & 0.18 & 0.125 & -- & -- & -- & -- & -- & -- \\
\hline 8/1/2008 9:00 PM & 0.366 & 20.1 & 0.197 & 0.130 & 19.4 & 0.18 & 0.125 & -- & -- & -- & -- & -- & -- \\
\hline 8/1/2008 10:00 PM & 0.366 & 20.1 & 0.197 & 0.130 & 19.4 & 0.18 & 0.125 & -- & -- & -- & -- & -- & -- \\
\hline 8/1/2008 11:00 PM & 0.366 & 20.1 & 0.197 & 0.130 & 19.4 & 0.18 & 0.125 & -- & -- & -- & -- & -- & -- \\
\hline 8/2/2008 12:00 AM & 0.366 & 20.1 & 0.197 & 0.130 & 19.4 & 0.18 & 0.125 & -- & -- & -- & -- & -- & -- \\
\hline 8/2/2008 1:00 AM & 0.367 & 20.1 & 0.197 & 0.130 & 19.3 & 0.18 & 0.125 & -- & -- & -- & -- & -- & -- \\
\hline 8/2/2008 2:00 AM & 0.367 & 20.2 & 0.197 & 0.130 & 19.4 & 0.18 & 0.125 & -- & -- & -- & -- & -- & -- \\
\hline 8/2/2008 3:00 AM & 0.368 & 20.2 & 0.197 & 0.130 & 19.3 & 0.18 & 0.125 & -- & -- & -- & -- & -- & -- \\
\hline 8/2/2008 4:00 AM & 0.368 & 20.2 & 0.197 & 0.130 & 19.4 & 0.18 & 0.125 & -- & -- & -- & -- & -- & -- \\
\hline 8/2/2008 5:00 AM & 0.368 & 20.2 & 0.196 & 0.130 & 19.4 & 0.18 & 0.125 & -- & -- & -- & -- & -- & -- \\
\hline 8/2/2008 6:00 AM & 0.369 & 20.2 & 0.196 & 0.129 & 19.3 & 0.18 & 0.125 & -- & -- & -- & -- & -- & -- \\
\hline 8/2/2008 7:00 AM & 0.369 & 20.2 & 0.196 & 0.130 & 19.3 & 0.18 & 0.125 & -- & -- & -- & -- & -- & -- \\
\hline 8/2/2008 8:00 AM & 0.369 & 20.3 & 0.196 & 0.130 & 19.4 & 0.18 & 0.125 & -- & -- & -- & -- & -- & -- \\
\hline 8/2/2008 9:00 AM & 0.369 & 20.2 & 0.196 & 0.130 & 19.3 & 0.18 & 0.125 & -- & -- & -- & -- & -- & -- \\
\hline 8/2/2008 10:00 AM & 0.368 & 20.2 & 0.196 & 0.130 & 19.3 & 0.18 & 0.125 & -- & -- & -- & -- & -- & -- \\
\hline 8/2/2008 11:00 AM & 0.367 & 20.2 & 0.196 & 0.130 & 19.3 & 0.18 & 0.125 & -- & -- & -- & -- & -- & -- \\
\hline 8/2/2008 12:00 PM & 0.366 & 20.3 & 0.197 & 0.130 & 19.3 & 0.18 & 0.125 & -- & -- & -- & -- & -- & -- \\
\hline 8/2/2008 1:00 PM & 0.366 & 20.3 & 0.197 & 0.130 & 19.3 & 0.18 & 0.125 & -- & -- & -- & -- & -- & -- \\
\hline 8/2/2008 2:00 PM & 0.366 & 20.2 & 0.196 & 0.129 & 19.3 & 0.18 & 0.125 & -- & -- & -- & -- & -- & -- \\
\hline 8/2/2008 3:00 PM & 0.366 & 20.2 & 0.196 & 0.129 & 19.3 & 0.18 & 0.125 & -- & -- & -- & -- & -- & -- \\
\hline 8/2/2008 4:00 PM & 0.366 & 20.2 & 0.196 & 0.130 & 19.3 & 0.18 & 0.125 & -- & -- & -- & -- & -- & -- \\
\hline 8/2/2008 5:00 PM & 0.366 & 20.2 & 0.196 & 0.129 & 19.3 & 0.18 & 0.125 & -- & -- & -- & -- & -- & -- \\
\hline 8/2/2008 6:00 PM & 0.366 & 20.2 & 0.196 & 0.129 & 19.3 & 0.18 & 0.125 & -- & -- & -- & -- & -- & -- \\
\hline
\end{tabular}


Appendix 3. Volumetric water content, temperature, and electrical-conductivity data collected at the sprinkler-irrigated site during 2008.-Continued

[Depth in feet below land surface; volumetric water content dimensionless; temperature in degrees Celsius; bulk electrical conductivity in decisiemens per meter; --, no data]

\begin{tabular}{|c|c|c|c|c|c|c|c|c|c|c|c|c|c|}
\hline \multirow[b]{3}{*}{$\begin{array}{l}\text { Measurement } \\
\text { date and time }\end{array}$} & \multicolumn{13}{|c|}{ Depth } \\
\hline & \multicolumn{2}{|c|}{2} & \multirow{2}{*}{$\begin{array}{c}3 \\
\text { Volumetric } \\
\text { water } \\
\text { content }\end{array}$} & \multicolumn{3}{|c|}{4} & \multirow{2}{*}{$\begin{array}{c}5 \\
\text { Volumetric } \\
\text { water } \\
\text { content }\end{array}$} & \multicolumn{2}{|l|}{7} & \multirow{2}{*}{$\begin{array}{c}9 \\
\text { Volumetric } \\
\text { water } \\
\text { content }\end{array}$} & \multicolumn{2}{|c|}{13} & \multirow{2}{*}{$\begin{array}{c}16.5 \\
\text { Volumetric } \\
\text { water } \\
\text { content }\end{array}$} \\
\hline & $\begin{array}{c}\text { Volumetric } \\
\text { water } \\
\text { content }\end{array}$ & $\begin{array}{l}\text { Temper- } \\
\text { ature }\end{array}$ & & $\begin{array}{c}\text { Volumetric } \\
\text { water } \\
\text { content }\end{array}$ & $\begin{array}{l}\text { Temper- } \\
\text { ature }\end{array}$ & $\begin{array}{c}\text { Bulk } \\
\text { electrical } \\
\text { conduct- } \\
\text { ivity }\end{array}$ & & $\begin{array}{c}\text { Volumetric } \\
\text { water } \\
\text { content }\end{array}$ & $\begin{array}{l}\text { Temper- } \\
\text { ature }\end{array}$ & & $\begin{array}{c}\text { Volumetric } \\
\text { water } \\
\text { content }\end{array}$ & $\begin{array}{l}\text { Temper- } \\
\text { ature }\end{array}$ & \\
\hline 8/2/2008 7:00 PM & 0.366 & 20.2 & 0.196 & 0.129 & 19.3 & 0.18 & 0.125 & -- & -- & -- & -- & -- & -- \\
\hline 8/2/2008 8:00 PM & 0.365 & 20.1 & 0.195 & 0.129 & 19.3 & 0.18 & 0.125 & -- & -- & -- & -- & -- & -- \\
\hline 8/2/2008 9:00 PM & 0.365 & 20.2 & 0.196 & 0.129 & 19.3 & 0.18 & 0.125 & -- & -- & -- & -- & -- & -- \\
\hline 8/2/2008 10:00 PM & 0.365 & 20.1 & 0.195 & 0.129 & 19.3 & 0.18 & 0.124 & -- & -- & -- & -- & -- & -- \\
\hline 8/2/2008 11:00 PM & 0.365 & 20.1 & 0.195 & 0.129 & 19.3 & 0.18 & 0.124 & -- & -- & -- & -- & -- & -- \\
\hline 8/3/2008 12:00 AM & 0.365 & 20.2 & 0.195 & 0.129 & 19.4 & 0.18 & 0.124 & -- & -- & -- & -- & -- & -- \\
\hline 8/3/2008 1:00 AM & 0.365 & 20.2 & 0.195 & 0.129 & 19.3 & 0.18 & 0.124 & -- & -- & -- & -- & -- & -- \\
\hline 8/3/2008 2:00 AM & 0.365 & 20.2 & 0.195 & 0.129 & 19.3 & 0.18 & 0.124 & -- & -- & -- & -- & -- & -- \\
\hline 8/3/2008 3:00 AM & 0.366 & 20.2 & 0.195 & 0.129 & 19.3 & 0.18 & 0.124 & -- & -- & -- & -- & -- & -- \\
\hline 8/3/2008 4:00 AM & 0.366 & 20.2 & 0.195 & 0.130 & 19.3 & 0.18 & 0.124 & -- & -- & -- & -- & -- & -- \\
\hline 8/3/2008 5:00 AM & 0.366 & 20.2 & 0.195 & 0.129 & 19.3 & 0.18 & 0.124 & -- & -- & -- & -- & -- & -- \\
\hline 8/3/2008 6:00 AM & 0.366 & 20.2 & 0.195 & 0.129 & 19.3 & 0.18 & 0.124 & -- & -- & -- & -- & -- & -- \\
\hline 8/3/2008 7:00 AM & 0.366 & 20.2 & 0.195 & 0.129 & 19.3 & 0.18 & 0.124 & -- & -- & -- & -- & -- & -- \\
\hline 8/3/2008 8:00 AM & 0.367 & 20.2 & 0.195 & 0.129 & 19.3 & 0.18 & 0.124 & -- & -- & -- & -- & -- & -- \\
\hline 8/3/2008 9:00 AM & 0.367 & 20.2 & 0.195 & 0.129 & 19.3 & 0.18 & 0.124 & -- & -- & -- & -- & -- & -- \\
\hline 8/3/2008 10:00 AM & 0.367 & 20.2 & 0.195 & 0.129 & 19.3 & 0.18 & 0.124 & -- & -- & -- & -- & -- & -- \\
\hline 8/3/2008 11:00 AM & 0.367 & 20.2 & 0.195 & 0.129 & 19.3 & 0.18 & 0.124 & -- & -- & -- & -- & -- & -- \\
\hline 8/3/2008 12:00 PM & 0.367 & 20.2 & 0.195 & 0.129 & 19.3 & 0.18 & 0.124 & -- & -- & -- & -- & -- & -- \\
\hline 8/3/2008 1:00 PM & 0.367 & 20.2 & 0.195 & 0.129 & 19.3 & 0.18 & 0.124 & -- & -- & -- & -- & -- & -- \\
\hline 8/3/2008 2:00 PM & 0.367 & 20.1 & 0.195 & 0.129 & 19.3 & 0.18 & 0.124 & -- & -- & -- & -- & -- & -- \\
\hline 8/3/2008 3:00 PM & 0.366 & 20.1 & 0.195 & 0.129 & 19.4 & 0.18 & 0.124 & -- & -- & -- & -- & -- & -- \\
\hline 8/3/2008 4:00 PM & 0.366 & 20.1 & 0.195 & 0.128 & 19.3 & 0.18 & 0.124 & -- & -- & -- & -- & -- & -- \\
\hline 8/3/2008 5:00 PM & 0.366 & 20.1 & 0.195 & 0.129 & 19.3 & 0.18 & 0.124 & -- & -- & -- & -- & -- & -- \\
\hline 8/3/2008 6:00 PM & 0.365 & 20.1 & 0.195 & 0.129 & 19.3 & 0.18 & 0.124 & -- & -- & -- & -- & -- & -- \\
\hline 8/3/2008 7:00 PM & 0.366 & 20.1 & 0.195 & 0.128 & 19.3 & 0.18 & 0.124 & -- & -- & -- & -- & -- & -- \\
\hline 8/3/2008 8:00 PM & 0.367 & 20.0 & 0.195 & 0.128 & 19.4 & 0.18 & 0.124 & -- & -- & -- & -- & -- & -- \\
\hline 8/3/2008 9:00 PM & 0.368 & 20.0 & 0.195 & 0.128 & 19.3 & 0.18 & 0.124 & -- & -- & -- & -- & -- & -- \\
\hline 8/3/2008 10:00 PM & 0.368 & 20.0 & 0.195 & 0.128 & 19.3 & 0.18 & 0.124 & -- & -- & -- & -- & -- & -- \\
\hline 8/3/2008 11:00 PM & 0.369 & 20.1 & 0.195 & 0.128 & 19.3 & 0.18 & 0.124 & -- & -- & -- & -- & -- & -- \\
\hline 8/4/2008 12:00 AM & 0.369 & 20.1 & 0.195 & 0.128 & 19.3 & 0.18 & 0.124 & -- & -- & -- & -- & -- & -- \\
\hline 8/4/2008 1:00 AM & 0.370 & 20.1 & 0.195 & 0.128 & 19.3 & 0.18 & 0.124 & -- & -- & -- & -- & -- & -- \\
\hline 8/4/2008 2:00 AM & 0.370 & 20.1 & 0.195 & 0.128 & 19.3 & 0.18 & 0.124 & -- & -- & -- & -- & -- & -- \\
\hline 8/4/2008 3:00 AM & 0.370 & 20.1 & 0.195 & 0.128 & 19.3 & 0.18 & 0.124 & -- & -- & -- & -- & -- & -- \\
\hline 8/4/2008 4:00 AM & 0.370 & 20.1 & 0.195 & 0.128 & 19.3 & 0.18 & 0.124 & -- & -- & -- & -- & -- & -- \\
\hline 8/4/2008 5:00 AM & 0.370 & 20.1 & 0.195 & 0.128 & 19.3 & 0.18 & 0.124 & -- & -- & -- & -- & -- & -- \\
\hline
\end{tabular}


Appendix 3. Volumetric water content, temperature, and electrical-conductivity data collected at the sprinkler-irrigated site during 2008.-Continued

[Depth in feet below land surface; volumetric water content dimensionless; temperature in degrees Celsius; bulk electrical conductivity in decisiemens per meter; --, no data]

\begin{tabular}{|c|c|c|c|c|c|c|c|c|c|c|c|c|c|}
\hline \multirow[b]{3}{*}{$\begin{array}{l}\text { Measurement } \\
\text { date and time }\end{array}$} & \multirow{2}{*}{\multicolumn{2}{|c|}{$\begin{array}{r} \\
2\end{array}$}} & \multirow[b]{3}{*}{$\begin{array}{c}3 \\
\text { Volumetric } \\
\text { water } \\
\text { content }\end{array}$} & \multicolumn{9}{|c|}{ Depth } & \multirow{3}{*}{$\begin{array}{c}16.5 \\
\text { Volumetric } \\
\text { water } \\
\text { content }\end{array}$} \\
\hline & & & & \multicolumn{3}{|c|}{4} & \multirow[b]{2}{*}{$\begin{array}{c}\mathbf{5} \\
\text { Volumetric } \\
\text { water } \\
\text { content }\end{array}$} & \multicolumn{2}{|l|}{7} & \multirow{2}{*}{$\begin{array}{c}9 \\
\text { Volumetric } \\
\text { water } \\
\text { content }\end{array}$} & \multicolumn{2}{|c|}{13} & \\
\hline & $\begin{array}{c}\text { Volumetric } \\
\text { water } \\
\text { content }\end{array}$ & $\begin{array}{l}\text { Temper- } \\
\text { ature }\end{array}$ & & $\begin{array}{c}\text { Volumetric } \\
\text { water } \\
\text { content }\end{array}$ & $\begin{array}{l}\text { Temper- } \\
\text { ature }\end{array}$ & $\begin{array}{c}\text { Bulk } \\
\text { electrical } \\
\text { conduct- } \\
\text { ivity }\end{array}$ & & $\begin{array}{c}\text { Volumetric } \\
\text { water } \\
\text { content }\end{array}$ & $\begin{array}{l}\text { Temper- } \\
\text { ature }\end{array}$ & & $\begin{array}{c}\text { Volumetric } \\
\text { water } \\
\text { content }\end{array}$ & $\begin{array}{l}\text { Temper- } \\
\text { ature }\end{array}$ & \\
\hline 8/4/2008 6:00 AM & 0.371 & 20.1 & 0.195 & 0.128 & 19.3 & 0.18 & 0.123 & -- & -- & -- & -- & -- & -- \\
\hline 8/4/2008 7:00 AM & 0.370 & 20.1 & 0.195 & 0.128 & 19.3 & 0.18 & 0.123 & -- & -- & -- & -- & -- & -- \\
\hline 8/4/2008 8:00 AM & 0.371 & 20.1 & 0.195 & 0.128 & 19.3 & 0.18 & 0.123 & -- & -- & -- & -- & -- & -- \\
\hline 8/4/2008 9:00 AM & 0.370 & 20.1 & 0.195 & 0.128 & 19.3 & 0.18 & 0.124 & -- & -- & -- & -- & -- & -- \\
\hline 8/4/2008 10:00 AM & 0.370 & 20.1 & 0.195 & 0.128 & 19.3 & 0.18 & 0.124 & -- & -- & -- & -- & -- & -- \\
\hline 8/4/2008 11:00 AM & 0.370 & 20.0 & 0.195 & 0.128 & 19.3 & 0.18 & 0.124 & -- & -- & -- & -- & -- & -- \\
\hline 8/4/2008 12:00 PM & 0.369 & 20.0 & 0.195 & 0.128 & 19.3 & 0.18 & 0.124 & -- & -- & -- & -- & -- & -- \\
\hline 8/4/2008 1:00 PM & 0.369 & 20.0 & 0.195 & 0.128 & 19.3 & 0.18 & 0.124 & -- & -- & -- & -- & -- & -- \\
\hline 8/4/2008 2:00 PM & 0.369 & 20.0 & 0.196 & 0.128 & 19.3 & 0.18 & 0.124 & -- & -- & -- & -- & -- & -- \\
\hline 8/4/2008 3:00 PM & 0.369 & 20.0 & 0.196 & 0.128 & 19.3 & 0.18 & 0.124 & -- & -- & -- & -- & -- & -- \\
\hline 8/4/2008 4:00 PM & 0.370 & 20.0 & 0.196 & 0.128 & 19.3 & 0.18 & 0.123 & -- & -- & -- & -- & -- & -- \\
\hline 8/4/2008 5:00 PM & 0.369 & 20.0 & 0.195 & 0.128 & 19.3 & 0.18 & 0.123 & -- & -- & -- & -- & -- & -- \\
\hline 8/4/2008 6:00 PM & 0.369 & 19.9 & 0.195 & 0.128 & 19.3 & 0.18 & 0.123 & -- & -- & -- & -- & -- & -- \\
\hline 8/4/2008 7:00 PM & 0.370 & 19.9 & 0.195 & 0.128 & 19.3 & 0.18 & 0.123 & -- & -- & -- & -- & -- & -- \\
\hline 8/4/2008 8:00 PM & 0.369 & 19.9 & 0.195 & 0.128 & 19.3 & 0.18 & 0.123 & -- & -- & -- & -- & -- & -- \\
\hline 8/4/2008 9:00 PM & 0.369 & 19.9 & 0.195 & 0.128 & 19.3 & 0.18 & 0.123 & -- & -- & -- & -- & -- & -- \\
\hline 8/4/2008 10:00 PM & 0.368 & 19.9 & 0.195 & 0.128 & 19.3 & 0.18 & 0.123 & -- & -- & -- & -- & -- & -- \\
\hline 8/4/2008 11:00 PM & 0.368 & 19.9 & 0.195 & 0.128 & 19.3 & 0.18 & 0.123 & -- & -- & -- & -- & -- & -- \\
\hline 8/5/2008 12:00 AM & 0.368 & 19.9 & 0.195 & 0.128 & 19.3 & 0.18 & 0.123 & -- & -- & -- & -- & -- & -- \\
\hline 8/5/2008 1:00 AM & 0.368 & 19.9 & 0.195 & 0.128 & 19.3 & 0.18 & 0.123 & -- & -- & -- & -- & -- & -- \\
\hline 8/5/2008 2:00 AM & 0.368 & 19.9 & 0.195 & 0.128 & 19.3 & 0.18 & 0.123 & -- & -- & -- & -- & -- & -- \\
\hline 8/5/2008 3:00 AM & 0.369 & 19.9 & 0.195 & 0.126 & 19.3 & 0.18 & 0.123 & -- & -- & -- & -- & -- & -- \\
\hline 8/5/2008 4:00 AM & 0.369 & 19.9 & 0.195 & 0.126 & 19.3 & 0.18 & 0.123 & -- & -- & -- & -- & -- & -- \\
\hline 8/5/2008 5:00 AM & 0.369 & 20.0 & 0.195 & 0.128 & 19.3 & 0.18 & 0.123 & -- & -- & -- & -- & -- & -- \\
\hline 8/5/2008 6:00 AM & 0.369 & 19.9 & 0.195 & 0.128 & 19.3 & 0.18 & 0.123 & -- & -- & -- & -- & -- & -- \\
\hline 8/5/2008 7:00 AM & 0.369 & 20.0 & 0.195 & 0.128 & 19.3 & 0.18 & 0.123 & -- & -- & -- & -- & -- & -- \\
\hline 8/5/2008 8:00 AM & 0.369 & 20.0 & 0.195 & 0.128 & 19.3 & 0.18 & 0.123 & -- & -- & -- & -- & -- & -- \\
\hline 8/5/2008 9:00 AM & 0.369 & 19.9 & 0.195 & 0.126 & 19.3 & 0.18 & 0.123 & -- & -- & -- & -- & -- & -- \\
\hline 8/5/2008 10:00 AM & 0.370 & 19.9 & 0.195 & 0.126 & 19.3 & 0.18 & 0.123 & -- & -- & -- & -- & -- & -- \\
\hline 8/5/2008 11:00 AM & 0.369 & 19.9 & 0.195 & 0.128 & 19.3 & 0.18 & 0.123 & -- & -- & -- & -- & -- & -- \\
\hline 8/5/2008 12:00 PM & 0.369 & 19.9 & 0.195 & 0.126 & 19.3 & 0.18 & 0.123 & -- & -- & -- & -- & -- & -- \\
\hline 8/5/2008 1:00 PM & 0.369 & 19.9 & 0.195 & 0.126 & 19.3 & 0.18 & 0.123 & -- & -- & -- & -- & -- & -- \\
\hline 8/5/2008 2:00 PM & 0.368 & 19.9 & 0.195 & 0.126 & 19.3 & 0.18 & 0.123 & -- & -- & -- & -- & -- & -- \\
\hline 8/5/2008 3:00 PM & 0.368 & 19.9 & 0.195 & 0.126 & 19.3 & 0.18 & 0.123 & -- & -- & -- & -- & -- & -- \\
\hline 8/5/2008 4:00 PM & 0.367 & 19.9 & 0.195 & 0.126 & 19.3 & 0.18 & 0.123 & -- & -- & -- & -- & -- & -- \\
\hline
\end{tabular}


Appendix 3. Volumetric water content, temperature, and electrical-conductivity data collected at the sprinkler-irrigated site during 2008.-Continued

[Depth in feet below land surface; volumetric water content dimensionless; temperature in degrees Celsius; bulk electrical conductivity in decisiemens per meter; --, no data]

\begin{tabular}{|c|c|c|c|c|c|c|c|c|c|c|c|c|c|}
\hline \multirow[b]{3}{*}{$\begin{array}{l}\text { Measurement } \\
\text { date and time }\end{array}$} & \multicolumn{13}{|c|}{ Depth } \\
\hline & \multicolumn{2}{|c|}{2} & \multirow{2}{*}{$\begin{array}{c}3 \\
\text { Volumetric } \\
\text { water } \\
\text { content }\end{array}$} & \multicolumn{3}{|c|}{4} & \multirow{2}{*}{$\begin{array}{c}5 \\
\text { Volumetric } \\
\text { water } \\
\text { content }\end{array}$} & \multicolumn{2}{|l|}{7} & \multirow{2}{*}{$\begin{array}{c}9 \\
\text { Volumetric } \\
\text { water } \\
\text { content }\end{array}$} & \multicolumn{2}{|c|}{13} & \multirow{2}{*}{$\begin{array}{c}16.5 \\
\text { Volumetric } \\
\text { water } \\
\text { content }\end{array}$} \\
\hline & $\begin{array}{c}\text { Volumetric } \\
\text { water } \\
\text { content }\end{array}$ & $\begin{array}{l}\text { Temper- } \\
\text { ature }\end{array}$ & & $\begin{array}{c}\text { Volumetric } \\
\text { water } \\
\text { content }\end{array}$ & $\begin{array}{l}\text { Temper- } \\
\text { ature }\end{array}$ & $\begin{array}{c}\text { Bulk } \\
\text { electrical } \\
\text { conduct- } \\
\text { ivity }\end{array}$ & & $\begin{array}{c}\text { Volumetric } \\
\text { water } \\
\text { content }\end{array}$ & $\begin{array}{l}\text { Temper- } \\
\text { ature }\end{array}$ & & $\begin{array}{c}\text { Volumetric } \\
\text { water } \\
\text { content }\end{array}$ & $\begin{array}{l}\text { Temper- } \\
\text { ature }\end{array}$ & \\
\hline 8/5/2008 5:00 PM & 0.367 & 19.9 & 0.195 & 0.126 & 19.3 & 0.18 & 0.123 & -- & -- & -- & -- & -- & -- \\
\hline 8/5/2008 6:00 PM & 0.366 & 19.9 & 0.195 & 0.126 & 19.3 & 0.18 & 0.123 & -- & -- & -- & -- & -- & -- \\
\hline 8/5/2008 7:00 PM & 0.366 & 19.8 & 0.195 & 0.126 & 19.3 & 0.18 & 0.123 & -- & -- & -- & -- & -- & -- \\
\hline 8/5/2008 8:00 PM & 0.366 & 19.8 & 0.195 & 0.126 & 19.3 & 0.18 & 0.123 & -- & -- & -- & -- & -- & -- \\
\hline 8/5/2008 9:00 PM & 0.365 & 19.8 & 0.195 & 0.126 & 19.3 & 0.18 & 0.123 & -- & -- & -- & -- & -- & -- \\
\hline 8/5/2008 10:00 PM & 0.366 & 19.9 & 0.195 & 0.126 & 19.3 & 0.18 & 0.123 & -- & -- & -- & -- & -- & -- \\
\hline 8/5/2008 11:00 PM & 0.366 & 19.9 & 0.194 & 0.126 & 19.3 & 0.18 & 0.123 & -- & -- & -- & -- & -- & -- \\
\hline 8/6/2008 12:00 AM & 0.366 & 19.9 & 0.194 & 0.126 & 19.3 & 0.18 & 0.123 & -- & -- & -- & -- & -- & -- \\
\hline 8/6/2008 1:00 AM & 0.366 & 19.9 & 0.194 & 0.126 & 19.3 & 0.18 & 0.123 & -- & -- & -- & -- & -- & -- \\
\hline 8/6/2008 2:00 AM & 0.366 & 19.9 & 0.194 & 0.126 & 19.3 & 0.18 & 0.123 & -- & -- & -- & -- & -- & -- \\
\hline 8/6/2008 3:00 AM & 0.366 & 19.9 & 0.194 & 0.126 & 19.3 & 0.18 & 0.123 & -- & -- & -- & -- & -- & -- \\
\hline 8/6/2008 4:00 AM & 0.366 & 19.9 & 0.194 & 0.126 & 19.3 & 0.18 & 0.123 & -- & -- & -- & -- & -- & -- \\
\hline 8/6/2008 5:00 AM & 0.367 & 20.0 & 0.194 & 0.126 & 19.3 & 0.18 & 0.123 & -- & -- & -- & -- & -- & -- \\
\hline 8/6/2008 6:00 AM & 0.367 & 20.0 & 0.194 & 0.126 & 19.3 & 0.18 & 0.123 & -- & -- & -- & -- & -- & -- \\
\hline 8/6/2008 7:00 AM & 0.367 & 20.0 & 0.194 & 0.126 & 19.3 & 0.18 & 0.123 & -- & -- & -- & -- & -- & -- \\
\hline 8/6/2008 8:00 AM & 0.366 & 20.0 & 0.194 & 0.126 & 19.3 & 0.18 & 0.123 & -- & -- & -- & -- & -- & -- \\
\hline 8/6/2008 9:00 AM & 0.367 & 20.0 & 0.194 & 0.126 & 19.3 & 0.18 & 0.123 & -- & -- & -- & -- & -- & -- \\
\hline 8/6/2008 10:00 AM & 0.367 & 19.9 & 0.194 & 0.126 & 19.3 & 0.18 & 0.123 & -- & -- & -- & -- & -- & -- \\
\hline 8/6/2008 11:00 AM & 0.367 & 19.9 & 0.194 & 0.126 & 19.3 & 0.18 & 0.123 & -- & -- & -- & -- & -- & -- \\
\hline 8/6/2008 12:00 PM & 0.367 & 19.9 & 0.194 & 0.126 & 19.3 & 0.18 & 0.123 & -- & -- & -- & -- & -- & -- \\
\hline 8/6/2008 1:00 PM & 0.366 & 19.9 & 0.194 & 0.126 & 19.3 & 0.18 & 0.123 & -- & -- & -- & -- & -- & -- \\
\hline 8/6/2008 2:00 PM & 0.366 & 19.9 & 0.195 & 0.125 & 19.3 & 0.18 & 0.123 & -- & -- & -- & -- & -- & -- \\
\hline 8/6/2008 3:00 PM & 0.366 & 19.9 & 0.195 & 0.126 & 19.3 & 0.18 & 0.123 & -- & -- & -- & -- & -- & -- \\
\hline 8/6/2008 4:00 PM & 0.365 & 19.8 & 0.194 & 0.126 & 19.3 & 0.18 & 0.123 & -- & -- & -- & -- & -- & -- \\
\hline 8/6/2008 5:00 PM & 0.365 & 19.9 & 0.194 & 0.126 & 19.3 & 0.17 & 0.123 & -- & -- & -- & -- & -- & -- \\
\hline 8/6/2008 6:00 PM & 0.365 & 19.8 & 0.194 & 0.126 & 19.3 & 0.17 & 0.123 & -- & -- & -- & -- & -- & -- \\
\hline 8/6/2008 7:00 PM & 0.365 & 19.9 & 0.194 & 0.126 & 19.3 & 0.17 & 0.123 & -- & -- & -- & -- & -- & -- \\
\hline 8/6/2008 8:00 PM & 0.449 & 20.0 & 0.225 & 0.126 & 19.3 & 0.17 & 0.123 & -- & -- & -- & -- & -- & -- \\
\hline 8/6/2008 9:00 PM & 0.486 & 20.2 & 0.394 & 0.126 & 19.3 & 0.17 & 0.123 & -- & -- & -- & -- & -- & -- \\
\hline 8/6/2008 10:00 PM & 0.488 & 20.2 & 0.405 & 0.136 & 19.3 & 0.20 & 0.123 & -- & -- & -- & -- & -- & -- \\
\hline 8/6/2008 11:00 PM & 0.488 & 20.3 & 0.411 & 0.198 & 19.3 & 0.33 & 0.124 & -- & -- & -- & -- & -- & -- \\
\hline 8/7/2008 12:00 AM & 0.490 & 20.3 & 0.416 & 0.231 & 19.3 & 0.41 & 0.124 & -- & -- & -- & -- & -- & -- \\
\hline 8/7/2008 1:00 AM & 0.490 & 20.3 & 0.417 & 0.247 & 19.3 & 0.49 & 0.126 & -- & -- & -- & -- & -- & -- \\
\hline 8/7/2008 2:00 AM & 0.490 & 20.3 & 0.415 & 0.243 & 19.3 & 0.49 & 0.129 & -- & -- & -- & -- & -- & -- \\
\hline 8/7/2008 3:00 AM & 0.490 & 20.3 & 0.408 & 0.232 & 19.3 & 0.46 & 0.134 & -- & -- & -- & -- & -- & -- \\
\hline
\end{tabular}


Appendix 3. Volumetric water content, temperature, and electrical-conductivity data collected at the sprinkler-irrigated site during 2008.-Continued

[Depth in feet below land surface; volumetric water content dimensionless; temperature in degrees Celsius; bulk electrical conductivity in decisiemens per meter; --, no data]

\begin{tabular}{|c|c|c|c|c|c|c|c|c|c|c|c|c|c|}
\hline \multirow[b]{3}{*}{$\begin{array}{l}\text { Measurement } \\
\text { date and time }\end{array}$} & \multicolumn{13}{|c|}{ Depth } \\
\hline & \multicolumn{2}{|l|}{2} & \multirow{2}{*}{$\begin{array}{c}3 \\
\begin{array}{c}\text { Volumetric } \\
\text { water } \\
\text { content }\end{array}\end{array}$} & \multicolumn{3}{|c|}{4} & \multirow{2}{*}{$\begin{array}{c}5 \\
\text { Volumetric } \\
\text { water } \\
\text { content }\end{array}$} & \multicolumn{2}{|l|}{7} & \multirow[b]{2}{*}{$\begin{array}{c}9 \\
\text { Volumetric } \\
\text { water } \\
\text { content }\end{array}$} & \multicolumn{2}{|c|}{13} & \multirow{2}{*}{$\begin{array}{c}16.5 \\
\text { Volumetric } \\
\text { water } \\
\text { content }\end{array}$} \\
\hline & $\begin{array}{c}\text { Volumetric } \\
\text { water } \\
\text { content }\end{array}$ & $\begin{array}{c}\text { Temper- } \\
\text { ature }\end{array}$ & & $\begin{array}{c}\text { Volumetric } \\
\text { water } \\
\text { content }\end{array}$ & $\begin{array}{l}\text { Temper- } \\
\text { ature }\end{array}$ & $\begin{array}{c}\text { Bulk } \\
\text { electrical } \\
\text { conduct- } \\
\text { ivity }\end{array}$ & & $\begin{array}{l}\text { Volumetric } \\
\text { water } \\
\text { content }\end{array}$ & $\begin{array}{l}\text { Temper- } \\
\text { ature }\end{array}$ & & $\begin{array}{c}\text { Volumetric } \\
\text { water } \\
\text { content }\end{array}$ & $\begin{array}{l}\text { Temper- } \\
\text { ature }\end{array}$ & \\
\hline 8/7/2008 4:00 AM & 0.486 & 20.2 & 0.392 & 0.220 & 19.3 & 0.43 & 0.138 & -- & -- & -- & -- & -- & -- \\
\hline 8/7/2008 5:00 AM & 0.474 & 20.2 & 0.378 & 0.210 & 19.3 & 0.40 & 0.142 & -- & -- & -- & -- & -- & -- \\
\hline 8/7/2008 6:00 AM & 0.468 & 20.2 & 0.353 & 0.201 & 19.4 & 0.38 & 0.144 & -- & -- & -- & -- & -- & -- \\
\hline 8/7/2008 7:00 AM & 0.462 & 20.2 & 0.302 & 0.195 & 19.3 & 0.36 & 0.145 & -- & -- & -- & -- & -- & -- \\
\hline 8/7/2008 8:00 AM & 0.458 & 20.1 & 0.275 & 0.190 & 19.3 & 0.34 & 0.147 & -- & -- & -- & -- & -- & -- \\
\hline 8/7/2008 9:00 AM & 0.455 & 20.1 & 0.268 & 0.186 & 19.4 & 0.33 & 0.147 & -- & -- & -- & -- & -- & -- \\
\hline 8/7/2008 10:00 AM & 0.450 & 20.0 & 0.262 & 0.183 & 19.3 & 0.32 & 0.148 & -- & -- & -- & -- & -- & -- \\
\hline 8/7/2008 11:00 AM & 0.447 & 20.0 & 0.256 & 0.180 & 19.3 & 0.31 & 0.149 & -- & -- & -- & -- & -- & -- \\
\hline 8/7/2008 12:00 PM & 0.444 & 20.0 & 0.253 & 0.178 & 19.4 & 0.30 & 0.149 & -- & -- & -- & -- & -- & -- \\
\hline 8/7/2008 1:00 PM & 0.441 & 19.9 & 0.249 & 0.175 & 19.4 & 0.29 & 0.149 & -- & -- & -- & -- & -- & -- \\
\hline 8/7/2008 2:00 PM & 0.437 & 19.9 & 0.246 & 0.173 & 19.3 & 0.29 & 0.149 & -- & -- & -- & -- & -- & -- \\
\hline 8/7/2008 3:00 PM & 0.435 & 19.9 & 0.244 & 0.172 & 19.3 & 0.28 & 0.150 & -- & -- & -- & -- & -- & -- \\
\hline 8/7/2008 4:00 PM & 0.433 & 19.9 & 0.241 & 0.171 & 19.4 & 0.28 & 0.150 & -- & -- & -- & -- & -- & -- \\
\hline 8/7/2008 5:00 PM & 0.430 & 19.9 & 0.239 & 0.169 & 19.3 & 0.28 & 0.150 & -- & -- & -- & -- & -- & -- \\
\hline 8/7/2008 6:00 PM & 0.428 & 19.8 & 0.238 & 0.168 & 19.4 & 0.27 & 0.150 & -- & -- & -- & -- & -- & -- \\
\hline 8/7/2008 7:00 PM & 0.425 & 19.8 & 0.236 & 0.168 & 19.4 & 0.27 & 0.150 & -- & -- & -- & -- & -- & -- \\
\hline 8/7/2008 8:00 PM & 0.423 & 19.8 & 0.234 & 0.166 & 19.4 & 0.27 & 0.150 & -- & -- & -- & -- & -- & -- \\
\hline 8/7/2008 9:00 PM & 0.422 & 19.8 & 0.234 & 0.166 & 19.4 & 0.26 & 0.150 & -- & -- & -- & -- & -- & -- \\
\hline 8/7/2008 10:00 PM & 0.422 & 19.8 & 0.233 & 0.163 & 19.4 & 0.26 & 0.150 & -- & -- & -- & -- & -- & -- \\
\hline 8/7/2008 11:00 PM & 0.421 & 19.8 & 0.232 & 0.163 & 19.3 & 0.26 & 0.150 & -- & -- & -- & -- & -- & -- \\
\hline 8/8/2008 12:00 AM & 0.421 & 19.8 & 0.231 & 0.162 & 19.3 & 0.26 & 0.150 & -- & -- & -- & -- & -- & -- \\
\hline 8/8/2008 1:00 AM & 0.420 & 19.8 & 0.230 & 0.162 & 19.3 & 0.26 & 0.150 & -- & -- & -- & -- & -- & -- \\
\hline 8/8/2008 2:00 AM & 0.419 & 19.8 & 0.229 & 0.161 & 19.4 & 0.26 & 0.150 & -- & -- & -- & -- & -- & -- \\
\hline 8/8/2008 3:00 AM & 0.418 & 19.8 & 0.228 & 0.162 & 19.4 & 0.25 & 0.150 & -- & -- & -- & -- & -- & -- \\
\hline 8/8/2008 4:00 AM & 0.418 & 19.8 & 0.228 & 0.160 & 19.3 & 0.25 & 0.150 & -- & -- & -- & -- & -- & -- \\
\hline 8/8/2008 5:00 AM & 0.417 & 19.9 & 0.228 & 0.161 & 19.3 & 0.25 & 0.150 & -- & -- & -- & -- & -- & -- \\
\hline 8/8/2008 6:00 AM & 0.416 & 19.8 & 0.227 & 0.160 & 19.3 & 0.25 & 0.149 & -- & -- & -- & -- & -- & -- \\
\hline 8/8/2008 7:00 AM & 0.416 & 19.8 & 0.227 & 0.159 & 19.4 & 0.25 & 0.149 & -- & -- & -- & -- & -- & -- \\
\hline 8/8/2008 8:00 AM & 0.416 & 19.8 & 0.226 & 0.159 & 19.3 & 0.25 & 0.149 & -- & -- & -- & -- & -- & -- \\
\hline 8/8/2008 9:00 AM & 0.415 & 19.8 & 0.226 & 0.159 & 19.3 & 0.25 & 0.149 & -- & -- & -- & -- & -- & -- \\
\hline 8/8/2008 10:00 AM & 0.413 & 19.8 & 0.225 & 0.158 & 19.3 & 0.25 & 0.149 & -- & -- & -- & -- & -- & -- \\
\hline 8/8/2008 11:00 AM & 0.413 & 19.8 & 0.225 & 0.158 & 19.3 & 0.24 & 0.149 & -- & -- & -- & -- & -- & -- \\
\hline 8/8/2008 12:00 PM & 0.412 & 19.8 & 0.224 & 0.157 & 19.4 & 0.24 & 0.149 & -- & -- & -- & -- & -- & -- \\
\hline 8/8/2008 1:00 PM & 0.412 & 19.8 & 0.224 & 0.157 & 19.3 & 0.24 & 0.149 & -- & -- & -- & -- & -- & -- \\
\hline 8/8/2008 2:00 PM & 0.411 & 19.8 & 0.224 & 0.157 & 19.3 & 0.24 & 0.149 & -- & -- & -- & -- & -- & -- \\
\hline
\end{tabular}


Appendix 3. Volumetric water content, temperature, and electrical-conductivity data collected at the sprinkler-irrigated site during 2008.-Continued

[Depth in feet below land surface; volumetric water content dimensionless; temperature in degrees Celsius; bulk electrical conductivity in decisiemens per meter; --, no data]

\begin{tabular}{|c|c|c|c|c|c|c|c|c|c|c|c|c|c|}
\hline \multirow[b]{3}{*}{$\begin{array}{l}\text { Measurement } \\
\text { date and time }\end{array}$} & \multicolumn{13}{|c|}{ Depth } \\
\hline & \multicolumn{2}{|c|}{2} & \multirow{2}{*}{$\begin{array}{c}3 \\
\begin{array}{c}\text { Volumetric } \\
\text { water } \\
\text { content }\end{array}\end{array}$} & \multicolumn{3}{|c|}{4} & \multirow{2}{*}{$\begin{array}{c}5 \\
\text { Volumetric } \\
\text { water } \\
\text { content }\end{array}$} & \multicolumn{2}{|l|}{7} & \multirow{2}{*}{$\begin{array}{c}9 \\
\text { Volumetric } \\
\text { water } \\
\text { content }\end{array}$} & \multicolumn{2}{|c|}{13} & \multirow{2}{*}{$\begin{array}{c}16.5 \\
\text { Volumetric } \\
\text { water } \\
\text { content }\end{array}$} \\
\hline & $\begin{array}{c}\text { Volumetric } \\
\text { water } \\
\text { content }\end{array}$ & $\begin{array}{l}\text { Temper- } \\
\text { ature }\end{array}$ & & $\begin{array}{c}\text { Volumetric } \\
\text { water } \\
\text { content }\end{array}$ & $\begin{array}{l}\text { Temper- } \\
\text { ature }\end{array}$ & $\begin{array}{c}\text { Bulk } \\
\text { electrical } \\
\text { conduct- } \\
\text { ivity }\end{array}$ & & $\begin{array}{c}\text { Volumetric } \\
\text { water } \\
\text { content }\end{array}$ & $\begin{array}{l}\text { Temper- } \\
\text { ature }\end{array}$ & & $\begin{array}{c}\text { Volumetric } \\
\text { water } \\
\text { content }\end{array}$ & $\begin{array}{l}\text { Temper- } \\
\text { ature }\end{array}$ & \\
\hline 8/8/2008 3:00 PM & 0.410 & 19.8 & 0.223 & 0.157 & 19.3 & 0.24 & 0.149 & -- & -- & -- & -- & -- & -- \\
\hline 8/8/2008 4:00 PM & 0.409 & 19.8 & 0.223 & 0.156 & 19.3 & 0.24 & 0.149 & -- & -- & -- & -- & -- & -- \\
\hline 8/8/2008 5:00 PM & 0.408 & 19.7 & 0.222 & 0.156 & 19.3 & 0.24 & 0.149 & -- & -- & -- & -- & -- & -- \\
\hline 8/8/2008 6:00 PM & 0.407 & 19.7 & 0.222 & 0.155 & 19.3 & 0.24 & 0.149 & -- & -- & -- & -- & -- & -- \\
\hline 8/8/2008 7:00 PM & 0.406 & 19.7 & 0.222 & 0.155 & 19.3 & 0.24 & 0.149 & -- & -- & -- & -- & -- & -- \\
\hline 8/8/2008 8:00 PM & 0.405 & 19.7 & 0.221 & 0.155 & 19.3 & 0.24 & 0.149 & -- & -- & -- & -- & -- & -- \\
\hline 8/8/2008 9:00 PM & 0.405 & 19.7 & 0.221 & 0.155 & 19.3 & 0.24 & 0.149 & -- & -- & -- & -- & -- & -- \\
\hline 8/8/2008 10:00 PM & 0.405 & 19.7 & 0.221 & 0.155 & 19.3 & 0.24 & 0.148 & -- & -- & -- & -- & -- & -- \\
\hline 8/8/2008 11:00 PM & 0.404 & 19.7 & 0.220 & 0.154 & 19.3 & 0.23 & 0.148 & -- & -- & -- & -- & -- & -- \\
\hline 8/9/2008 12:00 AM & 0.404 & 19.7 & 0.220 & 0.154 & 19.3 & 0.23 & 0.148 & -- & -- & -- & -- & -- & -- \\
\hline 8/9/2008 1:00 AM & 0.404 & 19.7 & 0.220 & 0.154 & 19.3 & 0.23 & 0.148 & -- & -- & -- & -- & -- & -- \\
\hline 8/9/2008 2:00 AM & 0.403 & 19.7 & 0.219 & 0.153 & 19.3 & 0.23 & 0.148 & -- & -- & -- & -- & -- & -- \\
\hline 8/9/2008 3:00 AM & 0.404 & 19.8 & 0.219 & 0.153 & 19.3 & 0.23 & 0.148 & -- & -- & -- & -- & -- & -- \\
\hline 8/9/2008 4:00 AM & 0.403 & 19.8 & 0.219 & 0.153 & 19.3 & 0.23 & 0.148 & -- & -- & -- & -- & -- & -- \\
\hline 8/9/2008 5:00 AM & 0.403 & 19.8 & 0.219 & 0.153 & 19.3 & 0.23 & 0.147 & -- & -- & -- & -- & -- & -- \\
\hline 8/9/2008 6:00 AM & 0.403 & 19.8 & 0.218 & 0.151 & 19.3 & 0.23 & 0.147 & -- & -- & -- & -- & -- & -- \\
\hline 8/9/2008 7:00 AM & 0.403 & 19.8 & 0.218 & 0.153 & 19.3 & 0.23 & 0.147 & -- & -- & -- & -- & -- & -- \\
\hline 8/9/2008 8:00 AM & 0.403 & 19.8 & 0.218 & 0.151 & 19.3 & 0.23 & 0.147 & -- & -- & -- & -- & -- & -- \\
\hline 8/9/2008 9:00 AM & 0.403 & 19.8 & 0.218 & 0.151 & 19.3 & 0.23 & 0.147 & -- & -- & -- & -- & -- & -- \\
\hline 8/9/2008 10:00 AM & 0.403 & 19.8 & 0.217 & 0.150 & 19.3 & 0.23 & 0.147 & -- & -- & -- & -- & -- & -- \\
\hline 8/9/2008 11:00 AM & 0.403 & 19.8 & 0.217 & 0.151 & 19.3 & 0.23 & 0.147 & -- & -- & -- & -- & -- & -- \\
\hline 8/9/2008 12:00 PM & 0.401 & 19.8 & 0.217 & 0.150 & 19.3 & 0.23 & 0.147 & -- & -- & -- & -- & -- & -- \\
\hline 8/9/2008 1:00 PM & 0.401 & 19.8 & 0.217 & 0.150 & 19.3 & 0.23 & 0.147 & -- & -- & -- & -- & -- & -- \\
\hline 8/9/2008 2:00 PM & 0.400 & 19.8 & 0.217 & 0.150 & 19.3 & 0.23 & 0.146 & -- & -- & -- & -- & -- & -- \\
\hline 8/9/2008 3:00 PM & 0.400 & 19.8 & 0.217 & 0.150 & 19.3 & 0.23 & 0.146 & -- & -- & -- & -- & -- & -- \\
\hline 8/9/2008 4:00 PM & 0.399 & 19.8 & 0.217 & 0.149 & 19.3 & 0.22 & 0.146 & -- & -- & -- & -- & -- & -- \\
\hline 8/9/2008 5:00 PM & 0.399 & 19.8 & 0.216 & 0.149 & 19.3 & 0.22 & 0.146 & -- & -- & -- & -- & -- & -- \\
\hline 8/9/2008 6:00 PM & 0.398 & 19.8 & 0.216 & 0.149 & 19.3 & 0.22 & 0.146 & -- & -- & -- & -- & -- & -- \\
\hline 8/9/2008 7:00 PM & 0.397 & 19.7 & 0.216 & 0.149 & 19.3 & 0.22 & 0.146 & -- & -- & -- & -- & -- & -- \\
\hline 8/9/2008 8:00 PM & 0.397 & 19.8 & 0.216 & 0.149 & 19.3 & 0.22 & 0.145 & -- & -- & -- & -- & -- & -- \\
\hline 8/9/2008 9:00 PM & 0.397 & 19.8 & 0.215 & 0.148 & 19.3 & 0.22 & 0.145 & -- & -- & -- & -- & -- & -- \\
\hline 8/9/2008 10:00 PM & 0.397 & 19.8 & 0.215 & 0.148 & 19.3 & 0.22 & 0.145 & -- & -- & -- & -- & -- & -- \\
\hline 8/9/2008 11:00 PM & 0.397 & 19.8 & 0.215 & 0.148 & 19.3 & 0.22 & 0.145 & -- & -- & -- & -- & -- & -- \\
\hline 8/10/2008 12:00 AM & 0.397 & 19.8 & 0.215 & 0.148 & 19.3 & 0.22 & 0.145 & -- & -- & -- & -- & -- & -- \\
\hline 8/10/2008 1:00 AM & 0.397 & 19.8 & 0.215 & 0.148 & 19.3 & 0.22 & 0.145 & -- & -- & -- & -- & -- & -- \\
\hline
\end{tabular}


Appendix 3. Volumetric water content, temperature, and electrical-conductivity data collected at the sprinkler-irrigated site during 2008.-Continued

[Depth in feet below land surface; volumetric water content dimensionless; temperature in degrees Celsius; bulk electrical conductivity in decisiemens per meter; --, no data]

\begin{tabular}{|c|c|c|c|c|c|c|c|c|c|c|c|c|c|}
\hline \multirow[b]{3}{*}{$\begin{array}{l}\text { Measurement } \\
\text { date and time }\end{array}$} & \multicolumn{13}{|c|}{ Depth } \\
\hline & \multicolumn{2}{|c|}{2} & \multirow{2}{*}{$\begin{array}{c}3 \\
\text { Volumetric } \\
\text { water } \\
\text { content }\end{array}$} & \multicolumn{3}{|c|}{4} & \multirow{2}{*}{$\begin{array}{c}5 \\
\text { Volumetric } \\
\text { water } \\
\text { content }\end{array}$} & \multicolumn{2}{|l|}{7} & \multirow{2}{*}{$\begin{array}{c}9 \\
\text { Volumetric } \\
\text { water } \\
\text { content }\end{array}$} & \multicolumn{2}{|c|}{13} & \multirow{2}{*}{$\begin{array}{c}16.5 \\
\text { Volumetric } \\
\text { water } \\
\text { content }\end{array}$} \\
\hline & $\begin{array}{c}\text { Volumetric } \\
\text { water } \\
\text { content }\end{array}$ & $\begin{array}{l}\text { Temper- } \\
\text { ature }\end{array}$ & & $\begin{array}{c}\text { Volumetric } \\
\text { water } \\
\text { content }\end{array}$ & $\begin{array}{l}\text { Temper- } \\
\text { ature }\end{array}$ & $\begin{array}{c}\text { Bulk } \\
\text { electrical } \\
\text { conduct- } \\
\text { ivity }\end{array}$ & & $\begin{array}{c}\text { Volumetric } \\
\text { water } \\
\text { content }\end{array}$ & $\begin{array}{l}\text { Temper- } \\
\text { ature }\end{array}$ & & $\begin{array}{c}\text { Volumetric } \\
\text { water } \\
\text { content }\end{array}$ & $\begin{array}{l}\text { Temper- } \\
\text { ature }\end{array}$ & \\
\hline 8/10/2008 2:00 AM & 0.396 & 19.8 & 0.215 & 0.147 & 19.3 & 0.22 & 0.145 & -- & $\overline{--}$ & $\overline{--}$ & -- & $\overline{--}$ & $\overline{--}$ \\
\hline 8/10/2008 3:00 AM & 0.396 & 19.8 & 0.214 & 0.147 & 19.3 & 0.22 & 0.144 & -- & -- & -- & -- & -- & -- \\
\hline 8/10/2008 4:00 AM & 0.396 & 19.9 & 0.214 & 0.147 & 19.3 & 0.22 & 0.144 & -- & -- & -- & -- & -- & -- \\
\hline 8/10/2008 5:00 AM & 0.396 & 19.9 & 0.214 & 0.147 & 19.3 & 0.22 & 0.144 & -- & -- & -- & -- & -- & -- \\
\hline 8/10/2008 6:00 AM & 0.395 & 19.9 & 0.214 & 0.147 & 19.3 & 0.22 & 0.144 & -- & -- & -- & -- & -- & -- \\
\hline 8/10/2008 7:00 AM & 0.396 & 19.9 & 0.214 & 0.147 & 19.3 & 0.22 & 0.144 & -- & -- & -- & -- & -- & -- \\
\hline 8/10/2008 8:00 AM & 0.395 & 19.9 & 0.213 & 0.146 & 19.3 & 0.22 & 0.144 & -- & -- & -- & -- & -- & -- \\
\hline 8/10/2008 9:00 AM & 0.395 & 19.9 & 0.213 & 0.146 & 19.3 & 0.22 & 0.144 & -- & -- & -- & -- & -- & -- \\
\hline 8/10/2008 10:00 AM & 0.396 & 19.9 & 0.213 & 0.146 & 19.3 & 0.22 & 0.144 & -- & -- & -- & -- & -- & -- \\
\hline 8/10/2008 11:00 AM & 0.396 & 19.9 & 0.213 & 0.146 & 19.3 & 0.22 & 0.143 & -- & -- & -- & -- & -- & -- \\
\hline 8/10/2008 12:00 PM & 0.396 & 19.9 & 0.213 & 0.146 & 19.3 & 0.22 & 0.143 & -- & -- & -- & -- & -- & -- \\
\hline 8/10/2008 1:00 PM & 0.396 & 19.9 & 0.213 & 0.146 & 19.3 & 0.22 & 0.143 & -- & -- & -- & -- & -- & -- \\
\hline 8/10/2008 2:00 PM & 0.396 & 19.8 & 0.213 & 0.146 & 19.3 & 0.21 & 0.143 & -- & -- & -- & -- & -- & -- \\
\hline 8/10/2008 3:00 PM & 0.395 & 19.8 & 0.213 & 0.145 & 19.3 & 0.21 & 0.143 & -- & -- & -- & -- & -- & -- \\
\hline 8/10/2008 4:00 PM & 0.394 & 19.8 & 0.212 & 0.145 & 19.3 & 0.21 & 0.143 & -- & -- & -- & -- & -- & -- \\
\hline 8/10/2008 5:00 PM & 0.393 & 19.8 & 0.212 & 0.145 & 19.3 & 0.21 & 0.143 & -- & -- & -- & -- & -- & -- \\
\hline 8/10/2008 6:00 PM & 0.393 & 19.8 & 0.212 & 0.145 & 19.3 & 0.21 & 0.143 & -- & -- & -- & -- & -- & -- \\
\hline 8/10/2008 7:00 PM & 0.392 & 19.8 & 0.212 & 0.145 & 19.3 & 0.21 & 0.143 & -- & -- & -- & -- & -- & -- \\
\hline 8/10/2008 8:00 PM & 0.392 & 19.8 & 0.211 & 0.145 & 19.3 & 0.21 & 0.143 & -- & -- & -- & -- & -- & -- \\
\hline 8/10/2008 9:00 PM & 0.392 & 19.8 & 0.211 & 0.145 & 19.3 & 0.21 & 0.143 & -- & -- & -- & -- & -- & -- \\
\hline 8/10/2008 10:00 PM & 0.392 & 19.9 & 0.211 & 0.144 & 19.3 & 0.21 & 0.143 & -- & -- & -- & -- & -- & -- \\
\hline 8/10/2008 11:00 PM & 0.391 & 19.9 & 0.211 & 0.145 & 19.3 & 0.21 & 0.143 & -- & -- & -- & -- & -- & -- \\
\hline 8/11/2008 12:00 AM & 0.392 & 19.9 & 0.211 & 0.144 & 19.3 & 0.21 & 0.143 & -- & -- & -- & -- & -- & -- \\
\hline 8/11/2008 1:00 AM & 0.391 & 19.9 & 0.211 & 0.144 & 19.3 & 0.21 & 0.143 & -- & -- & -- & -- & -- & -- \\
\hline 8/11/2008 2:00 AM & 0.392 & 19.9 & 0.211 & 0.144 & 19.3 & 0.21 & 0.143 & -- & -- & -- & -- & -- & -- \\
\hline 8/11/2008 3:00 AM & 0.391 & 19.9 & 0.211 & 0.143 & 19.2 & 0.21 & 0.142 & -- & -- & -- & -- & -- & -- \\
\hline 8/11/2008 4:00 AM & 0.391 & 19.9 & 0.211 & 0.143 & 19.3 & 0.21 & 0.142 & -- & -- & -- & -- & -- & -- \\
\hline 8/11/2008 5:00 AM & 0.391 & 19.9 & 0.211 & 0.143 & 19.3 & 0.21 & 0.142 & -- & -- & -- & -- & -- & -- \\
\hline 8/11/2008 6:00 AM & 0.391 & 19.9 & 0.211 & 0.143 & 19.3 & 0.21 & 0.142 & -- & -- & -- & -- & -- & -- \\
\hline 8/11/2008 7:00 AM & 0.391 & 19.9 & 0.211 & 0.143 & 19.3 & 0.21 & 0.142 & -- & -- & -- & -- & -- & -- \\
\hline 8/11/2008 8:00 AM & 0.391 & 19.9 & 0.211 & 0.143 & 19.3 & 0.21 & 0.142 & -- & -- & -- & -- & -- & -- \\
\hline 8/11/2008 9:00 AM & 0.391 & 19.9 & 0.211 & 0.143 & 19.3 & 0.21 & 0.142 & -- & -- & -- & -- & -- & -- \\
\hline 8/11/2008 10:00 AM & 0.391 & 19.9 & 0.211 & 0.143 & 19.3 & 0.21 & 0.142 & -- & -- & -- & -- & -- & -- \\
\hline 8/11/2008 11:00 AM & 0.391 & 19.8 & 0.211 & 0.143 & 19.3 & 0.21 & 0.142 & -- & -- & -- & -- & -- & -- \\
\hline 8/11/2008 12:00 PM & 0.390 & 19.8 & 0.210 & 0.143 & 19.3 & 0.21 & 0.141 & -- & -- & -- & -- & -- & -- \\
\hline
\end{tabular}


Appendix 3. Volumetric water content, temperature, and electrical-conductivity data collected at the sprinkler-irrigated site during 2008.-Continued

[Depth in feet below land surface; volumetric water content dimensionless; temperature in degrees Celsius; bulk electrical conductivity in decisiemens per meter; --, no data]

\begin{tabular}{|c|c|c|c|c|c|c|c|c|c|c|c|c|c|}
\hline \multirow[b]{3}{*}{$\begin{array}{l}\text { Measurement } \\
\text { date and time }\end{array}$} & \multicolumn{13}{|c|}{ Depth } \\
\hline & \multicolumn{2}{|c|}{2} & \multirow{2}{*}{$\begin{array}{c}3 \\
\begin{array}{c}\text { Volumetric } \\
\text { water } \\
\text { content }\end{array}\end{array}$} & \multicolumn{3}{|c|}{4} & \multirow{2}{*}{$\begin{array}{c}5 \\
\text { Volumetric } \\
\text { water } \\
\text { content }\end{array}$} & \multicolumn{2}{|l|}{7} & \multirow{2}{*}{$\begin{array}{c}9 \\
\text { Volumetric } \\
\text { water } \\
\text { content }\end{array}$} & \multicolumn{2}{|c|}{13} & \multirow{2}{*}{$\begin{array}{c}16.5 \\
\text { Volumetric } \\
\text { water } \\
\text { content }\end{array}$} \\
\hline & $\begin{array}{c}\text { Volumetric } \\
\text { water } \\
\text { content }\end{array}$ & $\begin{array}{l}\text { Temper- } \\
\text { ature }\end{array}$ & & $\begin{array}{c}\text { Volumetric } \\
\text { water } \\
\text { content }\end{array}$ & $\begin{array}{l}\text { Temper- } \\
\text { ature }\end{array}$ & $\begin{array}{c}\text { Bulk } \\
\text { electrical } \\
\text { conduct- } \\
\text { ivity }\end{array}$ & & $\begin{array}{c}\text { Volumetric } \\
\text { water } \\
\text { content }\end{array}$ & $\begin{array}{l}\text { Temper- } \\
\text { ature }\end{array}$ & & $\begin{array}{c}\text { Volumetric } \\
\text { water } \\
\text { content }\end{array}$ & $\begin{array}{l}\text { Temper- } \\
\text { ature }\end{array}$ & \\
\hline 8/11/2008 1:00 PM & 0.390 & 19.8 & 0.211 & 0.143 & 19.3 & 0.21 & 0.141 & -- & -- & -- & -- & -- & -- \\
\hline 8/11/2008 2:00 PM & 0.390 & 19.8 & 0.211 & 0.143 & 19.3 & 0.21 & 0.141 & -- & -- & -- & -- & -- & -- \\
\hline 8/11/2008 3:00 PM & 0.388 & 19.8 & 0.210 & 0.142 & 19.3 & 0.21 & 0.141 & -- & -- & -- & -- & -- & -- \\
\hline 8/11/2008 4:00 PM & 0.387 & 19.8 & 0.210 & 0.142 & 19.2 & 0.21 & 0.141 & -- & -- & -- & -- & -- & -- \\
\hline 8/11/2008 5:00 PM & 0.386 & 19.7 & 0.209 & 0.142 & 19.3 & 0.21 & 0.141 & -- & -- & -- & -- & -- & -- \\
\hline 8/11/2008 6:00 PM & 0.386 & 19.7 & 0.209 & 0.142 & 19.3 & 0.20 & 0.141 & -- & -- & -- & -- & -- & -- \\
\hline 8/11/2008 7:00 PM & 0.386 & 19.7 & 0.209 & 0.142 & 19.3 & 0.20 & 0.141 & -- & -- & -- & -- & -- & -- \\
\hline 8/11/2008 8:00 PM & 0.385 & 19.7 & 0.209 & 0.142 & 19.3 & 0.20 & 0.140 & -- & -- & -- & -- & -- & -- \\
\hline 8/11/2008 9:00 PM & 0.385 & 19.7 & 0.209 & 0.141 & 19.3 & 0.20 & 0.140 & -- & -- & -- & -- & -- & -- \\
\hline 8/11/2008 10:00 PM & 0.386 & 19.7 & 0.209 & 0.141 & 19.3 & 0.20 & 0.140 & -- & -- & -- & -- & -- & -- \\
\hline 8/11/2008 11:00 PM & 0.386 & 19.7 & 0.208 & 0.142 & 19.3 & 0.20 & 0.140 & -- & -- & -- & -- & -- & -- \\
\hline 8/12/2008 12:00 AM & 0.385 & 19.7 & 0.208 & 0.141 & 19.3 & 0.20 & 0.140 & -- & -- & -- & -- & -- & -- \\
\hline 8/12/2008 1:00 AM & 0.386 & 19.7 & 0.208 & 0.141 & 19.3 & 0.20 & 0.140 & -- & -- & -- & -- & -- & -- \\
\hline 8/12/2008 2:00 AM & 0.385 & 19.7 & 0.208 & 0.141 & 19.2 & 0.20 & 0.140 & -- & -- & -- & -- & -- & -- \\
\hline 8/12/2008 3:00 AM & 0.385 & 19.7 & 0.208 & 0.141 & 19.3 & 0.20 & 0.140 & -- & -- & -- & -- & -- & -- \\
\hline 8/12/2008 4:00 AM & 0.386 & 19.7 & 0.208 & 0.141 & 19.2 & 0.20 & 0.140 & -- & -- & -- & -- & -- & -- \\
\hline 8/12/2008 5:00 AM & 0.386 & 19.7 & 0.208 & 0.141 & 19.3 & 0.20 & 0.139 & -- & -- & -- & -- & -- & -- \\
\hline 8/12/2008 6:00 AM & 0.385 & 19.8 & 0.208 & 0.141 & 19.3 & 0.20 & 0.139 & -- & -- & -- & -- & -- & -- \\
\hline 8/12/2008 7:00 AM & 0.385 & 19.7 & 0.208 & 0.141 & 19.3 & 0.20 & 0.139 & -- & -- & -- & -- & -- & -- \\
\hline 8/12/2008 8:00 AM & 0.385 & 19.7 & 0.208 & 0.141 & 19.2 & 0.20 & 0.139 & -- & -- & -- & -- & -- & -- \\
\hline 8/12/2008 9:00 AM & 0.385 & 19.7 & 0.207 & 0.141 & 19.3 & 0.20 & 0.139 & -- & -- & -- & -- & -- & -- \\
\hline 8/12/2008 10:00 AM & 0.385 & 19.7 & 0.207 & 0.140 & 19.2 & 0.20 & 0.139 & -- & -- & -- & -- & -- & -- \\
\hline 8/12/2008 11:00 AM & 0.384 & 19.7 & 0.207 & 0.140 & 19.2 & 0.20 & 0.139 & -- & -- & -- & -- & -- & -- \\
\hline 8/12/2008 12:00 PM & 0.384 & 19.6 & 0.207 & 0.140 & 19.2 & 0.20 & 0.139 & -- & -- & -- & -- & -- & -- \\
\hline 8/12/2008 1:00 PM & 0.383 & 19.6 & 0.207 & 0.140 & 19.3 & 0.20 & 0.139 & -- & -- & -- & -- & -- & -- \\
\hline 8/12/2008 2:00 PM & 0.382 & 19.6 & 0.207 & 0.140 & 19.3 & 0.20 & 0.139 & -- & -- & -- & -- & -- & -- \\
\hline 8/12/2008 3:00 PM & 0.382 & 19.6 & 0.207 & 0.140 & 19.3 & 0.20 & 0.139 & -- & -- & -- & -- & -- & -- \\
\hline 8/12/2008 4:00 PM & 0.381 & 19.6 & 0.207 & 0.140 & 19.3 & 0.20 & 0.139 & -- & -- & -- & -- & -- & -- \\
\hline 8/12/2008 5:00 PM & 0.380 & 19.5 & 0.206 & 0.140 & 19.2 & 0.20 & 0.139 & -- & -- & -- & -- & -- & -- \\
\hline 8/12/2008 6:00 PM & 0.381 & 19.5 & 0.206 & 0.138 & 19.3 & 0.20 & 0.138 & -- & -- & -- & -- & -- & -- \\
\hline 8/12/2008 7:00 PM & 0.380 & 19.5 & 0.206 & 0.140 & 19.3 & 0.20 & 0.138 & -- & -- & -- & -- & -- & -- \\
\hline 8/12/2008 8:00 PM & 0.381 & 19.5 & 0.206 & 0.138 & 19.2 & 0.20 & 0.138 & -- & -- & -- & -- & -- & -- \\
\hline 8/12/2008 9:00 PM & 0.381 & 19.5 & 0.205 & 0.138 & 19.2 & 0.20 & 0.138 & -- & -- & -- & -- & -- & -- \\
\hline 8/12/2008 10:00 PM & 0.381 & 19.5 & 0.205 & 0.140 & 19.2 & 0.20 & 0.138 & -- & -- & -- & -- & -- & -- \\
\hline 8/12/2008 11:00 PM & 0.381 & 19.5 & 0.205 & 0.138 & 19.2 & 0.20 & 0.138 & -- & -- & -- & -- & -- & -- \\
\hline
\end{tabular}


Appendix 3. Volumetric water content, temperature, and electrical-conductivity data collected at the sprinkler-irrigated site during 2008.-Continued

[Depth in feet below land surface; volumetric water content dimensionless; temperature in degrees Celsius; bulk electrical conductivity in decisiemens per meter; --, no data]

\begin{tabular}{|c|c|c|c|c|c|c|c|c|c|c|c|c|c|}
\hline \multirow[b]{3}{*}{$\begin{array}{l}\text { Measurement } \\
\text { date and time }\end{array}$} & \multirow{2}{*}{\multicolumn{2}{|c|}{2}} & \multicolumn{10}{|c|}{ Depth } & \multirow{3}{*}{$\begin{array}{c}16.5 \\
\text { Volumetric } \\
\text { water } \\
\text { content }\end{array}$} \\
\hline & & & \multirow[b]{2}{*}{$\begin{array}{c}3 \\
\begin{array}{c}\text { Volumetric } \\
\text { water } \\
\text { content }\end{array}\end{array}$} & \multicolumn{3}{|c|}{4} & \multirow[b]{2}{*}{$\begin{array}{c}\mathbf{5} \\
\text { Volumetric } \\
\text { water } \\
\text { content }\end{array}$} & \multicolumn{2}{|c|}{7} & \multirow[b]{2}{*}{$\begin{array}{c}9 \\
\text { Volumetric } \\
\text { water } \\
\text { content }\end{array}$} & \multicolumn{2}{|c|}{13} & \\
\hline & $\begin{array}{c}\text { Volumetric } \\
\text { water } \\
\text { content }\end{array}$ & $\begin{array}{l}\text { Temper- } \\
\text { ature }\end{array}$ & & $\begin{array}{c}\text { Volumetric } \\
\text { water } \\
\text { content }\end{array}$ & $\begin{array}{l}\text { Temper- } \\
\text { ature }\end{array}$ & $\begin{array}{c}\text { Bulk } \\
\text { electrical } \\
\text { conduct- } \\
\text { ivity }\end{array}$ & & $\begin{array}{c}\text { Volumetric } \\
\text { water } \\
\text { content }\end{array}$ & $\begin{array}{l}\text { Temper- } \\
\text { ature }\end{array}$ & & $\begin{array}{c}\text { Volumetric } \\
\text { water } \\
\text { content }\end{array}$ & $\begin{array}{l}\text { Temper- } \\
\text { ature }\end{array}$ & \\
\hline $8 / 13 / 2008$ 12:00 AM & 0.381 & 19.5 & 0.205 & 0.138 & 19.2 & 0.20 & 0.138 & -- & -- & -- & -- & -- & -- \\
\hline 8/13/2008 1:00 AM & 0.381 & 19.5 & 0.205 & 0.138 & 19.2 & 0.20 & 0.138 & -- & -- & -- & -- & -- & -- \\
\hline 8/13/2008 2:00 AM & 0.381 & 19.5 & 0.205 & 0.138 & 19.3 & 0.20 & 0.138 & -- & -- & -- & -- & -- & -- \\
\hline 8/13/2008 3:00 AM & 0.381 & 19.5 & 0.205 & 0.138 & 19.2 & 0.20 & 0.138 & -- & -- & -- & -- & -- & -- \\
\hline 8/13/2008 4:00 AM & 0.381 & 19.5 & 0.205 & 0.138 & 19.2 & 0.20 & 0.138 & -- & -- & -- & -- & -- & -- \\
\hline 8/13/2008 5:00 AM & 0.381 & 19.5 & 0.205 & 0.138 & 19.2 & 0.20 & 0.138 & -- & -- & -- & -- & -- & -- \\
\hline 8/13/2008 6:00 AM & 0.381 & 19.5 & 0.205 & 0.138 & 19.2 & 0.20 & 0.138 & -- & -- & -- & -- & -- & -- \\
\hline 8/13/2008 7:00 AM & 0.381 & 19.5 & 0.205 & 0.138 & 19.2 & 0.20 & 0.138 & -- & -- & -- & -- & -- & -- \\
\hline 8/13/2008 8:00 AM & 0.381 & 19.5 & 0.205 & 0.137 & 19.2 & 0.20 & 0.138 & -- & -- & -- & -- & -- & -- \\
\hline 8/13/2008 9:00 AM & 0.381 & 19.5 & 0.205 & 0.137 & 19.2 & 0.20 & 0.138 & -- & -- & -- & -- & -- & -- \\
\hline 8/13/2008 10:00 AM & 0.380 & 19.5 & 0.205 & 0.137 & 19.2 & 0.20 & 0.138 & -- & -- & -- & -- & -- & -- \\
\hline 8/13/2008 11:00 AM & 0.380 & 19.5 & 0.205 & 0.137 & 19.2 & 0.19 & 0.138 & -- & -- & -- & -- & -- & -- \\
\hline 8/13/2008 12:00 PM & 0.380 & 19.5 & 0.205 & 0.137 & 19.2 & 0.19 & 0.138 & -- & -- & -- & -- & -- & -- \\
\hline 8/13/2008 1:00 PM & 0.379 & 19.5 & 0.205 & 0.137 & 19.2 & 0.19 & 0.138 & -- & -- & -- & -- & -- & -- \\
\hline 8/13/2008 2:00 PM & 0.379 & 19.5 & 0.205 & 0.137 & 19.2 & 0.19 & 0.138 & -- & -- & -- & -- & -- & -- \\
\hline 8/13/2008 3:00 PM & 0.378 & 19.4 & 0.205 & 0.137 & 19.2 & 0.19 & 0.138 & -- & -- & -- & -- & -- & -- \\
\hline 8/13/2008 4:00 PM & 0.378 & 19.4 & 0.205 & 0.137 & 19.2 & 0.19 & 0.138 & -- & -- & -- & -- & -- & -- \\
\hline 8/13/2008 5:00 PM & 0.378 & 19.4 & 0.204 & 0.137 & 19.2 & 0.19 & 0.138 & -- & -- & -- & -- & -- & -- \\
\hline 8/13/2008 6:00 PM & 0.378 & 19.4 & 0.204 & 0.137 & 19.2 & 0.19 & 0.137 & -- & -- & -- & -- & -- & -- \\
\hline 8/13/2008 7:00 PM & 0.378 & 19.4 & 0.204 & 0.137 & 19.2 & 0.19 & 0.137 & -- & -- & -- & -- & -- & -- \\
\hline 8/13/2008 8:00 PM & 0.376 & 19.4 & 0.204 & 0.136 & 19.2 & 0.19 & 0.137 & -- & -- & -- & -- & -- & -- \\
\hline 8/13/2008 9:00 PM & 0.378 & 19.4 & 0.204 & 0.137 & 19.2 & 0.19 & 0.137 & -- & -- & -- & -- & -- & -- \\
\hline 8/13/2008 10:00 PM & 0.378 & 19.4 & 0.204 & 0.136 & 19.2 & 0.19 & 0.137 & -- & -- & -- & -- & -- & -- \\
\hline 8/13/2008 11:00 PM & 0.378 & 19.4 & 0.203 & 0.136 & 19.2 & 0.19 & 0.137 & -- & -- & -- & -- & -- & -- \\
\hline 8/14/2008 12:00 AM & 0.378 & 19.4 & 0.203 & 0.136 & 19.2 & 0.19 & 0.137 & -- & -- & -- & -- & -- & -- \\
\hline 8/14/2008 1:00 AM & 0.378 & 19.4 & 0.203 & 0.136 & 19.2 & 0.19 & 0.137 & -- & -- & -- & -- & -- & -- \\
\hline 8/14/2008 2:00 AM & 0.378 & 19.4 & 0.203 & 0.136 & 19.2 & 0.19 & 0.137 & -- & -- & -- & -- & -- & -- \\
\hline 8/14/2008 3:00 AM & 0.378 & 19.4 & 0.203 & 0.136 & 19.2 & 0.19 & 0.137 & -- & -- & -- & -- & -- & -- \\
\hline 8/14/2008 4:00 AM & 0.378 & 19.4 & 0.203 & 0.136 & 19.2 & 0.19 & 0.137 & -- & -- & -- & -- & -- & -- \\
\hline 8/14/2008 5:00 AM & 0.378 & 19.4 & 0.203 & 0.136 & 19.2 & 0.19 & 0.137 & -- & -- & -- & -- & -- & -- \\
\hline 8/14/2008 6:00 AM & 0.378 & 19.4 & 0.203 & 0.136 & 19.2 & 0.19 & 0.137 & -- & -- & -- & -- & -- & -- \\
\hline 8/14/2008 7:00 AM & 0.378 & 19.4 & 0.203 & 0.135 & 19.2 & 0.19 & 0.136 & -- & -- & -- & -- & -- & -- \\
\hline 8/14/2008 8:00 AM & 0.378 & 19.4 & 0.203 & 0.136 & 19.2 & 0.19 & 0.137 & -- & -- & -- & -- & -- & -- \\
\hline 8/14/2008 9:00 AM & 0.378 & 19.4 & 0.203 & 0.135 & 19.2 & 0.19 & 0.137 & -- & -- & -- & -- & -- & -- \\
\hline 8/14/2008 10:00 AM & 0.378 & 19.4 & 0.203 & 0.135 & 19.2 & 0.19 & 0.137 & -- & -- & -- & -- & -- & -- \\
\hline
\end{tabular}


Appendix 3. Volumetric water content, temperature, and electrical-conductivity data collected at the sprinkler-irrigated site during 2008.-Continued

[Depth in feet below land surface; volumetric water content dimensionless; temperature in degrees Celsius; bulk electrical conductivity in decisiemens per meter; --, no data]

\begin{tabular}{|c|c|c|c|c|c|c|c|c|c|c|c|c|c|}
\hline \multirow[b]{3}{*}{$\begin{array}{l}\text { Measurement } \\
\text { date and time }\end{array}$} & \multicolumn{13}{|c|}{ Depth } \\
\hline & \multicolumn{2}{|c|}{2} & \multirow{2}{*}{$\begin{array}{c}3 \\
\text { Volumetric } \\
\text { water } \\
\text { content }\end{array}$} & \multicolumn{3}{|c|}{4} & \multirow{2}{*}{$\begin{array}{c}5 \\
\text { Volumetric } \\
\text { water } \\
\text { content }\end{array}$} & \multicolumn{2}{|c|}{7} & \multirow{2}{*}{$\begin{array}{c}9 \\
\text { Volumetric } \\
\text { water } \\
\text { content }\end{array}$} & \multicolumn{2}{|c|}{13} & \multirow{2}{*}{$\begin{array}{c}16.5 \\
\text { Volumetric } \\
\text { water } \\
\text { content }\end{array}$} \\
\hline & $\begin{array}{c}\text { Volumetric } \\
\text { water } \\
\text { content }\end{array}$ & $\begin{array}{l}\text { Temper- } \\
\text { ature }\end{array}$ & & $\begin{array}{c}\text { Volumetric } \\
\text { water } \\
\text { content }\end{array}$ & $\begin{array}{l}\text { Temper- } \\
\text { ature }\end{array}$ & $\begin{array}{c}\text { Bulk } \\
\text { electrical } \\
\text { conduct- } \\
\text { ivity }\end{array}$ & & $\begin{array}{c}\text { Volumetric } \\
\text { water } \\
\text { content }\end{array}$ & $\begin{array}{l}\text { Temper- } \\
\text { ature }\end{array}$ & & $\begin{array}{c}\text { Volumetric } \\
\text { water } \\
\text { content }\end{array}$ & $\begin{array}{l}\text { Temper- } \\
\text { ature }\end{array}$ & \\
\hline 8/14/2008 11:00 AM & 0.378 & 19.4 & 0.203 & 0.135 & 19.2 & 0.19 & 0.136 & -- & -- & -- & -- & -- & -- \\
\hline 8/14/2008 12:00 PM & 0.376 & 19.4 & 0.202 & 0.135 & 19.2 & 0.19 & 0.136 & -- & -- & -- & -- & -- & -- \\
\hline 8/14/2008 1:00 PM & 0.376 & 19.4 & 0.202 & 0.135 & 19.2 & 0.19 & 0.136 & -- & -- & -- & -- & -- & -- \\
\hline 8/14/2008 2:00 PM & 0.376 & 19.4 & 0.202 & 0.136 & 19.2 & 0.19 & 0.136 & -- & -- & -- & -- & -- & -- \\
\hline 8/14/2008 3:00 PM & 0.376 & 19.4 & 0.202 & 0.135 & 19.2 & 0.19 & 0.136 & -- & -- & -- & -- & -- & -- \\
\hline 8/14/2008 4:00 PM & 0.375 & 19.3 & 0.202 & 0.135 & 19.2 & 0.19 & 0.136 & -- & -- & -- & -- & -- & -- \\
\hline 8/14/2008 5:00 PM & 0.375 & 19.3 & 0.202 & 0.135 & 19.1 & 0.19 & 0.136 & -- & -- & -- & -- & -- & -- \\
\hline 8/14/2008 6:00 PM & 0.375 & 19.3 & 0.202 & 0.135 & 19.2 & 0.19 & 0.136 & -- & -- & -- & -- & -- & -- \\
\hline 8/14/2008 7:00 PM & 0.375 & 19.3 & 0.201 & 0.135 & 19.1 & 0.19 & 0.136 & -- & -- & -- & -- & -- & -- \\
\hline 8/14/2008 8:00 PM & 0.375 & 19.3 & 0.201 & 0.135 & 19.2 & 0.19 & 0.136 & -- & -- & -- & -- & -- & -- \\
\hline 8/14/2008 9:00 PM & 0.376 & 19.3 & 0.201 & 0.135 & 19.2 & 0.19 & 0.136 & -- & -- & -- & -- & -- & -- \\
\hline 8/14/2008 10:00 PM & 0.379 & 19.3 & 0.201 & 0.135 & 19.2 & 0.19 & 0.136 & -- & -- & -- & -- & -- & -- \\
\hline 8/14/2008 11:00 PM & 0.382 & 19.3 & 0.202 & 0.135 & 19.2 & 0.19 & 0.136 & -- & -- & -- & -- & -- & -- \\
\hline 8/15/2008 12:00 AM & 0.385 & 19.2 & 0.202 & 0.135 & 19.1 & 0.19 & 0.136 & -- & -- & -- & -- & -- & -- \\
\hline 8/15/2008 1:00 AM & 0.387 & 19.3 & 0.202 & 0.135 & 19.1 & 0.19 & 0.136 & -- & -- & -- & -- & -- & -- \\
\hline 8/15/2008 2:00 AM & 0.391 & 19.2 & 0.202 & 0.135 & 19.1 & 0.19 & 0.136 & -- & -- & -- & -- & -- & -- \\
\hline 8/15/2008 3:00 AM & 0.392 & 19.2 & 0.203 & 0.135 & 19.2 & 0.19 & 0.136 & -- & -- & -- & -- & -- & -- \\
\hline 8/15/2008 4:00 AM & 0.393 & 19.2 & 0.203 & 0.134 & 19.1 & 0.19 & 0.136 & -- & -- & -- & -- & -- & -- \\
\hline 8/15/2008 5:00 AM & 0.394 & 19.2 & 0.204 & 0.134 & 19.1 & 0.19 & 0.135 & -- & -- & -- & -- & -- & -- \\
\hline 8/15/2008 6:00 AM & 0.395 & 19.2 & 0.204 & 0.134 & 19.1 & 0.19 & 0.136 & -- & -- & -- & -- & -- & -- \\
\hline 8/15/2008 7:00 AM & 0.396 & 19.2 & 0.204 & 0.135 & 19.1 & 0.19 & 0.136 & -- & -- & -- & -- & -- & -- \\
\hline 8/15/2008 8:00 AM & 0.396 & 19.2 & 0.205 & 0.135 & 19.1 & 0.19 & 0.136 & -- & -- & -- & -- & -- & -- \\
\hline 8/15/2008 9:00 AM & 0.397 & 19.2 & 0.205 & 0.135 & 19.1 & 0.19 & 0.135 & -- & -- & -- & -- & -- & -- \\
\hline $8 / 15 / 2008$ 10:00 AM & 0.398 & 19.2 & 0.205 & 0.134 & 19.1 & 0.19 & 0.135 & -- & -- & -- & -- & -- & -- \\
\hline 8/15/2008 11:00 AM & 0.399 & 19.1 & 0.205 & 0.135 & 19.1 & 0.19 & 0.135 & -- & -- & -- & -- & -- & -- \\
\hline 8/15/2008 12:00 PM & 0.401 & 19.1 & 0.205 & 0.135 & 19.1 & 0.19 & 0.135 & -- & -- & -- & -- & -- & -- \\
\hline 8/15/2008 1:00 PM & 0.407 & 19.1 & 0.205 & 0.135 & 19.1 & 0.19 & 0.135 & -- & -- & -- & -- & -- & -- \\
\hline 8/15/2008 2:00 PM & 0.422 & 19.0 & 0.206 & 0.135 & 19.1 & 0.19 & 0.135 & -- & -- & -- & -- & -- & -- \\
\hline 8/15/2008 3:00 PM & 0.434 & 19.0 & 0.208 & 0.135 & 19.1 & 0.19 & 0.135 & -- & -- & -- & -- & -- & -- \\
\hline 8/15/2008 4:00 PM & 0.446 & 19.0 & 0.211 & 0.135 & 19.1 & 0.19 & 0.136 & -- & -- & -- & -- & -- & -- \\
\hline 8/15/2008 5:00 PM & 0.453 & 18.9 & 0.215 & 0.135 & 19.1 & 0.19 & 0.136 & -- & -- & -- & -- & -- & -- \\
\hline 8/15/2008 6:00 PM & 0.463 & 18.9 & 0.221 & 0.135 & 19.1 & 0.19 & 0.135 & -- & -- & -- & -- & -- & -- \\
\hline 8/15/2008 7:00 PM & 0.461 & 18.8 & 0.227 & 0.135 & 19.1 & 0.19 & 0.136 & -- & -- & -- & -- & -- & -- \\
\hline 8/15/2008 8:00 PM & 0.459 & 18.7 & 0.232 & 0.135 & 19.1 & 0.19 & 0.136 & -- & -- & -- & -- & -- & -- \\
\hline 8/15/2008 9:00 PM & 0.457 & 18.7 & 0.234 & 0.136 & 19.1 & 0.19 & 0.136 & -- & -- & -- & -- & -- & -- \\
\hline
\end{tabular}


Appendix 3. Volumetric water content, temperature, and electrical-conductivity data collected at the sprinkler-irrigated site during 2008.-Continued

[Depth in feet below land surface; volumetric water content dimensionless; temperature in degrees Celsius; bulk electrical conductivity in decisiemens per meter; --, no data]

\begin{tabular}{|c|c|c|c|c|c|c|c|c|c|c|c|c|c|}
\hline \multirow[b]{3}{*}{$\begin{array}{l}\text { Measurement } \\
\text { date and time }\end{array}$} & \multicolumn{13}{|c|}{ Depth } \\
\hline & \multicolumn{2}{|l|}{2} & \multirow{2}{*}{$\begin{array}{c}3 \\
\text { Volumetric } \\
\text { water } \\
\text { content }\end{array}$} & \multicolumn{3}{|c|}{4} & \multirow{2}{*}{$\begin{array}{c}\mathbf{5} \\
\text { Volumetric } \\
\text { water } \\
\text { content }\end{array}$} & \multicolumn{2}{|l|}{7} & \multirow{2}{*}{$\begin{array}{c}9 \\
\text { Volumetric } \\
\text { water } \\
\text { content }\end{array}$} & \multicolumn{2}{|c|}{13} & \multirow{2}{*}{$\begin{array}{c}16.5 \\
\text { Volumetric } \\
\text { water } \\
\text { content }\end{array}$} \\
\hline & $\begin{array}{c}\text { Volumetric } \\
\text { water } \\
\text { content }\end{array}$ & $\begin{array}{l}\text { Temper- } \\
\text { ature }\end{array}$ & & $\begin{array}{c}\text { Volumetric } \\
\text { water } \\
\text { content }\end{array}$ & $\begin{array}{l}\text { Temper- } \\
\text { ature }\end{array}$ & $\begin{array}{c}\text { Bulk } \\
\text { electrical } \\
\text { conduct- } \\
\text { ivity }\end{array}$ & & $\begin{array}{c}\text { Volumetric } \\
\text { water } \\
\text { content }\end{array}$ & $\begin{array}{l}\text { Temper- } \\
\text { ature }\end{array}$ & & $\begin{array}{c}\text { Volumetric } \\
\text { water } \\
\text { content }\end{array}$ & $\begin{array}{l}\text { Temper- } \\
\text { ature }\end{array}$ & \\
\hline 8/15/2008 10:00 PM & 0.454 & 18.6 & 0.235 & 0.137 & 19.1 & 0.19 & 0.136 & -- & -- & -- & -- & -- & -- \\
\hline 8/15/2008 11:00 PM & 0.450 & 18.6 & 0.235 & 0.138 & 19.1 & 0.19 & 0.136 & -- & -- & -- & -- & -- & -- \\
\hline 8/16/2008 12:00 AM & 0.447 & 18.5 & 0.235 & 0.140 & 19.1 & 0.20 & 0.136 & -- & -- & -- & -- & -- & -- \\
\hline 8/16/2008 1:00 AM & 0.445 & 18.5 & 0.234 & 0.141 & 19.1 & 0.20 & 0.137 & -- & -- & -- & -- & -- & -- \\
\hline 8/16/2008 2:00 AM & 0.443 & 18.4 & 0.234 & 0.143 & 19.1 & 0.20 & 0.137 & -- & -- & -- & -- & -- & -- \\
\hline 8/16/2008 3:00 AM & 0.441 & 18.4 & 0.234 & 0.144 & 19.1 & 0.21 & 0.138 & -- & -- & -- & -- & -- & -- \\
\hline 8/16/2008 4:00 AM & 0.438 & 18.4 & 0.233 & 0.145 & 19.1 & 0.21 & 0.138 & -- & -- & -- & -- & -- & -- \\
\hline 8/16/2008 5:00 AM & 0.436 & 18.3 & 0.232 & 0.146 & 19.1 & 0.21 & 0.138 & -- & -- & -- & -- & -- & -- \\
\hline 8/16/2008 6:00 AM & 0.433 & 18.3 & 0.232 & 0.148 & 19.1 & 0.21 & 0.139 & -- & -- & -- & -- & -- & -- \\
\hline 8/16/2008 7:00 AM & 0.432 & 18.2 & 0.231 & 0.148 & 19.1 & 0.22 & 0.139 & -- & -- & -- & -- & -- & -- \\
\hline 8/16/2008 8:00 AM & 0.436 & 18.2 & 0.231 & 0.149 & 19.1 & 0.22 & 0.140 & -- & -- & -- & -- & -- & -- \\
\hline 8/16/2008 9:00 AM & 0.443 & 18.1 & 0.232 & 0.149 & 19.1 & 0.22 & 0.141 & -- & -- & -- & -- & -- & -- \\
\hline 8/16/2008 10:00 AM & 0.453 & 18.1 & 0.234 & 0.150 & 19.1 & 0.22 & 0.141 & -- & -- & -- & -- & -- & -- \\
\hline 8/16/2008 11:00 AM & 0.485 & 18.0 & 0.239 & 0.150 & 19.1 & 0.22 & 0.142 & -- & -- & -- & -- & -- & -- \\
\hline 8/16/2008 12:00 PM & 0.491 & 17.9 & 0.272 & 0.154 & 19.0 & 0.23 & 0.143 & -- & -- & -- & -- & -- & -- \\
\hline 8/16/2008 1:00 PM & 0.491 & 17.8 & 0.305 & 0.159 & 19.0 & 0.24 & 0.143 & -- & -- & -- & -- & -- & -- \\
\hline 8/16/2008 2:00 PM & 0.492 & 17.7 & 0.393 & 0.173 & 19.1 & 0.27 & 0.144 & -- & -- & -- & -- & -- & -- \\
\hline 8/16/2008 3:00 PM & 0.491 & 17.7 & 0.360 & 0.193 & 19.1 & 0.31 & 0.146 & -- & -- & -- & -- & -- & -- \\
\hline 8/16/2008 4:00 PM & 0.475 & 17.6 & 0.301 & 0.206 & 19.0 & 0.33 & 0.149 & -- & -- & -- & -- & -- & -- \\
\hline 8/16/2008 5:00 PM & 0.467 & 17.6 & 0.281 & 0.210 & 19.1 & 0.36 & 0.154 & -- & -- & -- & -- & -- & -- \\
\hline 8/16/2008 6:00 PM & 0.459 & 17.6 & 0.273 & 0.210 & 19.0 & 0.36 & 0.159 & -- & -- & -- & -- & -- & -- \\
\hline 8/16/2008 7:00 PM & 0.455 & 17.6 & 0.267 & 0.207 & 19.0 & 0.36 & 0.163 & -- & -- & -- & -- & -- & -- \\
\hline 8/16/2008 8:00 PM & 0.451 & 17.5 & 0.264 & 0.204 & 19.0 & 0.35 & 0.166 & -- & -- & -- & -- & -- & -- \\
\hline 8/16/2008 9:00 PM & 0.448 & 17.5 & 0.261 & 0.200 & 19.0 & 0.34 & 0.168 & -- & -- & -- & -- & -- & -- \\
\hline 8/16/2008 10:00 PM & 0.446 & 17.5 & 0.257 & 0.197 & 19.0 & 0.33 & 0.170 & -- & -- & -- & -- & -- & -- \\
\hline 8/16/2008 11:00 PM & 0.443 & 17.5 & 0.255 & 0.195 & 19.0 & 0.32 & 0.171 & -- & -- & -- & -- & -- & -- \\
\hline 8/17/2008 12:00 AM & 0.441 & 17.4 & 0.252 & 0.193 & 19.0 & 0.32 & 0.171 & -- & -- & -- & -- & -- & -- \\
\hline 8/17/2008 1:00 AM & 0.437 & 17.4 & 0.250 & 0.190 & 19.0 & 0.31 & 0.172 & -- & -- & -- & -- & -- & -- \\
\hline 8/17/2008 2:00 AM & 0.436 & 17.4 & 0.246 & 0.186 & 19.0 & 0.30 & 0.172 & -- & -- & -- & -- & -- & -- \\
\hline 8/17/2008 3:00 AM & 0.434 & 17.4 & 0.245 & 0.184 & 19.0 & 0.29 & 0.172 & -- & -- & -- & -- & -- & -- \\
\hline 8/17/2008 4:00 AM & 0.432 & 17.4 & 0.242 & 0.182 & 19.0 & 0.29 & 0.172 & -- & -- & -- & -- & -- & -- \\
\hline 8/17/2008 5:00 AM & 0.430 & 17.4 & 0.239 & 0.181 & 18.9 & 0.28 & 0.172 & -- & -- & -- & -- & -- & -- \\
\hline 8/17/2008 6:00 AM & 0.430 & 17.3 & 0.239 & 0.179 & 18.9 & 0.28 & 0.172 & -- & -- & -- & -- & -- & -- \\
\hline 8/17/2008 7:00 AM & 0.429 & 17.3 & 0.238 & 0.176 & 18.9 & 0.27 & 0.171 & -- & -- & -- & -- & -- & -- \\
\hline 8/17/2008 8:00 AM & 0.426 & 17.3 & 0.236 & 0.175 & 18.9 & 0.27 & 0.171 & -- & -- & -- & -- & -- & -- \\
\hline
\end{tabular}


Appendix 3. Volumetric water content, temperature, and electrical-conductivity data collected at the sprinkler-irrigated site during 2008.-Continued

[Depth in feet below land surface; volumetric water content dimensionless; temperature in degrees Celsius; bulk electrical conductivity in decisiemens per meter; --, no data]

\begin{tabular}{|c|c|c|c|c|c|c|c|c|c|c|c|c|c|}
\hline \multirow[b]{3}{*}{$\begin{array}{l}\text { Measurement } \\
\text { date and time }\end{array}$} & \multicolumn{13}{|c|}{ Depth } \\
\hline & \multicolumn{2}{|c|}{2} & \multirow{2}{*}{$\begin{array}{c}3 \\
\begin{array}{c}\text { Volumetric } \\
\text { water } \\
\text { content }\end{array}\end{array}$} & \multicolumn{3}{|c|}{4} & \multirow{2}{*}{$\begin{array}{c}5 \\
\text { Volumetric } \\
\text { water } \\
\text { content }\end{array}$} & \multicolumn{2}{|l|}{7} & \multirow{2}{*}{$\begin{array}{c}9 \\
\begin{array}{c}\text { Volumetric } \\
\text { water } \\
\text { content }\end{array}\end{array}$} & \multicolumn{2}{|c|}{13} & \multirow{2}{*}{$\begin{array}{c}16.5 \\
\text { Volumetric } \\
\text { water } \\
\text { content }\end{array}$} \\
\hline & $\begin{array}{c}\text { Volumetric } \\
\text { water } \\
\text { content }\end{array}$ & $\begin{array}{l}\text { Temper- } \\
\text { ature }\end{array}$ & & $\begin{array}{c}\text { Volumetric } \\
\text { water } \\
\text { content }\end{array}$ & $\begin{array}{l}\text { Temper- } \\
\text { ature }\end{array}$ & $\begin{array}{c}\text { Bulk } \\
\text { electrical } \\
\text { conduct- } \\
\text { ivity }\end{array}$ & & $\begin{array}{l}\text { Volumetric } \\
\text { water } \\
\text { content }\end{array}$ & $\begin{array}{l}\text { Temper- } \\
\text { ature }\end{array}$ & & $\begin{array}{c}\text { Volumetric } \\
\text { water } \\
\text { content }\end{array}$ & $\begin{array}{l}\text { Temper- } \\
\text { ature }\end{array}$ & \\
\hline 8/17/2008 9:00 AM & 0.425 & 17.3 & 0.235 & 0.174 & 18.9 & 0.27 & 0.171 & -- & -- & -- & -- & -- & -- \\
\hline 8/17/2008 10:00 AM & 0.424 & 17.2 & 0.235 & 0.173 & 18.9 & 0.26 & 0.171 & -- & -- & -- & -- & -- & -- \\
\hline 8/17/2008 11:00 AM & 0.423 & 17.2 & 0.234 & 0.172 & 18.9 & 0.26 & 0.171 & -- & -- & -- & -- & -- & -- \\
\hline 8/17/2008 12:00 PM & 0.422 & 17.2 & 0.233 & 0.171 & 18.9 & 0.26 & 0.170 & -- & -- & -- & -- & -- & -- \\
\hline 8/17/2008 1:00 PM & 0.421 & 17.2 & 0.232 & 0.170 & 18.9 & 0.26 & 0.170 & -- & -- & -- & -- & -- & -- \\
\hline 8/17/2008 2:00 PM & 0.420 & 17.2 & 0.231 & 0.169 & 18.8 & 0.25 & 0.169 & -- & -- & -- & -- & -- & -- \\
\hline 8/17/2008 3:00 PM & 0.418 & 17.1 & 0.230 & 0.168 & 18.8 & 0.25 & 0.169 & -- & -- & -- & -- & -- & -- \\
\hline 8/17/2008 4:00 PM & 0.417 & 17.1 & 0.230 & 0.168 & 18.8 & 0.25 & 0.168 & -- & -- & -- & -- & -- & -- \\
\hline 8/17/2008 5:00 PM & 0.417 & 17.1 & 0.229 & 0.167 & 18.8 & 0.25 & 0.167 & -- & -- & -- & -- & -- & -- \\
\hline 8/17/2008 6:00 PM & 0.415 & 17.1 & 0.228 & 0.166 & 18.8 & 0.25 & 0.167 & -- & -- & -- & -- & -- & -- \\
\hline 8/17/2008 7:00 PM & 0.415 & 17.1 & 0.228 & 0.165 & 18.8 & 0.24 & 0.166 & -- & -- & -- & -- & -- & -- \\
\hline 8/17/2008 8:00 PM & 0.413 & 17.1 & 0.227 & 0.163 & 18.8 & 0.24 & 0.166 & -- & -- & -- & -- & -- & -- \\
\hline 8/17/2008 9:00 PM & 0.412 & 17.1 & 0.227 & 0.162 & 18.8 & 0.24 & 0.166 & -- & -- & -- & -- & -- & -- \\
\hline 8/17/2008 10:00 PM & 0.412 & 17.1 & 0.226 & 0.162 & 18.7 & 0.24 & 0.165 & -- & -- & -- & -- & -- & -- \\
\hline 8/17/2008 11:00 PM & 0.411 & 17.1 & 0.226 & 0.161 & 18.8 & 0.24 & 0.164 & -- & -- & -- & -- & -- & -- \\
\hline 8/18/2008 12:00 AM & 0.410 & 17.1 & 0.225 & 0.161 & 18.7 & 0.23 & 0.164 & -- & -- & -- & -- & -- & -- \\
\hline 8/18/2008 1:00 AM & 0.410 & 17.1 & 0.225 & 0.160 & 18.7 & 0.23 & 0.163 & -- & -- & -- & -- & -- & -- \\
\hline 8/18/2008 2:00 AM & 0.409 & 17.1 & 0.224 & 0.159 & 18.7 & 0.23 & 0.163 & -- & -- & -- & -- & -- & -- \\
\hline 8/18/2008 3:00 AM & 0.409 & 17.1 & 0.223 & 0.159 & 18.7 & 0.23 & 0.162 & -- & -- & -- & -- & -- & -- \\
\hline 8/18/2008 4:00 AM & 0.408 & 17.1 & 0.223 & 0.159 & 18.7 & 0.23 & 0.161 & -- & -- & -- & -- & -- & -- \\
\hline 8/18/2008 5:00 AM & 0.408 & 17.1 & 0.222 & 0.158 & 18.7 & 0.23 & 0.161 & -- & -- & -- & -- & -- & -- \\
\hline 8/18/2008 6:00 AM & 0.407 & 17.2 & 0.222 & 0.157 & 18.7 & 0.23 & 0.160 & -- & -- & -- & -- & -- & -- \\
\hline 8/18/2008 7:00 AM & 0.407 & 17.2 & 0.222 & 0.157 & 18.7 & 0.23 & 0.160 & -- & -- & -- & -- & -- & -- \\
\hline 8/18/2008 8:00 AM & 0.406 & 17.2 & 0.222 & 0.156 & 18.6 & 0.23 & 0.160 & -- & -- & -- & -- & -- & -- \\
\hline 8/18/2008 9:00 AM & 0.406 & 17.2 & 0.222 & 0.156 & 18.6 & 0.22 & 0.160 & -- & -- & -- & -- & -- & -- \\
\hline 8/18/2008 10:00 AM & 0.405 & 17.2 & 0.221 & 0.156 & 18.6 & 0.22 & 0.159 & -- & -- & -- & -- & -- & -- \\
\hline 8/18/2008 11:00 AM & 0.405 & 17.2 & 0.220 & 0.155 & 18.6 & 0.22 & 0.159 & -- & -- & -- & -- & -- & -- \\
\hline 8/18/2008 12:00 PM & 0.404 & 17.1 & 0.220 & 0.155 & 18.6 & 0.22 & 0.158 & -- & -- & -- & -- & -- & -- \\
\hline 8/18/2008 1:00 PM & 0.404 & 17.2 & 0.219 & 0.155 & 18.6 & 0.22 & 0.158 & -- & -- & -- & -- & -- & -- \\
\hline 8/18/2008 2:00 PM & 0.403 & 17.2 & 0.219 & 0.155 & 18.6 & 0.22 & 0.158 & -- & -- & -- & -- & -- & -- \\
\hline 8/18/2008 3:00 PM & 0.401 & 17.2 & 0.218 & 0.154 & 18.6 & 0.22 & 0.157 & -- & -- & -- & -- & -- & -- \\
\hline 8/18/2008 4:00 PM & 0.401 & 17.1 & 0.218 & 0.154 & 18.6 & 0.22 & 0.157 & -- & -- & -- & -- & -- & -- \\
\hline 8/18/2008 5:00 PM & 0.400 & 17.1 & 0.218 & 0.153 & 18.6 & 0.22 & 0.156 & -- & -- & -- & -- & -- & -- \\
\hline 8/18/2008 6:00 PM & 0.400 & 17.1 & 0.217 & 0.153 & 18.6 & 0.22 & 0.156 & -- & -- & -- & -- & -- & -- \\
\hline 8/18/2008 7:00 PM & 0.399 & 17.1 & 0.217 & 0.151 & 18.5 & 0.22 & 0.155 & -- & -- & -- & -- & -- & -- \\
\hline
\end{tabular}


Appendix 3. Volumetric water content, temperature, and electrical-conductivity data collected at the sprinkler-irrigated site during 2008.-Continued

[Depth in feet below land surface; volumetric water content dimensionless; temperature in degrees Celsius; bulk electrical conductivity in decisiemens per meter; --, no data]

\begin{tabular}{|c|c|c|c|c|c|c|c|c|c|c|c|c|c|}
\hline \multirow[b]{3}{*}{$\begin{array}{l}\text { Measurement } \\
\text { date and time }\end{array}$} & \multirow{2}{*}{\multicolumn{2}{|c|}{$\begin{array}{r} \\
2\end{array}$}} & \multicolumn{10}{|c|}{ Depth } & \multirow{3}{*}{$\begin{array}{c}16.5 \\
\text { Volumetric } \\
\text { water } \\
\text { content }\end{array}$} \\
\hline & & & \multirow[b]{2}{*}{$\begin{array}{c}3 \\
\begin{array}{c}\text { Volumetric } \\
\text { water } \\
\text { content }\end{array}\end{array}$} & \multicolumn{3}{|c|}{4} & \multirow[b]{2}{*}{$\begin{array}{c}\mathbf{5} \\
\text { Volumetric } \\
\text { water } \\
\text { content }\end{array}$} & \multicolumn{2}{|c|}{7} & \multirow{2}{*}{$\begin{array}{c}9 \\
\text { Volumetric } \\
\text { water } \\
\text { content }\end{array}$} & \multicolumn{2}{|c|}{13} & \\
\hline & $\begin{array}{c}\text { Volumetric } \\
\text { water } \\
\text { content }\end{array}$ & $\begin{array}{l}\text { Temper- } \\
\text { ature }\end{array}$ & & $\begin{array}{c}\text { Volumetric } \\
\text { water } \\
\text { content }\end{array}$ & $\begin{array}{l}\text { Temper- } \\
\text { ature }\end{array}$ & $\begin{array}{c}\text { Bulk } \\
\text { electrical } \\
\text { conduct- } \\
\text { ivity }\end{array}$ & & $\begin{array}{c}\text { Volumetric } \\
\text { water } \\
\text { content }\end{array}$ & $\begin{array}{l}\text { Temper- } \\
\text { ature }\end{array}$ & & $\begin{array}{c}\text { Volumetric } \\
\text { water } \\
\text { content }\end{array}$ & $\begin{array}{l}\text { Temper- } \\
\text { ature }\end{array}$ & \\
\hline 8/18/2008 8:00 PM & 0.399 & 17.1 & 0.217 & 0.151 & 18.5 & 0.22 & 0.155 & -- & -- & -- & -- & -- & -- \\
\hline 8/18/2008 9:00 PM & 0.398 & 17.1 & 0.217 & 0.151 & 18.5 & 0.21 & 0.155 & -- & -- & -- & -- & -- & -- \\
\hline 8/18/2008 10:00 PM & 0.398 & 17.1 & 0.217 & 0.150 & 18.5 & 0.21 & 0.155 & -- & -- & -- & -- & -- & -- \\
\hline 8/18/2008 11:00 PM & 0.398 & 17.2 & 0.217 & 0.150 & 18.5 & 0.21 & 0.155 & -- & -- & -- & -- & -- & -- \\
\hline 8/19/2008 12:00 AM & 0.398 & 17.2 & 0.217 & 0.150 & 18.5 & 0.21 & 0.154 & -- & -- & -- & -- & -- & -- \\
\hline 8/19/2008 1:00 AM & 0.397 & 17.2 & 0.216 & 0.149 & 18.5 & 0.21 & 0.154 & -- & -- & -- & -- & -- & -- \\
\hline 8/19/2008 2:00 AM & 0.397 & 17.2 & 0.216 & 0.149 & 18.5 & 0.21 & 0.153 & -- & -- & -- & -- & -- & -- \\
\hline 8/19/2008 3:00 AM & 0.397 & 17.2 & 0.216 & 0.149 & 18.5 & 0.21 & 0.153 & -- & -- & -- & -- & -- & -- \\
\hline 8/19/2008 4:00 AM & 0.397 & 17.3 & 0.216 & 0.149 & 18.5 & 0.21 & 0.153 & -- & -- & -- & -- & -- & -- \\
\hline 8/19/2008 5:00 AM & 0.396 & 17.3 & 0.215 & 0.149 & 18.5 & 0.21 & 0.152 & -- & -- & -- & -- & -- & -- \\
\hline 8/19/2008 6:00 AM & 0.396 & 17.3 & 0.215 & 0.149 & 18.5 & 0.21 & 0.152 & -- & -- & -- & -- & -- & -- \\
\hline 8/19/2008 7:00 AM & 0.396 & 17.3 & 0.215 & 0.149 & 18.4 & 0.21 & 0.152 & -- & -- & -- & -- & -- & -- \\
\hline 8/19/2008 8:00 AM & 0.395 & 17.3 & 0.215 & 0.148 & 18.4 & 0.21 & 0.151 & -- & -- & -- & -- & -- & -- \\
\hline 8/19/2008 9:00 AM & 0.395 & 17.3 & 0.215 & 0.148 & 18.4 & 0.21 & 0.151 & -- & -- & -- & -- & -- & -- \\
\hline 8/19/2008 10:00 AM & 0.395 & 17.3 & 0.214 & 0.148 & 18.4 & 0.21 & 0.151 & -- & -- & -- & -- & -- & -- \\
\hline 8/19/2008 11:00 AM & 0.395 & 17.3 & 0.214 & 0.148 & 18.4 & 0.21 & 0.151 & -- & -- & -- & -- & -- & -- \\
\hline 8/19/2008 12:00 PM & 0.395 & 17.3 & 0.214 & 0.148 & 18.4 & 0.21 & 0.150 & -- & -- & -- & -- & -- & -- \\
\hline 8/19/2008 1:00 PM & 0.395 & 17.3 & 0.213 & 0.147 & 18.4 & 0.21 & 0.150 & -- & -- & -- & -- & -- & -- \\
\hline 8/19/2008 2:00 PM & 0.394 & 17.3 & 0.213 & 0.147 & 18.4 & 0.21 & 0.150 & -- & -- & -- & -- & -- & -- \\
\hline 8/19/2008 3:00 PM & 0.394 & 17.3 & 0.213 & 0.147 & 18.4 & 0.21 & 0.150 & -- & -- & -- & -- & -- & -- \\
\hline 8/19/2008 4:00 PM & 0.393 & 17.3 & 0.213 & 0.147 & 18.4 & 0.20 & 0.150 & -- & -- & -- & -- & -- & -- \\
\hline 8/19/2008 5:00 PM & 0.393 & 17.3 & 0.212 & 0.146 & 18.4 & 0.20 & 0.149 & -- & -- & -- & -- & -- & -- \\
\hline 8/19/2008 6:00 PM & 0.392 & 17.3 & 0.212 & 0.146 & 18.4 & 0.20 & 0.149 & -- & -- & -- & -- & -- & -- \\
\hline 8/19/2008 7:00 PM & 0.391 & 17.3 & 0.212 & 0.146 & 18.4 & 0.20 & 0.149 & -- & -- & -- & -- & -- & -- \\
\hline 8/19/2008 8:00 PM & 0.391 & 17.3 & 0.212 & 0.146 & 18.3 & 0.20 & 0.149 & -- & -- & -- & -- & -- & -- \\
\hline 8/19/2008 9:00 PM & 0.391 & 17.3 & 0.211 & 0.145 & 18.4 & 0.20 & 0.149 & -- & -- & -- & -- & -- & -- \\
\hline 8/19/2008 10:00 PM & 0.391 & 17.3 & 0.212 & 0.145 & 18.4 & 0.20 & 0.149 & -- & -- & -- & -- & -- & -- \\
\hline 8/19/2008 11:00 PM & 0.390 & 17.3 & 0.211 & 0.145 & 18.3 & 0.20 & 0.148 & -- & -- & -- & -- & -- & -- \\
\hline 8/20/2008 12:00 AM & 0.391 & 17.3 & 0.211 & 0.145 & 18.3 & 0.20 & 0.148 & & -- & & & -- & \\
\hline 8/20/2008 1:00 AM & 0.391 & 17.3 & 0.211 & 0.145 & 18.4 & 0.20 & 0.148 & -- & -- & -- & -- & -- & -- \\
\hline 8/20/2008 2:00 AM & 0.391 & 17.3 & 0.211 & 0.145 & 18.3 & 0.20 & 0.148 & -- & -- & -- & -- & -- & -- \\
\hline 8/20/2008 3:00 AM & 0.391 & 17.4 & 0.211 & 0.145 & 18.3 & 0.20 & 0.148 & -- & -- & -- & -- & -- & -- \\
\hline 8/20/2008 4:00 AM & 0.391 & 17.4 & 0.211 & 0.144 & 18.3 & 0.20 & 0.147 & -- & -- & -- & -- & -- & -- \\
\hline 8/20/2008 5:00 AM & 0.390 & 17.4 & 0.211 & 0.144 & 18.3 & 0.20 & 0.147 & -- & -- & -- & -- & -- & -- \\
\hline 8/20/2008 6:00 AM & 0.390 & 17.4 & 0.211 & 0.144 & 18.3 & 0.20 & 0.147 & -- & -- & -- & -- & -- & -- \\
\hline
\end{tabular}


Appendix 3. Volumetric water content, temperature, and electrical-conductivity data collected at the sprinkler-irrigated site during 2008.-Continued

[Depth in feet below land surface; volumetric water content dimensionless; temperature in degrees Celsius; bulk electrical conductivity in decisiemens per meter; --, no data]

\begin{tabular}{|c|c|c|c|c|c|c|c|c|c|c|c|c|c|}
\hline \multirow[b]{3}{*}{$\begin{array}{l}\text { Measurement } \\
\text { date and time }\end{array}$} & \multicolumn{13}{|c|}{ Depth } \\
\hline & \multicolumn{2}{|c|}{2} & \multirow{2}{*}{$\begin{array}{c}3 \\
\text { Volumetric } \\
\text { water } \\
\text { content }\end{array}$} & \multicolumn{3}{|c|}{4} & \multirow{2}{*}{$\begin{array}{c}5 \\
\text { Volumetric } \\
\text { water } \\
\text { content }\end{array}$} & \multicolumn{2}{|c|}{7} & \multirow{2}{*}{$\begin{array}{c}9 \\
\text { Volumetric } \\
\text { water } \\
\text { content }\end{array}$} & \multicolumn{2}{|c|}{13} & \multirow{2}{*}{$\begin{array}{c}16.5 \\
\text { Volumetric } \\
\text { water } \\
\text { content }\end{array}$} \\
\hline & $\begin{array}{c}\text { Volumetric } \\
\text { water } \\
\text { content }\end{array}$ & $\begin{array}{l}\text { Temper- } \\
\text { ature }\end{array}$ & & $\begin{array}{c}\text { Volumetric } \\
\text { water } \\
\text { content }\end{array}$ & $\begin{array}{l}\text { Temper- } \\
\text { ature }\end{array}$ & $\begin{array}{c}\text { Bulk } \\
\text { electrical } \\
\text { conduct- } \\
\text { ivity }\end{array}$ & & $\begin{array}{c}\text { Volumetric } \\
\text { water } \\
\text { content }\end{array}$ & $\begin{array}{l}\text { Temper- } \\
\text { ature }\end{array}$ & & $\begin{array}{c}\text { Volumetric } \\
\text { water } \\
\text { content }\end{array}$ & $\begin{array}{l}\text { Temper- } \\
\text { ature }\end{array}$ & \\
\hline 8/20/2008 7:00 AM & 0.390 & 17.4 & 0.211 & 0.144 & 18.3 & 0.20 & 0.147 & -- & -- & -- & -- & -- & -- \\
\hline 8/20/2008 8:00 AM & 0.390 & 17.4 & 0.211 & 0.144 & 18.3 & 0.20 & 0.146 & -- & -- & -- & -- & -- & -- \\
\hline 8/20/2008 9:00 AM & 0.390 & 17.5 & 0.210 & 0.143 & 18.3 & 0.20 & 0.146 & -- & -- & -- & -- & -- & -- \\
\hline 8/20/2008 10:00 AM & 0.390 & 17.5 & 0.210 & 0.144 & 18.3 & 0.20 & 0.146 & -- & -- & -- & -- & -- & -- \\
\hline 8/20/2008 11:00 AM & 0.390 & 17.5 & 0.210 & 0.143 & 18.3 & 0.20 & 0.146 & -- & -- & -- & -- & -- & -- \\
\hline 8/20/2008 12:00 PM & 0.390 & 17.5 & 0.210 & 0.143 & 18.3 & 0.20 & 0.146 & -- & -- & -- & -- & -- & -- \\
\hline 8/20/2008 1:00 PM & 0.388 & 17.5 & 0.209 & 0.143 & 18.3 & 0.20 & 0.146 & -- & -- & -- & -- & -- & -- \\
\hline 8/20/2008 2:00 PM & 0.388 & 17.5 & 0.209 & 0.143 & 18.3 & 0.20 & 0.146 & -- & -- & -- & -- & -- & -- \\
\hline 8/20/2008 3:00 PM & 0.387 & 17.5 & 0.209 & 0.143 & 18.3 & 0.20 & 0.145 & -- & -- & -- & -- & -- & -- \\
\hline 8/20/2008 4:00 PM & 0.387 & 17.5 & 0.209 & 0.143 & 18.3 & 0.20 & 0.145 & -- & -- & -- & -- & -- & -- \\
\hline 8/20/2008 5:00 PM & 0.387 & 17.5 & 0.209 & 0.143 & 18.2 & 0.20 & 0.145 & -- & -- & -- & -- & -- & -- \\
\hline 8/20/2008 6:00 PM & 0.387 & 17.5 & 0.208 & 0.142 & 18.3 & 0.20 & 0.145 & -- & -- & -- & -- & -- & -- \\
\hline 8/20/2008 7:00 PM & 0.386 & 17.5 & 0.208 & 0.142 & 18.2 & 0.19 & 0.145 & -- & -- & -- & -- & -- & -- \\
\hline 8/20/2008 8:00 PM & 0.386 & 17.5 & 0.208 & 0.142 & 18.2 & 0.19 & 0.144 & -- & -- & -- & -- & -- & -- \\
\hline 8/20/2008 9:00 PM & 0.386 & 17.5 & 0.208 & 0.142 & 18.2 & 0.19 & 0.144 & -- & -- & -- & -- & -- & -- \\
\hline 8/20/2008 10:00 PM & 0.386 & 17.5 & 0.208 & 0.142 & 18.2 & 0.19 & 0.144 & -- & -- & -- & -- & -- & -- \\
\hline 8/20/2008 11:00 PM & 0.386 & 17.5 & 0.208 & 0.142 & 18.2 & 0.19 & 0.144 & -- & -- & -- & -- & -- & -- \\
\hline 8/21/2008 12:00 AM & 0.386 & 17.5 & 0.208 & 0.141 & 18.2 & 0.19 & 0.143 & -- & -- & -- & -- & -- & -- \\
\hline $8 / 21 / 2008$ 1:00 AM & 0.386 & 17.5 & 0.208 & 0.141 & 18.2 & 0.19 & 0.143 & -- & -- & -- & -- & -- & -- \\
\hline 8/21/2008 2:00 AM & 0.386 & 17.5 & 0.208 & 0.141 & 18.2 & 0.19 & 0.143 & -- & -- & -- & -- & -- & -- \\
\hline 8/21/2008 3:00 AM & 0.386 & 17.6 & 0.207 & 0.141 & 18.2 & 0.19 & 0.143 & -- & -- & -- & -- & -- & -- \\
\hline 8/21/2008 4:00 AM & 0.386 & 17.6 & 0.207 & 0.141 & 18.2 & 0.19 & 0.143 & -- & -- & -- & -- & -- & -- \\
\hline 8/21/2008 5:00 AM & 0.386 & 17.6 & 0.207 & 0.141 & 18.2 & 0.19 & 0.143 & -- & -- & -- & -- & -- & -- \\
\hline 8/21/2008 6:00 AM & 0.386 & 17.6 & 0.207 & 0.141 & 18.2 & 0.19 & 0.143 & -- & -- & -- & -- & -- & -- \\
\hline 8/21/2008 7:00 AM & 0.386 & 17.6 & 0.207 & 0.141 & 18.2 & 0.19 & 0.143 & -- & -- & -- & -- & -- & -- \\
\hline 8/21/2008 8:00 AM & 0.386 & 17.6 & 0.207 & 0.141 & 18.2 & 0.19 & 0.143 & -- & -- & -- & -- & -- & -- \\
\hline 8/21/2008 9:00 AM & 0.386 & 17.7 & 0.207 & 0.141 & 18.2 & 0.19 & 0.143 & -- & -- & -- & -- & -- & -- \\
\hline 8/21/2008 10:00 AM & 0.386 & 17.7 & 0.206 & 0.140 & 18.2 & 0.19 & 0.143 & -- & -- & -- & -- & -- & -- \\
\hline 8/21/2008 11:00 AM & 0.386 & 17.7 & 0.206 & 0.140 & 18.2 & 0.19 & 0.143 & -- & -- & -- & -- & -- & -- \\
\hline 8/21/2008 12:00 PM & 0.386 & 17.7 & 0.206 & 0.141 & 18.2 & 0.19 & 0.143 & -- & -- & -- & -- & -- & -- \\
\hline 8/21/2008 1:00 PM & 0.385 & 17.7 & 0.206 & 0.140 & 18.2 & 0.19 & 0.143 & -- & -- & -- & -- & -- & -- \\
\hline 8/21/2008 2:00 PM & 0.385 & 17.7 & 0.205 & 0.140 & 18.2 & 0.19 & 0.143 & -- & -- & -- & -- & -- & -- \\
\hline 8/21/2008 3:00 PM & 0.384 & 17.7 & 0.205 & 0.140 & 18.2 & 0.19 & 0.142 & -- & -- & -- & -- & -- & -- \\
\hline 8/21/2008 4:00 PM & 0.383 & 17.7 & 0.205 & 0.140 & 18.2 & 0.19 & 0.142 & -- & -- & -- & -- & -- & -- \\
\hline 8/21/2008 5:00 PM & 0.383 & 17.7 & 0.205 & 0.140 & 18.2 & 0.19 & 0.142 & -- & -- & -- & -- & -- & -- \\
\hline
\end{tabular}


Appendix 3. Volumetric water content, temperature, and electrical-conductivity data collected at the sprinkler-irrigated site during 2008.-Continued

[Depth in feet below land surface; volumetric water content dimensionless; temperature in degrees Celsius; bulk electrical conductivity in decisiemens per meter; --, no data]

\begin{tabular}{|c|c|c|c|c|c|c|c|c|c|c|c|c|c|}
\hline \multirow[b]{3}{*}{$\begin{array}{l}\text { Measurement } \\
\text { date and time }\end{array}$} & \multicolumn{13}{|c|}{ Depth } \\
\hline & \multicolumn{2}{|c|}{2} & \multirow{2}{*}{$\begin{array}{c}3 \\
\text { Volumetric } \\
\text { water } \\
\text { content }\end{array}$} & \multicolumn{3}{|c|}{4} & \multirow{2}{*}{$\begin{array}{c}\mathbf{5} \\
\begin{array}{c}\text { Volumetric } \\
\text { water } \\
\text { content }\end{array}\end{array}$} & \multicolumn{2}{|l|}{7} & \multirow{2}{*}{$\begin{array}{c}9 \\
\text { Volumetric } \\
\text { water } \\
\text { content }\end{array}$} & \multicolumn{2}{|c|}{13} & \multirow{2}{*}{$\begin{array}{c}16.5 \\
\text { Volumetric } \\
\text { water } \\
\text { content }\end{array}$} \\
\hline & $\begin{array}{c}\text { Volumetric } \\
\text { water } \\
\text { content }\end{array}$ & $\begin{array}{l}\text { Temper- } \\
\text { ature }\end{array}$ & & $\begin{array}{c}\text { Volumetric } \\
\text { water } \\
\text { content }\end{array}$ & $\begin{array}{l}\text { Temper- } \\
\text { ature }\end{array}$ & $\begin{array}{c}\text { Bulk } \\
\text { electrical } \\
\text { conduct- } \\
\text { ivity }\end{array}$ & & $\begin{array}{c}\text { Volumetric } \\
\text { water } \\
\text { content }\end{array}$ & $\begin{array}{l}\text { Temper- } \\
\text { ature }\end{array}$ & & $\begin{array}{c}\text { Volumetric } \\
\text { water } \\
\text { content }\end{array}$ & $\begin{array}{l}\text { Temper- } \\
\text { ature }\end{array}$ & \\
\hline 8/21/2008 6:00 PM & 0.383 & 17.7 & 0.205 & 0.140 & 18.2 & 0.19 & 0.142 & -- & -- & -- & -- & -- & -- \\
\hline 8/21/2008 7:00 PM & 0.382 & 17.7 & 0.205 & 0.140 & 18.2 & 0.19 & 0.142 & -- & -- & -- & -- & -- & -- \\
\hline 8/21/2008 8:00 PM & 0.382 & 17.7 & 0.205 & 0.140 & 18.2 & 0.19 & 0.142 & -- & -- & -- & -- & -- & -- \\
\hline 8/21/2008 9:00 PM & 0.382 & 17.7 & 0.205 & 0.138 & 18.2 & 0.19 & 0.141 & -- & -- & -- & -- & -- & -- \\
\hline 8/21/2008 10:00 PM & 0.382 & 17.7 & 0.205 & 0.138 & 18.2 & 0.19 & 0.141 & -- & -- & -- & -- & -- & -- \\
\hline 8/21/2008 11:00 PM & 0.382 & 17.7 & 0.205 & 0.138 & 18.2 & 0.19 & 0.141 & -- & -- & -- & -- & -- & -- \\
\hline 8/22/2008 12:00 AM & 0.382 & 17.7 & 0.205 & 0.138 & 18.2 & 0.19 & 0.141 & -- & -- & -- & -- & -- & -- \\
\hline 8/22/2008 1:00 AM & 0.382 & 17.7 & 0.205 & 0.138 & 18.2 & 0.19 & 0.141 & -- & -- & -- & -- & -- & -- \\
\hline 8/22/2008 2:00 AM & 0.382 & 17.7 & 0.205 & 0.138 & 18.2 & 0.19 & 0.141 & -- & -- & -- & -- & -- & -- \\
\hline 8/22/2008 3:00 AM & 0.382 & 17.7 & 0.205 & 0.138 & 18.2 & 0.19 & 0.140 & -- & -- & -- & -- & -- & -- \\
\hline 8/22/2008 4:00 AM & 0.382 & 17.8 & 0.205 & 0.138 & 18.2 & 0.19 & 0.140 & -- & -- & -- & -- & -- & -- \\
\hline 8/22/2008 5:00 AM & 0.382 & 17.8 & 0.205 & 0.138 & 18.2 & 0.19 & 0.140 & -- & -- & -- & -- & -- & -- \\
\hline 8/22/2008 6:00 AM & 0.382 & 17.8 & 0.204 & 0.138 & 18.2 & 0.19 & 0.140 & -- & -- & -- & -- & -- & -- \\
\hline 8/22/2008 7:00 AM & 0.382 & 17.8 & 0.204 & 0.137 & 18.2 & 0.19 & 0.140 & -- & -- & -- & -- & -- & -- \\
\hline 8/22/2008 8:00 AM & 0.382 & 17.8 & 0.204 & 0.137 & 18.1 & 0.19 & 0.140 & -- & -- & -- & -- & -- & -- \\
\hline 8/22/2008 9:00 AM & 0.383 & 17.9 & 0.204 & 0.137 & 18.2 & 0.19 & 0.140 & -- & -- & -- & -- & -- & -- \\
\hline 8/22/2008 10:00 AM & 0.382 & 17.9 & 0.203 & 0.137 & 18.2 & 0.19 & 0.140 & -- & -- & -- & -- & -- & -- \\
\hline 8/22/2008 11:00 AM & 0.381 & 17.9 & 0.203 & 0.137 & 18.2 & 0.19 & 0.140 & -- & -- & -- & -- & -- & -- \\
\hline 8/22/2008 12:00 PM & 0.381 & 17.9 & 0.203 & 0.137 & 18.2 & 0.19 & 0.139 & -- & -- & -- & -- & -- & -- \\
\hline 8/22/2008 1:00 PM & 0.380 & 17.9 & 0.203 & 0.137 & 18.1 & 0.18 & 0.139 & -- & -- & -- & -- & -- & -- \\
\hline 8/22/2008 2:00 PM & 0.379 & 17.9 & 0.203 & 0.137 & 18.1 & 0.18 & 0.139 & -- & -- & -- & -- & -- & -- \\
\hline 8/22/2008 3:00 PM & 0.379 & 17.9 & 0.203 & 0.137 & 18.1 & 0.18 & 0.139 & -- & -- & -- & -- & -- & -- \\
\hline 8/22/2008 4:00 PM & 0.379 & 17.9 & 0.203 & 0.137 & 18.2 & 0.18 & 0.139 & -- & -- & -- & -- & -- & -- \\
\hline 8/22/2008 5:00 PM & 0.379 & 17.9 & 0.202 & 0.136 & 18.1 & 0.18 & 0.139 & -- & -- & -- & -- & -- & -- \\
\hline 8/22/2008 6:00 PM & 0.379 & 17.9 & 0.202 & 0.136 & 18.1 & 0.18 & 0.139 & -- & -- & -- & -- & -- & -- \\
\hline 8/22/2008 7:00 PM & 0.379 & 17.8 & 0.202 & 0.136 & 18.2 & 0.18 & 0.139 & -- & -- & -- & -- & -- & -- \\
\hline 8/22/2008 8:00 PM & 0.379 & 17.8 & 0.202 & 0.136 & 18.2 & 0.18 & 0.138 & -- & -- & -- & -- & -- & -- \\
\hline 8/22/2008 9:00 PM & 0.379 & 17.8 & 0.202 & 0.136 & 18.2 & 0.18 & 0.138 & -- & -- & -- & -- & -- & -- \\
\hline 8/22/2008 10:00 PM & 0.379 & 17.8 & 0.202 & 0.136 & 18.1 & 0.18 & 0.138 & -- & -- & -- & -- & -- & -- \\
\hline 8/22/2008 11:00 PM & 0.379 & 17.8 & 0.202 & 0.136 & 18.1 & 0.18 & 0.138 & -- & -- & -- & -- & -- & -- \\
\hline 8/23/2008 12:00 AM & 0.379 & 17.8 & 0.202 & 0.136 & 18.2 & 0.18 & 0.138 & -- & -- & -- & -- & -- & -- \\
\hline 8/23/2008 1:00 AM & 0.378 & 17.9 & 0.202 & 0.136 & 18.1 & 0.18 & 0.138 & -- & -- & -- & -- & -- & -- \\
\hline 8/23/2008 2:00 AM & 0.378 & 17.9 & 0.202 & 0.136 & 18.1 & 0.18 & 0.138 & -- & -- & -- & -- & -- & -- \\
\hline 8/23/2008 3:00 AM & 0.379 & 17.9 & 0.202 & 0.136 & 18.1 & 0.18 & 0.138 & -- & -- & -- & -- & -- & -- \\
\hline 8/23/2008 4:00 AM & 0.379 & 17.9 & 0.202 & 0.136 & 18.1 & 0.18 & 0.138 & -- & -- & -- & -- & -- & -- \\
\hline
\end{tabular}


Appendix 3. Volumetric water content, temperature, and electrical-conductivity data collected at the sprinkler-irrigated site during 2008.-Continued

[Depth in feet below land surface; volumetric water content dimensionless; temperature in degrees Celsius; bulk electrical conductivity in decisiemens per meter; --, no data]

\begin{tabular}{|c|c|c|c|c|c|c|c|c|c|c|c|c|c|}
\hline \multirow[b]{3}{*}{$\begin{array}{l}\text { Measurement } \\
\text { date and time }\end{array}$} & \multicolumn{13}{|c|}{ Depth } \\
\hline & \multicolumn{2}{|c|}{2} & \multirow{2}{*}{$\begin{array}{c}3 \\
\text { Volumetric } \\
\text { water } \\
\text { content }\end{array}$} & \multicolumn{3}{|c|}{4} & \multirow{2}{*}{$\begin{array}{c}5 \\
\text { Volumetric } \\
\text { water } \\
\text { content }\end{array}$} & \multicolumn{2}{|l|}{7} & \multirow{2}{*}{$\begin{array}{c}9 \\
\text { Volumetric } \\
\text { water } \\
\text { content }\end{array}$} & \multicolumn{2}{|c|}{13} & \multirow{2}{*}{$\begin{array}{c}16.5 \\
\text { Volumetric } \\
\text { water } \\
\text { content }\end{array}$} \\
\hline & $\begin{array}{c}\text { Volumetric } \\
\text { water } \\
\text { content }\end{array}$ & $\begin{array}{l}\text { Temper- } \\
\text { ature }\end{array}$ & & $\begin{array}{c}\text { Volumetric } \\
\text { water } \\
\text { content }\end{array}$ & $\begin{array}{l}\text { Temper- } \\
\text { ature }\end{array}$ & $\begin{array}{c}\text { Bulk } \\
\text { electrical } \\
\text { conduct- } \\
\text { ivity }\end{array}$ & & $\begin{array}{c}\text { Volumetric } \\
\text { water } \\
\text { content }\end{array}$ & $\begin{array}{l}\text { Temper- } \\
\text { ature }\end{array}$ & & $\begin{array}{c}\text { Volumetric } \\
\text { water } \\
\text { content }\end{array}$ & $\begin{array}{l}\text { Temper- } \\
\text { ature }\end{array}$ & \\
\hline 8/23/2008 5:00 AM & 0.378 & 17.9 & 0.201 & 0.136 & 18.1 & 0.18 & 0.138 & -- & -- & -- & -- & -- & -- \\
\hline 8/23/2008 6:00 AM & 0.378 & 17.9 & 0.201 & 0.136 & 18.1 & 0.18 & 0.138 & -- & -- & -- & -- & -- & -- \\
\hline 8/23/2008 7:00 AM & 0.378 & 17.9 & 0.201 & 0.136 & 18.1 & 0.18 & 0.138 & -- & -- & -- & -- & -- & -- \\
\hline 8/23/2008 8:00 AM & 0.378 & 17.9 & 0.201 & 0.135 & 18.1 & 0.18 & 0.138 & -- & -- & -- & -- & -- & -- \\
\hline 8/23/2008 9:00 AM & 0.378 & 17.9 & 0.201 & 0.135 & 18.1 & 0.18 & 0.138 & -- & -- & -- & -- & -- & -- \\
\hline 8/23/2008 10:00 AM & 0.376 & 17.9 & 0.201 & 0.135 & 18.1 & 0.18 & 0.138 & -- & -- & -- & -- & -- & -- \\
\hline 8/23/2008 11:00 AM & 0.376 & 17.9 & 0.201 & 0.135 & 18.1 & 0.18 & 0.138 & -- & -- & -- & -- & -- & -- \\
\hline 8/23/2008 12:00 PM & 0.376 & 17.9 & 0.200 & 0.135 & 18.1 & 0.18 & 0.138 & -- & -- & -- & -- & -- & -- \\
\hline 8/23/2008 1:00 PM & 0.375 & 17.9 & 0.200 & 0.135 & 18.1 & 0.18 & 0.138 & -- & -- & -- & -- & -- & -- \\
\hline 8/23/2008 2:00 PM & 0.374 & 17.9 & 0.200 & 0.135 & 18.1 & 0.18 & 0.138 & -- & -- & -- & -- & -- & -- \\
\hline 8/23/2008 3:00 PM & 0.374 & 17.9 & 0.200 & 0.135 & 18.1 & 0.18 & 0.138 & -- & -- & -- & -- & -- & -- \\
\hline 8/23/2008 4:00 PM & 0.374 & 17.9 & 0.200 & 0.135 & 18.1 & 0.18 & 0.137 & -- & -- & -- & -- & -- & -- \\
\hline 8/23/2008 5:00 PM & 0.374 & 17.9 & 0.200 & 0.135 & 18.1 & 0.18 & 0.137 & -- & -- & -- & -- & -- & -- \\
\hline 8/23/2008 6:00 PM & 0.373 & 17.9 & 0.200 & 0.135 & 18.2 & 0.18 & 0.137 & -- & -- & -- & -- & -- & -- \\
\hline 8/23/2008 7:00 PM & 0.373 & 17.8 & 0.200 & 0.134 & 18.1 & 0.18 & 0.137 & -- & -- & -- & -- & -- & -- \\
\hline 8/23/2008 8:00 PM & 0.373 & 17.8 & 0.200 & 0.135 & 18.1 & 0.18 & 0.137 & -- & -- & -- & -- & -- & -- \\
\hline 8/23/2008 9:00 PM & 0.373 & 17.8 & 0.200 & 0.135 & 18.1 & 0.18 & 0.137 & -- & -- & -- & -- & -- & -- \\
\hline 8/23/2008 10:00 PM & 0.373 & 17.8 & 0.200 & 0.135 & 18.1 & 0.18 & 0.137 & -- & -- & -- & -- & -- & -- \\
\hline 8/23/2008 11:00 PM & 0.373 & 17.8 & 0.200 & 0.134 & 18.1 & 0.18 & 0.137 & -- & -- & -- & -- & -- & -- \\
\hline 8/24/2008 12:00 AM & 0.373 & 17.8 & 0.200 & 0.134 & 18.1 & 0.18 & 0.137 & -- & -- & -- & -- & -- & -- \\
\hline 8/24/2008 1:00 AM & 0.373 & 17.9 & 0.200 & 0.134 & 18.1 & 0.18 & 0.136 & -- & -- & -- & -- & -- & -- \\
\hline 8/24/2008 2:00 AM & 0.373 & 17.9 & 0.200 & 0.134 & 18.1 & 0.18 & 0.136 & -- & -- & -- & -- & -- & -- \\
\hline 8/24/2008 3:00 AM & 0.373 & 17.9 & 0.200 & 0.134 & 18.1 & 0.18 & 0.136 & -- & -- & -- & -- & -- & -- \\
\hline 8/24/2008 4:00 AM & 0.373 & 17.9 & 0.200 & 0.134 & 18.1 & 0.18 & 0.136 & -- & -- & -- & -- & -- & -- \\
\hline 8/24/2008 5:00 AM & 0.373 & 17.9 & 0.200 & 0.134 & 18.1 & 0.18 & 0.136 & -- & -- & -- & -- & -- & -- \\
\hline 8/24/2008 6:00 AM & 0.373 & 17.9 & 0.200 & 0.134 & 18.1 & 0.18 & 0.136 & -- & -- & -- & -- & -- & -- \\
\hline 8/24/2008 7:00 AM & 0.373 & 17.9 & 0.200 & 0.134 & 18.1 & 0.18 & 0.136 & -- & -- & -- & -- & -- & -- \\
\hline 8/24/2008 8:00 AM & 0.373 & 18.0 & 0.200 & 0.134 & 18.1 & 0.18 & 0.136 & -- & -- & -- & -- & -- & -- \\
\hline 8/24/2008 9:00 AM & 0.373 & 18.0 & 0.199 & 0.134 & 18.1 & 0.18 & 0.136 & -- & -- & -- & -- & -- & -- \\
\hline 8/24/2008 10:00 AM & 0.373 & 17.9 & 0.199 & 0.134 & 18.1 & 0.18 & 0.136 & -- & -- & -- & -- & -- & -- \\
\hline $8 / 24 / 2008$ 11:00 AM & 0.372 & 18.0 & 0.198 & 0.134 & 18.1 & 0.18 & 0.136 & -- & -- & -- & -- & -- & -- \\
\hline 8/24/2008 12:00 PM & 0.372 & 18.0 & 0.198 & 0.134 & 18.1 & 0.18 & 0.136 & -- & -- & -- & -- & -- & -- \\
\hline 8/24/2008 1:00 PM & 0.372 & 17.9 & 0.198 & 0.134 & 18.1 & 0.18 & 0.136 & -- & -- & -- & -- & -- & -- \\
\hline 8/24/2008 2:00 PM & 0.372 & 17.9 & 0.198 & 0.134 & 18.1 & 0.18 & 0.136 & -- & -- & -- & -- & -- & -- \\
\hline 8/24/2008 3:00 PM & 0.372 & 17.9 & 0.198 & 0.133 & 18.1 & 0.18 & 0.136 & -- & -- & -- & -- & -- & -- \\
\hline
\end{tabular}


Appendix 3. Volumetric water content, temperature, and electrical-conductivity data collected at the sprinkler-irrigated site during 2008.-Continued

[Depth in feet below land surface; volumetric water content dimensionless; temperature in degrees Celsius; bulk electrical conductivity in decisiemens per meter; --, no data]

\begin{tabular}{|c|c|c|c|c|c|c|c|c|c|c|c|c|c|}
\hline \multirow{3}{*}{$\begin{array}{l}\text { Measurement } \\
\text { date and time }\end{array}$} & \multicolumn{13}{|c|}{ Depth } \\
\hline & \multicolumn{2}{|c|}{2} & \multirow{2}{*}{$\begin{array}{c}3 \\
\text { Volumetric } \\
\text { water } \\
\text { content }\end{array}$} & \multicolumn{3}{|c|}{4} & \multirow{2}{*}{$\begin{array}{c}\mathbf{5} \\
\begin{array}{c}\text { Volumetric } \\
\text { water } \\
\text { content }\end{array}\end{array}$} & \multicolumn{2}{|l|}{7} & \multirow{2}{*}{$\begin{array}{c}9 \\
\text { Volumetric } \\
\text { water } \\
\text { content }\end{array}$} & \multicolumn{2}{|c|}{13} & \multirow{2}{*}{$\begin{array}{c}16.5 \\
\text { Volumetric } \\
\text { water } \\
\text { content }\end{array}$} \\
\hline & $\begin{array}{c}\text { Volumetric } \\
\text { water } \\
\text { content }\end{array}$ & $\begin{array}{c}\text { Temper- } \\
\text { ature }\end{array}$ & & $\begin{array}{l}\text { Volumetric } \\
\text { water } \\
\text { content }\end{array}$ & $\begin{array}{l}\text { Temper- } \\
\text { ature }\end{array}$ & $\begin{array}{c}\text { Bulk } \\
\text { electrical } \\
\text { conduct- } \\
\text { ivity }\end{array}$ & & $\begin{array}{l}\text { Volumetric } \\
\text { water } \\
\text { content }\end{array}$ & $\begin{array}{l}\text { Temper- } \\
\text { ature }\end{array}$ & & $\begin{array}{l}\text { Volumetric } \\
\text { water } \\
\text { content }\end{array}$ & $\begin{array}{l}\text { Temper- } \\
\text { ature }\end{array}$ & \\
\hline 8/24/2008 4:00 PM & 0.371 & 17.9 & 0.198 & 0.134 & 18.2 & 0.18 & 0.136 & $\overline{--}$ & $\overline{--}$ & $\overline{--}$ & $\overline{--}$ & $\overline{--}$ & -- \\
\hline 8/24/2008 5:00 PM & 0.372 & 17.9 & 0.198 & 0.133 & 18.1 & 0.18 & 0.135 & -- & -- & -- & -- & -- & -- \\
\hline 8/24/2008 6:00 PM & 0.372 & 17.9 & 0.198 & 0.133 & 18.1 & 0.18 & 0.135 & -- & -- & -- & -- & -- & -- \\
\hline 8/24/2008 7:00 PM & 0.371 & 17.9 & 0.198 & 0.133 & 18.1 & 0.18 & 0.135 & -- & -- & -- & -- & -- & -- \\
\hline 8/24/2008 8:00 PM & 0.372 & 17.9 & 0.198 & 0.134 & 18.1 & 0.18 & 0.135 & -- & -- & -- & -- & -- & -- \\
\hline 8/24/2008 9:00 PM & 0.371 & 17.9 & 0.198 & 0.133 & 18.1 & 0.18 & 0.135 & -- & -- & -- & -- & -- & -- \\
\hline 8/24/2008 10:00 PM & 0.372 & 17.9 & 0.198 & 0.133 & 18.1 & 0.18 & 0.135 & -- & -- & -- & -- & -- & -- \\
\hline 8/24/2008 11:00 PM & 0.372 & 17.9 & 0.198 & 0.133 & 18.1 & 0.18 & 0.135 & -- & -- & -- & -- & -- & -- \\
\hline 8/25/2008 12:00 AM & 0.372 & 17.9 & 0.198 & 0.133 & 18.1 & 0.18 & 0.135 & -- & -- & -- & -- & -- & -- \\
\hline 8/25/2008 1:00 AM & 0.372 & 18.0 & 0.198 & 0.133 & 18.1 & 0.18 & 0.135 & -- & -- & -- & -- & -- & -- \\
\hline 8/25/2008 2:00 AM & 0.372 & 18.0 & 0.198 & 0.133 & 18.1 & 0.18 & 0.134 & -- & -- & -- & -- & -- & -- \\
\hline 8/25/2008 3:00 AM & 0.371 & 18.0 & 0.198 & 0.133 & 18.1 & 0.18 & 0.135 & -- & -- & -- & -- & -- & -- \\
\hline 8/25/2008 4:00 AM & 0.371 & 18.0 & 0.198 & 0.133 & 18.1 & 0.18 & 0.134 & -- & -- & -- & -- & -- & -- \\
\hline 8/25/2008 5:00 AM & 0.371 & 18.0 & 0.198 & 0.133 & 18.1 & 0.18 & 0.134 & -- & -- & -- & -- & -- & -- \\
\hline 8/25/2008 6:00 AM & 0.372 & 18.0 & 0.198 & 0.133 & 18.1 & 0.18 & 0.134 & -- & -- & -- & -- & -- & -- \\
\hline 8/25/2008 7:00 AM & 0.372 & 18.1 & 0.198 & 0.133 & 18.1 & 0.18 & 0.134 & -- & -- & -- & -- & -- & -- \\
\hline 8/25/2008 8:00 AM & 0.373 & 18.1 & 0.198 & 0.133 & 18.1 & 0.18 & 0.134 & -- & -- & -- & -- & -- & -- \\
\hline 8/25/2008 9:00 AM & 0.373 & 18.1 & 0.198 & 0.133 & 18.1 & 0.18 & 0.134 & -- & -- & -- & -- & -- & -- \\
\hline $8 / 25 / 2008$ 10:00 AM & 0.373 & 18.1 & 0.197 & 0.133 & 18.1 & 0.18 & 0.134 & -- & -- & -- & -- & -- & -- \\
\hline 8/25/2008 11:00 AM & 0.372 & 18.1 & 0.197 & 0.133 & 18.1 & 0.18 & 0.134 & -- & -- & -- & -- & -- & -- \\
\hline 8/25/2008 12:00 PM & 0.371 & 18.1 & 0.197 & 0.133 & 18.1 & 0.18 & 0.134 & -- & -- & -- & -- & -- & -- \\
\hline 8/25/2008 1:00 PM & 0.371 & 18.1 & 0.197 & 0.133 & 18.1 & 0.18 & 0.134 & -- & -- & -- & -- & -- & -- \\
\hline 8/25/2008 2:00 PM & 0.371 & 18.1 & 0.197 & 0.132 & 18.1 & 0.18 & 0.134 & -- & -- & -- & -- & -- & -- \\
\hline 8/25/2008 3:00 PM & 0.371 & 18.1 & 0.197 & 0.132 & 18.1 & 0.18 & 0.134 & -- & -- & -- & -- & -- & -- \\
\hline 8/25/2008 4:00 PM & 0.372 & 18.1 & 0.197 & 0.132 & 18.1 & 0.18 & 0.134 & -- & -- & -- & -- & -- & -- \\
\hline 8/25/2008 5:00 PM & 0.372 & 18.0 & 0.197 & 0.132 & 18.1 & 0.17 & 0.134 & -- & -- & -- & -- & -- & -- \\
\hline 8/25/2008 6:00 PM & 0.372 & 18.0 & 0.197 & 0.132 & 18.1 & 0.17 & 0.133 & -- & -- & -- & -- & -- & -- \\
\hline 8/25/2008 7:00 PM & 0.372 & 18.0 & 0.197 & 0.132 & 18.1 & 0.17 & 0.133 & -- & -- & -- & -- & -- & -- \\
\hline 8/25/2008 8:00 PM & 0.371 & 18.0 & 0.197 & 0.132 & 18.1 & 0.17 & 0.133 & -- & -- & -- & -- & -- & -- \\
\hline 8/25/2008 9:00 PM & 0.371 & 18.0 & 0.196 & 0.132 & 18.1 & 0.17 & 0.133 & -- & -- & -- & -- & -- & -- \\
\hline 8/25/2008 10:00 PM & 0.371 & 18.0 & 0.196 & 0.133 & 18.1 & 0.17 & 0.133 & -- & -- & -- & -- & -- & -- \\
\hline 8/25/2008 11:00 PM & 0.371 & 18.0 & 0.196 & 0.132 & 18.1 & 0.17 & 0.133 & -- & -- & -- & -- & -- & -- \\
\hline 8/26/2008 12:00 AM & 0.371 & 18.0 & 0.197 & 0.132 & 18.1 & 0.17 & 0.133 & -- & -- & -- & -- & -- & -- \\
\hline $8 / 26 / 2008$ 1:00 AM & 0.371 & 18.0 & 0.197 & 0.132 & 18.1 & 0.17 & 0.133 & -- & -- & -- & -- & -- & -- \\
\hline 8/26/2008 2:00 AM & 0.371 & 18.1 & 0.197 & 0.132 & 18.1 & 0.17 & 0.133 & -- & -- & -- & -- & -- & -- \\
\hline
\end{tabular}


Appendix 3. Volumetric water content, temperature, and electrical-conductivity data collected at the sprinkler-irrigated site during 2008.-Continued

[Depth in feet below land surface; volumetric water content dimensionless; temperature in degrees Celsius; bulk electrical conductivity in decisiemens per meter; --, no data]

\begin{tabular}{|c|c|c|c|c|c|c|c|c|c|c|c|c|c|}
\hline \multirow[b]{3}{*}{$\begin{array}{l}\text { Measurement } \\
\text { date and time }\end{array}$} & \multicolumn{13}{|c|}{ Depth } \\
\hline & \multicolumn{2}{|c|}{2} & \multirow{2}{*}{$\begin{array}{c}3 \\
\text { Volumetric } \\
\text { water } \\
\text { content }\end{array}$} & \multicolumn{3}{|c|}{4} & \multirow{2}{*}{$\begin{array}{c}5 \\
\text { Volumetric } \\
\text { water } \\
\text { content }\end{array}$} & \multicolumn{2}{|l|}{7} & \multirow{2}{*}{$\begin{array}{c}9 \\
\text { Volumetric } \\
\text { water } \\
\text { content }\end{array}$} & \multicolumn{2}{|c|}{13} & \multirow{2}{*}{$\begin{array}{c}16.5 \\
\text { Volumetric } \\
\text { water } \\
\text { content }\end{array}$} \\
\hline & $\begin{array}{c}\text { Volumetric } \\
\text { water } \\
\text { content }\end{array}$ & $\begin{array}{l}\text { Temper- } \\
\text { ature }\end{array}$ & & $\begin{array}{c}\text { Volumetric } \\
\text { water } \\
\text { content }\end{array}$ & $\begin{array}{l}\text { Temper- } \\
\text { ature }\end{array}$ & $\begin{array}{c}\text { Bulk } \\
\text { electrical } \\
\text { conduct- } \\
\text { ivity }\end{array}$ & & $\begin{array}{c}\text { Volumetric } \\
\text { water } \\
\text { content }\end{array}$ & $\begin{array}{l}\text { Temper- } \\
\text { ature }\end{array}$ & & $\begin{array}{c}\text { Volumetric } \\
\text { water } \\
\text { content }\end{array}$ & $\begin{array}{l}\text { Temper- } \\
\text { ature }\end{array}$ & \\
\hline 8/26/2008 3:00 AM & 0.372 & 18.1 & 0.197 & 0.132 & 18.1 & 0.17 & 0.133 & -- & -- & -- & -- & -- & -- \\
\hline 8/26/2008 4:00 AM & 0.372 & 18.0 & 0.196 & 0.132 & 18.1 & 0.17 & 0.133 & -- & -- & -- & -- & -- & -- \\
\hline 8/26/2008 5:00 AM & 0.372 & 18.1 & 0.196 & 0.132 & 18.1 & 0.17 & 0.133 & -- & -- & -- & -- & -- & -- \\
\hline 8/26/2008 6:00 AM & 0.372 & 18.1 & 0.196 & 0.132 & 18.1 & 0.17 & 0.133 & -- & -- & -- & -- & -- & -- \\
\hline 8/26/2008 7:00 AM & 0.372 & 18.1 & 0.196 & 0.132 & 18.1 & 0.17 & 0.133 & -- & -- & -- & -- & -- & -- \\
\hline 8/26/2008 8:00 AM & 0.372 & 18.1 & 0.196 & 0.132 & 18.1 & 0.17 & 0.133 & -- & -- & -- & -- & -- & -- \\
\hline 8/26/2008 9:00 AM & 0.372 & 18.0 & 0.196 & 0.132 & 18.1 & 0.17 & 0.132 & -- & -- & -- & -- & -- & -- \\
\hline 8/26/2008 10:00 AM & 0.372 & 18.1 & 0.196 & 0.132 & 18.1 & 0.17 & 0.132 & -- & -- & -- & -- & -- & -- \\
\hline 8/26/2008 11:00 AM & 0.372 & 18.1 & 0.196 & 0.132 & 18.1 & 0.17 & 0.132 & -- & -- & -- & -- & -- & -- \\
\hline 8/26/2008 12:00 PM & 0.371 & 18.1 & 0.196 & 0.132 & 18.1 & 0.17 & 0.132 & -- & -- & -- & -- & -- & -- \\
\hline 8/26/2008 1:00 PM & 0.371 & 18.1 & 0.196 & 0.132 & 18.1 & 0.17 & 0.132 & -- & -- & -- & -- & -- & -- \\
\hline 8/26/2008 2:00 PM & 0.370 & 18.0 & 0.196 & 0.131 & 18.1 & 0.17 & 0.132 & -- & -- & -- & -- & -- & -- \\
\hline 8/26/2008 3:00 PM & 0.370 & 18.0 & 0.196 & 0.131 & 18.1 & 0.17 & 0.132 & -- & -- & -- & -- & -- & -- \\
\hline 8/26/2008 4:00 PM & 0.370 & 18.0 & 0.195 & 0.131 & 18.1 & 0.17 & 0.132 & -- & -- & -- & -- & -- & -- \\
\hline 8/26/2008 5:00 PM & 0.370 & 18.0 & 0.195 & 0.131 & 18.1 & 0.17 & 0.132 & -- & -- & -- & -- & -- & -- \\
\hline 8/26/2008 6:00 PM & 0.369 & 18.0 & 0.195 & 0.131 & 18.1 & 0.17 & 0.132 & -- & -- & -- & -- & -- & -- \\
\hline 8/26/2008 7:00 PM & 0.369 & 18.0 & 0.195 & 0.131 & 18.1 & 0.17 & 0.132 & -- & -- & -- & -- & -- & -- \\
\hline 8/26/2008 8:00 PM & 0.369 & 18.0 & 0.195 & 0.131 & 18.1 & 0.17 & 0.132 & -- & -- & -- & -- & -- & -- \\
\hline 8/26/2008 9:00 PM & 0.369 & 18.0 & 0.195 & 0.131 & 18.1 & 0.17 & 0.132 & -- & -- & -- & -- & -- & -- \\
\hline 8/26/2008 10:00 PM & 0.369 & 18.0 & 0.195 & 0.131 & 18.1 & 0.17 & 0.132 & -- & -- & -- & -- & -- & -- \\
\hline 8/26/2008 11:00 PM & 0.369 & 18.0 & 0.195 & 0.131 & 18.1 & 0.17 & 0.132 & -- & -- & -- & -- & -- & -- \\
\hline 8/27/2008 12:00 AM & 0.369 & 18.0 & 0.195 & 0.131 & 18.1 & 0.17 & 0.132 & -- & -- & -- & -- & -- & -- \\
\hline 8/27/2008 1:00 AM & 0.369 & 18.0 & 0.195 & 0.131 & 18.1 & 0.17 & 0.132 & -- & -- & -- & -- & -- & -- \\
\hline 8/27/2008 2:00 AM & 0.370 & 18.0 & 0.194 & 0.131 & 18.1 & 0.17 & 0.132 & -- & -- & -- & -- & -- & -- \\
\hline 8/27/2008 3:00 AM & 0.370 & 18.0 & 0.194 & 0.131 & 18.1 & 0.17 & 0.132 & -- & -- & -- & -- & -- & -- \\
\hline 8/27/2008 4:00 AM & 0.370 & 18.1 & 0.194 & 0.131 & 18.1 & 0.17 & 0.132 & -- & -- & -- & -- & -- & -- \\
\hline 8/27/2008 5:00 AM & 0.370 & 18.1 & 0.195 & 0.131 & 18.1 & 0.17 & 0.132 & -- & -- & -- & -- & -- & -- \\
\hline 8/27/2008 6:00 AM & 0.370 & 18.1 & 0.195 & 0.131 & 18.1 & 0.17 & 0.132 & -- & -- & -- & -- & -- & -- \\
\hline 8/27/2008 7:00 AM & 0.370 & 18.1 & 0.195 & 0.131 & 18.1 & 0.17 & 0.132 & -- & -- & -- & -- & -- & -- \\
\hline 8/27/2008 8:00 AM & 0.370 & 18.1 & 0.195 & 0.131 & 18.1 & 0.17 & 0.132 & -- & -- & -- & -- & -- & -- \\
\hline 8/27/2008 9:00 AM & 0.370 & 18.1 & 0.194 & 0.131 & 18.1 & 0.17 & 0.132 & -- & -- & -- & -- & -- & -- \\
\hline 8/27/2008 10:00 AM & 0.369 & 18.1 & 0.194 & 0.131 & 18.1 & 0.17 & 0.132 & -- & -- & -- & -- & -- & -- \\
\hline 8/27/2008 11:00 AM & 0.369 & 18.1 & 0.194 & 0.131 & 18.1 & 0.17 & 0.132 & -- & -- & -- & -- & -- & -- \\
\hline 8/27/2008 12:00 PM & 0.369 & 18.2 & 0.194 & 0.131 & 18.1 & 0.17 & 0.132 & -- & -- & -- & -- & -- & -- \\
\hline 8/27/2008 1:00 PM & 0.368 & 18.2 & 0.194 & 0.131 & 18.1 & 0.17 & 0.132 & -- & -- & -- & -- & -- & -- \\
\hline
\end{tabular}


Appendix 3. Volumetric water content, temperature, and electrical-conductivity data collected at the sprinkler-irrigated site during 2008.-Continued

[Depth in feet below land surface; volumetric water content dimensionless; temperature in degrees Celsius; bulk electrical conductivity in decisiemens per meter; --, no data]

\begin{tabular}{|c|c|c|c|c|c|c|c|c|c|c|c|c|c|}
\hline \multirow[b]{3}{*}{$\begin{array}{l}\text { Measurement } \\
\text { date and time }\end{array}$} & \multicolumn{13}{|c|}{ Depth } \\
\hline & \multicolumn{2}{|c|}{2} & \multirow{2}{*}{$\begin{array}{c}3 \\
\text { Volumetric } \\
\text { water } \\
\text { content }\end{array}$} & \multicolumn{3}{|c|}{4} & \multirow{2}{*}{$\begin{array}{c}5 \\
\text { Volumetric } \\
\text { water } \\
\text { content }\end{array}$} & \multicolumn{2}{|c|}{7} & \multirow{2}{*}{$\begin{array}{c}9 \\
\text { Volumetric } \\
\text { water } \\
\text { content }\end{array}$} & \multicolumn{2}{|c|}{13} & \multirow{2}{*}{$\begin{array}{c}16.5 \\
\text { Volumetric } \\
\text { water } \\
\text { content }\end{array}$} \\
\hline & $\begin{array}{c}\text { Volumetric } \\
\text { water } \\
\text { content }\end{array}$ & $\begin{array}{l}\text { Temper- } \\
\text { ature }\end{array}$ & & $\begin{array}{c}\text { Volumetric } \\
\text { water } \\
\text { content }\end{array}$ & $\begin{array}{l}\text { Temper- } \\
\text { ature }\end{array}$ & $\begin{array}{c}\text { Bulk } \\
\text { electrical } \\
\text { conduct- } \\
\text { ivity }\end{array}$ & & $\begin{array}{c}\text { Volumetric } \\
\text { water } \\
\text { content }\end{array}$ & $\begin{array}{l}\text { Temper- } \\
\text { ature }\end{array}$ & & $\begin{array}{c}\text { Volumetric } \\
\text { water } \\
\text { content }\end{array}$ & $\begin{array}{l}\text { Temper- } \\
\text { ature }\end{array}$ & \\
\hline 8/27/2008 2:00 PM & 0.368 & 18.2 & 0.194 & 0.131 & 18.1 & 0.17 & 0.132 & $\begin{array}{l}- \\
-\end{array}$ & $\begin{array}{l}- \\
-\end{array}$ & -- & $\begin{array}{l}- \\
-\end{array}$ & $\overline{--}$ & -- \\
\hline 8/27/2008 3:00 PM & 0.368 & 18.1 & 0.194 & 0.130 & 18.1 & 0.17 & 0.132 & -- & -- & -- & -- & -- & -- \\
\hline 8/27/2008 4:00 PM & 0.367 & 18.1 & 0.194 & 0.130 & 18.1 & 0.17 & 0.132 & -- & -- & -- & -- & -- & -- \\
\hline 8/27/2008 5:00 PM & 0.367 & 18.1 & 0.194 & 0.130 & 18.1 & 0.17 & 0.131 & -- & -- & -- & -- & -- & -- \\
\hline 8/27/2008 6:00 PM & 0.367 & 18.1 & 0.194 & 0.130 & 18.1 & 0.17 & 0.131 & -- & -- & -- & -- & -- & -- \\
\hline 8/27/2008 7:00 PM & 0.368 & 18.1 & 0.194 & 0.131 & 18.1 & 0.17 & 0.131 & -- & -- & -- & -- & -- & -- \\
\hline 8/27/2008 8:00 PM & 0.368 & 18.1 & 0.194 & 0.131 & 18.1 & 0.17 & 0.131 & -- & -- & -- & -- & -- & -- \\
\hline 8/27/2008 9:00 PM & 0.368 & 18.1 & 0.194 & 0.130 & 18.1 & 0.17 & 0.131 & -- & -- & -- & -- & -- & -- \\
\hline 8/27/2008 10:00 PM & 0.367 & 18.1 & 0.194 & 0.130 & 18.1 & 0.17 & 0.131 & -- & -- & -- & -- & -- & -- \\
\hline 8/27/2008 11:00 PM & 0.368 & 18.1 & 0.194 & 0.130 & 18.1 & 0.17 & 0.131 & -- & -- & -- & -- & -- & -- \\
\hline 8/28/2008 12:00 AM & 0.367 & 18.1 & 0.194 & 0.130 & 18.1 & 0.17 & 0.131 & -- & -- & -- & -- & -- & -- \\
\hline 8/28/2008 1:00 AM & 0.367 & 18.1 & 0.194 & 0.130 & 18.1 & 0.17 & 0.131 & -- & -- & -- & -- & -- & -- \\
\hline 8/28/2008 2:00 AM & 0.368 & 18.1 & 0.194 & 0.130 & 18.1 & 0.17 & 0.131 & -- & -- & -- & -- & -- & -- \\
\hline 8/28/2008 3:00 AM & 0.368 & 18.1 & 0.194 & 0.130 & 18.1 & 0.17 & 0.131 & -- & -- & -- & -- & -- & -- \\
\hline 8/28/2008 4:00 AM & 0.368 & 18.1 & 0.194 & 0.130 & 18.1 & 0.17 & 0.131 & -- & -- & -- & -- & -- & -- \\
\hline 8/28/2008 5:00 AM & 0.368 & 18.1 & 0.194 & 0.130 & 18.1 & 0.17 & 0.131 & -- & -- & -- & -- & -- & -- \\
\hline 8/28/2008 6:00 AM & 0.368 & 18.1 & 0.194 & 0.130 & 18.1 & 0.17 & 0.131 & -- & -- & -- & -- & -- & -- \\
\hline 8/28/2008 7:00 AM & 0.368 & 18.1 & 0.194 & 0.130 & 18.1 & 0.17 & 0.131 & -- & -- & -- & -- & -- & -- \\
\hline 8/28/2008 8:00 AM & 0.368 & 18.1 & 0.194 & 0.130 & 18.1 & 0.17 & 0.131 & -- & -- & -- & -- & -- & -- \\
\hline 8/28/2008 9:00 AM & 0.368 & 18.1 & 0.194 & 0.131 & 18.1 & 0.17 & 0.130 & -- & -- & -- & -- & -- & -- \\
\hline 8/28/2008 10:00 AM & 0.368 & 18.1 & 0.193 & 0.130 & 18.1 & 0.17 & 0.131 & -- & -- & -- & -- & -- & -- \\
\hline 8/28/2008 11:00 AM & 0.367 & 18.1 & 0.193 & 0.130 & 18.1 & 0.17 & 0.131 & -- & -- & -- & -- & -- & -- \\
\hline 8/28/2008 12:00 PM & 0.367 & 18.1 & 0.193 & 0.130 & 18.1 & 0.17 & 0.131 & -- & -- & -- & -- & -- & -- \\
\hline 8/28/2008 1:00 PM & 0.367 & 18.1 & 0.193 & 0.130 & 18.1 & 0.17 & 0.131 & -- & -- & -- & -- & -- & -- \\
\hline 8/28/2008 2:00 PM & 0.366 & 18.1 & 0.193 & 0.130 & 18.1 & 0.17 & 0.130 & -- & -- & -- & -- & -- & -- \\
\hline 8/28/2008 3:00 PM & 0.366 & 18.1 & 0.193 & 0.129 & 18.1 & 0.17 & 0.130 & -- & -- & -- & -- & -- & -- \\
\hline 8/28/2008 4:00 PM & 0.366 & 18.0 & 0.193 & 0.129 & 18.1 & 0.17 & 0.130 & -- & -- & -- & -- & -- & -- \\
\hline 8/28/2008 5:00 PM & 0.365 & 18.0 & 0.193 & 0.130 & 18.1 & 0.17 & 0.130 & -- & -- & -- & -- & -- & -- \\
\hline 8/28/2008 6:00 PM & 0.363 & 18.0 & 0.193 & 0.129 & 18.1 & 0.17 & 0.130 & -- & -- & -- & -- & -- & -- \\
\hline 8/28/2008 7:00 PM & 0.365 & 18.0 & 0.192 & 0.129 & 18.1 & 0.17 & 0.130 & -- & -- & -- & -- & -- & -- \\
\hline 8/28/2008 8:00 PM & 0.365 & 18.0 & 0.192 & 0.129 & 18.1 & 0.17 & 0.130 & -- & -- & -- & -- & -- & -- \\
\hline 8/28/2008 9:00 PM & 0.365 & 17.9 & 0.192 & 0.129 & 18.1 & 0.17 & 0.130 & -- & -- & -- & -- & -- & -- \\
\hline 8/28/2008 10:00 PM & 0.365 & 17.9 & 0.193 & 0.130 & 18.1 & 0.17 & 0.130 & -- & -- & -- & -- & -- & -- \\
\hline 8/28/2008 11:00 PM & 0.365 & 17.9 & 0.193 & 0.129 & 18.1 & 0.17 & 0.130 & -- & -- & -- & -- & -- & -- \\
\hline 8/29/2008 12:00 AM & 0.363 & 17.9 & 0.193 & 0.130 & 18.1 & 0.17 & 0.130 & -- & -- & -- & -- & -- & -- \\
\hline
\end{tabular}


Appendix 3. Volumetric water content, temperature, and electrical-conductivity data collected at the sprinkler-irrigated site during 2008.-Continued

[Depth in feet below land surface; volumetric water content dimensionless; temperature in degrees Celsius; bulk electrical conductivity in decisiemens per meter; --, no data]

\begin{tabular}{|c|c|c|c|c|c|c|c|c|c|c|c|c|c|}
\hline \multirow[b]{3}{*}{$\begin{array}{l}\text { Measurement } \\
\text { date and time }\end{array}$} & \multicolumn{13}{|c|}{ Depth } \\
\hline & \multicolumn{2}{|c|}{2} & \multirow{2}{*}{$\begin{array}{c}3 \\
\text { Volumetric } \\
\text { water } \\
\text { content }\end{array}$} & \multicolumn{3}{|c|}{4} & \multirow{2}{*}{$\begin{array}{c}5 \\
\text { Volumetric } \\
\text { water } \\
\text { content }\end{array}$} & \multicolumn{2}{|l|}{7} & \multirow{2}{*}{$\begin{array}{c}9 \\
\text { Volumetric } \\
\text { water } \\
\text { content }\end{array}$} & \multicolumn{2}{|c|}{13} & \multirow{2}{*}{$\begin{array}{c}16.5 \\
\text { Volumetric } \\
\text { water } \\
\text { content }\end{array}$} \\
\hline & $\begin{array}{c}\text { Volumetric } \\
\text { water } \\
\text { content }\end{array}$ & $\begin{array}{l}\text { Temper- } \\
\text { ature }\end{array}$ & & $\begin{array}{c}\text { Volumetric } \\
\text { water } \\
\text { content }\end{array}$ & $\begin{array}{l}\text { Temper- } \\
\text { ature }\end{array}$ & $\begin{array}{c}\text { Bulk } \\
\text { electrical } \\
\text { conduct- } \\
\text { ivity }\end{array}$ & & $\begin{array}{c}\text { Volumetric } \\
\text { water } \\
\text { content }\end{array}$ & $\begin{array}{l}\text { Temper- } \\
\text { ature }\end{array}$ & & $\begin{array}{c}\text { Volumetric } \\
\text { water } \\
\text { content }\end{array}$ & $\begin{array}{l}\text { Temper- } \\
\text { ature }\end{array}$ & \\
\hline 8/29/2008 1:00 AM & 0.363 & 17.9 & 0.192 & 0.129 & 18.1 & 0.17 & 0.130 & -- & -- & -- & -- & -- & -- \\
\hline 8/29/2008 2:00 AM & 0.365 & 17.9 & 0.192 & 0.129 & 18.1 & 0.17 & 0.130 & -- & -- & -- & -- & -- & -- \\
\hline 8/29/2008 3:00 AM & 0.365 & 17.9 & 0.192 & 0.129 & 18.1 & 0.17 & 0.130 & -- & -- & -- & -- & -- & -- \\
\hline 8/29/2008 4:00 AM & 0.365 & 17.9 & 0.192 & 0.129 & 18.1 & 0.17 & 0.130 & -- & -- & -- & -- & -- & -- \\
\hline 8/29/2008 5:00 AM & 0.365 & 17.9 & 0.192 & 0.129 & 18.1 & 0.17 & 0.130 & -- & -- & -- & -- & -- & -- \\
\hline 8/29/2008 6:00 AM & 0.363 & 17.9 & 0.192 & 0.129 & 18.1 & 0.17 & 0.130 & -- & -- & -- & -- & -- & -- \\
\hline 8/29/2008 7:00 AM & 0.365 & 17.9 & 0.192 & 0.129 & 18.1 & 0.17 & 0.130 & -- & -- & -- & -- & -- & -- \\
\hline 8/29/2008 8:00 AM & 0.365 & 17.9 & 0.192 & 0.129 & 18.1 & 0.17 & 0.130 & -- & -- & -- & -- & -- & -- \\
\hline 8/29/2008 9:00 AM & 0.365 & 17.8 & 0.192 & 0.129 & 18.1 & 0.17 & 0.130 & -- & -- & -- & -- & -- & -- \\
\hline 8/29/2008 10:00 AM & 0.363 & 17.9 & 0.192 & 0.129 & 18.1 & 0.17 & 0.130 & -- & -- & -- & -- & -- & -- \\
\hline 8/29/2008 11:00 AM & 0.363 & 17.9 & 0.192 & 0.129 & 18.1 & 0.17 & 0.130 & -- & -- & -- & -- & -- & -- \\
\hline 8/29/2008 12:00 PM & 0.363 & 17.8 & 0.191 & 0.129 & 18.1 & 0.17 & 0.130 & -- & -- & -- & -- & -- & -- \\
\hline 8/29/2008 1:00 PM & 0.363 & 17.8 & 0.191 & 0.129 & 18.1 & 0.17 & 0.130 & -- & -- & -- & -- & -- & -- \\
\hline 8/29/2008 2:00 PM & 0.363 & 17.8 & 0.191 & 0.129 & 18.1 & 0.17 & 0.130 & -- & -- & -- & -- & -- & -- \\
\hline 8/29/2008 3:00 PM & 0.362 & 17.8 & 0.191 & 0.129 & 18.1 & 0.17 & 0.129 & -- & -- & -- & -- & -- & -- \\
\hline 8/29/2008 4:00 PM & 0.361 & 17.8 & 0.191 & 0.128 & 18.1 & 0.17 & 0.129 & -- & -- & -- & -- & -- & -- \\
\hline 8/29/2008 5:00 PM & 0.362 & 17.7 & 0.191 & 0.128 & 18.1 & 0.17 & 0.129 & -- & -- & -- & -- & -- & -- \\
\hline 8/29/2008 6:00 PM & 0.361 & 17.7 & 0.191 & 0.128 & 18.1 & 0.17 & 0.129 & -- & -- & -- & -- & -- & -- \\
\hline 8/29/2008 7:00 PM & 0.361 & 17.7 & 0.191 & 0.128 & 18.1 & 0.17 & 0.129 & -- & -- & -- & -- & -- & -- \\
\hline 8/29/2008 8:00 PM & 0.361 & 17.7 & 0.191 & 0.129 & 18.1 & 0.17 & 0.129 & -- & -- & -- & -- & -- & -- \\
\hline 8/29/2008 9:00 PM & 0.360 & 17.7 & 0.191 & 0.129 & 18.1 & 0.17 & 0.129 & -- & -- & -- & -- & -- & -- \\
\hline 8/29/2008 10:00 PM & 0.360 & 17.7 & 0.191 & 0.129 & 18.1 & 0.17 & 0.129 & -- & -- & -- & -- & -- & -- \\
\hline 8/29/2008 11:00 PM & 0.360 & 17.7 & 0.190 & 0.128 & 18.1 & 0.17 & 0.129 & -- & -- & -- & -- & -- & -- \\
\hline 8/30/2008 12:00 AM & 0.360 & 17.7 & 0.190 & 0.128 & 18.1 & 0.17 & 0.129 & -- & -- & -- & -- & -- & -- \\
\hline 8/30/2008 1:00 AM & 0.360 & 17.7 & 0.190 & 0.128 & 18.1 & 0.17 & 0.129 & -- & -- & -- & -- & -- & -- \\
\hline 8/30/2008 2:00 AM & 0.360 & 17.7 & 0.190 & 0.129 & 18.1 & 0.17 & 0.129 & -- & -- & -- & -- & -- & -- \\
\hline 8/30/2008 3:00 AM & 0.361 & 17.7 & 0.190 & 0.128 & 18.1 & 0.17 & 0.129 & -- & -- & -- & -- & -- & -- \\
\hline 8/30/2008 4:00 AM & 0.361 & 17.7 & 0.191 & 0.128 & 18.1 & 0.17 & 0.129 & -- & -- & -- & -- & -- & -- \\
\hline 8/30/2008 5:00 AM & 0.361 & 17.7 & 0.191 & 0.129 & 18.1 & 0.17 & 0.129 & -- & -- & -- & -- & -- & -- \\
\hline 8/30/2008 6:00 AM & 0.361 & 17.7 & 0.190 & 0.128 & 18.1 & 0.17 & 0.129 & -- & -- & -- & -- & -- & -- \\
\hline 8/30/2008 7:00 AM & 0.360 & 17.7 & 0.190 & 0.128 & 18.1 & 0.17 & 0.129 & -- & -- & -- & -- & -- & -- \\
\hline 8/30/2008 8:00 AM & 0.361 & 17.8 & 0.190 & 0.128 & 18.1 & 0.17 & 0.129 & -- & -- & -- & -- & -- & -- \\
\hline 8/30/2008 9:00 AM & 0.361 & 17.8 & 0.190 & 0.128 & 18.1 & 0.17 & 0.129 & -- & -- & -- & -- & -- & -- \\
\hline 8/30/2008 10:00 AM & 0.361 & 17.8 & 0.190 & 0.129 & 18.1 & 0.17 & 0.129 & -- & -- & -- & -- & -- & -- \\
\hline 8/30/2008 11:00 AM & 0.361 & 17.8 & 0.190 & 0.128 & 18.1 & 0.17 & 0.129 & -- & -- & -- & -- & -- & -- \\
\hline
\end{tabular}


Appendix 3. Volumetric water content, temperature, and electrical-conductivity data collected at the sprinkler-irrigated site during 2008.-Continued

[Depth in feet below land surface; volumetric water content dimensionless; temperature in degrees Celsius; bulk electrical conductivity in decisiemens per meter; --, no data]

\begin{tabular}{|c|c|c|c|c|c|c|c|c|c|c|c|c|c|}
\hline \multirow[b]{3}{*}{$\begin{array}{l}\text { Measurement } \\
\text { date and time }\end{array}$} & \multicolumn{13}{|c|}{ Depth } \\
\hline & \multicolumn{2}{|c|}{2} & \multirow{2}{*}{$\begin{array}{c}3 \\
\text { Volumetric } \\
\text { water } \\
\text { content }\end{array}$} & \multicolumn{3}{|c|}{4} & \multirow{2}{*}{$\begin{array}{c}5 \\
\text { Volumetric } \\
\text { water } \\
\text { content }\end{array}$} & \multicolumn{2}{|c|}{7} & \multirow{2}{*}{$\begin{array}{c}9 \\
\text { Volumetric } \\
\text { water } \\
\text { content }\end{array}$} & \multicolumn{2}{|c|}{13} & \multirow{2}{*}{$\begin{array}{c}16.5 \\
\text { Volumetric } \\
\text { water } \\
\text { content }\end{array}$} \\
\hline & $\begin{array}{c}\text { Volumetric } \\
\text { water } \\
\text { content }\end{array}$ & $\begin{array}{l}\text { Temper- } \\
\text { ature }\end{array}$ & & $\begin{array}{c}\text { Volumetric } \\
\text { water } \\
\text { content }\end{array}$ & $\begin{array}{l}\text { Temper- } \\
\text { ature }\end{array}$ & $\begin{array}{c}\text { Bulk } \\
\text { electrical } \\
\text { conduct- } \\
\text { ivity }\end{array}$ & & $\begin{array}{c}\text { Volumetric } \\
\text { water } \\
\text { content }\end{array}$ & $\begin{array}{l}\text { Temper- } \\
\text { ature }\end{array}$ & & $\begin{array}{c}\text { Volumetric } \\
\text { water } \\
\text { content }\end{array}$ & $\begin{array}{l}\text { Temper- } \\
\text { ature }\end{array}$ & \\
\hline 8/30/2008 12:00 PM & 0.360 & 17.8 & 0.190 & 0.128 & 18.1 & 0.17 & 0.129 & $\begin{array}{l}- \\
-\end{array}$ & $\begin{array}{l}- \\
-\end{array}$ & -- & $\begin{array}{l}- \\
-\end{array}$ & $\overline{--}$ & -- \\
\hline 8/30/2008 1:00 PM & 0.360 & 17.8 & 0.190 & 0.128 & 18.1 & 0.17 & 0.129 & -- & -- & -- & -- & -- & -- \\
\hline 8/30/2008 2:00 PM & 0.359 & 17.8 & 0.190 & 0.128 & 18.1 & 0.17 & 0.128 & -- & -- & -- & -- & -- & -- \\
\hline 8/30/2008 3:00 PM & 0.359 & 17.8 & 0.189 & 0.126 & 18.1 & 0.17 & 0.128 & -- & -- & -- & -- & -- & -- \\
\hline 8/30/2008 4:00 PM & 0.358 & 17.8 & 0.189 & 0.126 & 18.1 & 0.17 & 0.128 & -- & -- & -- & -- & -- & -- \\
\hline 8/30/2008 5:00 PM & 0.357 & 17.8 & 0.189 & 0.126 & 18.1 & 0.17 & 0.128 & -- & -- & -- & -- & -- & -- \\
\hline 8/30/2008 6:00 PM & 0.358 & 17.8 & 0.189 & 0.126 & 18.1 & 0.16 & 0.128 & -- & -- & -- & -- & -- & -- \\
\hline 8/30/2008 7:00 PM & 0.358 & 17.8 & 0.188 & 0.128 & 18.1 & 0.17 & 0.128 & -- & -- & -- & -- & -- & -- \\
\hline 8/30/2008 8:00 PM & 0.358 & 17.8 & 0.189 & 0.128 & 18.1 & 0.17 & 0.128 & -- & -- & -- & -- & -- & -- \\
\hline 8/30/2008 9:00 PM & 0.358 & 17.8 & 0.189 & 0.128 & 18.1 & 0.17 & 0.128 & -- & -- & -- & -- & -- & -- \\
\hline 8/30/2008 10:00 PM & 0.358 & 17.8 & 0.189 & 0.126 & 18.1 & 0.17 & 0.128 & -- & -- & -- & -- & -- & -- \\
\hline 8/30/2008 11:00 PM & 0.358 & 17.8 & 0.189 & 0.128 & 18.0 & 0.17 & 0.128 & -- & -- & -- & -- & -- & -- \\
\hline 8/31/2008 12:00 AM & 0.359 & 17.9 & 0.189 & 0.128 & 18.0 & 0.17 & 0.128 & -- & -- & -- & -- & -- & -- \\
\hline 8/31/2008 1:00 AM & 0.360 & 17.9 & 0.189 & 0.126 & 18.1 & 0.17 & 0.128 & -- & -- & -- & -- & -- & -- \\
\hline 8/31/2008 2:00 AM & 0.361 & 17.9 & 0.189 & 0.126 & 18.0 & 0.17 & 0.128 & -- & -- & -- & -- & -- & -- \\
\hline 8/31/2008 3:00 AM & 0.361 & 17.9 & 0.189 & 0.126 & 18.1 & 0.17 & 0.128 & -- & -- & -- & -- & -- & -- \\
\hline 8/31/2008 4:00 AM & 0.362 & 18.0 & 0.189 & 0.126 & 18.0 & 0.17 & 0.128 & -- & -- & -- & -- & -- & -- \\
\hline 8/31/2008 5:00 AM & 0.362 & 18.0 & 0.189 & 0.128 & 18.0 & 0.17 & 0.128 & -- & -- & -- & -- & -- & -- \\
\hline 8/31/2008 6:00 AM & 0.362 & 18.0 & 0.189 & 0.126 & 18.0 & 0.17 & 0.128 & -- & -- & -- & -- & -- & -- \\
\hline 8/31/2008 7:00 AM & 0.362 & 18.0 & 0.189 & 0.126 & 18.0 & 0.17 & 0.128 & -- & -- & -- & -- & -- & -- \\
\hline 8/31/2008 8:00 AM & 0.363 & 18.1 & 0.189 & 0.126 & 18.0 & 0.16 & 0.128 & -- & -- & -- & -- & -- & -- \\
\hline 8/31/2008 9:00 AM & 0.366 & 18.1 & 0.189 & 0.128 & 18.0 & 0.16 & 0.128 & -- & -- & -- & -- & -- & -- \\
\hline 8/31/2008 10:00 AM & 0.366 & 18.1 & 0.189 & 0.126 & 18.0 & 0.16 & 0.128 & -- & -- & -- & -- & -- & -- \\
\hline 8/31/2008 11:00 AM & 0.367 & 18.1 & 0.189 & 0.128 & 18.0 & 0.16 & 0.128 & -- & -- & -- & -- & -- & -- \\
\hline 8/31/2008 12:00 PM & 0.366 & 18.1 & 0.189 & 0.126 & 18.0 & 0.16 & 0.128 & -- & -- & -- & -- & -- & -- \\
\hline 8/31/2008 1:00 PM & 0.366 & 18.1 & 0.189 & 0.126 & 18.0 & 0.16 & 0.128 & -- & -- & -- & -- & -- & -- \\
\hline 8/31/2008 2:00 PM & 0.366 & 18.1 & 0.189 & 0.126 & 18.0 & 0.16 & 0.127 & -- & -- & -- & -- & -- & -- \\
\hline 8/31/2008 3:00 PM & 0.366 & 18.0 & 0.189 & 0.126 & 18.0 & 0.16 & 0.127 & -- & -- & -- & -- & -- & -- \\
\hline 8/31/2008 4:00 PM & 0.366 & 18.0 & 0.189 & 0.126 & 18.0 & 0.16 & 0.127 & -- & -- & -- & -- & -- & -- \\
\hline 8/31/2008 5:00 PM & 0.367 & 18.0 & 0.189 & 0.126 & 18.0 & 0.16 & 0.127 & -- & -- & -- & -- & -- & -- \\
\hline 8/31/2008 6:00 PM & 0.367 & 18.0 & 0.189 & 0.126 & 18.0 & 0.16 & 0.127 & -- & -- & -- & -- & -- & -- \\
\hline 8/31/2008 7:00 PM & 0.366 & 18.0 & 0.189 & 0.126 & 18.0 & 0.16 & 0.127 & -- & -- & -- & -- & -- & -- \\
\hline 8/31/2008 8:00 PM & 0.366 & 18.0 & 0.189 & 0.126 & 18.0 & 0.16 & 0.127 & -- & -- & -- & -- & -- & -- \\
\hline 8/31/2008 9:00 PM & 0.366 & 18.0 & 0.189 & 0.126 & 18.0 & 0.16 & 0.127 & -- & -- & -- & -- & -- & -- \\
\hline 8/31/2008 10:00 PM & 0.366 & 18.0 & 0.189 & 0.126 & 18.0 & 0.16 & 0.127 & -- & -- & -- & -- & -- & -- \\
\hline
\end{tabular}


Appendix 3. Volumetric water content, temperature, and electrical-conductivity data collected at the sprinkler-irrigated site during 2008.-Continued

[Depth in feet below land surface; volumetric water content dimensionless; temperature in degrees Celsius; bulk electrical conductivity in decisiemens per meter; --, no data]

\begin{tabular}{|c|c|c|c|c|c|c|c|c|c|c|c|c|c|}
\hline \multirow[b]{3}{*}{$\begin{array}{l}\text { Measurement } \\
\text { date and time }\end{array}$} & \multicolumn{13}{|c|}{ Depth } \\
\hline & \multicolumn{2}{|l|}{2} & \multirow{2}{*}{$\begin{array}{c}3 \\
\begin{array}{c}\text { Volumetric } \\
\text { water } \\
\text { content }\end{array}\end{array}$} & \multicolumn{3}{|c|}{4} & \multirow{2}{*}{$\begin{array}{c}5 \\
\text { Volumetric } \\
\text { water } \\
\text { content }\end{array}$} & \multicolumn{2}{|l|}{7} & \multirow{2}{*}{$\begin{array}{c}9 \\
\text { Volumetric } \\
\text { water } \\
\text { content }\end{array}$} & \multicolumn{2}{|c|}{13} & \multirow{2}{*}{$\begin{array}{c}16.5 \\
\text { Volumetric } \\
\text { water } \\
\text { content }\end{array}$} \\
\hline & $\begin{array}{c}\text { Volumetric } \\
\text { water } \\
\text { content }\end{array}$ & $\begin{array}{l}\text { Temper- } \\
\text { ature }\end{array}$ & & $\begin{array}{c}\text { Volumetric } \\
\text { water } \\
\text { content }\end{array}$ & $\begin{array}{l}\text { Temper- } \\
\text { ature }\end{array}$ & $\begin{array}{c}\text { Bulk } \\
\text { electrical } \\
\text { conduct- } \\
\text { ivity }\end{array}$ & & $\begin{array}{c}\text { Volumetric } \\
\text { water } \\
\text { content }\end{array}$ & $\begin{array}{l}\text { Temper- } \\
\text { ature }\end{array}$ & & $\begin{array}{c}\text { Volumetric } \\
\text { water } \\
\text { content }\end{array}$ & $\begin{array}{l}\text { Temper- } \\
\text { ature }\end{array}$ & \\
\hline 8/31/2008 11:00 PM & 0.366 & 18.1 & 0.189 & 0.126 & 18.0 & 0.16 & 0.127 & -- & -- & -- & -- & -- & -- \\
\hline 9/1/2008 12:00 AM & 0.367 & 18.1 & 0.189 & 0.126 & 18.0 & 0.16 & 0.127 & -- & -- & -- & -- & -- & -- \\
\hline 9/1/2008 1:00 AM & 0.367 & 18.1 & 0.189 & 0.126 & 18.0 & 0.16 & 0.127 & -- & -- & -- & -- & -- & -- \\
\hline 9/1/2008 2:00 AM & 0.368 & 18.1 & 0.189 & 0.128 & 18.0 & 0.16 & 0.127 & -- & -- & -- & -- & -- & -- \\
\hline 9/1/2008 3:00 AM & 0.368 & 18.1 & 0.189 & 0.126 & 18.0 & 0.16 & 0.127 & -- & -- & -- & -- & -- & -- \\
\hline 9/1/2008 4:00 AM & 0.368 & 18.1 & 0.189 & 0.126 & 18.0 & 0.16 & 0.127 & -- & -- & -- & -- & -- & -- \\
\hline 9/1/2008 5:00 AM & 0.368 & 18.1 & 0.190 & 0.126 & 18.0 & 0.16 & 0.127 & -- & -- & -- & -- & -- & -- \\
\hline 9/1/2008 6:00 AM & 0.368 & 18.1 & 0.190 & 0.126 & 18.0 & 0.16 & 0.127 & -- & -- & -- & -- & -- & -- \\
\hline 9/1/2008 7:00 AM & 0.368 & 18.1 & 0.190 & 0.126 & 18.0 & 0.16 & 0.127 & -- & -- & -- & -- & -- & -- \\
\hline 9/1/2008 8:00 AM & 0.367 & 18.2 & 0.190 & 0.126 & 18.0 & 0.16 & 0.127 & -- & -- & -- & -- & -- & -- \\
\hline 9/1/2008 9:00 AM & 0.367 & 18.2 & 0.189 & 0.126 & 18.0 & 0.16 & 0.127 & -- & -- & -- & -- & -- & -- \\
\hline 9/1/2008 10:00 AM & 0.367 & 18.2 & 0.189 & 0.126 & 18.0 & 0.16 & 0.127 & -- & -- & -- & -- & -- & -- \\
\hline 9/1/2008 11:00 AM & 0.368 & 18.1 & 0.190 & 0.126 & 18.0 & 0.16 & 0.127 & -- & -- & -- & -- & -- & -- \\
\hline 9/1/2008 12:00 PM & 0.368 & 18.1 & 0.190 & 0.126 & 18.0 & 0.16 & 0.127 & -- & -- & -- & -- & -- & -- \\
\hline 9/1/2008 1:00 PM & 0.368 & 18.1 & 0.190 & 0.126 & 18.0 & 0.16 & 0.127 & -- & -- & -- & -- & -- & -- \\
\hline 9/1/2008 2:00 PM & 0.368 & 18.1 & 0.190 & 0.126 & 18.0 & 0.16 & 0.127 & -- & -- & -- & -- & -- & -- \\
\hline 9/1/2008 3:00 PM & 0.368 & 18.1 & 0.190 & 0.126 & 18.0 & 0.16 & 0.127 & -- & -- & -- & -- & -- & -- \\
\hline 9/1/2008 4:00 PM & 0.367 & 18.1 & 0.190 & 0.126 & 18.0 & 0.16 & 0.127 & -- & -- & -- & -- & -- & -- \\
\hline 9/1/2008 5:00 PM & 0.367 & 18.1 & 0.190 & 0.126 & 18.0 & 0.16 & 0.127 & -- & -- & -- & -- & -- & -- \\
\hline 9/1/2008 6:00 PM & 0.366 & 18.1 & 0.190 & 0.126 & 18.0 & 0.16 & 0.127 & -- & -- & -- & -- & -- & -- \\
\hline 9/1/2008 7:00 PM & 0.366 & 18.1 & 0.189 & 0.126 & 18.0 & 0.16 & 0.127 & -- & -- & -- & -- & -- & -- \\
\hline 9/1/2008 8:00 PM & 0.366 & 18.1 & 0.189 & 0.126 & 18.0 & 0.16 & 0.127 & -- & -- & -- & -- & -- & -- \\
\hline 9/1/2008 9:00 PM & 0.366 & 18.1 & 0.189 & 0.126 & 18.0 & 0.16 & 0.126 & -- & -- & -- & -- & -- & -- \\
\hline 9/1/2008 10:00 PM & 0.366 & 18.2 & 0.189 & 0.126 & 18.0 & 0.16 & 0.126 & -- & -- & -- & -- & -- & -- \\
\hline 9/1/2008 11:00 PM & 0.366 & 18.2 & 0.189 & 0.126 & 18.0 & 0.16 & 0.126 & -- & -- & -- & -- & -- & -- \\
\hline 9/2/2008 12:00 AM & 0.366 & 18.2 & 0.190 & 0.125 & 18.0 & 0.16 & 0.126 & -- & -- & -- & -- & -- & -- \\
\hline 9/2/2008 1:00 AM & 0.366 & 18.2 & 0.190 & 0.126 & 18.0 & 0.16 & 0.126 & -- & -- & -- & -- & -- & -- \\
\hline 9/2/2008 2:00 AM & 0.366 & 18.2 & 0.190 & 0.126 & 18.0 & 0.16 & 0.126 & -- & -- & -- & -- & -- & -- \\
\hline 9/2/2008 3:00 AM & 0.366 & 18.2 & 0.190 & 0.126 & 18.0 & 0.16 & 0.126 & -- & -- & -- & -- & -- & -- \\
\hline 9/2/2008 4:00 AM & 0.366 & 18.2 & 0.190 & 0.126 & 18.0 & 0.16 & 0.126 & -- & -- & -- & -- & -- & -- \\
\hline 9/2/2008 5:00 AM & 0.367 & 18.2 & 0.190 & 0.126 & 18.0 & 0.16 & 0.126 & -- & -- & -- & -- & -- & -- \\
\hline 9/2/2008 6:00 AM & 0.367 & 18.2 & 0.190 & 0.125 & 18.0 & 0.16 & 0.126 & -- & -- & -- & -- & -- & -- \\
\hline 9/2/2008 7:00 AM & 0.367 & 18.2 & 0.190 & 0.125 & 18.0 & 0.16 & 0.126 & -- & -- & -- & -- & -- & -- \\
\hline 9/2/2008 8:00 AM & 0.367 & 18.2 & 0.190 & 0.126 & 18.0 & 0.16 & 0.126 & -- & -- & -- & -- & -- & -- \\
\hline 9/2/2008 9:00 AM & 0.367 & 18.2 & 0.190 & 0.126 & 18.0 & 0.16 & 0.126 & -- & -- & -- & -- & -- & -- \\
\hline
\end{tabular}


Appendix 3. Volumetric water content, temperature, and electrical-conductivity data collected at the sprinkler-irrigated site during 2008.-Continued

[Depth in feet below land surface; volumetric water content dimensionless; temperature in degrees Celsius; bulk electrical conductivity in decisiemens per meter; --, no data]

\begin{tabular}{|c|c|c|c|c|c|c|c|c|c|c|c|c|c|}
\hline \multirow[b]{3}{*}{$\begin{array}{l}\text { Measurement } \\
\text { date and time }\end{array}$} & \multirow{2}{*}{\multicolumn{2}{|c|}{$\begin{array}{l} \\
2\end{array}$}} & \multicolumn{10}{|c|}{ Depth } & \multirow{3}{*}{$\begin{array}{c}16.5 \\
\text { Volumetric } \\
\text { water } \\
\text { content }\end{array}$} \\
\hline & & & 3 & \multicolumn{3}{|c|}{4} & \multirow[b]{2}{*}{$\begin{array}{c}\mathbf{5} \\
\text { Volumetric } \\
\text { water } \\
\text { content }\end{array}$} & \multicolumn{2}{|c|}{7} & \multirow[b]{2}{*}{$\begin{array}{c}9 \\
\text { Volumetric } \\
\text { water } \\
\text { content }\end{array}$} & \multicolumn{2}{|c|}{13} & \\
\hline & $\begin{array}{c}\text { Volumetric } \\
\text { water } \\
\text { content }\end{array}$ & $\begin{array}{l}\text { Temper- } \\
\text { ature }\end{array}$ & $\begin{array}{c}\text { Volumetric } \\
\text { water } \\
\text { content }\end{array}$ & $\begin{array}{c}\text { Volumetric } \\
\text { water } \\
\text { content }\end{array}$ & $\begin{array}{l}\text { Temper- } \\
\text { ature }\end{array}$ & $\begin{array}{c}\text { Bulk } \\
\text { electrical } \\
\text { conduct- } \\
\text { ivity }\end{array}$ & & $\begin{array}{c}\text { Volumetric } \\
\text { water } \\
\text { content }\end{array}$ & $\begin{array}{l}\text { Temper- } \\
\text { ature }\end{array}$ & & $\begin{array}{c}\text { Volumetric } \\
\text { water } \\
\text { content }\end{array}$ & $\begin{array}{l}\text { Temper- } \\
\text { ature }\end{array}$ & \\
\hline 9/2/2008 10:00 AM & 0.367 & 18.2 & 0.190 & 0.125 & 18.0 & 0.16 & 0.126 & -- & -- & -- & -- & -- & -- \\
\hline 9/2/2008 11:00 AM & 0.366 & 18.2 & 0.190 & 0.126 & 18.0 & 0.16 & 0.126 & -- & -- & -- & -- & -- & -- \\
\hline 9/2/2008 12:00 PM & 0.366 & 18.2 & 0.190 & 0.126 & 18.0 & 0.16 & 0.126 & -- & -- & -- & -- & -- & -- \\
\hline 9/2/2008 1:00 PM & 0.366 & 18.2 & 0.189 & 0.126 & 18.0 & 0.16 & 0.126 & -- & -- & -- & -- & -- & -- \\
\hline 9/2/2008 2:00 PM & 0.366 & 18.2 & 0.189 & 0.125 & 18.0 & 0.16 & 0.126 & -- & -- & -- & -- & -- & -- \\
\hline 9/2/2008 3:00 PM & 0.365 & 18.1 & 0.189 & 0.126 & 18.0 & 0.16 & 0.126 & -- & -- & -- & -- & -- & -- \\
\hline 9/2/2008 4:00 PM & 0.366 & 18.1 & 0.189 & 0.125 & 18.0 & 0.16 & 0.126 & -- & -- & -- & -- & -- & -- \\
\hline 9/2/2008 5:00 PM & 0.366 & 18.1 & 0.189 & 0.125 & 18.0 & 0.16 & 0.126 & -- & -- & -- & -- & -- & -- \\
\hline 9/2/2008 6:00 PM & 0.365 & 18.1 & 0.189 & 0.126 & 18.0 & 0.16 & 0.126 & -- & -- & -- & -- & -- & -- \\
\hline 9/2/2008 7:00 PM & 0.365 & 18.1 & 0.189 & 0.126 & 18.0 & 0.16 & 0.126 & -- & -- & -- & -- & -- & -- \\
\hline 9/2/2008 8:00 PM & 0.365 & 18.0 & 0.189 & 0.126 & 18.0 & 0.16 & 0.126 & -- & -- & -- & -- & -- & -- \\
\hline 9/2/2008 9:00 PM & 0.365 & 18.0 & 0.190 & 0.126 & 18.0 & 0.16 & 0.126 & -- & -- & -- & -- & -- & -- \\
\hline 9/2/2008 10:00 PM & 0.365 & 18.0 & 0.190 & 0.125 & 18.0 & 0.16 & 0.126 & -- & -- & -- & -- & -- & -- \\
\hline 9/2/2008 11:00 PM & 0.365 & 17.9 & 0.190 & 0.126 & 18.1 & 0.16 & 0.126 & -- & -- & -- & -- & -- & -- \\
\hline 9/3/2008 12:00 AM & 0.365 & 17.9 & 0.190 & 0.126 & 18.0 & 0.16 & 0.126 & -- & -- & -- & -- & -- & -- \\
\hline 9/3/2008 1:00 AM & 0.365 & 17.9 & 0.190 & 0.126 & 18.0 & 0.16 & 0.126 & -- & -- & -- & -- & -- & -- \\
\hline 9/3/2008 2:00 AM & 0.365 & 17.9 & 0.189 & 0.125 & 18.0 & 0.16 & 0.126 & -- & -- & -- & -- & -- & -- \\
\hline 9/3/2008 3:00 AM & 0.365 & 17.9 & 0.189 & 0.125 & 18.1 & 0.16 & 0.126 & -- & -- & -- & -- & -- & -- \\
\hline 9/3/2008 4:00 AM & 0.365 & 17.9 & 0.189 & 0.125 & 18.0 & 0.16 & 0.126 & -- & -- & -- & -- & -- & -- \\
\hline 9/3/2008 5:00 AM & 0.365 & 17.8 & 0.189 & 0.125 & 18.0 & 0.16 & 0.126 & -- & -- & -- & -- & -- & -- \\
\hline 9/3/2008 6:00 AM & 0.365 & 17.8 & 0.189 & 0.125 & 18.0 & 0.16 & 0.126 & -- & -- & -- & -- & -- & -- \\
\hline 9/3/2008 7:00 AM & 0.365 & 17.8 & 0.189 & 0.126 & 18.0 & 0.16 & 0.126 & -- & -- & -- & -- & -- & -- \\
\hline 9/3/2008 8:00 AM & 0.363 & 17.8 & 0.189 & 0.125 & 18.1 & 0.16 & 0.126 & -- & -- & -- & -- & -- & -- \\
\hline 9/3/2008 9:00 AM & 0.363 & 17.7 & 0.189 & 0.125 & 18.0 & 0.16 & 0.126 & -- & -- & -- & -- & -- & -- \\
\hline 9/3/2008 10:00 AM & 0.363 & 17.7 & 0.189 & 0.125 & 18.0 & 0.16 & 0.126 & -- & -- & -- & -- & -- & -- \\
\hline 9/3/2008 11:00 AM & 0.363 & 17.7 & 0.189 & 0.126 & 18.0 & 0.16 & 0.126 & -- & -- & -- & -- & -- & -- \\
\hline 9/3/2008 12:00 PM & 0.363 & 17.6 & 0.189 & 0.125 & 18.0 & 0.16 & 0.126 & -- & -- & -- & -- & -- & -- \\
\hline 9/3/2008 1:00 PM & 0.363 & 17.6 & 0.189 & 0.125 & 18.0 & 0.16 & 0.126 & -- & -- & -- & -- & -- & -- \\
\hline 9/3/2008 2:00 PM & 0.362 & 17.6 & 0.189 & 0.125 & 18.0 & 0.16 & 0.126 & -- & -- & -- & -- & -- & -- \\
\hline 9/3/2008 3:00 PM & 0.361 & 17.5 & 0.189 & 0.126 & 18.0 & 0.16 & 0.126 & -- & -- & -- & -- & -- & -- \\
\hline 9/3/2008 4:00 PM & 0.361 & 17.5 & 0.189 & 0.125 & 18.0 & 0.16 & 0.126 & -- & -- & -- & -- & -- & -- \\
\hline 9/3/2008 5:00 PM & 0.360 & 17.4 & 0.188 & 0.125 & 18.0 & 0.16 & 0.126 & -- & -- & -- & -- & -- & -- \\
\hline 9/3/2008 6:00 PM & 0.360 & 17.4 & 0.188 & 0.125 & 18.0 & 0.16 & 0.126 & -- & -- & -- & -- & -- & -- \\
\hline 9/3/2008 7:00 PM & 0.360 & 17.4 & 0.188 & 0.125 & 18.0 & 0.16 & 0.126 & -- & -- & -- & -- & -- & -- \\
\hline 9/3/2008 8:00 PM & 0.360 & 17.4 & 0.188 & 0.125 & 18.0 & 0.16 & 0.126 & -- & -- & -- & -- & -- & -- \\
\hline
\end{tabular}


Appendix 3. Volumetric water content, temperature, and electrical-conductivity data collected at the sprinkler-irrigated site during 2008.-Continued

[Depth in feet below land surface; volumetric water content dimensionless; temperature in degrees Celsius; bulk electrical conductivity in decisiemens per meter; --, no data]

\begin{tabular}{|c|c|c|c|c|c|c|c|c|c|c|c|c|c|}
\hline \multirow[b]{3}{*}{$\begin{array}{l}\text { Measurement } \\
\text { date and time }\end{array}$} & \multicolumn{13}{|c|}{ Depth } \\
\hline & \multicolumn{2}{|c|}{2} & \multirow{2}{*}{$\begin{array}{c}3 \\
\text { Volumetric } \\
\text { water } \\
\text { content }\end{array}$} & \multicolumn{3}{|c|}{4} & \multirow{2}{*}{$\begin{array}{c}5 \\
\text { Volumetric } \\
\text { water } \\
\text { content }\end{array}$} & \multicolumn{2}{|l|}{7} & \multirow{2}{*}{$\begin{array}{c}9 \\
\text { Volumetric } \\
\text { water } \\
\text { content }\end{array}$} & \multicolumn{2}{|c|}{13} & \multirow{2}{*}{$\begin{array}{c}16.5 \\
\text { Volumetric } \\
\text { water } \\
\text { content }\end{array}$} \\
\hline & $\begin{array}{c}\text { Volumetric } \\
\text { water } \\
\text { content }\end{array}$ & $\begin{array}{l}\text { Temper- } \\
\text { ature }\end{array}$ & & $\begin{array}{c}\text { Volumetric } \\
\text { water } \\
\text { content }\end{array}$ & $\begin{array}{l}\text { Temper- } \\
\text { ature }\end{array}$ & $\begin{array}{c}\text { Bulk } \\
\text { electrical } \\
\text { conduct- } \\
\text { ivity }\end{array}$ & & $\begin{array}{c}\text { Volumetric } \\
\text { water } \\
\text { content }\end{array}$ & $\begin{array}{l}\text { Temper- } \\
\text { ature }\end{array}$ & & $\begin{array}{c}\text { Volumetric } \\
\text { water } \\
\text { content }\end{array}$ & $\begin{array}{l}\text { Temper- } \\
\text { ature }\end{array}$ & \\
\hline 9/3/2008 9:00 PM & 0.359 & 17.3 & 0.189 & 0.125 & 18.0 & 0.16 & 0.126 & -- & -- & -- & -- & -- & -- \\
\hline 9/3/2008 10:00 PM & 0.359 & 17.3 & 0.189 & 0.125 & 18.0 & 0.16 & 0.126 & -- & -- & -- & -- & -- & -- \\
\hline 9/3/2008 11:00 PM & 0.359 & 17.3 & 0.189 & 0.125 & 18.0 & 0.16 & 0.126 & -- & -- & -- & -- & -- & -- \\
\hline 9/4/2008 12:00 AM & 0.359 & 17.3 & 0.189 & 0.125 & 18.0 & 0.16 & 0.126 & -- & -- & -- & -- & -- & -- \\
\hline 9/4/2008 1:00 AM & 0.359 & 17.3 & 0.188 & 0.125 & 18.0 & 0.16 & 0.126 & -- & -- & -- & -- & -- & -- \\
\hline 9/4/2008 2:00 AM & 0.359 & 17.2 & 0.188 & 0.125 & 18.0 & 0.16 & 0.126 & -- & -- & -- & -- & -- & -- \\
\hline 9/4/2008 3:00 AM & 0.359 & 17.3 & 0.188 & 0.125 & 18.0 & 0.16 & 0.126 & -- & -- & -- & -- & -- & -- \\
\hline 9/4/2008 4:00 AM & 0.360 & 17.2 & 0.188 & 0.125 & 18.0 & 0.16 & 0.126 & -- & -- & -- & -- & -- & -- \\
\hline 9/4/2008 5:00 AM & 0.360 & 17.2 & 0.188 & 0.125 & 18.0 & 0.16 & 0.126 & -- & -- & -- & -- & -- & -- \\
\hline 9/4/2008 6:00 AM & 0.359 & 17.2 & 0.188 & 0.125 & 18.0 & 0.16 & 0.126 & -- & -- & -- & -- & -- & -- \\
\hline 9/4/2008 7:00 AM & 0.359 & 17.2 & 0.188 & 0.125 & 18.0 & 0.16 & 0.126 & -- & -- & -- & -- & -- & -- \\
\hline 9/4/2008 8:00 AM & 0.360 & 17.2 & 0.188 & 0.125 & 18.0 & 0.16 & 0.126 & -- & -- & -- & -- & -- & -- \\
\hline 9/4/2008 9:00 AM & 0.359 & 17.2 & 0.188 & 0.125 & 18.0 & 0.16 & 0.126 & -- & -- & -- & -- & -- & -- \\
\hline 9/4/2008 10:00 AM & 0.359 & 17.2 & 0.188 & 0.125 & 18.0 & 0.16 & 0.126 & -- & -- & -- & -- & -- & -- \\
\hline 9/4/2008 11:00 AM & 0.359 & 17.2 & 0.188 & 0.125 & 18.0 & 0.16 & 0.126 & -- & -- & -- & -- & -- & -- \\
\hline 9/4/2008 12:00 PM & 0.359 & 17.2 & 0.188 & 0.125 & 18.0 & 0.16 & 0.126 & -- & -- & -- & -- & -- & -- \\
\hline 9/4/2008 1:00 PM & 0.359 & 17.1 & 0.188 & 0.125 & 18.0 & 0.16 & 0.126 & -- & -- & -- & -- & -- & -- \\
\hline 9/4/2008 2:00 PM & 0.358 & 17.1 & 0.188 & 0.125 & 18.0 & 0.16 & 0.126 & -- & -- & -- & -- & -- & -- \\
\hline 9/4/2008 3:00 PM & 0.358 & 17.1 & 0.188 & 0.124 & 18.0 & 0.16 & 0.126 & -- & -- & -- & -- & -- & -- \\
\hline 9/4/2008 4:00 PM & 0.357 & 17.0 & 0.188 & 0.124 & 17.9 & 0.16 & 0.126 & -- & -- & -- & -- & -- & -- \\
\hline 9/4/2008 5:00 PM & 0.357 & 17.0 & 0.188 & 0.124 & 18.0 & 0.16 & 0.126 & -- & -- & -- & -- & -- & -- \\
\hline 9/4/2008 6:00 PM & 0.356 & 17.0 & 0.188 & 0.124 & 18.0 & 0.16 & 0.126 & -- & -- & -- & -- & -- & -- \\
\hline 9/4/2008 7:00 PM & 0.356 & 17.0 & 0.188 & 0.124 & 17.9 & 0.16 & 0.126 & -- & -- & -- & -- & -- & -- \\
\hline 9/4/2008 8:00 PM & 0.356 & 16.9 & 0.188 & 0.125 & 17.9 & 0.16 & 0.126 & -- & -- & -- & -- & -- & -- \\
\hline 9/4/2008 9:00 PM & 0.357 & 17.0 & 0.188 & 0.125 & 17.9 & 0.16 & 0.126 & -- & -- & -- & -- & -- & -- \\
\hline 9/4/2008 10:00 PM & 0.357 & 16.9 & 0.188 & 0.124 & 17.9 & 0.16 & 0.126 & -- & -- & -- & -- & -- & -- \\
\hline 9/4/2008 11:00 PM & 0.356 & 16.9 & 0.188 & 0.124 & 17.9 & 0.16 & 0.126 & -- & -- & -- & -- & -- & -- \\
\hline 9/5/2008 12:00 AM & 0.356 & 16.9 & 0.188 & 0.124 & 17.9 & 0.16 & 0.126 & -- & -- & -- & -- & -- & -- \\
\hline 9/5/2008 1:00 AM & 0.356 & 16.9 & 0.188 & 0.124 & 17.9 & 0.16 & 0.126 & -- & -- & -- & -- & -- & -- \\
\hline 9/5/2008 2:00 AM & 0.355 & 16.9 & 0.188 & 0.124 & 17.9 & 0.16 & 0.126 & -- & -- & -- & -- & -- & -- \\
\hline 9/5/2008 3:00 AM & 0.355 & 16.9 & 0.188 & 0.124 & 17.9 & 0.16 & 0.126 & -- & -- & -- & -- & -- & -- \\
\hline 9/5/2008 4:00 AM & 0.355 & 16.9 & 0.188 & 0.124 & 17.9 & 0.16 & 0.126 & -- & -- & -- & -- & -- & -- \\
\hline 9/5/2008 5:00 AM & 0.355 & 16.9 & 0.188 & 0.124 & 17.9 & 0.16 & 0.126 & -- & -- & -- & -- & -- & -- \\
\hline 9/5/2008 6:00 AM & 0.355 & 16.9 & 0.188 & 0.124 & 17.9 & 0.16 & 0.126 & -- & -- & -- & -- & -- & -- \\
\hline 9/5/2008 7:00 AM & 0.355 & 16.9 & 0.188 & 0.124 & 17.9 & 0.16 & 0.126 & -- & -- & -- & -- & -- & -- \\
\hline
\end{tabular}


Appendix 3. Volumetric water content, temperature, and electrical-conductivity data collected at the sprinkler-irrigated site during 2008.-Continued

[Depth in feet below land surface; volumetric water content dimensionless; temperature in degrees Celsius; bulk electrical conductivity in decisiemens per meter; --, no data]

\begin{tabular}{|c|c|c|c|c|c|c|c|c|c|c|c|c|c|}
\hline \multirow[b]{3}{*}{$\begin{array}{l}\text { Measurement } \\
\text { date and time }\end{array}$} & \multirow{2}{*}{\multicolumn{2}{|c|}{2}} & \multicolumn{10}{|c|}{ Depth } & \multirow{3}{*}{$\begin{array}{c}16.5 \\
\text { Volumetric } \\
\text { water } \\
\text { content }\end{array}$} \\
\hline & & & \multirow[b]{2}{*}{$\begin{array}{c}3 \\
\begin{array}{c}\text { Volumetric } \\
\text { water } \\
\text { content }\end{array}\end{array}$} & \multicolumn{3}{|c|}{4} & \multirow[b]{2}{*}{$\begin{array}{c}\mathbf{5} \\
\text { Volumetric } \\
\text { water } \\
\text { content }\end{array}$} & \multicolumn{2}{|c|}{7} & \multirow{2}{*}{$\begin{array}{c}9 \\
\text { Volumetric } \\
\text { water } \\
\text { content }\end{array}$} & \multicolumn{2}{|c|}{13} & \\
\hline & $\begin{array}{c}\text { Volumetric } \\
\text { water } \\
\text { content }\end{array}$ & $\begin{array}{l}\text { Temper- } \\
\text { ature }\end{array}$ & & $\begin{array}{c}\text { Volumetric } \\
\text { water } \\
\text { content }\end{array}$ & $\begin{array}{l}\text { Temper- } \\
\text { ature }\end{array}$ & $\begin{array}{c}\text { Bulk } \\
\text { electrical } \\
\text { conduct- } \\
\text { ivity }\end{array}$ & & $\begin{array}{c}\text { Volumetric } \\
\text { water } \\
\text { content }\end{array}$ & $\begin{array}{l}\text { Temper- } \\
\text { ature }\end{array}$ & & $\begin{array}{c}\text { Volumetric } \\
\text { water } \\
\text { content }\end{array}$ & $\begin{array}{l}\text { Temper- } \\
\text { ature }\end{array}$ & \\
\hline 9/5/2008 8:00 AM & 0.355 & 16.9 & 0.188 & 0.125 & 17.9 & 0.16 & 0.126 & -- & -- & -- & -- & -- & -- \\
\hline 9/5/2008 9:00 AM & 0.355 & 16.9 & 0.188 & 0.124 & 17.9 & 0.16 & 0.126 & -- & -- & -- & -- & -- & -- \\
\hline 9/5/2008 10:00 AM & 0.355 & 16.9 & 0.188 & 0.124 & 17.9 & 0.16 & 0.126 & -- & -- & -- & -- & -- & -- \\
\hline 9/5/2008 11:00 AM & 0.355 & 16.9 & 0.188 & 0.124 & 17.9 & 0.16 & 0.126 & -- & -- & -- & -- & -- & -- \\
\hline 9/5/2008 12:00 PM & 0.355 & 16.9 & 0.188 & 0.125 & 17.8 & 0.16 & 0.126 & -- & -- & -- & -- & -- & -- \\
\hline 9/5/2008 1:00 PM & 0.355 & 16.8 & 0.188 & 0.124 & 17.8 & 0.16 & 0.126 & -- & -- & -- & -- & -- & -- \\
\hline 9/5/2008 2:00 PM & 0.355 & 16.8 & 0.188 & 0.124 & 17.8 & 0.16 & 0.126 & -- & -- & -- & -- & -- & -- \\
\hline 9/5/2008 3:00 PM & 0.355 & 16.8 & 0.188 & 0.124 & 17.8 & 0.16 & 0.126 & -- & -- & -- & -- & -- & -- \\
\hline 9/5/2008 4:00 PM & 0.355 & 16.8 & 0.188 & 0.124 & 17.8 & 0.16 & 0.126 & -- & -- & -- & -- & -- & -- \\
\hline 9/5/2008 5:00 PM & 0.355 & 16.8 & 0.188 & 0.124 & 17.8 & 0.16 & 0.126 & -- & -- & -- & -- & -- & -- \\
\hline 9/5/2008 6:00 PM & 0.355 & 16.8 & 0.188 & 0.124 & 17.8 & 0.16 & 0.126 & -- & -- & -- & -- & -- & -- \\
\hline 9/5/2008 7:00 PM & 0.354 & 16.7 & 0.188 & 0.124 & 17.8 & 0.16 & 0.125 & -- & -- & -- & -- & -- & -- \\
\hline 9/5/2008 8:00 PM & 0.354 & 16.7 & 0.187 & 0.124 & 17.8 & 0.16 & 0.125 & -- & -- & -- & -- & -- & -- \\
\hline 9/5/2008 9:00 PM & 0.354 & 16.7 & 0.187 & 0.124 & 17.8 & 0.16 & 0.125 & -- & -- & -- & -- & -- & -- \\
\hline 9/5/2008 10:00 PM & 0.355 & 16.7 & 0.187 & 0.124 & 17.8 & 0.16 & 0.125 & -- & -- & -- & -- & -- & -- \\
\hline 9/5/2008 11:00 PM & 0.354 & 16.7 & 0.188 & 0.124 & 17.8 & 0.16 & 0.125 & -- & -- & -- & -- & -- & -- \\
\hline 9/6/2008 12:00 AM & 0.354 & 16.7 & 0.187 & 0.124 & 17.8 & 0.16 & 0.125 & -- & -- & -- & -- & -- & -- \\
\hline 9/6/2008 1:00 AM & 0.354 & 16.7 & 0.187 & 0.124 & 17.8 & 0.16 & 0.125 & -- & -- & -- & -- & -- & -- \\
\hline 9/6/2008 2:00 AM & 0.354 & 16.6 & 0.187 & 0.124 & 17.8 & 0.16 & 0.125 & -- & -- & -- & -- & -- & -- \\
\hline 9/6/2008 3:00 AM & 0.354 & 16.6 & 0.187 & 0.124 & 17.8 & 0.16 & 0.125 & -- & -- & -- & -- & -- & -- \\
\hline 9/6/2008 4:00 AM & 0.354 & 16.6 & 0.187 & 0.124 & 17.8 & 0.16 & 0.125 & -- & -- & -- & -- & -- & -- \\
\hline 9/6/2008 5:00 AM & 0.354 & 16.6 & 0.187 & 0.124 & 17.8 & 0.16 & 0.125 & -- & -- & -- & -- & -- & -- \\
\hline 9/6/2008 6:00 AM & 0.354 & 16.6 & 0.187 & 0.124 & 17.7 & 0.16 & 0.125 & -- & -- & -- & -- & -- & -- \\
\hline 9/6/2008 7:00 AM & 0.354 & 16.6 & 0.187 & 0.124 & 17.8 & 0.16 & 0.125 & -- & -- & -- & -- & -- & -- \\
\hline 9/6/2008 8:00 AM & 0.354 & 16.5 & 0.187 & 0.124 & 17.8 & 0.16 & 0.125 & -- & -- & -- & -- & -- & -- \\
\hline 9/6/2008 9:00 AM & 0.355 & 16.5 & 0.187 & 0.124 & 17.7 & 0.16 & 0.125 & -- & -- & -- & -- & -- & -- \\
\hline 9/6/2008 10:00 AM & 0.355 & 16.5 & 0.187 & 0.124 & 17.8 & 0.16 & 0.125 & -- & -- & -- & -- & -- & -- \\
\hline 9/6/2008 11:00 AM & 0.355 & 16.5 & 0.187 & 0.124 & 17.7 & 0.16 & 0.125 & -- & -- & -- & -- & -- & -- \\
\hline 9/6/2008 12:00 PM & 0.354 & 16.5 & 0.187 & 0.123 & 17.7 & 0.16 & 0.125 & -- & -- & -- & -- & -- & -- \\
\hline 9/6/2008 1:00 PM & 0.354 & 16.4 & 0.186 & 0.124 & 17.7 & 0.16 & 0.125 & -- & -- & -- & -- & -- & -- \\
\hline 9/6/2008 2:00 PM & 0.354 & 16.4 & 0.186 & 0.124 & 17.7 & 0.16 & 0.125 & -- & -- & -- & -- & -- & -- \\
\hline 9/6/2008 3:00 PM & 0.353 & 16.4 & 0.186 & 0.123 & 17.7 & 0.16 & 0.125 & -- & -- & -- & -- & -- & -- \\
\hline 9/6/2008 4:00 PM & 0.353 & 16.4 & 0.186 & 0.123 & 17.7 & 0.16 & 0.125 & -- & -- & -- & -- & -- & -- \\
\hline 9/6/2008 5:00 PM & 0.353 & 16.4 & 0.186 & 0.123 & 17.7 & 0.16 & 0.125 & -- & -- & -- & -- & -- & -- \\
\hline 9/6/2008 6:00 PM & 0.353 & 16.4 & 0.186 & 0.123 & 17.7 & 0.16 & 0.125 & -- & -- & -- & -- & -- & -- \\
\hline
\end{tabular}


Appendix 3. Volumetric water content, temperature, and electrical-conductivity data collected at the sprinkler-irrigated site during 2008.-Continued

[Depth in feet below land surface; volumetric water content dimensionless; temperature in degrees Celsius; bulk electrical conductivity in decisiemens per meter; --, no data]

\begin{tabular}{|c|c|c|c|c|c|c|c|c|c|c|c|c|c|}
\hline \multirow[b]{3}{*}{$\begin{array}{l}\text { Measurement } \\
\text { date and time }\end{array}$} & \multicolumn{13}{|c|}{ Depth } \\
\hline & \multicolumn{2}{|c|}{2} & \multirow{2}{*}{$\begin{array}{c}3 \\
\text { Volumetric } \\
\text { water } \\
\text { content }\end{array}$} & \multicolumn{3}{|c|}{4} & \multirow{2}{*}{$\begin{array}{c}5 \\
\text { Volumetric } \\
\text { water } \\
\text { content }\end{array}$} & \multicolumn{2}{|l|}{7} & \multirow{2}{*}{$\begin{array}{c}9 \\
\text { Volumetric } \\
\text { water } \\
\text { content }\end{array}$} & \multicolumn{2}{|c|}{13} & \multirow{2}{*}{$\begin{array}{c}16.5 \\
\text { Volumetric } \\
\text { water } \\
\text { content }\end{array}$} \\
\hline & $\begin{array}{c}\text { Volumetric } \\
\text { water } \\
\text { content }\end{array}$ & $\begin{array}{l}\text { Temper- } \\
\text { ature }\end{array}$ & & $\begin{array}{c}\text { Volumetric } \\
\text { water } \\
\text { content }\end{array}$ & $\begin{array}{l}\text { Temper- } \\
\text { ature }\end{array}$ & $\begin{array}{c}\text { Bulk } \\
\text { electrical } \\
\text { conduct- } \\
\text { ivity }\end{array}$ & & $\begin{array}{c}\text { Volumetric } \\
\text { water } \\
\text { content }\end{array}$ & $\begin{array}{l}\text { Temper- } \\
\text { ature }\end{array}$ & & $\begin{array}{c}\text { Volumetric } \\
\text { water } \\
\text { content }\end{array}$ & $\begin{array}{l}\text { Temper- } \\
\text { ature }\end{array}$ & \\
\hline 9/6/2008 7:00 PM & 0.351 & 16.3 & 0.186 & 0.123 & 17.7 & 0.16 & 0.124 & -- & -- & -- & -- & -- & -- \\
\hline 9/6/2008 8:00 PM & 0.351 & 16.3 & 0.186 & 0.123 & 17.7 & 0.16 & 0.125 & -- & -- & -- & -- & -- & -- \\
\hline 9/6/2008 9:00 PM & 0.351 & 16.3 & 0.186 & 0.123 & 17.7 & 0.16 & 0.125 & -- & -- & -- & -- & -- & -- \\
\hline 9/6/2008 10:00 PM & 0.351 & 16.3 & 0.186 & 0.123 & 17.7 & 0.16 & 0.125 & -- & -- & -- & -- & -- & -- \\
\hline 9/6/2008 11:00 PM & 0.351 & 16.3 & 0.186 & 0.123 & 17.7 & 0.16 & 0.125 & -- & -- & -- & -- & -- & -- \\
\hline 9/7/2008 12:00 AM & 0.351 & 16.3 & 0.186 & 0.123 & 17.7 & 0.16 & 0.125 & -- & -- & -- & -- & -- & -- \\
\hline 9/7/2008 1:00 AM & 0.351 & 16.3 & 0.186 & 0.124 & 17.7 & 0.16 & 0.125 & -- & -- & -- & -- & -- & -- \\
\hline 9/7/2008 2:00 AM & 0.351 & 16.3 & 0.186 & 0.123 & 17.7 & 0.16 & 0.125 & -- & -- & -- & -- & -- & -- \\
\hline 9/7/2008 3:00 AM & 0.351 & 16.3 & 0.186 & 0.123 & 17.6 & 0.16 & 0.125 & -- & -- & -- & -- & -- & -- \\
\hline 9/7/2008 4:00 AM & 0.351 & 16.3 & 0.185 & 0.124 & 17.6 & 0.16 & 0.125 & -- & -- & -- & -- & -- & -- \\
\hline 9/7/2008 5:00 AM & 0.351 & 16.3 & 0.186 & 0.124 & 17.6 & 0.16 & 0.124 & -- & -- & -- & -- & -- & -- \\
\hline 9/7/2008 6:00 AM & 0.351 & 16.3 & 0.186 & 0.123 & 17.6 & 0.16 & 0.124 & -- & -- & -- & -- & -- & -- \\
\hline 9/7/2008 7:00 AM & 0.351 & 16.3 & 0.186 & 0.123 & 17.6 & 0.16 & 0.124 & -- & -- & -- & -- & -- & -- \\
\hline 9/7/2008 8:00 AM & 0.351 & 16.3 & 0.186 & 0.123 & 17.6 & 0.16 & 0.124 & -- & -- & -- & -- & -- & -- \\
\hline 9/7/2008 9:00 AM & 0.351 & 16.3 & 0.186 & 0.123 & 17.6 & 0.16 & 0.124 & -- & -- & -- & -- & -- & -- \\
\hline 9/7/2008 10:00 AM & 0.350 & 16.3 & 0.186 & 0.123 & 17.6 & 0.16 & 0.125 & -- & -- & -- & -- & -- & -- \\
\hline 9/7/2008 11:00 AM & 0.350 & 16.3 & 0.186 & 0.123 & 17.6 & 0.16 & 0.124 & -- & -- & -- & -- & -- & -- \\
\hline 9/7/2008 12:00 PM & 0.350 & 16.3 & 0.186 & 0.124 & 17.6 & 0.16 & 0.124 & -- & -- & -- & -- & -- & -- \\
\hline 9/7/2008 1:00 PM & 0.350 & 16.3 & 0.185 & 0.123 & 17.6 & 0.16 & 0.124 & -- & -- & -- & -- & -- & -- \\
\hline 9/7/2008 2:00 PM & 0.350 & 16.3 & 0.185 & 0.123 & 17.6 & 0.16 & 0.124 & -- & -- & -- & -- & -- & -- \\
\hline 9/7/2008 3:00 PM & 0.350 & 16.3 & 0.185 & 0.123 & 17.6 & 0.16 & 0.124 & -- & -- & -- & -- & -- & -- \\
\hline 9/7/2008 4:00 PM & 0.350 & 16.3 & 0.185 & 0.123 & 17.6 & 0.16 & 0.124 & -- & -- & -- & -- & -- & -- \\
\hline 9/7/2008 5:00 PM & 0.350 & 16.2 & 0.185 & 0.123 & 17.6 & 0.16 & 0.124 & -- & -- & -- & -- & -- & -- \\
\hline 9/7/2008 6:00 PM & 0.349 & 16.2 & 0.185 & 0.123 & 17.6 & 0.16 & 0.124 & -- & -- & -- & -- & -- & -- \\
\hline 9/7/2008 7:00 PM & 0.349 & 16.2 & 0.185 & 0.123 & 17.5 & 0.16 & 0.124 & -- & -- & -- & -- & -- & -- \\
\hline 9/7/2008 8:00 PM & 0.349 & 16.2 & 0.185 & 0.123 & 17.5 & 0.16 & 0.124 & -- & -- & -- & -- & -- & -- \\
\hline 9/7/2008 9:00 PM & 0.349 & 16.2 & 0.185 & 0.122 & 17.5 & 0.16 & 0.124 & -- & -- & -- & -- & -- & -- \\
\hline 9/7/2008 10:00 PM & 0.349 & 16.2 & 0.185 & 0.122 & 17.5 & 0.16 & 0.124 & -- & -- & -- & -- & -- & -- \\
\hline 9/7/2008 11:00 PM & 0.349 & 16.2 & 0.185 & 0.123 & 17.5 & 0.16 & 0.124 & -- & -- & -- & -- & -- & -- \\
\hline 9/8/2008 12:00 AM & 0.349 & 16.2 & 0.185 & 0.123 & 17.5 & 0.16 & 0.124 & -- & -- & -- & -- & -- & -- \\
\hline 9/8/2008 1:00 AM & 0.349 & 16.2 & 0.185 & 0.123 & 17.5 & 0.16 & 0.124 & -- & -- & -- & -- & -- & -- \\
\hline 9/8/2008 2:00 AM & 0.349 & 16.2 & 0.185 & 0.122 & 17.5 & 0.16 & 0.124 & -- & -- & -- & -- & -- & -- \\
\hline 9/8/2008 3:00 AM & 0.349 & 16.2 & 0.185 & 0.123 & 17.5 & 0.16 & 0.124 & -- & -- & -- & -- & -- & -- \\
\hline 9/8/2008 4:00 AM & 0.349 & 16.2 & 0.185 & 0.123 & 17.5 & 0.16 & 0.124 & -- & -- & -- & -- & -- & -- \\
\hline 9/8/2008 5:00 AM & 0.349 & 16.2 & 0.185 & 0.123 & 17.5 & 0.16 & 0.124 & -- & -- & -- & -- & -- & -- \\
\hline
\end{tabular}


Appendix 3. Volumetric water content, temperature, and electrical-conductivity data collected at the sprinkler-irrigated site during 2008.-Continued

[Depth in feet below land surface; volumetric water content dimensionless; temperature in degrees Celsius; bulk electrical conductivity in decisiemens per meter; --, no data]

\begin{tabular}{|c|c|c|c|c|c|c|c|c|c|c|c|c|c|}
\hline \multirow[b]{3}{*}{$\begin{array}{l}\text { Measurement } \\
\text { date and time }\end{array}$} & \multirow{2}{*}{\multicolumn{2}{|c|}{$\begin{array}{r} \\
2\end{array}$}} & \multirow[b]{3}{*}{$\begin{array}{c}3 \\
\text { Volumetric } \\
\text { water } \\
\text { content }\end{array}$} & \multicolumn{9}{|c|}{ Depth } & \multirow{3}{*}{$\begin{array}{c}16.5 \\
\text { Volumetric } \\
\text { water } \\
\text { content }\end{array}$} \\
\hline & & & & \multicolumn{3}{|c|}{4} & \multirow[b]{2}{*}{$\begin{array}{c}\mathbf{5} \\
\text { Volumetric } \\
\text { water } \\
\text { content }\end{array}$} & \multicolumn{2}{|l|}{7} & \multirow{2}{*}{$\begin{array}{c}9 \\
\text { Volumetric } \\
\text { water } \\
\text { content }\end{array}$} & \multicolumn{2}{|c|}{13} & \\
\hline & $\begin{array}{c}\text { Volumetric } \\
\text { water } \\
\text { content }\end{array}$ & $\begin{array}{l}\text { Temper- } \\
\text { ature }\end{array}$ & & $\begin{array}{c}\text { Volumetric } \\
\text { water } \\
\text { content }\end{array}$ & $\begin{array}{l}\text { Temper- } \\
\text { ature }\end{array}$ & $\begin{array}{c}\text { Bulk } \\
\text { electrical } \\
\text { conduct- } \\
\text { ivity }\end{array}$ & & $\begin{array}{c}\text { Volumetric } \\
\text { water } \\
\text { content }\end{array}$ & $\begin{array}{l}\text { Temper- } \\
\text { ature }\end{array}$ & & $\begin{array}{c}\text { Volumetric } \\
\text { water } \\
\text { content }\end{array}$ & $\begin{array}{l}\text { Temper- } \\
\text { ature }\end{array}$ & \\
\hline 9/8/2008 6:00 AM & 0.349 & 16.2 & 0.185 & 0.123 & 17.5 & 0.16 & 0.123 & -- & -- & -- & -- & -- & -- \\
\hline 9/8/2008 7:00 AM & 0.349 & 16.2 & 0.185 & 0.123 & 17.4 & 0.16 & 0.123 & -- & -- & -- & -- & -- & -- \\
\hline 9/8/2008 8:00 AM & 0.349 & 16.2 & 0.185 & 0.123 & 17.5 & 0.16 & 0.124 & -- & -- & -- & -- & -- & -- \\
\hline 9/8/2008 9:00 AM & 0.349 & 16.3 & 0.185 & 0.122 & 17.5 & 0.16 & 0.123 & -- & -- & -- & -- & -- & -- \\
\hline 9/8/2008 10:00 AM & 0.349 & 16.2 & 0.185 & 0.123 & 17.4 & 0.16 & 0.123 & -- & -- & -- & -- & -- & -- \\
\hline 9/8/2008 11:00 AM & 0.349 & 16.2 & 0.185 & 0.122 & 17.4 & 0.16 & 0.124 & -- & -- & -- & -- & -- & -- \\
\hline 9/8/2008 12:00 PM & 0.349 & 16.2 & 0.185 & 0.123 & 17.4 & 0.16 & 0.124 & -- & -- & -- & -- & -- & -- \\
\hline 9/8/2008 1:00 PM & 0.349 & 16.2 & 0.185 & 0.123 & 17.4 & 0.16 & 0.124 & -- & -- & -- & -- & -- & -- \\
\hline 9/8/2008 2:00 PM & 0.349 & 16.2 & 0.185 & 0.123 & 17.4 & 0.16 & 0.124 & -- & -- & -- & -- & -- & -- \\
\hline 9/8/2008 3:00 PM & 0.349 & 16.2 & 0.185 & 0.123 & 17.4 & 0.16 & 0.124 & -- & -- & -- & -- & -- & -- \\
\hline 9/8/2008 4:00 PM & 0.349 & 16.2 & 0.185 & 0.123 & 17.4 & 0.16 & 0.124 & -- & -- & -- & -- & -- & -- \\
\hline 9/8/2008 5:00 PM & 0.349 & 16.2 & 0.185 & 0.123 & 17.4 & 0.16 & 0.124 & -- & -- & -- & -- & -- & -- \\
\hline 9/8/2008 6:00 PM & 0.349 & 16.2 & 0.184 & 0.122 & 17.4 & 0.16 & 0.124 & -- & -- & -- & -- & -- & -- \\
\hline 9/8/2008 7:00 PM & 0.348 & 16.1 & 0.184 & 0.122 & 17.4 & 0.16 & 0.124 & -- & -- & -- & -- & -- & -- \\
\hline 9/8/2008 8:00 PM & 0.348 & 16.1 & 0.184 & 0.122 & 17.4 & 0.16 & 0.124 & -- & -- & -- & -- & -- & -- \\
\hline 9/8/2008 9:00 PM & 0.348 & 16.1 & 0.184 & 0.122 & 17.4 & 0.16 & 0.124 & -- & -- & -- & -- & -- & -- \\
\hline 9/8/2008 10:00 PM & 0.348 & 16.1 & 0.184 & 0.123 & 17.4 & 0.16 & 0.124 & -- & -- & -- & -- & -- & -- \\
\hline 9/8/2008 11:00 PM & 0.348 & 16.1 & 0.184 & 0.122 & 17.4 & 0.16 & 0.124 & -- & -- & -- & -- & -- & -- \\
\hline 9/9/2008 12:00 AM & 0.348 & 16.0 & 0.184 & 0.122 & 17.4 & 0.16 & 0.123 & -- & -- & -- & -- & -- & -- \\
\hline 9/9/2008 1:00 AM & 0.347 & 16.0 & 0.184 & 0.122 & 17.4 & 0.16 & 0.123 & -- & -- & -- & -- & -- & -- \\
\hline 9/9/2008 2:00 AM & 0.347 & 16.0 & 0.184 & 0.122 & 17.4 & 0.16 & 0.123 & -- & -- & -- & -- & -- & -- \\
\hline 9/9/2008 3:00 AM & 0.347 & 16.0 & 0.184 & 0.122 & 17.3 & 0.16 & 0.123 & -- & -- & -- & -- & -- & -- \\
\hline 9/9/2008 4:00 AM & 0.348 & 16.0 & 0.184 & 0.122 & 17.3 & 0.16 & 0.123 & -- & -- & -- & -- & -- & -- \\
\hline 9/9/2008 5:00 AM & 0.348 & 15.9 & 0.184 & 0.122 & 17.3 & 0.16 & 0.123 & -- & -- & -- & -- & -- & -- \\
\hline 9/9/2008 6:00 AM & 0.348 & 15.9 & 0.184 & 0.123 & 17.3 & 0.16 & 0.123 & -- & -- & -- & -- & -- & -- \\
\hline 9/9/2008 7:00 AM & 0.348 & 15.9 & 0.184 & 0.122 & 17.3 & 0.16 & 0.123 & -- & -- & -- & -- & -- & -- \\
\hline 9/9/2008 8:00 AM & 0.348 & 15.9 & 0.184 & 0.122 & 17.3 & 0.16 & 0.123 & -- & -- & -- & -- & -- & -- \\
\hline 9/9/2008 9:00 AM & 0.348 & 15.9 & 0.184 & 0.122 & 17.3 & 0.16 & 0.123 & -- & -- & -- & -- & -- & -- \\
\hline 9/9/2008 10:00 AM & 0.348 & 15.8 & 0.184 & 0.122 & 17.3 & 0.16 & 0.123 & -- & -- & -- & -- & -- & -- \\
\hline 9/9/2008 11:00 AM & 0.347 & 15.8 & 0.184 & 0.122 & 17.3 & 0.16 & 0.123 & -- & -- & -- & -- & -- & -- \\
\hline 9/9/2008 12:00 PM & 0.347 & 15.8 & 0.184 & 0.122 & 17.3 & 0.16 & 0.123 & -- & -- & -- & -- & -- & -- \\
\hline 9/9/2008 1:00 PM & 0.347 & 15.8 & 0.184 & 0.122 & 17.3 & 0.16 & 0.123 & -- & -- & -- & -- & -- & -- \\
\hline 9/9/2008 2:00 PM & 0.347 & 15.7 & 0.184 & 0.122 & 17.3 & 0.16 & 0.123 & -- & -- & -- & -- & -- & -- \\
\hline 9/9/2008 3:00 PM & 0.347 & 15.7 & 0.183 & 0.122 & 17.3 & 0.16 & 0.123 & -- & -- & -- & -- & -- & -- \\
\hline 9/9/2008 4:00 PM & 0.347 & 15.7 & 0.183 & 0.122 & 17.3 & 0.16 & 0.123 & -- & -- & -- & -- & -- & -- \\
\hline
\end{tabular}


Appendix 3. Volumetric water content, temperature, and electrical-conductivity data collected at the sprinkler-irrigated site during 2008.-Continued

[Depth in feet below land surface; volumetric water content dimensionless; temperature in degrees Celsius; bulk electrical conductivity in decisiemens per meter; --, no data]

\begin{tabular}{|c|c|c|c|c|c|c|c|c|c|c|c|c|c|}
\hline \multirow[b]{3}{*}{$\begin{array}{l}\text { Measurement } \\
\text { date and time }\end{array}$} & \multicolumn{13}{|c|}{ Depth } \\
\hline & \multicolumn{2}{|c|}{2} & \multirow{2}{*}{$\begin{array}{c}3 \\
\text { Volumetric } \\
\text { water } \\
\text { content }\end{array}$} & \multicolumn{3}{|c|}{4} & \multirow{2}{*}{$\begin{array}{c}5 \\
\text { Volumetric } \\
\text { water } \\
\text { content }\end{array}$} & \multicolumn{2}{|c|}{7} & \multirow{2}{*}{$\begin{array}{c}9 \\
\begin{array}{c}\text { Volumetric } \\
\text { water } \\
\text { content }\end{array}\end{array}$} & \multicolumn{2}{|c|}{13} & \multirow{2}{*}{$\begin{array}{c}16.5 \\
\text { Volumetric } \\
\text { water } \\
\text { content }\end{array}$} \\
\hline & $\begin{array}{c}\text { Volumetric } \\
\text { water } \\
\text { content }\end{array}$ & $\begin{array}{l}\text { Temper- } \\
\text { ature }\end{array}$ & & $\begin{array}{c}\text { Volumetric } \\
\text { water } \\
\text { content }\end{array}$ & $\begin{array}{l}\text { Temper- } \\
\text { ature }\end{array}$ & $\begin{array}{c}\text { Bulk } \\
\text { electrical } \\
\text { conduct- } \\
\text { ivity }\end{array}$ & & $\begin{array}{c}\text { Volumetric } \\
\text { water } \\
\text { content }\end{array}$ & $\begin{array}{l}\text { Temper- } \\
\text { ature }\end{array}$ & & $\begin{array}{c}\text { Volumetric } \\
\text { water } \\
\text { content }\end{array}$ & $\begin{array}{l}\text { Temper- } \\
\text { ature }\end{array}$ & \\
\hline 9/9/2008 5:00 PM & 0.346 & 15.7 & 0.183 & 0.122 & 17.3 & 0.16 & 0.123 & -- & -- & -- & -- & -- & -- \\
\hline 9/9/2008 6:00 PM & 0.346 & 15.7 & 0.183 & 0.122 & 17.3 & 0.16 & 0.123 & -- & -- & -- & -- & -- & -- \\
\hline 9/9/2008 7:00 PM & 0.346 & 15.6 & 0.184 & 0.122 & 17.3 & 0.16 & 0.123 & -- & -- & -- & -- & -- & -- \\
\hline 9/9/2008 8:00 PM & 0.345 & 15.6 & 0.184 & 0.122 & 17.3 & 0.16 & 0.123 & -- & -- & -- & -- & -- & -- \\
\hline 9/9/2008 9:00 PM & 0.345 & 15.7 & 0.184 & 0.122 & 17.2 & 0.16 & 0.123 & -- & -- & -- & -- & -- & -- \\
\hline 9/9/2008 10:00 PM & 0.345 & 15.6 & 0.184 & 0.122 & 17.3 & 0.16 & 0.123 & -- & -- & -- & -- & -- & -- \\
\hline 9/9/2008 11:00 PM & 0.345 & 15.7 & 0.184 & 0.122 & 17.3 & 0.16 & 0.123 & -- & -- & -- & -- & -- & -- \\
\hline 9/10/2008 12:00 AM & 0.345 & 15.6 & 0.183 & 0.122 & 17.2 & 0.16 & 0.123 & -- & -- & -- & -- & -- & -- \\
\hline 9/10/2008 1:00 AM & 0.345 & 15.6 & 0.183 & 0.122 & 17.2 & 0.16 & 0.123 & -- & -- & -- & -- & -- & -- \\
\hline 9/10/2008 2:00 AM & 0.345 & 15.7 & 0.183 & 0.122 & 17.2 & 0.16 & 0.123 & -- & -- & -- & -- & -- & -- \\
\hline 9/10/2008 3:00 AM & 0.345 & 15.7 & 0.183 & 0.122 & 17.2 & 0.16 & 0.123 & -- & -- & -- & -- & -- & -- \\
\hline 9/10/2008 4:00 AM & 0.345 & 15.7 & 0.183 & 0.122 & 17.2 & 0.16 & 0.122 & -- & -- & -- & -- & -- & -- \\
\hline 9/10/2008 5:00 AM & 0.345 & 15.7 & 0.183 & 0.121 & 17.2 & 0.16 & 0.122 & -- & -- & -- & -- & -- & -- \\
\hline 9/10/2008 6:00 AM & 0.345 & 15.7 & 0.183 & 0.121 & 17.2 & 0.16 & 0.122 & -- & -- & -- & -- & -- & -- \\
\hline 9/10/2008 7:00 AM & 0.345 & 15.7 & 0.183 & 0.122 & 17.2 & 0.16 & 0.122 & -- & -- & -- & -- & -- & -- \\
\hline 9/10/2008 8:00 AM & 0.346 & 15.7 & 0.183 & 0.122 & 17.2 & 0.16 & 0.122 & -- & -- & -- & -- & -- & -- \\
\hline 9/10/2008 9:00 AM & 0.346 & 15.7 & 0.183 & 0.122 & 17.2 & 0.16 & 0.123 & -- & -- & -- & -- & -- & -- \\
\hline 9/10/2008 10:00 AM & 0.346 & 15.7 & 0.183 & 0.122 & 17.2 & 0.16 & 0.123 & -- & -- & -- & -- & -- & -- \\
\hline 9/10/2008 11:00 AM & 0.346 & 15.7 & 0.183 & 0.122 & 17.1 & 0.16 & 0.123 & -- & -- & -- & -- & -- & -- \\
\hline 9/10/2008 12:00 PM & 0.346 & 15.7 & 0.183 & 0.122 & 17.2 & 0.16 & 0.123 & -- & -- & -- & -- & -- & -- \\
\hline 9/10/2008 1:00 PM & 0.346 & 15.7 & 0.183 & 0.121 & 17.1 & 0.16 & 0.122 & -- & -- & -- & -- & -- & -- \\
\hline 9/10/2008 2:00 PM & 0.346 & 15.7 & 0.183 & 0.121 & 17.1 & 0.16 & 0.122 & -- & -- & -- & -- & -- & -- \\
\hline 9/10/2008 3:00 PM & 0.346 & 15.7 & 0.183 & 0.121 & 17.1 & 0.16 & 0.122 & -- & -- & -- & -- & -- & -- \\
\hline 9/10/2008 4:00 PM & 0.345 & 15.7 & 0.183 & 0.121 & 17.1 & 0.16 & 0.122 & -- & -- & -- & -- & -- & -- \\
\hline 9/10/2008 5:00 PM & 0.345 & 15.6 & 0.183 & 0.121 & 17.1 & 0.16 & 0.122 & -- & -- & -- & -- & -- & -- \\
\hline 9/10/2008 6:00 PM & 0.345 & 15.7 & 0.183 & 0.121 & 17.1 & 0.16 & 0.122 & -- & -- & -- & -- & -- & -- \\
\hline 9/10/2008 7:00 PM & 0.345 & 15.7 & 0.183 & 0.121 & 17.1 & 0.16 & 0.122 & -- & -- & -- & -- & -- & -- \\
\hline 9/10/2008 8:00 PM & 0.344 & 15.7 & 0.183 & 0.121 & 17.1 & 0.16 & 0.122 & -- & -- & -- & -- & -- & -- \\
\hline 9/10/2008 9:00 PM & 0.344 & 15.7 & 0.183 & 0.120 & 17.1 & 0.16 & 0.122 & -- & -- & -- & -- & -- & -- \\
\hline 9/10/2008 10:00 PM & 0.344 & 15.7 & 0.183 & 0.121 & 17.1 & 0.16 & 0.122 & -- & -- & -- & -- & -- & -- \\
\hline 9/10/2008 11:00 PM & 0.344 & 15.7 & 0.183 & 0.121 & 17.1 & 0.16 & 0.122 & -- & -- & -- & -- & -- & -- \\
\hline 9/11/2008 12:00 AM & 0.344 & 15.7 & 0.183 & 0.120 & 17.1 & 0.16 & 0.122 & -- & -- & -- & -- & -- & -- \\
\hline 9/11/2008 1:00 AM & 0.345 & 15.7 & 0.183 & 0.121 & 17.1 & 0.16 & 0.122 & -- & -- & -- & -- & -- & -- \\
\hline 9/11/2008 2:00 AM & 0.345 & 15.8 & 0.183 & 0.121 & 17.1 & 0.16 & 0.122 & -- & -- & -- & -- & -- & -- \\
\hline 9/11/2008 3:00 AM & 0.345 & 15.7 & 0.183 & 0.121 & 17.1 & 0.16 & 0.122 & -- & -- & -- & -- & -- & -- \\
\hline
\end{tabular}


Appendix 3. Volumetric water content, temperature, and electrical-conductivity data collected at the sprinkler-irrigated site during 2008.-Continued

[Depth in feet below land surface; volumetric water content dimensionless; temperature in degrees Celsius; bulk electrical conductivity in decisiemens per meter; --, no data]

\begin{tabular}{|c|c|c|c|c|c|c|c|c|c|c|c|c|c|}
\hline \multirow[b]{3}{*}{$\begin{array}{l}\text { Measurement } \\
\text { date and time }\end{array}$} & \multicolumn{13}{|c|}{ Depth } \\
\hline & \multicolumn{2}{|c|}{2} & \multirow{2}{*}{$\begin{array}{c}3 \\
\text { Volumetric } \\
\text { water } \\
\text { content }\end{array}$} & \multicolumn{3}{|c|}{4} & \multirow{2}{*}{$\begin{array}{c}5 \\
\text { Volumetric } \\
\text { water } \\
\text { content }\end{array}$} & \multicolumn{2}{|l|}{7} & \multirow{2}{*}{$\begin{array}{c}9 \\
\text { Volumetric } \\
\text { water } \\
\text { content }\end{array}$} & \multicolumn{2}{|c|}{13} & \multirow{2}{*}{$\begin{array}{c}16.5 \\
\text { Volumetric } \\
\text { water } \\
\text { content }\end{array}$} \\
\hline & $\begin{array}{c}\text { Volumetric } \\
\text { water } \\
\text { content }\end{array}$ & $\begin{array}{l}\text { Temper- } \\
\text { ature }\end{array}$ & & $\begin{array}{c}\text { Volumetric } \\
\text { water } \\
\text { content }\end{array}$ & $\begin{array}{l}\text { Temper- } \\
\text { ature }\end{array}$ & $\begin{array}{c}\text { Bulk } \\
\text { electrical } \\
\text { conduct- } \\
\text { ivity }\end{array}$ & & $\begin{array}{c}\text { Volumetric } \\
\text { water } \\
\text { content }\end{array}$ & $\begin{array}{l}\text { Temper- } \\
\text { ature }\end{array}$ & & $\begin{array}{c}\text { Volumetric } \\
\text { water } \\
\text { content }\end{array}$ & $\begin{array}{l}\text { Temper- } \\
\text { ature }\end{array}$ & \\
\hline 9/11/2008 4:00 AM & 0.345 & 15.8 & 0.183 & 0.121 & 17.1 & 0.16 & 0.122 & -- & -- & -- & -- & -- & -- \\
\hline 9/11/2008 5:00 AM & 0.345 & 15.8 & 0.183 & 0.121 & 17.1 & 0.16 & 0.122 & -- & -- & -- & -- & -- & -- \\
\hline 9/11/2008 6:00 AM & 0.345 & 15.8 & 0.183 & 0.121 & 17.0 & 0.16 & 0.122 & -- & -- & -- & -- & -- & -- \\
\hline 9/11/2008 7:00 AM & 0.345 & 15.8 & 0.183 & 0.121 & 17.1 & 0.16 & 0.122 & -- & -- & -- & -- & -- & -- \\
\hline 9/11/2008 8:00 AM & 0.345 & 15.8 & 0.183 & 0.121 & 17.0 & 0.16 & 0.122 & -- & -- & -- & -- & -- & -- \\
\hline 9/11/2008 9:00 AM & 0.345 & 15.8 & 0.183 & 0.121 & 17.0 & 0.16 & 0.122 & -- & -- & -- & -- & -- & -- \\
\hline 9/11/2008 10:00 AM & 0.345 & 15.8 & 0.183 & 0.121 & 17.0 & 0.16 & 0.122 & -- & -- & -- & -- & -- & -- \\
\hline 9/11/2008 11:00 AM & 0.345 & 15.8 & 0.183 & 0.121 & 17.0 & 0.16 & 0.122 & -- & -- & -- & -- & -- & -- \\
\hline 9/11/2008 12:00 PM & 0.345 & 15.8 & 0.183 & 0.121 & 17.0 & 0.16 & 0.122 & -- & -- & -- & -- & -- & -- \\
\hline 9/11/2008 1:00 PM & 0.345 & 15.8 & 0.183 & 0.121 & 17.0 & 0.16 & 0.122 & -- & -- & -- & -- & -- & -- \\
\hline 9/11/2008 2:00 PM & 0.345 & 15.8 & 0.183 & 0.121 & 17.0 & 0.16 & 0.122 & -- & -- & -- & -- & -- & -- \\
\hline 9/11/2008 3:00 PM & 0.345 & 15.8 & 0.183 & 0.121 & 17.0 & 0.16 & 0.122 & -- & -- & -- & -- & -- & -- \\
\hline 9/11/2008 4:00 PM & 0.345 & 15.8 & 0.183 & 0.121 & 17.0 & 0.16 & 0.122 & -- & -- & -- & -- & -- & -- \\
\hline 9/11/2008 5:00 PM & 0.345 & 15.8 & 0.183 & 0.121 & 17.0 & 0.16 & 0.122 & -- & -- & -- & -- & -- & -- \\
\hline 9/11/2008 6:00 PM & 0.345 & 15.8 & 0.183 & 0.121 & 17.0 & 0.16 & 0.121 & -- & -- & -- & -- & -- & -- \\
\hline 9/11/2008 7:00 PM & 0.345 & 15.8 & 0.183 & 0.121 & 17.0 & 0.16 & 0.122 & -- & -- & -- & -- & -- & -- \\
\hline 9/11/2008 8:00 PM & 0.346 & 15.7 & 0.183 & 0.121 & 17.0 & 0.16 & 0.122 & -- & -- & -- & -- & -- & -- \\
\hline 9/11/2008 9:00 PM & 0.346 & 15.7 & 0.183 & 0.120 & 17.0 & 0.16 & 0.122 & -- & -- & -- & -- & -- & -- \\
\hline 9/11/2008 10:00 PM & 0.346 & 15.7 & 0.182 & 0.121 & 17.0 & 0.16 & 0.121 & -- & -- & -- & -- & -- & -- \\
\hline 9/11/2008 11:00 PM & 0.346 & 15.7 & 0.182 & 0.121 & 17.0 & 0.16 & 0.121 & -- & -- & -- & -- & -- & -- \\
\hline 9/12/2008 12:00 AM & 0.346 & 15.7 & 0.182 & 0.121 & 17.0 & 0.16 & 0.121 & -- & -- & -- & -- & -- & -- \\
\hline 9/12/2008 1:00 AM & 0.346 & 15.7 & 0.182 & 0.121 & 17.0 & 0.16 & 0.121 & -- & -- & -- & -- & -- & -- \\
\hline 9/12/2008 2:00 AM & 0.346 & 15.7 & 0.182 & 0.121 & 17.0 & 0.16 & 0.121 & -- & -- & -- & -- & -- & -- \\
\hline 9/12/2008 3:00 AM & 0.347 & 15.7 & 0.182 & 0.121 & 17.0 & 0.16 & 0.121 & -- & -- & -- & -- & -- & -- \\
\hline 9/12/2008 4:00 AM & 0.348 & 15.7 & 0.182 & 0.121 & 16.9 & 0.16 & 0.121 & -- & -- & -- & -- & -- & -- \\
\hline 9/12/2008 5:00 AM & 0.349 & 15.7 & 0.182 & 0.121 & 16.9 & 0.16 & 0.121 & -- & -- & -- & -- & -- & -- \\
\hline 9/12/2008 6:00 AM & 0.354 & 15.7 & 0.182 & 0.121 & 16.9 & 0.16 & 0.121 & -- & -- & -- & -- & -- & -- \\
\hline 9/12/2008 7:00 AM & 0.360 & 15.7 & 0.182 & 0.121 & 16.9 & 0.16 & 0.121 & -- & -- & -- & -- & -- & -- \\
\hline 9/12/2008 8:00 AM & 0.376 & 15.7 & 0.182 & 0.121 & 17.0 & 0.16 & 0.122 & -- & -- & -- & -- & -- & -- \\
\hline 9/12/2008 9:00 AM & 0.396 & 15.6 & 0.182 & 0.121 & 16.9 & 0.16 & 0.122 & -- & -- & -- & -- & -- & -- \\
\hline 9/12/2008 10:00 AM & 0.403 & 15.6 & 0.182 & 0.121 & 16.9 & 0.16 & 0.122 & -- & -- & -- & -- & -- & -- \\
\hline 9/12/2008 11:00 AM & 0.405 & 15.6 & 0.183 & 0.121 & 17.0 & 0.16 & 0.122 & -- & -- & -- & -- & -- & -- \\
\hline 9/12/2008 12:00 PM & 0.410 & 15.6 & 0.183 & 0.121 & 16.9 & 0.16 & 0.122 & -- & -- & -- & -- & -- & -- \\
\hline 9/12/2008 1:00 PM & 0.417 & 15.6 & 0.183 & 0.121 & 16.9 & 0.16 & 0.122 & -- & -- & -- & -- & -- & -- \\
\hline 9/12/2008 2:00 PM & 0.418 & 15.6 & 0.185 & 0.121 & 16.9 & 0.16 & 0.122 & -- & -- & -- & -- & -- & -- \\
\hline
\end{tabular}


Appendix 3. Volumetric water content, temperature, and electrical-conductivity data collected at the sprinkler-irrigated site during 2008.-Continued

[Depth in feet below land surface; volumetric water content dimensionless; temperature in degrees Celsius; bulk electrical conductivity in decisiemens per meter; --, no data]

\begin{tabular}{|c|c|c|c|c|c|c|c|c|c|c|c|c|c|}
\hline \multirow[b]{3}{*}{$\begin{array}{l}\text { Measurement } \\
\text { date and time }\end{array}$} & \multicolumn{13}{|c|}{ Depth } \\
\hline & \multicolumn{2}{|c|}{2} & \multirow{2}{*}{$\begin{array}{c}3 \\
\text { Volumetric } \\
\text { water } \\
\text { content }\end{array}$} & \multicolumn{3}{|c|}{4} & \multirow{2}{*}{$\begin{array}{c}5 \\
\text { Volumetric } \\
\text { water } \\
\text { content }\end{array}$} & \multicolumn{2}{|l|}{7} & \multirow{2}{*}{$\begin{array}{c}9 \\
\text { Volumetric } \\
\text { water } \\
\text { content }\end{array}$} & \multicolumn{2}{|c|}{13} & \multirow{2}{*}{$\begin{array}{c}16.5 \\
\text { Volumetric } \\
\text { water } \\
\text { content }\end{array}$} \\
\hline & $\begin{array}{c}\text { Volumetric } \\
\text { water } \\
\text { content }\end{array}$ & $\begin{array}{l}\text { Temper- } \\
\text { ature }\end{array}$ & & $\begin{array}{c}\text { Volumetric } \\
\text { water } \\
\text { content }\end{array}$ & $\begin{array}{l}\text { Temper- } \\
\text { ature }\end{array}$ & $\begin{array}{c}\text { Bulk } \\
\text { electrical } \\
\text { conduct- } \\
\text { ivity }\end{array}$ & & $\begin{array}{c}\text { Volumetric } \\
\text { water } \\
\text { content }\end{array}$ & $\begin{array}{l}\text { Temper- } \\
\text { ature }\end{array}$ & & $\begin{array}{c}\text { Volumetric } \\
\text { water } \\
\text { content }\end{array}$ & $\begin{array}{l}\text { Temper- } \\
\text { ature }\end{array}$ & \\
\hline 9/12/2008 3:00 PM & 0.417 & 15.5 & 0.188 & 0.121 & 16.9 & 0.16 & 0.122 & -- & -- & -- & -- & -- & -- \\
\hline 9/12/2008 4:00 PM & 0.415 & 15.5 & 0.191 & 0.120 & 16.9 & 0.16 & 0.122 & -- & -- & -- & -- & -- & -- \\
\hline 9/12/2008 5:00 PM & 0.412 & 15.5 & 0.194 & 0.121 & 16.9 & 0.16 & 0.122 & -- & -- & -- & -- & -- & -- \\
\hline 9/12/2008 6:00 PM & 0.411 & 15.5 & 0.196 & 0.121 & 16.9 & 0.16 & 0.122 & -- & -- & -- & -- & -- & -- \\
\hline 9/12/2008 7:00 PM & 0.409 & 15.4 & 0.198 & 0.121 & 16.9 & 0.16 & 0.122 & -- & -- & -- & -- & -- & -- \\
\hline 9/12/2008 8:00 PM & 0.407 & 15.5 & 0.199 & 0.121 & 16.9 & 0.16 & 0.122 & -- & -- & -- & -- & -- & -- \\
\hline 9/12/2008 9:00 PM & 0.406 & 15.4 & 0.200 & 0.120 & 16.9 & 0.16 & 0.122 & -- & -- & -- & -- & -- & -- \\
\hline 9/12/2008 10:00 PM & 0.404 & 15.4 & 0.200 & 0.121 & 16.9 & 0.16 & 0.121 & -- & -- & -- & -- & -- & -- \\
\hline 9/12/2008 11:00 PM & 0.403 & 15.4 & 0.201 & 0.121 & 16.9 & 0.16 & 0.121 & -- & -- & -- & -- & -- & -- \\
\hline 9/13/2008 12:00 AM & 0.401 & 15.4 & 0.201 & 0.121 & 16.9 & 0.16 & 0.121 & -- & -- & -- & -- & -- & -- \\
\hline 9/13/2008 1:00 AM & 0.400 & 15.4 & 0.201 & 0.121 & 16.9 & 0.16 & 0.121 & -- & -- & -- & -- & -- & -- \\
\hline 9/13/2008 2:00 AM & 0.399 & 15.4 & 0.202 & 0.121 & 16.9 & 0.16 & 0.121 & -- & -- & -- & -- & -- & -- \\
\hline 9/13/2008 3:00 AM & 0.398 & 15.4 & 0.202 & 0.121 & 16.9 & 0.16 & 0.121 & -- & -- & -- & -- & -- & -- \\
\hline 9/13/2008 4:00 AM & 0.397 & 15.4 & 0.202 & 0.121 & 16.8 & 0.16 & 0.121 & -- & -- & -- & -- & -- & -- \\
\hline 9/13/2008 5:00 AM & 0.396 & 15.4 & 0.202 & 0.121 & 16.9 & 0.16 & 0.121 & -- & -- & -- & -- & -- & -- \\
\hline 9/13/2008 6:00 AM & 0.395 & 15.4 & 0.202 & 0.121 & 16.8 & 0.16 & 0.121 & -- & -- & -- & -- & -- & -- \\
\hline 9/13/2008 7:00 AM & 0.395 & 15.3 & 0.202 & 0.122 & 16.8 & 0.16 & 0.121 & -- & -- & -- & -- & -- & -- \\
\hline 9/13/2008 8:00 AM & 0.394 & 15.3 & 0.202 & 0.122 & 16.8 & 0.16 & 0.121 & -- & -- & -- & -- & -- & -- \\
\hline 9/13/2008 9:00 AM & 0.393 & 15.3 & 0.202 & 0.122 & 16.8 & 0.16 & 0.121 & -- & -- & -- & -- & -- & -- \\
\hline 9/13/2008 10:00 AM & 0.392 & 15.3 & 0.202 & 0.122 & 16.8 & 0.16 & 0.122 & -- & -- & -- & -- & -- & -- \\
\hline 9/13/2008 11:00 AM & 0.392 & 15.3 & 0.202 & 0.122 & 16.8 & 0.16 & 0.122 & -- & -- & -- & -- & -- & -- \\
\hline 9/13/2008 12:00 PM & 0.391 & 15.3 & 0.202 & 0.123 & 16.8 & 0.16 & 0.122 & -- & -- & -- & -- & -- & -- \\
\hline 9/13/2008 1:00 PM & 0.391 & 15.3 & 0.202 & 0.122 & 16.8 & 0.16 & 0.122 & -- & -- & -- & -- & -- & -- \\
\hline 9/13/2008 2:00 PM & 0.390 & 15.2 & 0.201 & 0.123 & 16.8 & 0.16 & 0.122 & -- & -- & -- & -- & -- & -- \\
\hline 9/13/2008 3:00 PM & 0.388 & 15.2 & 0.201 & 0.122 & 16.8 & 0.16 & 0.122 & -- & -- & -- & -- & -- & -- \\
\hline 9/13/2008 4:00 PM & 0.387 & 15.2 & 0.201 & 0.122 & 16.8 & 0.16 & 0.122 & -- & -- & -- & -- & -- & -- \\
\hline 9/13/2008 5:00 PM & 0.386 & 15.2 & 0.202 & 0.123 & 16.8 & 0.16 & 0.122 & -- & -- & -- & -- & -- & -- \\
\hline 9/13/2008 6:00 PM & 0.385 & 15.2 & 0.201 & 0.123 & 16.8 & 0.16 & 0.122 & -- & -- & -- & -- & -- & -- \\
\hline 9/13/2008 7:00 PM & 0.384 & 15.1 & 0.201 & 0.123 & 16.8 & 0.16 & 0.122 & -- & -- & -- & -- & -- & -- \\
\hline 9/13/2008 8:00 PM & 0.384 & 15.1 & 0.201 & 0.123 & 16.8 & 0.16 & 0.122 & -- & -- & -- & -- & -- & -- \\
\hline 9/13/2008 9:00 PM & 0.383 & 15.1 & 0.201 & 0.124 & 16.8 & 0.16 & 0.123 & -- & -- & -- & -- & -- & -- \\
\hline 9/13/2008 10:00 PM & 0.383 & 15.1 & 0.201 & 0.124 & 16.8 & 0.16 & 0.123 & -- & -- & -- & -- & -- & -- \\
\hline 9/13/2008 11:00 PM & 0.383 & 15.1 & 0.201 & 0.123 & 16.8 & 0.16 & 0.123 & -- & -- & -- & -- & -- & -- \\
\hline 9/14/2008 12:00 AM & 0.383 & 15.1 & 0.201 & 0.124 & 16.8 & 0.16 & 0.123 & -- & -- & -- & -- & -- & -- \\
\hline 9/14/2008 1:00 AM & 0.382 & 15.1 & 0.201 & 0.124 & 16.7 & 0.16 & 0.123 & -- & -- & -- & -- & -- & -- \\
\hline
\end{tabular}


Appendix 3. Volumetric water content, temperature, and electrical-conductivity data collected at the sprinkler-irrigated site during 2008.-Continued

[Depth in feet below land surface; volumetric water content dimensionless; temperature in degrees Celsius; bulk electrical conductivity in decisiemens per meter; --, no data]

\begin{tabular}{|c|c|c|c|c|c|c|c|c|c|c|c|c|c|}
\hline \multirow[b]{3}{*}{$\begin{array}{l}\text { Measurement } \\
\text { date and time }\end{array}$} & \multirow{2}{*}{\multicolumn{2}{|c|}{2}} & \multirow{3}{*}{$\begin{array}{c}3 \\
\text { Volumetric } \\
\text { water } \\
\text { content }\end{array}$} & \multicolumn{9}{|c|}{ Depth } & \multirow{3}{*}{$\begin{array}{c}16.5 \\
\text { Volumetric } \\
\text { water } \\
\text { content }\end{array}$} \\
\hline & & & & \multicolumn{3}{|c|}{4} & \multirow[b]{2}{*}{$\begin{array}{c}\mathbf{5} \\
\text { Volumetric } \\
\text { water } \\
\text { content }\end{array}$} & \multicolumn{2}{|l|}{7} & \multirow[b]{2}{*}{$\begin{array}{c}9 \\
\text { Volumetric } \\
\text { water } \\
\text { content }\end{array}$} & \multicolumn{2}{|c|}{13} & \\
\hline & $\begin{array}{c}\text { Volumetric } \\
\text { water } \\
\text { content }\end{array}$ & $\begin{array}{l}\text { Temper- } \\
\text { ature }\end{array}$ & & $\begin{array}{c}\text { Volumetric } \\
\text { water } \\
\text { content }\end{array}$ & $\begin{array}{l}\text { Temper- } \\
\text { ature }\end{array}$ & $\begin{array}{c}\text { Bulk } \\
\text { electrical } \\
\text { conduct- } \\
\text { ivity }\end{array}$ & & $\begin{array}{l}\text { Volumetric } \\
\text { water } \\
\text { content }\end{array}$ & $\begin{array}{l}\text { Temper- } \\
\text { ature }\end{array}$ & & $\begin{array}{c}\text { Volumetric } \\
\text { water } \\
\text { content }\end{array}$ & $\begin{array}{l}\text { Temper- } \\
\text { ature }\end{array}$ & \\
\hline 9/14/2008 2:00 AM & 0.382 & 15.1 & 0.201 & 0.124 & 16.7 & 0.16 & 0.123 & -- & -- & -- & -- & -- & -- \\
\hline 9/14/2008 3:00 AM & 0.382 & 15.2 & 0.201 & 0.124 & 16.7 & 0.16 & 0.123 & -- & -- & -- & -- & -- & -- \\
\hline 9/14/2008 4:00 AM & 0.382 & 15.2 & 0.201 & 0.125 & 16.7 & 0.16 & 0.123 & -- & -- & -- & -- & -- & -- \\
\hline 9/14/2008 5:00 AM & 0.381 & 15.2 & 0.200 & 0.125 & 16.7 & 0.16 & 0.123 & -- & -- & -- & -- & -- & -- \\
\hline 9/14/2008 6:00 AM & 0.381 & 15.2 & 0.200 & 0.125 & 16.7 & 0.16 & 0.123 & -- & -- & -- & -- & -- & -- \\
\hline 9/14/2008 7:00 AM & 0.380 & 15.2 & 0.200 & 0.125 & 16.7 & 0.16 & 0.123 & -- & -- & -- & -- & -- & -- \\
\hline 9/14/2008 8:00 AM & 0.380 & 15.2 & 0.200 & 0.125 & 16.7 & 0.16 & 0.123 & -- & -- & -- & -- & -- & -- \\
\hline 9/14/2008 9:00 AM & 0.380 & 15.2 & 0.200 & 0.125 & 16.7 & 0.16 & 0.123 & -- & -- & -- & -- & -- & -- \\
\hline 9/14/2008 10:00 AM & 0.380 & 15.2 & 0.200 & 0.125 & 16.7 & 0.16 & 0.124 & -- & -- & -- & -- & -- & -- \\
\hline 9/14/2008 11:00 AM & 0.380 & 15.2 & 0.200 & 0.125 & 16.7 & 0.16 & 0.124 & -- & -- & -- & -- & -- & -- \\
\hline 9/14/2008 12:00 PM & 0.380 & 15.2 & 0.200 & 0.125 & 16.7 & 0.16 & 0.124 & -- & -- & -- & -- & -- & -- \\
\hline 9/14/2008 1:00 PM & 0.379 & 15.2 & 0.200 & 0.126 & 16.7 & 0.16 & 0.124 & -- & -- & -- & -- & -- & -- \\
\hline 9/14/2008 2:00 PM & 0.379 & 15.2 & 0.200 & 0.125 & 16.7 & 0.16 & 0.124 & -- & -- & -- & -- & -- & -- \\
\hline 9/14/2008 3:00 PM & 0.378 & 15.2 & 0.200 & 0.125 & 16.7 & 0.16 & 0.124 & -- & -- & -- & -- & -- & -- \\
\hline 9/14/2008 4:00 PM & 0.378 & 15.2 & 0.200 & 0.125 & 16.7 & 0.17 & 0.124 & -- & -- & -- & -- & -- & -- \\
\hline 9/14/2008 5:00 PM & 0.378 & 15.2 & 0.200 & 0.126 & 16.7 & 0.17 & 0.124 & -- & -- & -- & -- & -- & -- \\
\hline 9/14/2008 6:00 PM & 0.376 & 15.2 & 0.200 & 0.125 & 16.6 & 0.17 & 0.124 & -- & -- & -- & -- & -- & -- \\
\hline 9/14/2008 7:00 PM & 0.376 & 15.2 & 0.200 & 0.125 & 16.6 & 0.17 & 0.125 & -- & -- & -- & -- & -- & -- \\
\hline 9/14/2008 8:00 PM & 0.376 & 15.2 & 0.200 & 0.126 & 16.6 & 0.17 & 0.125 & -- & -- & -- & -- & -- & -- \\
\hline 9/14/2008 9:00 PM & 0.375 & 15.2 & 0.200 & 0.126 & 16.6 & 0.17 & 0.124 & -- & -- & -- & -- & -- & -- \\
\hline 9/14/2008 10:00 PM & 0.375 & 15.2 & 0.200 & 0.126 & 16.6 & 0.17 & 0.124 & -- & -- & -- & -- & -- & -- \\
\hline 9/14/2008 11:00 PM & 0.375 & 15.2 & 0.199 & 0.126 & 16.6 & 0.17 & 0.125 & -- & -- & -- & -- & -- & -- \\
\hline 9/15/2008 12:00 AM & 0.375 & 15.2 & 0.199 & 0.126 & 16.6 & 0.17 & 0.125 & -- & -- & -- & -- & -- & -- \\
\hline 9/15/2008 1:00 AM & 0.375 & 15.2 & 0.199 & 0.126 & 16.6 & 0.17 & 0.125 & -- & -- & -- & -- & -- & -- \\
\hline 9/15/2008 2:00 AM & 0.375 & 15.2 & 0.199 & 0.126 & 16.6 & 0.17 & 0.125 & -- & -- & -- & -- & -- & -- \\
\hline 9/15/2008 3:00 AM & 0.374 & 15.2 & 0.199 & 0.126 & 16.6 & 0.17 & 0.125 & -- & -- & -- & -- & -- & -- \\
\hline 9/15/2008 4:00 AM & 0.374 & 15.2 & 0.199 & 0.126 & 16.6 & 0.17 & 0.125 & -- & -- & -- & -- & -- & -- \\
\hline 9/15/2008 5:00 AM & 0.374 & 15.2 & 0.199 & 0.126 & 16.6 & 0.17 & 0.125 & -- & -- & -- & -- & -- & -- \\
\hline 9/15/2008 6:00 AM & 0.374 & 15.2 & 0.198 & 0.126 & 16.6 & 0.17 & 0.126 & -- & -- & -- & -- & -- & -- \\
\hline 9/15/2008 7:00 AM & 0.374 & 15.2 & 0.199 & 0.126 & 16.6 & 0.17 & 0.126 & -- & -- & -- & -- & -- & -- \\
\hline 9/15/2008 8:00 AM & 0.373 & 15.2 & 0.199 & 0.126 & 16.6 & 0.17 & 0.125 & -- & -- & -- & -- & -- & -- \\
\hline 9/15/2008 9:00 AM & 0.373 & 15.2 & 0.199 & 0.128 & 16.6 & 0.17 & 0.125 & -- & -- & -- & -- & -- & -- \\
\hline 9/15/2008 10:00 AM & 0.373 & 15.2 & 0.199 & 0.128 & 16.6 & 0.17 & 0.125 & -- & -- & -- & -- & -- & -- \\
\hline 9/15/2008 11:00 AM & 0.373 & 15.1 & 0.199 & 0.128 & 16.6 & 0.17 & 0.126 & -- & -- & -- & -- & -- & -- \\
\hline 9/15/2008 12:00 PM & 0.372 & 15.1 & 0.199 & 0.126 & 16.5 & 0.17 & 0.126 & -- & -- & -- & -- & -- & -- \\
\hline
\end{tabular}


Appendix 3. Volumetric water content, temperature, and electrical-conductivity data collected at the sprinkler-irrigated site during 2008.-Continued

[Depth in feet below land surface; volumetric water content dimensionless; temperature in degrees Celsius; bulk electrical conductivity in decisiemens per meter; --, no data]

\begin{tabular}{|c|c|c|c|c|c|c|c|c|c|c|c|c|c|}
\hline \multirow{3}{*}{$\begin{array}{l}\text { Measurement } \\
\text { date and time }\end{array}$} & \multicolumn{13}{|c|}{ Depth } \\
\hline & \multicolumn{2}{|c|}{2} & \multirow{2}{*}{$\begin{array}{c}3 \\
\text { Volumetric } \\
\text { water } \\
\text { content }\end{array}$} & \multicolumn{3}{|c|}{4} & \multirow{2}{*}{$\begin{array}{c}5 \\
\text { Volumetric } \\
\text { water } \\
\text { content }\end{array}$} & \multicolumn{2}{|l|}{7} & \multirow{2}{*}{$\begin{array}{c}9 \\
\text { Volumetric } \\
\text { water } \\
\text { content }\end{array}$} & \multicolumn{2}{|c|}{13} & \multirow{2}{*}{$\begin{array}{c}16.5 \\
\text { Volumetric } \\
\text { water } \\
\text { content }\end{array}$} \\
\hline & $\begin{array}{l}\text { Volumetric } \\
\text { water } \\
\text { content }\end{array}$ & $\begin{array}{l}\text { Temper- } \\
\text { ature }\end{array}$ & & $\begin{array}{l}\text { Volumetric } \\
\text { water } \\
\text { content }\end{array}$ & $\begin{array}{l}\text { Temper- } \\
\text { ature }\end{array}$ & $\begin{array}{l}\text { Bulk } \\
\text { electrical } \\
\text { conduct- } \\
\text { ivity }\end{array}$ & & $\begin{array}{l}\text { Volumetric } \\
\text { water } \\
\text { content }\end{array}$ & $\begin{array}{l}\text { Temper- } \\
\text { ature }\end{array}$ & & $\begin{array}{l}\text { Volumetric } \\
\text { water } \\
\text { content }\end{array}$ & $\begin{array}{l}\text { Temper- } \\
\text { ature }\end{array}$ & \\
\hline 9/15/2008 1:00 PM & 0.372 & 15.1 & 0.198 & 0.126 & 16.6 & 0.17 & 0.126 & -- & -- & -- & -- & -- & -- \\
\hline 9/15/2008 2:00 PM & 0.372 & 15.1 & 0.198 & 0.126 & 16.6 & 0.17 & 0.126 & -- & -- & -- & -- & -- & -- \\
\hline 9/15/2008 3:00 PM & 0.372 & 15.1 & 0.198 & 0.126 & 16.5 & 0.17 & 0.126 & -- & -- & -- & -- & -- & -- \\
\hline 9/15/2008 4:00 PM & 0.371 & 15.0 & 0.198 & 0.126 & 16.5 & 0.17 & 0.126 & -- & -- & -- & -- & -- & -- \\
\hline 9/15/2008 5:00 PM & 0.371 & 15.0 & 0.198 & 0.126 & 16.5 & 0.17 & 0.126 & -- & -- & -- & -- & -- & -- \\
\hline 9/15/2008 6:00 PM & 0.371 & 15.0 & 0.198 & 0.126 & 16.5 & 0.17 & 0.126 & -- & -- & -- & -- & -- & -- \\
\hline 9/15/2008 7:00 PM & 0.371 & 15.0 & 0.198 & 0.126 & 16.5 & 0.17 & 0.126 & -- & -- & -- & -- & -- & -- \\
\hline 9/15/2008 8:00 PM & 0.371 & 15.0 & 0.197 & 0.128 & 16.5 & 0.17 & 0.126 & -- & -- & -- & -- & -- & -- \\
\hline 9/15/2008 9:00 PM & 0.371 & 14.9 & 0.197 & 0.126 & 16.5 & 0.17 & 0.126 & -- & -- & -- & -- & -- & -- \\
\hline 9/15/2008 10:00 PM & 0.370 & 14.9 & 0.197 & 0.126 & 16.5 & 0.17 & 0.126 & -- & -- & -- & -- & -- & -- \\
\hline 9/15/2008 11:00 PM & 0.370 & 15.0 & 0.197 & 0.126 & 16.5 & 0.17 & 0.126 & -- & -- & -- & -- & -- & -- \\
\hline 9/16/2008 12:00 AM & 0.370 & 14.9 & 0.197 & 0.126 & 16.5 & 0.17 & 0.126 & -- & -- & -- & -- & -- & -- \\
\hline 9/16/2008 1:00 AM & 0.370 & 15.0 & 0.197 & 0.126 & 16.5 & 0.17 & 0.126 & -- & -- & -- & -- & -- & -- \\
\hline 9/16/2008 2:00 AM & 0.370 & 15.0 & 0.196 & 0.126 & 16.5 & 0.17 & 0.126 & -- & -- & -- & -- & -- & -- \\
\hline 9/16/2008 3:00 AM & 0.370 & 15.0 & 0.197 & 0.126 & 16.5 & 0.17 & 0.126 & -- & -- & -- & -- & -- & -- \\
\hline 9/16/2008 4:00 AM & 0.370 & 15.0 & 0.196 & 0.126 & 16.5 & 0.17 & 0.126 & -- & -- & -- & -- & -- & -- \\
\hline 9/16/2008 5:00 AM & 0.370 & 15.0 & 0.197 & 0.126 & 16.5 & 0.17 & 0.126 & -- & -- & -- & -- & -- & -- \\
\hline 9/16/2008 6:00 AM & 0.370 & 15.0 & 0.196 & 0.128 & 16.5 & 0.17 & 0.126 & -- & -- & -- & -- & -- & -- \\
\hline 9/16/2008 7:00 AM & 0.369 & 15.0 & 0.196 & 0.126 & 16.5 & 0.17 & 0.126 & -- & -- & -- & -- & -- & -- \\
\hline 9/16/2008 8:00 AM & 0.369 & 15.0 & 0.196 & 0.126 & 16.5 & 0.17 & 0.126 & -- & -- & -- & -- & -- & -- \\
\hline 9/16/2008 9:00 AM & 0.369 & 15.0 & 0.196 & 0.126 & 16.5 & 0.17 & 0.126 & -- & -- & -- & -- & -- & -- \\
\hline 9/16/2008 10:00 AM & 0.369 & 15.0 & 0.196 & 0.126 & 16.5 & 0.17 & 0.126 & -- & -- & -- & -- & -- & -- \\
\hline 9/16/2008 11:00 AM & 0.369 & 15.0 & 0.196 & 0.126 & 16.5 & 0.17 & 0.126 & -- & -- & -- & -- & -- & -- \\
\hline 9/16/2008 12:00 PM & 0.368 & 15.0 & 0.196 & 0.126 & 16.5 & 0.17 & 0.126 & -- & -- & -- & -- & -- & -- \\
\hline 9/16/2008 1:00 PM & 0.368 & 15.0 & 0.196 & 0.126 & 16.4 & 0.17 & 0.126 & -- & -- & -- & -- & -- & -- \\
\hline 9/16/2008 2:00 PM & 0.368 & 15.0 & 0.195 & 0.126 & 16.4 & 0.17 & 0.126 & -- & -- & -- & -- & -- & -- \\
\hline 9/16/2008 3:00 PM & 0.368 & 14.9 & 0.195 & 0.126 & 16.4 & 0.17 & 0.126 & -- & -- & -- & -- & -- & -- \\
\hline 9/16/2008 4:00 PM & 0.368 & 14.9 & 0.195 & 0.126 & 16.4 & 0.17 & 0.126 & -- & -- & -- & -- & -- & -- \\
\hline 9/16/2008 5:00 PM & 0.367 & 14.9 & 0.196 & 0.126 & 16.4 & 0.17 & 0.126 & -- & -- & -- & -- & -- & -- \\
\hline 9/16/2008 6:00 PM & 0.367 & 14.9 & 0.195 & 0.126 & 16.4 & 0.17 & 0.126 & -- & -- & -- & -- & -- & -- \\
\hline 9/16/2008 7:00 PM & 0.366 & 14.9 & 0.195 & 0.126 & 16.4 & 0.17 & 0.126 & -- & -- & -- & -- & -- & -- \\
\hline 9/16/2008 8:00 PM & 0.366 & 14.9 & 0.195 & 0.126 & 16.4 & 0.17 & 0.126 & -- & -- & -- & -- & -- & -- \\
\hline 9/16/2008 9:00 PM & 0.366 & 14.9 & 0.195 & 0.126 & 16.4 & 0.17 & 0.126 & -- & -- & -- & -- & -- & -- \\
\hline 9/16/2008 10:00 PM & 0.366 & 14.9 & 0.195 & 0.126 & 16.4 & 0.17 & 0.126 & -- & -- & -- & -- & -- & -- \\
\hline 9/16/2008 11:00 PM & 0.366 & 14.9 & 0.195 & 0.126 & 16.4 & 0.17 & 0.126 & -- & -- & -- & -- & -- & -- \\
\hline
\end{tabular}


Appendix 3. Volumetric water content, temperature, and electrical-conductivity data collected at the sprinkler-irrigated site during 2008.-Continued

[Depth in feet below land surface; volumetric water content dimensionless; temperature in degrees Celsius; bulk electrical conductivity in decisiemens per meter; --, no data]

\begin{tabular}{|c|c|c|c|c|c|c|c|c|c|c|c|c|c|}
\hline \multirow[b]{3}{*}{$\begin{array}{l}\text { Measurement } \\
\text { date and time }\end{array}$} & \multirow{2}{*}{\multicolumn{2}{|c|}{2}} & \multirow{3}{*}{$\begin{array}{c}3 \\
\text { Volumetric } \\
\text { water } \\
\text { content }\end{array}$} & \multicolumn{9}{|c|}{ Depth } & \multirow{3}{*}{$\begin{array}{c}16.5 \\
\text { Volumetric } \\
\text { water } \\
\text { content }\end{array}$} \\
\hline & & & & \multicolumn{3}{|c|}{4} & \multirow[b]{2}{*}{$\begin{array}{c}\mathbf{5} \\
\text { Volumetric } \\
\text { water } \\
\text { content }\end{array}$} & \multicolumn{2}{|l|}{7} & \multirow{2}{*}{$\begin{array}{c}9 \\
\text { Volumetric } \\
\text { water } \\
\text { content }\end{array}$} & \multicolumn{2}{|c|}{13} & \\
\hline & $\begin{array}{c}\text { Volumetric } \\
\text { water } \\
\text { content }\end{array}$ & $\begin{array}{l}\text { Temper- } \\
\text { ature }\end{array}$ & & $\begin{array}{c}\text { Volumetric } \\
\text { water } \\
\text { content }\end{array}$ & $\begin{array}{l}\text { Temper- } \\
\text { ature }\end{array}$ & $\begin{array}{c}\text { Bulk } \\
\text { electrical } \\
\text { conduct- } \\
\text { ivity }\end{array}$ & & $\begin{array}{l}\text { Volumetric } \\
\text { water } \\
\text { content }\end{array}$ & $\begin{array}{l}\text { Temper- } \\
\text { ature }\end{array}$ & & $\begin{array}{c}\text { Volumetric } \\
\text { water } \\
\text { content }\end{array}$ & $\begin{array}{l}\text { Temper- } \\
\text { ature }\end{array}$ & \\
\hline 9/17/2008 12:00 AM & 0.366 & 14.9 & 0.195 & 0.126 & 16.4 & 0.17 & 0.126 & -- & -- & -- & -- & -- & -- \\
\hline 9/17/2008 1:00 AM & 0.366 & 14.9 & 0.195 & 0.126 & 16.4 & 0.17 & 0.126 & -- & -- & -- & -- & -- & -- \\
\hline 9/17/2008 2:00 AM & 0.366 & 14.9 & 0.194 & 0.126 & 16.4 & 0.17 & 0.126 & -- & -- & -- & -- & -- & -- \\
\hline 9/17/2008 3:00 AM & 0.366 & 14.9 & 0.194 & 0.126 & 16.4 & 0.17 & 0.126 & -- & -- & -- & -- & -- & -- \\
\hline 9/17/2008 4:00 AM & 0.366 & 15.0 & 0.194 & 0.126 & 16.4 & 0.17 & 0.126 & -- & -- & -- & -- & -- & -- \\
\hline 9/17/2008 5:00 AM & 0.366 & 15.0 & 0.194 & 0.126 & 16.4 & 0.17 & 0.126 & -- & -- & -- & -- & -- & -- \\
\hline 9/17/2008 6:00 AM & 0.366 & 15.0 & 0.194 & 0.126 & 16.4 & 0.17 & 0.126 & -- & -- & -- & -- & -- & -- \\
\hline 9/17/2008 7:00 AM & 0.366 & 15.0 & 0.194 & 0.126 & 16.4 & 0.17 & 0.126 & -- & -- & -- & -- & -- & -- \\
\hline 9/17/2008 8:00 AM & 0.365 & 15.0 & 0.194 & 0.126 & 16.4 & 0.17 & 0.126 & -- & -- & -- & -- & -- & -- \\
\hline 9/17/2008 9:00 AM & 0.365 & 15.0 & 0.194 & 0.126 & 16.4 & 0.17 & 0.126 & -- & -- & -- & -- & -- & -- \\
\hline 9/17/2008 10:00 AM & 0.365 & 15.0 & 0.194 & 0.126 & 16.4 & 0.17 & 0.126 & -- & -- & -- & -- & -- & -- \\
\hline 9/17/2008 11:00 AM & 0.365 & 15.0 & 0.194 & 0.126 & 16.3 & 0.17 & 0.126 & -- & -- & -- & -- & -- & -- \\
\hline 9/17/2008 12:00 PM & 0.365 & 15.0 & 0.194 & 0.126 & 16.3 & 0.17 & 0.126 & -- & -- & 0.120 & -- & -- & -- \\
\hline 9/17/2008 1:00 PM & 0.365 & 15.0 & 0.194 & 0.126 & 16.3 & 0.17 & 0.126 & -- & -- & 0.120 & -- & -- & -- \\
\hline 9/17/2008 2:00 PM & 0.365 & 15.0 & 0.194 & 0.125 & 16.4 & 0.17 & 0.126 & -- & -- & 0.120 & -- & -- & -- \\
\hline 9/17/2008 3:00 PM & 0.363 & 15.0 & 0.194 & 0.126 & 16.3 & 0.17 & 0.126 & -- & -- & 0.120 & -- & -- & -- \\
\hline 9/17/2008 4:00 PM & 0.363 & 15.0 & 0.194 & 0.125 & 16.3 & 0.16 & 0.126 & -- & -- & 0.120 & -- & -- & -- \\
\hline 9/17/2008 5:00 PM & 0.363 & 15.0 & 0.194 & 0.126 & 16.3 & 0.16 & 0.126 & -- & -- & 0.120 & -- & -- & -- \\
\hline 9/17/2008 6:00 PM & 0.363 & 15.0 & 0.194 & 0.126 & 16.3 & 0.17 & 0.126 & -- & -- & 0.120 & -- & -- & -- \\
\hline 9/17/2008 7:00 PM & 0.363 & 15.0 & 0.194 & 0.125 & 16.3 & 0.17 & 0.126 & -- & -- & 0.120 & -- & -- & -- \\
\hline 9/17/2008 8:00 PM & 0.363 & 15.0 & 0.194 & 0.126 & 16.3 & 0.17 & 0.126 & -- & -- & 0.121 & -- & -- & -- \\
\hline 9/17/2008 9:00 PM & 0.363 & 14.9 & 0.194 & 0.126 & 16.3 & 0.17 & 0.126 & -- & -- & 0.121 & -- & -- & -- \\
\hline 9/17/2008 10:00 PM & 0.363 & 15.0 & 0.194 & 0.126 & 16.3 & 0.17 & 0.126 & -- & -- & 0.121 & -- & -- & -- \\
\hline 9/17/2008 11:00 PM & 0.363 & 15.0 & 0.194 & 0.126 & 16.3 & 0.17 & 0.126 & -- & -- & 0.121 & -- & -- & -- \\
\hline 9/18/2008 12:00 AM & 0.362 & 15.0 & 0.193 & 0.125 & 16.3 & 0.17 & 0.126 & -- & -- & 0.121 & -- & -- & -- \\
\hline 9/18/2008 1:00 AM & 0.362 & 15.0 & 0.194 & 0.125 & 16.3 & 0.17 & 0.126 & -- & -- & 0.120 & -- & -- & -- \\
\hline 9/18/2008 2:00 AM & 0.362 & 15.0 & 0.193 & 0.125 & 16.3 & 0.17 & 0.126 & -- & -- & 0.117 & -- & -- & -- \\
\hline 9/18/2008 3:00 AM & 0.362 & 15.1 & 0.193 & 0.126 & 16.3 & 0.16 & 0.126 & -- & -- & 0.120 & -- & -- & -- \\
\hline 9/18/2008 4:00 AM & 0.362 & 15.1 & 0.193 & 0.125 & 16.3 & 0.16 & 0.126 & -- & -- & 0.122 & -- & -- & -- \\
\hline 9/18/2008 5:00 AM & 0.362 & 15.1 & 0.193 & 0.125 & 16.3 & 0.16 & 0.126 & -- & -- & 0.124 & -- & -- & -- \\
\hline 9/18/2008 6:00 AM & 0.362 & 15.1 & 0.193 & 0.125 & 16.3 & 0.16 & 0.126 & -- & -- & 0.125 & -- & -- & -- \\
\hline 9/18/2008 7:00 AM & 0.361 & 15.1 & 0.193 & 0.125 & 16.3 & 0.16 & 0.126 & -- & -- & 0.124 & -- & -- & -- \\
\hline 9/18/2008 8:00 AM & 0.361 & 15.1 & 0.193 & 0.125 & 16.3 & 0.16 & 0.126 & -- & -- & 0.123 & -- & -- & -- \\
\hline 9/18/2008 9:00 AM & 0.361 & 15.1 & 0.193 & 0.125 & 16.3 & 0.16 & 0.126 & -- & -- & 0.123 & -- & -- & -- \\
\hline 9/18/2008 10:00 AM & 0.362 & 15.1 & 0.193 & 0.126 & 16.3 & 0.16 & 0.126 & -- & -- & 0.126 & -- & -- & -- \\
\hline
\end{tabular}


Appendix 3. Volumetric water content, temperature, and electrical-conductivity data collected at the sprinkler-irrigated site during 2008.-Continued

[Depth in feet below land surface; volumetric water content dimensionless; temperature in degrees Celsius; bulk electrical conductivity in decisiemens per meter; --, no data]

\begin{tabular}{|c|c|c|c|c|c|c|c|c|c|c|c|c|c|}
\hline \multirow[b]{3}{*}{$\begin{array}{l}\text { Measurement } \\
\text { date and time }\end{array}$} & \multicolumn{13}{|c|}{ Depth } \\
\hline & \multicolumn{2}{|c|}{2} & \multirow{2}{*}{$\begin{array}{c}3 \\
\text { Volumetric } \\
\text { water } \\
\text { content }\end{array}$} & \multicolumn{3}{|c|}{4} & \multirow{2}{*}{$\begin{array}{c}5 \\
\text { Volumetric } \\
\text { water } \\
\text { content }\end{array}$} & \multicolumn{2}{|l|}{7} & \multirow{2}{*}{$\begin{array}{c}9 \\
\text { Volumetric } \\
\text { water } \\
\text { content }\end{array}$} & \multicolumn{2}{|c|}{13} & \multirow{2}{*}{$\begin{array}{c}16.5 \\
\text { Volumetric } \\
\text { water } \\
\text { content }\end{array}$} \\
\hline & $\begin{array}{c}\text { Volumetric } \\
\text { water } \\
\text { content }\end{array}$ & $\begin{array}{l}\text { Temper- } \\
\text { ature }\end{array}$ & & $\begin{array}{c}\text { Volumetric } \\
\text { water } \\
\text { content }\end{array}$ & $\begin{array}{l}\text { Temper- } \\
\text { ature }\end{array}$ & $\begin{array}{c}\text { Bulk } \\
\text { electrical } \\
\text { conduct- } \\
\text { ivity }\end{array}$ & & $\begin{array}{l}\text { Volumetric } \\
\text { water } \\
\text { content }\end{array}$ & $\begin{array}{l}\text { Temper- } \\
\text { ature }\end{array}$ & & $\begin{array}{c}\text { Volumetric } \\
\text { water } \\
\text { content }\end{array}$ & $\begin{array}{l}\text { Temper- } \\
\text { ature }\end{array}$ & \\
\hline 9/18/2008 11:00 AM & 0.361 & 15.1 & 0.193 & 0.125 & 16.3 & 0.16 & 0.126 & -- & -- & 0.126 & -- & -- & -- \\
\hline 9/18/2008 12:00 PM & 0.362 & 15.2 & 0.192 & 0.125 & 16.3 & 0.16 & 0.126 & -- & -- & 0.126 & -- & -- & -- \\
\hline 9/18/2008 1:00 PM & 0.362 & 15.1 & 0.192 & 0.125 & 16.2 & 0.16 & 0.126 & -- & -- & 0.125 & -- & -- & -- \\
\hline 9/18/2008 2:00 PM & 0.361 & 15.1 & 0.192 & 0.125 & 16.3 & 0.16 & 0.126 & -- & -- & 0.121 & -- & -- & -- \\
\hline 9/18/2008 3:00 PM & 0.361 & 15.2 & 0.192 & 0.125 & 16.2 & 0.17 & 0.126 & -- & -- & 0.121 & -- & -- & -- \\
\hline 9/18/2008 4:00 PM & 0.361 & 15.1 & 0.192 & 0.125 & 16.3 & 0.16 & 0.126 & -- & -- & 0.121 & -- & -- & -- \\
\hline 9/18/2008 5:00 PM & 0.360 & 15.1 & 0.192 & 0.125 & 16.2 & 0.17 & 0.126 & -- & -- & 0.121 & -- & -- & -- \\
\hline 9/18/2008 6:00 PM & 0.360 & 15.1 & 0.192 & 0.125 & 16.2 & 0.17 & 0.126 & -- & -- & 0.121 & -- & -- & -- \\
\hline 9/18/2008 7:00 PM & 0.360 & 15.1 & 0.192 & 0.125 & 16.2 & 0.17 & 0.125 & -- & -- & 0.121 & -- & -- & -- \\
\hline 9/18/2008 8:00 PM & 0.360 & 15.1 & 0.192 & 0.125 & 16.2 & 0.17 & 0.126 & -- & -- & 0.121 & -- & -- & -- \\
\hline 9/18/2008 9:00 PM & 0.360 & 15.1 & 0.192 & 0.125 & 16.2 & 0.16 & 0.126 & -- & -- & 0.121 & -- & -- & -- \\
\hline 9/18/2008 10:00 PM & 0.360 & 15.1 & 0.192 & 0.125 & 16.2 & 0.17 & 0.126 & -- & -- & 0.121 & -- & -- & -- \\
\hline 9/18/2008 11:00 PM & 0.359 & 15.1 & 0.192 & 0.125 & 16.2 & 0.17 & 0.125 & -- & -- & 0.121 & -- & -- & -- \\
\hline 9/19/2008 12:00 AM & 0.359 & 15.1 & 0.192 & 0.125 & 16.2 & 0.17 & 0.125 & -- & -- & 0.121 & -- & -- & -- \\
\hline 9/19/2008 1:00 AM & 0.359 & 15.2 & 0.192 & 0.125 & 16.2 & 0.17 & 0.125 & -- & -- & 0.124 & -- & -- & -- \\
\hline 9/19/2008 2:00 AM & 0.359 & 15.2 & 0.192 & 0.125 & 16.2 & 0.17 & 0.125 & -- & -- & 0.124 & -- & -- & -- \\
\hline 9/19/2008 3:00 AM & 0.360 & 15.2 & 0.192 & 0.125 & 16.2 & 0.16 & 0.125 & -- & -- & 0.125 & -- & -- & -- \\
\hline 9/19/2008 4:00 AM & 0.360 & 15.2 & 0.192 & 0.125 & 16.2 & 0.16 & 0.125 & -- & -- & 0.126 & -- & -- & -- \\
\hline 9/19/2008 5:00 AM & 0.360 & 15.2 & 0.192 & 0.125 & 16.2 & 0.16 & 0.125 & -- & -- & 0.125 & -- & -- & -- \\
\hline 9/19/2008 6:00 AM & 0.360 & 15.2 & 0.192 & 0.125 & 16.2 & 0.16 & 0.125 & -- & -- & 0.124 & -- & -- & -- \\
\hline 9/19/2008 7:00 AM & 0.360 & 15.2 & 0.191 & 0.125 & 16.2 & 0.16 & 0.125 & -- & -- & 0.124 & -- & -- & -- \\
\hline 9/19/2008 8:00 AM & 0.359 & 15.3 & 0.191 & 0.125 & 16.2 & 0.16 & 0.125 & -- & -- & 0.124 & -- & -- & -- \\
\hline 9/19/2008 9:00 AM & 0.359 & 15.2 & 0.191 & 0.125 & 16.2 & 0.16 & 0.125 & -- & -- & 0.126 & -- & -- & -- \\
\hline 9/19/2008 10:00 AM & 0.360 & 15.2 & 0.191 & 0.125 & 16.2 & 0.16 & 0.125 & -- & -- & 0.127 & -- & -- & -- \\
\hline 9/19/2008 11:00 AM & 0.359 & 15.2 & 0.191 & 0.125 & 16.2 & 0.16 & 0.125 & -- & -- & 0.128 & -- & -- & -- \\
\hline 9/19/2008 12:00 PM & 0.359 & 15.2 & 0.191 & 0.125 & 16.2 & 0.16 & 0.125 & -- & -- & 0.126 & -- & -- & -- \\
\hline 9/19/2008 1:00 PM & 0.359 & 15.2 & 0.191 & 0.125 & 16.2 & 0.16 & 0.125 & -- & -- & 0.121 & -- & -- & -- \\
\hline 9/19/2008 2:00 PM & 0.359 & 15.2 & 0.191 & 0.124 & 16.2 & 0.16 & 0.125 & -- & -- & 0.121 & -- & -- & -- \\
\hline 9/19/2008 3:00 PM & 0.359 & 15.2 & 0.191 & 0.124 & 16.2 & 0.16 & 0.125 & -- & -- & 0.121 & -- & -- & -- \\
\hline 9/19/2008 4:00 PM & 0.358 & 15.2 & 0.191 & 0.125 & 16.2 & 0.16 & 0.125 & -- & -- & 0.121 & -- & -- & -- \\
\hline 9/19/2008 5:00 PM & 0.358 & 15.2 & 0.190 & 0.124 & 16.2 & 0.16 & 0.125 & -- & -- & 0.121 & -- & -- & -- \\
\hline 9/19/2008 6:00 PM & 0.358 & 15.2 & 0.190 & 0.124 & 16.2 & 0.16 & 0.125 & -- & -- & 0.121 & -- & -- & -- \\
\hline 9/19/2008 7:00 PM & 0.358 & 15.2 & 0.191 & 0.124 & 16.2 & 0.16 & 0.125 & -- & -- & 0.121 & -- & -- & -- \\
\hline 9/19/2008 8:00 PM & 0.358 & 15.2 & 0.191 & 0.124 & 16.2 & 0.16 & 0.125 & -- & -- & 0.121 & -- & -- & -- \\
\hline 9/19/2008 9:00 PM & 0.357 & 15.2 & 0.191 & 0.124 & 16.2 & 0.16 & 0.125 & -- & -- & 0.121 & -- & -- & -- \\
\hline
\end{tabular}


Appendix 3. Volumetric water content, temperature, and electrical-conductivity data collected at the sprinkler-irrigated site during 2008.-Continued

[Depth in feet below land surface; volumetric water content dimensionless; temperature in degrees Celsius; bulk electrical conductivity in decisiemens per meter; --, no data]

\begin{tabular}{|c|c|c|c|c|c|c|c|c|c|c|c|c|c|}
\hline \multirow[b]{3}{*}{$\begin{array}{l}\text { Measurement } \\
\text { date and time }\end{array}$} & \multicolumn{13}{|c|}{ Depth } \\
\hline & \multicolumn{2}{|l|}{2} & \multirow{2}{*}{$\begin{array}{c}3 \\
\text { Volumetric } \\
\text { water } \\
\text { content }\end{array}$} & \multicolumn{3}{|c|}{4} & \multirow{2}{*}{$\begin{array}{c}\mathbf{5} \\
\text { Volumetric } \\
\text { water } \\
\text { content }\end{array}$} & \multicolumn{2}{|c|}{7} & \multirow{2}{*}{$\begin{array}{c}9 \\
\text { Volumetric } \\
\text { water } \\
\text { content }\end{array}$} & \multicolumn{2}{|c|}{13} & \multirow{2}{*}{$\begin{array}{c}16.5 \\
\text { Volumetric } \\
\text { water } \\
\text { content }\end{array}$} \\
\hline & $\begin{array}{c}\text { Volumetric } \\
\text { water } \\
\text { content }\end{array}$ & $\begin{array}{l}\text { Temper- } \\
\text { ature }\end{array}$ & & $\begin{array}{c}\text { Volumetric } \\
\text { water } \\
\text { content }\end{array}$ & $\begin{array}{l}\text { Temper- } \\
\text { ature }\end{array}$ & $\begin{array}{c}\text { Bulk } \\
\text { electrical } \\
\text { conduct- } \\
\text { ivity }\end{array}$ & & $\begin{array}{c}\text { Volumetric } \\
\text { water } \\
\text { content }\end{array}$ & $\begin{array}{l}\text { Temper- } \\
\text { ature }\end{array}$ & & $\begin{array}{c}\text { Volumetric } \\
\text { water } \\
\text { content }\end{array}$ & $\begin{array}{l}\text { Temper- } \\
\text { ature }\end{array}$ & \\
\hline 9/19/2008 10:00 PM & 0.357 & 15.2 & 0.190 & 0.124 & 16.2 & 0.16 & 0.125 & -- & -- & 0.119 & -- & -- & -- \\
\hline 9/19/2008 11:00 PM & 0.357 & 15.2 & 0.190 & 0.124 & 16.2 & 0.16 & 0.125 & -- & -- & 0.125 & -- & -- & -- \\
\hline 9/20/2008 12:00 AM & 0.357 & 15.2 & 0.190 & 0.124 & 16.2 & 0.16 & 0.125 & & -- & 0.126 & & -- & \\
\hline 9/20/2008 1:00 AM & 0.357 & 15.2 & 0.190 & 0.124 & 16.2 & 0.16 & 0.125 & -- & -- & 0.127 & -- & -- & -- \\
\hline 9/20/2008 2:00 AM & 0.357 & 15.2 & 0.190 & 0.124 & 16.2 & 0.16 & 0.124 & -- & -- & 0.126 & -- & -- & -- \\
\hline 9/20/2008 3:00 AM & 0.357 & 15.3 & 0.190 & 0.124 & 16.2 & 0.16 & 0.125 & -- & -- & 0.126 & -- & -- & -- \\
\hline 9/20/2008 4:00 AM & 0.357 & 15.3 & 0.190 & 0.124 & 16.2 & 0.16 & 0.124 & -- & -- & 0.125 & -- & -- & -- \\
\hline 9/20/2008 5:00 AM & 0.357 & 15.3 & 0.190 & 0.124 & 16.2 & 0.16 & 0.124 & -- & -- & 0.125 & -- & -- & -- \\
\hline 9/20/2008 6:00 AM & 0.358 & 15.3 & 0.190 & 0.124 & 16.2 & 0.16 & 0.124 & -- & -- & 0.124 & -- & -- & -- \\
\hline 9/20/2008 7:00 AM & 0.358 & 15.3 & 0.190 & 0.124 & 16.2 & 0.16 & 0.125 & -- & -- & 0.124 & -- & -- & -- \\
\hline 9/20/2008 8:00 AM & 0.358 & 15.4 & 0.190 & 0.124 & 16.2 & 0.16 & 0.124 & -- & -- & 0.124 & -- & -- & -- \\
\hline 9/20/2008 9:00 AM & 0.357 & 15.4 & 0.190 & 0.125 & 16.2 & 0.16 & 0.124 & -- & -- & 0.124 & -- & -- & -- \\
\hline 9/20/2008 10:00 AM & 0.358 & 15.4 & 0.190 & 0.124 & 16.2 & 0.16 & 0.125 & -- & -- & 0.125 & -- & -- & -- \\
\hline 9/20/2008 11:00 AM & 0.358 & 15.3 & 0.190 & 0.124 & 16.2 & 0.16 & 0.125 & -- & -- & 0.126 & -- & -- & -- \\
\hline 9/20/2008 12:00 PM & 0.357 & 15.3 & 0.190 & 0.124 & 16.2 & 0.16 & 0.125 & -- & -- & 0.127 & -- & -- & -- \\
\hline 9/20/2008 1:00 PM & 0.357 & 15.3 & 0.189 & 0.124 & 16.2 & 0.16 & 0.125 & -- & -- & 0.126 & -- & -- & -- \\
\hline 9/20/2008 2:00 PM & 0.357 & 15.3 & 0.189 & 0.124 & 16.2 & 0.16 & 0.125 & -- & -- & 0.123 & -- & -- & -- \\
\hline 9/20/2008 3:00 PM & 0.357 & 15.3 & 0.189 & 0.124 & 16.2 & 0.16 & 0.125 & -- & -- & 0.121 & -- & -- & -- \\
\hline 9/20/2008 4:00 PM & 0.357 & 15.3 & 0.189 & 0.124 & 16.2 & 0.16 & 0.124 & -- & -- & 0.121 & -- & -- & -- \\
\hline 9/20/2008 5:00 PM & 0.356 & 15.3 & 0.189 & 0.124 & 16.2 & 0.16 & 0.124 & -- & -- & 0.121 & -- & -- & -- \\
\hline 9/20/2008 6:00 PM & 0.356 & 15.3 & 0.189 & 0.124 & 16.2 & 0.16 & 0.124 & -- & -- & 0.121 & -- & -- & -- \\
\hline 9/20/2008 7:00 PM & 0.356 & 15.3 & 0.189 & 0.124 & 16.2 & 0.16 & 0.124 & -- & -- & 0.121 & -- & -- & -- \\
\hline 9/20/2008 8:00 PM & 0.356 & 15.3 & 0.189 & 0.124 & 16.2 & 0.16 & 0.124 & -- & -- & 0.121 & -- & -- & -- \\
\hline 9/20/2008 9:00 PM & 0.355 & 15.3 & 0.189 & 0.124 & 16.2 & 0.16 & 0.124 & -- & -- & 0.121 & -- & -- & -- \\
\hline 9/20/2008 10:00 PM & 0.355 & 15.3 & 0.189 & 0.124 & 16.2 & 0.16 & 0.124 & -- & -- & 0.121 & -- & -- & -- \\
\hline 9/20/2008 11:00 PM & 0.355 & 15.3 & 0.189 & 0.124 & 16.2 & 0.16 & 0.124 & -- & -- & 0.121 & -- & -- & -- \\
\hline 9/21/2008 12:00 AM & 0.355 & 15.3 & 0.189 & 0.124 & 16.2 & 0.16 & 0.124 & -- & -- & 0.121 & -- & -- & -- \\
\hline 9/21/2008 1:00 AM & 0.355 & 15.3 & 0.189 & 0.124 & 16.2 & 0.16 & 0.124 & -- & -- & 0.121 & -- & -- & -- \\
\hline 9/21/2008 2:00 AM & 0.355 & 15.3 & 0.189 & 0.124 & 16.2 & 0.16 & 0.124 & -- & -- & 0.121 & -- & -- & -- \\
\hline 9/21/2008 3:00 AM & 0.355 & 15.4 & 0.189 & 0.124 & 16.2 & 0.16 & 0.124 & -- & -- & 0.120 & -- & -- & -- \\
\hline 9/21/2008 4:00 AM & 0.355 & 15.4 & 0.189 & 0.124 & 16.1 & 0.16 & 0.124 & -- & -- & 0.118 & -- & -- & -- \\
\hline 9/21/2008 5:00 AM & 0.355 & 15.4 & 0.189 & 0.123 & 16.2 & 0.16 & 0.124 & -- & -- & 0.119 & -- & -- & -- \\
\hline 9/21/2008 6:00 AM & 0.355 & 15.4 & 0.189 & 0.124 & 16.1 & 0.16 & 0.124 & -- & -- & 0.121 & -- & -- & -- \\
\hline 9/21/2008 7:00 AM & 0.355 & 15.4 & 0.188 & 0.124 & 16.2 & 0.16 & 0.124 & -- & -- & 0.121 & -- & -- & -- \\
\hline 9/21/2008 8:00 AM & 0.356 & 15.4 & 0.188 & 0.124 & 16.1 & 0.16 & 0.124 & -- & -- & 0.122 & -- & -- & -- \\
\hline
\end{tabular}


Appendix 3. Volumetric water content, temperature, and electrical-conductivity data collected at the sprinkler-irrigated site during 2008.-Continued

[Depth in feet below land surface; volumetric water content dimensionless; temperature in degrees Celsius; bulk electrical conductivity in decisiemens per meter; --, no data]

\begin{tabular}{|c|c|c|c|c|c|c|c|c|c|c|c|c|c|}
\hline \multirow[b]{3}{*}{$\begin{array}{l}\text { Measurement } \\
\text { date and time }\end{array}$} & \multicolumn{13}{|c|}{ Depth } \\
\hline & \multicolumn{2}{|c|}{2} & \multirow{2}{*}{$\begin{array}{c}3 \\
\text { Volumetric } \\
\text { water } \\
\text { content }\end{array}$} & \multicolumn{3}{|c|}{4} & \multirow{2}{*}{$\begin{array}{c}5 \\
\text { Volumetric } \\
\text { water } \\
\text { content }\end{array}$} & \multicolumn{2}{|l|}{7} & \multirow{2}{*}{$\begin{array}{c}9 \\
\text { Volumetric } \\
\text { water } \\
\text { content }\end{array}$} & \multicolumn{2}{|c|}{13} & \multirow{2}{*}{$\begin{array}{c}16.5 \\
\text { Volumetric } \\
\text { water } \\
\text { content }\end{array}$} \\
\hline & $\begin{array}{c}\text { Volumetric } \\
\text { water } \\
\text { content }\end{array}$ & $\begin{array}{l}\text { Temper- } \\
\text { ature }\end{array}$ & & $\begin{array}{c}\text { Volumetric } \\
\text { water } \\
\text { content }\end{array}$ & $\begin{array}{l}\text { Temper- } \\
\text { ature }\end{array}$ & $\begin{array}{c}\text { Bulk } \\
\text { electrical } \\
\text { conduct- } \\
\text { ivity }\end{array}$ & & $\begin{array}{l}\text { Volumetric } \\
\text { water } \\
\text { content }\end{array}$ & $\begin{array}{l}\text { Temper- } \\
\text { ature }\end{array}$ & & $\begin{array}{c}\text { Volumetric } \\
\text { water } \\
\text { content }\end{array}$ & $\begin{array}{l}\text { Temper- } \\
\text { ature }\end{array}$ & \\
\hline 9/21/2008 9:00 AM & 0.355 & 15.4 & 0.188 & 0.124 & 16.2 & 0.16 & 0.124 & -- & -- & 0.124 & -- & -- & -- \\
\hline 9/21/2008 10:00 AM & 0.355 & 15.4 & 0.188 & 0.124 & 16.2 & 0.16 & 0.124 & -- & -- & 0.124 & -- & -- & -- \\
\hline 9/21/2008 11:00 AM & 0.355 & 15.4 & 0.188 & 0.124 & 16.2 & 0.16 & 0.124 & -- & -- & 0.122 & -- & -- & -- \\
\hline 9/21/2008 12:00 PM & 0.356 & 15.4 & 0.188 & 0.124 & 16.2 & 0.16 & 0.124 & -- & -- & 0.122 & -- & -- & -- \\
\hline 9/21/2008 1:00 PM & 0.356 & 15.4 & 0.188 & 0.124 & 16.2 & 0.16 & 0.124 & -- & -- & 0.121 & -- & -- & -- \\
\hline 9/21/2008 2:00 PM & 0.356 & 15.4 & 0.188 & 0.124 & 16.1 & 0.16 & 0.124 & -- & -- & 0.121 & -- & -- & -- \\
\hline 9/21/2008 3:00 PM & 0.355 & 15.4 & 0.188 & 0.123 & 16.2 & 0.16 & 0.124 & -- & -- & 0.121 & -- & -- & -- \\
\hline 9/21/2008 4:00 PM & 0.355 & 15.4 & 0.188 & 0.123 & 16.1 & 0.16 & 0.124 & -- & -- & 0.121 & -- & -- & -- \\
\hline 9/21/2008 5:00 PM & 0.355 & 15.4 & 0.188 & 0.123 & 16.1 & 0.16 & 0.124 & -- & -- & 0.121 & -- & -- & -- \\
\hline 9/21/2008 6:00 PM & 0.355 & 15.4 & 0.188 & 0.123 & 16.1 & 0.16 & 0.124 & -- & -- & 0.121 & -- & -- & -- \\
\hline 9/21/2008 7:00 PM & 0.355 & 15.4 & 0.188 & 0.123 & 16.2 & 0.16 & 0.124 & -- & -- & 0.121 & -- & -- & -- \\
\hline 9/21/2008 8:00 PM & 0.354 & 15.4 & 0.188 & 0.123 & 16.2 & 0.16 & 0.124 & -- & -- & 0.121 & -- & -- & -- \\
\hline 9/21/2008 9:00 PM & 0.354 & 15.4 & 0.188 & 0.123 & 16.1 & 0.16 & 0.124 & -- & -- & 0.121 & -- & -- & -- \\
\hline 9/21/2008 10:00 PM & 0.354 & 15.4 & 0.188 & 0.123 & 16.2 & 0.16 & 0.124 & -- & -- & 0.121 & -- & -- & -- \\
\hline 9/21/2008 11:00 PM & 0.354 & 15.4 & 0.188 & 0.123 & 16.2 & 0.16 & 0.124 & -- & -- & 0.121 & -- & -- & -- \\
\hline 9/22/2008 12:00 AM & 0.354 & 15.4 & 0.188 & 0.123 & 16.2 & 0.16 & 0.123 & -- & -- & 0.121 & -- & -- & -- \\
\hline 9/22/2008 1:00 AM & 0.354 & 15.5 & 0.188 & 0.123 & 16.2 & 0.16 & 0.123 & -- & -- & 0.121 & -- & -- & -- \\
\hline 9/22/2008 2:00 AM & 0.354 & 15.5 & 0.188 & 0.123 & 16.1 & 0.16 & 0.123 & -- & -- & 0.119 & -- & -- & -- \\
\hline 9/22/2008 3:00 AM & 0.354 & 15.5 & 0.188 & 0.123 & 16.1 & 0.16 & 0.124 & -- & -- & 0.128 & -- & -- & -- \\
\hline 9/22/2008 4:00 AM & 0.354 & 15.5 & 0.188 & 0.123 & 16.2 & 0.16 & 0.123 & -- & -- & 0.143 & -- & -- & -- \\
\hline 9/22/2008 5:00 AM & 0.354 & 15.6 & 0.188 & 0.124 & 16.1 & 0.16 & 0.123 & -- & -- & 0.133 & -- & -- & -- \\
\hline 9/22/2008 6:00 AM & 0.355 & 15.6 & 0.188 & 0.123 & 16.2 & 0.16 & 0.123 & -- & -- & 0.134 & -- & -- & -- \\
\hline 9/22/2008 7:00 AM & 0.354 & 15.6 & 0.188 & 0.123 & 16.2 & 0.16 & 0.124 & -- & -- & 0.134 & -- & -- & -- \\
\hline 9/22/2008 8:00 AM & 0.355 & 15.6 & 0.188 & 0.123 & 16.1 & 0.16 & 0.123 & -- & -- & 0.137 & -- & -- & -- \\
\hline 9/22/2008 9:00 AM & 0.355 & 15.6 & 0.188 & 0.123 & 16.1 & 0.16 & 0.123 & -- & -- & 0.138 & -- & -- & -- \\
\hline 9/22/2008 10:00 AM & 0.355 & 15.6 & 0.188 & 0.123 & 16.1 & 0.16 & 0.124 & -- & -- & 0.152 & -- & -- & -- \\
\hline 9/22/2008 11:00 AM & 0.355 & 15.6 & 0.188 & 0.123 & 16.1 & 0.16 & 0.124 & -- & -- & 0.149 & -- & -- & -- \\
\hline 9/22/2008 12:00 PM & 0.355 & 15.6 & 0.188 & 0.123 & 16.1 & 0.16 & 0.124 & -- & -- & 0.143 & -- & -- & -- \\
\hline 9/22/2008 1:00 PM & 0.355 & 15.6 & 0.188 & 0.123 & 16.1 & 0.16 & 0.123 & -- & -- & 0.136 & -- & -- & -- \\
\hline 9/22/2008 2:00 PM & 0.355 & 15.5 & 0.188 & 0.123 & 16.1 & 0.16 & 0.123 & -- & -- & 0.124 & -- & -- & -- \\
\hline 9/22/2008 3:00 PM & 0.354 & 15.5 & 0.188 & 0.123 & 16.1 & 0.16 & 0.123 & -- & -- & 0.121 & -- & -- & -- \\
\hline 9/22/2008 4:00 PM & 0.354 & 15.5 & 0.188 & 0.123 & 16.1 & 0.16 & 0.123 & -- & -- & 0.121 & -- & -- & -- \\
\hline 9/22/2008 5:00 PM & 0.354 & 15.5 & 0.187 & 0.123 & 16.2 & 0.16 & 0.123 & -- & -- & 0.121 & -- & -- & -- \\
\hline 9/22/2008 6:00 PM & 0.353 & 15.5 & 0.187 & 0.123 & 16.2 & 0.16 & 0.123 & -- & -- & 0.121 & -- & -- & -- \\
\hline 9/22/2008 7:00 PM & 0.353 & 15.5 & 0.188 & 0.123 & 16.1 & 0.16 & 0.123 & -- & -- & 0.121 & -- & -- & -- \\
\hline
\end{tabular}


Appendix 3. Volumetric water content, temperature, and electrical-conductivity data collected at the sprinkler-irrigated site during 2008.-Continued

[Depth in feet below land surface; volumetric water content dimensionless; temperature in degrees Celsius; bulk electrical conductivity in decisiemens per meter; --, no data]

\begin{tabular}{|c|c|c|c|c|c|c|c|c|c|c|c|c|c|}
\hline \multirow[b]{3}{*}{$\begin{array}{l}\text { Measurement } \\
\text { date and time }\end{array}$} & \multicolumn{13}{|c|}{ Depth } \\
\hline & \multicolumn{2}{|l|}{2} & \multirow[b]{2}{*}{$\begin{array}{c}3 \\
\begin{array}{c}\text { Volumetric } \\
\text { water } \\
\text { content }\end{array}\end{array}$} & \multicolumn{3}{|c|}{4} & \multirow{2}{*}{$\begin{array}{c}\mathbf{5} \\
\text { Volumetric } \\
\text { water } \\
\text { content }\end{array}$} & \multicolumn{2}{|c|}{7} & \multirow{2}{*}{$\begin{array}{c}9 \\
\text { Volumetric } \\
\text { water } \\
\text { content }\end{array}$} & \multicolumn{2}{|c|}{13} & \multirow{2}{*}{$\begin{array}{c}16.5 \\
\text { Volumetric } \\
\text { water } \\
\text { content }\end{array}$} \\
\hline & $\begin{array}{c}\text { Volumetric } \\
\text { water } \\
\text { content }\end{array}$ & $\begin{array}{l}\text { Temper- } \\
\text { ature }\end{array}$ & & $\begin{array}{c}\text { Volumetric } \\
\text { water } \\
\text { content }\end{array}$ & $\begin{array}{l}\text { Temper- } \\
\text { ature }\end{array}$ & $\begin{array}{c}\text { Bulk } \\
\text { electrical } \\
\text { conduct- } \\
\text { ivity } \\
\end{array}$ & & $\begin{array}{c}\text { Volumetric } \\
\text { water } \\
\text { content }\end{array}$ & $\begin{array}{l}\text { Temper- } \\
\text { ature }\end{array}$ & & $\begin{array}{c}\text { Volumetric } \\
\text { water } \\
\text { content }\end{array}$ & $\begin{array}{l}\text { Temper- } \\
\text { ature }\end{array}$ & \\
\hline 9/22/2008 8:00 PM & 0.353 & 15.5 & 0.188 & 0.123 & 16.2 & 0.16 & 0.123 & -- & -- & 0.121 & -- & -- & -- \\
\hline 9/22/2008 9:00 PM & 0.353 & 15.5 & 0.188 & 0.123 & 16.1 & 0.16 & 0.123 & -- & -- & 0.121 & -- & -- & -- \\
\hline 9/22/2008 10:00 PM & 0.353 & 15.5 & 0.188 & 0.123 & 16.1 & 0.16 & 0.123 & -- & -- & 0.121 & -- & -- & -- \\
\hline 9/22/2008 11:00 PM & 0.353 & 15.5 & 0.187 & 0.123 & 16.2 & 0.16 & 0.123 & -- & -- & 0.121 & -- & -- & -- \\
\hline 9/23/2008 12:00 AM & 0.353 & 15.5 & 0.187 & 0.123 & 16.2 & 0.16 & 0.123 & -- & -- & 0.121 & -- & -- & -- \\
\hline 9/23/2008 1:00 AM & 0.353 & 15.5 & 0.187 & 0.123 & 16.1 & 0.16 & 0.123 & -- & -- & 0.120 & -- & -- & -- \\
\hline 9/23/2008 2:00 AM & 0.353 & 15.5 & 0.187 & 0.123 & 16.1 & 0.16 & 0.123 & -- & -- & 0.122 & -- & -- & -- \\
\hline 9/23/2008 3:00 AM & 0.353 & 15.6 & 0.187 & 0.123 & 16.1 & 0.16 & 0.123 & -- & -- & 0.133 & -- & -- & -- \\
\hline 9/23/2008 4:00 AM & 0.353 & 15.6 & 0.187 & 0.123 & 16.1 & 0.16 & 0.123 & -- & -- & 0.138 & -- & -- & -- \\
\hline 9/23/2008 5:00 AM & 0.353 & 15.6 & 0.187 & 0.123 & 16.1 & 0.16 & 0.123 & -- & -- & 0.142 & -- & -- & -- \\
\hline 9/23/2008 6:00 AM & 0.353 & 15.6 & 0.187 & 0.123 & 16.1 & 0.16 & 0.123 & -- & -- & 0.143 & -- & -- & -- \\
\hline 9/23/2008 7:00 AM & 0.353 & 15.6 & 0.187 & 0.123 & 16.2 & 0.16 & 0.123 & -- & -- & 0.143 & -- & -- & -- \\
\hline 9/23/2008 8:00 AM & 0.353 & 15.6 & 0.187 & 0.123 & 16.2 & 0.16 & 0.123 & -- & -- & 0.144 & -- & -- & -- \\
\hline 9/23/2008 9:00 AM & 0.353 & 15.6 & 0.187 & 0.122 & 16.2 & 0.16 & 0.123 & -- & -- & 0.146 & -- & -- & -- \\
\hline 9/23/2008 10:00 AM & 0.353 & 15.6 & 0.187 & 0.123 & 16.2 & 0.16 & 0.123 & -- & -- & 0.143 & -- & -- & -- \\
\hline 9/23/2008 11:00 AM & 0.353 & 15.6 & 0.187 & 0.122 & 16.1 & 0.16 & 0.123 & -- & -- & 0.141 & -- & -- & -- \\
\hline 9/23/2008 12:00 PM & 0.353 & 15.6 & 0.187 & 0.123 & 16.1 & 0.16 & 0.123 & -- & -- & 0.137 & -- & -- & -- \\
\hline 9/23/2008 1:00 PM & 0.353 & 15.6 & 0.186 & 0.123 & 16.1 & 0.16 & 0.123 & -- & -- & 0.128 & -- & -- & -- \\
\hline 9/23/2008 2:00 PM & 0.353 & 15.6 & 0.186 & 0.123 & 16.1 & 0.16 & 0.123 & -- & -- & 0.121 & -- & -- & -- \\
\hline 9/23/2008 3:00 PM & 0.353 & 15.6 & 0.187 & 0.122 & 16.2 & 0.16 & 0.123 & -- & -- & 0.121 & -- & -- & -- \\
\hline 9/23/2008 4:00 PM & 0.353 & 15.5 & 0.187 & 0.122 & 16.1 & 0.16 & 0.123 & -- & -- & 0.121 & -- & -- & -- \\
\hline 9/23/2008 5:00 PM & 0.351 & 15.5 & 0.187 & 0.122 & 16.1 & 0.16 & 0.123 & -- & -- & 0.121 & -- & -- & -- \\
\hline 9/23/2008 6:00 PM & 0.351 & 15.5 & 0.187 & 0.122 & 16.1 & 0.16 & 0.123 & -- & -- & 0.121 & -- & -- & -- \\
\hline 9/23/2008 7:00 PM & 0.350 & 15.5 & 0.187 & 0.122 & 16.2 & 0.16 & 0.123 & -- & -- & 0.121 & -- & -- & -- \\
\hline 9/23/2008 8:00 PM & 0.350 & 15.5 & 0.187 & 0.122 & 16.2 & 0.16 & 0.123 & -- & -- & 0.121 & -- & -- & -- \\
\hline 9/23/2008 9:00 PM & 0.350 & 15.5 & 0.187 & 0.122 & 16.1 & 0.16 & 0.123 & -- & -- & 0.121 & -- & -- & -- \\
\hline 9/23/2008 10:00 PM & 0.350 & 15.5 & 0.186 & 0.122 & 16.1 & 0.16 & 0.122 & -- & -- & 0.119 & -- & -- & -- \\
\hline 9/23/2008 11:00 PM & 0.350 & 15.5 & 0.186 & 0.122 & 16.2 & 0.16 & 0.122 & -- & -- & 0.119 & -- & -- & -- \\
\hline 9/24/2008 12:00 AM & 0.350 & 15.5 & 0.186 & 0.122 & 16.2 & 0.16 & 0.123 & -- & -- & 0.122 & -- & -- & -- \\
\hline 9/24/2008 1:00 AM & 0.350 & 15.5 & 0.186 & 0.122 & 16.1 & 0.16 & 0.123 & -- & -- & 0.122 & -- & -- & -- \\
\hline 9/24/2008 2:00 AM & 0.350 & 15.5 & 0.186 & 0.122 & 16.1 & 0.16 & 0.122 & -- & -- & 0.121 & -- & -- & -- \\
\hline 9/24/2008 3:00 AM & 0.349 & 15.5 & 0.186 & 0.122 & 16.2 & 0.16 & 0.122 & -- & -- & 0.125 & -- & -- & -- \\
\hline 9/24/2008 4:00 AM & 0.349 & 15.5 & 0.186 & 0.123 & 16.2 & 0.16 & 0.122 & -- & -- & 0.128 & -- & -- & -- \\
\hline 9/24/2008 5:00 AM & 0.349 & 15.5 & 0.186 & 0.122 & 16.1 & 0.16 & 0.122 & -- & -- & 0.132 & -- & -- & -- \\
\hline 9/24/2008 6:00 AM & 0.349 & 15.5 & 0.186 & 0.123 & 16.2 & 0.16 & 0.122 & -- & -- & 0.133 & -- & -- & -- \\
\hline
\end{tabular}


Appendix 3. Volumetric water content, temperature, and electrical-conductivity data collected at the sprinkler-irrigated site during 2008.-Continued

[Depth in feet below land surface; volumetric water content dimensionless; temperature in degrees Celsius; bulk electrical conductivity in decisiemens per meter; --, no data]

\begin{tabular}{|c|c|c|c|c|c|c|c|c|c|c|c|c|c|}
\hline \multirow[b]{3}{*}{$\begin{array}{l}\text { Measurement } \\
\text { date and time }\end{array}$} & \multicolumn{13}{|c|}{ Depth } \\
\hline & \multicolumn{2}{|c|}{2} & \multirow{2}{*}{$\begin{array}{c}3 \\
\text { Volumetric } \\
\text { water } \\
\text { content }\end{array}$} & \multicolumn{3}{|c|}{4} & \multirow{2}{*}{$\begin{array}{c}5 \\
\text { Volumetric } \\
\text { water } \\
\text { content }\end{array}$} & \multicolumn{2}{|l|}{7} & \multirow{2}{*}{$\begin{array}{c}9 \\
\text { Volumetric } \\
\text { water } \\
\text { content }\end{array}$} & \multicolumn{2}{|c|}{13} & \multirow{2}{*}{$\begin{array}{c}16.5 \\
\text { Volumetric } \\
\text { water } \\
\text { content }\end{array}$} \\
\hline & $\begin{array}{c}\text { Volumetric } \\
\text { water } \\
\text { content }\end{array}$ & $\begin{array}{l}\text { Temper- } \\
\text { ature }\end{array}$ & & $\begin{array}{c}\text { Volumetric } \\
\text { water } \\
\text { content }\end{array}$ & $\begin{array}{l}\text { Temper- } \\
\text { ature }\end{array}$ & $\begin{array}{c}\text { Bulk } \\
\text { electrical } \\
\text { conduct- } \\
\text { ivity }\end{array}$ & & $\begin{array}{l}\text { Volumetric } \\
\text { water } \\
\text { content }\end{array}$ & $\begin{array}{l}\text { Temper- } \\
\text { ature }\end{array}$ & & $\begin{array}{c}\text { Volumetric } \\
\text { water } \\
\text { content }\end{array}$ & $\begin{array}{l}\text { Temper- } \\
\text { ature }\end{array}$ & \\
\hline 9/24/2008 7:00 AM & 0.349 & 15.5 & 0.186 & 0.122 & 16.2 & 0.16 & 0.122 & -- & -- & 0.135 & -- & -- & -- \\
\hline 9/24/2008 8:00 AM & 0.349 & 15.5 & 0.186 & 0.122 & 16.1 & 0.16 & 0.122 & -- & -- & 0.138 & -- & -- & -- \\
\hline 9/24/2008 9:00 AM & 0.349 & 15.5 & 0.186 & 0.122 & 16.1 & 0.16 & 0.122 & -- & -- & 0.144 & -- & -- & -- \\
\hline 9/24/2008 10:00 AM & 0.349 & 15.5 & 0.186 & 0.122 & 16.1 & 0.16 & 0.122 & -- & -- & 0.143 & -- & -- & -- \\
\hline 9/24/2008 11:00 AM & 0.349 & 15.5 & 0.186 & 0.122 & 16.2 & 0.16 & 0.122 & -- & -- & 0.139 & -- & -- & -- \\
\hline 9/24/2008 12:00 PM & 0.349 & 15.4 & 0.186 & 0.122 & 16.1 & 0.16 & 0.122 & -- & -- & 0.138 & -- & -- & -- \\
\hline 9/24/2008 1:00 PM & 0.349 & 15.4 & 0.185 & 0.122 & 16.2 & 0.16 & 0.122 & -- & -- & 0.131 & -- & -- & -- \\
\hline 9/24/2008 2:00 PM & 0.349 & 15.4 & 0.185 & 0.122 & 16.2 & 0.16 & 0.122 & -- & -- & 0.126 & -- & -- & -- \\
\hline 9/24/2008 3:00 PM & 0.349 & 15.4 & 0.185 & 0.122 & 16.1 & 0.16 & 0.122 & -- & -- & 0.122 & -- & -- & -- \\
\hline 9/24/2008 4:00 PM & 0.348 & 15.4 & 0.185 & 0.122 & 16.2 & 0.16 & 0.122 & -- & -- & 0.121 & -- & -- & -- \\
\hline 9/24/2008 5:00 PM & 0.348 & 15.3 & 0.185 & 0.122 & 16.1 & 0.16 & 0.122 & -- & -- & 0.121 & -- & -- & -- \\
\hline 9/24/2008 6:00 PM & 0.348 & 15.3 & 0.185 & 0.122 & 16.2 & 0.16 & 0.122 & -- & -- & 0.121 & -- & -- & -- \\
\hline 9/24/2008 7:00 PM & 0.347 & 15.3 & 0.186 & 0.122 & 16.1 & 0.16 & 0.122 & -- & -- & 0.121 & -- & -- & -- \\
\hline 9/24/2008 8:00 PM & 0.347 & 15.3 & 0.186 & 0.122 & 16.2 & 0.16 & 0.122 & -- & -- & 0.121 & -- & -- & -- \\
\hline 9/24/2008 9:00 PM & 0.347 & 15.3 & 0.185 & 0.121 & 16.1 & 0.16 & 0.122 & -- & -- & 0.121 & -- & -- & -- \\
\hline 9/24/2008 10:00 PM & 0.347 & 15.3 & 0.185 & 0.122 & 16.1 & 0.16 & 0.122 & -- & -- & 0.120 & -- & -- & -- \\
\hline 9/24/2008 11:00 PM & 0.347 & 15.3 & 0.185 & 0.122 & 16.1 & 0.16 & 0.122 & -- & -- & 0.121 & -- & -- & -- \\
\hline 9/25/2008 12:00 AM & 0.347 & 15.3 & 0.185 & 0.122 & 16.2 & 0.16 & 0.122 & -- & -- & 0.131 & -- & -- & -- \\
\hline 9/25/2008 1:00 AM & 0.347 & 15.3 & 0.185 & 0.122 & 16.1 & 0.16 & 0.122 & -- & -- & 0.133 & -- & -- & -- \\
\hline 9/25/2008 2:00 AM & 0.347 & 15.3 & 0.185 & 0.121 & 16.1 & 0.16 & 0.122 & -- & -- & 0.136 & -- & -- & -- \\
\hline 9/25/2008 3:00 AM & 0.347 & 15.3 & 0.185 & 0.122 & 16.1 & 0.16 & 0.122 & -- & -- & 0.138 & -- & -- & -- \\
\hline 9/25/2008 4:00 AM & 0.347 & 15.3 & 0.185 & 0.122 & 16.1 & 0.16 & 0.122 & -- & -- & 0.141 & -- & -- & -- \\
\hline 9/25/2008 5:00 AM & 0.347 & 15.4 & 0.185 & 0.122 & 16.1 & 0.16 & 0.122 & -- & -- & 0.143 & -- & -- & -- \\
\hline 9/25/2008 6:00 AM & 0.347 & 15.3 & 0.185 & 0.122 & 16.2 & 0.16 & 0.122 & -- & -- & 0.141 & -- & -- & -- \\
\hline 9/25/2008 7:00 AM & 0.347 & 15.3 & 0.185 & 0.121 & 16.1 & 0.16 & 0.122 & -- & -- & 0.135 & -- & -- & -- \\
\hline 9/25/2008 8:00 AM & 0.347 & 15.3 & 0.185 & 0.121 & 16.1 & 0.16 & 0.122 & -- & -- & 0.131 & -- & -- & -- \\
\hline 9/25/2008 9:00 AM & 0.347 & 15.3 & 0.185 & 0.122 & 16.1 & 0.16 & 0.122 & -- & -- & 0.128 & -- & -- & -- \\
\hline 9/25/2008 10:00 AM & 0.347 & 15.3 & 0.185 & 0.122 & 16.1 & 0.16 & 0.122 & -- & -- & 0.129 & -- & -- & -- \\
\hline 9/25/2008 11:00 AM & 0.347 & 15.3 & 0.185 & 0.122 & 16.1 & 0.16 & 0.122 & -- & -- & 0.145 & -- & -- & -- \\
\hline 9/25/2008 12:00 PM & 0.347 & 15.3 & 0.185 & 0.122 & 16.1 & 0.16 & 0.122 & -- & -- & 0.137 & -- & -- & -- \\
\hline 9/25/2008 1:00 PM & 0.347 & 15.3 & 0.184 & 0.121 & 16.2 & 0.16 & 0.122 & -- & -- & 0.132 & -- & -- & -- \\
\hline 9/25/2008 2:00 PM & 0.347 & 15.3 & 0.184 & 0.122 & 16.1 & 0.16 & 0.122 & -- & -- & 0.125 & -- & -- & -- \\
\hline 9/25/2008 3:00 PM & 0.347 & 15.3 & 0.184 & 0.122 & 16.1 & 0.16 & 0.122 & -- & -- & 0.122 & -- & -- & -- \\
\hline 9/25/2008 4:00 PM & 0.346 & 15.2 & 0.184 & 0.122 & 16.1 & 0.16 & 0.122 & -- & -- & 0.121 & -- & -- & -- \\
\hline 9/25/2008 5:00 PM & 0.346 & 15.2 & 0.184 & 0.121 & 16.1 & 0.16 & 0.121 & -- & -- & 0.121 & -- & -- & -- \\
\hline
\end{tabular}


Appendix 3. Volumetric water content, temperature, and electrical-conductivity data collected at the sprinkler-irrigated site during 2008.-Continued

[Depth in feet below land surface; volumetric water content dimensionless; temperature in degrees Celsius; bulk electrical conductivity in decisiemens per meter; --, no data]

\begin{tabular}{|c|c|c|c|c|c|c|c|c|c|c|c|c|c|}
\hline \multirow[b]{3}{*}{$\begin{array}{l}\text { Measurement } \\
\text { date and time }\end{array}$} & \multicolumn{13}{|c|}{ Depth } \\
\hline & \multicolumn{2}{|l|}{2} & \multirow{2}{*}{$\begin{array}{c}3 \\
\text { Volumetric } \\
\text { water } \\
\text { content }\end{array}$} & \multicolumn{3}{|c|}{4} & \multirow{2}{*}{$\begin{array}{c}\mathbf{5} \\
\text { Volumetric } \\
\text { water } \\
\text { content }\end{array}$} & \multicolumn{2}{|l|}{7} & \multirow{2}{*}{$\begin{array}{c}9 \\
\text { Volumetric } \\
\text { water } \\
\text { content }\end{array}$} & \multicolumn{2}{|c|}{13} & \multirow{2}{*}{$\begin{array}{c}16.5 \\
\text { Volumetric } \\
\text { water } \\
\text { content }\end{array}$} \\
\hline & $\begin{array}{c}\text { Volumetric } \\
\text { water } \\
\text { content }\end{array}$ & $\begin{array}{l}\text { Temper- } \\
\text { ature }\end{array}$ & & $\begin{array}{c}\text { Volumetric } \\
\text { water } \\
\text { content }\end{array}$ & $\begin{array}{l}\text { Temper- } \\
\text { ature }\end{array}$ & $\begin{array}{c}\text { Bulk } \\
\text { electrical } \\
\text { conduct- } \\
\text { ivity }\end{array}$ & & $\begin{array}{l}\text { Volumetric } \\
\text { water } \\
\text { content }\end{array}$ & $\begin{array}{l}\text { Temper- } \\
\text { ature }\end{array}$ & & $\begin{array}{l}\text { Volumetric } \\
\text { water } \\
\text { content }\end{array}$ & $\begin{array}{l}\text { Temper- } \\
\text { ature }\end{array}$ & \\
\hline 9/25/2008 6:00 PM & 0.346 & 15.2 & 0.184 & 0.122 & 16.1 & 0.16 & 0.121 & -- & -- & 0.121 & -- & -- & -- \\
\hline 9/25/2008 7:00 PM & 0.345 & 15.2 & 0.185 & 0.121 & 16.1 & 0.16 & 0.121 & -- & -- & 0.121 & -- & -- & -- \\
\hline 9/25/2008 8:00 PM & 0.345 & 15.2 & 0.184 & 0.121 & 16.1 & 0.16 & 0.121 & -- & -- & 0.121 & -- & -- & -- \\
\hline 9/25/2008 9:00 PM & 0.345 & 15.2 & 0.184 & 0.121 & 16.1 & 0.16 & 0.121 & -- & -- & 0.121 & -- & -- & -- \\
\hline 9/25/2008 10:00 PM & 0.345 & 15.2 & 0.184 & 0.121 & 16.1 & 0.16 & 0.121 & -- & -- & 0.119 & -- & -- & -- \\
\hline 9/25/2008 11:00 PM & 0.345 & 15.2 & 0.184 & 0.121 & 16.1 & 0.16 & 0.121 & -- & -- & 0.123 & -- & -- & -- \\
\hline 9/26/2008 12:00 AM & 0.345 & 15.2 & 0.184 & 0.121 & 16.1 & 0.16 & 0.121 & -- & -- & 0.128 & -- & -- & -- \\
\hline 9/26/2008 1:00 AM & 0.345 & 15.2 & 0.184 & 0.121 & 16.1 & 0.16 & 0.121 & -- & -- & 0.130 & -- & -- & -- \\
\hline 9/26/2008 2:00 AM & 0.345 & 15.2 & 0.184 & 0.121 & 16.1 & 0.16 & 0.121 & -- & -- & 0.133 & -- & -- & -- \\
\hline 9/26/2008 3:00 AM & 0.345 & 15.2 & 0.184 & 0.121 & 16.1 & 0.16 & 0.121 & -- & -- & 0.135 & -- & -- & -- \\
\hline 9/26/2008 4:00 AM & 0.345 & 15.2 & 0.184 & 0.121 & 16.1 & 0.16 & 0.121 & -- & -- & 0.138 & -- & -- & -- \\
\hline 9/26/2008 5:00 AM & 0.345 & 15.3 & 0.184 & 0.121 & 16.1 & 0.16 & 0.121 & -- & -- & 0.138 & -- & -- & -- \\
\hline 9/26/2008 6:00 AM & 0.345 & 15.3 & 0.184 & 0.121 & 16.1 & 0.16 & 0.121 & -- & -- & 0.139 & -- & -- & -- \\
\hline 9/26/2008 7:00 AM & 0.345 & 15.3 & 0.184 & 0.121 & 16.1 & 0.16 & 0.121 & -- & -- & 0.140 & -- & -- & -- \\
\hline 9/26/2008 8:00 AM & 0.345 & 15.3 & 0.184 & 0.121 & 16.1 & 0.16 & 0.121 & -- & -- & 0.141 & -- & -- & -- \\
\hline 9/26/2008 9:00 AM & 0.345 & 15.3 & 0.184 & 0.121 & 16.1 & 0.16 & 0.121 & -- & -- & 0.142 & -- & -- & -- \\
\hline 9/26/2008 10:00 AM & 0.345 & 15.3 & 0.184 & 0.121 & 16.1 & 0.16 & 0.121 & -- & -- & 0.142 & -- & -- & -- \\
\hline 9/26/2008 11:00 AM & 0.345 & 15.3 & 0.184 & 0.121 & 16.1 & 0.16 & 0.121 & -- & -- & 0.138 & -- & -- & -- \\
\hline 9/26/2008 12:00 PM & 0.345 & 15.3 & 0.184 & 0.121 & 16.1 & 0.16 & 0.121 & -- & -- & 0.132 & -- & -- & -- \\
\hline 9/26/2008 1:00 PM & 0.345 & 15.2 & 0.184 & 0.121 & 16.1 & 0.16 & 0.121 & -- & -- & 0.124 & -- & -- & -- \\
\hline 9/26/2008 2:00 PM & 0.345 & 15.2 & 0.183 & 0.121 & 16.1 & 0.16 & 0.121 & -- & -- & 0.121 & -- & -- & -- \\
\hline 9/26/2008 3:00 PM & 0.345 & 15.2 & 0.183 & 0.121 & 16.1 & 0.16 & 0.121 & -- & -- & 0.121 & -- & -- & -- \\
\hline 9/26/2008 4:00 PM & 0.344 & 15.2 & 0.183 & 0.121 & 16.1 & 0.16 & 0.121 & -- & -- & 0.121 & -- & -- & -- \\
\hline 9/26/2008 5:00 PM & 0.344 & 15.2 & 0.183 & 0.120 & 16.1 & 0.16 & 0.121 & -- & -- & 0.121 & -- & -- & -- \\
\hline 9/26/2008 6:00 PM & 0.344 & 15.2 & 0.184 & 0.121 & 16.1 & 0.16 & 0.121 & -- & -- & 0.121 & -- & -- & -- \\
\hline 9/26/2008 7:00 PM & 0.344 & 15.2 & 0.184 & 0.121 & 16.1 & 0.16 & 0.121 & -- & -- & 0.121 & -- & -- & -- \\
\hline 9/26/2008 8:00 PM & 0.344 & 15.2 & 0.184 & 0.121 & 16.1 & 0.16 & 0.121 & -- & -- & 0.121 & -- & -- & -- \\
\hline 9/26/2008 9:00 PM & 0.344 & 15.2 & 0.183 & 0.121 & 16.1 & 0.16 & 0.121 & -- & -- & 0.121 & -- & -- & -- \\
\hline 9/26/2008 10:00 PM & 0.344 & 15.2 & 0.183 & 0.121 & 16.1 & 0.16 & 0.121 & -- & -- & 0.121 & -- & -- & -- \\
\hline 9/26/2008 11:00 PM & 0.344 & 15.2 & 0.183 & 0.121 & 16.1 & 0.16 & 0.121 & -- & -- & 0.121 & -- & -- & -- \\
\hline 9/27/2008 12:00 AM & 0.344 & 15.2 & 0.183 & 0.121 & 16.1 & 0.16 & 0.121 & -- & -- & 0.121 & -- & -- & -- \\
\hline 9/27/2008 1:00 AM & 0.344 & 15.2 & 0.183 & 0.121 & 16.1 & 0.16 & 0.121 & -- & -- & 0.121 & -- & -- & -- \\
\hline 9/27/2008 2:00 AM & 0.343 & 15.2 & 0.183 & 0.121 & 16.1 & 0.16 & 0.121 & -- & -- & 0.121 & -- & -- & -- \\
\hline 9/27/2008 3:00 AM & 0.343 & 15.2 & 0.183 & 0.121 & 16.1 & 0.16 & 0.121 & -- & -- & 0.121 & -- & -- & -- \\
\hline 9/27/2008 4:00 AM & 0.343 & 15.2 & 0.183 & 0.121 & 16.1 & 0.16 & 0.121 & -- & -- & 0.121 & -- & -- & -- \\
\hline
\end{tabular}


Appendix 3. Volumetric water content, temperature, and electrical-conductivity data collected at the sprinkler-irrigated site during 2008.-Continued

[Depth in feet below land surface; volumetric water content dimensionless; temperature in degrees Celsius; bulk electrical conductivity in decisiemens per meter; --, no data]

\begin{tabular}{|c|c|c|c|c|c|c|c|c|c|c|c|c|c|}
\hline \multirow[b]{3}{*}{$\begin{array}{l}\text { Measurement } \\
\text { date and time }\end{array}$} & \multicolumn{13}{|c|}{ Depth } \\
\hline & \multicolumn{2}{|c|}{2} & \multirow{2}{*}{$\begin{array}{c}3 \\
\text { Volumetric } \\
\text { water } \\
\text { content }\end{array}$} & \multicolumn{3}{|c|}{4} & \multirow{2}{*}{$\begin{array}{c}5 \\
\text { Volumetric } \\
\text { water } \\
\text { content }\end{array}$} & \multicolumn{2}{|c|}{7} & \multirow{2}{*}{$\begin{array}{c}9 \\
\text { Volumetric } \\
\text { water } \\
\text { content }\end{array}$} & \multicolumn{2}{|c|}{13} & \multirow{2}{*}{$\begin{array}{c}16.5 \\
\text { Volumetric } \\
\text { water } \\
\text { content }\end{array}$} \\
\hline & $\begin{array}{c}\text { Volumetric } \\
\text { water } \\
\text { content }\end{array}$ & $\begin{array}{l}\text { Temper- } \\
\text { ature }\end{array}$ & & $\begin{array}{c}\text { Volumetric } \\
\text { water } \\
\text { content }\end{array}$ & $\begin{array}{l}\text { Temper- } \\
\text { ature }\end{array}$ & $\begin{array}{c}\text { Bulk } \\
\text { electrical } \\
\text { conduct- } \\
\text { ivity }\end{array}$ & & $\begin{array}{c}\text { Volumetric } \\
\text { water } \\
\text { content }\end{array}$ & $\begin{array}{l}\text { Temper- } \\
\text { ature }\end{array}$ & & $\begin{array}{c}\text { Volumetric } \\
\text { water } \\
\text { content }\end{array}$ & $\begin{array}{l}\text { Temper- } \\
\text { ature }\end{array}$ & \\
\hline 9/27/2008 5:00 AM & 0.344 & 15.3 & 0.183 & 0.121 & 16.1 & 0.16 & 0.121 & -- & -- & 0.120 & -- & -- & -- \\
\hline 9/27/2008 6:00 AM & 0.344 & 15.3 & 0.183 & 0.121 & 16.0 & 0.16 & 0.121 & -- & -- & 0.119 & -- & -- & -- \\
\hline 9/27/2008 7:00 AM & 0.344 & 15.3 & 0.183 & 0.121 & 16.1 & 0.16 & 0.121 & -- & -- & 0.116 & -- & -- & -- \\
\hline 9/27/2008 8:00 AM & 0.344 & 15.3 & 0.183 & 0.121 & 16.1 & 0.16 & 0.121 & -- & -- & 0.121 & -- & -- & -- \\
\hline 9/27/2008 9:00 AM & 0.344 & 15.3 & 0.183 & 0.120 & 16.1 & 0.16 & 0.121 & -- & -- & 0.127 & -- & -- & -- \\
\hline 9/27/2008 10:00 AM & 0.344 & 15.3 & 0.183 & 0.121 & 16.1 & 0.16 & 0.121 & -- & -- & 0.128 & -- & -- & -- \\
\hline 9/27/2008 11:00 AM & 0.344 & 15.3 & 0.183 & 0.120 & 16.1 & 0.16 & 0.121 & -- & -- & 0.126 & -- & -- & -- \\
\hline 9/27/2008 12:00 PM & 0.344 & 15.3 & 0.183 & 0.120 & 16.1 & 0.16 & 0.121 & -- & -- & 0.122 & -- & -- & -- \\
\hline 9/27/2008 1:00 PM & 0.344 & 15.3 & 0.183 & 0.120 & 16.0 & 0.16 & 0.121 & -- & -- & 0.120 & -- & -- & -- \\
\hline 9/27/2008 2:00 PM & 0.344 & 15.3 & 0.183 & 0.120 & 16.1 & 0.16 & 0.121 & -- & -- & 0.121 & -- & -- & -- \\
\hline 9/27/2008 3:00 PM & 0.344 & 15.2 & 0.183 & 0.120 & 16.0 & 0.16 & 0.121 & -- & -- & 0.121 & -- & -- & -- \\
\hline 9/27/2008 4:00 PM & 0.343 & 15.2 & 0.183 & 0.121 & 16.0 & 0.16 & 0.121 & -- & -- & 0.121 & -- & -- & -- \\
\hline 9/27/2008 5:00 PM & 0.343 & 15.2 & 0.183 & 0.120 & 16.0 & 0.16 & 0.121 & -- & -- & 0.121 & -- & -- & -- \\
\hline 9/27/2008 6:00 PM & 0.343 & 15.2 & 0.183 & 0.120 & 16.0 & 0.16 & 0.121 & -- & -- & 0.121 & -- & -- & -- \\
\hline 9/27/2008 7:00 PM & 0.343 & 15.2 & 0.183 & 0.120 & 16.0 & 0.16 & 0.121 & -- & -- & 0.121 & -- & -- & -- \\
\hline 9/27/2008 8:00 PM & 0.343 & 15.2 & 0.183 & 0.120 & 16.0 & 0.16 & 0.121 & -- & -- & 0.121 & -- & -- & -- \\
\hline 9/27/2008 9:00 PM & 0.343 & 15.2 & 0.183 & 0.121 & 16.0 & 0.16 & 0.121 & -- & -- & 0.121 & -- & -- & -- \\
\hline 9/27/2008 10:00 PM & 0.343 & 15.2 & 0.183 & 0.121 & 16.0 & 0.16 & 0.121 & -- & -- & 0.121 & -- & -- & -- \\
\hline 9/27/2008 11:00 PM & 0.343 & 15.2 & 0.183 & 0.120 & 16.0 & 0.16 & 0.121 & -- & -- & 0.121 & -- & -- & -- \\
\hline 9/28/2008 12:00 AM & 0.343 & 15.2 & 0.183 & 0.120 & 16.0 & 0.16 & 0.121 & -- & -- & 0.121 & -- & -- & -- \\
\hline 9/28/2008 1:00 AM & 0.342 & 15.2 & 0.183 & 0.120 & 16.1 & 0.16 & 0.121 & -- & -- & 0.120 & -- & -- & -- \\
\hline 9/28/2008 2:00 AM & 0.342 & 15.3 & 0.183 & 0.120 & 16.0 & 0.16 & 0.121 & -- & -- & 0.117 & -- & -- & -- \\
\hline 9/28/2008 3:00 AM & 0.342 & 15.3 & 0.183 & 0.120 & 16.0 & 0.16 & 0.121 & -- & -- & 0.122 & -- & -- & -- \\
\hline 9/28/2008 4:00 AM & 0.343 & 15.3 & 0.183 & 0.120 & 16.0 & 0.16 & 0.121 & -- & -- & 0.130 & -- & -- & -- \\
\hline 9/28/2008 5:00 AM & 0.343 & 15.3 & 0.183 & 0.120 & 16.0 & 0.16 & 0.121 & -- & -- & 0.136 & -- & -- & -- \\
\hline 9/28/2008 6:00 AM & 0.343 & 15.3 & 0.183 & 0.120 & 16.0 & 0.16 & 0.121 & -- & -- & 0.135 & -- & -- & -- \\
\hline 9/28/2008 7:00 AM & 0.343 & 15.3 & 0.183 & 0.120 & 16.0 & 0.16 & 0.121 & -- & -- & 0.134 & -- & -- & -- \\
\hline 9/28/2008 8:00 AM & 0.343 & 15.3 & 0.183 & 0.120 & 16.0 & 0.16 & 0.121 & -- & -- & 0.134 & -- & -- & -- \\
\hline 9/28/2008 9:00 AM & 0.343 & 15.3 & 0.183 & 0.120 & 16.0 & 0.16 & 0.121 & -- & -- & 0.134 & -- & -- & -- \\
\hline 9/28/2008 10:00 AM & 0.343 & 15.3 & 0.183 & 0.120 & 16.0 & 0.16 & 0.121 & -- & -- & 0.134 & -- & -- & -- \\
\hline 9/28/2008 11:00 AM & 0.343 & 15.3 & 0.183 & 0.120 & 16.0 & 0.16 & 0.121 & -- & -- & 0.132 & -- & -- & -- \\
\hline 9/28/2008 12:00 PM & 0.343 & 15.3 & 0.183 & 0.120 & 16.0 & 0.16 & 0.121 & -- & -- & 0.126 & -- & -- & -- \\
\hline 9/28/2008 1:00 PM & 0.343 & 15.2 & 0.183 & 0.120 & 16.0 & 0.16 & 0.121 & -- & -- & 0.121 & -- & -- & -- \\
\hline 9/28/2008 2:00 PM & 0.342 & 15.2 & 0.183 & 0.120 & 16.0 & 0.16 & 0.121 & -- & -- & 0.121 & -- & -- & -- \\
\hline 9/28/2008 3:00 PM & 0.342 & 15.2 & 0.183 & 0.120 & 16.0 & 0.16 & 0.121 & -- & -- & 0.121 & -- & -- & -- \\
\hline
\end{tabular}


Appendix 3. Volumetric water content, temperature, and electrical-conductivity data collected at the sprinkler-irrigated site during 2008.-Continued

[Depth in feet below land surface; volumetric water content dimensionless; temperature in degrees Celsius; bulk electrical conductivity in decisiemens per meter; --, no data]

\begin{tabular}{|c|c|c|c|c|c|c|c|c|c|c|c|c|c|}
\hline \multirow[b]{3}{*}{$\begin{array}{l}\text { Measurement } \\
\text { date and time }\end{array}$} & \multirow{2}{*}{\multicolumn{2}{|c|}{2}} & \multirow{3}{*}{$\begin{array}{c}3 \\
\text { Volumetric } \\
\text { water } \\
\text { content }\end{array}$} & \multicolumn{9}{|c|}{ Depth } & \multirow{3}{*}{$\begin{array}{c}16.5 \\
\text { Volumetric } \\
\text { water } \\
\text { content }\end{array}$} \\
\hline & & & & \multicolumn{3}{|c|}{4} & \multirow[b]{2}{*}{$\begin{array}{c}\mathbf{5} \\
\text { Volumetric } \\
\text { water } \\
\text { content }\end{array}$} & \multicolumn{2}{|l|}{7} & \multirow{2}{*}{$\begin{array}{c}9 \\
\text { Volumetric } \\
\text { water } \\
\text { content }\end{array}$} & \multicolumn{2}{|c|}{13} & \\
\hline & $\begin{array}{c}\text { Volumetric } \\
\text { water } \\
\text { content }\end{array}$ & $\begin{array}{l}\text { Temper- } \\
\text { ature }\end{array}$ & & $\begin{array}{c}\text { Volumetric } \\
\text { water } \\
\text { content }\end{array}$ & $\begin{array}{l}\text { Temper- } \\
\text { ature }\end{array}$ & $\begin{array}{c}\text { Bulk } \\
\text { electrical } \\
\text { conduct- } \\
\text { ivity }\end{array}$ & & $\begin{array}{l}\text { Volumetric } \\
\text { water } \\
\text { content }\end{array}$ & $\begin{array}{l}\text { Temper- } \\
\text { ature }\end{array}$ & & $\begin{array}{c}\text { Volumetric } \\
\text { water } \\
\text { content }\end{array}$ & $\begin{array}{l}\text { Temper- } \\
\text { ature }\end{array}$ & \\
\hline 9/28/2008 4:00 PM & 0.342 & 15.2 & 0.183 & 0.120 & 16.0 & 0.16 & 0.121 & -- & -- & 0.121 & -- & -- & -- \\
\hline 9/28/2008 5:00 PM & 0.342 & 15.2 & 0.183 & 0.120 & 16.0 & 0.16 & 0.121 & -- & -- & 0.121 & -- & -- & -- \\
\hline 9/28/2008 6:00 PM & 0.342 & 15.2 & 0.183 & 0.120 & 16.0 & 0.16 & 0.121 & -- & -- & 0.121 & -- & -- & -- \\
\hline 9/28/2008 7:00 PM & 0.341 & 15.2 & 0.183 & 0.120 & 16.0 & 0.16 & 0.121 & -- & -- & 0.121 & -- & -- & -- \\
\hline 9/28/2008 8:00 PM & 0.341 & 15.1 & 0.183 & 0.120 & 16.0 & 0.16 & 0.121 & -- & -- & 0.121 & -- & -- & -- \\
\hline 9/28/2008 9:00 PM & 0.341 & 15.1 & 0.183 & 0.120 & 16.0 & 0.16 & 0.121 & -- & -- & 0.121 & -- & -- & -- \\
\hline 9/28/2008 10:00 PM & 0.341 & 15.2 & 0.183 & 0.120 & 16.0 & 0.16 & 0.121 & -- & -- & 0.121 & -- & -- & -- \\
\hline 9/28/2008 11:00 PM & 0.341 & 15.2 & 0.183 & 0.120 & 16.0 & 0.16 & 0.121 & -- & -- & 0.121 & -- & -- & -- \\
\hline 9/29/2008 12:00 AM & 0.341 & 15.2 & 0.183 & 0.120 & 16.0 & 0.16 & 0.121 & -- & -- & 0.121 & -- & -- & -- \\
\hline 9/29/2008 1:00 AM & 0.341 & 15.2 & 0.183 & 0.120 & 16.0 & 0.16 & 0.121 & -- & -- & 0.121 & -- & -- & -- \\
\hline 9/29/2008 2:00 AM & 0.341 & 15.2 & 0.183 & 0.120 & 16.0 & 0.16 & 0.120 & -- & -- & 0.121 & -- & -- & -- \\
\hline 9/29/2008 3:00 AM & 0.341 & 15.2 & 0.183 & 0.120 & 16.0 & 0.16 & 0.120 & -- & -- & 0.121 & -- & -- & -- \\
\hline 9/29/2008 4:00 AM & 0.341 & 15.2 & 0.182 & 0.120 & 16.0 & 0.16 & 0.120 & -- & -- & 0.121 & -- & -- & -- \\
\hline 9/29/2008 5:00 AM & 0.341 & 15.2 & 0.182 & 0.120 & 16.0 & 0.16 & 0.121 & -- & -- & 0.121 & -- & -- & -- \\
\hline 9/29/2008 6:00 AM & 0.341 & 15.2 & 0.182 & 0.120 & 16.0 & 0.16 & 0.120 & -- & -- & 0.121 & -- & -- & -- \\
\hline 9/29/2008 7:00 AM & 0.341 & 15.3 & 0.182 & 0.120 & 16.0 & 0.16 & 0.120 & -- & -- & 0.121 & -- & -- & -- \\
\hline 9/29/2008 8:00 AM & 0.341 & 15.2 & 0.182 & 0.119 & 16.0 & 0.16 & 0.120 & -- & -- & 0.121 & -- & -- & -- \\
\hline 9/29/2008 9:00 AM & 0.341 & 15.2 & 0.182 & 0.119 & 16.0 & 0.16 & 0.121 & -- & -- & 0.121 & -- & -- & -- \\
\hline 9/29/2008 10:00 AM & 0.341 & 15.3 & 0.182 & 0.120 & 16.0 & 0.16 & 0.120 & -- & -- & 0.121 & -- & -- & -- \\
\hline 9/29/2008 11:00 AM & 0.341 & 15.3 & 0.183 & 0.119 & 16.0 & 0.16 & 0.121 & -- & -- & 0.121 & -- & -- & -- \\
\hline 9/29/2008 12:00 PM & 0.341 & 15.2 & 0.183 & 0.120 & 16.0 & 0.16 & 0.120 & -- & -- & 0.121 & -- & -- & -- \\
\hline 9/29/2008 1:00 PM & 0.341 & 15.2 & 0.183 & 0.120 & 16.0 & 0.16 & 0.121 & -- & -- & 0.121 & -- & -- & -- \\
\hline 9/29/2008 2:00 PM & 0.341 & 15.2 & 0.182 & 0.120 & 16.0 & 0.16 & 0.121 & -- & -- & 0.121 & -- & -- & -- \\
\hline 9/29/2008 3:00 PM & 0.341 & 15.2 & 0.182 & 0.119 & 16.0 & 0.16 & 0.121 & -- & -- & 0.121 & -- & -- & -- \\
\hline 9/29/2008 4:00 PM & 0.341 & 15.2 & 0.182 & 0.119 & 16.0 & 0.16 & 0.121 & -- & -- & 0.121 & -- & -- & -- \\
\hline 9/29/2008 5:00 PM & 0.340 & 15.2 & 0.182 & 0.119 & 16.0 & 0.15 & 0.121 & -- & -- & 0.121 & -- & -- & -- \\
\hline 9/29/2008 6:00 PM & 0.340 & 15.2 & 0.182 & 0.119 & 16.0 & 0.15 & 0.121 & -- & -- & 0.121 & -- & -- & -- \\
\hline 9/29/2008 7:00 PM & 0.340 & 15.1 & 0.182 & 0.119 & 16.0 & 0.16 & 0.121 & -- & -- & 0.121 & -- & -- & -- \\
\hline 9/29/2008 8:00 PM & 0.338 & 15.1 & 0.182 & 0.120 & 16.0 & 0.16 & 0.121 & -- & -- & 0.121 & -- & -- & -- \\
\hline 9/29/2008 9:00 PM & 0.338 & 15.1 & 0.182 & 0.119 & 16.0 & 0.16 & 0.120 & -- & -- & 0.121 & -- & -- & -- \\
\hline 9/29/2008 10:00 PM & 0.338 & 15.1 & 0.182 & 0.120 & 16.0 & 0.16 & 0.120 & -- & -- & 0.121 & -- & -- & -- \\
\hline 9/29/2008 11:00 PM & 0.338 & 15.1 & 0.182 & 0.119 & 16.0 & 0.16 & 0.120 & -- & -- & 0.121 & -- & -- & -- \\
\hline 9/30/2008 12:00 AM & 0.338 & 15.1 & 0.182 & 0.119 & 16.0 & 0.16 & 0.120 & -- & -- & 0.121 & -- & -- & -- \\
\hline 9/30/2008 1:00 AM & 0.338 & 15.1 & 0.182 & 0.119 & 16.0 & 0.16 & 0.120 & -- & -- & 0.121 & -- & -- & -- \\
\hline 9/30/2008 2:00 AM & 0.338 & 15.1 & 0.182 & 0.119 & 16.0 & 0.16 & 0.120 & -- & -- & 0.121 & -- & -- & -- \\
\hline
\end{tabular}


Appendix 3. Volumetric water content, temperature, and electrical-conductivity data collected at the sprinkler-irrigated site during 2008.-Continued

[Depth in feet below land surface; volumetric water content dimensionless; temperature in degrees Celsius; bulk electrical conductivity in decisiemens per meter; --, no data]

\begin{tabular}{|c|c|c|c|c|c|c|c|c|c|c|c|c|c|}
\hline \multirow[b]{3}{*}{$\begin{array}{l}\text { Measurement } \\
\text { date and time }\end{array}$} & \multicolumn{13}{|c|}{ Depth } \\
\hline & \multicolumn{2}{|c|}{2} & \multirow{2}{*}{$\begin{array}{c}3 \\
\text { Volumetric } \\
\text { water } \\
\text { content }\end{array}$} & \multicolumn{3}{|c|}{4} & \multirow{2}{*}{$\begin{array}{c}5 \\
\text { Volumetric } \\
\text { water } \\
\text { content }\end{array}$} & \multicolumn{2}{|l|}{7} & \multirow{2}{*}{$\begin{array}{c}9 \\
\text { Volumetric } \\
\text { water } \\
\text { content }\end{array}$} & \multicolumn{2}{|c|}{13} & \multirow{2}{*}{$\begin{array}{c}16.5 \\
\text { Volumetric } \\
\text { water } \\
\text { content }\end{array}$} \\
\hline & $\begin{array}{c}\text { Volumetric } \\
\text { water } \\
\text { content }\end{array}$ & $\begin{array}{l}\text { Temper- } \\
\text { ature }\end{array}$ & & $\begin{array}{c}\text { Volumetric } \\
\text { water } \\
\text { content }\end{array}$ & $\begin{array}{l}\text { Temper- } \\
\text { ature }\end{array}$ & $\begin{array}{c}\text { Bulk } \\
\text { electrical } \\
\text { conduct- } \\
\text { ivity }\end{array}$ & & $\begin{array}{l}\text { Volumetric } \\
\text { water } \\
\text { content }\end{array}$ & $\begin{array}{l}\text { Temper- } \\
\text { ature }\end{array}$ & & $\begin{array}{c}\text { Volumetric } \\
\text { water } \\
\text { content }\end{array}$ & $\begin{array}{l}\text { Temper- } \\
\text { ature }\end{array}$ & \\
\hline 9/30/2008 3:00 AM & 0.338 & 15.1 & 0.181 & 0.119 & 16.0 & 0.16 & 0.120 & -- & -- & 0.121 & -- & -- & -- \\
\hline 9/30/2008 4:00 AM & 0.338 & 15.1 & 0.181 & 0.119 & 16.0 & 0.16 & 0.120 & -- & -- & 0.121 & -- & -- & -- \\
\hline 9/30/2008 5:00 AM & 0.338 & 15.1 & 0.181 & 0.119 & 16.0 & 0.15 & 0.120 & -- & -- & 0.121 & -- & -- & -- \\
\hline 9/30/2008 6:00 AM & 0.338 & 15.1 & 0.181 & 0.119 & 16.0 & 0.16 & 0.120 & -- & -- & 0.121 & -- & -- & -- \\
\hline 9/30/2008 7:00 AM & 0.338 & 15.1 & 0.181 & 0.119 & 16.0 & 0.16 & 0.120 & -- & -- & 0.121 & -- & -- & -- \\
\hline 9/30/2008 8:00 AM & 0.338 & 15.1 & 0.182 & 0.119 & 16.0 & 0.16 & 0.120 & -- & -- & 0.121 & -- & -- & -- \\
\hline 9/30/2008 9:00 AM & 0.338 & 15.1 & 0.181 & 0.119 & 16.0 & 0.16 & 0.120 & -- & -- & 0.121 & -- & -- & -- \\
\hline 9/30/2008 10:00 AM & 0.338 & 15.1 & 0.182 & 0.119 & 16.0 & 0.16 & 0.120 & -- & -- & 0.121 & -- & -- & -- \\
\hline 9/30/2008 11:00 AM & 0.338 & 15.1 & 0.182 & 0.119 & 16.0 & 0.16 & 0.120 & -- & -- & 0.121 & -- & -- & -- \\
\hline 9/30/2008 12:00 PM & 0.338 & 15.0 & 0.182 & 0.120 & 16.0 & 0.16 & 0.120 & -- & -- & 0.121 & -- & -- & -- \\
\hline 9/30/2008 1:00 PM & 0.338 & 15.0 & 0.181 & 0.119 & 16.0 & 0.16 & 0.120 & -- & -- & 0.121 & -- & -- & -- \\
\hline 9/30/2008 2:00 PM & 0.338 & 15.0 & 0.181 & 0.119 & 15.9 & 0.16 & 0.120 & -- & -- & 0.121 & -- & -- & -- \\
\hline 9/30/2008 3:00 PM & 0.338 & 15.0 & 0.181 & 0.119 & 15.9 & 0.15 & 0.120 & -- & -- & 0.121 & -- & -- & -- \\
\hline 9/30/2008 4:00 PM & 0.337 & 15.0 & 0.181 & 0.119 & 15.9 & 0.15 & 0.120 & -- & -- & 0.121 & -- & -- & -- \\
\hline 9/30/2008 5:00 PM & 0.337 & 14.9 & 0.181 & 0.120 & 15.9 & 0.15 & 0.120 & -- & -- & 0.121 & -- & -- & -- \\
\hline 9/30/2008 6:00 PM & 0.337 & 14.9 & 0.181 & 0.119 & 16.0 & 0.15 & 0.120 & -- & -- & 0.121 & -- & -- & -- \\
\hline 9/30/2008 7:00 PM & 0.337 & 14.9 & 0.181 & 0.119 & 15.9 & 0.15 & 0.120 & -- & -- & 0.121 & -- & -- & -- \\
\hline 9/30/2008 8:00 PM & 0.337 & 14.9 & 0.181 & 0.119 & 16.0 & 0.15 & 0.120 & -- & -- & 0.121 & -- & -- & -- \\
\hline 9/30/2008 9:00 PM & 0.337 & 14.8 & 0.181 & 0.119 & 15.9 & 0.15 & 0.120 & -- & -- & 0.121 & -- & -- & -- \\
\hline 9/30/2008 10:00 PM & 0.337 & 14.8 & 0.181 & 0.119 & 15.9 & 0.15 & 0.120 & -- & -- & 0.121 & -- & -- & -- \\
\hline 9/30/2008 11:00 PM & 0.337 & 14.8 & 0.181 & 0.119 & 15.9 & 0.15 & 0.120 & -- & -- & 0.121 & -- & -- & -- \\
\hline 10/1/2008 12:00 AM & 0.337 & 14.9 & 0.181 & 0.119 & 15.9 & 0.15 & 0.120 & -- & -- & 0.121 & -- & -- & -- \\
\hline 10/1/2008 1:00 AM & 0.337 & 14.9 & 0.181 & 0.119 & 15.9 & 0.15 & 0.120 & -- & -- & 0.121 & -- & -- & -- \\
\hline 10/1/2008 2:00 AM & 0.337 & 14.9 & 0.181 & 0.119 & 15.9 & 0.15 & 0.120 & -- & -- & 0.121 & -- & -- & -- \\
\hline 10/1/2008 3:00 AM & 0.337 & 14.9 & 0.181 & 0.119 & 15.9 & 0.15 & 0.120 & -- & -- & 0.121 & -- & -- & -- \\
\hline 10/1/2008 4:00 AM & 0.337 & 14.9 & 0.181 & 0.119 & 15.9 & 0.15 & 0.120 & -- & -- & 0.121 & -- & -- & -- \\
\hline 10/1/2008 5:00 AM & 0.337 & 14.9 & 0.181 & 0.119 & 15.9 & 0.15 & 0.120 & -- & -- & 0.121 & -- & -- & -- \\
\hline 10/1/2008 6:00 AM & 0.337 & 14.9 & 0.180 & 0.119 & 15.9 & 0.15 & 0.120 & -- & -- & 0.121 & -- & -- & -- \\
\hline 10/1/2008 7:00 AM & 0.337 & 14.9 & 0.180 & 0.119 & 15.9 & 0.15 & 0.120 & -- & -- & 0.121 & -- & -- & -- \\
\hline 10/1/2008 8:00 AM & 0.337 & 14.9 & 0.180 & 0.119 & 15.9 & 0.15 & 0.120 & -- & -- & 0.121 & -- & -- & -- \\
\hline 10/1/2008 9:00 AM & 0.337 & 14.9 & 0.180 & 0.119 & 15.9 & 0.15 & 0.120 & -- & -- & 0.121 & -- & -- & -- \\
\hline 10/1/2008 10:00 AM & 0.337 & 14.9 & 0.181 & 0.119 & 15.9 & 0.15 & 0.120 & -- & -- & 0.121 & -- & -- & -- \\
\hline 10/1/2008 11:00 AM & 0.337 & 14.9 & 0.181 & 0.119 & 15.9 & 0.15 & 0.120 & -- & -- & 0.121 & -- & -- & -- \\
\hline 10/1/2008 12:00 PM & 0.337 & 14.9 & 0.181 & 0.119 & 15.9 & 0.15 & 0.120 & -- & -- & 0.121 & -- & -- & -- \\
\hline 10/1/2008 1:00 PM & 0.337 & 14.8 & 0.181 & 0.119 & 15.9 & 0.15 & 0.120 & -- & -- & 0.121 & -- & -- & -- \\
\hline
\end{tabular}


Appendix 3. Volumetric water content, temperature, and electrical-conductivity data collected at the sprinkler-irrigated site during 2008.-Continued

[Depth in feet below land surface; volumetric water content dimensionless; temperature in degrees Celsius; bulk electrical conductivity in decisiemens per meter; --, no data]

\begin{tabular}{|c|c|c|c|c|c|c|c|c|c|c|c|c|c|}
\hline \multirow[b]{3}{*}{$\begin{array}{l}\text { Measurement } \\
\text { date and time }\end{array}$} & \multirow{2}{*}{\multicolumn{2}{|c|}{2}} & \multirow{3}{*}{$\begin{array}{c}3 \\
\text { Volumetric } \\
\text { water } \\
\text { content }\end{array}$} & \multicolumn{9}{|c|}{ Depth } & \multirow{3}{*}{$\begin{array}{c}16.5 \\
\text { Volumetric } \\
\text { water } \\
\text { content }\end{array}$} \\
\hline & & & & \multicolumn{3}{|c|}{4} & \multirow[b]{2}{*}{$\begin{array}{c}\mathbf{5} \\
\text { Volumetric } \\
\text { water } \\
\text { content }\end{array}$} & \multicolumn{2}{|l|}{7} & \multirow{2}{*}{$\begin{array}{c}9 \\
\text { Volumetric } \\
\text { water } \\
\text { content }\end{array}$} & \multicolumn{2}{|c|}{13} & \\
\hline & $\begin{array}{c}\text { Volumetric } \\
\text { water } \\
\text { content }\end{array}$ & $\begin{array}{l}\text { Temper- } \\
\text { ature }\end{array}$ & & $\begin{array}{c}\text { Volumetric } \\
\text { water } \\
\text { content }\end{array}$ & $\begin{array}{l}\text { Temper- } \\
\text { ature }\end{array}$ & $\begin{array}{c}\text { Bulk } \\
\text { electrical } \\
\text { conduct- } \\
\text { ivity }\end{array}$ & & $\begin{array}{l}\text { Volumetric } \\
\text { water } \\
\text { content }\end{array}$ & $\begin{array}{l}\text { Temper- } \\
\text { ature }\end{array}$ & & $\begin{array}{c}\text { Volumetric } \\
\text { water } \\
\text { content }\end{array}$ & $\begin{array}{l}\text { Temper- } \\
\text { ature }\end{array}$ & \\
\hline 10/1/2008 2:00 PM & 0.337 & 14.8 & 0.181 & 0.119 & 15.9 & 0.15 & 0.120 & -- & -- & 0.121 & -- & -- & -- \\
\hline 10/1/2008 3:00 PM & 0.337 & 14.8 & 0.181 & 0.119 & 15.9 & 0.15 & 0.120 & -- & -- & 0.121 & -- & -- & -- \\
\hline 10/1/2008 4:00 PM & 0.337 & 14.8 & 0.180 & 0.119 & 15.9 & 0.15 & 0.120 & -- & -- & 0.121 & -- & -- & -- \\
\hline 10/1/2008 5:00 PM & 0.336 & 14.8 & 0.180 & 0.119 & 15.9 & 0.15 & 0.120 & -- & -- & 0.121 & -- & -- & -- \\
\hline 10/1/2008 6:00 PM & 0.336 & 14.7 & 0.181 & 0.119 & 15.9 & 0.15 & 0.120 & -- & -- & 0.121 & -- & -- & -- \\
\hline 10/1/2008 7:00 PM & 0.335 & 14.8 & 0.181 & 0.119 & 15.9 & 0.15 & 0.119 & -- & -- & 0.121 & -- & -- & -- \\
\hline 10/1/2008 8:00 PM & 0.335 & 14.7 & 0.181 & 0.119 & 15.9 & 0.15 & 0.120 & -- & -- & 0.121 & -- & -- & -- \\
\hline 10/1/2008 9:00 PM & 0.335 & 14.7 & 0.180 & 0.119 & 15.9 & 0.15 & 0.120 & -- & -- & 0.121 & -- & -- & -- \\
\hline 10/1/2008 10:00 PM & 0.335 & 14.7 & 0.180 & 0.119 & 15.9 & 0.15 & 0.119 & -- & -- & 0.121 & -- & -- & -- \\
\hline 10/1/2008 11:00 PM & 0.335 & 14.7 & 0.180 & 0.119 & 15.9 & 0.15 & 0.119 & -- & -- & 0.121 & -- & -- & -- \\
\hline 10/2/2008 12:00 AM & 0.335 & 14.7 & 0.180 & 0.119 & 15.9 & 0.15 & 0.119 & -- & -- & 0.121 & -- & -- & -- \\
\hline 10/2/2008 1:00 AM & 0.335 & 14.7 & 0.180 & 0.119 & 15.9 & 0.15 & 0.119 & -- & -- & 0.121 & -- & -- & -- \\
\hline 10/2/2008 2:00 AM & 0.335 & 14.7 & 0.180 & 0.119 & 15.9 & 0.15 & 0.119 & -- & -- & 0.121 & -- & -- & -- \\
\hline 10/2/2008 3:00 AM & 0.335 & 14.8 & 0.180 & 0.119 & 15.9 & 0.15 & 0.119 & -- & -- & 0.121 & -- & -- & -- \\
\hline 10/2/2008 4:00 AM & 0.334 & 14.8 & 0.180 & 0.119 & 15.9 & 0.15 & 0.119 & -- & -- & 0.121 & -- & -- & -- \\
\hline 10/2/2008 5:00 AM & 0.335 & 14.8 & 0.180 & 0.118 & 15.9 & 0.15 & 0.119 & -- & -- & 0.121 & -- & -- & -- \\
\hline 10/2/2008 6:00 AM & 0.335 & 14.8 & 0.180 & 0.119 & 15.9 & 0.15 & 0.119 & -- & -- & 0.121 & -- & -- & -- \\
\hline 10/2/2008 7:00 AM & 0.335 & 14.8 & 0.180 & 0.118 & 15.9 & 0.15 & 0.119 & -- & -- & 0.121 & -- & -- & -- \\
\hline 10/2/2008 8:00 AM & 0.335 & 14.8 & 0.180 & 0.119 & 15.9 & 0.15 & 0.120 & -- & -- & 0.121 & -- & -- & -- \\
\hline 10/2/2008 9:00 AM & 0.335 & 14.8 & 0.180 & 0.118 & 15.9 & 0.15 & 0.119 & -- & -- & 0.121 & -- & -- & -- \\
\hline 10/2/2008 10:00 AM & 0.335 & 14.8 & 0.180 & 0.119 & 15.8 & 0.15 & 0.119 & -- & -- & 0.121 & -- & -- & -- \\
\hline 10/2/2008 11:00 AM & 0.335 & 14.8 & 0.180 & 0.119 & 15.8 & 0.15 & 0.119 & -- & -- & 0.121 & -- & -- & -- \\
\hline 10/2/2008 12:00 PM & 0.335 & 14.8 & 0.180 & 0.119 & 15.8 & 0.15 & 0.119 & -- & -- & 0.121 & -- & -- & -- \\
\hline 10/2/2008 1:00 PM & 0.335 & 14.8 & 0.180 & 0.119 & 15.8 & 0.15 & 0.120 & -- & -- & 0.121 & -- & -- & -- \\
\hline 10/2/2008 2:00 PM & 0.335 & 14.8 & 0.180 & 0.119 & 15.8 & 0.15 & 0.119 & -- & -- & 0.121 & -- & -- & -- \\
\hline 10/2/2008 3:00 PM & 0.335 & 14.8 & 0.180 & 0.119 & 15.8 & 0.15 & 0.119 & -- & -- & 0.121 & -- & -- & -- \\
\hline 10/2/2008 4:00 PM & 0.335 & 14.8 & 0.180 & 0.118 & 15.8 & 0.15 & 0.119 & -- & -- & 0.121 & -- & -- & -- \\
\hline 10/2/2008 5:00 PM & 0.334 & 14.7 & 0.180 & 0.118 & 15.8 & 0.15 & 0.119 & -- & -- & 0.121 & -- & -- & -- \\
\hline 10/2/2008 6:00 PM & 0.334 & 14.7 & 0.180 & 0.119 & 15.8 & 0.15 & 0.119 & -- & -- & 0.121 & -- & -- & -- \\
\hline 10/2/2008 7:00 PM & 0.334 & 14.7 & 0.180 & 0.118 & 15.8 & 0.15 & 0.119 & -- & -- & 0.121 & -- & -- & -- \\
\hline 10/2/2008 8:00 PM & 0.334 & 14.7 & 0.180 & 0.118 & 15.8 & 0.15 & 0.119 & -- & -- & 0.121 & -- & -- & -- \\
\hline 10/2/2008 9:00 PM & 0.334 & 14.7 & 0.180 & 0.118 & 15.8 & 0.15 & 0.119 & -- & -- & 0.121 & -- & -- & -- \\
\hline 10/2/2008 10:00 PM & 0.334 & 14.7 & 0.180 & 0.118 & 15.8 & 0.15 & 0.119 & -- & -- & 0.121 & -- & -- & -- \\
\hline 10/2/2008 11:00 PM & 0.334 & 14.7 & 0.180 & 0.118 & 15.8 & 0.15 & 0.119 & -- & -- & 0.121 & -- & -- & -- \\
\hline 10/3/2008 12:00 AM & 0.334 & 14.7 & 0.180 & 0.118 & 15.8 & 0.15 & 0.119 & -- & -- & 0.121 & -- & -- & -- \\
\hline
\end{tabular}


Appendix 3. Volumetric water content, temperature, and electrical-conductivity data collected at the sprinkler-irrigated site during 2008.-Continued

[Depth in feet below land surface; volumetric water content dimensionless; temperature in degrees Celsius; bulk electrical conductivity in decisiemens per meter; --, no data]

\begin{tabular}{|c|c|c|c|c|c|c|c|c|c|c|c|c|c|}
\hline \multirow[b]{3}{*}{$\begin{array}{l}\text { Measurement } \\
\text { date and time }\end{array}$} & \multicolumn{13}{|c|}{ Depth } \\
\hline & \multicolumn{2}{|c|}{2} & \multirow{2}{*}{$\begin{array}{c}3 \\
\text { Volumetric } \\
\text { water } \\
\text { content }\end{array}$} & \multicolumn{3}{|c|}{4} & \multirow{2}{*}{$\begin{array}{c}5 \\
\text { Volumetric } \\
\text { water } \\
\text { content }\end{array}$} & \multicolumn{2}{|l|}{7} & \multirow{2}{*}{$\begin{array}{c}9 \\
\text { Volumetric } \\
\text { water } \\
\text { content }\end{array}$} & \multicolumn{2}{|c|}{13} & \multirow{2}{*}{$\begin{array}{c}16.5 \\
\text { Volumetric } \\
\text { water } \\
\text { content }\end{array}$} \\
\hline & $\begin{array}{c}\text { Volumetric } \\
\text { water } \\
\text { content }\end{array}$ & $\begin{array}{l}\text { Temper- } \\
\text { ature }\end{array}$ & & $\begin{array}{c}\text { Volumetric } \\
\text { water } \\
\text { content }\end{array}$ & $\begin{array}{l}\text { Temper- } \\
\text { ature }\end{array}$ & $\begin{array}{c}\text { Bulk } \\
\text { electrical } \\
\text { conduct- } \\
\text { ivity }\end{array}$ & & $\begin{array}{c}\text { Volumetric } \\
\text { water } \\
\text { content }\end{array}$ & $\begin{array}{l}\text { Temper- } \\
\text { ature }\end{array}$ & & $\begin{array}{c}\text { Volumetric } \\
\text { water } \\
\text { content }\end{array}$ & $\begin{array}{l}\text { Temper- } \\
\text { ature }\end{array}$ & \\
\hline 10/3/2008 1:00 AM & 0.334 & 14.7 & 0.179 & 0.118 & 15.8 & 0.15 & 0.119 & -- & -- & 0.121 & -- & -- & -- \\
\hline 10/3/2008 2:00 AM & 0.334 & 14.7 & 0.179 & 0.118 & 15.8 & 0.15 & 0.119 & -- & -- & 0.121 & -- & -- & -- \\
\hline 10/3/2008 3:00 AM & 0.334 & 14.7 & 0.179 & 0.118 & 15.8 & 0.15 & 0.119 & -- & -- & 0.121 & -- & -- & -- \\
\hline 10/3/2008 4:00 AM & 0.334 & 14.7 & 0.179 & 0.118 & 15.8 & 0.15 & 0.119 & -- & -- & 0.121 & -- & -- & -- \\
\hline 10/3/2008 5:00 AM & 0.334 & 14.7 & 0.179 & 0.118 & 15.8 & 0.15 & 0.119 & -- & -- & 0.121 & -- & -- & -- \\
\hline 10/3/2008 6:00 AM & 0.334 & 14.7 & 0.179 & 0.119 & 15.8 & 0.15 & 0.119 & -- & -- & 0.121 & -- & -- & -- \\
\hline 10/3/2008 7:00 AM & 0.334 & 14.7 & 0.179 & 0.119 & 15.8 & 0.15 & 0.119 & -- & -- & 0.121 & -- & -- & -- \\
\hline 10/3/2008 8:00 AM & 0.334 & 14.7 & 0.179 & 0.118 & 15.8 & 0.15 & 0.119 & -- & -- & 0.121 & -- & -- & -- \\
\hline 10/3/2008 9:00 AM & 0.334 & 14.7 & 0.179 & 0.118 & 15.8 & 0.15 & 0.119 & -- & -- & 0.121 & -- & -- & -- \\
\hline 10/3/2008 10:00 AM & 0.334 & 14.7 & 0.179 & 0.118 & 15.8 & 0.15 & 0.119 & -- & -- & 0.121 & -- & -- & -- \\
\hline 10/3/2008 11:00 AM & 0.334 & 14.7 & 0.179 & 0.118 & 15.8 & 0.15 & 0.119 & -- & -- & 0.121 & -- & -- & -- \\
\hline 10/3/2008 12:00 PM & 0.333 & 14.7 & 0.179 & 0.119 & 15.8 & 0.15 & 0.119 & -- & -- & 0.121 & -- & -- & -- \\
\hline 10/3/2008 1:00 PM & 0.333 & 14.7 & 0.179 & 0.118 & 15.8 & 0.15 & 0.119 & -- & -- & 0.121 & -- & -- & -- \\
\hline 10/3/2008 2:00 PM & 0.333 & 14.7 & 0.179 & 0.118 & 15.8 & 0.15 & 0.119 & -- & -- & 0.121 & -- & -- & -- \\
\hline 10/3/2008 3:00 PM & 0.333 & 14.7 & 0.179 & 0.119 & 15.8 & 0.15 & 0.119 & -- & -- & 0.121 & -- & -- & -- \\
\hline 10/3/2008 4:00 PM & 0.333 & 14.6 & 0.179 & 0.118 & 15.8 & 0.15 & 0.119 & -- & -- & 0.121 & -- & -- & -- \\
\hline 10/3/2008 5:00 PM & 0.333 & 14.6 & 0.179 & 0.118 & 15.8 & 0.15 & 0.119 & -- & -- & 0.121 & -- & -- & -- \\
\hline 10/3/2008 6:00 PM & 0.332 & 14.6 & 0.179 & 0.118 & 15.8 & 0.15 & 0.119 & -- & -- & 0.121 & -- & -- & -- \\
\hline 10/3/2008 7:00 PM & 0.332 & 14.6 & 0.179 & 0.118 & 15.8 & 0.15 & 0.119 & -- & -- & 0.121 & -- & -- & -- \\
\hline 10/3/2008 8:00 PM & 0.332 & 14.5 & 0.179 & 0.119 & 15.8 & 0.15 & 0.119 & -- & -- & 0.121 & -- & -- & -- \\
\hline 10/3/2008 9:00 PM & 0.331 & 14.6 & 0.179 & 0.118 & 15.8 & 0.15 & 0.119 & -- & -- & 0.121 & -- & -- & -- \\
\hline 10/3/2008 10:00 PM & 0.332 & 14.6 & 0.179 & 0.118 & 15.8 & 0.15 & 0.119 & -- & -- & 0.121 & -- & -- & -- \\
\hline 10/3/2008 11:00 PM & 0.332 & 14.6 & 0.179 & 0.118 & 15.7 & 0.15 & 0.119 & -- & -- & 0.121 & -- & -- & -- \\
\hline 10/4/2008 12:00 AM & 0.331 & 14.6 & 0.179 & 0.119 & 15.8 & 0.15 & 0.119 & -- & -- & 0.121 & -- & -- & -- \\
\hline 10/4/2008 1:00 AM & 0.332 & 14.6 & 0.179 & 0.119 & 15.8 & 0.15 & 0.119 & -- & -- & 0.121 & -- & -- & -- \\
\hline 10/4/2008 2:00 AM & 0.332 & 14.6 & 0.179 & 0.118 & 15.8 & 0.15 & 0.118 & -- & -- & 0.121 & -- & -- & -- \\
\hline 10/4/2008 3:00 AM & 0.332 & 14.6 & 0.179 & 0.118 & 15.8 & 0.15 & 0.118 & -- & -- & 0.121 & -- & -- & -- \\
\hline 10/4/2008 4:00 AM & 0.332 & 14.7 & 0.178 & 0.118 & 15.8 & 0.15 & 0.118 & -- & -- & 0.121 & -- & -- & -- \\
\hline 10/4/2008 5:00 AM & 0.332 & 14.6 & 0.178 & 0.118 & 15.7 & 0.15 & 0.118 & -- & -- & 0.121 & -- & -- & -- \\
\hline 10/4/2008 6:00 AM & 0.332 & 14.7 & 0.179 & 0.118 & 15.7 & 0.15 & 0.118 & -- & -- & 0.121 & -- & -- & -- \\
\hline 10/4/2008 7:00 AM & 0.332 & 14.7 & 0.178 & 0.118 & 15.7 & 0.15 & 0.118 & -- & -- & 0.121 & -- & -- & -- \\
\hline 10/4/2008 8:00 AM & 0.332 & 14.7 & 0.179 & 0.118 & 15.7 & 0.15 & 0.118 & -- & -- & 0.121 & -- & -- & -- \\
\hline 10/4/2008 9:00 AM & 0.332 & 14.7 & 0.178 & 0.118 & 15.7 & 0.15 & 0.118 & -- & -- & 0.121 & -- & -- & -- \\
\hline 10/4/2008 10:00 AM & 0.332 & 14.6 & 0.179 & 0.118 & 15.7 & 0.15 & 0.118 & -- & -- & 0.121 & -- & -- & -- \\
\hline 10/4/2008 11:00 AM & 0.332 & 14.7 & 0.179 & 0.118 & 15.7 & 0.15 & 0.119 & -- & -- & 0.121 & -- & -- & -- \\
\hline
\end{tabular}


Appendix 3. Volumetric water content, temperature, and electrical-conductivity data collected at the sprinkler-irrigated site during 2008.-Continued

[Depth in feet below land surface; volumetric water content dimensionless; temperature in degrees Celsius; bulk electrical conductivity in decisiemens per meter; --, no data]

\begin{tabular}{|c|c|c|c|c|c|c|c|c|c|c|c|c|c|}
\hline \multirow[b]{3}{*}{$\begin{array}{l}\text { Measurement } \\
\text { date and time }\end{array}$} & \multirow{2}{*}{\multicolumn{2}{|c|}{2}} & \multirow{3}{*}{$\begin{array}{c}3 \\
\text { Volumetric } \\
\text { water } \\
\text { content }\end{array}$} & \multicolumn{9}{|c|}{ Depth } & \multirow{3}{*}{$\begin{array}{c}16.5 \\
\text { Volumetric } \\
\text { water } \\
\text { content }\end{array}$} \\
\hline & & & & \multicolumn{3}{|c|}{4} & \multirow[b]{2}{*}{$\begin{array}{c}\mathbf{5} \\
\text { Volumetric } \\
\text { water } \\
\text { content }\end{array}$} & \multicolumn{2}{|l|}{7} & \multirow{2}{*}{$\begin{array}{c}9 \\
\text { Volumetric } \\
\text { water } \\
\text { content }\end{array}$} & \multicolumn{2}{|c|}{13} & \\
\hline & $\begin{array}{c}\text { Volumetric } \\
\text { water } \\
\text { content }\end{array}$ & $\begin{array}{l}\text { Temper- } \\
\text { ature }\end{array}$ & & $\begin{array}{c}\text { Volumetric } \\
\text { water } \\
\text { content }\end{array}$ & $\begin{array}{l}\text { Temper- } \\
\text { ature }\end{array}$ & $\begin{array}{c}\text { Bulk } \\
\text { electrical } \\
\text { conduct- } \\
\text { ivity }\end{array}$ & & $\begin{array}{l}\text { Volumetric } \\
\text { water } \\
\text { content }\end{array}$ & $\begin{array}{l}\text { Temper- } \\
\text { ature }\end{array}$ & & $\begin{array}{c}\text { Volumetric } \\
\text { water } \\
\text { content }\end{array}$ & $\begin{array}{l}\text { Temper- } \\
\text { ature }\end{array}$ & \\
\hline 10/4/2008 12:00 PM & 0.333 & 14.7 & 0.179 & 0.118 & 15.7 & 0.15 & 0.119 & -- & -- & 0.121 & -- & -- & -- \\
\hline 10/4/2008 1:00 PM & 0.333 & 14.6 & 0.179 & 0.118 & 15.7 & 0.15 & 0.119 & -- & -- & 0.121 & -- & -- & -- \\
\hline 10/4/2008 2:00 PM & 0.332 & 14.6 & 0.178 & 0.118 & 15.7 & 0.15 & 0.119 & -- & -- & 0.121 & -- & -- & -- \\
\hline 10/4/2008 3:00 PM & 0.332 & 14.6 & 0.178 & 0.118 & 15.7 & 0.15 & 0.119 & -- & -- & 0.121 & -- & -- & -- \\
\hline 10/4/2008 4:00 PM & 0.332 & 14.6 & 0.179 & 0.118 & 15.7 & 0.15 & 0.119 & -- & -- & 0.121 & -- & -- & -- \\
\hline 10/4/2008 5:00 PM & 0.332 & 14.6 & 0.179 & 0.118 & 15.7 & 0.15 & 0.119 & -- & -- & 0.121 & -- & -- & -- \\
\hline 10/4/2008 6:00 PM & 0.331 & 14.6 & 0.179 & 0.118 & 15.7 & 0.15 & 0.119 & -- & -- & 0.121 & -- & -- & -- \\
\hline 10/4/2008 7:00 PM & 0.331 & 14.6 & 0.179 & 0.118 & 15.7 & 0.15 & 0.119 & -- & -- & 0.121 & -- & -- & -- \\
\hline 10/4/2008 8:00 PM & 0.331 & 14.5 & 0.178 & 0.118 & 15.7 & 0.15 & 0.119 & -- & -- & 0.121 & -- & -- & -- \\
\hline 10/4/2008 9:00 PM & 0.331 & 14.5 & 0.178 & 0.118 & 15.7 & 0.15 & 0.119 & -- & -- & 0.121 & -- & -- & -- \\
\hline 10/4/2008 10:00 PM & 0.331 & 14.5 & 0.178 & 0.118 & 15.7 & 0.15 & 0.119 & -- & -- & 0.121 & -- & -- & -- \\
\hline 10/4/2008 11:00 PM & 0.331 & 14.5 & 0.178 & 0.118 & 15.7 & 0.15 & 0.119 & -- & -- & 0.121 & -- & -- & -- \\
\hline 10/5/2008 12:00 AM & 0.331 & 14.6 & 0.178 & 0.118 & 15.7 & 0.15 & 0.118 & -- & -- & 0.121 & -- & -- & -- \\
\hline 10/5/2008 1:00 AM & 0.331 & 14.6 & 0.178 & 0.118 & 15.7 & 0.15 & 0.118 & -- & -- & 0.121 & -- & -- & -- \\
\hline 10/5/2008 2:00 AM & 0.331 & 14.6 & 0.178 & 0.118 & 15.7 & 0.15 & 0.118 & -- & -- & 0.121 & -- & -- & -- \\
\hline 10/5/2008 3:00 AM & 0.331 & 14.6 & 0.179 & 0.118 & 15.7 & 0.15 & 0.118 & -- & -- & 0.121 & -- & -- & -- \\
\hline 10/5/2008 4:00 AM & 0.331 & 14.6 & 0.178 & 0.118 & 15.7 & 0.15 & 0.118 & -- & -- & 0.121 & -- & -- & -- \\
\hline 10/5/2008 5:00 AM & 0.331 & 14.6 & 0.178 & 0.118 & 15.7 & 0.15 & 0.118 & -- & -- & 0.121 & -- & -- & -- \\
\hline 10/5/2008 6:00 AM & 0.331 & 14.6 & 0.178 & 0.118 & 15.7 & 0.15 & 0.118 & -- & -- & 0.121 & -- & -- & -- \\
\hline 10/5/2008 7:00 AM & 0.331 & 14.6 & 0.179 & 0.118 & 15.7 & 0.15 & 0.118 & -- & -- & 0.121 & -- & -- & -- \\
\hline 10/5/2008 8:00 AM & 0.331 & 14.6 & 0.178 & 0.118 & 15.7 & 0.15 & 0.118 & -- & -- & 0.121 & -- & -- & -- \\
\hline 10/5/2008 9:00 AM & 0.331 & 14.6 & 0.178 & 0.118 & 15.7 & 0.15 & 0.118 & -- & -- & 0.121 & -- & -- & -- \\
\hline 10/5/2008 10:00 AM & 0.331 & 14.6 & 0.179 & 0.118 & 15.7 & 0.15 & 0.119 & -- & -- & 0.121 & -- & -- & -- \\
\hline 10/5/2008 11:00 AM & 0.331 & 14.6 & 0.178 & 0.118 & 15.7 & 0.15 & 0.119 & -- & -- & 0.121 & -- & -- & -- \\
\hline 10/5/2008 12:00 PM & 0.332 & 14.6 & 0.179 & 0.118 & 15.7 & 0.15 & 0.118 & -- & -- & 0.121 & -- & -- & -- \\
\hline 10/5/2008 1:00 PM & 0.332 & 14.6 & 0.179 & 0.118 & 15.7 & 0.15 & 0.119 & -- & -- & 0.121 & -- & -- & -- \\
\hline 10/5/2008 2:00 PM & 0.332 & 14.6 & 0.178 & 0.118 & 15.7 & 0.15 & 0.119 & -- & -- & 0.121 & -- & -- & -- \\
\hline 10/5/2008 3:00 PM & 0.331 & 14.6 & 0.178 & 0.118 & 15.7 & 0.15 & 0.119 & -- & -- & 0.121 & -- & -- & -- \\
\hline 10/5/2008 4:00 PM & 0.331 & 14.6 & 0.178 & 0.118 & 15.7 & 0.15 & 0.119 & -- & -- & 0.121 & -- & -- & -- \\
\hline 10/5/2008 5:00 PM & 0.331 & 14.6 & 0.178 & 0.118 & 15.6 & 0.15 & 0.119 & -- & -- & 0.121 & -- & -- & -- \\
\hline 10/5/2008 6:00 PM & 0.331 & 14.6 & 0.178 & 0.118 & 15.7 & 0.15 & 0.119 & -- & -- & 0.121 & -- & -- & -- \\
\hline 10/5/2008 7:00 PM & 0.330 & 14.6 & 0.178 & 0.118 & 15.7 & 0.15 & 0.119 & -- & -- & 0.121 & -- & -- & -- \\
\hline 10/5/2008 8:00 PM & 0.330 & 14.5 & 0.178 & 0.118 & 15.7 & 0.15 & 0.118 & -- & -- & 0.121 & -- & -- & -- \\
\hline 10/5/2008 9:00 PM & 0.330 & 14.5 & 0.178 & 0.118 & 15.7 & 0.15 & 0.118 & -- & -- & 0.121 & -- & -- & -- \\
\hline 10/5/2008 10:00 PM & 0.330 & 14.6 & 0.178 & 0.118 & 15.6 & 0.15 & 0.118 & -- & -- & 0.153 & -- & -- & -- \\
\hline
\end{tabular}


Appendix 3. Volumetric water content, temperature, and electrical-conductivity data collected at the sprinkler-irrigated site during 2008.-Continued

[Depth in feet below land surface; volumetric water content dimensionless; temperature in degrees Celsius; bulk electrical conductivity in decisiemens per meter; --, no data]

\begin{tabular}{|c|c|c|c|c|c|c|c|c|c|c|c|c|c|}
\hline \multirow[b]{3}{*}{$\begin{array}{l}\text { Measurement } \\
\text { date and time }\end{array}$} & \multicolumn{13}{|c|}{ Depth } \\
\hline & \multicolumn{2}{|l|}{2} & \multirow{2}{*}{$\begin{array}{c}3 \\
\text { Volumetric } \\
\text { water } \\
\text { content }\end{array}$} & \multicolumn{3}{|c|}{4} & \multirow{2}{*}{$\begin{array}{c}5 \\
\text { Volumetric } \\
\text { water } \\
\text { content }\end{array}$} & \multicolumn{2}{|l|}{7} & \multirow{2}{*}{$\begin{array}{c}9 \\
\text { Volumetric } \\
\text { water } \\
\text { content }\end{array}$} & \multicolumn{2}{|c|}{13} & \multirow{2}{*}{$\begin{array}{c}16.5 \\
\text { Volumetric } \\
\text { water } \\
\text { content }\end{array}$} \\
\hline & $\begin{array}{c}\text { Volumetric } \\
\text { water } \\
\text { content }\end{array}$ & $\begin{array}{l}\text { Temper- } \\
\text { ature }\end{array}$ & & $\begin{array}{c}\text { Volumetric } \\
\text { water } \\
\text { content }\end{array}$ & $\begin{array}{l}\text { Temper- } \\
\text { ature }\end{array}$ & $\begin{array}{c}\text { Bulk } \\
\text { electrical } \\
\text { conduct- } \\
\text { ivity }\end{array}$ & & $\begin{array}{c}\text { Volumetric } \\
\text { water } \\
\text { content }\end{array}$ & $\begin{array}{l}\text { Temper- } \\
\text { ature }\end{array}$ & & $\begin{array}{c}\text { Volumetric } \\
\text { water } \\
\text { content }\end{array}$ & $\begin{array}{l}\text { Temper- } \\
\text { ature }\end{array}$ & \\
\hline 10/5/2008 11:00 PM & 0.330 & 14.6 & 0.178 & 0.118 & 15.6 & 0.15 & 0.118 & -- & -- & 0.152 & -- & -- & -- \\
\hline 10/6/2008 12:00 AM & 0.330 & 14.6 & 0.178 & 0.118 & 15.6 & 0.15 & 0.118 & -- & -- & 0.131 & -- & -- & -- \\
\hline 10/6/2008 1:00 AM & 0.331 & 14.6 & 0.178 & 0.118 & 15.6 & 0.15 & 0.118 & -- & -- & 0.126 & -- & -- & -- \\
\hline 10/6/2008 2:00 AM & 0.331 & 14.6 & 0.178 & 0.118 & 15.7 & 0.15 & 0.118 & -- & -- & 0.124 & -- & -- & -- \\
\hline 10/6/2008 3:00 AM & 0.331 & 14.6 & 0.178 & 0.118 & 15.6 & 0.15 & 0.118 & -- & -- & 0.125 & -- & -- & -- \\
\hline 10/6/2008 4:00 AM & 0.331 & 14.6 & 0.178 & 0.118 & 15.6 & 0.15 & 0.118 & -- & -- & 0.120 & -- & -- & -- \\
\hline 10/6/2008 5:00 AM & 0.331 & 14.7 & 0.178 & 0.118 & 15.6 & 0.15 & 0.118 & -- & -- & 0.115 & -- & -- & -- \\
\hline 10/6/2008 6:00 AM & 0.331 & 14.7 & 0.177 & 0.118 & 15.6 & 0.15 & 0.118 & -- & -- & 0.113 & -- & -- & -- \\
\hline 10/6/2008 7:00 AM & 0.331 & 14.6 & 0.178 & 0.118 & 15.6 & 0.15 & 0.118 & -- & -- & 0.109 & -- & -- & -- \\
\hline 10/6/2008 8:00 AM & 0.331 & 14.6 & 0.178 & 0.118 & 15.6 & 0.15 & 0.118 & -- & -- & 0.110 & -- & -- & -- \\
\hline 10/6/2008 9:00 AM & 0.331 & 14.7 & 0.178 & 0.118 & 15.7 & 0.15 & 0.118 & -- & -- & 0.112 & -- & -- & -- \\
\hline 10/6/2008 10:00 AM & 0.331 & 14.7 & 0.178 & 0.117 & 15.6 & 0.15 & 0.118 & -- & -- & 0.122 & -- & -- & -- \\
\hline 10/6/2008 11:00 AM & 0.331 & 14.6 & 0.178 & 0.118 & 15.6 & 0.15 & 0.118 & -- & -- & 0.151 & -- & -- & -- \\
\hline 10/6/2008 12:00 PM & 0.331 & 14.7 & 0.178 & 0.117 & 15.6 & 0.15 & 0.118 & -- & -- & 0.143 & -- & -- & -- \\
\hline 10/6/2008 1:00 PM & 0.331 & 14.6 & 0.178 & 0.118 & 15.6 & 0.15 & 0.118 & -- & -- & 0.132 & -- & -- & -- \\
\hline 10/6/2008 2:00 PM & 0.331 & 14.6 & 0.178 & 0.117 & 15.6 & 0.15 & 0.118 & -- & -- & 0.128 & -- & -- & -- \\
\hline 10/6/2008 3:00 PM & 0.331 & 14.7 & 0.178 & 0.118 & 15.6 & 0.15 & 0.118 & -- & -- & 0.121 & -- & -- & -- \\
\hline 10/6/2008 4:00 PM & 0.331 & 14.6 & 0.178 & 0.118 & 15.6 & 0.15 & 0.118 & -- & -- & 0.121 & -- & -- & -- \\
\hline 10/6/2008 5:00 PM & 0.330 & 14.6 & 0.178 & 0.117 & 15.6 & 0.15 & 0.118 & -- & -- & 0.121 & -- & -- & -- \\
\hline 10/6/2008 6:00 PM & 0.330 & 14.6 & 0.178 & 0.117 & 15.6 & 0.15 & 0.118 & -- & -- & 0.121 & -- & -- & -- \\
\hline 10/6/2008 7:00 PM & 0.330 & 14.6 & 0.178 & 0.117 & 15.6 & 0.15 & 0.118 & -- & -- & 0.121 & -- & -- & -- \\
\hline 10/6/2008 8:00 PM & 0.330 & 14.6 & 0.178 & 0.117 & 15.6 & 0.15 & 0.118 & -- & -- & 0.121 & -- & -- & -- \\
\hline 10/6/2008 9:00 PM & 0.330 & 14.6 & 0.178 & 0.117 & 15.6 & 0.15 & 0.118 & -- & -- & 0.121 & -- & -- & -- \\
\hline 10/6/2008 10:00 PM & 0.330 & 14.6 & 0.177 & 0.117 & 15.6 & 0.15 & 0.118 & -- & -- & 0.121 & -- & -- & -- \\
\hline 10/6/2008 11:00 PM & 0.330 & 14.6 & 0.178 & 0.117 & 15.6 & 0.15 & 0.118 & -- & -- & 0.120 & -- & -- & -- \\
\hline 10/7/2008 12:00 AM & 0.329 & 14.5 & 0.177 & 0.117 & 15.6 & 0.15 & 0.118 & -- & -- & 0.120 & -- & -- & -- \\
\hline 10/7/2008 1:00 AM & 0.329 & 14.6 & 0.177 & 0.118 & 15.6 & 0.15 & 0.118 & -- & -- & 0.120 & -- & -- & -- \\
\hline 10/7/2008 2:00 AM & 0.329 & 14.6 & 0.177 & 0.117 & 15.6 & 0.15 & 0.118 & -- & -- & 0.120 & -- & -- & -- \\
\hline 10/7/2008 3:00 AM & 0.329 & 14.5 & 0.177 & 0.117 & 15.6 & 0.15 & 0.118 & -- & -- & 0.120 & -- & -- & -- \\
\hline 10/7/2008 4:00 AM & 0.329 & 14.5 & 0.177 & 0.118 & 15.6 & 0.15 & 0.118 & -- & -- & 0.120 & -- & -- & -- \\
\hline 10/7/2008 5:00 AM & 0.329 & 14.6 & 0.177 & 0.117 & 15.6 & 0.15 & 0.118 & -- & -- & 0.120 & -- & -- & -- \\
\hline 10/7/2008 6:00 AM & 0.329 & 14.5 & 0.177 & 0.117 & 15.6 & 0.15 & 0.118 & -- & -- & 0.119 & -- & -- & -- \\
\hline 10/7/2008 7:00 AM & 0.329 & 14.5 & 0.177 & 0.117 & 15.6 & 0.15 & 0.118 & -- & -- & 0.119 & -- & -- & -- \\
\hline 10/7/2008 8:00 AM & 0.330 & 14.5 & 0.177 & 0.117 & 15.6 & 0.15 & 0.118 & -- & -- & 0.119 & -- & -- & -- \\
\hline 10/7/2008 9:00 AM & 0.329 & 14.5 & 0.177 & 0.117 & 15.6 & 0.15 & 0.118 & -- & -- & 0.121 & -- & -- & -- \\
\hline
\end{tabular}


Appendix 3. Volumetric water content, temperature, and electrical-conductivity data collected at the sprinkler-irrigated site during 2008.-Continued

[Depth in feet below land surface; volumetric water content dimensionless; temperature in degrees Celsius; bulk electrical conductivity in decisiemens per meter; --, no data]

\begin{tabular}{|c|c|c|c|c|c|c|c|c|c|c|c|c|c|}
\hline \multirow[b]{3}{*}{$\begin{array}{l}\text { Measurement } \\
\text { date and time }\end{array}$} & \multicolumn{13}{|c|}{ Depth } \\
\hline & \multicolumn{2}{|l|}{2} & \multirow{2}{*}{$\begin{array}{c}3 \\
\text { Volumetric } \\
\text { water } \\
\text { content }\end{array}$} & \multicolumn{3}{|c|}{4} & \multirow{2}{*}{$\begin{array}{c}5 \\
\text { Volumetric } \\
\text { water } \\
\text { content }\end{array}$} & \multicolumn{2}{|l|}{7} & \multirow{2}{*}{$\begin{array}{c}9 \\
\text { Volumetric } \\
\text { water } \\
\text { content }\end{array}$} & \multicolumn{2}{|c|}{13} & \multirow{2}{*}{$\begin{array}{c}16.5 \\
\text { Volumetric } \\
\text { water } \\
\text { content }\end{array}$} \\
\hline & $\begin{array}{c}\text { Volumetric } \\
\text { water } \\
\text { content }\end{array}$ & $\begin{array}{c}\text { Temper- } \\
\text { ature }\end{array}$ & & $\begin{array}{c}\text { Volumetric } \\
\text { water } \\
\text { content }\end{array}$ & $\begin{array}{l}\text { Temper- } \\
\text { ature }\end{array}$ & $\begin{array}{c}\text { Bulk } \\
\text { electrical } \\
\text { conduct- } \\
\text { ivity }\end{array}$ & & $\begin{array}{l}\text { Volumetric } \\
\text { water } \\
\text { content }\end{array}$ & $\begin{array}{l}\text { Temper- } \\
\text { ature }\end{array}$ & & $\begin{array}{c}\text { Volumetric } \\
\text { water } \\
\text { content }\end{array}$ & $\begin{array}{l}\text { Temper- } \\
\text { ature }\end{array}$ & \\
\hline 10/7/2008 10:00 AM & 0.330 & 14.5 & 0.177 & 0.118 & 15.6 & 0.15 & 0.118 & -- & -- & 0.121 & -- & -- & -- \\
\hline 10/7/2008 11:00 AM & 0.330 & 14.5 & 0.177 & 0.117 & 15.6 & 0.15 & 0.118 & -- & -- & 0.119 & -- & -- & -- \\
\hline 10/7/2008 12:00 PM & 0.330 & 14.4 & 0.178 & 0.117 & 15.6 & 0.15 & 0.118 & -- & -- & 0.120 & -- & -- & -- \\
\hline 10/7/2008 1:00 PM & 0.330 & 14.4 & 0.178 & 0.117 & 15.6 & 0.15 & 0.118 & -- & -- & 0.120 & -- & -- & -- \\
\hline 10/7/2008 2:00 PM & 0.330 & 14.4 & 0.178 & 0.117 & 15.6 & 0.15 & 0.118 & -- & -- & 0.120 & -- & -- & -- \\
\hline 10/7/2008 3:00 PM & 0.330 & 14.3 & 0.177 & 0.117 & 15.6 & 0.15 & 0.118 & -- & -- & 0.120 & -- & -- & -- \\
\hline 10/7/2008 4:00 PM & 0.330 & 14.3 & 0.177 & 0.117 & 15.6 & 0.15 & 0.118 & -- & -- & 0.120 & -- & -- & -- \\
\hline 10/7/2008 5:00 PM & 0.330 & 14.3 & 0.178 & 0.117 & 15.6 & 0.15 & 0.118 & -- & -- & 0.120 & -- & -- & -- \\
\hline 10/7/2008 6:00 PM & 0.331 & 14.3 & 0.177 & 0.117 & 15.6 & 0.15 & 0.118 & -- & -- & 0.120 & -- & -- & -- \\
\hline 10/7/2008 7:00 PM & 0.331 & 14.2 & 0.177 & 0.117 & 15.6 & 0.15 & 0.118 & -- & -- & 0.120 & -- & -- & -- \\
\hline 10/7/2008 8:00 PM & 0.330 & 14.2 & 0.177 & 0.117 & 15.6 & 0.15 & 0.117 & -- & -- & 0.120 & -- & -- & -- \\
\hline 10/7/2008 9:00 PM & 0.330 & 14.2 & 0.177 & 0.117 & 15.6 & 0.15 & 0.117 & -- & -- & 0.120 & -- & -- & -- \\
\hline 10/7/2008 10:00 PM & 0.330 & 14.1 & 0.177 & 0.117 & 15.6 & 0.15 & 0.117 & -- & -- & 0.120 & -- & -- & -- \\
\hline 10/7/2008 11:00 PM & 0.329 & 14.2 & 0.177 & 0.117 & 15.6 & 0.15 & 0.118 & -- & -- & 0.120 & -- & -- & -- \\
\hline 10/8/2008 12:00 AM & 0.329 & 14.2 & 0.177 & 0.117 & 15.6 & 0.15 & 0.118 & -- & -- & 0.120 & -- & -- & -- \\
\hline 10/8/2008 1:00 AM & 0.330 & 14.1 & 0.177 & 0.117 & 15.6 & 0.15 & 0.118 & -- & -- & 0.120 & -- & -- & -- \\
\hline 10/8/2008 2:00 AM & 0.330 & 14.1 & 0.177 & 0.117 & 15.6 & 0.15 & 0.118 & -- & -- & 0.120 & -- & -- & -- \\
\hline 10/8/2008 3:00 AM & 0.330 & 14.2 & 0.177 & 0.117 & 15.6 & 0.15 & 0.118 & -- & -- & 0.120 & -- & -- & -- \\
\hline 10/8/2008 4:00 AM & 0.330 & 14.2 & 0.177 & 0.117 & 15.6 & 0.15 & 0.118 & -- & -- & 0.120 & -- & -- & -- \\
\hline 10/8/2008 5:00 AM & 0.330 & 14.1 & 0.177 & 0.117 & 15.6 & 0.15 & 0.118 & -- & -- & 0.120 & -- & -- & -- \\
\hline 10/8/2008 6:00 AM & 0.330 & 14.2 & 0.177 & 0.117 & 15.6 & 0.15 & 0.118 & -- & -- & 0.120 & -- & -- & -- \\
\hline 10/8/2008 7:00 AM & 0.330 & 14.1 & 0.177 & 0.117 & 15.6 & 0.15 & 0.117 & -- & -- & 0.120 & -- & -- & -- \\
\hline 10/8/2008 8:00 AM & 0.329 & 14.1 & 0.177 & 0.117 & 15.6 & 0.15 & 0.118 & -- & -- & 0.120 & -- & -- & -- \\
\hline 10/8/2008 9:00 AM & 0.329 & 14.1 & 0.177 & 0.117 & 15.5 & 0.15 & 0.118 & -- & -- & 0.120 & -- & -- & -- \\
\hline 10/8/2008 10:00 AM & 0.330 & 14.1 & 0.177 & 0.117 & 15.6 & 0.15 & 0.117 & -- & -- & 0.120 & -- & -- & -- \\
\hline 10/8/2008 11:00 AM & 0.330 & 14.1 & 0.177 & 0.117 & 15.5 & 0.15 & 0.118 & -- & -- & 0.120 & -- & -- & -- \\
\hline 10/8/2008 12:00 PM & 0.330 & 14.1 & 0.177 & 0.117 & 15.5 & 0.15 & 0.118 & -- & -- & 0.120 & -- & -- & -- \\
\hline 10/8/2008 1:00 PM & 0.330 & 14.0 & 0.177 & 0.117 & 15.5 & 0.15 & 0.118 & -- & -- & 0.120 & -- & -- & -- \\
\hline 10/8/2008 2:00 PM & 0.329 & 14.0 & 0.177 & 0.117 & 15.5 & 0.15 & 0.118 & -- & -- & 0.120 & -- & -- & -- \\
\hline 10/8/2008 3:00 PM & 0.329 & 14.0 & 0.177 & 0.117 & 15.5 & 0.15 & 0.118 & -- & -- & 0.120 & -- & -- & -- \\
\hline 10/8/2008 4:00 PM & 0.329 & 14.0 & 0.177 & 0.117 & 15.5 & 0.15 & 0.118 & -- & -- & 0.120 & -- & -- & -- \\
\hline 10/8/2008 5:00 PM & 0.329 & 14.0 & 0.177 & 0.117 & 15.5 & 0.15 & 0.118 & -- & -- & 0.120 & -- & -- & -- \\
\hline 10/8/2008 6:00 PM & 0.328 & 13.9 & 0.177 & 0.117 & 15.5 & 0.15 & 0.118 & -- & -- & 0.120 & -- & -- & -- \\
\hline 10/8/2008 7:00 PM & 0.328 & 13.9 & 0.177 & 0.117 & 15.5 & 0.15 & 0.118 & -- & -- & 0.120 & -- & -- & -- \\
\hline 10/8/2008 8:00 PM & 0.329 & 13.9 & 0.177 & 0.117 & 15.5 & 0.15 & 0.117 & -- & -- & 0.120 & -- & -- & -- \\
\hline
\end{tabular}


Appendix 3. Volumetric water content, temperature, and electrical-conductivity data collected at the sprinkler-irrigated site during 2008.-Continued

[Depth in feet below land surface; volumetric water content dimensionless; temperature in degrees Celsius; bulk electrical conductivity in decisiemens per meter; --, no data]

\begin{tabular}{|c|c|c|c|c|c|c|c|c|c|c|c|c|c|}
\hline \multirow[b]{3}{*}{$\begin{array}{l}\text { Measurement } \\
\text { date and time }\end{array}$} & \multicolumn{13}{|c|}{ Depth } \\
\hline & \multicolumn{2}{|l|}{2} & \multirow{2}{*}{$\begin{array}{c}3 \\
\text { Volumetric } \\
\text { water } \\
\text { content }\end{array}$} & \multicolumn{3}{|c|}{4} & \multirow{2}{*}{$\begin{array}{c}5 \\
\text { Volumetric } \\
\text { water } \\
\text { content }\end{array}$} & \multicolumn{2}{|l|}{7} & \multirow{2}{*}{$\begin{array}{c}9 \\
\text { Volumetric } \\
\text { water } \\
\text { content }\end{array}$} & \multicolumn{2}{|c|}{13} & \multirow{2}{*}{$\begin{array}{c}16.5 \\
\text { Volumetric } \\
\text { water } \\
\text { content }\end{array}$} \\
\hline & $\begin{array}{c}\text { Volumetric } \\
\text { water } \\
\text { content }\end{array}$ & $\begin{array}{l}\text { Temper- } \\
\text { ature }\end{array}$ & & $\begin{array}{c}\text { Volumetric } \\
\text { water } \\
\text { content }\end{array}$ & $\begin{array}{l}\text { Temper- } \\
\text { ature }\end{array}$ & $\begin{array}{c}\text { Bulk } \\
\text { electrical } \\
\text { conduct- } \\
\text { ivity }\end{array}$ & & $\begin{array}{c}\text { Volumetric } \\
\text { water } \\
\text { content }\end{array}$ & $\begin{array}{l}\text { Temper- } \\
\text { ature }\end{array}$ & & $\begin{array}{c}\text { Volumetric } \\
\text { water } \\
\text { content }\end{array}$ & $\begin{array}{l}\text { Temper- } \\
\text { ature }\end{array}$ & \\
\hline 10/8/2008 9:00 PM & 0.329 & 13.9 & 0.177 & 0.117 & 15.5 & 0.15 & 0.117 & -- & -- & 0.120 & -- & -- & -- \\
\hline 10/8/2008 10:00 PM & 0.328 & 13.9 & 0.177 & 0.117 & 15.5 & 0.15 & 0.117 & -- & -- & 0.120 & -- & -- & -- \\
\hline 10/8/2008 11:00 PM & 0.328 & 13.8 & 0.177 & 0.117 & 15.5 & 0.15 & 0.117 & -- & -- & 0.120 & -- & -- & -- \\
\hline 10/9/2008 12:00 AM & 0.329 & 13.9 & 0.177 & 0.117 & 15.5 & 0.15 & 0.117 & -- & -- & 0.120 & -- & -- & -- \\
\hline 10/9/2008 1:00 AM & 0.328 & 13.9 & 0.177 & 0.117 & 15.5 & 0.15 & 0.117 & -- & -- & 0.120 & -- & -- & -- \\
\hline 10/9/2008 2:00 AM & 0.328 & 13.9 & 0.177 & 0.117 & 15.5 & 0.15 & 0.117 & -- & -- & 0.120 & -- & -- & -- \\
\hline 10/9/2008 3:00 AM & 0.328 & 13.9 & 0.177 & 0.117 & 15.5 & 0.15 & 0.117 & -- & -- & 0.120 & -- & -- & -- \\
\hline 10/9/2008 4:00 AM & 0.328 & 14.0 & 0.177 & 0.117 & 15.5 & 0.15 & 0.117 & -- & -- & 0.120 & -- & -- & -- \\
\hline 10/9/2008 5:00 AM & 0.328 & 14.0 & 0.177 & 0.117 & 15.5 & 0.15 & 0.117 & -- & -- & 0.120 & -- & -- & -- \\
\hline 10/9/2008 6:00 AM & 0.328 & 14.0 & 0.177 & 0.117 & 15.5 & 0.15 & 0.117 & -- & -- & 0.120 & -- & -- & -- \\
\hline 10/9/2008 7:00 AM & 0.328 & 14.0 & 0.177 & 0.117 & 15.5 & 0.15 & 0.117 & -- & -- & 0.120 & -- & -- & -- \\
\hline 10/9/2008 8:00 AM & 0.328 & 14.0 & 0.177 & 0.118 & 15.5 & 0.15 & 0.117 & -- & -- & 0.120 & -- & -- & -- \\
\hline 10/9/2008 9:00 AM & 0.328 & 14.0 & 0.177 & 0.117 & 15.4 & 0.15 & 0.117 & -- & -- & 0.120 & -- & -- & -- \\
\hline 10/9/2008 10:00 AM & 0.328 & 14.0 & 0.177 & 0.117 & 15.4 & 0.15 & 0.117 & -- & -- & 0.120 & -- & -- & -- \\
\hline 10/9/2008 11:00 AM & 0.328 & 14.0 & 0.177 & 0.117 & 15.5 & 0.15 & 0.117 & -- & -- & 0.120 & -- & -- & -- \\
\hline 10/9/2008 12:00 PM & 0.328 & 14.0 & 0.177 & 0.117 & 15.4 & 0.15 & 0.117 & -- & -- & 0.120 & -- & -- & -- \\
\hline 10/9/2008 1:00 PM & 0.328 & 14.0 & 0.177 & 0.117 & 15.4 & 0.15 & 0.117 & -- & -- & 0.120 & -- & -- & -- \\
\hline 10/9/2008 2:00 PM & 0.328 & 13.9 & 0.177 & 0.117 & 15.4 & 0.15 & 0.117 & -- & -- & 0.120 & -- & -- & -- \\
\hline 10/9/2008 3:00 PM & 0.328 & 13.9 & 0.177 & 0.117 & 15.4 & 0.15 & 0.117 & -- & -- & 0.120 & -- & -- & -- \\
\hline 10/9/2008 4:00 PM & 0.328 & 13.9 & 0.177 & 0.117 & 15.4 & 0.15 & 0.117 & -- & -- & 0.120 & -- & -- & -- \\
\hline 10/9/2008 5:00 PM & 0.328 & 13.9 & 0.177 & 0.117 & 15.4 & 0.15 & 0.117 & -- & -- & 0.120 & -- & -- & -- \\
\hline 10/9/2008 6:00 PM & 0.328 & 13.9 & 0.177 & 0.118 & 15.4 & 0.15 & 0.117 & -- & -- & 0.120 & -- & -- & -- \\
\hline 10/9/2008 7:00 PM & 0.328 & 13.8 & 0.177 & 0.118 & 15.4 & 0.15 & 0.117 & -- & -- & 0.120 & -- & -- & -- \\
\hline 10/9/2008 8:00 PM & 0.328 & 13.8 & 0.177 & 0.117 & 15.4 & 0.15 & 0.117 & -- & -- & 0.120 & -- & -- & -- \\
\hline 10/9/2008 9:00 PM & 0.328 & 13.8 & 0.177 & 0.117 & 15.4 & 0.15 & 0.117 & -- & -- & 0.120 & -- & -- & -- \\
\hline 10/9/2008 10:00 PM & 0.328 & 13.8 & 0.177 & 0.117 & 15.4 & 0.15 & 0.117 & -- & -- & 0.120 & -- & -- & -- \\
\hline 10/9/2008 11:00 PM & 0.328 & 13.8 & 0.177 & 0.118 & 15.4 & 0.15 & 0.117 & -- & -- & 0.120 & -- & -- & -- \\
\hline 10/10/2008 12:00 AM & 0.328 & 13.8 & 0.177 & 0.118 & 15.4 & 0.15 & 0.117 & -- & -- & 0.120 & -- & -- & -- \\
\hline 10/10/2008 1:00 AM & 0.328 & 13.8 & 0.177 & 0.117 & 15.4 & 0.15 & 0.117 & -- & -- & 0.120 & -- & -- & -- \\
\hline 10/10/2008 2:00 AM & 0.328 & 13.7 & 0.177 & 0.117 & 15.4 & 0.15 & 0.117 & -- & -- & 0.120 & -- & -- & -- \\
\hline 10/10/2008 3:00 AM & 0.328 & 13.7 & 0.177 & 0.117 & 15.4 & 0.15 & 0.117 & -- & -- & 0.120 & -- & -- & -- \\
\hline 10/10/2008 4:00 AM & 0.328 & 13.7 & 0.177 & 0.117 & 15.4 & 0.15 & 0.117 & -- & -- & 0.120 & -- & -- & -- \\
\hline 10/10/2008 5:00 AM & 0.328 & 13.7 & 0.177 & 0.117 & 15.4 & 0.15 & 0.117 & -- & -- & 0.120 & -- & -- & -- \\
\hline 10/10/2008 6:00 AM & 0.328 & 13.7 & 0.177 & 0.117 & 15.4 & 0.15 & 0.117 & -- & -- & 0.120 & -- & -- & -- \\
\hline 10/10/2008 7:00 AM & 0.328 & 13.7 & 0.177 & 0.117 & 15.3 & 0.15 & 0.117 & -- & -- & 0.120 & -- & -- & -- \\
\hline
\end{tabular}


Appendix 3. Volumetric water content, temperature, and electrical-conductivity data collected at the sprinkler-irrigated site during 2008.-Continued

[Depth in feet below land surface; volumetric water content dimensionless; temperature in degrees Celsius; bulk electrical conductivity in decisiemens per meter; --, no data]

\begin{tabular}{|c|c|c|c|c|c|c|c|c|c|c|c|c|c|}
\hline \multirow[b]{3}{*}{$\begin{array}{l}\text { Measurement } \\
\text { date and time }\end{array}$} & \multicolumn{13}{|c|}{ Depth } \\
\hline & \multicolumn{2}{|l|}{2} & \multirow{2}{*}{$\begin{array}{c}3 \\
\begin{array}{c}\text { Volumetric } \\
\text { water } \\
\text { content }\end{array}\end{array}$} & \multicolumn{3}{|c|}{4} & \multirow{2}{*}{$\begin{array}{c}5 \\
\begin{array}{c}\text { Volumetric } \\
\text { water } \\
\text { content }\end{array}\end{array}$} & \multicolumn{2}{|l|}{7} & \multirow{2}{*}{$\begin{array}{c}9 \\
\text { Volumetric } \\
\text { water } \\
\text { content }\end{array}$} & \multicolumn{2}{|c|}{13} & \multirow{2}{*}{$\begin{array}{c}16.5 \\
\text { Volumetric } \\
\text { water } \\
\text { content }\end{array}$} \\
\hline & $\begin{array}{c}\text { Volumetric } \\
\text { water } \\
\text { content }\end{array}$ & $\begin{array}{l}\text { Temper- } \\
\text { ature }\end{array}$ & & $\begin{array}{c}\text { Volumetric } \\
\text { water } \\
\text { content }\end{array}$ & $\begin{array}{l}\text { Temper- } \\
\text { ature }\end{array}$ & $\begin{array}{c}\text { Bulk } \\
\text { electrical } \\
\text { conduct- } \\
\text { ivity }\end{array}$ & & $\begin{array}{c}\text { Volumetric } \\
\text { water } \\
\text { content }\end{array}$ & $\begin{array}{l}\text { Temper- } \\
\text { ature }\end{array}$ & & $\begin{array}{c}\text { Volumetric } \\
\text { water } \\
\text { content }\end{array}$ & $\begin{array}{l}\text { Temper- } \\
\text { ature }\end{array}$ & \\
\hline 10/10/2008 8:00 AM & 0.328 & 13.7 & 0.177 & 0.116 & 15.4 & 0.15 & 0.117 & -- & -- & 0.120 & -- & - & -- \\
\hline 10/10/2008 9:00 AM & 0.328 & 13.7 & 0.177 & 0.117 & 15.4 & 0.15 & 0.117 & -- & -- & 0.120 & -- & -- & -- \\
\hline 10/10/2008 10:00 AM & 0.328 & 13.7 & 0.177 & 0.117 & 15.4 & 0.15 & 0.117 & -- & -- & 0.120 & -- & -- & -- \\
\hline 10/10/2008 11:00 AM & 0.329 & 13.6 & 0.177 & 0.117 & 15.4 & 0.15 & 0.117 & -- & -- & 0.120 & -- & -- & -- \\
\hline 10/10/2008 12:00 PM & 0.329 & 13.7 & 0.177 & 0.117 & 15.4 & 0.15 & 0.117 & -- & -- & 0.120 & -- & -- & -- \\
\hline 10/10/2008 1:00 PM & 0.329 & 13.6 & 0.177 & 0.117 & 15.4 & 0.15 & 0.117 & -- & -- & 0.120 & -- & -- & -- \\
\hline 10/10/2008 2:00 PM & 0.329 & 13.6 & 0.177 & 0.117 & 15.3 & 0.15 & 0.117 & -- & -- & 0.120 & -- & -- & -- \\
\hline 10/10/2008 3:00 PM & 0.329 & 13.5 & 0.177 & 0.117 & 15.3 & 0.15 & 0.117 & -- & -- & 0.120 & -- & -- & -- \\
\hline 10/10/2008 4:00 PM & 0.329 & 13.5 & 0.177 & 0.117 & 15.3 & 0.15 & 0.117 & -- & -- & 0.120 & -- & -- & -- \\
\hline 10/10/2008 5:00 PM & 0.329 & 13.5 & 0.177 & 0.117 & 15.3 & 0.15 & 0.117 & -- & -- & 0.120 & -- & -- & -- \\
\hline 10/10/2008 6:00 PM & 0.329 & 13.5 & 0.177 & 0.117 & 15.3 & 0.15 & 0.117 & -- & -- & 0.120 & -- & -- & -- \\
\hline 10/10/2008 7:00 PM & 0.329 & 13.5 & 0.177 & 0.117 & 15.3 & 0.15 & 0.117 & -- & -- & 0.120 & -- & -- & -- \\
\hline 10/10/2008 8:00 PM & 0.329 & 13.5 & 0.177 & 0.117 & 15.3 & 0.15 & 0.117 & -- & -- & 0.120 & -- & -- & -- \\
\hline 10/10/2008 9:00 PM & 0.328 & 13.5 & 0.177 & 0.117 & 15.3 & 0.15 & 0.117 & -- & -- & 0.120 & -- & -- & -- \\
\hline 10/10/2008 10:00 PM & 0.329 & 13.4 & 0.177 & 0.117 & 15.3 & 0.15 & 0.117 & -- & -- & 0.120 & -- & -- & -- \\
\hline 10/10/2008 11:00 PM & 0.328 & 13.5 & 0.177 & 0.116 & 15.3 & 0.15 & 0.117 & -- & -- & 0.120 & -- & -- & -- \\
\hline 10/11/2008 12:00 AM & 0.328 & 13.4 & 0.177 & 0.117 & 15.3 & 0.15 & 0.117 & -- & -- & 0.120 & -- & -- & -- \\
\hline 10/11/2008 1:00 AM & 0.328 & 13.4 & 0.177 & 0.117 & 15.3 & 0.15 & 0.117 & -- & -- & 0.120 & -- & -- & -- \\
\hline 10/11/2008 2:00 AM & 0.328 & 13.4 & 0.176 & 0.117 & 15.3 & 0.15 & 0.117 & -- & -- & 0.120 & -- & -- & -- \\
\hline 10/11/2008 3:00 AM & 0.328 & 13.4 & 0.176 & 0.117 & 15.3 & 0.15 & 0.117 & -- & -- & 0.120 & -- & -- & -- \\
\hline 10/11/2008 4:00 AM & 0.328 & 13.4 & 0.176 & 0.116 & 15.3 & 0.15 & 0.117 & -- & -- & 0.120 & -- & -- & -- \\
\hline 10/11/2008 5:00 AM & 0.328 & 13.4 & 0.176 & 0.117 & 15.3 & 0.15 & 0.117 & -- & -- & 0.120 & -- & -- & -- \\
\hline 10/11/2008 6:00 AM & 0.328 & 13.4 & 0.176 & 0.117 & 15.3 & 0.15 & 0.117 & -- & -- & 0.120 & -- & -- & -- \\
\hline 10/11/2008 7:00 AM & 0.328 & 13.4 & 0.177 & 0.116 & 15.3 & 0.15 & 0.117 & -- & -- & 0.128 & -- & -- & -- \\
\hline 10/11/2008 8:00 AM & 0.326 & 13.4 & 0.176 & 0.117 & 15.3 & 0.15 & 0.117 & -- & -- & 0.125 & -- & -- & -- \\
\hline 10/11/2008 9:00 AM & 0.326 & 13.4 & 0.177 & 0.117 & 15.3 & 0.15 & 0.117 & -- & -- & 0.118 & -- & -- & -- \\
\hline 10/11/2008 10:00 AM & 0.326 & 13.3 & 0.177 & 0.116 & 15.2 & 0.15 & 0.117 & -- & -- & 0.114 & -- & -- & -- \\
\hline 10/11/2008 11:00 AM & 0.326 & 13.3 & 0.177 & 0.116 & 15.2 & 0.15 & 0.117 & -- & -- & 0.114 & -- & -- & -- \\
\hline 10/11/2008 12:00 PM & 0.326 & 13.3 & 0.177 & 0.117 & 15.2 & 0.15 & 0.117 & -- & -- & 0.112 & -- & -- & -- \\
\hline 10/11/2008 1:00 PM & 0.326 & 13.3 & 0.177 & 0.117 & 15.2 & 0.15 & 0.117 & -- & -- & 0.115 & -- & -- & -- \\
\hline 10/11/2008 2:00 PM & 0.326 & 13.2 & 0.177 & 0.116 & 15.2 & 0.15 & 0.117 & -- & -- & 0.110 & -- & -- & -- \\
\hline 10/11/2008 3:00 PM & 0.326 & 13.2 & 0.177 & 0.116 & 15.2 & 0.15 & 0.117 & -- & -- & 0.105 & -- & -- & -- \\
\hline 10/11/2008 4:00 PM & 0.326 & 13.2 & 0.177 & 0.116 & 15.2 & 0.15 & 0.117 & -- & -- & 0.101 & -- & -- & -- \\
\hline 10/11/2008 5:00 PM & 0.326 & 13.2 & 0.177 & 0.117 & 15.2 & 0.15 & 0.117 & -- & -- & 0.098 & -- & -- & -- \\
\hline 10/11/2008 6:00 PM & 0.326 & 13.1 & 0.176 & 0.117 & 15.2 & 0.15 & 0.117 & -- & -- & 0.105 & -- & -- & -- \\
\hline
\end{tabular}


Appendix 3. Volumetric water content, temperature, and electrical-conductivity data collected at the sprinkler-irrigated site during 2008.-Continued

[Depth in feet below land surface; volumetric water content dimensionless; temperature in degrees Celsius; bulk electrical conductivity in decisiemens per meter; --, no data]

\begin{tabular}{|c|c|c|c|c|c|c|c|c|c|c|c|c|c|}
\hline \multirow[b]{3}{*}{$\begin{array}{l}\text { Measurement } \\
\text { date and time }\end{array}$} & \multicolumn{13}{|c|}{ Depth } \\
\hline & \multicolumn{2}{|c|}{2} & \multirow{2}{*}{$\begin{array}{c}3 \\
\text { Volumetric } \\
\text { water } \\
\text { content }\end{array}$} & \multicolumn{3}{|c|}{4} & \multirow{2}{*}{$\begin{array}{c}5 \\
\text { Volumetric } \\
\text { water } \\
\text { content }\end{array}$} & \multicolumn{2}{|l|}{7} & \multirow{2}{*}{$\begin{array}{c}9 \\
\text { Volumetric } \\
\text { water } \\
\text { content }\end{array}$} & \multicolumn{2}{|c|}{13} & \multirow{2}{*}{$\begin{array}{c}16.5 \\
\text { Volumetric } \\
\text { water } \\
\text { content }\end{array}$} \\
\hline & $\begin{array}{c}\text { Volumetric } \\
\text { water } \\
\text { content }\end{array}$ & $\begin{array}{l}\text { Temper- } \\
\text { ature }\end{array}$ & & $\begin{array}{c}\text { Volumetric } \\
\text { water } \\
\text { content }\end{array}$ & $\begin{array}{l}\text { Temper- } \\
\text { ature }\end{array}$ & $\begin{array}{c}\text { Bulk } \\
\text { electrical } \\
\text { conduct- } \\
\text { ivity }\end{array}$ & & $\begin{array}{c}\text { Volumetric } \\
\text { water } \\
\text { content }\end{array}$ & $\begin{array}{l}\text { Temper- } \\
\text { ature }\end{array}$ & & $\begin{array}{c}\text { Volumetric } \\
\text { water } \\
\text { content }\end{array}$ & $\begin{array}{l}\text { Temper- } \\
\text { ature }\end{array}$ & \\
\hline 10/11/2008 7:00 PM & 0.326 & 13.1 & 0.176 & 0.117 & 15.2 & 0.14 & 0.117 & -- & -- & 0.106 & -- & -- & -- \\
\hline 10/11/2008 8:00 PM & 0.326 & 13.1 & 0.176 & 0.117 & 15.2 & 0.14 & 0.117 & -- & -- & 0.108 & -- & -- & -- \\
\hline 10/11/2008 9:00 PM & 0.326 & 13.1 & 0.176 & 0.117 & 15.2 & 0.14 & 0.117 & -- & -- & 0.111 & -- & -- & -- \\
\hline 10/11/2008 10:00 PM & 0.326 & 13.1 & 0.176 & 0.117 & 15.2 & 0.14 & 0.117 & -- & -- & 0.111 & -- & -- & -- \\
\hline 10/11/2008 11:00 PM & 0.326 & 13.1 & 0.176 & 0.116 & 15.2 & 0.14 & 0.117 & -- & -- & 0.114 & -- & -- & -- \\
\hline 10/12/2008 12:00 AM & 0.326 & 13.0 & 0.176 & 0.117 & 15.2 & 0.14 & 0.117 & -- & -- & 0.116 & -- & -- & -- \\
\hline 10/12/2008 1:00 AM & 0.328 & 13.0 & 0.176 & 0.117 & 15.2 & 0.14 & 0.117 & -- & -- & 0.116 & -- & -- & -- \\
\hline 10/12/2008 2:00 AM & 0.328 & 13.0 & 0.176 & 0.117 & 15.1 & 0.14 & 0.117 & -- & -- & 0.115 & -- & -- & -- \\
\hline 10/12/2008 3:00 AM & 0.329 & 13.0 & 0.176 & 0.117 & 15.1 & 0.14 & 0.117 & -- & -- & 0.114 & -- & -- & -- \\
\hline 10/12/2008 4:00 AM & 0.329 & 12.9 & 0.176 & 0.116 & 15.2 & 0.14 & 0.117 & -- & -- & 0.115 & -- & -- & -- \\
\hline 10/12/2008 5:00 AM & 0.329 & 12.9 & 0.176 & 0.116 & 15.2 & 0.14 & 0.117 & -- & -- & 0.115 & -- & -- & -- \\
\hline 10/12/2008 6:00 AM & 0.330 & 12.9 & 0.176 & 0.116 & 15.1 & 0.14 & 0.117 & -- & -- & 0.116 & -- & -- & -- \\
\hline 10/12/2008 7:00 AM & 0.330 & 12.9 & 0.176 & 0.116 & 15.1 & 0.14 & 0.117 & -- & -- & 0.116 & -- & -- & -- \\
\hline 10/12/2008 8:00 AM & 0.330 & 12.8 & 0.176 & 0.116 & 15.1 & 0.14 & 0.117 & -- & -- & 0.116 & -- & -- & -- \\
\hline 10/12/2008 9:00 AM & 0.330 & 12.8 & 0.176 & 0.116 & 15.1 & 0.14 & 0.117 & -- & -- & 0.115 & -- & -- & -- \\
\hline 10/12/2008 10:00 AM & 0.330 & 12.8 & 0.176 & 0.116 & 15.1 & 0.14 & 0.117 & -- & -- & 0.116 & -- & -- & -- \\
\hline 10/12/2008 11:00 AM & 0.329 & 12.8 & 0.177 & 0.116 & 15.1 & 0.14 & 0.116 & -- & -- & 0.116 & -- & -- & -- \\
\hline 10/12/2008 12:00 PM & 0.330 & 12.8 & 0.176 & 0.116 & 15.1 & 0.14 & 0.116 & -- & -- & 0.117 & -- & -- & -- \\
\hline 10/12/2008 1:00 PM & 0.329 & 12.7 & 0.176 & 0.116 & 15.1 & 0.14 & 0.117 & -- & -- & 0.119 & -- & -- & -- \\
\hline 10/12/2008 2:00 PM & 0.329 & 12.7 & 0.177 & 0.116 & 15.1 & 0.14 & 0.116 & -- & -- & 0.119 & -- & -- & -- \\
\hline 10/12/2008 3:00 PM & 0.329 & 12.7 & 0.177 & 0.116 & 15.1 & 0.14 & 0.116 & -- & -- & 0.121 & -- & -- & -- \\
\hline 10/12/2008 4:00 PM & 0.329 & 12.7 & 0.176 & 0.116 & 15.1 & 0.14 & 0.116 & -- & -- & 0.121 & -- & -- & -- \\
\hline 10/12/2008 5:00 PM & 0.328 & 12.6 & 0.176 & 0.116 & 15.1 & 0.14 & 0.116 & -- & -- & 0.121 & -- & -- & -- \\
\hline 10/12/2008 6:00 PM & 0.328 & 12.6 & 0.176 & 0.116 & 15.1 & 0.14 & 0.116 & -- & -- & 0.119 & -- & -- & -- \\
\hline 10/12/2008 7:00 PM & 0.328 & 12.6 & 0.176 & 0.116 & 15.0 & 0.14 & 0.116 & -- & -- & 0.120 & -- & -- & -- \\
\hline 10/12/2008 8:00 PM & 0.328 & 12.6 & 0.176 & 0.116 & 15.0 & 0.14 & 0.116 & -- & -- & 0.115 & -- & -- & -- \\
\hline 10/12/2008 9:00 PM & 0.328 & 12.6 & 0.176 & 0.116 & 15.0 & 0.14 & 0.117 & -- & -- & 0.113 & -- & -- & -- \\
\hline 10/12/2008 10:00 PM & 0.328 & 12.6 & 0.176 & 0.116 & 15.0 & 0.14 & 0.117 & -- & -- & 0.111 & -- & -- & -- \\
\hline 10/12/2008 11:00 PM & 0.326 & 12.5 & 0.176 & 0.116 & 15.0 & 0.14 & 0.116 & -- & -- & 0.109 & -- & -- & -- \\
\hline 10/13/2008 12:00 AM & 0.328 & 12.5 & 0.176 & 0.116 & 15.0 & 0.14 & 0.116 & -- & -- & 0.107 & -- & -- & -- \\
\hline 10/13/2008 1:00 AM & 0.326 & 12.5 & 0.176 & 0.116 & 15.0 & 0.14 & 0.116 & -- & -- & 0.106 & -- & -- & -- \\
\hline 10/13/2008 2:00 AM & 0.328 & 12.5 & 0.176 & 0.116 & 15.0 & 0.14 & 0.116 & -- & -- & 0.105 & -- & -- & -- \\
\hline 10/13/2008 3:00 AM & 0.328 & 12.5 & 0.176 & 0.116 & 15.0 & 0.14 & 0.116 & -- & -- & 0.106 & -- & -- & -- \\
\hline 10/13/2008 4:00 AM & 0.328 & 12.5 & 0.176 & 0.116 & 15.0 & 0.14 & 0.116 & -- & -- & 0.109 & -- & -- & -- \\
\hline 10/13/2008 5:00 AM & 0.328 & 12.5 & 0.176 & 0.116 & 15.0 & 0.14 & 0.116 & -- & -- & 0.109 & -- & -- & -- \\
\hline
\end{tabular}


Appendix 3. Volumetric water content, temperature, and electrical-conductivity data collected at the sprinkler-irrigated site during 2008.-Continued

[Depth in feet below land surface; volumetric water content dimensionless; temperature in degrees Celsius; bulk electrical conductivity in decisiemens per meter; --, no data]

\begin{tabular}{|c|c|c|c|c|c|c|c|c|c|c|c|c|c|}
\hline \multirow{3}{*}{$\begin{array}{l}\text { Measurement } \\
\text { date and time }\end{array}$} & \multicolumn{13}{|c|}{ Depth } \\
\hline & \multicolumn{2}{|c|}{2} & \multirow{2}{*}{$\begin{array}{c}3 \\
\begin{array}{c}\text { Volumetric } \\
\text { water } \\
\text { content }\end{array}\end{array}$} & \multicolumn{3}{|c|}{4} & \multirow{2}{*}{$\begin{array}{c}\mathbf{5} \\
\text { Volumetric } \\
\text { water } \\
\text { content }\end{array}$} & \multicolumn{2}{|l|}{7} & \multirow{2}{*}{$\begin{array}{c}9 \\
\text { Volumetric } \\
\text { water } \\
\text { content }\end{array}$} & \multicolumn{2}{|c|}{13} & \multirow{2}{*}{$\begin{array}{c}16.5 \\
\text { Volumetric } \\
\text { water } \\
\text { content }\end{array}$} \\
\hline & $\begin{array}{c}\text { Volumetric } \\
\text { water } \\
\text { content }\end{array}$ & $\begin{array}{l}\text { Temper- } \\
\text { ature }\end{array}$ & & $\begin{array}{l}\text { Volumetric } \\
\text { water } \\
\text { content }\end{array}$ & $\begin{array}{l}\text { Temper- } \\
\text { ature }\end{array}$ & $\begin{array}{l}\text { Bulk } \\
\text { electrical } \\
\text { conduct- } \\
\quad \text { ivity }\end{array}$ & & $\begin{array}{l}\text { Volumetric } \\
\text { water } \\
\text { content }\end{array}$ & $\begin{array}{l}\text { Temper- } \\
\text { ature }\end{array}$ & & $\begin{array}{l}\text { Volumetric } \\
\text { water } \\
\text { content }\end{array}$ & $\begin{array}{l}\text { Temper- } \\
\text { ature }\end{array}$ & \\
\hline 10/13/2008 6:00 AM & 0.326 & 12.4 & 0.176 & 0.116 & 15.0 & 0.14 & 0.116 & $\overline{--}$ & -- & 0.109 & -- & -- & $\overline{--}$ \\
\hline 10/13/2008 7:00 AM & 0.328 & 12.4 & 0.176 & 0.116 & 15.0 & 0.14 & 0.116 & -- & -- & 0.109 & -- & -- & -- \\
\hline 10/13/2008 8:00 AM & 0.328 & 12.4 & 0.176 & 0.116 & 15.0 & 0.14 & 0.116 & -- & -- & 0.111 & -- & -- & -- \\
\hline 10/13/2008 9:00 AM & 0.328 & 12.4 & 0.176 & 0.116 & 14.9 & 0.14 & 0.116 & -- & -- & 0.112 & -- & -- & -- \\
\hline 10/13/2008 10:00 AM & 0.328 & 12.3 & 0.176 & 0.116 & 14.9 & 0.14 & 0.116 & -- & -- & 0.111 & -- & -- & -- \\
\hline 10/13/2008 11:00 AM & 0.326 & 12.3 & 0.176 & 0.116 & 14.9 & 0.14 & 0.116 & -- & -- & 0.112 & -- & -- & -- \\
\hline 10/13/2008 12:00 PM & 0.325 & 12.3 & 0.176 & 0.116 & 14.9 & 0.14 & 0.116 & -- & -- & 0.115 & -- & -- & -- \\
\hline 10/13/2008 1:00 PM & 0.325 & 12.2 & 0.176 & 0.116 & 14.9 & 0.14 & 0.116 & -- & -- & 0.119 & -- & -- & -- \\
\hline 10/13/2008 2:00 PM & 0.325 & 12.2 & 0.176 & 0.116 & 14.9 & 0.14 & 0.116 & -- & -- & 0.117 & -- & -- & -- \\
\hline 10/13/2008 3:00 PM & 0.325 & 12.2 & 0.176 & 0.116 & 14.9 & 0.14 & 0.116 & -- & -- & 0.119 & -- & -- & -- \\
\hline 10/13/2008 4:00 PM & 0.326 & 12.1 & 0.176 & 0.116 & 14.9 & 0.14 & 0.116 & -- & -- & 0.117 & -- & -- & -- \\
\hline 10/13/2008 5:00 PM & 0.326 & 12.1 & 0.176 & 0.116 & 14.9 & 0.14 & 0.116 & -- & -- & 0.118 & -- & -- & -- \\
\hline 10/13/2008 6:00 PM & 0.328 & 12.1 & 0.176 & 0.116 & 14.9 & 0.14 & 0.116 & -- & -- & 0.118 & -- & -- & -- \\
\hline 10/13/2008 7:00 PM & 0.328 & 12.1 & 0.175 & 0.116 & 14.9 & 0.14 & 0.116 & -- & -- & 0.118 & -- & -- & -- \\
\hline 10/13/2008 8:00 PM & 0.329 & 12.0 & 0.175 & 0.116 & 14.8 & 0.14 & 0.116 & -- & -- & 0.118 & -- & -- & -- \\
\hline 10/13/2008 9:00 PM & 0.328 & 12.0 & 0.176 & 0.116 & 14.8 & 0.14 & 0.116 & -- & -- & 0.117 & -- & -- & -- \\
\hline 10/13/2008 10:00 PM & 0.328 & 12.0 & 0.176 & 0.116 & 14.8 & 0.14 & 0.116 & -- & -- & 0.116 & -- & -- & -- \\
\hline 10/13/2008 11:00 PM & 0.328 & 12.0 & 0.176 & 0.116 & 14.8 & 0.14 & 0.116 & -- & -- & 0.117 & -- & -- & -- \\
\hline 10/14/2008 12:00 AM & 0.326 & 12.0 & 0.176 & 0.116 & 14.8 & 0.14 & 0.116 & -- & -- & 0.119 & -- & -- & -- \\
\hline 10/14/2008 1:00 AM & 0.328 & 11.9 & 0.176 & 0.116 & 14.8 & 0.14 & 0.116 & -- & -- & 0.119 & -- & -- & -- \\
\hline 10/14/2008 2:00 AM & 0.326 & 11.9 & 0.176 & 0.116 & 14.8 & 0.14 & 0.116 & -- & -- & 0.119 & -- & -- & -- \\
\hline 10/14/2008 3:00 AM & 0.326 & 11.9 & 0.176 & 0.116 & 14.8 & 0.14 & 0.116 & -- & -- & 0.118 & -- & -- & -- \\
\hline 10/14/2008 4:00 AM & 0.328 & 11.9 & 0.176 & 0.116 & 14.8 & 0.14 & 0.116 & -- & -- & 0.117 & -- & -- & -- \\
\hline 10/14/2008 5:00 AM & 0.326 & 11.9 & 0.176 & 0.116 & 14.8 & 0.14 & 0.116 & -- & -- & 0.117 & -- & -- & -- \\
\hline 10/14/2008 6:00 AM & 0.326 & 11.9 & 0.176 & 0.116 & 14.8 & 0.14 & 0.116 & -- & -- & 0.118 & -- & -- & -- \\
\hline 10/14/2008 7:00 AM & 0.326 & 11.8 & 0.176 & 0.116 & 14.8 & 0.14 & 0.116 & -- & -- & 0.118 & -- & -- & -- \\
\hline 10/14/2008 8:00 AM & 0.326 & 11.8 & 0.176 & 0.116 & 14.7 & 0.14 & 0.116 & -- & -- & 0.118 & -- & -- & -- \\
\hline 10/14/2008 9:00 AM & 0.326 & 11.8 & 0.176 & 0.116 & 14.7 & 0.14 & 0.116 & -- & -- & 0.117 & -- & -- & -- \\
\hline $10 / 14 / 2008$ 10:00 AM & 0.326 & 11.8 & 0.176 & 0.116 & 14.7 & 0.14 & 0.116 & -- & -- & 0.115 & -- & -- & -- \\
\hline $10 / 14 / 2008$ 11:00 AM & 0.326 & 11.7 & 0.175 & 0.116 & 14.7 & 0.14 & 0.116 & -- & -- & 0.116 & -- & -- & -- \\
\hline 10/14/2008 12:00 PM & 0.326 & 11.7 & 0.176 & 0.116 & 14.7 & 0.14 & 0.116 & -- & -- & 0.118 & -- & -- & -- \\
\hline 10/14/2008 1:00 PM & 0.326 & 11.7 & 0.176 & 0.116 & 14.7 & 0.14 & 0.116 & -- & -- & 0.118 & -- & -- & -- \\
\hline $10 / 14 / 2008$ 2:00 PM & 0.326 & 11.7 & 0.176 & 0.116 & 14.7 & 0.14 & 0.116 & -- & -- & 0.116 & -- & -- & -- \\
\hline 10/14/2008 3:00 PM & 0.326 & 11.6 & 0.176 & 0.116 & 14.7 & 0.14 & 0.116 & -- & -- & 0.117 & -- & -- & -- \\
\hline 10/14/2008 4:00 PM & 0.326 & 11.6 & 0.176 & 0.116 & 14.7 & 0.14 & 0.116 & -- & -- & 0.117 & -- & -- & -- \\
\hline
\end{tabular}


Appendix 3. Volumetric water content, temperature, and electrical-conductivity data collected at the sprinkler-irrigated site during 2008.-Continued

[Depth in feet below land surface; volumetric water content dimensionless; temperature in degrees Celsius; bulk electrical conductivity in decisiemens per meter; --, no data]

\begin{tabular}{|c|c|c|c|c|c|c|c|c|c|c|c|c|c|}
\hline \multirow[b]{3}{*}{$\begin{array}{l}\text { Measurement } \\
\text { date and time }\end{array}$} & \multicolumn{13}{|c|}{ Depth } \\
\hline & \multicolumn{2}{|c|}{2} & \multirow{2}{*}{$\begin{array}{c}3 \\
\text { Volumetric } \\
\text { water } \\
\text { content }\end{array}$} & \multicolumn{3}{|c|}{4} & \multirow{2}{*}{$\begin{array}{c}5 \\
\text { Volumetric } \\
\text { water } \\
\text { content }\end{array}$} & \multicolumn{2}{|l|}{7} & \multirow{2}{*}{$\begin{array}{c}9 \\
\text { Volumetric } \\
\text { water } \\
\text { content }\end{array}$} & \multicolumn{2}{|c|}{13} & \multirow{2}{*}{$\begin{array}{c}16.5 \\
\text { Volumetric } \\
\text { water } \\
\text { content }\end{array}$} \\
\hline & $\begin{array}{c}\text { Volumetric } \\
\text { water } \\
\text { content }\end{array}$ & $\begin{array}{l}\text { Temper- } \\
\text { ature }\end{array}$ & & $\begin{array}{c}\text { Volumetric } \\
\text { water } \\
\text { content }\end{array}$ & $\begin{array}{l}\text { Temper- } \\
\text { ature }\end{array}$ & $\begin{array}{c}\text { Bulk } \\
\text { electrical } \\
\text { conduct- } \\
\text { ivity }\end{array}$ & & $\begin{array}{c}\text { Volumetric } \\
\text { water } \\
\text { content }\end{array}$ & $\begin{array}{l}\text { Temper- } \\
\text { ature }\end{array}$ & & $\begin{array}{c}\text { Volumetric } \\
\text { water } \\
\text { content }\end{array}$ & $\begin{array}{l}\text { Temper- } \\
\text { ature }\end{array}$ & \\
\hline 10/14/2008 5:00 PM & 0.326 & 11.6 & 0.176 & 0.116 & 14.7 & 0.14 & 0.116 & -- & -- & 0.117 & -- & -- & -- \\
\hline 10/14/2008 6:00 PM & 0.326 & 11.5 & 0.175 & 0.116 & 14.6 & 0.14 & 0.116 & -- & -- & 0.117 & -- & -- & -- \\
\hline 10/14/2008 7:00 PM & 0.328 & 11.5 & 0.175 & 0.116 & 14.6 & 0.14 & 0.116 & -- & -- & 0.117 & -- & -- & -- \\
\hline 10/14/2008 8:00 PM & 0.328 & 11.5 & 0.175 & 0.116 & 14.6 & 0.14 & 0.116 & -- & -- & 0.117 & -- & -- & -- \\
\hline 10/14/2008 9:00 PM & 0.328 & 11.4 & 0.175 & 0.115 & 14.6 & 0.14 & 0.116 & -- & -- & 0.116 & -- & -- & -- \\
\hline 10/14/2008 10:00 PM & 0.329 & 11.4 & 0.175 & 0.116 & 14.6 & 0.14 & 0.116 & -- & -- & 0.116 & -- & -- & -- \\
\hline 10/14/2008 11:00 PM & 0.328 & 11.4 & 0.175 & 0.116 & 14.6 & 0.14 & 0.116 & -- & -- & 0.116 & -- & -- & -- \\
\hline 10/15/2008 12:00 AM & 0.328 & 11.4 & 0.175 & 0.116 & 14.6 & 0.14 & 0.116 & -- & -- & 0.115 & -- & -- & -- \\
\hline 10/15/2008 1:00 AM & 0.328 & 11.4 & 0.175 & 0.116 & 14.6 & 0.14 & 0.115 & -- & -- & 0.115 & -- & -- & -- \\
\hline 10/15/2008 2:00 AM & 0.328 & 11.4 & 0.175 & 0.115 & 14.6 & 0.14 & 0.116 & -- & -- & 0.115 & -- & -- & -- \\
\hline 10/15/2008 3:00 AM & 0.328 & 11.4 & 0.175 & 0.116 & 14.6 & 0.14 & 0.116 & -- & -- & 0.114 & -- & -- & -- \\
\hline 10/15/2008 4:00 AM & 0.328 & 11.4 & 0.175 & 0.116 & 14.6 & 0.14 & 0.116 & -- & -- & 0.114 & -- & -- & -- \\
\hline 10/15/2008 5:00 AM & 0.328 & 11.3 & 0.175 & 0.115 & 14.5 & 0.14 & 0.115 & -- & -- & 0.113 & -- & -- & -- \\
\hline 10/15/2008 6:00 AM & 0.329 & 11.3 & 0.175 & 0.115 & 14.5 & 0.14 & 0.115 & -- & -- & 0.112 & -- & -- & -- \\
\hline 10/15/2008 7:00 AM & 0.329 & 11.3 & 0.175 & 0.116 & 14.5 & 0.14 & 0.115 & -- & -- & 0.111 & -- & -- & -- \\
\hline 10/15/2008 8:00 AM & 0.329 & 11.3 & 0.175 & 0.115 & 14.5 & 0.14 & 0.115 & -- & -- & 0.112 & -- & -- & -- \\
\hline 10/15/2008 9:00 AM & 0.329 & 11.3 & 0.175 & 0.115 & 14.5 & 0.14 & 0.115 & -- & -- & 0.112 & -- & -- & -- \\
\hline 10/15/2008 10:00 AM & 0.329 & 11.3 & 0.175 & 0.116 & 14.5 & 0.14 & 0.116 & -- & -- & 0.113 & -- & -- & -- \\
\hline 10/15/2008 11:00 AM & 0.329 & 11.3 & 0.175 & 0.116 & 14.5 & 0.14 & 0.115 & -- & -- & 0.114 & -- & -- & -- \\
\hline 10/15/2008 12:00 PM & 0.330 & 11.3 & 0.175 & 0.116 & 14.5 & 0.14 & 0.115 & -- & -- & 0.115 & -- & -- & -- \\
\hline 10/15/2008 1:00 PM & 0.331 & 11.3 & 0.175 & 0.115 & 14.5 & 0.14 & 0.116 & -- & -- & 0.115 & -- & -- & -- \\
\hline 10/15/2008 2:00 PM & 0.332 & 11.3 & 0.175 & 0.116 & 14.5 & 0.14 & 0.116 & -- & -- & 0.115 & -- & -- & -- \\
\hline 10/15/2008 3:00 PM & 0.330 & 11.3 & 0.175 & 0.116 & 14.4 & 0.14 & 0.116 & -- & -- & 0.118 & -- & -- & -- \\
\hline 10/15/2008 4:00 PM & 0.330 & 11.3 & 0.175 & 0.115 & 14.4 & 0.14 & 0.116 & -- & -- & 0.119 & -- & -- & -- \\
\hline 10/15/2008 5:00 PM & 0.331 & 11.2 & 0.175 & 0.116 & 14.4 & 0.14 & 0.115 & -- & -- & 0.119 & -- & -- & -- \\
\hline 10/15/2008 6:00 PM & 0.332 & 11.2 & 0.175 & 0.115 & 14.4 & 0.14 & 0.115 & -- & -- & 0.119 & -- & -- & -- \\
\hline 10/15/2008 7:00 PM & 0.331 & 11.2 & 0.175 & 0.115 & 14.4 & 0.14 & 0.115 & -- & -- & 0.119 & -- & -- & -- \\
\hline 10/15/2008 8:00 PM & 0.330 & 11.2 & 0.175 & 0.115 & 14.4 & 0.14 & 0.116 & -- & -- & 0.118 & -- & -- & -- \\
\hline 10/15/2008 9:00 PM & 0.330 & 11.2 & 0.175 & 0.115 & 14.4 & 0.14 & 0.116 & -- & -- & 0.118 & -- & -- & -- \\
\hline 10/15/2008 10:00 PM & 0.329 & 11.2 & 0.175 & 0.115 & 14.4 & 0.14 & 0.116 & -- & -- & 0.118 & -- & -- & -- \\
\hline 10/15/2008 11:00 PM & 0.329 & 11.2 & 0.175 & 0.115 & 14.4 & 0.14 & 0.115 & -- & -- & 0.117 & -- & -- & -- \\
\hline 10/16/2008 12:00 AM & 0.329 & 11.2 & 0.175 & 0.115 & 14.4 & 0.14 & 0.115 & -- & -- & 0.115 & -- & -- & -- \\
\hline 10/16/2008 1:00 AM & 0.329 & 11.2 & 0.175 & 0.115 & 14.3 & 0.14 & 0.115 & -- & -- & 0.115 & -- & -- & -- \\
\hline 10/16/2008 2:00 AM & 0.329 & 11.2 & 0.175 & 0.115 & 14.3 & 0.14 & 0.115 & -- & -- & 0.115 & -- & -- & -- \\
\hline 10/16/2008 3:00 AM & 0.330 & 11.2 & 0.175 & 0.115 & 14.3 & 0.14 & 0.115 & -- & -- & 0.115 & -- & -- & -- \\
\hline
\end{tabular}


Appendix 3. Volumetric water content, temperature, and electrical-conductivity data collected at the sprinkler-irrigated site during 2008.-Continued

[Depth in feet below land surface; volumetric water content dimensionless; temperature in degrees Celsius; bulk electrical conductivity in decisiemens per meter; --, no data]

\begin{tabular}{|c|c|c|c|c|c|c|c|c|c|c|c|c|c|}
\hline \multirow[b]{3}{*}{$\begin{array}{l}\text { Measurement } \\
\text { date and time }\end{array}$} & \multicolumn{13}{|c|}{ Depth } \\
\hline & \multicolumn{2}{|l|}{2} & \multirow{2}{*}{$\begin{array}{c}3 \\
\text { Volumetric } \\
\text { water } \\
\text { content }\end{array}$} & \multicolumn{3}{|c|}{4} & \multirow{2}{*}{$\begin{array}{c}5 \\
\text { Volumetric } \\
\text { water } \\
\text { content }\end{array}$} & \multicolumn{2}{|l|}{7} & \multirow{2}{*}{$\begin{array}{c}9 \\
\text { Volumetric } \\
\text { water } \\
\text { content }\end{array}$} & \multicolumn{2}{|c|}{13} & \multirow{2}{*}{$\begin{array}{c}16.5 \\
\text { Volumetric } \\
\text { water } \\
\text { content }\end{array}$} \\
\hline & $\begin{array}{c}\text { Volumetric } \\
\text { water } \\
\text { content }\end{array}$ & $\begin{array}{l}\text { Temper- } \\
\text { ature }\end{array}$ & & $\begin{array}{c}\text { Volumetric } \\
\text { water } \\
\text { content }\end{array}$ & $\begin{array}{l}\text { Temper- } \\
\text { ature }\end{array}$ & $\begin{array}{c}\text { Bulk } \\
\text { electrical } \\
\text { conduct- } \\
\text { ivity }\end{array}$ & & $\begin{array}{l}\text { Volumetric } \\
\text { water } \\
\text { content }\end{array}$ & $\begin{array}{l}\text { Temper- } \\
\text { ature }\end{array}$ & & $\begin{array}{c}\text { Volumetric } \\
\text { water } \\
\text { content }\end{array}$ & $\begin{array}{l}\text { Temper- } \\
\text { ature }\end{array}$ & \\
\hline 10/16/2008 4:00 AM & 0.330 & 11.2 & 0.175 & 0.115 & 14.3 & 0.14 & 0.115 & -- & -- & 0.115 & -- & -- & -- \\
\hline 10/16/2008 5:00 AM & 0.330 & 11.2 & 0.175 & 0.115 & 14.3 & 0.14 & 0.115 & -- & -- & 0.115 & -- & -- & -- \\
\hline 10/16/2008 6:00 AM & 0.330 & 11.2 & 0.175 & 0.115 & 14.3 & 0.14 & 0.115 & -- & -- & 0.115 & -- & -- & -- \\
\hline 10/16/2008 7:00 AM & 0.330 & 11.2 & 0.174 & 0.115 & 14.3 & 0.14 & 0.115 & -- & -- & 0.115 & -- & -- & -- \\
\hline 10/16/2008 8:00 AM & 0.330 & 11.2 & 0.175 & 0.115 & 14.3 & 0.14 & 0.115 & -- & -- & 0.115 & -- & -- & -- \\
\hline 10/16/2008 9:00 AM & 0.330 & 11.2 & 0.175 & 0.115 & 14.3 & 0.14 & 0.115 & -- & -- & 0.115 & -- & -- & -- \\
\hline 10/16/2008 10:00 AM & 0.330 & 11.2 & 0.175 & 0.116 & 14.2 & 0.14 & 0.115 & -- & -- & 0.115 & -- & -- & -- \\
\hline 10/16/2008 11:00 AM & 0.330 & 11.2 & 0.175 & 0.115 & 14.2 & 0.14 & 0.115 & -- & -- & 0.116 & -- & -- & -- \\
\hline 10/16/2008 12:00 PM & 0.331 & 11.2 & 0.175 & 0.115 & 14.2 & 0.14 & 0.115 & -- & -- & 0.118 & -- & -- & -- \\
\hline 10/16/2008 1:00 PM & 0.331 & 11.2 & 0.175 & 0.115 & 14.2 & 0.14 & 0.115 & -- & -- & 0.119 & -- & -- & -- \\
\hline 10/16/2008 2:00 PM & 0.331 & 11.2 & 0.175 & 0.115 & 14.2 & 0.14 & 0.115 & -- & -- & 0.120 & -- & -- & -- \\
\hline 10/16/2008 3:00 PM & 0.331 & 11.1 & 0.175 & 0.115 & 14.2 & 0.14 & 0.115 & -- & -- & 0.119 & -- & -- & -- \\
\hline 10/16/2008 4:00 PM & 0.330 & 11.1 & 0.175 & 0.115 & 14.2 & 0.14 & 0.115 & -- & -- & 0.119 & -- & -- & -- \\
\hline 10/16/2008 5:00 PM & 0.328 & 11.1 & 0.175 & 0.115 & 14.2 & 0.14 & 0.115 & -- & -- & 0.119 & -- & -- & -- \\
\hline 10/16/2008 6:00 PM & 0.325 & 11.1 & 0.175 & 0.115 & 14.2 & 0.14 & 0.115 & -- & -- & 0.119 & -- & -- & -- \\
\hline 10/16/2008 7:00 PM & 0.324 & 11.1 & 0.175 & 0.115 & 14.2 & 0.14 & 0.115 & -- & -- & 0.119 & -- & -- & -- \\
\hline 10/16/2008 8:00 PM & 0.323 & 11.0 & 0.175 & 0.115 & 14.1 & 0.14 & 0.115 & -- & -- & 0.119 & -- & -- & -- \\
\hline 10/16/2008 9:00 PM & 0.322 & 11.1 & 0.175 & 0.115 & 14.1 & 0.14 & 0.115 & -- & -- & 0.119 & -- & -- & -- \\
\hline 10/16/2008 10:00 PM & 0.321 & 11.1 & 0.175 & 0.115 & 14.1 & 0.14 & 0.115 & -- & -- & 0.119 & -- & -- & -- \\
\hline 10/16/2008 11:00 PM & 0.321 & 11.1 & 0.175 & 0.115 & 14.1 & 0.14 & 0.115 & -- & -- & 0.119 & -- & -- & -- \\
\hline 10/17/2008 12:00 AM & 0.322 & 11.1 & 0.175 & 0.115 & 14.1 & 0.14 & 0.115 & -- & -- & 0.119 & -- & -- & -- \\
\hline 10/17/2008 1:00 AM & 0.323 & 11.1 & 0.174 & 0.115 & 14.1 & 0.14 & 0.115 & -- & -- & 0.119 & -- & -- & -- \\
\hline 10/17/2008 2:00 AM & 0.324 & 11.0 & 0.174 & 0.115 & 14.1 & 0.14 & 0.115 & -- & -- & 0.119 & -- & -- & -- \\
\hline 10/17/2008 3:00 AM & 0.324 & 11.1 & 0.174 & 0.115 & 14.1 & 0.14 & 0.115 & -- & -- & 0.119 & -- & -- & -- \\
\hline 10/17/2008 4:00 AM & 0.325 & 11.1 & 0.174 & 0.115 & 14.1 & 0.14 & 0.115 & -- & -- & 0.119 & -- & -- & -- \\
\hline 10/17/2008 5:00 AM & 0.326 & 11.1 & 0.174 & 0.115 & 14.1 & 0.14 & 0.115 & -- & -- & 0.119 & -- & -- & -- \\
\hline 10/17/2008 6:00 AM & 0.326 & 11.1 & 0.174 & 0.115 & 14.1 & 0.14 & 0.115 & -- & -- & 0.118 & -- & -- & -- \\
\hline 10/17/2008 7:00 AM & 0.328 & 11.2 & 0.174 & 0.115 & 14.0 & 0.14 & 0.115 & -- & -- & 0.118 & -- & -- & -- \\
\hline 10/17/2008 8:00 AM & 0.329 & 11.2 & 0.174 & 0.115 & 14.0 & 0.14 & 0.115 & -- & -- & 0.115 & -- & -- & -- \\
\hline 10/17/2008 9:00 AM & 0.330 & 11.2 & 0.174 & 0.115 & 14.0 & 0.14 & 0.115 & -- & -- & 0.116 & -- & -- & -- \\
\hline 10/17/2008 10:00 AM & 0.331 & 11.2 & 0.174 & 0.115 & 14.0 & 0.14 & 0.115 & -- & -- & 0.117 & -- & -- & -- \\
\hline 10/17/2008 11:00 AM & 0.331 & 11.2 & 0.174 & 0.115 & 14.0 & 0.14 & 0.115 & -- & -- & 0.118 & -- & -- & -- \\
\hline 10/17/2008 12:00 PM & 0.331 & 11.2 & 0.174 & 0.115 & 14.0 & 0.14 & 0.115 & -- & -- & 0.117 & -- & -- & -- \\
\hline 10/17/2008 1:00 PM & 0.331 & 11.2 & 0.175 & 0.115 & 14.0 & 0.14 & 0.115 & -- & -- & 0.119 & -- & -- & -- \\
\hline 10/17/2008 2:00 PM & 0.331 & 11.2 & 0.175 & 0.115 & 14.0 & 0.14 & 0.115 & -- & -- & 0.119 & -- & -- & -- \\
\hline
\end{tabular}


Appendix 3. Volumetric water content, temperature, and electrical-conductivity data collected at the sprinkler-irrigated site during 2008.-Continued

[Depth in feet below land surface; volumetric water content dimensionless; temperature in degrees Celsius; bulk electrical conductivity in decisiemens per meter; --, no data]

\begin{tabular}{|c|c|c|c|c|c|c|c|c|c|c|c|c|c|}
\hline \multirow[b]{3}{*}{$\begin{array}{l}\text { Measurement } \\
\text { date and time }\end{array}$} & \multicolumn{13}{|c|}{ Depth } \\
\hline & \multicolumn{2}{|c|}{2} & \multirow{2}{*}{$\begin{array}{c}3 \\
\text { Volumetric } \\
\text { water } \\
\text { content }\end{array}$} & \multicolumn{3}{|c|}{4} & \multirow{2}{*}{$\begin{array}{c}5 \\
\text { Volumetric } \\
\text { water } \\
\text { content }\end{array}$} & \multicolumn{2}{|l|}{7} & \multirow{2}{*}{$\begin{array}{c}9 \\
\text { Volumetric } \\
\text { water } \\
\text { content }\end{array}$} & \multicolumn{2}{|c|}{13} & \multirow{2}{*}{$\begin{array}{c}16.5 \\
\text { Volumetric } \\
\text { water } \\
\text { content }\end{array}$} \\
\hline & $\begin{array}{c}\text { Volumetric } \\
\text { water } \\
\text { content }\end{array}$ & $\begin{array}{l}\text { Temper- } \\
\text { ature }\end{array}$ & & $\begin{array}{c}\text { Volumetric } \\
\text { water } \\
\text { content }\end{array}$ & $\begin{array}{l}\text { Temper- } \\
\text { ature }\end{array}$ & $\begin{array}{c}\text { Bulk } \\
\text { electrical } \\
\text { conduct- } \\
\text { ivity }\end{array}$ & & $\begin{array}{c}\text { Volumetric } \\
\text { water } \\
\text { content }\end{array}$ & $\begin{array}{l}\text { Temper- } \\
\text { ature }\end{array}$ & & $\begin{array}{c}\text { Volumetric } \\
\text { water } \\
\text { content }\end{array}$ & $\begin{array}{l}\text { Temper- } \\
\text { ature }\end{array}$ & \\
\hline 10/17/2008 3:00 PM & 0.331 & 11.2 & 0.175 & 0.116 & 14.0 & 0.14 & 0.115 & -- & -- & 0.119 & -- & -- & -- \\
\hline 10/17/2008 4:00 PM & 0.331 & 11.2 & 0.175 & 0.115 & 14.0 & 0.14 & 0.115 & -- & -- & 0.119 & -- & -- & -- \\
\hline 10/17/2008 5:00 PM & 0.332 & 11.2 & 0.175 & 0.115 & 14.0 & 0.14 & 0.115 & -- & -- & 0.119 & -- & -- & -- \\
\hline 10/17/2008 6:00 PM & 0.331 & 11.2 & 0.175 & 0.115 & 14.0 & 0.14 & 0.115 & -- & -- & 0.119 & -- & -- & -- \\
\hline 10/17/2008 7:00 PM & 0.331 & 11.2 & 0.174 & 0.115 & 14.0 & 0.14 & 0.115 & -- & -- & 0.119 & -- & -- & -- \\
\hline 10/17/2008 8:00 PM & 0.331 & 11.2 & 0.174 & 0.115 & 13.9 & 0.14 & 0.115 & -- & -- & 0.119 & -- & -- & -- \\
\hline 10/17/2008 9:00 PM & 0.330 & 11.2 & 0.174 & 0.115 & 14.0 & 0.14 & 0.115 & -- & -- & 0.119 & -- & -- & -- \\
\hline 10/17/2008 10:00 PM & 0.331 & 11.2 & 0.174 & 0.115 & 13.9 & 0.14 & 0.115 & -- & -- & 0.118 & -- & -- & -- \\
\hline 10/17/2008 11:00 PM & 0.331 & 11.2 & 0.174 & 0.115 & 13.9 & 0.14 & 0.115 & -- & -- & 0.118 & -- & -- & -- \\
\hline 10/18/2008 12:00 AM & 0.331 & 11.3 & 0.174 & 0.115 & 13.9 & 0.14 & 0.115 & -- & -- & 0.117 & -- & -- & -- \\
\hline 10/18/2008 1:00 AM & 0.332 & 11.3 & 0.174 & 0.115 & 13.9 & 0.14 & 0.115 & -- & -- & 0.115 & -- & -- & -- \\
\hline 10/18/2008 2:00 AM & 0.331 & 11.3 & 0.174 & 0.115 & 13.9 & 0.14 & 0.115 & -- & -- & 0.117 & -- & -- & -- \\
\hline 10/18/2008 3:00 AM & 0.331 & 11.3 & 0.174 & 0.115 & 13.9 & 0.14 & 0.115 & -- & -- & 0.117 & -- & -- & -- \\
\hline 10/18/2008 4:00 AM & 0.331 & 11.3 & 0.174 & 0.115 & 13.9 & 0.14 & 0.115 & -- & -- & 0.117 & -- & -- & -- \\
\hline 10/18/2008 5:00 AM & 0.330 & 11.3 & 0.174 & 0.115 & 13.9 & 0.14 & 0.115 & -- & -- & 0.117 & -- & -- & -- \\
\hline 10/18/2008 6:00 AM & 0.330 & 11.3 & 0.174 & 0.115 & 13.9 & 0.14 & 0.115 & -- & -- & 0.117 & -- & -- & -- \\
\hline 10/18/2008 7:00 AM & 0.329 & 11.3 & 0.174 & 0.115 & 13.9 & 0.14 & 0.115 & -- & -- & 0.116 & -- & -- & -- \\
\hline 10/18/2008 8:00 AM & 0.329 & 11.3 & 0.174 & 0.115 & 13.9 & 0.14 & 0.115 & -- & -- & 0.115 & -- & -- & -- \\
\hline 10/18/2008 9:00 AM & 0.329 & 11.4 & 0.174 & 0.115 & 13.8 & 0.14 & 0.115 & -- & -- & 0.116 & -- & -- & -- \\
\hline 10/18/2008 10:00 AM & 0.328 & 11.4 & 0.174 & 0.115 & 13.9 & 0.14 & 0.115 & -- & -- & 0.117 & -- & -- & -- \\
\hline 10/18/2008 11:00 AM & 0.330 & 11.4 & 0.174 & 0.115 & 13.8 & 0.14 & 0.115 & -- & -- & 0.119 & -- & -- & -- \\
\hline 10/18/2008 12:00 PM & 0.330 & 11.4 & 0.174 & 0.115 & 13.8 & 0.14 & 0.115 & -- & -- & 0.121 & -- & -- & -- \\
\hline 10/18/2008 1:00 PM & 0.330 & 11.4 & 0.174 & 0.115 & 13.8 & 0.14 & 0.115 & -- & -- & 0.121 & -- & -- & -- \\
\hline 10/18/2008 2:00 PM & 0.330 & 11.4 & 0.174 & 0.115 & 13.8 & 0.14 & 0.115 & -- & -- & 0.120 & -- & -- & -- \\
\hline 10/18/2008 3:00 PM & 0.330 & 11.3 & 0.174 & 0.115 & 13.8 & 0.14 & 0.115 & -- & -- & 0.118 & -- & -- & -- \\
\hline 10/18/2008 4:00 PM & 0.331 & 11.3 & 0.174 & 0.115 & 13.8 & 0.14 & 0.115 & -- & -- & 0.119 & -- & -- & -- \\
\hline 10/18/2008 5:00 PM & 0.331 & 11.3 & 0.174 & 0.115 & 13.8 & 0.14 & 0.115 & -- & -- & 0.119 & -- & -- & -- \\
\hline 10/18/2008 6:00 PM & 0.331 & 11.3 & 0.174 & 0.115 & 13.8 & 0.14 & 0.115 & -- & -- & 0.119 & -- & -- & -- \\
\hline 10/18/2008 7:00 PM & 0.330 & 11.3 & 0.174 & 0.115 & 13.8 & 0.14 & 0.115 & -- & -- & 0.118 & -- & -- & -- \\
\hline 10/18/2008 8:00 PM & 0.330 & 11.3 & 0.174 & 0.115 & 13.8 & 0.14 & 0.115 & -- & -- & 0.118 & -- & -- & -- \\
\hline 10/18/2008 9:00 PM & 0.330 & 11.3 & 0.174 & 0.115 & 13.8 & 0.14 & 0.115 & -- & -- & 0.118 & -- & -- & -- \\
\hline 10/18/2008 10:00 PM & 0.330 & 11.3 & 0.174 & 0.113 & 13.8 & 0.14 & 0.115 & -- & -- & 0.118 & -- & -- & -- \\
\hline 10/18/2008 11:00 PM & 0.330 & 11.3 & 0.174 & 0.115 & 13.8 & 0.14 & 0.115 & -- & -- & 0.118 & -- & -- & -- \\
\hline 10/19/2008 12:00 AM & 0.330 & 11.3 & 0.174 & 0.115 & 13.8 & 0.14 & 0.115 & -- & -- & 0.118 & -- & -- & -- \\
\hline 10/19/2008 1:00 AM & 0.329 & 11.3 & 0.174 & 0.115 & 13.8 & 0.14 & 0.115 & -- & -- & 0.117 & -- & -- & -- \\
\hline
\end{tabular}


Appendix 3. Volumetric water content, temperature, and electrical-conductivity data collected at the sprinkler-irrigated site during 2008.-Continued

[Depth in feet below land surface; volumetric water content dimensionless; temperature in degrees Celsius; bulk electrical conductivity in decisiemens per meter; --, no data]

\begin{tabular}{|c|c|c|c|c|c|c|c|c|c|c|c|c|c|}
\hline \multirow[b]{3}{*}{$\begin{array}{l}\text { Measurement } \\
\text { date and time }\end{array}$} & \multicolumn{13}{|c|}{ Depth } \\
\hline & \multicolumn{2}{|l|}{2} & \multirow{2}{*}{$\begin{array}{c}3 \\
\text { Volumetric } \\
\text { water } \\
\text { content }\end{array}$} & \multicolumn{3}{|c|}{4} & \multirow{2}{*}{$\begin{array}{c}5 \\
\text { Volumetric } \\
\text { water } \\
\text { content }\end{array}$} & \multicolumn{2}{|l|}{7} & \multirow{2}{*}{$\begin{array}{c}9 \\
\text { Volumetric } \\
\text { water } \\
\text { content }\end{array}$} & \multicolumn{2}{|c|}{13} & \multirow{2}{*}{$\begin{array}{c}16.5 \\
\text { Volumetric } \\
\text { water } \\
\text { content }\end{array}$} \\
\hline & $\begin{array}{c}\text { Volumetric } \\
\text { water } \\
\text { content }\end{array}$ & $\begin{array}{l}\text { Temper- } \\
\text { ature }\end{array}$ & & $\begin{array}{c}\text { Volumetric } \\
\text { water } \\
\text { content }\end{array}$ & $\begin{array}{l}\text { Temper- } \\
\text { ature }\end{array}$ & $\begin{array}{c}\text { Bulk } \\
\text { electrical } \\
\text { conduct- } \\
\text { ivity }\end{array}$ & & $\begin{array}{l}\text { Volumetric } \\
\text { water } \\
\text { content }\end{array}$ & $\begin{array}{l}\text { Temper- } \\
\text { ature }\end{array}$ & & $\begin{array}{c}\text { Volumetric } \\
\text { water } \\
\text { content }\end{array}$ & $\begin{array}{l}\text { Temper- } \\
\text { ature }\end{array}$ & \\
\hline 10/19/2008 2:00 AM & 0.329 & 11.3 & 0.174 & 0.115 & 13.8 & 0.14 & 0.115 & -- & -- & 0.115 & -- & -- & -- \\
\hline 10/19/2008 3:00 AM & 0.328 & 11.4 & 0.174 & 0.115 & 13.8 & 0.14 & 0.115 & -- & -- & 0.115 & -- & -- & -- \\
\hline 10/19/2008 4:00 AM & 0.328 & 11.4 & 0.174 & 0.115 & 13.8 & 0.14 & 0.115 & -- & -- & 0.117 & -- & -- & -- \\
\hline 10/19/2008 5:00 AM & 0.328 & 11.4 & 0.174 & 0.115 & 13.8 & 0.14 & 0.115 & -- & -- & 0.118 & -- & -- & -- \\
\hline 10/19/2008 6:00 AM & 0.328 & 11.4 & 0.174 & 0.115 & 13.7 & 0.14 & 0.115 & -- & -- & 0.118 & -- & -- & -- \\
\hline 10/19/2008 7:00 AM & 0.328 & 11.4 & 0.174 & 0.113 & 13.7 & 0.14 & 0.115 & -- & -- & 0.118 & -- & -- & -- \\
\hline 10/19/2008 8:00 AM & 0.328 & 11.4 & 0.174 & 0.115 & 13.7 & 0.14 & 0.115 & -- & -- & 0.118 & -- & -- & -- \\
\hline 10/19/2008 9:00 AM & 0.328 & 11.4 & 0.174 & 0.115 & 13.7 & 0.14 & 0.115 & -- & -- & 0.118 & -- & -- & -- \\
\hline 10/19/2008 10:00 AM & 0.328 & 11.4 & 0.174 & 0.115 & 13.7 & 0.14 & 0.115 & -- & -- & 0.119 & -- & -- & -- \\
\hline 10/19/2008 11:00 AM & 0.328 & 11.4 & 0.174 & 0.115 & 13.7 & 0.14 & 0.115 & -- & -- & 0.119 & -- & -- & -- \\
\hline 10/19/2008 12:00 PM & 0.328 & 11.4 & 0.174 & 0.115 & 13.7 & 0.14 & 0.115 & -- & -- & 0.119 & -- & -- & -- \\
\hline 10/19/2008 1:00 PM & 0.328 & 11.5 & 0.174 & 0.115 & 13.7 & 0.14 & 0.115 & -- & -- & 0.118 & -- & -- & -- \\
\hline 10/19/2008 2:00 PM & 0.328 & 11.4 & 0.174 & 0.115 & 13.7 & 0.14 & 0.115 & -- & -- & 0.119 & -- & -- & -- \\
\hline 10/19/2008 3:00 PM & 0.328 & 11.4 & 0.174 & 0.115 & 13.7 & 0.14 & 0.115 & -- & -- & 0.118 & -- & -- & -- \\
\hline 10/19/2008 4:00 PM & 0.328 & 11.4 & 0.174 & 0.115 & 13.7 & 0.14 & 0.115 & -- & -- & 0.118 & -- & -- & -- \\
\hline 10/19/2008 5:00 PM & 0.328 & 11.4 & 0.174 & 0.115 & 13.7 & 0.14 & 0.115 & -- & -- & 0.118 & -- & -- & -- \\
\hline 10/19/2008 6:00 PM & 0.328 & 11.4 & 0.174 & 0.115 & 13.7 & 0.14 & 0.115 & -- & -- & 0.118 & -- & -- & -- \\
\hline 10/19/2008 7:00 PM & 0.329 & 11.4 & 0.174 & 0.115 & 13.7 & 0.14 & 0.115 & -- & -- & 0.118 & -- & -- & -- \\
\hline 10/19/2008 8:00 PM & 0.329 & 11.4 & 0.174 & 0.113 & 13.7 & 0.14 & 0.115 & -- & -- & 0.118 & -- & -- & -- \\
\hline 10/19/2008 9:00 PM & 0.329 & 11.4 & 0.174 & 0.115 & 13.7 & 0.14 & 0.115 & -- & -- & 0.118 & -- & -- & -- \\
\hline 10/19/2008 10:00 PM & 0.329 & 11.4 & 0.174 & 0.115 & 13.7 & 0.14 & 0.115 & -- & -- & 0.118 & -- & -- & -- \\
\hline 10/19/2008 11:00 PM & 0.328 & 11.5 & 0.174 & 0.113 & 13.6 & 0.14 & 0.115 & -- & -- & 0.118 & -- & -- & -- \\
\hline 10/20/2008 12:00 AM & 0.328 & 11.5 & 0.174 & 0.115 & 13.7 & 0.14 & 0.115 & & -- & 0.118 & & -- & \\
\hline 10/20/2008 1:00 AM & 0.328 & 11.5 & 0.174 & 0.113 & 13.7 & 0.14 & 0.115 & -- & -- & 0.118 & -- & -- & -- \\
\hline 10/20/2008 2:00 AM & 0.328 & 11.5 & 0.174 & 0.113 & 13.7 & 0.14 & 0.115 & -- & -- & 0.118 & -- & -- & -- \\
\hline 10/20/2008 3:00 AM & 0.326 & 11.5 & 0.174 & 0.113 & 13.7 & 0.14 & 0.115 & -- & -- & 0.118 & -- & -- & -- \\
\hline 10/20/2008 4:00 AM & 0.326 & 11.5 & 0.174 & 0.113 & 13.6 & 0.14 & 0.115 & -- & -- & 0.118 & -- & -- & -- \\
\hline 10/20/2008 5:00 AM & 0.328 & 11.5 & 0.174 & 0.115 & 13.7 & 0.14 & 0.115 & -- & -- & 0.118 & -- & -- & -- \\
\hline 10/20/2008 6:00 AM & 0.326 & 11.6 & 0.174 & 0.113 & 13.6 & 0.14 & 0.115 & -- & -- & 0.118 & -- & -- & -- \\
\hline 10/20/2008 7:00 AM & 0.326 & 11.6 & 0.174 & 0.113 & 13.6 & 0.14 & 0.115 & -- & -- & 0.118 & -- & -- & -- \\
\hline 10/20/2008 8:00 AM & 0.328 & 11.6 & 0.174 & 0.115 & 13.7 & 0.14 & 0.115 & -- & -- & 0.118 & -- & -- & -- \\
\hline 10/20/2008 9:00 AM & 0.326 & 11.6 & 0.174 & 0.113 & 13.7 & 0.14 & 0.115 & -- & -- & 0.118 & -- & -- & -- \\
\hline 10/20/2008 10:00 AM & 0.326 & 11.6 & 0.174 & 0.115 & 13.6 & 0.14 & 0.115 & -- & -- & 0.118 & -- & -- & -- \\
\hline 10/20/2008 11:00 AM & 0.326 & 11.6 & 0.174 & 0.115 & 13.6 & 0.14 & 0.115 & -- & -- & 0.118 & -- & -- & -- \\
\hline 10/20/2008 12:00 PM & 0.326 & 11.7 & 0.174 & 0.115 & 13.6 & 0.14 & 0.115 & -- & -- & 0.118 & -- & -- & -- \\
\hline
\end{tabular}


Appendix 3. Volumetric water content, temperature, and electrical-conductivity data collected at the sprinkler-irrigated site during 2008.-Continued

[Depth in feet below land surface; volumetric water content dimensionless; temperature in degrees Celsius; bulk electrical conductivity in decisiemens per meter; --, no data]

\begin{tabular}{|c|c|c|c|c|c|c|c|c|c|c|c|c|c|}
\hline \multirow[b]{3}{*}{$\begin{array}{l}\text { Measurement } \\
\text { date and time }\end{array}$} & \multicolumn{13}{|c|}{ Depth } \\
\hline & \multicolumn{2}{|c|}{2} & \multirow{2}{*}{$\begin{array}{c}3 \\
\text { Volumetric } \\
\text { water } \\
\text { content }\end{array}$} & \multicolumn{3}{|c|}{4} & \multirow{2}{*}{$\begin{array}{c}5 \\
\text { Volumetric } \\
\text { water } \\
\text { content }\end{array}$} & \multicolumn{2}{|l|}{7} & \multirow{2}{*}{$\begin{array}{c}9 \\
\text { Volumetric } \\
\text { water } \\
\text { content }\end{array}$} & \multicolumn{2}{|c|}{13} & \multirow{2}{*}{$\begin{array}{c}16.5 \\
\text { Volumetric } \\
\text { water } \\
\text { content }\end{array}$} \\
\hline & $\begin{array}{c}\text { Volumetric } \\
\text { water } \\
\text { content }\end{array}$ & $\begin{array}{l}\text { Temper- } \\
\text { ature }\end{array}$ & & $\begin{array}{c}\text { Volumetric } \\
\text { water } \\
\text { content }\end{array}$ & $\begin{array}{l}\text { Temper- } \\
\text { ature }\end{array}$ & $\begin{array}{c}\text { Bulk } \\
\text { electrical } \\
\text { conduct- } \\
\text { ivity }\end{array}$ & & $\begin{array}{c}\text { Volumetric } \\
\text { water } \\
\text { content }\end{array}$ & $\begin{array}{l}\text { Temper- } \\
\text { ature }\end{array}$ & & $\begin{array}{c}\text { Volumetric } \\
\text { water } \\
\text { content }\end{array}$ & $\begin{array}{l}\text { Temper- } \\
\text { ature }\end{array}$ & \\
\hline 10/20/2008 1:00 PM & 0.326 & 11.7 & 0.174 & 0.115 & 13.6 & 0.14 & 0.115 & -- & -- & 0.118 & -- & -- & -- \\
\hline 10/20/2008 2:00 PM & 0.326 & 11.7 & 0.174 & 0.113 & 13.6 & 0.14 & 0.115 & -- & -- & 0.118 & -- & -- & -- \\
\hline 10/20/2008 3:00 PM & 0.326 & 11.7 & 0.174 & 0.113 & 13.6 & 0.14 & 0.115 & -- & -- & 0.118 & -- & -- & -- \\
\hline 10/20/2008 4:00 PM & 0.326 & 11.7 & 0.174 & 0.113 & 13.6 & 0.14 & 0.115 & -- & -- & 0.118 & -- & -- & -- \\
\hline 10/20/2008 5:00 PM & 0.326 & 11.7 & 0.174 & 0.113 & 13.6 & 0.14 & 0.115 & -- & -- & 0.118 & -- & -- & -- \\
\hline 10/20/2008 6:00 PM & 0.326 & 11.7 & 0.174 & 0.115 & 13.6 & 0.14 & 0.115 & -- & -- & 0.118 & -- & -- & -- \\
\hline 10/20/2008 7:00 PM & 0.326 & 11.7 & 0.174 & 0.113 & 13.6 & 0.14 & 0.115 & -- & -- & 0.118 & -- & -- & -- \\
\hline 10/20/2008 8:00 PM & 0.326 & 11.7 & 0.174 & 0.113 & 13.6 & 0.14 & 0.115 & -- & -- & 0.118 & -- & -- & -- \\
\hline 10/20/2008 9:00 PM & 0.326 & 11.7 & 0.174 & 0.113 & 13.6 & 0.14 & 0.115 & -- & -- & 0.118 & -- & -- & -- \\
\hline 10/20/2008 10:00 PM & 0.326 & 11.7 & 0.174 & 0.113 & 13.6 & 0.14 & 0.115 & -- & -- & 0.118 & -- & -- & -- \\
\hline 10/20/2008 11:00 PM & 0.326 & 11.7 & 0.174 & 0.113 & 13.6 & 0.14 & 0.115 & -- & -- & 0.118 & -- & -- & -- \\
\hline 10/21/2008 12:00 AM & 0.326 & 11.7 & 0.174 & 0.113 & 13.6 & 0.14 & 0.115 & -- & -- & 0.118 & -- & -- & -- \\
\hline 10/21/2008 1:00 AM & 0.326 & 11.7 & 0.174 & 0.113 & 13.6 & 0.14 & 0.115 & -- & -- & 0.118 & -- & -- & -- \\
\hline 10/21/2008 2:00 AM & 0.325 & 11.8 & 0.174 & 0.113 & 13.6 & 0.14 & 0.115 & -- & -- & 0.118 & -- & -- & -- \\
\hline 10/21/2008 3:00 AM & 0.326 & 11.8 & 0.174 & 0.115 & 13.6 & 0.14 & 0.115 & -- & -- & 0.118 & -- & -- & -- \\
\hline 10/21/2008 4:00 AM & 0.326 & 11.8 & 0.174 & 0.115 & 13.6 & 0.14 & 0.115 & -- & -- & 0.118 & -- & -- & -- \\
\hline 10/21/2008 5:00 AM & 0.325 & 11.8 & 0.174 & 0.115 & 13.6 & 0.14 & 0.115 & -- & -- & 0.115 & -- & -- & -- \\
\hline 10/21/2008 6:00 AM & 0.326 & 11.8 & 0.174 & 0.115 & 13.6 & 0.14 & 0.115 & -- & -- & 0.116 & -- & -- & -- \\
\hline 10/21/2008 7:00 AM & 0.326 & 11.8 & 0.174 & 0.113 & 13.6 & 0.14 & 0.115 & -- & -- & 0.116 & -- & -- & -- \\
\hline 10/21/2008 8:00 AM & 0.326 & 11.8 & 0.174 & 0.115 & 13.6 & 0.14 & 0.115 & -- & -- & 0.115 & -- & -- & -- \\
\hline 10/21/2008 9:00 AM & 0.326 & 11.8 & 0.174 & 0.113 & 13.6 & 0.14 & 0.115 & -- & -- & 0.115 & -- & -- & -- \\
\hline 10/21/2008 10:00 AM & 0.326 & 11.8 & 0.174 & 0.115 & 13.6 & 0.14 & 0.115 & -- & -- & 0.115 & -- & -- & -- \\
\hline 10/21/2008 11:00 AM & 0.326 & 11.9 & 0.174 & 0.113 & 13.6 & 0.14 & 0.115 & -- & -- & 0.115 & -- & -- & -- \\
\hline 10/21/2008 12:00 PM & 0.326 & 11.8 & 0.174 & 0.115 & 13.6 & 0.14 & 0.115 & -- & -- & 0.116 & -- & -- & -- \\
\hline 10/21/2008 1:00 PM & 0.326 & 11.9 & 0.174 & 0.113 & 13.6 & 0.14 & 0.115 & -- & -- & 0.117 & -- & -- & -- \\
\hline 10/21/2008 2:00 PM & 0.326 & 11.8 & 0.174 & 0.115 & 13.6 & 0.14 & 0.115 & -- & -- & 0.118 & -- & -- & -- \\
\hline 10/21/2008 3:00 PM & 0.326 & 11.8 & 0.174 & 0.115 & 13.6 & 0.14 & 0.115 & -- & -- & 0.118 & -- & -- & -- \\
\hline 10/21/2008 4:00 PM & 0.326 & 11.8 & 0.174 & 0.115 & 13.6 & 0.14 & 0.115 & -- & -- & 0.121 & -- & -- & -- \\
\hline 10/21/2008 5:00 PM & 0.326 & 11.8 & 0.174 & 0.115 & 13.6 & 0.14 & 0.115 & -- & -- & 0.124 & -- & -- & -- \\
\hline 10/21/2008 6:00 PM & 0.326 & 11.8 & 0.174 & 0.115 & 13.6 & 0.14 & 0.115 & -- & -- & 0.118 & -- & -- & -- \\
\hline $10 / 21 / 2008$ 7:00 PM & 0.325 & 11.8 & 0.174 & 0.113 & 13.6 & 0.14 & 0.115 & -- & -- & 0.113 & -- & -- & -- \\
\hline 10/21/2008 8:00 PM & 0.326 & 11.8 & 0.174 & 0.115 & 13.6 & 0.14 & 0.115 & -- & -- & 0.119 & -- & -- & -- \\
\hline 10/21/2008 9:00 PM & 0.326 & 11.8 & 0.174 & 0.115 & 13.6 & 0.14 & 0.115 & -- & -- & 0.121 & -- & -- & -- \\
\hline 10/21/2008 10:00 PM & 0.326 & 11.8 & 0.174 & 0.113 & 13.6 & 0.14 & 0.115 & -- & -- & 0.123 & -- & -- & -- \\
\hline 10/21/2008 11:00 PM & 0.326 & 11.7 & 0.174 & 0.113 & 13.6 & 0.14 & 0.115 & -- & -- & 0.122 & -- & -- & -- \\
\hline
\end{tabular}


Appendix 3. Volumetric water content, temperature, and electrical-conductivity data collected at the sprinkler-irrigated site during 2008.-Continued

[Depth in feet below land surface; volumetric water content dimensionless; temperature in degrees Celsius; bulk electrical conductivity in decisiemens per meter; --, no data]

\begin{tabular}{|c|c|c|c|c|c|c|c|c|c|c|c|c|c|}
\hline \multirow[b]{3}{*}{$\begin{array}{l}\text { Measurement } \\
\text { date and time }\end{array}$} & \multicolumn{13}{|c|}{ Depth } \\
\hline & \multicolumn{2}{|l|}{2} & \multirow{2}{*}{$\begin{array}{c}3 \\
\text { Volumetric } \\
\text { water } \\
\text { content }\end{array}$} & \multicolumn{3}{|c|}{4} & \multirow{2}{*}{$\begin{array}{c}5 \\
\text { Volumetric } \\
\text { water } \\
\text { content }\end{array}$} & \multicolumn{2}{|l|}{7} & \multirow{2}{*}{$\begin{array}{c}9 \\
\text { Volumetric } \\
\text { water } \\
\text { content }\end{array}$} & \multicolumn{2}{|c|}{13} & \multirow{2}{*}{$\begin{array}{c}16.5 \\
\text { Volumetric } \\
\text { water } \\
\text { content }\end{array}$} \\
\hline & $\begin{array}{c}\text { Volumetric } \\
\text { water } \\
\text { content }\end{array}$ & $\begin{array}{l}\text { Temper- } \\
\text { ature }\end{array}$ & & $\begin{array}{c}\text { Volumetric } \\
\text { water } \\
\text { content }\end{array}$ & $\begin{array}{l}\text { Temper- } \\
\text { ature }\end{array}$ & $\begin{array}{c}\text { Bulk } \\
\text { electrical } \\
\text { conduct- } \\
\text { ivity }\end{array}$ & & $\begin{array}{l}\text { Volumetric } \\
\text { water } \\
\text { content }\end{array}$ & $\begin{array}{l}\text { Temper- } \\
\text { ature }\end{array}$ & & $\begin{array}{c}\text { Volumetric } \\
\text { water } \\
\text { content }\end{array}$ & $\begin{array}{l}\text { Temper- } \\
\text { ature }\end{array}$ & \\
\hline 10/22/2008 12:00 AM & 0.326 & 11.7 & 0.174 & 0.113 & 13.6 & 0.14 & 0.115 & -- & -- & 0.123 & -- & -- & -- \\
\hline 10/22/2008 1:00 AM & 0.326 & 11.7 & 0.174 & 0.113 & 13.6 & 0.14 & 0.115 & -- & -- & 0.123 & -- & -- & -- \\
\hline 10/22/2008 2:00 AM & 0.326 & 11.8 & 0.174 & 0.113 & 13.6 & 0.14 & 0.115 & -- & -- & 0.124 & -- & -- & -- \\
\hline 10/22/2008 3:00 AM & 0.326 & 11.7 & 0.174 & 0.113 & 13.6 & 0.14 & 0.115 & -- & -- & 0.120 & -- & -- & -- \\
\hline 10/22/2008 4:00 AM & 0.326 & 11.7 & 0.174 & 0.113 & 13.6 & 0.14 & 0.115 & -- & -- & 0.119 & -- & -- & -- \\
\hline 10/22/2008 5:00 AM & 0.326 & 11.7 & 0.174 & 0.113 & 13.6 & 0.14 & 0.115 & -- & -- & 0.118 & -- & -- & -- \\
\hline 10/22/2008 6:00 AM & 0.326 & 11.7 & 0.174 & 0.113 & 13.6 & 0.14 & 0.115 & -- & -- & 0.118 & -- & -- & -- \\
\hline 10/22/2008 7:00 AM & 0.326 & 11.7 & 0.174 & 0.113 & 13.6 & 0.14 & 0.115 & -- & -- & 0.117 & -- & -- & -- \\
\hline 10/22/2008 8:00 AM & 0.326 & 11.7 & 0.174 & 0.113 & 13.6 & 0.14 & 0.115 & -- & -- & 0.117 & -- & -- & -- \\
\hline 10/22/2008 9:00 AM & 0.326 & 11.6 & 0.174 & 0.113 & 13.6 & 0.14 & 0.115 & -- & -- & 0.117 & -- & -- & -- \\
\hline 10/22/2008 10:00 AM & 0.328 & 11.6 & 0.174 & 0.115 & 13.6 & 0.14 & 0.115 & -- & -- & 0.117 & -- & -- & -- \\
\hline 10/22/2008 11:00 AM & 0.328 & 11.6 & 0.174 & 0.113 & 13.6 & 0.14 & 0.115 & -- & -- & 0.118 & -- & -- & -- \\
\hline 10/22/2008 12:00 PM & 0.328 & 11.6 & 0.174 & 0.113 & 13.6 & 0.14 & 0.115 & -- & -- & 0.117 & -- & -- & -- \\
\hline 10/22/2008 1:00 PM & 0.326 & 11.6 & 0.174 & 0.115 & 13.6 & 0.14 & 0.115 & -- & -- & 0.118 & -- & -- & -- \\
\hline 10/22/2008 2:00 PM & 0.328 & 11.5 & 0.174 & 0.115 & 13.5 & 0.14 & 0.115 & -- & -- & 0.118 & -- & -- & -- \\
\hline 10/22/2008 3:00 PM & 0.328 & 11.5 & 0.174 & 0.113 & 13.5 & 0.14 & 0.115 & -- & -- & 0.118 & -- & -- & -- \\
\hline 10/22/2008 4:00 PM & 0.328 & 11.5 & 0.174 & 0.113 & 13.6 & 0.14 & 0.115 & -- & -- & 0.118 & -- & -- & -- \\
\hline 10/22/2008 5:00 PM & 0.328 & 11.4 & 0.174 & 0.113 & 13.6 & 0.14 & 0.115 & -- & -- & 0.118 & -- & -- & -- \\
\hline 10/22/2008 6:00 PM & 0.328 & 11.4 & 0.174 & 0.113 & 13.5 & 0.14 & 0.115 & -- & -- & 0.118 & -- & -- & -- \\
\hline 10/22/2008 7:00 PM & 0.328 & 11.4 & 0.174 & 0.113 & 13.6 & 0.14 & 0.115 & -- & -- & 0.118 & -- & -- & -- \\
\hline 10/22/2008 8:00 PM & 0.328 & 11.4 & 0.174 & 0.113 & 13.5 & 0.14 & 0.115 & -- & -- & 0.118 & -- & -- & -- \\
\hline 10/22/2008 9:00 PM & 0.329 & 11.4 & 0.174 & 0.113 & 13.5 & 0.14 & 0.115 & -- & -- & 0.118 & -- & -- & -- \\
\hline 10/22/2008 10:00 PM & 0.329 & 11.3 & 0.174 & 0.113 & 13.6 & 0.14 & 0.114 & -- & -- & 0.118 & -- & -- & -- \\
\hline 10/22/2008 11:00 PM & 0.330 & 11.3 & 0.174 & 0.115 & 13.6 & 0.14 & 0.114 & -- & -- & 0.117 & -- & -- & -- \\
\hline 10/23/2008 12:00 AM & 0.331 & 11.3 & 0.174 & 0.113 & 13.5 & 0.14 & 0.114 & -- & -- & 0.117 & -- & -- & -- \\
\hline 10/23/2008 1:00 AM & 0.332 & 11.3 & 0.174 & 0.113 & 13.5 & 0.14 & 0.114 & -- & -- & 0.117 & -- & -- & -- \\
\hline 10/23/2008 2:00 AM & 0.331 & 11.3 & 0.174 & 0.113 & 13.5 & 0.14 & 0.114 & -- & -- & 0.117 & -- & -- & -- \\
\hline 10/23/2008 3:00 AM & 0.332 & 11.3 & 0.174 & 0.113 & 13.5 & 0.14 & 0.114 & -- & -- & 0.117 & -- & -- & -- \\
\hline 10/23/2008 4:00 AM & 0.331 & 11.2 & 0.174 & 0.113 & 13.5 & 0.14 & 0.114 & -- & -- & 0.117 & -- & -- & -- \\
\hline 10/23/2008 5:00 AM & 0.331 & 11.2 & 0.174 & 0.113 & 13.5 & 0.14 & 0.114 & -- & -- & 0.117 & -- & -- & -- \\
\hline 10/23/2008 6:00 AM & 0.330 & 11.2 & 0.174 & 0.113 & 13.5 & 0.14 & 0.114 & -- & -- & 0.117 & -- & -- & -- \\
\hline 10/23/2008 7:00 AM & 0.329 & 11.2 & 0.174 & 0.113 & 13.5 & 0.14 & 0.114 & -- & -- & 0.117 & -- & -- & -- \\
\hline 10/23/2008 8:00 AM & 0.328 & 11.1 & 0.174 & 0.113 & 13.5 & 0.14 & 0.114 & -- & -- & 0.117 & -- & -- & -- \\
\hline 10/23/2008 9:00 AM & 0.325 & 11.1 & 0.174 & 0.113 & 13.5 & 0.14 & 0.114 & -- & -- & 0.117 & -- & -- & -- \\
\hline 10/23/2008 10:00 AM & 0.324 & 11.0 & 0.174 & 0.113 & 13.5 & 0.14 & 0.115 & -- & -- & 0.117 & -- & -- & -- \\
\hline
\end{tabular}


Appendix 3. Volumetric water content, temperature, and electrical-conductivity data collected at the sprinkler-irrigated site during 2008.-Continued

[Depth in feet below land surface; volumetric water content dimensionless; temperature in degrees Celsius; bulk electrical conductivity in decisiemens per meter; --, no data]

\begin{tabular}{|c|c|c|c|c|c|c|c|c|c|c|c|c|c|}
\hline \multirow[b]{3}{*}{$\begin{array}{l}\text { Measurement } \\
\text { date and time }\end{array}$} & \multicolumn{13}{|c|}{ Depth } \\
\hline & \multicolumn{2}{|c|}{2} & \multirow{2}{*}{$\begin{array}{c}3 \\
\text { Volumetric } \\
\text { water } \\
\text { content }\end{array}$} & \multicolumn{3}{|c|}{4} & \multirow{2}{*}{$\begin{array}{c}5 \\
\text { Volumetric } \\
\text { water } \\
\text { content }\end{array}$} & \multicolumn{2}{|l|}{7} & \multirow{2}{*}{$\begin{array}{c}9 \\
\text { Volumetric } \\
\text { water } \\
\text { content }\end{array}$} & \multicolumn{2}{|c|}{13} & \multirow{2}{*}{$\begin{array}{c}16.5 \\
\text { Volumetric } \\
\text { water } \\
\text { content }\end{array}$} \\
\hline & $\begin{array}{c}\text { Volumetric } \\
\text { water } \\
\text { content }\end{array}$ & $\begin{array}{l}\text { Temper- } \\
\text { ature }\end{array}$ & & $\begin{array}{c}\text { Volumetric } \\
\text { water } \\
\text { content }\end{array}$ & $\begin{array}{l}\text { Temper- } \\
\text { ature }\end{array}$ & $\begin{array}{c}\text { Bulk } \\
\text { electrical } \\
\text { conduct- } \\
\text { ivity }\end{array}$ & & $\begin{array}{c}\text { Volumetric } \\
\text { water } \\
\text { content }\end{array}$ & $\begin{array}{l}\text { Temper- } \\
\text { ature }\end{array}$ & & $\begin{array}{c}\text { Volumetric } \\
\text { water } \\
\text { content }\end{array}$ & $\begin{array}{l}\text { Temper- } \\
\text { ature }\end{array}$ & \\
\hline 10/23/2008 11:00 AM & 0.323 & 11.0 & 0.174 & 0.113 & 13.5 & 0.14 & 0.115 & -- & -- & 0.117 & -- & -- & -- \\
\hline 10/23/2008 12:00 PM & 0.323 & 11.0 & 0.174 & 0.113 & 13.5 & 0.14 & 0.115 & -- & -- & 0.118 & -- & -- & -- \\
\hline 10/23/2008 1:00 PM & 0.322 & 11.0 & 0.174 & 0.113 & 13.5 & 0.14 & 0.115 & -- & -- & 0.118 & -- & -- & -- \\
\hline 10/23/2008 2:00 PM & 0.320 & 10.9 & 0.174 & 0.115 & 13.5 & 0.14 & 0.115 & -- & -- & 0.118 & -- & -- & -- \\
\hline 10/23/2008 3:00 PM & 0.318 & 10.9 & 0.174 & 0.113 & 13.5 & 0.14 & 0.115 & -- & -- & 0.118 & -- & -- & -- \\
\hline 10/23/2008 4:00 PM & 0.315 & 10.8 & 0.174 & 0.115 & 13.5 & 0.14 & 0.115 & -- & -- & 0.118 & -- & -- & -- \\
\hline 10/23/2008 5:00 PM & 0.309 & 10.8 & 0.174 & 0.113 & 13.5 & 0.14 & 0.115 & -- & -- & 0.118 & -- & -- & -- \\
\hline 10/23/2008 6:00 PM & 0.305 & 10.8 & 0.173 & 0.113 & 13.5 & 0.14 & 0.115 & -- & -- & 0.117 & -- & -- & -- \\
\hline 10/23/2008 7:00 PM & 0.300 & 10.7 & 0.174 & 0.113 & 13.5 & 0.14 & 0.115 & -- & -- & 0.117 & -- & -- & -- \\
\hline 10/23/2008 8:00 PM & 0.298 & 10.7 & 0.174 & 0.115 & 13.5 & 0.14 & 0.114 & -- & -- & 0.117 & -- & -- & -- \\
\hline 10/23/2008 9:00 PM & 0.295 & 10.7 & 0.174 & 0.115 & 13.5 & 0.14 & 0.114 & -- & -- & 0.117 & -- & -- & -- \\
\hline 10/23/2008 10:00 PM & 0.293 & 10.7 & 0.174 & 0.113 & 13.5 & 0.14 & 0.114 & -- & -- & 0.117 & -- & -- & -- \\
\hline 10/23/2008 11:00 PM & 0.292 & 10.7 & 0.173 & 0.113 & 13.4 & 0.14 & 0.114 & -- & -- & 0.117 & -- & -- & -- \\
\hline 10/24/2008 12:00 AM & 0.292 & 10.7 & 0.173 & 0.113 & 13.4 & 0.14 & 0.114 & -- & -- & 0.117 & -- & -- & -- \\
\hline 10/24/2008 1:00 AM & 0.292 & 10.6 & 0.173 & 0.113 & 13.5 & 0.14 & 0.114 & -- & -- & 0.117 & -- & -- & -- \\
\hline 10/24/2008 2:00 AM & 0.292 & 10.6 & 0.173 & 0.113 & 13.4 & 0.14 & 0.114 & -- & -- & 0.117 & -- & -- & -- \\
\hline 10/24/2008 3:00 AM & 0.292 & 10.7 & 0.173 & 0.113 & 13.5 & 0.14 & 0.114 & -- & -- & 0.117 & -- & -- & -- \\
\hline 10/24/2008 4:00 AM & 0.292 & 10.6 & 0.173 & 0.113 & 13.4 & 0.14 & 0.114 & -- & -- & 0.117 & -- & -- & -- \\
\hline 10/24/2008 5:00 AM & 0.293 & 10.6 & 0.173 & 0.113 & 13.4 & 0.14 & 0.114 & -- & -- & 0.117 & -- & -- & -- \\
\hline 10/24/2008 6:00 AM & 0.293 & 10.6 & 0.173 & 0.113 & 13.4 & 0.14 & 0.114 & -- & -- & 0.117 & -- & -- & -- \\
\hline 10/24/2008 7:00 AM & 0.294 & 10.6 & 0.173 & 0.113 & 13.4 & 0.14 & 0.114 & -- & -- & 0.117 & -- & -- & -- \\
\hline 10/24/2008 8:00 AM & 0.296 & 10.6 & 0.173 & 0.113 & 13.4 & 0.14 & 0.114 & -- & -- & 0.117 & -- & -- & -- \\
\hline 10/24/2008 9:00 AM & 0.296 & 10.6 & 0.173 & 0.113 & 13.4 & 0.14 & 0.114 & -- & -- & 0.117 & -- & -- & -- \\
\hline 10/24/2008 10:00 AM & 0.296 & 10.6 & 0.174 & 0.113 & 13.4 & 0.14 & 0.115 & -- & -- & 0.117 & -- & -- & -- \\
\hline 10/24/2008 11:00 AM & 0.295 & 10.6 & 0.173 & 0.113 & 13.4 & 0.14 & 0.115 & -- & -- & 0.117 & -- & -- & -- \\
\hline 10/24/2008 12:00 PM & 0.295 & 10.5 & 0.174 & 0.113 & 13.4 & 0.14 & 0.114 & -- & -- & 0.117 & -- & -- & -- \\
\hline 10/24/2008 1:00 PM & 0.297 & 10.5 & 0.174 & 0.113 & 13.4 & 0.14 & 0.115 & -- & -- & 0.117 & -- & -- & -- \\
\hline 10/24/2008 2:00 PM & 0.300 & 10.5 & 0.174 & 0.113 & 13.4 & 0.14 & 0.115 & -- & -- & 0.117 & -- & -- & -- \\
\hline 10/24/2008 3:00 PM & 0.309 & 10.4 & 0.174 & 0.113 & 13.4 & 0.14 & 0.114 & -- & -- & 0.117 & -- & -- & -- \\
\hline $10 / 24 / 2008$ 4:00 PM & 0.315 & 10.4 & 0.174 & 0.113 & 13.3 & 0.14 & 0.114 & -- & -- & 0.117 & -- & -- & -- \\
\hline 10/24/2008 5:00 PM & 0.319 & 10.4 & 0.174 & 0.113 & 13.3 & 0.14 & 0.115 & -- & -- & 0.117 & -- & -- & -- \\
\hline 10/24/2008 6:00 PM & 0.322 & 10.3 & 0.174 & 0.113 & 13.3 & 0.14 & 0.115 & -- & -- & 0.117 & -- & -- & -- \\
\hline 10/24/2008 7:00 PM & 0.324 & 10.4 & 0.173 & 0.113 & 13.3 & 0.14 & 0.115 & -- & -- & 0.117 & -- & -- & -- \\
\hline 10/24/2008 8:00 PM & 0.324 & 10.4 & 0.173 & 0.113 & 13.3 & 0.14 & 0.114 & -- & -- & 0.117 & -- & -- & -- \\
\hline 10/24/2008 9:00 PM & 0.324 & 10.3 & 0.173 & 0.113 & 13.3 & 0.14 & 0.114 & -- & -- & 0.117 & -- & -- & -- \\
\hline
\end{tabular}


Appendix 3. Volumetric water content, temperature, and electrical-conductivity data collected at the sprinkler-irrigated site during 2008.-Continued

[Depth in feet below land surface; volumetric water content dimensionless; temperature in degrees Celsius; bulk electrical conductivity in decisiemens per meter; --, no data]

\begin{tabular}{|c|c|c|c|c|c|c|c|c|c|c|c|c|c|}
\hline \multirow[b]{3}{*}{$\begin{array}{l}\text { Measurement } \\
\text { date and time }\end{array}$} & \multicolumn{13}{|c|}{ Depth } \\
\hline & \multicolumn{2}{|l|}{2} & \multirow{2}{*}{$\begin{array}{c}3 \\
\text { Volumetric } \\
\text { water } \\
\text { content }\end{array}$} & \multicolumn{3}{|c|}{4} & \multirow{2}{*}{$\begin{array}{c}5 \\
\text { Volumetric } \\
\text { water } \\
\text { content }\end{array}$} & \multicolumn{2}{|l|}{7} & \multirow{2}{*}{$\begin{array}{c}9 \\
\text { Volumetric } \\
\text { water } \\
\text { content }\end{array}$} & \multicolumn{2}{|c|}{13} & \multirow{2}{*}{$\begin{array}{c}16.5 \\
\text { Volumetric } \\
\text { water } \\
\text { content }\end{array}$} \\
\hline & $\begin{array}{c}\text { Volumetric } \\
\text { water } \\
\text { content }\end{array}$ & $\begin{array}{l}\text { Temper- } \\
\text { ature }\end{array}$ & & $\begin{array}{c}\text { Volumetric } \\
\text { water } \\
\text { content }\end{array}$ & $\begin{array}{l}\text { Temper- } \\
\text { ature }\end{array}$ & $\begin{array}{c}\text { Bulk } \\
\text { electrical } \\
\text { conduct- } \\
\text { ivity }\end{array}$ & & $\begin{array}{l}\text { Volumetric } \\
\text { water } \\
\text { content }\end{array}$ & $\begin{array}{l}\text { Temper- } \\
\text { ature }\end{array}$ & & $\begin{array}{c}\text { Volumetric } \\
\text { water } \\
\text { content }\end{array}$ & $\begin{array}{l}\text { Temper- } \\
\text { ature }\end{array}$ & \\
\hline 10/24/2008 10:00 PM & 0.325 & 10.3 & 0.173 & 0.113 & 13.3 & 0.14 & 0.114 & -- & -- & 0.117 & -- & -- & -- \\
\hline 10/24/2008 11:00 PM & 0.324 & 10.3 & 0.173 & 0.113 & 13.3 & 0.14 & 0.114 & -- & -- & 0.117 & -- & -- & -- \\
\hline 10/25/2008 12:00 AM & 0.325 & 10.3 & 0.173 & 0.113 & 13.3 & 0.14 & 0.114 & -- & -- & 0.117 & -- & -- & -- \\
\hline 10/25/2008 1:00 AM & 0.324 & 10.3 & 0.173 & 0.113 & 13.3 & 0.14 & 0.114 & -- & -- & 0.117 & -- & -- & -- \\
\hline 10/25/2008 2:00 AM & 0.324 & 10.3 & 0.173 & 0.113 & 13.3 & 0.14 & 0.114 & -- & -- & 0.117 & -- & -- & -- \\
\hline 10/25/2008 3:00 AM & 0.324 & 10.4 & 0.173 & 0.113 & 13.3 & 0.14 & 0.114 & -- & -- & 0.117 & -- & -- & -- \\
\hline 10/25/2008 4:00 AM & 0.324 & 10.4 & 0.173 & 0.113 & 13.3 & 0.14 & 0.114 & -- & -- & 0.117 & -- & -- & -- \\
\hline 10/25/2008 5:00 AM & 0.324 & 10.4 & 0.173 & 0.113 & 13.2 & 0.14 & 0.114 & -- & -- & 0.117 & -- & -- & -- \\
\hline 10/25/2008 6:00 AM & 0.324 & 10.4 & 0.172 & 0.113 & 13.2 & 0.14 & 0.114 & -- & -- & 0.117 & -- & -- & -- \\
\hline 10/25/2008 7:00 AM & 0.324 & 10.4 & 0.172 & 0.113 & 13.2 & 0.14 & 0.114 & -- & -- & 0.117 & -- & -- & -- \\
\hline 10/25/2008 8:00 AM & 0.324 & 10.4 & 0.172 & 0.113 & 13.2 & 0.14 & 0.114 & -- & -- & 0.117 & -- & -- & -- \\
\hline 10/25/2008 9:00 AM & 0.324 & 10.4 & 0.173 & 0.113 & 13.2 & 0.14 & 0.114 & -- & -- & 0.117 & -- & -- & -- \\
\hline 10/25/2008 10:00 AM & 0.324 & 10.4 & 0.173 & 0.113 & 13.2 & 0.14 & 0.114 & -- & -- & 0.117 & -- & -- & -- \\
\hline 10/25/2008 11:00 AM & 0.323 & 10.4 & 0.173 & 0.113 & 13.2 & 0.14 & 0.115 & -- & -- & 0.117 & -- & -- & -- \\
\hline 10/25/2008 12:00 PM & 0.322 & 10.3 & 0.173 & 0.113 & 13.2 & 0.14 & 0.115 & -- & -- & 0.117 & -- & -- & -- \\
\hline 10/25/2008 1:00 PM & 0.321 & 10.3 & 0.173 & 0.113 & 13.2 & 0.14 & 0.115 & -- & -- & 0.117 & -- & -- & -- \\
\hline 10/25/2008 2:00 PM & 0.321 & 10.3 & 0.173 & 0.115 & 13.2 & 0.14 & 0.115 & -- & -- & 0.117 & -- & -- & -- \\
\hline 10/25/2008 3:00 PM & 0.322 & 10.3 & 0.173 & 0.113 & 13.2 & 0.14 & 0.114 & -- & -- & 0.117 & -- & -- & -- \\
\hline 10/25/2008 4:00 PM & 0.323 & 10.3 & 0.173 & 0.113 & 13.2 & 0.14 & 0.115 & -- & -- & 0.117 & -- & -- & -- \\
\hline 10/25/2008 5:00 PM & 0.324 & 10.3 & 0.173 & 0.113 & 13.2 & 0.14 & 0.115 & -- & -- & 0.117 & -- & -- & -- \\
\hline 10/25/2008 6:00 PM & 0.324 & 10.3 & 0.173 & 0.113 & 13.1 & 0.14 & 0.115 & -- & -- & 0.117 & -- & -- & -- \\
\hline 10/25/2008 7:00 PM & 0.324 & 10.3 & 0.172 & 0.113 & 13.1 & 0.14 & 0.115 & -- & -- & 0.117 & -- & -- & -- \\
\hline 10/25/2008 8:00 PM & 0.324 & 10.2 & 0.173 & 0.113 & 13.1 & 0.14 & 0.115 & -- & -- & 0.117 & -- & -- & -- \\
\hline 10/25/2008 9:00 PM & 0.324 & 10.3 & 0.173 & 0.113 & 13.1 & 0.14 & 0.115 & -- & -- & 0.117 & -- & -- & -- \\
\hline 10/25/2008 10:00 PM & 0.324 & 10.2 & 0.172 & 0.113 & 13.1 & 0.14 & 0.114 & -- & -- & 0.117 & -- & -- & -- \\
\hline 10/25/2008 11:00 PM & 0.324 & 10.3 & 0.173 & 0.113 & 13.1 & 0.14 & 0.114 & -- & -- & 0.117 & -- & -- & -- \\
\hline 10/26/2008 12:00 AM & 0.324 & 10.2 & 0.173 & 0.113 & 13.1 & 0.14 & 0.114 & -- & -- & 0.117 & -- & -- & -- \\
\hline 10/26/2008 1:00 AM & 0.324 & 10.3 & 0.173 & 0.113 & 13.1 & 0.14 & 0.114 & -- & -- & 0.117 & -- & -- & -- \\
\hline 10/26/2008 2:00 AM & 0.324 & 10.3 & 0.172 & 0.113 & 13.1 & 0.14 & 0.114 & -- & -- & 0.117 & -- & -- & -- \\
\hline 10/26/2008 3:00 AM & 0.324 & 10.3 & 0.173 & 0.113 & 13.1 & 0.14 & 0.114 & -- & -- & 0.117 & -- & -- & -- \\
\hline 10/26/2008 4:00 AM & 0.324 & 10.3 & 0.173 & 0.113 & 13.1 & 0.14 & 0.114 & -- & -- & 0.117 & -- & -- & -- \\
\hline 10/26/2008 5:00 AM & 0.324 & 10.3 & 0.173 & 0.113 & 13.1 & 0.14 & 0.114 & -- & -- & 0.117 & -- & -- & -- \\
\hline 10/26/2008 6:00 AM & 0.324 & 10.3 & 0.173 & 0.113 & 13.1 & 0.14 & 0.114 & -- & -- & 0.117 & -- & -- & -- \\
\hline 10/26/2008 7:00 AM & 0.324 & 10.3 & 0.173 & 0.113 & 13.1 & 0.14 & 0.114 & -- & -- & 0.117 & -- & -- & -- \\
\hline 10/26/2008 8:00 AM & 0.324 & 10.4 & 0.172 & 0.113 & 13.0 & 0.14 & 0.114 & -- & -- & 0.117 & -- & -- & -- \\
\hline
\end{tabular}


Appendix 3. Volumetric water content, temperature, and electrical-conductivity data collected at the sprinkler-irrigated site during 2008.-Continued

[Depth in feet below land surface; volumetric water content dimensionless; temperature in degrees Celsius; bulk electrical conductivity in decisiemens per meter; --, no data]

\begin{tabular}{|c|c|c|c|c|c|c|c|c|c|c|c|c|c|}
\hline \multirow[b]{3}{*}{$\begin{array}{l}\text { Measurement } \\
\text { date and time }\end{array}$} & \multicolumn{13}{|c|}{ Depth } \\
\hline & \multicolumn{2}{|c|}{2} & \multirow{2}{*}{$\begin{array}{c}3 \\
\begin{array}{c}\text { Volumetric } \\
\text { water } \\
\text { content }\end{array}\end{array}$} & \multicolumn{3}{|c|}{4} & \multirow{2}{*}{$\begin{array}{c}\mathbf{5} \\
\text { Volumetric } \\
\text { water } \\
\text { content }\end{array}$} & \multicolumn{2}{|l|}{7} & \multirow{2}{*}{$\begin{array}{c}9 \\
\text { Volumetric } \\
\text { water } \\
\text { content }\end{array}$} & \multicolumn{2}{|c|}{13} & \multirow{2}{*}{$\begin{array}{c}16.5 \\
\text { Volumetric } \\
\text { water } \\
\text { content }\end{array}$} \\
\hline & $\begin{array}{c}\text { Volumetric } \\
\text { water } \\
\text { content }\end{array}$ & $\begin{array}{l}\text { Temper- } \\
\text { ature }\end{array}$ & & $\begin{array}{c}\text { Volumetric } \\
\text { water } \\
\text { content }\end{array}$ & $\begin{array}{l}\text { Temper- } \\
\text { ature }\end{array}$ & $\begin{array}{c}\text { Bulk } \\
\text { electrical } \\
\text { conduct- } \\
\text { ivity }\end{array}$ & & $\begin{array}{c}\text { Volumetric } \\
\text { water } \\
\text { content }\end{array}$ & $\begin{array}{l}\text { Temper- } \\
\text { ature }\end{array}$ & & $\begin{array}{c}\text { Volumetric } \\
\text { water } \\
\text { content }\end{array}$ & $\begin{array}{l}\text { Temper- } \\
\text { ature }\end{array}$ & \\
\hline 10/26/2008 9:00 AM & 0.324 & 10.4 & 0.172 & 0.113 & 13.1 & 0.14 & 0.114 & -- & -- & 0.117 & -- & -- & -- \\
\hline 10/26/2008 10:00 AM & 0.324 & 10.4 & 0.173 & 0.113 & 13.0 & 0.14 & 0.114 & -- & -- & 0.117 & -- & -- & -- \\
\hline 10/26/2008 11:00 AM & 0.323 & 10.4 & 0.173 & 0.113 & 13.0 & 0.14 & 0.114 & -- & -- & 0.117 & -- & -- & -- \\
\hline 10/26/2008 12:00 PM & 0.323 & 10.3 & 0.172 & 0.113 & 13.0 & 0.14 & 0.114 & -- & -- & 0.117 & -- & -- & -- \\
\hline 10/26/2008 1:00 PM & 0.323 & 10.3 & 0.172 & 0.113 & 13.0 & 0.14 & 0.114 & -- & -- & 0.117 & -- & -- & -- \\
\hline 10/26/2008 2:00 PM & 0.323 & 10.3 & 0.172 & 0.113 & 13.0 & 0.14 & 0.114 & -- & -- & 0.117 & -- & -- & -- \\
\hline 10/26/2008 3:00 PM & 0.323 & 10.3 & 0.172 & 0.113 & 13.0 & 0.14 & 0.114 & -- & -- & 0.117 & -- & -- & -- \\
\hline 10/26/2008 4:00 PM & 0.324 & 10.3 & 0.173 & 0.113 & 13.0 & 0.14 & 0.114 & -- & -- & 0.117 & -- & -- & -- \\
\hline 10/26/2008 5:00 PM & 0.324 & 10.3 & 0.173 & 0.113 & 13.0 & 0.14 & 0.114 & -- & -- & 0.117 & -- & -- & -- \\
\hline 10/26/2008 6:00 PM & 0.324 & 10.3 & 0.173 & 0.113 & 13.0 & 0.14 & 0.114 & -- & -- & 0.117 & -- & -- & -- \\
\hline 10/26/2008 7:00 PM & 0.324 & 10.3 & 0.172 & 0.113 & 13.0 & 0.14 & 0.114 & -- & -- & 0.117 & -- & -- & -- \\
\hline 10/26/2008 8:00 PM & 0.324 & 10.3 & 0.172 & 0.113 & 13.0 & 0.14 & 0.114 & -- & -- & 0.117 & -- & -- & -- \\
\hline 10/26/2008 9:00 PM & 0.324 & 10.3 & 0.172 & 0.113 & 13.0 & 0.14 & 0.114 & -- & -- & 0.117 & -- & -- & -- \\
\hline 10/26/2008 10:00 PM & 0.324 & 10.3 & 0.172 & 0.113 & 13.0 & 0.14 & 0.114 & -- & -- & 0.117 & -- & -- & -- \\
\hline 10/26/2008 11:00 PM & 0.324 & 10.3 & 0.172 & 0.113 & 13.0 & 0.14 & 0.114 & -- & -- & 0.117 & -- & -- & -- \\
\hline 10/27/2008 12:00 AM & 0.324 & 10.3 & 0.172 & 0.113 & 13.0 & 0.14 & 0.114 & -- & -- & 0.117 & -- & -- & -- \\
\hline 10/27/2008 1:00 AM & 0.324 & 10.3 & 0.172 & 0.113 & 12.9 & 0.14 & 0.114 & -- & -- & 0.117 & -- & -- & -- \\
\hline 10/27/2008 2:00 AM & 0.324 & 10.3 & 0.172 & 0.113 & 12.9 & 0.14 & 0.114 & -- & -- & 0.117 & -- & -- & -- \\
\hline 10/27/2008 3:00 AM & 0.324 & 10.3 & 0.172 & 0.113 & 12.9 & 0.14 & 0.114 & -- & -- & 0.117 & -- & -- & -- \\
\hline $10 / 27 / 2008$ 4:00 AM & 0.324 & 10.3 & 0.172 & 0.113 & 12.9 & 0.14 & 0.114 & -- & -- & 0.117 & -- & -- & -- \\
\hline 10/27/2008 5:00 AM & 0.324 & 10.3 & 0.172 & 0.113 & 12.9 & 0.14 & 0.114 & -- & -- & 0.117 & -- & -- & -- \\
\hline 10/27/2008 6:00 AM & 0.324 & 10.3 & 0.172 & 0.113 & 12.9 & 0.14 & 0.114 & -- & -- & 0.117 & -- & -- & -- \\
\hline 10/27/2008 7:00 AM & 0.324 & 10.3 & 0.172 & 0.113 & 12.9 & 0.14 & 0.114 & -- & -- & 0.117 & -- & -- & -- \\
\hline 10/27/2008 8:00 AM & 0.324 & 10.3 & 0.172 & 0.113 & 12.9 & 0.14 & 0.114 & -- & -- & 0.117 & -- & -- & -- \\
\hline 10/27/2008 9:00 AM & 0.324 & 10.3 & 0.172 & 0.113 & 12.9 & 0.14 & 0.114 & -- & -- & 0.117 & -- & -- & -- \\
\hline 10/27/2008 10:00 AM & 0.323 & 10.2 & 0.172 & 0.113 & 12.9 & 0.14 & 0.114 & -- & -- & 0.117 & -- & -- & -- \\
\hline 10/27/2008 11:00 AM & 0.323 & 10.2 & 0.172 & 0.113 & 12.9 & 0.14 & 0.114 & -- & -- & 0.117 & -- & -- & -- \\
\hline 10/27/2008 12:00 PM & 0.323 & 10.2 & 0.172 & 0.113 & 12.9 & 0.14 & 0.114 & -- & -- & 0.117 & -- & -- & 0.004 \\
\hline 10/27/2008 1:00 PM & 0.323 & 10.2 & 0.172 & 0.113 & 12.9 & 0.14 & 0.114 & -- & -- & 0.117 & -- & -- & 0.067 \\
\hline 10/27/2008 2:00 PM & 0.322 & 10.2 & 0.172 & 0.113 & 12.9 & 0.14 & 0.114 & -- & -- & 0.117 & -- & -- & 0.019 \\
\hline 10/27/2008 3:00 PM & 0.322 & 10.1 & 0.172 & 0.113 & 12.9 & 0.14 & 0.114 & -- & -- & 0.117 & -- & -- & -- \\
\hline 10/27/2008 4:00 PM & 0.321 & 10.1 & 0.172 & 0.113 & 12.9 & 0.14 & 0.114 & -- & -- & 0.117 & -- & -- & 0.021 \\
\hline 10/27/2008 5:00 PM & 0.319 & 10.0 & 0.172 & 0.113 & 12.9 & 0.14 & 0.114 & -- & -- & 0.117 & -- & -- & 0.050 \\
\hline 10/27/2008 6:00 PM & 0.317 & 10.0 & 0.172 & 0.113 & 12.8 & 0.14 & 0.114 & -- & -- & 0.117 & -- & -- & -- \\
\hline 10/27/2008 7:00 PM & 0.315 & 10.0 & 0.172 & 0.113 & 12.9 & 0.14 & 0.114 & -- & -- & 0.117 & -- & -- & -- \\
\hline
\end{tabular}


Appendix 3. Volumetric water content, temperature, and electrical-conductivity data collected at the sprinkler-irrigated site during 2008.-Continued

[Depth in feet below land surface; volumetric water content dimensionless; temperature in degrees Celsius; bulk electrical conductivity in decisiemens per meter; --, no data]

\begin{tabular}{|c|c|c|c|c|c|c|c|c|c|c|c|c|c|}
\hline \multirow[b]{3}{*}{$\begin{array}{l}\text { Measurement } \\
\text { date and time }\end{array}$} & \multicolumn{13}{|c|}{ Depth } \\
\hline & \multicolumn{2}{|l|}{2} & \multirow{2}{*}{$\begin{array}{c}3 \\
\text { Volumetric } \\
\text { water } \\
\text { content }\end{array}$} & \multicolumn{3}{|c|}{4} & \multirow{2}{*}{$\begin{array}{c}5 \\
\text { Volumetric } \\
\text { water } \\
\text { content }\end{array}$} & \multicolumn{2}{|c|}{7} & \multirow{2}{*}{$\begin{array}{c}9 \\
\text { Volumetric } \\
\text { water } \\
\text { content }\end{array}$} & \multicolumn{2}{|c|}{13} & \multirow{2}{*}{$\begin{array}{c}16.5 \\
\text { Volumetric } \\
\text { water } \\
\text { content }\end{array}$} \\
\hline & $\begin{array}{c}\text { Volumetric } \\
\text { water } \\
\text { content }\end{array}$ & $\begin{array}{c}\text { Temper- } \\
\text { ature }\end{array}$ & & $\begin{array}{c}\text { Volumetric } \\
\text { water } \\
\text { content }\end{array}$ & $\begin{array}{c}\text { Temper- } \\
\text { ature }\end{array}$ & $\begin{array}{c}\text { Bulk } \\
\text { electrical } \\
\text { conduct- } \\
\text { ivity }\end{array}$ & & $\begin{array}{c}\text { Volumetric } \\
\text { water } \\
\text { content }\end{array}$ & $\begin{array}{c}\text { Temper- } \\
\text { ature }\end{array}$ & & $\begin{array}{c}\text { Volumetric } \\
\text { water } \\
\text { content }\end{array}$ & $\begin{array}{c}\text { Temper- } \\
\text { ature }\end{array}$ & \\
\hline 10/27/2008 8:00 PM & 0.312 & 10.0 & 0.172 & 0.113 & 12.9 & 0.14 & 0.114 & -- & -- & 0.117 & -- & -- & -- \\
\hline 10/27/2008 9:00 PM & 0.310 & 9.9 & 0.172 & 0.113 & 12.8 & 0.14 & 0.114 & -- & -- & 0.116 & -- & -- & -- \\
\hline 10/27/2008 10:00 PM & 0.309 & 9.9 & 0.172 & 0.113 & 12.8 & 0.14 & 0.114 & -- & -- & 0.116 & -- & -- & -- \\
\hline 10/27/2008 11:00 PM & 0.308 & 9.9 & 0.172 & 0.113 & 12.8 & 0.14 & 0.114 & -- & -- & 0.116 & -- & -- & -- \\
\hline 10/28/2008 12:00 AM & 0.307 & 9.9 & 0.172 & 0.113 & 12.8 & 0.14 & 0.114 & -- & -- & 0.116 & -- & -- & -- \\
\hline 10/28/2008 1:00 AM & 0.307 & 9.8 & 0.172 & 0.113 & 12.8 & 0.14 & 0.114 & -- & -- & 0.116 & -- & -- & -- \\
\hline 10/28/2008 2:00 AM & 0.307 & 9.8 & 0.172 & 0.113 & 12.8 & 0.14 & 0.114 & -- & -- & 0.116 & -- & -- & -- \\
\hline 10/28/2008 3:00 AM & 0.307 & 9.8 & 0.172 & 0.113 & 12.8 & 0.14 & 0.114 & -- & -- & 0.116 & -- & -- & -- \\
\hline 10/28/2008 4:00 AM & 0.307 & 9.8 & 0.171 & 0.113 & 12.8 & 0.14 & 0.114 & -- & -- & 0.116 & -- & -- & -- \\
\hline 10/28/2008 5:00 AM & 0.307 & 9.8 & 0.171 & 0.113 & 12.8 & 0.14 & 0.114 & -- & -- & 0.116 & -- & -- & -- \\
\hline 10/28/2008 6:00 AM & 0.307 & 9.8 & 0.171 & 0.113 & 12.8 & 0.14 & 0.114 & -- & -- & 0.116 & -- & -- & -- \\
\hline 10/28/2008 7:00 AM & 0.308 & 9.8 & 0.171 & 0.113 & 12.8 & 0.14 & 0.114 & -- & -- & 0.116 & -- & -- & -- \\
\hline 10/28/2008 8:00 AM & 0.308 & 9.7 & 0.172 & 0.113 & 12.8 & 0.14 & 0.114 & -- & -- & 0.116 & -- & -- & -- \\
\hline 10/28/2008 9:00 AM & 0.309 & 9.7 & 0.172 & 0.113 & 12.8 & 0.14 & 0.114 & -- & -- & 0.116 & -- & -- & -- \\
\hline 10/28/2008 10:00 AM & 0.309 & 9.7 & 0.172 & 0.113 & 12.8 & 0.14 & 0.114 & -- & -- & 0.116 & -- & -- & -- \\
\hline 10/28/2008 11:00 AM & 0.308 & 9.7 & 0.172 & 0.113 & 12.8 & 0.14 & 0.114 & -- & -- & 0.117 & -- & -- & -- \\
\hline
\end{tabular}


Appendix 4. Volumetric water content, temperature, and electrical-conductivity data collected at the sprinkler-irrigated site during 2009.

[Depth in feet below land surface; volumetric water content dimensionless; temperature in degrees Celsius; bulk electrical conductivity in decisiemens per meter; --, no data]

\begin{tabular}{|c|c|c|c|c|c|c|c|c|c|c|c|c|c|c|}
\hline \multirow[b]{3}{*}{$\begin{array}{l}\text { Measurement } \\
\text { date and time }\end{array}$} & \multicolumn{14}{|c|}{ Depth } \\
\hline & \multirow{2}{*}{$\begin{array}{c}1 \\
\text { Volumetric } \\
\text { water } \\
\text { content }\end{array}$} & \multicolumn{2}{|l|}{2} & \multirow{2}{*}{$\begin{array}{c}3 \\
\text { Volumetric } \\
\text { water } \\
\text { content }\end{array}$} & \multicolumn{3}{|c|}{4} & \multirow{2}{*}{$\begin{array}{c}5 \\
\text { Volumetric } \\
\text { water } \\
\text { content }\end{array}$} & \multicolumn{2}{|l|}{7} & \multirow{2}{*}{$\begin{array}{c}9 \\
\text { Volumetric } \\
\text { water } \\
\text { content }\end{array}$} & \multicolumn{2}{|c|}{13} & \multirow{2}{*}{$\begin{array}{c}16.5 \\
\text { Volumetric } \\
\text { water } \\
\text { content }\end{array}$} \\
\hline & & $\begin{array}{c}\text { Volumetric } \\
\text { water } \\
\text { content }\end{array}$ & $\begin{array}{l}\text { Temper- } \\
\text { ature }\end{array}$ & & $\begin{array}{c}\text { Volumetric } \\
\text { water } \\
\text { content }\end{array}$ & $\begin{array}{l}\text { Temper- } \\
\text { ature }\end{array}$ & $\begin{array}{c}\text { Bulk } \\
\text { electrical } \\
\text { conduct- } \\
\text { ivity }\end{array}$ & & $\begin{array}{c}\text { Volumetric } \\
\text { water } \\
\text { content }\end{array}$ & $\begin{array}{l}\text { Temper- } \\
\text { ature }\end{array}$ & & $\begin{array}{l}\text { Volumetric } \\
\text { water } \\
\text { content }\end{array}$ & $\begin{array}{l}\text { Temper- } \\
\text { ature }\end{array}$ & \\
\hline 6/15/2009 8:00 PM & -- & 0.359 & 16.6 & 0.188 & 0.122 & 15.2 & 0.13 & 0.123 & 0.174 & 12.5 & 0.117 & 0.317 & 10.5 & 0.476 \\
\hline 6/16/2009 12:00 AM & -- & 0.359 & 16.6 & 0.188 & 0.122 & 15.2 & 0.13 & 0.123 & 0.173 & 12.5 & 0.117 & 0.319 & 10.6 & 0.476 \\
\hline 6/16/2009 4:00 AM & -- & 0.358 & 16.7 & 0.188 & 0.122 & 15.2 & 0.13 & 0.122 & 0.174 & 12.5 & 0.117 & 0.321 & 10.6 & 0.476 \\
\hline 6/16/2009 8:00 AM & -- & 0.359 & 16.8 & 0.188 & 0.122 & 15.2 & 0.13 & 0.122 & 0.173 & 12.5 & 0.117 & 0.323 & 10.6 & 0.476 \\
\hline 6/16/2009 12:00 PM & -- & 0.359 & 16.8 & 0.188 & 0.122 & 15.2 & 0.13 & 0.123 & 0.174 & 12.5 & 0.117 & 0.326 & 10.6 & 0.476 \\
\hline 6/16/2009 4:00 PM & -- & 0.358 & 16.7 & 0.188 & 0.122 & 15.2 & 0.13 & 0.123 & 0.174 & 12.5 & 0.117 & 0.332 & 10.6 & 0.476 \\
\hline 6/16/2009 8:00 PM & -- & 0.358 & 16.7 & 0.188 & 0.122 & 15.2 & 0.13 & 0.122 & 0.174 & 12.5 & 0.117 & 0.334 & 10.5 & 0.476 \\
\hline 6/17/2009 12:00 AM & -- & 0.358 & 16.8 & 0.188 & 0.122 & 15.3 & 0.13 & 0.122 & 0.174 & 12.5 & 0.117 & 0.334 & 10.6 & 0.476 \\
\hline 6/17/2009 4:00 AM & -- & 0.359 & 17.0 & 0.188 & 0.122 & 15.3 & 0.13 & 0.122 & 0.173 & 12.5 & 0.117 & 0.337 & 10.7 & 0.476 \\
\hline 6/17/2009 8:00 AM & -- & 0.360 & 17.2 & 0.188 & 0.122 & 15.3 & 0.13 & 0.122 & 0.173 & 12.6 & 0.117 & 0.338 & 10.7 & 0.476 \\
\hline 6/17/2009 12:00 PM & -- & 0.360 & 17.2 & 0.188 & 0.122 & 15.3 & 0.13 & 0.122 & 0.174 & 12.6 & 0.117 & 0.341 & 10.7 & 0.476 \\
\hline 6/17/2009 4:00 PM & -- & 0.360 & 17.2 & 0.188 & 0.122 & 15.3 & 0.13 & 0.122 & 0.174 & 12.6 & 0.117 & 0.344 & 10.7 & 0.476 \\
\hline 6/17/2009 8:00 PM & -- & 0.360 & 17.2 & 0.188 & 0.122 & 15.3 & 0.13 & 0.122 & 0.174 & 12.6 & 0.117 & 0.345 & 10.7 & 0.476 \\
\hline 6/18/2009 12:00 AM & -- & 0.360 & 17.4 & 0.188 & 0.122 & 15.4 & 0.13 & 0.122 & 0.174 & 12.6 & 0.117 & 0.348 & 10.7 & 0.476 \\
\hline 6/18/2009 4:00 AM & -- & 0.360 & 17.6 & 0.188 & 0.122 & 15.4 & 0.13 & 0.122 & 0.174 & 12.6 & 0.117 & 0.349 & 10.7 & 0.476 \\
\hline 6/18/2009 8:00 AM & -- & 0.361 & 17.7 & 0.188 & 0.122 & 15.4 & 0.13 & 0.122 & 0.174 & 12.6 & 0.116 & 0.348 & 10.7 & 0.476 \\
\hline 6/18/2009 12:00 PM & -- & 0.362 & 17.8 & 0.188 & 0.122 & 15.4 & 0.13 & 0.122 & 0.174 & 12.6 & 0.117 & 0.350 & 10.7 & 0.476 \\
\hline 6/18/2009 4:00 PM & -- & 0.362 & 17.8 & 0.188 & 0.122 & 15.4 & 0.13 & 0.122 & 0.174 & 12.6 & 0.117 & 0.350 & 10.6 & 0.476 \\
\hline 6/18/2009 8:00 PM & -- & 0.362 & 17.8 & 0.188 & 0.122 & 15.5 & 0.13 & 0.122 & 0.174 & 12.7 & 0.117 & 0.350 & 10.7 & 0.476 \\
\hline 6/19/2009 12:00 AM & -- & 0.362 & 17.9 & 0.188 & 0.122 & 15.5 & 0.13 & 0.122 & 0.174 & 12.6 & 0.117 & 0.349 & 10.8 & 0.476 \\
\hline 6/19/2009 4:00 AM & -- & 0.362 & 18.1 & 0.188 & 0.122 & 15.5 & 0.13 & 0.121 & 0.174 & 12.7 & 0.117 & 0.349 & 10.8 & 0.476 \\
\hline 6/19/2009 8:00 AM & -- & 0.362 & 18.3 & 0.188 & 0.122 & 15.6 & 0.13 & 0.121 & 0.174 & 12.7 & 0.116 & 0.349 & 10.8 & 0.476 \\
\hline 6/19/2009 12:00 PM & -- & 0.363 & 18.3 & 0.188 & 0.122 & 15.6 & 0.13 & 0.122 & 0.174 & 12.7 & 0.117 & 0.349 & 10.7 & 0.476 \\
\hline 6/19/2009 4:00 PM & -- & 0.363 & 18.3 & 0.188 & 0.122 & 15.6 & 0.13 & 0.122 & 0.174 & 12.7 & 0.117 & 0.350 & 10.7 & 0.476 \\
\hline 6/19/2009 8:00 PM & -- & 0.362 & 18.3 & 0.188 & 0.122 & 15.7 & 0.13 & 0.122 & 0.174 & 12.7 & 0.117 & 0.350 & 10.7 & 0.476 \\
\hline 6/20/2009 12:00 AM & -- & 0.362 & 18.4 & 0.188 & 0.122 & 15.7 & 0.13 & 0.121 & 0.174 & 12.7 & 0.116 & 0.350 & 10.8 & 0.476 \\
\hline 6/20/2009 4:00 AM & -- & 0.363 & 18.6 & 0.188 & 0.122 & 15.7 & 0.13 & 0.121 & 0.174 & 12.7 & 0.116 & 0.350 & 10.8 & 0.476 \\
\hline 6/20/2009 8:00 AM & -- & 0.363 & 18.7 & 0.188 & 0.122 & 15.8 & 0.13 & 0.121 & 0.174 & 12.7 & 0.116 & 0.350 & 10.8 & 0.476 \\
\hline 6/20/2009 12:00 PM & -- & 0.363 & 18.8 & 0.188 & 0.122 & 15.8 & 0.13 & 0.121 & 0.174 & 12.8 & 0.116 & 0.351 & 10.8 & 0.476 \\
\hline 6/20/2009 4:00 PM & -- & 0.363 & 18.8 & 0.188 & 0.122 & 15.8 & 0.13 & 0.121 & 0.174 & 12.8 & 0.116 & 0.350 & 10.8 & 0.476 \\
\hline 6/20/2009 8:00 PM & -- & 0.363 & 18.7 & 0.188 & 0.122 & 15.9 & 0.13 & 0.121 & 0.174 & 12.8 & 0.116 & 0.350 & 10.8 & 0.476 \\
\hline 6/21/2009 12:00 AM & -- & 0.362 & 18.7 & 0.188 & 0.122 & 15.9 & 0.13 & 0.121 & 0.174 & 12.8 & 0.116 & 0.349 & 10.8 & 0.476 \\
\hline 6/21/2009 4:00 AM & -- & 0.362 & 18.8 & 0.188 & 0.122 & 15.9 & 0.13 & 0.121 & 0.174 & 12.8 & 0.116 & 0.350 & 10.8 & 0.476 \\
\hline 6/21/2009 8:00 AM & -- & 0.362 & 18.7 & 0.188 & 0.122 & 16.0 & 0.13 & 0.121 & 0.174 & 12.8 & 0.116 & 0.350 & 10.9 & 0.476 \\
\hline 6/21/2009 12:00 PM & -- & 0.362 & 18.7 & 0.188 & 0.122 & 16.0 & 0.13 & 0.121 & 0.174 & 12.8 & 0.116 & 0.351 & 10.8 & 0.476 \\
\hline 6/21/2009 4:00 PM & -- & 0.362 & 18.6 & 0.188 & 0.122 & 16.1 & 0.13 & 0.121 & 0.174 & 12.9 & 0.116 & 0.350 & 10.8 & 0.476 \\
\hline 6/21/2009 8:00 PM & -- & 0.362 & 18.5 & 0.188 & 0.122 & 16.1 & 0.13 & 0.121 & 0.174 & 12.9 & 0.116 & 0.351 & 10.9 & 0.476 \\
\hline
\end{tabular}


Appendix 4. Volumetric water content, temperature, and electrical-conductivity data collected at the sprinkler-irrigated site during 2009.-Continued

[Depth in feet below land surface; volumetric water content dimensionless; temperature in degrees Celsius; bulk electrical conductivity in decisiemens per meter; --, no data]

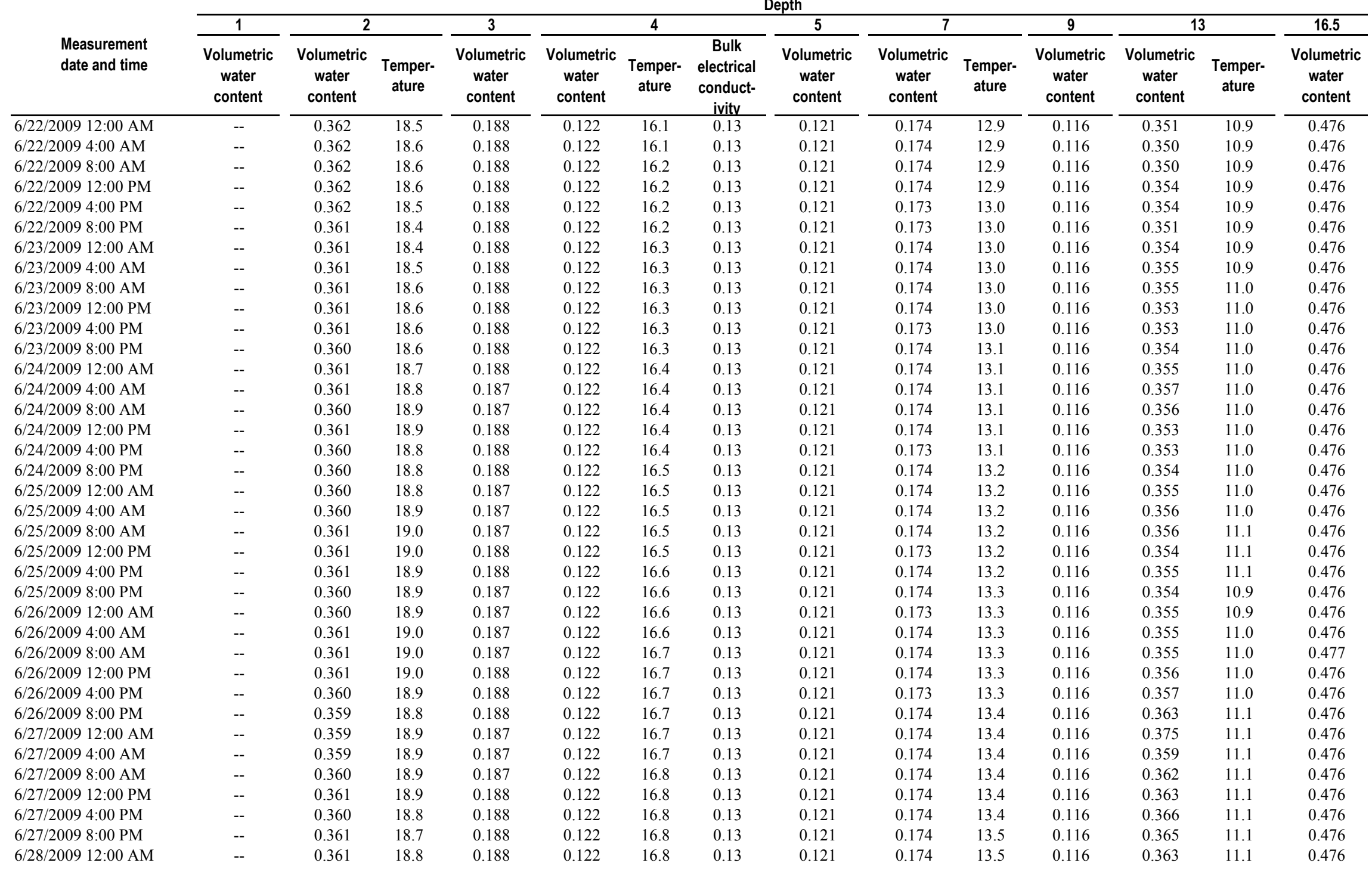


Appendix 4. Volumetric water content, temperature, and electrical-conductivity data collected at the sprinkler-irrigated site during 2009.-Continued

[Depth in feet below land surface; volumetric water content dimensionless; temperature in degrees Celsius; bulk electrical conductivity in decisiemens per meter; --, no data]

\begin{tabular}{|c|c|c|c|c|c|c|c|c|c|c|c|c|c|c|}
\hline \multirow[b]{3}{*}{$\begin{array}{l}\text { Measurement } \\
\text { date and time }\end{array}$} & \multicolumn{14}{|c|}{ Depth } \\
\hline & \multirow{2}{*}{$\begin{array}{c}1 \\
\text { Volumetric } \\
\text { water } \\
\text { content }\end{array}$} & \multicolumn{2}{|c|}{2} & \multirow{2}{*}{$\begin{array}{c}3 \\
\begin{array}{c}\text { Volumetric } \\
\text { water } \\
\text { content }\end{array}\end{array}$} & \multicolumn{3}{|c|}{4} & \multirow{2}{*}{$\begin{array}{c}5 \\
\text { Volumetric } \\
\text { water } \\
\text { content }\end{array}$} & \multicolumn{2}{|l|}{7} & \multirow{2}{*}{$\begin{array}{c}9 \\
\text { Volumetric } \\
\text { water } \\
\text { content }\end{array}$} & \multicolumn{2}{|c|}{13} & \multirow{2}{*}{$\begin{array}{c}16.5 \\
\text { Volumetric } \\
\text { water } \\
\text { content }\end{array}$} \\
\hline & & $\begin{array}{c}\text { Volumetric } \\
\text { water } \\
\text { content }\end{array}$ & $\begin{array}{l}\text { Temper- } \\
\text { ature }\end{array}$ & & $\begin{array}{c}\text { Volumetric } \\
\text { water } \\
\text { content }\end{array}$ & $\begin{array}{l}\text { Temper- } \\
\text { ature }\end{array}$ & $\begin{array}{c}\text { Bulk } \\
\text { electrical } \\
\text { conduct- } \\
\text { ivity }\end{array}$ & & $\begin{array}{c}\text { Volumetric } \\
\text { water } \\
\text { content }\end{array}$ & $\begin{array}{l}\text { Temper- } \\
\text { ature }\end{array}$ & & $\begin{array}{l}\text { Volumetric } \\
\text { water } \\
\text { content }\end{array}$ & $\begin{array}{l}\text { Temper- } \\
\text { ature }\end{array}$ & \\
\hline 6/28/2009 4:00 AM & -- & 0.360 & 18.9 & 0.187 & 0.122 & 16.8 & 0.13 & 0.121 & 0.174 & 13.5 & 0.116 & 0.363 & 11.1 & 0.476 \\
\hline 6/28/2009 8:00 AM & -- & 0.360 & 18.9 & 0.187 & 0.122 & 16.8 & 0.13 & 0.121 & 0.174 & 13.5 & 0.116 & 0.362 & 11.1 & 0.476 \\
\hline 6/28/2009 12:00 PM & -- & 0.361 & 18.9 & 0.188 & 0.122 & 16.9 & 0.13 & 0.121 & 0.174 & 13.5 & 0.116 & 0.365 & 11.1 & 0.476 \\
\hline 6/28/2009 4:00 PM & -- & 0.360 & 18.8 & 0.188 & 0.122 & 16.9 & 0.13 & 0.121 & 0.174 & 13.5 & 0.116 & 0.368 & 11.1 & 0.476 \\
\hline 6/28/2009 8:00 PM & -- & 0.361 & 18.7 & 0.188 & 0.122 & 16.9 & 0.13 & 0.121 & 0.174 & 13.5 & 0.116 & 0.369 & 11.1 & 0.476 \\
\hline 6/29/2009 12:00 AM & -- & 0.361 & 18.7 & 0.187 & 0.122 & 16.9 & 0.13 & 0.121 & 0.174 & 13.6 & 0.116 & 0.367 & 11.1 & 0.476 \\
\hline 6/29/2009 4:00 AM & -- & 0.362 & 18.8 & 0.187 & 0.122 & 16.9 & 0.13 & 0.121 & 0.174 & 13.6 & 0.115 & 0.365 & 11.1 & 0.476 \\
\hline 6/29/2009 8:00 AM & -- & 0.362 & 18.9 & 0.188 & 0.122 & 16.9 & 0.13 & 0.121 & 0.174 & 13.6 & 0.115 & 0.365 & 11.1 & 0.476 \\
\hline 6/29/2009 12:00 PM & -- & 0.365 & 18.9 & 0.188 & 0.122 & 16.9 & 0.13 & 0.121 & 0.174 & 13.6 & 0.115 & 0.365 & 11.2 & 0.476 \\
\hline 6/29/2009 4:00 PM & -- & 0.363 & 18.8 & 0.188 & 0.122 & 16.9 & 0.13 & 0.121 & 0.174 & 13.6 & 0.115 & 0.366 & 11.2 & 0.476 \\
\hline 6/29/2009 8:00 PM & -- & 0.365 & 18.7 & 0.188 & 0.122 & 16.9 & 0.13 & 0.121 & 0.174 & 13.6 & 0.116 & 0.369 & 11.2 & 0.476 \\
\hline 6/30/2009 12:00 AM & -- & 0.365 & 18.8 & 0.188 & 0.122 & 16.9 & 0.13 & 0.121 & 0.173 & 13.7 & 0.116 & 0.373 & 11.2 & 0.476 \\
\hline 6/30/2009 4:00 AM & -- & 0.363 & 18.8 & 0.188 & 0.122 & 17.0 & 0.13 & 0.121 & 0.175 & 13.7 & 0.115 & 0.369 & 11.2 & 0.476 \\
\hline 6/30/2009 8:00 AM & -- & 0.363 & 18.9 & 0.188 & 0.122 & 17.0 & 0.13 & 0.121 & 0.174 & 13.7 & 0.115 & 0.370 & 11.2 & 0.476 \\
\hline 6/30/2009 12:00 PM & -- & 0.363 & 18.8 & 0.188 & 0.123 & 17.0 & 0.13 & 0.121 & 0.174 & 13.7 & 0.116 & 0.370 & 11.2 & 0.476 \\
\hline 6/30/2009 4:00 PM & -- & 0.363 & 18.7 & 0.188 & 0.123 & 17.0 & 0.13 & 0.121 & 0.174 & 13.7 & 0.115 & 0.372 & 11.2 & 0.476 \\
\hline 6/30/2009 8:00 PM & -- & 0.365 & 18.7 & 0.188 & 0.123 & 17.0 & 0.13 & 0.121 & 0.173 & 13.7 & 0.115 & 0.378 & 11.2 & 0.476 \\
\hline 7/1/2009 12:00 AM & -- & 0.365 & 18.7 & 0.188 & 0.123 & 17.0 & 0.13 & 0.121 & 0.173 & 13.8 & 0.115 & 0.383 & 11.2 & 0.476 \\
\hline 7/1/2009 4:00 AM & -- & 0.366 & 18.8 & 0.188 & 0.123 & 17.0 & 0.13 & 0.121 & 0.173 & 13.8 & 0.115 & 0.374 & 11.2 & 0.476 \\
\hline 7/1/2009 8:00 AM & -- & 0.366 & 18.8 & 0.188 & 0.123 & 17.0 & 0.13 & 0.121 & 0.174 & 13.8 & 0.115 & 0.384 & 11.2 & 0.476 \\
\hline 7/1/2009 12:00 PM & -- & 0.366 & 18.8 & 0.188 & 0.123 & 17.0 & 0.13 & 0.121 & 0.174 & 13.8 & 0.116 & 0.387 & 11.2 & 0.476 \\
\hline 7/1/2009 4:00 PM & -- & 0.365 & 18.8 & 0.188 & 0.123 & 17.0 & 0.13 & 0.121 & 0.173 & 13.8 & 0.115 & 0.386 & 11.2 & 0.476 \\
\hline 7/1/2009 8:00 PM & -- & 0.366 & 18.7 & 0.188 & 0.123 & 17.1 & 0.13 & 0.121 & 0.174 & 13.8 & 0.116 & 0.393 & 11.3 & 0.476 \\
\hline 7/2/2009 12:00 AM & -- & 0.366 & 18.7 & 0.188 & 0.123 & 17.1 & 0.13 & 0.121 & 0.174 & 13.8 & 0.116 & 0.390 & 11.3 & 0.477 \\
\hline 7/2/2009 4:00 AM & -- & 0.366 & 18.8 & 0.188 & 0.123 & 17.1 & 0.13 & 0.121 & 0.173 & 13.8 & 0.115 & 0.391 & 11.3 & 0.477 \\
\hline 7/2/2009 8:00 AM & -- & 0.365 & 18.8 & 0.188 & 0.123 & 17.1 & 0.13 & 0.121 & 0.174 & 13.9 & 0.115 & 0.398 & 11.3 & 0.477 \\
\hline 7/2/2009 12:00 PM & -- & 0.363 & 18.8 & 0.188 & 0.123 & 17.1 & 0.13 & 0.121 & 0.174 & 13.9 & 0.115 & 0.398 & 11.3 & 0.476 \\
\hline 7/2/2009 4:00 PM & -- & 0.365 & 18.7 & 0.188 & 0.123 & 17.1 & 0.13 & 0.121 & 0.173 & 13.9 & 0.115 & 0.393 & 11.3 & 0.476 \\
\hline 7/2/2009 8:00 PM & -- & 0.365 & 18.7 & 0.188 & 0.123 & 17.1 & 0.13 & 0.121 & 0.174 & 13.9 & 0.116 & 0.394 & 11.3 & 0.476 \\
\hline 7/3/2009 12:00 AM & -- & 0.363 & 18.7 & 0.188 & 0.123 & 17.1 & 0.13 & 0.121 & 0.174 & 13.9 & 0.116 & 0.388 & 11.3 & 0.476 \\
\hline 7/3/2009 4:00 AM & -- & 0.363 & 18.7 & 0.188 & 0.123 & 17.1 & 0.13 & 0.121 & 0.174 & 13.9 & 0.115 & 0.388 & 11.3 & 0.477 \\
\hline 7/3/2009 8:00 AM & -- & 0.363 & 18.8 & 0.188 & 0.123 & 17.1 & 0.13 & 0.121 & 0.174 & 14.0 & 0.115 & 0.391 & 11.3 & 0.477 \\
\hline 7/3/2009 12:00 PM & -- & 0.363 & 18.7 & 0.188 & 0.123 & 17.1 & 0.13 & 0.121 & 0.174 & 14.0 & 0.115 & 0.386 & 11.3 & 0.476 \\
\hline 7/3/2009 4:00 PM & -- & 0.363 & 18.7 & 0.188 & 0.123 & 17.1 & 0.13 & 0.121 & 0.174 & 14.0 & 0.115 & 0.400 & 11.3 & 0.476 \\
\hline 7/3/2009 8:00 PM & -- & 0.362 & 18.7 & 0.188 & 0.123 & 17.1 & 0.13 & 0.121 & 0.173 & 14.0 & 0.116 & 0.401 & 11.3 & 0.476 \\
\hline 7/4/2009 12:00 AM & -- & 0.362 & 18.7 & 0.188 & 0.123 & 17.2 & 0.13 & 0.121 & 0.174 & 14.0 & 0.116 & 0.381 & 11.3 & 0.476 \\
\hline 7/4/2009 4:00 AM & -- & 0.362 & 18.8 & 0.188 & 0.123 & 17.1 & 0.13 & 0.121 & 0.174 & 14.0 & 0.116 & 0.371 & 11.3 & 0.476 \\
\hline
\end{tabular}


Appendix 4. Volumetric water content, temperature, and electrical-conductivity data collected at the sprinkler-irrigated site during 2009.-Continued

Depth in feet below land surface; volumetric water content dimensionless; temperature in degrees Celsius; bulk electrical conductivity in decisiemens per meter; --, no data]

\begin{tabular}{|c|c|c|c|c|c|c|c|c|c|c|c|c|c|c|}
\hline \multirow[b]{3}{*}{$\begin{array}{l}\text { Measurement } \\
\text { date and time }\end{array}$} & \multicolumn{14}{|c|}{ Depth } \\
\hline & \multirow[b]{2}{*}{$\begin{array}{c}1 \\
\text { Volumetric } \\
\text { water } \\
\text { content }\end{array}$} & \multicolumn{2}{|l|}{2} & \multirow[b]{2}{*}{$\begin{array}{c}3 \\
\text { Volumetric } \\
\text { water } \\
\text { content }\end{array}$} & \multicolumn{3}{|c|}{4} & \multirow[b]{2}{*}{$\begin{array}{c}5 \\
\text { Volumetric } \\
\text { water } \\
\text { content }\end{array}$} & \multicolumn{2}{|l|}{7} & \multirow{2}{*}{$\begin{array}{c}9 \\
\text { Volumetric } \\
\text { water } \\
\text { content }\end{array}$} & \multicolumn{2}{|c|}{13} & \multirow[b]{2}{*}{$\begin{array}{c}16.5 \\
\text { Volumetric } \\
\text { water } \\
\text { content }\end{array}$} \\
\hline & & $\begin{array}{c}\text { Volumetric } \\
\text { water } \\
\text { content }\end{array}$ & $\begin{array}{l}\text { Temper- } \\
\text { ature }\end{array}$ & & $\begin{array}{c}\begin{array}{c}\text { Volumetric } \\
\text { water } \\
\text { content }\end{array} \\
\end{array}$ & $\begin{array}{l}\text { Temper- } \\
\text { ature }\end{array}$ & $\begin{array}{c}\text { Bulk } \\
\text { electrical } \\
\text { conduct- } \\
\text { ivity }\end{array}$ & & $\begin{array}{l}\text { Volumetric } \\
\text { water } \\
\text { content }\end{array}$ & $\begin{array}{l}\text { Temper- } \\
\text { ature }\end{array}$ & & $\begin{array}{c}\text { Volumetric } \\
\text { water } \\
\text { content }\end{array}$ & $\begin{array}{l}\text { Temper- } \\
\text { ature }\end{array}$ & \\
\hline$\overline{7 / 4 / 20098: 00 \mathrm{AM}}$ & -- & 0.361 & 18.8 & 0.188 & 0.123 & 17.2 & 0.13 & 0.121 & 0.174 & 14.0 & 0.116 & 0.363 & 11.4 & 0.476 \\
\hline 7/4/2009 12:00 PM & -- & 0.360 & 18.8 & 0.188 & 0.123 & 17.2 & 0.13 & 0.121 & 0.174 & 14.1 & 0.116 & 0.368 & 11.4 & 0.476 \\
\hline 7/4/2009 4:00 PM & -- & 0.361 & 18.8 & 0.187 & 0.123 & 17.2 & 0.13 & 0.121 & 0.174 & 14.1 & 0.116 & 0.388 & 11.4 & 0.476 \\
\hline 7/4/2009 8:00 PM & -- & 0.361 & 18.7 & 0.187 & 0.123 & 17.2 & 0.13 & 0.121 & 0.174 & 14.1 & 0.116 & 0.380 & 11.4 & 0.477 \\
\hline 7/5/2009 12:00 AM & -- & 0.361 & 18.7 & 0.187 & 0.123 & 17.2 & 0.13 & 0.121 & 0.174 & 14.1 & 0.115 & 0.385 & 11.4 & 0.476 \\
\hline 7/5/2009 4:00 AM & -- & 0.360 & 18.7 & 0.187 & 0.123 & 17.2 & 0.13 & 0.121 & 0.174 & 14.1 & 0.115 & 0.378 & 11.4 & 0.476 \\
\hline 7/5/2009 8:00 AM & -- & 0.360 & 18.6 & 0.187 & 0.123 & 17.2 & 0.13 & 0.121 & 0.174 & 14.1 & 0.115 & 0.382 & 11.4 & 0.476 \\
\hline 7/5/2009 12:00 PM & -- & 0.360 & 18.6 & 0.187 & 0.123 & 17.2 & 0.13 & 0.121 & 0.174 & 14.1 & 0.115 & 0.381 & 11.4 & 0.477 \\
\hline 7/5/2009 4:00 PM & -- & 0.360 & 18.5 & 0.187 & 0.123 & 17.2 & 0.13 & 0.121 & 0.174 & 14.1 & 0.115 & 0.391 & 11.4 & 0.477 \\
\hline 7/5/2009 8:00 PM & -- & 0.358 & 18.4 & 0.187 & 0.123 & 17.2 & 0.13 & 0.121 & 0.174 & 14.1 & 0.115 & 0.387 & 11.4 & 0.477 \\
\hline 7/6/2009 12:00 AM & -- & 0.359 & 18.3 & 0.186 & 0.122 & 17.2 & 0.13 & 0.121 & 0.174 & 14.2 & 0.115 & 0.381 & 11.4 & 0.477 \\
\hline 7/6/2009 4:00 AM & -- & 0.358 & 18.4 & 0.186 & 0.122 & 17.2 & 0.13 & 0.121 & 0.174 & 14.2 & 0.115 & 0.380 & 11.4 & 0.477 \\
\hline 7/6/2009 8:00 AM & -- & 0.358 & 18.4 & 0.186 & 0.122 & 17.2 & 0.13 & 0.121 & 0.174 & 14.2 & 0.115 & 0.378 & 11.4 & 0.477 \\
\hline 7/6/2009 12:00 PM & -- & 0.358 & 18.4 & 0.186 & 0.122 & 17.2 & 0.13 & 0.121 & 0.174 & 14.2 & 0.115 & 0.382 & 11.4 & 0.477 \\
\hline 7/6/2009 4:00 PM & -- & 0.358 & 18.3 & 0.186 & 0.122 & 17.2 & 0.13 & 0.121 & 0.174 & 14.2 & 0.115 & 0.396 & 11.5 & 0.477 \\
\hline 7/6/2009 8:00 PM & -- & 0.357 & 18.2 & 0.186 & 0.122 & 17.2 & 0.13 & 0.121 & 0.174 & 14.2 & 0.115 & 0.382 & 11.5 & 0.477 \\
\hline 7/7/2009 12:00 AM & -- & 0.356 & 18.2 & 0.186 & 0.122 & 17.2 & 0.13 & 0.121 & 0.174 & 14.2 & 0.115 & 0.388 & 11.5 & 0.477 \\
\hline 7/7/2009 4:00 AM & -- & 0.356 & 18.2 & 0.186 & 0.122 & 17.2 & 0.13 & 0.121 & 0.174 & 14.2 & 0.115 & 0.388 & 11.5 & 0.477 \\
\hline 7/7/2009 8:00 AM & -- & 0.356 & 18.3 & 0.186 & 0.122 & 17.2 & 0.13 & 0.121 & 0.174 & 14.3 & 0.115 & 0.386 & 11.5 & 0.477 \\
\hline 7/7/2009 12:00 PM & -- & 0.356 & 18.2 & 0.186 & 0.122 & 17.2 & 0.13 & 0.121 & 0.174 & 14.3 & 0.115 & 0.400 & 11.5 & 0.477 \\
\hline 7/7/2009 4:00 PM & 0.145 & 0.355 & 18.2 & 0.185 & 0.122 & 17.2 & 0.13 & 0.121 & 0.174 & 14.3 & 0.115 & 0.369 & 11.5 & 0.476 \\
\hline 7/7/2009 8:00 PM & 0.146 & 0.355 & 18.1 & 0.185 & 0.122 & 17.2 & 0.13 & 0.121 & 0.174 & 14.3 & 0.116 & 0.390 & 11.5 & 0.477 \\
\hline 7/8/2009 12:00 AM & 0.146 & 0.354 & 18.1 & 0.185 & 0.122 & 17.2 & 0.13 & 0.121 & 0.174 & 14.3 & 0.116 & 0.385 & 11.5 & 0.477 \\
\hline 7/8/2009 4:00 AM & 0.147 & 0.355 & 18.1 & 0.185 & 0.122 & 17.2 & 0.13 & 0.121 & 0.174 & 14.3 & 0.116 & 0.385 & 11.5 & 0.477 \\
\hline 7/8/2009 8:00 AM & 0.147 & 0.354 & 18.2 & 0.185 & 0.122 & 17.2 & 0.13 & 0.121 & 0.174 & 14.3 & 0.116 & 0.381 & 11.5 & 0.477 \\
\hline 7/8/2009 12:00 PM & 0.146 & 0.354 & 18.2 & 0.185 & 0.122 & 17.2 & 0.13 & 0.121 & 0.174 & 14.3 & 0.116 & 0.393 & 11.5 & 0.477 \\
\hline 7/8/2009 4:00 PM & 0.145 & 0.353 & 18.1 & 0.185 & 0.122 & 17.2 & 0.13 & 0.121 & 0.174 & 14.3 & 0.116 & 0.399 & 11.5 & 0.477 \\
\hline 7/8/2009 8:00 PM & 0.144 & 0.353 & 18.1 & 0.184 & 0.122 & 17.2 & 0.13 & 0.121 & 0.174 & 14.4 & 0.116 & 0.394 & 11.6 & 0.477 \\
\hline 7/9/2009 12:00 AM & 0.146 & 0.353 & 18.1 & 0.184 & 0.122 & 17.2 & 0.13 & 0.121 & 0.175 & 14.4 & 0.116 & 0.390 & 11.5 & 0.477 \\
\hline 7/9/2009 4:00 AM & 0.147 & 0.353 & 18.2 & 0.184 & 0.122 & 17.2 & 0.13 & 0.121 & 0.174 & 14.4 & 0.116 & 0.391 & 11.6 & 0.477 \\
\hline 7/9/2009 8:00 AM & 0.147 & 0.353 & 18.2 & 0.184 & 0.122 & 17.2 & 0.13 & 0.121 & 0.174 & 14.4 & 0.116 & 0.403 & 11.6 & 0.476 \\
\hline 7/9/2009 12:00 PM & 0.146 & 0.354 & 18.3 & 0.184 & 0.122 & 17.2 & 0.13 & 0.121 & 0.174 & 14.4 & 0.116 & 0.416 & 11.6 & 0.476 \\
\hline 7/9/2009 4:00 PM & 0.145 & 0.353 & 18.2 & 0.184 & 0.122 & 17.2 & 0.13 & 0.121 & 0.174 & 14.4 & 0.116 & 0.387 & 11.6 & 0.476 \\
\hline 7/9/2009 8:00 PM & 0.145 & 0.351 & 18.2 & 0.184 & 0.122 & 17.2 & 0.13 & 0.121 & 0.174 & 14.4 & 0.116 & 0.350 & 11.6 & 0.477 \\
\hline 7/10/2009 12:00 AM & 0.146 & 0.350 & 18.2 & 0.184 & 0.122 & 17.2 & 0.13 & 0.120 & 0.174 & 14.4 & 0.116 & 0.415 & 11.6 & 0.476 \\
\hline 7/10/2009 4:00 AM & 0.146 & 0.350 & 18.2 & 0.184 & 0.122 & 17.2 & 0.13 & 0.120 & 0.174 & 14.4 & 0.116 & 0.410 & 11.6 & 0.476 \\
\hline 7/10/2009 8:00 AM & 0.146 & 0.350 & 18.2 & 0.184 & 0.122 & 17.2 & 0.13 & 0.120 & 0.174 & 14.4 & 0.116 & 0.405 & 11.6 & 0.476 \\
\hline
\end{tabular}


Appendix 4. Volumetric water content, temperature, and electrical-conductivity data collected at the sprinkler-irrigated site during 2009.-Continued

[Depth in feet below land surface; volumetric water content dimensionless; temperature in degrees Celsius; bulk electrical conductivity in decisiemens per meter; --, no data]

\begin{tabular}{|c|c|c|c|c|c|c|c|c|c|c|c|c|c|c|}
\hline \multirow[b]{3}{*}{$\begin{array}{l}\text { Measurement } \\
\text { date and time }\end{array}$} & \multicolumn{14}{|c|}{ Depth } \\
\hline & \multirow{2}{*}{$\begin{array}{c}1 \\
\text { Volumetric } \\
\text { water } \\
\text { content }\end{array}$} & \multicolumn{2}{|c|}{2} & \multirow{2}{*}{$\begin{array}{c}3 \\
\text { Volumetric } \\
\text { water } \\
\text { content }\end{array}$} & \multicolumn{3}{|c|}{4} & \multirow{2}{*}{$\begin{array}{c}5 \\
\text { Volumetric } \\
\text { water } \\
\text { content }\end{array}$} & \multicolumn{2}{|l|}{7} & \multirow{2}{*}{$\begin{array}{c}9 \\
\text { Volumetric } \\
\text { water } \\
\text { content }\end{array}$} & \multicolumn{2}{|c|}{13} & \multirow{2}{*}{$\begin{array}{c}16.5 \\
\text { Volumetric } \\
\text { water } \\
\text { content }\end{array}$} \\
\hline & & $\begin{array}{c}\text { Volumetric } \\
\text { water } \\
\text { content }\end{array}$ & $\begin{array}{l}\text { Temper- } \\
\text { ature }\end{array}$ & & $\begin{array}{c}\text { Volumetric } \\
\text { water } \\
\text { content }\end{array}$ & $\begin{array}{l}\text { Temper- } \\
\text { ature }\end{array}$ & $\begin{array}{c}\text { Bulk } \\
\text { electrical } \\
\text { conduct- } \\
\text { ivity }\end{array}$ & & $\begin{array}{c}\text { Volumetric } \\
\text { water } \\
\text { content }\end{array}$ & $\begin{array}{l}\text { Temper- } \\
\text { ature }\end{array}$ & & $\begin{array}{c}\text { Volumetric } \\
\text { water } \\
\text { content }\end{array}$ & $\begin{array}{l}\text { Temper- } \\
\text { ature }\end{array}$ & \\
\hline 7/10/2009 12:00 PM & 0.145 & 0.351 & 18.3 & 0.183 & 0.122 & 17.2 & 0.13 & 0.120 & 0.174 & 14.4 & 0.116 & 0.382 & 11.6 & 0.476 \\
\hline 7/10/2009 4:00 PM & 0.145 & 0.351 & 18.2 & 0.183 & 0.122 & 17.2 & 0.13 & 0.120 & 0.174 & 14.4 & 0.116 & 0.393 & 11.7 & 0.476 \\
\hline 7/10/2009 8:00 PM & 0.146 & 0.349 & 18.2 & 0.183 & 0.122 & 17.2 & 0.13 & 0.120 & 0.174 & 14.4 & 0.116 & 0.382 & 11.7 & 0.476 \\
\hline 7/11/2009 12:00 AM & 0.146 & 0.349 & 18.2 & 0.183 & 0.121 & 17.2 & 0.13 & 0.120 & 0.174 & 14.5 & 0.116 & 0.376 & 11.6 & 0.477 \\
\hline 7/11/2009 4:00 AM & 0.144 & 0.349 & 18.3 & 0.183 & 0.121 & 17.2 & 0.13 & 0.120 & 0.175 & 14.5 & 0.116 & 0.385 & 11.7 & 0.477 \\
\hline 7/11/2009 8:00 AM & 0.149 & 0.350 & 18.3 & 0.183 & 0.121 & 17.2 & 0.13 & 0.120 & 0.174 & 14.5 & 0.116 & 0.385 & 11.7 & 0.477 \\
\hline 7/11/2009 12:00 PM & 0.192 & 0.350 & 18.4 & 0.183 & 0.122 & 17.2 & 0.13 & 0.120 & 0.174 & 14.5 & 0.116 & 0.386 & 11.7 & 0.477 \\
\hline 7/11/2009 4:00 PM & 0.190 & 0.350 & 18.3 & 0.183 & 0.122 & 17.2 & 0.13 & 0.120 & 0.174 & 14.5 & 0.116 & 0.380 & 11.7 & 0.477 \\
\hline 7/11/2009 8:00 PM & 0.187 & 0.350 & 18.3 & 0.183 & 0.121 & 17.2 & 0.13 & 0.120 & 0.174 & 14.5 & 0.116 & 0.403 & 11.7 & 0.477 \\
\hline 7/12/2009 12:00 AM & 0.185 & 0.350 & 18.3 & 0.183 & 0.121 & 17.2 & 0.13 & 0.120 & 0.174 & 14.5 & 0.116 & 0.403 & 11.7 & 0.477 \\
\hline 7/12/2009 4:00 AM & 0.183 & 0.350 & 18.4 & 0.183 & 0.121 & 17.2 & 0.13 & 0.120 & 0.174 & 14.5 & 0.116 & 0.410 & 11.7 & 0.477 \\
\hline 7/12/2009 8:00 AM & 0.183 & 0.351 & 18.5 & 0.183 & 0.121 & 17.2 & 0.13 & 0.120 & 0.174 & 14.5 & 0.116 & 0.415 & 11.7 & 0.477 \\
\hline 7/12/2009 12:00 PM & 0.182 & 0.351 & 18.5 & 0.183 & 0.121 & 17.2 & 0.13 & 0.120 & 0.174 & 14.5 & 0.116 & 0.403 & 11.7 & 0.477 \\
\hline 7/12/2009 4:00 PM & 0.180 & 0.353 & 18.5 & 0.183 & 0.121 & 17.2 & 0.13 & 0.120 & 0.174 & 14.5 & 0.116 & 0.381 & 11.7 & 0.477 \\
\hline 7/12/2009 8:00 PM & 0.179 & 0.351 & 18.4 & 0.183 & 0.121 & 17.2 & 0.13 & 0.120 & 0.174 & 14.5 & 0.116 & 0.387 & 11.7 & 0.477 \\
\hline 7/13/2009 12:00 AM & 0.177 & 0.350 & 18.4 & 0.183 & 0.121 & 17.2 & 0.13 & 0.120 & 0.174 & 14.6 & 0.116 & 0.388 & 11.8 & 0.477 \\
\hline 7/13/2009 4:00 AM & 0.176 & 0.351 & 18.5 & 0.183 & 0.121 & 17.2 & 0.13 & 0.120 & 0.175 & 14.6 & 0.116 & 0.403 & 11.7 & 0.477 \\
\hline 7/13/2009 8:00 AM & 0.175 & 0.351 & 18.5 & 0.183 & 0.121 & 17.2 & 0.13 & 0.119 & 0.175 & 14.6 & 0.116 & 0.399 & 11.8 & 0.477 \\
\hline 7/13/2009 12:00 PM & 0.174 & 0.351 & 18.5 & 0.183 & 0.121 & 17.2 & 0.13 & 0.120 & 0.174 & 14.6 & 0.116 & 0.395 & 11.8 & 0.477 \\
\hline 7/13/2009 4:00 PM & 0.173 & 0.351 & 18.5 & 0.183 & 0.121 & 17.2 & 0.13 & 0.120 & 0.174 & 14.6 & 0.116 & 0.395 & 11.8 & 0.477 \\
\hline 7/13/2009 8:00 PM & 0.172 & 0.350 & 18.4 & 0.183 & 0.121 & 17.3 & 0.13 & 0.120 & 0.174 & 14.6 & 0.116 & 0.401 & 11.8 & 0.477 \\
\hline 7/14/2009 12:00 AM & 0.171 & 0.349 & 18.4 & 0.183 & 0.121 & 17.3 & 0.13 & 0.119 & 0.174 & 14.6 & 0.116 & 0.407 & 11.8 & 0.477 \\
\hline 7/14/2009 4:00 AM & 0.170 & 0.348 & 18.4 & 0.183 & 0.121 & 17.3 & 0.13 & 0.119 & 0.174 & 14.6 & 0.116 & 0.400 & 11.8 & 0.477 \\
\hline 7/14/2009 8:00 AM & 0.180 & 0.349 & 18.4 & 0.183 & 0.121 & 17.3 & 0.13 & 0.119 & 0.174 & 14.6 & 0.116 & 0.385 & 11.8 & 0.478 \\
\hline 7/14/2009 12:00 PM & 0.194 & 0.350 & 18.4 & 0.183 & 0.121 & 17.3 & 0.13 & 0.120 & 0.174 & 14.6 & 0.116 & 0.384 & 11.8 & 0.478 \\
\hline 7/14/2009 4:00 PM & 0.190 & 0.348 & 18.3 & 0.183 & 0.121 & 17.3 & 0.13 & 0.120 & 0.174 & 14.6 & 0.116 & 0.394 & 11.8 & 0.478 \\
\hline 7/14/2009 8:00 PM & 0.187 & 0.348 & 18.3 & 0.183 & 0.121 & 17.3 & 0.13 & 0.119 & 0.174 & 14.6 & 0.116 & 0.397 & 11.8 & 0.478 \\
\hline 7/15/2009 12:00 AM & 0.184 & 0.348 & 18.3 & 0.182 & 0.121 & 17.3 & 0.13 & 0.119 & 0.175 & 14.6 & 0.116 & 0.401 & 11.8 & 0.478 \\
\hline 7/15/2009 4:00 AM & 0.183 & 0.348 & 18.3 & 0.183 & 0.120 & 17.3 & 0.13 & 0.119 & 0.175 & 14.6 & 0.116 & 0.400 & 11.8 & 0.478 \\
\hline 7/15/2009 8:00 AM & 0.182 & 0.349 & 18.4 & 0.182 & 0.120 & 17.3 & 0.13 & 0.119 & 0.175 & 14.6 & 0.117 & 0.397 & 11.9 & 0.478 \\
\hline 7/15/2009 12:00 PM & 0.180 & 0.348 & 18.3 & 0.182 & 0.120 & 17.3 & 0.13 & 0.119 & 0.174 & 14.6 & 0.117 & 0.401 & 11.9 & 0.478 \\
\hline 7/15/2009 4:00 PM & 0.178 & 0.348 & 18.2 & 0.182 & 0.120 & 17.3 & 0.13 & 0.119 & 0.174 & 14.7 & 0.117 & 0.409 & 11.9 & 0.478 \\
\hline 7/15/2009 8:00 PM & 0.177 & 0.346 & 18.2 & 0.182 & 0.121 & 17.3 & 0.13 & 0.119 & 0.174 & 14.7 & 0.117 & 0.415 & 11.9 & 0.478 \\
\hline 7/16/2009 12:00 AM & 0.176 & 0.346 & 18.1 & 0.182 & 0.120 & 17.3 & 0.13 & 0.119 & 0.175 & 14.7 & 0.117 & 0.419 & 11.9 & 0.478 \\
\hline 7/16/2009 4:00 AM & 0.175 & 0.347 & 18.2 & 0.182 & 0.120 & 17.3 & 0.13 & 0.119 & 0.174 & 14.7 & 0.117 & 0.419 & 11.9 & 0.478 \\
\hline 7/16/2009 8:00 AM & 0.174 & 0.346 & 18.2 & 0.182 & 0.120 & 17.3 & 0.13 & 0.119 & 0.175 & 14.7 & 0.117 & 0.419 & 11.9 & 0.478 \\
\hline 7/16/2009 12:00 PM & 0.173 & 0.347 & 18.2 & 0.182 & 0.120 & 17.3 & 0.13 & 0.119 & 0.174 & 14.7 & 0.117 & 0.424 & 11.9 & 0.478 \\
\hline
\end{tabular}


Appendix 4. Volumetric water content, temperature, and electrical-conductivity data collected at the sprinkler-irrigated site during 2009.-Continued

[Depth in feet below land surface; volumetric water content dimensionless; temperature in degrees Celsius; bulk electrical conductivity in decisiemens per meter; --, no data]

\begin{tabular}{|c|c|c|c|c|c|c|c|c|c|c|c|c|c|c|}
\hline \multirow[b]{3}{*}{$\begin{array}{l}\text { Measurement } \\
\text { date and time }\end{array}$} & \multirow[b]{3}{*}{$\begin{array}{c}1 \\
\text { Volumetric } \\
\text { water } \\
\text { content }\end{array}$} & & & \multirow[b]{3}{*}{$\begin{array}{c}3 \\
\text { Volumetric } \\
\text { water } \\
\text { content }\end{array}$} & \multirow{2}{*}{\multicolumn{3}{|c|}{4}} & \multirow{3}{*}{$\begin{array}{c}\text { epth } \\
\text { Volumetric } \\
\text { water } \\
\text { content }\end{array}$} & \multirow{2}{*}{\multicolumn{2}{|c|}{7}} & \multirow{3}{*}{$\begin{array}{c}9 \\
\text { Volumetric } \\
\text { water } \\
\text { content }\end{array}$} & \multirow{2}{*}{\multicolumn{2}{|c|}{13}} & \multirow[b]{3}{*}{$\begin{array}{c}16.5 \\
\text { Volumetric } \\
\text { water } \\
\text { content }\end{array}$} \\
\hline & & \multicolumn{2}{|c|}{2} & & & & & & & & & & & \\
\hline & & $\begin{array}{c}\text { Volumetric } \\
\text { water } \\
\text { content }\end{array}$ & $\begin{array}{l}\text { Temper- } \\
\text { ature }\end{array}$ & & $\begin{array}{c}\text { Volumetric } \\
\text { water } \\
\text { content }\end{array}$ & $\begin{array}{l}\text { Temper- } \\
\text { ature }\end{array}$ & $\begin{array}{c}\text { Bulk } \\
\text { electrical } \\
\text { conduct- } \\
\text { ivity }\end{array}$ & & $\begin{array}{c}\text { Volumetric } \\
\text { water } \\
\text { content }\end{array}$ & $\begin{array}{l}\text { Temper- } \\
\text { ature }\end{array}$ & & $\begin{array}{c}\text { Volumetric } \\
\text { water } \\
\text { content }\end{array}$ & $\begin{array}{l}\text { Temper- } \\
\text { ature }\end{array}$ & \\
\hline 7/16/2009 4:00 PM & 0.155 & 0.345 & 18.2 & 0.182 & 0.120 & 17.3 & 0.13 & 0.119 & 0.171 & 14.5 & 0.117 & 0.423 & 11.9 & 0.478 \\
\hline 7/16/2009 8:00 PM & 0.144 & 0.344 & 18.1 & 0.181 & 0.120 & 17.3 & 0.13 & 0.119 & 0.174 & 14.7 & 0.117 & 0.417 & 11.9 & 0.478 \\
\hline 7/17/2009 12:00 AM & 0.169 & 0.344 & 18.0 & 0.182 & 0.120 & 17.3 & 0.13 & 0.119 & 0.175 & 14.7 & 0.117 & 0.413 & 11.9 & 0.478 \\
\hline 7/17/2009 4:00 AM & 0.168 & 0.344 & 18.1 & 0.181 & 0.120 & 17.3 & 0.13 & 0.119 & 0.175 & 14.7 & 0.117 & 0.409 & 11.9 & 0.478 \\
\hline 7/17/2009 8:00 AM & 0.166 & 0.344 & 18.1 & 0.181 & 0.120 & 17.3 & 0.13 & 0.119 & 0.175 & 14.7 & 0.117 & 0.358 & 12.0 & 0.478 \\
\hline 7/17/2009 12:00 PM & 0.166 & 0.344 & 18.1 & 0.181 & 0.120 & 17.3 & 0.13 & 0.119 & 0.175 & 14.7 & 0.117 & 0.354 & 11.9 & 0.478 \\
\hline 7/17/2009 4:00 PM & 0.166 & 0.343 & 18.1 & 0.181 & 0.120 & 17.3 & 0.13 & 0.119 & 0.175 & 14.7 & 0.117 & 0.391 & 11.9 & 0.478 \\
\hline 7/17/2009 8:00 PM & 0.164 & 0.342 & 18.1 & 0.181 & 0.120 & 17.3 & 0.13 & 0.119 & 0.175 & 14.7 & 0.117 & 0.382 & 11.9 & 0.478 \\
\hline 7/18/2009 12:00 AM & 0.160 & 0.341 & 18.1 & 0.181 & 0.120 & 17.3 & 0.13 & 0.119 & 0.176 & 14.7 & 0.117 & 0.380 & 12.0 & 0.478 \\
\hline 7/18/2009 4:00 AM & 0.162 & 0.342 & 18.1 & 0.181 & 0.120 & 17.3 & 0.13 & 0.119 & 0.176 & 14.7 & 0.117 & 0.385 & 12.0 & 0.478 \\
\hline 7/18/2009 8:00 AM & 0.169 & 0.342 & 18.2 & 0.181 & 0.120 & 17.3 & 0.13 & 0.119 & 0.176 & 14.7 & 0.117 & 0.392 & 12.0 & 0.478 \\
\hline 7/18/2009 12:00 PM & 0.176 & 0.342 & 18.2 & 0.180 & 0.120 & 17.3 & 0.13 & 0.119 & 0.175 & 14.7 & 0.117 & 0.381 & 12.0 & 0.478 \\
\hline 7/18/2009 4:00 PM & 0.177 & 0.341 & 18.2 & 0.180 & 0.120 & 17.3 & 0.13 & 0.118 & 0.175 & 14.7 & 0.117 & 0.380 & 12.0 & 0.478 \\
\hline 7/18/2009 8:00 PM & 0.176 & 0.341 & 18.1 & 0.180 & 0.120 & 17.3 & 0.13 & 0.118 & 0.175 & 14.7 & 0.117 & 0.381 & 12.0 & 0.478 \\
\hline 7/19/2009 12:00 AM & 0.174 & 0.341 & 18.1 & 0.180 & 0.120 & 17.3 & 0.13 & 0.118 & 0.175 & 14.7 & 0.117 & 0.379 & 12.0 & 0.478 \\
\hline 7/19/2009 4:00 AM & 0.173 & 0.341 & 18.2 & 0.180 & 0.120 & 17.3 & 0.13 & 0.118 & 0.175 & 14.7 & 0.117 & 0.379 & 12.0 & 0.478 \\
\hline 7/19/2009 8:00 AM & 0.172 & 0.341 & 18.2 & 0.180 & 0.119 & 17.3 & 0.13 & 0.118 & 0.176 & 14.7 & 0.117 & 0.380 & 12.0 & 0.478 \\
\hline 7/19/2009 12:00 PM & 0.171 & 0.342 & 18.2 & 0.179 & 0.120 & 17.3 & 0.13 & 0.118 & 0.175 & 14.7 & 0.117 & 0.380 & 12.0 & 0.478 \\
\hline 7/19/2009 4:00 PM & 0.210 & 0.340 & 18.2 & 0.179 & 0.120 & 17.3 & 0.13 & 0.119 & 0.176 & 14.7 & 0.117 & 0.379 & 12.0 & 0.478 \\
\hline 7/19/2009 8:00 PM & 0.221 & 0.338 & 18.2 & 0.179 & 0.120 & 17.3 & 0.13 & 0.119 & 0.176 & 14.7 & 0.117 & 0.379 & 12.0 & 0.478 \\
\hline 7/20/2009 12:00 AM & 0.209 & 0.340 & 18.2 & 0.179 & 0.120 & 17.3 & 0.13 & 0.118 & 0.176 & 14.7 & 0.117 & 0.379 & 12.0 & 0.478 \\
\hline 7/20/2009 4:00 AM & 0.205 & 0.340 & 18.2 & 0.179 & 0.119 & 17.3 & 0.13 & 0.118 & 0.178 & 14.8 & 0.117 & 0.376 & 12.0 & 0.478 \\
\hline 7/20/2009 8:00 AM & 0.201 & 0.341 & 18.3 & 0.179 & 0.120 & 17.3 & 0.13 & 0.118 & 0.176 & 14.7 & 0.117 & 0.375 & 12.0 & 0.478 \\
\hline 7/20/2009 12:00 PM & 0.198 & 0.343 & 18.3 & 0.179 & 0.120 & 17.3 & 0.13 & 0.118 & 0.176 & 14.7 & 0.117 & 0.376 & 12.0 & 0.478 \\
\hline 7/20/2009 4:00 PM & 0.192 & 0.342 & 18.3 & 0.179 & 0.119 & 17.3 & 0.13 & 0.118 & 0.176 & 14.8 & 0.117 & 0.378 & 12.1 & 0.478 \\
\hline 7/20/2009 8:00 PM & 0.188 & 0.342 & 18.2 & 0.179 & 0.119 & 17.3 & 0.13 & 0.118 & 0.176 & 14.8 & 0.117 & 0.378 & 12.1 & 0.478 \\
\hline 7/21/2009 12:00 AM & 0.187 & 0.341 & 18.2 & 0.179 & 0.119 & 17.3 & 0.13 & 0.118 & 0.175 & 14.8 & 0.117 & 0.378 & 12.1 & 0.478 \\
\hline 7/21/2009 4:00 AM & 0.250 & 0.344 & 18.3 & 0.179 & 0.119 & 17.3 & 0.13 & 0.118 & 0.176 & 14.8 & 0.117 & 0.376 & 12.1 & 0.478 \\
\hline 7/21/2009 8:00 AM & 0.216 & 0.357 & 18.3 & 0.180 & 0.119 & 17.3 & 0.13 & 0.118 & 0.176 & 14.8 & 0.117 & 0.376 & 12.1 & 0.479 \\
\hline 7/21/2009 12:00 PM & 0.211 & 0.362 & 18.3 & 0.181 & 0.119 & 17.3 & 0.13 & 0.118 & 0.175 & 14.8 & 0.117 & 0.378 & 12.1 & 0.479 \\
\hline 7/21/2009 4:00 PM & 0.205 & 0.365 & 18.3 & 0.182 & 0.119 & 17.3 & 0.13 & 0.118 & 0.175 & 14.8 & 0.117 & 0.378 & 12.1 & 0.479 \\
\hline 7/21/2009 8:00 PM & 0.200 & 0.365 & 18.2 & 0.183 & 0.119 & 17.3 & 0.13 & 0.118 & 0.175 & 14.8 & 0.117 & 0.378 & 12.1 & 0.479 \\
\hline 7/22/2009 12:00 AM & 0.198 & 0.363 & 18.2 & 0.183 & 0.119 & 17.3 & 0.13 & 0.118 & 0.175 & 14.8 & 0.117 & 0.379 & 12.1 & 0.478 \\
\hline 7/22/2009 4:00 AM & 0.196 & 0.365 & 18.2 & 0.184 & 0.119 & 17.3 & 0.13 & 0.118 & 0.175 & 14.8 & 0.117 & 0.376 & 12.1 & 0.478 \\
\hline 7/22/2009 8:00 AM & 0.195 & 0.365 & 18.2 & 0.185 & 0.119 & 17.3 & 0.13 & 0.118 & 0.175 & 14.8 & 0.117 & 0.375 & 12.1 & 0.478 \\
\hline 7/22/2009 12:00 PM & 0.194 & 0.365 & 18.2 & 0.186 & 0.120 & 17.3 & 0.13 & 0.118 & 0.175 & 14.8 & 0.117 & 0.378 & 12.1 & 0.479 \\
\hline 7/22/2009 4:00 PM & 0.190 & 0.363 & 18.1 & 0.187 & 0.120 & 17.4 & 0.13 & 0.118 & 0.175 & 14.8 & 0.117 & 0.378 & 12.1 & 0.479 \\
\hline
\end{tabular}


Appendix 4. Volumetric water content, temperature, and electrical-conductivity data collected at the sprinkler-irrigated site during 2009.-Continued

[Depth in feet below land surface; volumetric water content dimensionless; temperature in degrees Celsius; bulk electrical conductivity in decisiemens per meter; --, no data]

\begin{tabular}{|c|c|c|c|c|c|c|c|c|c|c|c|c|c|c|}
\hline \multirow[b]{3}{*}{$\begin{array}{l}\text { Measurement } \\
\text { date and time }\end{array}$} & \multicolumn{14}{|c|}{ Depth } \\
\hline & \multirow{2}{*}{$\begin{array}{c}1 \\
\text { Volumetric } \\
\text { water } \\
\text { content }\end{array}$} & \multicolumn{2}{|c|}{2} & \multirow{2}{*}{$\begin{array}{c}3 \\
\text { Volumetric } \\
\text { water } \\
\text { content }\end{array}$} & \multicolumn{3}{|c|}{4} & \multirow{2}{*}{$\begin{array}{c}5 \\
\text { Volumetric } \\
\text { water } \\
\text { content }\end{array}$} & \multicolumn{2}{|l|}{7} & \multirow{2}{*}{$\begin{array}{c}9 \\
\text { Volumetric } \\
\text { water } \\
\text { content }\end{array}$} & \multicolumn{2}{|c|}{13} & \multirow{2}{*}{$\begin{array}{c}16.5 \\
\text { Volumetric } \\
\text { water } \\
\text { content }\end{array}$} \\
\hline & & $\begin{array}{c}\text { Volumetric } \\
\text { water } \\
\text { content }\end{array}$ & $\begin{array}{l}\text { Temper- } \\
\text { ature }\end{array}$ & & $\begin{array}{c}\text { Volumetric } \\
\text { water } \\
\text { content }\end{array}$ & $\begin{array}{l}\text { Temper- } \\
\text { ature }\end{array}$ & $\begin{array}{c}\text { Bulk } \\
\text { electrical } \\
\text { conduct- } \\
\text { ivity }\end{array}$ & & $\begin{array}{c}\text { Volumetric } \\
\text { water } \\
\text { content }\end{array}$ & $\begin{array}{l}\text { Temper- } \\
\text { ature }\end{array}$ & & $\begin{array}{c}\text { Volumetric } \\
\text { water } \\
\text { content }\end{array}$ & $\begin{array}{l}\text { Temper- } \\
\text { ature }\end{array}$ & \\
\hline 7/22/2009 8:00 PM & 0.188 & 0.363 & 18.0 & 0.187 & 0.120 & 17.4 & 0.13 & 0.118 & 0.175 & 14.8 & 0.117 & 0.378 & 12.1 & 0.479 \\
\hline 7/23/2009 12:00 AM & 0.187 & 0.362 & 18.0 & 0.188 & 0.120 & 17.4 & 0.13 & 0.118 & 0.175 & 14.8 & 0.117 & 0.378 & 12.2 & 0.479 \\
\hline 7/23/2009 4:00 AM & 0.186 & 0.363 & 18.1 & 0.188 & 0.120 & 17.4 & 0.13 & 0.118 & 0.175 & 14.7 & 0.117 & 0.378 & 12.2 & 0.479 \\
\hline 7/23/2009 8:00 AM & 0.212 & 0.363 & 18.1 & 0.188 & 0.120 & 17.4 & 0.13 & 0.118 & 0.175 & 14.8 & 0.117 & 0.379 & 12.2 & 0.479 \\
\hline 7/23/2009 12:00 PM & 0.242 & 0.369 & 18.1 & 0.188 & 0.120 & 17.4 & 0.13 & 0.119 & 0.175 & 14.8 & 0.117 & 0.380 & 12.2 & 0.479 \\
\hline 7/23/2009 4:00 PM & 0.216 & 0.373 & 18.1 & 0.189 & 0.120 & 17.4 & 0.13 & 0.119 & 0.175 & 14.7 & 0.117 & 0.380 & 12.2 & 0.478 \\
\hline 7/23/2009 8:00 PM & 0.206 & 0.375 & 18.0 & 0.191 & 0.121 & 17.4 & 0.13 & 0.119 & 0.175 & 14.7 & 0.118 & 0.380 & 12.2 & 0.478 \\
\hline 7/24/2009 12:00 AM & 0.203 & 0.374 & 18.0 & 0.192 & 0.120 & 17.4 & 0.13 & 0.119 & 0.175 & 14.8 & 0.118 & 0.381 & 12.2 & 0.479 \\
\hline 7/24/2009 4:00 AM & 0.201 & 0.374 & 18.0 & 0.193 & 0.121 & 17.4 & 0.13 & 0.119 & 0.175 & 14.7 & 0.118 & 0.380 & 12.2 & 0.479 \\
\hline 7/24/2009 8:00 AM & 0.199 & 0.375 & 18.1 & 0.194 & 0.121 & 17.4 & 0.13 & 0.119 & 0.175 & 14.7 & 0.118 & 0.380 & 12.2 & 0.479 \\
\hline 7/24/2009 12:00 PM & 0.197 & 0.374 & 18.1 & 0.194 & 0.121 & 17.4 & 0.13 & 0.119 & 0.175 & 14.8 & 0.118 & 0.380 & 12.2 & 0.479 \\
\hline 7/24/2009 4:00 PM & 0.193 & 0.373 & 18.1 & 0.194 & 0.121 & 17.4 & 0.13 & 0.120 & 0.176 & 14.8 & 0.118 & 0.381 & 12.2 & 0.479 \\
\hline 7/24/2009 8:00 PM & 0.189 & 0.372 & 18.0 & 0.194 & 0.122 & 17.4 & 0.13 & 0.120 & 0.175 & 14.8 & 0.118 & 0.381 & 12.2 & 0.479 \\
\hline 7/25/2009 12:00 AM & 0.188 & 0.372 & 18.0 & 0.194 & 0.122 & 17.4 & 0.13 & 0.120 & 0.175 & 14.8 & 0.118 & 0.380 & 12.2 & 0.479 \\
\hline 7/25/2009 4:00 AM & 0.188 & 0.371 & 18.1 & 0.194 & 0.122 & 17.4 & 0.14 & 0.120 & 0.175 & 14.8 & 0.118 & 0.380 & 12.2 & 0.479 \\
\hline 7/25/2009 8:00 AM & 0.186 & 0.371 & 18.2 & 0.194 & 0.122 & 17.4 & 0.14 & 0.120 & 0.175 & 14.8 & 0.118 & 0.379 & 12.2 & 0.479 \\
\hline 7/25/2009 12:00 PM & 0.186 & 0.372 & 18.2 & 0.194 & 0.122 & 17.4 & 0.14 & 0.120 & 0.175 & 14.8 & 0.118 & 0.380 & 12.2 & 0.479 \\
\hline 7/25/2009 4:00 PM & 0.183 & 0.371 & 18.2 & 0.194 & 0.123 & 17.4 & 0.14 & 0.121 & 0.176 & 14.8 & 0.118 & 0.380 & 12.3 & 0.479 \\
\hline 7/25/2009 8:00 PM & 0.188 & 0.370 & 18.2 & 0.193 & 0.123 & 17.4 & 0.14 & 0.121 & 0.175 & 14.8 & 0.118 & 0.380 & 12.2 & 0.479 \\
\hline 7/26/2009 12:00 AM & 0.238 & 0.370 & 18.2 & 0.193 & 0.123 & 17.4 & 0.14 & 0.121 & 0.175 & 14.8 & 0.118 & 0.382 & 12.2 & 0.479 \\
\hline 7/26/2009 4:00 AM & 0.230 & 0.372 & 18.3 & 0.194 & 0.123 & 17.4 & 0.14 & 0.121 & 0.175 & 14.8 & 0.118 & 0.381 & 12.2 & 0.479 \\
\hline 7/26/2009 8:00 AM & 0.217 & 0.375 & 18.3 & 0.194 & 0.123 & 17.4 & 0.14 & 0.121 & 0.176 & 14.8 & 0.118 & 0.380 & 12.2 & 0.479 \\
\hline 7/26/2009 12:00 PM & 0.211 & 0.376 & 18.3 & 0.194 & 0.122 & 17.4 & 0.14 & 0.121 & 0.176 & 14.8 & 0.118 & 0.380 & 12.3 & 0.479 \\
\hline 7/26/2009 4:00 PM & 0.205 & 0.376 & 18.3 & 0.194 & 0.123 & 17.4 & 0.14 & 0.121 & 0.176 & 14.8 & 0.119 & 0.380 & 12.3 & 0.479 \\
\hline 7/26/2009 8:00 PM & 0.201 & 0.376 & 18.2 & 0.195 & 0.123 & 17.4 & 0.14 & 0.121 & 0.176 & 14.8 & 0.118 & 0.380 & 12.3 & 0.479 \\
\hline 7/27/2009 12:00 AM & 0.200 & 0.376 & 18.2 & 0.195 & 0.123 & 17.4 & 0.14 & 0.121 & 0.176 & 14.8 & 0.119 & 0.381 & 12.3 & 0.479 \\
\hline 7/27/2009 4:00 AM & 0.199 & 0.376 & 18.3 & 0.195 & 0.123 & 17.4 & 0.14 & 0.121 & 0.176 & 14.8 & 0.119 & 0.381 & 12.3 & 0.479 \\
\hline 7/27/2009 8:00 AM & 0.197 & 0.375 & 18.4 & 0.195 & 0.123 & 17.4 & 0.14 & 0.121 & 0.176 & 14.8 & 0.119 & 0.380 & 12.3 & 0.479 \\
\hline 7/27/2009 12:00 PM & 0.195 & 0.378 & 18.4 & 0.195 & 0.123 & 17.4 & 0.14 & 0.121 & 0.178 & 14.8 & 0.119 & 0.379 & 12.3 & 0.479 \\
\hline 7/27/2009 4:00 PM & 0.192 & 0.375 & 18.4 & 0.195 & 0.123 & 17.4 & 0.14 & 0.121 & 0.178 & 14.8 & 0.119 & 0.379 & 12.3 & 0.479 \\
\hline 7/27/2009 8:00 PM & 0.188 & 0.373 & 18.3 & 0.195 & 0.124 & 17.4 & 0.14 & 0.122 & 0.178 & 14.8 & 0.119 & 0.380 & 12.3 & 0.479 \\
\hline 7/28/2009 12:00 AM & 0.189 & 0.373 & 18.3 & 0.195 & 0.124 & 17.4 & 0.14 & 0.122 & 0.176 & 14.8 & 0.119 & 0.381 & 12.3 & 0.479 \\
\hline 7/28/2009 4:00 AM & 0.188 & 0.373 & 18.4 & 0.195 & 0.124 & 17.4 & 0.14 & 0.122 & 0.176 & 14.8 & 0.119 & 0.379 & 12.3 & 0.479 \\
\hline 7/28/2009 8:00 AM & 0.188 & 0.373 & 18.3 & 0.195 & 0.124 & 17.4 & 0.14 & 0.122 & 0.176 & 14.8 & 0.119 & 0.379 & 12.3 & 0.479 \\
\hline 7/28/2009 12:00 PM & 0.190 & 0.372 & 18.3 & 0.195 & 0.124 & 17.5 & 0.14 & 0.122 & 0.178 & 14.8 & 0.119 & 0.381 & 12.3 & 0.479 \\
\hline 7/28/2009 4:00 PM & 0.189 & 0.372 & 18.3 & 0.194 & 0.124 & 17.5 & 0.14 & 0.122 & 0.178 & 14.8 & 0.119 & 0.382 & 12.3 & 0.479 \\
\hline 7/28/2009 8:00 PM & 0.188 & 0.371 & 18.2 & 0.194 & 0.124 & 17.5 & 0.14 & 0.122 & 0.178 & 14.8 & 0.119 & 0.382 & 12.3 & 0.479 \\
\hline
\end{tabular}


Appendix 4. Volumetric water content, temperature, and electrical-conductivity data collected at the sprinkler-irrigated site during 2009.-Continued [Depth in feet below land surface; volumetric water content dimensionless; temperature in degrees Celsius; bulk electrical conductivity in decisiemens per meter; --, no data]

\begin{tabular}{|c|c|c|c|c|c|c|c|c|c|c|c|c|c|c|}
\hline \multirow[b]{3}{*}{$\begin{array}{l}\text { Measurement } \\
\text { date and time }\end{array}$} & \multicolumn{14}{|c|}{ Depth } \\
\hline & \multirow{2}{*}{$\begin{array}{c}1 \\
\text { Volumetric } \\
\text { water } \\
\text { content }\end{array}$} & \multicolumn{2}{|c|}{2} & \multirow{2}{*}{$\begin{array}{c}3 \\
\text { Volumetric } \\
\text { water } \\
\text { content }\end{array}$} & \multicolumn{3}{|c|}{4} & \multirow{2}{*}{$\begin{array}{c}5 \\
\text { Volumetric } \\
\text { water } \\
\text { content } \\
\end{array}$} & \multicolumn{2}{|l|}{7} & \multirow{2}{*}{$\begin{array}{c}9 \\
\text { Volumetric } \\
\text { water } \\
\text { content }\end{array}$} & \multicolumn{2}{|c|}{13} & \multirow{2}{*}{$\begin{array}{c}16.5 \\
\text { Volumetric } \\
\text { water } \\
\text { content }\end{array}$} \\
\hline & & $\begin{array}{c}\text { Volumetric } \\
\text { water } \\
\text { content }\end{array}$ & $\begin{array}{l}\text { Temper- } \\
\text { ature }\end{array}$ & & $\begin{array}{c}\text { Volumetric } \\
\text { water } \\
\text { content }\end{array}$ & $\begin{array}{l}\text { Temper- } \\
\text { ature }\end{array}$ & $\begin{array}{c}\text { Bulk } \\
\text { electrical } \\
\text { conduct- } \\
\text { ivity }\end{array}$ & & $\begin{array}{c}\text { Volumetric } \\
\text { water } \\
\text { content }\end{array}$ & $\begin{array}{l}\text { Temper- } \\
\text { ature }\end{array}$ & & $\begin{array}{l}\text { Volumetric } \\
\text { water } \\
\text { content }\end{array}$ & $\begin{array}{l}\text { Temper- } \\
\text { ature }\end{array}$ & \\
\hline$\overline{7 / 29 / 2009 ~ 12: 00 \mathrm{AM}}$ & 0.187 & 0.370 & 18.2 & 0.194 & 0.124 & 17.5 & 0.14 & 0.122 & 0.178 & 14.8 & 0.120 & 0.384 & 12.3 & 0.479 \\
\hline 7/29/2009 4:00 AM & 0.184 & 0.369 & 18.2 & 0.194 & 0.124 & 17.5 & 0.14 & 0.122 & 0.178 & 14.8 & 0.120 & 0.383 & 12.3 & 0.479 \\
\hline 7/29/2009 8:00 AM & 0.187 & 0.369 & 18.2 & 0.194 & 0.124 & 17.5 & 0.14 & 0.123 & 0.178 & 14.8 & 0.120 & 0.380 & 12.4 & 0.479 \\
\hline 7/29/2009 12:00 PM & 0.192 & 0.369 & 18.2 & 0.194 & 0.124 & 17.5 & 0.14 & 0.123 & 0.178 & 14.8 & 0.120 & 0.379 & 12.4 & 0.480 \\
\hline 7/29/2009 4:00 PM & 0.194 & 0.369 & 18.1 & 0.194 & 0.124 & 17.5 & 0.14 & 0.123 & 0.179 & 14.8 & 0.120 & 0.379 & 12.4 & 0.479 \\
\hline 7/29/2009 8:00 PM & 0.261 & 0.369 & 18.0 & 0.194 & 0.124 & 17.5 & 0.14 & 0.123 & 0.178 & 14.9 & 0.120 & 0.379 & 12.4 & 0.479 \\
\hline 7/30/2009 12:00 AM & 0.228 & 0.372 & 18.0 & 0.194 & 0.124 & 17.5 & 0.14 & 0.123 & 0.178 & 14.9 & 0.120 & 0.380 & 12.4 & 0.479 \\
\hline 7/30/2009 4:00 AM & 0.229 & 0.374 & 17.9 & 0.194 & 0.124 & 17.5 & 0.14 & 0.123 & 0.178 & 14.9 & 0.121 & 0.380 & 12.4 & 0.480 \\
\hline 7/30/2009 8:00 AM & 0.219 & 0.378 & 17.8 & 0.195 & 0.124 & 17.5 & 0.14 & 0.123 & 0.178 & 14.9 & 0.121 & 0.381 & 12.4 & 0.479 \\
\hline 7/30/2009 12:00 PM & 0.216 & 0.381 & 17.8 & 0.196 & 0.124 & 17.5 & 0.14 & 0.123 & 0.178 & 14.9 & 0.121 & 0.380 & 12.4 & 0.479 \\
\hline 7/30/2009 4:00 PM & 0.211 & 0.381 & 17.7 & 0.197 & 0.124 & 17.5 & 0.14 & 0.123 & 0.178 & 14.9 & 0.121 & 0.381 & 12.4 & 0.480 \\
\hline 7/30/2009 8:00 PM & 0.208 & 0.381 & 17.6 & 0.197 & 0.124 & 17.5 & 0.14 & 0.123 & 0.178 & 14.9 & 0.121 & 0.380 & 12.4 & 0.479 \\
\hline 7/31/2009 12:00 AM & 0.205 & 0.379 & 17.5 & 0.198 & 0.124 & 17.5 & 0.14 & 0.123 & 0.178 & 14.9 & 0.121 & 0.380 & 12.4 & 0.480 \\
\hline 7/31/2009 4:00 AM & 0.203 & 0.380 & 17.4 & 0.199 & 0.124 & 17.5 & 0.14 & 0.123 & 0.178 & 14.9 & 0.121 & 0.381 & 12.5 & 0.479 \\
\hline 7/31/2009 8:00 AM & 0.200 & 0.379 & 17.4 & 0.199 & 0.124 & 17.4 & 0.14 & 0.123 & 0.178 & 14.9 & 0.121 & 0.380 & 12.5 & 0.480 \\
\hline 7/31/2009 12:00 PM & 0.198 & 0.379 & 17.3 & 0.199 & 0.125 & 17.4 & 0.14 & 0.123 & 0.179 & 14.9 & 0.121 & 0.380 & 12.4 & 0.480 \\
\hline 7/31/2009 4:00 PM & 0.194 & 0.378 & 17.2 & 0.199 & 0.125 & 17.4 & 0.14 & 0.124 & 0.179 & 14.9 & 0.121 & 0.382 & 12.5 & 0.479 \\
\hline 7/31/2009 8:00 PM & 0.190 & 0.376 & 17.1 & 0.198 & 0.125 & 17.4 & 0.14 & 0.124 & 0.178 & 14.9 & 0.121 & 0.381 & 12.5 & 0.479 \\
\hline 8/1/2009 12:00 AM & 0.192 & 0.376 & 17.1 & 0.198 & 0.125 & 17.4 & 0.14 & 0.124 & 0.178 & 14.9 & 0.121 & 0.381 & 12.5 & 0.479 \\
\hline 8/1/2009 4:00 AM & 0.192 & 0.375 & 17.1 & 0.198 & 0.125 & 17.4 & 0.14 & 0.124 & 0.178 & 14.9 & 0.121 & 0.381 & 12.5 & 0.479 \\
\hline 8/1/2009 8:00 AM & 0.191 & 0.375 & 17.1 & 0.198 & 0.126 & 17.4 & 0.14 & 0.124 & 0.178 & 14.9 & 0.121 & 0.381 & 12.5 & 0.479 \\
\hline 8/1/2009 12:00 PM & 0.188 & 0.374 & 17.0 & 0.198 & 0.126 & 17.3 & 0.14 & 0.125 & 0.179 & 14.9 & 0.121 & 0.382 & 12.5 & 0.479 \\
\hline 8/1/2009 4:00 PM & 0.186 & 0.373 & 17.0 & 0.197 & 0.126 & 17.3 & 0.14 & 0.126 & 0.179 & 14.9 & 0.121 & 0.383 & 12.5 & 0.479 \\
\hline 8/1/2009 8:00 PM & 0.183 & 0.371 & 17.0 & 0.197 & 0.126 & 17.3 & 0.14 & 0.126 & 0.179 & 14.9 & 0.121 & 0.382 & 12.5 & 0.479 \\
\hline 8/2/2009 12:00 AM & 0.182 & 0.370 & 17.0 & 0.197 & 0.126 & 17.3 & 0.14 & 0.126 & 0.179 & 14.9 & 0.121 & 0.381 & 12.5 & 0.479 \\
\hline 8/2/2009 4:00 AM & 0.182 & 0.370 & 17.0 & 0.196 & 0.126 & 17.3 & 0.14 & 0.126 & 0.179 & 14.9 & 0.121 & 0.382 & 12.5 & 0.479 \\
\hline 8/2/2009 8:00 AM & 0.183 & 0.370 & 17.1 & 0.196 & 0.126 & 17.3 & 0.14 & 0.126 & 0.178 & 14.9 & 0.121 & 0.382 & 12.5 & 0.479 \\
\hline 8/2/2009 12:00 PM & 0.182 & 0.370 & 17.1 & 0.196 & 0.126 & 17.2 & 0.14 & 0.126 & 0.179 & 14.9 & 0.121 & 0.381 & 12.5 & 0.479 \\
\hline 8/2/2009 4:00 PM & 0.180 & 0.369 & 17.1 & 0.195 & 0.126 & 17.2 & 0.14 & 0.126 & 0.180 & 15.0 & 0.121 & 0.382 & 12.5 & 0.479 \\
\hline 8/2/2009 8:00 PM & 0.179 & 0.367 & 17.0 & 0.195 & 0.126 & 17.2 & 0.14 & 0.126 & 0.179 & 15.0 & 0.122 & 0.382 & 12.5 & 0.479 \\
\hline 8/3/2009 12:00 AM & 0.177 & 0.367 & 17.1 & 0.195 & 0.126 & 17.2 & 0.14 & 0.126 & 0.179 & 14.9 & 0.122 & 0.382 & 12.5 & 0.479 \\
\hline 8/3/2009 4:00 AM & 0.177 & 0.367 & 17.2 & 0.194 & 0.126 & 17.2 & 0.14 & 0.126 & 0.179 & 15.0 & 0.122 & 0.382 & 12.5 & 0.479 \\
\hline 8/3/2009 8:00 AM & 0.177 & 0.367 & 17.3 & 0.194 & 0.126 & 17.2 & 0.14 & 0.126 & 0.179 & 14.9 & 0.122 & 0.384 & 12.5 & 0.479 \\
\hline 8/3/2009 12:00 PM & 0.180 & 0.366 & 17.3 & 0.194 & 0.126 & 17.2 & 0.14 & 0.126 & 0.179 & 15.0 & 0.122 & 0.386 & 12.5 & 0.480 \\
\hline 8/3/2009 4:00 PM & 0.183 & 0.365 & 17.3 & 0.194 & 0.126 & 17.1 & 0.14 & 0.126 & 0.180 & 15.0 & 0.122 & 0.386 & 12.5 & 0.479 \\
\hline 8/3/2009 8:00 PM & 0.183 & 0.363 & 17.3 & 0.194 & 0.125 & 17.1 & 0.14 & 0.126 & 0.179 & 15.0 & 0.122 & 0.387 & 12.6 & 0.479 \\
\hline 8/4/2009 12:00 AM & 0.183 & 0.363 & 17.3 & 0.194 & 0.125 & 17.1 & 0.14 & 0.126 & 0.179 & 14.9 & 0.122 & 0.387 & 12.6 & 0.479 \\
\hline
\end{tabular}


Appendix 4. Volumetric water content, temperature, and electrical-conductivity data collected at the sprinkler-irrigated site during 2009.-Continued

[Depth in feet below land surface; volumetric water content dimensionless; temperature in degrees Celsius; bulk electrical conductivity in decisiemens per meter; --, no data]

\begin{tabular}{|c|c|c|c|c|c|c|c|c|c|c|c|c|c|c|}
\hline \multirow[b]{3}{*}{$\begin{array}{l}\text { Measurement } \\
\text { date and time }\end{array}$} & \multicolumn{14}{|c|}{ Depth } \\
\hline & \multirow{2}{*}{$\begin{array}{c}1 \\
\text { Volumetric } \\
\text { water } \\
\text { content }\end{array}$} & \multicolumn{2}{|l|}{2} & \multirow{2}{*}{$\begin{array}{c}\frac{3}{\text { Volumetric }} \\
\text { water } \\
\text { content }\end{array}$} & \multicolumn{3}{|c|}{4} & \multirow{2}{*}{$\begin{array}{c}5 \\
\text { Volumetric } \\
\text { water } \\
\text { content }\end{array}$} & \multicolumn{2}{|l|}{7} & \multirow{2}{*}{$\begin{array}{c}9 \\
\text { Volumetric } \\
\text { water } \\
\text { content }\end{array}$} & \multicolumn{2}{|c|}{13} & \multirow{2}{*}{$\begin{array}{c}16.5 \\
\text { Volumetric } \\
\text { water } \\
\text { content }\end{array}$} \\
\hline & & $\begin{array}{c}\text { Volumetric } \\
\text { water } \\
\text { content }\end{array}$ & $\begin{array}{l}\text { Temper- } \\
\text { ature }\end{array}$ & & $\begin{array}{c}\text { Volumetric } \\
\text { water } \\
\text { content }\end{array}$ & $\begin{array}{l}\text { Temper- } \\
\text { ature }\end{array}$ & $\begin{array}{c}\text { Bulk } \\
\text { electrical } \\
\text { conduct- } \\
\text { ivity }\end{array}$ & & $\begin{array}{l}\text { Volumetric } \\
\text { water } \\
\text { content }\end{array}$ & $\begin{array}{l}\text { Temper- } \\
\text { ature }\end{array}$ & & $\begin{array}{l}\text { Volumetric } \\
\text { water } \\
\text { content }\end{array}$ & $\begin{array}{l}\text { Temper- } \\
\text { ature }\end{array}$ & \\
\hline 8/4/2009 4:00 AM & 0.183 & 0.362 & 17.4 & 0.194 & 0.125 & 17.1 & 0.14 & 0.126 & 0.179 & 15.0 & 0.122 & 0.385 & 12.6 & 0.479 \\
\hline 8/4/2009 8:00 AM & 0.183 & 0.363 & 17.4 & 0.194 & 0.125 & 17.1 & 0.14 & 0.126 & 0.179 & 15.0 & 0.122 & 0.384 & 12.6 & 0.479 \\
\hline 8/4/2009 12:00 PM & 0.183 & 0.363 & 17.4 & 0.193 & 0.125 & 17.1 & 0.14 & 0.126 & 0.180 & 15.0 & 0.122 & 0.384 & 12.6 & 0.479 \\
\hline 8/4/2009 4:00 PM & 0.182 & 0.362 & 17.4 & 0.193 & 0.125 & 17.1 & 0.14 & 0.126 & 0.180 & 15.0 & 0.122 & 0.385 & 12.6 & 0.479 \\
\hline 8/4/2009 8:00 PM & 0.180 & 0.361 & 17.4 & 0.193 & 0.125 & 17.1 & 0.14 & 0.126 & 0.180 & 15.0 & 0.122 & 0.384 & 12.6 & 0.479 \\
\hline 8/5/2009 12:00 AM & 0.179 & 0.361 & 17.4 & 0.193 & 0.124 & 17.1 & 0.14 & 0.126 & 0.179 & 14.9 & 0.122 & 0.385 & 12.6 & 0.479 \\
\hline 8/5/2009 4:00 AM & 0.178 & 0.361 & 17.4 & 0.192 & 0.124 & 17.1 & 0.14 & 0.126 & 0.179 & 14.9 & 0.122 & 0.385 & 12.6 & 0.479 \\
\hline 8/5/2009 8:00 AM & 0.177 & 0.361 & 17.5 & 0.192 & 0.124 & 17.1 & 0.14 & 0.126 & 0.179 & 14.9 & 0.122 & 0.385 & 12.6 & 0.480 \\
\hline 8/5/2009 12:00 PM & 0.177 & 0.361 & 17.5 & 0.192 & 0.124 & 17.1 & 0.14 & 0.126 & 0.179 & 14.9 & 0.122 & 0.385 & 12.6 & 0.479 \\
\hline 8/5/2009 4:00 PM & 0.177 & 0.359 & 17.5 & 0.192 & 0.124 & 17.1 & 0.14 & 0.126 & 0.180 & 15.0 & 0.122 & 0.385 & 12.6 & 0.479 \\
\hline 8/5/2009 8:00 PM & 0.176 & 0.358 & 17.4 & 0.191 & 0.124 & 17.1 & 0.14 & 0.125 & 0.179 & 15.0 & 0.122 & 0.385 & 12.6 & 0.479 \\
\hline 8/6/2009 12:00 AM & 0.175 & 0.358 & 17.5 & 0.191 & 0.124 & 17.1 & 0.14 & 0.125 & 0.179 & 14.9 & 0.122 & 0.385 & 12.6 & 0.480 \\
\hline 8/6/2009 4:00 AM & 0.182 & 0.358 & 17.5 & 0.191 & 0.124 & 17.1 & 0.14 & 0.125 & 0.179 & 14.9 & 0.122 & 0.385 & 12.6 & 0.480 \\
\hline 8/6/2009 8:00 AM & 0.209 & 0.359 & 17.6 & 0.191 & 0.123 & 17.1 & 0.14 & 0.125 & 0.179 & 14.9 & 0.122 & 0.385 & 12.6 & 0.480 \\
\hline 8/6/2009 12:00 PM & 0.205 & 0.360 & 17.6 & 0.190 & 0.123 & 17.1 & 0.14 & 0.125 & 0.179 & 15.0 & 0.122 & 0.385 & 12.6 & 0.480 \\
\hline 8/6/2009 4:00 PM & 0.201 & 0.360 & 17.7 & 0.190 & 0.124 & 17.1 & 0.14 & 0.125 & 0.180 & 15.0 & 0.122 & 0.385 & 12.6 & 0.480 \\
\hline 8/6/2009 8:00 PM & 0.197 & 0.360 & 17.7 & 0.190 & 0.123 & 17.1 & 0.14 & 0.125 & 0.179 & 14.9 & 0.122 & 0.385 & 12.6 & 0.480 \\
\hline 8/7/2009 12:00 AM & 0.195 & 0.360 & 17.7 & 0.190 & 0.123 & 17.1 & 0.14 & 0.124 & 0.179 & 14.9 & 0.122 & 0.385 & 12.7 & 0.480 \\
\hline 8/7/2009 4:00 AM & 0.194 & 0.361 & 17.8 & 0.190 & 0.123 & 17.1 & 0.14 & 0.124 & 0.179 & 14.9 & 0.122 & 0.385 & 12.7 & 0.479 \\
\hline 8/7/2009 8:00 AM & 0.194 & 0.361 & 17.8 & 0.190 & 0.123 & 17.1 & 0.14 & 0.124 & 0.179 & 14.9 & 0.122 & 0.385 & 12.7 & 0.479 \\
\hline 8/7/2009 12:00 PM & 0.193 & 0.362 & 17.8 & 0.190 & 0.123 & 17.2 & 0.14 & 0.124 & 0.179 & 14.9 & 0.122 & 0.386 & 12.7 & 0.480 \\
\hline 8/7/2009 4:00 PM & 0.189 & 0.361 & 17.8 & 0.190 & 0.123 & 17.2 & 0.14 & 0.124 & 0.180 & 15.0 & 0.122 & 0.386 & 12.7 & 0.479 \\
\hline 8/7/2009 8:00 PM & 0.186 & 0.360 & 17.8 & 0.190 & 0.123 & 17.2 & 0.14 & 0.124 & 0.180 & 15.0 & 0.122 & 0.385 & 12.6 & 0.479 \\
\hline 8/8/2009 12:00 AM & 0.184 & 0.360 & 17.8 & 0.190 & 0.123 & 17.2 & 0.14 & 0.124 & 0.179 & 14.9 & 0.123 & 0.386 & 12.7 & 0.480 \\
\hline 8/8/2009 4:00 АМ & 0.183 & 0.360 & 17.9 & 0.190 & 0.123 & 17.2 & 0.14 & 0.124 & 0.179 & 14.9 & 0.123 & 0.386 & 12.7 & 0.480 \\
\hline 8/8/2009 8:00 AM & 0.183 & 0.360 & 17.9 & 0.190 & 0.123 & 17.2 & 0.14 & 0.124 & 0.179 & 14.9 & 0.123 & 0.386 & 12.7 & 0.479 \\
\hline 8/8/2009 12:00 PM & 0.183 & 0.360 & 18.0 & 0.189 & 0.123 & 17.2 & 0.14 & 0.124 & 0.179 & 14.9 & 0.123 & 0.383 & 12.7 & 0.479 \\
\hline 8/8/2009 4:00 PM & 0.181 & 0.359 & 18.0 & 0.189 & 0.123 & 17.2 & 0.14 & 0.124 & 0.180 & 15.0 & 0.123 & 0.384 & 12.7 & 0.479 \\
\hline 8/8/2009 8:00 PM & 0.179 & 0.358 & 17.9 & 0.189 & 0.123 & 17.2 & 0.14 & 0.124 & 0.179 & 14.9 & 0.123 & 0.385 & 12.7 & 0.479 \\
\hline 8/9/2009 12:00 AM & 0.204 & 0.358 & 17.9 & 0.189 & 0.123 & 17.2 & 0.14 & 0.124 & 0.179 & 14.9 & 0.123 & 0.386 & 12.7 & 0.479 \\
\hline 8/9/2009 4:00 AM & 0.322 & 0.393 & 17.9 & 0.190 & 0.122 & 17.2 & 0.14 & 0.124 & 0.179 & 14.9 & 0.123 & 0.386 & 12.7 & 0.480 \\
\hline 8/9/2009 8:00 AM & 0.247 & 0.406 & 17.9 & 0.196 & 0.122 & 17.2 & 0.14 & 0.124 & 0.179 & 14.9 & 0.123 & 0.386 & 12.7 & 0.480 \\
\hline 8/9/2009 12:00 PM & 0.225 & 0.405 & 17.8 & 0.201 & 0.123 & 17.2 & 0.14 & 0.124 & 0.179 & 14.9 & 0.122 & 0.385 & 12.7 & 0.479 \\
\hline 8/9/2009 4:00 PM & 0.211 & 0.401 & 17.7 & 0.204 & 0.123 & 17.2 & 0.14 & 0.124 & 0.179 & 15.0 & 0.122 & 0.385 & 12.7 & 0.479 \\
\hline 8/9/2009 8:00 PM & 0.205 & 0.397 & 17.5 & 0.204 & 0.124 & 17.3 & 0.14 & 0.124 & 0.179 & 15.0 & 0.122 & 0.385 & 12.7 & 0.479 \\
\hline 8/10/2009 12:00 AM & 0.201 & 0.394 & 17.5 & 0.204 & 0.125 & 17.3 & 0.14 & 0.124 & 0.179 & 14.9 & 0.123 & 0.386 & 12.7 & 0.479 \\
\hline 8/10/2009 4:00 AM & 0.200 & 0.393 & 17.5 & 0.204 & 0.126 & 17.3 & 0.14 & 0.125 & 0.179 & 15.0 & 0.123 & 0.386 & 12.7 & 0.480 \\
\hline
\end{tabular}


Appendix 4. Volumetric water content, temperature, and electrical-conductivity data collected at the sprinkler-irrigated site during 2009.-Continued

Depth in feet below land surface; volumetric water content dimensionless; temperature in degrees Celsius; bulk electrical conductivity in decisiemens per meter; --, no data]

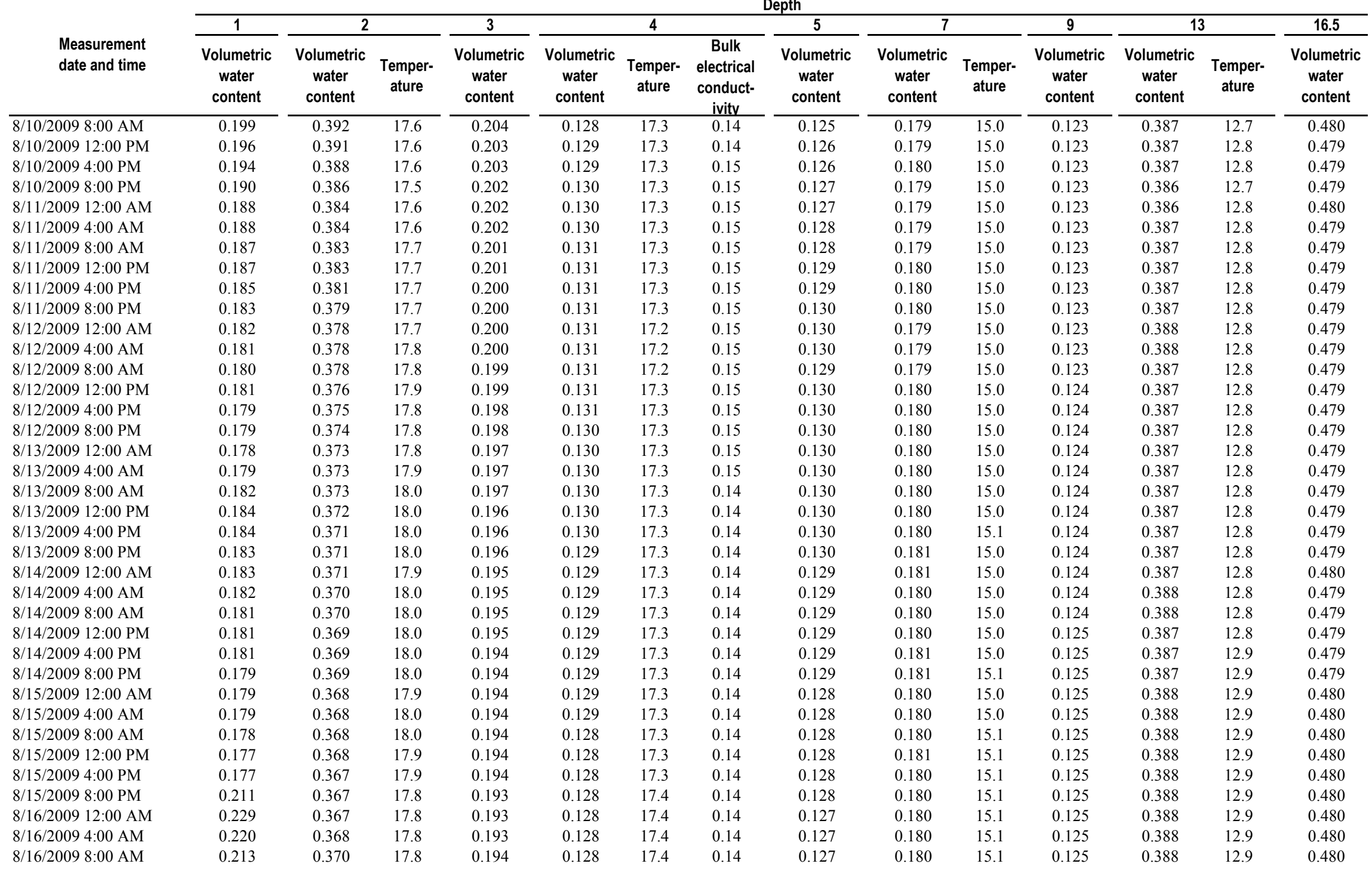


Appendix 4. Volumetric water content, temperature, and electrical-conductivity data collected at the sprinkler-irrigated site during 2009.-Continued

[Depth in feet below land surface; volumetric water content dimensionless; temperature in degrees Celsius; bulk electrical conductivity in decisiemens per meter; --, no data]

\begin{tabular}{|c|c|c|c|c|c|c|c|c|c|c|c|c|c|c|}
\hline \multirow[b]{3}{*}{$\begin{array}{l}\text { Measurement } \\
\text { date and time }\end{array}$} & \multicolumn{14}{|c|}{ Depth } \\
\hline & \multirow{2}{*}{$\begin{array}{c}1 \\
\text { Volumetric } \\
\text { water } \\
\text { content }\end{array}$} & \multicolumn{2}{|l|}{2} & \multirow{2}{*}{$\begin{array}{c}3 \\
\text { Volumetric } \\
\text { water } \\
\text { content }\end{array}$} & \multicolumn{3}{|c|}{4} & \multirow{2}{*}{$\begin{array}{c}5 \\
\text { Volumetric } \\
\text { water } \\
\text { content } \\
\end{array}$} & \multicolumn{2}{|l|}{7} & \multirow{2}{*}{$\begin{array}{c}9 \\
\text { Volumetric } \\
\text { water } \\
\text { content }\end{array}$} & \multicolumn{2}{|c|}{13} & \multirow{2}{*}{$\begin{array}{c}16.5 \\
\text { Volumetric } \\
\text { water } \\
\text { content }\end{array}$} \\
\hline & & $\begin{array}{c}\text { Volumetric } \\
\text { water } \\
\text { content }\end{array}$ & $\begin{array}{l}\text { Temper- } \\
\text { ature }\end{array}$ & & $\begin{array}{c}\text { Volumetric } \\
\text { water } \\
\text { content }\end{array}$ & $\begin{array}{l}\text { Temper- } \\
\text { ature }\end{array}$ & $\begin{array}{c}\text { Bulk } \\
\text { electrical } \\
\text { conduct- } \\
\text { ivity }\end{array}$ & & $\begin{array}{l}\text { Volumetric } \\
\text { water } \\
\text { content }\end{array}$ & $\begin{array}{l}\text { Temper- } \\
\text { ature }\end{array}$ & & $\begin{array}{c}\text { Volumetric } \\
\text { water } \\
\text { content }\end{array}$ & $\begin{array}{l}\text { Temper- } \\
\text { ature }\end{array}$ & \\
\hline 8/16/2009 12:00 PM & 0.207 & 0.371 & 17.8 & 0.194 & 0.128 & 17.4 & 0.14 & 0.127 & 0.181 & 15.1 & 0.125 & 0.390 & 12.9 & 0.480 \\
\hline 8/16/2009 4:00 PM & 0.200 & 0.371 & 17.7 & 0.194 & 0.128 & 17.4 & 0.14 & 0.127 & 0.180 & 15.1 & 0.124 & 0.388 & 12.9 & 0.479 \\
\hline 8/16/2009 8:00 PM & 0.195 & 0.369 & 17.5 & 0.193 & 0.128 & 17.4 & 0.14 & 0.127 & 0.181 & 15.1 & 0.125 & 0.388 & 12.9 & 0.479 \\
\hline 8/17/2009 12:00 AM & 0.194 & 0.368 & 17.5 & 0.194 & 0.128 & 17.4 & 0.14 & 0.127 & 0.180 & 15.1 & 0.125 & 0.390 & 12.9 & 0.480 \\
\hline 8/17/2009 4:00 AM & 0.193 & 0.368 & 17.5 & 0.194 & 0.128 & 17.4 & 0.14 & 0.126 & 0.180 & 15.1 & 0.125 & 0.390 & 12.9 & 0.480 \\
\hline 8/17/2009 8:00 AM & 0.192 & 0.369 & 17.5 & 0.194 & 0.128 & 17.4 & 0.14 & 0.126 & 0.180 & 15.1 & 0.125 & 0.390 & 12.9 & 0.480 \\
\hline 8/17/2009 12:00 PM & 0.190 & 0.369 & 17.4 & 0.194 & 0.128 & 17.4 & 0.14 & 0.127 & 0.181 & 15.1 & 0.125 & 0.391 & 12.9 & 0.480 \\
\hline 8/17/2009 4:00 PM & 0.188 & 0.368 & 17.3 & 0.193 & 0.128 & 17.4 & 0.14 & 0.127 & 0.181 & 15.1 & 0.125 & 0.391 & 12.9 & 0.480 \\
\hline 8/17/2009 8:00 PM & 0.186 & 0.367 & 17.3 & 0.193 & 0.128 & 17.3 & 0.14 & 0.127 & 0.180 & 15.1 & 0.125 & 0.391 & 12.9 & 0.480 \\
\hline 8/18/2009 12:00 AM & 0.185 & 0.367 & 17.2 & 0.193 & 0.128 & 17.3 & 0.14 & 0.126 & 0.180 & 15.1 & 0.125 & 0.391 & 12.9 & 0.480 \\
\hline 8/18/2009 4:00 AM & 0.184 & 0.366 & 17.3 & 0.193 & 0.126 & 17.3 & 0.14 & 0.126 & 0.180 & 15.1 & 0.125 & 0.391 & 13.0 & 0.480 \\
\hline 8/18/2009 8:00 AM & 0.183 & 0.366 & 17.3 & 0.193 & 0.126 & 17.3 & 0.14 & 0.126 & 0.180 & 15.1 & 0.125 & 0.392 & 12.9 & 0.480 \\
\hline 8/18/2009 12:00 PM & 0.199 & 0.366 & 17.3 & 0.192 & 0.126 & 17.3 & 0.14 & 0.126 & 0.181 & 15.1 & 0.125 & 0.392 & 13.0 & 0.480 \\
\hline 8/18/2009 4:00 PM & 0.210 & 0.366 & 17.2 & 0.192 & 0.128 & 17.3 & 0.14 & 0.126 & 0.181 & 15.1 & 0.125 & 0.390 & 12.9 & 0.480 \\
\hline 8/18/2009 8:00 PM & 0.205 & 0.367 & 17.2 & 0.192 & 0.126 & 17.3 & 0.14 & 0.126 & 0.181 & 15.1 & 0.125 & 0.388 & 12.9 & 0.480 \\
\hline 8/19/2009 12:00 AM & 0.202 & 0.367 & 17.2 & 0.192 & 0.126 & 17.3 & 0.14 & 0.126 & 0.180 & 15.1 & 0.125 & 0.390 & 13.0 & 0.480 \\
\hline 8/19/2009 4:00 AM & 0.223 & 0.368 & 17.2 & 0.192 & 0.126 & 17.3 & 0.14 & 0.126 & 0.180 & 15.1 & 0.126 & 0.390 & 13.0 & 0.480 \\
\hline 8/19/2009 8:00 AM & 0.221 & 0.370 & 17.3 & 0.192 & 0.126 & 17.3 & 0.14 & 0.126 & 0.180 & 15.1 & 0.126 & 0.388 & 13.0 & 0.480 \\
\hline 8/19/2009 12:00 PM & 0.212 & 0.372 & 17.2 & 0.193 & 0.126 & 17.3 & 0.14 & 0.126 & 0.181 & 15.1 & 0.126 & 0.390 & 13.0 & 0.480 \\
\hline 8/19/2009 4:00 PM & 0.204 & 0.373 & 17.1 & 0.193 & 0.126 & 17.2 & 0.14 & 0.126 & 0.180 & 15.1 & 0.126 & 0.388 & 13.0 & 0.480 \\
\hline 8/19/2009 8:00 PM & 0.199 & 0.372 & 17.1 & 0.193 & 0.126 & 17.2 & 0.14 & 0.126 & 0.181 & 15.1 & 0.126 & 0.388 & 13.0 & 0.480 \\
\hline 8/20/2009 12:00 AM & 0.196 & 0.372 & 17.1 & 0.193 & 0.126 & 17.2 & 0.14 & 0.126 & 0.180 & 15.1 & 0.126 & 0.391 & 13.0 & 0.480 \\
\hline 8/20/2009 4:00 AM & 0.195 & 0.372 & 17.1 & 0.194 & 0.126 & 17.2 & 0.14 & 0.126 & 0.180 & 15.1 & 0.126 & 0.391 & 13.0 & 0.480 \\
\hline 8/20/2009 8:00 AM & 0.217 & 0.372 & 17.2 & 0.194 & 0.126 & 17.2 & 0.14 & 0.126 & 0.180 & 15.1 & 0.126 & 0.391 & 13.0 & 0.480 \\
\hline 8/20/2009 12:00 PM & 0.249 & 0.373 & 17.1 & 0.194 & 0.128 & 17.2 & 0.14 & 0.126 & 0.181 & 15.1 & 0.126 & 0.391 & 13.0 & 0.480 \\
\hline 8/20/2009 4:00 PM & 0.216 & 0.375 & 17.1 & 0.194 & 0.128 & 17.2 & 0.14 & 0.126 & 0.180 & 15.1 & 0.126 & 0.390 & 13.0 & 0.480 \\
\hline 8/20/2009 8:00 PM & 0.206 & 0.376 & 17.0 & 0.194 & 0.128 & 17.2 & 0.14 & 0.126 & 0.181 & 15.1 & 0.126 & 0.391 & 13.0 & 0.480 \\
\hline 8/21/2009 12:00 AM & 0.202 & 0.376 & 17.0 & 0.194 & 0.126 & 17.2 & 0.14 & 0.126 & 0.181 & 15.1 & 0.126 & 0.391 & 13.0 & 0.480 \\
\hline 8/21/2009 4:00 AM & 0.200 & 0.378 & 17.1 & 0.194 & 0.128 & 17.2 & 0.14 & 0.126 & 0.180 & 15.1 & 0.126 & 0.391 & 13.0 & 0.480 \\
\hline 8/21/2009 8:00 AM & 0.199 & 0.378 & 17.2 & 0.195 & 0.128 & 17.2 & 0.14 & 0.126 & 0.180 & 15.1 & 0.126 & 0.392 & 13.0 & 0.480 \\
\hline 8/21/2009 12:00 PM & 0.197 & 0.378 & 17.1 & 0.195 & 0.128 & 17.2 & 0.14 & 0.126 & 0.181 & 15.1 & 0.126 & 0.394 & 13.0 & 0.480 \\
\hline 8/21/2009 4:00 PM & 0.194 & 0.376 & 17.1 & 0.195 & 0.129 & 17.2 & 0.14 & 0.127 & 0.180 & 15.1 & 0.126 & 0.393 & 13.0 & 0.480 \\
\hline 8/21/2009 8:00 PM & 0.190 & 0.374 & 17.0 & 0.195 & 0.129 & 17.1 & 0.14 & 0.127 & 0.181 & 15.1 & 0.126 & 0.392 & 13.1 & 0.480 \\
\hline 8/22/2009 12:00 AM & 0.188 & 0.373 & 17.0 & 0.195 & 0.129 & 17.1 & 0.14 & 0.127 & 0.180 & 15.1 & 0.126 & 0.392 & 13.0 & 0.480 \\
\hline 8/22/2009 4:00 AM & 0.188 & 0.373 & 17.1 & 0.195 & 0.129 & 17.1 & 0.14 & 0.126 & 0.180 & 15.1 & 0.126 & 0.396 & 13.0 & 0.480 \\
\hline 8/22/2009 8:00 AM & 0.188 & 0.373 & 17.1 & 0.194 & 0.129 & 17.1 & 0.14 & 0.127 & 0.180 & 15.1 & 0.126 & 0.398 & 13.1 & 0.480 \\
\hline 8/22/2009 12:00 PM & 0.187 & 0.373 & 17.1 & 0.194 & 0.129 & 17.1 & 0.14 & 0.127 & 0.180 & 15.1 & 0.126 & 0.397 & 13.0 & 0.480 \\
\hline
\end{tabular}


Appendix 4. Volumetric water content, temperature, and electrical-conductivity data collected at the sprinkler-irrigated site during 2009.-Continued [Depth in feet below land surface; volumetric water content dimensionless; temperature in degrees Celsius; bulk electrical conductivity in decisiemens per meter; --, no data]

\begin{tabular}{|c|c|c|c|c|c|c|c|c|c|c|c|c|c|c|}
\hline \multirow[b]{3}{*}{$\begin{array}{l}\text { Measurement } \\
\text { date and time }\end{array}$} & \multirow[b]{3}{*}{$\begin{array}{c}1 \\
\text { Volumetric } \\
\text { water } \\
\text { content }\end{array}$} & & & \multirow[b]{3}{*}{$\begin{array}{c}3 \\
\text { Volumetric } \\
\text { water } \\
\text { content }\end{array}$} & \multirow{2}{*}{\multicolumn{3}{|c|}{4}} & \multirow{3}{*}{$\begin{array}{c}\text { epth } \\
5 \\
\text { Volumetric } \\
\text { water } \\
\text { content }\end{array}$} & \multirow{2}{*}{\multicolumn{2}{|c|}{7}} & \multirow{3}{*}{$\begin{array}{c}9 \\
\text { Volumetric } \\
\text { water } \\
\text { content }\end{array}$} & \multirow{2}{*}{\multicolumn{2}{|c|}{13}} & \multirow[b]{3}{*}{$\begin{array}{c}16.5 \\
\text { Volumetric } \\
\text { water } \\
\text { content }\end{array}$} \\
\hline & & \multicolumn{2}{|c|}{2} & & & & & & & & & & & \\
\hline & & $\begin{array}{c}\text { Volumetric } \\
\text { water } \\
\text { content }\end{array}$ & $\begin{array}{l}\text { Temper- } \\
\text { ature }\end{array}$ & & $\begin{array}{c}\text { Volumetric } \\
\text { water } \\
\text { content }\end{array}$ & $\begin{array}{l}\text { Temper- } \\
\text { ature }\end{array}$ & $\begin{array}{c}\text { Bulk } \\
\text { electrical } \\
\text { conduct- } \\
\text { ivity }\end{array}$ & & $\begin{array}{c}\text { Volumetric } \\
\text { water } \\
\text { content }\end{array}$ & $\begin{array}{l}\text { Temper- } \\
\text { ature }\end{array}$ & & $\begin{array}{c}\text { Volumetric } \\
\text { water } \\
\text { content }\end{array}$ & $\begin{array}{l}\text { Temper- } \\
\text { ature }\end{array}$ & \\
\hline 8/22/2009 4:00 PM & 0.186 & 0.372 & 17.1 & 0.194 & 0.129 & 17.1 & 0.14 & 0.127 & 0.180 & 15.1 & 0.126 & 0.394 & 13.1 & 0.480 \\
\hline 8/22/2009 8:00 PM & 0.183 & 0.371 & 17.0 & 0.194 & 0.129 & 17.1 & 0.14 & 0.127 & 0.181 & 15.1 & 0.126 & 0.397 & 13.1 & 0.480 \\
\hline 8/23/2009 12:00 AM & 0.183 & 0.370 & 17.1 & 0.194 & 0.129 & 17.1 & 0.14 & 0.127 & 0.181 & 15.2 & 0.126 & 0.399 & 13.1 & 0.480 \\
\hline 8/23/2009 4:00 AM & 0.194 & 0.370 & 17.2 & 0.193 & 0.129 & 17.1 & 0.14 & 0.127 & 0.181 & 15.1 & 0.126 & 0.398 & 13.1 & 0.480 \\
\hline 8/23/2009 8:00 AM & 0.205 & 0.370 & 17.2 & 0.193 & 0.129 & 17.1 & 0.14 & 0.127 & 0.181 & 15.1 & 0.126 & 0.396 & 13.1 & 0.481 \\
\hline 8/23/2009 12:00 PM & 0.204 & 0.370 & 17.3 & 0.193 & 0.129 & 17.1 & 0.14 & 0.127 & 0.181 & 15.1 & 0.126 & 0.399 & 13.1 & 0.480 \\
\hline 8/23/2009 4:00 PM & 0.200 & 0.370 & 17.3 & 0.193 & 0.128 & 17.1 & 0.14 & 0.127 & 0.180 & 15.1 & 0.126 & 0.403 & 13.1 & 0.480 \\
\hline 8/23/2009 8:00 PM & 0.196 & 0.370 & 17.3 & 0.193 & 0.129 & 17.1 & 0.14 & 0.127 & 0.181 & 15.1 & 0.127 & 0.405 & 13.1 & 0.480 \\
\hline 8/24/2009 12:00 AM & 0.194 & 0.370 & 17.4 & 0.192 & 0.128 & 17.1 & 0.14 & 0.127 & 0.181 & 15.1 & 0.127 & 0.411 & 13.1 & 0.480 \\
\hline 8/24/2009 4:00 AM & 0.194 & 0.371 & 17.5 & 0.193 & 0.128 & 17.1 & 0.14 & 0.127 & 0.181 & 15.1 & 0.127 & 0.420 & 13.1 & 0.480 \\
\hline 8/24/2009 8:00 AM & 0.193 & 0.372 & 17.6 & 0.193 & 0.128 & 17.1 & 0.14 & 0.127 & 0.181 & 15.1 & 0.127 & 0.412 & 13.1 & 0.480 \\
\hline 8/24/2009 12:00 PM & 0.193 & 0.372 & 17.7 & 0.193 & 0.128 & 17.1 & 0.14 & 0.127 & 0.181 & 15.1 & 0.127 & 0.410 & 13.1 & 0.480 \\
\hline 8/24/2009 4:00 PM & 0.190 & 0.372 & 17.7 & 0.193 & 0.128 & 17.1 & 0.14 & 0.127 & 0.181 & 15.1 & 0.127 & 0.409 & 13.1 & 0.480 \\
\hline 8/24/2009 8:00 PM & 0.197 & 0.371 & 17.7 & 0.193 & 0.128 & 17.1 & 0.14 & 0.127 & 0.181 & 15.1 & 0.127 & 0.411 & 13.1 & 0.480 \\
\hline 8/25/2009 12:00 AM & 0.199 & 0.371 & 17.7 & 0.193 & 0.128 & 17.1 & 0.14 & 0.127 & 0.181 & 15.1 & 0.127 & 0.410 & 13.1 & 0.480 \\
\hline 8/25/2009 4:00 AM & 0.199 & 0.371 & 17.7 & 0.193 & 0.128 & 17.1 & 0.14 & 0.126 & 0.181 & 15.1 & 0.127 & 0.410 & 13.1 & 0.480 \\
\hline 8/25/2009 8:00 AM & 0.247 & 0.372 & 17.8 & 0.193 & 0.128 & 17.1 & 0.14 & 0.126 & 0.181 & 15.1 & 0.127 & 0.409 & 13.1 & 0.480 \\
\hline 8/25/2009 12:00 PM & 0.246 & 0.378 & 17.7 & 0.193 & 0.128 & 17.1 & 0.14 & 0.127 & 0.181 & 15.1 & 0.127 & 0.409 & 13.1 & 0.480 \\
\hline 8/25/2009 4:00 PM & 0.219 & 0.382 & 17.7 & 0.194 & 0.128 & 17.1 & 0.14 & 0.127 & 0.181 & 15.1 & 0.126 & 0.408 & 13.1 & 0.480 \\
\hline 8/25/2009 8:00 PM & 0.212 & 0.383 & 17.7 & 0.194 & 0.128 & 17.1 & 0.14 & 0.126 & 0.181 & 15.1 & 0.127 & 0.408 & 13.2 & 0.480 \\
\hline 8/26/2009 12:00 AM & 0.209 & 0.384 & 17.7 & 0.195 & 0.128 & 17.2 & 0.14 & 0.126 & 0.181 & 15.1 & 0.127 & 0.408 & 13.2 & 0.480 \\
\hline 8/26/2009 4:00 AM & 0.206 & 0.384 & 17.7 & 0.196 & 0.129 & 17.2 & 0.14 & 0.126 & 0.181 & 15.1 & 0.127 & 0.408 & 13.2 & 0.480 \\
\hline 8/26/2009 8:00 AM & 0.205 & 0.384 & 17.8 & 0.197 & 0.129 & 17.2 & 0.14 & 0.127 & 0.181 & 15.1 & 0.127 & 0.408 & 13.2 & 0.480 \\
\hline 8/26/2009 12:00 PM & 0.203 & 0.384 & 17.8 & 0.197 & 0.129 & 17.2 & 0.14 & 0.127 & 0.181 & 15.1 & 0.127 & 0.408 & 13.2 & 0.480 \\
\hline 8/26/2009 4:00 PM & 0.200 & 0.383 & 17.7 & 0.197 & 0.130 & 17.2 & 0.14 & 0.127 & 0.181 & 15.1 & 0.126 & 0.409 & 13.2 & 0.480 \\
\hline 8/26/2009 8:00 PM & 0.196 & 0.381 & 17.7 & 0.197 & 0.130 & 17.2 & 0.15 & 0.128 & 0.181 & 15.1 & 0.127 & 0.409 & 13.2 & 0.480 \\
\hline 8/27/2009 12:00 AM & 0.194 & 0.381 & 17.7 & 0.197 & 0.130 & 17.2 & 0.15 & 0.127 & 0.181 & 15.1 & 0.127 & 0.410 & 13.2 & 0.480 \\
\hline 8/27/2009 4:00 AM & 0.193 & 0.380 & 17.7 & 0.197 & 0.130 & 17.2 & 0.15 & 0.128 & 0.181 & 15.1 & 0.127 & 0.410 & 13.2 & 0.480 \\
\hline 8/27/2009 8:00 AM & 0.192 & 0.380 & 17.7 & 0.196 & 0.130 & 17.2 & 0.15 & 0.128 & 0.181 & 15.1 & 0.127 & 0.409 & 13.2 & 0.480 \\
\hline 8/27/2009 12:00 PM & 0.192 & 0.380 & 17.7 & 0.196 & 0.130 & 17.2 & 0.15 & 0.128 & 0.181 & 15.1 & 0.127 & 0.399 & 13.2 & 0.480 \\
\hline 8/27/2009 4:00 PM & 0.189 & 0.378 & 17.6 & 0.196 & 0.131 & 17.2 & 0.15 & 0.129 & 0.181 & 15.1 & 0.127 & 0.398 & 13.2 & 0.480 \\
\hline 8/27/2009 8:00 PM & 0.187 & 0.376 & 17.6 & 0.195 & 0.131 & 17.2 & 0.15 & 0.129 & 0.181 & 15.1 & 0.128 & 0.397 & 13.2 & 0.481 \\
\hline 8/28/2009 12:00 AM & 0.185 & 0.374 & 17.6 & 0.195 & 0.130 & 17.2 & 0.15 & 0.128 & 0.181 & 15.1 & 0.128 & 0.397 & 13.2 & 0.480 \\
\hline 8/28/2009 4:00 AM & 0.184 & 0.374 & 17.6 & 0.194 & 0.130 & 17.3 & 0.15 & 0.128 & 0.181 & 15.1 & 0.128 & 0.397 & 13.1 & 0.481 \\
\hline 8/28/2009 8:00 AM & 0.183 & 0.374 & 17.6 & 0.194 & 0.130 & 17.3 & 0.15 & 0.128 & 0.181 & 15.1 & 0.128 & 0.398 & 13.2 & 0.480 \\
\hline 8/28/2009 12:00 PM & 0.183 & 0.374 & 17.5 & 0.194 & 0.130 & 17.3 & 0.15 & 0.129 & 0.181 & 15.1 & 0.128 & 0.398 & 13.2 & 0.481 \\
\hline 8/28/2009 4:00 PM & 0.182 & 0.372 & 17.5 & 0.194 & 0.130 & 17.2 & 0.15 & 0.129 & 0.181 & 15.2 & 0.128 & 0.399 & 13.2 & 0.481 \\
\hline
\end{tabular}


Appendix 4. Volumetric water content, temperature, and electrical-conductivity data collected at the sprinkler-irrigated site during 2009.-Continued

[Depth in feet below land surface; volumetric water content dimensionless; temperature in degrees Celsius; bulk electrical conductivity in decisiemens per meter; --, no data]

\begin{tabular}{|c|c|c|c|c|c|c|c|c|c|c|c|c|c|c|}
\hline \multirow[b]{3}{*}{$\begin{array}{l}\text { Measurement } \\
\text { date and time }\end{array}$} & \multicolumn{14}{|c|}{ Depth } \\
\hline & \multirow{2}{*}{$\begin{array}{c}1 \\
\text { Volumetric } \\
\text { water } \\
\text { content }\end{array}$} & \multicolumn{2}{|l|}{2} & \multirow{2}{*}{$\begin{array}{c}3 \\
\text { Volumetric } \\
\text { water } \\
\text { content }\end{array}$} & \multicolumn{3}{|c|}{4} & \multirow{2}{*}{$\begin{array}{c}5 \\
\text { Volumetric } \\
\text { water } \\
\text { content } \\
\end{array}$} & \multicolumn{2}{|l|}{7} & \multirow{2}{*}{$\begin{array}{c}9 \\
\text { Volumetric } \\
\text { water } \\
\text { content }\end{array}$} & \multicolumn{2}{|c|}{13} & \multirow{2}{*}{$\begin{array}{c}16.5 \\
\text { Volumetric } \\
\text { water } \\
\text { content }\end{array}$} \\
\hline & & $\begin{array}{c}\text { Volumetric } \\
\text { water } \\
\text { content }\end{array}$ & $\begin{array}{l}\text { Temper- } \\
\text { ature }\end{array}$ & & $\begin{array}{c}\text { Volumetric } \\
\text { water } \\
\text { content }\end{array}$ & $\begin{array}{l}\text { Temper- } \\
\text { ature }\end{array}$ & $\begin{array}{c}\text { Bulk } \\
\text { electrical } \\
\text { conduct- } \\
\text { ivity }\end{array}$ & & $\begin{array}{l}\text { Volumetric } \\
\text { water } \\
\text { content }\end{array}$ & $\begin{array}{l}\text { Temper- } \\
\text { ature }\end{array}$ & & $\begin{array}{c}\text { Volumetric } \\
\text { water } \\
\text { content }\end{array}$ & $\begin{array}{l}\text { Temper- } \\
\text { ature }\end{array}$ & \\
\hline 8/28/2009 8:00 PM & 0.194 & 0.370 & 17.4 & 0.194 & 0.130 & 17.3 & 0.14 & 0.129 & 0.181 & 15.1 & 0.128 & 0.399 & 13.2 & 0.481 \\
\hline 8/29/2009 12:00 AM & 0.239 & 0.370 & 17.4 & 0.194 & 0.129 & 17.3 & 0.14 & 0.128 & 0.181 & 15.1 & 0.128 & 0.398 & 13.2 & 0.481 \\
\hline 8/29/2009 4:00 AM & 0.218 & 0.371 & 17.4 & 0.194 & 0.130 & 17.3 & 0.14 & 0.128 & 0.181 & 15.1 & 0.128 & 0.398 & 13.2 & 0.480 \\
\hline 8/29/2009 8:00 AM & 0.211 & 0.373 & 17.5 & 0.194 & 0.130 & 17.3 & 0.14 & 0.128 & 0.181 & 15.1 & 0.128 & 0.399 & 13.2 & 0.480 \\
\hline 8/29/2009 12:00 PM & 0.205 & 0.373 & 17.4 & 0.194 & 0.129 & 17.3 & 0.14 & 0.128 & 0.181 & 15.2 & 0.128 & 0.399 & 13.2 & 0.481 \\
\hline 8/29/2009 4:00 PM & 0.201 & 0.373 & 17.4 & 0.194 & 0.129 & 17.2 & 0.14 & 0.128 & 0.181 & 15.2 & 0.128 & 0.399 & 13.2 & 0.481 \\
\hline 8/29/2009 8:00 PM & 0.198 & 0.373 & 17.3 & 0.194 & 0.129 & 17.2 & 0.14 & 0.128 & 0.181 & 15.2 & 0.128 & 0.399 & 13.2 & 0.481 \\
\hline 8/30/2009 12:00 AM & 0.196 & 0.373 & 17.3 & 0.194 & 0.129 & 17.2 & 0.14 & 0.128 & 0.182 & 15.2 & 0.128 & 0.404 & 13.2 & 0.480 \\
\hline 8/30/2009 4:00 AM & 0.195 & 0.373 & 17.3 & 0.194 & 0.129 & 17.2 & 0.14 & 0.128 & 0.182 & 15.2 & 0.128 & 0.400 & 13.2 & 0.480 \\
\hline 8/30/2009 8:00 AM & 0.194 & 0.372 & 17.4 & 0.194 & 0.130 & 17.2 & 0.14 & 0.128 & 0.181 & 15.2 & 0.128 & 0.399 & 13.2 & 0.480 \\
\hline 8/30/2009 12:00 PM & 0.193 & 0.372 & 17.4 & 0.194 & 0.130 & 17.2 & 0.14 & 0.128 & 0.182 & 15.2 & 0.128 & 0.403 & 13.2 & 0.481 \\
\hline 8/30/2009 4:00 PM & 0.192 & 0.372 & 17.3 & 0.194 & 0.129 & 17.2 & 0.14 & 0.128 & 0.182 & 15.2 & 0.128 & 0.401 & 13.2 & 0.480 \\
\hline 8/30/2009 8:00 PM & 0.191 & 0.372 & 17.3 & 0.194 & 0.130 & 17.2 & 0.14 & 0.128 & 0.182 & 15.2 & 0.129 & 0.399 & 13.2 & 0.480 \\
\hline 8/31/2009 12:00 AM & 0.194 & 0.372 & 17.3 & 0.194 & 0.129 & 17.2 & 0.14 & 0.128 & 0.182 & 15.2 & 0.129 & 0.404 & 13.3 & 0.480 \\
\hline 8/31/2009 4:00 AM & 0.197 & 0.371 & 17.3 & 0.194 & 0.129 & 17.2 & 0.14 & 0.127 & 0.182 & 15.2 & 0.129 & 0.405 & 13.2 & 0.480 \\
\hline 8/31/2009 8:00 AM & 0.198 & 0.372 & 17.2 & 0.194 & 0.129 & 17.2 & 0.14 & 0.127 & 0.181 & 15.2 & 0.129 & 0.398 & 13.2 & 0.480 \\
\hline 8/31/2009 12:00 PM & 0.239 & 0.372 & 17.2 & 0.193 & 0.130 & 17.2 & 0.14 & 0.128 & 0.182 & 15.2 & 0.129 & 0.398 & 13.2 & 0.480 \\
\hline 8/31/2009 4:00 PM & 0.223 & 0.373 & 17.1 & 0.193 & 0.129 & 17.2 & 0.14 & 0.128 & 0.182 & 15.2 & 0.129 & 0.398 & 13.2 & 0.480 \\
\hline 8/31/2009 8:00 PM & 0.214 & 0.375 & 17.0 & 0.194 & 0.129 & 17.2 & 0.14 & 0.128 & 0.182 & 15.2 & 0.129 & 0.399 & 13.2 & 0.480 \\
\hline 9/1/2009 12:00 AM & 0.211 & 0.376 & 17.0 & 0.194 & 0.129 & 17.2 & 0.14 & 0.128 & 0.182 & 15.2 & 0.129 & 0.400 & 13.2 & 0.480 \\
\hline 9/1/2009 4:00 AM & 0.207 & 0.378 & 17.1 & 0.194 & 0.129 & 17.2 & 0.14 & 0.127 & 0.182 & 15.2 & 0.130 & 0.400 & 13.2 & 0.480 \\
\hline 9/1/2009 8:00 AM & 0.205 & 0.379 & 17.1 & 0.194 & 0.130 & 17.2 & 0.14 & 0.128 & 0.182 & 15.2 & 0.130 & 0.422 & 13.3 & 0.480 \\
\hline 9/1/2009 12:00 PM & 0.203 & 0.379 & 17.1 & 0.195 & 0.130 & 17.2 & 0.14 & 0.128 & 0.182 & 15.2 & 0.130 & 0.419 & 13.3 & 0.480 \\
\hline 9/1/2009 4:00 PM & 0.200 & 0.378 & 17.0 & 0.195 & 0.130 & 17.2 & 0.15 & 0.128 & 0.182 & 15.2 & 0.130 & 0.412 & 13.3 & 0.481 \\
\hline 9/1/2009 8:00 PM & 0.209 & 0.376 & 17.0 & 0.194 & 0.130 & 17.2 & 0.15 & 0.128 & 0.182 & 15.2 & 0.130 & 0.398 & 13.3 & 0.481 \\
\hline 9/2/2009 12:00 AM & 0.211 & 0.376 & 17.0 & 0.195 & 0.130 & 17.2 & 0.15 & 0.128 & 0.182 & 15.2 & 0.130 & 0.401 & 13.3 & 0.481 \\
\hline 9/2/2009 4:00 AM & 0.209 & 0.379 & 17.1 & 0.195 & 0.130 & 17.1 & 0.15 & 0.128 & 0.182 & 15.2 & 0.130 & 0.405 & 13.3 & 0.481 \\
\hline 9/2/2009 8:00 AM & 0.206 & 0.379 & 17.2 & 0.195 & 0.130 & 17.1 & 0.15 & 0.128 & 0.182 & 15.2 & 0.130 & 0.405 & 13.3 & 0.481 \\
\hline 9/2/2009 12:00 PM & 0.205 & 0.379 & 17.2 & 0.195 & 0.130 & 17.1 & 0.15 & 0.129 & 0.182 & 15.2 & 0.130 & 0.401 & 13.3 & 0.481 \\
\hline 9/2/2009 4:00 PM & 0.200 & 0.379 & 17.2 & 0.195 & 0.130 & 17.1 & 0.15 & 0.129 & 0.182 & 15.2 & 0.130 & 0.403 & 13.3 & 0.481 \\
\hline 9/2/2009 8:00 PM & 0.196 & 0.378 & 17.1 & 0.195 & 0.131 & 17.1 & 0.15 & 0.129 & 0.182 & 15.2 & 0.131 & 0.400 & 13.3 & 0.481 \\
\hline 9/3/2009 12:00 AM & 0.194 & 0.376 & 17.2 & 0.195 & 0.130 & 17.1 & 0.15 & 0.129 & 0.182 & 15.2 & 0.131 & 0.401 & 13.3 & 0.481 \\
\hline 9/3/2009 4:00 AM & 0.194 & 0.376 & 17.2 & 0.195 & 0.131 & 17.1 & 0.15 & 0.129 & 0.182 & 15.2 & 0.131 & 0.401 & 13.3 & 0.481 \\
\hline 9/3/2009 8:00 AM & 0.193 & 0.376 & 17.3 & 0.195 & 0.130 & 17.1 & 0.15 & 0.129 & 0.182 & 15.2 & 0.131 & 0.401 & 13.3 & 0.481 \\
\hline 9/3/2009 12:00 PM & 0.193 & 0.375 & 17.3 & 0.195 & 0.131 & 17.1 & 0.15 & 0.129 & 0.182 & 15.2 & 0.131 & 0.405 & 13.3 & 0.481 \\
\hline 9/3/2009 4:00 PM & 0.190 & 0.374 & 17.2 & 0.194 & 0.131 & 17.1 & 0.15 & 0.129 & 0.182 & 15.2 & 0.131 & 0.405 & 13.3 & 0.481 \\
\hline 9/3/2009 8:00 PM & 0.188 & 0.373 & 17.2 & 0.194 & 0.130 & 17.1 & 0.15 & 0.129 & 0.182 & 15.2 & 0.132 & 0.404 & 13.3 & 0.481 \\
\hline
\end{tabular}


Appendix 4. Volumetric water content, temperature, and electrical-conductivity data collected at the sprinkler-irrigated site during 2009.-Continued

[Depth in feet below land surface; volumetric water content dimensionless; temperature in degrees Celsius; bulk electrical conductivity in decisiemens per meter; --, no data]

\begin{tabular}{|c|c|c|c|c|c|c|c|c|c|c|c|c|c|c|}
\hline \multirow[b]{3}{*}{$\begin{array}{l}\text { Measurement } \\
\text { date and time }\end{array}$} & \multirow[b]{3}{*}{$\begin{array}{c}1 \\
\text { Volumetric } \\
\text { water } \\
\text { content }\end{array}$} & & & \multirow{3}{*}{$\begin{array}{c}3 \\
\text { Volumetric } \\
\text { water } \\
\text { content }\end{array}$} & \multirow{2}{*}{\multicolumn{3}{|c|}{4}} & \multirow{3}{*}{$\begin{array}{c}\text { epth } \\
\text { Volumetric } \\
\text { water } \\
\text { content }\end{array}$} & \multirow{2}{*}{\multicolumn{2}{|c|}{7}} & \multirow{3}{*}{$\begin{array}{c}9 \\
\text { Volumetric } \\
\text { water } \\
\text { content }\end{array}$} & \multirow{2}{*}{\multicolumn{2}{|c|}{13}} & \multirow[b]{3}{*}{$\begin{array}{c}16.5 \\
\text { Volumetric } \\
\text { water } \\
\text { content }\end{array}$} \\
\hline & & \multicolumn{2}{|c|}{2} & & & & & & & & & & & \\
\hline & & $\begin{array}{c}\text { Volumetric } \\
\text { water } \\
\text { content }\end{array}$ & $\begin{array}{l}\text { Temper- } \\
\text { ature }\end{array}$ & & $\begin{array}{c}\text { Volumetric } \\
\text { water } \\
\text { content }\end{array}$ & $\begin{array}{l}\text { Temper- } \\
\text { ature }\end{array}$ & $\begin{array}{c}\text { Bulk } \\
\text { electrical } \\
\text { conduct- } \\
\text { ivity }\end{array}$ & & $\begin{array}{c}\text { Volumetric } \\
\text { water } \\
\text { content }\end{array}$ & $\begin{array}{l}\text { Temper- } \\
\text { ature }\end{array}$ & & $\begin{array}{c}\text { Volumetric } \\
\text { water } \\
\text { content }\end{array}$ & $\begin{array}{l}\text { Temper- } \\
\text { ature }\end{array}$ & \\
\hline 9/4/2009 12:00 AM & 0.186 & 0.373 & 17.2 & 0.194 & 0.130 & 17.1 & 0.15 & 0.129 & 0.183 & 15.2 & 0.132 & 0.405 & 13.3 & 0.481 \\
\hline 9/4/2009 4:00 AM & 0.186 & 0.373 & 17.3 & 0.194 & 0.130 & 17.1 & 0.15 & 0.129 & 0.182 & 15.2 & 0.132 & 0.405 & 13.3 & 0.481 \\
\hline 9/4/2009 8:00 AM & 0.185 & 0.372 & 17.3 & 0.194 & 0.130 & 17.1 & 0.15 & 0.129 & 0.182 & 15.2 & 0.132 & 0.409 & 13.3 & 0.481 \\
\hline 9/4/2009 12:00 PM & 0.185 & 0.372 & 17.3 & 0.194 & 0.130 & 17.1 & 0.15 & 0.129 & 0.183 & 15.2 & 0.132 & 0.407 & 13.3 & 0.481 \\
\hline 9/4/2009 4:00 PM & 0.184 & 0.371 & 17.2 & 0.194 & 0.130 & 17.1 & 0.14 & 0.129 & 0.182 & 15.2 & 0.132 & 0.403 & 13.4 & 0.481 \\
\hline 9/4/2009 8:00 PM & 0.211 & 0.371 & 17.2 & 0.193 & 0.130 & 17.1 & 0.14 & 0.129 & 0.183 & 15.2 & 0.132 & 0.405 & 13.3 & 0.481 \\
\hline 9/5/2009 12:00 AM & 0.228 & 0.371 & 17.2 & 0.193 & 0.130 & 17.1 & 0.14 & 0.129 & 0.183 & 15.2 & 0.132 & 0.407 & 13.3 & 0.481 \\
\hline 9/5/2009 4:00 AM & 0.216 & 0.372 & 17.2 & 0.193 & 0.130 & 17.1 & 0.14 & 0.128 & 0.183 & 15.2 & 0.132 & 0.403 & 13.4 & 0.481 \\
\hline 9/5/2009 8:00 AM & 0.211 & 0.373 & 17.3 & 0.194 & 0.130 & 17.1 & 0.14 & 0.128 & 0.183 & 15.2 & 0.132 & 0.404 & 13.4 & 0.481 \\
\hline 9/5/2009 12:00 PM & 0.207 & 0.374 & 17.3 & 0.194 & 0.130 & 17.1 & 0.14 & 0.128 & 0.183 & 15.2 & 0.132 & 0.403 & 13.4 & 0.481 \\
\hline 9/5/2009 4:00 PM & 0.203 & 0.374 & 17.2 & 0.194 & 0.129 & 17.1 & 0.14 & 0.129 & 0.183 & 15.2 & 0.132 & 0.405 & 13.4 & 0.481 \\
\hline 9/5/2009 8:00 PM & 0.199 & 0.374 & 17.2 & 0.194 & 0.130 & 17.1 & 0.14 & 0.128 & 0.183 & 15.2 & 0.132 & 0.409 & 13.4 & 0.481 \\
\hline 9/6/2009 12:00 AM & 0.197 & 0.374 & 17.2 & 0.194 & 0.130 & 17.1 & 0.14 & 0.128 & 0.183 & 15.2 & 0.132 & 0.403 & 13.4 & 0.481 \\
\hline 9/6/2009 4:00 AM & 0.197 & 0.373 & 17.3 & 0.194 & 0.130 & 17.1 & 0.14 & 0.128 & 0.183 & 15.2 & 0.132 & 0.404 & 13.4 & 0.481 \\
\hline 9/6/2009 8:00 AM & 0.196 & 0.373 & 17.3 & 0.194 & 0.130 & 17.1 & 0.14 & 0.128 & 0.183 & 15.2 & 0.132 & 0.413 & 13.4 & 0.481 \\
\hline 9/6/2009 12:00 PM & 0.194 & 0.373 & 17.3 & 0.194 & 0.130 & 17.1 & 0.14 & 0.128 & 0.183 & 15.2 & 0.132 & 0.415 & 13.4 & 0.481 \\
\hline 9/6/2009 4:00 PM & 0.236 & 0.373 & 17.2 & 0.194 & 0.130 & 17.1 & 0.14 & 0.128 & 0.183 & 15.2 & 0.132 & 0.413 & 13.4 & 0.481 \\
\hline 9/6/2009 8:00 PM & 0.222 & 0.375 & 17.2 & 0.194 & 0.129 & 17.1 & 0.14 & 0.128 & 0.183 & 15.2 & 0.132 & 0.413 & 13.4 & 0.481 \\
\hline 9/7/2009 12:00 AM & 0.215 & 0.378 & 17.2 & 0.194 & 0.130 & 17.1 & 0.14 & 0.128 & 0.183 & 15.2 & 0.132 & 0.412 & 13.4 & 0.481 \\
\hline 9/7/2009 4:00 AM & 0.211 & 0.379 & 17.3 & 0.194 & 0.130 & 17.1 & 0.14 & 0.128 & 0.183 & 15.2 & 0.132 & 0.406 & 13.4 & 0.481 \\
\hline 9/7/2009 8:00 AM & 0.207 & 0.379 & 17.3 & 0.195 & 0.130 & 17.1 & 0.14 & 0.128 & 0.183 & 15.2 & 0.133 & 0.397 & 13.4 & 0.481 \\
\hline 9/7/2009 12:00 PM & 0.276 & 0.386 & 17.3 & 0.196 & 0.130 & 17.1 & 0.15 & 0.129 & 0.183 & 15.2 & 0.133 & 0.413 & 13.4 & 0.481 \\
\hline 9/7/2009 4:00 PM & 0.224 & 0.394 & 17.3 & 0.199 & 0.130 & 17.1 & 0.15 & 0.129 & 0.183 & 15.2 & 0.133 & 0.417 & 13.4 & 0.481 \\
\hline 9/7/2009 8:00 PM & 0.212 & 0.393 & 17.3 & 0.200 & 0.131 & 17.1 & 0.15 & 0.129 & 0.183 & 15.2 & 0.133 & 0.411 & 13.4 & 0.481 \\
\hline 9/8/2009 12:00 AM & 0.209 & 0.391 & 17.3 & 0.201 & 0.131 & 17.1 & 0.15 & 0.129 & 0.183 & 15.2 & 0.133 & 0.388 & 13.4 & 0.481 \\
\hline 9/8/2009 4:00 AM & 0.206 & 0.391 & 17.4 & 0.201 & 0.132 & 17.1 & 0.15 & 0.129 & 0.183 & 15.2 & 0.133 & 0.396 & 13.4 & 0.481 \\
\hline 9/8/2009 8:00 AM & 0.204 & 0.390 & 17.4 & 0.201 & 0.133 & 17.1 & 0.15 & 0.130 & 0.183 & 15.2 & 0.133 & 0.396 & 13.4 & 0.481 \\
\hline 9/8/2009 12:00 PM & 0.203 & 0.390 & 17.4 & 0.201 & 0.133 & 17.1 & 0.15 & 0.131 & 0.183 & 15.2 & 0.133 & 0.401 & 13.4 & 0.481 \\
\hline 9/8/2009 4:00 PM & 0.199 & 0.387 & 17.4 & 0.200 & 0.134 & 17.1 & 0.15 & 0.131 & 0.183 & 15.2 & 0.134 & 0.399 & 13.4 & 0.481 \\
\hline 9/8/2009 8:00 PM & 0.196 & 0.385 & 17.4 & 0.200 & 0.134 & 17.1 & 0.15 & 0.131 & 0.183 & 15.2 & 0.134 & 0.403 & 13.4 & 0.481 \\
\hline 9/9/2009 12:00 AM & 0.194 & 0.384 & 17.4 & 0.200 & 0.134 & 17.1 & 0.15 & 0.132 & 0.183 & 15.2 & 0.134 & 0.403 & 13.4 & 0.481 \\
\hline 9/9/2009 4:00 AM & 0.193 & 0.383 & 17.4 & 0.200 & 0.134 & 17.1 & 0.15 & 0.132 & 0.184 & 15.2 & 0.134 & 0.406 & 13.4 & 0.481 \\
\hline 9/9/2009 8:00 AM & 0.192 & 0.382 & 17.5 & 0.200 & 0.134 & 17.1 & 0.15 & 0.132 & 0.184 & 15.2 & 0.134 & 0.408 & 13.4 & 0.481 \\
\hline 9/9/2009 12:00 PM & 0.192 & 0.382 & 17.4 & 0.199 & 0.134 & 17.1 & 0.15 & 0.132 & 0.184 & 15.2 & 0.135 & 0.412 & 13.4 & 0.481 \\
\hline 9/9/2009 4:00 PM & 0.191 & 0.381 & 17.4 & 0.199 & 0.134 & 17.1 & 0.15 & 0.132 & 0.184 & 15.2 & 0.135 & 0.406 & 13.4 & 0.481 \\
\hline 9/9/2009 8:00 PM & 0.189 & 0.380 & 17.3 & 0.198 & 0.134 & 17.1 & 0.15 & 0.132 & 0.185 & 15.2 & 0.136 & 0.400 & 13.4 & 0.481 \\
\hline 9/10/2009 12:00 AM & 0.188 & 0.379 & 17.4 & 0.198 & 0.134 & 17.1 & 0.15 & 0.132 & 0.185 & 15.2 & 0.137 & 0.399 & 13.4 & 0.481 \\
\hline
\end{tabular}


Appendix 4. Volumetric water content, temperature, and electrical-conductivity data collected at the sprinkler-irrigated site during 2009.-Continued

[Depth in feet below land surface; volumetric water content dimensionless; temperature in degrees Celsius; bulk electrical conductivity in decisiemens per meter; --, no data]

\begin{tabular}{|c|c|c|c|c|c|c|c|c|c|c|c|c|c|c|}
\hline \multirow[b]{3}{*}{$\begin{array}{l}\text { Measurement } \\
\text { date and time }\end{array}$} & \multirow[b]{3}{*}{$\begin{array}{c}1 \\
\text { Volumetric } \\
\text { water } \\
\text { content }\end{array}$} & \multirow{2}{*}{\multicolumn{2}{|c|}{2}} & \multirow[b]{3}{*}{$\begin{array}{c}3 \\
\text { Volumetric } \\
\text { water } \\
\text { content }\end{array}$} & & & & \multirow{3}{*}{$\begin{array}{c}5 \\
\text { Volumetric } \\
\text { water } \\
\text { content }\end{array}$} & \multirow{2}{*}{\multicolumn{2}{|c|}{7}} & \multirow[b]{3}{*}{$\begin{array}{c}9 \\
\text { Volumetric } \\
\text { water } \\
\text { content }\end{array}$} & \multirow{2}{*}{\multicolumn{2}{|c|}{13}} & \\
\hline & & & & & \multicolumn{3}{|c|}{4} & & & & & & & \multirow{2}{*}{$\begin{array}{c}16.5 \\
\text { Volumetric } \\
\text { water } \\
\text { content }\end{array}$} \\
\hline & & $\begin{array}{c}\text { Volumetric } \\
\text { water } \\
\text { content }\end{array}$ & $\begin{array}{l}\text { Temper- } \\
\text { ature }\end{array}$ & & $\begin{array}{c}\text { Volumetric } \\
\text { water } \\
\text { content }\end{array}$ & $\begin{array}{l}\text { Temper- } \\
\text { ature }\end{array}$ & $\begin{array}{c}\text { Bulk } \\
\text { electrical } \\
\text { conduct- } \\
\text { ivity }\end{array}$ & & $\begin{array}{l}\text { Volumetric } \\
\text { water } \\
\text { content }\end{array}$ & $\begin{array}{l}\text { Temper- } \\
\text { ature }\end{array}$ & & $\begin{array}{l}\text { Volumetric } \\
\text { water } \\
\text { content }\end{array}$ & $\begin{array}{l}\text { Temper- } \\
\text { ature }\end{array}$ & \\
\hline 9/10/2009 4:00 AM & 0.188 & 0.379 & 17.4 & 0.198 & 0.134 & 17.1 & 0.15 & 0.132 & 0.186 & 15.2 & 0.137 & 0.398 & 13.4 & 0.481 \\
\hline 9/10/2009 8:00 AM & 0.187 & 0.378 & 17.5 & 0.197 & 0.134 & 17.1 & 0.15 & 0.132 & 0.186 & 15.2 & 0.137 & 0.428 & 13.5 & 0.481 \\
\hline 9/10/2009 12:00 PM & 0.186 & 0.376 & 17.4 & 0.197 & 0.133 & 17.1 & 0.15 & 0.132 & 0.185 & 15.2 & 0.138 & 0.410 & 13.5 & 0.481 \\
\hline 9/10/2009 4:00 PM & 0.186 & 0.375 & 17.4 & 0.196 & 0.133 & 17.1 & 0.15 & 0.132 & 0.186 & 15.2 & 0.138 & 0.393 & 13.5 & 0.481 \\
\hline 9/10/2009 8:00 PM & 0.185 & 0.375 & 17.4 & 0.196 & 0.133 & 17.2 & 0.15 & 0.132 & 0.186 & 15.2 & 0.138 & 0.391 & 13.4 & 0.481 \\
\hline 9/11/2009 12:00 AM & 0.183 & 0.373 & 17.4 & 0.196 & 0.133 & 17.2 & 0.15 & 0.132 & 0.186 & 15.2 & 0.138 & 0.387 & 13.4 & 0.481 \\
\hline 9/11/2009 4:00 AM & 0.183 & 0.373 & 17.4 & 0.195 & 0.133 & 17.2 & 0.15 & 0.132 & 0.186 & 15.2 & 0.139 & 0.386 & 13.4 & 0.481 \\
\hline 9/11/2009 8:00 AM & 0.183 & 0.374 & 17.5 & 0.195 & 0.133 & 17.2 & 0.15 & 0.132 & 0.186 & 15.2 & 0.139 & 0.387 & 13.4 & 0.481 \\
\hline 9/11/2009 12:00 PM & 0.182 & 0.373 & 17.4 & 0.195 & 0.133 & 17.1 & 0.15 & 0.132 & 0.186 & 15.2 & 0.139 & 0.386 & 13.4 & 0.481 \\
\hline 9/11/2009 4:00 PM & 0.181 & 0.372 & 17.4 & 0.194 & 0.133 & 17.2 & 0.15 & 0.132 & 0.186 & 15.2 & 0.139 & 0.388 & 13.4 & 0.481 \\
\hline 9/11/2009 8:00 PM & 0.179 & 0.371 & 17.3 & 0.194 & 0.132 & 17.2 & 0.15 & 0.132 & 0.186 & 15.2 & 0.140 & 0.388 & 13.4 & 0.481 \\
\hline 9/12/2009 12:00 AM & 0.251 & 0.370 & 17.3 & 0.194 & 0.132 & 17.2 & 0.15 & 0.131 & 0.186 & 15.2 & 0.140 & 0.384 & 13.5 & 0.481 \\
\hline 9/12/2009 4:00 AM & 0.233 & 0.371 & 17.3 & 0.194 & 0.132 & 17.2 & 0.15 & 0.131 & 0.187 & 15.2 & 0.140 & 0.385 & 13.5 & 0.481 \\
\hline 9/12/2009 8:00 AM & 0.214 & 0.373 & 17.3 & 0.194 & 0.132 & 17.2 & 0.15 & 0.131 & 0.187 & 15.2 & 0.140 & 0.387 & 13.5 & 0.481 \\
\hline 9/12/2009 12:00 PM & 0.208 & 0.373 & 17.2 & 0.194 & 0.132 & 17.2 & 0.15 & 0.131 & 0.187 & 15.2 & 0.141 & 0.390 & 13.5 & 0.481 \\
\hline 9/12/2009 4:00 PM & 0.207 & 0.373 & 17.2 & 0.194 & 0.132 & 17.2 & 0.15 & 0.131 & 0.187 & 15.2 & 0.141 & 0.390 & 13.5 & 0.481 \\
\hline 9/12/2009 8:00 PM & 0.243 & 0.373 & 17.1 & 0.194 & 0.131 & 17.2 & 0.15 & 0.131 & 0.187 & 15.2 & 0.141 & 0.388 & 13.5 & 0.481 \\
\hline 9/13/2009 12:00 AM & 0.234 & 0.373 & 17.0 & 0.194 & 0.131 & 17.2 & 0.15 & 0.131 & 0.187 & 15.2 & 0.141 & 0.392 & 13.5 & 0.481 \\
\hline 9/13/2009 4:00 AM & 0.226 & 0.374 & 16.9 & 0.194 & 0.132 & 17.2 & 0.15 & 0.131 & 0.187 & 15.2 & 0.142 & 0.393 & 13.5 & 0.481 \\
\hline 9/13/2009 8:00 AM & 0.218 & 0.375 & 16.7 & 0.195 & 0.132 & 17.2 & 0.15 & 0.131 & 0.187 & 15.2 & 0.142 & 0.392 & 13.5 & 0.481 \\
\hline 9/13/2009 12:00 PM & 0.211 & 0.376 & 16.6 & 0.195 & 0.132 & 17.1 & 0.15 & 0.131 & 0.187 & 15.2 & 0.142 & 0.392 & 13.5 & 0.481 \\
\hline 9/13/2009 4:00 PM & 0.207 & 0.376 & 16.5 & 0.195 & 0.132 & 17.1 & 0.15 & 0.131 & 0.187 & 15.2 & 0.142 & 0.391 & 13.5 & 0.481 \\
\hline 9/13/2009 8:00 PM & 0.203 & 0.375 & 16.4 & 0.196 & 0.132 & 17.1 & 0.15 & 0.131 & 0.187 & 15.2 & 0.143 & 0.391 & 13.5 & 0.481 \\
\hline 9/14/2009 12:00 AM & 0.201 & 0.375 & 16.3 & 0.196 & 0.132 & 17.1 & 0.15 & 0.131 & 0.188 & 15.2 & 0.143 & 0.388 & 13.5 & 0.481 \\
\hline 9/14/2009 4:00 AM & 0.200 & 0.374 & 16.4 & 0.196 & 0.132 & 17.1 & 0.15 & 0.131 & 0.188 & 15.2 & 0.143 & 0.393 & 13.5 & 0.481 \\
\hline 9/14/2009 8:00 AM & 0.200 & 0.374 & 16.4 & 0.196 & 0.132 & 17.1 & 0.15 & 0.131 & 0.188 & 15.2 & 0.143 & 0.391 & 13.5 & 0.481 \\
\hline 9/14/2009 12:00 PM & 0.198 & 0.374 & 16.4 & 0.196 & 0.132 & 17.0 & 0.15 & 0.131 & 0.188 & 15.2 & 0.143 & 0.393 & 13.5 & 0.481 \\
\hline 9/14/2009 4:00 PM & 0.195 & 0.374 & 16.3 & 0.195 & 0.132 & 17.0 & 0.15 & 0.131 & 0.187 & 15.2 & 0.143 & 0.392 & 13.5 & 0.481 \\
\hline 9/14/2009 8:00 PM & 0.194 & 0.372 & 16.3 & 0.195 & 0.132 & 17.0 & 0.15 & 0.131 & 0.188 & 15.2 & 0.143 & 0.390 & 13.5 & 0.481 \\
\hline 9/15/2009 12:00 AM & 0.192 & 0.371 & 16.3 & 0.195 & 0.132 & 17.0 & 0.15 & 0.131 & 0.188 & 15.2 & 0.143 & 0.388 & 13.5 & 0.481 \\
\hline 9/15/2009 4:00 AM & 0.192 & 0.371 & 16.4 & 0.195 & 0.132 & 17.0 & 0.15 & 0.131 & 0.188 & 15.2 & 0.143 & 0.388 & 13.5 & 0.481 \\
\hline 9/15/2009 8:00 AM & 0.191 & 0.372 & 16.4 & 0.194 & 0.132 & 17.0 & 0.15 & 0.131 & 0.188 & 15.2 & 0.143 & 0.390 & 13.5 & 0.481 \\
\hline 9/15/2009 12:00 PM & 0.190 & 0.372 & 16.5 & 0.194 & 0.132 & 16.9 & 0.15 & 0.131 & 0.188 & 15.2 & 0.143 & 0.388 & 13.5 & 0.481 \\
\hline 9/15/2009 4:00 PM & 0.189 & 0.371 & 16.4 & 0.194 & 0.132 & 16.9 & 0.15 & 0.131 & 0.188 & 15.2 & 0.143 & 0.388 & 13.5 & 0.481 \\
\hline 9/15/2009 8:00 PM & 0.188 & 0.370 & 16.4 & 0.194 & 0.132 & 16.9 & 0.15 & 0.131 & 0.188 & 15.2 & 0.143 & 0.386 & 13.5 & 0.481 \\
\hline 9/16/2009 12:00 AM & 0.186 & 0.369 & 16.4 & 0.194 & 0.131 & 16.9 & 0.15 & 0.131 & 0.188 & 15.2 & 0.143 & 0.386 & 13.5 & 0.481 \\
\hline 9/16/2009 4:00 AM & 0.186 & 0.369 & 16.5 & 0.194 & 0.132 & 16.9 & 0.15 & 0.130 & 0.190 & 15.2 & 0.143 & 0.387 & 13.5 & 0.481 \\
\hline
\end{tabular}


Appendix 4. Volumetric water content, temperature, and electrical-conductivity data collected at the sprinkler-irrigated site during 2009.-Continued

Depth in feet below land surface; volumetric water content dimensionless; temperature in degrees Celsius; bulk electrical conductivity in decisiemens per meter; --, no data]

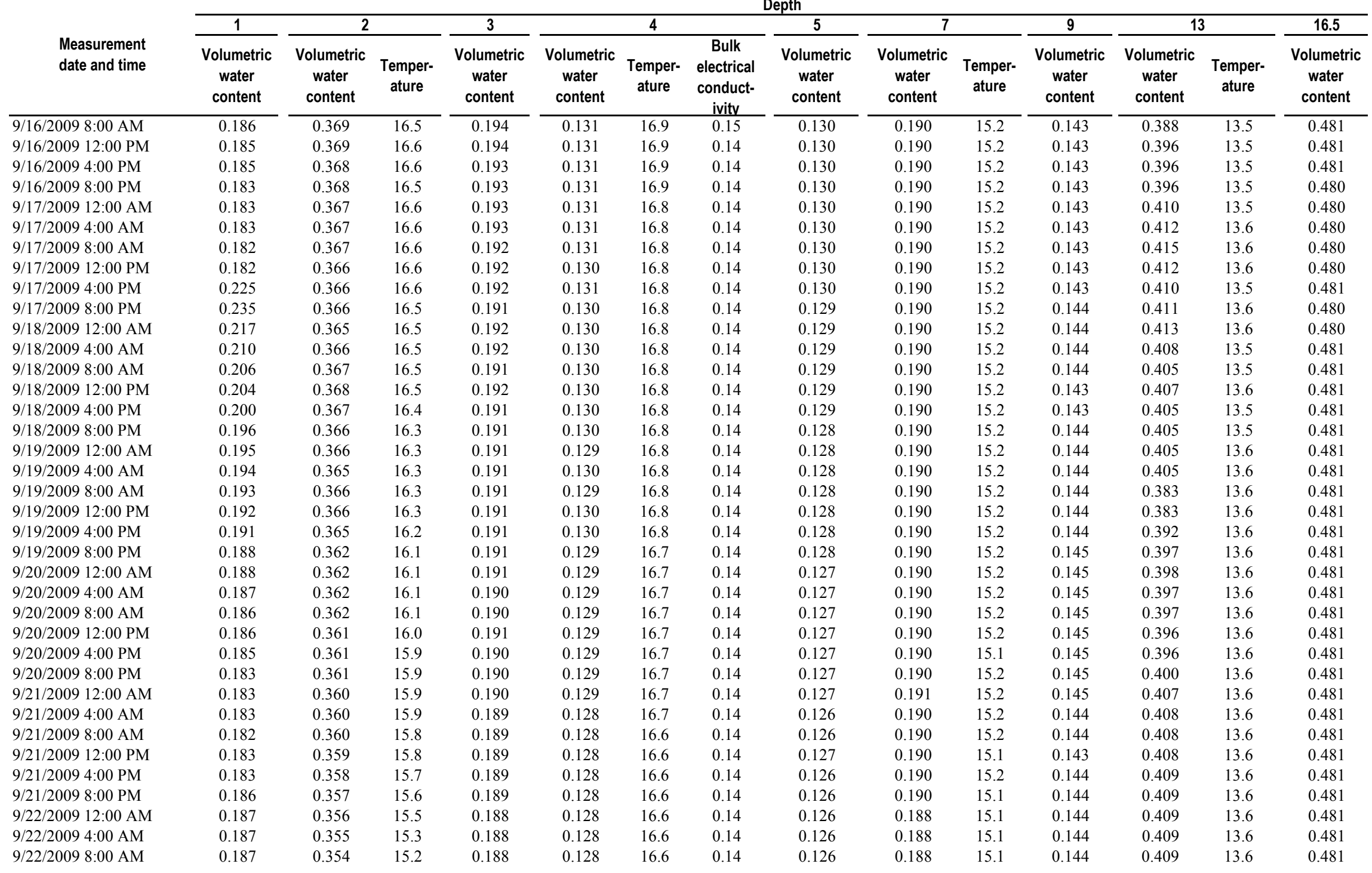


Appendix 4. Volumetric water content, temperature, and electrical-conductivity data collected at the sprinkler-irrigated site during 2009.-Continued

[Depth in feet below land surface; volumetric water content dimensionless; temperature in degrees Celsius; bulk electrical conductivity in decisiemens per meter; --, no data]

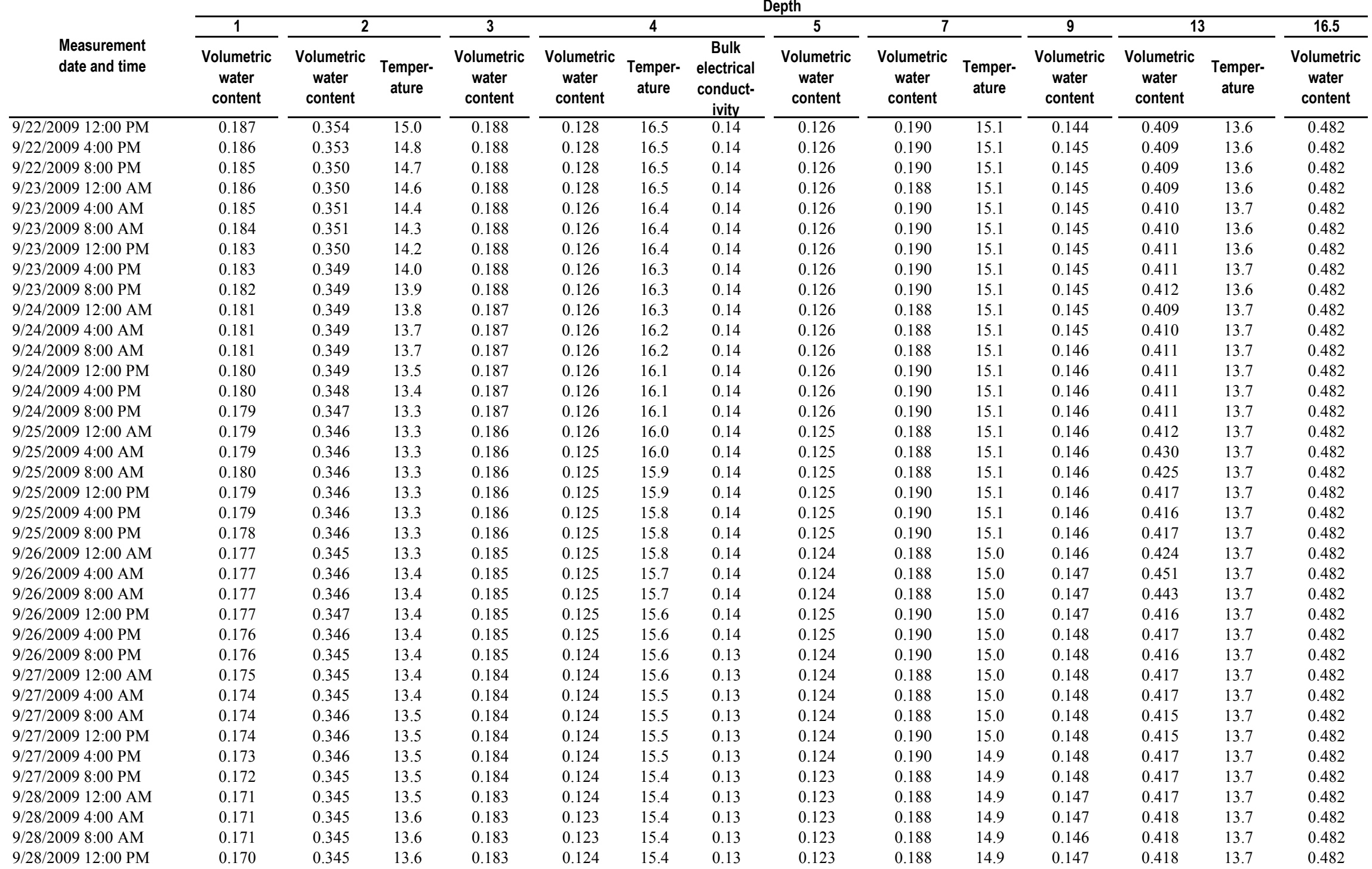


Appendix 4. Volumetric water content, temperature, and electrical-conductivity data collected at the sprinkler-irrigated site during 2009.-Continued

Depth in feet below land surface; volumetric water content dimensionless; temperature in degrees Celsius; bulk electrical conductivity in decisiemens per meter; --, no data]

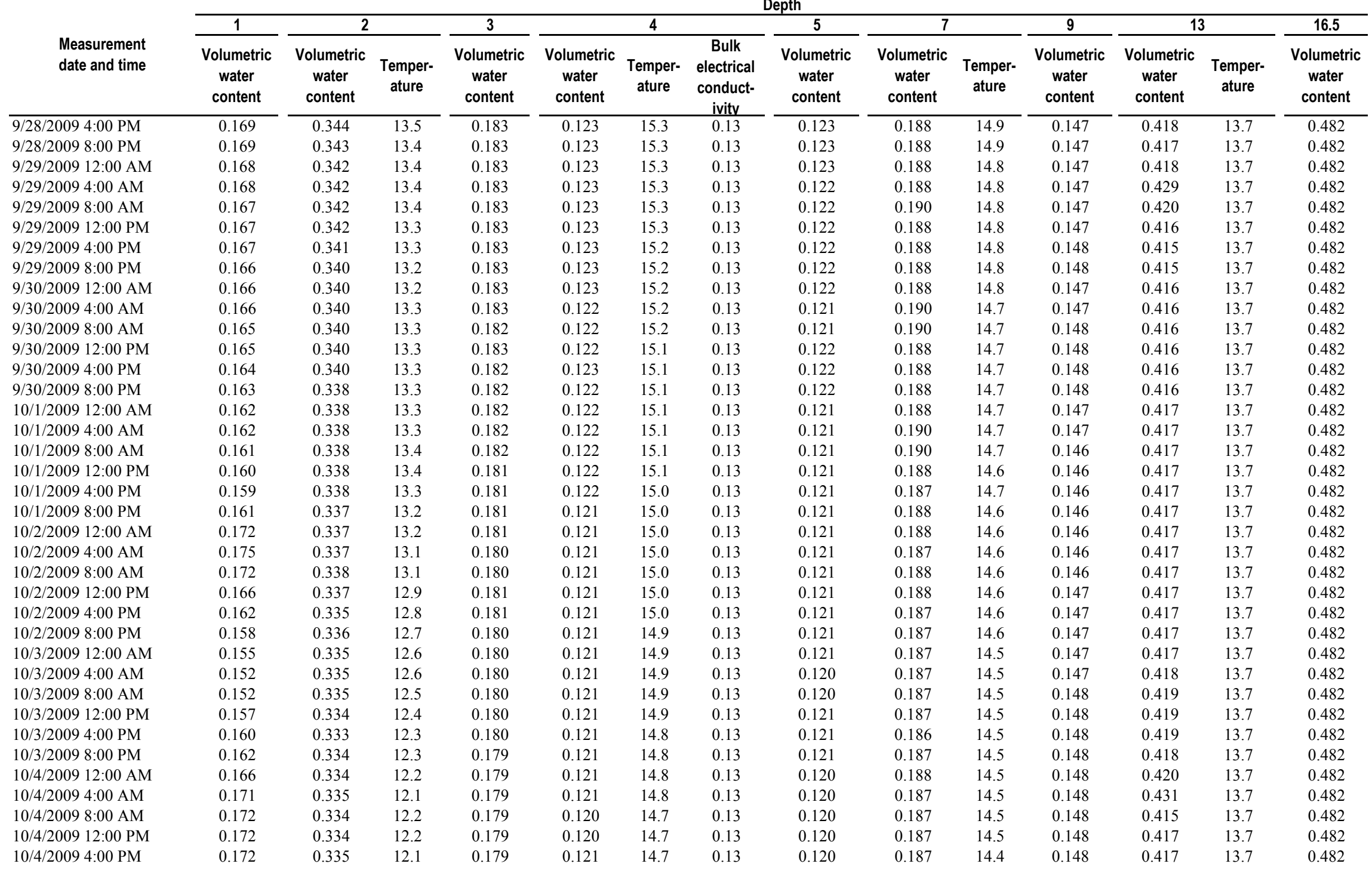


Appendix 4. Volumetric water content, temperature, and electrical-conductivity data collected at the sprinkler-irrigated site during 2009.-Continued

[Depth in feet below land surface; volumetric water content dimensionless; temperature in degrees Celsius; bulk electrical conductivity in decisiemens per meter; --, no data]

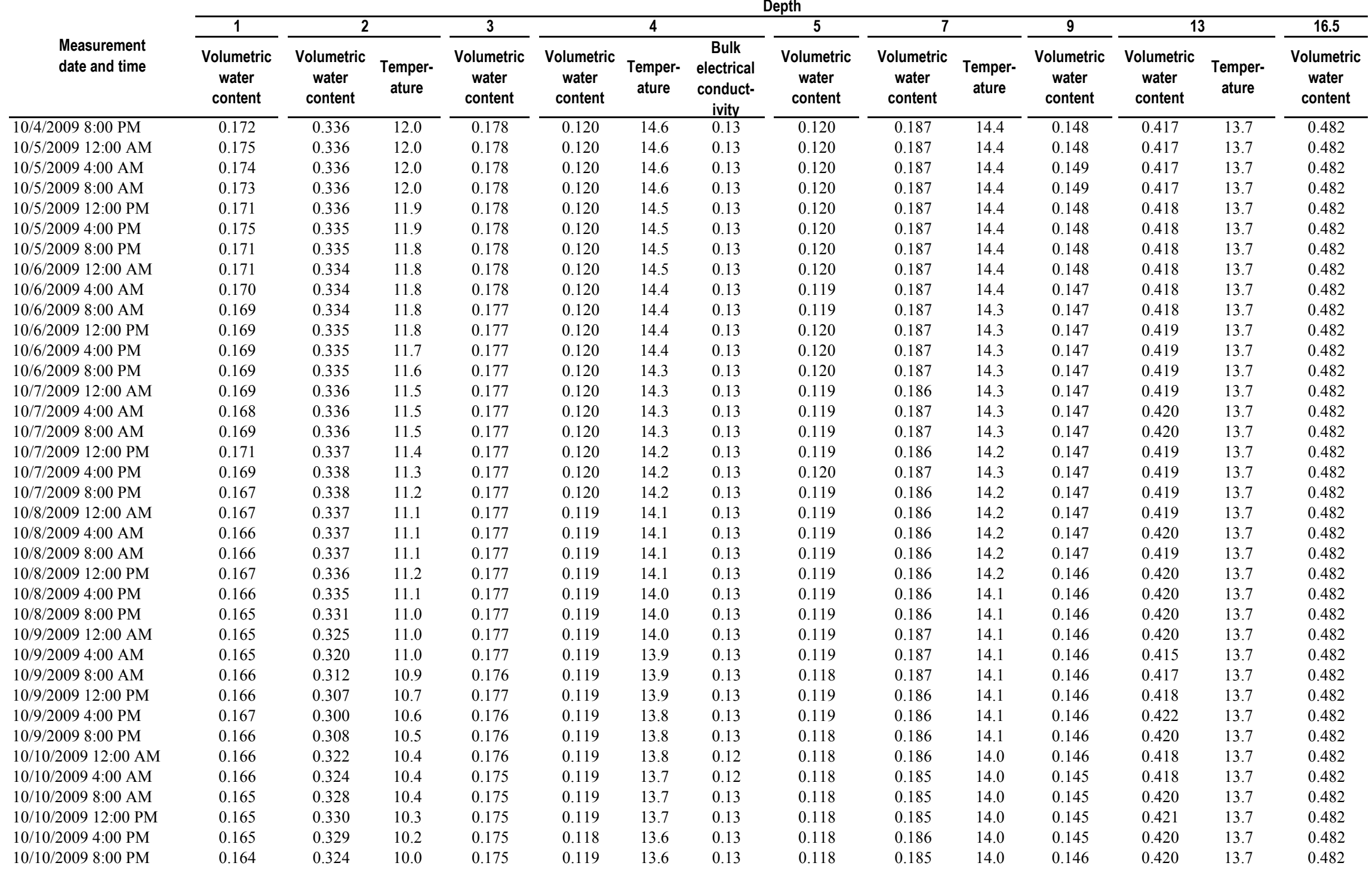


Appendix 4. Volumetric water content, temperature, and electrical-conductivity data collected at the sprinkler-irrigated site during 2009.-Continued

[Depth in feet below land surface; volumetric water content dimensionless; temperature in degrees Celsius; bulk electrical conductivity in decisiemens per meter; --, no data]

\begin{tabular}{|c|c|c|c|c|c|c|c|c|c|c|c|c|c|c|}
\hline \multirow[b]{3}{*}{$\begin{array}{l}\text { Measurement } \\
\text { date and time }\end{array}$} & \multicolumn{14}{|c|}{ Depth } \\
\hline & \multirow{2}{*}{$\begin{array}{c}1 \\
\text { Volumetric } \\
\text { water } \\
\text { content } \\
\end{array}$} & \multicolumn{2}{|c|}{2} & \multirow{2}{*}{$\begin{array}{c}3 \\
\begin{array}{c}\text { Volumetric } \\
\text { water } \\
\text { content }\end{array} \\
\end{array}$} & \multicolumn{3}{|c|}{4} & \multirow{2}{*}{$\begin{array}{c}5 \\
\text { Volumetric } \\
\text { water } \\
\text { content } \\
\end{array}$} & \multicolumn{2}{|l|}{7} & \multirow{2}{*}{$\begin{array}{c}9 \\
\text { Volumetric } \\
\text { water } \\
\text { content } \\
\end{array}$} & \multicolumn{2}{|c|}{13} & \multirow{2}{*}{$\begin{array}{c}16.5 \\
\text { Volumetric } \\
\text { water } \\
\text { content } \\
\end{array}$} \\
\hline & & $\begin{array}{c}\text { Volumetric } \\
\text { water } \\
\text { content }\end{array}$ & $\begin{array}{l}\text { Temper- } \\
\text { ature }\end{array}$ & & $\begin{array}{c}\text { Volumetric } \\
\text { water } \\
\text { content }\end{array}$ & $\begin{array}{l}\text { Temper- } \\
\text { ature }\end{array}$ & $\begin{array}{c}\text { Bulk } \\
\text { electrical } \\
\text { conduct- } \\
\text { ivity }\end{array}$ & & $\begin{array}{c}\text { Volumetric } \\
\text { water } \\
\text { content }\end{array}$ & $\begin{array}{l}\text { Temper- } \\
\text { ature }\end{array}$ & & $\begin{array}{c}\text { Volumetric } \\
\text { water } \\
\text { content }\end{array}$ & $\begin{array}{l}\text { Temper- } \\
\text { ature }\end{array}$ & \\
\hline 10/11/2009 12:00 AM & 0.164 & 0.318 & 9.9 & 0.175 & 0.119 & 13.6 & 0.13 & 0.118 & 0.185 & 13.9 & 0.145 & 0.421 & 13.7 & 0.482 \\
\hline 10/11/2009 4:00 AM & 0.164 & 0.312 & 9.7 & 0.175 & 0.118 & 13.5 & 0.12 & 0.118 & 0.185 & 13.9 & 0.145 & 0.421 & 13.7 & 0.482 \\
\hline 10/11/2009 8:00 AM & 0.163 & 0.319 & 9.6 & 0.175 & 0.118 & 13.5 & 0.12 & 0.118 & 0.185 & 13.9 & 0.146 & 0.421 & 13.7 & 0.482 \\
\hline 10/11/2009 12:00 PM & 0.163 & 0.321 & 9.4 & 0.175 & 0.118 & 13.5 & 0.12 & 0.118 & 0.185 & 13.9 & 0.146 & 0.421 & 13.7 & 0.482 \\
\hline 10/11/2009 4:00 PM & 0.163 & 0.322 & 9.3 & 0.175 & 0.119 & 13.4 & 0.12 & 0.118 & 0.186 & 13.9 & 0.146 & 0.421 & 13.7 & 0.482 \\
\hline 10/11/2009 8:00 PM & 0.163 & 0.320 & 9.1 & 0.175 & 0.118 & 13.4 & 0.12 & 0.118 & 0.186 & 13.9 & 0.146 & 0.421 & 13.7 & 0.482 \\
\hline 10/12/2009 12:00 AM & 0.163 & 0.317 & 9.0 & 0.174 & 0.118 & 13.3 & 0.12 & 0.118 & 0.185 & 13.9 & 0.146 & 0.421 & 13.7 & 0.482 \\
\hline 10/12/2009 4:00 AM & 0.163 & 0.315 & 9.0 & 0.174 & 0.118 & 13.3 & 0.12 & 0.118 & 0.185 & 13.8 & 0.146 & 0.421 & 13.7 & 0.482 \\
\hline 10/12/2009 8:00 AM & 0.163 & 0.313 & 8.9 & 0.174 & 0.118 & 13.2 & 0.12 & 0.118 & 0.185 & 13.8 & 0.145 & 0.421 & 13.7 & 0.482 \\
\hline 10/12/2009 12:00 PM & 0.163 & 0.312 & 8.8 & 0.174 & 0.118 & 13.2 & 0.12 & 0.118 & 0.185 & 13.8 & 0.145 & 0.421 & 13.7 & 0.482 \\
\hline 10/12/2009 4:00 PM & 0.164 & 0.312 & 8.8 & 0.174 & 0.118 & 13.1 & 0.12 & 0.118 & 0.186 & 13.8 & 0.145 & 0.421 & 13.7 & 0.482 \\
\hline 10/12/2009 8:00 PM & 0.164 & 0.315 & 8.7 & 0.174 & 0.118 & 13.1 & 0.12 & 0.118 & 0.185 & 13.8 & 0.145 & 0.421 & 13.7 & 0.482 \\
\hline 10/13/2009 12:00 AM & 0.164 & 0.315 & 8.7 & 0.174 & 0.118 & 13.1 & 0.12 & 0.118 & 0.184 & 13.8 & 0.145 & 0.421 & 13.6 & 0.482 \\
\hline 10/13/2009 4:00 AM & 0.164 & 0.315 & 8.7 & 0.174 & 0.118 & 13.0 & 0.12 & 0.118 & 0.184 & 13.8 & 0.145 & 0.421 & 13.7 & 0.482 \\
\hline 10/13/2009 8:00 AM & 0.164 & 0.315 & 8.7 & 0.174 & 0.118 & 13.0 & 0.12 & 0.118 & 0.184 & 13.7 & 0.145 & 0.421 & 13.7 & 0.482 \\
\hline 10/13/2009 12:00 PM & 0.164 & 0.315 & 8.7 & 0.174 & 0.118 & 12.9 & 0.12 & 0.118 & 0.185 & 13.7 & 0.145 & 0.421 & 13.6 & 0.482 \\
\hline 10/13/2009 4:00 PM & 0.164 & 0.313 & 8.7 & 0.174 & 0.118 & 12.9 & 0.12 & 0.118 & 0.186 & 13.7 & 0.145 & 0.421 & 13.7 & 0.482 \\
\hline 10/13/2009 8:00 PM & 0.164 & 0.315 & 8.7 & 0.174 & 0.118 & 12.8 & 0.12 & 0.118 & 0.185 & 13.7 & 0.145 & 0.421 & 13.6 & 0.482 \\
\hline 10/14/2009 12:00 AM & 0.165 & 0.315 & 8.7 & 0.173 & 0.118 & 12.8 & 0.12 & 0.118 & 0.184 & 13.7 & 0.145 & 0.421 & 13.6 & 0.482 \\
\hline 10/14/2009 4:00 AM & 0.165 & 0.313 & 8.7 & 0.174 & 0.118 & 12.7 & 0.12 & 0.118 & 0.184 & 13.7 & 0.145 & 0.424 & 13.6 & 0.482 \\
\hline 10/14/2009 8:00 AM & 0.166 & 0.313 & 8.7 & 0.173 & 0.118 & 12.7 & 0.12 & 0.117 & 0.184 & 13.6 & 0.145 & 0.422 & 13.6 & 0.482 \\
\hline 10/14/2009 12:00 PM & 0.165 & 0.312 & 8.7 & 0.174 & 0.118 & 12.6 & 0.12 & 0.117 & 0.184 & 13.6 & 0.145 & 0.418 & 13.6 & 0.482 \\
\hline 10/14/2009 4:00 PM & 0.166 & 0.312 & 8.8 & 0.174 & 0.118 & 12.6 & 0.12 & 0.117 & 0.185 & 13.6 & 0.145 & 0.420 & 13.6 & 0.482 \\
\hline 10/14/2009 8:00 PM & 0.166 & 0.312 & 8.8 & 0.174 & 0.118 & 12.6 & 0.12 & 0.117 & 0.184 & 13.6 & 0.145 & 0.423 & 13.6 & 0.482 \\
\hline 10/15/2009 12:00 AM & 0.166 & 0.312 & 8.8 & 0.173 & 0.118 & 12.5 & 0.12 & 0.117 & 0.184 & 13.6 & 0.144 & 0.419 & 13.6 & 0.482 \\
\hline 10/15/2009 4:00 AM & 0.166 & 0.315 & 8.9 & 0.173 & 0.118 & 12.5 & 0.12 & 0.117 & 0.185 & 13.5 & 0.144 & 0.418 & 13.6 & 0.482 \\
\hline 10/15/2009 8:00 AM & 0.166 & 0.317 & 9.0 & 0.173 & 0.118 & 12.5 & 0.12 & 0.117 & 0.186 & 13.5 & 0.144 & 0.422 & 13.6 & 0.482 \\
\hline
\end{tabular}


Publishing support provided by:

Denver Publishing Service Center

For more information concerning this publication, contact:

Director, USGS Colorado Water Science Center

Box 25046, Mail Stop 415

Denver, CO 80225

(303) 236-4882

Or visit the Colorado Water Science Center Web site at: http://co.water.usgs.gov/ 


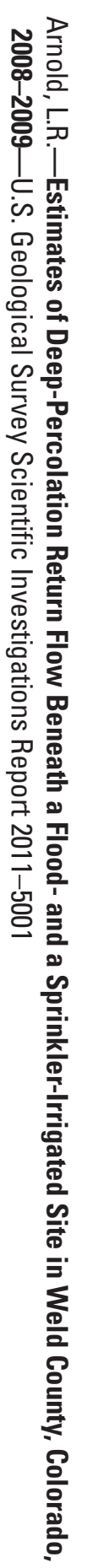

